\title{
Optically Active Flavaglines-Inspired Molecules by a Palladium- Catalyzed Decarboxylative Dearomative Asymmetric Allylic Alkylation
}

\author{
Meng-Yue Cao, ${ }^{\dagger} \S$ Bin-Jie Ma, ${ }^{\dagger}$ Zhi-Qi Lao,${ }^{\dagger}$ H. Wang,${ }^{\S}$ Jing Wang, ${ }^{\dagger}$ Juan Liu, ${ }^{\dagger}$ Kuan Xing,${ }^{\dagger}$ Yu- \\ Hao Huang, ${ }^{\dagger}$ Kang-Ji Gan, ${ }^{\dagger, \S}$ Wei Gao, ${ }^{\dagger}$ Huaiming Wang, ${ }^{\dagger}$ Xin Hong, ${ }^{\S}$ and Hai-Hua Lu, ${ }^{*}, \dagger, \S$ \\ ${ }^{\dagger}$ Key Laboratory of Precise Synthesis of Functional Molecules of Zhejiang Province, School of Science, \\ Westlake University, 18 Shi-longshan Road, Hangzhou 310024, China; Institute of Natural Sciences, Westlake \\ Institute for Advanced Study, Hangzhou 310024, China \\ Institute of Advanced Synthesis (IAS), Nanjing Tech University, 30 South Puzhu Road, Nanjing 211816, China \\ ${ }^{\S}$ Department of Chemistry, Zhejiang University, 866 Yuhangtang Road, Hangzhou 310058, China
}

\section{TABLE OF CONTENTS}

PAGE

1 General Methods

2 Condition Optimization Data for the the Pd-Catalyzed AAA 3

3 Experimental Procedures and Characterizations 9

3.1 Synthesis of New Bisphosphine Ligands

3.2 General Procedure for the Preparation of Substrates 9

3.3 10: General Procedure for the Pd-Catalyzed AAA 23

3.4 Synthesis of Flavaglines $(\mathbf{1 8}, 20,21$ and 22)

3.5 Formal Synthesis of rocaglamide (5)

4 Preliminary Results of Biological Studies 84

5 References 85

6 NMR Spectra 86

7 HPLC Spectra 259 


\section{General Methods}

All reactions were carried out under positive pressure of nitrogen unless otherwise noted. Anhydrous dichloromethane (DCM) was distilled from calcium hydride $(5 \% \mathrm{w} / \mathrm{v})$ under positive pressure of nitrogen. Anhydrous tetrahydrofuran (THF) was distilled over sodium/benzophenone ketyl under positive pressure of nitrogen. Other commercially available solvents or reagents were used without further purification unless otherwise noted. Reactions were monitored by thin layer chromatography (TLC) using precoated silica gel plates. Flash column chromatography was performed over silica gel (200-300 mesh).

NMR spectra were recorded on Bruker AVANCE NEO (500 and 600MHz), Bruker AVANCE 400 $\mathrm{MHz}$ or Jeol $400 \mathrm{MHz}$ spectrometers using residual solvent peaks as an internal standard (e.g. $\mathrm{CDCl}_{3}$ @ $7.26 \mathrm{ppm}{ }^{1} \mathrm{H}$ NMR, $77.00 \mathrm{ppm}{ }^{13} \mathrm{C}$ NMR). The following abbreviations (or combinations thereof) were used to explain the multiplicities: $\mathrm{s}=$ singlet, $\mathrm{d}=$ doublet, $\mathrm{t}=$ triplet, $\mathrm{q}=$ quartet, $\mathrm{dd}=$ doublet of doublets, $\mathrm{m}=$ multiplet, $\mathrm{br}=$ broad. HRMS was recorded on Waters LCT Premier ESI-TOF. The enantiomeric excesses (ee) of the products were determined by chiral stationary phase HPLC with Chiralpak (ADH, ODH, IA-3, ID-3, IC-3, IBN-3, AD-3, IG-3). Optical rotations were measured with Rudolph Autopol IVT. The single crystal X-ray diffraction studies were carried out on a Bruker D8 Venture diffractometer. 


\section{Condition Optimization Data for the the Pd-Catalyzed AAA}

Table S1. Ligand Effect.
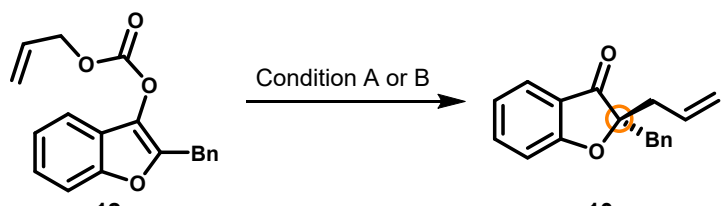

$12 a$

$10 \mathrm{a}$

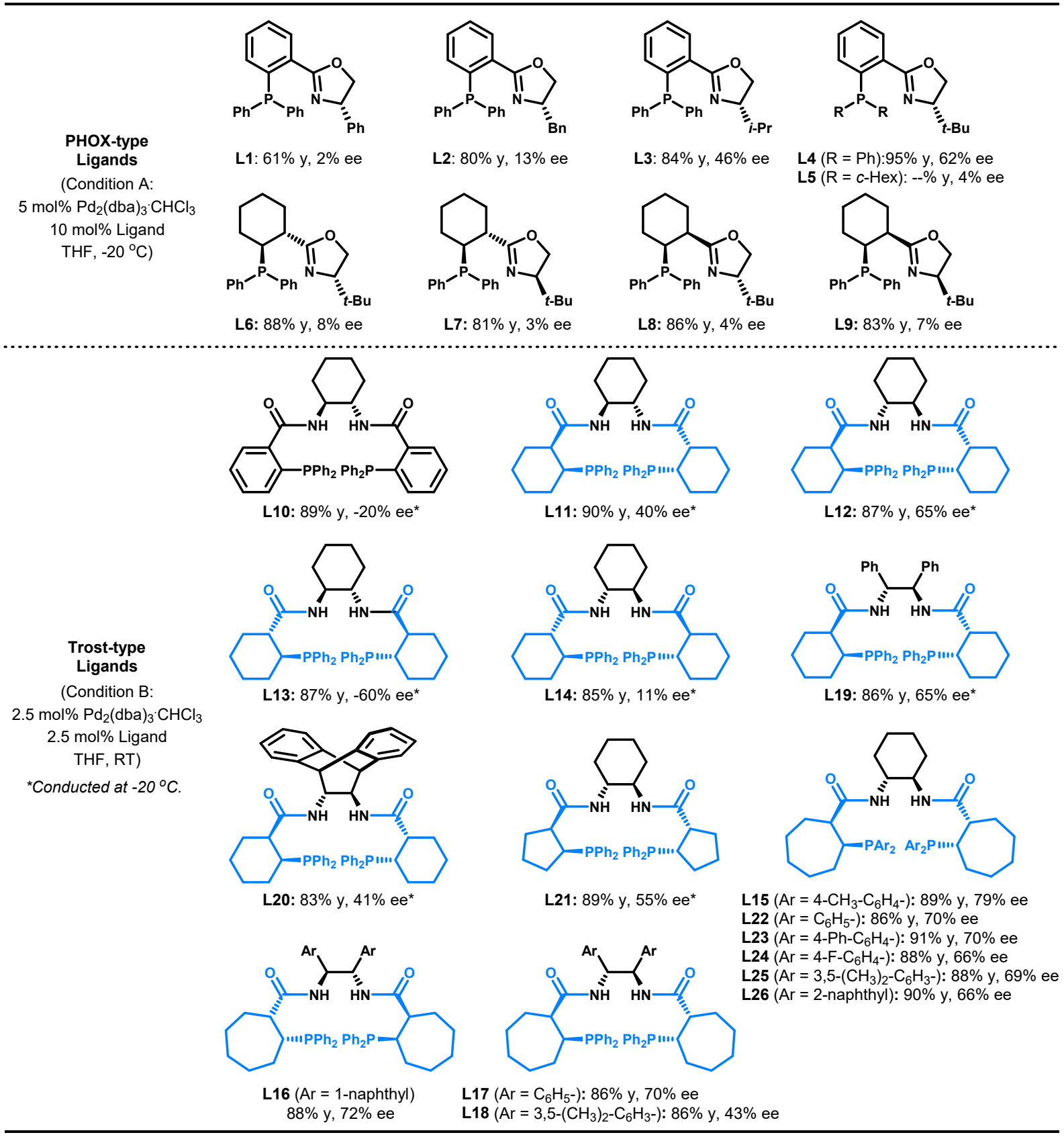


Table S2. Solvent Screening by Using $\mathbf{L 1 2}$.
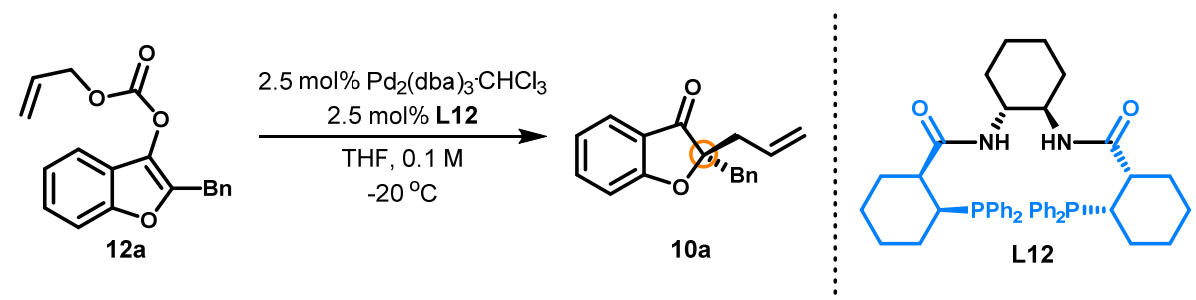

\begin{tabular}{cccccccc}
\hline entry & solvent & yield (\%) & ee (\%) & entry & solvent & yield (\%) & ee (\%) \\
\hline 1 & $\mathrm{MeOH}$ & 85 & 0 & 10 & dioxane & 86 & 11 \\
2 & $\mathrm{EtOAc}$ & 82 & 58 & 11 & anisole & 90 & 39 \\
3 & $\mathrm{THF}$ & 88 & 65 & 12 & $\mathrm{CPME}$ & 85 & 58 \\
4 & $\mathrm{DCM}$ & 84 & 41 & 13 & ${ }^{n} \mathrm{Bu}_{2} \mathrm{O}$ & 78 & 39 \\
5 & Toluene & 88 & 64 & $14^{a}$ & $\mathrm{CHCl}_{3}$ & 98 & 13 \\
6 & Hexane & 87 & 58 & $15^{a}$ & $\mathrm{CH}_{3} \mathrm{CN}$ & 98 & 39 \\
7 & Cyclohexane & 90 & 59 & $16^{a}$ & DMF & 95 & 26 \\
8 & $\mathrm{TBME}$ & 90 & 64 & $17^{a}$ & DMSO & 80 & 4 \\
9 & $\mathrm{Et}_{2} \mathrm{O}$ & 92 & 59 & $18^{a}$ & $2: 1$ hexane/THF & 95 & 68 \\
\hline
\end{tabular}

${ }^{a}$ Run at room temperature.

Table S3. The Influence of Temperature with $2.5 \mathrm{~mol} \%$ of Catalyst.
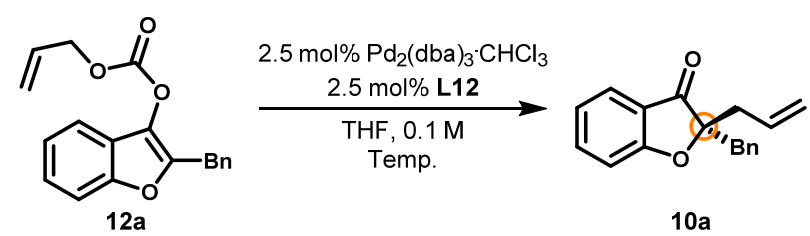

$10 a$

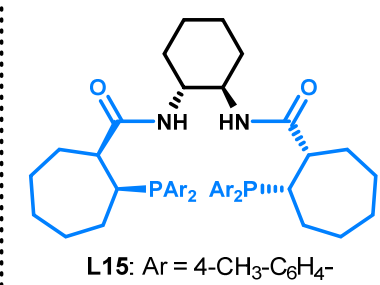

\begin{tabular}{ccccc}
\hline entry & Temp. $\left({ }^{\circ} \mathbf{C}\right)$ & time $(\mathbf{h})$ & yield $(\mathbf{\%})$ & ee (\%) \\
\hline 1 & RT & 2 & 90 & 79 \\
2 & -20 & 6 & 91 & 89 \\
3 & -25 & 6 & 89 & 87 \\
4 & -30 & 6 & 89 & 89 \\
5 & -35 & 6 & 82 & 88 \\
6 & -40 & 6 & 89 & 89 \\
7 & -80 & 6 & 92 & 87 \\
\hline
\end{tabular}


Table S4. The Effect of Catalyst Loading with Best Ligand L15.
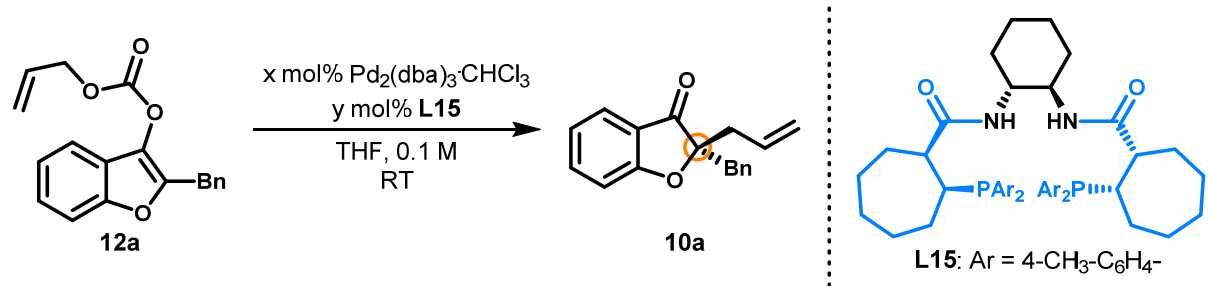

\begin{tabular}{ccccc}
\hline entry & $\mathbf{x}$ & $\mathbf{y}$ & yield $\mathbf{( \% )}$ & ee (\%) \\
\hline 1 & 2.5 & 2.5 & 89 & 79 \\
2 & 2.5 & 2.0 & 86 & 79 \\
3 & 2.5 & 1.5 & 88 & 78 \\
4 & 2.5 & 1.0 & 87 & 79 \\
5 & 2.5 & 0.5 & 90 & 82 \\
6 & 2.0 & 0.5 & 92 & 79 \\
7 & 1.5 & 0.5 & 91 & 78 \\
8 & 1.0 & 0.5 & 93 & 78 \\
9 & 0.5 & 0.5 & 91 & 78 \\
10 & 1.0 & 0.2 & 92 & 78 \\
11 & 0.8 & 0.2 & 89 & 80 \\
12 & 0.2 & 0.2 & 92 & 80 \\
\hline
\end{tabular}

Table S5. More on The Effect of Temperature with Best Ligand L15.
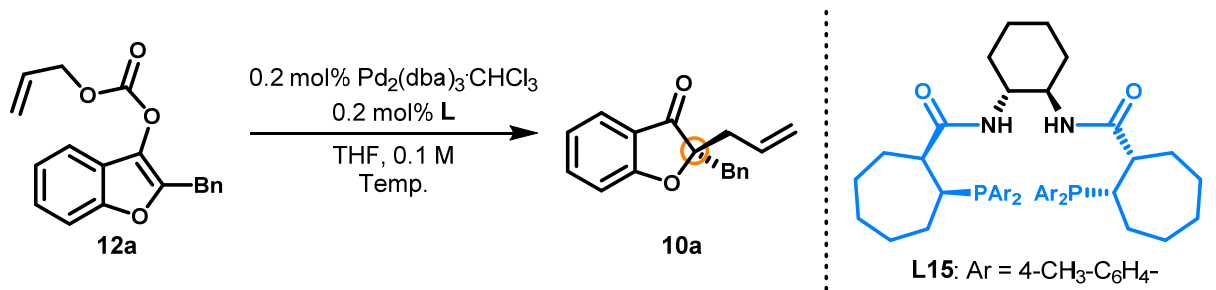

\begin{tabular}{ccccc}
\hline entry & temp. $\left({ }^{\circ} \mathbf{C}\right)$ & time $(\mathbf{h})$ & yield $(\mathbf{\%})$ & ee (\%) \\
\hline 1 & 0 & 6 & 92 & 90 \\
2 & -5 & 6 & 88 & 91 \\
3 & -10 & 6 & 91 & 89 \\
4 & -15 & 6 & 76 & 90 \\
5 & -20 & 8 & 89 & 92 \\
6 & -40 & 12 & 88 & 91 \\
7 & -50 & 12 & 65 & 87 \\
\hline
\end{tabular}


Table S6. Ligand Effect for substrate 12g' with aryl substituent at $\mathrm{C}_{3 \mathrm{a}}$.
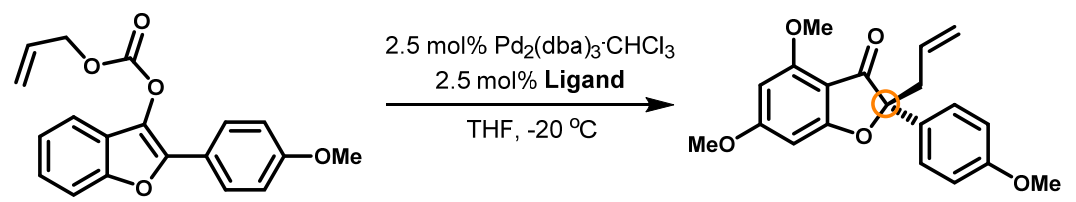

$12 g^{\prime}$

$10 g^{\prime}$

\begin{tabular}{cccccccc}
\hline entry & Ligand & yield (\%) & ee (\%) & entry & Ligand & yield (\%) & ee (\%) \\
\hline 1 & L1 & 90 & 2 & 10 & $\mathbf{L 1 5}$ & 88 & 43 \\
2 & L2 & 46 & 10 & 11 & $\mathbf{L 1 6}$ & 92 & 64 \\
3 & L3 & 94 & 1 & 12 & $\mathbf{L 1 7}$ & 93 & 56 \\
4 & L4 & 91 & 3 & 13 & $\mathbf{L 1 8}$ & 90 & 15 \\
5 & $\mathbf{L 6}$ & 88 & -8 & 14 & $\mathbf{L 2 3}$ & 90 & 37 \\
6 & L7 & 87 & -10 & 15 & $\mathbf{L 2 4}$ & 90 & 43 \\
7 & L8 & 90 & -13 & 16 & $\mathbf{L 2 5}$ & 87 & 30 \\
8 & L9 & 42 & 0 & 17 & $\mathbf{L 2 6}$ & 91 & 35 \\
9 & L10 & 93 & 2 & & & & \\
\hline
\end{tabular}

Table S7. Solvent Effect.

\begin{tabular}{|c|c|c|c|c|c|}
\hline & $\begin{array}{l}\mathrm{R}=\mathrm{OMe} \\
(\mathrm{R}=\mathrm{H})\end{array}$ & & & $\begin{array}{c}10 \mathrm{~g}^{\prime}(\mathrm{R}=\mathrm{OMe}) \\
10 \mathrm{~h}^{\prime}(\mathrm{R}=\mathrm{H})\end{array}$ & \\
\hline entry & $\mathrm{R}$ & solvent & time (h) & yield $(\%)$ & ee $(\%)$ \\
\hline 1 & $\mathrm{OMe}$ & THF & 15 & 92 & 64 \\
\hline 2 & $\mathrm{H}$ & THF & 18 & 93 & 70 \\
\hline 3 & $\mathrm{H}$ & TBME & 18 & 92 & 29 \\
\hline 4 & $\mathrm{H}$ & $\mathrm{Et}_{2} \mathrm{O}$ & 18 & 91 & 42 \\
\hline 5 & $\mathrm{H}$ & 2-Me-THF & 18 & 92 & 42 \\
\hline 6 & $\mathrm{H}$ & EtOAc & 18 & 90 & 31 \\
\hline 7 & $\mathrm{H}$ & DCM & 18 & 94 & 8 \\
\hline 8 & $\mathrm{H}$ & Toluene & 18 & 92 & 40 \\
\hline $9^{a}$ & $\mathrm{H}$ & THF & 18 & 93 & 72 \\
\hline $10^{b}$ & $\mathrm{H}$ & THF & 18 & 92 & 73 \\
\hline $11^{c}$ & $\mathrm{H}$ & THF & 18 & 93 & 74 \\
\hline $12^{d}$ & $\mathrm{H}$ & THF & 18 & 90 & 73 \\
\hline
\end{tabular}

${ }^{a}$ With $1.5 \mathrm{~mol} \% \mathrm{Pd}_{2}(\mathrm{dba})_{3} \cdot \mathrm{CHCl}_{3} / \mathrm{L16} .{ }^{b}$ With $1.0 \mathrm{~mol} \% \mathrm{Pd}_{2}\left(\mathrm{dba}_{3} \cdot \mathrm{CHCl}_{3} / \mathrm{L16}\right.$.

${ }^{c}$ With $0.5 \mathrm{~mol} \% \mathrm{Pd}_{2}(\mathrm{dba})_{3} \cdot \mathrm{CHCl}_{3} / \mathrm{L16} .{ }^{d}$ With $0.2 \mathrm{~mol} \% \mathrm{Pd}_{2}\left(\mathrm{dba}_{3} \cdot \mathrm{CHCl}_{3} / \mathrm{L} 16\right.$.

$\mathrm{CPME}=$ cyclopentyl methyl ether. TBME $=$ tert-butyl methyl ether. 
Table S8. Additive Effect.

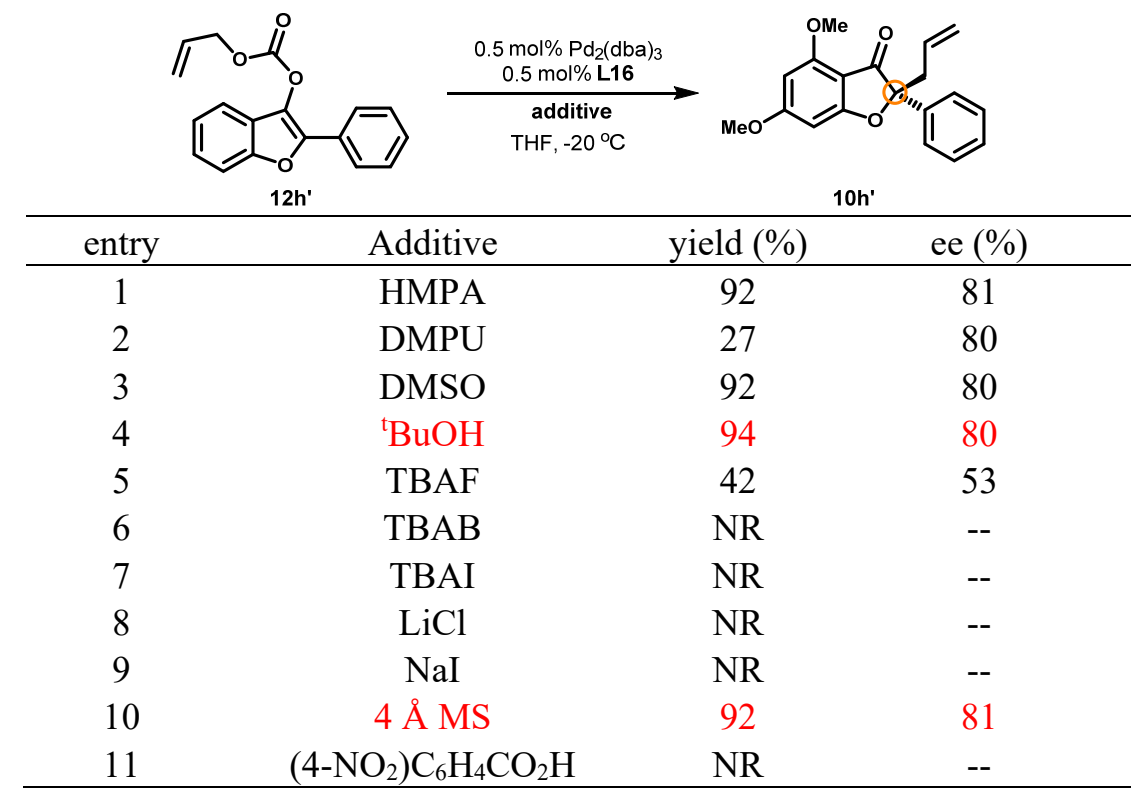

Table S9. The Combination Effect of Additive and Temperature.

\begin{tabular}{|c|c|c|c|c|c|}
\hline \multirow[b]{2}{*}{ entry } & \multicolumn{2}{|c|}{$\begin{array}{c}12 \mathrm{~g}^{\prime}(\mathrm{R}=\mathrm{OMe}) \\
12 \mathrm{~h}^{\prime}(\mathrm{R}=\mathrm{H})\end{array}$} & \multicolumn{2}{|c|}{$\begin{array}{c}10 \mathbf{g}^{\prime}(\mathrm{R}=\mathrm{OMe}) \\
10 \mathrm{~h}^{\prime}(\mathrm{R}=\mathrm{H})\end{array}$} & \multirow[b]{2}{*}{ ee $(\%)$} \\
\hline & $\mathrm{R}$ & additive & $\mathrm{x}$ & yield (\%) & \\
\hline 1 & $\mathrm{H}$ & ${ }^{t} \mathrm{BuOH}$ & 0.1 & 90 & 79 \\
\hline 2 & $\mathrm{H}$ & ${ }^{t} \mathrm{BuOH}$ & 0.5 & 94 & 82 \\
\hline 3 & $\mathrm{H}$ & ${ }^{t} \mathrm{BuOH}$ & 1.0 & 92 & 79 \\
\hline 4 & $\mathrm{H}$ & ${ }^{\mathrm{t}} \mathrm{BuOH}$ & 2.0 & 90 & 77 \\
\hline 5 & $\mathrm{H}$ & ${ }^{\mathrm{t}} \mathrm{BuOH}$ & 3.0 & 93 & 75 \\
\hline 6 & $\mathrm{H}$ & $4 \AA \mathrm{MS}$ & $50 \mathrm{mg}$ & 92 & 88 \\
\hline 7 & $\mathrm{H}$ & $4 \AA \mathrm{MS}+{ }^{t} \mathrm{BuOH}$ & $\begin{array}{c}50 \mathrm{mg} \\
+1.0\end{array}$ & 91 & 87 \\
\hline $8^{a}$ & $\mathrm{H}$ & $4 \AA \mathrm{MS}$ & $50 \mathrm{mg}$ & 93 & 88 \\
\hline $9^{b}$ & $\mathrm{H}$ & $4 \AA \mathrm{MS}$ & $50 \mathrm{mg}$ & 83 & 90 \\
\hline $10^{c}$ & $\mathrm{H}$ & $4 \AA \mathrm{MS}$ & $50 \mathrm{mg}$ & 64 & 90 \\
\hline 11 & $\mathrm{OMe}$ & $4 \AA \mathrm{MS}$ & $50 \mathrm{mg}$ & 94 & 85 \\
\hline $12^{b}$ & $\mathrm{OMe}$ & $4 \AA \mathrm{MS}$ & $50 \mathrm{mg}$ & 90 & 89 \\
\hline $13^{c}$ & $\mathrm{OMe}$ & $4 \AA \mathrm{MS}$ & $50 \mathrm{mg}$ & 92 & 90 \\
\hline $14^{d}$ & $\mathrm{OMe}$ & $4 \AA \mathrm{MS}$ & $50 \mathrm{mg}$ & 92 & 86 \\
\hline
\end{tabular}

${ }^{a}$ Run at $-35{ }^{\circ} \mathrm{C} .{ }^{b}$ Run at $-45{ }^{\circ} \mathrm{C}$. ${ }^{c}$ Run at $-50{ }^{\circ} \mathrm{C}$. ${ }^{d}$ With $0.5 \mathrm{~mol} \% \mathrm{Pd}_{2}(\mathrm{dba})_{3} / \mathrm{Ligand}$ 


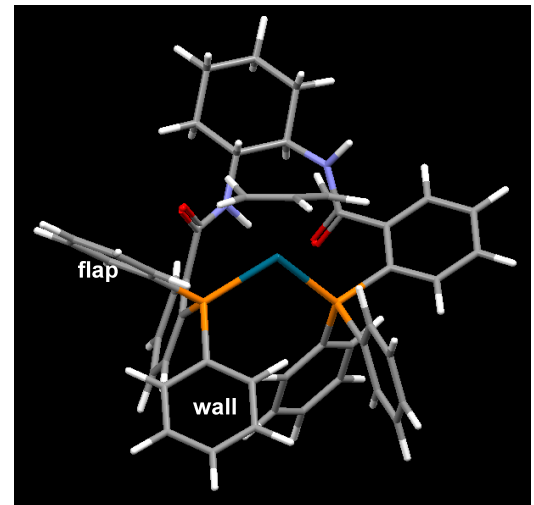

Complex with (R, R)-Trost ligand

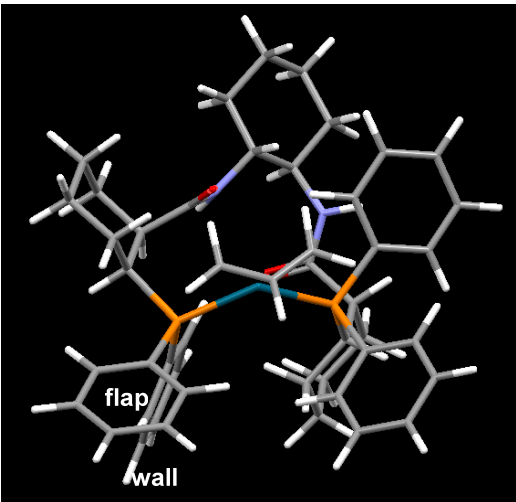

Complex with L11

Note: All DFT calculations were performed using Gaussian 09 program. ${ }^{[1 a]}$ Geometry optimizations were carried out using B3LYP[1b-d]-D3 (Becke-Johnson damping function) ${ }^{[1 \mathrm{le}, 1 \mathrm{f}]}$ functional, with def2-SVP basis set $^{[\mathrm{lg}]}$ for all elements.

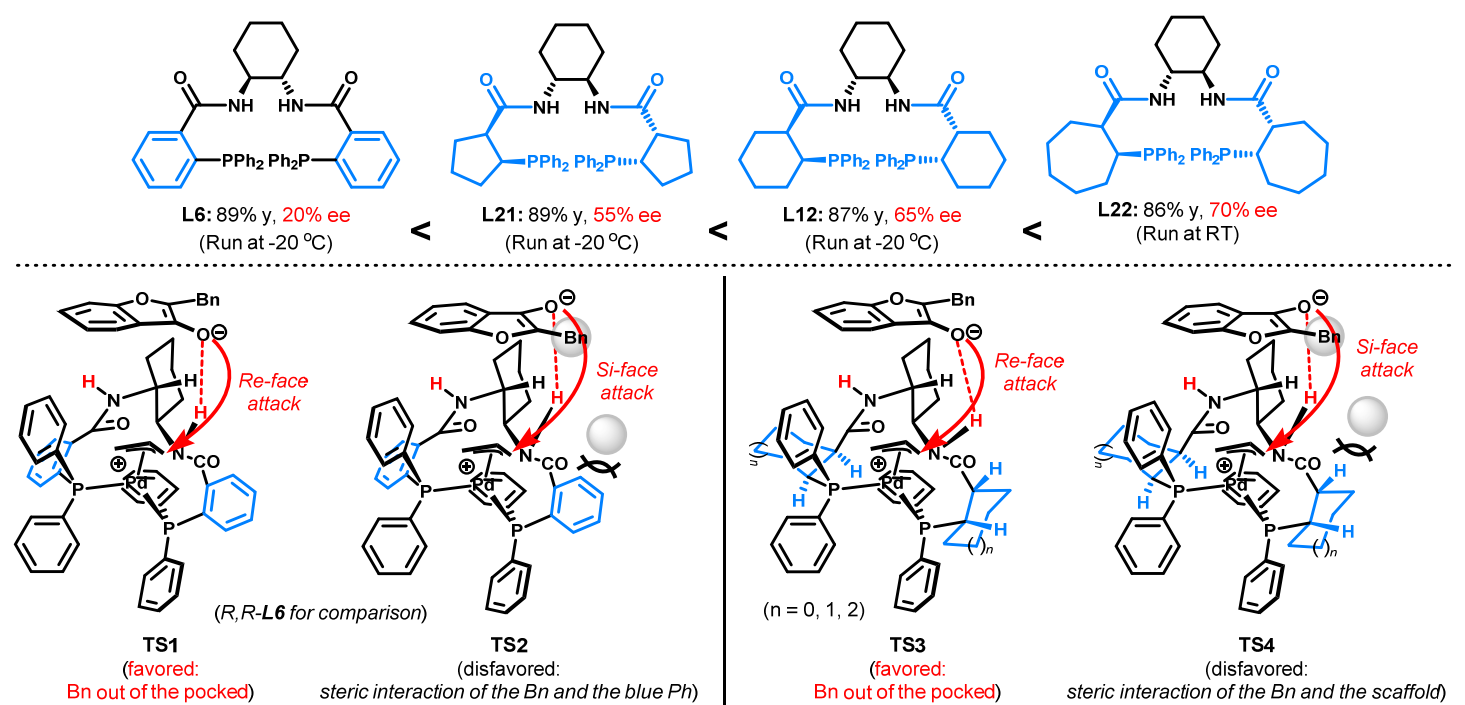

Scheme S1. Prelimenary Computational Results and Proposed Transition States for the Rationale of Stereochemical Outcome. 


\section{Experimental Procedures and Characterizations}

\subsection{Synthesis of New Bisphosphine Ligands}

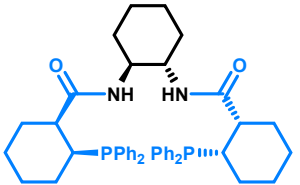

L11

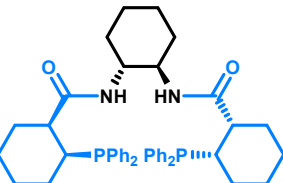

L12

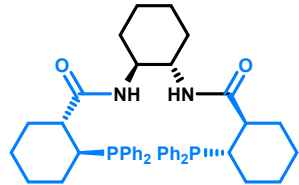

L13

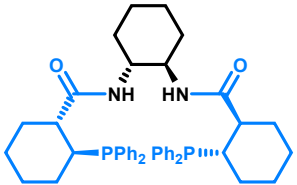

L14

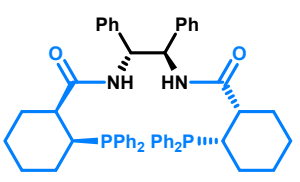

L19

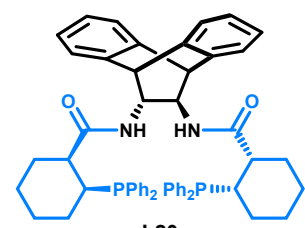

L20

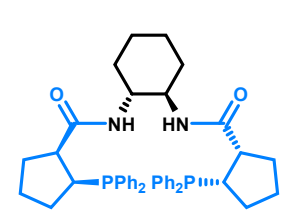

L21
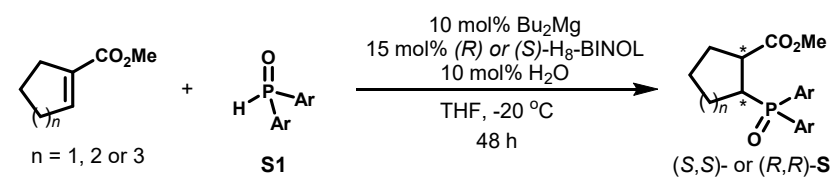

$(\mathrm{S}, \mathrm{S})$ - or $(R, R)$-S2

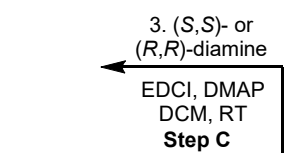

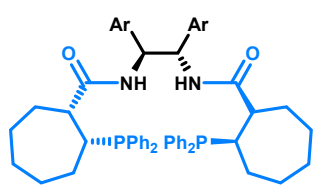

L16: Ar = 1-naphthyl

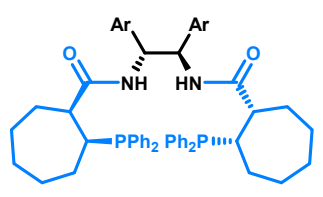

$\mathrm{L} 17\left(\mathrm{Ar}=\mathrm{C}_{6} \mathrm{H}_{5-}\right)$ L18 $\left(\mathrm{Ar}=3,5-\left(\mathrm{CH}_{3}\right)_{2}-\mathrm{C}_{6} \mathrm{H}_{3}-\right)$

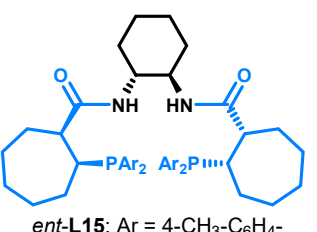

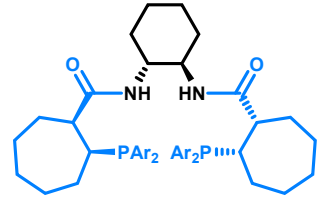

L15: $\mathrm{Ar}=4-\mathrm{CH}_{3}-\mathrm{C}_{6} \mathrm{H}_{4}$ L22: $\mathrm{Ar}=\mathrm{C}_{6} \mathrm{H}_{5}-$ L23: $\mathrm{Ar}=4-\mathrm{Ph}-\mathrm{C}_{6} \mathrm{H}_{4}$

$=4-\mathrm{F}-\mathrm{C}_{6} \mathrm{H}_{4}$

L25: $\mathrm{Ar}=3,5-\left(\mathrm{CH}_{3}\right)_{2}-\mathrm{C}_{6} \mathrm{H}_{3}$ -

L26: $\mathrm{Ar}=2$-naphthyl

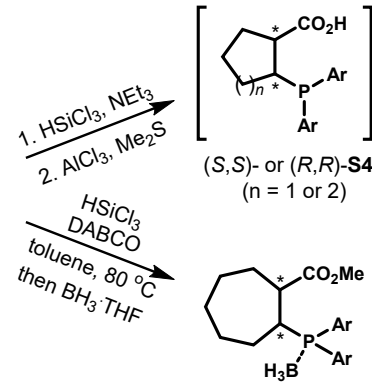

$(\mathrm{S}, \mathrm{S})$ - or $(R, R)-\mathrm{S} 3$ 1. $\mathrm{AICl}_{3}, \mathrm{Me}_{2} \mathrm{~S}$

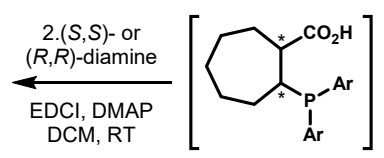

Scheme S2. Synthesis of New Bisphosphine Ligands

$(S, S)$ - or $(\boldsymbol{R}, \boldsymbol{R})-\mathrm{S} 2:$ General Procedure ${ }^{2}$

Under nitrogen atmosphere, a mixture of $\mathrm{H}_{2} \mathrm{O}(10 \mathrm{~mol} \%)$ in THF $(1 \mathrm{~mL} / \mathrm{mmol})$ was added to an ovendried flask charged with $(R)$ or $(S)-\mathrm{H}_{8}$-BINOL $(15 \mathrm{~mol} \%)$. After stirred at RT for $5 \mathrm{~min}, \mathrm{MgBu}_{2}((10$ mol\%), 1.0 $\mathrm{M}$ in heptane) and diarylphosphine oxide (1.0 equiv.) were sequentially added and stirred for another 5 min before cooled to $-20^{\circ} \mathrm{C}$. $\alpha, \beta$-Unsaturated ester (1.2 equiv.) was then added, and the reaction was stirred at $-20^{\circ} \mathrm{C}$ for $2-3$ days (Note: the purity of the diarylphosphine oxide is very important for this reaction). The reaction mixture was quenched with sat. $\mathrm{NH}_{4} \mathrm{Cl}$, diluted with $\mathrm{H}_{2} \mathrm{O}$ and extracted with DCM. The combined organic phase was dried over anhydrous $\mathrm{Na}_{2} \mathrm{SO}_{4}$. After filtration and concentration, the residue was purified by silica gel column chromatography to give the product. The enantiomeric excess was determined by the chiral HPLC analysis. 


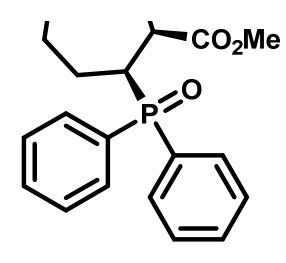

S2a Methyl (1S,2S)-2-(diphenylphosphoryl)cycloheptane-1-carboxylate (S2a). With diphenylphosphine oxide $(2.0 \mathrm{~g}, 10 \mathrm{mmol})$ to afford $\mathbf{S 2 a}$ as colorless oil $(3.4 \mathrm{~g}, 95 \%$ yield, $11.5: 1 \mathrm{dr}$ and $95 \%$ ee).

$\underline{R}_{f} \quad 0.45\left(\mathrm{DCM} / \mathrm{EtOAc}=3: 1, \mathrm{KMnO}_{4}\right)$

Opt. Rot. $\quad[\alpha]^{20}=-67.0(\mathrm{c}=0.1, \mathrm{MeOH})$

${ }^{1} \mathrm{H} \mathrm{NMR} \quad\left(400 \mathrm{MHz}, \mathrm{CDCl}_{3}\right)$

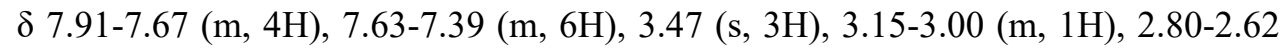
$(\mathrm{m}, 1 \mathrm{H}), 2.27-2.14(\mathrm{~m}, 1 \mathrm{H}), 2.08-1.98(\mathrm{~m}, 1 \mathrm{H}), 1.94-1.84(\mathrm{~m}, 2 \mathrm{H}), 1.73(\mathrm{~s}, 3 \mathrm{H})$, $1.59-1.49(\mathrm{~m}, 2 \mathrm{H}), 1.41-1.28(\mathrm{~m}, 1 \mathrm{H})$.

${ }^{13} \mathrm{C} \mathrm{NMR} \quad\left(100 \mathrm{MHz}, \mathrm{CDCl}_{3}\right)$

$\delta 176.2,132.9,132.6,131.9,131.7,131.7,131.6,131.6,131.2,131.1,128.9,128.7$, $128.4,128.3,51.8,42.2,38.6,37.9,30.5,30.4,29.7,29.6,25.5$.

${ }^{31} \mathrm{P} \mathrm{NMR} \quad\left(162 \mathrm{MHz}, \mathrm{CDCl}_{3}\right)$ $\delta 38.03$.

HRMS (ESI) for: $\mathrm{C}_{21} \mathrm{H}_{26} \mathrm{O}_{3} \mathrm{P}[\mathrm{M}+\mathrm{H}]^{+}$: calcd 357.4098, found 357.4082 .

HPLC chiralpak AD-H column $(250 * 4.6 \mathrm{~mm} / 5 \mu \mathrm{m}): n$-hexane $/ i$-PrOH $(90 / 10), 1.0 \mathrm{~mL} / \mathrm{min}$ $230 \mathrm{~nm}, 21.33 \mathrm{~min}$ (major enantiomer), $17.71 \mathrm{~min}$ (minor enantiomer).<smiles>CO[Sb](C)(=O)(O)c1ccc(P(=O)(c2ccc(C)cc2)C2CCCCC[C@H]2C(C)=O)cc1</smiles>

Methyl (1S,2S)-2-(di-p-tolylphosphoryl)cycloheptane-1-carboxylate (S2b). With $\mathrm{d} i$ - $p$-tolylphosphine oxide $(2.3 \mathrm{~g}, 10 \mathrm{mmol})$ to yield $\mathbf{S 2 b}$ as colorless oil $(3.6 \mathrm{~g}, 95 \%$ yield, $6.3: 1 \mathrm{dr}$ and $95 \%$ ee).

$\underline{R}_{f} \quad 0.40\left(\mathrm{DCM} / \mathrm{EtOAc}=3: 1, \mathrm{KMnO}_{4}\right)$

Opt. Rot. $\quad[\alpha]^{20}{ }_{\mathrm{D}}=-29.0(\mathrm{c}=0.1, \mathrm{MeOH})$

${ }^{1} \mathrm{H} \mathrm{NMR} \quad\left(500 \mathrm{MHz}, \mathrm{CDCl}_{3}\right)$

$\delta 7.72-7.61(\mathrm{~m}, 4 \mathrm{H}), 7.31-7.22(\mathrm{~m}, 4 \mathrm{H}), 3.49(\mathrm{~s}, 3 \mathrm{H}), 3.08-3.02(\mathrm{~m}, 1 \mathrm{H}), 2.71-$ $2.58(\mathrm{~m}, 1 \mathrm{H}), 2.38(\mathrm{~d}, J=5.0 \mathrm{~Hz}, 6 \mathrm{H}), 2.26-2.13(\mathrm{~m}, 1 \mathrm{H}), 2.09-1.97(\mathrm{~m}, 1 \mathrm{H})$, $1.92-1.84(\mathrm{~m}, 1 \mathrm{H}), 1.77-1.72(\mathrm{~m}, 4 \mathrm{H}), 1.61-1.46(\mathrm{~m}, 2 \mathrm{H}), 1.39-1.28(\mathrm{~m}, 1 \mathrm{H})$.

${ }^{13} \mathrm{C} \mathrm{NMR} \quad\left(125 \mathrm{MHz}, \mathrm{CDCl}_{3}\right)$

$\delta 174.5,141.8,131.3,131.2,130.9,130.9,130.8,130.7,130.3,129.7,129.6,129.3$, 129.2, 129.1, 128.8, 51.4, 41.4, 41.0, 40.9, 31.9, 31.8, 29.1, 29.0, 28.7, 25.2, 23.5, 21.5 .

${ }^{31} \mathrm{P} \mathrm{NMR} \quad\left(202 \mathrm{MHz}, \mathrm{CDCl}_{3}\right)$

$\delta 35.18$.

HRMS (ESI) for: $\mathrm{C}_{23} \mathrm{H}_{30} \mathrm{O}_{3} \mathrm{P}[\mathrm{M}+\mathrm{H}]^{+}$: calcd 385.4638 , found 385.4662 .

HPLC Daicel Chiralpak IA-3 column, $n$-hexane/i-PrOH (70/30), $1 \mathrm{~mL} / \mathrm{min}, 210 \mathrm{~nm}$, $25.948 \mathrm{~min}$ (major enantiomer), $17.754 \mathrm{~min}$ (minor enantiomer). 
<smiles>CC(=O)C1CCCCCC1P(=O)(c1ccc(-c2ccccc2)cc1)c1ccc(-c2ccccc2)cc1</smiles>

Methyl (1S,2S)-2-(di([1,1'-biphenyl]-4-yl)phosphoryl)cycloheptane-1carboxylate (S2c). With di([1,1'-biphenyl]-4-yl)phosphine oxide (3.5 g, $10 \mathrm{mmol})$ to yield S2c as white solid (4.7 g, 92\% yield, 13:1 dr and 96\% ee).

$\underline{R}_{f} \quad 0.50\left(\mathrm{DCM} / \mathrm{EtOAc}=3: 1, \mathrm{KMnO}_{4}\right)$

Opt. Rot. $[\alpha]^{20}=-62.0(\mathrm{c}=0.1, \mathrm{MeOH})$

${ }^{1} \mathrm{H} \mathrm{NMR} \quad\left(500 \mathrm{MHz}, \mathrm{CDCl}_{3}\right)$

$\delta 7.98-7.84(\mathrm{~m}, 4 \mathrm{H}), 7.74-7.74(\mathrm{~m}, 4 \mathrm{H}), 7.64-7.57(\mathrm{~m}, 4 \mathrm{H}), 7.63-7.58(\mathrm{~m}, 4 \mathrm{H})$, $7.42-7.34(\mathrm{~m}, 2 \mathrm{H}), 3.51(\mathrm{~s}, 3 \mathrm{H}), 3.22-3.08(\mathrm{~m}, 1 \mathrm{H}), 2.82-2.72(\mathrm{~m}, 1 \mathrm{H}), 2.32-$ $2.17(\mathrm{~m}, 2 \mathrm{H}), 2.14-2.05(\mathrm{~m}, 1 \mathrm{H}), 1.97-1.87(\mathrm{~m}, 2 \mathrm{H}), 1.84-1.72(\mathrm{~m}, 2 \mathrm{H}), 1.65-$ $1.51(\mathrm{~m}, 2 \mathrm{H}), 1.48-1.36(\mathrm{~m}, 1 \mathrm{H})$.

${ }^{13} \mathrm{C} \mathrm{NMR} \quad\left(125 \mathrm{MHz}, \mathrm{CDCl}_{3}\right)$

$\delta 174.5,144.4,144.3,139.9,132.0,131.9,131.8,131.5,131.4,131.3,131.3,130.6$, $128.9,128.1,127.3,127.3,127.1,51.5,41.2,40.9,31.9,29.1,28.7,25.3,23.7$.

${ }^{31} \mathrm{P} \mathrm{NMR} \quad\left(202 \mathrm{MHz}, \mathrm{CDCl}_{3}\right)$

$\delta 34.78$.

HRMS (ESI) for: $\mathrm{C}_{33} \mathrm{H}_{34} \mathrm{O}_{3} \mathrm{P}[\mathrm{M}+\mathrm{H}]^{+}$: calcd 509.6058, found 509.6067 .

HPLC Daicel Chiralpak IC-3 column, $n$-hexane $/ i$-PrOH (70/30), $1 \mathrm{~mL} / \mathrm{min} 210 \mathrm{~nm} 66.044$ min (major enantiomer), 35.329 min (minor enantiomer).<smiles>CC(=O)OC1CCCCCC1P(=O)(c1ccc(F)cc1)c1ccc(F)cc1</smiles>

S2d

Methyl

(1S,2S)-2-(bis(4-fluorophenyl)phosphoryl)cycloheptane-1carboxylate (S2d). With bis(4-fluorophenyl)phosphine oxide $(2.4 \mathrm{~g}, 10 \mathrm{mmol})$ to yield S2d as white solid (4.1 g, 93\% yield, 7:1 dr and 95\% ee).

$\underline{R}_{f} \quad 0.40\left(\mathrm{DCM} / \mathrm{EtOAc}=5: 1, \mathrm{KMnO}_{4}\right)$

Opt. Rot. $[\alpha]^{20}{ }_{\mathrm{D}}=-41.0(\mathrm{c}=0.1, \mathrm{MeOH})$

$\underline{{ }^{1} \mathrm{H} \mathrm{NMR}} \quad\left(400 \mathrm{MHz}, \mathrm{CDCl}_{3}\right)$

$\delta 7.99-7.68(\mathrm{~m}, 4 \mathrm{H}), 7.26-7.08(\mathrm{~m}, 4 \mathrm{H}), 3.82-3.73(\mathrm{~m}, 1 \mathrm{H}), 3.52(\mathrm{~s}, 3 \mathrm{H}), 3.11-$ $3.04(\mathrm{~m}, 1 \mathrm{H}), 2.69-2.63(\mathrm{~m}, 1 \mathrm{H}), 2.26-2.14(\mathrm{~m}, 1 \mathrm{H}), 2.10-2.01(\mathrm{~m}, 1 \mathrm{H}), 1.95-$ $1.90(\mathrm{~m}, 1 \mathrm{H}), 1.82-1.73(\mathrm{~m}, 3 \mathrm{H}), 1.62-1.51(\mathrm{~m}, 2 \mathrm{H}), 1.41-1.31(\mathrm{~m}, 1 \mathrm{H})$.

${ }^{13} \mathrm{C} \mathrm{NMR} \quad\left(100 \mathrm{MHz}, \mathrm{CDCl}_{3}\right)$

$\delta 174.4,166.2,163.7,133.9,133.8,133.7,133.5,133.4,133.3,116.3,116.2,116.1$, $116.0,115.9,68.1,51.7,41.8,41.1,31.8,31.7,29.2,29.0,28.7,25.7,25.2,23.7$.

${ }^{31} \mathrm{P} \mathrm{NMR} \quad\left(162 \mathrm{MHz}, \mathrm{CDCl}_{3}\right)$

$\delta 34.60$. 


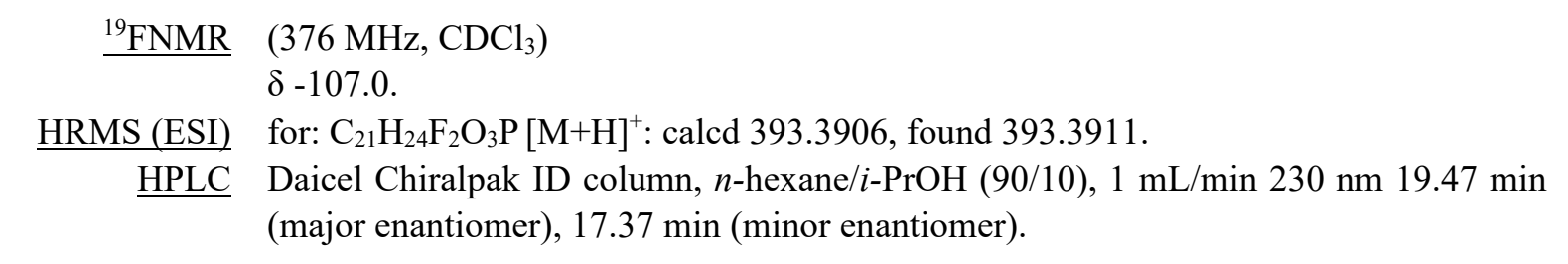<smiles>CC(=O)[C@H]1CCCCC[C@@H]1P(=O)(c1cc(C)cc(C)c1)c1cc(C)cc(C)c1</smiles>
solid (3.7 g, 91\% yield, 5.6:1 dr and 96\% ee).

$\underline{R}_{f} \quad 0.4\left(\mathrm{DCM} / \mathrm{EtOAc}=4: 1, \mathrm{KMnO}_{4}\right)$

Opt. Rot. $[\alpha]^{20}{ }_{\mathrm{D}}=-72.0(\mathrm{c}=0.1, \mathrm{MeOH})$

${ }^{1} \mathrm{H} \mathrm{NMR} \quad\left(500 \mathrm{MHz}, \mathrm{CDCl}_{3}\right)$

$\delta 7.39(\mathrm{dd}, J=25.7,11.0 \mathrm{~Hz}, 4 \mathrm{H}), 7.11(\mathrm{~s}, 2 \mathrm{H}), 3.51(\mathrm{~s}, 3 \mathrm{H}), 3.04-2.98(\mathrm{~m}, 1 \mathrm{H})$, $2.72-2.54(\mathrm{~m}, 1 \mathrm{H}), 2.34(\mathrm{~d}, J=2.9 \mathrm{~Hz}, 12 \mathrm{H}), 2.25-2.16(\mathrm{~m}, 1 \mathrm{H}), 2.11-1.98(\mathrm{~m}$, $1 \mathrm{H}), 1.92-1.83(\mathrm{~m}, 1 \mathrm{H}), 1.82-1.65(\mathrm{~s}, 4 \mathrm{H}), 1.63-1.49(\mathrm{~m}, 2 \mathrm{H}), 1.42-1.30(\mathrm{~m}$, $1 \mathrm{H})$.

${ }^{13} \mathrm{C} \mathrm{NMR} \quad\left(125 \mathrm{MHz}, \mathrm{CDCl}_{3}\right)$

$\delta 174.5,138.2,138.0,133.3,133.2,133.2,133.2,133.2,132.6,132.4,131.9,128.7$, $128.7,128.5,128.4,77.3,51.4,41.0,40.5,32.0,31.9,29.1,29.0,28.8,25.3,23.4$, 21.4 .

${ }^{31} \mathrm{P} \mathrm{NMR} \quad\left(202 \mathrm{MHz}, \mathrm{CDCl}_{3}\right)$ $\delta 35.14$

HRMS (ESI) for: $\mathrm{C}_{25} \mathrm{H}_{34} \mathrm{O}_{3} \mathrm{P}[\mathrm{M}+\mathrm{H}]^{+}$: calcd 413.5178, found 413.5174 .

HPLC Daicel Chiralpak ID-3 column, $n$-hexane $/ i$-PrOH (90/10), $0.5 \mathrm{~mL} / \mathrm{min} 210 \mathrm{~nm}$ 38.927 min (major enantiomer), $36.043 \mathrm{~min}$ (minor enantiomer).

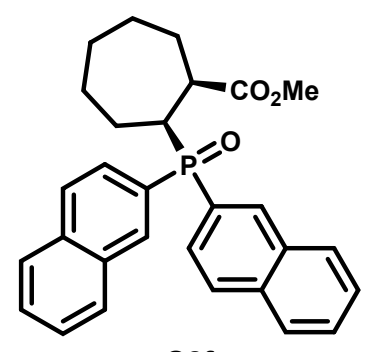

S2f

Methyl (1S,2S)-2-(di(naphthalen-2-yl)phosphoryl)cycloheptane-1carboxylate (S2f). With di(naphthalen-2-yl)phosphine oxide (3.0 g, $10 \mathrm{mmol})$ to yield S2f as white solid (4.0 g, 88\% yield, $7.7: 1 \mathrm{dr}$ and $99 \%$ ee).

$\underline{R}_{f} \quad 0.3\left(\mathrm{DCM} / \mathrm{EtOAc}=5: 1, \mathrm{KMnO}_{4}\right)$

Opt. Rot. $[\alpha]^{20}{ }_{\mathrm{D}}=-52.0(\mathrm{c}=0.1, \mathrm{MeOH})$

$\underline{{ }^{1} \mathrm{H} \mathrm{NMR}} \quad\left(500 \mathrm{MHz}, \mathrm{CDCl}_{3}\right)$

$\delta 8.54(\mathrm{~d}, J=10.5 \mathrm{~Hz}, 1 \mathrm{H}), 8.43(\mathrm{~d}, J=12.5 \mathrm{~Hz}, 1 \mathrm{H}), 8.05-7.73(\mathrm{~m}, 8 \mathrm{H}), 7.67-$ 
$7.47(\mathrm{~m}, 4 \mathrm{H}), 3.43(\mathrm{~s}, 3 \mathrm{H}), 3.18-3.10(\mathrm{~m}, 1 \mathrm{H}), 3.00-2.84(\mathrm{~m}, 1 \mathrm{H}), 2.36-2.25(\mathrm{~m}$, $1 \mathrm{H}), 2.13-1.98(\mathrm{~m}, 1 \mathrm{H}), 1.96-1.89(\mathrm{~m}, 2 \mathrm{H}), 1.83-1.69(\mathrm{~m}, 3 \mathrm{H}), 1.63-1.53(\mathrm{~m}$, $2 \mathrm{H}), 1.49-1.33(\mathrm{~m}, 1 \mathrm{H})$.

${ }^{13} \mathrm{C} \mathrm{NMR} \quad\left(125 \mathrm{MHz}, \mathrm{CDCl}_{3}\right)$ $\delta 174.5,134.5,133.9,133.8,133.2,133.1,132.7,132.6,130.4,129.9,129.7,129.1$, $128.9,128.5,128.4,128.3,128.2,128.1,127.8,126.9,125.9,125.8,125.7,125.6$, $51.5,41.2,40.7,31.9,31.8,29.1,29.0,28.7,25.4,25.3,23.7$.

${ }^{31} \mathrm{P} \mathrm{NMR} \quad\left(202 \mathrm{MHz}, \mathrm{CDCl}_{3}\right)$ $\delta 34.93$

HRMS (ESI) for: $\mathrm{C}_{29} \mathrm{H}_{30} \mathrm{O}_{3} \mathrm{P}[\mathrm{M}+\mathrm{H}]^{+}$: calcd 457.5298, found 457.5288 .

HPLC Daicel Chiralpak IC-3 column, $n$-hexane $/ i$-PrOH (70/30), 1 mL/min $254 \mathrm{~nm} 87.703$ min (major enantiomer), $60.879 \mathrm{~min}$ (minor enantiomer).

\section{$(S, S)$ - or $(\boldsymbol{R}, \boldsymbol{R})-\mathrm{S3}$ : General Procedure ${ }^{3}$}

A solution of $\mathbf{S 2}$ (1.0 equiv.) and 1,4-diazabicyclo[2.2.2]octane (DABCO, 3.0 equiv.) in anhydrous toluene $(10 \mathrm{~mL} / \mathrm{mmol})$ was cooled to $0{ }^{\circ} \mathrm{C}$ and treated drop-wise with trichlorosilane (2.0 equiv.). Then the solution was stirred at $80^{\circ} \mathrm{C}$ under $\mathrm{N}_{2}$ over $18 \mathrm{~h} . \mathrm{BH}_{3} \cdot \mathrm{THF}$ (5.0 equiv.) was then added to the mixture at $0{ }^{\circ} \mathrm{C}$ slowly, the reaction mixture was stirred at $0{ }^{\circ} \mathrm{C}$ for another $2 \mathrm{~h}$. MeOH was added to quenched the excess reagents, removal of all volatiles and purification of the residue by column chromatography on silica gel gave the desired product.

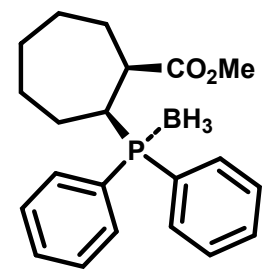

S3a

Methyl (1S,2S)-2-(diphenylphosphanyl)cycloheptane-1-carboxylate-borane complex (S3a). With S2a (3.6 g, $10 \mathrm{mmol})$ to yield $\mathbf{S 3 a}$ as a white solid (1.5 g, $42 \%$ yield).

$\underline{R}_{f} \quad 0.4\left(\mathrm{Hex} / \mathrm{EtOAc}=20: 1, \mathrm{KMnO}_{4}\right)$

Opt. Rot. $[\alpha]^{20}{ }_{\mathrm{D}}=-60.0(\mathrm{c}=0.1, \mathrm{MeOH})$

${ }^{1} \mathrm{H} \mathrm{NMR} \quad\left(400 \mathrm{MHz}, \mathrm{CDCl}_{3}\right)$ $\delta 7.91-7.71(\mathrm{~m}, 2 \mathrm{H}), 7.69-7.61(\mathrm{~m}, 2 \mathrm{H}), 7.54-7.39(\mathrm{~m}, 6 \mathrm{H}), 3.35(\mathrm{~s}, 3 \mathrm{H}), 3.27-$ $3.10(\mathrm{~m}, 1 \mathrm{H}), 2.86-2.64(\mathrm{~m}, 1 \mathrm{H}), 2.35-2.17(\mathrm{~m}, 1 \mathrm{H}), 2.16-2.03(\mathrm{~m}, 1 \mathrm{H}), 1.89-$ $1.75(\mathrm{~m}, 2 \mathrm{H}), 1.67-1.53(\mathrm{~m}, 5 \mathrm{H}), 1.50-1.36(\mathrm{~m}, 1 \mathrm{H})$.

${ }^{13} \mathrm{C} \mathrm{NMR} \quad\left(100 \mathrm{MHz}, \mathrm{CDCl}_{3}\right)$

$\delta 173.8,133.7,133.6,132.4,132.3,131.4,131.1,129.9,129.3,128.90,128.8,128.6$, $128.5,128.3,127.8,51.3,42.1,37.9,37.6,33.4,33.3,28.6,28.5,27.7,25.6,25.1$.

${ }^{31} \mathrm{P} \mathrm{NMR} \quad\left(162 \mathrm{MHz}, \mathrm{CDCl}_{3}\right)$ $\delta 26.70$

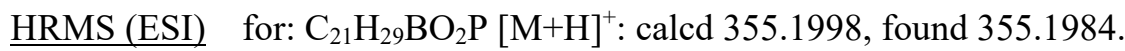


<smiles>CC(=O)C1CCCCCC1[PH](C)(c1ccc(C)cc1)c1ccc([N+](=O)[O-])cc1</smiles>

S3b

Methyl (1S,2S)-2-(di-p-tolylphosphanyl)cycloheptane-1-carboxylateborane complex (S3b). With S2b (3.8 g, $10 \mathrm{mmol})$ to yield $\mathbf{S 3 b}$ as a white solid (1.8 g, 48\% yield).

$\underline{R}_{f} \quad 0.35\left(\mathrm{Hex} / \mathrm{EtOAc}=20: 1, \mathrm{KMnO}_{4}\right)$

Opt. Rot. $[\alpha]^{20}{ }_{\mathrm{D}}=-49.0(\mathrm{c}=0.1, \mathrm{MeOH})$

${ }^{1} \mathrm{H} \mathrm{NMR} \quad\left(500 \mathrm{MHz}, \mathrm{CDCl}_{3}\right)$

$\delta 7.70-7.62(\mathrm{~m}, 2 \mathrm{H}), 7.54(\mathrm{dd}, J=9.8,8.2 \mathrm{~Hz}, 2 \mathrm{H}), 7.30-7.17(\mathrm{~m}, 4 \mathrm{H}), 3.38(\mathrm{~s}$, $3 \mathrm{H}), 3.20-3.11(\mathrm{~m}, 1 \mathrm{H}), 2.84-2.61(\mathrm{~m}, 1 \mathrm{H}), 2.37(\mathrm{~d}, J=7.3 \mathrm{~Hz}, 6 \mathrm{H}), 2.31-2.17$ $(\mathrm{m}, 1 \mathrm{H}), 2.14-1.97(\mathrm{~m}, 1 \mathrm{H}), 1.85-1.73(\mathrm{~m}, 2 \mathrm{H}), 1.70-1.55(\mathrm{~m}, 5 \mathrm{H}), 1.44(\mathrm{~s}, 1 \mathrm{H})$.

${ }^{13} \mathrm{C} \mathrm{NMR} \quad\left(125 \mathrm{MHz}, \mathrm{CDCl}_{3}\right)$

$\delta 173.8,141.5,141.2,133.5,133.4,132.3,132.2,129.5,129.5,129.3,129.2,126.5$, $126.0,125.0,124.5,51.2,42.1,37.9,37.6,33.4,33.3,28.5,28.4,27.6,25.5,24.9$, 21.4 .

${ }^{31} \mathrm{P} \mathrm{NMR} \quad\left(202 \mathrm{MHz}, \mathrm{CDCl}_{3}\right)$ $\delta 24.67$

HRMS (ESI) for: $\mathrm{C}_{23} \mathrm{H}_{33} \mathrm{BO}_{2} \mathrm{P}[\mathrm{M}+\mathrm{H}]^{+}$: calcd 383.2988, found 383.2983.<smiles>CC(=O)C1CCCCCC1[PH](c1ccccc1)(c1ccccc1)c1ccc(-c2ccccc2)cc1</smiles>

S3c

Methyl (1S,2S)-2-(di([1,1'-biphenyl]-4-yl)phosphanyl)cycloheptane-1carboxylate-borane complex (S3c). With S2c $(5.1 \mathrm{~g}, 10 \mathrm{mmol})$ to yield $\mathbf{S 3 c}$ as a white solid $(2.1 \mathrm{~g}, 41 \%$ yield).

$\underline{R}_{f} \quad 0.25\left(\mathrm{Hex} / \mathrm{EtOAc}=20: 1, \mathrm{KMnO}_{4}\right)$

Opt. Rot. $[\alpha]^{24}=-26.0(\mathrm{c}=0.1, \mathrm{MeOH})$

${ }^{1} \mathrm{H} \mathrm{NMR} \quad\left(500 \mathrm{MHz}, \mathrm{CDCl}_{3}\right)$

$\delta 7.97-7.85(\mathrm{~m}, 2 \mathrm{H}), 7.78(\mathrm{t}, J=8.8 \mathrm{~Hz}, 2 \mathrm{H}), 7.37-7.66(\mathrm{~m}, 4 \mathrm{H}), 7.65-7.58(\mathrm{~m}$, $4 \mathrm{H}), 7.51-7.44(\mathrm{~m}, 4 \mathrm{H}), 7.44-7.36(\mathrm{~m}, 2 \mathrm{H}), 3.41(\mathrm{~s}, 3 \mathrm{H}), 3.33-3.20(\mathrm{~m}, 1 \mathrm{H}), 2.93-$ $2.76(\mathrm{~m}, 1 \mathrm{H}), 2.41-2.27(\mathrm{~m}, 1 \mathrm{H}), 2.20-2.10(\mathrm{~m}, 1 \mathrm{H}), 1.98-1.88(\mathrm{~m}, 1 \mathrm{H}), 1.88-$ $1.79(\mathrm{~m}, 1 \mathrm{H}), 1.81-1.72(\mathrm{~m}, 1 \mathrm{H}), 1.72-1.62(\mathrm{~m}, 4 \mathrm{H}), 1.58-1.47(\mathrm{~m}, 1 \mathrm{H})$.

${ }^{13} \mathrm{C} \mathrm{NMR} \quad\left(125 \mathrm{MHz}, \mathrm{CDCl}_{3}\right)$

$\delta 173.8,144.1,143.8,139.9,134.1,134.0,132.8,129.0,128.4,128.2,128.1,128.0$, $127.5,127.4,127.2,127.1,126.9,126.5,51.3,42.2,38.0,37.8,33.4,33.3,28.6,28.5$, 27.6, 25.6, 25.2.

${ }^{31} \mathrm{P} \mathrm{NMR} \quad\left(202 \mathrm{MHz}, \mathrm{CDCl}_{3}\right)$

$\delta 25.22$

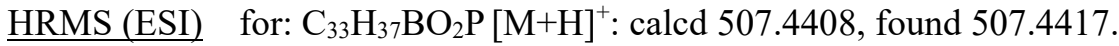




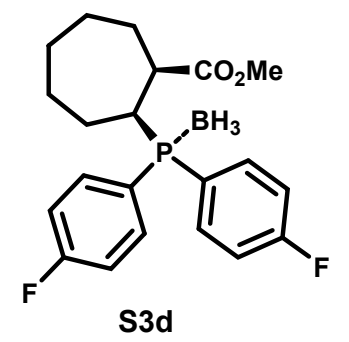

Methyl (1S,2S)-2-(bis(4-fluorophenyl)phosphanyl)cycloheptane-1carboxylate-borane complex (S3d). With S2d (3.9 g, $10 \mathrm{mmol})$ to yield S3d as a white solid (1.8 g, $47 \%$ yield).

$\underline{R}_{f} \quad 0.40\left(\mathrm{Hex} / \mathrm{EtOAc}=20: 1, \mathrm{KMnO}_{4}\right)$

Opt. Rot. $\quad[\alpha]^{20}{ }_{\mathrm{D}}=-42.0(\mathrm{c}=0.1, \mathrm{MeOH})$

${ }^{1} \mathrm{H} \mathrm{NMR} \quad\left(500 \mathrm{MHz}, \mathrm{CDCl}_{3}\right)$

$\delta 7.88(\mathrm{t}, J=9.7 \mathrm{~Hz}, 1 \mathrm{H}), 7.76(\mathrm{t}, J=8.9 \mathrm{~Hz}, 2 \mathrm{H}), 7.72-7.56(\mathrm{~m}, 5 \mathrm{H}), 7.50-7.42(\mathrm{~m}$, $2 \mathrm{H}), 7.43-7.33(\mathrm{~m}, 1 \mathrm{H}), 7.21-7.10(\mathrm{~m}, 1 \mathrm{H}), 3.40(\mathrm{~d}, J=9.8 \mathrm{~Hz}, 3 \mathrm{H}), 3.31-3.10(\mathrm{~s}$, $1 \mathrm{H}), 2.89-2.60(\mathrm{~m}, 1 \mathrm{H}), 2.40-2.18(\mathrm{~m}, 1 \mathrm{H}), 2.16-2.04(\mathrm{~m}, 1 \mathrm{H}), 1.97-1.70(\mathrm{~m}$, $3 \mathrm{H}), 1.68-1.47(\mathrm{~m}, 5 \mathrm{H})$.

${ }^{13} \mathrm{C} \mathrm{NMR} \quad\left(125 \mathrm{MHz}, \mathrm{CDCl}_{3}\right)$

$\delta 173.8,173.8,144.0,143.8,139.9,136.0,135.9,135.8,134.6,134.5,134.5,134.1$, $134.0,132.8,132.8,129.0,128.4,128.2,128.1,128.0,127.5,127.4,127.2,127.1$, $126.9,126.5,116.4,116.3,116.2,116.15,116.10,116.0,115.9,115.8,51.4,51.3,42.2$, $38.32,38.1,38.0,37.8,33.3,33.2,33.1,33.0,28.5,28.4,27.6,25.5,25.3,25.2,25.1$.

${ }^{31} \mathrm{P} \mathrm{NMR} \quad\left(202 \mathrm{MHz}, \mathrm{CDCl}_{3}\right)$

$\delta 25.35$.

${ }^{19} \mathrm{~F} \mathrm{NMR} \quad\left(470 \mathrm{MHz}, \mathrm{CDCl}_{3}\right)$ $\delta-107.37,-107.96$.

HRMS (ESI) for: $\mathrm{C}_{21} \mathrm{H}_{27} \mathrm{BF}_{2} \mathrm{O}_{2} \mathrm{P}[\mathrm{M}+\mathrm{H}]^{+}$: calcd 391.2256, found 391.2239.

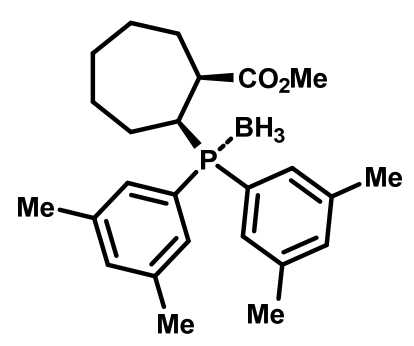

S3e

Methyl (1S,2S)-2-(bis(3,5-dimethylphenyl)phosphanyl)cycloheptane-1carboxylate-borane complex (S3e). With S2e $(4.1 \mathrm{~g}, 10 \mathrm{mmol})$ to yield S3e as a white solid $(2.1 \mathrm{~g}, 51 \%$ yield).

$\underline{R}_{f} \quad 0.35\left(\mathrm{Hex} / \mathrm{EtOAc}=20: 1, \mathrm{KMnO}_{4}\right)$

Opt. Rot. $\quad[\alpha]^{20}{ }_{\mathrm{D}}=-53.0(\mathrm{c}=0.1, \mathrm{MeOH})$

${ }^{1} \mathrm{H} \mathrm{NMR} \quad\left(500 \mathrm{MHz}, \mathrm{CDCl}_{3}\right)$

$\delta 7.35(\mathrm{~d}, J=10.5 \mathrm{~Hz}, 2 \mathrm{H}), 7.24(\mathrm{~d}, J=10.3 \mathrm{~Hz}, 2 \mathrm{H}), 7.09(\mathrm{~d}, J=13.4 \mathrm{~Hz}, 2 \mathrm{H}), 3.38$ $(\mathrm{s}, 3 \mathrm{H}), 3.15-3.08(\mathrm{~m}, 1 \mathrm{H}), 2.77-2.67(\mathrm{~m}, 1 \mathrm{H}), 2.33(\mathrm{~d}, J=9.9 \mathrm{~Hz}, 12 \mathrm{H}), 2.29-2.20$ $(\mathrm{m}, 1 \mathrm{H}), 2.13-2.02(\mathrm{~m}, 1 \mathrm{H}), 1.83-1.69(\mathrm{~m}, 3 \mathrm{H}), 1.66-1.59(\mathrm{~m}, 4 \mathrm{H}), 1.53-1.42(\mathrm{~m}$, $1 \mathrm{H})$.

${ }^{13} \mathrm{C} \mathrm{NMR} \quad\left(125 \mathrm{MHz}, \mathrm{CDCl}_{3}\right)$

$\delta 173.8,138.3,138.2,138.0,137.9,133.0,132.8,131.0,130.9,129.94,129.9,129.4$, 129.0, 128.1, 127.6, 51.1, 42.0, 37.4, 37.2, 33.6, 33.5, 28.5, 28.4, 27.6, 25.7, 24.9, 21.4. 


\author{
${ }^{31} \mathrm{P} \mathrm{NMR} \quad\left(202 \mathrm{MHz}, \mathrm{CDCl}_{3}\right)$ \\ $\delta 25.41$ \\ HRMS (ESI) for: $\mathrm{C}_{25} \mathrm{H}_{37} \mathrm{BO}_{2} \mathrm{P}[\mathrm{M}+\mathrm{H}]^{+}$: calcd 411.3528, found 411.3519 .
}

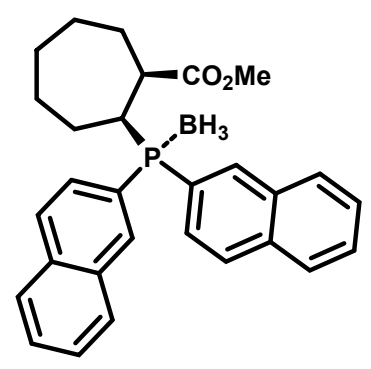

S3f

Methyl (1S,2S)-2-(di(naphthalen-2-yl)phosphanyl)cycloheptane-1carboxylate-borane complex (S3f). With S2f $(4.6 \mathrm{~g}, 10 \mathrm{mmol})$ to yield $\mathbf{S 3 f}$ as a white solid $(1.7 \mathrm{~g}, 38 \%$ yield).

$\underline{R}_{f} \quad 0.30\left(\mathrm{Hex} / \mathrm{EtOAc}=20: 1, \mathrm{KMnO}_{4}\right)$

Opt. Rot. $[\alpha]^{20}{ }_{\mathrm{D}}=-38.0(\mathrm{c}=0.1, \mathrm{MeOH})$

${ }^{1} \mathrm{H} \mathrm{NMR} \quad\left(500 \mathrm{MHz}, \mathrm{CDCl}_{3}\right)$

$\delta 8.45(\mathrm{~d}, J=12.6 \mathrm{~Hz}, 1 \mathrm{H}), 8.23(\mathrm{~d}, J=11.9 \mathrm{~Hz}, 1 \mathrm{H}), 7.96-7.81(\mathrm{~m}, 6 \mathrm{H}), 7.81-7.74$ $(\mathrm{m}, 1 \mathrm{H}), 7.70(\mathrm{t}, J=7.7 \mathrm{~Hz}, 1 \mathrm{H}), 7.61-7.50(\mathrm{~m}, 4 \mathrm{H}), 3.30-3.25(\mathrm{~m}, 1 \mathrm{H}), 3.24(\mathrm{~s}$, $3 \mathrm{H}), 3.02-2.94(\mathrm{~m}, 1 \mathrm{H}), 2.45-2.28(\mathrm{~m}, 1 \mathrm{H}), 2.17-2.04(\mathrm{~m}, 1 \mathrm{H}), 2.01-1.88(\mathrm{~m}$, $1 \mathrm{H}), 1.91-1.78(\mathrm{~m}, 1 \mathrm{H}), 1.78-1.59(\mathrm{~m}, 5 \mathrm{H}), 1.56-1.47(\mathrm{~m}, 1 \mathrm{H})$.

${ }^{13} \mathrm{C} \mathrm{NMR} \quad\left(125 \mathrm{MHz}, \mathrm{CDCl}_{3}\right)$

$\delta 173.8,135.9,135.8,134.3,134.2,134 ., 132.8,132.8,132.74,132.68,128.8,128.7$, $128.6,128.5,128.1,128.1,128.07,127.98,127.8,127.2,127.1,126.9,126.5,125.5$, 125.0, 51.2, 42.2, 37.9, 37.6, 33.4, 33.3, 28.6, 28.5, 27.6, 25.6, 25.2.

${ }^{31} \mathrm{P}$ NMR $\quad\left(202 \mathrm{MHz}, \mathrm{CDCl}_{3}\right)$ $\delta 26.85$

HRMS (ESI) for: $\mathrm{C}_{29} \mathrm{H}_{33} \mathrm{BO}_{2} \mathrm{P}[\mathrm{M}+\mathrm{H}]^{+}$: calcd 455.3648, found 455.3651 .

\title{
L2-L6: General Procedure
}

A solution of $\mathbf{S 2}$ (1.0 equiv.) in anhydrous toluene $(10 \mathrm{~mL} / \mathrm{mmol})$ was cooled to $0{ }^{\circ} \mathrm{C}$ and treated dropwise with trichlorosilane (2.0 equiv.) and $\mathrm{NEt}_{3}$ (3.0 equiv.). Then the solution was stirred at $100{ }^{\circ} \mathrm{C}$ under $\mathrm{N}_{2}$ over $18 \mathrm{~h}$. The mixture was then cooled to $0{ }^{\circ} \mathrm{C}$ and quenched with $10 \% \mathrm{NaOH}$ solution. The filtrate was extracted with DCM quickly after filtration. The combined organic phase was dried over anhydrous $\mathrm{Na}_{2} \mathrm{SO}_{4}$. After filtration and concentration, the residue was purified by silica gel column chromatography to give the product for the next step.

A well-dried $100 \mathrm{~mL}$ round-bottomed flask was charged with the ester (1.0 equiv.) obtained above and $\mathrm{AlCl}_{3}$ (10.0 equiv.) under $\mathrm{N}_{2}$. The flask was cooled to $0{ }^{\circ} \mathrm{C}$ for $5 \mathrm{~min}$ and treated drop-wise with $\mathrm{Me}_{2} \mathrm{~S}(10$ $\mathrm{mL} / \mathrm{mmol}$ ). Then the mixture was stirred at $0{ }^{\circ} \mathrm{C}$ for $4 \mathrm{~h}$. The reaction mixture was added slowly to icecooled $1.0 \mathrm{M} \mathrm{HCl}(20 \mathrm{~mL} / \mathrm{mmol})$ at $0{ }^{\circ} \mathrm{C}$ with vigorous stirring. The aqueous phase was extracted with DCM quickly. The combined organic phases were dried over $\mathrm{Na}_{2} \mathrm{SO}_{4}$, filtered and concentrated under $\mathrm{N}_{2}$ to afford the crude $\mathbf{S} \mathbf{4}$ as a off-white solid. ${ }^{4}$

To a solution of crude S4 (2.5 equiv.), N,N-dimethyl-4-aminopyridine (1.0 equiv.) and 3(((ethylimino)methylene)amino)-N,N-dimethylpropan-1-amine (3.5 equiv.) in dry DCM $(10 \mathrm{~mL} / \mathrm{mmol})$, was added $(1 R, 2 R)$-trans-1,2-diamino cyclohexane (1.0 equiv.) under $\mathrm{N}_{2}$. The mixture was stirred at room 
temperature overnight. $10 \%$ hydrochloric acid was added, and the aqueous layer was extracted with DCM three times, washed with $10 \% \mathrm{NaOH}$, dried with $\mathrm{Na}_{2} \mathrm{SO}_{4}$ and concentrated in vacuo quickly, the residue was purified by silica gel column chromatography under $\mathrm{N}_{2}$ to give the product as a white solid. (silica gel was pretreated with a mixture of hexanes and $\mathrm{Et}_{3} \mathrm{~N}$, the elution was pre-cooled to $0{ }^{\circ} \mathrm{C}$ )

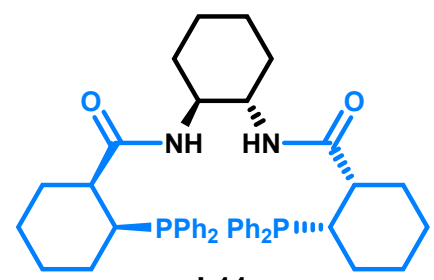

L11

$(1 S, 1 ' S, 2 S, 2 ' S)-\mathrm{N}, \mathrm{N}^{\prime}-((1 S, 2 S)$-cyclohexane-1,2-diyl)bis(2(diphenylphosphanyl)cyclohexane-1-carboxamide) (L11). With $\quad(1 S, 2 S)-2-$ (diphenylphosphanyl)cyclohexane-1-carboxylic acid $(624.7 \mathrm{mg}, 2 \mathrm{mmol})$ and $(1 S, 2 S)$-cyclohexane-1,2diamine (91.4 mg, $0.8 \mathrm{mmol}$ ) to yield $\mathbf{L 1 1}$ as a white solid (269.9 $\mathrm{mg}$, $48 \%$ yield for two steps).

$\underline{R}_{f} \quad 0.30\left(\mathrm{Hex} / \mathrm{EtOAc}=3: 1, \mathrm{KMnO}_{4}\right)$

Opt. Rot. $[\alpha]^{20}{ }_{\mathrm{D}}=-126.0(\mathrm{c}=0.1, \mathrm{MeOH})$

${ }^{1} \mathrm{H} \mathrm{NMR} \quad\left(400 \mathrm{MHz}, \mathrm{CDCl}_{3}\right)$

$\delta 7.55-7.45(\mathrm{~m}, 8 \mathrm{H}), 7.37-7.32(\mathrm{~m}, 6 \mathrm{H}), 7.31-7.26(\mathrm{~m}, 6 \mathrm{H}), 5.49(\mathrm{~d}, J=7.9 \mathrm{~Hz}$, 2H), $3.60-3.38(\mathrm{~m}, 2 \mathrm{H}), 2.48-2.39(\mathrm{~m}, 2 \mathrm{H}), 2.19-2.14(\mathrm{~m}, 2 \mathrm{H}), 2.13-2.01(\mathrm{~m}$, $4 \mathrm{H}), 1.84-1.55(\mathrm{~m}, 10 \mathrm{H}), 1.42-1.33(\mathrm{~m}, 4 \mathrm{H}), 1.29-1.21(\mathrm{~m}, 4 \mathrm{H})$.

${ }^{13} \mathrm{C} \mathrm{NMR} \quad\left(100 \mathrm{MHz}, \mathrm{CDCl}_{3}\right)$ $\delta 174.2,137.3,137.2,136.8,136.6,134.1,133.9,133.8,133.6,129.4,128.83,128.75$, $128.7,128.4,128.3,52.9,41.9,41.8,38.1,38.0,32.8,30.3,30.2,26.5,26.5,24.8$, 24.7, 24.5, 21.8.

${ }^{31} \mathrm{P} \mathrm{NMR} \quad\left(162 \mathrm{MHz}, \mathrm{CDCl}_{3}\right)$

$\delta-11.86$

HRMS (ESI) for: $\mathrm{C}_{44} \mathrm{H}_{52} \mathrm{~N}_{2} \mathrm{NaO}_{2} \mathrm{P}_{2}[\mathrm{M}+\mathrm{Na}]^{+}$: calcd 725.3402, found 725.3406.

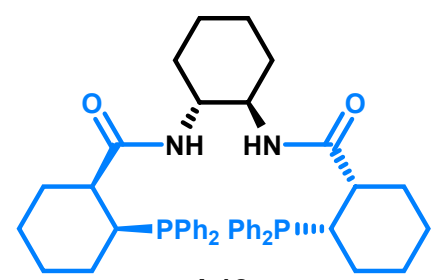

L12

$(1 S, 1 ' S, 2 S, 2 ' S)-\mathrm{N}, \mathrm{N}^{\prime}-((1 R, 2 R)$-cyclohexane-1,2-diyl)bis(2(diphenylphosphanyl)cyclohexane-1-carboxamide) (L12). With (1S,2S)-2(diphenylphosphanyl)cyclohexane-1-carboxylic acid $(624.7 \mathrm{mg}, 2 \mathrm{mmol})$ and $(1 R, 2 R)$-cyclohexane-1,2diamine (91.4 mg, $0.8 \mathrm{mmol}$ ) to yield $\mathbf{L 1 2}$ as a white solid (236.2 $\mathrm{mg}, 42 \%$ yield for two steps).

$\underline{R}_{f} \quad 0.30\left(\mathrm{Hex} / \mathrm{EtOAc}=3: 1, \mathrm{KMnO}_{4}\right)$

Opt. Rot. $\quad[\alpha]^{20}{ }_{\mathrm{D}}=-69.0(\mathrm{c}=0.1, \mathrm{MeOH})$

${ }^{1} \mathrm{H} \mathrm{NMR} \quad\left(400 \mathrm{MHz}, \mathrm{CDCl}_{3}\right)$

$\delta 7.57-7.36(\mathrm{~m}, 8 \mathrm{H}), 7.35-7.25(\mathrm{~m}, 6 \mathrm{H}), 7.24-7.07(\mathrm{~m}, 6 \mathrm{H}), 6.16(\mathrm{~s}, 2 \mathrm{H}), 2.53-$ $2.32(\mathrm{~m}, 2 \mathrm{H}), 2.84-2.60(\mathrm{~m}, 2 \mathrm{H}), 2.57-2.35(\mathrm{~m}, 2 \mathrm{H}), 2.12-2.03(\mathrm{~m}, 2 \mathrm{H}), 1.84-$ $1.56(\mathrm{~m}, 12 \mathrm{H}), 1.52-1.39(\mathrm{~m}, 4 \mathrm{H}), 1.33-1.23(\mathrm{~m}, 3 \mathrm{H}), 1.21-1.13(\mathrm{~m}, 2 \mathrm{H})$.

${ }^{13} \mathrm{C} \mathrm{NMR} \quad\left(100 \mathrm{MHz}, \mathrm{CDCl}_{3}\right)$

$\delta 174.1,137.9,137.7,137.2,137.1,134.0,133.9,133.8,133.6,128.7,128.6,128.3$, $128.2,53.7,44.2,43.9,37.2,37.1,32.0,29.0,28.8,26.6,26.5,25.4,25.3,24.6,23.2$. 
${ }^{31} \mathrm{P} \mathrm{NMR} \quad\left(162 \mathrm{MHz}, \mathrm{CDCl}_{3}\right)$

$\delta$-12.94.

HRMS (ESI) for: $\mathrm{C}_{44} \mathrm{H}_{52} \mathrm{~N}_{2} \mathrm{NaO}_{2} \mathrm{P}_{2}[\mathrm{M}+\mathrm{Na}]^{+}$: calcd 725.3402, found 725.3408 .

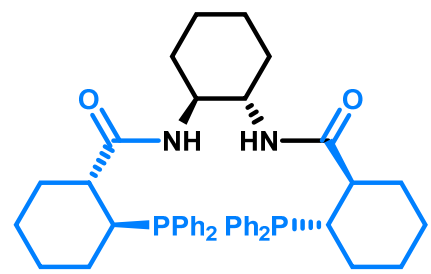

L13

$\left(1 R, 1^{\prime} R, 2 S, 2^{\prime} S\right)-\mathrm{N}, \mathrm{N}^{\prime}-((1 S, 2 S)$-cyclohexane-1,2-diyl)bis(2-

(diphenylphosphanyl)cyclohexane-1-carboxamide) (L13). With (1R,2S)-2-

(diphenylphosphanyl)cyclohexane-1-carboxylic acid $(624.7 \mathrm{mg}, 2 \mathrm{mmol})$ and $(1 S, 2 S)$-cyclohexane-1,2diamine (91.4 mg, $0.8 \mathrm{mmol}$ ) to yield $\mathbf{L 1 3}$ as a white solid (213.7 $\mathrm{mg}, 38 \%$ yield for two steps).

$\underline{R}_{f} \quad 0.30\left(\mathrm{Hex} / \mathrm{EtOAc}=3: 1, \mathrm{KMnO}_{4}\right)$

Opt. Rot. $\quad[\alpha]^{20}=145.4(\mathrm{c}=0.1, \mathrm{MeOH})$

$\underline{{ }^{1} \mathrm{H} \mathrm{NMR}} \quad\left(400 \mathrm{MHz}, \mathrm{CDCl}_{3}\right)$

$\delta 7.54-7.26(\mathrm{~m}, 14 \mathrm{H}), 7.19(\mathrm{~m}, 6 \mathrm{H}), 6.22(\mathrm{~s}, 2 \mathrm{H}), 3.70(\mathrm{~m}, 2 \mathrm{H}), 2.70(\mathrm{~m}, 2 \mathrm{H}), 2.13$ $(\mathrm{m}, 2 \mathrm{H}), 1.96-1.87(\mathrm{~m}, 2 \mathrm{H}), 1.80-1.51(\mathrm{~m}, 14 \mathrm{H}), 1.36-1.10(\mathrm{~m}, 8 \mathrm{H})$.

${ }^{13} \mathrm{C} \mathrm{NMR} \quad\left(100 \mathrm{MHz}, \mathrm{CDCl}_{3}\right)$

$\delta 175.6,136.8,136.7,136.1,136.0,135.1,134.9,132.3,132.1,129.0,128.4,128.3$, $128.2,128.1,127.7,53.8,47.2,47.0,35.3,35.1,32.5,29.9,29.8,26.6,25.1,24.8$, 24.7.

${ }^{31} \mathrm{P} \mathrm{NMR} \quad\left(162 \mathrm{MHz}, \mathrm{CDCl}_{3}\right)$ $\delta-4.93$

HRMS (ESI) for: $\mathrm{C}_{44} \mathrm{H}_{52} \mathrm{~N}_{2} \mathrm{NaO}_{2} \mathrm{P}_{2}[\mathrm{M}+\mathrm{Na}]^{+}$: calcd 725.3402, found 725.3416.

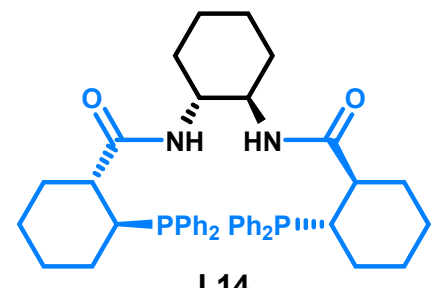

L14

$\left(1 R, 1^{\prime} R, 2 S, 2^{\prime} S\right)-\mathrm{N}, \mathrm{N}^{\prime}-((1 R, 2 R)$-cyclohexane-1,2-diyl)bis(2(diphenylphosphanyl) cyclohexane-1-carboxamide) (L14). With (1R,2S)-2(diphenylphosphanyl)cyclohexane-1-carboxylic acid $(624.7 \mathrm{mg}, 2 \mathrm{mmol})$ and $(1 R, 2 R)$-cyclohexane-1,2diamine (91.4 mg, $0.8 \mathrm{mmol}$ ) to yield $\mathbf{L 1 4}$ as a white solid (225.0 $\mathrm{mg}, 40 \%$ yield for two steps).

$\underline{R}_{f} \quad 0.30\left(\mathrm{Hex} / \mathrm{EtOAc}=3: 1, \mathrm{KMnO}_{4}\right)$

Opt. Rot. $[\alpha]^{20}=55.0(\mathrm{c}=0.1, \mathrm{MeOH})$

${ }^{1} \mathrm{H} \mathrm{NMR} \quad\left(400 \mathrm{MHz}, \mathrm{CDCl}_{3}\right)$

$\delta 7.56-7.40(\mathrm{~m}, 8 \mathrm{H}), 7.39-7.25(\mathrm{~m}, 12 \mathrm{H}), 6.08(\mathrm{~s}, 2 \mathrm{H}), 3.57-3.37(\mathrm{~m}, 2 \mathrm{H}), 2.72-$ $2.48(\mathrm{~m}, 2 \mathrm{H}), 2.02-1.83(\mathrm{~m}, 4 \mathrm{H}), 1.81-1.57(\mathrm{~m}, 12 \mathrm{H}), 1.37-0.80(\mathrm{~m}, 10 \mathrm{H})$.

${ }^{13} \mathrm{C} \mathrm{NMR} \quad\left(100 \mathrm{MHz}, \mathrm{CDCl}_{3}\right)$ $\delta 175.4,137.0,136.9,135.8,135.6,135.5,135.3,132.4,132.3,129.3,128.3,128.2$, $128.2,127.8,53.7,49.1,48.9,35.7,35.6,32.5,31.5,31.3,27.4,26.1,25.2,24.7$.

${ }^{31} \mathrm{P} \mathrm{NMR} \quad\left(162 \mathrm{MHz}, \mathrm{CDCl}_{3}\right)$ $\delta-1.42$

$\underline{\mathrm{HRMS}(\mathrm{ESI})}$ for: $\mathrm{C}_{44} \mathrm{H}_{52} \mathrm{~N}_{2} \mathrm{NaO}_{2} \mathrm{P}_{2}[\mathrm{M}+\mathrm{Na}]^{+}$: calcd 725.3402 , found 725.3403 . 


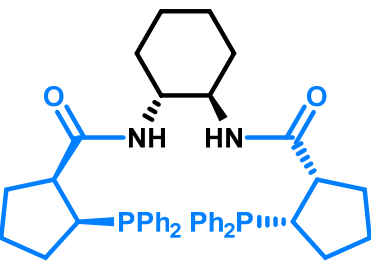

L21
$(1 S, 1 ' S, 2 S, 2 ' S)-\mathrm{N}, \mathrm{N}^{\prime}-((1 R, 2 R)$-cyclohexane-1,2-diyl)bis(2-

$\begin{array}{lcccc}\text { (diphenylphosphanyl) } & \text { cyclohexane-1-carboxamide) } & \text { (L21). With } & (1 S, 2 S)-2-\end{array}$ diamine (91.4 mg, $0.8 \mathrm{mmol}$ ) to yield $\mathbf{L 2 1}$ as a white solid (334.7 $\mathrm{mg}, 62 \%$ yield for two steps).

$\underline{R}_{f} 0.30\left(\mathrm{Hex} / \mathrm{EtOAc}=3: 1, \mathrm{KMnO}_{4}\right)$

Opt. Rot. $[\alpha]^{20}=-4.0(\mathrm{c}=0.1, \mathrm{MeOH})$

${ }^{1} \mathrm{H} \mathrm{NMR} \quad\left(500 \mathrm{MHz}, \mathrm{CDCl}_{3}\right)$

$\delta 7.81-7.71(\mathrm{~m}, 4 \mathrm{H}), 7.68-7.60(\mathrm{~m}, 4 \mathrm{H}), 7.25-7.10(\mathrm{~m}, 12 \mathrm{H}), 6.02(\mathrm{~d}, J=4.9 \mathrm{~Hz}$, $2 \mathrm{H}), 3.76-3.58(\mathrm{~m}, 2 \mathrm{H}), 2.97-2.85(\mathrm{~m}, 2 \mathrm{H}), 2.82-2.68(\mathrm{~m}, 2 \mathrm{H}), 2.45-2.32(\mathrm{~m}$, $2 \mathrm{H}), 2.14-1.98(\mathrm{~m}, 6 \mathrm{H}), 1.96-1.86(\mathrm{~m}, 2 \mathrm{H}), 1.76-1.62(\mathrm{~m}, 2 \mathrm{H}), 1.60-1.49(\mathrm{~m}$, $2 \mathrm{H}), 1.46-1.36(\mathrm{~m}, 2 \mathrm{H}), 1.09-0.87(\mathrm{~m}, 4 \mathrm{H})$.

${ }^{13} \mathrm{C} \mathrm{NMR} \quad\left(125 \mathrm{MHz}, \mathrm{CDCl}_{3}\right)$ $\delta 174.3,134.0,133.9,133.1,132.9,128.8,128.3,128.3,128.2,128.2,68.0,53.7$, $49.6,49.5,40.6,32.2,30.3,30.3,30.0,29.9,25.6,24.6,24.5,24.5$.

${ }^{31} \mathrm{P} \mathrm{NMR} \quad\left(202 \mathrm{MHz}, \mathrm{CDCl}_{3}\right)$ $\delta-13.86$.

HRMS (ESI) for: $\mathrm{C}_{44} \mathrm{H}_{48} \mathrm{~N}_{2} \mathrm{NaO}_{2} \mathrm{P}_{2}[\mathrm{M}+\mathrm{Na}]^{+}$: calcd 697.3089, found 697.3074.

\section{L7-L12: General Procedure}

A well-dried $100 \mathrm{~mL}$ round-bottomed flask was charged with $\mathrm{AlCl}_{3}$ (10.0 equiv.) under $\mathrm{N}_{2}$. The flask was cooled to $-10{ }^{\circ} \mathrm{C}$ for $5 \mathrm{~min}$ and treated drop-wise with $\mathrm{Me}_{2} \mathrm{~S}(8 \mathrm{~mL} / \mathrm{mmol})$. A solution of $\mathbf{S 3}(1.0$ equiv.) in $\mathrm{Me}_{2} \mathrm{~S}(2 \mathrm{~mL} / \mathrm{mmol})$ was added to the reaction mixture by injection pump through $30 \mathrm{~min}$, Then the mixture was stirred at $-10{ }^{\circ} \mathrm{C}$ for $20 \mathrm{~h}$. The reaction mixture was added slowly to ice-cooled $1.0 \mathrm{M}$ $\mathrm{HCl}(20 \mathrm{~mL} / \mathrm{mmol})$ at $0{ }^{\circ} \mathrm{C}$ with vigorous stirring. The organic phase was drawn off with a syringe and the aqueous phase was extracted with DCM under $\mathrm{N}_{2}$. The combined organic phases were dried over $\mathrm{Na}_{2} \mathrm{SO}_{4}$, filtered and concentrated under $\mathrm{N}_{2}$ to afford the crude acid as a off-white solid.

A well-dried $100 \mathrm{~mL}$ round-bottomed flask was charged with crude acid (2.5 equiv.), 3(((ethylimino)methylene)amino)-N,N-dimethylpropan-1-amine $\quad(3.5 \quad$ equiv.), $\quad \mathrm{N}, \mathrm{N}-$ dimethyl-4aminopyridine (1.0 equiv.) under $\mathrm{N}_{2}$. The flask was cooled to $0{ }^{\circ} \mathrm{C}$, treated with anhydrous DCM (10 $\mathrm{mL} / \mathrm{mmol})$ and a solution of $(1 R, 2 R)$-trans-1,2-diamino cyclohexane (1.0 equiv.) in DCM, then the mixture was stirred at $\mathrm{RT}$ for $24 \mathrm{~h}$. Ice-cooled $1.0 \mathrm{M} \mathrm{HCl}$ was added slowly to the reaction at $0{ }^{\circ} \mathrm{C}$ with vigorous stirring. The organic phase was drawn off with a syringe and the aqueous phase was extracted with DCM under $\mathrm{N}_{2}$. The combined organic phases were dried over $\mathrm{Na}_{2} \mathrm{SO}_{4}$, filtered and concentrated under $\mathrm{N}_{2}$ to afford the crude product as yellow solid. Purification of the residue by column chromatography on silica gel (pentane/diethyl ether/acetone) under $\mathrm{N}_{2}$ gave the product as a white solid. 


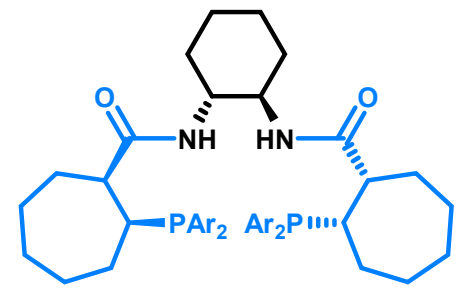

L22: $\mathrm{Ar}=\mathrm{C}_{6} \mathrm{H}_{5}$

$(1 S, 1 ' S, 2 S, 2 ' S)-\mathrm{N}, \mathrm{N}^{\prime}-((1 R, 2 R)$-cyclohexane-1,2-diyl)bis(2(diphenylphosphanyl)cycloheptane-1-carboxamide) (L22). With methyl (1S,2S)-2(diphenylphosphanyl)cycloheptane-1-carboxylate-borane complex S3a $(1.78 \mathrm{~g}, 5 \mathrm{mmol})$ and $(1 R, 2 R)$ cyclohexane-1,2-diamine (228.5 mg, $2.0 \mathrm{mmol}$ ) to yield $\mathbf{L 2 2}$ as a white solid (307.0 $\mathrm{mg}, 21 \%$ yield for two steps).

$\underline{R}_{f} \quad 0.30\left(\mathrm{Hex} / \mathrm{EtOAc}=3: 1, \mathrm{KMnO}_{4}\right)$

Opt. Rot. $\quad[\alpha]^{20}{ }_{\mathrm{D}}=-98.0(\mathrm{c}=0.1, \mathrm{MeOH})$

${ }^{1} \mathrm{H} \mathrm{NMR} \quad\left(500 \mathrm{MHz}, \mathrm{CDCl}_{3}\right)$

$\delta 7.58-7.42(\mathrm{~m}, 8 \mathrm{H}), 7.37-7.14(\mathrm{~m}, 12 \mathrm{H}), 6.40(\mathrm{~s}, 2 \mathrm{H}), 3.65-3.48(\mathrm{~m}, 2 \mathrm{H}), 2.87-$ $2.74(\mathrm{~m}, 2 \mathrm{H}), 2.61-2.49(\mathrm{~m}, 2 \mathrm{H}), 2.18-2.07(\mathrm{~m}, 2 \mathrm{H}), 2.04-1.95(\mathrm{~m}, 2 \mathrm{H}), 1.91-$ $1.80(\mathrm{~m}, 2 \mathrm{H}), 1.78-1.58(\mathrm{~m}, 8 \mathrm{H}), 1.53-1.43(\mathrm{~m}, 6 \mathrm{H}), 1.41-1.31(\mathrm{~m}, 4 \mathrm{H}), 1.30-$ $1.24(\mathrm{~m}, 2 \mathrm{H}), 1.15-1.02(\mathrm{~m}, 2 \mathrm{H})$.

${ }^{13} \mathrm{C} \mathrm{NMR} \quad\left(125 \mathrm{MHz}, \mathrm{CDCl}_{3}\right)$

$\delta 174.3,138.0,137.9,137.8,137.7,133.8,133.6,128.9,128.8,128.4,128.4,128.28$, 128.2, 53.9, 45.1, 45.0, 37.6, 37.4, 32.1, 31.8, 31.7, 28.5, 28.4, 28.0, 27.9, 27.8, 26.1, 24.6.

${ }^{31} \mathrm{P} \mathrm{NMR} \quad\left(202 \mathrm{MHz}, \mathrm{CDCl}_{3}\right)$ $\delta-8.39$.

HRMS (ESI) for: $\mathrm{C}_{46} \mathrm{H}_{56} \mathrm{~N}_{2} \mathrm{NaO}_{2} \mathrm{P}_{2}[\mathrm{M}+\mathrm{Na}]^{+}$: calcd 753.3715, found 753.3708.

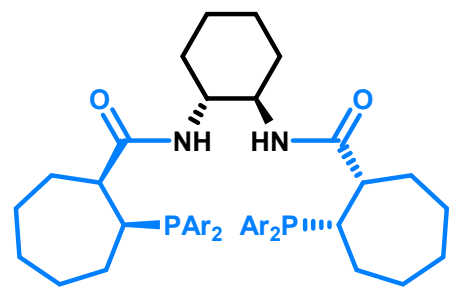

L15: $\mathrm{Ar}=4-\mathrm{CH}_{3}-\mathrm{C}_{6} \mathrm{H}_{4}$

$\left(1 S, 1^{\prime} S, 2 S, 2 ' S\right)-\mathrm{N}, \mathrm{N}^{\prime}-((1 R, 2 R)$-cyclohexane-1,2-diyl)bis(2-(di-ptolylphosphanyl) cycloheptane-1-carboxamide) (L15). With methyl (1S,2S)-2-(di-ptolylphosphanyl)cycloheptane-1-carboxylate-borane complex S3b (1.92 g, $5 \mathrm{mmol})$ and $(1 R, 2 R)-$ cyclohexane-1,2-diamine (228.5 mg, $2.0 \mathrm{mmol}$ ) to yield $\mathbf{L 1 5}$ as a white solid (440.7 $\mathrm{mg}, 28 \%$ yield for two steps).

$\underline{R}_{f} \quad 0.30\left(\mathrm{Hex} / \mathrm{EtOAc}=3: 1, \mathrm{KMnO}_{4}\right)$

Opt. Rot. $[\alpha]^{20}=-39.0(\mathrm{c}=0.1, \mathrm{MeOH})$

$\underline{{ }^{1} \mathrm{H} \mathrm{NMR}} \quad\left(500 \mathrm{MHz}, \mathrm{CDCl}_{3}\right)$

$\delta 7.40(\mathrm{t}, J=7.4 \mathrm{~Hz}, 8 \mathrm{H}), 7.07(\mathrm{dd}, J=26.3,7.8 \mathrm{~Hz}, 8 \mathrm{H}), 6.45(\mathrm{~s}, 2 \mathrm{H}), 3.67-3.50$ $(\mathrm{m}, 2 \mathrm{H}), 2.84-2.77(\mathrm{~m}, 2 \mathrm{H}), 2.60-2.50(\mathrm{~m}, 2 \mathrm{H}), 2.27(\mathrm{~d}, J=32.2 \mathrm{~Hz}, 12 \mathrm{H}), 2.19-$ $2.06(\mathrm{~m}, 2 \mathrm{H}), 1.96(\mathrm{~d}, J=12.9 \mathrm{~Hz}, 2 \mathrm{H}), 1.88-1.80(\mathrm{~m}, 2 \mathrm{H}), 1.78-1.56(\mathrm{~m}, 10 \mathrm{H})$, $1.53-1.41(\mathrm{~m}, 6 \mathrm{H}), 1.39-1.30(\mathrm{~m}, 4 \mathrm{H}), 1.15-1.01(\mathrm{~m}, 2 \mathrm{H})$.

${ }^{13} \mathrm{C} \mathrm{NMR} \quad\left(125 \mathrm{MHz}, \mathrm{CDCl}_{3}\right)$

$\delta 174.3,138.7,138.7,134.5,134.4,134.3,134.2,133.7,133.7,133.6,129.2,129.2$, $129.1,53.8,45.0,44.9,37.6,37.5,32.0,31.7,31.6,28.5,28.4,28.2,28.0,27.7,26.1$, 
24.6, 21.3.

${ }^{31} \mathrm{P}$ NMR $\quad\left(202 \mathrm{MHz}, \mathrm{CDCl}_{3}\right)$

$\delta-10.97$.

HRMS (ESI) for: $\mathrm{C}_{50} \mathrm{H}_{64} \mathrm{~N}_{2} \mathrm{NaO}_{2} \mathrm{P}_{2}[\mathrm{M}+\mathrm{Na}]^{+}$: calcd 809.4341, found 809.4331.

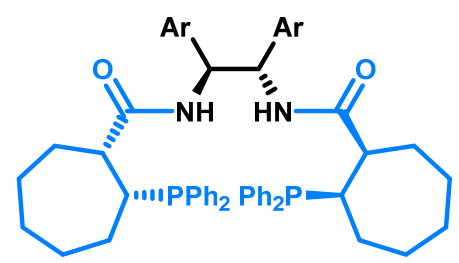

L16 (Ar = 1-naphthyl)

$\left(1 R, 1^{\prime} R, 2 R, 2^{\prime} R\right)-\mathrm{N}^{\prime} \mathrm{N}^{\prime}-((1 S, 2 S)-1,2-\mathrm{di}($ naphthalen-1-yl)ethane-1,2diyl)bis(2-(diphenylphosphanyl)cycloheptane-1-carboxamide) (L16). With methyl $(1 R, 2 R)-2$ (diphenylphosphanyl)cycloheptane-1-carboxylate-borane complex ent-S3b $(1.77 \mathrm{~g}, 5 \mathrm{mmol})$ and $(1 \mathrm{~S}, 2 \mathrm{~S})$ 1,2-di(naphthalen-1-yl)ethane-1,2-diamine (624.8 mg, $2.0 \mathrm{mmol}$ ) to yield $\mathbf{L 1 6}$ as a white solid $(510.7 \mathrm{mg}$, $28 \%$ yield for two steps).

$\underline{R}_{f} \quad 0.25\left(\mathrm{Hex} / \mathrm{EtOAc}=3: 1, \mathrm{KMnO}_{4}\right)$

Opt. Rot. $\quad[\alpha]^{20}=236.0(\mathrm{c}=0.1, \mathrm{MeOH})$

$\underline{{ }^{1} \mathrm{H} \mathrm{NMR}} \quad\left(500 \mathrm{MHz}, \mathrm{CDCl}_{3}\right)$

$\delta 7.98(\mathrm{~s}, 2 \mathrm{H}), 7.71-7.00(\mathrm{~m}, 32 \mathrm{H}), 6.41(\mathrm{~s}, 2 \mathrm{H}), 2.74-2.50(\mathrm{~m}, 4 \mathrm{H}), 2.22-2.07$ $(\mathrm{m}, 2 \mathrm{H}), 1.87-1.06(\mathrm{~m}, 20 \mathrm{H})$.

${ }^{13} \mathrm{C} \mathrm{NMR} \quad\left(125 \mathrm{MHz}, \mathrm{CDCl}_{3}\right)$ $\delta 137.5,133.7,133.6,133.6,133.5,128.9,128.8,128.3,128.3,126.1,125.4,125.1$, $45.1,45.0,37.8,37.7,32.5,28.6,28.6,27.8,27.4,27.2,25.9$.

${ }^{31} \mathrm{P} \mathrm{NMR} \quad\left(202 \mathrm{MHz}, \mathrm{CDCl}_{3}\right)$ -8.25 .

HRMS (ESI) for: $\mathrm{C}_{62} \mathrm{H}_{62} \mathrm{~N}_{2} \mathrm{NaO}_{2} \mathrm{P}_{2}[\mathrm{M}+\mathrm{Na}]^{+}$: calcd 951.1193, found 951.1172.

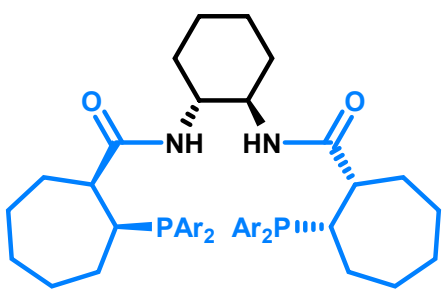

L23: $\mathrm{Ar}=4-\mathrm{Ph}-\mathrm{C}_{6} \mathrm{H}_{4}{ }^{-}$

$\left(1 S, 1^{\prime} S, 2 S, 2 ' S\right)-\mathrm{N}, \mathrm{N}^{\prime}-((1 R, 2 R)$-cyclohexane-1,2-diyl)bis(2-(di([1,1'biphenyl]-4-yl)phosphanyl) cycloheptane-1-carboxamide) (L23). With methyl (1S,2S)-2-(di([1,1'biphenyl]-4-yl)phosphanyl) cycloheptane-1-carboxylate-borane complex S3c $(1.5 \mathrm{~g}, 3 \mathrm{mmol})$ and $(1 R, 2 R)$-cyclohexane-1,2-diamine $(137.1 \mathrm{mg}, 1.2 \mathrm{mmol})$ to yield $\mathbf{L 2 3}$ as a white solid $(285.7 \mathrm{mg}, 23 \%$ yield for two steps).

$\underline{R}_{f} 0.30\left(\mathrm{Hex} / \mathrm{EtOAc}=3: 1, \mathrm{KMnO}_{4}\right)$

Opt. Rot. $\quad[\alpha]^{20}{ }_{\mathrm{D}}=-34.0(\mathrm{c}=0.1, \mathrm{MeOH})$

${ }^{1} \mathrm{H} \mathrm{NMR} \quad\left(500 \mathrm{MHz}, \mathrm{CDCl}_{3}\right)$

$\delta 7.64-7.56(\mathrm{~m}, 8 \mathrm{H}), 7.56-7.43(\mathrm{~m}, 16 \mathrm{H}), 7.44-7.27(\mathrm{~m}, 12 \mathrm{H}), 6.37(\mathrm{~s}, 2 \mathrm{H}), 3.61$ (s, 2H), $2.96-2.84(\mathrm{~m}, 2 \mathrm{H}), 2.73-2.63(\mathrm{~m}, 2 \mathrm{H}), 2.23-2.13(\mathrm{~m}, 2 \mathrm{H}), 2.04-1.92(\mathrm{~m}$, $4 \mathrm{H}), 1.87-1.73(\mathrm{~m}, 4 \mathrm{H}), 1.72-1.61(\mathrm{~m}, 4 \mathrm{H}), 1.57-1.52(\mathrm{~m}, 4 \mathrm{H}), 1.48-1.37(\mathrm{~m}$, $4 \mathrm{H}), 1.34-1.18(\mathrm{~m}, 4 \mathrm{H}), 1.14-1.01(\mathrm{~m}, 2 \mathrm{H})$.

${ }^{13} \mathrm{C} \mathrm{NMR} \quad\left(125 \mathrm{MHz}, \mathrm{CDCl}_{3}\right)$

$\delta 174.4,141.6,141.5,140.5,140.4,136.9,136.7,136.6,134.3,134.2,134.0,128.8$, 
$127.5,127.4,127.1,127.1,127.0,126.9,53.9,45.3,45.1,37.8,37.7,32.2,31.8,31.7$, 28.7, 28.6, 28.2, 28.1, 27.9, 26.0, 24.6.

${ }^{31} \mathrm{P} \mathrm{NMR} \quad\left(202 \mathrm{MHz}, \mathrm{CDCl}_{3}\right)$

$\delta-10.46$.

HRMS (ESI) for: $\mathrm{C}_{70} \mathrm{H}_{72} \mathrm{~N}_{2} \mathrm{NaO}_{2} \mathrm{P}_{2}[\mathrm{M}+\mathrm{Na}]^{+}$: calcd 1057.4967, found 1057.4963.

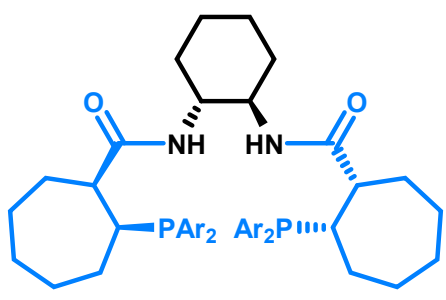

L24: $\mathrm{Ar}=4-\mathrm{F}-\mathrm{C}_{6} \mathrm{H}_{4}-$

$(1 S, 1 ' S, 2 S, 2 ' S)-\mathrm{N}, \mathrm{N}^{\prime}-((1 R, 2 R)$-cyclohexane-1,2-diyl)bis(2-(bis(4-

fluorophenyl)phosphanyl) cycloheptane-1-carboxamide) (L24). With methyl (1S,2S)-2-(bis(4fluorophenyl)phosphanyl) cycloheptane-1-carboxylate-borane complex S3d (1.5 g, $4 \mathrm{mmol})$ and $(1 R, 2 R)$ cyclohexane-1,2-diamine (182.8 $\mathrm{mg}, 1.6 \mathrm{mmol})$ to yield $\mathbf{L 2 4}$ as a white solid $(359.7 .7 \mathrm{mg}, 28 \%$ yield for two steps).

$\underline{R}_{f} \quad 0.30\left(\mathrm{Hex} / \mathrm{EtOAc}=3: 1, \mathrm{KMnO}_{4}\right)$

Opt. Rot. $[\alpha]_{\mathrm{D}}^{20}=-42.0(\mathrm{c}=0.1, \mathrm{MeOH})$

${ }^{1} \mathrm{H} \mathrm{NMR} \quad\left(500 \mathrm{MHz}, \mathrm{CDCl}_{3}\right)$

$\delta 7.52-7.38(\mathrm{~m}, 8 \mathrm{H}), 7.07-6.87(\mathrm{~m}, 8 \mathrm{H}), 6.23(\mathrm{~s}, 2 \mathrm{H}), 3.53(\mathrm{~s}, 2 \mathrm{H}), 2.77-2.66(\mathrm{~m}$, $2 \mathrm{H}), 2.57-2.47(\mathrm{~m}, 2 \mathrm{H}), 2.14-2.03(\mathrm{~m}, 2 \mathrm{H}), 2.10-1.93(\mathrm{~m}, 2 \mathrm{H}), 1.92-1.81(\mathrm{~m}$, 2H), $1.79-1.59(\mathrm{~m}, 8 \mathrm{H}), 1.55-1.48(\mathrm{~m}, 4 \mathrm{H}), 1.37-1.23(\mathrm{~m}, 8 \mathrm{H}), 1.10-0.99(\mathrm{~m}$, $2 \mathrm{H})$.

${ }^{13} \mathrm{C} \mathrm{NMR} \quad\left(125 \mathrm{MHz}, \mathrm{CDCl}_{3}\right)$

$\delta 174.3,164.4,162.4,135.7,135.6,135.5,135.4,135.4,135.3,135.2,135.2,133.3$, 133.3, 133.2, 133.2, 133.1, 133.1, 115.8, 115.7, 115.7, 115.6, 115.5, 115.4, 54.0, 45.2, $45.1,38.2,38.1,32.2,31.6,31.5,28.7,28.0,28.0,27.9,27.7,25.8,24.5$.

${ }^{31} \mathrm{P} \mathrm{NMR} \quad\left(202 \mathrm{MHz}, \mathrm{CDCl}_{3}\right)$

$\delta-11.81$.

HRMS (ESI) for: $\mathrm{C}_{46} \mathrm{H}_{52} \mathrm{~F}_{2} \mathrm{~N}_{2} \mathrm{NaO}_{2} \mathrm{P}_{2}[\mathrm{M}+\mathrm{Na}]^{+}$: calcd 825.3338, found 825.3341.

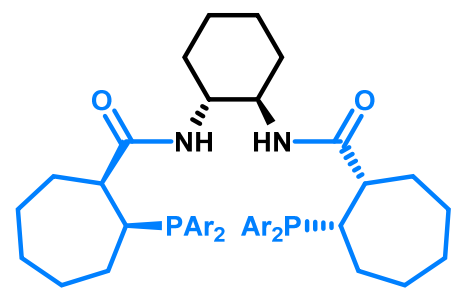

L25: $\mathrm{Ar}=3,5-\left(\mathrm{CH}_{3}\right)_{2}-\mathrm{C}_{6} \mathrm{H}_{3}-$

$\left(1 S, 1^{\prime} S, 2 S, 2 ' S\right)-\mathrm{N}, \mathrm{N}^{\prime}-((1 R, 2 R)$-cyclohexane-1,2-diyl)bis(2-(bis(3,5dimethylphenyl) phosphanyl)cycloheptane-1-carboxamide) (L25). With methyl (1S,2S)-2-(bis(3,5dimethylphenyl)phosphanyl) cycloheptane-1-carboxylate-borane complex S3e $(1.2 \mathrm{~g}, 3 \mathrm{mmol})$ and $(1 R, 2 R)$-cyclohexane-1,2-diamine (137.1 $\mathrm{mg}, 1.2 \mathrm{mmol}$ ) to yield $\mathbf{L 2 5}$ as a white solid (263.0 $\mathrm{mg}, 26 \%$ yield for two steps).

$$
\underline{R}_{f} \quad 0.30\left(\mathrm{Hex} / \mathrm{EtOAc}=3: 1, \mathrm{KMnO}_{4}\right)
$$

Opt. Rot. $[\alpha]^{20}=-46.6(\mathrm{c}=0.1, \mathrm{MeOH})$

$\underline{{ }^{1} \mathrm{H} \mathrm{NMR}} \quad\left(500 \mathrm{MHz}, \mathrm{CDCl}_{3}\right)$

$\delta 7.13(\mathrm{~d}, J=7.9 \mathrm{~Hz}, 8 \mathrm{H}), 6.90(\mathrm{~d}, J=18.4 \mathrm{~Hz}, 4 \mathrm{H}), 6.61(\mathrm{t}, J=5.5 \mathrm{~Hz}, 2 \mathrm{H}), 3.65-$ $3.52(\mathrm{~m}, 2 \mathrm{H}), 2.88-2.78(\mathrm{~m}, 2 \mathrm{H}), 2.60-2.48(\mathrm{~m}, 2 \mathrm{H}), 2.35-2.19(\mathrm{~m}, 24 \mathrm{H}), 2.19-$ 
$2.09(\mathrm{~m}, 2 \mathrm{H}), 1.98(\mathrm{~d}, J=12.8 \mathrm{~Hz}, 2 \mathrm{H}), 1.77-1.62(\mathrm{~m}, 8 \mathrm{H}), 1.47-1.25(\mathrm{~m}, 14 \mathrm{H})$, $1.16-1.05(\mathrm{~m}, 2 \mathrm{H})$.

${ }^{13} \mathrm{C} \mathrm{NMR} \quad\left(125 \mathrm{MHz}, \mathrm{CDCl}_{3}\right)$

$\delta 174.2,137.7,137.6,137.5,137.5,137.3,137.2,137.2,137.0,131.5,131.5,131.3$, $131.3,130.7,130.7,53.8,44.8,44.7,37.1,37.0,34.1,31.9,31.7,31.6,28.3,28.3$, 28.1, 27.4, 26.3, 24.5, 22.4, 21.3, 14.1 .

${ }^{31} \mathrm{P} \mathrm{NMR} \quad\left(202 \mathrm{MHz}, \mathrm{CDCl}_{3}\right)$

$\delta-8.52$.

HRMS (ESI) for: $\mathrm{C}_{54} \mathrm{H}_{72} \mathrm{~N}_{2} \mathrm{NaO}_{2} \mathrm{P}_{2}[\mathrm{M}+\mathrm{Na}]^{+}$: calcd 865.4967, found 865.4978.

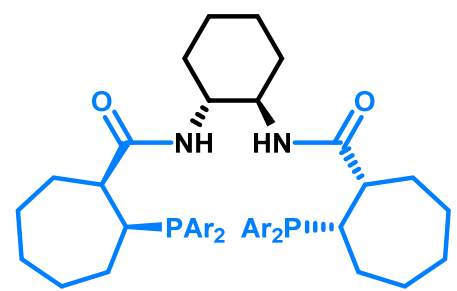

L26: $\mathrm{Ar}=2$-naphthyl
$(1 S, 1 ' S, 2 S, 2 ' S)-\mathrm{N}, \mathrm{N}^{\prime}-((1 R, 2 R)$-cyclohexane-1,2-diyl)bis(2(di(naphthalen-2-yl)phosphanyl) cycloheptane-1-carboxamide) (L26). With methyl (1S,2S)-2(di(naphthalen-2-yl)phosphanyl) cycloheptane-1-carboxylate-borane complex S3f $(1.4 \mathrm{~g}, 3 \mathrm{mmol})$ and (1R,2R)-cyclohexane-1,2-diamine (137.1 $\mathrm{mg}, 1.2 \mathrm{mmol})$ to yield $\mathbf{L 2 6}$ as a white solid (279.3 $\mathrm{mg}, 25 \%$ yield for two steps).

$$
\underline{R}_{f} \quad 0.25\left(\mathrm{Hex} / \mathrm{EtOAc}=3: 1, \mathrm{KMnO}_{4}\right)
$$

Opt. Rot. $[\alpha]^{20}=-80.0(\mathrm{c}=0.1, \mathrm{MeOH})$

$\underline{{ }^{1} \mathrm{H} \mathrm{NMR}} \quad\left(500 \mathrm{MHz}, \mathrm{CDCl}_{3}\right)$

$\delta 8.07(\mathrm{~d}, J=9.2 \mathrm{~Hz}, 4 \mathrm{H}), 7.83-7.69(\mathrm{~m}, 6 \mathrm{H}), 7.67-7.55(\mathrm{~m}, 6 \mathrm{H}), 7.54-7.41(\mathrm{~m}$, $8 \mathrm{H}), 7.36(\mathrm{~d}, J=9.6 \mathrm{~Hz}, 4 \mathrm{H}), 6.24(\mathrm{~s}, 2 \mathrm{H}), 3.573 .49(\mathrm{~m}, 2 \mathrm{H}), 3.12-3.03(\mathrm{~m}, 2 \mathrm{H})$, $2.73-2.60(\mathrm{~m}, 2 \mathrm{H}), 2.21-2,10(\mathrm{~m}, 2 \mathrm{H}), 2.08-1.96(\mathrm{~m}, 2 \mathrm{H}), 1.84-1.75(\mathrm{~m}, 6 \mathrm{H})$, $1.71-1.63(\mathrm{~m}, 2 \mathrm{H}), 1.59-1.31(\mathrm{~m}, 14 \mathrm{H}), 1.17-1.07(\mathrm{~m}, 2 \mathrm{H})$.

${ }^{13} \mathrm{C} \mathrm{NMR} \quad\left(125 \mathrm{MHz}, \mathrm{CDCl}_{3}\right)$

$\delta 174.6,135.5,135.4,135.3,135.1,135.0,134.8,134.3,134.1,133.4,133.2,133.1$, $133.1,133.0,129.7,129.6,129.5,129.4,128.2,127.9,127.8,127.7,127.7,127.6$, $127.5,126.6,126.5,126.1,53.9,45.6,45.5,37.6,37.5,32.0,31.6,31.6,28.7,28.6$, $28.4,28.2,28.0,26.0,24.5$.

${ }^{31} \mathrm{P}$ NMR $\quad\left(202 \mathrm{MHz}, \mathrm{CDCl}_{3}\right)$ $\delta-7.97$.

HRMS (ESI) for: $\mathrm{C}_{62} \mathrm{H}_{56} \mathrm{~N}_{2} \mathrm{NaO}_{2} \mathrm{P}_{2}[\mathrm{M}+\mathrm{Na}]^{+}$: calcd 945.3715, found 945.3708.

3.2 Preparation of Substrates

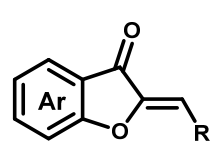

S6

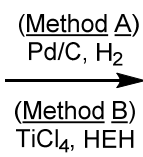

$\frac{(\text { Method }}{\mathrm{TiCl}_{4}, \mathrm{HEH}}$

s7

Scheme S3. Preparation of Substrates

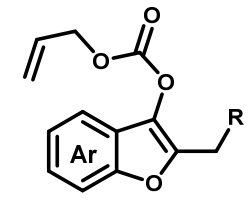

12

S7: General Procedure 
Method A. A solution of the condensation product (1.0 equiv.) in $\mathrm{MeOH}$ and dioxane $(10 \mathrm{~mL} / \mathrm{mmol}$, MeOH:dioxane $=5: 1$ ) was added $10 \% \mathrm{Pd} / \mathrm{C}$, then the reaction mixture was charged with $\mathrm{H}_{2}$ three times and stirred at RT for 1-18 h. The reaction mixture was filtered, concentrated and subjected to the purification by column chromatography on silica gel to afford the desired product $\mathbf{S}$.

Method B. A solution of the condensation product (1.0 equiv.) and hantzsch ester (1.5 equiv.) in THF $(10 \mathrm{~mL} / \mathrm{mmol})$ was added $\mathrm{TiCl}_{4}(1.2$ equiv., $1 \mathrm{M}$ in DCM$)$ at $0{ }^{\circ} \mathrm{C}$ slowly, then the mixture was stirred at that temperature for $2 \mathrm{~h}$. The reaction mixture was directly concentrated and subjected to the purification by column chromatography on silica gel to afford the desired product $\mathbf{S} 7$.

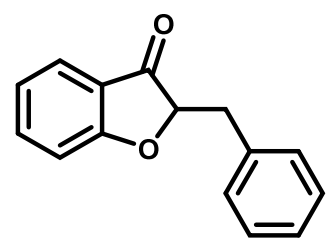

S7a 2-Benzylbenzofuran-3(2H)-one (S7a). Using Method A to afford S7a as a white solid (2.8 g, 58\% yield for two steps).

$\underline{R}_{f} \quad 0.35\left(\mathrm{Hex} / \mathrm{EtOAc}=20: 1, \mathrm{KMnO}_{4}\right)$

${ }^{1} \mathrm{H} \mathrm{NMR} \quad\left(400 \mathrm{MHz}, \mathrm{CDCl}_{3}\right)$ $\delta 7.69-7.43(\mathrm{~m}, 2 \mathrm{H}), 7.37-7.20(\mathrm{~m}, 5 \mathrm{H}), 7.14-6.95(\mathrm{~m}, 2 \mathrm{H}), 4.83-4.75(\mathrm{~m}, 1 \mathrm{H})$, $3.38(\mathrm{dd}, J=14.7,3.6 \mathrm{~Hz}, 1 \mathrm{H}), 3.00(\mathrm{dd}, J=14.7,8.6 \mathrm{~Hz}, 1 \mathrm{H})$.

${ }^{13} \mathrm{C} \mathrm{NMR} \quad\left(100 \mathrm{MHz}, \mathrm{CDCl}_{3}\right)$

$\delta 201.2,172.8,138.2,136.2,129.5,128.6,127.0,124.4,122.0,121.1,113.7,85.9,37.5$.

$\underline{\mathrm{HRMS}(\mathrm{ESI})}$ for: $\mathrm{C}_{15} \mathrm{H}_{13} \mathrm{O}_{2}[\mathrm{M}+\mathrm{H}]^{+}$: calcd 225.0916, found 225.0922 .<smiles>CC1Oc2ccccc2C1=O</smiles>

S7c

Methyl 4-((3-oxo-2,3-dihydrobenzofuran-2-yl)methyl)benzoate (S7c). Using Method B to afford S7c as a white solid ( $3.0 \mathrm{~g}, 54 \%$ yield for two steps).

$\underline{R}_{f} \quad 0.25\left(\mathrm{Hex} / \mathrm{EtOAc}=20: 1, \mathrm{KMnO}_{4}\right)$

${ }^{1} \mathrm{H} \mathrm{NMR} \quad\left(400 \mathrm{MHz}, \mathrm{CDCl}_{3}\right)$

$\delta 8.02-7.82(\mathrm{~m}, 2 \mathrm{H}), 7.71-7.48(\mathrm{~m}, 2 \mathrm{H}), 7.43-7.27(\mathrm{~m}, 2 \mathrm{H}), 7.15-6.95(\mathrm{~m}, 2 \mathrm{H})$,

$4.79(\mathrm{dd}, J=8.1,3.8 \mathrm{~Hz}, 1 \mathrm{H}), 3.89(\mathrm{~s}, 3 \mathrm{H}), 3.41(\mathrm{dd}, J=14.7,3.8 \mathrm{~Hz}, 1 \mathrm{H}), 3.07$ (dd, $J$

$=14.6,8.1 \mathrm{~Hz}, 1 \mathrm{H})$.

${ }^{13} \mathrm{C} \mathrm{NMR} \quad\left(100 \mathrm{MHz}, \mathrm{CDCl}_{3}\right)$

$\delta 200.8,172.7,167.0,141.3,138.3,129.8,129.6,129.0,124.4,122.2,120.9,113.6$, $85.2,52.1,37.4$.

HRMS (ESI) for: $\mathrm{C}_{17} \mathrm{H}_{15} \mathrm{O}_{4}[\mathrm{M}+\mathrm{H}]^{+}$: calcd 283.0970, found 283.0982 . 


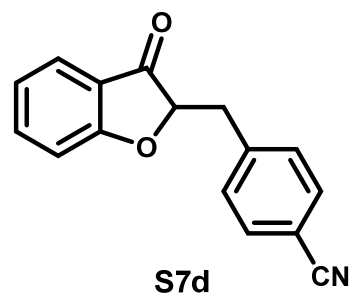

4-((3-Oxo-2,3-dihydrobenzofuran-2-yl)methyl)benzonitrile (S7d). Using Method B to yield $\mathbf{S 7 d}$ as a white solid (1.6 g, 32\% yield for two steps).

$\underline{R}_{f} \quad 0.30\left(\mathrm{Hex} / \mathrm{EtOAc}=10: 1, \mathrm{KMnO}_{4}\right)$

$\underline{{ }^{1} \mathrm{H} \mathrm{NMR}} \quad\left(500 \mathrm{MHz}, \mathrm{CDCl}_{3}\right)$

$\delta 7.70-7.52(\mathrm{~m}, 4 \mathrm{H}), 7.41(\mathrm{~d}, J=8.2 \mathrm{~Hz}, 2 \mathrm{H}), 7.17-6.99(\mathrm{~m}, 2 \mathrm{H}), 4.78(\mathrm{dd}, J=8.0$, $3.9 \mathrm{~Hz}, 1 \mathrm{H}), 3.41(\mathrm{dd}, J=14.7,3.9 \mathrm{~Hz}, 1 \mathrm{H}), 3.10(\mathrm{dd}, J=14.7,8.0 \mathrm{~Hz}, 1 \mathrm{H})$.

${ }^{13} \mathrm{C} \mathrm{NMR} \quad\left(125 \mathrm{MHz}, \mathrm{CDCl}_{3}\right)$

$\delta 200.3,172.5,141.4,138.4,132.2,130.3,124.4,122.3,120.8,118.8,113.5,111.0$, 84.6, 37.3.

HRMS (ESI) for: $\mathrm{C}_{16} \mathrm{H}_{12} \mathrm{NO}_{2}[\mathrm{M}+\mathrm{H}]^{+}$: calcd 250.0868, found 250.0887 .<smiles>CC1Oc2ccccc2C1=O</smiles>

s7e

2-(4-(Trifluoromethyl)benzyl)benzofuran-3(2H)-one (S7e). Using Method B to yield S7e as a white solid (3.3 g, 56\% yield for two steps).

$\underline{R}_{f} \quad 0.35\left(\mathrm{Hex} / \mathrm{EtOAc}=20: 1, \mathrm{KMnO}_{4}\right)$

${ }^{1} \mathrm{H} \mathrm{NMR} \quad\left(500 \mathrm{MHz}, \mathrm{CDCl}_{3}\right)$

$\delta 7.68-7.47(\mathrm{~m}, 4 \mathrm{H}), 7.42(\mathrm{~d}, J=8.0 \mathrm{~Hz}, 2 \mathrm{H}), 7.21-6.96(\mathrm{~m}, 2 \mathrm{H}), 4.78(\mathrm{~d}, J=12.0$

$\mathrm{Hz}, 1 \mathrm{H}), 3.41(\mathrm{dd}, J=14.7,3.6 \mathrm{~Hz}, 1 \mathrm{H}), 3.13-3.00(\mathrm{~m}, 1 \mathrm{H})$.

${ }^{13} \mathrm{C} \mathrm{NMR} \quad\left(125 \mathrm{MHz}, \mathrm{CDCl}_{3}\right)$

$\delta 200.6,172.6,140.1,138.3,131.1,129.8,129.5,129.2,125.4,124.4,123.1,122.2$, $120.8,113.5,85.1,37.1$.

${ }^{19} \mathrm{~F} \mathrm{NMR} \quad\left(470 \mathrm{MHz}, \mathrm{CDCl}_{3}\right)$

$\delta-62.52$.

HRMS (ESI) for: $\mathrm{C}_{16} \mathrm{H}_{12} \mathrm{~F}_{3} \mathrm{O}_{2}[\mathrm{M}+\mathrm{H}]^{+}$: calcd 293.0789, found 293.0784 .<smiles>O=C1c2ccccc2OC1Cc1ccc(F)cc1</smiles>

2-(4-Fluorobenzyl)benzofuran-3(2H)-one (S7f). Using Method A to yield S7f as a white solid (2.3 g, $48 \%$ yield for two steps).

$\underline{R}_{f} \quad 0.40\left(\mathrm{Hex} / \mathrm{EtOAc}=20: 1, \mathrm{KMnO}_{4}\right)$

${ }^{1} \mathrm{H} \mathrm{NMR} \quad\left(400 \mathrm{MHz}, \mathrm{CDCl}_{3}\right)$

$\delta 7.64-7.49(\mathrm{~m}, 2 \mathrm{H}), 7.32-7.16(\mathrm{~m}, 2 \mathrm{H}), 7.14-6.86(\mathrm{~m}, 4 \mathrm{H}), 4.73(\mathrm{dd}, J=8.0,3.8$ $\mathrm{Hz}, 1 \mathrm{H}), 3.31$ (dd, $J=14.7,3.8 \mathrm{~Hz}, 1 \mathrm{H}), 2.99$ (dd, $J=14.7,8.0 \mathrm{~Hz}, 1 \mathrm{H})$. 
${ }^{13} \mathrm{C} \mathrm{NMR} \quad\left(100 \mathrm{MHz}, \mathrm{CDCl}_{3}\right)$

$\delta$ 201.03, 172.70, 163.22, 160.78, 138.25, 131.59, 131.55, 131.11, 131.03, 124.36, $122.07,121.05,115.45,115.24,113.55,85.63,36.58$.

${ }^{19} \mathrm{~F} \mathrm{NMR} \quad\left(470 \mathrm{MHz}, \mathrm{CDCl}_{3}\right)$

$\delta-115.98$.

$\underline{\text { HRMS (ESI) }}$ for: $\mathrm{C}_{15} \mathrm{H}_{12} \mathrm{FO}_{2}[\mathrm{M}+\mathrm{H}]^{+}$: calcd 243.0821, found 243.0810.<smiles>CC1Oc2ccccc2C1=O</smiles>

S7g

2-(4-Chlorobenzyl)benzofuran-3(2H)-one (S7g). Using Method A to yield S7g as a white solid ( $3.2 \mathrm{~g}, 61 \%$ yield for two steps).

$\underline{R}_{f} \quad 0.30\left(\mathrm{Hex} / \mathrm{EtOAc}=20: 1, \mathrm{KMnO}_{4}\right)$

${ }^{1} \mathrm{H} \mathrm{NMR} \quad\left(500 \mathrm{MHz}, \mathrm{CDCl}_{3}\right)$

$\delta 7.59-7.41(\mathrm{~m}, 2 \mathrm{H}), 7.17-7.07(\mathrm{~m}, 4 \mathrm{H}), 7.02-6.83(\mathrm{~m}, 2 \mathrm{H}), 4.64(\mathrm{dd}, J=8.1,3.8$ $\mathrm{Hz}, 1 \mathrm{H}), 3.22(\mathrm{dd}, J=14.7,3.8 \mathrm{~Hz}, 1 \mathrm{H}), 2.89(\mathrm{dd}, J=14.7,8.1 \mathrm{~Hz}, 1 \mathrm{H})$.

${ }^{13} \mathrm{C} \mathrm{NMR} \quad\left(125 \mathrm{MHz}, \mathrm{CDCl}_{3}\right)$

$\delta 200.6,172.5,138.1,134.2,132.7,130.7,128.5,124.2,121.9,120.8,113.4,85.2,36.5$.

HRMS (ESI) for: $\mathrm{C}_{15} \mathrm{H}_{12} \mathrm{ClO}_{2}[\mathrm{M}+\mathrm{H}]^{+}$: calcd 259.0526, found 259.0531 .<smiles>COc1ccc(CC2Oc3ccccc3C2=O)cc1</smiles>

S7h as a white solid (2.6 g, 52\% yield for two steps).

$\underline{R}_{f} \quad 0.30\left(\mathrm{Hex} / \mathrm{EtOAc}=20: 1, \mathrm{KMnO}_{4}\right)$

${ }^{1} \mathrm{H} \mathrm{NMR} \quad\left(400 \mathrm{MHz}, \mathrm{CDCl}_{3}\right)$

$\delta 7.66-7.48(\mathrm{~m}, 2 \mathrm{H}), 7.20(\mathrm{~d}, J=8.6 \mathrm{~Hz}, 2 \mathrm{H}), 7.11-6.97(\mathrm{~m}, 2 \mathrm{H}), 6.90-6.69(\mathrm{~m}$, $2 \mathrm{H}), 4.83-4.65(\mathrm{~m}, 1 \mathrm{H}), 3.75(\mathrm{~s}, 3 \mathrm{H}), 3.40-3.23(\mathrm{~m}, 1 \mathrm{H}), 2.94(\mathrm{~d}, J=6.4 \mathrm{~Hz}, 1 \mathrm{H})$.

${ }^{13} \mathrm{C} \mathrm{NMR} \quad\left(100 \mathrm{MHz}, \mathrm{CDCl}_{3}\right)$

$\delta 201.3,172.8,158.6,138.1,130.5,128.0,124.4,122.0,121.1,113.9,113.6,86.1,55.3$, 36.6.

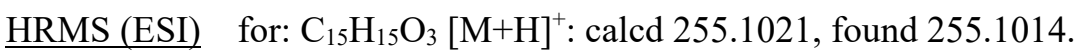

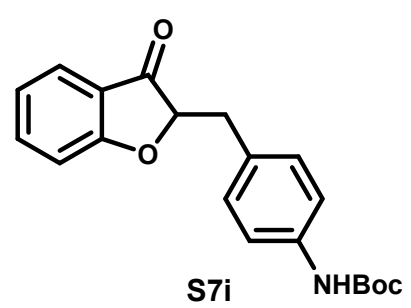

tert-Butyl

(4-((3-oxo-2,3-dihydrobenzofuran-2-

yl)methyl)phenyl)carbamate (S7i). Using Method B to yield S7i as a white solid (3.4 g, 50\% yield for two steps).

$$
\underline{R}_{f} \quad 0.25\left(\mathrm{Hex} / \mathrm{EtOAc}=15: 1, \mathrm{KMnO}_{4}\right)
$$


豆 NMR $\quad\left(500 \mathrm{MHz}, \mathrm{CDCl}_{3}\right)$

$\delta 7.59-7.38(\mathrm{~m}, 2 \mathrm{H}), 7.25-7.08(\mathrm{~m}, 4 \mathrm{H}), 7.06-6.82(\mathrm{~m}, 2 \mathrm{H}), 6.44(\mathrm{~s}, 1 \mathrm{H}), 4.66(\mathrm{dd}$, $J=8.2,3.8 \mathrm{~Hz}, 1 \mathrm{H}), 3.24(\mathrm{~d}, J=3.8 \mathrm{~Hz}, 1 \mathrm{H}), 2.88(\mathrm{dd}, J=14.8,8.2 \mathrm{~Hz}, 1 \mathrm{H}), 1.42(\mathrm{~s}$, 9H).

${ }^{13} \mathrm{C} \mathrm{NMR} \quad\left(125 \mathrm{MHz}, \mathrm{CDCl}_{3}\right)$

$\delta 201.2,172.7,138.1,137.2,130.4,130.0,124.3,121.9,121.0,118.6,113.5,85.8,36.7$, 28.4 .

$\underline{\text { HRMS (ESI) }}$ for: $\mathrm{C}_{20} \mathrm{H}_{21} \mathrm{NNaO}_{4}[\mathrm{M}+\mathrm{Na}]^{+}$: calcd 362.1368, found 362.1351 .

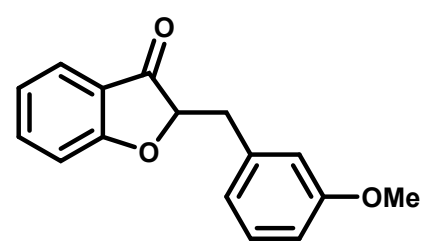

S7j

2-(3-Methoxybenzyl)benzofuran-3(2H)-one (S7j). Using Method B to yield $\mathbf{S} 7 \mathbf{j}$ as a white solid (2.2 $\mathrm{g}, 43 \%$ yield for two steps).

$\underline{R}_{f} \quad 0.30\left(\mathrm{Hex} / \mathrm{EtOAc}=20: 1, \mathrm{KMnO}_{4}\right)$

${ }^{1} \mathrm{H} \mathrm{NMR} \quad\left(400 \mathrm{MHz}, \mathrm{CDCl}_{3}\right)$

$\delta 7.67-7.53(\mathrm{~m}, 2 \mathrm{H}), 7.20(\mathrm{t}, J=7.9 \mathrm{~Hz}, 1 \mathrm{H}), 7.12-7.00(\mathrm{~m}, 2 \mathrm{H}), 6.93-6.84(\mathrm{~m}$, $2 \mathrm{H}), 6.80-6.70(\mathrm{~m}, 1 \mathrm{H}), 4.78(\mathrm{dd}, J=8.8,3.5 \mathrm{~Hz}, 1 \mathrm{H}), 3.78(\mathrm{~s}, 3 \mathrm{H}), 3.34(\mathrm{dd}, J=14.7$, $3.5 \mathrm{~Hz}, 1 \mathrm{H}), 2.95(\mathrm{dd}, J=14.7,8.9 \mathrm{~Hz}, 1 \mathrm{H})$.

${ }^{13} \mathrm{C} \mathrm{NMR} \quad\left(100 \mathrm{MHz}, \mathrm{CDCl}_{3}\right)$

$\delta 201.0,172.7,159.6,138.1,137.7,129.5,124.3,121.9,121.7,120.9,115.0,113.6$, $112.4,85.8,55.2,37.5$.

HRMS (ESI) for: $\mathrm{C}_{15} \mathrm{H}_{15} \mathrm{O}_{3}[\mathrm{M}+\mathrm{H}]^{+}$: calcd 255.1021, found 255.1014 .

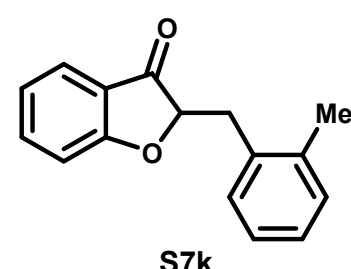

S7k

2-(2-Methylbenzyl)benzofuran-3(2H)-one (S7k). Using Method B to yield S7k as a white solid (2.1 $\mathrm{g}, 45 \%$ yield for two steps).

$\underline{R}_{f} \quad 0.35\left(\mathrm{Hex} / \mathrm{EtOAc}=20: 1, \mathrm{KMnO}_{4}\right)$

$\underline{{ }^{1} \mathrm{H} \mathrm{NMR}} \quad\left(500 \mathrm{MHz}, \mathrm{CDCl}_{3}\right)$

$\delta 7.65(\mathrm{dd}, J=7.9,1.6 \mathrm{~Hz}, 1 \mathrm{H}), 7.60-7.52(\mathrm{~m}, 1 \mathrm{H}), 7.31-7.26(\mathrm{~m}, 1 \mathrm{H}), 7.18-7.11$ $(\mathrm{m}, 3 \mathrm{H}), 7.10-7.01(\mathrm{~m}, 2 \mathrm{H}), 4.76(\mathrm{dd}, J=9.7,3.4 \mathrm{~Hz}, 1 \mathrm{H}), 3.35(\mathrm{dd}, J=15.0,3.4 \mathrm{~Hz}$, $1 \mathrm{H}), 2.91(\mathrm{dd}, J=15.0,9.7 \mathrm{~Hz}, 1 \mathrm{H}), 2.36(\mathrm{~s}, 3 \mathrm{H})$.

${ }^{13} \mathrm{C} \mathrm{NMR} \quad\left(125 \mathrm{MHz}, \mathrm{CDCl}_{3}\right)$

$\delta$ 201.2, 172.6, 138.1, 136.8, 134.8, 130.5, 129.8, 127.1, 126.1, 124.4, 122.0, 120.9, $113.6,85.6,34.7,19.8$.

$\underline{\mathrm{HRMS}(\mathrm{ESI})}$ for: $\mathrm{C}_{16} \mathrm{H}_{15} \mathrm{O}_{2}[\mathrm{M}+\mathrm{H}]^{+}$: calcd 239.1072, found 239.1068 . 


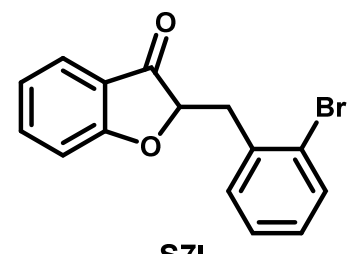

S7I

2-(2-Bromobenzyl)benzofuran-3(2H)-one (S7l) . Using Method A to yield S7l as a yellow solid (2.9 $\mathrm{g}, 48 \%$ yield for two steps).

$\underline{R_{f}} \quad 0.40\left(\mathrm{Hex} / \mathrm{EtOAc}=20: 1, \mathrm{KMnO}_{4}\right)$

${ }^{1} \mathrm{H} \mathrm{NMR} \quad\left(400 \mathrm{MHz}, \mathrm{CDCl}_{3}\right)$

$\delta 7.70-7.49(\mathrm{~m}, 2 \mathrm{H}), 7.33-7.19(\mathrm{~m}, 4 \mathrm{H}), 7.14-6.95(\mathrm{~m}, 2 \mathrm{H}), 4.77(\mathrm{dd}, J=8.7,3.6$

$\mathrm{Hz}, 1 \mathrm{H}), 3.36(\mathrm{dd}, J=14.7,3.6 \mathrm{~Hz}, 1 \mathrm{H}), 2.98$ (dd, $J=14.7,8.7 \mathrm{~Hz}, 1 \mathrm{H})$.

${ }^{13} \mathrm{C} \mathrm{NMR} \quad\left(100 \mathrm{MHz}, \mathrm{CDCl}_{3}\right)$

$\delta 201.2,172.8,138.2,136.2,129.5,128.6,127.1,124.4,122.0,121.1,113.7,85.9,37.5$.

HRMS (ESI) for: $\mathrm{C}_{15} \mathrm{H}_{12} \mathrm{BrO}_{2}[\mathrm{M}+\mathrm{H}]^{+}$: calcd 303.0021 , found 303.0029 .

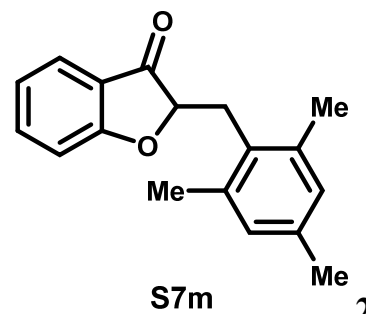

2-(2,4,6-Trimethylbenzyl)benzofuran-3(2H)-one $(\mathbf{S 7 m})$. Using Method A to yield $\mathbf{S 7 m}$ as a white solid (1.7 g, 32\% yield for two steps).

$\underline{R}_{f} \quad 0.50\left(\mathrm{Hex} / \mathrm{EtOAc}=20: 1, \mathrm{KMnO}_{4}\right)$

${ }^{1} \mathrm{H} \mathrm{NMR} \quad\left(500 \mathrm{MHz}, \mathrm{CDCl}_{3}\right)$

$\delta 7.82-7.45(\mathrm{~m}, 2 \mathrm{H}), 7.11(\mathrm{t}, J=7.2 \mathrm{~Hz}, 2 \mathrm{H}), 6.92(\mathrm{~s}, 2 \mathrm{H}), 4.71(\mathrm{dd}, J=11.2,3.2 \mathrm{~Hz}$, $1 \mathrm{H}), 3.31(\mathrm{dd}, J=14.8,3.2 \mathrm{~Hz}, 1 \mathrm{H}), 2.96(\mathrm{dd}, J=14.8,11.1 \mathrm{~Hz}, 1 \mathrm{H}), 2.33$ (d, $J=33.5$ $\mathrm{Hz}, 9 \mathrm{H})$.

${ }^{13} \mathrm{C} \mathrm{NMR} \quad\left(125 \mathrm{MHz}, \mathrm{CDCl}_{3}\right)$

$\delta 201.2,172.5,138.1,137.0,136.4,130.5,129.3,124.5,121.9,120.6,113.5,85.7,31.1$, $20.9,20.3$.

$\underline{\mathrm{HRMS}(\mathrm{ESI})}$ for: $\mathrm{C}_{18} \mathrm{H}_{19} \mathrm{O}_{2}[\mathrm{M}+\mathrm{H}]^{+}$: calcd 267.1385, found 267.1398.

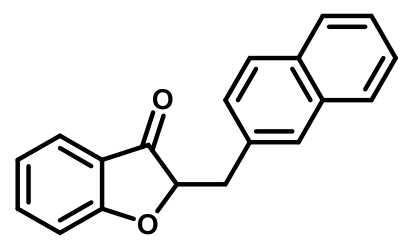

S7n 2-(Naphthalen-2-ylmethyl)benzofuran-3(2H)-one (S7n) . Using Method A to yield $\mathbf{S 7 n}$ as a white solid (2.9 $\mathrm{g}, 50 \%$ yield for two steps).

$\underline{R_{f}} \quad 0.40\left(\mathrm{Hex} / \mathrm{EtOAc}=20: 1, \mathrm{KMnO}_{4}\right)$

${ }^{1} \mathrm{H} \mathrm{NMR} \quad\left(400 \mathrm{MHz}, \mathrm{CDCl}_{3}\right)$

$\delta 7.87-7.72(\mathrm{~m}, 4 \mathrm{H}), 7.65(\mathrm{~d}, J=8.4 \mathrm{~Hz}, 1 \mathrm{H}), 7.61-7.52(\mathrm{~m}, 1 \mathrm{H}), 7.52-7.40(\mathrm{~m}$, $3 \mathrm{H}), 7.16-6.97(\mathrm{~m}, 2 \mathrm{H}), 4.88(\mathrm{dd}, J=8.7,3.6 \mathrm{~Hz}, 1 \mathrm{H}), 3.54(\mathrm{dd}, J=14.7,3.6 \mathrm{~Hz}, 1 \mathrm{H})$, $3.16(\mathrm{dd}, J=14.7,8.7 \mathrm{~Hz}, 1 \mathrm{H})$.

${ }^{13} \mathrm{C} \mathrm{NMR} \quad\left(100 \mathrm{MHz}, \mathrm{CDCl}_{3}\right)$

$\delta$ 201.1, 172.7, 138.1, 133.7, 133.5, 132.5, 128.1, 128.1, 127.7, 127.7, 127.6, 126.1, 
$125.7,124.4,122.0,121.0,113.6,85.8,37.6$.

HRMS (ESI) for: $\mathrm{C}_{19} \mathrm{H}_{15} \mathrm{O}_{2}[\mathrm{M}+\mathrm{H}]^{+}$: calcd 275.1072, found 275.1093.

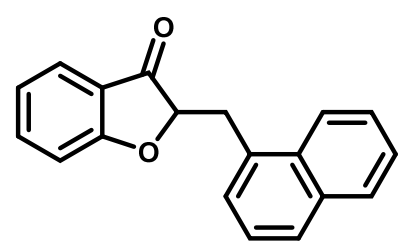

S7o

2-(Naphthalen-1-ylmethyl)benzofuran-3(2H)-one (S7o). Using Method B to yield S7o as a white solid (2.4 g, $42 \%$ yield for two steps).

$\underline{R}_{f} \quad 0.40\left(\mathrm{Hex} / \mathrm{EtOAc}=20: 1, \mathrm{KMnO}_{4}\right)$

${ }^{1} \mathrm{H} \mathrm{NMR} \quad\left(500 \mathrm{MHz}, \mathrm{CDCl}_{3}\right)$

$\delta 8.15(\mathrm{~d}, J=8.5 \mathrm{~Hz}, 1 \mathrm{H}), 7.88(\mathrm{~d}, J=8.7 \mathrm{~Hz}, 1 \mathrm{H}), 7.80(\mathrm{~d}, J=8.1 \mathrm{~Hz}, 1 \mathrm{H}), 7.70(\mathrm{dd}, J$ $=8.0,1.3 \mathrm{~Hz}, 1 \mathrm{H}), 7.64-7.54(\mathrm{~m}, 2 \mathrm{H}), 7.55-7.49(\mathrm{~m}, 2 \mathrm{H}), 7.49-7.41(\mathrm{~m}, 1 \mathrm{H}), 7.08$ $(\mathrm{dd}, J=6.4,1.4 \mathrm{~Hz}, 2 \mathrm{H}), 4.94(\mathrm{dd}, J=9.8,3.2 \mathrm{~Hz}, 1 \mathrm{H}), 3.93(\mathrm{dd}, J=15.1,3.1 \mathrm{~Hz}, 1 \mathrm{H})$, $3.28(\mathrm{dd}, J=15.2,9.8 \mathrm{~Hz}, 1 \mathrm{H})$.

${ }^{13} \mathrm{C} \mathrm{NMR} \quad\left(125 \mathrm{MHz}, \mathrm{CDCl}_{3}\right)$

$\delta 201.2,172.6,138.1,133.9,132.6,131.9,128.9,127.9,127.6,126.3,125.8,125.5$, $124.5,123.6,122.0,120.8,113.7,85.5,34.8$.

HRMS (ESI) for: $\mathrm{C}_{19} \mathrm{H}_{15} \mathrm{O}_{2}[\mathrm{M}+\mathrm{H}]^{+}$: calcd 275.1072, found 275.1091.<smiles>Cn1cc(CC2Oc3ccccc3C2=O)c2ccccc21</smiles>

2-((1-Methyl-1H-indol-3-yl)methyl)benzofuran-3(2H)-one (S7p). Using Method B to yield S7p as orange solid (1.6 g, 28\% yield for two steps).

$\underline{R}_{f} \quad 0.30\left(\mathrm{Hex} / \mathrm{EtOAc}=10: 1, \mathrm{KMnO}_{4}\right)$

${ }^{1} \mathrm{H} \mathrm{NMR} \quad\left(400 \mathrm{MHz}, \mathrm{CDCl}_{3}\right)$

$\delta 7.69(\mathrm{dt}, J=7.9,1.0 \mathrm{~Hz}, 1 \mathrm{H}), 7.64-7.59(\mathrm{~m}, 1 \mathrm{H}), 7.58-7.50(\mathrm{~m}, 1 \mathrm{H}), 7.28-7.17$ $(\mathrm{m}, 2 \mathrm{H}), 7.15-7.05(\mathrm{~m}, 2 \mathrm{H}), 7.04-6.97(\mathrm{~m}, 2 \mathrm{H}), 4.83(\mathrm{dd}, J=8.4,3.7 \mathrm{~Hz}, 1 \mathrm{H}), 3.71$ $(\mathrm{s}, 3 \mathrm{H}), 3.56-3.46(\mathrm{~m}, 1 \mathrm{H}), 3.20-3.08(\mathrm{~m}, 1 \mathrm{H})$.

${ }^{13} \mathrm{C} \mathrm{NMR} \quad\left(100 \mathrm{MHz}, \mathrm{CDCl}_{3}\right)$

$\delta 201.6,172.8,137.9,136.9,127.8,124.3,121.8,121.1,119.1,113.5,109.2,108.8$, $86.0,32.7,27.3$.

$\underline{\operatorname{HRMS}(\mathrm{ESI})}$ for: $\mathrm{C}_{18} \mathrm{H}_{16} \mathrm{NO}_{2}[\mathrm{M}+\mathrm{H}]^{+}$: calcd 278.1181, found 278.1187.<smiles>O=C1c2ccccc2OC1Cc1ccco1</smiles>

S7q 2-(Furan-3-ylmethyl)benzofuran-3(2H)-one (S7q). Using Method A to yield S7q as a yellow oil (1.4 g, 33\% yield for two steps).

$\underline{R}_{f} \quad 0.30\left(\mathrm{Hex} / \mathrm{EtOAc}=15: 1, \mathrm{KMnO}_{4}\right)$ 


\section{${ }^{1} \mathrm{H} \mathrm{NMR} \quad\left(400 \mathrm{MHz}, \mathrm{CDCl}_{3}\right)$}

$\delta 7.79-7.49(\mathrm{~m}, 2 \mathrm{H}), 7.31(\mathrm{dd}, J=1.9,0.9 \mathrm{~Hz}, 1 \mathrm{H}), 7.18-7.00(\mathrm{~m}, 2 \mathrm{H}), 6.40-6.04$ $(\mathrm{m}, 2 \mathrm{H}), 4.83(\mathrm{dd}, J=8.4,3.8 \mathrm{~Hz}, 1 \mathrm{H}), 3.42-3.35(\mathrm{~m}, 1 \mathrm{H}), 3.06(\mathrm{dd}, J=15.8,8.3 \mathrm{~Hz}$, $1 \mathrm{H})$.

${ }^{13} \mathrm{C} \mathrm{NMR} \quad\left(100 \mathrm{MHz}, \mathrm{CDCl}_{3}\right)$ $\delta$ 200.7, 172.8, 150.2, 142.0, 138.3, 124.5, 122.2, 120.8, 113.7, 110.5, 107.7, 83.6, 30.3. $\underline{\text { HRMS (ESI) }}$ for: $\mathrm{C}_{13} \mathrm{H}_{11} \mathrm{O}_{3}[\mathrm{M}+\mathrm{H}]^{+}$: calcd 215.0708, found 215.0715 .

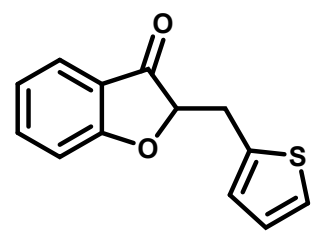

S7r 2-(Thiophen-3-ylmethyl)benzofuran-3(2H)-one (S7r) . Using Method B to yield S7r as a orange oil (1.4 g, 30\% yield for two steps).

${ }^{1} \mathrm{H} \mathrm{NMR} \quad\left(400 \mathrm{MHz}, \mathrm{CDCl}_{3}\right)$ $\delta 7.70-7.50(\mathrm{~m}, 2 \mathrm{H}), 7.16-7.10(\mathrm{~m}, 2 \mathrm{H}), 7.09-7.01(\mathrm{~m}, 1 \mathrm{H}), 6.99-6.84(\mathrm{~m}, 2 \mathrm{H})$, 4.77 (dd, $J=7.7,3.7 \mathrm{~Hz}, 1 \mathrm{H}), 3.59-5.55(\mathrm{~m}, 1 \mathrm{H}), 3.30-3.25(\mathrm{~m}, 1 \mathrm{H})$.

${ }^{13} \mathrm{C} \mathrm{NMR} \quad\left(100 \mathrm{MHz}, \mathrm{CDCl}_{3}\right)$ $\delta$ 200.6, 172.9, 138.3, 137.3, 126.9, 124.8, 124.4, 122.1, 121.1, 113.7, 85.2, 31.7.

HRMS (ESI) for: $\mathrm{C}_{13} \mathrm{H}_{11} \mathrm{O}_{2} \mathrm{~S}[\mathrm{M}+\mathrm{H}]^{+}$: calcd 231.0480, found 231.0496.

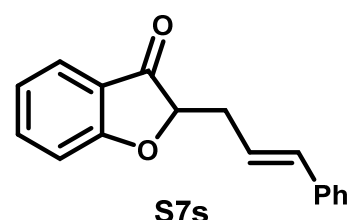

(E)-2-(3-Phenylprop-1-en-1-yl)benzofuran-3(2H)-one (S7s). Using Method B to yield $\mathbf{S 7 s}$ as a white solid ( $0.9 \mathrm{~g}, 18 \%$ yield for two steps).

$\underline{R}_{f} \quad 0.30\left(\mathrm{Hex} / \mathrm{EtOAc}=20: 1, \mathrm{KMnO}_{4}\right)$

${ }^{1} \mathrm{H} \mathrm{NMR} \quad\left(500 \mathrm{MHz}, \mathrm{CDCl}_{3}\right)$ $\delta 7.69-7.42(\mathrm{~m}, 2 \mathrm{H}), 7.30-7.17(\mathrm{~m}, 4 \mathrm{H}), 7.17-7.10(\mathrm{~m}, 1 \mathrm{H}), 7.07(\mathrm{~d}, J=8.4 \mathrm{~Hz}$, $1 \mathrm{H}), 7.04-6.97(\mathrm{~m}, 1 \mathrm{H}), 6.50(\mathrm{dt}, J=16.1,1.5 \mathrm{~Hz}, 1 \mathrm{H}), 6.19-6.11(\mathrm{~m}, 1 \mathrm{H}), 4.61(\mathrm{dd}$, $J=7.9,4.2 \mathrm{~Hz}, 1 \mathrm{H}), 2.93-2.84(\mathrm{~m}, 1 \mathrm{H}), 2.65-2.55(\mathrm{~m}, 1 \mathrm{H})$.

${ }^{13} \mathrm{C} \mathrm{NMR} \quad\left(125 \mathrm{MHz}, \mathrm{CDCl}_{3}\right)$ $\delta 201.3,172.8,138.1,137.0,133.9,128.5,127.5,126.3,124.3,123.3,122.0,121.0$, 113.6, 84.9, 34.9.

$\underline{\mathrm{HRMS}(\mathrm{ESI})}$ for: $\mathrm{C}_{17} \mathrm{H}_{15} \mathrm{O}_{2}[\mathrm{M}+\mathrm{H}]^{+}$: calcd 251.1072, found 251.1077 .

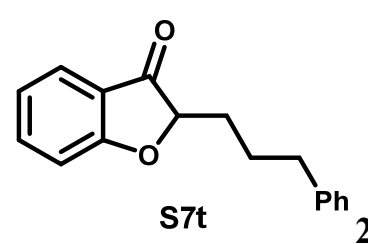
as a white solid (1.9 g, 39\% yield for two steps).

2-(3-Phenylpropyl)benzofuran-3(2H)-one (S7t). Using Method A to yield S7t $\underline{R}_{f} \quad 0.32\left(\mathrm{Hex} / \mathrm{EtOAc}=20: 1, \mathrm{KMnO}_{4}\right)$

$\underline{{ }^{1} \mathrm{H} \mathrm{NMR}} \quad\left(500 \mathrm{MHz}, \mathrm{CDCl}_{3}\right)$ 
$\delta 7.61-7.49(\mathrm{~m}, 2 \mathrm{H}), 7.23-7.16(\mathrm{~m}, 2 \mathrm{H}), 7.15-6.94(\mathrm{~m}, 5 \mathrm{H}), 4.51(\mathrm{~d}, J=7.3 \mathrm{~Hz}$, $1 \mathrm{H}), 2.66-2.54(\mathrm{~m}, 2 \mathrm{H}), 2.05-1.91(\mathrm{~m}, 1 \mathrm{H}), 1.86-1.65(\mathrm{~m}, 3 \mathrm{H})$.

${ }^{13} \mathrm{C} \mathrm{NMR} \quad\left(125 \mathrm{MHz}, \mathrm{CDCl}_{3}\right)$

$\delta 202.0,172.8,141.6,138.0,128.4,125.9,124.3,121.8,121.1,113.5,85.4,35.6,31.0$, 26.6 .

$\underline{\text { HRMS (ESI) }}$ for: $\mathrm{C}_{17} \mathrm{H}_{17} \mathrm{O}_{2}[\mathrm{M}+\mathrm{H}]^{+}$: calcd 253.1229, found 253.1218 .

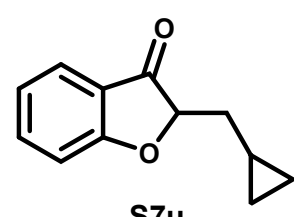

2-(Cyclopropylmethyl)benzofuran-3(2H)-one (S7u). Using Method B to yield $\mathbf{S 7} \mathbf{u}$ as a yellow oil (1.8 $\mathrm{g}, 48 \%$ yield for two steps).

$\underline{R}_{f} \quad 0.30\left(\mathrm{Hex} / \mathrm{EtOAc}=30: 1, \mathrm{KMnO}_{4}\right)$

$\underline{{ }^{1} \mathrm{H} \mathrm{NMR}} \quad\left(500 \mathrm{MHz}, \mathrm{CDCl}_{3}\right)$

$\delta 7.71-7.49(\mathrm{~m}, 2 \mathrm{H}), 7.16-6.94(\mathrm{~m}, 2 \mathrm{H}), 4.60(\mathrm{dd}, J=7.5,4.2 \mathrm{~Hz}, 1 \mathrm{H}), 1.90-1.62$ $(\mathrm{m}, 2 \mathrm{H}), 0.98-0.83(\mathrm{~m}, 1 \mathrm{H}), 0.50-0.36(\mathrm{~m}, 2 \mathrm{H}), 0.25-0.09(\mathrm{~m}, 2 \mathrm{H})$.

${ }^{13} \mathrm{C} \mathrm{NMR} \quad\left(125 \mathrm{MHz}, \mathrm{CDCl}_{3}\right)$

HRMS (ESI) $\delta 202.1,172.8,137.9,121.7,121.1,113.4,86.0,36.3,6.9,5.0,4.1$.

for: $\mathrm{C}_{12} \mathrm{H}_{13} \mathrm{O}_{2}[\mathrm{M}+\mathrm{H}]^{+}$: calcd 189.0916, found 189.0934 .

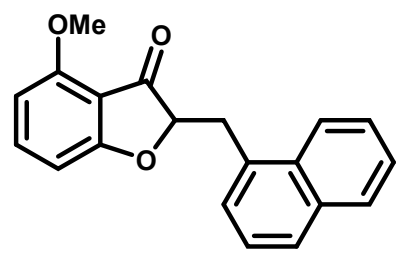

S7v

Using Method B to yield $\mathbf{S 7 v}$ as a yellow solid (1.3 g, 44\% yield for two steps).

$\underline{R}_{f} \quad 0.35\left(\mathrm{Hex} / \mathrm{EtOAc}=15: 1, \mathrm{KMnO}_{4}\right)$

$\underline{{ }^{1} \mathrm{H} \mathrm{NMR}} \quad\left(500 \mathrm{MHz}, \mathrm{CDCl}_{3}\right)$

$\delta 8.13(\mathrm{~d}, J=8.4 \mathrm{~Hz}, 1 \mathrm{H}), 7.83(\mathrm{dd}, J=42.0,8.1 \mathrm{~Hz}, 2 \mathrm{H}), 7.61-7.39(\mathrm{~m}, 5 \mathrm{H}), 6.61(\mathrm{~d}$, $J=8.3 \mathrm{~Hz}, 1 \mathrm{H}), 6.45(d, \mathrm{~J}=8.1 \mathrm{~Hz}, 1 \mathrm{H}), 4.91(\mathrm{dd}, J=10.0,3.0 \mathrm{~Hz}, 1 \mathrm{H}), 3.97(\mathrm{~s}, 3 \mathrm{H})$, $3.95-3.87(\mathrm{~m}, 1 \mathrm{H}), 3.26(\mathrm{dd}, J=15.2,9.9 \mathrm{~Hz}, 1 \mathrm{H})$.

${ }^{13} \mathrm{C} \mathrm{NMR} \quad\left(125 \mathrm{MHz}, \mathrm{CDCl}_{3}\right)$

$\delta 198.4,173.7,158.3,139.7,133.9,132.7,132.0,128.9,127.8,127.6,126.2,125.7$, $125.5,123.7,110.1,105.4,103.2,85.4,56.1,34.9$.

$\underline{\text { HRMS (ESI) }}$ for: $\mathrm{C}_{20} \mathrm{H}_{17} \mathrm{O}_{3}[\mathrm{M}+\mathrm{H}]^{+}$: calcd 305.1178, found 305.1183 .

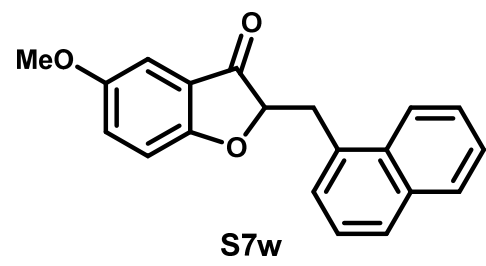

5-Methoxy-2-(naphthalen-1-ylmethyl)benzofuran-3(2H)-one (S7w).

Using Method B to yield $\mathbf{S 7 w}$ as a yellow solid (1.5 g, 50\% yield for two steps).

$\underline{R}_{f} \quad 0.35\left(\mathrm{Hex} / \mathrm{EtOAc}=15: 1, \mathrm{KMnO}_{4}\right)$

${ }^{1} \mathrm{H} \mathrm{NMR} \quad\left(500 \mathrm{MHz}, \mathrm{CDCl}_{3}\right)$

$\delta 8.14(\mathrm{~d}, J=8.5 \mathrm{~Hz}, 1 \mathrm{H}), 7.83(\mathrm{dd}, J=42.0,8.1 \mathrm{~Hz}, 2 \mathrm{H}), 7.62-7.40(\mathrm{~m}, 4 \mathrm{H}), 7.22$ 
$(\mathrm{dd}, J=9.0,2.8 \mathrm{~Hz}, 1 \mathrm{H}), 7.10-6.95(\mathrm{~m}, 2 \mathrm{H}), 4.95(\mathrm{dd}, J=9.8,3.1 \mathrm{~Hz}, 1 \mathrm{H}), 3.91(\mathrm{dd}, J$ $=15.1,3.2 \mathrm{~Hz}, 1 \mathrm{H}), 3.80(\mathrm{~s}, 3 \mathrm{H}), 3.27(\mathrm{dd}, J=15.1,9.8 \mathrm{~Hz}, 1 \mathrm{H})$.

${ }^{13} \mathrm{C} \mathrm{NMR} \quad\left(125 \mathrm{MHz}, \mathrm{CDCl}_{3}\right)$

$\delta 201.5,168.1,155.0,133.9,132.6,131.9,128.9,128.3,127.9,127.5,126.2,125.7$, $125.4,123.6,120.6,114.5,104.0,86.2,55.9,34.8$.

HRMS (ESI) for: $\mathrm{C}_{20} \mathrm{H}_{17} \mathrm{O}_{3}[\mathrm{M}+\mathrm{H}]^{+}$: calcd 305.1178 , found 305.1188 .<smiles>COc1ccc2c(c1)OC(Cc1cccc3ccccc13)C2=O</smiles>

6-Methoxy-2-(naphthalen-1-ylmethyl)benzofuran-3(2H)-one (S7x). Using Method B to yield $\mathbf{S 7} \mathbf{x}$ as a yellow oil (1.3 $\mathrm{g}, 44 \%$ yield for two steps).

$\underline{R}_{f} \quad 0.35\left(\mathrm{Hex} / \mathrm{EtOAc}=15: 1, \mathrm{KMnO}_{4}\right)$

${ }^{1} \mathrm{H} \mathrm{NMR} \quad\left(500 \mathrm{MHz}, \mathrm{CDCl}_{3}\right)$

$\delta 8.23-7.99(\mathrm{~m}, 1 \mathrm{H}), 7.91-7.76(\mathrm{~m}, 2 \mathrm{H}), 7.63-7.40(\mathrm{~m}, 5 \mathrm{H}), 6.64(\mathrm{dd}, J=8.6,2.1$

$\mathrm{Hz}, 1 \mathrm{H}), 6.48(\mathrm{~d}, J=2.1 \mathrm{~Hz}, 1 \mathrm{H}), 4.95(\mathrm{dd}, J=9.9,3.0 \mathrm{~Hz}, 1 \mathrm{H}), 3.93(\mathrm{dd}, J=15.2,3.0$

$\mathrm{Hz}, 1 \mathrm{H}), 3.84(\mathrm{~s}, 3 \mathrm{H}), 3.24(\mathrm{dd}, J=15.1,9.9 \mathrm{~Hz}, 1 \mathrm{H})$.

${ }^{13} \mathrm{C} \mathrm{NMR} \quad\left(125 \mathrm{MHz}, \mathrm{CDCl}_{3}\right)$

$\delta 198.7,175.1,168.4,133.9,132.8,131.9,128.9,127.8,127.5,126.2,125.7,125.5$, $125.4,123.6,113.9,111.8,96.3,86.4,55.9,34.9$.

HRMS (ESI) for: $\mathrm{C}_{20} \mathrm{H}_{17} \mathrm{O}_{3}[\mathrm{M}+\mathrm{H}]^{+}$: calcd 305.1178, found 305.1176.<smiles>O=C1c2ccc(Br)cc2OC1Cc1cccc2ccccc12</smiles>

S7y

6-Bromo-2-(naphthalen-1-ylmethyl)benzofuran-3(2H)-one (S7y).

Using Method B to yield $\mathbf{S 7 y}$ as a white solid (1.3 $\mathrm{g}, 38 \%$ yield for two steps).

$\underline{R}_{f} \quad 0.35\left(\mathrm{Hex} / \mathrm{EtOAc}=15: 1, \mathrm{KMnO}_{4}\right)$

${ }^{1} \mathrm{H} \mathrm{NMR} \quad\left(500 \mathrm{MHz}, \mathrm{CDCl}_{3}\right)$

$\delta 8.15-7.92(\mathrm{~m}, 1 \mathrm{H}), 7.90-7.65(\mathrm{~m}, 2 \mathrm{H}), 7.59-7.31(\mathrm{~m}, 5 \mathrm{H}), 7.26-7.03(\mathrm{~m}, 2 \mathrm{H})$, 4.87 (dd, $J=9.5,3.3 \mathrm{~Hz}, 1 \mathrm{H}), 3.83(\mathrm{dd}, J=15.1,3.3 \mathrm{~Hz}, 1 \mathrm{H}), 3.20(\mathrm{dd}, J=15.2,9.5$ $\mathrm{Hz}, 1 \mathrm{H})$.

${ }^{13} \mathrm{C} \mathrm{NMR} \quad\left(125 \mathrm{MHz}, \mathrm{CDCl}_{3}\right)$

$\delta 199.8,172.7,133.9,133.0,132.1,131.8,129.0,128.1,127.7,125.9,125.8,125.4$, $125.2,123.5,119.8,117.2,86.2,34.7$.

HRMS (ESI) for: $\mathrm{C}_{19} \mathrm{H}_{14} \mathrm{BrO}_{2}[\mathrm{M}+\mathrm{H}]^{+}$: calcd 353.0177, found 353.0185 .

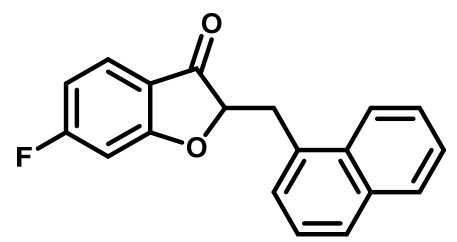

S7z

6-Fluoro-2-(naphthalen-1-ylmethyl)benzofuran-3(2H)-one

$(\mathbf{S 7 z})$.

Using Method B to yield $\mathbf{S 7 z}$ as a yellow solid (1.3 g, 48\% yield for two steps). 
$\begin{array}{rl}\underline{R}_{f} & 0.35\left(\mathrm{Hex} / \mathrm{EtOAc}=20: 1, \mathrm{KMnO}_{4}\right) \\ { }^{1} \mathrm{H} \mathrm{NMR} & \left(500 \mathrm{MHz}, \mathrm{CDCl}_{3}\right)\end{array}$

$\delta 8.10(\mathrm{dd}, J=8.5,1.2 \mathrm{~Hz}, 1 \mathrm{H}), 7.85(\mathrm{dd}, J=8.1,1.4 \mathrm{~Hz}, 1 \mathrm{H}), 7.77(\mathrm{~d}, J=8.0 \mathrm{~Hz}, 1 \mathrm{H})$, $7.65(\mathrm{dd}, J=8.5,5.8 \mathrm{~Hz}, 1 \mathrm{H}), 7.58-7.52(\mathrm{~m}, 1 \mathrm{H}), 7.52-7.37(\mathrm{~m}, 3 \mathrm{H}), 6.87-6.59(\mathrm{~m}$, $2 \mathrm{H}), 4.96(\mathrm{dd}, J=9.6,3.2 \mathrm{~Hz}, 1 \mathrm{H}), 3.90(\mathrm{dd}, J=15.2,3.2 \mathrm{~Hz}, 1 \mathrm{H}), 3.26(\mathrm{dd}, J=15.2$, $9.6 \mathrm{~Hz}, 1 \mathrm{H})$.

${ }^{13} \mathrm{C} \mathrm{NMR} \quad\left(125 \mathrm{MHz}, \mathrm{CDCl}_{3}\right)$

$\delta 199.1,174.1,174.0,170.5,168.4,134.0,132.2,131.9,129.0,128.0,127.7,126.4$, 126.4, 126.3, 125.8, 125.4, 123.5, 117.5, 111.1, 110.9, 101.0, 100.8, 86.7, 77.4, 77.1, $76.8,34.7$.

${ }^{19} \mathrm{~F} \mathrm{NMR} \quad(470 \mathrm{MHz}, \mathrm{CDCl} 3)$

$\delta$ - 96.69 .

HRMS (ESI) for: $\mathrm{C}_{19} \mathrm{H}_{14} \mathrm{FO}_{2}[\mathrm{M}+\mathrm{H}]^{+}$: calcd 293.0978, found 293.0967<smiles>O=C1c2ccc(N3CCOCC3)cc2OC1Cc1cccc2ccccc12</smiles>

S7a'

6-Morpholino-2-(naphthalen-1-ylmethyl)benzofuran-3(2H)-one

(S7a'). Using Method B to yield S7a' as a white solid (1.5 g, 44\% yield for two steps).

$\underline{R}_{f} 0.20\left(\mathrm{Hex} / \mathrm{EtOAc}=10: 1, \mathrm{KMnO}_{4}\right)$

${ }^{1} \mathrm{H} \mathrm{NMR} \quad\left(500 \mathrm{MHz}, \mathrm{CDCl}_{3}\right)$

$\delta 8.15(\mathrm{~d}, J=8.5 \mathrm{~Hz}, 1 \mathrm{H}), 7.93-7.72(\mathrm{~m}, 2 \mathrm{H}), 7.59-7.41(\mathrm{~m}, 5 \mathrm{H}), 6.60(\mathrm{~d}, J=11.0$ $\mathrm{Hz}, 1 \mathrm{H}), 6.31(\mathrm{~s}, 1 \mathrm{H}), 4.92(\mathrm{dd}, J=10.1,2.9 \mathrm{~Hz}, 1 \mathrm{H}), 4.03-3.69(\mathrm{~m}, 5 \mathrm{H}), 3.46-3.08$ $(\mathrm{m}, 5 \mathrm{H})$.

${ }^{13} \mathrm{C} \mathrm{NMR} \quad\left(125 \mathrm{MHz}, \mathrm{CDCl}_{3}\right)$

$\delta 198.0,174.9,158.6,133.9,133.1,131.9,128.9,127.7,127.4,126.2,125.7,125.5$, $123.7,111.4,109.5,95.4,86.3,66.4,47.4,35.1$.

HRMS (ESI) for: $\mathrm{C}_{23} \mathrm{H}_{22} \mathrm{NO}_{3}[\mathrm{M}+\mathrm{H}]^{+}$: calcd 360.1600 , found 360.1622

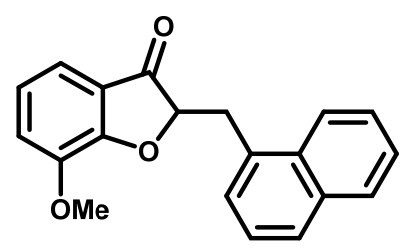

S7b'

7-Methoxy-2-(naphthalen-1-ylmethyl)benzofuran-3(2H)-one (S7b').

Using Method A to yield $\mathbf{S 7 b}$ ' as a white solid (1.2 g, 41\% yield for two steps).

$\underline{R}_{f} 0.30\left(\mathrm{Hex} / \mathrm{EtOAc}=15: 1, \mathrm{KMnO}_{4}\right)$

${ }^{1} \mathrm{H} \mathrm{NMR} \quad\left(500 \mathrm{MHz}, \mathrm{CDCl}_{3}\right)$

$\delta 8.16(\mathrm{~d}, J=8.5 \mathrm{~Hz}, 1 \mathrm{H}), 7.86(\mathrm{~d}, J=8.0 \mathrm{~Hz}, 1 \mathrm{H}), 7.77(\mathrm{~d}, J=6.7 \mathrm{~Hz}, 1 \mathrm{H}), 7.58-7.47$ (m, 3H), $7.43(\mathrm{dd}, J=8.1,7.2 \mathrm{~Hz}, 1 \mathrm{H}), 7.28-7.25(\mathrm{~m}, 1 \mathrm{H}), 7.16-6.94(\mathrm{~m}, 2 \mathrm{H}), 5.00$ (dd, $J=9.1,3.6 \mathrm{~Hz}, 1 \mathrm{H}), 3.95-3.80(\mathrm{~m}, 4 \mathrm{H}), 3.47-3.27(\mathrm{~m}, 1 \mathrm{H})$.

${ }^{13} \mathrm{C} \mathrm{NMR} \quad\left(125 \mathrm{MHz}, \mathrm{CDCl}_{3}\right)$

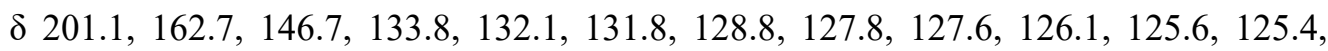
$123.5,122.4,122.0,118.9,115.4,85.7,56.2,34.6$.

$\underline{\text { HRMS (ESI) }}$ for: $\mathrm{C}_{20} \mathrm{H}_{17} \mathrm{O}_{3}[\mathrm{M}+\mathrm{H}]^{+}$: calcd 305.1178, found 305.1169. 


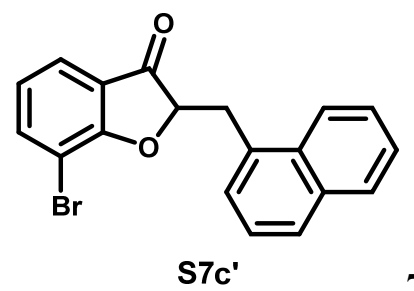

7-Bromo-2-(naphthalen-1-ylmethyl)benzofuran-3(2H)-one (S7c'). Using Method B to yield $\mathbf{S 7} \mathbf{c}^{\prime}$ as a white solid (1.4 g, $42 \%$ yield for two steps).

$\underline{R}_{f} \quad 0.35\left(\mathrm{Hex} / \mathrm{EtOAc}=15: 1, \mathrm{KMnO}_{4}\right)$

${ }^{1} \mathrm{H} \mathrm{NMR} \quad\left(500 \mathrm{MHz}, \mathrm{CDCl}_{3}\right)$

$\delta 8.19-7.98(\mathrm{~m}, 1 \mathrm{H}), 7.86-7.60(\mathrm{~m}, 3 \mathrm{H}), 7.58-7.30(\mathrm{~m}, 5 \mathrm{H}), 6.86(\mathrm{t}, J=7.7 \mathrm{~Hz}$, $1 \mathrm{H}), 4.94(\mathrm{dd}, J=8.8,3.6 \mathrm{~Hz}, 1 \mathrm{H}), 3.83(\mathrm{dd}, J=15.1,3.6 \mathrm{~Hz}, 1 \mathrm{H}), 3.32(\mathrm{dd}, J=15.1$, $8.8 \mathrm{~Hz}, 1 \mathrm{H})$.

${ }^{13} \mathrm{C} \mathrm{NMR} \quad\left(125 \mathrm{MHz}, \mathrm{CDCl}_{3}\right)$

$\delta 200.4,169.1,140.5,133.9,132.0,131.8,128.8,128.0,128.0,126.3,125.7,125.4$, $123.8,123.3,123.2,122.4,106.8,86.4,34.6$.

HRMS (ESI) for: $\mathrm{C}_{19} \mathrm{H}_{14} \mathrm{BrO}_{2}[\mathrm{M}+\mathrm{H}]^{+}$: calcd 353.0177 , found 353.0175

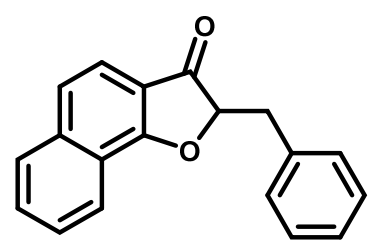

S7d' 2-Benzylnaphtho[1,2-b]furan-3(2H)-one (S7d'). Using Method B to yield S7d' as a white solid (1.2 g, $45 \%$ yield for two steps).

$\underline{R}_{f} \quad 0.30\left(\mathrm{Hex} / \mathrm{EtOAc}=20: 1, \mathrm{KMnO}_{4}\right)$

${ }^{1} \mathrm{H} \mathrm{NMR} \quad\left(500 \mathrm{MHz}, \mathrm{CDCl}_{3}\right)$

$\delta 8.76(\mathrm{~d}, \mathrm{~J}=8.2 \mathrm{~Hz}, 1 \mathrm{H}), 8.03(\mathrm{~d}, \mathrm{~J}=9.0 \mathrm{~Hz}, 1 \mathrm{H}), 7.81(\mathrm{~d}, \mathrm{~J}=8.1 \mathrm{~Hz}, 1 \mathrm{H}), 7.66(\mathrm{t}, \mathrm{J}=$ $8.1 \mathrm{~Hz}, 1 \mathrm{H}), 7.47(\mathrm{t}, \mathrm{J}=8.0 \mathrm{~Hz}, 1 \mathrm{H}), 7.37(\mathrm{~d}, \mathrm{~J}=7.3 \mathrm{~Hz}, 2 \mathrm{H}), 7.30(\mathrm{dd}, \mathrm{J}=8.4,6.8 \mathrm{~Hz}$, $2 \mathrm{H}), 7.23(\mathrm{dd}, \mathrm{J}=8.2,4.6 \mathrm{~Hz}, 2 \mathrm{H}), 4.90(\mathrm{dd}, \mathrm{J}=8.9,3.6 \mathrm{~Hz}, 1 \mathrm{H}), 3.47(\mathrm{dd}, \mathrm{J}=14.8,3.6$ $\mathrm{Hz}, 1 \mathrm{H}), 3.06(\mathrm{dd}, \mathrm{J}=14.8,8.9 \mathrm{~Hz}, 1 \mathrm{H})$.

${ }^{13} \mathrm{C} \mathrm{NMR} \quad\left(125 \mathrm{MHz}, \mathrm{CDCl}_{3}\right)$

$\delta 200.4,175.3,140.0,136.3,129.9,129.4,129.2,129.2,128.5,127.0,125.4,123.2$, $114.0,113.1,86.8,37.6$.

HRMS (ESI) for: $\mathrm{C}_{23} \mathrm{H}_{17} \mathrm{O}_{2}[\mathrm{M}+\mathrm{H}]^{+}$: calcd 325.1229 , found 325.1218

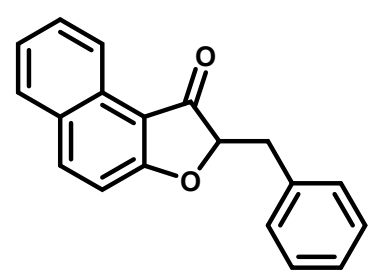

S7e' 2-Benzylnaphtho[2,1-b]furan-1(2H)-one (S7e'). Using Method A to yield S7e' as a white solid (1.3 g, $46 \%$ yield for two steps).

$\underline{R}_{f} \quad 0.30\left(\mathrm{Hex} / \mathrm{EtOAc}=20: 1, \mathrm{KMnO}_{4}\right)$

${ }^{1} \mathrm{H} \mathrm{NMR} \quad\left(500 \mathrm{MHz}, \mathrm{CDCl}_{3}\right)$

$\delta 8.24(\mathrm{~d}, J=8.2 \mathrm{~Hz}, 1 \mathrm{H}), 7.86(\mathrm{~d}, J=8.1 \mathrm{~Hz}, 1 \mathrm{H}), 7.70(\mathrm{t}, J=7.6 \mathrm{~Hz}, 1 \mathrm{H}), 7.59(\mathrm{t}, J=$

$7.6 \mathrm{~Hz}, 1 \mathrm{H}), 7.53(\mathrm{~d}, J=8.5 \mathrm{~Hz}, 1 \mathrm{H}), 7.45-7.33(\mathrm{~m}, 3 \mathrm{H}), 7.33-7.17(\mathrm{~m}, 3 \mathrm{H}), 5.13-$ 
$4.89(\mathrm{~m}, 1 \mathrm{H}), 3.47(\mathrm{dd}, J=14.7,3.5 \mathrm{~Hz}, 1 \mathrm{H}), 3.07(\mathrm{dd}, J=14.7,8.8 \mathrm{~Hz}, 1 \mathrm{H})$.

${ }^{13} \mathrm{C} \mathrm{NMR} \quad\left(125 \mathrm{MHz}, \mathrm{CDCl}_{3}\right)$

$\delta$ 200.2, 173.4, 138.7, 136.2, 130.7, 129.5, 128.5, 128.5, 126.9, 126.7, 122.5, 122.4,

$121.7,118.8,115.7,87.2,37.7$.

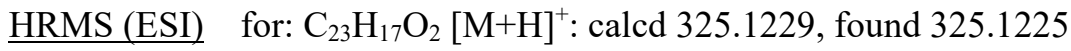

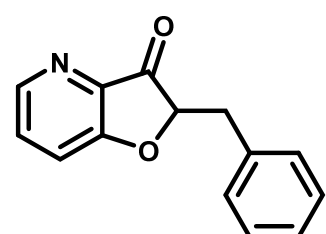

S7f' 2-Benzylfuro[2,3-b]pyridin-3(2H)-one (S7f'). Using Method A to yield S7f' as a yellow oil ( $1.0 \mathrm{~g}, 45 \%$ yield for two steps).

$\underline{R}_{f} \quad 0.25\left(\mathrm{Hex} / \mathrm{EtOAc}=8: 1, \mathrm{KMnO}_{4}\right)$

$\underline{{ }^{1} \mathrm{H} \mathrm{NMR}} \quad\left(400 \mathrm{MHz}, \mathrm{CDCl}_{3}\right)$

$\delta 7.69-7.49(\mathrm{~m}, 2 \mathrm{H}), 7.33-7.19(\mathrm{~m}, 4 \mathrm{H}), 7.17-6.95(\mathrm{~m}, 2 \mathrm{H}), 4.77(\mathrm{dd}, J=8.8,3.5$

$\mathrm{Hz}, 1 \mathrm{H}), 3.36$ (dd, $J=14.7,3.5 \mathrm{~Hz}, 1 \mathrm{H}), 2.98$ (dd, $J=14.7,8.7 \mathrm{~Hz}, 1 \mathrm{H})$.

${ }^{13} \mathrm{C} \mathrm{NMR} \quad\left(100 \mathrm{MHz}, \mathrm{CDCl}_{3}\right)$

$\delta 201.2,172.8,138.2,136.2,129.5,128.6,127.1,124.4,122.0,121.0,113.7,85.9,37.5$.

HRMS (ESI) for: $\mathrm{C}_{14} \mathrm{H}_{12} \mathrm{NO}_{2}[\mathrm{M}+\mathrm{H}]^{+}$: calcd 226.0868, found 226.0865 .

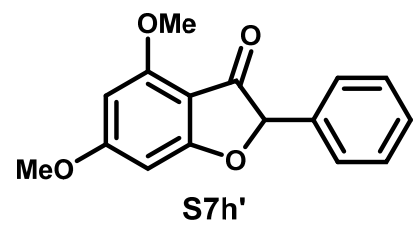

4,6-Dimethoxy-2-phenylbenzofuran-3(2H)-one (S7h').

A solution of 3,5-dimethoxyphenol $(1.5 \mathrm{~g}, 10 \mathrm{mmol})$ and ethyl 2-bromo-2-phenylacetate (1.05 equiv.) in acetone $(0.2 \mathrm{M})$ was added $\mathrm{K}_{2} \mathrm{CO}_{3}\left(5.0\right.$ equiv.). After being refluxed at $65{ }^{\circ} \mathrm{C}$ overnight, the mixture was filtered and concentrated in vacuo. Which was used directly without further purification.

To a stirred solution of the ester (crude, obtain above) in THF/MeOH/ $\mathrm{H}_{2} \mathrm{O}$ (THF/MeOH$/ \mathrm{H}_{2} \mathrm{O}=2: 2: 1$, $0.5 \mathrm{M}$ ) was added $\mathrm{LiOH}_{\mathrm{H}} \mathrm{O}$ ( $\mathrm{O}(10.0$ equiv.). The resulting mixture was stirred at RT overnight. THF was removed in vacuo and the resulting mixture was acidified with $6 \mathrm{M} \mathrm{HCl}(10 \mathrm{~mL}$, sat. aq. $)$ to $\mathrm{Ph}=1$ while the product precipitated. Filtration and drying in vacuo gave the acid. Which was used directly without further purification.

To a stirred solution of the acid (crude, obtain above) and $\mathrm{ZnCl}_{2}$ (1.5 equiv.) was added Phosphorus oxychloride $(0.2 \mathrm{M})$. The resulting mixture was stirred at RT overnight. Then the mixture was diluted with DCM $(20 \mathrm{~mL})$ and poured into ice $(100 \mathrm{~g})$ with vigorous agitation. The organic layers were separated and the aqueous layer was extracted with DCM $(3 \times 20 \mathrm{~mL})$. The combined organic layers were washed with brine, dried with $\mathrm{Na}_{2} \mathrm{SO}_{4}$ and concentrated in vacuo. The desired product $\mathbf{S} 7 \mathbf{h}^{\prime}$ was purified using flash chromatography (petroleum ether/EA=2:1) as a white solid $(1.7 \mathrm{~g}, 62 \%$ for 3 steps).

\footnotetext{
$\underline{R}_{f} \quad 0.25\left(\mathrm{Hex} / \mathrm{EtOAc}=3: 1, \mathrm{KMnO}_{4}\right)$

$\underline{{ }^{1} \mathrm{H} \mathrm{NMR}} \quad\left(500 \mathrm{MHz}, \mathrm{CDCl}_{3}\right)$

$\delta 7.46-7.28(\mathrm{~m}, 5 \mathrm{H}), 6.26(\mathrm{~s}, 1 \mathrm{H}), 6.04(\mathrm{~s}, 1 \mathrm{H}), 5.48(\mathrm{~s}, 1 \mathrm{H}), 3.89(\mathrm{~s}, 6 \mathrm{H})$.

${ }^{13} \mathrm{C} \mathrm{NMR} \quad\left(125 \mathrm{MHz}, \mathrm{CDCl}_{3}\right)$

$\delta 194.4,176.1,170.2,159.6,134.7,128.8,128.8,126.3,103.9,93.4,89.0,86.8,56.2$.

HRMS (ESI) for: $\mathrm{C}_{16} \mathrm{H}_{15} \mathrm{O} 4[\mathrm{M}+\mathrm{H}]^{+}$: calcd 271.0790, found 271.1008 .
} 


\section{2: General Procedure}

To a solution of NaHMDS (1.2 equiv., $2 \mathrm{M}$ in THF) in THF $(10 \mathrm{~mL} / \mathrm{mmol})$ at $-78{ }^{\circ} \mathrm{C}$ was added TMEDA (3.0 equiv.). A solution of $\mathbf{S 7}$ (1.0 equiv.) in THF was added drop-wise. After $1 \mathrm{~h}$ at $-78{ }^{\circ} \mathrm{C}$, allyl chloroformate (3.0 equiv.) was added, stirred for further $45 \mathrm{~min}$ before it was quenched with a saturated aqueous solution of $\mathrm{NH}_{4} \mathrm{Cl}$. The aqueous phase was extracted with EtOAc and the combined organic phases were dried over $\mathrm{Na}_{2} \mathrm{SO}_{4}$, filtered and evaporated under reduced pressure to afford a crude residue, which was purified by flash column chromatography over silica gel to afford the corresponding allyl enol carbonate 12.

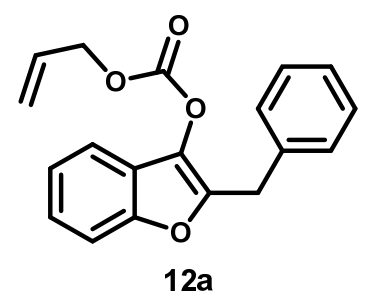

Allyl (2-benzylbenzofuran-3-yl) carbonate (12a). Following the General Procedure to yield 12a as a white solid (1.5 g, 98\% yield).

$\underline{R}_{f} \quad 0.50\left(\mathrm{Hex} / \mathrm{EtOAc}=20: 1, \mathrm{KMnO}_{4}\right)$

${ }^{1} \mathrm{H} \mathrm{NMR} \quad\left(400 \mathrm{MHz}, \mathrm{CDCl}_{3}\right)$

$\delta 7.36-7.29(\mathrm{~m}, 1 \mathrm{H}), 7.29-7.23(\mathrm{~m}, 1 \mathrm{H}), 7.18(\mathrm{~d}, J=3.1 \mathrm{~Hz}, 4 \mathrm{H}), 7.16-7.08(\mathrm{~m}$, $3 \mathrm{H}), 5.96-5.81(\mathrm{~m}, 1 \mathrm{H}), 5.44-5.13(\mathrm{~m}, 2 \mathrm{H}), 4.64(\mathrm{dt}, J=5.9,1.3 \mathrm{~Hz}, 2 \mathrm{H}), 4.00(\mathrm{~s}$, $2 \mathrm{H})$.

${ }^{13} \mathrm{C} \mathrm{NMR} \quad\left(100 \mathrm{MHz}, \mathrm{CDCl}_{3}\right)$

$\delta 152.8,152.4,146.0,136.5,131.1,130.9,128.9,128.7,126.9,124.5,123.0,122.7$, $119.9,118.2,111.8,69.8,31.9$.

HRMS (ESI) for: $\mathrm{C}_{19} \mathrm{H}_{16} \mathrm{NaO}_{4}[\mathrm{M}+\mathrm{Na}]^{+}$: calcd 331.0946, found 331.0954 .

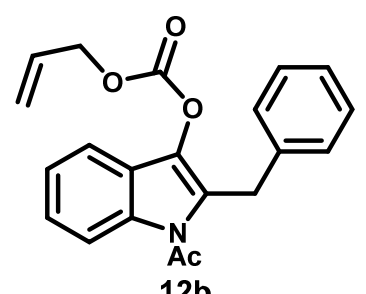

1-Acetyl-2-benzyl-1H-indol-3-yl allyl carbonate (12b). Following the General Procedure to yield $\mathbf{1 2 b}$ as a yellow solid (1.3 g, $82 \%$ yield), while its starting material was prepared according to a known procedure. ${ }^{5}$

$R_{f} \quad 0.40\left(\mathrm{Hex} / \mathrm{EtOAc}=10: 1, \mathrm{KMnO}_{4}\right)$

${ }^{1} \mathrm{H} \mathrm{NMR} \quad\left(500 \mathrm{MHz}, \mathrm{CDCl}_{3}\right)$

$\delta 7.83(\mathrm{~d}, J=7.6 \mathrm{~Hz}, 1 \mathrm{H}), 7.36(\mathrm{~d}, J=8.9 \mathrm{~Hz}, 1 \mathrm{H}), 7.25-7.18(\mathrm{~m}, 2 \mathrm{H}), 7.16(\mathrm{t}, J=7.4$

$\mathrm{Hz}, 2 \mathrm{H}), 7.11-7.04(\mathrm{~m}, 3 \mathrm{H}), 5.93-5.84(\mathrm{~m}, 1 \mathrm{H}), 5.33(\mathrm{~d}, J=21.2 \mathrm{~Hz}, 1 \mathrm{H}), 5.24(\mathrm{~d}, J$

$=11.6 \mathrm{~Hz}, 1 \mathrm{H}), 4.66(\mathrm{~d}, J=5.9 \mathrm{~Hz}, 2 \mathrm{H}), 4.30(\mathrm{~s}, 2 \mathrm{H}), 2.48(\mathrm{~s}, 3 \mathrm{H})$.

${ }^{13} \mathrm{C} \mathrm{NMR} \quad\left(125 \mathrm{MHz}, \mathrm{CDCl}_{3}\right)$

$\delta 169.8,153.1,138.1,134.9,133.8,130.9,128.6,128.2,126.5,125.2,123.5,123.1$, $119.9,117.54,115.4,69.8,31.4,27.2$.

HRMS (ESI) for: $\mathrm{C}_{21} \mathrm{H}_{20} \mathrm{NO}_{4}[\mathrm{M}+\mathrm{H}]^{+}$: calcd 350.1392, found 350.1379 . 


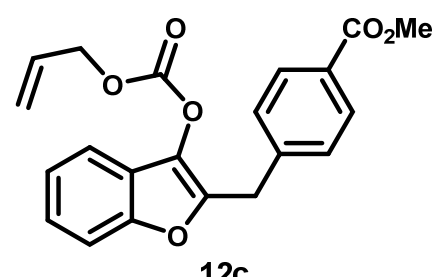

$12 \mathrm{c}$

Methyl

4-((3-(((allyloxy)carbonyl)oxy)benzofuran-2-

yl)methyl)benzoate (12c). Following the General Procedure to yield 12c as a white solid $(1.7 \mathrm{~g}, 92 \%$ yield).

$R_{f} \quad 0.30\left(\mathrm{Hex} / \mathrm{EtOAc}=20: 1, \mathrm{KMnO}_{4}\right)$

${ }^{1} \mathrm{H} \mathrm{NMR} \quad\left(400 \mathrm{MHz}, \mathrm{CDCl}_{3}\right)$

$\delta 8.03-7.90(\mathrm{~m}, 2 \mathrm{H}), 7.45-7.40(\mathrm{~m}, 1 \mathrm{H}), 7.39-7.32(\mathrm{~m}, 3 \mathrm{H}), 7.29-7.19(\mathrm{~m}, 2 \mathrm{H})$, $6.10-5.91(\mathrm{~m}, 1 \mathrm{H}), 5.53-5.29(\mathrm{~m}, 2 \mathrm{H}), 4.75(\mathrm{~d}, J=5.9 \mathrm{~Hz}, 2 \mathrm{H}), 4.15(\mathrm{~s}, 2 \mathrm{H}), 3.89(\mathrm{~s}$, $3 \mathrm{H})$.

${ }^{13} \mathrm{C} \mathrm{NMR} \quad\left(100 \mathrm{MHz}, \mathrm{CDCl}_{3}\right)$

$\delta 167.0,152.8,152.5,145.0,141.8,131.5,130.9,130.1,129.0,128.9,124.7,123.2$, 122.6, 120.1, 118.4, 111.8, 69.9, 52.2, 31.9.

HRMS (ESI) for: $\mathrm{C}_{21} \mathrm{H}_{18} \mathrm{NaO}_{6}[\mathrm{M}+\mathrm{Na}]^{+}$: calcd 389.1001, found 389.1027 .<smiles>C=CCOC(=O)Oc1c(Cc2ccc(C#N)cc2)oc2ccccc12</smiles>

Allyl (2-(4-cyanobenzyl)benzofuran-3-yl) carbonate (12d). Following the General Procedure to yield $\mathbf{1 2 d}$ as a yellow solid (1.6 g, 95\% yield).

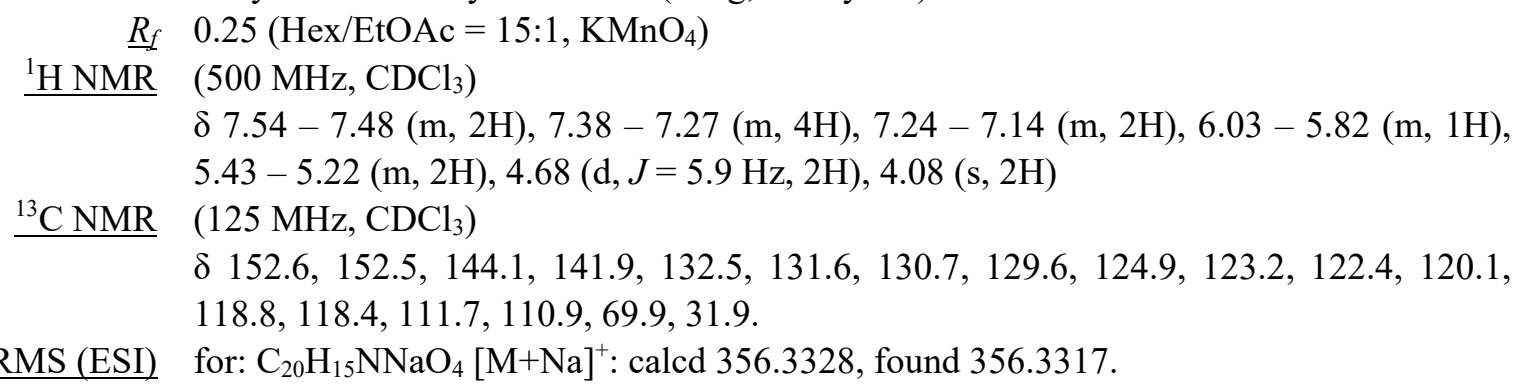<smiles>C=CCOC(=O)Oc1c(Cc2ccc(C(F)(F)F)cc2)oc2ccccc12</smiles>

Allyl (2-(4-(trifluoromethyl)benzyl)benzofuran-3-yl) carbonate (12e). Following the General Procedure to yield $\mathbf{1 2 e}$ as a white solid (1.8 g, 95\% yield).

$\underline{R}_{f} \quad 0.45\left(\mathrm{Hex} / \mathrm{EtOAc}=20: 1, \mathrm{KMnO}_{4}\right)$

$\underline{{ }^{1} \mathrm{H} \mathrm{NMR}} \quad\left(500 \mathrm{MHz}, \mathrm{CDCl}_{3}\right)$

$\delta 7.49(\mathrm{~d}, J=8.1 \mathrm{~Hz}, 2 \mathrm{H}), 7.43-7.26(\mathrm{~m}, 4 \mathrm{H}), 7.26-7.09(\mathrm{~m}, 2 \mathrm{H}), 6.06-5.79(\mathrm{~m}$, $1 \mathrm{H}), 5.47-5.20(\mathrm{~m}, 2 \mathrm{H}), 4.68(\mathrm{~d}, J=5.9 \mathrm{~Hz}, 2 \mathrm{H}), 4.09(\mathrm{~s}, 2 \mathrm{H})$. 
${ }^{13} \mathrm{C} \mathrm{NMR} \quad\left(125 \mathrm{MHz}, \mathrm{CDCl}_{3}\right)$

$\delta 152.7,152.4,144.7,140.4,131.4,130.7,129.4,129.2,129.1,125.6,125.6,125.6$, $125.5,125.3,124.7,123.1,123.1,122.5,120.0,118.3,111.7,69.8,31.7$.

${ }^{19} \mathrm{~F} \mathrm{NMR} \quad\left(470 \mathrm{MHz}, \mathrm{CDCl}_{3}\right)$

$\delta-62.48$.

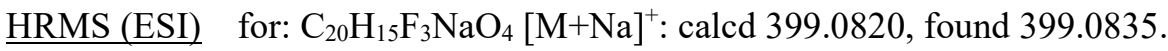

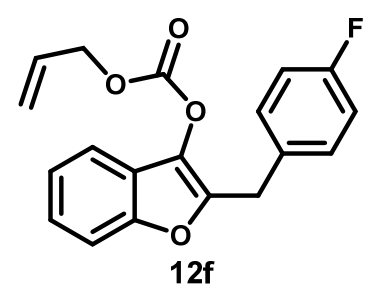

Allyl (2-(4-fluorobenzyl)benzofuran-3-yl) carbonate (12f). Following the General Procedure to yield $\mathbf{1 2 f}$ as a white solid (1.5 g, 90\% yield).

$\underline{R}_{f} \quad 0.45\left(\mathrm{Hex} / \mathrm{EtOAc}=20: 1, \mathrm{KMnO}_{4}\right)$

${ }^{1} \mathrm{H} \mathrm{NMR} \quad\left(400 \mathrm{MHz}, \mathrm{CDCl}_{3}\right)$

反 $7.42-7.37(\mathrm{~m}, 1 \mathrm{H}), 7.36-7.31(\mathrm{~m}, 1 \mathrm{H}), 7.30-7.26(\mathrm{~m}, 2 \mathrm{H}), 7.26-7.23(\mathrm{~m}, 1 \mathrm{H})$,

$7.23-7.18(\mathrm{~m}, 3 \mathrm{H}), 6.09-5.87(\mathrm{~m}, 1 \mathrm{H}), 5.53-5.22(\mathrm{~m}, 2 \mathrm{H}), 4.72(\mathrm{~d}, J=5.9 \mathrm{~Hz}, 2 \mathrm{H})$, $4.08(\mathrm{~s}, 2 \mathrm{H})$.

${ }^{13} \mathrm{C} \mathrm{NMR} \quad\left(100 \mathrm{MHz}, \mathrm{CDCl}_{3}\right)$

$\delta 152.8,152.5,146.0,136.5,131.2,131.0,128.9,128.8,126.9,124.5,123.1,122.7$, $119.9,118.3,111.8,69.8,31.9$.

${ }^{19} \mathrm{~F} \mathrm{NMR} \quad\left(470 \mathrm{MHz}, \mathrm{CDCl}_{3}\right)$

$\delta-116.18$.

HRMS (ESI) for: $\mathrm{C}_{19} \mathrm{H}_{15} \mathrm{FNaO}_{4}[\mathrm{M}+\mathrm{Na}]^{+}$: calcd 349.0852, found 349.0859 .

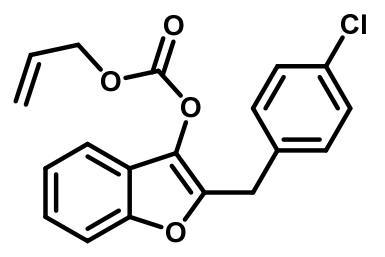

12g

Allyl (2-(4-chlorobenzyl)benzofuran-3-yl) carbonate (12g). Following the General Procedure to yield $\mathbf{1 2 g}$ as a white solid (1.6 g, 92\% yield).

$\underline{R}_{f} \quad 0.30\left(\mathrm{Hex} / \mathrm{EtOAc}=20: 1, \mathrm{KMnO}_{4}\right)$

${ }^{1} \mathrm{H} \mathrm{NMR} \quad\left(600 \mathrm{MHz}, \mathrm{CDCl}_{3}\right)$

$\delta 7.39-7.25(\mathrm{~m}, 2 \mathrm{H}), 7.28-7.09(\mathrm{~m}, 6 \mathrm{H}), 6.03-5.81(\mathrm{~m}, 1 \mathrm{H}), 5.45-5.25(\mathrm{~m}, 2 \mathrm{H})$, $4.72-4.62(\mathrm{~m}, 2 \mathrm{H}), 4.00(\mathrm{~s}, 2 \mathrm{H})$.

${ }^{13} \mathrm{C} \mathrm{NMR} \quad\left(150 \mathrm{MHz}, \mathrm{CDCl}_{3}\right)$

$\delta 152.7,152.4,145.3,134.8,132.7,131.2,130.8,130.2,128.8,124.6,123.1,122.5$, $120.0,118.2,111.7,69.8,31.2$.

$\underline{\text { HRMS (ESI) }}$ for: $\mathrm{C}_{19} \mathrm{H}_{15} \mathrm{ClNaO}_{4}[\mathrm{M}+\mathrm{Na}]^{+}$: calcd 365.0557, found 365.0551. 


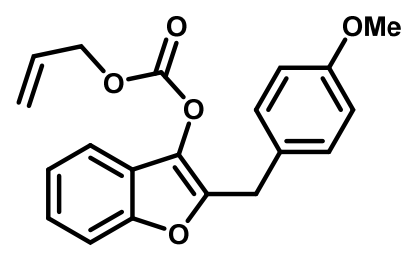

$12 \mathrm{~h}$

Allyl (2-(4-methoxybenzyl)benzofuran-3-yl) carbonate (12h). Following the General Procedure to yield $\mathbf{1 2 h}$ as a white solid (1.6 g, 93\% yield).

$\underline{R_{f}} \quad 0.35\left(\mathrm{Hex} / \mathrm{EtOAc}=20: 1, \mathrm{KMnO}_{4}\right)$

${ }^{1} \mathrm{H} \mathrm{NMR} \quad\left(400 \mathrm{MHz}, \mathrm{CDCl}_{3}\right)$

$\delta 7.45-7.34(\mathrm{~m}, 2 \mathrm{H}), 7.28-7.18(\mathrm{~m}, 4 \mathrm{H}), 6.88-6.81(\mathrm{~m}, 2 \mathrm{H}), 6.12-5.89(\mathrm{~m}, 1 \mathrm{H})$,

$5.54-5.25(\mathrm{~m}, 2 \mathrm{H}), 4.76(\mathrm{dt}, J=5.8,1.4 \mathrm{~Hz}, 2 \mathrm{H}), 4.05(\mathrm{~s}, 2 \mathrm{H}), 3.78(\mathrm{~s}, 3 \mathrm{H})$.

${ }^{13} \mathrm{C} \mathrm{NMR} \quad\left(100 \mathrm{MHz}, \mathrm{CDCl}_{3}\right)$

$\delta 158.6,152.9,152.4,146.4,131.0,130.9,129.9,128.5,124.4,123.0,122.8,119.9$, 118.2, 114.1, 111.8, 69.8, 55.4, 31.1.

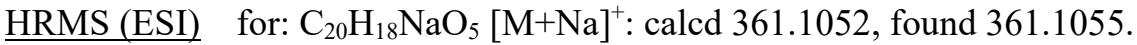

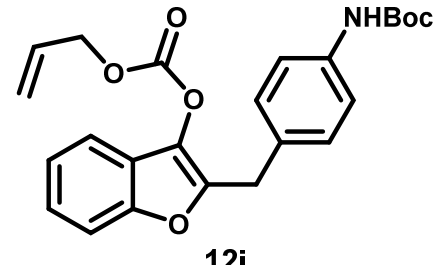

$12 \mathbf{i}$

Tert-butyl

(4-((3-(((Allyloxy)carbonyl)oxy)benzofuran-2-

yl)methyl)phenyl)carbamate (12i). Following the General Procedure to yield 12i as a yellow solid (1.9 g, $88 \%$ yield).

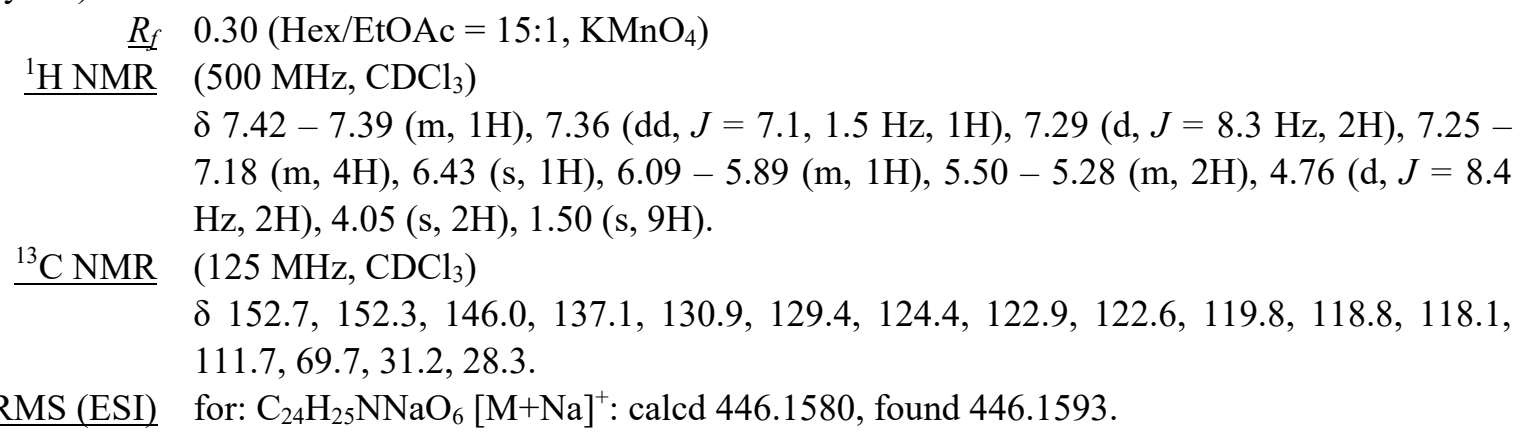<smiles>C=CCOC(=O)Oc1c(Cc2cccc(OC)c2)oc2ccccc12</smiles>

$12 \mathrm{j}$

Allyl (2-(3-methoxybenzyl)benzofuran-3-yl) carbonate (12j). Following the General Procedure to yield $\mathbf{1 2} \mathbf{j}$ as a colorless oil (1.5 g, 91\% yield).

$\underline{R}_{f} \quad 0.35\left(\mathrm{Hex} / \mathrm{EtOAc}=20: 1, \mathrm{KMnO}_{4}\right)$

${ }^{1} \mathrm{H} \mathrm{NMR} \quad\left(400 \mathrm{MHz}, \mathrm{CDCl}_{3}\right)$

$\delta 7.54-7.33(\mathrm{~m}, 2 \mathrm{H}), 7.29-7.13(\mathrm{~m}, 3 \mathrm{H}), 6.92-6.75(\mathrm{~m}, 3 \mathrm{H}), 6.09-5.88(\mathrm{~m}, 1 \mathrm{H})$, $5.49-5.28(\mathrm{~m}, 2 \mathrm{H}), 4.82-4.65(\mathrm{~m}, 2 \mathrm{H}), 4.08(\mathrm{~s}, 2 \mathrm{H}), 3.77(\mathrm{~s}, 3 \mathrm{H})$.

${ }^{13} \mathrm{C} \mathrm{NMR} \quad\left(100 \mathrm{MHz}, \mathrm{CDCl}_{3}\right)$

$\delta 159.9,152.8,152.5,145.9,138.0,131.2,131.0,129.7,124.5,123.1,122.7,121.2$, 
$119.9,118.2,114.5,112.5,111.8,69.8,55.3,31.9$.

HRMS (ESI) for: $\mathrm{C}_{20} \mathrm{H}_{18} \mathrm{NaO}_{5}[\mathrm{M}+\mathrm{Na}]^{+}$: calcd 361.1052 , found 361.1057 .

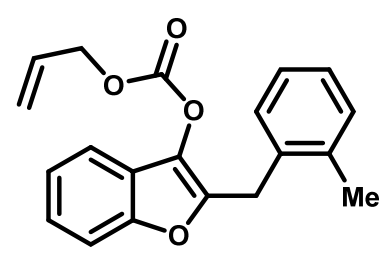

$12 k$

Allyl (2-(2-methylbenzyl)benzofuran-3-yl) carbonate (12k). Following the General Procedure to yield $\mathbf{1 2 k}$ as a white solid (1.5 g, 96\% yield).

$\underline{R}_{f} \quad 0.40\left(\mathrm{Hex} / \mathrm{EtOAc}=20: 1, \mathrm{KMnO}_{4}\right)$

${ }^{1} \mathrm{H} \mathrm{NMR} \quad\left(500 \mathrm{MHz}, \mathrm{CDCl}_{3}\right)$

$\delta 7.34-7.25(\mathrm{~m}, 2 \mathrm{H}), 7.20-7.11(\mathrm{~m}, 3 \mathrm{H}), 7.11-7.04(\mathrm{~m}, 3 \mathrm{H}), 5.97-5.85(\mathrm{~m}, 1 \mathrm{H})$, $5.40-5.23(\mathrm{~m}, 2 \mathrm{H}), 4.64(\mathrm{~d}, J=5.9 \mathrm{~Hz}, 2 \mathrm{H}), 4.02(\mathrm{~s}, 2 \mathrm{H}), 2.28(\mathrm{~s}, 3 \mathrm{H})$.

${ }^{13} \mathrm{C} \mathrm{NMR} \quad\left(125 \mathrm{MHz}, \mathrm{CDCl}_{3}\right)$

$\delta 152.6,152.3,145.5,136.6,134.5,131.0,130.9,130.3,129.6,127.1,126.2,124.3$, $122.9,122.7,119.8,118.1,111.7,77.3,77.2,77.0,76.8,69.7,29.7,19.6$.

$\underline{\mathrm{HRMS}(\mathrm{ESI})}$ for: $\mathrm{C}_{20} \mathrm{H}_{18} \mathrm{NaO}_{4}[\mathrm{M}+\mathrm{Na}]^{+}$: calcd 345.1103 , found 345.1114 .

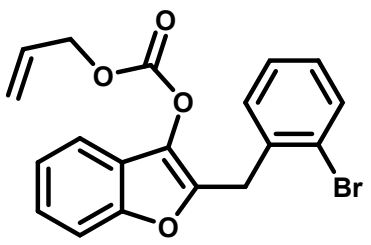

12 I

Allyl (2-(2-bromobenzyl)benzofuran-3-yl) carbonate (12l). Following the General Procedure to yield $\mathbf{1 2 l}$ as a white solid (1.7 g, $90 \%$ yield).

$\underline{R_{f}} \quad 0.45\left(\mathrm{Hex} / \mathrm{EtOAc}=20: 1, \mathrm{KMnO}_{4}\right)$

${ }^{1} \mathrm{H} \mathrm{NMR} \quad\left(400 \mathrm{MHz}, \mathrm{CDCl}_{3}\right)$

$\delta 7.42-7.32(\mathrm{~m}, 2 \mathrm{H}), 7.31-7.26(\mathrm{~m}, 2 \mathrm{H}), 7.25-7.15(\mathrm{~m}, 4 \mathrm{H}), 6.05-5.87(\mathrm{~m}, 1 \mathrm{H})$, $5.50-5.22(\mathrm{~m}, 2 \mathrm{H}), 4.72(\mathrm{dt}, J=5.9,1.3 \mathrm{~Hz}, 2 \mathrm{H}), 4.08(\mathrm{~s}, 2 \mathrm{H})$.

${ }^{13} \mathrm{C} \mathrm{NMR} \quad\left(100 \mathrm{MHz}, \mathrm{CDCl}_{3}\right)$

$\delta 152.8,152.5,146.0,136.5,131.2,131.0,128.9,128.8,126.9,124.5,123.1,122.7$, 120.0, 118.3, 111.8, 69.8, 31.9.

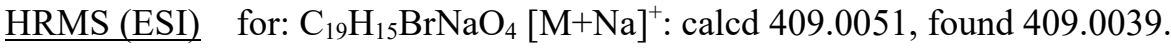

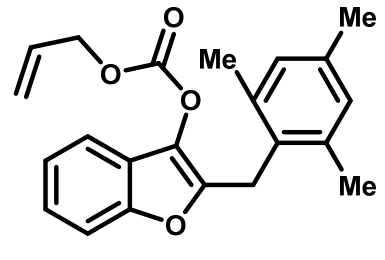

$12 \mathrm{~m}$

Allyl (2-(2,4,6-trimethylbenzyl)benzofuran-3-yl) carbonate (12m). Following the General Procedure to yield $\mathbf{1 2 m}$ as a white solid (1.7 g, 96\% yield).

$\underline{R}_{f} \quad 0.55\left(\mathrm{Hex} / \mathrm{EtOAc}=20: 1, \mathrm{KMnO}_{4}\right)$

${ }^{1} \mathrm{H} \mathrm{NMR} \quad\left(600 \mathrm{MHz}, \mathrm{CDCl}_{3}\right)$

$\delta 7.37-7.31(\mathrm{~m}, 2 \mathrm{H}), 7.24-7.17(\mathrm{~m}, 2 \mathrm{H}), 6.88(\mathrm{~s}, 2 \mathrm{H}), 6.03-5.91(\mathrm{~m}, 1 \mathrm{H}), 5.46-$

$5.31(\mathrm{~m}, 2 \mathrm{H}), 4.66(\mathrm{dt}, J=5.9,1.3 \mathrm{~Hz}, 2 \mathrm{H}), 4.09(\mathrm{~s}, 2 \mathrm{H}), 2.31(\mathrm{~d}, J=53.6 \mathrm{~Hz}, 9 \mathrm{H})$. 
${ }^{13} \mathrm{C} \mathrm{NMR} \quad\left(150 \mathrm{MHz}, \mathrm{CDCl}_{3}\right)$

$\delta 152.6,152.1,145.4,137.3,136.3,131.0,130.4,129.4,128.9,124.1,122.8,122.8$, 119.6, 117.9, 111.7, 69.5, 26.1, 20.9, 20.2.

HRMS (ESI) for: $\mathrm{C}_{22} \mathrm{H}_{22} \mathrm{NaO}_{4}[\mathrm{M}+\mathrm{Na}]^{+}$: calcd 373.1416 , found 373.1419 .<smiles>C=CCOC(=O)Oc1c(Cc2ccc3ccccc3c2)oc2ccccc12</smiles>

Allyl (2-(naphthalen-2-ylmethyl)benzofuran-3-yl) carbonate (12n). Following the General Procedure to yield 12n as a yellow solid (1.6 g, 90\% yield).

$\underline{R}_{f} \quad 0.50\left(\mathrm{Hex} / \mathrm{EtOAc}=20: 1, \mathrm{KMnO}_{4}\right)$

${ }^{1} \mathrm{H} \mathrm{NMR} \quad\left(400 \mathrm{MHz}, \mathrm{CDCl}_{3}\right)$

$\delta 7.84-7.72(\mathrm{~m}, 4 \mathrm{H}), 7.50-7.35(\mathrm{~m}, 5 \mathrm{H}), 7.29-7.21(\mathrm{~m}, 2 \mathrm{H}), 6.02-5.84(\mathrm{~m}, 1 \mathrm{H})$, $5.48-5.27(\mathrm{~m}, 2 \mathrm{H}), 4.70(\mathrm{dt}, J=5.8,1.3 \mathrm{~Hz}, 2 \mathrm{H}), 4.29(\mathrm{~s}, 2 \mathrm{H})$.

${ }^{13} \mathrm{C} \mathrm{NMR} \quad\left(100 \mathrm{MHz}, \mathrm{CDCl}_{3}\right)$

$\delta 152.8,152.5,146.0,133.9,133.7,132.5,131.3,130.9,128.5,127.8,127.4,127.2$, $126.3,125.8,124.6,123.1,122.8,120.0,118.3,111.9,69.8,32.2$.

HRMS (ESI) for: $\mathrm{C}_{23} \mathrm{H}_{18} \mathrm{NaO}_{4}[\mathrm{M}+\mathrm{Na}]^{+}$: calcd 381.1103 , found 381.1106 .<smiles>C=CCOC(=O)Oc1c(Cc2cccc3ccccc23)oc2ccccc12</smiles>

Allyl (2-(naphthalen-1-ylmethyl)benzofuran-3-yl) carbonate (12o). Following the General Procedure to yield $\mathbf{1 2 0}$ as a white solid (1.6 g, 90\% yield).

$\underline{R}_{f} \quad 0.50\left(\mathrm{Hex} / \mathrm{EtOAc}=20: 1, \mathrm{KMnO}_{4}\right)$

$\underline{{ }^{1} \mathrm{H} \mathrm{NMR}} \quad\left(400 \mathrm{MHz}, \mathrm{CDCl}_{3}\right)$

$\delta 8.11(\mathrm{dd}, J=7.8,1.3 \mathrm{~Hz}, 1 \mathrm{H}), 7.89-7.74(\mathrm{~m}, 2 \mathrm{H}), 7.56-7.46(\mathrm{~m}, 2 \mathrm{H}), 7.44-7.38$ $(\mathrm{m}, 3 \mathrm{H}), 7.38-7.33(\mathrm{~m}, 1 \mathrm{H}), 7.27-7.18(\mathrm{~m}, 2 \mathrm{H}), 6.05-5.88(\mathrm{~m}, 1 \mathrm{H}), 5.56-5.21(\mathrm{~m}$, $2 \mathrm{H}), 4.69(\mathrm{dt}, J=5.9,1.4 \mathrm{~Hz}, 2 \mathrm{H}), 4.56(\mathrm{~s}, 2 \mathrm{H})$.

${ }^{13} \mathrm{C} \mathrm{NMR} \quad\left(100 \mathrm{MHz}, \mathrm{CDCl}_{3}\right)$

$\delta 152.6,152.4,145.6,133.9,132.3,132.0,131.2,130.9,128.8,127.9,127.2,126.4$, 125.9, 125.6, 124.5, 123.6, 123.1, 122.8, 119.9, 118.3, 111.8, 69.8, 29.6.

for: $\mathrm{C}_{23} \mathrm{H}_{18} \mathrm{NaO}_{4}[\mathrm{M}+\mathrm{Na}]^{+}$: calcd 381.1103, found 381.1108.

$\underline{\text { HRMS (ESI) }}$

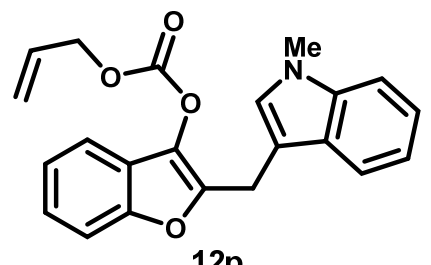

$12 p$

Allyl (2-((1-methyl-1H-indol-3-yl)methyl)benzofuran-3-yl) carbonate (12p). Following the General Procedure to yield 12p as a yellow solid (1.5 g, 85\% yield).

$\underline{R}_{f} \quad 0.35\left(\mathrm{Hex} / \mathrm{EtOAc}=15: 1, \mathrm{KMnO}_{4}\right)$ 
鼻 NMR $\quad\left(400 \mathrm{MHz}, \mathrm{CDCl}_{3}\right)$

$\delta 7.66(\mathrm{dt}, J=7.9,1.0 \mathrm{~Hz}, 1 \mathrm{H}), 7.50-7.33(\mathrm{~m}, 2 \mathrm{H}), 7.33-7.19(\mathrm{~m}, 4 \mathrm{H}), 7.17-7.09$ $(\mathrm{m}, 1 \mathrm{H}), 6.97(\mathrm{~s}, 1 \mathrm{H}), 6.07-5.93(\mathrm{~m}, 1 \mathrm{H}), 5.51-5.32(\mathrm{~m}, 2 \mathrm{H}), 4.74(\mathrm{dt}, J=5.8,1.4 \mathrm{~Hz}$, $2 \mathrm{H}), 4.26(\mathrm{~d}, J=0.9 \mathrm{~Hz}, 2 \mathrm{H}), 3.73(\mathrm{~s}, 3 \mathrm{H})$.

${ }^{13} \mathrm{C} \mathrm{NMR} \quad\left(100 \mathrm{MHz}, \mathrm{CDCl}_{3}\right)$ $\delta 152.9,152.3,146.6,137.1,131.1,130.5,127.7,127.5,124.3,123.0,122.9,121.9$, $119.9,119.2,119.0,118.2,111.8,109.4,109.2,69.8,32.8,21.9$.

HRMS (ESI) for: $\mathrm{C}_{22} \mathrm{H}_{19} \mathrm{NNaO}_{4}[\mathrm{M}+\mathrm{Na}]^{+}$: calcd 384.1212, found 384.1215 .<smiles>C=CCOC(=O)Oc1c(Cc2ccco2)oc2ccccc12</smiles>

Allyl (2-(furan-3-ylmethyl)benzofuran-3-yl) carbonate (12q). Following the General Procedure to yield $\mathbf{1 2 q}$ as a yellow oil (1.3 g, 86\% yield).

$\underline{R}_{f} \quad 0.35\left(\mathrm{Hex} / \mathrm{EtOAc}=15: 1, \mathrm{KMnO}_{4}\right)$

${ }^{1} \mathrm{H} \mathrm{NMR} \quad\left(400 \mathrm{MHz}, \mathrm{CDCl}_{3}\right)$

$\delta 7.44-7.38(\mathrm{~m}, 2 \mathrm{H}), 7.33(\mathrm{dd}, J=1.8,0.8 \mathrm{~Hz}, 1 \mathrm{H}), 7.30-7.19(\mathrm{~m}, 2 \mathrm{H}), 6.30(\mathrm{dd}, J=$ $3.2,1.9 \mathrm{~Hz}, 1 \mathrm{H}), 6.23-6.10(\mathrm{~m}, 1 \mathrm{H}), 6.10-5.89(\mathrm{~m}, 1 \mathrm{H}), 5.55-5.27(\mathrm{~m}, 2 \mathrm{H}), 4.86-$ $4.70(\mathrm{~m}, 2 \mathrm{H}), 4.14(\mathrm{~s}, 2 \mathrm{H})$.

${ }^{13} \mathrm{C} \mathrm{NMR} \quad\left(100 \mathrm{MHz}, \mathrm{CDCl}_{3}\right)$

$\delta 152.7,152.4,149.6,143.2,142.0,131.3,131.0,124.7,123.1,122.6,119.9,118.4$, $111.8,110.6,107.2,69.8,25.2$.

HRMS (ESI) for: $\mathrm{C}_{17} \mathrm{H}_{14} \mathrm{NaO}_{5}[\mathrm{M}+\mathrm{Na}]^{+}$: calcd 322.0772 , found 322.0785 .

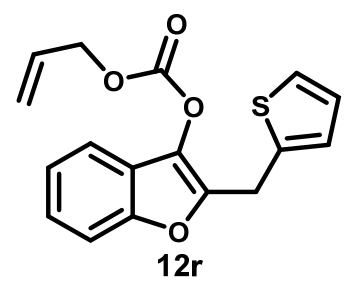

Allyl (2-(thiophen-3-ylmethyl)benzofuran-3-yl) carbonate (12r). Following the General Procedure to yield $12 \mathrm{r}$ as a yellow solid (1.4 $\mathrm{g}, 88 \%$ yield).

$\underline{R}_{f} \quad 0.30\left(\mathrm{Hex} / \mathrm{EtOAc}=15: 1, \mathrm{KMnO}_{4}\right)$

${ }^{1} \mathrm{H} \mathrm{NMR} \quad\left(400 \mathrm{MHz}, \mathrm{CDCl}_{3}\right)$

$\delta 7.46-7.37(\mathrm{~m}, 2 \mathrm{H}), 7.30-7.21(\mathrm{~m}, 2 \mathrm{H}), 7.20-7.15(\mathrm{~m}, 1 \mathrm{H}), 6.97-6.91(\mathrm{~m}, 2 \mathrm{H})$, $6.11-5.91(\mathrm{~m}, 1 \mathrm{H}), 5.59-5.24(\mathrm{~m}, 2 \mathrm{H}), 4.78(\mathrm{dt}, J=5.9,1.3 \mathrm{~Hz}, 2 \mathrm{H}), 4.30(\mathrm{~s}, 2 \mathrm{H})$.

${ }^{13} \mathrm{C} \mathrm{NMR} \quad\left(100 \mathrm{MHz}, \mathrm{CDCl}_{3}\right)$

$\delta 152.7,152.4,144.9,138.3,130.9,130.9,127.1,126.2,124.7,124.6,123.2,122.6$, $120.0,118.5,111.9,69.9,26.3$.

$\underline{\text { HRMS (ESI) }}$ for: $\mathrm{C}_{17} \mathrm{H}_{14} \mathrm{NaO}_{4} \mathrm{~S}[\mathrm{M}+\mathrm{Na}]^{+}$: calcd 377.0510, found 377.0513 . 


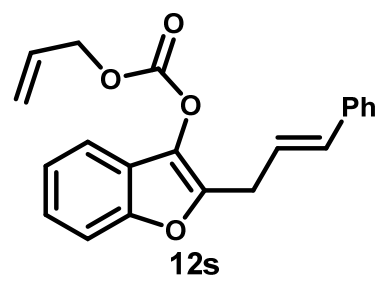

(E)-Allyl (2-(3-phenylprop-1-en-1-yl)benzofuran-3-yl) carbonate (12s). Following the General Procedure to yield $\mathbf{1 2 s}$ as a white solid (1.6 $\mathrm{g}, 95 \%$ yield).

$\underline{R}_{f} \quad 0.40\left(\mathrm{Hex} / \mathrm{EtOAc}=20: 1, \mathrm{KMnO}_{4}\right)$

${ }^{1} \mathrm{H} \mathrm{NMR} \quad\left(500 \mathrm{MHz}, \mathrm{CDCl}_{3}\right)$

$\delta 7.39-7.25(\mathrm{~m}, 4 \mathrm{H}), 7.25-7.09(\mathrm{~m}, 5 \mathrm{H}), 6.49(\mathrm{~d}, J=15.8 \mathrm{~Hz}, 1 \mathrm{H}), 6.35-6.15(\mathrm{~m}$, $1 \mathrm{H}), 5.96-5.78(\mathrm{~m}, 1 \mathrm{H}), 5.38-5.16(\mathrm{~m}, 2 \mathrm{H}), 4.62(\mathrm{~d}, J=5.9 \mathrm{~Hz}, 2 \mathrm{H}), 3.61(\mathrm{~d}, J=8.0$ $\mathrm{Hz}, 2 \mathrm{H})$.

${ }^{13} \mathrm{C} \mathrm{NMR} \quad\left(125 \mathrm{MHz}, \mathrm{CDCl}_{3}\right)$

$\delta 152.8,152.3,145.4,137.0,132.9,130.9,130.9,128.6,127.5,126.3,124.4,123.5$, 123.0, 122.8, 119.8, 118.1, 111.7, 69.7, 29.5.

HRMS (ESI) for: $\mathrm{C}_{21} \mathrm{H}_{18} \mathrm{NaO}_{4}[\mathrm{M}+\mathrm{Na}]^{+}$: calcd 357.1103, found 357.1108.

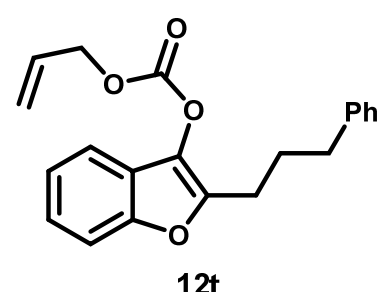

12t Allyl (2-(3-phenylpropyl)benzofuran-3-yl) carbonate (12t). Following the General Procedure to yield $\mathbf{1 2 t}$ as a colorless oil (1.6 $\mathrm{g}, 94 \%$ yield).

$\underline{R}_{f} \quad 0.40\left(\mathrm{Hex} / \mathrm{EtOAc}=20: 1, \mathrm{KMnO}_{4}\right)$

${ }^{1} \mathrm{H} \mathrm{NMR} \quad\left(500 \mathrm{MHz}, \mathrm{CDCl}_{3}\right)$

$\delta 7.31(\mathrm{dt}, J=7.6,1.4 \mathrm{~Hz}, 2 \mathrm{H}), 7.23-7.06(\mathrm{~m}, 7 \mathrm{H}), 5.98-5.87(\mathrm{~m}, 1 \mathrm{H}), 5.4-5.23(\mathrm{~m}$, 2H), 4.69 (dt, $J=5.8,1.4 \mathrm{~Hz}, 2 \mathrm{H}), 2.70$ (t, $J=7.4 \mathrm{~Hz}, 2 \mathrm{H}), 2.61$ (t, $J=7.6 \mathrm{~Hz}, 2 \mathrm{H}$ ), $2.04-1.95(\mathrm{~m}, 2 \mathrm{H})$.

${ }^{13} \mathrm{C} \mathrm{NMR} \quad\left(125 \mathrm{MHz}, \mathrm{CDCl}_{3}\right)$

$\delta 152.8,152.2,147.6,141.6,131.0,130.6,128.5,128.4,125.9,124.1,122.9,122.8$, $119.8,118.0,111.5,69.7,35.2,28.8,25.0$.

$\underline{\text { HRMS (ESI) }}$ for: $\mathrm{C}_{21} \mathrm{H}_{20} \mathrm{NaO}_{4}[\mathrm{M}+\mathrm{Na}]^{+}$: calcd 359.1259, found 359.1273 .

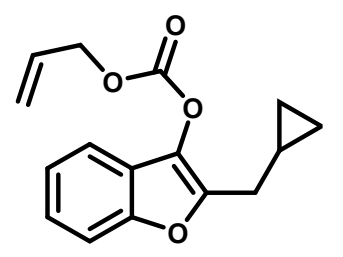

12u Allyl (2-(cyclopropylmethyl)benzofuran-3-yl) carbonate (12u). Following the General Procedure to yield $12 \mathrm{u}$ as a yellow oil ( $1.3 \mathrm{~g}, 98 \%$ yield).

$\underline{R}_{f} \quad 0.40\left(\mathrm{Hex} / \mathrm{EtOAc}=30: 1, \mathrm{KMnO}_{4}\right)$

${ }^{1} \mathrm{H} \mathrm{NMR} \quad\left(500 \mathrm{MHz}, \mathrm{CDCl}_{3}\right)$

d $7.42-7.24(\mathrm{~m}, 2 \mathrm{H}), 7.24-7.09(\mathrm{~m}, 2 \mathrm{H}), 6.00-5.88(\mathrm{~m}, 1 \mathrm{H}), 5.48-5.22(\mathrm{~m}, 2 \mathrm{H})$, $4.71(\mathrm{~d}, J=5.9 \mathrm{~Hz}, 2 \mathrm{H}), 2.61(\mathrm{~d}, J=6.9 \mathrm{~Hz}, 2 \mathrm{H}), 1.14-0.96(\mathrm{~m}, 1 \mathrm{H}), 0.57-0.36(\mathrm{~m}$, $2 \mathrm{H}), 0.36-0.07(\mathrm{~m}, 2 \mathrm{H})$.

${ }^{13} \mathrm{C} \mathrm{NMR} \quad\left(125 \mathrm{MHz}, \mathrm{CDCl}_{3}\right)$ 
$\delta 152.9,152.2,147.6,130.9,130.4,124.1,122.8,122.8,119.8,118.0,111.6,69.6,30.4$, 9.0, 4.6.

HRMS (ESI) for: $\mathrm{C}_{16} \mathrm{H}_{16} \mathrm{NaO}_{4}[\mathrm{M}+\mathrm{Na}]^{+}$: calcd 295.0946, found 295.0951 .<smiles>C=CCOC(=O)Oc1c(Cc2cccc3ccccc23)oc2cccc(O)c12</smiles>

$12 \mathrm{v}$

Allyl (4-methoxy-2-(naphthalen-1-ylmethyl)benzofuran-3-yl) carbonate (12v). Following the General Procedure to yield $12 \mathrm{v}$ as a white solid (1.8 g, 92\% yield).

$\underline{R}_{f} \quad 0.40\left(\mathrm{Hex} / \mathrm{EtOAc}=15: 1, \mathrm{KMnO}_{4}\right)$

${ }^{1} \mathrm{H} \mathrm{NMR} \quad\left(500 \mathrm{MHz}, \mathrm{CDCl}_{3}\right)$

$\delta 8.15(\mathrm{~d}, J=8.4 \mathrm{~Hz}, 1 \mathrm{H}), 7.94-7.75(\mathrm{~m}, 2 \mathrm{H}), 7.62-7.37(\mathrm{~m}, 4 \mathrm{H}), 7.15(\mathrm{t}, J=8.2 \mathrm{~Hz}$, $1 \mathrm{H}), 6.98(\mathrm{~d}, J=8.3 \mathrm{~Hz}, 1 \mathrm{H}), 6.63(\mathrm{~d}, J=8.0 \mathrm{~Hz}, 1 \mathrm{H}), 6.12-5.92(\mathrm{~m}, 1 \mathrm{H}), 5.55-5.24$ $(\mathrm{m}, 2 \mathrm{H}), 4.73(\mathrm{~d}, J=8.3 \mathrm{~Hz}, 2 \mathrm{H}), 4.54(\mathrm{~s}, 2 \mathrm{H}), 3.87(\mathrm{~s}, 3 \mathrm{H})$.

${ }^{13} \mathrm{C} \mathrm{NMR} \quad\left(125 \mathrm{MHz}, \mathrm{CDCl}_{3}\right)$

$\delta 153.7,153.1,152.9,144.0,133.9,132.5,131.9,131.2,130.8,128.7,127.8,127.1$, $126.3,125.8,125.6,125.2,123.6,119.3,112.8,105.0,103.9,69.5,55.7,29.1$.

HRMS (ESI) for: $\mathrm{C}_{24} \mathrm{H}_{20} \mathrm{NaO}_{5}[\mathrm{M}+\mathrm{Na}]^{+}$: calcd 411.1208, found 411.1213.

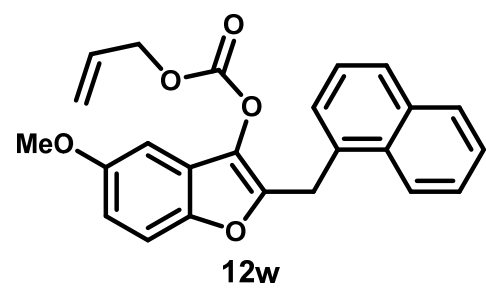

Allyl (5-methoxy-2-(naphthalen-1-ylmethyl)benzofuran-3-yl) carbonate (12w). Following the General Procedure to yield $\mathbf{1 2 w}$ as a yellow solid (1.8 $\mathrm{g}, 92 \%$ yield).

$\underline{R}_{f} \quad 0.40\left(\mathrm{Hex} / \mathrm{EtOAc}=15: 1, \mathrm{KMnO}_{4}\right)$

${ }^{1} \mathrm{H} \mathrm{NMR} \quad\left(500 \mathrm{MHz}, \mathrm{CDCl}_{3}\right)$

$\delta 8.00(\mathrm{~d}, J=8.4 \mathrm{~Hz}, 1 \mathrm{H}), 7.84-7.60(\mathrm{~m}, 2 \mathrm{H}), 7.49-7.25(\mathrm{~m}, 4 \mathrm{H}), 7.22-7.07(\mathrm{~m}$, $1 \mathrm{H}), 6.80-6.68(\mathrm{~m}, 2 \mathrm{H}), 5.97-5.79(\mathrm{~m}, 1 \mathrm{H}), 5.45-5.14(\mathrm{~m}, 2 \mathrm{H}), 4.70-4.55(\mathrm{~m}, 2 \mathrm{H})$, $4.44(\mathrm{~s}, 2 \mathrm{H}), 3.72(\mathrm{~s}, 3 \mathrm{H})$.

${ }^{13} \mathrm{C} \mathrm{NMR} \quad\left(125 \mathrm{MHz}, \mathrm{CDCl}_{3}\right)$

$\delta 156.1,152.5,147.3,146.4,133.8,132.2,131.9,131.2,130.9,128.7,127.8,127.1$, $126.3,125.8,125.6,123.6,123.1,119.8,113.3,112.4,100.5,69.7,56.0,29.7$.

HRMS (ESI) for: $\mathrm{C}_{24} \mathrm{H}_{20} \mathrm{NaO}_{5}[\mathrm{M}+\mathrm{Na}]^{+}$: calcd 411.1208, found 411.1204.

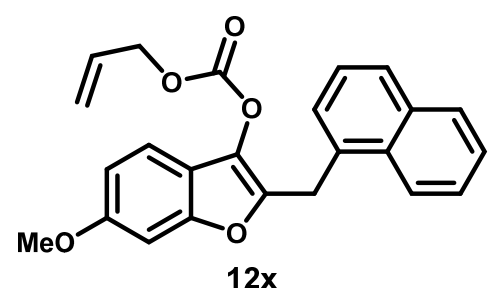

Allyl (6-methoxy-2-(naphthalen-1-ylmethyl)benzofuran-3-yl) carbonate (12x). Following the General Procedure to yield 12x as a white solid ( $1.9 \mathrm{~g}, 97 \%$ yield).

$\underline{R}_{f} \quad 0.40\left(\mathrm{Hex} / \mathrm{EtOAc}=15: 1, \mathrm{KMnO}_{4}\right)$

${ }^{1} \mathrm{H} \mathrm{NMR} \quad\left(500 \mathrm{MHz}, \mathrm{CDCl}_{3}\right)$ 
$\delta 8.02(\mathrm{dd}, J=8.6,1 \mathrm{H}), 7.84-7.61(\mathrm{~m}, 2 \mathrm{H}), 7.54-7.26(\mathrm{~m}, 4 \mathrm{H}), 7.18(\mathrm{~d}, J=8.5 \mathrm{~Hz}$, $1 \mathrm{H}), 6.85-6.72(\mathrm{~m}, 2 \mathrm{H}), 5.97-5.77(\mathrm{~m}, 1 \mathrm{H}), 5.42-5.17(\mathrm{~m}, 2 \mathrm{H}), 4.69-4.53(\mathrm{~m}, 2 \mathrm{H})$, $4.43(\mathrm{~s}, 2 \mathrm{H}), 3.68(\mathrm{~s}, 3 \mathrm{H})$.

${ }^{13} \mathrm{C} \mathrm{NMR} \quad\left(125 \mathrm{MHz}, \mathrm{CDCl}_{3}\right)$

$\delta 158.1,153.4,152.6,144.3,133.8,132.5,131.9,131.1,130.9,128.7,127.8,127.0$, $126.3,125.7,125.6,123.6,119.8,118.5,116.1,112.114,96.4,69.6,55.7,29.4$.

for: $\mathrm{C}_{24} \mathrm{H}_{20} \mathrm{NaO}_{5}[\mathrm{M}+\mathrm{Na}]^{+}$: calcd 411.1208, found 411.1207.

\section{$\underline{\operatorname{HRMS}(\mathrm{ESI})}$}

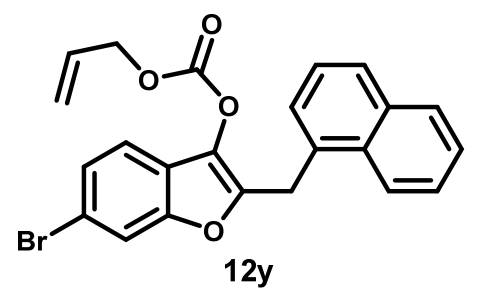

Allyl (6-bromo-2-(naphthalen-1-ylmethyl)benzofuran-3-yl) carbonate (12y). Following the General Procedure to yield 12y as a white solid (1.9 g, 89\% yield).

$\underline{R}_{f} \quad 0.35\left(\mathrm{Hex} / \mathrm{EtOAc}=20: 1, \mathrm{KMnO}_{4}\right)$

${ }^{1} \mathrm{H} \mathrm{NMR} \quad\left(500 \mathrm{MHz}, \mathrm{CDCl}_{3}\right)$

$\delta 7.97(\mathrm{~d}, J=8.1 \mathrm{~Hz}, 1 \mathrm{H}), 7.72(\mathrm{dd}, J=39.7,7.8 \mathrm{~Hz}, 2 \mathrm{H}), 7.56-7.07(\mathrm{~m}, 7 \mathrm{H}), 5.96-$ $5.73(\mathrm{~m}, 1 \mathrm{H}), 5.40-5.15(\mathrm{~m}, 2 \mathrm{H}), 4.58(\mathrm{~d}, J=5.9 \mathrm{~Hz}, 2 \mathrm{H}), 4.43(\mathrm{~s}, 2 \mathrm{H})$.

${ }^{13} \mathrm{C} \mathrm{NMR} \quad\left(125 \mathrm{MHz}, \mathrm{CDCl}_{3}\right)$

$\delta 152.5,152.4,146.4,133.9,131.9,131.8,131.0,130.8,128.8,128.0,127.2,126.4$, $125.9,125.6,123.4,121.9,120.0,119.3,117.7,115.2,69.8,29.5$.

HRMS (ESI) for: $\mathrm{C}_{23} \mathrm{H}_{17} \mathrm{BrNaO}_{4}[\mathrm{M}+\mathrm{Na}]^{+}$: calcd 459.0208, found 459.0211.

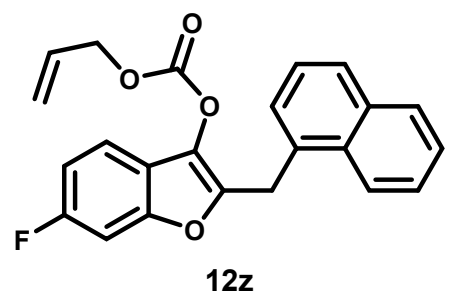

(12z). Following the General Procedure to yield $12 \mathrm{z}$ as a white solid (1.7 g, 92\% yield).

$\underline{R}_{f} \quad 0.30\left(\mathrm{Hex} / \mathrm{EtOAc}=20: 1, \mathrm{KMnO}_{4}\right)$

${ }^{1} \mathrm{H} \mathrm{NMR} \quad\left(500 \mathrm{MHz}, \mathrm{CDCl}_{3}\right)$

$\delta 8.12(\mathrm{~d}, J=8.2 \mathrm{~Hz}, 1 \mathrm{H}), 8.00-7.70(\mathrm{~m}, 2 \mathrm{H}), 7.59-7.35(\mathrm{~m}, 4 \mathrm{H}), 7.15(\mathrm{t}, J=7.9 \mathrm{~Hz}$, $1 \mathrm{H}), 7.01(\mathrm{~d}, J=7.9 \mathrm{~Hz}, 1 \mathrm{H}), 6.78(\mathrm{~d}, J=7.9 \mathrm{~Hz}, 1 \mathrm{H}), 6.04-5.85(\mathrm{~m}, 1 \mathrm{H}), 5.36(\mathrm{dd}, J$ $=33.9,13.5 \mathrm{~Hz}, 2 \mathrm{H}), 4.77-4.52(\mathrm{~m}, 4 \mathrm{H}), 3.97(\mathrm{~s}, 3 \mathrm{H})$.

${ }^{13} \mathrm{C} \mathrm{NMR} \quad\left(125 \mathrm{MHz}, \mathrm{CDCl}_{3}\right)$

$\delta 152.4,145.7,145.3,141.7,133.8,132.0,131.9,131.6,130.9,128.7,127.8,127.1$, $126.3,125.7,125.6,124.5,123.8,123.5,119.7,110.5,106.8,69.6,56.1,29.6$.

$\left(470 \mathrm{MHz}, \mathrm{CDCl}_{3}\right)$

${ }^{19} \mathrm{~F} \mathrm{NMR} \quad \delta-117.09$.

$\underline{\text { HRMS (ESI) }}$ for: $\mathrm{C}_{23} \mathrm{H}_{17} \mathrm{FNaO}_{4}[\mathrm{M}+\mathrm{Na}]^{+}$: calcd 399.1009, found 399.1018 . 
<smiles>C=CCOC(=O)Oc1c(Cc2cccc3ccccc23)oc2cc(N3CCOCC3)ccc12</smiles>

Allyl (6-morpholino-2-(naphthalen-1-ylmethyl)benzofuran-3-yl) carbonate(12a'). Following the General Procedure to yield 12a' as a white solid (2.1g, 95\% yield).

$\underline{R}_{f} \quad 0.35\left(\mathrm{Hex} / \mathrm{EtOAc}=10: 1, \mathrm{KMnO}_{4}\right)$

${ }^{1} \mathrm{H} \mathrm{NMR} \quad\left(500 \mathrm{MHz}, \mathrm{CDCl}_{3}\right)$

$\delta 8.13(\mathrm{~d}, J=8.3 \mathrm{~Hz}, 1 \mathrm{H}), 7.83(\mathrm{dd}, J=44.5,7.5 \mathrm{~Hz}, 2 \mathrm{H}), 7.65-7.27(\mathrm{~m}, 5 \mathrm{H}), 6.98-$ $6.74(\mathrm{~m}, 2 \mathrm{H}), 6.06-5.88(\mathrm{~m}, 1 \mathrm{H}), 5.55-5.25(\mathrm{~m}, 2 \mathrm{H}), 4.71(\mathrm{~d}, J=5.8 \mathrm{~Hz}, 2 \mathrm{H}), 4.54$ $(\mathrm{s}, 2 \mathrm{H}), 3.95-3.73(\mathrm{~m}, 4 \mathrm{H}), 3.23-2.99(\mathrm{~m}, 4 \mathrm{H})$.

${ }^{13} \mathrm{C} \mathrm{NMR} \quad\left(125 \mathrm{MHz}, \mathrm{CDCl}_{3}\right)$

$\delta 153.7,152.6,149.8,144.0,133.8,132.5,131.9,131.1,130.9,128.7,127.7,127.0$, $126.3,125.8,125.6,123.6,119.7,118.4,115.8,113.6,99.0,77.4,77.1,76.9,69.6,66.9$, $50.4,29.4$.

HRMS (ESI) for: $\mathrm{C}_{27} \mathrm{H}_{25} \mathrm{NNaO}_{5}[\mathrm{M}+\mathrm{Na}]^{+}$: calcd 466.1630, found 466.1637 .

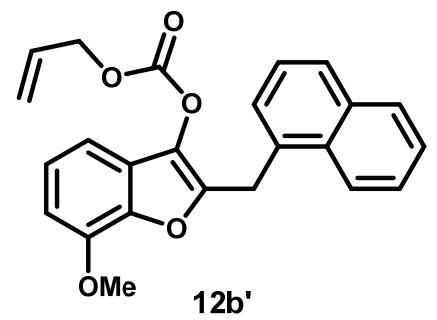

Allyl (7-methoxy-2-(naphthalen-1-ylmethyl)benzofuran-3-yl)

carbonate (12b'). Following the General Procedure to yield 12b' as a white solid (1.7 g, 90\% yield).

$\underline{R}_{f} \quad 0.40\left(\mathrm{Hex} / \mathrm{EtOAc}=15: 1, \mathrm{KMnO}_{4}\right)$

${ }^{1} \mathrm{H} \mathrm{NMR} \quad\left(500 \mathrm{MHz}, \mathrm{CDCl}_{3}\right)$

$\delta 8.11(\mathrm{~d}, J=8.2 \mathrm{~Hz}, 1 \mathrm{H}), 7.96-7.72(\mathrm{~m}, 2 \mathrm{H}), 7.61-7.36(\mathrm{~m}, 4 \mathrm{H}), 7.14(\mathrm{~d}, J=7.9$

$\mathrm{Hz}, 1 \mathrm{H}), 7.01(\mathrm{~d}, J=7.9 \mathrm{~Hz}, 1 \mathrm{H}), 6.78(\mathrm{~d}, J=7.9 \mathrm{~Hz}, 1 \mathrm{H}), 6.00-5.85(\mathrm{~m}, 1 \mathrm{H}), 5.35$ $(\mathrm{dd}, J=33.9,13.5 \mathrm{~Hz}, 2 \mathrm{H}), 4.61(\mathrm{~s}, 4 \mathrm{H}), 3.96(\mathrm{~s}, 3 \mathrm{H})$.

${ }^{13} \mathrm{C} \mathrm{NMR} \quad\left(125 \mathrm{MHz}, \mathrm{CDCl}_{3}\right)$

$\delta 152.4,145.7,145.3,141.7,133.8,132.0,131.9,131.6,130.9,128.7,127.8,127.1$, $126.3,125.7,125.6,124.5,123.8,123.5,119.7,110.5,106.8,69.6,56.1,29.6$.

HRMS (ESI) for: $\mathrm{C}_{24} \mathrm{H}_{20} \mathrm{NaO}_{5}[\mathrm{M}+\mathrm{Na}]^{+}$: calcd 411.1208, found 411.1215 .

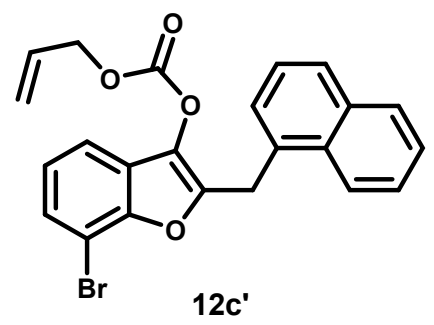

carbonate(12c'). Following the General Procedure to yield 12c' as a white solid (1.5 g, 80\% yield).

$\underline{R}_{f} \quad 0.30\left(\mathrm{Hex} / \mathrm{EtOAc}=20: 1, \mathrm{KMnO}_{4}\right)$

${ }^{1} \mathrm{H} \mathrm{NMR} \quad\left(500 \mathrm{MHz}, \mathrm{CDCl}_{3}\right)$ 
$\delta 8.19(\mathrm{~d}, J=1.2 \mathrm{~Hz}, 1 \mathrm{H}), 8.00-7.78(\mathrm{~m}, 2 \mathrm{H}), 7.69-7.33(\mathrm{~m}, 6 \mathrm{H}), 7.13(\mathrm{t}, J=7.8 \mathrm{~Hz}$, $1 \mathrm{H}), 6.01-5.92(\mathrm{~m}, 1 \mathrm{H}), 5.53-5.21(\mathrm{~m}, 2 \mathrm{H}), 4.81-4.52(\mathrm{~m}, 4 \mathrm{H})$.

${ }^{13} \mathrm{C} \mathrm{NMR} \quad\left(125 \mathrm{MHz}, \mathrm{CDCl}_{3}\right)$

$\delta 152.3,149.3,146.9,133.1,131.9,131.7,131.6,130.8,128.8,128.0,127.6,127.2$, 126.4, 125.9, 125.6, 124.3, 123.6, 119.9, 117.6, 104.7, 69.8, 29.7.

HRMS (ESI) for: $\mathrm{C}_{23} \mathrm{H}_{17} \mathrm{BrNaO}_{4}[\mathrm{M}+\mathrm{Na}]^{+}$: calcd 459.0208, found 459.0204.<smiles>C=CCOC(=O)Oc1c(Cc2ccccc2)oc2c1ccc1ccccc12</smiles>

General Procedure to yield 12d' as a white solid (1.7 g, 96\% yield).

$\underline{R}_{f} \quad 0.40\left(\mathrm{Hex} / \mathrm{EtOAc}=20: 1, \mathrm{KMnO}_{4}\right)$

$\underline{{ }^{1} \mathrm{H} \mathrm{NMR}} \quad\left(500 \mathrm{MHz}, \mathrm{CDCl}_{3}\right)$

$\delta 8.15(\mathrm{~d}, J=8.3 \mathrm{~Hz}, 1 \mathrm{H}), 7.82(\mathrm{~d}, J=8.2 \mathrm{~Hz}, 1 \mathrm{H}), 7.58(\mathrm{~d}, J=8.5 \mathrm{~Hz}, 1 \mathrm{H}), 7.50-$

$7.36(\mathrm{~m}, 3 \mathrm{H}), 7.30-7.13(\mathrm{~m}, 4 \mathrm{H}), 6.00-5.85(\mathrm{~m}, 1 \mathrm{H}), 5.50-5.25(\mathrm{~m}, 2 \mathrm{H}), 4.70(\mathrm{~d}, J$

$=5.9 \mathrm{~Hz}, 2 \mathrm{H}), 4.15(\mathrm{~s}, 2 \mathrm{H})$.

${ }^{13} \mathrm{C} \mathrm{NMR} \quad\left(125 \mathrm{MHz}, \mathrm{CDCl}_{3}\right)$

$\delta 152.8,147.8,145.0,136.8,132.1,131.4,130.9,128.8,128.6,128.4,126.7,126.5$, $125.3,123.7,121.3,119.8,119.7,118.2,116.6,69.7,32.0$.

HRMS (ESI) for: $\mathrm{C}_{23} \mathrm{H}_{18} \mathrm{NaO}_{4}[\mathrm{M}+\mathrm{Na}]^{+}$: calcd 381.1103 , found 381.1107 .

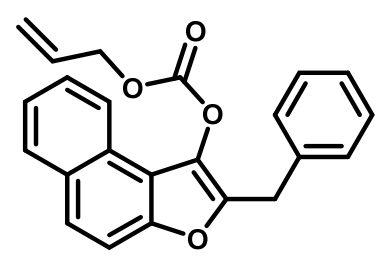

$12 \mathrm{e}$

Allyl (2-benzylnaphtho[2,1-b]furan-1-yl) carbonate(12e'). Following the General Procedure to yield $12 \mathrm{e}^{\prime}$ as a white solid (1.7 g, 96\% yield).

$\underline{R}_{f} \quad 0.40\left(\mathrm{Hex} / \mathrm{EtOAc}=20: 1, \mathrm{KMnO}_{4}\right)$

${ }^{1} \mathrm{H} \mathrm{NMR} \quad\left(500 \mathrm{MHz}, \mathrm{CDCl}_{3}\right)$

$\delta 7.97(\mathrm{~d}, J=8.3 \mathrm{~Hz}, 1 \mathrm{H}), 7.76(\mathrm{~d}, J=8.2 \mathrm{~Hz}, 1 \mathrm{H}), 7.52(\mathrm{~d}, J=9.0 \mathrm{~Hz}, 1 \mathrm{H}), 7.43-$

$7.28(\mathrm{~m}, 3 \mathrm{H}), 7.20-7.13(\mathrm{~m}, 3 \mathrm{H}), 7.12-7.05(\mathrm{~m}, 2 \mathrm{H}), 5.95-5.75(\mathrm{~m}, 1 \mathrm{H}), 5.42-5.08$ $(\mathrm{m}, 2 \mathrm{H}), 4.75-4.52(\mathrm{~m}, 2 \mathrm{H}), 4.02(\mathrm{~s}, 2 \mathrm{H})$.

${ }^{13} \mathrm{C} \mathrm{NMR} \quad\left(125 \mathrm{MHz}, \mathrm{CDCl}_{3}\right)$

$\delta 153.0,150.0,145.7,136.5,132.8,130.9,130.6,128.9,128.8,128.6,126.8,126.6$, $126.5,125.5,124.7,123.0,120.0,116.5,112.6,69.9,32.1$.

HRMS (ESI) for: $\mathrm{C}_{23} \mathrm{H}_{18} \mathrm{NaO}_{4}[\mathrm{M}+\mathrm{Na}]^{+}$: calcd 381.1103, found 381.1115 . 


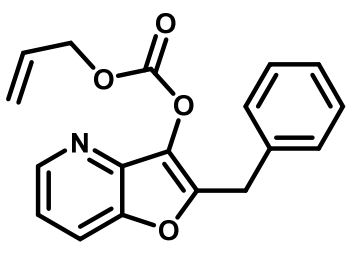

12f' Allyl (2-benzylfuro[2,3-b]pyridin-3-yl) carbonate(12f'). Following the General Procedure to yield $\mathbf{1 2 f ^ { \prime }}$ as a white solid (1.4 g, 88\% yield).

$\underline{R_{f}} \quad 0.20\left(\mathrm{Hex} / \mathrm{EtOAc}=15: 1, \mathrm{KMnO}_{4}\right)$

${ }^{1} \mathrm{H} \mathrm{NMR} \quad\left(400 \mathrm{MHz}, \mathrm{CDCl}_{3}\right)$

$\delta 7.51-7.11(\mathrm{~m}, 9 \mathrm{H}), 6.07-5.90(\mathrm{~m}, 1 \mathrm{H}), 5.52-5.28(\mathrm{~m}, 2 \mathrm{H}), 4.75(\mathrm{~d}, J=5.9 \mathrm{~Hz}$, $2 \mathrm{H}), 4.10(\mathrm{~d}, J=1.2 \mathrm{~Hz}, 2 \mathrm{H})$.

${ }^{13} \mathrm{C} \mathrm{NMR} \quad\left(100 \mathrm{MHz}, \mathrm{CDCl}_{3}\right)$

$\delta 152.8,152.4,146.0,136.4,131.1,130.9,128.8,128.7,126.9,124.4,123.0,122.7$, 119.9, 118.2, 111.7, 69.7, 31.9.

HRMS (ESI) for: $\mathrm{C}_{18} \mathrm{H}_{15} \mathrm{NNaO}_{4}[\mathrm{M}+\mathrm{Na}]^{+}$: calcd 332.0899, found 332.0903 .

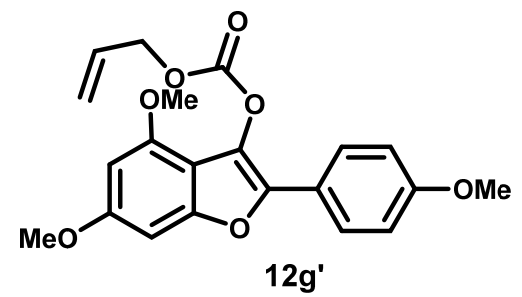

Allyl (4,6-dimethoxy-2-(4-methoxyphenyl)benzofuran-3-yl) carbonate (12g'). Following the General Procedure to yield 12g as a white solid (1.5 g, 86\% yield).

$\underline{R}_{f} \quad 0.40\left(\mathrm{Hex} / \mathrm{EtOAc}=3: 1, \mathrm{KMnO}_{4}\right)$

${ }^{1} \mathrm{H} \mathrm{NMR} \quad\left(400 \mathrm{MHz}, \mathrm{CDCl}_{3}\right)$

$\delta 7.77(\mathrm{~d}, J=8.9 \mathrm{~Hz}, 2 \mathrm{H}), 6.98(\mathrm{~d}, J=8.9 \mathrm{~Hz}, 2 \mathrm{H}), 6.62(\mathrm{~s}, 1 \mathrm{H}), 6.31(\mathrm{~s}, 1 \mathrm{H}), 6.13-$ $5.94(\mathrm{~m}, 1 \mathrm{H}), 5.58-5.21(\mathrm{~m}, 2 \mathrm{H}), 4.80(\mathrm{~d}, J=5.7 \mathrm{~Hz}, 2 \mathrm{H}), 3.85$ (s, 9H).

${ }^{13} \mathrm{C} \mathrm{NMR} \quad\left(100 \mathrm{MHz}, \mathrm{CDCl}_{3}\right)$

$\delta 159.6,159.4,153.8,153.1,141.6,131.3,129.2,126.4,122.1,119.4,114.3,107.8$, 95.0, 88.7, 69.7, 55.9, 55.4, 31.7.

$\underline{\mathrm{HRMS}(\mathrm{ESI})}$ for: $\mathrm{C}_{21} \mathrm{H}_{21} \mathrm{O}_{7}[\mathrm{M}+\mathrm{H}]^{+}$: calcd 385.1287, found 385.1292.<smiles>C=CCOC(=O)Oc1c(-c2ccccc2)oc2cc(OC)cc(OC)c12</smiles>

Allyl (4,6-dimethoxy-2-phenylbenzofuran-3-yl) carbonate (12h'). Following the General Procedure to yield 12h' as a white solid (1.4 g, 88\% yield).

$\underline{R}_{f} \quad 0.45\left(\mathrm{Hex} / \mathrm{EtOAc}=3: 1, \mathrm{KMnO}_{4}\right)$

${ }^{1} \mathrm{H} \mathrm{NMR} \quad\left(500 \mathrm{MHz}, \mathrm{CDCl}_{3}\right)$

$\delta 7.83(\mathrm{~d}, J=8.4 \mathrm{~Hz}, 2 \mathrm{H}), 7.43(\mathrm{t}, J=7.8 \mathrm{~Hz}, 2 \mathrm{H}), 7.30(\mathrm{t}, J=7.4 \mathrm{~Hz}, 1 \mathrm{H}), 6.63(\mathrm{~s}$, $1 \mathrm{H}), 6.31(\mathrm{~s}, 1 \mathrm{H}), 6.11-5.96(\mathrm{~m}, 1 \mathrm{H}), 5.51-5.23(\mathrm{~m}, 2 \mathrm{H}), 4.80(\mathrm{~d}, J=5.7 \mathrm{~Hz}, 2 \mathrm{H})$, $3.85(\mathrm{~d}, J=3.0 \mathrm{~Hz}, 6 \mathrm{H})$.

${ }^{13} \mathrm{C} \mathrm{NMR} \quad\left(125 \mathrm{MHz}, \mathrm{CDCl}_{3}\right)$

$\delta 159.9,153.9,153.3,152.7,141.2,131.2,130.5,129.2,128.7,127.8,124.7,119.3$, 
107.6, 95.01, 88.5, 69.6.

$\underline{\text { HRMS (ESI) }}$ for: $\mathrm{C}_{20} \mathrm{H}_{19} \mathrm{O}_{6}[\mathrm{M}+\mathrm{H}]^{+}$: calcd 355.1182 , found 355.1185 .

3.3 10: General Procedure for the Pd-Catalyzed AAA
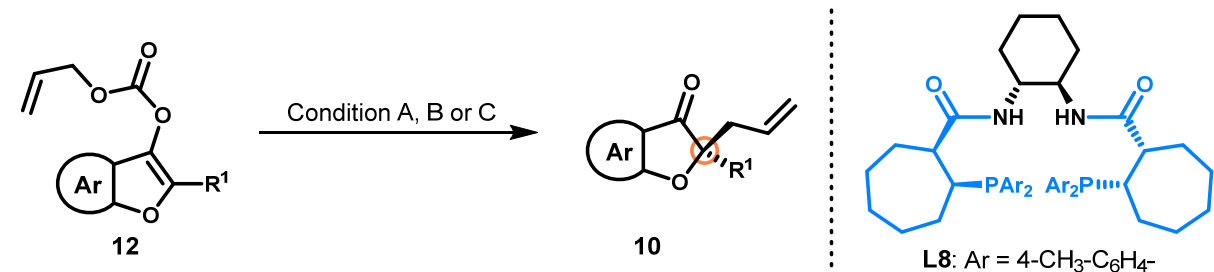

Condition A. A solution of $\mathrm{Pd}_{2}(\mathrm{dba})_{3}(18.3 \mathrm{mg}, 0.02 \mathrm{mmol})$ and $\mathbf{L 1 5}(15.7 \mathrm{mg}, 0.02 \mathrm{mmol})$ in anhydrous THF $(10 \mathrm{~mL})$ was prepared in the glove box and stored at $-20^{\circ} \mathrm{C.} 12(0.5 \mathrm{mmol})$ was dissolved in $1.5 \mathrm{~mL}$ anhydrous THF and stirred at $-20^{\circ} \mathrm{C}$ for $15 \mathrm{~min}$ under $\mathrm{N}_{2}$, then the pre-cooled catalyst solution $(0.5 \mathrm{~mL})$ was added to the reaction mixture drop-wise. The reaction mixture was then stirred at $-20{ }^{\circ} \mathrm{C}$ overnight. The reaction mixture was evaporated under reduced pressure after complete consumption of the starting material, then the residue was purified by flash column chromatography over silica gel to afford the corresponding chiral products $\mathbf{1 0 .}$

Condition B. A solution of $\mathrm{Pd}_{2}(\mathrm{dba})_{3}(18.3 \mathrm{mg}, 0.02 \mathrm{mmol})$ and $15(15.7 \mathrm{mg}, 0.02 \mathrm{mmol})$ in anhydrous THF $(10 \mathrm{~mL})$ was prepared in the glove box and stored at $-20^{\circ} \mathrm{C} .12(0.5 \mathrm{mmol})$ was dissolved in $4 \mathrm{~mL}$ anhydrous THF and stirred at $-20{ }^{\circ} \mathrm{C}$ for 15 min under $\mathrm{N}_{2}$, then the pre-cooled catalyst solution $(1.0 \mathrm{~mL})$ was added to the reaction mixture drop-wise. The reaction mixture was then stirred at $-20{ }^{\circ} \mathrm{C}$ overnight. The reaction mixture was evaporated under reduced pressure after complete consumption of the starting material, then the residue was purified by flash column chromatography over silica gel to afford the corresponding chiral products $\mathbf{1 0}$.

Condition C. A solution of $\operatorname{Pd}_{2}(\mathrm{dba})_{3}(18.3 \mathrm{mg}, 0.02 \mathrm{mmol})$ and $\mathbf{L 1 5}(15.7 \mathrm{mg}, 0.02 \mathrm{mmol})$ in anhydrous THF $(10 \mathrm{~mL})$ was prepared in the glove box and stored at $-20^{\circ} \mathrm{C} .12(0.5 \mathrm{mmol})$ in anhydrous THF ( $4 \mathrm{~mL}$ ) was stirred at $-5^{\circ} \mathrm{C}$ for $15 \mathrm{~min}$ under $\mathrm{N}_{2}$, then the pre-cooled catalyst solution $(1.0 \mathrm{~mL})$ was added to the reaction mixture drop-wise. The reaction mixture was then stirred at $-5{ }^{\circ} \mathrm{C}$ overnight. The reaction mixture was evaporated under reduced pressure after complete consumption of the starting material, then the residue was purified by flash column chromatography over silica gel to afford the corresponding chiral products $\mathbf{1 0}$.

Condition D. A solution of $\operatorname{Pd}_{2}(\mathrm{dba})_{3}(9.2 \mathrm{mg}, 0.01 \mathrm{mmol})$ and $\mathbf{L 1 6}(9.2 \mathrm{mg}, 0.01 \mathrm{mmol})$ in anhydrous THF $(2 \mathrm{~mL})$ was prepared in the glove box and stored at $-20^{\circ} \mathrm{C} .12(0.1 \mathrm{mmol})$ and $4 \AA \mathrm{MS}(50 \mathrm{mg})$ (in anhydrous THF $(0.8 \mathrm{~mL})$ was stirred at -20 or -45 or $-50{ }^{\circ} \mathrm{C}$ for 15 min under $\mathrm{N}_{2}$, then the pre-cooled catalyst solution $(0.2 \mathrm{~mL})$ was added to the reaction mixture drop-wise. The reaction mixture was then stirred at -20 or -45 or $-50{ }^{\circ} \mathrm{C}$ overnight. The reaction mixture was evaporated under reduced pressure after complete consumption of the starting material, then the residue was purified by flash column chromatography over silica gel to afford the corresponding chiral products $\mathbf{1 0}$.

Multigram Scale for 12a: To a solution of $\mathrm{Pd}_{2}(\mathrm{dba})_{3}(36.6 \mathrm{mg}, 0.04 \mathrm{mmol})$ in $15 \mathrm{~mL}$ anhydrous THF was added ent-L8 (31.4 $\mathrm{mg}, 0.04 \mathrm{mmol}$ ) in the glove box, and the mixture was stirred at RT for $5 \mathrm{~min}$. This solution was then cooled to $-20^{\circ} \mathrm{C}$ and transferred via cannula to a solution of 12a (6.2 g, $\left.20 \mathrm{mmol}\right)$ in anhydrous THF $(60 \mathrm{~mL})$ at $-20{ }^{\circ} \mathrm{C}$. The reaction mixture was then stirred at $-20{ }^{\circ} \mathrm{C}$ for $24 \mathrm{~h}$. The reaction mixture was evaporated under reduced pressure directly after complete consumption of the 
starting material, the residue was purified by flash column chromatography over silica gel to afford 10a as a colorless oil ( $4.97 \mathrm{~g}, 94 \%$ yield, $91 \%$ ee).<smiles>C=CC[C@]1(c2ccccc2)Oc2ccccc2C1=O</smiles>

10a (S)-2-Allyl-2-benzylbenzofuran-3(2H)-one (10a). Using Condition A to yield 10a as a colorless oil (117.6 mg, 89\% yield, 92\% ee).

$\underline{R}_{f} \quad 0.50(\mathrm{Hex} / \mathrm{EtOAc}=20: 1$, Vanillin $)$

Opt. Rot. $\quad[\alpha]^{20}{ }_{\mathrm{D}}=-50.0(\mathrm{c}=0.1, \mathrm{MeOH})$

${ }^{1} \mathrm{H} \mathrm{NMR} \quad\left(400 \mathrm{MHz}, \mathrm{CDCl}_{3}\right)$

$\delta 7.36-7.22(\mathrm{~m}, 2 \mathrm{H}), 7.21-6.80(\mathrm{~m}, 7 \mathrm{H}), 6.00-5.78(\mathrm{~m}, 1 \mathrm{H}), 5.39-5.16(\mathrm{~m}, 2 \mathrm{H})$, $4.64(\mathrm{~d}, J=5.8 \mathrm{~Hz}, 2 \mathrm{H}), 4.00(\mathrm{~s}, 2 \mathrm{H})$.

${ }^{13} \mathrm{C} \mathrm{NMR} \quad\left(100 \mathrm{MHz}, \mathrm{CDCl}_{3}\right)$

$\delta$ 203.4, 171.8, 138.1, 134.5, 130.7, 130.5, 128.1, 126.9, 124.1, 121.6, 121.5, 119.9, $113.1,91.9,41.8,40.2$.

$\underline{\text { HRMS (ESI) }}$ for: $\mathrm{C}_{18} \mathrm{H}_{17} \mathrm{O}_{2}[\mathrm{M}+\mathrm{H}]^{+}$: calcd 265.1229, found 265.1236.

HPLC Daicel Chiralpak IBN-3 column, $n$-hexane $/ i$-PrOH (99.6/0.4), $0.5 \mathrm{~mL} / \mathrm{min} 210 \mathrm{~nm}$ $16.969 \mathrm{~min}$ (major enantiomer), $15.970 \mathrm{~min}$ (minor enantiomer).<smiles></smiles>

10b (S)-1-Acetyl-2-allyl-2-benzylindolin-3-one (10b). Using Condition D at $-20{ }^{\circ} \mathrm{C}$ to yield $10 b$ as yellow oil ( $27.2 \mathrm{mg}, 89 \%$ yield, $81 \%$ ee).

$\underline{R}_{f} \quad 0.30(\mathrm{Hex} / \mathrm{EtOAc}=10: 1$, Vanillin)

Opt. Rot. $[\alpha]^{20}=-42.0(\mathrm{c}=0.1, \mathrm{MeOH})$

$\underline{{ }^{1} \mathrm{H} \mathrm{NMR}} \quad\left(500 \mathrm{MHz}, \mathrm{CDCl}_{3}\right)$

$\delta 7.63(\mathrm{~d}, J=7.3 \mathrm{~Hz}, 1 \mathrm{H}), 7.42(\mathrm{t}, J=7.5 \mathrm{~Hz}, 1 \mathrm{H}), 7.21-6.81(\mathrm{~m}, 7 \mathrm{H}), 5.40-5.25(\mathrm{~m}$, $1 \mathrm{H}), 5.07(\mathrm{~d}, J=16.9 \mathrm{~Hz}, 1 \mathrm{H}), 4.86(\mathrm{~d}, J=9.5 \mathrm{~Hz}, 1 \mathrm{H}), 3.76(\mathrm{~d}, J=12.8 \mathrm{~Hz}, 1 \mathrm{H}), 3.45$ $-3.18(\mathrm{~m}, 2 \mathrm{H}), 2.96-2.73(\mathrm{~s}, 1 \mathrm{H}), 2.46(\mathrm{~s}, 3 \mathrm{H})$.

${ }^{13} \mathrm{C} \mathrm{NMR} \quad\left(125 \mathrm{MHz}, \mathrm{CDCl}_{3}\right)$

$\delta 202.0,168.6,152.5,136.7,135.1,131.2,129.4,127.8,126.6,124.1,123.1,119.4$, 114.7, 41.56, 40.4, 27.1 .

HRMS (ESI) for: $\mathrm{C}_{20} \mathrm{H}_{20} \mathrm{NO}_{2}[\mathrm{M}+\mathrm{H}]^{+}$: calcd 306.1494, found 306.1562 .

HPLC Daicel Chiralpak AD-H column, $n$-hexane/i-PrOH (95/5), $0.5 \mathrm{~mL} / \mathrm{min} 210 \mathrm{~nm} 23.788$ $\min$ (major enantiomer), $19.623 \mathrm{~min}$ (minor enantiomer). 
<smiles>C=CC[C@]1(Cc2ccc(C(C)=O)cc2)Oc2ccccc2C1=O</smiles>

Using Condition A to yield 10c as a colorless oil (159.6 mg, 99\% yield, $91 \%$ ee).

$\underline{R}_{f} \quad 0.30(\mathrm{Hex} / \mathrm{EtOAc}=20: 1$, Vanillin $)$

Opt. Rot. $\quad[\alpha]^{20}{ }_{\mathrm{D}}=-34.0(\mathrm{c}=0.1, \mathrm{MeOH})$

${ }^{1} \mathrm{H} \mathrm{NMR} \quad\left(400 \mathrm{MHz}, \mathrm{CDCl}_{3}\right)$

$\delta 7.86-7.71(\mathrm{~m}, 2 \mathrm{H}), 7.55-7.34(\mathrm{~m}, 2 \mathrm{H}), 7.23(\mathrm{~d}, J=8.3 \mathrm{~Hz}, 2 \mathrm{H}), 7.06-6.80(\mathrm{~m}$, $2 \mathrm{H}), 5.70-5.52(\mathrm{~m}, 1 \mathrm{H}), 5.21-4.93(\mathrm{~m}, 2 \mathrm{H}), 3.82(\mathrm{~s}, 3 \mathrm{H}), 3.28-3.03(\mathrm{~m}, 2 \mathrm{H}), 2.73-$ $2.43(\mathrm{~m}, 2 \mathrm{H})$.

${ }^{13} \mathrm{C} \mathrm{NMR} \quad\left(100 \mathrm{MHz}, \mathrm{CDCl}_{3}\right)$

$\delta 202.8,171.6,167.0,140.0,138.2,130.4,130.3,129.3,128.7,124.1,121.7,121.3$, $120.1,113.0,91.5,52.0,41.5,40.3$.

HRMS (ESI) for: $\mathrm{C}_{20} \mathrm{H}_{19} \mathrm{O}_{4}[\mathrm{M}+\mathrm{H}]^{+}$: calcd 323.1283, found 323.1291 .

HPLC Daicel Chiralpak OD-H column, $n$-hexane/i-PrOH (99/1), $0.5 \mathrm{~mL} / \mathrm{min} 210 \mathrm{~nm} 13.624$ min (major enantiomer), 12.549 min (minor enantiomer).<smiles>C=CC[C@@]1(c2ccc(C#N)cc2)Oc2ccccc2C1=O</smiles>

\section{(S)-4-((2-Allyl-3-oxo-2,3-dihydrobenzofuran-2-yl)methyl)benzonitrile}

(10d).

Using Condition A to yield $\mathbf{1 0 d}$ as a white solid $(131.6 \mathrm{mg}, 91 \%$ yield, $90 \%$ ee).

$\underline{R}_{f} \quad 0.25(\mathrm{Hex} / \mathrm{EtOAc}=15: 1$, Vanillin $)$

Opt. Rot. $[\alpha]^{20}{ }_{\mathrm{D}}=-1.0(\mathrm{c}=0.1, \mathrm{MeOH})$

${ }^{1} \mathrm{H} \mathrm{NMR} \quad\left(500 \mathrm{MHz}, \mathrm{CDCl}_{3}\right)$

$\delta 7.50-7.28(\mathrm{~m}, 4 \mathrm{H}), 7.20(\mathrm{~d}, J=8.3 \mathrm{~Hz}, 2 \mathrm{H}), 7.02-6.79(\mathrm{~m}, 2 \mathrm{H}), 5.68-5.46(\mathrm{~m}$, $1 \mathrm{H}), 5.14-4.88(\mathrm{~m}, 2 \mathrm{H}), 3.22-2.98(\mathrm{~m}, 2 \mathrm{H}), 2.64-2.39(\mathrm{~m}, 2 \mathrm{H})$.

${ }^{13} \mathrm{C} \mathrm{NMR} \quad\left(125 \mathrm{MHz}, \mathrm{CDCl}_{3}\right)$

$\delta 202.5,171.5,140.0,138.4,131.7,131.1,130.1,124.1,121.9,121.2,120.3,118.7$, $112.9,110.8,91.2,41.5,40.3$.

HRMS (ESI) for: $\mathrm{C}_{19} \mathrm{H}_{16} \mathrm{NO}_{2}[\mathrm{M}+\mathrm{H}]^{+}$: calcd 290.1181, found 290.1183 .

HPLC Daicel Chiralpak AD-H column, $n$-hexane $/ i$-PrOH (95/5), $1 \mathrm{~mL} / \mathrm{min} 250 \mathrm{~nm} 15.341 \mathrm{~min}$ (major enantiomer), $12.175 \mathrm{~min}$ (minor enantiomer). 
<smiles>C=CC[C@]1(c2ccc(C(F)(F)F)cc2)Oc2ccccc2C1=O</smiles>

Condition A to yield 10 e as a yellow oil (152.9 $\mathrm{mg}, 92 \%$ yield, $90 \%$ ee).

$\underline{R}_{f} \quad 0.45(\mathrm{Hex} / \mathrm{EtOAc}=20: 1$, Vanillin $)$

Opt. Rot. $[\alpha]^{20}{ }_{\mathrm{D}}=-26.0(\mathrm{c}=0.1, \mathrm{MeOH})$

${ }^{1} \mathrm{H} \mathrm{NMR} \quad\left(500 \mathrm{MHz}, \mathrm{CDCl}_{3}\right)$

$\delta 7.53-7.38(\mathrm{~m}, 2 \mathrm{H}), 7.33(\mathrm{~d}, J=7.9 \mathrm{~Hz}, 2 \mathrm{H}), 7.25-7.18(\mathrm{~m}, 2 \mathrm{H}), 7.00-6.82(\mathrm{~m}$, $2 \mathrm{H}), 5.67-5.46(\mathrm{~m}, 1 \mathrm{H}), 5.02(\mathrm{dd}, J=48.8,13.6 \mathrm{~Hz}, 2 \mathrm{H}), 3.16-3.01(\mathrm{~m}, 2 \mathrm{H}), 2.65-$ $2.43(\mathrm{~m}, 2 \mathrm{H})$.

${ }^{13} \mathrm{C} \mathrm{NMR} \quad\left(125 \mathrm{MHz}, \mathrm{CDCl}_{3}\right)$

$\delta 202.8,171.6,138.5,138.3,130.7,130.3,129.3,129.0,124.9,124.9,124.9,124.8$, $124.1,121.8,121.2,120.1,113.0,91.3,41.3,40.2$.

${ }^{19} \mathrm{~F} \mathrm{NMR} \quad\left(470 \mathrm{MHz}, \mathrm{CDCl}_{3}\right)$

$\delta-62.58$.

HRMS (ESI) for: $\mathrm{C}_{19} \mathrm{H}_{16} \mathrm{FO}_{3}[\mathrm{M}+\mathrm{H}]^{+}$: calcd 333.1102, found 333.1095 .

HPLC Daicel Chiralpak AD-3 column, $n$-hexane/i-PrOH (99/1), $0.5 \mathrm{~mL} / \mathrm{min} 210 \mathrm{~nm} 17.134$ min (major enantiomer), 14.864 min (minor enantiomer).<smiles>C=CCC1(Cc2ccc(F)cc2)Oc2ccccc2C1=O</smiles>

$10 f$

(S)-2-Allyl -2-(4-fluorobenzyl)benzofuran-3(2H)-one (10f). Using Condition A to yield $10 \mathrm{f}$ as a yellow oil (129.9 $\mathrm{mg}, 92 \%$ yield, $90 \%$ ee).

$\underline{R}_{f} \quad 0.45(\mathrm{Hex} / \mathrm{EtOAc}=20: 1$, Vanillin)

Opt. Rot. $[\alpha]^{20}=-39.0(\mathrm{c}=0.1, \mathrm{MeOH})$

$\underline{{ }^{1} \mathrm{H} \mathrm{NMR}} \quad\left(400 \mathrm{MHz}, \mathrm{CDCl}_{3}\right)$

$\delta 7.56-7.40(\mathrm{~m}, 2 \mathrm{H}), 7.19-7.05(\mathrm{~m}, 2 \mathrm{H}), 7.05-6.88(\mathrm{~m}, 2 \mathrm{H}), 6.81(\mathrm{t}, J=8.7 \mathrm{~Hz}$, 2H), $5.70-5.52$ (m, 1H), $5.08(\mathrm{dd}, J=42.9,13.6 \mathrm{~Hz}, 2 \mathrm{H}), 3.09$ (s, 2H), $2.70-2.48(\mathrm{~m}$, $2 \mathrm{H})$.

${ }^{13} \mathrm{C} \mathrm{NMR} \quad\left(100 \mathrm{MHz}, \mathrm{CDCl}_{3}\right)$

$\delta 203.3,171.7,163.1,160.7,138.3,131.9,131.8,130.5,130.1,130.1,124.1,121.7$, $121.5,120.1,115.0,114.8,113.1,91.8,40.9,40.3$.

${ }^{19} \mathrm{~F} \mathrm{NMR} \quad\left(470 \mathrm{MHz}, \mathrm{CDCl}_{3}\right)$

$\delta-116.05$.

HRMS (ESI) for: $\mathrm{C}_{18} \mathrm{H}_{16} \mathrm{FO}_{2}[\mathrm{M}+\mathrm{H}]^{+}$: calcd 283.1134, found 283.1131 .

HPLC Daicel Chiralpak AD-H column, $n$-hexane/i-PrOH (99/1), 0.5 mL/min $210 \mathrm{~nm} 25.639$ min (major enantiomer), 22.815 min (minor enantiomer). 
<smiles>C=CC[C@@]1(c2ccc(Cl)cc2)Oc2ccccc2C1=O</smiles>

yield $10 \mathrm{~g}$ as a colorless oil (138.9 $\mathrm{mg}, 93 \%$ yield, $90 \%$ ee).

$\underline{R}_{f} \quad 0.30(\mathrm{Hex} / \mathrm{EtOAc}=20: 1$, Vanillin $)$

Opt. Rot. $\quad[\alpha]^{20}{ }_{\mathrm{D}}=-50.0(\mathrm{c}=0.1, \mathrm{MeOH})$

$\underline{{ }^{1} \mathrm{H} \mathrm{NMR}} \quad\left(500 \mathrm{MHz}, \mathrm{CDCl}_{3}\right)$

$\delta 7.61-7.44(\mathrm{~m}, 2 \mathrm{H}), 7.10(\mathrm{~s}, 4 \mathrm{H}), 7.04-6.90(\mathrm{~m}, 2 \mathrm{H}), 5.71-5.54(\mathrm{~m}, 1 \mathrm{H}), 5.20-$ $4.94(\mathrm{~m}, 2 \mathrm{H}), 3.08(\mathrm{~d}, J=3.0 \mathrm{~Hz}, 2 \mathrm{H}), 2.70-2.48(\mathrm{~m}, 2 \mathrm{H})$.

${ }^{13} \mathrm{C} \mathrm{NMR} \quad\left(125 \mathrm{MHz}, \mathrm{CDCl}_{3}\right)$

$\delta$ 203.0, 171.6, 138.2, 132.9, 132.8, 131.6, 130.4, 128.1, 128.0, 124.1, 121.7, 121.4, 120.0, 113.0, 91.6, 40.9, 40.2.

HRMS (ESI) for: $\mathrm{C}_{18} \mathrm{H}_{16} \mathrm{ClO}_{2}[\mathrm{M}+\mathrm{H}]^{+}$: calcd 299.0839, found 299.0830 .

$\underline{\text { HPLC }}$ Daicel Chiralpak AD-H column, $n$-hexane $/ i$-PrOH (99.5/0.5), $0.5 \mathrm{~mL} / \mathrm{min} 250 \mathrm{~nm}$ $25.754 \mathrm{~min}$ (major enantiomer), $23.530 \mathrm{~min}$ (minor enantiomer).<smiles>C=CC[C@@]1(c2ccc(OC)cc2)Oc2ccccc2C1=O</smiles>

(S)-2-Allyl-2-(4-methoxybenzyl)benzofuran-3(2H)-one (10h). Using Condition B to yield $\mathbf{1 0 h}$ as a colorless oil ( $145.7 \mathrm{mg}, 99 \%$ yield, $91 \%$ ee).

$\underline{R}_{f} \quad 0.35(\mathrm{Hex} / \mathrm{EtOAc}=20: 1$, Vanillin)

Opt. Rot. $\alpha]^{20}{ }_{\mathrm{D}}=-31.0(\mathrm{c}=0.1, \mathrm{MeOH})$

${ }^{1} \mathrm{H} \mathrm{NMR} \quad\left(400 \mathrm{MHz}, \mathrm{CDCl}_{3}\right)$

$\delta 7.58-7.38(\mathrm{~m}, 2 \mathrm{H}), 7.14-6.84(\mathrm{~m}, 4 \mathrm{H}), 6.68(\mathrm{~d}, J=8.7 \mathrm{~Hz}, 2 \mathrm{H}), 5.71-5.53(\mathrm{~m}$, $1 \mathrm{H}), 5.20-4.93(\mathrm{~m}, 2 \mathrm{H}), 3.70(\mathrm{~s}, 3 \mathrm{H}), 3.05(\mathrm{~s}, 2 \mathrm{H}), 2.68-2.49(\mathrm{~m}, 2 \mathrm{H})$.

${ }^{13} \mathrm{C} \mathrm{NMR} \quad\left(100 \mathrm{MHz}, \mathrm{CDCl}_{3}\right)$

$\delta 203.6,171.8,158.5,138.1,131.4,130.7,126.5,124.1,121.6,119.9,113.5,113.1$, $92.1,55.2,40.9,40.2$.

HRMS (ESI) for: $\mathrm{C}_{19} \mathrm{H}_{19} \mathrm{O}_{3}[\mathrm{M}+\mathrm{H}]^{+}$: calcd 295.1334, found 295.1338 .

HPLC Daicel Chiralpak OD-H column, $n$-hexane/i-PrOH (99/1), 0.5 mL/min $210 \mathrm{~nm} 23.343$ min (major enantiomer), $21.681 \mathrm{~min}$ (minor enantiomer). 
<smiles>C=CC[C@@]1(c2ccc([N+](=O)[O-])cc2)Oc2ccccc2C1=O</smiles>
yl)methyl)phenyl)carbamate(10i). Using Condition B to yield 10i as a yellow solid (170.0 $\mathrm{mg}, 88 \%$ yield, $90 \%$ ee).

$\underline{R}_{f} \quad 0.30(\mathrm{Hex} / \mathrm{EtOAc}=15: 1$, Vanillin $)$

Opt. Rot. $[\alpha]^{20}{ }_{\mathrm{D}}=-29.0(\mathrm{c}=0.1, \mathrm{MeOH})$

$\underline{{ }^{1} \mathrm{H} \mathrm{NMR}} \quad\left(500 \mathrm{MHz}, \mathrm{CDCl}_{3}\right)$ $\delta 7.52(\mathrm{~s}, 2 \mathrm{H}), 7.15(\mathrm{~s}, 4 \mathrm{H}), 7.01(\mathrm{~d}, J=11.3 \mathrm{~Hz}, 1 \mathrm{H}), 6.92(\mathrm{~d}, J=7.7 \mathrm{~Hz}, 1 \mathrm{H}), 6.33(\mathrm{~s}$, $1 \mathrm{H}), 5.71-5.52(\mathrm{~m}, 1 \mathrm{H}), 5.00(\mathrm{~d}, J=10.2 \mathrm{~Hz}, 2 \mathrm{H}), 3.05(\mathrm{~d}, J=2.7 \mathrm{~Hz}, 2 \mathrm{H}), 2.54(\mathrm{~s}$, $2 \mathrm{H}), 1.48(\mathrm{~s}, 9 \mathrm{H})$.

${ }^{13} \mathrm{C} \mathrm{NMR} \quad\left(125 \mathrm{MHz}, \mathrm{CDCl}_{3}\right)$ $\delta 203.3,171.7,152.6,138.0,137.1,130.9,130.6,128.9,124.0,121.5,121.4,119.8$, $118.0,113.1,91.9,41.0,40.1,28.3$.

HRMS (ESI) for: $\mathrm{C}_{23} \mathrm{H}_{26} \mathrm{NO}_{4}[\mathrm{M}+\mathrm{H}]^{+}$: calcd 380.1862 , found 380.1857 .

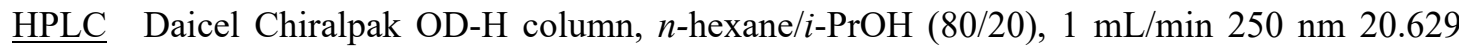
$\min$ (major enantiomer), 15.188 min (minor enantiomer).

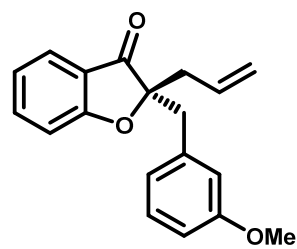

$10 \mathrm{j}$

(S)-2-Allyl-2-(3-methoxybenzyl)benzofuran-3(2H)-one (10j). Using Condition B to yield $\mathbf{1 0 j}$ as a colorless oil ( $145.7 \mathrm{mg}, 99 \%$ yield, $91 \%$ ee $)$.

$\underline{R}_{f} \quad 0.35(\mathrm{Hex} / \mathrm{EtOAc}=20: 1$, Vanillin)

Opt. Rot. $[\alpha]^{20}=-25.0(\mathrm{c}=0.1, \mathrm{MeOH})$

${ }^{1} \mathrm{H} \mathrm{NMR} \quad\left(400 \mathrm{MHz}, \mathrm{CDCl}_{3}\right)$

$\delta 7.51(\mathrm{t}, J=8.2 \mathrm{~Hz}, 2 \mathrm{H}), 7.14-6.88(\mathrm{~m}, 3 \mathrm{H}), 6.82-6.59(\mathrm{~m}, 3 \mathrm{H}), 5.73-5.52(\mathrm{~m}$, $1 \mathrm{H}), 5.23-4.92(\mathrm{~m}, 2 \mathrm{H}), 3.71(\mathrm{~s}, 3 \mathrm{H}), 3.08(\mathrm{~d}, J=3.1 \mathrm{~Hz}, 2 \mathrm{H}), 2.68-2.53(\mathrm{~m}, 2 \mathrm{H})$.

${ }^{13} \mathrm{C} \mathrm{NMR} \quad\left(100 \mathrm{MHz}, \mathrm{CDCl}_{3}\right)$

$\delta 203.2,171.7,159.2,138.0,135.9,130.6,128.9,124.1,122.8,121.5,121.5,119.8$, $115.8,113.0,112.6,91.8,55.1,41.7,40.1$.

HRMS (ESI) for: $\mathrm{C}_{18} \mathrm{H}_{19} \mathrm{O}_{3}[\mathrm{M}+\mathrm{H}]^{+}$: calcd 295.1334, found 295.1344 .

HPLC Daicel Chiralpak OD-H column, $n$-hexane/i-PrOH (99/1), $0.5 \mathrm{~mL} / \mathrm{min} 210 \mathrm{~nm} 18.113$ $\min$ (major enantiomer), $21.104 \mathrm{~min}$ (minor enantiomer). 


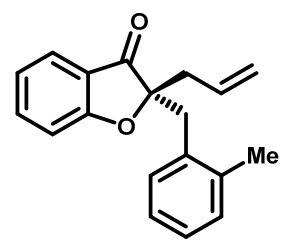

10k

(S)-2-Allyl-2-(2-methylbenzyl)benzofuran-3(2H)-one (10k). Using Condition A to yield 10k as a colorless oil (135.0 $\mathrm{mg}, 97 \%$ yield, $92 \%$ ee).

$\underline{R}_{f} \quad 0.40(\mathrm{Hex} / \mathrm{EtOAc}=20: 1$, Vanillin $)$

Opt. Rot. $[\alpha]^{20}{ }_{\mathrm{D}}=-135.0(\mathrm{c}=0.1, \mathrm{MeOH})$

${ }^{1} \mathrm{H} \mathrm{NMR} \quad\left(500 \mathrm{MHz}, \mathrm{CDCl}_{3}\right)$

$\delta 7.43(\mathrm{t}, J=7.1 \mathrm{~Hz}, 2 \mathrm{H}), 7.05-6.70(\mathrm{~m}, 6 \mathrm{H}), 5.64-5.47(\mathrm{~m}, 1 \mathrm{H}), 4.99(\mathrm{dd}, J=57.7$, $13.6 \mathrm{~Hz}, 2 \mathrm{H}), 3.08-2.92(\mathrm{~m}, 2 \mathrm{H}), 2.63-2.44(\mathrm{~m}, 2 \mathrm{H}), 2.16(\mathrm{~s}, 3 \mathrm{H})$.

${ }^{13} \mathrm{C} \mathrm{NMR} \quad\left(125 \mathrm{MHz}, \mathrm{CDCl}_{3}\right)$

$\delta$ 203.3, 171.7, 137.9, 137.5, 134.3, 131.2, 130.6, 127.9, 127.6, 127.4, 124.0, 121.5, $121.5,119.8,113.0,91.9,41.7,40.0,21.3$.

HRMS (ESI) for: $\mathrm{C}_{19} \mathrm{H}_{19} \mathrm{O}_{2}[\mathrm{M}+\mathrm{H}]^{+}$: calcd 279.1385, found 279.1382 .

HPLC Daicel Chiralpak IG-3 column, $n$-hexane $/ i$-PrOH (98/2), $1 \mathrm{~mL} / \mathrm{min} 210 \mathrm{~nm} 8.466 \mathrm{~min}$ (major enantiomer), $8.136 \mathrm{~min}$ (minor enantiomer).<smiles>C=CCC1(CC=C)Oc2ccccc2C1=O</smiles>

101

(S)-2-Allyl-2-(2-bromobenzyl)benzofuran-3(2H)-one (10I). Using Condition B to yield $\mathbf{1 0 1}$ as a yellow oil (140.7 $\mathrm{mg}, 82 \%$ yield, $93 \%$ ee).

$\underline{R}_{f} \quad 0.45(\mathrm{Hex} / \mathrm{EtOAc}=20: 1$, Vanillin $)$

Opt. Rot. $[\alpha]^{20}{ }_{\mathrm{D}}=-60.0(\mathrm{c}=0.1, \mathrm{MeOH})$

${ }^{1} \mathrm{H} \mathrm{NMR} \quad\left(400 \mathrm{MHz}, \mathrm{CDCl}_{3}\right)$

$\delta 7.65-7.43(\mathrm{~m}, 3 \mathrm{H}), 7.24(\mathrm{~s}, 1 \mathrm{H}), 7.20-6.89(\mathrm{~m}, 4 \mathrm{H}), 5.63(\mathrm{t}, J=7.2 \mathrm{~Hz}, 1 \mathrm{H}), 5.20-$ $4.92(\mathrm{~m}, 2 \mathrm{H}), 3.48(\mathrm{~d}, J=14.2 \mathrm{~Hz}, 1 \mathrm{H}), 3.20(\mathrm{~d}, J=14.2 \mathrm{~Hz}, 1 \mathrm{H}), 2.81-2.50(\mathrm{~m}, 2 \mathrm{H})$.

${ }^{13} \mathrm{C} \mathrm{NMR} \quad\left(100 \mathrm{MHz}, \mathrm{CDCl}_{3}\right)$

$\delta$ 203.1, 171.6, 138.1, 134.5, 133.0, 132.1, 130.4, 128.6, 127.0, 126.0, 124.1, 121.7, $121.2,120.0,113.1,91.9,40.7,39.6$.

HRMS (ESI) for: $\mathrm{C}_{18} \mathrm{H}_{16} \mathrm{BrO}_{2}[\mathrm{M}+\mathrm{H}]^{+}$: calcd 343.0334, found 343.0337 .

HPLC Daicel Chiralpak IC-3 column, $n$-hexane $/ i$-PrOH (99.5/0.5), 1 mL/min $210 \mathrm{~nm} 12.968$ $\min$ (major enantiomer), $14.438 \mathrm{~min}$ (minor enantiomer).

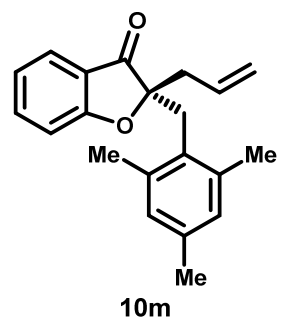

(S)-2-Allyl-2-(2,4,6-trimethylbenzyl)benzofuran-3(2H)-one $\quad(10 \mathrm{~m}) . \quad$ Using Condition A to yield $10 \mathrm{~m}$ as a white solid (145.5 $\mathrm{mg}, 95 \%$ yield, $92 \%$ ee).

$\underline{R}_{f} \quad 0.55(\mathrm{Hex} /$ EtOAc $=20: 1$, Vanillin $)$ 
Opt. Rot. $\quad[\alpha]^{20}{ }_{\mathrm{D}}=-121.0(\mathrm{c}=0.1, \mathrm{MeOH})$

${ }^{1} \mathrm{H} \mathrm{NMR} \quad\left(500 \mathrm{MHz}, \mathrm{CDCl}_{3}\right)$

$\delta 7.64-7.45(\mathrm{~m}, 2 \mathrm{H}), 6.99(\mathrm{t}, J=7.6 \mathrm{~Hz}, 2 \mathrm{H}), 6.76(\mathrm{~s}, 2 \mathrm{H}), 5.64-5.44(\mathrm{~m}, 1 \mathrm{H}), 5.10$ $(\mathrm{d}, J=17.0 \mathrm{~Hz}, 1 \mathrm{H}), 4.96(\mathrm{~d}, J=10.2 \mathrm{~Hz}, 1 \mathrm{H}), 3.30-3.06(\mathrm{~m}, 2 \mathrm{H}), 2.80-2.50(\mathrm{~m}$, $2 \mathrm{H}), 2.24(\mathrm{~d}, J=65.1 \mathrm{~Hz}, 9 \mathrm{H})$.

${ }^{13} \mathrm{C} \mathrm{NMR} \quad\left(125 \mathrm{MHz}, \mathrm{CDCl}_{3}\right)$

$\delta 204.0,171.5,138.0,138.0,136.0,130.7,129.1,128.9,124.1,121.5,121.2,119.8$, $112.9,93.1,40.0,34.8,21.1,20.8$.

HRMS (ESI) for: $\mathrm{C}_{21} \mathrm{H}_{23} \mathrm{O}_{2}[\mathrm{M}+\mathrm{H}]^{+}$: calcd 307.1698, found 307.1688.

HPLC Daicel Chiralpak IA-3 column, $n$-hexane $i$-PrOH (99.5/0.5), $0.5 \mathrm{~mL} / \mathrm{min} 210 \mathrm{~nm} 12.591$ min (major enantiomer), 11.999 min (minor enantiomer).<smiles>C=CC[C@]1(Cc2ccc3ccccc3c2)Oc2ccccc2C1=O</smiles>

(S)-2-Allyl-2-((1-methyl-1H-indol-3-yl)methyl)benzofuran-3(2H)-one

(10n).

Using Condition A to yield 10n as a colorless oil (143.0 mg, 91\% yield, $91 \%$ ee).

$\underline{R}_{f} \quad 0.50(\mathrm{Hex} / \mathrm{EtOAc}=20: 1$, Vanillin $)$

Opt. Rot. $[\alpha]^{20}{ }_{\mathrm{D}}=-100.0(\mathrm{c}=0.1, \mathrm{MeOH})$

$\underline{{ }^{1} \mathrm{H} \mathrm{NMR}} \quad\left(400 \mathrm{MHz}, \mathrm{CDCl}_{3}\right)$

$\delta 7.80-7.59(\mathrm{~m}, 4 \mathrm{H}), 7.56-7.31(\mathrm{~m}, 5 \mathrm{H}), 7.04(\mathrm{~d}, J=8.3 \mathrm{~Hz}, 1 \mathrm{H}), 6.90(\mathrm{t}, J=7.4 \mathrm{~Hz}$, $1 \mathrm{H}), 5.75-5.57(\mathrm{~m}, 1 \mathrm{H}), 5.22-4.95(\mathrm{~m}, 2 \mathrm{H}), 3.29(\mathrm{~s}, 2 \mathrm{H}), 2.81-2.53(\mathrm{~m}, 2 \mathrm{H})$.

${ }^{13} \mathrm{C} \mathrm{NMR} \quad\left(100 \mathrm{MHz}, \mathrm{CDCl}_{3}\right)$

$\delta$ 203.3, 171.7, 138.0, 133.2, 132.3, 132.1, 130.6, 129.2, 128.5, 127.7, 127.5, 127.5, 125.8, 125.6, 124.1, 121.6, 121.4, 119.9, 113.0, 92.0, 41.8, 40.3.

for: $\mathrm{C}_{22} \mathrm{H}_{19} \mathrm{O}_{2}[\mathrm{M}+\mathrm{H}]^{+}$: calcd 315.1385, found 315.1375.

HRMS (ESI) Daicel Chiralpak ID-3 column, $n$-hexane/i-PrOH (99/1), $0.5 \mathrm{~mL} / \mathrm{min} 210 \mathrm{~nm} 19.658$

$\underline{\text { HPLC }} \min$ (major enantiomer), $25.978 \mathrm{~min}$ (minor enantiomer).<smiles>C=CCC1(Cc2cccc3ccccc23)Oc2ccccc2C1=O</smiles>

(S)-2-Allyl-2-(naphthalen-1-ylmethyl)benzofuran-3(2H)-one

Condition A to yield $\mathbf{1 0 o}$ as a white solid (154.0 $\mathrm{mg}, 98 \%$ yield, $96 \% \mathrm{ee})$.

(10o). Using $\underline{R}_{f} \quad 0.50(\mathrm{Hex} / \mathrm{EtOAc}=20: 1$, Vanillin $)$

Opt. Rot. $[\alpha]^{20}=-37.0(\mathrm{c}=0.1, \mathrm{MeOH})$

$\underline{{ }^{1} \mathrm{H} \mathrm{NMR}} \quad\left(500 \mathrm{MHz}, \mathrm{CDCl}_{3}\right)$

$\delta 8.14(\mathrm{~d}, J=8.6 \mathrm{~Hz} 1 \mathrm{H}), 7.74(\mathrm{~d}, J=8.5 \mathrm{~Hz}, 2 \mathrm{H}), 7.55-7.26(\mathrm{~m}, 6 \mathrm{H}), 6.94-6.74(\mathrm{~m}$, $2 \mathrm{H}), 5.62(\mathrm{dd}, J=17.2,7.1 \mathrm{~Hz}, 1 \mathrm{H}), 5.20-4.98(\mathrm{~m}, 2 \mathrm{H}), 3.70(\mathrm{~d}, J=14.5 \mathrm{~Hz}, 1 \mathrm{H})$, $3.49(\mathrm{~d}, J=14.5 \mathrm{~Hz}, 1 \mathrm{H}), 2.86-2.61(\mathrm{~m}, 2 \mathrm{H})$. 
${ }^{13} \mathrm{C} \mathrm{NMR} \quad\left(125 \mathrm{MHz}, \mathrm{CDCl}_{3}\right)$

$\delta 203.5,171.6,137.9,133.7,132.8,131.0,130.6,129.1,128.4,127.8,125.7,125.4$, $125.0,125.0,124.0,121.5,121.3,120.0,112.9,92.4,40.1,37.9$.

for: $\mathrm{C}_{22} \mathrm{H}_{19} \mathrm{O}_{2}[\mathrm{M}+\mathrm{H}]^{+}$: calcd 315.1385 , found 315.1377 .

HRMS (ESI) Daicel Chiralpak IA-3 column, $n$-hexane $/ i$-PrOH (95/5), $0.3 \mathrm{~mL} / \mathrm{min} 210 \mathrm{~nm} 18.729$

$\underline{\text { HPLC }} \min$ (major enantiomer), $17.860 \mathrm{~min}$ (minor enantiomer).<smiles></smiles>

10p (S)-2-Allyl-2-((1-methyl-1H-indol-3-yl)methyl)benzofuran-3(2H)-one (10p). Using Condition B to yield 10p as a yellow oil (157.1 $\mathrm{mg}, 99 \%$ yield, $90 \%$ ee).

$\underline{R}_{f} \quad 0.35(\mathrm{Hex} / \mathrm{EtOAc}=15: 1$, Vanillin $)$

Opt. Rot. $[\alpha]^{20}=-104.0(\mathrm{c}=0.1, \mathrm{MeOH})$

$\underline{{ }^{1} \mathrm{H} \mathrm{NMR}} \quad\left(400 \mathrm{MHz}, \mathrm{CDCl}_{3}\right)$

$\delta 7.60(\mathrm{~d}, J=7.8 \mathrm{~Hz}, 1 \mathrm{H}), 7.47-7.29(\mathrm{~m}, 2 \mathrm{H}), 7.13-6.98(\mathrm{~m}, 3 \mathrm{H}), 6.96-6.75(\mathrm{~m}$, $3 \mathrm{H}), 5.63-5.38(\mathrm{~m}, 1 \mathrm{H}), 5.10-4.80(\mathrm{~m}, 2 \mathrm{H}), 3.57(\mathrm{~s}, 3 \mathrm{H}), 3.32-2.98(\mathrm{~m}, 2 \mathrm{H}), 2.59$ $(\mathrm{d}, J=7.2 \mathrm{~Hz}, 2 \mathrm{H})$.

${ }^{13} \mathrm{C} \mathrm{NMR} \quad\left(100 \mathrm{MHz}, \mathrm{CDCl}_{3}\right)$

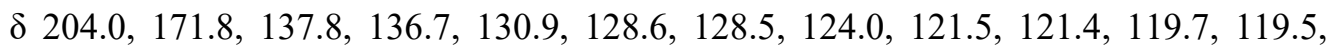
$119.0,113.1,109.0,107.4,92.7,39.8,32.6,31.5$.

HRMS (ESI) for: $\mathrm{C}_{21} \mathrm{H}_{20} \mathrm{NO}_{2}[\mathrm{M}+\mathrm{H}]^{+}$: calcd 318.1494, found 318.2998.

HPLC Daicel Chiralpak AD-H column, $n$-hexane $/ i-\operatorname{PrOH}(99.4 / 0.6), 0.5 \mathrm{~mL} / \mathrm{min} 210 \mathrm{~nm}$ $19.685 \mathrm{~min}$ (major enantiomer), $17.348 \mathrm{~min}$ (minor enantiomer).<smiles>C=CC[C@]1(Cc2ccco2)Oc2ccccc2C1=O</smiles>

$10 \mathrm{q}$

(S)-2-Allyl-2-(furan-2-ylmethyl)benzofuran-3(2H)-one (10q). Using Condition B to yield $\mathbf{1 0 q}$ as a yellow oil (120.8 $\mathrm{mg}, 95 \%$ yield, $90 \%$ ee).

$\underline{R}_{f} \quad 0.35$ (Hex/EtOAc $=15: 1$, Vanillin)

Opt. Rot. $[\alpha]^{20}=-16.0(\mathrm{c}=0.1, \mathrm{MeOH})$

${ }^{1} \mathrm{H} \mathrm{NMR} \quad\left(400 \mathrm{MHz}, \mathrm{CDCl}_{3}\right)$

$\delta 7.68-7.45(\mathrm{~m}, 2 \mathrm{H}), 7.18(\mathrm{~d}, J=2.5 \mathrm{~Hz}, 1 \mathrm{H}), 7.11-6.95(\mathrm{~m}, 2 \mathrm{H}), 6.29-5.93(\mathrm{~m}$, $2 \mathrm{H}), 5.80-5.48(\mathrm{~m}, 1 \mathrm{H}), 5.23-4.84(\mathrm{~m}, 2 \mathrm{H}), 3.29-2.97(\mathrm{~m}, 2 \mathrm{H}), 2.61(\mathrm{dd}, J=7.2$, $3.2 \mathrm{~Hz}, 2 \mathrm{H})$.

${ }^{13} \mathrm{C} \mathrm{NMR} \quad\left(100 \mathrm{MHz}, \mathrm{CDCl}_{3}\right)$

$\delta 202.7,171.7,149.0,141.8,138.1,130.3,124.2,121.7,121.0,120.1,113.2,110.2$, $108.7,90.6,39.8,34.2$.

HRMS (ESI) for: $\mathrm{C}_{16} \mathrm{H}_{15} \mathrm{O}_{3}[\mathrm{M}+\mathrm{H}]^{+}$: calcd 255.1021, found 255.1003 .

$\underline{\text { HPLC }}$ Daicel Chiralpak AD-H column, $n$-hexane $/ i$-PrOH (99.4/0.6), $0.5 \mathrm{~mL} / \mathrm{min} 210 \mathrm{~nm}$ $20.794 \mathrm{~min}$ (major enantiomer), $22.955 \mathrm{~min}$ (minor enantiomer). 


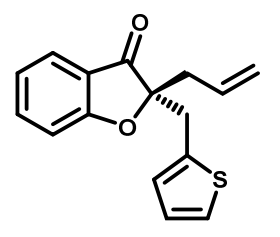

10r (S)-2-Allyl-2-(thiophen-2-ylmethyl)benzofuran-3(2H)-one (10r). Using Condition B to yield 10r as a white solid (127.1 mg, 94\% yield, 92\% ee).

$\underline{R}_{f} \quad 0.30(\mathrm{Hex} / \mathrm{EtOAc}=15: 1$, Vanillin)

Opt. Rot. $[\alpha]^{20}{ }_{\mathrm{D}}=-39.0(\mathrm{c}=0.1, \mathrm{MeOH})$

${ }^{1} \mathrm{H} \mathrm{NMR} \quad\left(500 \mathrm{MHz}, \mathrm{CDCl}_{3}\right)$

$\delta 7.63-7.47(\mathrm{~m}, 2 \mathrm{H}), 7.16-6.91(\mathrm{~m}, 3 \mathrm{H}), 6.80(\mathrm{dt}, J=8.5,3.9 \mathrm{~Hz}, 2 \mathrm{H}), 5.74-5.57$ $(\mathrm{m}, 1 \mathrm{H}), 5.25-4.97(\mathrm{~m}, 2 \mathrm{H}), 3.34(\mathrm{~d}, J=2.5 \mathrm{~Hz}, 2 \mathrm{H}), 2.62(\mathrm{~d}, J=8.2 \mathrm{~Hz}, 2 \mathrm{H})$.

${ }^{13} \mathrm{C} \mathrm{NMR} \quad\left(125 \mathrm{MHz}, \mathrm{CDCl}_{3}\right)$

$\delta 202.8,171.9,138.1,135.6,130.4,127.7,126.5,125.0,124.1,121.7,121.4,120.0$, $113.3,91.2,39.9,35.8$.

$\underline{\mathrm{HRMS}(\mathrm{ESI})}$ for: $\mathrm{C}_{16} \mathrm{H}_{150} \mathrm{O}_{2} \mathrm{~S}[\mathrm{M}+\mathrm{H}]^{+}$: calcd 271.0793, found 271.0778.

$\underline{\text { HPLC }}$ Daicel Chiralpak IC-3 column, $n$-hexane/ $i$-PrOH (90/10), 0.5 mL/min $210 \mathrm{~nm} 11.093$ min (major enantiomer), 10.036 min (minor enantiomer).<smiles>C=CC[C@]1(/C=C/c2ccccc2)Oc2ccccc2C1=O</smiles>

10s (S)-2-Allyl-2-cinnamylbenzofuran-3(2H)-one (10s). Using Condition A to yield 10s as a colorless oil (136.5 $\mathrm{mg}$, 94\% yield, 90\% ee).

$\underline{R}_{f} \quad 0.40(\mathrm{Hex} / \mathrm{EtOAc}=20: 1$, Vanillin)

Opt. Rot. $[\alpha]^{20}=-32.0(\mathrm{c}=0.1, \mathrm{MeOH})$

${ }^{1} \mathrm{H} \mathrm{NMR} \quad\left(500 \mathrm{MHz}, \mathrm{CDCl}_{3}\right)$

$\delta 7.77-7.51(\mathrm{~m}, 2 \mathrm{H}), 7.32-7.18(\mathrm{~m}, 5 \mathrm{H}), 7.14(\mathrm{~d}, J=8.4 \mathrm{~Hz}, 1 \mathrm{H}), 7.10-7.03(\mathrm{~m}$, $1 \mathrm{H}), 6.51(\mathrm{~d}, J=15.8 \mathrm{~Hz}, 1 \mathrm{H}), 6.22-5.94(\mathrm{~m}, 1 \mathrm{H}), 5.75-5.63(\mathrm{~m}, 1 \mathrm{H}), 5.24-4.98$ $(\mathrm{m}, 2 \mathrm{H}), 2.87-2.57(\mathrm{~m}, 4 \mathrm{H})$.

${ }^{13} \mathrm{C} \mathrm{NMR} \quad\left(125 \mathrm{MHz}, \mathrm{CDCl}_{3}\right)$

$\delta$ 203.3, 171.7, 138.2, 137.0, 134.6, 130.5, 128.5, 127.4, 126.3, 124.3, 122.1, 121.8, $121.3,119.9,113.3,91.5,39.9,39.2$.

$\underline{\mathrm{HRMS}(\mathrm{ESI})}$ for: $\mathrm{C}_{20} \mathrm{H}_{19} \mathrm{O}_{2}[\mathrm{M}+\mathrm{H}]^{+}$: calcd 291.1385, found 295.1391.

HPLC Daicel Chiralpak IA-3 column, $n$-hexane/ $i$-PrOH (99.4/0.6), 0.5 mL/min $250 \mathrm{~nm} 18.599$ min (major enantiomer), 24.889 min (minor enantiomer).<smiles>C=CC[C@]1(CCCc2ccccc2)Oc2ccccc2C1=O</smiles>

10t (R)-2-Allyl-2-(3-phenylpropyl)benzofuran-3(2H)-one (10t). Using Condition B to yield 10t as a colorless oil (141.8 $\mathrm{mg}, 97 \%$ yield, $90 \%$ ee).

$\underline{R}_{f} \quad 0.40(\mathrm{Hex} / \mathrm{EtOAc}=20: 1$, Vanillin $)$

Opt. Rot. $\quad[\alpha]^{20}{ }_{\mathrm{D}}=8.0(\mathrm{c}=0.1, \mathrm{MeOH})$

$\underline{{ }^{1} \mathrm{H} \mathrm{NMR}} \quad\left(500 \mathrm{MHz}, \mathrm{CDCl}_{3}\right)$ 
$\delta 7.60-7.45(\mathrm{~m}, 2 \mathrm{H}), 7.20-7.12(\mathrm{~m}, 2 \mathrm{H}), 7.11-6.89(\mathrm{~m}, 5 \mathrm{H}), 5.70-5.40(\mathrm{~m}, 1 \mathrm{H})$, $4.98(\mathrm{dd}, J=48.8,13.6 \mathrm{~Hz}, 2 \mathrm{H}), 2.53-2.42(\mathrm{~m}, 4 \mathrm{H}), 1.84(\mathrm{t}, J=8.3 \mathrm{~Hz}, 2 \mathrm{H}), 1.58-$ $1.48(\mathrm{~m}, 1 \mathrm{H}), 1.43-1.30(\mathrm{~m}, 1 \mathrm{H})$.

${ }^{13} \mathrm{C} \mathrm{NMR} \quad\left(125 \mathrm{MHz}, \mathrm{CDCl}_{3}\right)$

$\delta 203.8,171.9,141.6,138.1,130.7,128.4,128.3,125.9,124.2,121.6,121.5,119.6$, 113.2, 92.1, 40.6, 35.9, 35.5, 24.8 .

HRMS (ESI) for: $\mathrm{C}_{20} \mathrm{H}_{21} \mathrm{O}_{2}[\mathrm{M}+\mathrm{H}]^{+}$: calcd 293.1542, found 293.1552 .

HPLC Daicel Chiralpak IC-3 column, $n$-hexane $/ i-\mathrm{PrOH}$ (99/1), $0.5 \mathrm{~mL} / \mathrm{min} 210 \mathrm{~nm} 24.211$ $\min$ (major enantiomer), $18.998 \mathrm{~min}$ (minor enantiomer).<smiles>C=CC[C@]1(CC2CC2)Oc2ccccc2C1=O</smiles>

(S)-2-Allyl-2-(cyclopropylmethyl)benzofuran-3(2H)-one (10u). Using Condition B to yield $10 \mathrm{u}$ as a colorless oil ( $103.9 \mathrm{mg}, 91 \%$ yield, $90 \%$ ee).

$\underline{R}_{f} \quad 0.40(\mathrm{Hex} / \mathrm{EtOAc}=30: 1$, Vanillin $)$

Opt. Rot. $[\alpha]^{20}{ }_{\mathrm{D}}=-38.0(\mathrm{c}=0.1, \mathrm{MeOH})$

$\underline{{ }^{1} \mathrm{H} \mathrm{NMR}} \quad\left(500 \mathrm{MHz}, \mathrm{CDCl}_{3}\right)$

$\delta 7.73-7.52(\mathrm{~m}, 2 \mathrm{H}), 7.21-6.94(\mathrm{~m}, 2 \mathrm{H}), 5.75-5.52(\mathrm{~m}, 1 \mathrm{H}), 5.24-4.91(\mathrm{~m}, 2 \mathrm{H})$,

$2.72-2.41(\mathrm{~m}, 2 \mathrm{H}), 1.92(\mathrm{dd}, J=14.4,6.3 \mathrm{~Hz}, 1 \mathrm{H}), 1.75-1.60(\mathrm{~m}, 1 \mathrm{H}), 0.70-0.48$ $(\mathrm{m}, 1 \mathrm{H}), 0.39-0.16(\mathrm{~m}, 2 \mathrm{H}), 0.17--0.05(\mathrm{~m}, 2 \mathrm{H})$.

${ }^{13} \mathrm{C} \mathrm{NMR} \quad\left(125 \mathrm{MHz}, \mathrm{CDCl}_{3}\right)$

$\delta$ 204.1, 172.0, 137.9, 130.8, 124.1, 121.8, 121.5, 119.5, 113.1, 92.7, 40.6, 40.4, 5.3, 4.4, 4.3.

HRMS (ESI) for: $\mathrm{C}_{15} \mathrm{H}_{17} \mathrm{O}_{2}[\mathrm{M}+\mathrm{H}]^{+}$: calcd 229.1229, found 229.1217 .

HPLC Daicel Chiralpak IC-3 column, $n$-hexane $/ i-P r O H ~(99.35 / 0.65), 0.5 \mathrm{~mL} / \mathrm{min} 210 \mathrm{~nm}$ $18.407 \mathrm{~min}$ (major enantiomer), $19.467 \mathrm{~min}$ (minor enantiomer).<smiles>C=CC[C@]1(Cc2cccc3ccccc23)Oc2cccc(OC)c2C1=O</smiles>

Using Condition A to yield 10v as a colorless oil ( $170.5 \mathrm{mg}, 99 \%$ yield, $91 \% \mathrm{ee})$.

$\underline{R}_{f} \quad 0.40$ (Hex/EtOAc $=15: 1$, Vanillin)

Opt. Rot. $[\alpha]^{20}=-231.0(\mathrm{c}=0.1, \mathrm{MeOH})$

$\underline{{ }^{1} \mathrm{H} \mathrm{NMR}} \quad\left(500 \mathrm{MHz}, \mathrm{CDCl}_{3}\right)$

$\delta 8.14(\mathrm{~d}, J=8.5 \mathrm{~Hz}, 1 \mathrm{H}), 7.71(\mathrm{dd}, J=47.1,8.1 \mathrm{~Hz}, 2 \mathrm{H}), 7.55-7.48(\mathrm{~m}, 1 \mathrm{H}), 7.47-$ $7.36(\mathrm{~m}, 2 \mathrm{H}), 7.36-7.28(\mathrm{~m}, 2 \mathrm{H}), 6.32(\mathrm{dd}, J=47.2,8.2 \mathrm{~Hz}, 2 \mathrm{H}), 5.72-5.59(\mathrm{~m}, 1 \mathrm{H})$, $5.23-4.94(\mathrm{~m}, 2 \mathrm{H}), 3.86(\mathrm{~s}, 3 \mathrm{H}), 3.75-3.25(\mathrm{~m}, 2 \mathrm{H}), 2.82-2.54(\mathrm{~m}, 2 \mathrm{H})$.

${ }^{13} \mathrm{C} \mathrm{NMR} \quad\left(125 \mathrm{MHz}, \mathrm{CDCl}_{3}\right)$

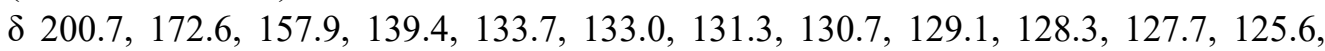
$125.4,125.1,125.0,119.9,110.6,104.8,102.7,92.3,56.0,40.0,37.9$.

HRMS (ESI) for: $\mathrm{C}_{23} \mathrm{H}_{21} \mathrm{O}_{3}[\mathrm{M}+\mathrm{H}]^{+}$: calcd 345.1491, found 345.1521.

HPLC Daicel Chiralpak OD-H column, $n$-hexane/i-PrOH (95/5), 0.5 mL/min $210 \mathrm{~nm} 27.511$ 
min (major enantiomer), 43.943 min (minor enantiomer).<smiles>C=CC[C@]1(Cc2cccc3ccccc23)Oc2ccc(OC)cc2C1=O</smiles>

(S)-2-Allyl-5-methoxy-2-(naphthalen-1-ylmethyl)benzofuran-3(2H)-one (10w). Using Condition A to yield $\mathbf{1 0 w}$ as a yellow solid (168.8 $\mathrm{mg}$, 98\% yield, $91 \%$ ee).

$\underline{R_{f}} \quad 0.40(\mathrm{Hex} / \mathrm{EtOAc}=15: 1$, Vanillin)

Opt. Rot. $\quad[\alpha]^{20}{ }_{\mathrm{D}}=-302.0(\mathrm{c}=0.1, \mathrm{MeOH})$

$\underline{{ }^{1} \mathrm{H} \mathrm{NMR}} \quad\left(500 \mathrm{MHz}, \mathrm{CDCl}_{3}\right)$

$\delta 8.08(\mathrm{~d}, J=8.6 \mathrm{~Hz}, 1 \mathrm{H}), 7.62(\mathrm{dd}, J=50.3,8.1 \mathrm{~Hz}, 2 \mathrm{H}), 7.51-7.12(\mathrm{~m}, 4 \mathrm{H}), 6.96$ $(\mathrm{dd}, J=9.0,2.9 \mathrm{~Hz}, 1 \mathrm{H}), 6.84-6.55(\mathrm{~m}, 2 \mathrm{H}), 5.68-5.48(\mathrm{~m}, 1 \mathrm{H}), 5.20-4.82(\mathrm{~m}, 2 \mathrm{H})$, $3.61(\mathrm{~d}, J=12.6 \mathrm{~Hz}, 4 \mathrm{H}), 3.41(\mathrm{~d}, J=14.4 \mathrm{~Hz}, 1 \mathrm{H}), 2.75-2.47(\mathrm{~m}, 2 \mathrm{H})$.

${ }^{13} \mathrm{C} \mathrm{NMR} \quad\left(125 \mathrm{MHz}, \mathrm{CDCl}_{3}\right)$

$\delta 203.7,167.0,154.5,133.7,132.8,131.1,130.7,129.0,128.4,128.1,127.8,125.6$, $125.4,125.0,125.0,121.1,119.8,113.7,103.7,93.1,55.8,40.2,37.9$.

HRMS (ESI) for: $\mathrm{C}_{23} \mathrm{H}_{21} \mathrm{O}_{3}[\mathrm{M}+\mathrm{H}]^{+}$: calcd 345.1491, found 345.1492.

HPLC Daicel Chiralpak OD-H column, $n$-hexane/i-PrOH (95/5), 0.5 mL/min $210 \mathrm{~nm} 18.349$ min (major enantiomer), $20.719 \mathrm{~min}$ (minor enantiomer).<smiles>C=CC[C@]1(Cc2cccc3ccccc23)Oc2cc(OC)ccc2C1=O</smiles>

10x

(S)-2-Allyl-6-methoxy-2-(naphthalen-1-ylmethyl)benzofuran-3(2H)-one

(10x). Using Condition A to yield 10x as a yellow solid (170.8 $\mathrm{mg}, 99 \%$ yield, $91 \%$ ee).

$\underline{R}_{f} \quad 0.40(\mathrm{Hex} / \mathrm{EtOAc}=15: 1$, Vanillin)

Opt. Rot. $\quad[\alpha]^{20}{ }_{\mathrm{D}}=-86.0(\mathrm{c}=0.1, \mathrm{MeOH})$

$\underline{{ }^{1} \mathrm{H} \mathrm{NMR}} \quad\left(600 \mathrm{MHz}, \mathrm{CDCl}_{3}\right)$

$\delta 8.15(\mathrm{~d}, J=8.5 \mathrm{~Hz}, 1 \mathrm{H}), 7.71(\mathrm{dd}, J=60.6,8.1 \mathrm{~Hz}, 2 \mathrm{H}), 7.55-7.40(\mathrm{~m}, 2 \mathrm{H}), 7.34$ $(\mathrm{dd}, J=38.9,8.2 \mathrm{~Hz}, 3 \mathrm{H}), 6.45(\mathrm{dd}, J=8.6,2.1 \mathrm{~Hz}, 1 \mathrm{H}), 6.22(\mathrm{~s}, 1 \mathrm{H}), 5.74-5.56(\mathrm{~m}$, $1 \mathrm{H}), 5.25-4.94(\mathrm{~m}, 2 \mathrm{H}), 3.69(\mathrm{~d}, J=14.6 \mathrm{~Hz}, 4 \mathrm{H}), 3.47(\mathrm{~d}, J=14.6 \mathrm{~Hz}, 1 \mathrm{H}), 2.81-$ $2.54(\mathrm{~m}, 2 \mathrm{H})$.

${ }^{13} \mathrm{C} \mathrm{NMR} \quad\left(150 \mathrm{MHz}, \mathrm{CDCl}_{3}\right)$

$\delta 200.8,174.0,168 ., 133.7,132.9,131.2,130.8,129.0,128.4,127.7,125.6,125.3$, $125.1,125.0,125.0,119.8,114.5,111.3,95.5,93.3,55.7,40.1,37.8$.

HRMS (ESI) for: $\mathrm{C}_{23} \mathrm{H}_{21} \mathrm{O}_{3}[\mathrm{M}+\mathrm{H}]^{+}$: calcd 345.1491, found 345.1544.

HPLC Daicel Chiralpak AD-H column, $n$-hexane $/ i-\mathrm{PrOH}(95 / 5), 1 \mathrm{~mL} / \mathrm{min} 210 \mathrm{~nm} 13.434 \mathrm{~min}$ (major enantiomer), $11.781 \mathrm{~min}$ (minor enantiomer). 
<smiles>C=CCC1(Cc2cccc3ccccc23)Oc2cc(Br)ccc2C1=O</smiles>

10y (S)-2-Allyl-6-bromo-2-(naphthalen-1-ylmethyl)benzofuran-3(2H)-one (10y). Using Condition A to yield 10y as a white solid (192.7 $\mathrm{mg}, 98 \%$ yield, $94 \%$ ee).

$\underline{R}_{f} \quad 0.35(\mathrm{Hex} / \mathrm{EtOAc}=20: 1$, Vanillin $)$

Opt. Rot. $[\alpha]^{20}=-78.0(\mathrm{c}=0.1, \mathrm{MeOH})$

$\underline{{ }^{1} \mathrm{H} \mathrm{NMR}} \quad\left(500 \mathrm{MHz}, \mathrm{CDCl}_{3}\right)$ $\delta 8.12(\mathrm{~d}, J=8.5 \mathrm{~Hz}, 1 \mathrm{H}), 7.76(\mathrm{~d}, J=8.1 \mathrm{~Hz}, 1 \mathrm{H}), 7.66(\mathrm{~d}, J=8.0 \mathrm{~Hz}, 1 \mathrm{H}), 7.58-$ $7.49(\mathrm{~m}, 1 \mathrm{H}), 7.49-7.40(\mathrm{~m}, 1 \mathrm{H}), 7.37-7.27(\mathrm{~m}, 3 \mathrm{H}), 7.05-6.97(\mathrm{~m}, 2 \mathrm{H}), 5.73-$ $5.51(\mathrm{~m}, 1 \mathrm{H}), 5.23-4.94(\mathrm{~m}, 2 \mathrm{H}), 3.70(\mathrm{~d}, J=14.5 \mathrm{~Hz}, 1 \mathrm{H}), 3.50(\mathrm{~d}, J=14.6 \mathrm{~Hz}$, $1 \mathrm{H}), 2.90-2.53(\mathrm{~m}, 2 \mathrm{H})$.

${ }^{13} \mathrm{C} \mathrm{NMR} \quad\left(125 \mathrm{MHz}, \mathrm{CDCl}_{3}\right)$

$\delta$ 202.1, 171.6, 133.7, 132.7, 132.7, 130.6, 130.2, 129.0, 128.5, 128.0, 125.8, 125.5, $125.4,125.0,124.8,124.7,120.3,120.3,116.3,93.5,40.2,37.8$.

HRMS (ESI) for: $\mathrm{C}_{22} \mathrm{H}_{18} \mathrm{BrO}_{2}[\mathrm{M}+\mathrm{H}]^{+}$: calcd 393.0493, found 393.0478.

HPLC Daicel Chiralpak AD-H column, $n$-hexane $/ i-\mathrm{PrOH}(95 / 5), 0.5 \mathrm{~mL} / \mathrm{min} 210 \mathrm{~nm} 12.888$ min (major enantiomer), 10.363 min (minor enantiomer).<smiles>C=CC[C@]1(Cc2cccc3ccccc23)Oc2cc(F)ccc2C1=O</smiles>

$10 z$

(S)-2-Allyl-6-fluoro-2-(naphthalen-1-ylmethyl)benzofuran-3(2H)-one (10z).

Using Condition C to yield 10z as a yellow solid ( $162.9 \mathrm{mg}, 98 \%$ yield, $94 \%$ ee).

$\underline{R}_{f} \quad 0.30(\mathrm{Hex} / \mathrm{EtOAc}=20: 1$, Vanillin $)$

Opt. Rot. $\quad[\alpha]^{20}{ }_{D}=-180.0(\mathrm{c}=0.1, \mathrm{MeOH})$

${ }^{1} \mathrm{H} \mathrm{NMR} \quad\left(500 \mathrm{MHz}, \mathrm{CDCl}_{3}\right)$

$\delta 8.05(\mathrm{~d}, J=8.5 \mathrm{~Hz}, 1 \mathrm{H}), 7.67(\mathrm{~d}, J=8.1 \mathrm{~Hz}, 1 \mathrm{H}), 7.56(\mathrm{~d}, J=8.1 \mathrm{~Hz}, 1 \mathrm{H}), 7.48-$ $7.40(\mathrm{~m}, 1 \mathrm{H}), 7.39-7.32(\mathrm{~m}, 2 \mathrm{H}), 7.26(\mathrm{~d}, J=7.8 \mathrm{~Hz}, 1 \mathrm{H}), 7.23-7.16(\mathrm{~m}, 1 \mathrm{H}), 6.49$ $(\mathrm{t}, J=8.7 \mathrm{~Hz}, 1 \mathrm{H}), 6.37(\mathrm{~d}, J=11.3 \mathrm{~Hz}, 1 \mathrm{H}), 5.68-5.47(\mathrm{~m}, 1 \mathrm{H}), 5.17-4.87(\mathrm{~m}, 2 \mathrm{H})$, $3.63(\mathrm{~d}, J=14.5 \mathrm{~Hz}, 1 \mathrm{H}), 3.42(\mathrm{~d}, J=14.5 \mathrm{~Hz}, 1 \mathrm{H}), 2.78-2.47(\mathrm{~m}, 2 \mathrm{H})$.

${ }^{13} \mathrm{C} \mathrm{NMR} \quad\left(125 \mathrm{MHz}, \mathrm{CDCl}_{3}\right)$

$\delta$ 201.3, 173.0, 172.9, 170.2, 168.2, 133.7, 132.7, 130.7, 130.3, 129.0, 128.4, 127.9, $125.9,125.8,125.7,125.5,125.0,124.8,120.2,118.0,110.5,110.3,100.1,99.9,93.9$, $40.2,37.8$

${ }^{19}$ F NMR $\quad(470 \mathrm{MHz}, \mathrm{CDCl} 3)$

$\delta-97.15$.

HRMS (ESI) for: $\mathrm{C}_{22} \mathrm{H}_{18} \mathrm{FO}_{2}[\mathrm{M}+\mathrm{H}]^{+}$: calcd 333.1291, found 333.1279.

HPLC Daicel Chiralpak AD-H column, $n$-hexane/i-PrOH (95/5), $0.5 \mathrm{~mL} / \mathrm{min} 210 \mathrm{~nm} 13.758$ min (major enantiomer), 11.597 min (minor enantiomer). 
<smiles>C=CC[C@]1(Cc2cccc3ccccc23)Oc2cc(N3CCOCC3)ccc2C1=O</smiles>

$10 a^{\prime}$

(S)-2-Allyl-6-morpholino-2-(naphthalen-1-ylmethyl)benzofuran-3(2H)one (10a'). Using Condition A to yield S10a' as a yellow solid (195.8 mg, 98\% yield, 90\% ee).

$\underline{R}_{f} \quad 0.35(\mathrm{Hex} / \mathrm{EtOAc}=10: 1$, Vanillin $)$

Opt. Rot. $\quad[\alpha]^{20}{ }_{\mathrm{D}}=-236.0(\mathrm{c}=0.1, \mathrm{MeOH})$

$\underline{\mathrm{H} \mathrm{NMR}} \quad\left(500 \mathrm{MHz}, \mathrm{CDCl}_{3}\right)$

$\delta 8.25-8.05(\mathrm{~m}, 1 \mathrm{H}), 7.88-7.61(\mathrm{~m}, 2 \mathrm{H}), 7.58-7.17(\mathrm{~m}, 5 \mathrm{H}), 6.44(\mathrm{q}, J=3.1 \mathrm{~Hz}$, $1 \mathrm{H}), 6.09(\mathrm{q}, J=2.9 \mathrm{~Hz}, 1 \mathrm{H}), 5.73-5.55(\mathrm{~m}, 1 \mathrm{H}), 5.05(\mathrm{dd}, J=58.0,13.5 \mathrm{~Hz}, 2 \mathrm{H})$, $3.77(\mathrm{t}, J=4.6 \mathrm{~Hz}, 3 \mathrm{H}), 3.71-3.61(\mathrm{~m}, 1 \mathrm{H}), 3.51-3.40(\mathrm{~m}, 1 \mathrm{H}), 3.31-3.18(\mathrm{~m}, 3 \mathrm{H})$, $2.85-2.47(\mathrm{~m}, 2 \mathrm{H})$.

${ }^{13} \mathrm{C} \mathrm{NMR} \quad\left(125 \mathrm{MHz}, \mathrm{CDCl}_{3}\right)$ $\delta$ 200.0, 173.7, 158.4, 133.7, 133.0, 131.6, 131.1, 129.1, 128.4, 127.6, 125.6, 125.3, 125.2, 125.1, 119.5, 112.1, 109.2, 94.7, 93.0, 66.4, 47.3, 40.1, 37.9.

HRMS (ESI) for: $\mathrm{C}_{26} \mathrm{H}_{26} \mathrm{NO}_{3}[\mathrm{M}+\mathrm{H}]^{+}$: calcd 400.1913, found 400.1994 .

HPLC Daicel Chiralpak AD-H column, $n$-hexane $/ i-\mathrm{PrOH}(80 / 20), 1 \mathrm{~mL} / \mathrm{min} 210 \mathrm{~nm} 12.639$ min (major enantiomer), 8.825 min (minor enantiomer).<smiles>C=CC[C@]1(Cc2cccc3ccccc23)Oc2c(OC)cccc2C1=O</smiles>

$10 b^{\prime}$

(S)-2-allyl-7-methoxy-2-(naphthalen-1-ylmethyl)benzofuran-3(2H)-one (10b'). Using Condition B to yield 10b' as a colorless oil (168.8 $\mathrm{mg}, 98 \%$ yield, $90 \%$ ee).

$\underline{R}_{f} \quad 0.40(\mathrm{Hex} / \mathrm{EtOAc}=15: 1$, Vanillin)

Opt. Rot. $[\alpha]^{20}{ }_{\mathrm{D}}=-62.0(\mathrm{c}=0.1, \mathrm{MeOH})$

${ }^{1} \mathrm{H} \mathrm{NMR} \quad\left(500 \mathrm{MHz}, \mathrm{CDCl}_{3}\right)$

$\delta 8.21(\mathrm{dd}, J=8.5,1.2 \mathrm{~Hz}, 1 \mathrm{H}), 7.80-7.59(\mathrm{~m}, 2 \mathrm{H}), 7.58-7.34(\mathrm{~m}, 3 \mathrm{H}), 7.31-7.25$ $(\mathrm{m}, 1 \mathrm{H}), 7.02(\mathrm{dd}, J=7.7,1.2 \mathrm{~Hz}, 1 \mathrm{H}), 6.95-6.68(\mathrm{~m}, 2 \mathrm{H}), 5.74-5.57(\mathrm{~m}, 1 \mathrm{H}), 5.22-$ $4.94(\mathrm{~m}, 2 \mathrm{H}), 3.73(\mathrm{~d}, J=15.0 \mathrm{~Hz}, 4 \mathrm{H}), 3.53(\mathrm{~d}, J=14.5 \mathrm{~Hz}, 1 \mathrm{H}), 2.88-2.65(\mathrm{~m}, 2 \mathrm{H})$.

${ }^{13} \mathrm{C} \mathrm{NMR} \quad\left(125 \mathrm{MHz}, \mathrm{CDCl}_{3}\right)$

$\delta$ 203.3, 161.8, 146.2, 133.7, 132.8, 130.9, 130.5, 129.0, 128.3, 127.8, 125.7, 125.3, 125.0, 124.9, 122.7, 121.9, 120.0, 119.0, 115.3, 93.1, 56.4, 40.1, 38.1.

HRMS (ESI) for: $\mathrm{C}_{23} \mathrm{H}_{21} \mathrm{O}_{3}[\mathrm{M}+\mathrm{H}]^{+}$: calcd 345.1491, found 345.1483.

HPLC Daicel Chiralpak IC-3 column, $n$-hexane $/ i-\mathrm{PrOH}(95 / 5), 0.5 \mathrm{~mL} / \mathrm{min} 210 \mathrm{~nm} 14.917$ min (major enantiomer), 15.932 min (minor enantiomer). 
<smiles>C=CCC1(Cc2cccc3ccccc23)Oc2c(Br)cccc2C1=O</smiles>

$10 c^{\prime}$

(S)-2-Allyl-7-bromo-2-(naphthalen-1-ylmethyl)benzofuran-3(2H)-one (10c'). Using Condition C to yield 10c' as a colorless oil (194.7 $\mathrm{mg}, 99 \%$ yield, $92 \%$ ee).

$\underline{R}_{f} \quad 0.30(\mathrm{Hex} / \mathrm{EtOAc}=20: 1$, Vanillin $)$

Opt. Rot. $[\alpha]^{20}{ }_{\mathrm{D}}=-9.0(\mathrm{c}=0.1, \mathrm{MeOH})$

$\underline{{ }^{1} \mathrm{H} \mathrm{NMR}} \quad\left(500 \mathrm{MHz}, \mathrm{CDCl}_{3}\right)$

$\delta 8.13(\mathrm{~d}, J=8.5 \mathrm{~Hz}, 1 \mathrm{H}), 7.65(\mathrm{~d}, J=8.1 \mathrm{~Hz}, 1 \mathrm{H}), 7.55(\mathrm{~d}, J=8.2 \mathrm{~Hz}, 1 \mathrm{H}), 7.50-$ $7.42(\mathrm{~m}, 2 \mathrm{H}), 7.39-7.32(\mathrm{~m}, 1 \mathrm{H}), 7.32-7.24(\mathrm{~m}, 2 \mathrm{H}), 7.22-7.15(\mathrm{~m}, 1 \mathrm{H}), 6.65(\mathrm{t}, J=$ $7.7 \mathrm{~Hz}, 1 \mathrm{H}), 5.68-5.48(\mathrm{~m}, 1 \mathrm{H}), 5.15-4.94(\mathrm{~m}, 2 \mathrm{H}), 3.57(\mathrm{dd}, J=98.6,14.5 \mathrm{~Hz}, 2 \mathrm{H})$, $2.80-2.58(\mathrm{~m}, 2 \mathrm{H})$.

${ }^{13} \mathrm{C} \mathrm{NMR} \quad\left(125 \mathrm{MHz}, \mathrm{CDCl}_{3}\right)$

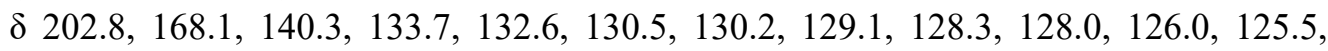
$125.1,124.9,122.9,122.8,122.6,120.4,106.2,93.8,40.1,38.0$.

for: $\mathrm{C}_{22} \mathrm{H}_{18} \mathrm{BrO}_{2}[\mathrm{M}+\mathrm{H}]^{+}$: calcd 393.0493, found 393.0483.

HRMS (ESI) Daicel Chiralpak OD-H column, $n$-hexane $/ i$-PrOH (95/5), $0.5 \mathrm{~mL} / \mathrm{min} 210 \mathrm{~nm} 21.950$ HPLC $\min$ (major enantiomer), 17.393 min (minor enantiomer).<smiles>C=CC[C@]1(c2ccccc2)Oc2c(ccc3ccccc23)C1=O</smiles>

to yield 10d' as a white solid ( $154.0 \mathrm{mg}$, $98 \%$ yield, $91 \%$ ee).

$\underline{R}_{f} \quad 0.40(\mathrm{Hex} / \mathrm{EtOAc}=20: 1$, Vanillin $)$

Opt. Rot. $\quad[\alpha]^{20}{ }_{\mathrm{D}}=-220.0(\mathrm{c}=0.1, \mathrm{MeOH})$

${ }^{1} \mathrm{H} \mathrm{NMR} \quad\left(500 \mathrm{MHz}, \mathrm{CDCl}_{3}\right)$

$\delta 8.27(\mathrm{~d}, J=8.2 \mathrm{~Hz}, 1 \mathrm{H}), 7.82(\mathrm{~d}, J=8.2 \mathrm{~Hz}, 1 \mathrm{H}), 7.76-7.54(\mathrm{~m}, 2 \mathrm{H}), 7.41(\mathrm{~d}, J=$ $8.5 \mathrm{~Hz}, 1 \mathrm{H}), 7.32(\mathrm{~d}, J=8.5 \mathrm{~Hz}, 1 \mathrm{H}), 7.26-7.20(\mathrm{~m}, 2 \mathrm{H}), 7.18-6.98(\mathrm{~m}, 3 \mathrm{H}), 5.71-$ $5.57(\mathrm{~m}, 1 \mathrm{H}), 5.15-5.09(\mathrm{~m}, 1 \mathrm{H}), 5.02-4.92(\mathrm{~m}, 1 \mathrm{H}), 3.34-3.05(\mathrm{~m}, 2 \mathrm{H}), 2.80-2.52$ $(\mathrm{m}, 2 \mathrm{H})$.

${ }^{13} \mathrm{C} \mathrm{NMR} \quad\left(125 \mathrm{MHz}, \mathrm{CDCl}_{3}\right)$

$\delta 202.2,172.3,138.7,134.5,130.6,130.5,130.3,128.4,128.0,126.8,126.6,122.3$, $122.1,121.4,119.8,118.7,116.2,93.4,41.9,40.0$.

HRMS (ESI) for: $\mathrm{C}_{22} \mathrm{H}_{19} \mathrm{O}_{2}[\mathrm{M}+\mathrm{H}]^{+}$: calcd 315.1385 , found 315.1421 .

HPLC Daicel Chiralpak OD-H column, $n$-hexane $/ i$-PrOH (99.4/0.6), 1 mL/min $210 \mathrm{~nm} 16.647$ min (major enantiomer), $15.688 \mathrm{~min}$ (minor enantiomer).

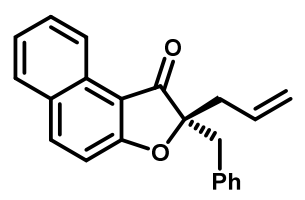

10e' $\quad(S)$-2-Allyl-2-benzylnaphtho[2,1-b]furan-1(2H)-one (10e'). Using Condition A to yield 10e' as a white solid (154.3 $\mathrm{mg}, 98 \%$ yield, 94\% ee).

$\underline{R}_{f} \quad 0.40(\mathrm{Hex} / \mathrm{EtOAc}=20: 1$, Vanillin $)$ 
Opt. Rot. $\quad[\alpha]^{20}{ }_{\mathrm{D}}=111.0(\mathrm{c}=0.1, \mathrm{MeOH})$

${ }^{1} \mathrm{H} \mathrm{NMR} \quad\left(500 \mathrm{MHz}, \mathrm{CDCl}_{3}\right)$

$\delta 8.69(\mathrm{~d}, J=8.3 \mathrm{~Hz}, 1 \mathrm{H}), 7.97(\mathrm{~d}, J=9.0 \mathrm{~Hz}, 1 \mathrm{H}), 7.76(\mathrm{~d}, J=8.1 \mathrm{~Hz}, 1 \mathrm{H}), 7.62(\mathrm{t}, J$ $=8.2 \mathrm{~Hz}, 1 \mathrm{H}), 7.43(\mathrm{t}, J=8.1 \mathrm{~Hz}, 1 \mathrm{H}), 7.23(\mathrm{~d}, J=7.1 \mathrm{~Hz}, 2 \mathrm{H}), 7.19-7.10(\mathrm{~m}, 3 \mathrm{H})$, $7.07(\mathrm{t}, J=7.3 \mathrm{~Hz}, 1 \mathrm{H}), 5.74-5.55(\mathrm{~m}, 1 \mathrm{H}), 5.24-4.90(\mathrm{~m}, 2 \mathrm{H}), 3.19(\mathrm{~s}, 2 \mathrm{H}), 2.81-$ $2.57(\mathrm{~m}, 2 \mathrm{H})$.

${ }^{13} \mathrm{C} \mathrm{NMR} \quad\left(125 \mathrm{MHz}, \mathrm{CDCl}_{3}\right)$

$\delta 202.5,174.2,139.9,134.5,130.7,130.3,129.7,129.0,128.9,128.4,128.0,126.8$, $125.2,123.1,119.8,113.7,113.5,92.7,41.8,40.1$.

HRMS (ESI) for: $\mathrm{C}_{22} \mathrm{H}_{19} \mathrm{O}_{2}[\mathrm{M}+\mathrm{H}]^{+}$: calcd 315.1385, found 315.1416 .

HPLC Daicel Chiralpak OD-H column, $n$-hexane $/ i$-PrOH (99.4/0.6), $1 \mathrm{~mL} / \mathrm{min} 210 \mathrm{~nm} 13.937$ min (major enantiomer), 12.544 min (minor enantiomer).

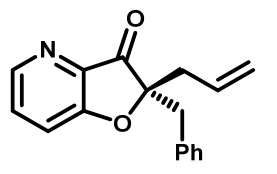

$10 f^{\prime}$

(S)-2-Allyl-2-benzylfuro[3,2-b]pyridin-3(2H)-one (10f'). Using Condition A to yield $\mathbf{1 0 f ^ { \prime }}$ as a colorless oil (119.4 $\mathrm{mg}, 90 \%$ yield, $90 \%$ ee).

$\underline{R}_{f} \quad 0.20(\mathrm{Hex} / \mathrm{EtOAc}=15: 1$, Vanillin $)$

Opt. Rot. $[\alpha]^{20}=-24.0(\mathrm{c}=0.1, \mathrm{MeOH})$

$\underline{{ }^{1} \mathrm{H} \mathrm{NMR}} \quad\left(400 \mathrm{MHz}, \mathrm{CDCl}_{3}\right)$

$\delta 7.58-7.42(\mathrm{~m}, 2 \mathrm{H}), 7.24-7.06(\mathrm{~m}, 4 \mathrm{H}), 7.01(\mathrm{~d}, J=8.2 \mathrm{~Hz}, 1 \mathrm{H}), 6.93(\mathrm{t}, J=7.4 \mathrm{~Hz}$, $1 \mathrm{H}), 5.71-5.52(\mathrm{~m}, 1 \mathrm{H}), 5.21-4.95(\mathrm{~m}, 2 \mathrm{H}), 3.11(\mathrm{~d}, J=2.8 \mathrm{~Hz}, 2 \mathrm{H}), 2.74-2.51(\mathrm{~m}$, $2 \mathrm{H})$.

${ }^{13} \mathrm{C} \mathrm{NMR} \quad\left(100 \mathrm{MHz}, \mathrm{CDCl}_{3}\right)$

$\delta 203.3,171.7,138.0,134.4,130.6,130.4,130.4,128.0,126.8,124.0,121.5,119.8$, 113.0, 91.8, 41.7, 40.1.

HRMS (ESI) for: $\mathrm{C}_{17} \mathrm{H}_{16} \mathrm{NO}_{2}[\mathrm{M}+\mathrm{H}]^{+}$: calcd 266.1181, found 266.1249.

HPLC Daicel Chiralpak OD-H column, $n$-hexane $/ i$-PrOH (99.4/0.6), $0.5 \mathrm{~mL} / \mathrm{min} 210 \mathrm{~nm}$ $16.800 \mathrm{~min}$ (major enantiomer), $15.603 \mathrm{~min}$ (minor enantiomer).<smiles>C=CC[C@]1(c2ccc(OC)cc2)Oc2cc(OC)cc(OC)c2C1=O</smiles>

(S)-2-allyl-4,6-dimethoxy-2-(4-methoxyphenyl)benzofuran-3(2H)-one

(10f'). Using Condition D at $-50{ }^{\circ} \mathrm{C}$ to yield $\mathbf{1 0 g}$ ' as a white solid (31.3 $\mathrm{mg}, 83 \%$ yield, $90 \%$ ee).

$\underline{R}_{f} \quad 0.20(\mathrm{Hex} / \mathrm{EtOAc}=3: 1$, Vanillin $)$

Opt. Rot. $[\alpha]^{20}{ }_{\mathrm{D}}=-349.6(\mathrm{c}=0.1, \mathrm{MeOH})$

$\underline{{ }^{1} \mathrm{H} \mathrm{NMR}} \quad\left(500 \mathrm{MHz}, \mathrm{CDCl}_{3}\right)$

$\delta 7.51(\mathrm{~d}, J=9.0 \mathrm{~Hz}, 2 \mathrm{H}), 6.85(\mathrm{~d}, J=9.0 \mathrm{~Hz}, 2 \mathrm{H}), 6.27(\mathrm{~s}, 1 \mathrm{H}), 5.99(\mathrm{~s}, 1 \mathrm{H}), 5.74-$ $5.57(\mathrm{~m}, 1 \mathrm{H}), 5.21-4.96(\mathrm{~m}, 2 \mathrm{H}), 3.87(\mathrm{~d}, J=10.1 \mathrm{~Hz}, 6 \mathrm{H}), 3.77(\mathrm{~s}, 3 \mathrm{H}), 2.89(\mathrm{~d}, J=$ $9.2 \mathrm{~Hz}, 2 \mathrm{H})$.

${ }^{13} \mathrm{C} \mathrm{NMR} \quad\left(125 \mathrm{MHz}, \mathrm{CDCl}_{3}\right)$

$\delta 196.3,174.5,170.1,159.4,131.3,129.8,126.3,119.7,113.8,103.7,93.1,92.1,88.8$, $56.1,55.3,42.6$.

HRMS (ESI) for: $\mathrm{C}_{20} \mathrm{H}_{21} \mathrm{O}_{5}[\mathrm{M}+\mathrm{H}]^{+}$: calcd 341.1389, found 341.1458. 
HPLC Daicel Chiralpak OD-H column, $n$-hexane/i-PrOH (80/20), $1 \mathrm{~mL} / \mathrm{min} 210 \mathrm{~nm} 20.787$ $\min$ (major enantiomer), $14.378 \mathrm{~min}$ (minor enantiomer).

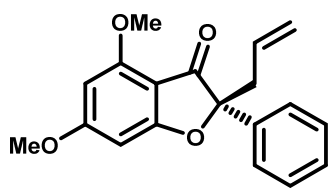

$10 \mathrm{~h}$

(S)-2-allyl-4,6-dimethoxy-2-phenylbenzofuran-3(2H)-one (10h'). Using Condition D at $-45^{\circ} \mathrm{C}$ to yield $\mathbf{1 0 h}$ ' as a colorless oil $(25.7 \mathrm{mg}, 92 \%$ yield, $90 \%$ ee).

$\underline{R}_{f} \quad 0.30(\mathrm{Hex} / \mathrm{EtOAc}=3: 1$, Vanillin $)$

Opt. Rot. $\quad[\alpha]^{20}{ }_{\mathrm{D}}=-218.8(\mathrm{c}=0.1, \mathrm{MeOH})$

${ }^{1} \mathrm{H} \mathrm{NMR} \quad\left(500 \mathrm{MHz}, \mathrm{CDCl}_{3}\right)$

$\delta 7.60(\mathrm{~d}, J=7.3 \mathrm{~Hz}, 2 \mathrm{H}), 7.35-7.24(\mathrm{~m}, 3 \mathrm{H}), 6.29(\mathrm{~s}, 1 \mathrm{H}), 6.00(\mathrm{~s}, 1 \mathrm{H}), 5.75-5.56$ $(\mathrm{m}, 1 \mathrm{H}), 5.23-4.93(\mathrm{~m}, 2 \mathrm{H}), 3.87(\mathrm{~d}, J=13.6 \mathrm{~Hz}, 6 \mathrm{H}), 2.93(\mathrm{~d}, J=8.0 \mathrm{~Hz}, 2 \mathrm{H})$.

${ }^{13} \mathrm{C} \mathrm{NMR} \quad\left(125 \mathrm{MHz}, \mathrm{CDCl}_{3}\right)$

$\delta 195.9,174.5,170.0,159.3,137.6,131.1,128.4,127.9,124.9,119.7,103.7,93.1,92.1$, $88.7,56.0,56.0,42.7$.

HRMS (ESI) for: $\mathrm{C}_{19} \mathrm{H}_{19} \mathrm{O}_{4}[\mathrm{M}+\mathrm{H}]^{+}$: calcd 311.1283, found 311.1334 .

HPLC Daicel Chiralpak OD-H column, $n$-hexane $/ i$-PrOH (80/20), 1.0 mL/min $210 \mathrm{~nm} 7.394$ min (major enantiomer), 6.457 min (minor enantiomer). 

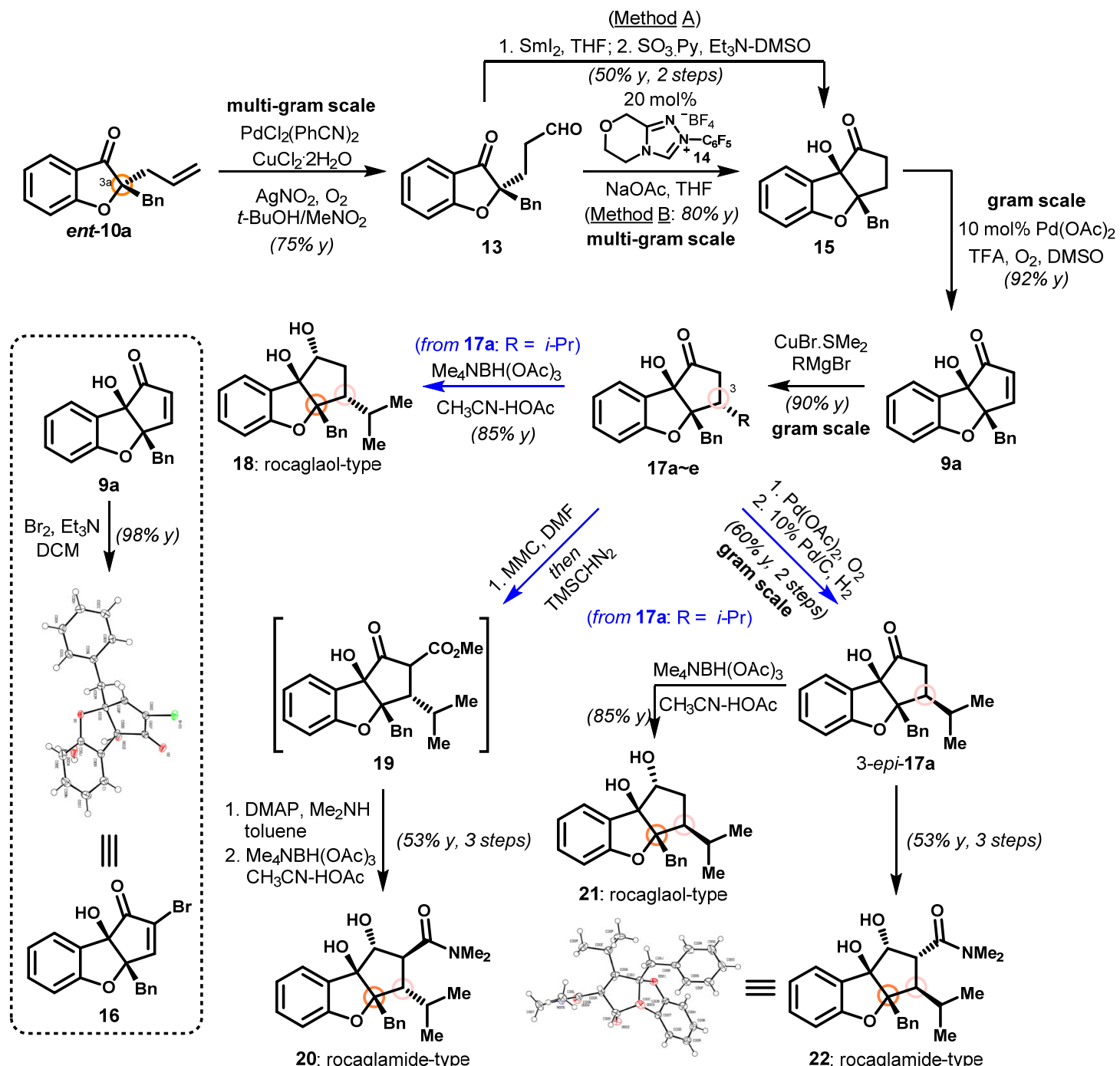

Scheme S4. Synthesis of Flavaglines 18 and 20-22.

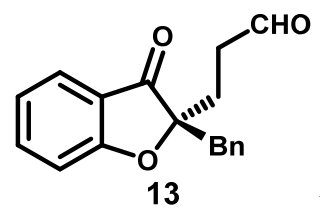

\section{3: 2R-2- Benzyl -2-(2-propen-1-yl)-3(2H)-benzofuranone}

A solution of $\mathrm{PdCl}_{2}\left(\mathrm{PhCN}_{2}\right)_{2}\left(0.12\right.$ equiv.), $\mathrm{CuCl}_{2} \cdot 2 \mathrm{H}_{2} \mathrm{O}$ (0.12 equiv.) and $\mathrm{AgNO}_{2}$ (0.06 equiv.) in $t$ $\mathrm{BuOH}(0.1 \mathrm{M})$ and $\mathrm{MeNO}_{2}(1.5 \mathrm{M})$ was sparged for 15 min with oxygen (1 atm, balloon) in a $500 \mathrm{~mL}$ flask. The alkene ent-10a $(4.75 \mathrm{~g}, 18 \mathrm{mmol})$ was added dropwise subsequently. The solution was saturated with oxygen by an additional $2 \mathrm{~min}$ of sparging. The reaction was then allowed to stir at $23{ }^{\circ} \mathrm{C}$ for $24 \mathrm{~h}$ under $1 \mathrm{~atm}$ oxygen ( $1 \mathrm{~atm}$, balloon). After the reaction was completed, the reaction mixture was concentrated. Next, the resulting mixture was added to water $(100 \mathrm{~mL})$ and extracted three times with TBME $(100 \mathrm{~mL} * 3)$. The combined organic layers were subsequently washed with a saturated solution of 
$\mathrm{NaHCO}_{3}$ and dried over $\mathrm{Na}_{2} \mathrm{SO}_{4}$. The solvent was removed under reduced pressure and the desired aldehyde product was purified using flash chromatography (petroleum ether/TBME $=4: 1$ ) as a white solid $(3.78 \mathrm{~g}, 75 \%)$.

$\underline{R}_{f} \quad 0.40\left(\mathrm{Hex} / \mathrm{EtOAc}=4: 1, \mathrm{KMnO}_{4}\right)$

Opt. Rot. $\alpha]^{20}=63.0(\mathrm{c}=0.1, \mathrm{MeOH})$

${ }^{1} \mathrm{H} \mathrm{NMR} \quad\left(500 \mathrm{MHz}, \mathrm{CDCl}_{3}\right)$

$\delta 9.63(\mathrm{~s}, 1 \mathrm{H}), 7.58-7.47(\mathrm{~m}, 2 \mathrm{H}), 7.19-7.08(\mathrm{~m}, 5 \mathrm{H}), 7.04-6.93(\mathrm{~m}, 2 \mathrm{H}), 3.10(\mathrm{~d}, J$ $=1.8 \mathrm{~Hz}, 2 \mathrm{H}), 2.50-2.09(\mathrm{~m}, 4 \mathrm{H})$.

${ }^{13} \mathrm{C} \mathrm{NMR} \quad\left(125 \mathrm{MHz}, \mathrm{CDCl}_{3}\right)$

$\delta 203.0,200.3,171.6,138.4,134.0,130.3,128.1,127.0,124.1,121.9,121.3,113.1$, $91.4,42.2,37.7,28.0$.

HRMS (ESI) for: $\mathrm{C}_{18} \mathrm{H}_{17} \mathrm{O}_{3}[\mathrm{M}+\mathrm{H}]^{+}$: calcd 281.1178, found 281.1182 .

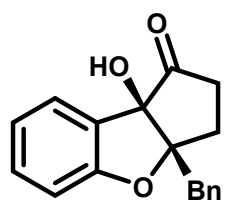

15

15: $\quad(3 \mathrm{a} R, \quad 8 \mathrm{~b} R)-2,3,3 \mathrm{a}, 8 \mathrm{~b}-$-Tetrahydro-8b-hydroxy-3a-benzyl-1 $H$-cyclopenta[ $b]$

benzofuran-1-one

Method A. A solution of $\mathbf{1 3}(2.8 \mathrm{~g}, 10 \mathrm{mmol})$ in degassed THF (1M) was added dropwise to $\mathrm{SmI}_{2}(2.2$ equiv., $0.1 \mathrm{M}$ in THF) at $-78{ }^{\circ} \mathrm{C}$ under $\mathrm{N}_{2}$. After being stirred at $-78{ }^{\circ} \mathrm{C}$ for $4 \mathrm{~h}$, the mixture was warmed to $\mathrm{RT}$, quenched with $10 \% \mathrm{HCl}$ solution and extracted with EA for three times. The combined organic layers were dried over $\mathrm{Na}_{2} \mathrm{SO}_{4}$. The solvent was removed under reduced pressure and the desired product was purified using flash chromatography (petroleum ether/TBME $=3: 1$ ) to provide diol as a white solid (1.65 g, 59\%).

To a solution of diol $(1.4 \mathrm{~g}, 5 \mathrm{mmol})$ in dry DCM $(0.125 \mathrm{M})$ was added DMSO $(20 \mathrm{~mL}), \mathrm{NEt}_{3}(6.0$ equiv.) at $0{ }^{\circ} \mathrm{C}$ under $\mathrm{N}_{2}$. Then a solution of pyridine sulfur trioxide (5.5 equiv.) in DMSO $(20 \mathrm{~mL})$ was added slowly. The mixture was stirred at $0{ }^{\circ} \mathrm{C}$ for $1 \mathrm{~h}$ followed by qunenched with $10 \% \mathrm{HCl}$ solution at $10{ }^{\circ} \mathrm{C}$. Then the mixture was extracted by TBME for 5 times. The combined organic layers were dried over $\mathrm{Na}_{2} \mathrm{SO}_{4}$. The solvent was removed under reduced pressure and the desired product was purified using flash chromatography (petroleum ether/TBME $=3: 1$ ) to generate the desired product $\mathbf{1 5}$ as a white solid (1.19 g, 50\% for 2 steps).

Method B. To a solution of NHC ( 0.2 equiv.), $\mathrm{NaOAc}\left(0.3\right.$ equiv.) in THF $(0.1 \mathrm{M})$ under $\mathrm{N}_{2}$ was added $13(8.4 \mathrm{~g}, 30 \mathrm{mmol})$ by injection pump through $2 \mathrm{~h}$. The reaction mixture was stirred for another $2 \mathrm{~h}$ at room temperature followed by direct concentration. The crude mixture was purified using flash chromatography (petroleum ether/TBME $=3: 1)$ to generate the desired product $\mathbf{1 5}$ as a white solid $(6.72 \mathrm{~g}$, $80 \%)$.

$\underline{R}_{f} \quad 0.38(\mathrm{Hex} / \mathrm{EtOAc}=3: 1$, Vanillin)

Opt. Rot. $\quad \alpha]^{20}{ }_{\mathrm{D}}=247.0(\mathrm{c}=0.1, \mathrm{MeOH})$

${ }^{1} \mathrm{H} \mathrm{NMR} \quad\left(500 \mathrm{MHz}, \mathrm{CDCl}_{3}\right)$

$\delta 7.40-7.11(\mathrm{~m}, 7 \mathrm{H}), 6.92-6.77(\mathrm{~m}, 2 \mathrm{H}), 3.18(\mathrm{~d}, J=14.2 \mathrm{~Hz}, 1 \mathrm{H}), 3.01(\mathrm{dd}, J=$ $14.3,1.6 \mathrm{~Hz}, 1 \mathrm{H}), 2.43-2.28(\mathrm{~m}, 1 \mathrm{H}), 2.27-2.08(\mathrm{~m}, 1 \mathrm{H}), 2.06-1.75(\mathrm{~m}, 2 \mathrm{H})$.

${ }^{13} \mathrm{C} \mathrm{NMR} \quad\left(125 \mathrm{MHz}, \mathrm{CDCl}_{3}\right)$

$\delta 213.3,213.3,159.5,135.7,131.7,130.7,128.4,127.0,126.4,124.4,121.6,111.2$, 
95.5, 86.4, 38.6, 34.3, 30.6.

HRMS (ESI) for: $\mathrm{C}_{18} \mathrm{H}_{16} \mathrm{NaO}_{3}[\mathrm{M}+\mathrm{Na}]^{+}$: calcd 303.0997, found 303.0980.<smiles>O=C1C=CC2(Br)Oc3ccccc3[C@]12O</smiles>

9a 9a: $(3 \mathrm{a} S, 8 \mathrm{~b} R)-3 \mathrm{a}-\mathrm{benzyl}-8 \mathrm{~b}-$ hydroxy-3a,8b-dihydro-1 $H$-cyclopenta[ $b]$ benzofuran-1-one

A solution of $15(1.7 \mathrm{~g}, 6.1 \mathrm{mmol}), \mathrm{Pd}(\mathrm{OAc})_{2}(0.1$ equiv.) and TFA (1.0 equiv.) in DMSO (1M) was sparged for $10 \mathrm{~min}$ with oxygen ( $1 \mathrm{~atm}$, balloon). The reaction was then allowed to stir at $80{ }^{\circ} \mathrm{C}$ for $24 \mathrm{~h}$ under $1 \mathrm{~atm}$ oxygen ( $1 \mathrm{~atm}$, balloon). The reaction mixture was poured into ice-cold water and extracted by TBME for three times. The combined organic layers were dried over $\mathrm{Na}_{2} \mathrm{SO}_{4}$. The solvent was removed under reduced pressure and the desired product was purified using flash chromatography (petroleum ether/EA=10:1) as a white solid (1.55 g, 92\%).

$\underline{R}_{f} \quad 0.35\left(\mathrm{Hex} / \mathrm{EtOAc}=3: 1, \mathrm{KMnO}_{4}\right)$

Opt. Rot. $\quad \alpha]^{20}{ }_{\mathrm{D}}=162.2(\mathrm{c}=0.1, \mathrm{MeOH})$

${ }^{1} \mathrm{H} \mathrm{NMR} \quad\left(500 \mathrm{MHz}, \mathrm{CDCl}_{3}\right)$

$\delta 7.45-7.27(\mathrm{~m}, 7 \mathrm{H}), 7.22(\mathrm{~d}, J=6.1 \mathrm{~Hz}, 1 \mathrm{H}), 6.97(\mathrm{t}, J=7.5 \mathrm{~Hz}, 1 \mathrm{H}), 6.89(\mathrm{~d}, J=8.2$ $\mathrm{Hz}, 1 \mathrm{H}), 6.32(\mathrm{~d}, J=6.1 \mathrm{~Hz}, 1 \mathrm{H}), 3.47(\mathrm{~d}, J=14.6 \mathrm{~Hz}, 2 \mathrm{H}), 3.08(\mathrm{~d}, J=14.6 \mathrm{~Hz}, 1 \mathrm{H})$.

${ }^{13} \mathrm{C} \mathrm{NMR} \quad\left(125 \mathrm{MHz}, \mathrm{CDCl}_{3}\right)$

$\delta 202.9,158.5,158.1,135.2,132.8,131.8,130.6,128.5,127.1,126.3,124.7,121.8$, 111.3, 94.6, 84.6, 38.9.

HRMS (ESI) for: $\mathrm{C}_{18} \mathrm{H}_{15} \mathrm{O}_{3}[\mathrm{M}+\mathrm{H}]^{+}$: calcd 279.1021, found 279.1004 .<smiles>O=C1C(Br)=CC2(Br)Oc3ccccc3C12O</smiles>

16
16:

(3a $S, 8 \mathrm{~b} R$ )-3a-benzyl-2-bromo-8b-hydroxy-3a,8b-dihydro- $1 H$ -

cyclopenta[b]benzofuran-1-one

A solution of $9 \mathrm{a}(139.2 \mathrm{mg}, 0.5 \mathrm{mmol})$ in dry $\mathrm{CH}_{2} \mathrm{Cl}_{2}$ was added $\mathrm{Br}_{2}\left(1.05\right.$ equiv.) at $0{ }^{\circ} \mathrm{C}$. The reaction was allowed to stir at that temperature for $30 \mathrm{~min}$. Then the reaction mixture was concentrated and dissolved in TBME $(0.2 \mathrm{M}) . \mathrm{Et}_{3} \mathrm{~N}$ was added dropwise to this mixture at $0{ }^{\circ} \mathrm{C}$, then the mixture was stirred at $0{ }^{\circ} \mathrm{C}$ overnight. The solvent was removed under reduced pressure and the desired product was purified using flash chromatography (petroleum ether/EA=10:1) as a white solid (175.0 $\mathrm{mg}, 98 \%$ ).

$\underline{R}_{f} \quad 0.50\left(\mathrm{Hex} / \mathrm{EtOAc}=3: 1, \mathrm{KMnO}_{4}\right)$

Opt. Rot. $\alpha]^{20}=54.0(\mathrm{c}=0.1, \mathrm{MeOH})$

${ }^{1} \mathrm{H} \mathrm{NMR} \quad\left(500 \mathrm{MHz}, \mathrm{CDCl}_{3}\right)$

$\delta 7.44-7.31(\mathrm{~m}, 8 \mathrm{H}), 7.03-6.97(\mathrm{~m}, 1 \mathrm{H}), 6.91(\mathrm{~d}, J=8.2 \mathrm{~Hz}, 1 \mathrm{H}), 3.46(\mathrm{~d}, J=14.6$ $\mathrm{Hz}, 1 \mathrm{H}), 3.28(\mathrm{~s}, 1 \mathrm{H}), 3.09$ (d, $J=14.7 \mathrm{~Hz}, 1 \mathrm{H})$. 
${ }^{13} \mathrm{C} \mathrm{NMR} \quad\left(125 \mathrm{MHz}, \mathrm{CDCl}_{3}\right)$

$\delta 196.0,158.1,156.2,134.6,132.3,130.5,128.7,127.3,126.6,125.4,124.8,122.2$, $111.5,94.2,83.6,38.9$.

HRMS (ESI) for: $\mathrm{C}_{18} \mathrm{H}_{14} \mathrm{BrO}_{3}[\mathrm{M}+\mathrm{H}]^{+}$: calcd 357.0126, found 357.0142 .

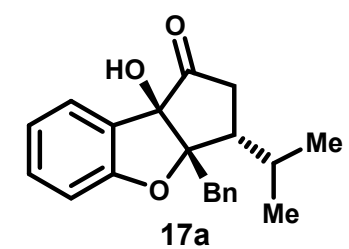

cyclopenta[$[b]$ benzofuran-1-one

17b: $(3 R, 3 \mathrm{a} S, 8 \mathrm{~b} R)-3 \mathrm{a}-$ benzyl-8b-hydroxy-3-isopropyl-2,3,3a,8b-tetrahydro- $1 H$ -

To a stirred solution of $\mathrm{CuBr} \cdot \mathrm{Me}_{2} \mathrm{~S}\left(2.0\right.$ equiv.) and $9 \mathrm{a}(2.78 \mathrm{~g}, 10 \mathrm{mmol})$ in dry $\mathrm{THF}(0.1 \mathrm{M})$ at $-78^{\circ} \mathrm{C}$ was added $i$ - $\mathrm{PrMgBr}$ (2.05 equiv., $3.0 \mathrm{M}$ in THF) dropwise. The resulting mixture was stirred at $-78{ }^{\circ} \mathrm{C}$ for $2.0 \mathrm{~h}$ before it was warmed to $0{ }^{\circ} \mathrm{C}$ for additional $1.0 \mathrm{~h}$. Then the resulting mixture was quenched with $\mathrm{NH}_{4} \mathrm{Cl}(25 \mathrm{~mL}$, sat. aq.). The layers were separated and the aqueous layer was extracted with EtOAc $(3 \times$ $25 \mathrm{~mL}$ ). The combined organic layers were dried with $\mathrm{Na}_{2} \mathrm{SO}_{4}$ and concentrated in vacuo. The desired product $\mathbf{1 7} \mathbf{b}$ was purified using flash chromatography (petroleum ether/EA=10:1) as a colorless oil (2.90 g, $90 \%,>19: 1 \mathrm{dr})$.

$\underline{R}_{f} \quad 0.45\left(\mathrm{Hex} / \mathrm{EtOAc}=3: 1, \mathrm{KMnO}_{4}\right)$

Opt. Rot. $\alpha]^{20}{ }_{\mathrm{D}}=52.0(\mathrm{c}=0.1, \mathrm{MeOH})$

${ }^{1} \mathrm{H} \mathrm{NMR} \quad\left(500 \mathrm{MHz}, \mathrm{CDCl}_{3}\right)$

$\delta 7.31-7.20(\mathrm{~m}, 2 \mathrm{H}), 7.18-7.05(\mathrm{~m}, 4 \mathrm{H}), 7.01(\mathrm{dd}, J=7.5,1.4 \mathrm{~Hz}, 1 \mathrm{H}), 6.79-6.61$ $(\mathrm{m}, 2 \mathrm{H}), 3.42(\mathrm{~s}, 1 \mathrm{H}), 3.11(\mathrm{~s}, 2 \mathrm{H}), 2.30-2.10(\mathrm{~m}, 2 \mathrm{H}), 2.10-2.01(\mathrm{~m}, 1 \mathrm{H}), 1.73-$ $1.62(\mathrm{~m}, 1 \mathrm{H}), 0.83(\mathrm{~d}, J=6.8 \mathrm{~Hz}, 3 \mathrm{H}), 0.69(\mathrm{~d}, J=6.7 \mathrm{~Hz}, 3 \mathrm{H})$.

${ }^{13} \mathrm{C} \mathrm{NMR} \quad\left(125 \mathrm{MHz}, \mathrm{CDCl}_{3}\right)$

$\delta 212.0,160.3,136.1,131.4,130.9,128.0,126.6,126.5,123.9,121.1,110.1,95.7,87.7$, 49.2, 40.8, 36.7, 27.9, 23.3, 20.0.

HRMS (ESI) for: $\mathrm{C}_{21} \mathrm{H}_{22} \mathrm{NaO}_{3}[\mathrm{M}+\mathrm{Na}]^{+}$: calcd 345.1467, found 345.1463 .

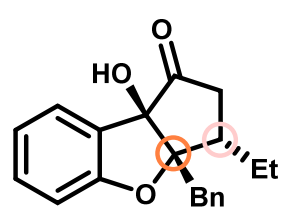

$( \pm)-17 b$

$( \pm)-17 b:$

(3S,3aS,8bR)-3a-benzyl-3-ethyl-8b-hydroxy-2,3,3a, 8b-tetrahydro- $1 H$ -

cyclopenta[b]benzofuran-1-one

To a stirred solution of $\mathrm{CuBr} \cdot \mathrm{Me}_{2} \mathrm{~S}(2.0$ equiv.) and $9 \mathrm{a}(278.3 \mathrm{mg}, 1 \mathrm{mmol})$ in dry THF $(0.1 \mathrm{M})$ at -78 ${ }^{\circ} \mathrm{C}$ was added $\mathrm{EtMgBr}\left(2.05\right.$ equiv., $3.0 \mathrm{M}$ in THF) dropwise. The resulting mixture was stirred at $-78{ }^{\circ} \mathrm{C}$ for $2.0 \mathrm{~h}$ before it was warmed to $0^{\circ} \mathrm{C}$ for additional $1.0 \mathrm{~h}$. Then the resulting mixture was quenched with $\mathrm{NH}_{4} \mathrm{Cl}(5 \mathrm{~mL}$, sat. aq.). The layers were separated and the aqueous layer was extracted with EtOAc $(3 \times 5$ $\mathrm{mL}$ ). The combined organic layers were dried with $\mathrm{Na}_{2} \mathrm{SO}_{4}$ and concentrated in vacuo. The desired product $17 \mathbf{c}$ was purified using flash chromatography (petroleum ether/EA=6:1) as a colorless oil (277.2 mg, 90\%, > 19:1 dr).

$$
\underline{R}_{f} \quad 0.6\left(\mathrm{Hex} / \mathrm{EtOAc}=4: 1, \mathrm{KMnO}_{4}\right)
$$


${ }^{1} \mathrm{H} \mathrm{NMR} \quad\left(500 \mathrm{MHz}, \mathrm{CDCl}_{3}\right)$

$\delta 7.39-7.34(\mathrm{~m}, 2 \mathrm{H}), 7.28(\mathrm{t}, J=1.6 \mathrm{~Hz}, 1 \mathrm{H}), 7.25-7.19(\mathrm{~m}, 3 \mathrm{H}), 7.14(\mathrm{dd}, J=7.5$, $1.3 \mathrm{~Hz}, 1 \mathrm{H}), 6.90-6.82(\mathrm{~m}, 2 \mathrm{H}), 3.37(\mathrm{~s}, 1 \mathrm{H}), 3.23(\mathrm{~d}, J=14.4 \mathrm{~Hz}, 1 \mathrm{H}), 3.03(\mathrm{~d}, J=$ $14.4 \mathrm{~Hz}, 1 \mathrm{H}), 2.52-2.40(\mathrm{~m}, 1 \mathrm{H}), 2.30-2.15(\mathrm{~m}, 2 \mathrm{H}), 1.41-1.22(\mathrm{~m}, 2 \mathrm{H}), 0.76(\mathrm{t}, J=$ $7.5 \mathrm{~Hz}, 3 \mathrm{H})$.

${ }^{13} \mathrm{C} \mathrm{NMR} \quad\left(125 \mathrm{MHz}, \mathrm{CDCl}_{3}\right)$

$\delta \quad 212.1,160.2,135.9,131.4,130.8,128.0,126.6,126.6,123.9,121.2,110.2,95.2$, $87.6,45.6,40.1,39.8,23.1,12.5$.

HRMS (ESI) for: $\mathrm{C}_{20} \mathrm{H}_{20} \mathrm{NaO}_{3}[\mathrm{M}+\mathrm{Na}]^{+}$: calcd 331.1310, found 331.1318 .

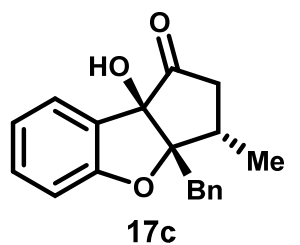

17c: $\quad(3 S, 3 \mathrm{a} S, 8 \mathrm{~b} R)-3 \mathrm{a}-$-benzyl-8b-hydroxy-3-methyl-2,3,3a,8b-tetrahydro-1 $H$ -

cyclopenta[b] benzofuran-1-one

To a stirred solution of $\mathrm{CuBr} \cdot \mathrm{Me}_{2} \mathrm{~S}(2.0$ equiv. $)$ and $9 \mathrm{a}(2.78 \mathrm{~g}, 10 \mathrm{mmol})$ in dry THF $(0.1 \mathrm{M})$ at $-78^{\circ} \mathrm{C}$ was added $\mathrm{MeMgBr}$ (1.05 equiv., 3.0 $\mathrm{M}$ in diethyl ether) dropwise. The resulting mixture was stirred at $78{ }^{\circ} \mathrm{C}$ for $2.0 \mathrm{~h}$ before it was warmed to $0{ }^{\circ} \mathrm{C}$ for additional $1.0 \mathrm{~h}$. Then the resulting mixture was quenched with $\mathrm{NH}_{4} \mathrm{Cl}(25 \mathrm{~mL}$, sat. aq.). The layers were separated and the aqueous layer was extracted with EtOAc $(3 \times 25 \mathrm{~mL})$. The combined organic layers were dried with $\mathrm{Na}_{2} \mathrm{SO}_{4}$ and concentrated in vасио. The desired product $\mathbf{1 7 c}$ was purified using flash chromatography (petroleum ether/EA=10:1) as a colorless oil $(2.68 \mathrm{~g}, 91 \%)$.

$\underline{R}_{f} \quad 0.50\left(\mathrm{Hex} / \mathrm{EtOAc}=3: 1, \mathrm{KMnO}_{4}\right)$

Opt. Rot. $\quad \alpha]^{20}{ }_{\mathrm{D}}=367.0(\mathrm{c}=0.1, \mathrm{MeOH})$

${ }^{1} \mathrm{H} \mathrm{NMR} \quad\left(500 \mathrm{MHz}, \mathrm{CDCl}_{3}\right)$

$\delta 7.30-7.26(\mathrm{~m}, 2 \mathrm{H}), 7.25-7.02(\mathrm{~m}, 5 \mathrm{H}), 6.84-6.68(\mathrm{~m}, 2 \mathrm{H}), 3.57(\mathrm{~s}, 1 \mathrm{H}), 3.14(\mathrm{~d}, \mathrm{~J}$

$=14.4 \mathrm{~Hz}, 1 \mathrm{H}), 2.92(\mathrm{~d}, \mathrm{~J}=14.4 \mathrm{~Hz}, 1 \mathrm{H}), 2.44-2.05(\mathrm{~m}, 3 \mathrm{H}), 0.77(\mathrm{~d}, \mathrm{~J}=6.7 \mathrm{~Hz}, 3 \mathrm{H})$.

${ }^{13} \mathrm{C} \mathrm{NMR} \quad\left(125 \mathrm{MHz}, \mathrm{CDCl}_{3}\right)$

$\delta 212.4,160.3,136.0,131.4,130.9,130.7,128.1,126.7,126.7,124.1,121.3,110.3$, 95.5, 87.6, 42.3, 40.0, 38.6, 14.5 .

HRMS (ESI) for: $\mathrm{C}_{19} \mathrm{H}_{18} \mathrm{NaO}_{3}[\mathrm{M}+\mathrm{Na}]^{+}$: calcd 317.1154 , found 317.1158 .

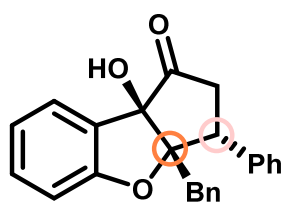

$( \pm)-17 d \quad( \pm)-\quad 17 d: \quad(3 R, 3 \mathrm{a} S, 8 \mathrm{~b} R)-3 \mathrm{a}-$ benzyl-8b-hydroxy-3-phenyl-2,3,3a,8b-tetrahydro-1 $H$ -

cyclopenta $[b]$ benzofuran-1-one

To a stirred solution of $\mathrm{CuBr} \cdot \mathrm{Me}_{2} \mathrm{~S}$ (2.0 equiv.) and $9 \mathrm{a}(278.3 \mathrm{mg}, 1 \mathrm{mmol})$ in dry THF $(0.1 \mathrm{M})$ at -78 ${ }^{\circ} \mathrm{C}$ was added $\mathrm{PhMgBr}$ (2.05 equiv., $3.0 \mathrm{M}$ in THF) dropwise. The resulting mixture was stirred at $-78^{\circ} \mathrm{C}$ for $2.0 \mathrm{~h}$ before it was warmed to $0{ }^{\circ} \mathrm{C}$ for additional $1.0 \mathrm{~h}$. Then the resulting mixture was quenched with $\mathrm{NH}_{4} \mathrm{Cl}(5 \mathrm{~mL}$, sat. aq. $)$. The layers were separated and the aqueous layer was extracted with EtOAc $(3 \times 5$ 
$\mathrm{mL}$ ). The combined organic layers were dried with $\mathrm{Na}_{2} \mathrm{SO}_{4}$ and concentrated in vacuo. The desired product $\mathbf{1 7 d}$ was purified using flash chromatography (petroleum ether/EA=5:1) as a colorless oil (309.7 $\mathrm{mg}, 87 \%,) 19: 1 \mathrm{dr})$.

$\underline{R}_{f} \quad 0.50\left(\mathrm{Hex} / \mathrm{EtOAc}=4: 1, \mathrm{KMnO}_{4}\right)$

${ }^{1} \mathrm{H} \mathrm{NMR} \quad\left(500 \mathrm{MHz}, \mathrm{CDCl}_{3}\right)$

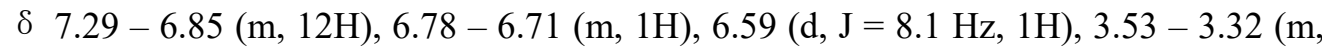
2H), $3.22(\mathrm{~d}, \mathrm{~J}=14.5 \mathrm{~Hz}, 1 \mathrm{H}), 3.09(\mathrm{~d}, \mathrm{~J}=14.5 \mathrm{~Hz}, 1 \mathrm{H}), 2.94-2.67(\mathrm{~m}, 1 \mathrm{H}), 2.59-$ $2.32(\mathrm{~m}, 1 \mathrm{H})$.

${ }^{13} \mathrm{C} \mathrm{NMR} \quad\left(125 \mathrm{MHz}, \mathrm{CDCl}_{3}\right)$

${ }^{1} \delta 211.4,160.1,136.6,135.4,131.5,131.2,129.4,127.9,127.3,126.6,126.5,123.8$, $121.4,110.2,95.9,87.3,49.2,41.2,39.6$

HRMS (ESI) for: $\mathrm{C}_{24} \mathrm{H}_{20} \mathrm{O}_{3} \mathrm{Na}[\mathrm{M}+\mathrm{Na}]^{+}$: calcd 379.1310, found 379.1352 .

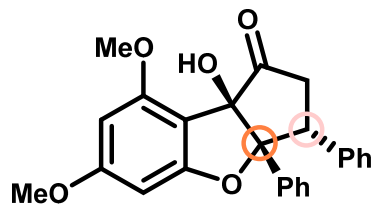

(士)-17e

$( \pm)-17 \mathrm{e}: \quad(3 R, 3 \mathrm{a} R, 8 \mathrm{~b} R)-8 \mathrm{~b}$-hydroxy-6,8-dimethoxy-3,3a-diphenyl-2,3,3a,8btetrahydro-1 $H$-cyclopenta[$[b]$ benzofuran-1-one

To a stirred solution of $\mathrm{CuBr} \cdot \mathrm{Me}_{2} \mathrm{~S}$ (2.0 equiv.) and $\mathbf{S 8}(162.2 \mathrm{mg}, 0.5 \mathrm{mmol})$ in dry THF $(0.1 \mathrm{M})$ at $40{ }^{\circ} \mathrm{C}$ was added $\mathrm{PhMgBr}$ (2.05 equiv., $3.0 \mathrm{M}$ in THF) dropwise. The resulting mixture was stirred at -40 ${ }^{\circ} \mathrm{C}$ for $2.0 \mathrm{~h}$ before it was warmed to $0{ }^{\circ} \mathrm{C}$ for additional $1.0 \mathrm{~h}$. Then the resulting mixture was quenched with $\mathrm{NH}_{4} \mathrm{Cl}(10 \mathrm{~mL}$, sat. aq.). The layers were separated and the aqueous layer was extracted with EtOAc $(3 \times 10 \mathrm{~mL})$. The combined organic layers were dried with $\mathrm{Na}_{2} \mathrm{SO}_{4}$ and concentrated in vacuo. The desired product $( \pm)$-17e was purified using flash chromatography (petroleum ether/EA=3:1) as a colorless oil $(249.2 \mathrm{mg}, 62 \%)$.

$\underline{R}_{f} \quad 0.45\left(\mathrm{Hex} / \mathrm{EtOAc}=2: 1, \mathrm{KMnO}_{4}\right)$

${ }^{1} \mathrm{H} \mathrm{NMR} \quad\left(500 \mathrm{MHz}, \mathrm{CDCl}_{3}\right)$

$\delta 7.37-7.30(\mathrm{~m}, 2 \mathrm{H}), 7.33-7.25(\mathrm{~m}, 2 \mathrm{H}), 7.27-7.19(\mathrm{~m}, 1 \mathrm{H}), 7.12-7.18(\mathrm{~m}, 3 \mathrm{H})$, $7.01-6.93(\mathrm{~m}, 2 \mathrm{H}), 6.09(\mathrm{~d}, J=1.9 \mathrm{~Hz}, 1 \mathrm{H}), 5.94(\mathrm{~d}, J=1.9 \mathrm{~Hz}, 1 \mathrm{H}), 3.99(\mathrm{dd}, J=$ 14.9, $6.8 \mathrm{~Hz}, 1 \mathrm{H}), 3.71(\mathrm{~s}, 3 \mathrm{H}), 3.70(\mathrm{~s}, 3 \mathrm{H}), 3.44(\mathrm{~s}, 1 \mathrm{H}), 3.24$ (dd, $J=15.7,14.9 \mathrm{~Hz}$, $1 \mathrm{H}), 2.65(\mathrm{dd}, J=15.8,6.8 \mathrm{~Hz}, 1 \mathrm{H})$.

${ }^{13} \mathrm{C} \mathrm{NMR} \quad(125 \mathrm{MHz}, \mathrm{CDCl} 3)$

$\delta 209.3,164.3,162.2,158.1,137.0,135.7,128.7,128.0,127.9,127.3,126.8,104.7$, 98.2, 92.4, 88.4, 87.0, 55.6, 55.5, 50.8, 39.1.

HRMS (ESI) for: $\mathrm{C}_{25} \mathrm{H}_{23} \mathrm{O}_{5}[\mathrm{M}+\mathrm{H}]^{+}$: calcd 403.1545, found 403.1564 .

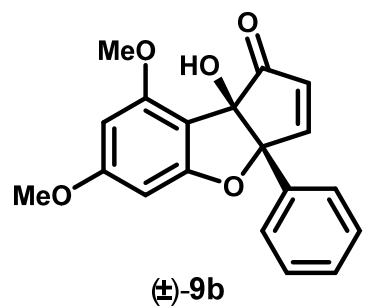

cyclopenta $[b]$ benzofuran-1-one

$( \pm)-9 \mathbf{b}: \quad(3 \mathrm{a} R, 8 \mathrm{~b} R)-8 \mathrm{~b}$-hydroxy-6,8-dimethoxy-3a-phenyl-3a,8b-dihydro-1 $H$ - 
A solution of $\mathbf{S 1 1 b}$ (326.3 g, $1.0 \mathrm{mmol}), \mathrm{Pd}(\mathrm{OAc})_{2}(0.5$ equiv.) and TFA (1.0 equiv.) in DMSO (1M) was sparged for $10 \mathrm{~min}$ with oxygen $\left(1 \mathrm{~atm}\right.$, balloon). The reaction was then allowed to stir at $80^{\circ} \mathrm{C}$ for 5 $\mathrm{h}$ under $1 \mathrm{~atm}$ oxygen ( $1 \mathrm{~atm}$, balloon). The reaction mixture was poured into ice-cold water and extracted by TBME for three times. The combined organic layers were dried over $\mathrm{Na}_{2} \mathrm{SO}_{4}$. The solvent was removed under reduced pressure and the desired product was purified using flash chromatography (petroleum ether/EA=3:1) as a colorless oil $(136.1 \mathrm{mg}, 42 \%)$.

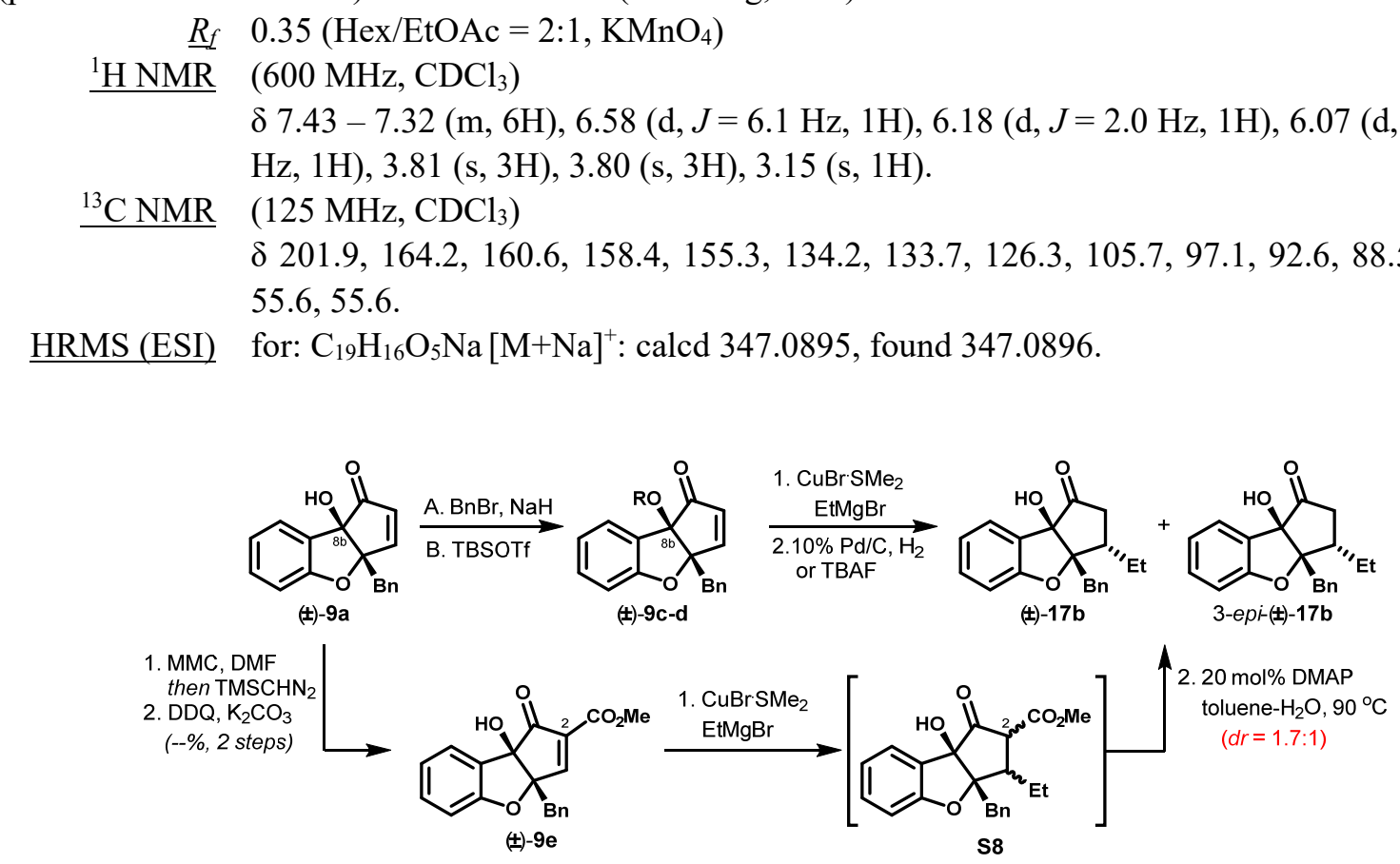

Scheme S5. Preliminary Results of Face Selecitivity

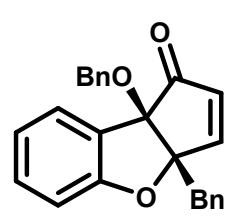

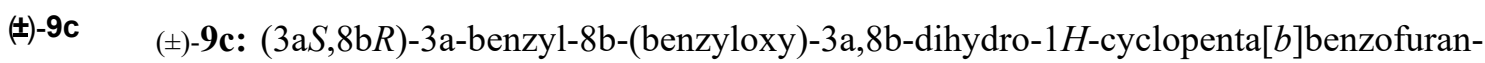
1-one

To a stirred solution of $( \pm)-9 \mathrm{a}(278.1 \mathrm{mg}, 1 \mathrm{mmol})$ in dry DMF $(0.15 \mathrm{M})$ at $0{ }^{\circ} \mathrm{C}$ was added $\mathrm{NaH}(2.5$ equiv.), then the resulting mixture was stirred at $0{ }^{\circ} \mathrm{C}$ for $1.0 \mathrm{~h}$ before it was added $\mathrm{BnBr}$ (2.5 equiv.) slowly. The reaction mixture was stirred at RT for another $4.0 \mathrm{~h}$. Then the resulting mixture was quenched with $\mathrm{NH}_{4} \mathrm{Cl}(10 \mathrm{~mL}$, sat. aq.). The layers were separated and the aqueous layer was extracted with EtOAc $(3 \times 10 \mathrm{~mL})$. The combined organic layers were dried with $\mathrm{Na}_{2} \mathrm{SO}_{4}$ and concentrated in vacuo. The desired product $( \pm)-\mathbf{9 c}$ was purified using flash chromatography (petroleum ether/EA=10:1) as a colorless oil (324.8 mg, $88 \%$ )

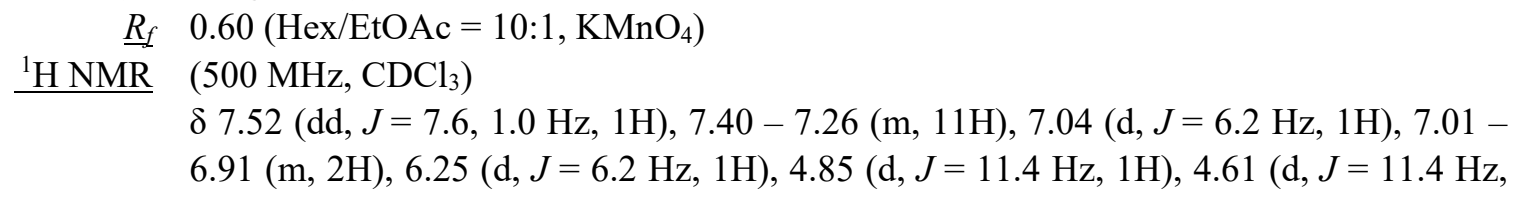


$1 \mathrm{H}), 3.48(\mathrm{~d}, J=14.5 \mathrm{~Hz}, 1 \mathrm{H}), 3.19$ (d, $J=14.5 \mathrm{~Hz}, 1 \mathrm{H})$.

${ }^{13} \mathrm{C} \mathrm{NMR} \quad\left(125 \mathrm{MHz}, \mathrm{CDCl}_{3}\right)$

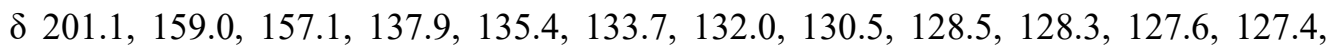
$127.1,126.5,122.6,121.4,111.7,95.5,88.6,68.5,39.5$.

HRMS (ESI) for: $\mathrm{C}_{25} \mathrm{H}_{21} \mathrm{O}_{3}[\mathrm{M}+\mathrm{H}]^{+}$: calcd 369.1491, found 369.1520<smiles>O=C1C=CC2(Br)Oc3ccccc3C12Br</smiles>

(t)-9d

$( \pm)-9 d: \quad(3 \mathrm{a} S, 8 \mathrm{~b} R)-3 \mathrm{a}-$-benzyl-8b-((tert-butyldimethylsilyl)oxy)-3a,8b-dihydro-1 $H$ cyclopenta[b]benzofuran-1-one

To a stirred solution of $( \pm)-9 \mathrm{a}(278.1 \mathrm{mg}, 1 \mathrm{mmol})$ in dry DCM $(0.1 \mathrm{M})$ at $0{ }^{\circ} \mathrm{C}$ was added TBSOTf (1.2 equiv.) and DIPEA (2.2 equiv.) dropwise. The resulting mixture was warmed to RT and stirred at RT for $2.0 \mathrm{~h}$. The reaction mixture was evaporated under reduced pressure after complete consumption of the starting material. The desired product $( \pm)-9 d$ was purified using flash chromatography (petroleum ether/EA=20:1) as a white solid $(287.7 \mathrm{mg}, 73 \%)$.

$\underline{R}_{f} \quad 0.50\left(\mathrm{Hex} / \mathrm{EtOAc}=20: 1, \mathrm{KMnO}_{4}\right)$

${ }^{1} \mathrm{H} \mathrm{NMR} \quad\left(500 \mathrm{MHz}, \mathrm{CDCl}_{3}\right)$

$\delta 7.46-7.26(\mathrm{~m}, 7 \mathrm{H}), 6.99(\mathrm{~d}, J=6.2 \mathrm{~Hz}, 1 \mathrm{H}), 6.90(\mathrm{dd}, J=30.4,8.3 \mathrm{~Hz}, 2 \mathrm{H}), 6.23(\mathrm{~d}$, $J=6.2 \mathrm{~Hz}, 1 \mathrm{H}), 3.42(\mathrm{~d}, J=14.6 \mathrm{~Hz}, 1 \mathrm{H}), 2.91(\mathrm{~d}, J=14.5 \mathrm{~Hz}, 1 \mathrm{H}), 0.89$ (s, 9H), 0.13 $(\mathrm{d}, J=51.7 \mathrm{~Hz}, 6 \mathrm{H})$.

${ }^{13} \mathrm{C} \mathrm{NMR} \quad\left(125 \mathrm{MHz}, \mathrm{CDCl}_{3}\right)$

$\delta$ 204.2, 160.9, 159.0, 138.2, 135.9, 134.1, 133.0, 131.0, 129.5, 128.8, 128.7, 123.6, $113.8,98.1,88.8,42.9,28.5,21.2,0.2$.

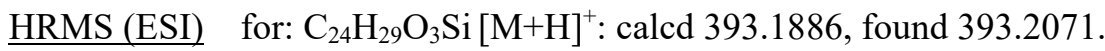<smiles>CC(=O)C1=CC2(Br)Oc3ccccc3C2(O)C1=O</smiles>

cyclopenta $[b]$ benzofuran-2-carboxylate

( \pm -9e: Methyl $(3 \mathrm{a} S, 8 \mathrm{~b} R)-3 \mathrm{a}-\mathrm{benzyl}-8 \mathrm{~b}-$ hydroxy-1-oxo-3a,8b-dihydro-1H-

To a stirred solution of $15(200 \mathrm{mg}, 0.56 \mathrm{mmol})$ in DMF $(1.0 \mathrm{M})$ at $25^{\circ} \mathrm{C}$ was added Stile's reagent (3.0 equiv., 2.0 M in DMF) dropwise. The resulting mixture was warmed to $80^{\circ} \mathrm{C}$ and stirred for $3 \mathrm{~h}$. After cooling to $0{ }^{\circ} \mathrm{C}$ with ice-water, the mixture was diluted with ice-cold $\mathrm{Et}_{2} \mathrm{O}(5.0 \mathrm{~mL})$, quenched with ice-cold $\mathrm{HCl}(2.0 \mathrm{~mL}, 6 \mathrm{M})$ and extracted by ice-cold $\mathrm{Et}_{2} \mathrm{O}$ for three times. The combined organic layers were dried with $\mathrm{Na}_{2} \mathrm{SO}_{4}$ and concentrated to $2.5 \mathrm{~mL}$ in vacuo at $5{ }^{\circ} \mathrm{C}$ followed with the addition of $\mathrm{MeOH}$ $(0.2 \mathrm{~mL})$ and $\mathrm{TMSCHN}_{2}$ (1.0 equiv., $2.0 \mathrm{M}$ in hexane). The resulting mixture was stirred for $1.0 \mathrm{~h}$ and then concentrated in vacuo. Which was used directly without further purification.

To a stirred solution of the ester (crude, obtain above) in dry THF $(0.08 \mathrm{M})$ at $25^{\circ} \mathrm{C}$ was added DDQ (2.5 equiv.) and $\mathrm{K}_{2} \mathrm{CO}_{3}$ (2.5 equiv.). The resulting mixture was stirred for $2.0 \mathrm{~h}$ before it was quenched 
with $\mathrm{NaHCO}_{3}(10 \mathrm{~mL}$, sat. aq.). The layers were separated and the aqueous layer was extracted with EtOAc $(3 \times 10 \mathrm{~mL})$. The combined organic layers were washed with brine, dried with $\mathrm{Na}_{2} \mathrm{SO}_{4}$ and concentrated in vacuo. The desired product $( \pm)$-9e was purified using flash chromatography (petroleum ether/EA=2:1) as a colorless oil ( $68 \mathrm{mg}, 39 \%$ for two steps).

$\underline{R}_{f} \quad 0.40\left(\mathrm{Hex} / \mathrm{EtOAc}=2: 1, \mathrm{KMnO}_{4}\right)$

${ }^{1} \mathrm{H} \mathrm{NMR} \quad\left(500 \mathrm{MHz}, \mathrm{CDCl}_{3}\right)$

$\delta 7.79(\mathrm{~s}, 1 \mathrm{H}), 7.42-7.33(\mathrm{~m}, 5 \mathrm{H}), 7.30(\mathrm{~d}, J=14.3 \mathrm{~Hz}, 2 \mathrm{H}), 7.00-6.93(\mathrm{~m}, 1 \mathrm{H}), 6.88$ $(\mathrm{d}, J=8.0,1 \mathrm{H}), 3.76(\mathrm{~s}, 4 \mathrm{H}), 3.50(\mathrm{~d}, J=14.7 \mathrm{~Hz}, 1 \mathrm{H}), 3.10(\mathrm{~d}, J=14.7 \mathrm{~Hz}, 1 \mathrm{H})$.

${ }^{13} \mathrm{C} \mathrm{NMR} \quad\left(125 \mathrm{MHz}, \mathrm{CDCl}_{3}\right)$

$\delta 197.0,163.6,161.1,158.1,135.1,134.5,132.0,130.5,128.7,127.3,125.6,124.9$, $122.2,111.3,92.3,86.0,52.5,38.4$.

HRMS (ESI) for: $\mathrm{C}_{20} \mathrm{H}_{17} \mathrm{O}_{5}[\mathrm{M}+\mathrm{H}]^{+}$: calcd 337.1076, found 337.1082.

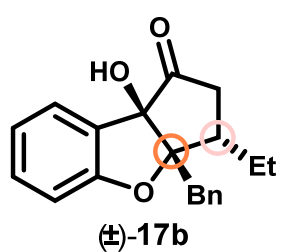

4.9:1 dr

[from (+)-9c]

$( \pm)-17 b: \quad(3 S, 3 \mathrm{a} S, 8 \mathrm{~b} R)-3 \mathrm{a}-\mathrm{b}$ enzyl-3-ethyl-8b-hydroxy-2,3,3a,8b-tetrahydro-1 $H$ -

cyclopenta[b]benzofuran-1-one

To a stirred solution of $\mathrm{CuBr} \cdot \mathrm{Me}_{2} \mathrm{~S}$ (2.0 equiv.) and ( \pm )-9c $(184.6 \mathrm{mg}, 0.5 \mathrm{mmol})$ in dry THF $(0.1 \mathrm{M})$ at $-78^{\circ} \mathrm{C}$ was added EtMgBr $(2.05$ equiv., $3.0 \mathrm{M}$ in THF) dropwise. The resulting mixture was stirred at $78{ }^{\circ} \mathrm{C}$ for $2.0 \mathrm{~h}$ before it was warmed to $0{ }^{\circ} \mathrm{C}$ for additional $1.0 \mathrm{~h}$. Then the resulting mixture was quenched with $\mathrm{NH}_{4} \mathrm{Cl}(5 \mathrm{~mL}$, sat. aq.). The layers were separated, and the aqueous layer was extracted with EtOAc $(3 \times 5 \mathrm{~mL})$. The combined organic layers were dried with $\mathrm{Na}_{2} \mathrm{SO}_{4}$ and concentrated in vacuo. Which was used directly without further purification.

To a stirred solution of the addition product (crude, obtain above) in $\mathrm{MeOH}(0.1 \mathrm{M})$ was added $10 \%$ $\mathrm{Pd} / \mathrm{C}(30 \mathrm{mg})$ and $\mathrm{Pd}(\mathrm{OH})_{2}(30 \mathrm{mg})$. The resulting mixture was stirred at $\mathrm{RT}$ under $\mathrm{H}_{2}$. Then the resulting mixture was filtered and concentrated in vacuo. The desired product $( \pm)-\mathbf{1 7} \mathbf{b}$ was purified using flash chromatography (petroleum ether/EA=4:1) as a colorless oil ( $92.4 \mathrm{mg}, 60 \%$ for 2 steps, $4: 1 \mathrm{dr}$ ).

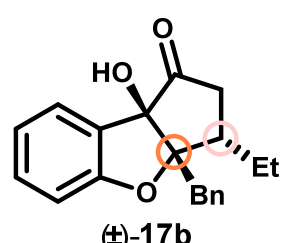

3.5: $1 \mathrm{dr}$

[from ( \pm )-9d]

$( \pm)-17 b: \quad(3 S, 3 \mathrm{a} S, 8 \mathrm{~b} R)-3 \mathrm{a}-\mathrm{b} e n z y l-3$-ethyl-8b-hydroxy-2,3,3a,8b-tetrahydro-1 $H$ -

cyclopenta[b]benzofuran-1-one

To a stirred solution of $\mathrm{CuBr} \cdot \mathrm{Me}_{2} \mathrm{~S}$ (2.0 equiv.) and ( \pm )-9e (196.6 mg, $\left.0.5 \mathrm{mmol}\right)$ in dry THF $(0.1 \mathrm{M})$ at $-78^{\circ} \mathrm{C}$ was added EtMgBr $(2.05$ equiv., $3.0 \mathrm{M}$ in THF) dropwise. The resulting mixture was stirred at $78{ }^{\circ} \mathrm{C}$ for $2.0 \mathrm{~h}$ before it was warmed to $0{ }^{\circ} \mathrm{C}$ for additional $1.0 \mathrm{~h}$. Then the resulting mixture was quenched with $\mathrm{NH}_{4} \mathrm{Cl}$ ( $5 \mathrm{~mL}$, sat. aq.). The layers were separated and the aqueous layer was extracted 
with EtOAc $(3 \times 5 \mathrm{~mL})$. The combined organic layers were dried with $\mathrm{Na}_{2} \mathrm{SO}_{4}$ and concentrated in vacuo. Which was used directly without further purification.

To a stirred solution of the addition product (crude, obtain above) in dry THF $(0.1 \mathrm{M})$ at $0{ }^{\circ} \mathrm{C}$ was added TBAF (5.0 equiv., 1.0 M in THF). The resulting mixture was stirred at RT for $1 \mathrm{~h}$. Then the resulting mixture was concentrated in vacuo. The desired product $( \pm)-\mathbf{1 7 b}$ was purified using flash chromatography (petroleum ether/EA=4:1) as a colorless oil ( $58.5 \mathrm{mg}, 38 \%$ for $2 \mathrm{steps}, 3.5: 1 \mathrm{dr}$ ).

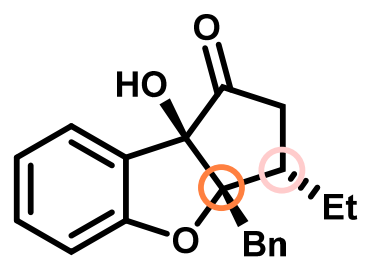

$( \pm)-17 b$

1.9: $1 \mathrm{dr}$

[from ( $(\mathbf{\pm})-9 e$ ]

cyclopenta[$[b]$ benzofuran-1-one

$( \pm)-17 b: \quad(3 S, 3 \mathrm{a} S, 8 \mathrm{~b} R)-3 \mathrm{a}-\mathrm{b} e n z y l-3-e t h y l-8 \mathrm{~b}-h y d r o x y-2,3,3 \mathrm{a}, 8 \mathrm{~b}-$ tetrahydro-1 $H$ -

To a stirred solution of $\mathrm{CuBr} \cdot \mathrm{Me}_{2} \mathrm{~S}(0.2$ equiv. $)$ and $( \pm)-9 \mathrm{e}(67 \mathrm{mg}, 0.2 \mathrm{mmol})$ in dry THF $(0.1 \mathrm{M})$ at $78{ }^{\circ} \mathrm{C}$ was added $\mathrm{EtMgBr}$ (2.2 equiv., $1.0 \mathrm{M}$ in THF) dropwise. The resulting mixture was stirred at -78 ${ }^{\circ} \mathrm{C}$ for $1.0 \mathrm{~h}$ before it was warmed to $0{ }^{\circ} \mathrm{C}$ for additional $1.0 \mathrm{~h}$. Then the resulting mixture was quenched with $\mathrm{NH}_{4} \mathrm{Cl}(5 \mathrm{~mL}$, sat. aq.). The layers were separated and the aqueous layer was extracted with EtOAc $(3 \times 5 \mathrm{~mL})$. The combined organic layers were dried with $\mathrm{Na}_{2} \mathrm{SO}_{4}$ and concentrated in vacuo. Which was used directly without further purification.

To a stirred solution of the ester (crude, obtain above) in $\mathrm{PhMe}(0.08 \mathrm{M})$ at $25{ }^{\circ} \mathrm{C}$ was added $\mathrm{H}_{2} \mathrm{O}(10$ equiv.) and DMAP (0.2 equiv.). The resulting mixture was warmed to $90{ }^{\circ} \mathrm{C}$ and stirred for $3.0 \mathrm{~h}$ before cooled down. It was directly purified using flash chromatography (petroleum ether/EA=4:1) to obtain $( \pm)$ $\mathbf{1 7 b}$ ( $23.4 \mathrm{mg}, 38 \%$ for two steps) and ( \pm )-epi-17b (13.5 mg, 22\% for two steps) as colorless oil. $\mathrm{dr}=1.9: 1$ was determined by crude NMR.

Data of $( \pm)-e p i-17 \mathbf{b}$ :

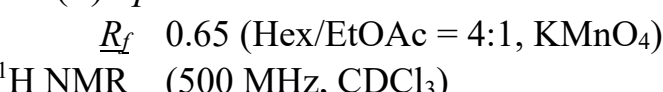

$\delta 7.48-7.43(\mathrm{~m}, 2 \mathrm{H}), 7.25-7.15(\mathrm{~m}, 5 \mathrm{H}), 6.88(\mathrm{td}, J=7.5,0.9 \mathrm{~Hz}, 1 \mathrm{H}), 6.81(\mathrm{dt}, J=$ 7.6, $1.0 \mathrm{~Hz}, 1 \mathrm{H}), 3.28-3.14(\mathrm{~m}, 2 \mathrm{H}), 3.04(\mathrm{~s}, 1 \mathrm{H}), 2.72(\mathrm{~m}, 1 \mathrm{H}), 2.43-2.33(\mathrm{~m}, 1 \mathrm{H})$, $2.22(\mathrm{dd}, J=17.0,4.5 \mathrm{~Hz}, 1 \mathrm{H}), 1.93-1.80(\mathrm{~m}, 1 \mathrm{H}), 1.19(\mathrm{~m}, 1 \mathrm{H}), 0.89(\mathrm{t}, J=7.3 \mathrm{~Hz}$, $3 \mathrm{H})$.

${ }^{13} \mathrm{C} \mathrm{NMR} \quad\left(125 \mathrm{MHz}, \mathrm{CDCl}_{3}\right)$

$\delta 212.9,159.0,135.8,131.1,130.8,127.9,126.9,126.4,123.9,121.4,110.6,98.5,86.2$, $44.2,39.0,35.4,22.1,11.9$.

HRMS (ESI) for: $\mathrm{C}_{20} \mathrm{H}_{20} \mathrm{NaO}_{3}[\mathrm{M}+\mathrm{Na}]^{+}: 331.1310$, found 331.1338 . 


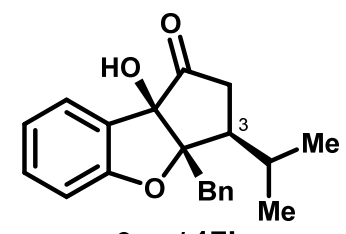

3-epi-17b

3-epi-17b: $(3 S, 3 \mathrm{a} S, 8 \mathrm{~b} R)-3 \mathrm{a}-\mathrm{benzyl}-8 \mathrm{~b}-\mathrm{hydroxy}-3$-isopropyl-2,3,3a,8b-tetrahydro-1 $H$ cyclopenta[b]benzo-furan-1-one

To a stirred solution of $\mathbf{1 7 b}(2.90 \mathrm{~g}, 9.0 \mathrm{mmol})$ in DMSO $(1.0 \mathrm{M})$ at $25^{\circ} \mathrm{C}$ was added $\mathrm{Pd}(\mathrm{OAc})_{2}(0.2$ equiv.). The resulting mixture was sparged for $10 \mathrm{~min}$ with oxygen (1 atm, balloon). Then the reaction was allowed to stir at $100{ }^{\circ} \mathrm{C}$ for $24 \mathrm{~h}$ under $1 \mathrm{~atm}$ oxygen $(1 \mathrm{~atm}$, balloon) before it was quenched with ice-cold water and extracted by TBME for three times. The combined organic layers were dried with $\mathrm{Na}_{2} \mathrm{SO}_{4}$ and concentrated in vacuo. Which was used directly without further purification.

To a stirred solution of the enone (crude, obtain above) in $\mathrm{MeOH}(0.2 \mathrm{M})$ at $25{ }^{\circ} \mathrm{C}$ was added $\mathrm{Pd} / \mathrm{C}$ (580 mg, $10 \mathrm{wt} \%$ ). The resulting mixture was sparged for $10 \mathrm{~min}$ with $\mathrm{H}_{2}$ (1 atm, balloon). Then the reaction was allowed to stir at $50{ }^{\circ} \mathrm{C}$ for $12 \mathrm{~h}$ under $1 \mathrm{~atm} \mathrm{H}_{2}$ (1 atm, balloon) before it was filtered through a short pad of celite. The filtrate was collected and concentrated in vacuo. Flash column chromatography (silica gel, petroleum ether/EA=10:1) afforded the desired product 3-epi-17b $(1.74 \mathrm{~g}$, $60 \%$ for two steps) as a colorless oil, along with $17 \mathbf{b}$ ( $0.87 \mathrm{~g}, 30 \%$ for two steps) recovered.

$\underline{R}_{f} \quad 0.50\left(\mathrm{Hex} / \mathrm{EtOAc}=3: 1, \mathrm{KMnO}_{4}\right)$

Opt. Rot. $\quad \alpha]^{20}{ }_{\mathrm{D}}=171.0(\mathrm{c}=0.1, \mathrm{MeOH})$

${ }^{1} \mathrm{H} \mathrm{NMR} \quad\left(500 \mathrm{MHz}, \mathrm{CDCl}_{3}\right)$

$\delta 7.40(\mathrm{~d}, J=7.3 \mathrm{~Hz}, 2 \mathrm{H}), 7.18-7.12(\mathrm{~m}, 4 \mathrm{H}), 7.09(\mathrm{t}, J=7.3 \mathrm{~Hz}, 1 \mathrm{H}), 6.84-6.74(\mathrm{~m}$, 2H), $3.35(\mathrm{~d}, J=14.8 \mathrm{~Hz}, 1 \mathrm{H}), 3.19(\mathrm{~d}, J=14.8 \mathrm{~Hz}, 1 \mathrm{H}), 2.68(\mathrm{~s}, 1 \mathrm{H}), 2.55-2.45(\mathrm{~m}$, $1 \mathrm{H}), 2.13-2.00(\mathrm{~m}, 3 \mathrm{H}), 1.04(\mathrm{~d}, J=6.0 \mathrm{~Hz}, 3 \mathrm{H}), 0.77$ (d, $J=6.4 \mathrm{~Hz}, 3 \mathrm{H})$.

${ }^{13} \mathrm{C} \mathrm{NMR} \quad\left(125 \mathrm{MHz}, \mathrm{CDCl}_{3}\right)$ $\delta 212.7,158.8,135.9,131.5,131.0,128.0,127.0,126.5,124.3,121.4,110.9,98.3,87.5$, $48.88,38.8,35.0,28.1,22.2,20.2$.

HRMS (ESI) for: $\mathrm{C}_{21} \mathrm{H}_{22} \mathrm{NaO}_{3}[\mathrm{M}+\mathrm{Na}]^{+}$: calcd 345.1467, found 345.1458 .

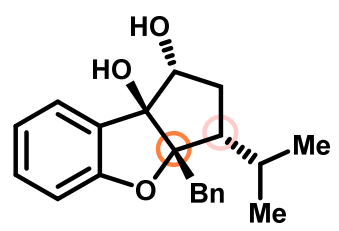

18: rocaglaol-type

18:

$(1 R, 3 R, 3 \mathrm{a} S, 8 \mathrm{~b} S)$-3a-benzyl-3-isopropyl-1,2,3,3a-tetrahydro-8b $H$ -

cyclopenta[$[b]$ benzofuran- $1,8 \mathrm{~b}$-diol

To a stirred solution of $\mathbf{1 7 b}(2.90 \mathrm{~g}, 9.0 \mathrm{mmol})$ in $\mathrm{MeCN}$ and $\mathrm{AcOH}(10: 1)(0.2 \mathrm{M})$ at $25^{\circ} \mathrm{C}$ was added $\mathrm{Me}_{4} \mathrm{NBH}(\mathrm{OAc})_{3}$ (3.0 equiv.). The resulting mixture was stirred at $25^{\circ} \mathrm{C}$ for $3.0 \mathrm{~h}$ before it was quenched with $\mathrm{Na}_{2} \mathrm{CO}_{3}(30 \mathrm{~mL}$, sat. aq.). The layers were separated and the aqueous layer was extracted with EtOAc $(3 \times 30 \mathrm{~mL})$. The combined organic layers were dried with $\mathrm{Na}_{2} \mathrm{SO}_{4}$ and concentrated in vacuo. The desired product 18 was purified using flash chromatography (petroleum ether/EA=5:1) as a white solid $(2.50 \mathrm{~g}, 85 \%)$.

$\underline{R}_{f} \quad 0.40\left(\mathrm{Hex} / \mathrm{EtOAc}=2: 1, \mathrm{KMnO}_{4}\right)$

Opt. Rot. $\quad \alpha]^{20}{ }_{\mathrm{D}}=-10.0(\mathrm{c}=0.1, \mathrm{MeOH})$

${ }^{1} \mathrm{H} \mathrm{NMR} \quad\left(500 \mathrm{MHz}, \mathrm{CDCl}_{3}\right)$ 
$\delta 7.32(\mathrm{dd}, J=20.0,7.4 \mathrm{~Hz}, 3 \mathrm{H}), 7.21-7.06(\mathrm{~m}, 4 \mathrm{H}), 6.81-6.72(\mathrm{~m}, 2 \mathrm{H}), 4.19(\mathrm{dd}, J=$ $11.9,6.0 \mathrm{~Hz}, 1 \mathrm{H}), 3.23(\mathrm{~d}, J=14.4 \mathrm{~Hz}, 1 \mathrm{H}), 2.97(\mathrm{~d}, J=14.4 \mathrm{~Hz}, 1 \mathrm{H}), 1.88(\mathrm{~s}, 1 \mathrm{H})$, $1.82(\mathrm{dt}, J=12.0,6.2 \mathrm{~Hz}, 1 \mathrm{H}), 1.71(\mathrm{dt}, J=12.6,6.2 \mathrm{~Hz}, 1 \mathrm{H}), 1.68-1.55(\mathrm{~m}, 1 \mathrm{H}), 0.95$ $(\mathrm{d}, J=12.1 \mathrm{~Hz}, 1 \mathrm{H}), 0.91(\mathrm{~d}, J=6.7 \mathrm{~Hz}, 3 \mathrm{H}), 0.72(\mathrm{~d}, J=6.7 \mathrm{~Hz}, 3 \mathrm{H})$.

${ }^{13} \mathrm{C} \mathrm{NMR} \quad\left(125 \mathrm{MHz}, \mathrm{CDCl}_{3}\right)$

$\delta 160.5,136.8,131.1,130.8,128.1,126.9,126.6,126.5,120.5,109.6,96.8,90.6,78.0$, 49.2, 41.7, 33.0, 27.9, 23.4, 20.7.

HRMS (ESI) for: $\mathrm{C}_{21} \mathrm{H}_{24} \mathrm{NaO}_{3}[\mathrm{M}+\mathrm{Na}]^{+}$: calcd 347.1623, found 347.1588.<smiles>CNC(=O)C1[C@H](C(C)[N+](=O)[O-])[C@@H](O)C2(O)c3ccccc3OC12O</smiles>

20: rocaglamide-type 20: $(1 R, 2 S, 3 S, 3 \mathrm{a} S, 8 \mathrm{~b} S)$-3a-benzyl-1,8b-dihydroxy-3-isopropyl-N,N-dimethyl2,3,3a,8b-tetrahydro-1H-cyclopenta[ $b]$ benzofuran-2-carboxamide

To a stirred solution of $\mathbf{1 7 b}(322 \mathrm{mg}, 1.0 \mathrm{mmol})$ in DMF $(1.0 \mathrm{M})$ at $25^{\circ} \mathrm{C}$ was added Stile's reagent (3.0 equiv., 2.0M in DMF) dropwise. The resulting mixture was warmed to $100^{\circ} \mathrm{C}$ and stirred for $12 \mathrm{~h}$. After cooling to $0{ }^{\circ} \mathrm{C}$ with ice-water, the mixture was diluted with ice-cold $\mathrm{Et}_{2} \mathrm{O}(5.0 \mathrm{~mL})$, quenched with ice-cold $\mathrm{HCl}(2.0 \mathrm{~mL}, 6 \mathrm{M})$ and extracted by ice-cold $\mathrm{Et}_{2} \mathrm{O}$ for three times. The combined organic layers were dried with $\mathrm{Na}_{2} \mathrm{SO}_{4}$ and concentrated to $2.5 \mathrm{~mL}$ in vacuo at $5{ }^{\circ} \mathrm{C}$ followed with the addition of $\mathrm{MeOH}$ $(0.2 \mathrm{~mL})$ and $\mathrm{TMSCHN}_{2}$ (1.0 equiv., $2.0 \mathrm{M}$ in hexane). The resulting mixture was stirred for $1.0 \mathrm{~h}$ and then concentrated in vacuo. Which was used directly without further purification.

To a stirred solution of the ester (crude, obtain above) in dry toluene $(0.08 \mathrm{M})$ at $25{ }^{\circ} \mathrm{C}$ was added $\operatorname{DMAP}\left(0.3\right.$ equiv.) and $\mathrm{Me}_{2} \mathrm{NH}$ (10.0 equiv. $2.0 \mathrm{M}$ in THF). The resulting mixture was warmed to $100^{\circ} \mathrm{C}$ and stirred for $3.0 \mathrm{~h}$. Then the mixture was concentrated in vacuo and could be used directly without further purification.

To a stirred solution of the amide (crude, obtain above) in $\mathrm{MeCN}$ and $\mathrm{AcOH}(10: 1)(0.2 \mathrm{M})$ at $25{ }^{\circ} \mathrm{C}$ was added $\mathrm{Me}_{4} \mathrm{NBH}(\mathrm{OAc})_{3}\left(5.0\right.$ equiv.). The resulting mixture was stirred at $25^{\circ} \mathrm{C}$ for $3.0 \mathrm{~h}$ before it was quenched with $\mathrm{Na}_{2} \mathrm{CO}_{3}(10 \mathrm{~mL}$, sat. aq.). The layers were separated and the aqueous layer was extracted with EtOAc $(3 \times 10 \mathrm{~mL})$. The combined organic layers were dried with $\mathrm{Na}_{2} \mathrm{SO}_{4}$ and concentrated in vacuo. The desired product $\mathbf{2 0}$ was purified using flash chromatography (petroleum ether/EA=1:2) as a white solid ( $210 \mathrm{mg}, 53 \%$ for three steps).

$\underline{R}_{f} \quad 0.50\left(\mathrm{Hex} / \mathrm{EtOAc}=1: 9, \mathrm{KMnO}_{4}\right)$

Opt. Rot. $\alpha]^{20}=-23.0(\mathrm{c}=0.1, \mathrm{MeOH})$

${ }^{1} \mathrm{H} \mathrm{NMR} \quad\left(500 \mathrm{MHz}, \mathrm{CDCl}_{3}\right)$

$\delta 7.50(\mathrm{dd}, J=7.6,1.1 \mathrm{~Hz}, 1 \mathrm{H}), 7.38(\mathrm{~d}, J=6.9 \mathrm{~Hz}, 2 \mathrm{H}), 7.23-7.07(\mathrm{~m}, 4 \mathrm{H}), 6.84(\mathrm{t}, J$ $=6.9 \mathrm{~Hz}, 1 \mathrm{H}), 6.76(\mathrm{~d}, J=8.1 \mathrm{~Hz}, 1 \mathrm{H}), 4.46(\mathrm{~d}, J=10.5 \mathrm{~Hz}, 1 \mathrm{H}), 3.16(\mathrm{~d}, J=14.2 \mathrm{~Hz}$, 1H), 3.03 (d, $J=14.2 \mathrm{~Hz}, 1 \mathrm{H}), 2.89$ (d, $J=11.0 \mathrm{~Hz}, 6 \mathrm{H}), 2.62(\mathrm{t}, J=10.9 \mathrm{~Hz}, 1 \mathrm{H}), 2.51$ $(\mathrm{dd}, J=11.3,3.9 \mathrm{~Hz}, 1 \mathrm{H}), 1.40-1.26(\mathrm{~m}, 1 \mathrm{H}), 0.53(\mathrm{dd}, J=11.3,6.8 \mathrm{~Hz}, 6 \mathrm{H})$.

${ }^{13} \mathrm{C} \mathrm{NMR} \quad\left(125 \mathrm{MHz}, \mathrm{CDCl}_{3}\right)$

$\delta 174.4,160.3,136.8,131.1,130.6,127.9,127.5,127.3,126.4,120.6,109.8,95.5,89.1$, 81.72, 52.1, 45.3, 42.2, 37.6, 36.4, 27.3, 23.2, 20.2 .

HRMS (ESI) for: $\mathrm{C}_{24} \mathrm{H}_{29} \mathrm{NNaO}_{4}[\mathrm{M}+\mathrm{Na}]^{+}$: calcd 418.1994, found 418.2023. 


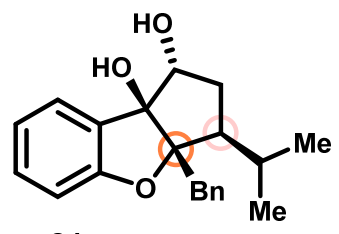

21: rocaglaol-type

21:

$(1 R, 3 S, 3 \mathrm{a} S, 8 \mathrm{~b} S)$-3a-benzyl-3-isopropyl-1,2,3,3a-tetrahydro- $8 \mathrm{~b} H$ -

cyclopenta[$[b]$ benzo furan-1,8b-diol

To a stirred solution of 3-epi-17b $(2.90 \mathrm{~g}, 9.0 \mathrm{mmol})$ in $\mathrm{MeCN}$ and $\mathrm{AcOH}(10: 1)(0.2 \mathrm{M})$ at $25^{\circ} \mathrm{C}$ was added $\mathrm{Me}_{4} \mathrm{NBH}(\mathrm{OAc})_{3}\left(3.0\right.$ equiv.). The resulting mixture was stirred at $25{ }^{\circ} \mathrm{C}$ for $3.0 \mathrm{~h}$ before it was quenched with $\mathrm{Na}_{2} \mathrm{CO}_{3}(30 \mathrm{~mL}$, sat. aq.). The layers were separated and the aqueous layer was extracted with EtOAc $(3 \times 30 \mathrm{~mL})$. The combined organic layers were dried with $\mathrm{Na}_{2} \mathrm{SO}_{4}$ and concentrated in vacuo. The desired product 21 was purified using flash chromatography (petroleum ether/EA=5:1) as a white solid $(2.50 \mathrm{~g}, 85 \%)$.

$\underline{R}_{f} \quad 0.45\left(\mathrm{Hex} / \mathrm{EtOAc}=2: 1, \mathrm{KMnO}_{4}\right)$

Opt. Rot. $\alpha]^{20}{ }_{\mathrm{D}}=14.0(\mathrm{c}=0.1, \mathrm{MeOH})$

${ }^{1} \mathrm{H} \mathrm{NMR} \quad\left(500 \mathrm{MHz}, \mathrm{CDCl}_{3}\right)$

$\delta 7.36-7.30(\mathrm{~m}, 2 \mathrm{H}), 7.12(\mathrm{~d}, J=8.2 \mathrm{~Hz}, 1 \mathrm{H}), 7.06(\mathrm{~d}, J=14.4 \mathrm{~Hz}, 3 \mathrm{H}), 7.01(\mathrm{~s}, 1 \mathrm{H})$, $6.74(\mathrm{~d}, J=18.0 \mathrm{~Hz}, 2 \mathrm{H}), 4.25(\mathrm{t}, J=4.8 \mathrm{~Hz}, 1 \mathrm{H}), 3.29(\mathrm{~d}, J=14.8 \mathrm{~Hz}, 1 \mathrm{H}), 2.92(\mathrm{~d}, J$ $=14.8 \mathrm{~Hz}, 1 \mathrm{H}), 2.14-2.06(\mathrm{~m}, 1 \mathrm{H}), 1.97-1.78(\mathrm{~m}, 3 \mathrm{H}), 1.73-1.63(\mathrm{~m}, 1 \mathrm{H}), 1.06(\mathrm{~d}$, $J=6.5 \mathrm{~Hz}, 3 \mathrm{H}), 0.91(\mathrm{~d}, J=6.5 \mathrm{~Hz}, 3 \mathrm{H})$.

${ }^{13} \mathrm{C} \mathrm{NMR} \quad\left(125 \mathrm{MHz}, \mathrm{CDCl}_{3}\right)$

$\delta 159.5,137.1,130.9,130.6,128.3,127.3,126.5,125.3,120.5,110.6,100.1,94.2,78.8$, 54.7, 35.7, 35.0, 27.2, 22.6, 21.6.

HRMS (ESI) for: $\mathrm{C}_{21} \mathrm{H}_{24} \mathrm{NaO}_{3}[\mathrm{M}+\mathrm{Na}]^{+}$: calcd 347.1623, found 347.1569 .

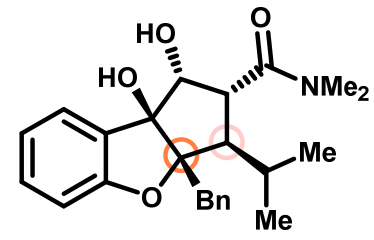

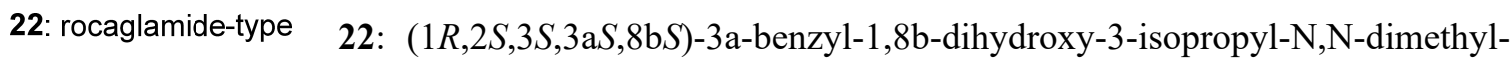
2,3,3a,8b-tetrahydro- $1 H$-cyclopenta $[b]$ benzofuran-2-carboxamide

To a stirred solution of 3-epi-17b $(322 \mathrm{mg}, 1.0 \mathrm{mmol})$ in DMF $(1.0 \mathrm{M})$ at $25{ }^{\circ} \mathrm{C}$ was added Stile's reagent (3.0 equiv., 2.0M in DMF) dropwise. The resulting mixture was warmed to $100{ }^{\circ} \mathrm{C}$ and stirred for $12 \mathrm{~h}$. After cooling to $0{ }^{\circ} \mathrm{C}$ with ice-water, the mixture was diluted with ice-cold $\mathrm{Et}_{2} \mathrm{O}(5.0 \mathrm{~mL})$, quenched with ice-cold $\mathrm{HCl}(2.0 \mathrm{~mL}, 6 \mathrm{M})$ and extracted by ice-cold $\mathrm{Et}_{2} \mathrm{O}$ for three times. The combined organic layers were dried with $\mathrm{Na}_{2} \mathrm{SO}_{4}$ and concentrated to $2.5 \mathrm{~mL}$ in vacuo at $5{ }^{\circ} \mathrm{C}$ followed with the addition of $\mathrm{MeOH}(0.2 \mathrm{~mL})$ and $\mathrm{TMSCHN}_{2}$ (1.0 equiv., $2.0 \mathrm{M}$ in hexane). The resulting mixture was stirred for $1.0 \mathrm{~h}$ and then concentrated in vacuo. Which was used directly without further purification.

To a stirred solution of the ester (crude, obtain above) in dry toluene $(0.08 \mathrm{M})$ at $25^{\circ} \mathrm{C}$ was added DMAP (0.3 equiv.) and $\mathrm{Me}_{2} \mathrm{NH}$ (10.0 equiv., $2.0 \mathrm{M}$ in THF). The resulting mixture was warmed to 100 ${ }^{\circ} \mathrm{C}$ and stirred for $3.0 \mathrm{~h}$. Then the mixture was concentrated in vacuo and could be used directly without further purification.

To a stirred solution of the amide (crude, obtain above) in $\mathrm{MeCN}$ and $\mathrm{AcOH}(10: 1)(0.2 \mathrm{M})$ at $25{ }^{\circ} \mathrm{C}$ was added $\mathrm{Me}_{4} \mathrm{NBH}(\mathrm{OAc})_{3}\left(5.0\right.$ equiv.). The resulting mixture was stirred at $25^{\circ} \mathrm{C}$ for $3.0 \mathrm{~h}$ before it was 
quenched with $\mathrm{Na}_{2} \mathrm{CO}_{3}(10 \mathrm{~mL}$, sat. aq.). The layers were separated and the aqueous layer was extracted with EtOAc $(3 \times 10 \mathrm{~mL})$. The combined organic layers were dried with $\mathrm{Na}_{2} \mathrm{SO}_{4}$ and concentrated in vacuo. The desired product 22 was purified using flash chromatography (petroleum ether/EA=1:2) as a white solid (210 $\mathrm{mg}, 53 \%$ for three steps).

$\underline{R}_{f} \quad 0.60\left(\mathrm{Hex} / \mathrm{EtOAc}=1: 9, \mathrm{KMnO}_{4}\right)$

Opt. Rot. $\alpha]^{20}=17.0(\mathrm{c}=0.1, \mathrm{MeOH})$

${ }^{1} \mathrm{H} \mathrm{NMR} \quad\left(500 \mathrm{MHz}, \mathrm{CDCl}_{3}\right)$

$\delta 7.39(\mathrm{~d}, J=7.2 \mathrm{~Hz}, 2 \mathrm{H}), 7.17-7.05(\mathrm{~m}, 4 \mathrm{H}), 7.05-6.99(\mathrm{~m}, 1 \mathrm{H}), 6.78-6.72(\mathrm{~m}$, 2H), $4.23(\mathrm{~d}, J=5.8 \mathrm{~Hz}, 1 \mathrm{H}), 3.40(\mathrm{~d}, J=14.4 \mathrm{~Hz}, 1 \mathrm{H}), 3.17(\mathrm{dd}, J=12.7,5.8 \mathrm{~Hz}, 1 \mathrm{H})$, $3.07(\mathrm{~s}, 3 \mathrm{H}), 2.93(\mathrm{~d}, J=14.5 \mathrm{~Hz}, 1 \mathrm{H}), 2.88(\mathrm{~s}, 3 \mathrm{H}), 2.67(\mathrm{dd}, J=12.7,8.7 \mathrm{~Hz}, 1 \mathrm{H})$, $2.07-1.97(\mathrm{~m}, 1 \mathrm{H}), 1.12(\mathrm{~d}, J=6.5 \mathrm{~Hz}, 3 \mathrm{H}), 0.81(\mathrm{~d}, J=6.7 \mathrm{~Hz}, 3 \mathrm{H})$.

${ }^{13} \mathrm{C} \mathrm{NMR} \quad\left(125 \mathrm{MHz}, \mathrm{CDCl}_{3}\right)$

$\delta 173.1,159.4,136.9,130.8,130.6,128.3,126.6,126.0,120.5,110.5,98.5,93.4,78.4$, $57.2,44.7,37.6,36.1,36.1,27.5,22.9,21.0$.

HRMS (ESI) for: $\mathrm{C}_{24} \mathrm{H}_{29} \mathrm{NNaO}_{4}[\mathrm{M}+\mathrm{Na}]^{+}$: calcd 418.1994, found 418.2044 .

3.5 Formal Synthesis of rocaglamide (5)

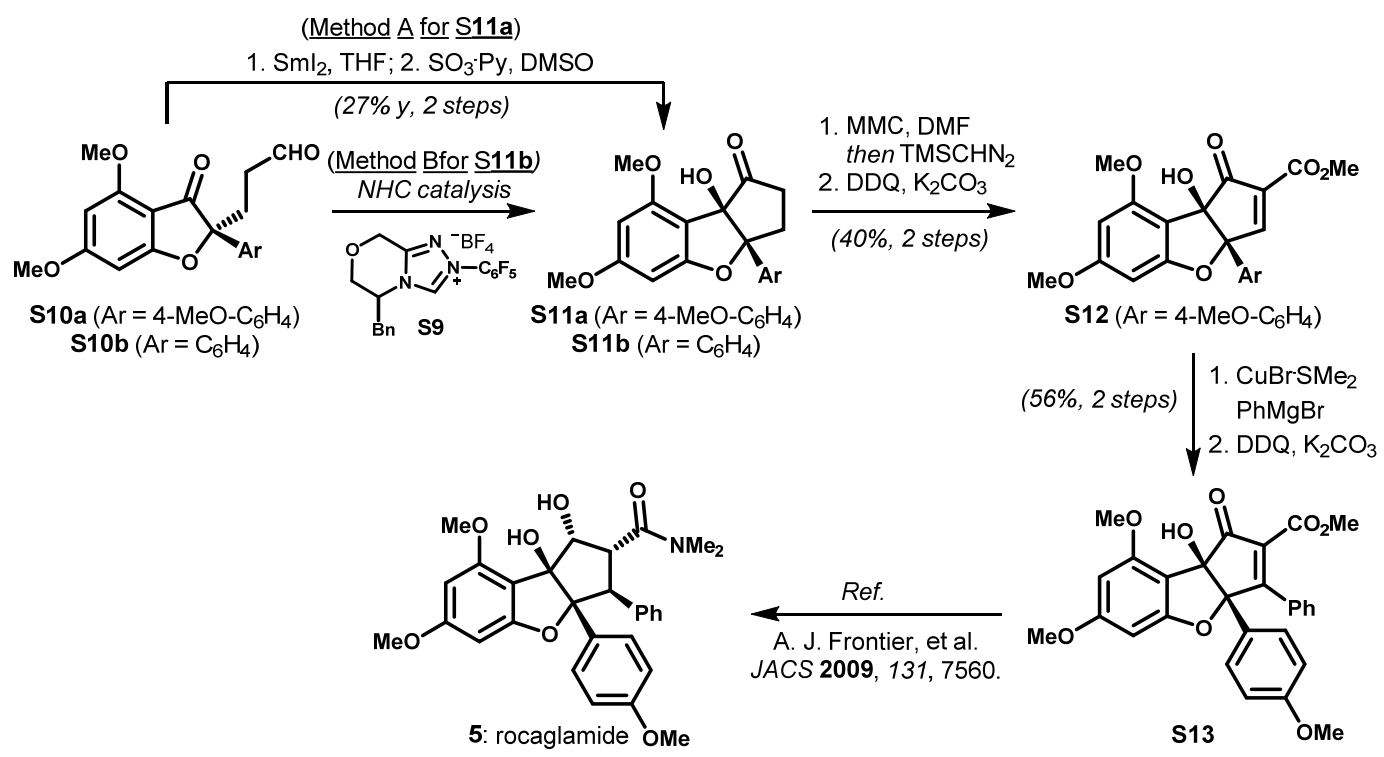

Scheme S6. Formal Synthesis of $( \pm)$-rocaglamide.

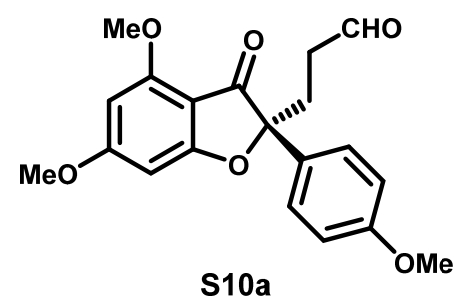

dihydrobenzofuran-2-yl)propanal

$( \pm)-$ S10a: $\quad( \pm)-(R)-3-(4,6-d i m e t h o x y-2-(4-m e t h o x y p h e n y l)-3-o x o-2,3-$ 
A solution of $\mathrm{PdCl}_{2}\left(\mathrm{PhCN}_{2}\right)_{2}\left(0.12\right.$ equiv.), $\mathrm{CuCl}_{2} \cdot 2 \mathrm{H}_{2} \mathrm{O}$ (0.12 equiv.) and $\mathrm{AgNO}_{2}$ (0.06 equiv.) in $t$ $\mathrm{BuOH}(0.1 \mathrm{M})$ and $\mathrm{MeNO}_{2}(1.5 \mathrm{M})$ was sparged for 15 min with oxygen $(1 \mathrm{~atm}$, balloon) in a $50 \mathrm{~mL}$ flask. The alkene $( \pm)-10 g^{\prime}(340.4 \mathrm{mg}, 1.0 \mathrm{mmol})$ was added dropwise subsequently. The solution was saturated with oxygen by an additional 2 min of sparging. The reaction was then allowed to stir at $23{ }^{\circ} \mathrm{C}$ for $24 \mathrm{~h}$ under $1 \mathrm{~atm}$ oxygen ( $1 \mathrm{~atm}$, balloon). After the reaction was completed, the reaction mixture was concentrated. Next, the resulting mixture was added to water $(20 \mathrm{~mL})$ and extracted three times with TBME $(20 \mathrm{~mL} \times 3)$. The combined organic layers were subsequently washed with a saturated solution of $\mathrm{NaHCO}_{3}$ and dried over $\mathrm{Na}_{2} \mathrm{SO}_{4}$. The solvent was removed under reduced pressure and the desired aldehyde product was purified using flash chromatography (petroleum ether/EA=3:1) as a white solid (306.5 mg, 86\%).

$\underline{R}_{f} \quad 0.30\left(\mathrm{Hex} / \mathrm{EtOAc}=3: 1, \mathrm{KMnO}_{4}\right)$

${ }^{1} \mathrm{H} \mathrm{NMR} \quad\left(500 \mathrm{MHz}, \mathrm{CDCl}_{3}\right)$

$\delta 9.63(\mathrm{~s}, 1 \mathrm{H}), 7.58-7.47(\mathrm{~m}, 2 \mathrm{H}), 7.19-7.08(\mathrm{~m}, 5 \mathrm{H}), 7.04-6.93(\mathrm{~m}, 2 \mathrm{H}), 3.10(\mathrm{~d}, J$

$=1.8 \mathrm{~Hz}, 2 \mathrm{H}), 2.50-2.09(\mathrm{~m}, 4 \mathrm{H})$.

${ }^{13} \mathrm{C} \mathrm{NMR} \quad\left(125 \mathrm{MHz}, \mathrm{CDCl}_{3}\right)$

$\delta 200.9,196.0,174.2,170.2,159.5,129.1,126.2,114.0,103.5,93.3,91.6,88.8,56.1$, $55.3,38.3,30.8$.

HRMS (ESI) for: $\mathrm{C}_{19} \mathrm{H}_{19} \mathrm{O}_{4}[\mathrm{M}+\mathrm{H}]^{+}$: calcd 311.1283, found 311.1334 .<smiles>COc1cc(OC)c2c(c1)O[C@](CCC=O)(c1ccccc1)C2=O</smiles>

S10b

S10b: (R)-3-(4,6-dimethoxy-3-oxo-2-phenyl-2,3-dihydro benzofuran-2-

yl)propanal

A solution of $\mathrm{PdCl}_{2}(\mathrm{PhCN})_{2}$ (0.12 equiv.), $\mathrm{CuCl}_{2} \cdot 2 \mathrm{H}_{2} \mathrm{O}$ (0.12 equiv.) and $\mathrm{AgNO}_{2}$ (0.06 equiv.) in $t$ $\mathrm{BuOH}(0.1 \mathrm{M})$ and $\mathrm{MeNO}_{2}(1.5 \mathrm{M})$ was sparged for 15 min with oxygen $(1 \mathrm{~atm}$, balloon) in a $50 \mathrm{~mL}$ flask. The alkene $( \pm)-10 h '(310.4 \mathrm{mg}, 1.0 \mathrm{mmol})$ was added dropwise subsequently. The solution was saturated with oxygen by an additional 2 min of sparging. The reaction was then allowed to stir at $23{ }^{\circ} \mathrm{C}$ for $24 \mathrm{~h}$ under $1 \mathrm{~atm}$ oxygen ( $1 \mathrm{~atm}$, balloon). After the reaction was completed, the reaction mixture was concentrated. Next, the resulting mixture was added to water $(20 \mathrm{~mL})$ and extracted three times with TBME $(20 \mathrm{~mL} \times 3)$. The combined organic layers were subsequently washed with a saturated solution of $\mathrm{NaHCO}_{3}$ and dried over $\mathrm{Na}_{2} \mathrm{SO}_{4}$. The solvent was removed under reduced pressure and the desired aldehyde product was purified using flash chromatography (petroleum ether/EA=3:1) as a white solid (254.6 mg, 78\%).

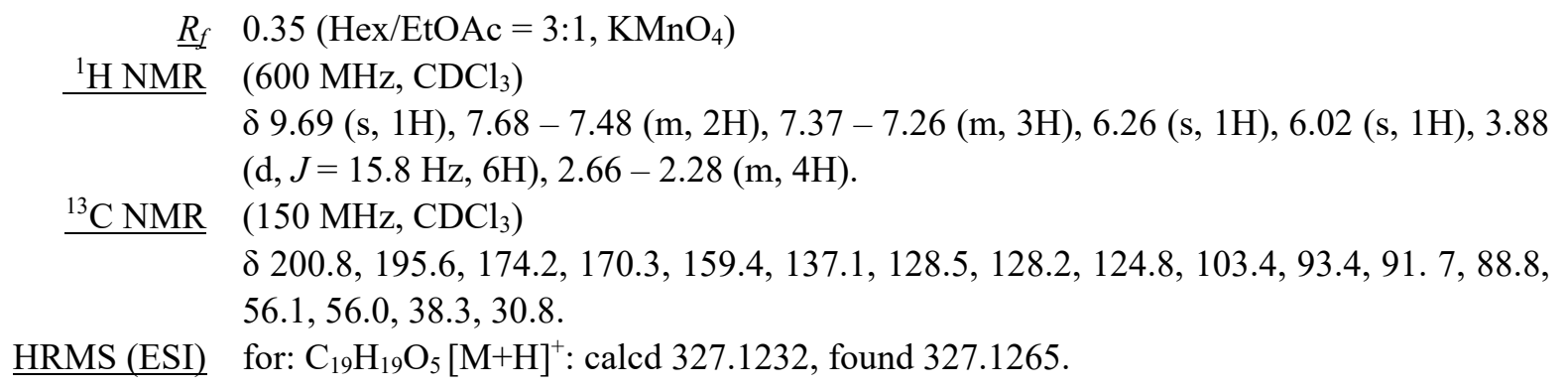




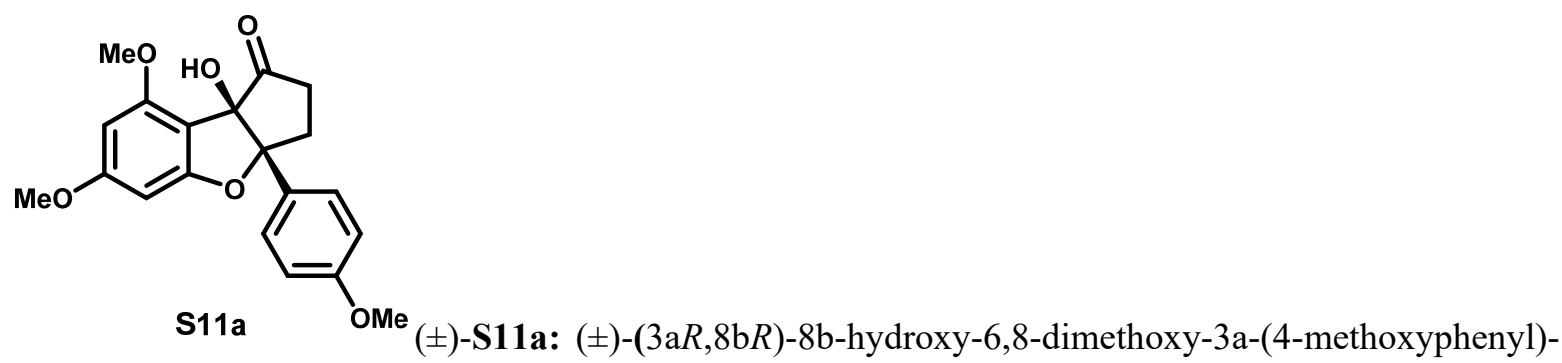
2,3,3a,8b-tetrahydro- $1 H$-cyclopenta[ $b]$ benzofuran-1-one

A solution of $\mathbf{S 1 0 a}(356.4 \mathrm{mg}, 1.0 \mathrm{mmol})$ in degassed THF $(1 \mathrm{M})$ was added dropwise to $\mathrm{SmI}_{2}(2.2$ equiv., $0.1 \mathrm{M}$ in THF) at $-78{ }^{\circ} \mathrm{C}$ under $\mathrm{N}_{2}$. After being stirred at $-78{ }^{\circ} \mathrm{C}$ for $1.5 \mathrm{~h}$, the mixture was purified quickly using reduced pressure column chromatography (petroleum ether/ $\mathrm{DCM} / \mathrm{EtOAc}=2: 3: 1$ ) to provide diol as a yellow solid (213.8 $\mathrm{mg}, 60 \%)$.

To a solution of diol (obtain above)) in dry DCM $(0.125 \mathrm{M})$ was added DMSO $(3 \mathrm{~mL}), \mathrm{NEt}_{3}(6.0$ equiv.) at $0{ }^{\circ} \mathrm{C}$ under $\mathrm{N}_{2}$. Then a solution of pyridine sulfur trioxide (5.5 equiv.) in DMSO $(5 \mathrm{~mL})$ was added slowly. The mixture was stirred at $0{ }^{\circ} \mathrm{C}$ for $6 \mathrm{~h}$ followed by qunenched with $10 \% \mathrm{HCl}$ solution at $10{ }^{\circ} \mathrm{C}$. Then the mixture was extracted by TBME for 5 times. The combined organic layers were dried over $\mathrm{Na}_{2} \mathrm{SO}_{4}$. The solvent was removed under reduced pressure and the desired product was purified using flash chromatography (petroleum ether/ EtOAc $=3: 1$ ) to generate the desired product S11a as a yellow solid ( $96.2 \mathrm{mg}, 27 \%$ for 2 steps).

$\underline{R}_{f} \quad 0.60(\mathrm{Hex} / \mathrm{EtOAc}=2: 3: 1$, Vanillin $)$

${ }^{1} \mathrm{H} \mathrm{NMR} \quad\left(500 \mathrm{MHz}, \mathrm{CDCl}_{3}\right)$

$\delta 7.34-7.27(\mathrm{~m}, 2 \mathrm{H}), 6.95-6.85(\mathrm{~m}, 2 \mathrm{H}), 6.20(\mathrm{~s}, 1 \mathrm{H}), 6.03(\mathrm{~s}, 1 \mathrm{H}), 3.87-3.71(\mathrm{~m}$, $9 \mathrm{H}), 2.93-2.75(\mathrm{~m}, 1 \mathrm{H}), 2.72-2.61(\mathrm{~m}, 1 \mathrm{H}), 2.59-2.47(\mathrm{~m}, 2 \mathrm{H})$.

${ }^{13} \mathrm{C} \mathrm{NMR} \quad\left(125 \mathrm{MHz}, \mathrm{CDCl}_{3}\right)$

$\delta 211.6,164.4,162.2,159.4,158.3,129.8,127.4,113.6,105.5,97.1,92.4,88.8,86.7$, $55.7,55.5,55.3,34.2,33.9$.

HRMS (ESI) for: $\mathrm{C}_{20} \mathrm{H}_{20} \mathrm{NaO}_{6}[\mathrm{M}+\mathrm{Na}]^{+}$: calcd 379.1158 , found 379.1153 .<smiles>COc1cc(OC)c2c(c1)O[C@]1(c3ccccc3)CCC(=O)[C@@]21O</smiles>

$1 H$-cyclopenta[$[b]$ benzofuran-1-one

S11b: $(3 \mathrm{a} R, 8 \mathrm{~b} R)-8 \mathrm{~b}-$ hydroxy-6,8-dimethoxy-3a-phenyl-2,3,3a,8b-tetrahydro-

Method B. To a solution of NHC S9 (0.3 equiv.), NaOAc (1.0 equiv.) in dry DCM ( $0.1 \mathrm{M})$ under $\mathrm{N}_{2}$ was added S11b $(65.2 \mathrm{mg}, 0.2 \mathrm{mmol})$ slowly. The reaction mixture was stirred overnight at room temperature followed by direct concentration. The crude mixture was purified using flash chromatography (petroleum ether/TBME=1:1) to generate the desired product $\mathbf{S 1 1} \mathbf{b}$ as a yellow oil (22.8 $\mathrm{mg}, 35 \%)$.

\footnotetext{
$\underline{R}_{f} \quad 0.60(\mathrm{Hex} / \mathrm{TBME}=1: 1$, Vanillin $)$

${ }^{1} \mathrm{H} \mathrm{NMR} \quad\left(500 \mathrm{MHz}, \mathrm{CDCl}_{3}\right)$
} 
$\delta 7.44-7.28(\mathrm{~m}, 5 \mathrm{H}), 6.22(\mathrm{~s}, 1 \mathrm{H}), 6.03(\mathrm{~s}, 1 \mathrm{H}), 3.79(\mathrm{~d}, J=26.9 \mathrm{~Hz}, 6 \mathrm{H}), 2.90-2.79$ $(\mathrm{m}, 1 \mathrm{H}), 2.74-2.63(\mathrm{~m}, 1 \mathrm{H}), 2.62-2.46(\mathrm{~m}, 2 \mathrm{H})$.

${ }^{13} \mathrm{C} \mathrm{NMR} \quad\left(150 \mathrm{MHz}, \mathrm{CDCl}_{3}\right)$

$\delta 211.5,164.4,162.1,158.2,137.9,128.2,126.0,105.4,97.0,92.4,88.8,86.8,55.5$, HRMS (ESI) 34.2 .

for: $\mathrm{C}_{19} \mathrm{H}_{18} \mathrm{NaO}_{5}[\mathrm{M}+\mathrm{Na}]^{+}$: calcd 349.1052, found 349.1013.

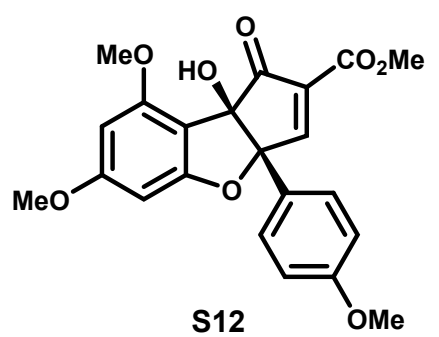

$( \pm)-$ S12: $\quad( \pm)$-Methyl $\quad(3 \mathrm{a} R, 8 \mathrm{~b} R)-8 \mathrm{~b}-$ hydroxy-6,8-dimethoxy-3a-(4methoxyphenyl)-1-oxo-3a,8b-dihydro-1 $H$-cyclopenta[ $b]$ benzofuran-2-carboxylate

To a stirred solution of S11a $(200 \mathrm{mg}, 0.56 \mathrm{mmol})$ in DMF $(1.0 \mathrm{M})$ at $25{ }^{\circ} \mathrm{C}$ was added Stile's reagent (3.0 equiv., $2.0 \mathrm{M}$ in DMF) dropwise. The resulting mixture was warmed to $80^{\circ} \mathrm{C}$ and stirred for $3 \mathrm{~h}$. After cooling to $0{ }^{\circ} \mathrm{C}$ with ice-water, the mixture was diluted with ice-cold $\mathrm{Et}_{2} \mathrm{O}(5.0 \mathrm{~mL})$, quenched with ice-cold $\mathrm{HCl}(2.0 \mathrm{~mL}, 6 \mathrm{M})$ and extracted by ice-cold $\mathrm{Et}_{2} \mathrm{O}$ for three times. The combined organic layers were dried with $\mathrm{Na}_{2} \mathrm{SO}_{4}$ and concentrated to $2.5 \mathrm{~mL}$ in vacuo at $5{ }^{\circ} \mathrm{C}$ followed with the addition of $\mathrm{MeOH}$ $(0.2 \mathrm{~mL})$ and $\mathrm{TMSCHN}_{2}$ (1.0 equiv., $2.0 \mathrm{M}$ in hexane). The resulting mixture was stirred for $1.0 \mathrm{~h}$ and then concentrated in vacuo. Which was used directly without further purification.

To a stirred solution of the ester (crude, obtain above) in dry THF $(0.08 \mathrm{M})$ at $25^{\circ} \mathrm{C}$ was added DDQ (2.5 equiv.) and $\mathrm{K}_{2} \mathrm{CO}_{3}$ (2.5 equiv.). The resulting mixture was stirred for $2.0 \mathrm{~h}$ before it was quenched with $\mathrm{NaHCO}_{3}(10 \mathrm{~mL}$, sat. aq.). The layers were separated and the aqueous layer was extracted with EtOAc $(3 \times 10 \mathrm{~mL})$. The combined organic layers were washed with brine, dried with $\mathrm{Na}_{2} \mathrm{SO}_{4}$ and concentrated in vacuo. The desired product $\mathbf{S 1 2}$ was purified using flash chromatography (petroleum ether/EA $=2: 1)$ as a colorless oil ( $68 \mathrm{mg}, 39 \%$ for two steps).

$\underline{R}_{f} \quad 0.40\left(\mathrm{Hex} / \mathrm{EtOAc}=23: 1, \mathrm{KMnO}_{4}\right)$

${ }^{1} \mathrm{H} \mathrm{NMR} \quad\left(500 \mathrm{MHz}, \mathrm{CDCl}_{3}\right)$

$\delta 7.99(\mathrm{~s}, 1 \mathrm{H}), 7.23(\mathrm{~d}, J=8.9 \mathrm{~Hz}, 2 \mathrm{H}), 6.93(\mathrm{~d}, J=8.9 \mathrm{~Hz}, 2 \mathrm{H}), 6.16(\mathrm{~d}, J=2.0 \mathrm{~Hz}$, $1 \mathrm{H}), 6.06(\mathrm{~d}, J=2.0 \mathrm{~Hz}, 1 \mathrm{H}), 3.87(\mathrm{~s}, 3 \mathrm{H}), 3.81(\mathrm{~s}, 3 \mathrm{H}), 3.80(\mathrm{~s}, 3 \mathrm{H}), 3.79(\mathrm{~s}, 3 \mathrm{H}), 3.29$ (s, 1H).

${ }^{13} \mathrm{C} \mathrm{NMR} \quad\left(125 \mathrm{MHz}, \mathrm{CDCl}_{3}\right)$

$\delta 195.9,164.5,161.5,160.8,160.6,160.1,158.5,135.6,127.8,125.0,114.0,105.2$, 94.5, 92.8, 88.5, 86.5, 55.7, 55.6, 55.3, 52.5.

$\underline{\text { HRMS (ESI) }}$ for: $\mathrm{C}_{22} \mathrm{H}_{21} \mathrm{O}_{8}[\mathrm{M}+\mathrm{H}]^{+}$: calcd 413.1236, found 413.2680. 


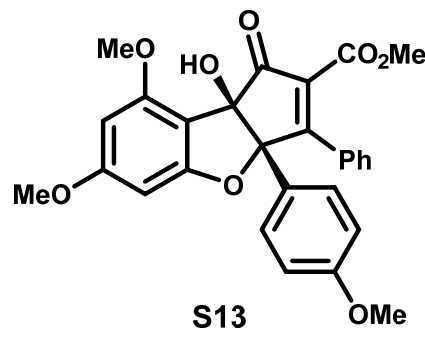

$( \pm)-$ S13: $\quad( \pm)$-Methyl $\quad(3 \mathrm{a} S, 8 \mathrm{~b} R)-8 \mathrm{~b}-$ hydroxy-6,8-dimethoxy-3a-(4methoxyphenyl)-1-oxo-3-phenyl-3a,8b-dihydro- $1 H$-cyclopenta[b]benzofuran-2-carboxylate

To a stirred solution of $\mathrm{CuBr} \cdot \mathrm{Me}_{2} \mathrm{~S}(0.2$ equiv. $)$ and $\mathbf{S 1 2}(68 \mathrm{mg}, 0.17 \mathrm{mmol})$ in dry THF $(0.1 \mathrm{M})$ at $78{ }^{\circ} \mathrm{C}$ was added $\mathrm{PhMgBr}(2.2$ equiv., $1.0 \mathrm{M}$ in THF) dropwise. The resulting mixture was stirred at -78 ${ }^{\circ} \mathrm{C}$ for $1.0 \mathrm{~h}$ before it was warmed to $0{ }^{\circ} \mathrm{C}$ for additional $1.0 \mathrm{~h}$. Then the resulting mixture was quenched with $\mathrm{NH}_{4} \mathrm{Cl}(5 \mathrm{~mL}$, sat. aq.). The layers were separated and the aqueous layer was extracted with EtOAc $(3 \times 5 \mathrm{~mL})$. The combined organic layers were dried with $\mathrm{Na}_{2} \mathrm{SO}_{4}$ and concentrated in vacuo. Which was used directly without further purification.

To a stirred solution of the ester (crude, obtain above) in dry THF $(0.08 \mathrm{M})$ at $25{ }^{\circ} \mathrm{C}$ was added DDQ (2.5 equiv.) and $\mathrm{K}_{2} \mathrm{CO}_{3}$ (2.5 equiv.). The resulting mixture was stirred for $2.0 \mathrm{~h}$ before it was quenched with $\mathrm{NaHCO}_{3}(10 \mathrm{~mL}$, sat. aq.). The layers were separated and the aqueous layer was extracted with EtOAc $(3 \times 10 \mathrm{~mL})$. The combined organic layers were washed with brine, dried with $\mathrm{Na}_{2} \mathrm{SO}_{4}$ and concentrated in vacuo. The desired product $\mathbf{S 1 3}$ was purified using flash chromatography (petroleum ether/EA=2:1) as a white solid ( $46 \mathrm{mg}, 56 \%$ for two steps).

$\underline{R}_{f} \quad 0.40\left(\mathrm{Hex} / \mathrm{EtOAc}=2: 1, \mathrm{KMnO}_{4}\right)$

Opt. Rot. $\quad \alpha]^{20}{ }_{\mathrm{D}}=162.2(\mathrm{c}=0.1, \mathrm{MeOH})$

${ }^{1} \mathrm{H} \mathrm{NMR} \quad\left(500 \mathrm{MHz}, \mathrm{CDCl}_{3}\right)$

$\delta 7.41-7.27(\mathrm{~m}, 7 \mathrm{H}), 6.93(\mathrm{~d}, J=8.9 \mathrm{~Hz}, 2 \mathrm{H}), 6.13(\mathrm{~d}, J=2.0 \mathrm{~Hz}, 1 \mathrm{H}), 6.08(\mathrm{~d}, J=2.0$ $\mathrm{Hz}, 1 \mathrm{H}), 3.82(\mathrm{~s}, 3 \mathrm{H}), 3.81(\mathrm{~s}, 3 \mathrm{H}), 3.79(\mathrm{~s}, 3 \mathrm{H}), 3.77(\mathrm{~s}, 3 \mathrm{H}), 3.18(\mathrm{~s}, 1 \mathrm{H})$.

${ }^{13} \mathrm{C} \mathrm{NMR} \quad\left(125 \mathrm{MHz}, \mathrm{CDCl}_{3}\right)$

$\delta 196.2,166.1,164.3,164.1,160.9,159.8,158.5,133.1,131.3,130.9,129.5,128.4$, $127.5,125.9,114.1,105.8,98.6,92.9,88.7,86.9,55.7,55.7,55.2,52.6$.

HRMS (ESI) for: $\mathrm{C}_{28} \mathrm{H}_{24} \mathrm{O}_{8} \mathrm{Na}[\mathrm{M}+\mathrm{Na}]^{+}$: calcd 511.1369, found 511.1327. 


\section{Preliminary Results of Biological Studies}
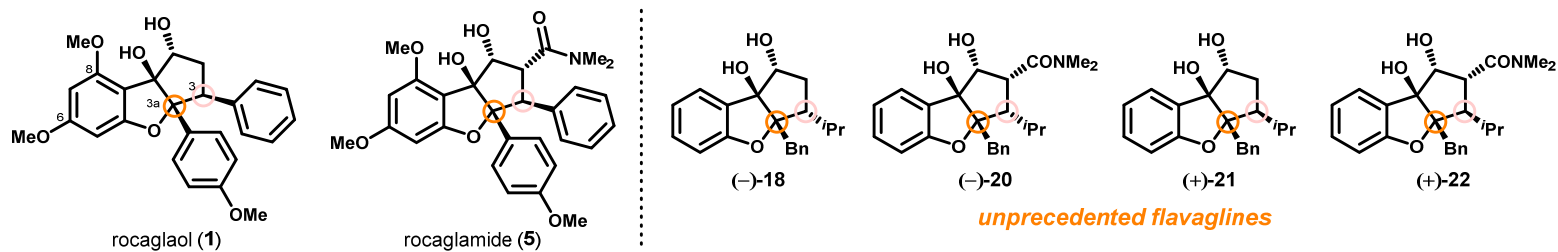

\section{Hela cell}

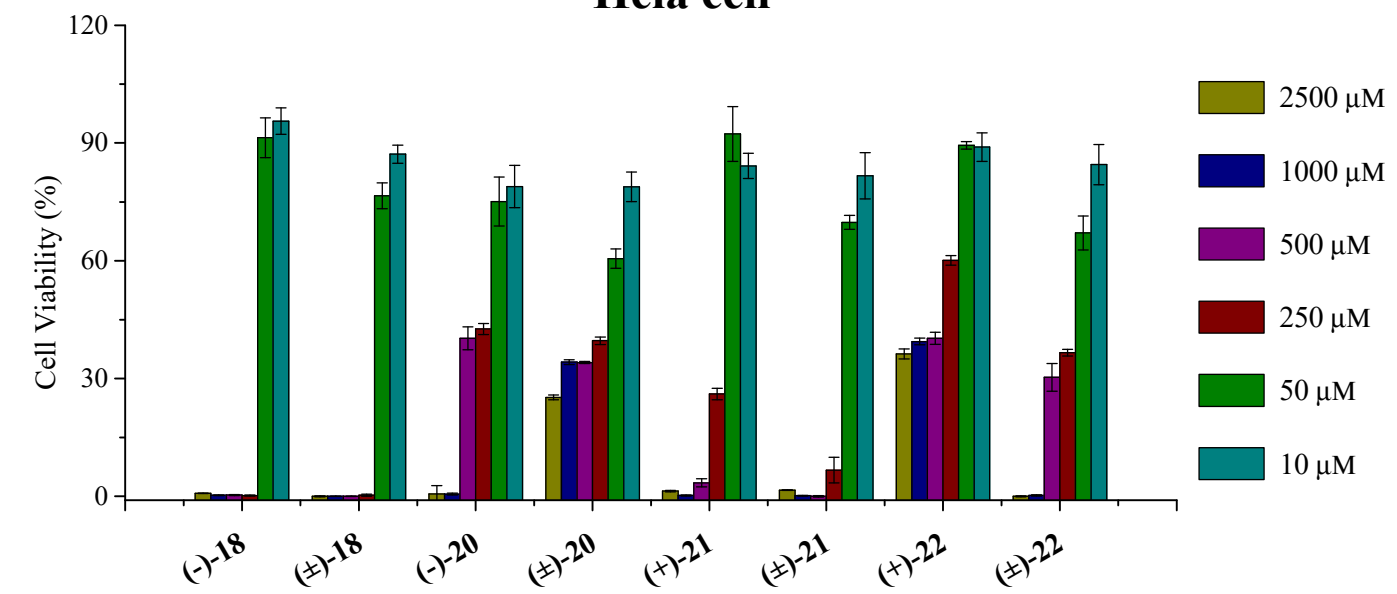

\begin{tabular}{c|cccccccc}
\hline Compound & $\mathbf{( - ) - 1 8}$ & $\mathbf{(} \pm)-\mathbf{1 8}$ & $\mathbf{( - )}-\mathbf{2 0}$ & $\mathbf{( \pm ) - 2 0}$ & $\mathbf{( + ) - 2 1}$ & $\mathbf{( \pm ) - 2 1}$ & $\mathbf{( + ) - 2 2}$ & $\mathbf{( \pm ) - 2 2}$ \\
\hline $\mathrm{IC}_{50}(\mu \mathrm{M})$ & 79.2 & 71.2 & 156.3 & 142.4 & 153.0 & 72.8 & 518.4 & 97.9 \\
\hline
\end{tabular}

Figure 1S. Inhibition of Cell Viability by the Non-natural Flavagline Analogs on Hela cells (IC50, $\mu$ M). 


\section{References}

(1) (a) M. J. Frisch, G. W. Trucks, H. B. Schlegel, G. E. Scuseria, M. A. Robb, J. R. Cheeseman, G. Scalmani, V. Barone, G. A. Petersson, H. Nakatsuji, X. Li, M. Caricato, A. V. Marenich, J. Bloino, B. G. Janesko, R. Gomperts, B. Mennucci, H. P. Hratchian, J. V. Ortiz, A. F. Izmaylov, J. L. Sonnenberg, D. Williams-Young, F. Ding, F. Lipparini, F. Egidi, J. Goings, B. Peng, A. Petrone, T. Henderson, D. Ranasinghe, V. G. Zakrzewski, J. Gao, N. Rega, G. Zheng, W. Liang, M. Hada, M. Ehara, K. Toyota, R. Fukuda, J. Hasegawa, M. Ishida, T. Nakajima, Y. Honda, O. Kitao, H. Nakai, T. Vreven, K. Throssell, J. A. Montgomery, Jr., J. E. Peralta, F. Ogliaro, M. J. Bearpark, J. J. Heyd, E. N. Brothers, K. N. Kudin, V. N. Staroverov, T. A. Keith, R. Kobayashi, J. Normand, K. Raghavachari, A. P. Rendell, J. C. Burant, S. S. Iyengar, J. Tomasi, M. Cossi, J. M. Millam, M. Klene, C. Adamo, R. Cammi, J. W. Ochterski, R. L. Martin, K. Morokuma, O. Farkas, J. B. Foresman, and D. J. Fox, Gaussian 09, revision C.01; Gaussian Inc.: Wallingford, CT, 2016. (b) C. Lee, W. Yang, R. G. Parr, Phys. Rev. B. 1988, 37, 785. (c) A. D. Becke, J. Chem. Phys. 1993, 98, 5648. (d) P. J. Stephens, F. J. Devlin, C. F. Chabalowski, M. J. Frisch, J. Phys. Chem. 1994, 98, 11623. (e) S. Grimme, J. Antony, S. Ehrlich, H. Krieg, J. Chem. Phys. 2010, 132, 154104. (f) S. Grimme, S. Ehrlich, L. Goerigk, J. Comput. Chem. 2011, 32, 1456. (g) A. Schäfer, H. Horn, R. Ahlrichs, J. Chem. Phys. 1992, 97, 2571.

(2) Cao, M.-Y.; Xu, Z.-M.; Gao, W.; Liu, J.; Tan, F.; Lu, H.-H. Catalytic Asymmetric Synthesis of a New Class of CyPHOX Ligands. Tetrahedron 2019, 75, 3282-3291.

(3) M. Hatano, T. Horibe, K. Ishihara. Chiral Magnesium (II) Binaphtholates as Cooperative Brønsted/Lewis Acid-Base Catalysts for the Highly Enantioselective Addition of Phosphorus Nucleophiles to $\alpha, \beta$-Unsaturated Esters and Ketones. Angew. Chem. Int. Ed. 2013, 52, 4549-4553.

(4) Node, M.; Nishide, K.; Sai, M.; Fuji, K.; Fujita, E. Hard acid and soft nucleophile systems. 3. Dealkylation of esters with aluminum halide-thiol and aluminum halide-sulfide systems. J. Org. Chem. 1981, 46, 1991-199.

(5) Guo, J.; Lin, Z.-H.; Chen, K.-B.; Xie, Y.; Chan, A. S. C.; Weng, J.; Lu, G. Asymmetric amination of 2-substituted indolin-3-ones catalyzed by natural cinchona alkaloids Org. Chem. Front. 2017, 4, 14001406.

(6) PHOX ligand $\mathbf{L 5}$ was prepared following a known method: Garcia-Fortanet, J.; Buchwald, S. L. Asymmetric palladium-catalyzed intramolecular $\alpha$-arylation of aldehydes. Angew. Chem. Int. Ed. 2008, $47,8108-8111$. 


\section{NMR Spectra}

Methyl (1S,2S)-2-(diphenylphosphoryl)cycloheptane-1-carboxylate (S2a)

매 NMR $\left(400 \mathrm{MHz}, \mathrm{CDCl}_{3} @ 7.26\right.$ ppm)

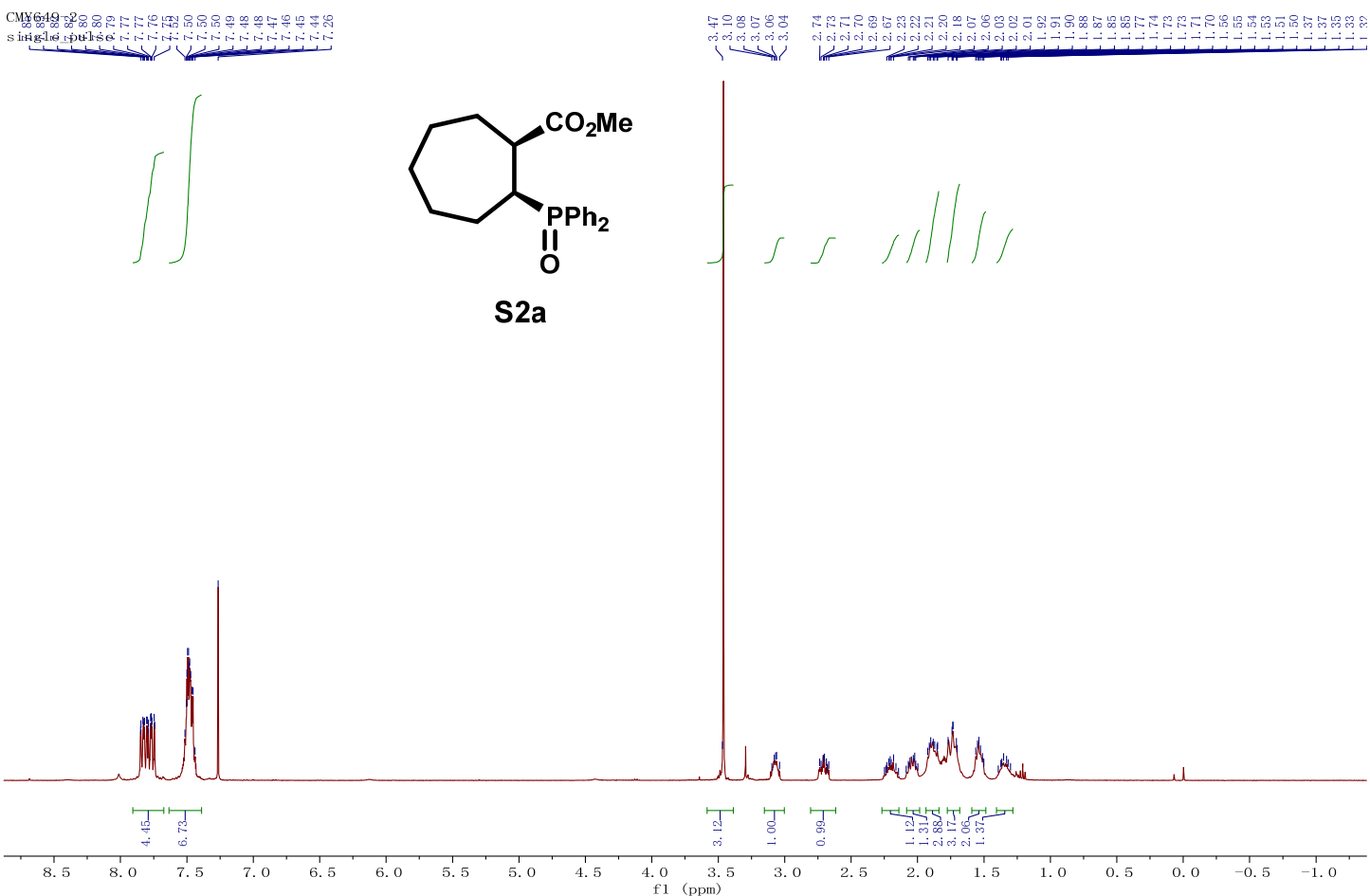

$\underline{{ }^{13} \mathrm{C} \mathrm{NMR}}\left(100 \mathrm{MHz}, \mathrm{CDCl}_{3} @ 77\right.$ ppm)

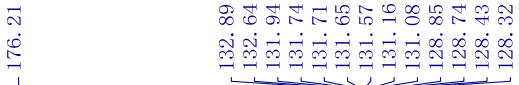

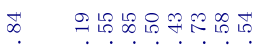

एँ भुष
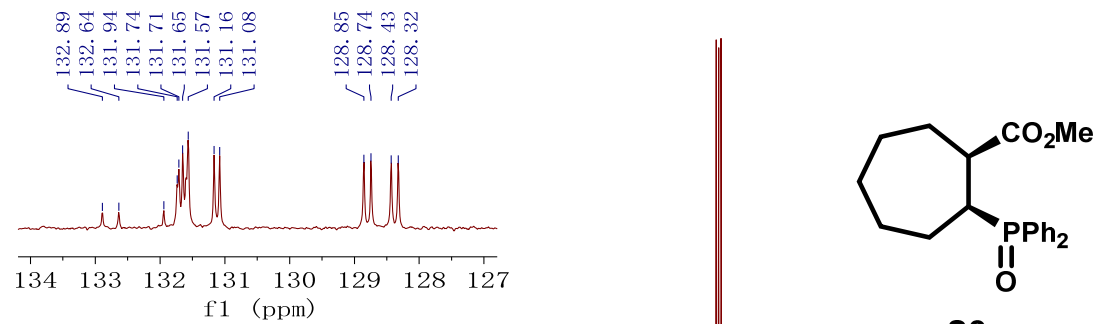

S2a

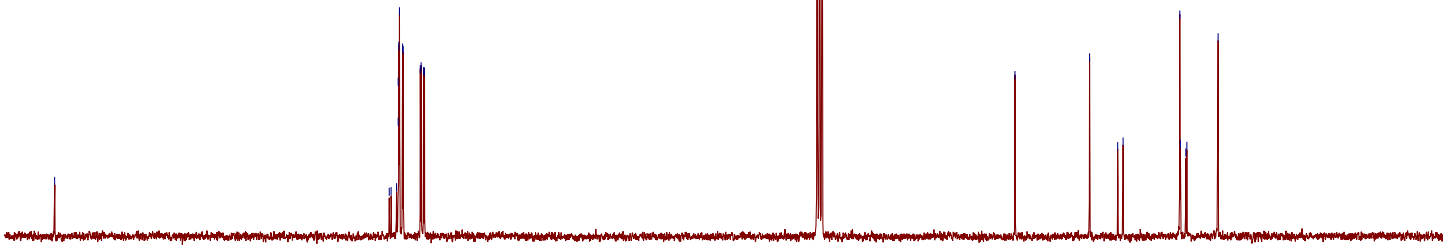

$\begin{array}{lllllllllllllllllll}180 & 170 & 160 & 150 & 140 & 130 & 120 & 110 & 100 & 90 & 80 & 70 & 60 & 50 & 40 & 30 & 20 & 10 & 0\end{array}$ 
31P NMR (162 MHz, $\left.\mathrm{CDCl}_{3}\right)$

CMY649-1

single pulse decoupled gated NOI
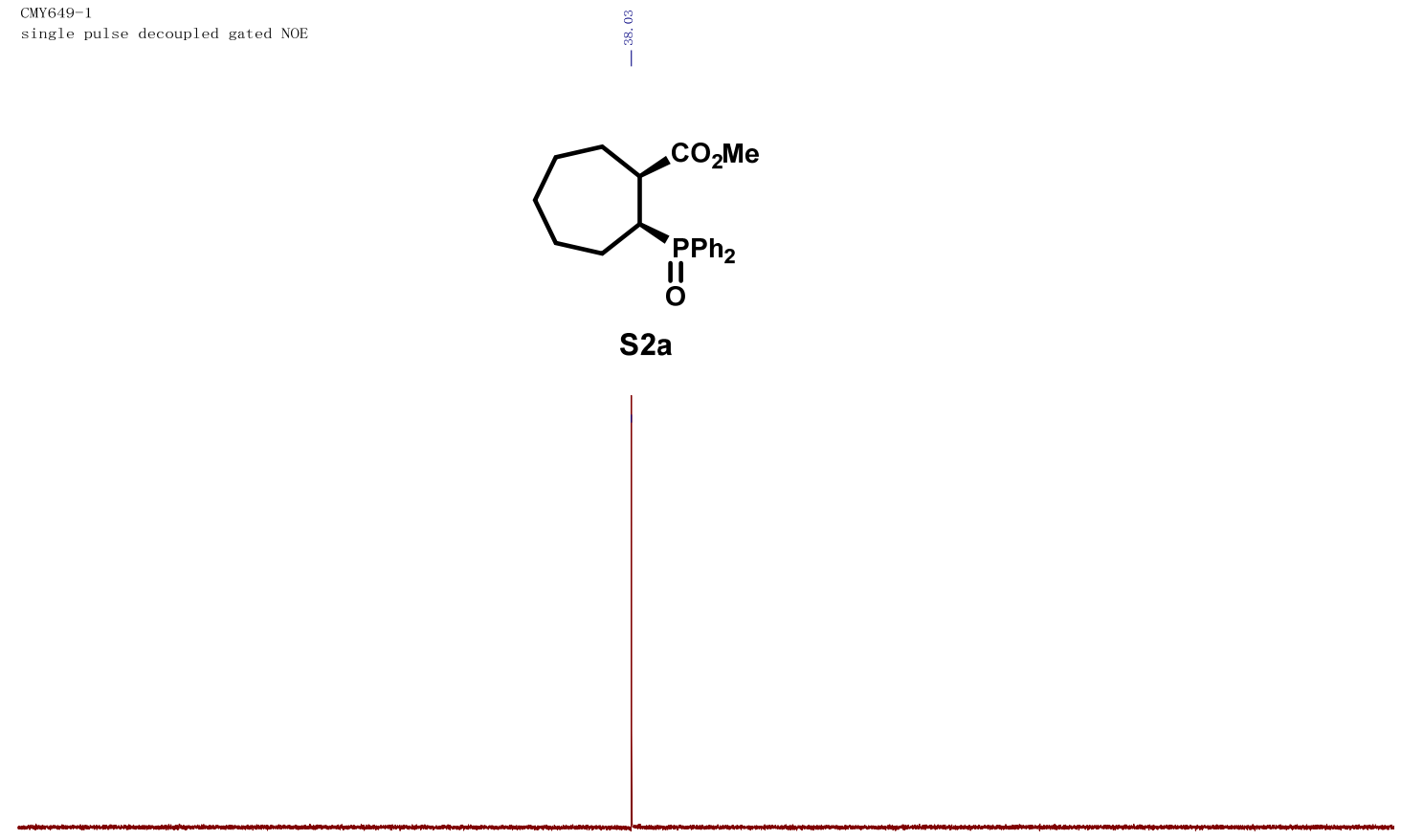

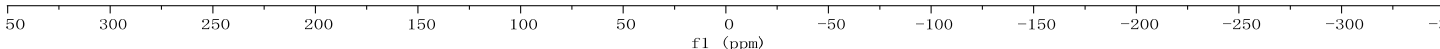

Methyl (1S,2S)-2-(di-p-tolylphosphoryl)cycloheptane-1-carboxylate (S2b)

$\underline{{ }^{1} \mathrm{H} \text { NMR }}\left(500 \mathrm{MHz}, \mathrm{CDCl}_{3} @ 7.26 \mathrm{ppm}\right)$ 

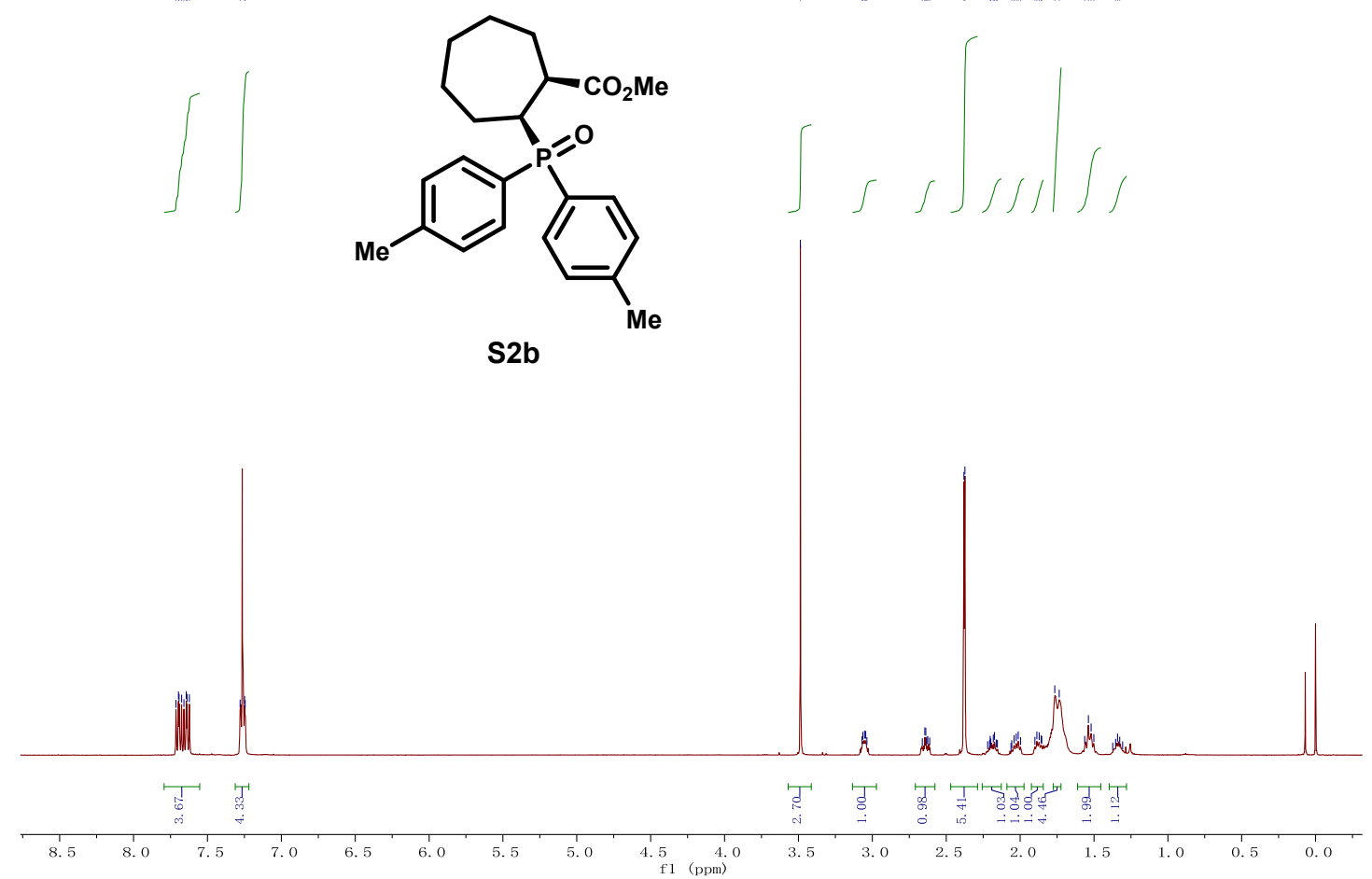

S2b

${ }^{13} \mathrm{C} \mathrm{NMR}\left(125 \mathrm{MHz}, \mathrm{CDCl}_{3} @ 77\right.$ ppm)

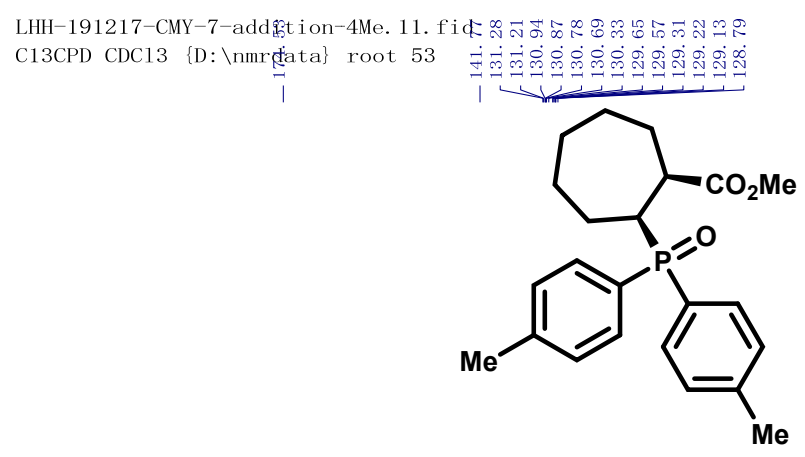

ำ

ப் मं

$S 2 b$

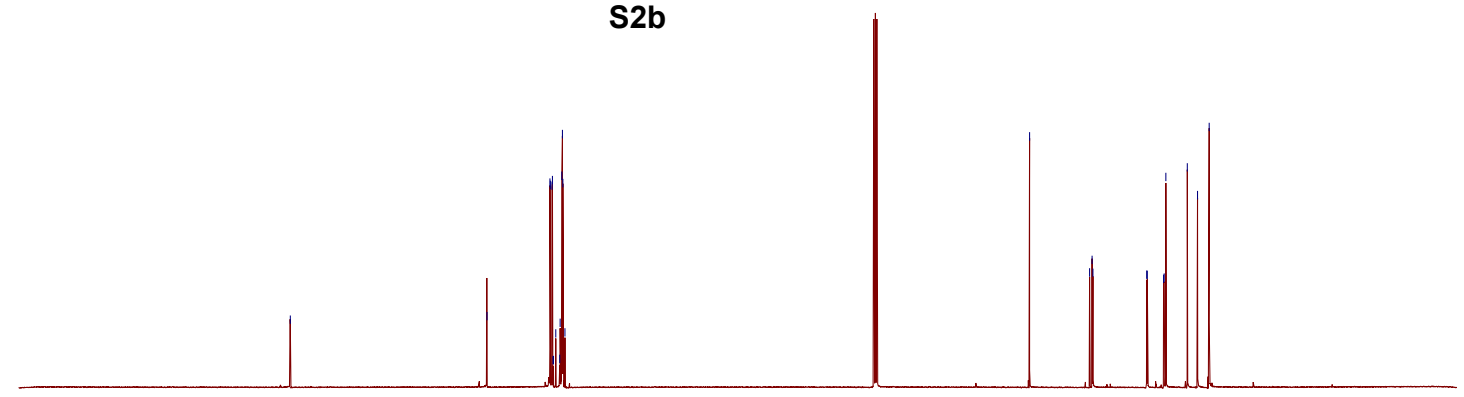

$\begin{array}{lllllllllllllllllllllll}210 & 200 & 190 & 180 & 170 & 160 & 150 & 140 & 130 & 120 & 110 & 100 & 90 & 80 & 70 & 60 & 50 & 40 & 30 & 20 & 10 & 0 & -10\end{array}$ f1 (ppm)

31P NMR (202 MHz, $\left.\mathrm{CDCl}_{3}\right)$ 

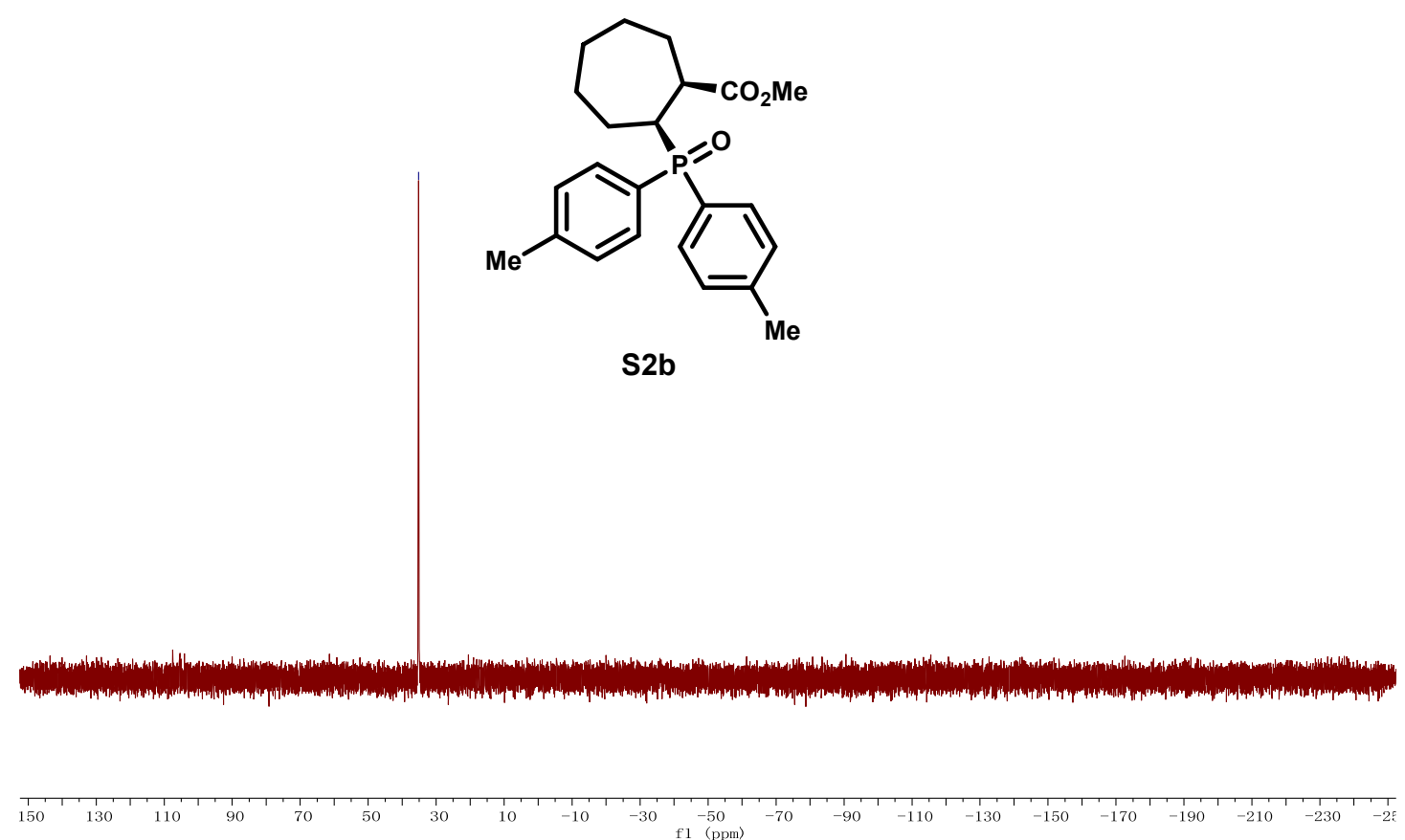

Methyl (1S,2S)-2-(di([1,1'-biphenyl]-4-yl)phosphoryl)cycloheptane-1-carboxylate (S2c)

${ }^{1} \mathrm{H}$ NMR $\left(500 \mathrm{MHz}, \mathrm{CDCl}_{3} @ 7.26\right.$ ppm)

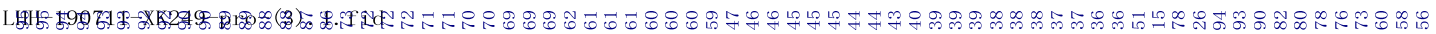

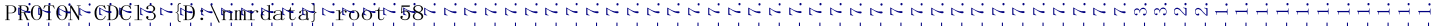

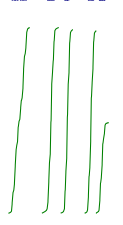<smiles>CC(=O)C1CCCCCC1P(=O)(c1ccccc1)c1ccc(-c2ccccc2)cc1</smiles>

S2c

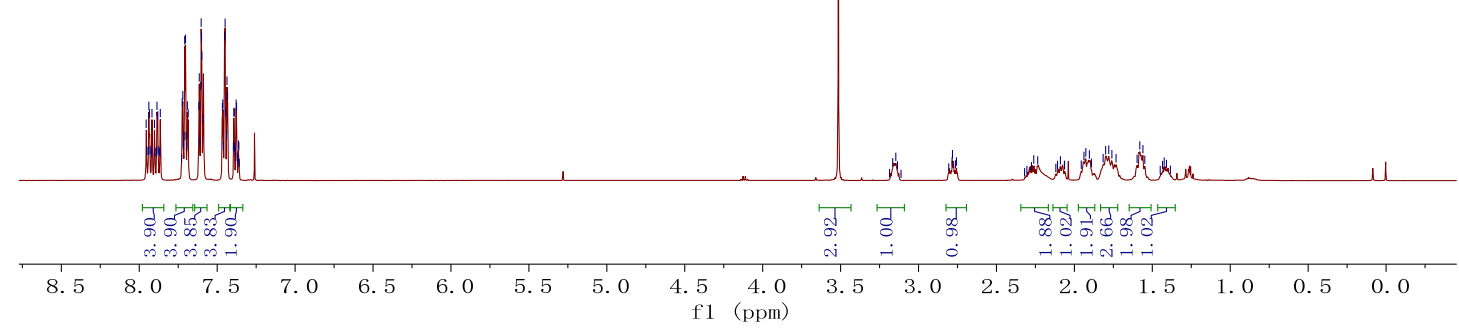




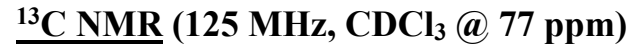

LHH-190711-XK249-pro-(3).3. fi

勿

|
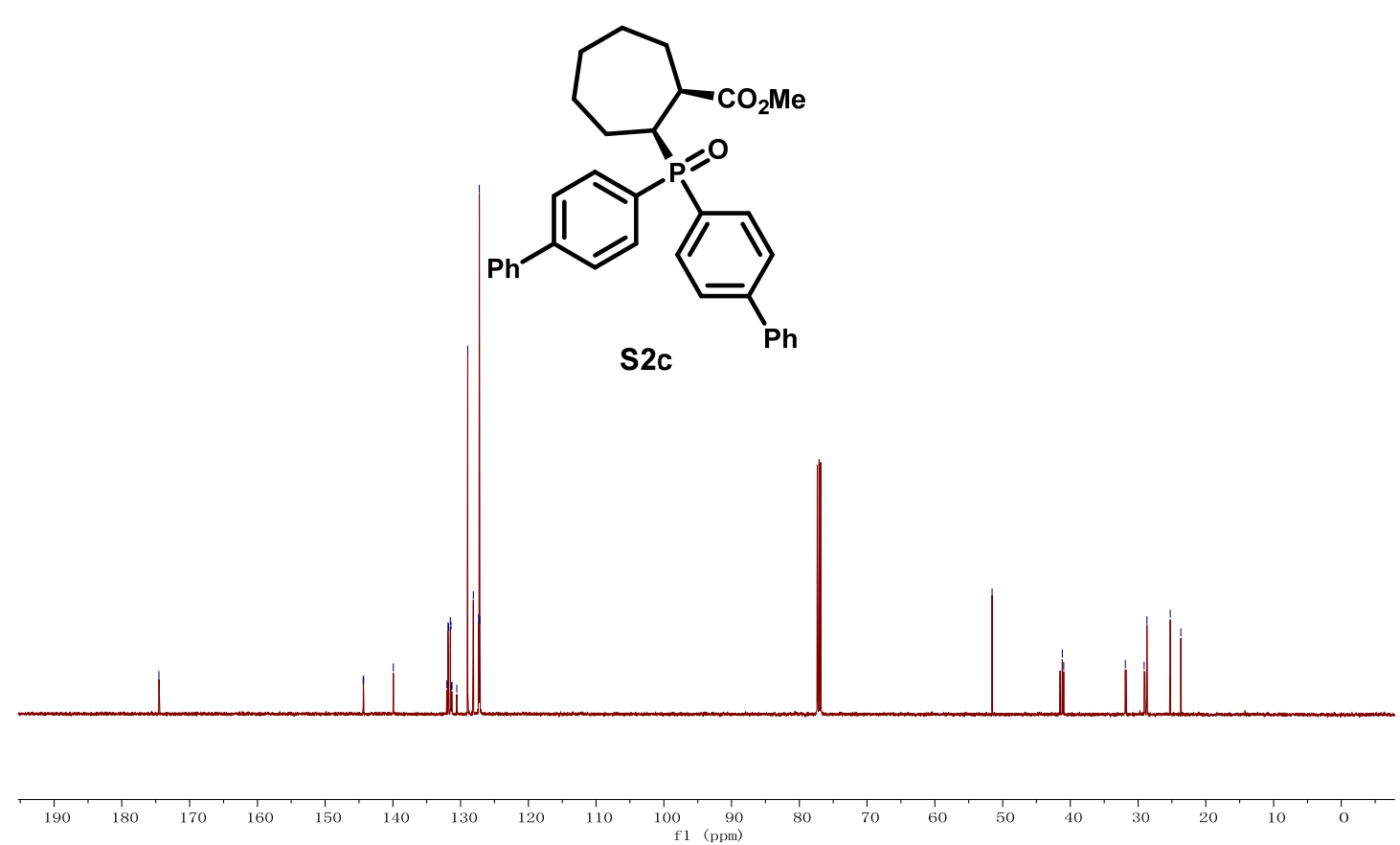

31P NMR (202 MHz, $\left.\mathrm{CDCl}_{3}\right)$

LHH-190711-CMY1122-4-Ph-pure. 2. fid

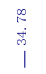

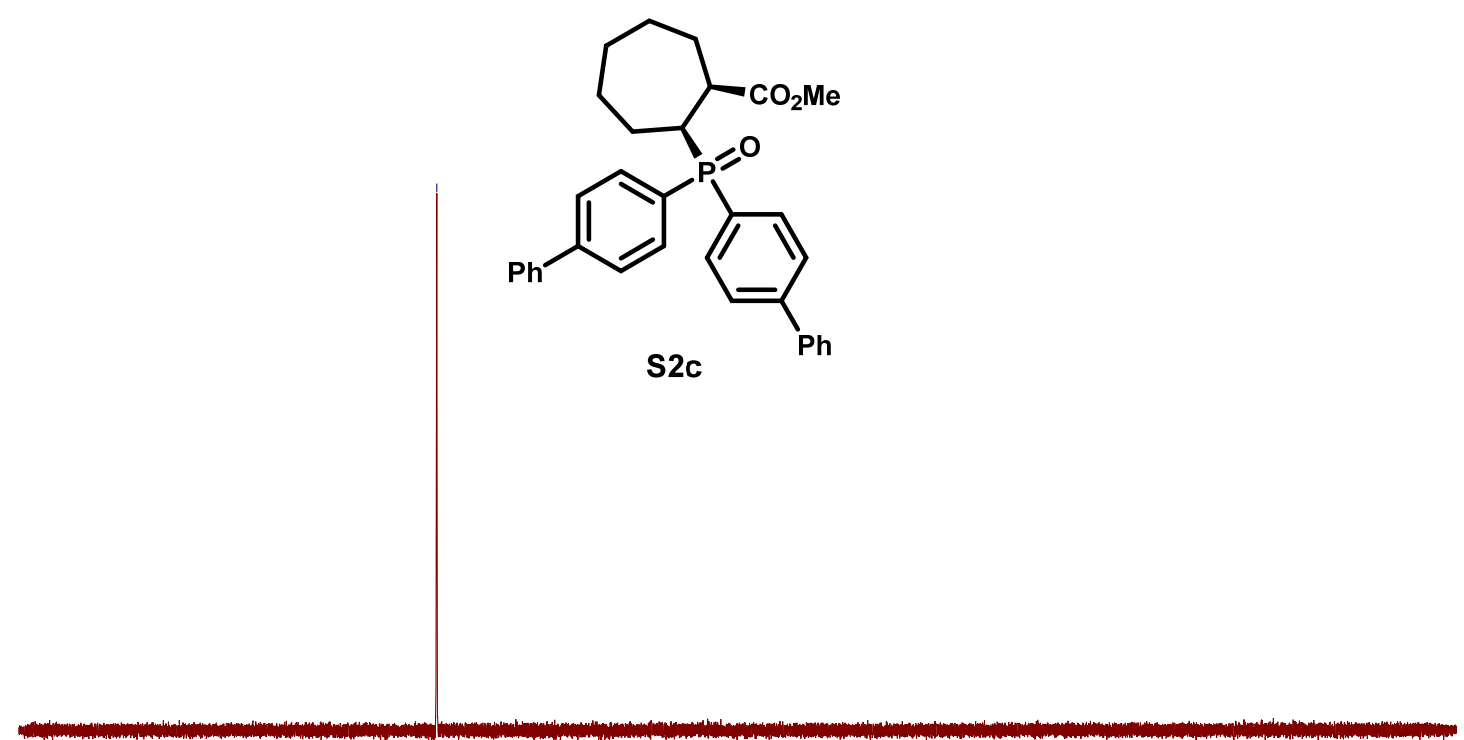

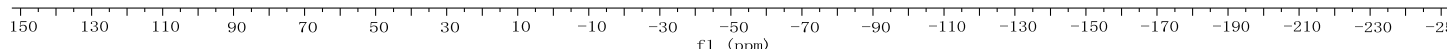

Methyl (1S,2S)-2-(bis(4-fluorophenyl)phosphoryl)cycloheptane-1-carboxylate (S2d) 
$\underline{{ }^{1} \mathrm{H} \text { NMR }}\left(400 \mathrm{MHz}, \mathrm{CDCl}_{3} @ 7.26\right.$ ppm)

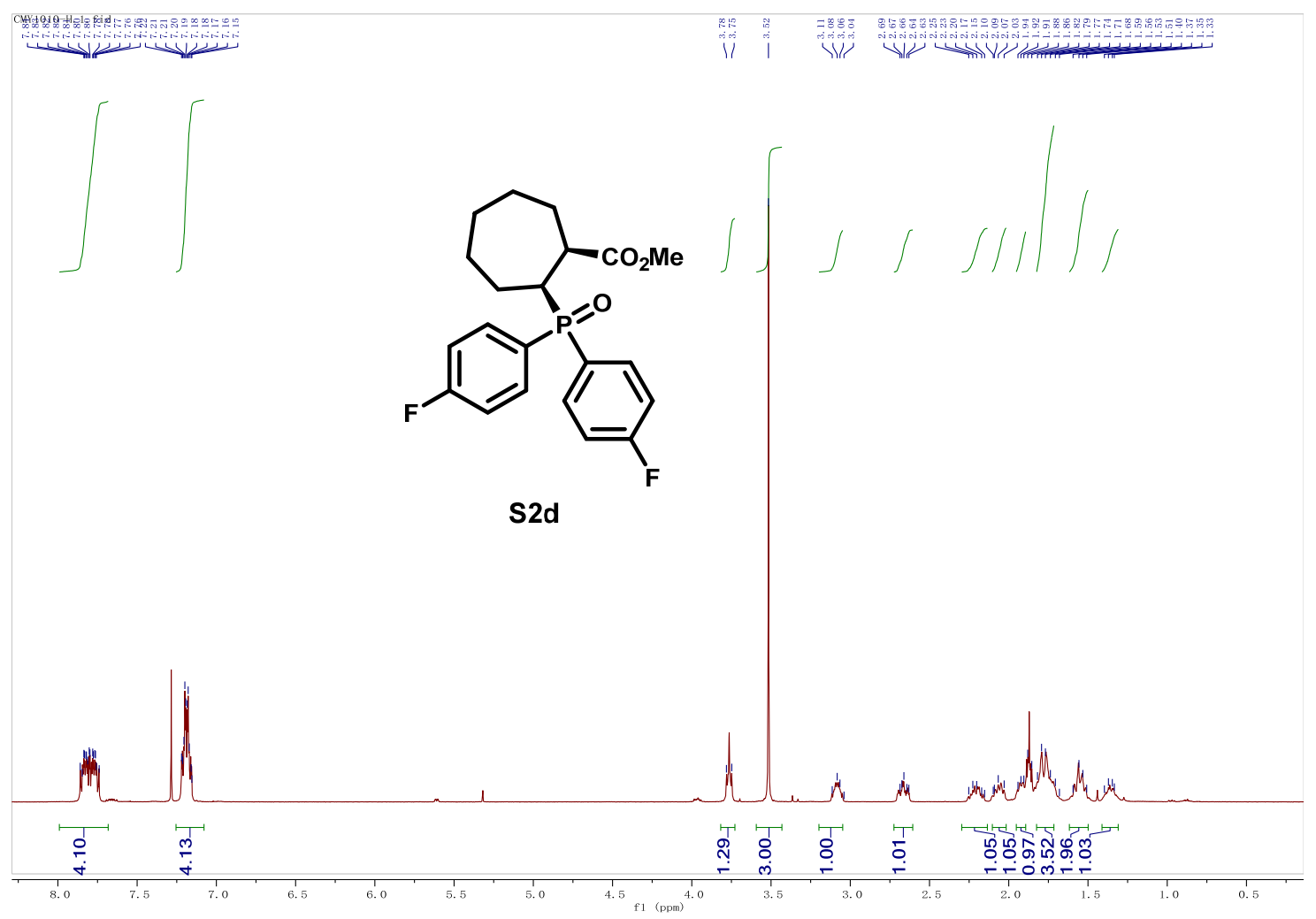

13. NMR (100 MHz, CDCl $@ 77$ ppm)

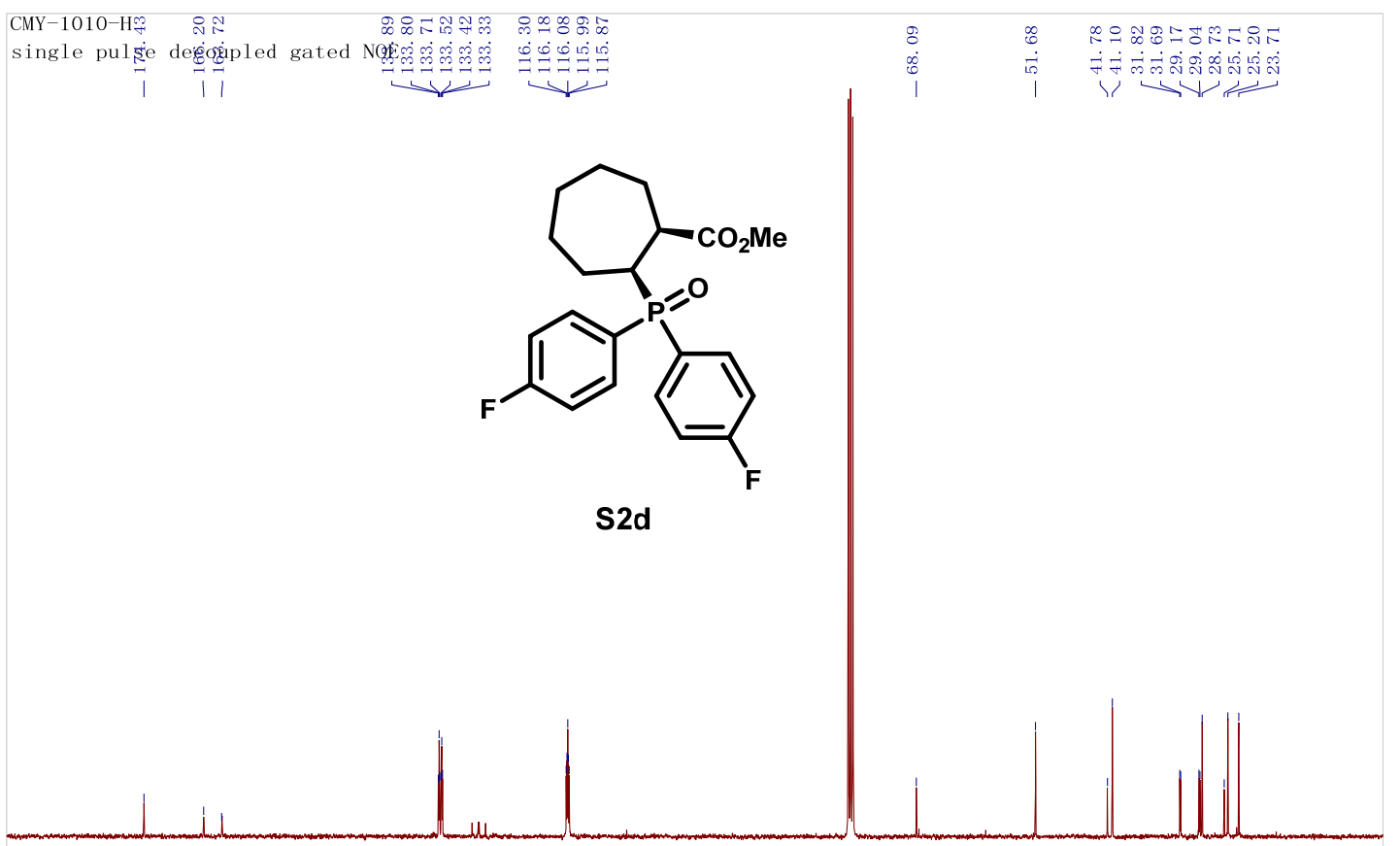

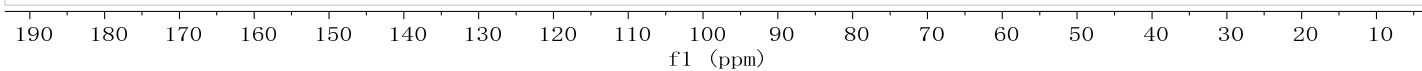




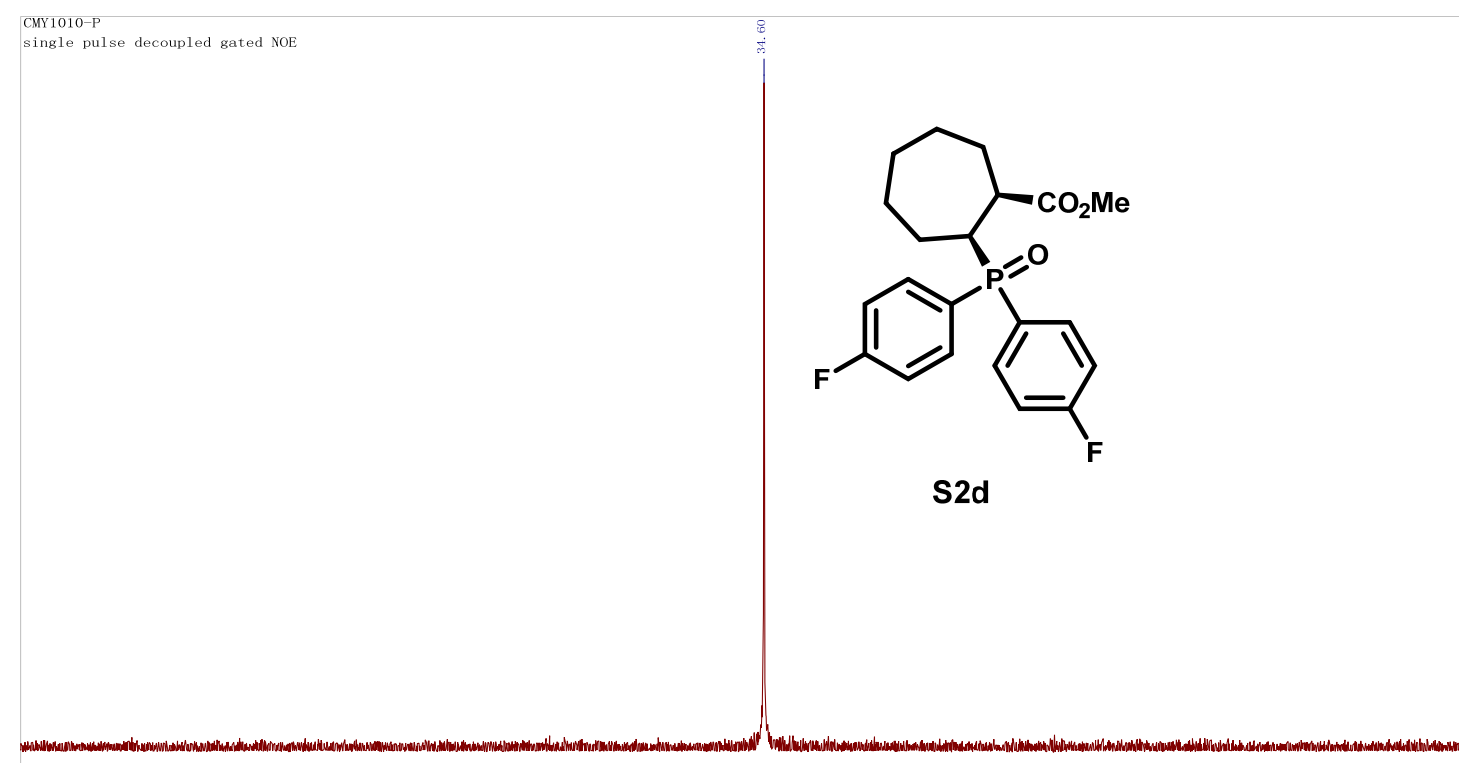

${ }^{19}$ F NMR (376 MHz, CDCl 3 )

CMY1010-F. 7. fid

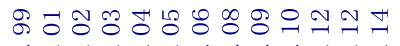

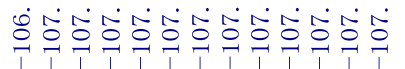

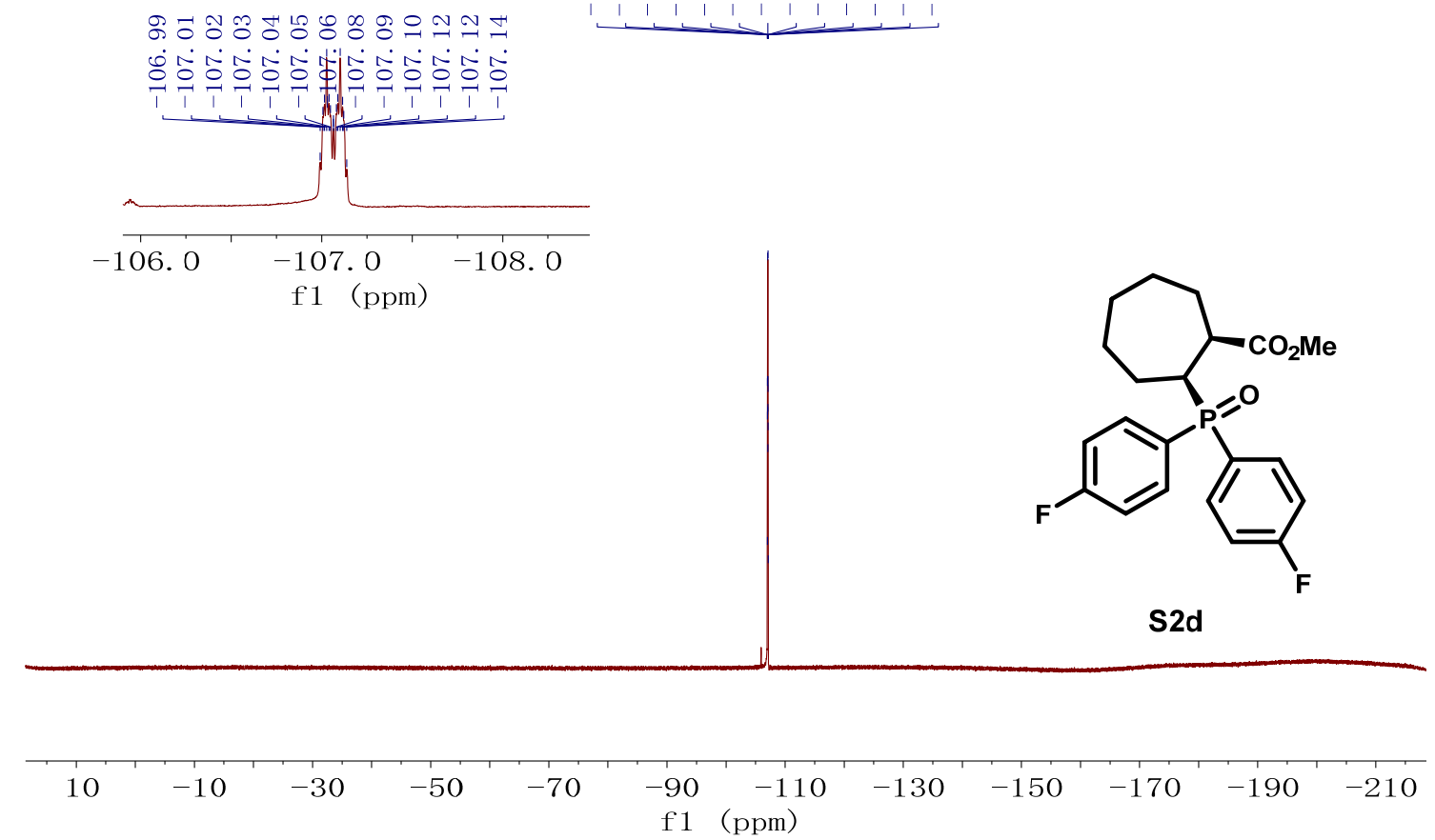

Methyl (1S,2S)-2-(bis(3,5-dimethylphenyl)phosphoryl)cycloheptane-1-carboxylate (S2e) 
$\underline{{ }^{1} \mathrm{H} \text { NMR }}\left(500 \mathrm{MHz}, \mathrm{CDCl}_{3} @ 7.26\right.$ ppm)

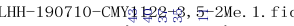

PROTON CDC13 \{ \{ $\underbrace{}_{\text {N Nrimidata }}$ root 3

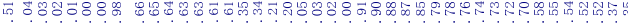

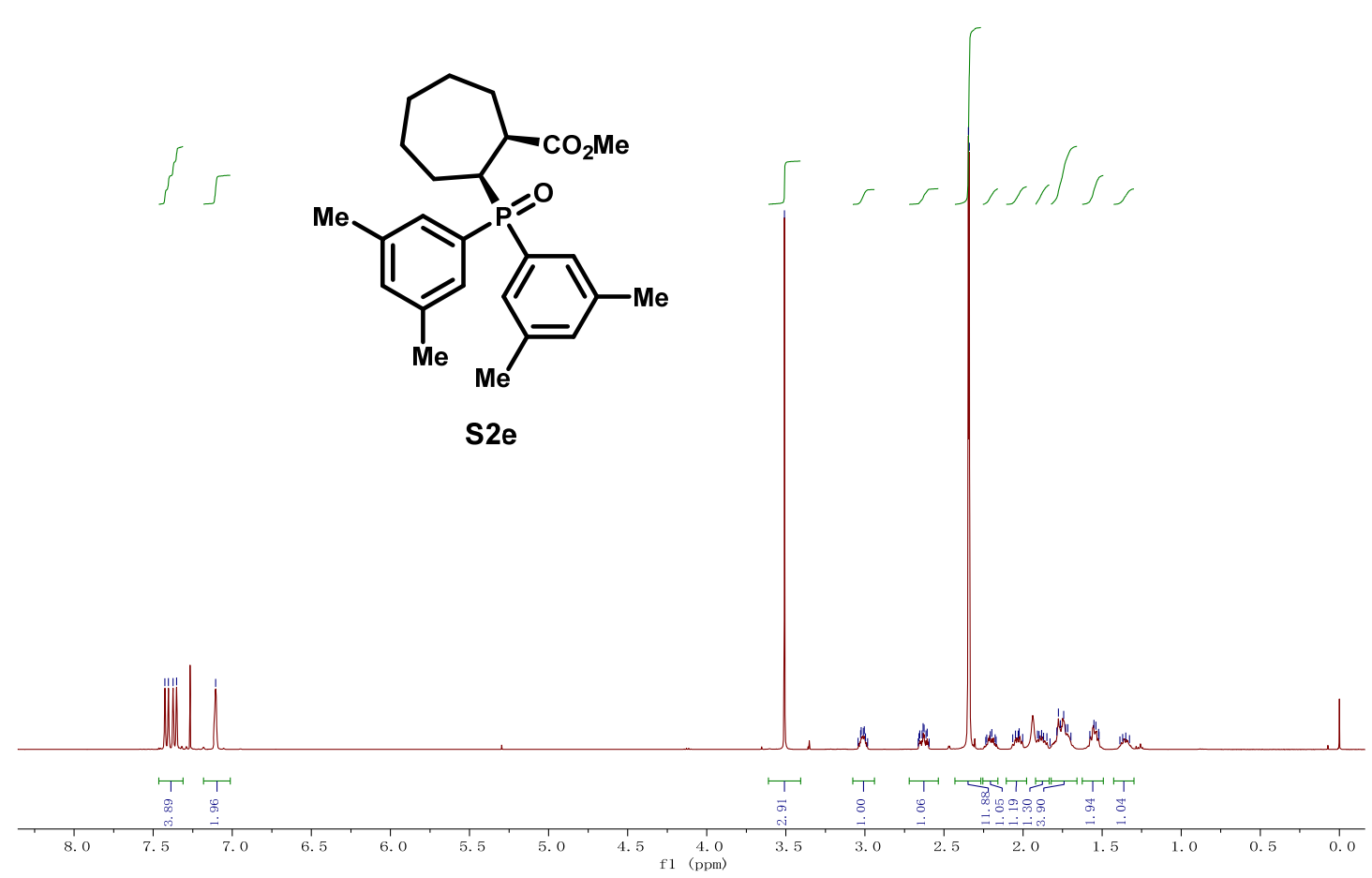

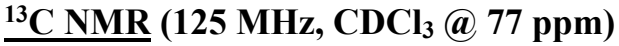

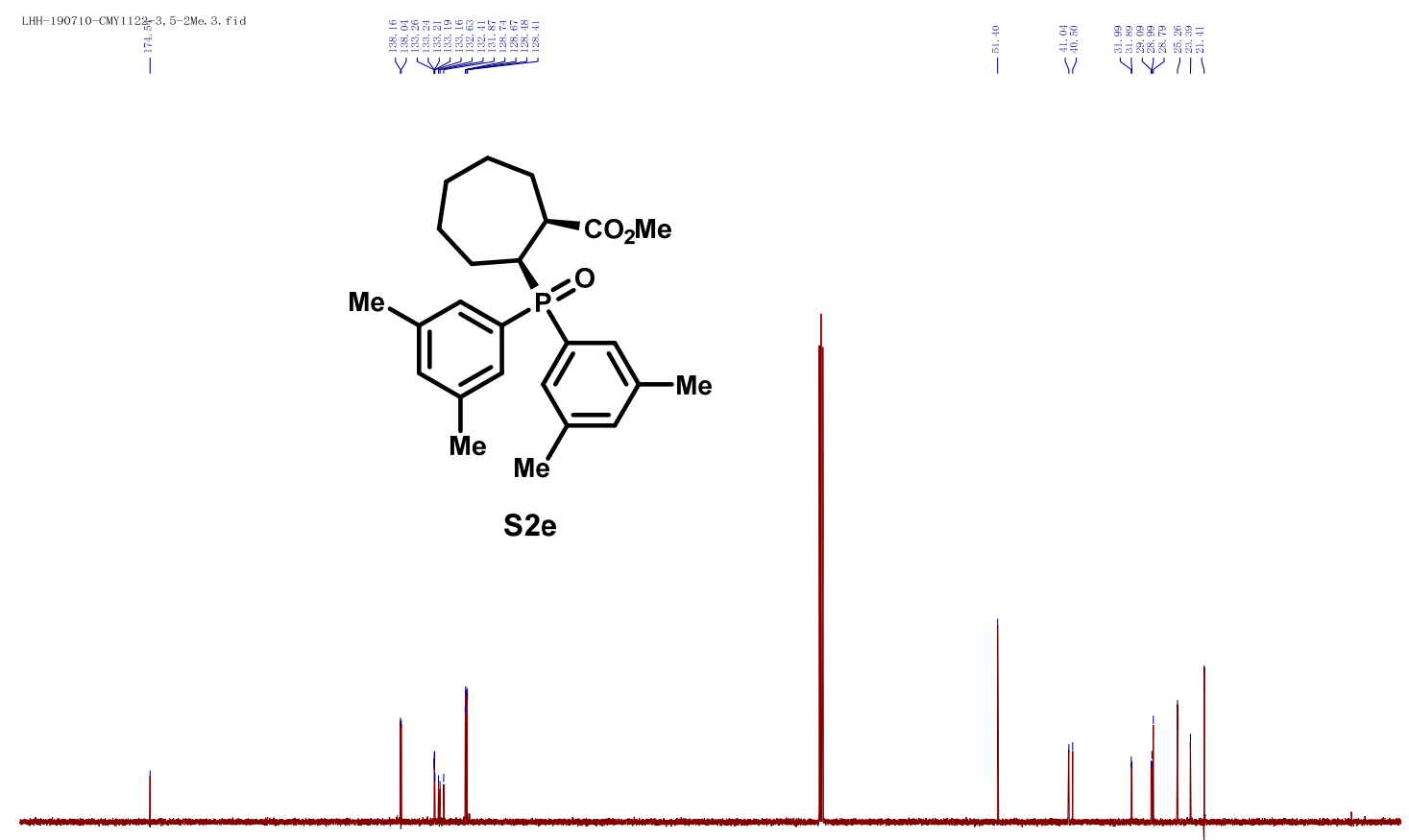


LHH-190710-CMY1122-3, 5-2Me. 2. fid 31 CDC13 \{D: \nmrdata\} root 3

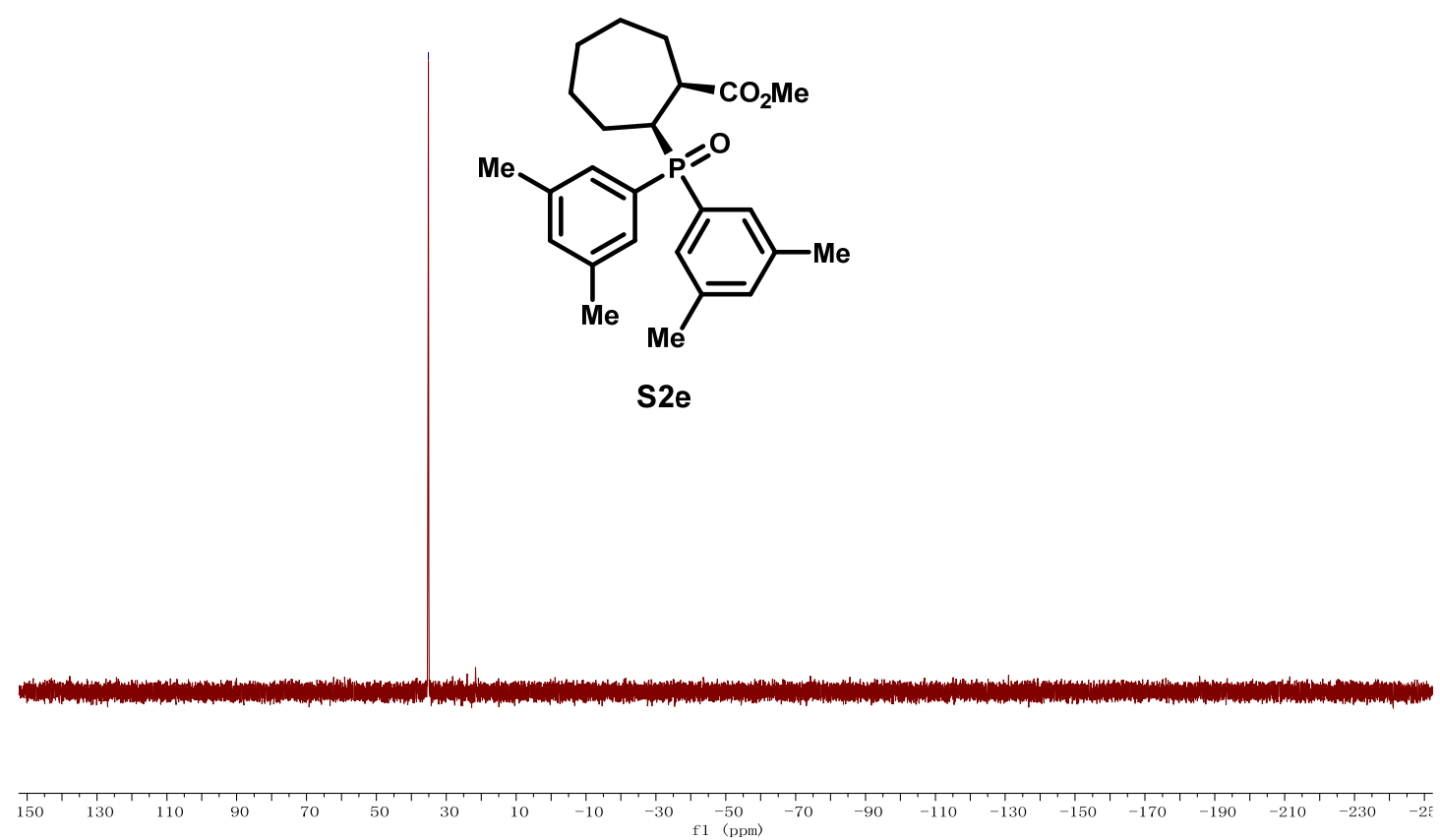

Methyl (1S,2S)-2-(di(naphthalen-2-yl)phosphoryl)cycloheptane-1-carboxylate (S2f)

$\underline{{ }^{1} \mathrm{H} \text { NMR }}\left(500 \mathrm{MHz}, \mathrm{CDCl}_{3} @ 7.26\right.$ ppm) 


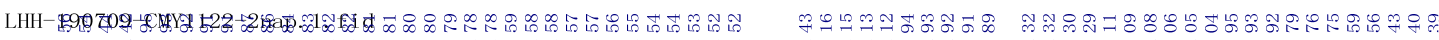

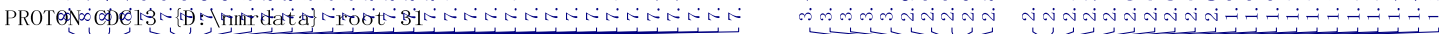

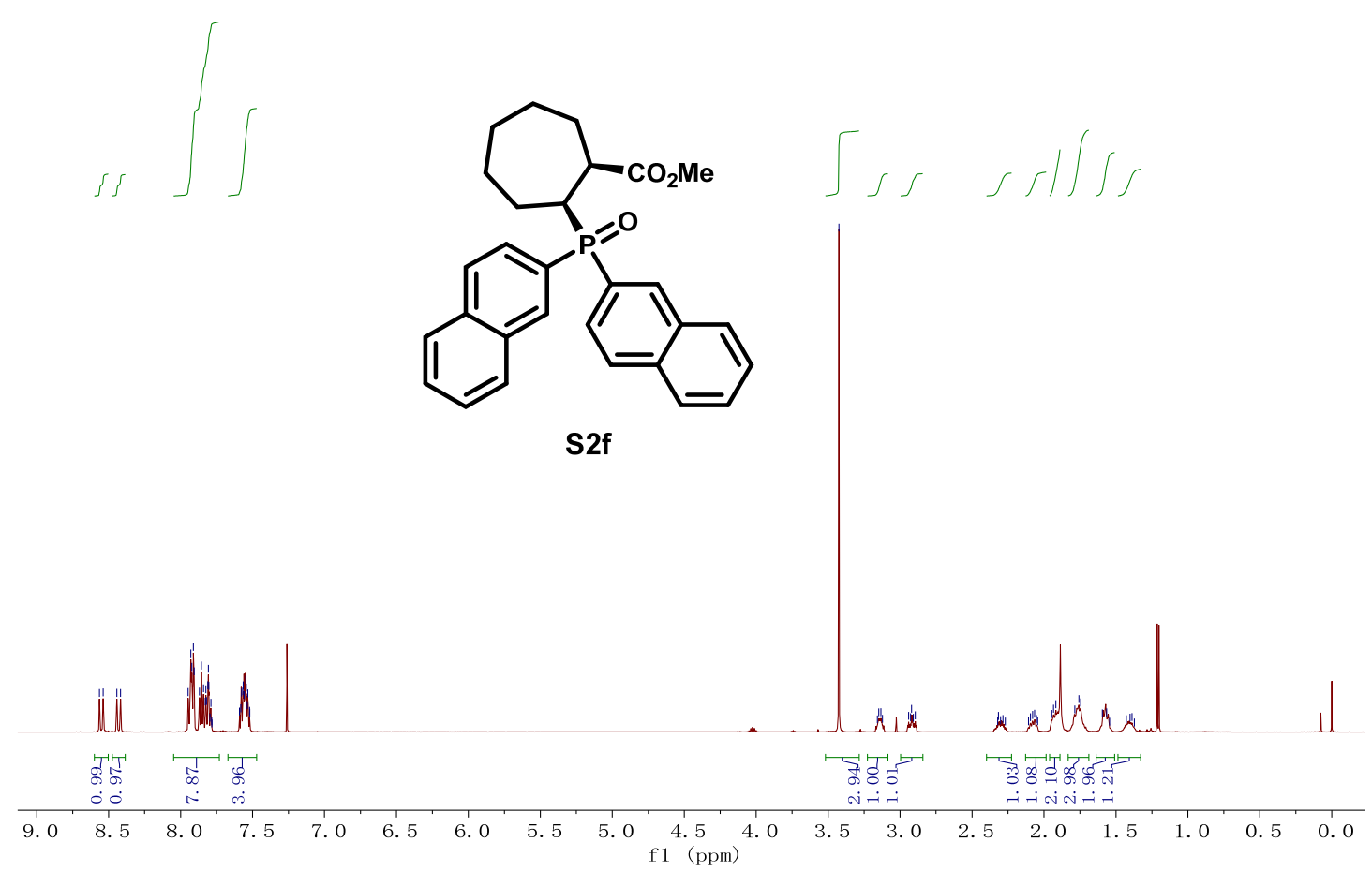

$\underline{{ }^{13} \mathrm{C} \mathrm{NMR}}\left(125 \mathrm{MHz}, \mathrm{CDCl}_{3} @ 77\right.$ ppm)

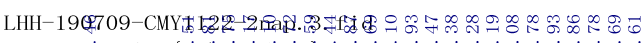

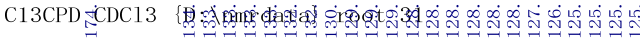

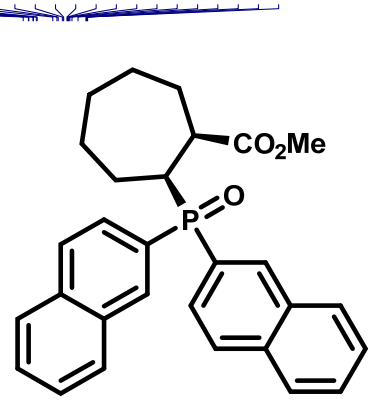

S2f

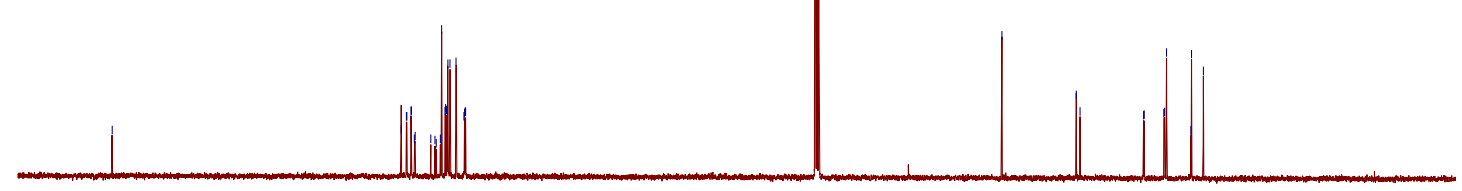

$\begin{array}{lllllllllll}180 & 170 & 160 & 150 & 140 & 130 & 120 & 110 & 100 & 90 & 80\end{array}$

31P NMR (202 MHz, $\left.\mathrm{CDCl}_{3}\right)$ 


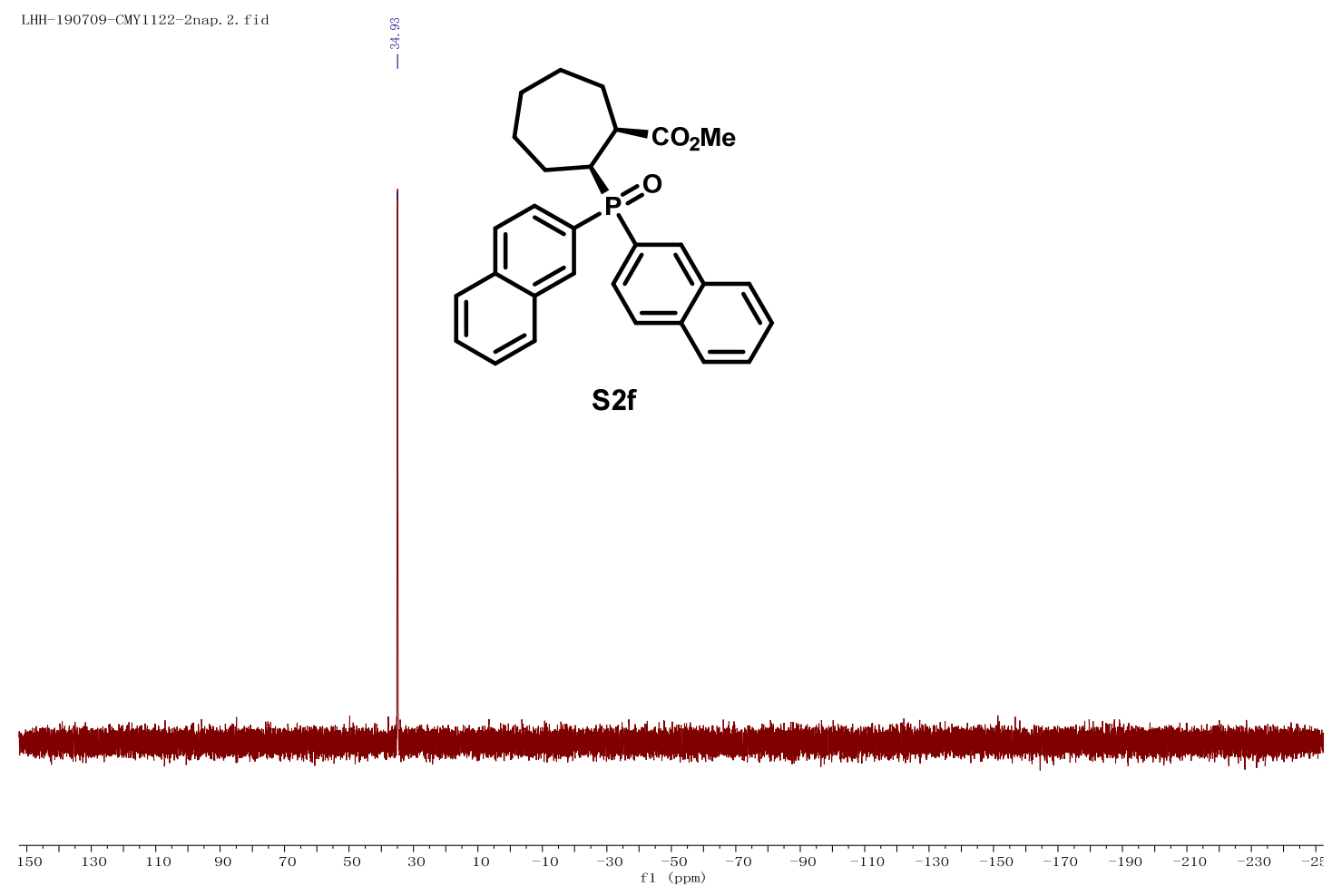

Methyl (1S,2S)-2-(diphenylphosphanyl)cycloheptane-1-carboxylate-borane complex (S3a)

$\underline{{ }^{1} \mathrm{H} \text { NMR }}\left(400 \mathrm{MHz}, \mathrm{CDCl}_{3} @ 7.26\right.$ ppm)

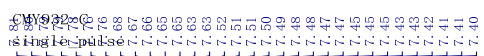

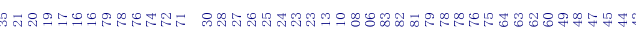

$\iint$<smiles>CC(=O)C1CCCCCC1[PH](C)(c1ccccc1)c1ccccc1</smiles>

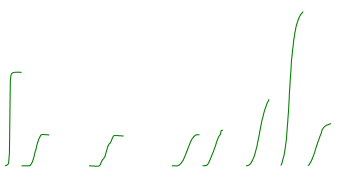

S3a

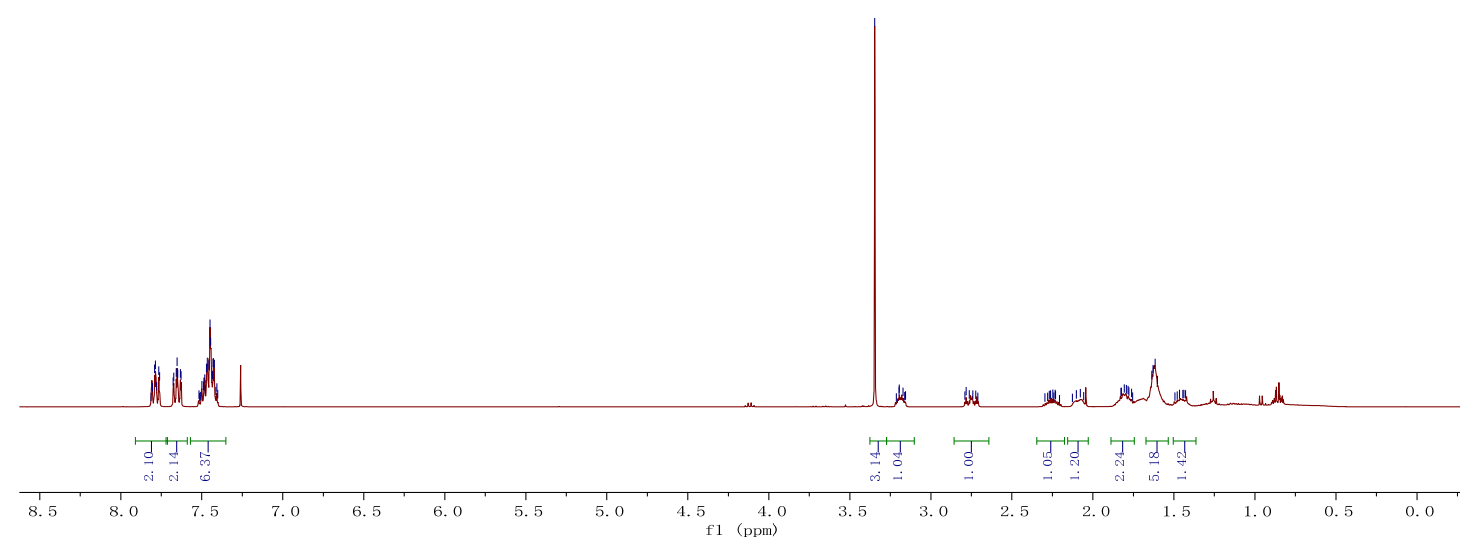


${ }^{13} \mathrm{C}$ NMR $\left(100 \mathrm{MHz}, \mathrm{CDCl}_{3} @ 77\right.$ ppm)
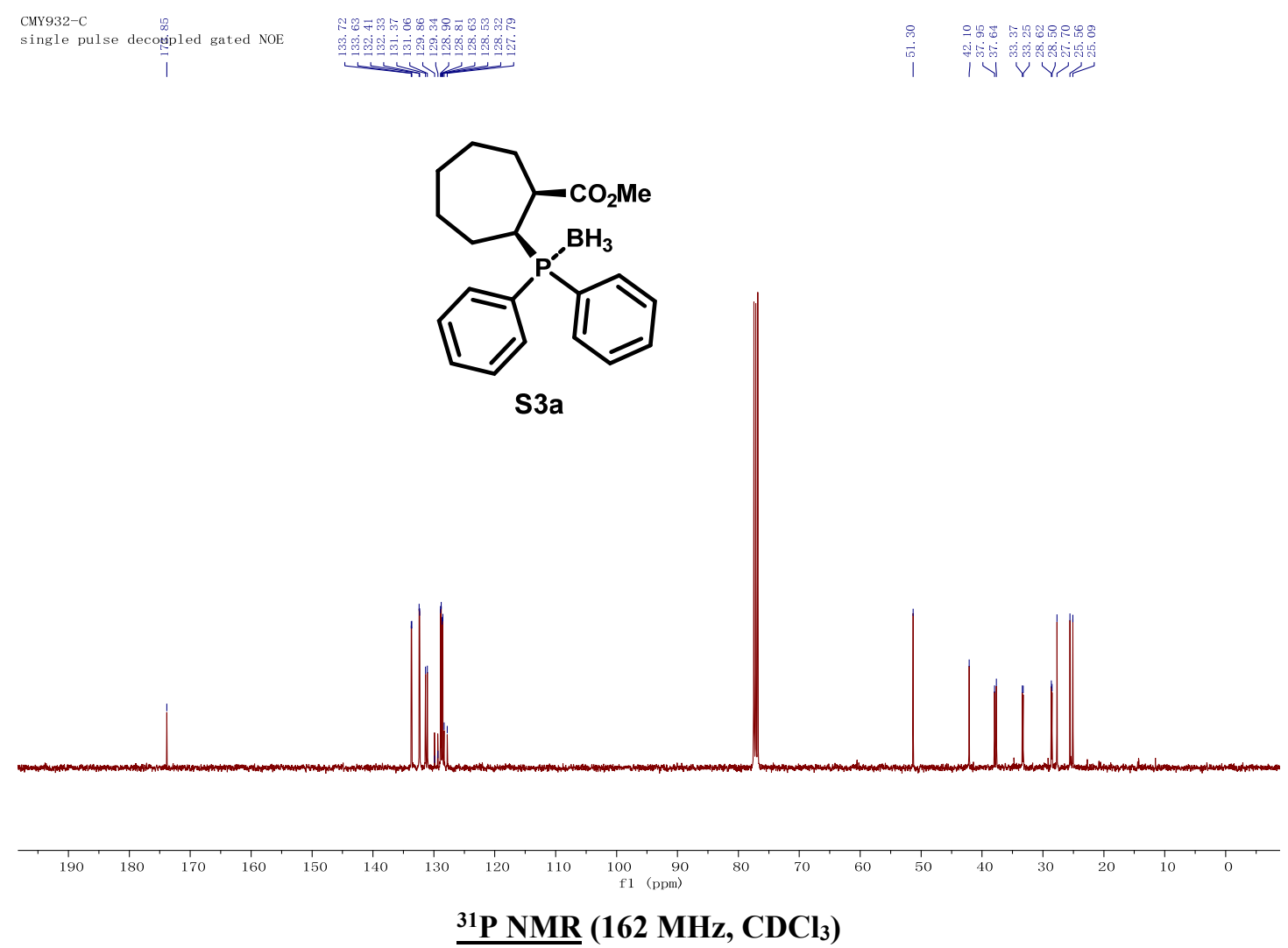

CMY932-C

single_pulse

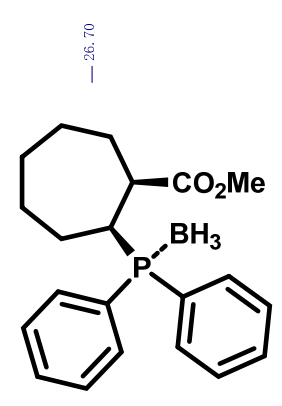

s3a

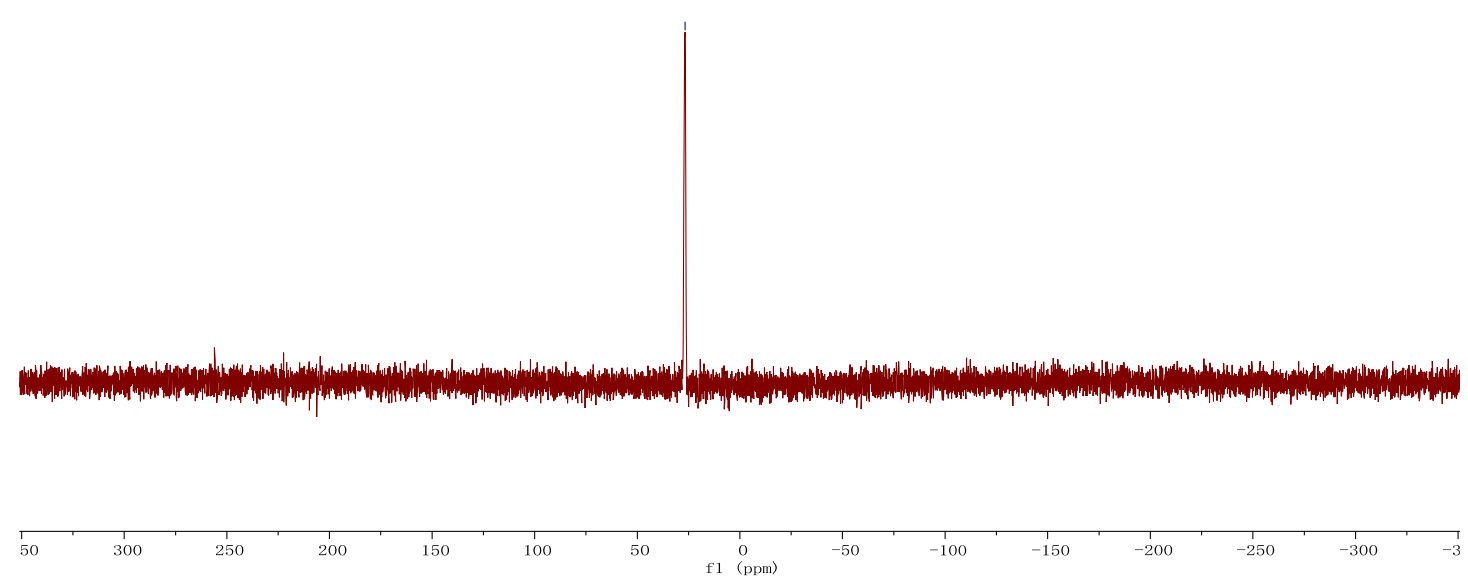

Methyl (1S,2S)-2-(di-p-tolylphosphanyl)cycloheptane-1-carboxylate-borane complex (S3b) 
$\underline{{ }^{1} \mathrm{H} \text { NMR }}\left(500 \mathrm{MHz}, \mathrm{CDCl}_{3} @ 7.26 \mathrm{ppm}\right)$

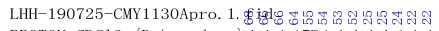

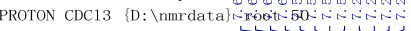

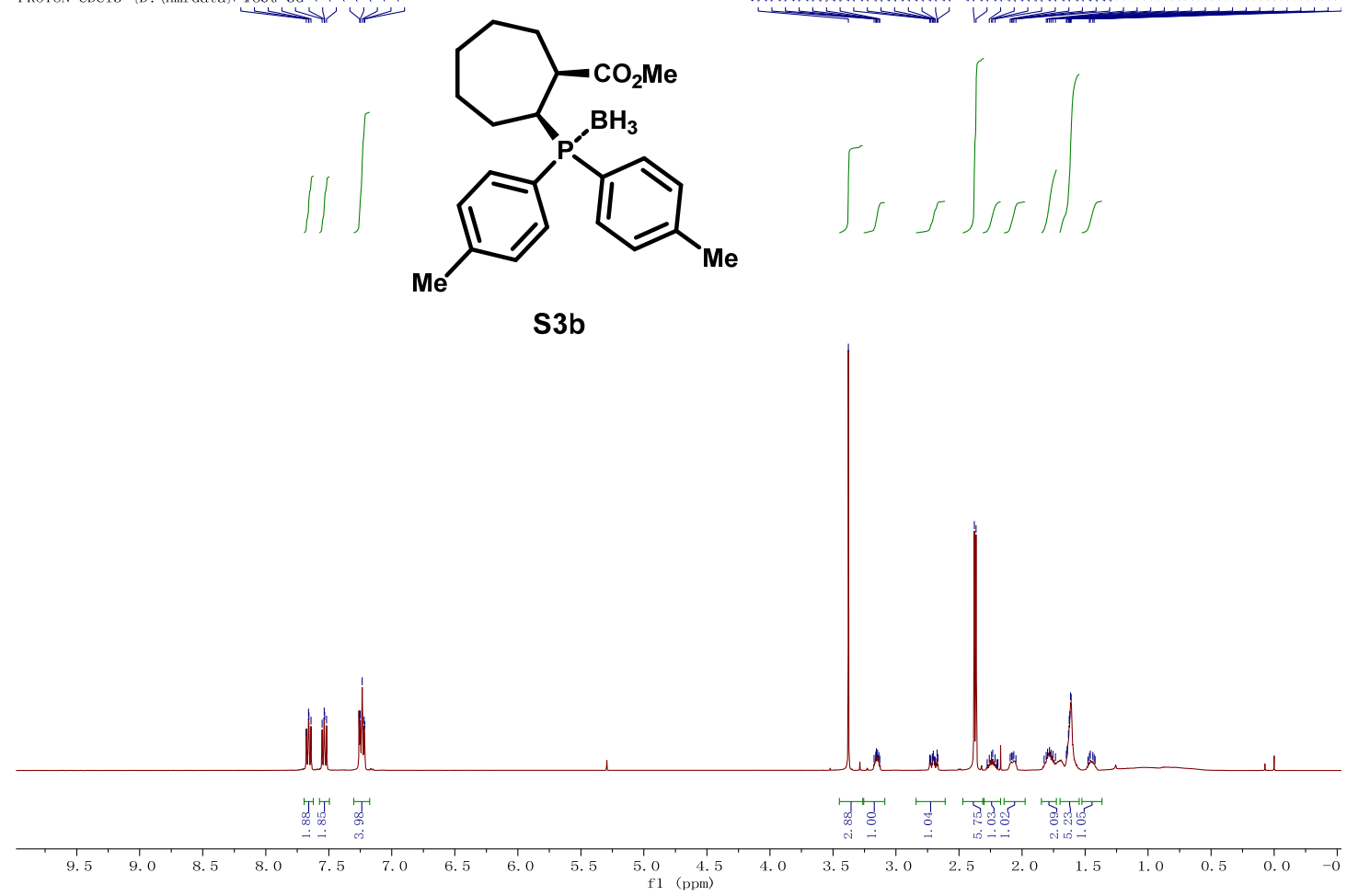

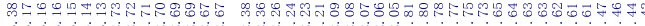

${ }^{13} \mathrm{C}$ NMR (125 MHz, $\mathrm{CDCl}_{3} @ 77$ ppm)

LHH-190725-CMY1130Apro. 2. fid以 C13CPD CDC13 $\{\mathrm{D}: \backslash$ nmrdata\} root

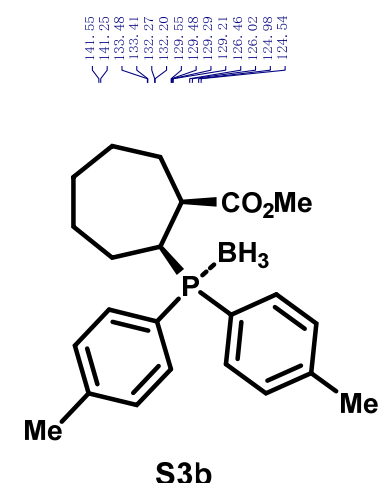

药

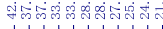

iv VII I

33

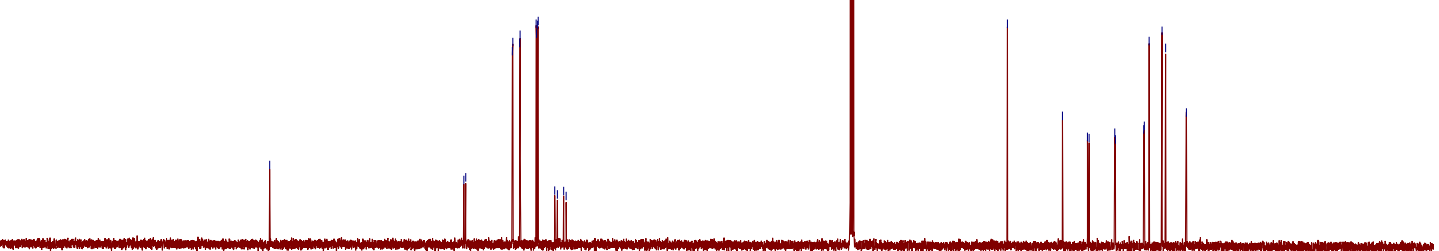

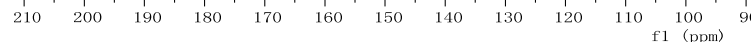


LHH-190724CMY1130Apro. 1. fid
P31 CDC13 $\{$ D: \nmrdata\} root 41
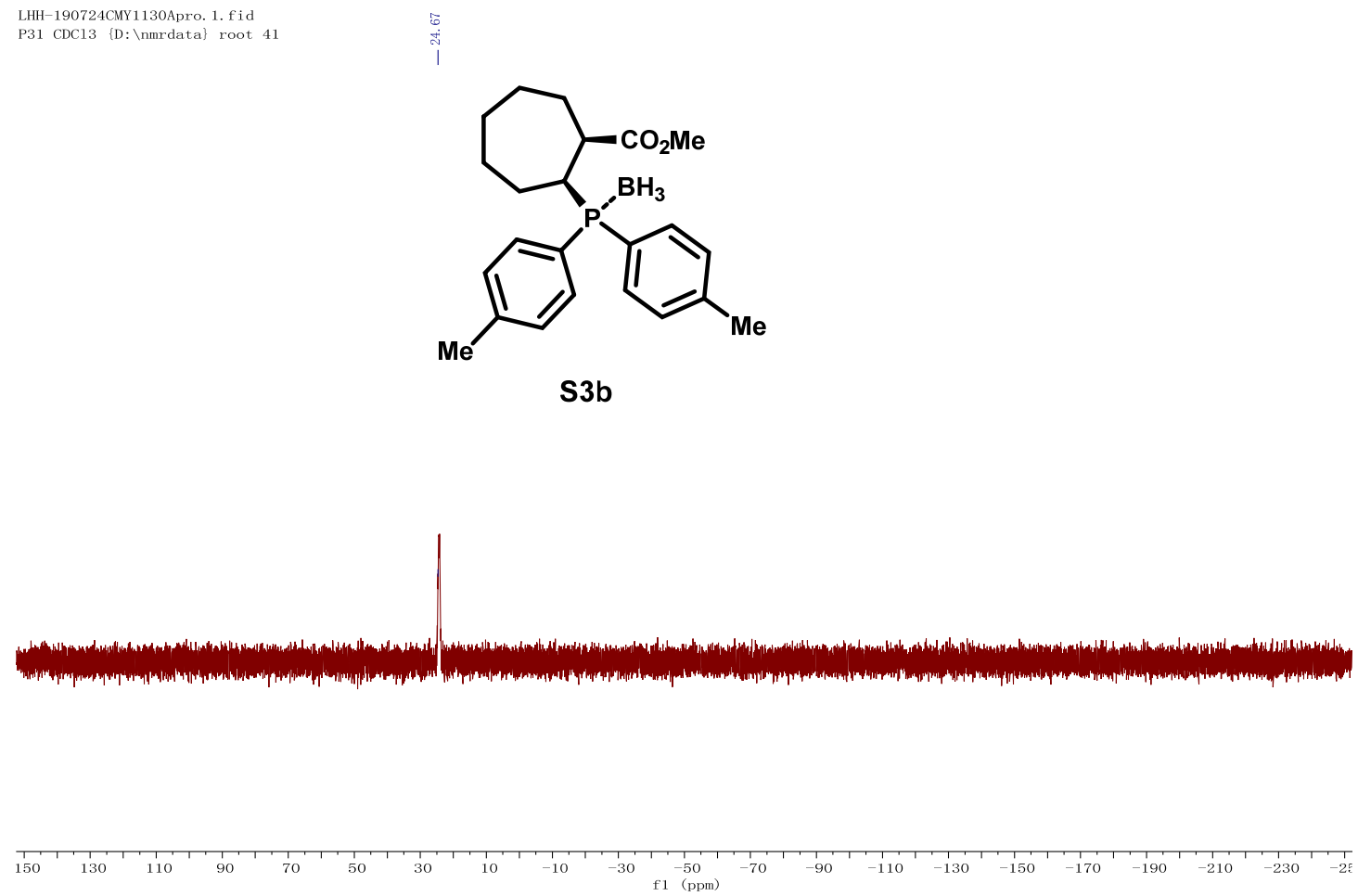

Methyl (1S,2S)-2-(di([1,1'-biphenyl]-4-yl)phosphanyl)cycloheptane-1-carboxylate-borane complex (S3c)

$\underline{{ }^{1} \mathrm{H} \text { NMR }}\left(500 \mathrm{MHz}, \mathrm{CDCl}_{3} @ 7.26\right.$ ppm)

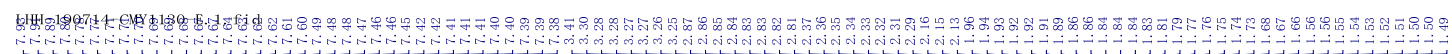

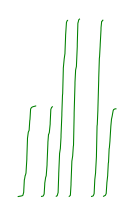<smiles>CC(=O)C1CCCCCC1[PH](c1ccccc1)(c1ccccc1)c1ccc(-c2ccccc2)cc1</smiles>

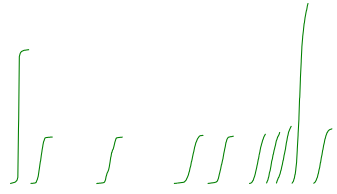

S3c

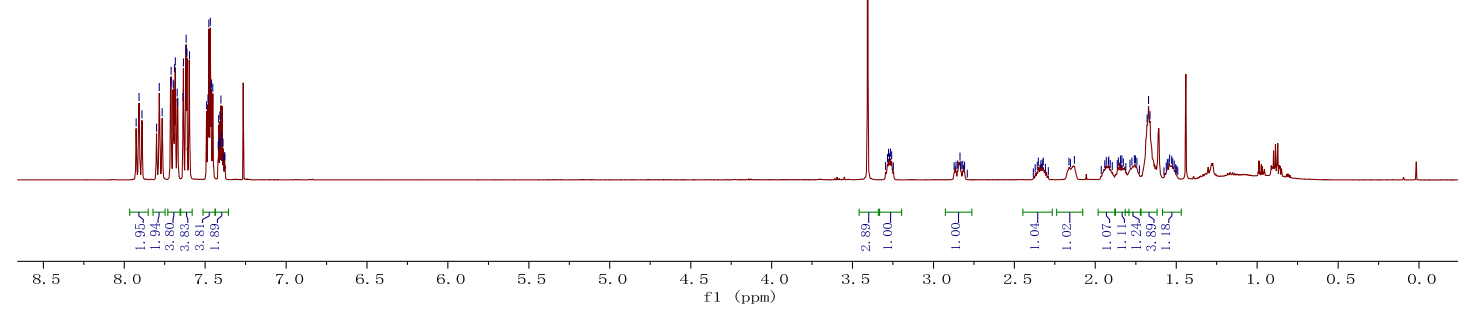




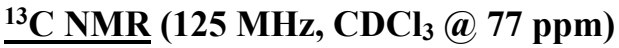
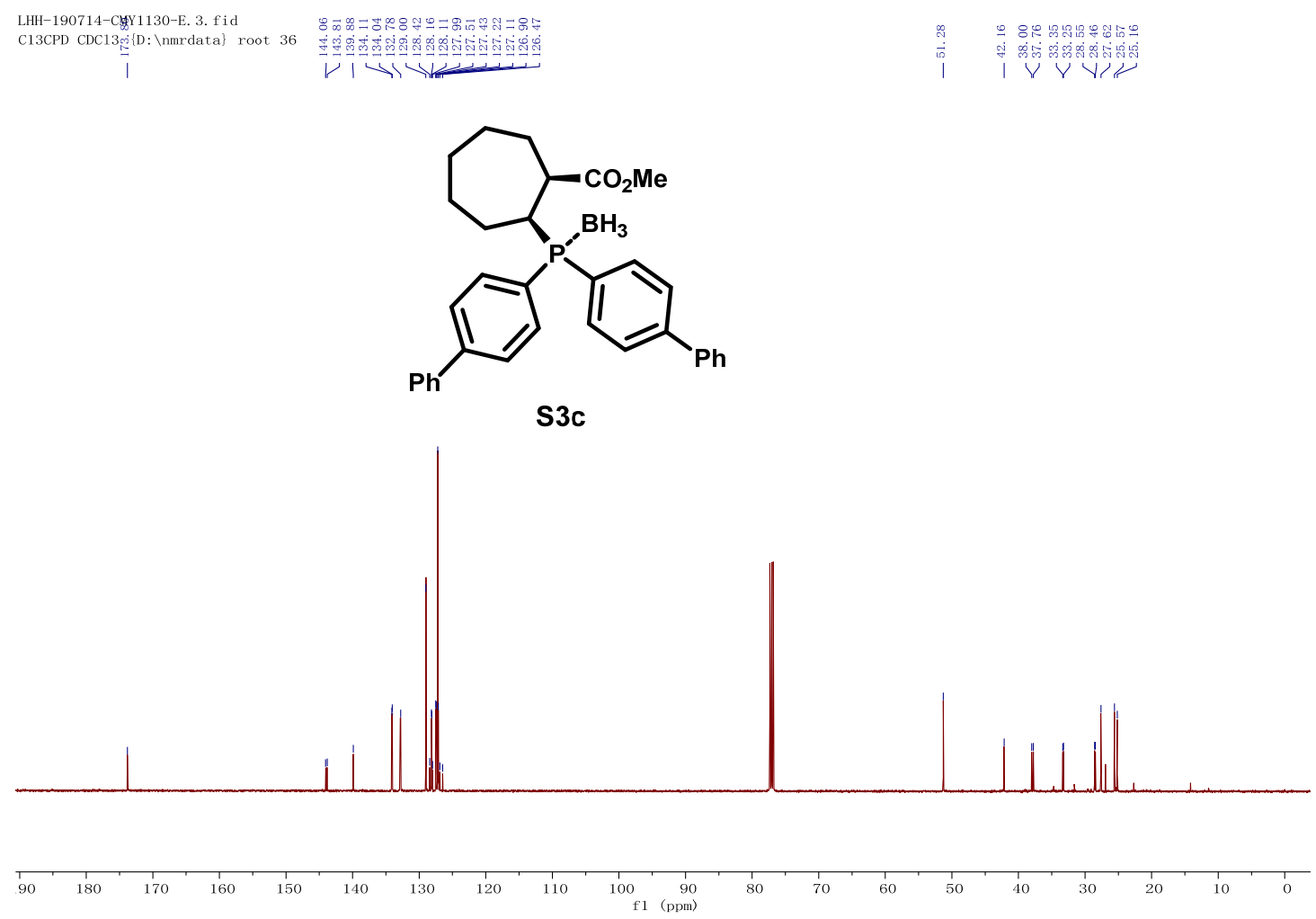

31P NMR (202 MHz, $\left.\mathrm{CDCl}_{3}\right)$

LHH-190714-CMY1130-E. 2. fid P31 CDC13 \{D: \nmrdata\} root 36

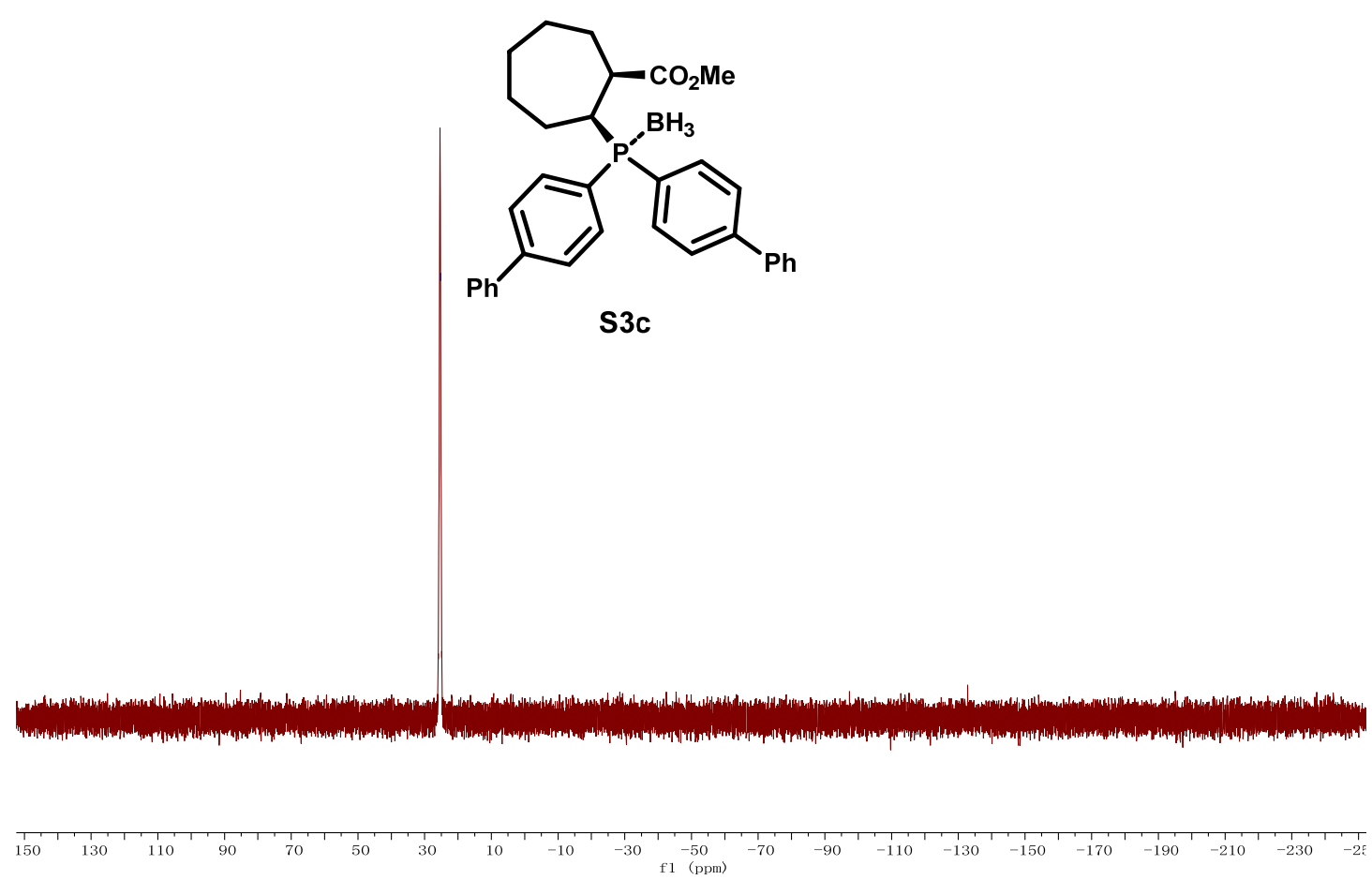

Methyl (1S,2S)-2-(bis(4-fluorophenyl)phosphanyl)cycloheptane-1-carboxylate-borane complex (S3d) S100 


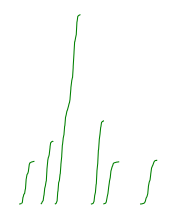<smiles>CC(=O)C1CCCCC1[P+](c1ccccc1)(c1ccc(F)cc1)c1ccc(F)cc1</smiles>

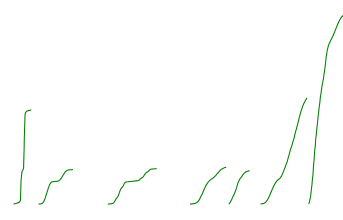

S3d
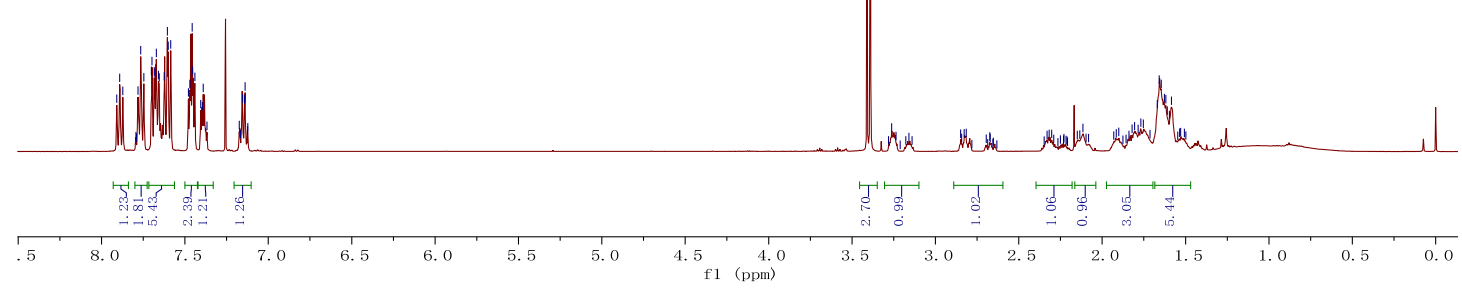

${ }^{13} \mathrm{C}$ NMR (125 MHz, CDCl 3 @ 77 ppm)

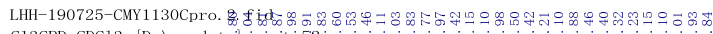

13 CPD CDC13 \{D: \nmrdateil fiopiti

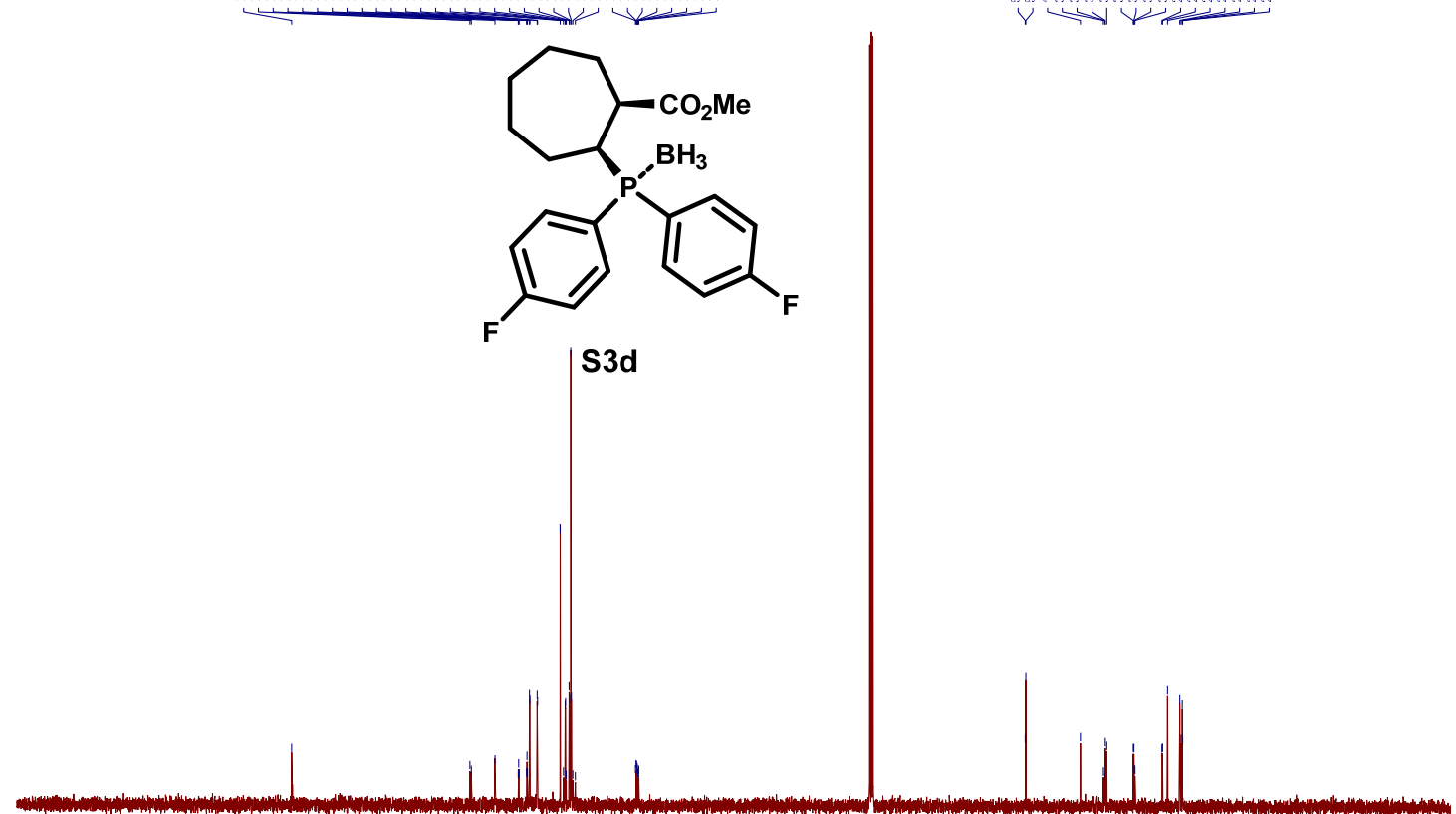

31P NMR (202 MHz, $\left.\mathrm{CDCl}_{3}\right)$

S101 


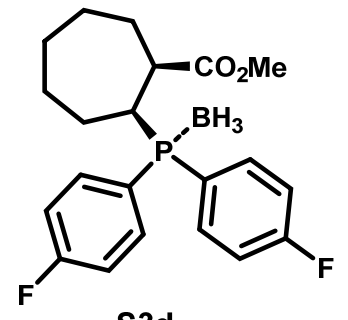

S3d

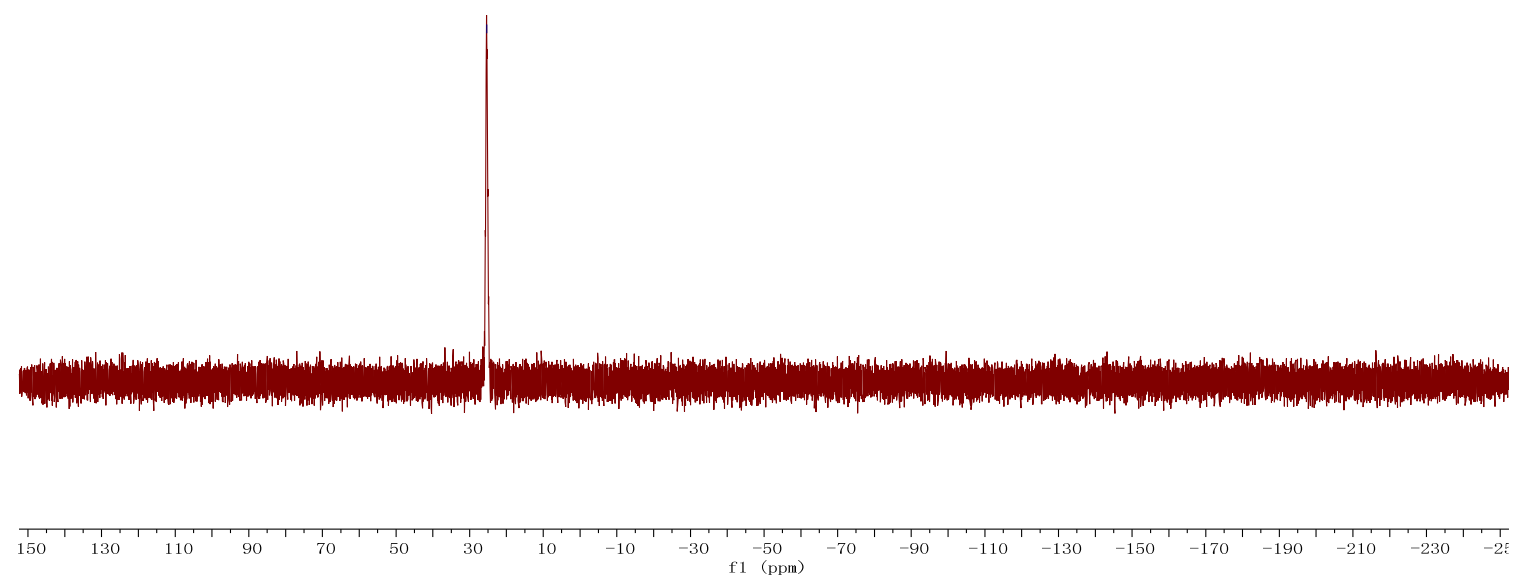

$\underline{{ }^{19} \mathrm{~F} \text { NMR }}\left(470 \mathrm{MHz}, \mathrm{CDCl}_{3}\right)$

LHH-191212-CMY1130-C. 2. fid F19 CDC13 $\{\mathrm{D}: \backslash$ nmrdata $\}$ root 8<smiles>CC(=O)C1CCCCCC1P(O)(c1ccccc1)(c1ccc(F)cc1)c1ccc(F)cc1</smiles>

S3d

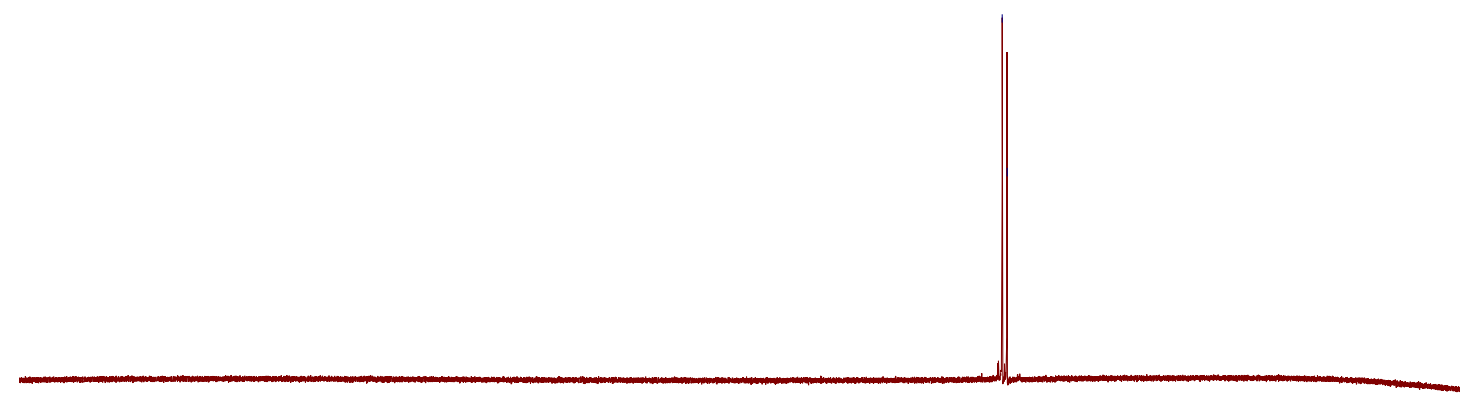

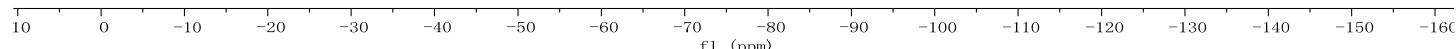


Methyl (1S,2S)-2-(bis(3,5-dimethylphenyl)phosphanyl)cycloheptane-1-carboxylate-borane complex (S3e)

느 NMR $\left(500 \mathrm{MHz}, \mathrm{CDCl}_{3} @ 7.26\right.$ ppm)

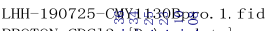

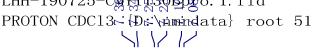

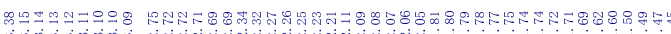

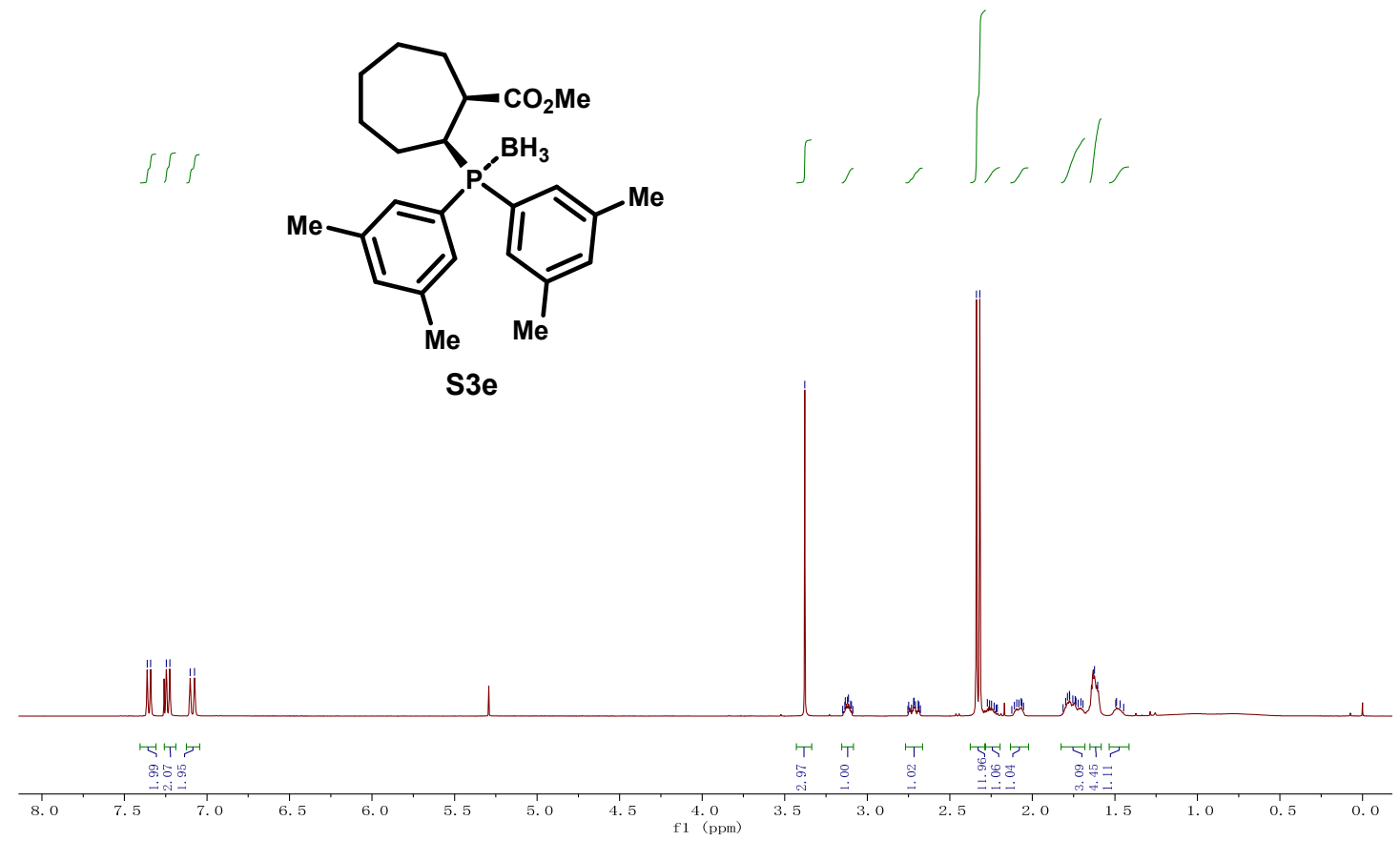

${ }^{13} \mathrm{C}$ NMR $\left(125 \mathrm{MHz}, \mathrm{CDCl}_{3} @ 77\right.$ ppm)

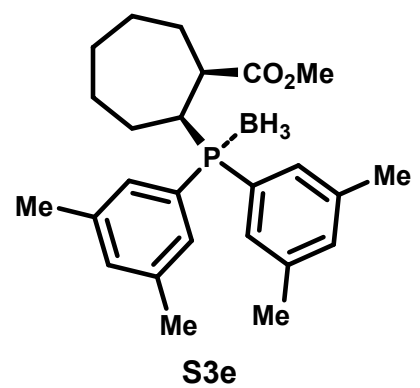

S3e

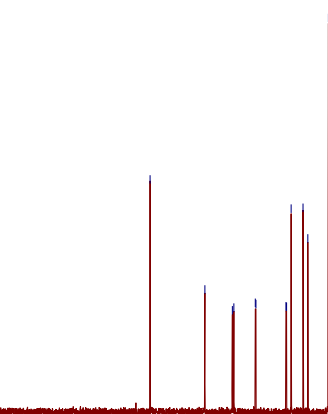




\section{P NMR (202 MHz, $\left.\mathrm{CDCl}_{3}\right)$}

LHH-191212-CMY1130-B. 3. fid P31 CDC13 [D: \nmrdata\} root 7<smiles>CC(=O)C1CCCCCC1CO</smiles><smiles>Cc1cc(C)cc(CPc2cc(C)cc(C)c2)c1</smiles>

S3e

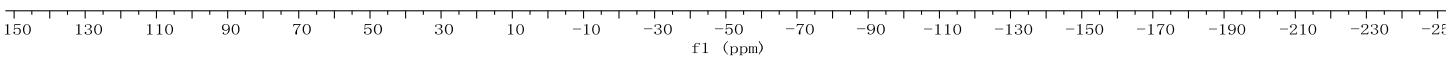

Methyl (1S,2S)-2-(di(naphthalen-2-yl)phosphanyl)cycloheptane-1-carboxylate-borane complex (S3f)

$\underline{{ }^{1} \mathrm{H} \text { NMR }}\left(500 \mathrm{MHz}, \mathrm{CDCl}_{3} @ 7.26\right.$ ppm)

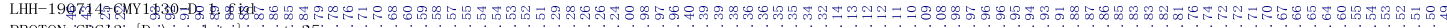

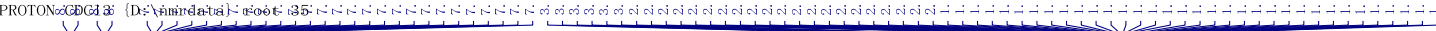

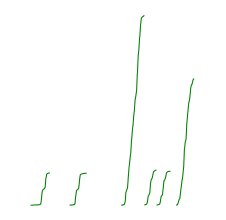<smiles>CC(=O)C1CCCCCC1[PH](C)(c1ccc2ccccc2c1)c1ccc2ccccc2c1</smiles>

S3f
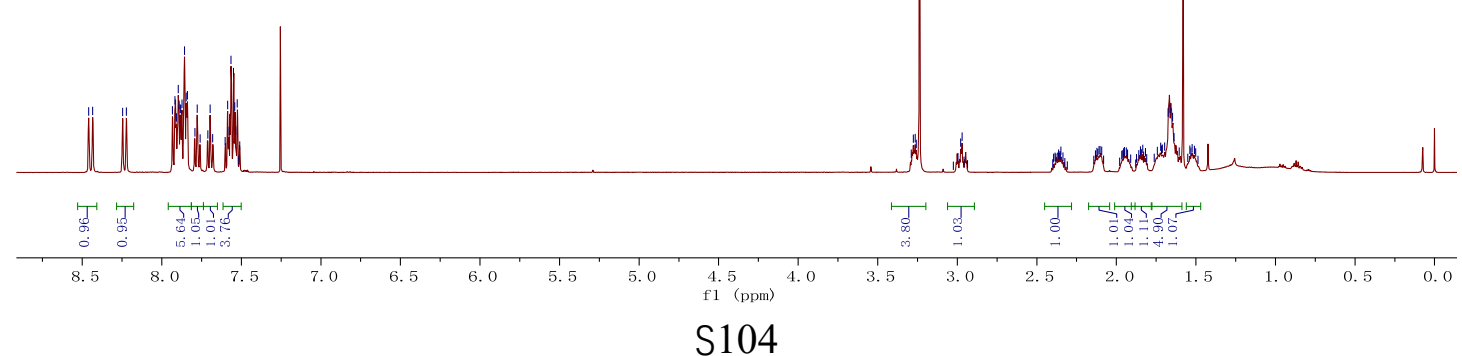


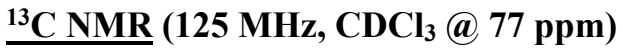

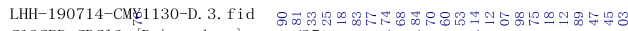

C13CPD CDC13

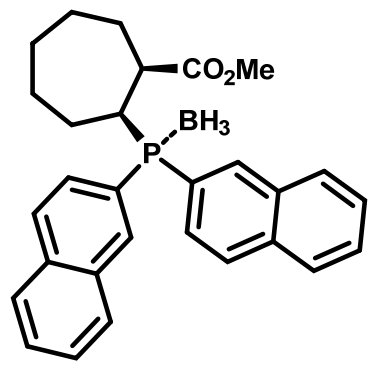

S3f
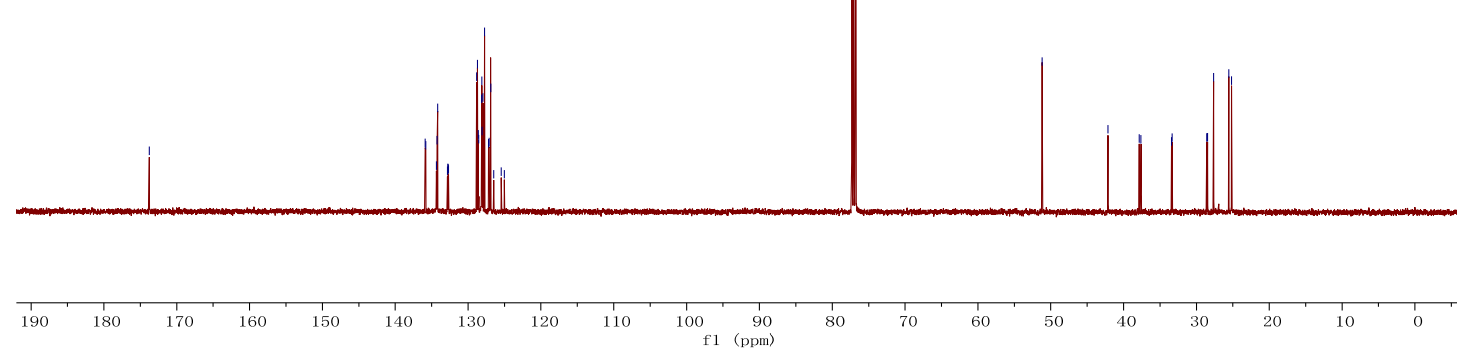

31P NMR (202 MHz, $\left.\mathrm{CDCl}_{3}\right)$

LHH-190714-CMY1130-D. 2. fid

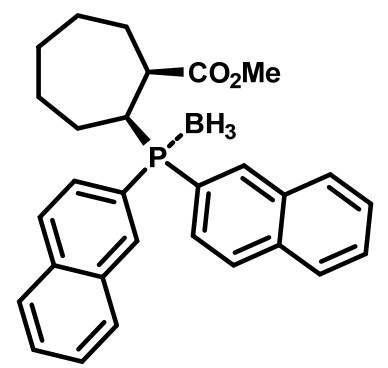

S3f

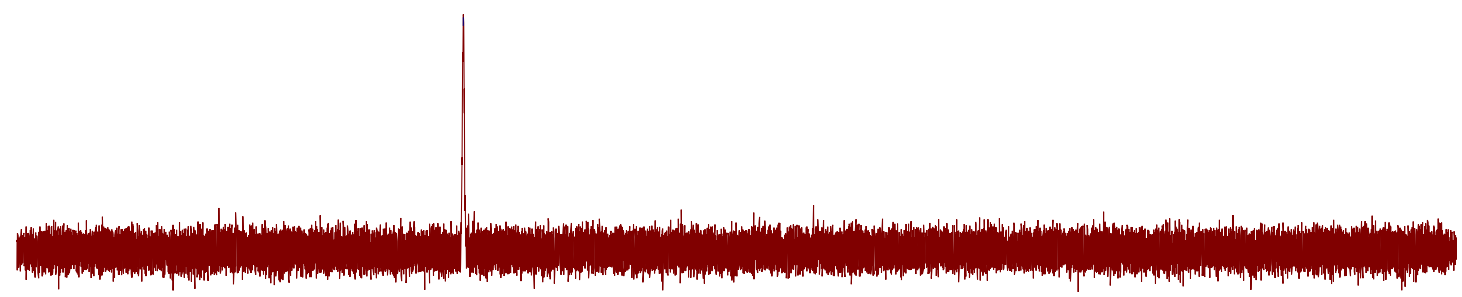

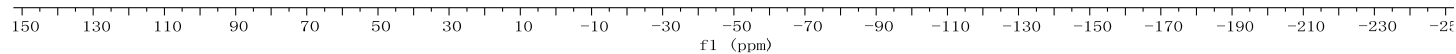


(1S,1'S,2S,2'S)-N,N'-((1S,2S)-Cyclohexane-1,2-diyl)bis(2-(diphenylphosphanyl)cyclohexane-1carboxamide) (L11)

$\underline{{ }^{1} \mathrm{H} \text { NMR }}\left(400 \mathrm{MHz}, \mathrm{CDCl}_{3} @ 7.26\right.$ ppm)

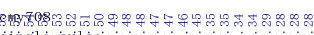

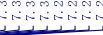

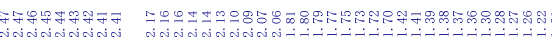
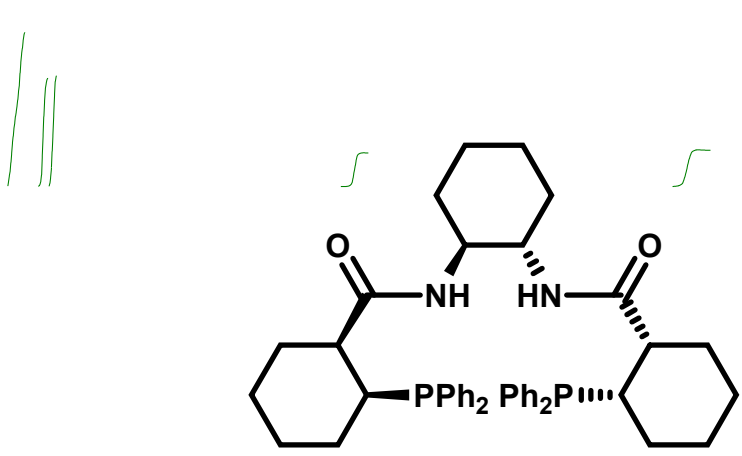

促

L11

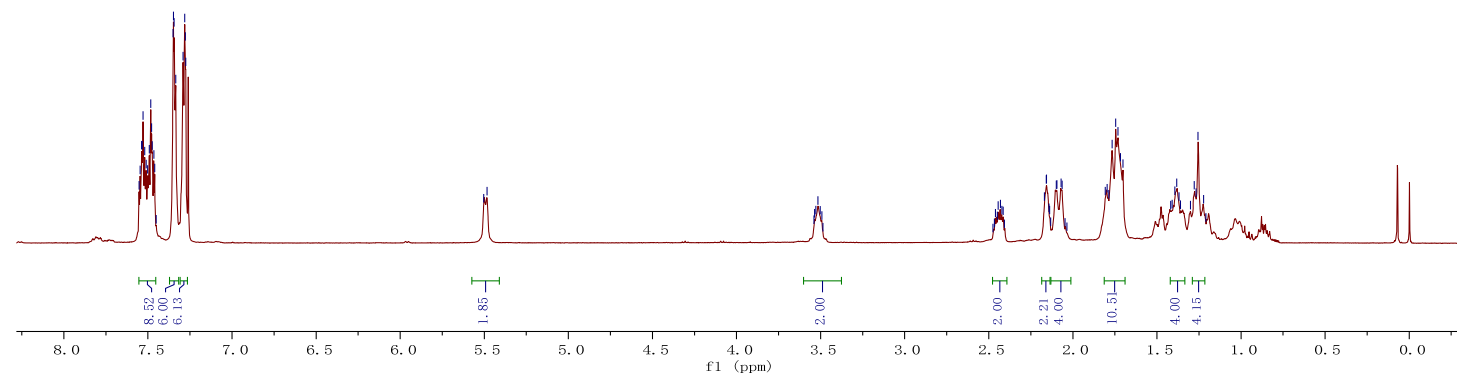

$\underline{{ }^{13} \mathrm{C} \mathrm{NMR}}\left(100 \mathrm{MHz}, \mathrm{CDCl}_{3} @ 77\right.$ ppm)

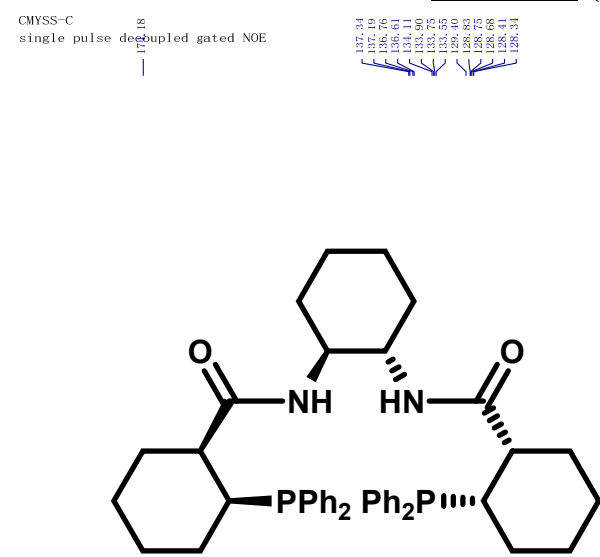

L11

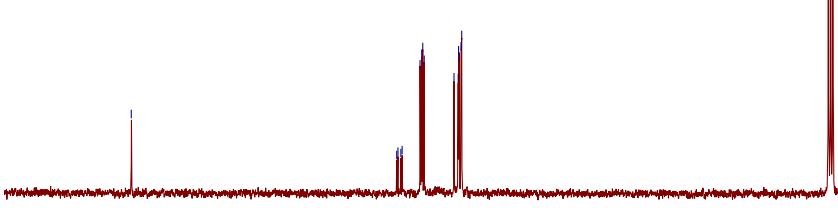

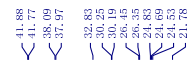

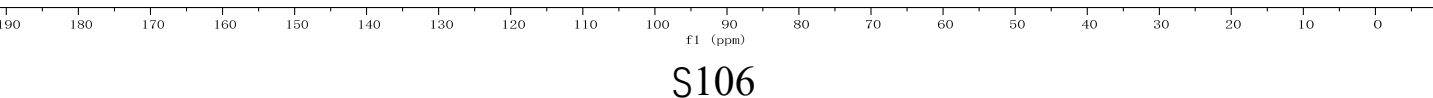



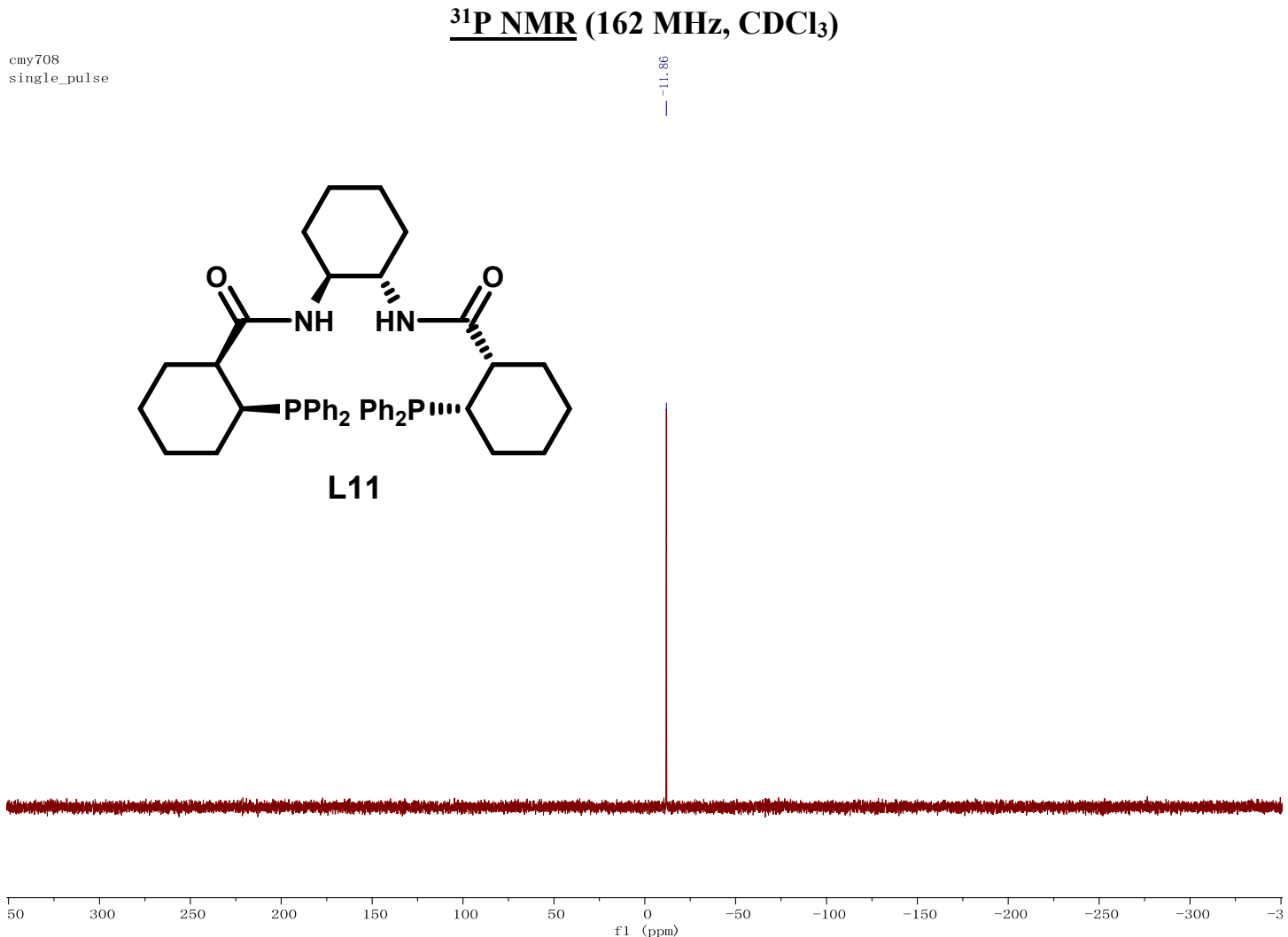

(1S,1'S,2S,2'S)-N,N'-((1R,2R)-Cyclohexane-1,2-diyl)bis(2-(diphenylphosphanyl)cyclohexane-1carboxamide) (L12)

$\underline{{ }^{1} \mathrm{H} \text { NMR }}\left(400 \mathrm{MHz}, \mathrm{CDCl}_{3} @ 7.26\right.$ ppm)

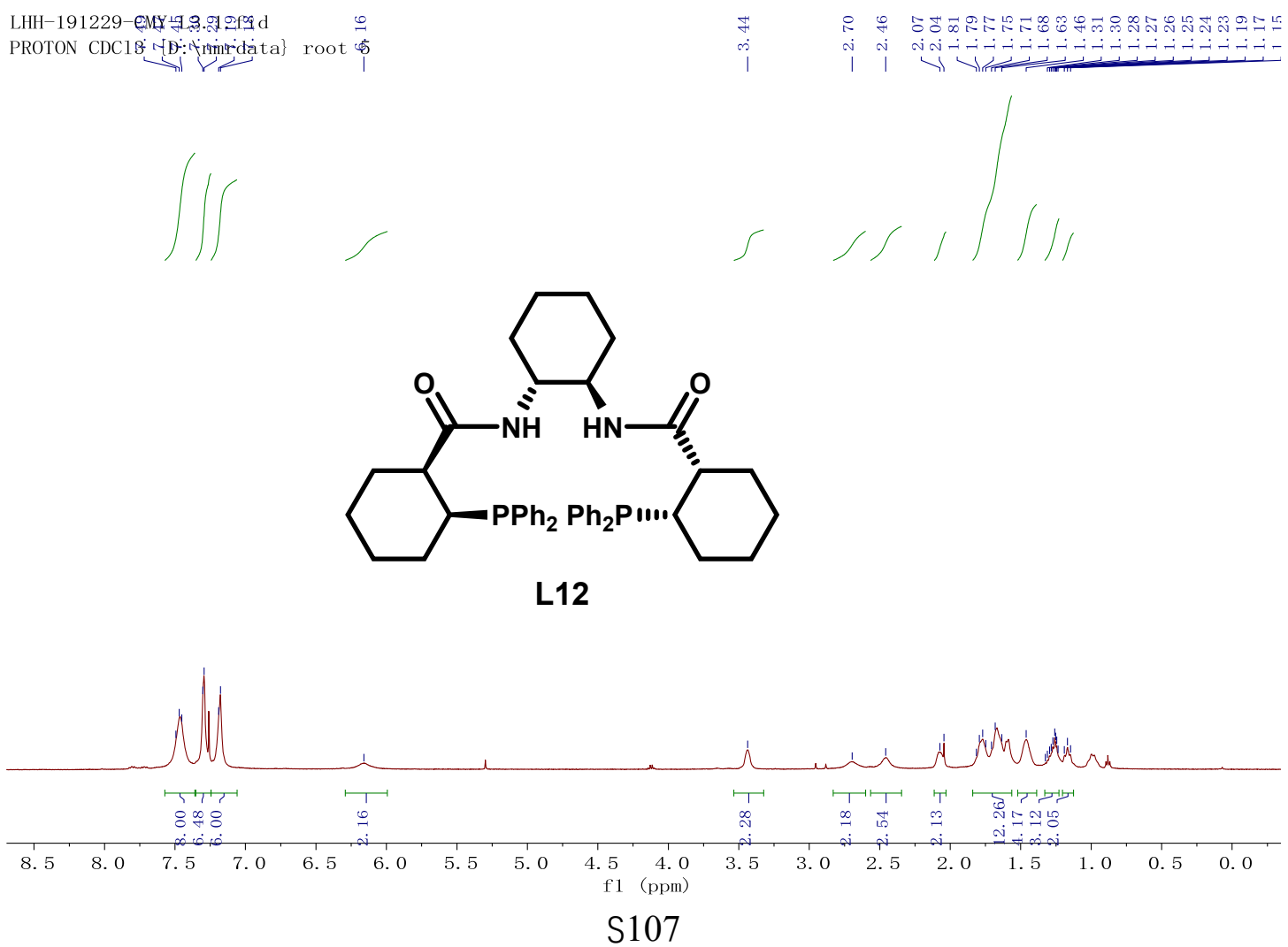


${ }^{13} \mathrm{C}$ NMR (100 MHz, CDCl 377 ppm)

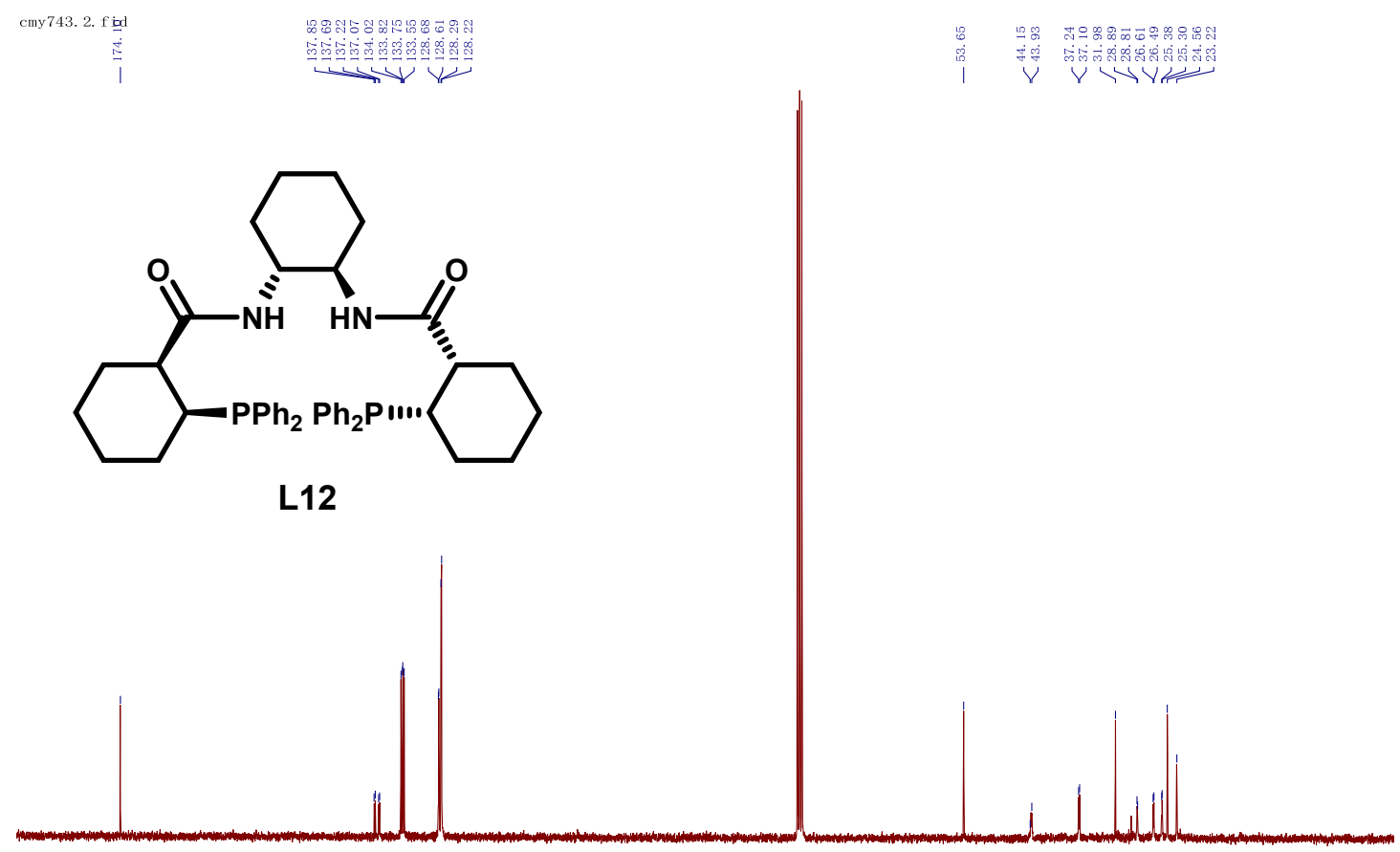

31P NMR (162 MHz, $\left.\mathrm{CDCl}_{3}\right)$

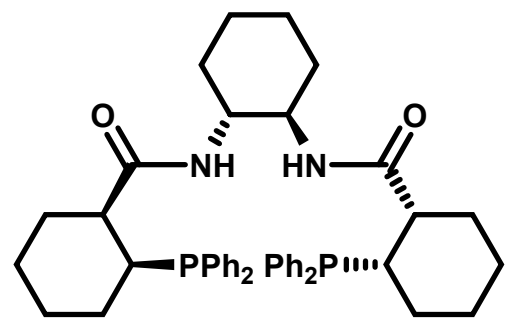

L12

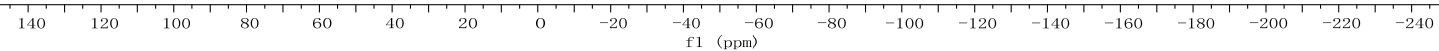


$\left(1 R, 1^{\prime} R, 2 S, 2^{\prime} S\right)$-N,N'-((1S,2S)-Cyclohexane-1,2-diyl)bis(2-(diphenylphosphanyl)cyclohexane-1carboxamide) (L13)

느 NMR $\left(400 \mathrm{MHz}, \mathrm{CDCl}_{3} @ 7.26\right.$ ppm)

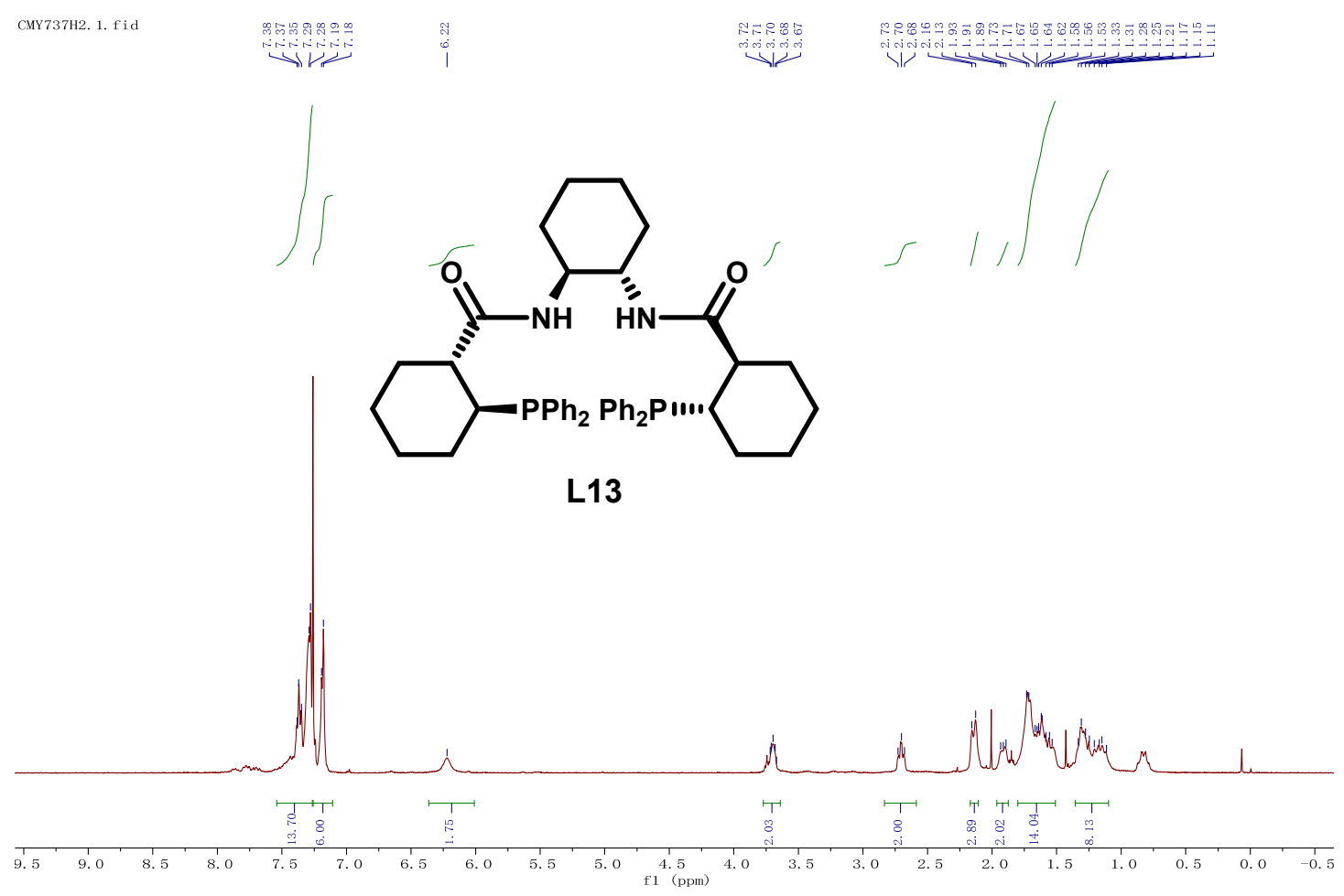

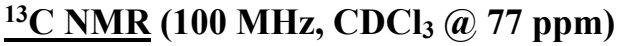

CMY737-7.5

single pulse decoupled gated NOE

$\longrightarrow$ ㄴ.

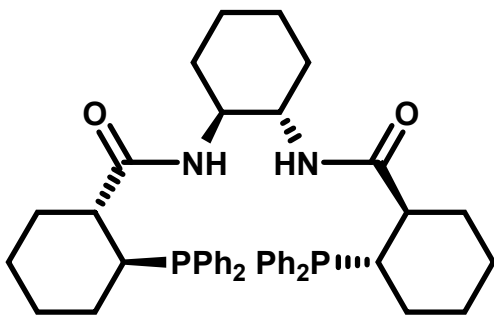

L13

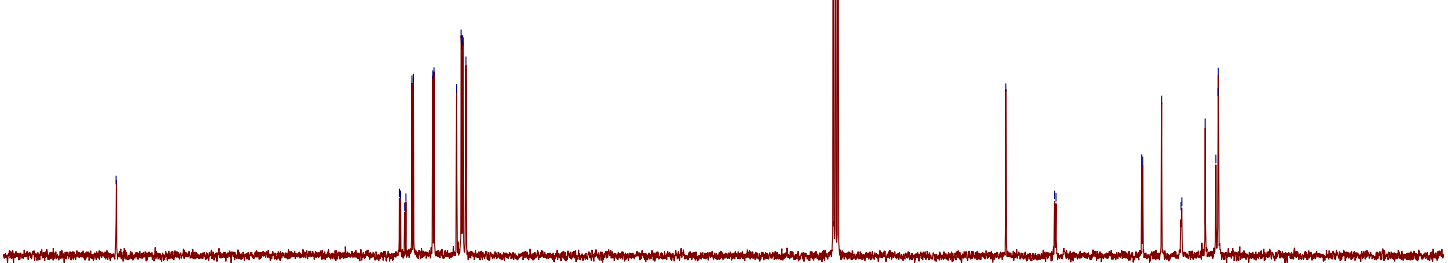

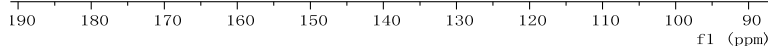




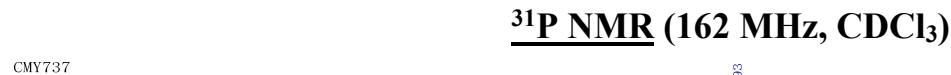

CMY737
single_pulse

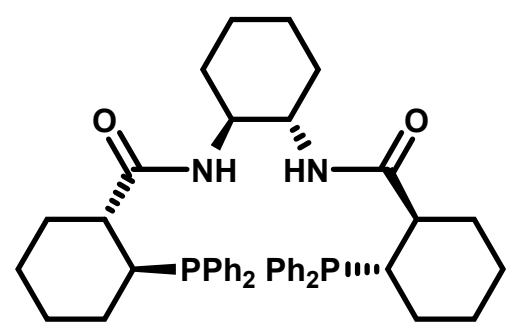

L13
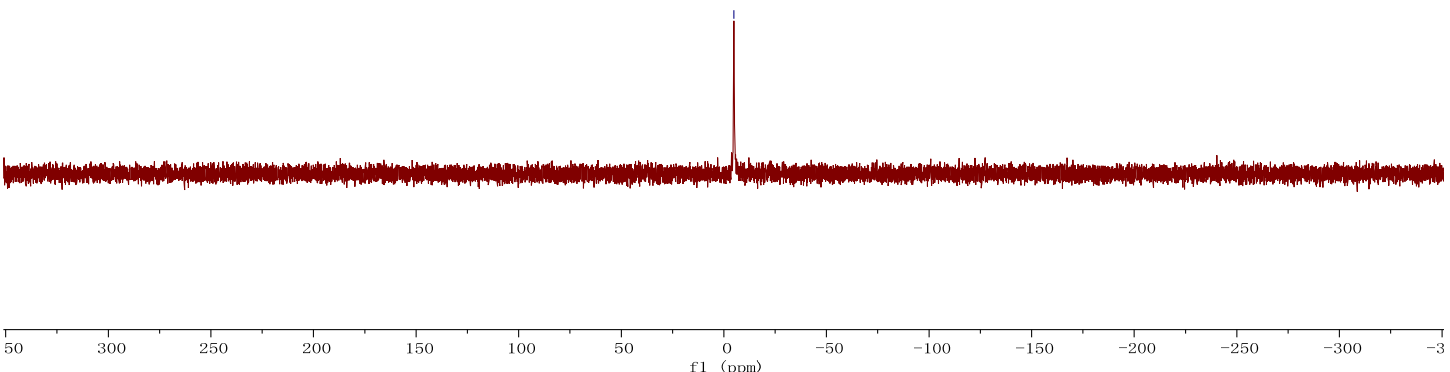

$\left(1 R, 1^{\prime} R, 2 S, 2 ' S\right)$-N,N'-((1R,2R)-Cyclohexane-1,2-diyl)bis(2-(diphenylphosphanyl) cyclohexane-1carboxamide) (L14)

$\underline{{ }^{1} \mathrm{H} \text { NMR }}\left(400 \mathrm{MHz}, \mathrm{CDCl}_{3} @ 7.26\right.$ ppm)

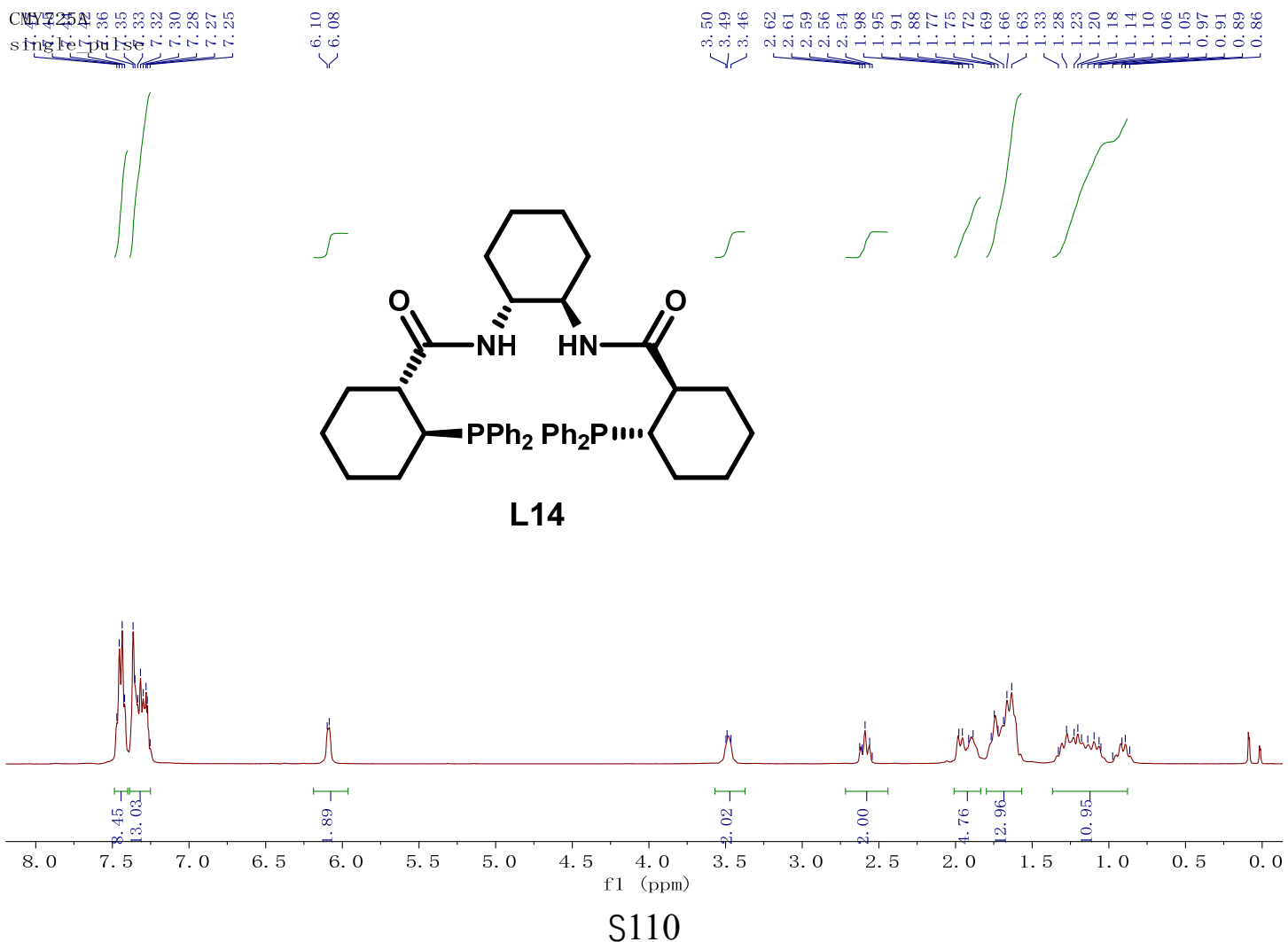


${ }^{13} \mathrm{C}$ NMR (100 MHz, CDCl 3 @ 77 ppm)

CMY-725-

single the decoupled sog

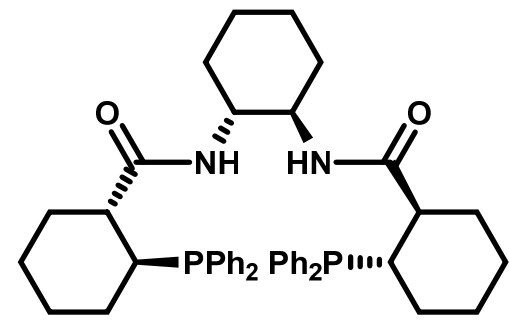

L14

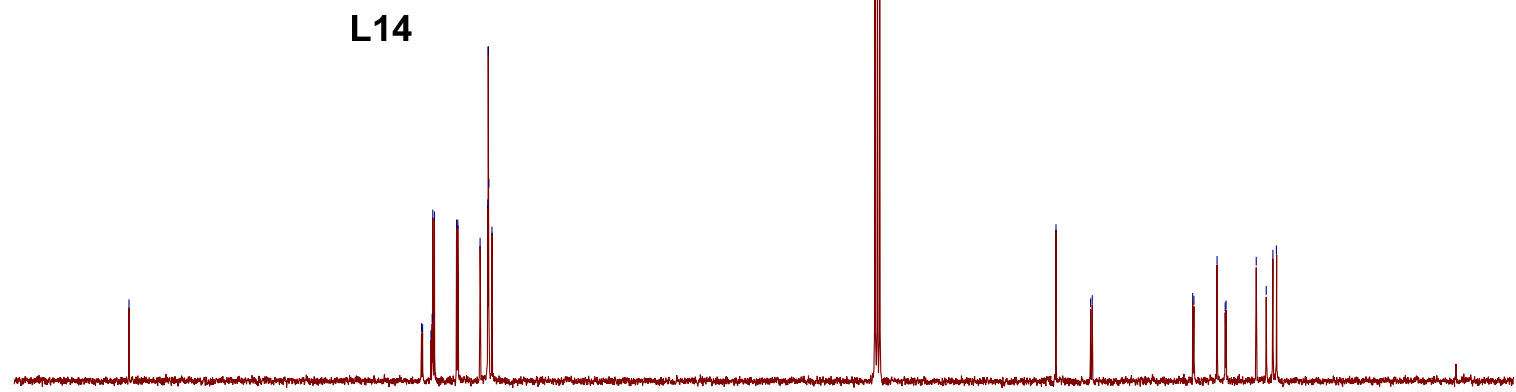

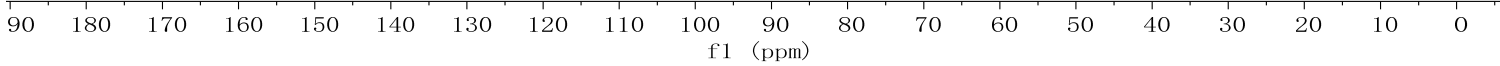

31P NMR (162 MHz, $\mathrm{CDCl}_{3}$ )

CMY725A

single pulse decoupled gated NOE

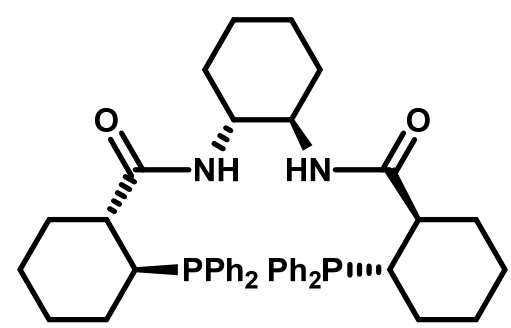

L14

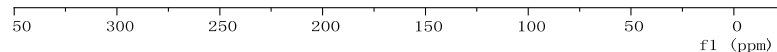


(1S,1'S,2S,2'S)-N,N'-((1R,2R)-Cyclohexane-1,2-diyl)bis(2-(diphenylphosphanyl) cyclohexane-1carboxamide) (L21)

$\underline{{ }^{1} \mathrm{H} \text { NMR }}\left(500 \mathrm{MHz}, \mathrm{CDCl}_{3} @ 7.26\right.$ ppm)

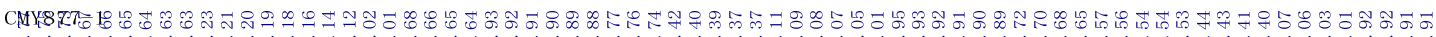
stristion putser

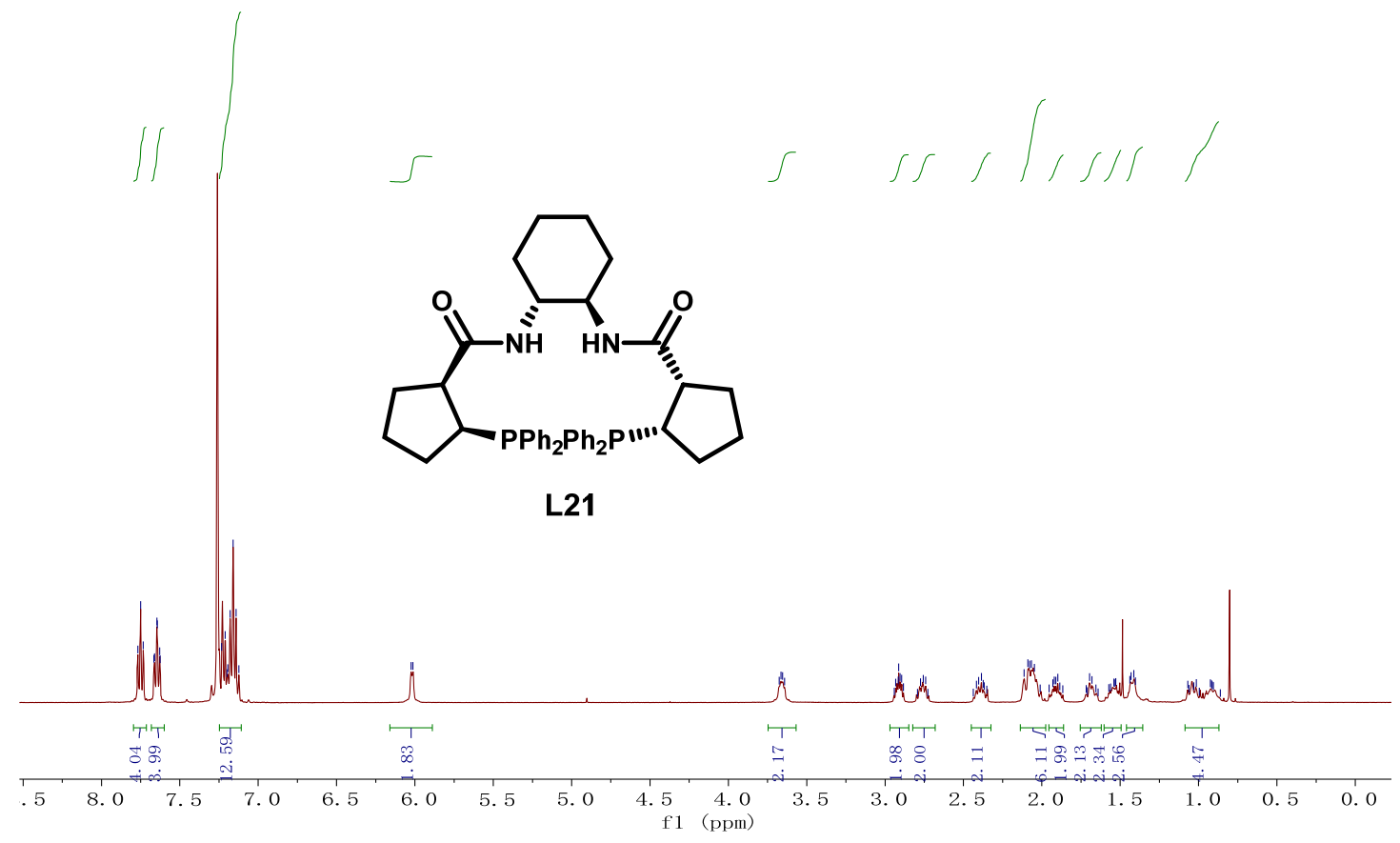

${ }^{13} \mathrm{C}$ NMR (125 MHz, CDCl $3 @ 77$ ppm)

LHH-191229-CMY-FIVE-1 igand\%

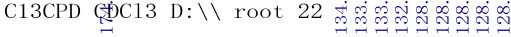

।

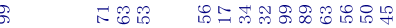

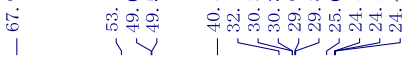

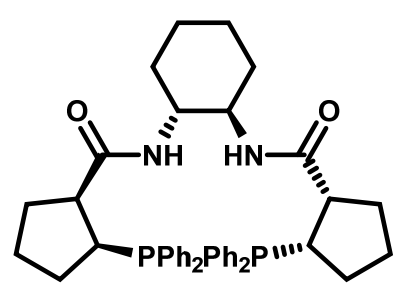

L21

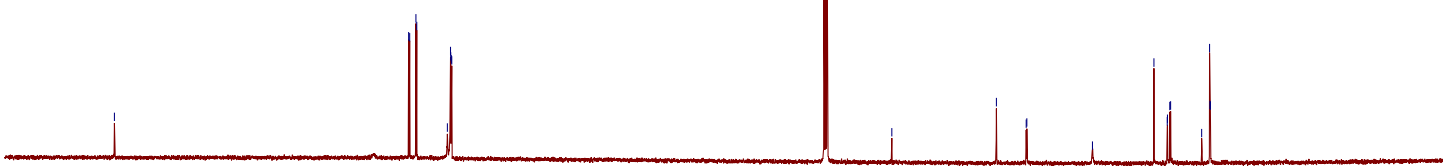

$\begin{array}{llllllllll}180 & 170 & 160 & 150 & 140 & 130 & 120 & 110 & 100 & 90 \\ & & & & & & & & & \end{array}$ 
LHH-191217-CMYL6. 11. fid

P31 CDC13 \{D: \nmrdata\} root 50

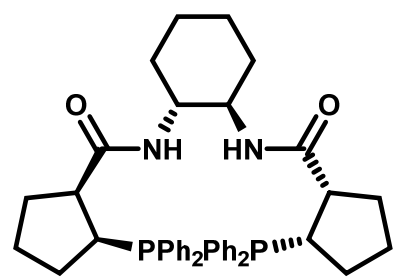

L21

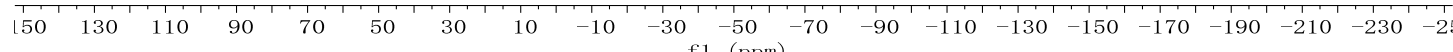

(1S,1'S,2S,2'S)-N,N'-((1R,2R)-Cyclohexane-1,2-diyl)bis(2-(diphenylphosphanyl)cycloheptane-1carboxamide) (L22)

1H NMR (500 MHz, CDCl 3 @ 7.26 ppm)

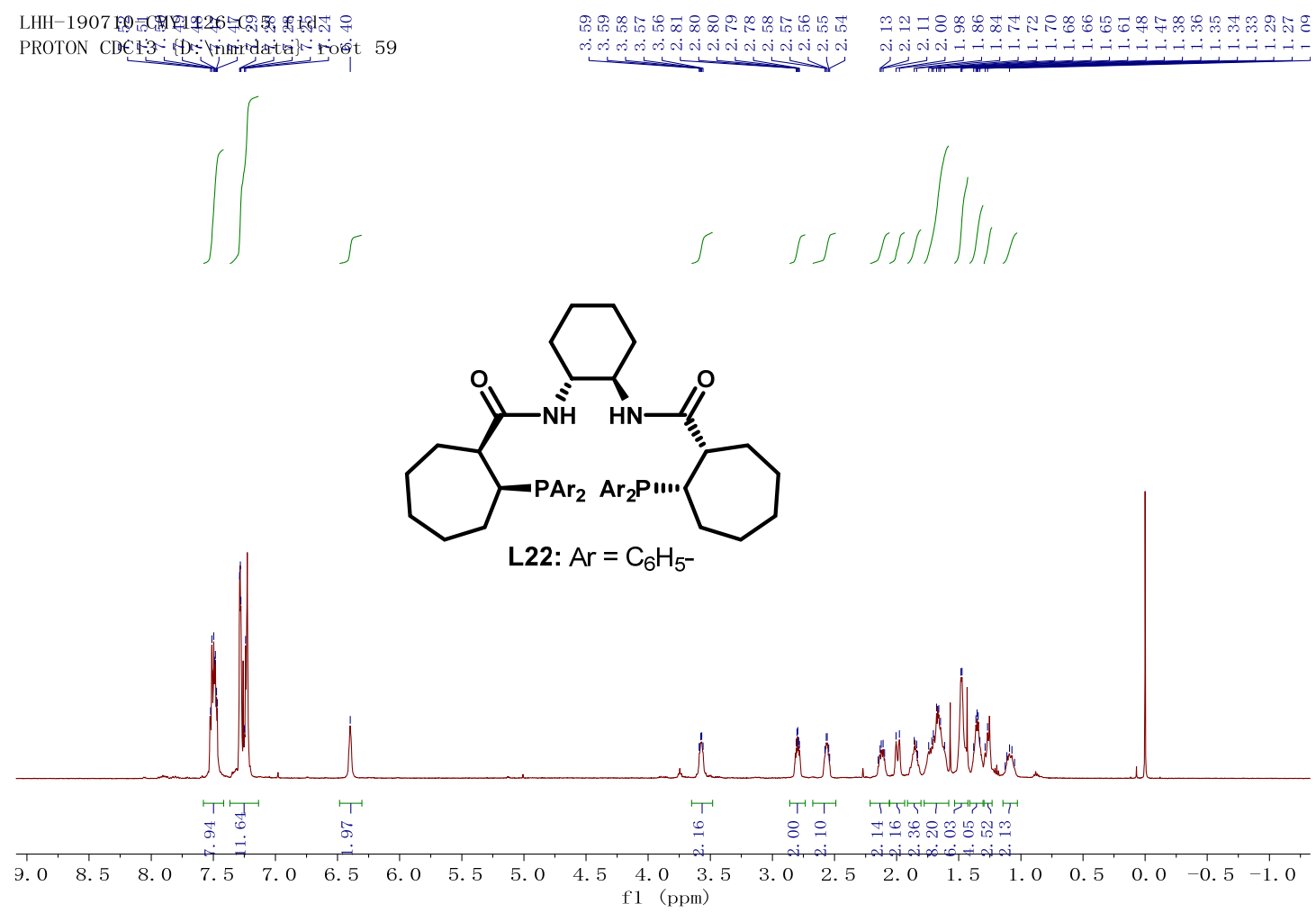


${ }^{13} \mathrm{C}$ NMR (125 MHz, CDCl $@ 77$ ppm)

LHH-190710-CMR1 126-C. 2. fid

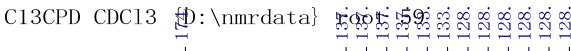

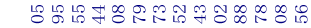

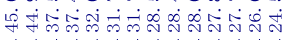

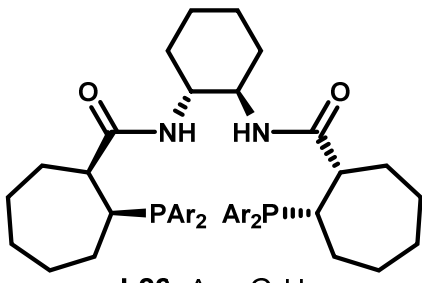

L22: $\mathrm{Ar}=\mathrm{C}_{6} \mathrm{H}_{5-}$

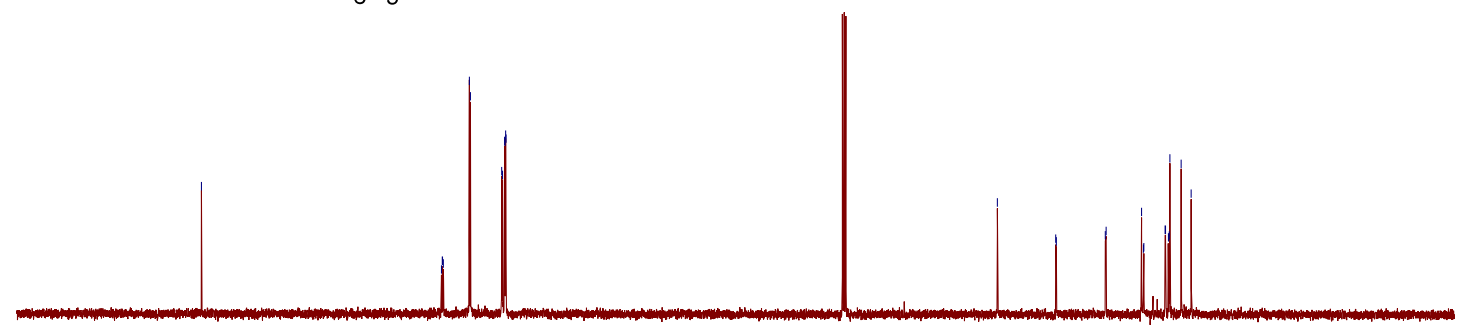

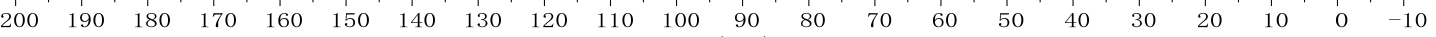

f1 (ppm)

31P NMR (202 MHz, $\left.\mathrm{CDCl}_{3}\right)$

LHH-190710-CMY1126-C. 1. fid

P31 CDC13 \{D: \nmrdata $\}$ root 59

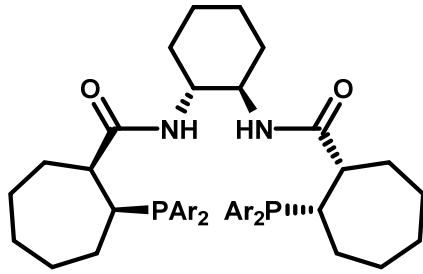

L22: $\mathrm{Ar}=\mathrm{C}_{6} \mathrm{H}_{5}-$

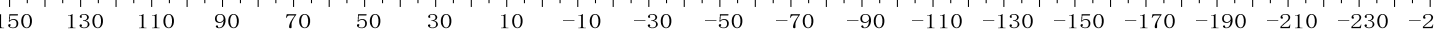
f1 (ppm) 
(1S,1'S,2S,2'S)-N,N'-((1R,2R)-Cyclohexane-1,2-diyl)bis(2-(di-p-tolylphosphanyl) cycloheptane-1carboxamide) (L15)

$\underline{{ }^{1} \mathrm{H} \text { NMR }}\left(500 \mathrm{MHz}, \mathrm{CDCl}_{3} @ 7.26\right.$ ppm)

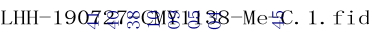

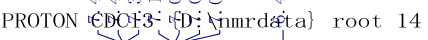

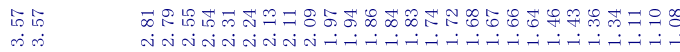

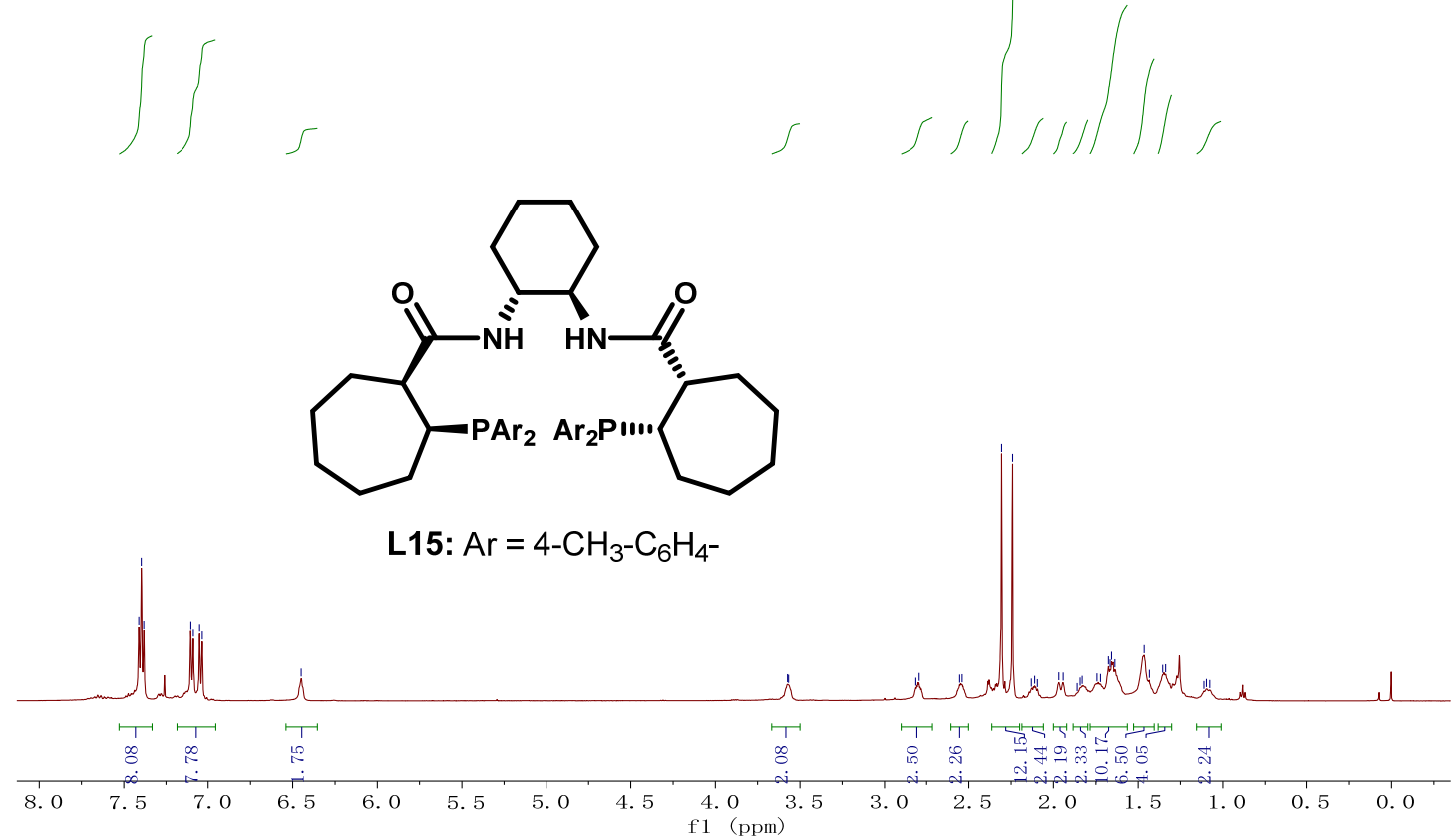

${ }^{13} \mathrm{C}$ NMR (125 MHz, CDCl 3 @ 77 ppm)

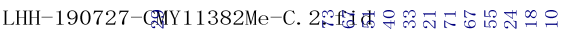

C13CPD CDCl3 $\{\mathrm{D}: \backslash$ nmrdata\}

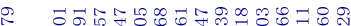

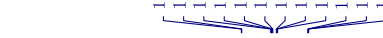

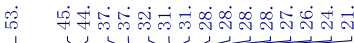

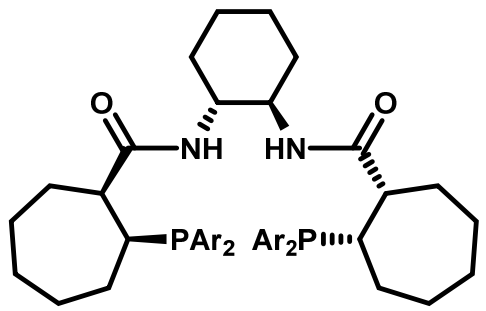

L15: $\mathrm{Ar}=4-\mathrm{CH}_{3}-\mathrm{C}_{6} \mathrm{H}_{4}$

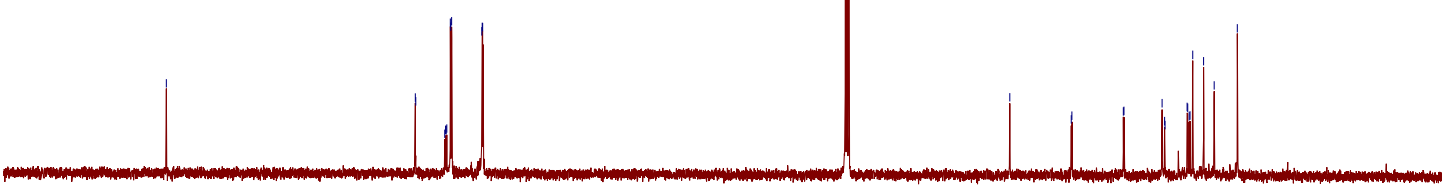

$\begin{array}{llllllllllllllllllll}190 & 180 & 170 & 160 & 150 & 140 & 130 & 120 & 110 & 100 & 90 & 80 & 70 & 60 & 50 & 40 & 30 & 20 & 10 & 0\end{array}$ 
LHH-190727-CMY1138-Me-C. 2. fid P31 CDC13 \{D: \nmrdata\} root 14

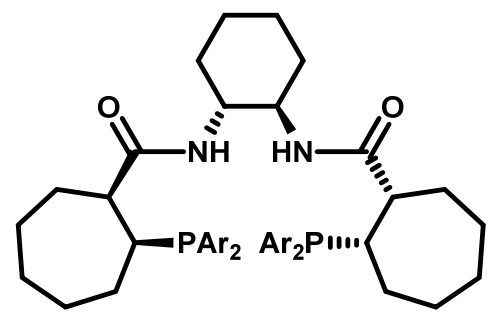

L15: $\mathrm{Ar}=4-\mathrm{CH}_{3}-\mathrm{C}_{6} \mathrm{H}_{4}-$

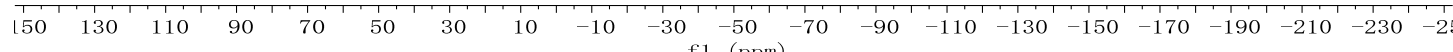

(1S,1'S,2S,2'S)-N,N'-((1R,2R)-1,2-Di(naphthalen-1-yl)ethane-1,2-diyl)bis(2-(diphenylphosphanyl) cycloptane-1-carboxamide) (L16)

1H NMR (500 MHz, $\mathrm{CDCl}_{3} @ 7.26$ ppm)
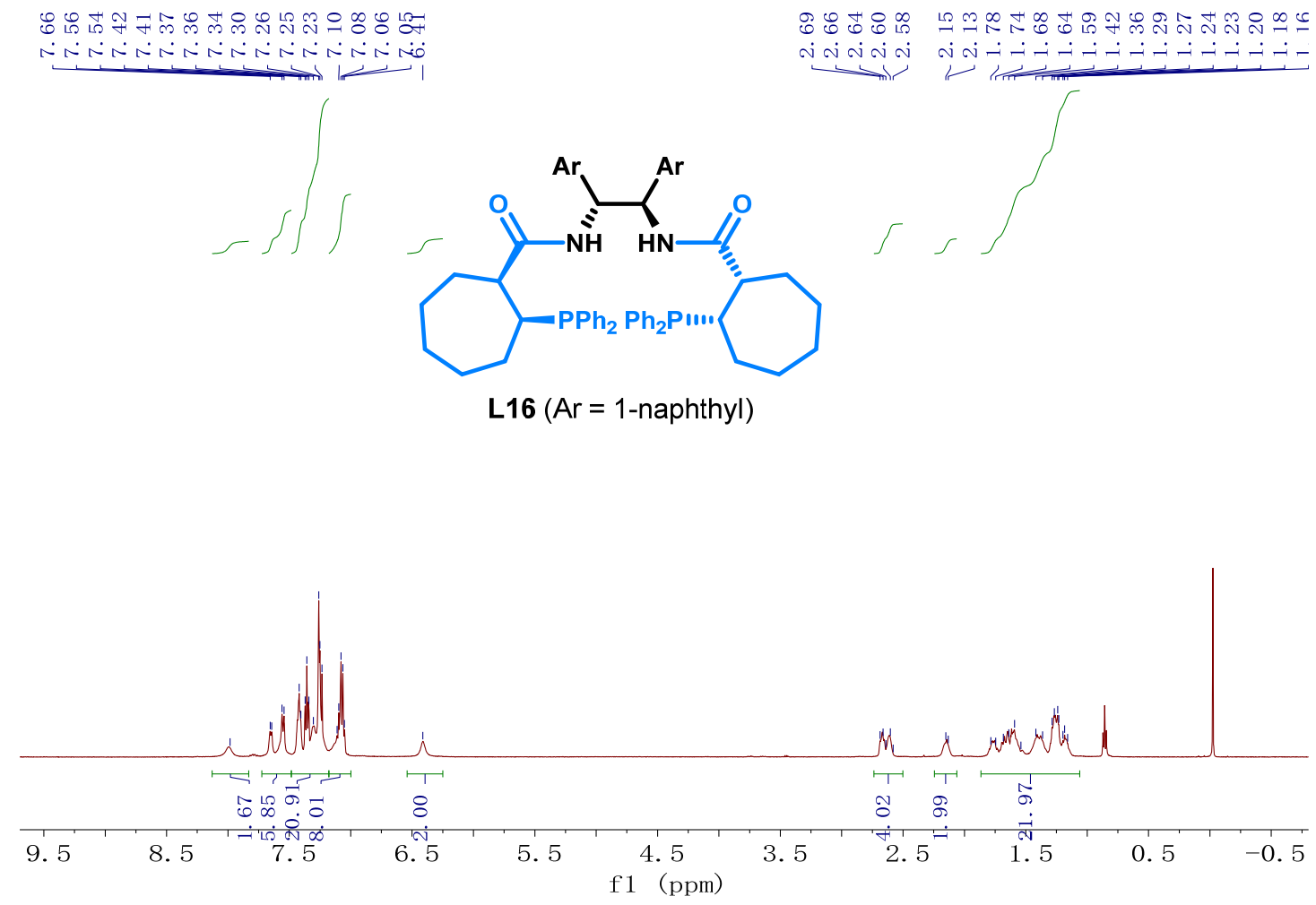
${ }^{13} \mathrm{C}$ NMR (125 MHz, CDCl $@ 77$ ppm)

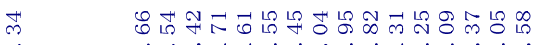

泀

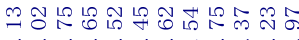

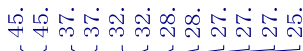

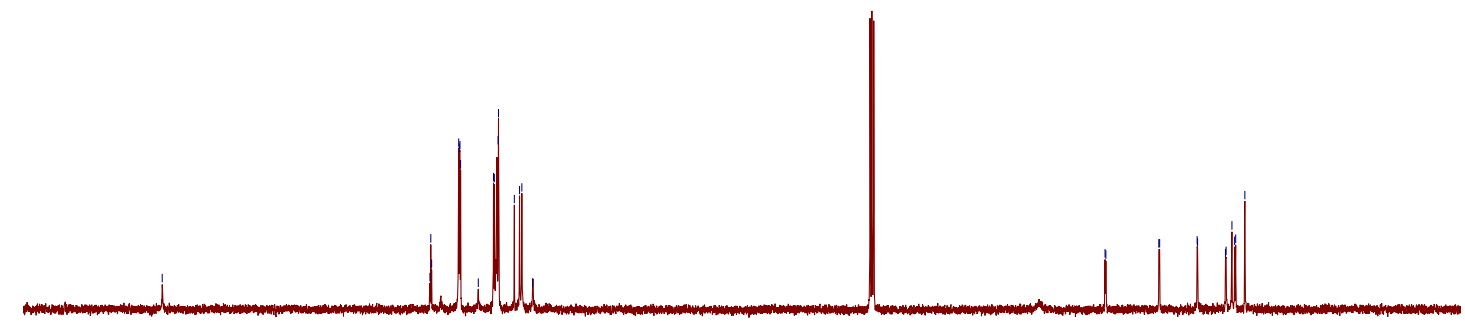

$\begin{array}{llllllllllllllllllll}190 & 180 & 170 & 160 & 150 & 140 & 130 & 120 & 110 & 100 & 90 & 80 & 70 & 60 & 50 & 40 & 30 & 20 & 10 & 0\end{array}$

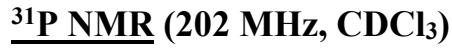

$\stackrel{\llcorner}{\infty}$

$\infty$

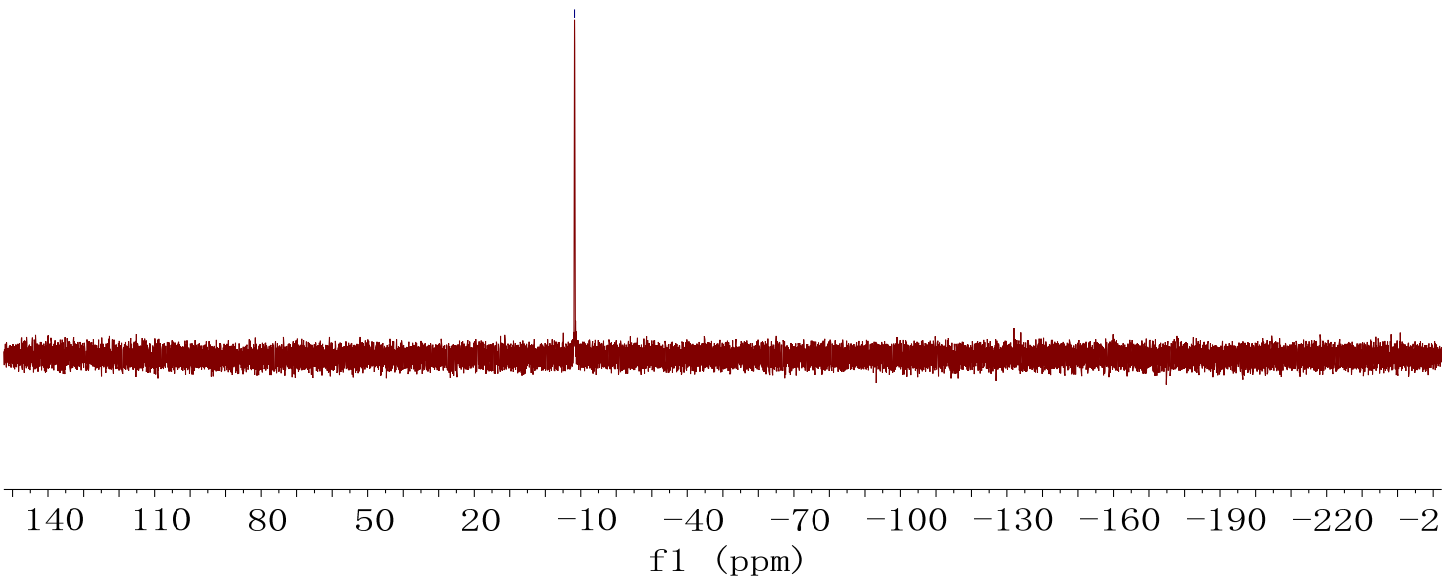


(1S,1'S,2S,2'S)-N,N'-((1R,2R)-Cyclohexane-1,2-diyl)bis(2-(di([1,1'-biphenyl]-4-yl)phosphanyl) cycloheptane-1-carboxamide) (L23)

$\underline{{ }^{1} \mathrm{H} \text { NMR }}\left(500 \mathrm{MHz}, \mathrm{CDCl}_{3} @ 7.26 \mathrm{ppm}\right)$

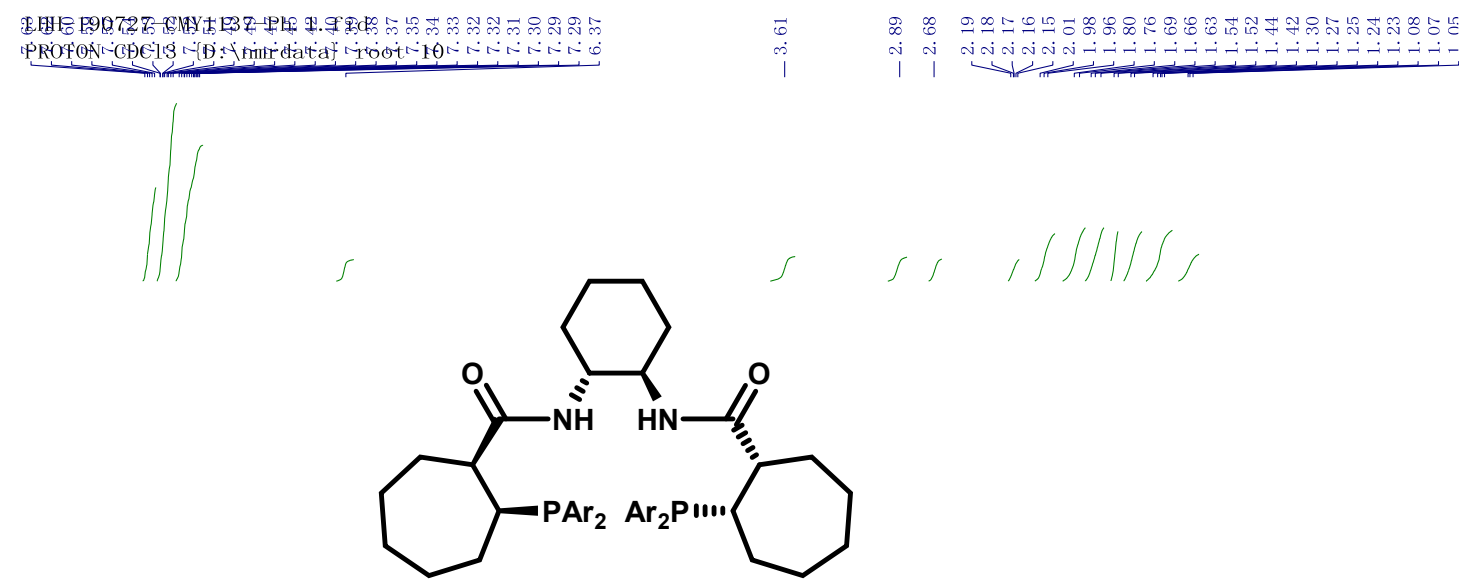

L23: $\mathrm{Ar}=4-\mathrm{Ph}-\mathrm{C}_{6} \mathrm{H}_{4}$

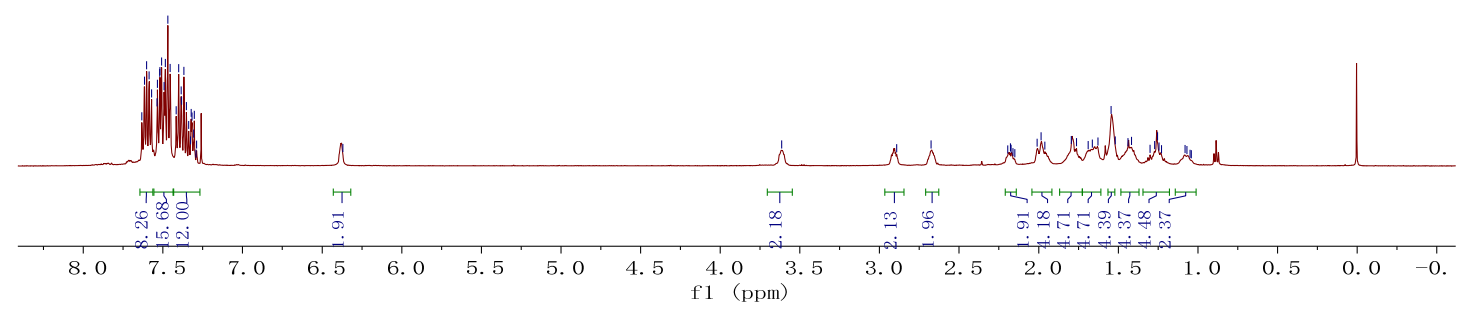

$\underline{{ }^{13} \mathrm{C} \mathrm{NMR}}$ (125 MHz, CDCl $@ 77$ ppm) 


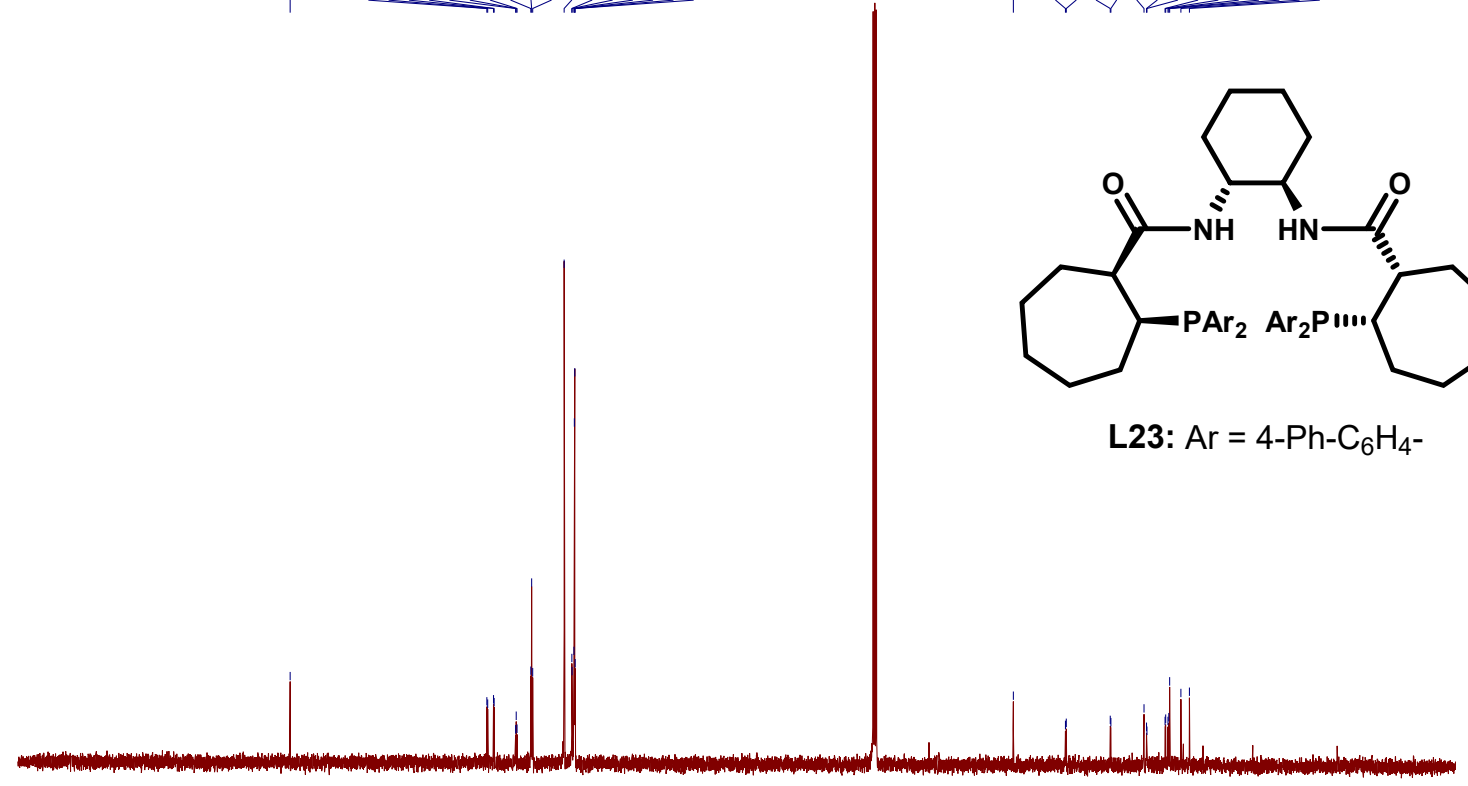

$\begin{array}{llllllllllllllllllllllll}210 & 200 & 190 & 180 & 170 & 160 & 150 & 140 & 130 & 120 & 110 & 100 & 90 & 80 & 70 & 60 & 50 & 40 & 30 & 20 & 10 & 0 & -10\end{array}$

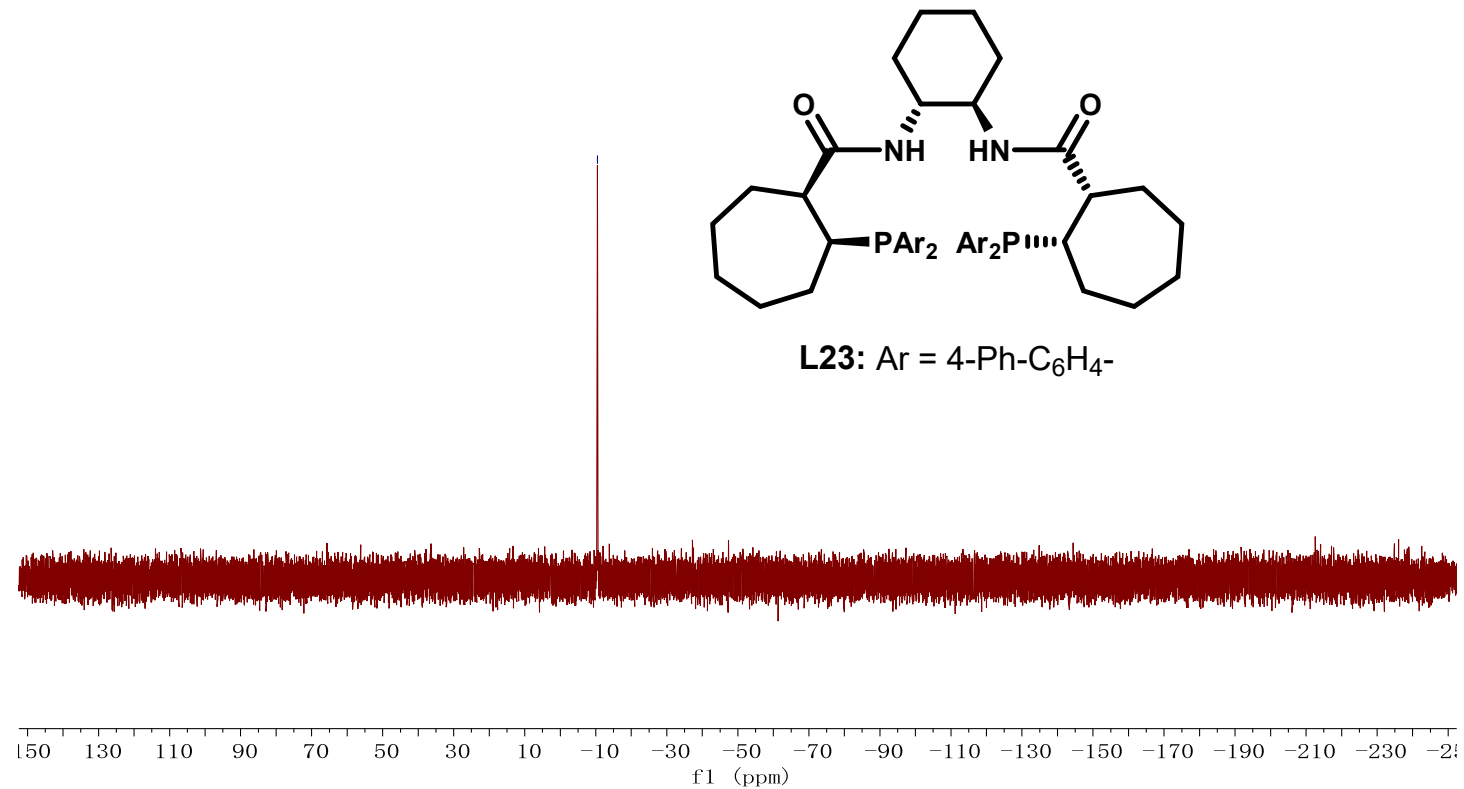

(1S,1'S,2S,2'S)-N,N'-((1R,2R)-Cyclohexane-1,2-diyl)bis(2-(bis(4-fluorophenyl)phosphanyl) cycloheptane-1-carboxamide) (L24) 
$\underline{{ }^{1} \mathrm{H} \text { NMR }}\left(500 \mathrm{MHz}, \mathrm{CDCl}_{3} @ 7.26 \mathrm{ppm}\right)$

LHH-

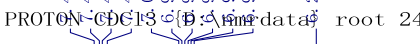

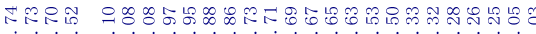
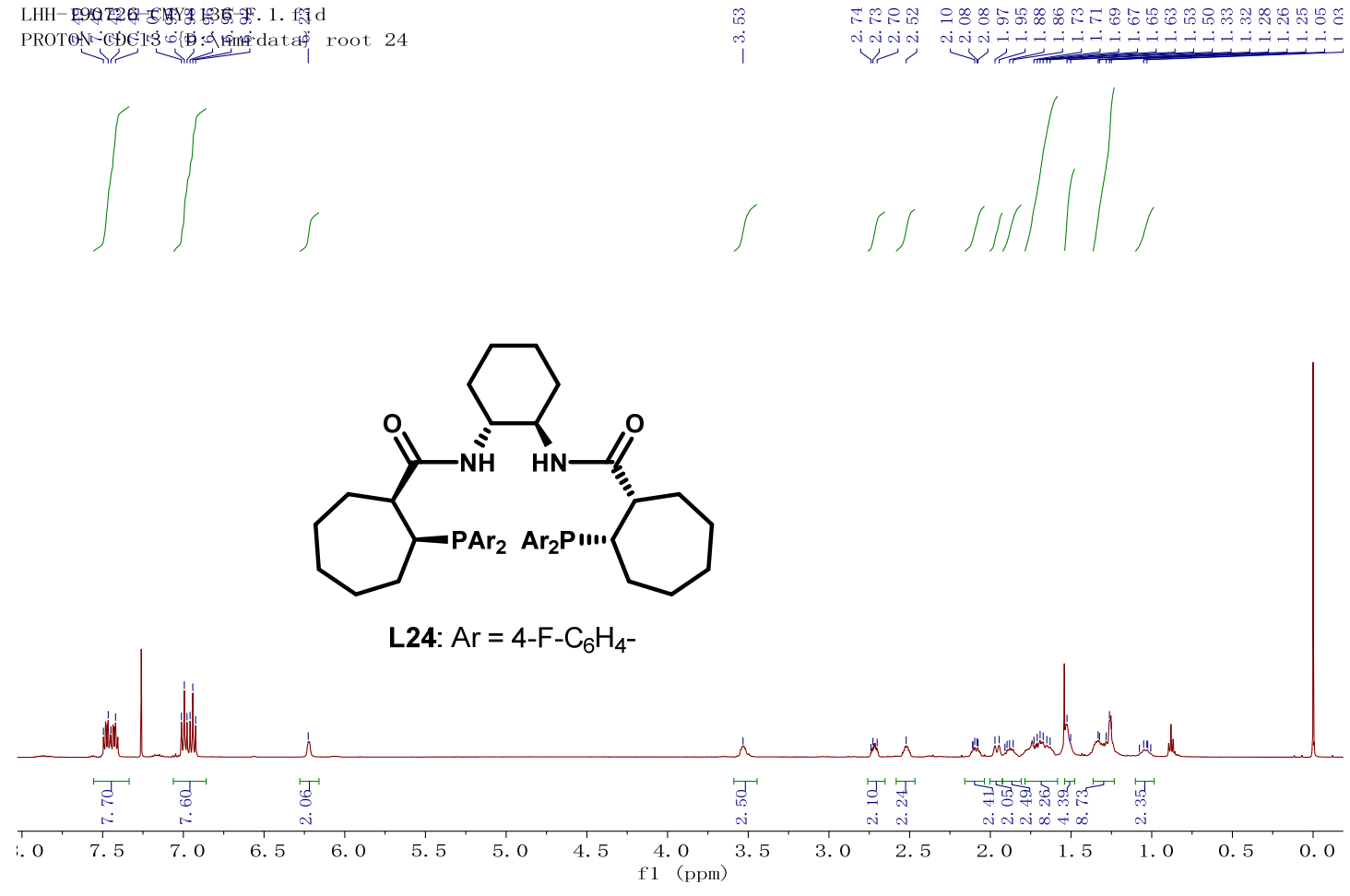

L24: $\mathrm{Ar}=4-\mathrm{F}-\mathrm{C}_{6} \mathrm{H}_{4}$

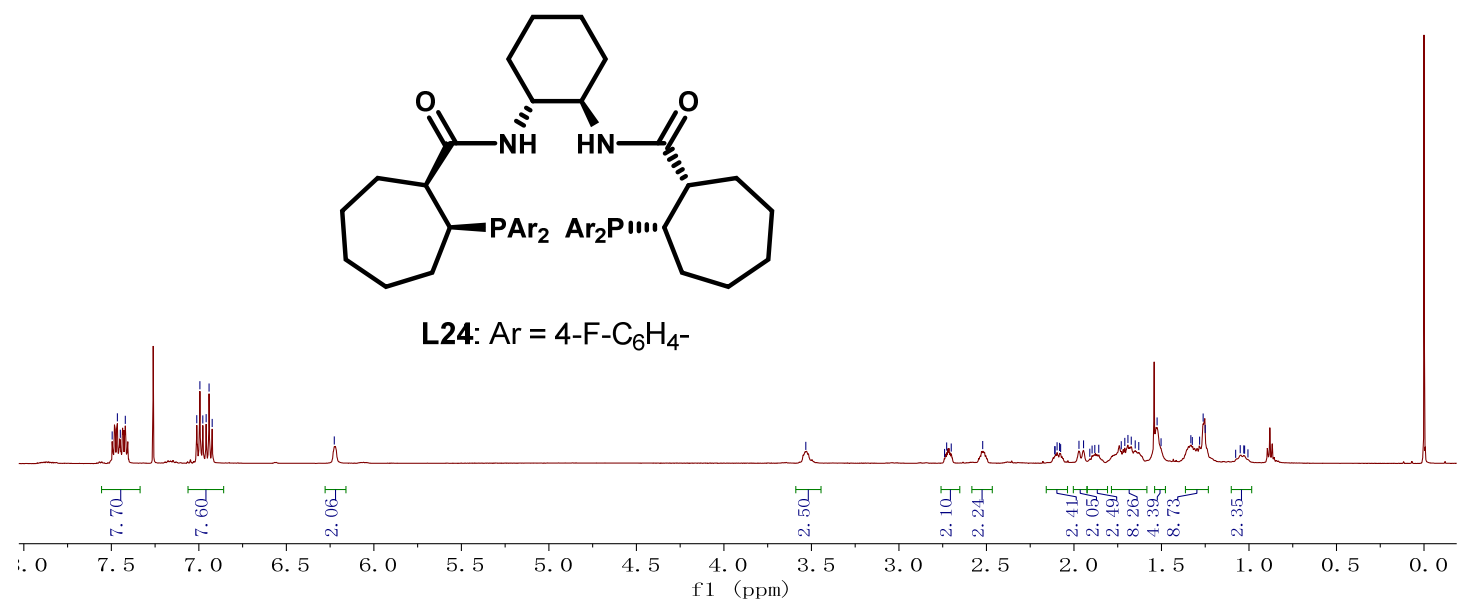

${ }^{13} \mathrm{C}$ NMR (125 MHz, CDCl 3 @7 ppm)
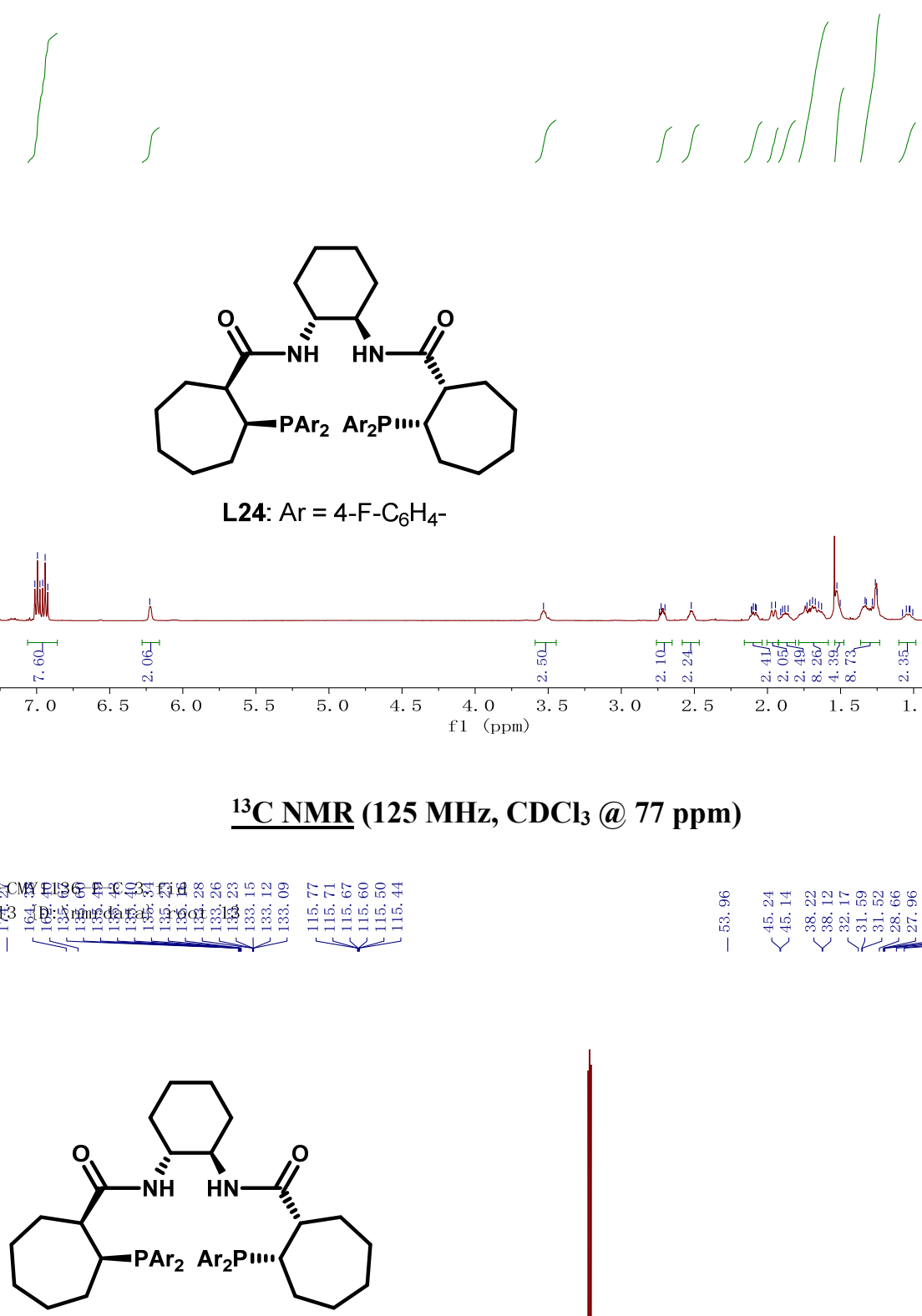

L24: $\mathrm{Ar}=4-\mathrm{F}-\mathrm{C}_{6} \mathrm{H}_{4}^{-}$

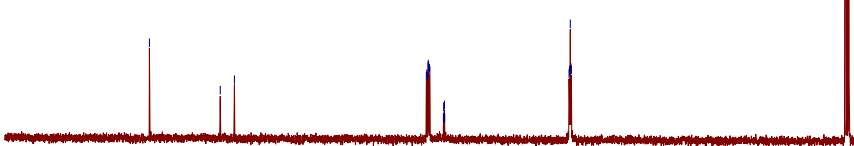

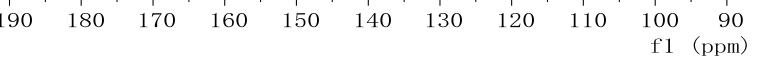

31P NMR (202 MHz, $\left.\mathrm{CDCl}_{3}\right)$ 


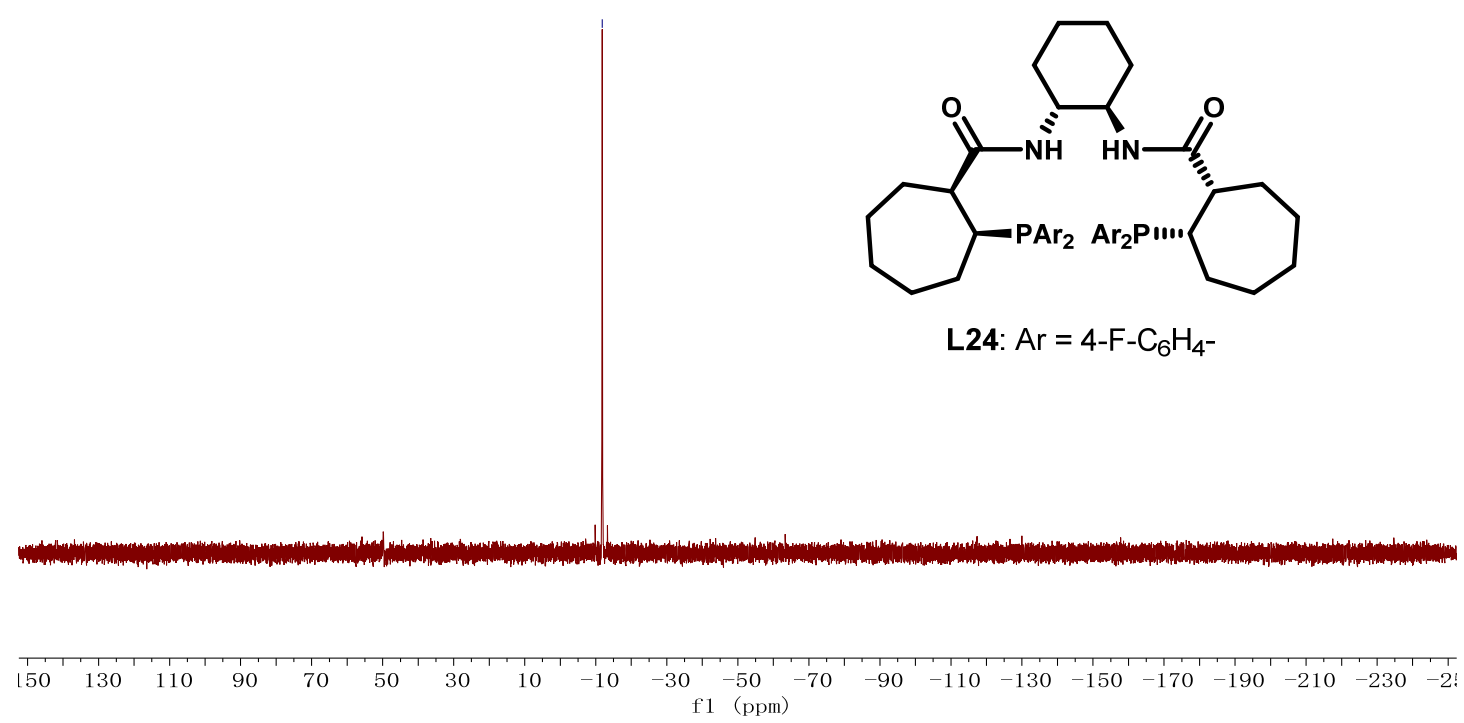

(1S,1'S,2S,2'S)-N,N'-((1R,2R)- Cyclohexane-1,2-diyl)bis(2-(bis(3,5-dimethylphenyl) phosphanyl)cycloheptane-1-carboxamide) (L25)

$\underline{{ }^{1} \mathrm{H} \text { NMR }}\left(500 \mathrm{MHz}, \mathrm{CDCl}_{3} @ 7.26\right.$ ppm)

LHH-190726 $=$ CHAl PROTON CDÉrB⿵ं

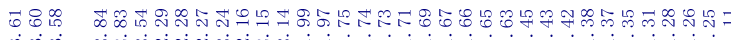
๗ู๗ n 1 , rat

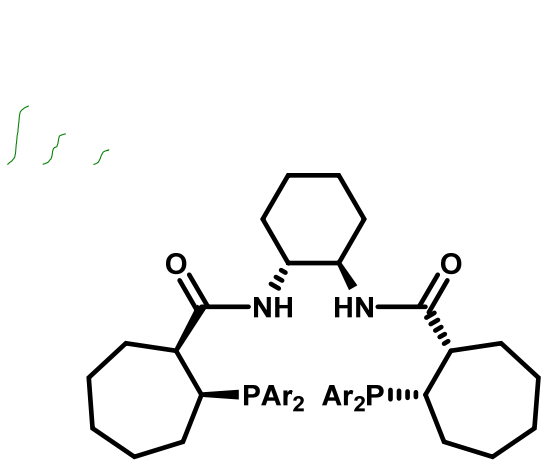

L25: $\mathrm{Ar}=3,5-\left(\mathrm{CH}_{3}\right)_{2}-\mathrm{C}_{6} \mathrm{H}_{3-}$

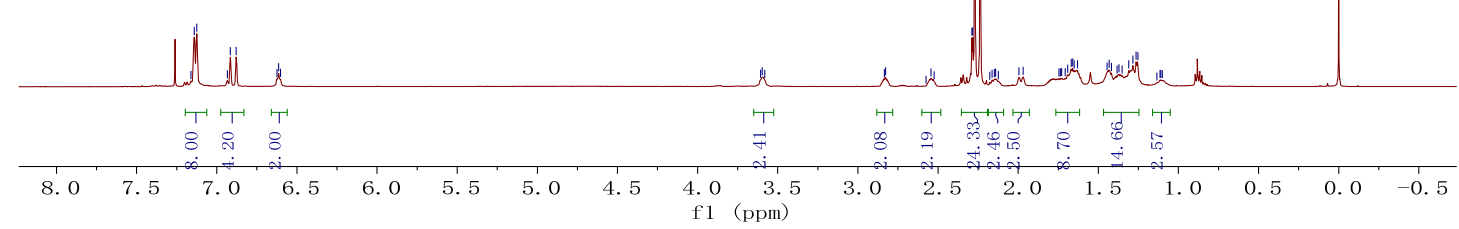




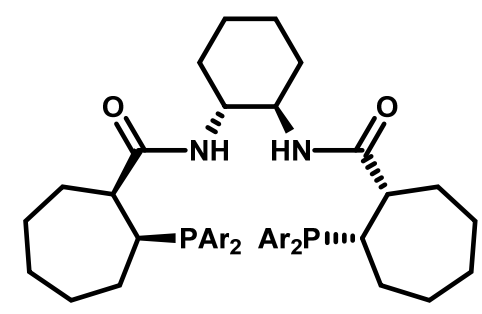

L25: $\mathrm{Ar}=3,5-\left(\mathrm{CH}_{3}\right)_{2}-\mathrm{C}_{6} \mathrm{H}_{3}-$

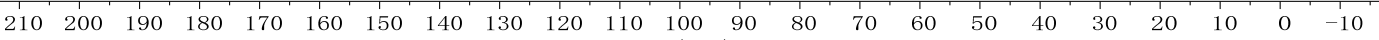
f1 (ppm)

31P NMR (202 MHz, $\left.\mathrm{CDCl}_{3}\right)$

LHH-190729-CMY1133-2Me-C. 1. fid P31 CDC13 \{D: \nmrdata $\}$ root 51

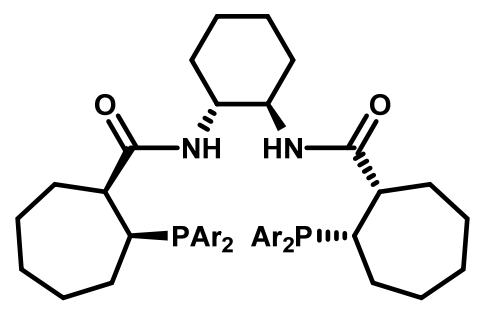

L25: $\mathrm{Ar}=3,5-\left(\mathrm{CH}_{3}\right)_{2}-\mathrm{C}_{6} \mathrm{H}_{3}-$

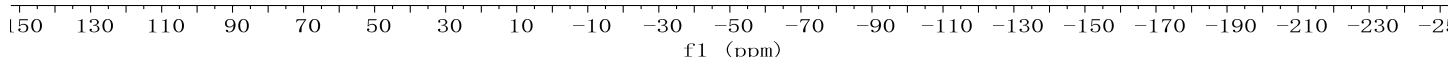
(1S,1'S,2S,2'S)-N,N'-((1R,2R)- Cyclohexane-1,2-diyl)bis(2-(di(naphthalen-2-yl)phosphanyl) cycloheptane-1-carboxamide) (L26) 
매 NMR (500 MHz, $\mathrm{CDCl}_{3} @ 7.26$ ppm)

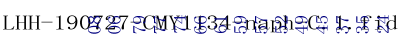
PROTON EBCH

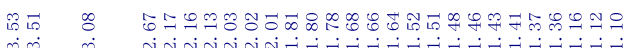

is
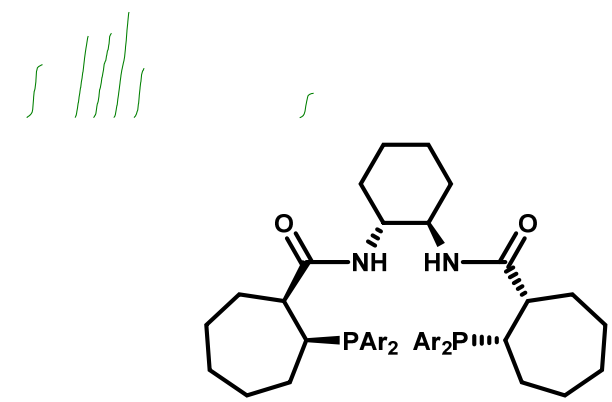

L26: Ar = 2-naphthyl-

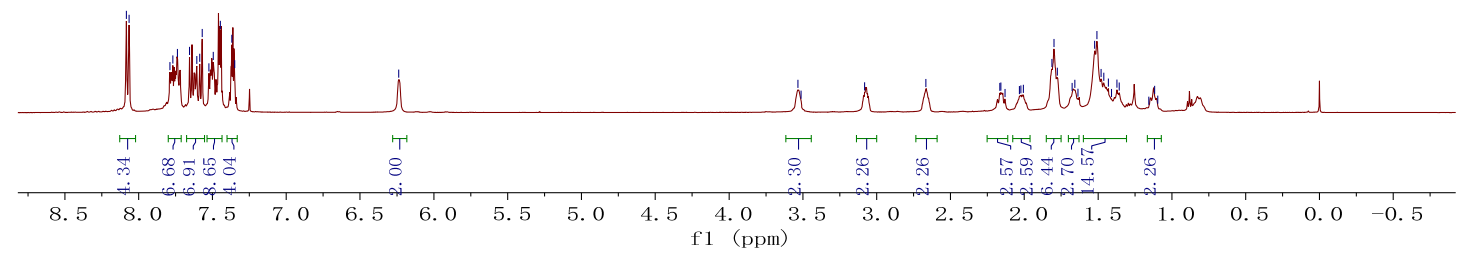

${ }^{13} \mathrm{C}$ NMR (125 MHz, CDCl 3 @ 77 ppm)

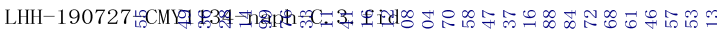

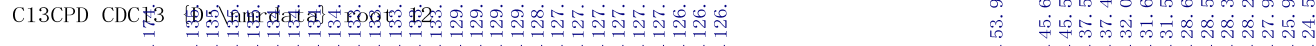

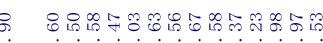

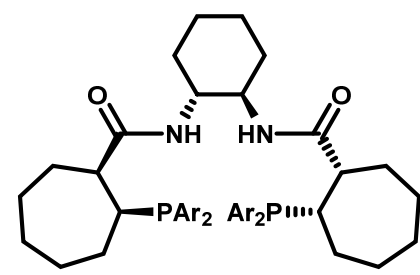

L26: Ar = 2-naphthyl-

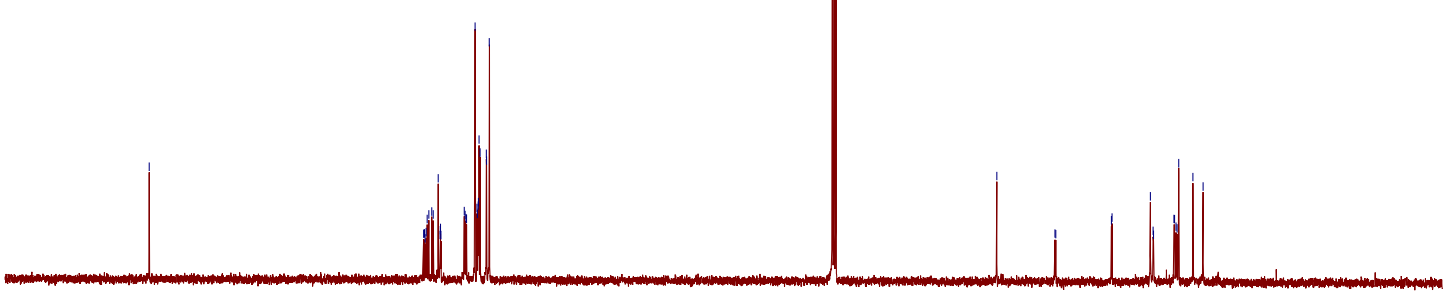

$\begin{array}{llllllllllllllllllll}190 & 180 & 170 & 160 & 150 & 140 & 130 & 120 & 110 & 100 & 90 & 80 & 70 & 60 & 50 & 40 & 30 & 20 & 10 & 0\end{array}$

31P NMR (202 MHz, $\left.\mathrm{CDCl}_{3}\right)$

S123 


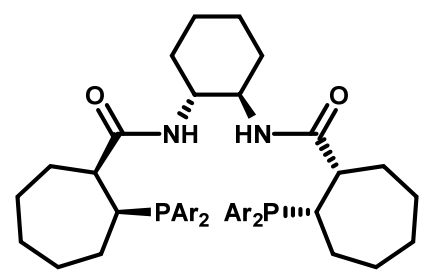

L26: $\mathrm{Ar}=$ 2-naphthyl-

2-Benzylbenzofuran-3(2H)-one (S7a)

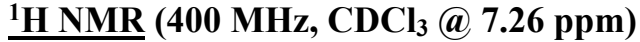

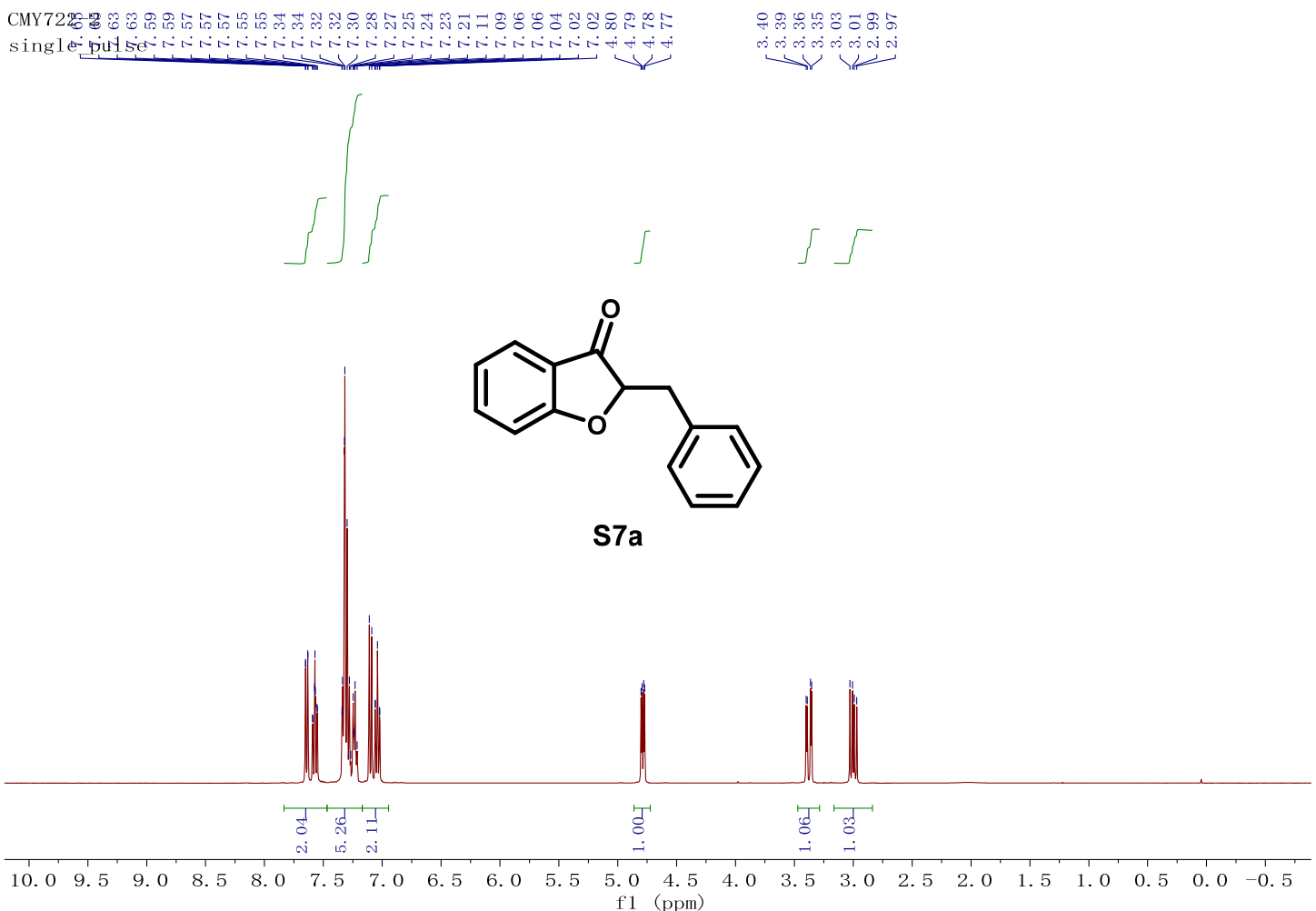



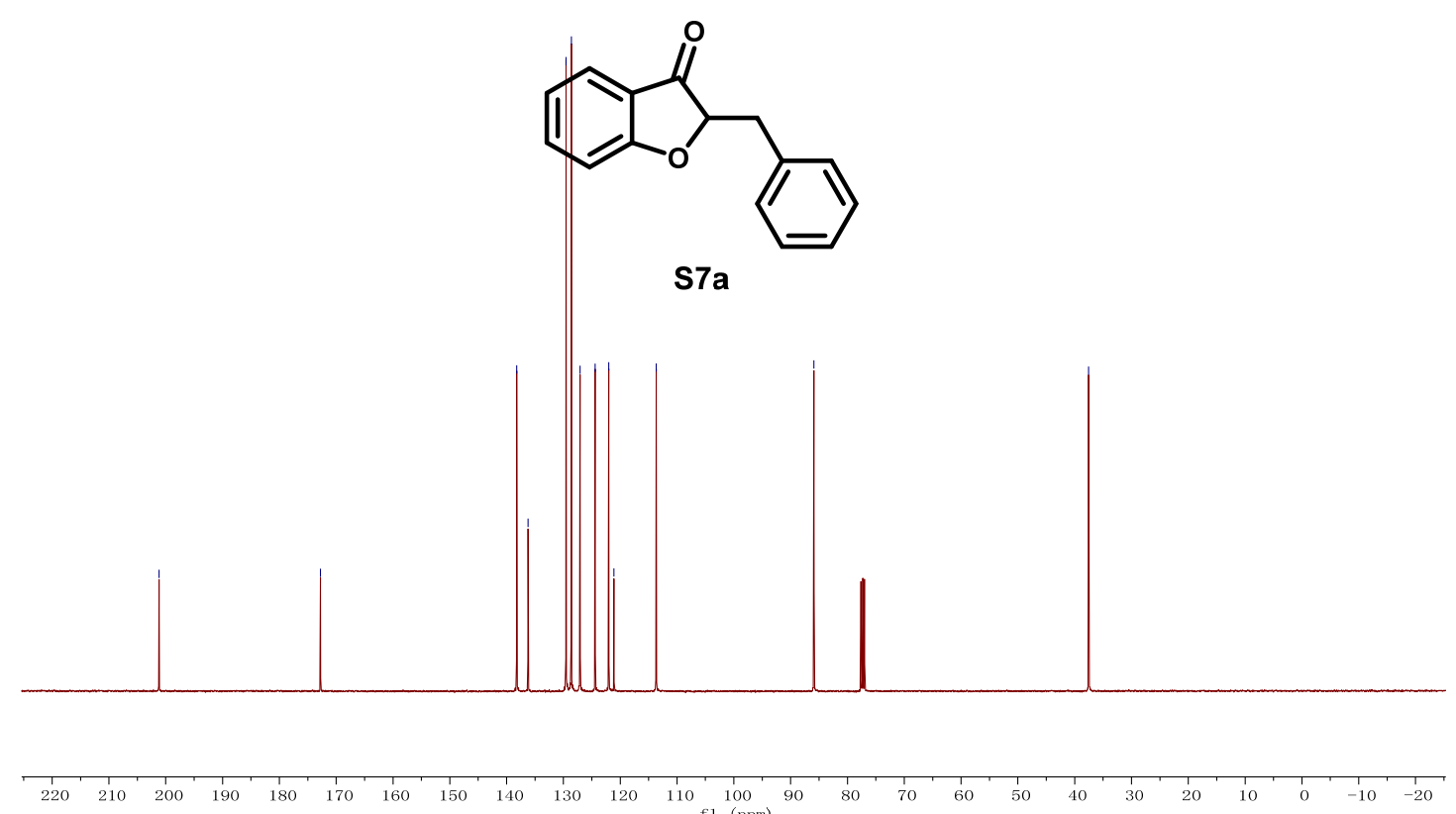

Methyl 4-((3-oxo-2,3-dihydrobenzofuran-2-yl)methyl)benzoate (S7c)

$\underline{{ }^{1} \mathrm{H} \text { NMR }}\left(400 \mathrm{MHz}, \mathrm{CDCl}_{3} @ 7.26\right.$ ppm)

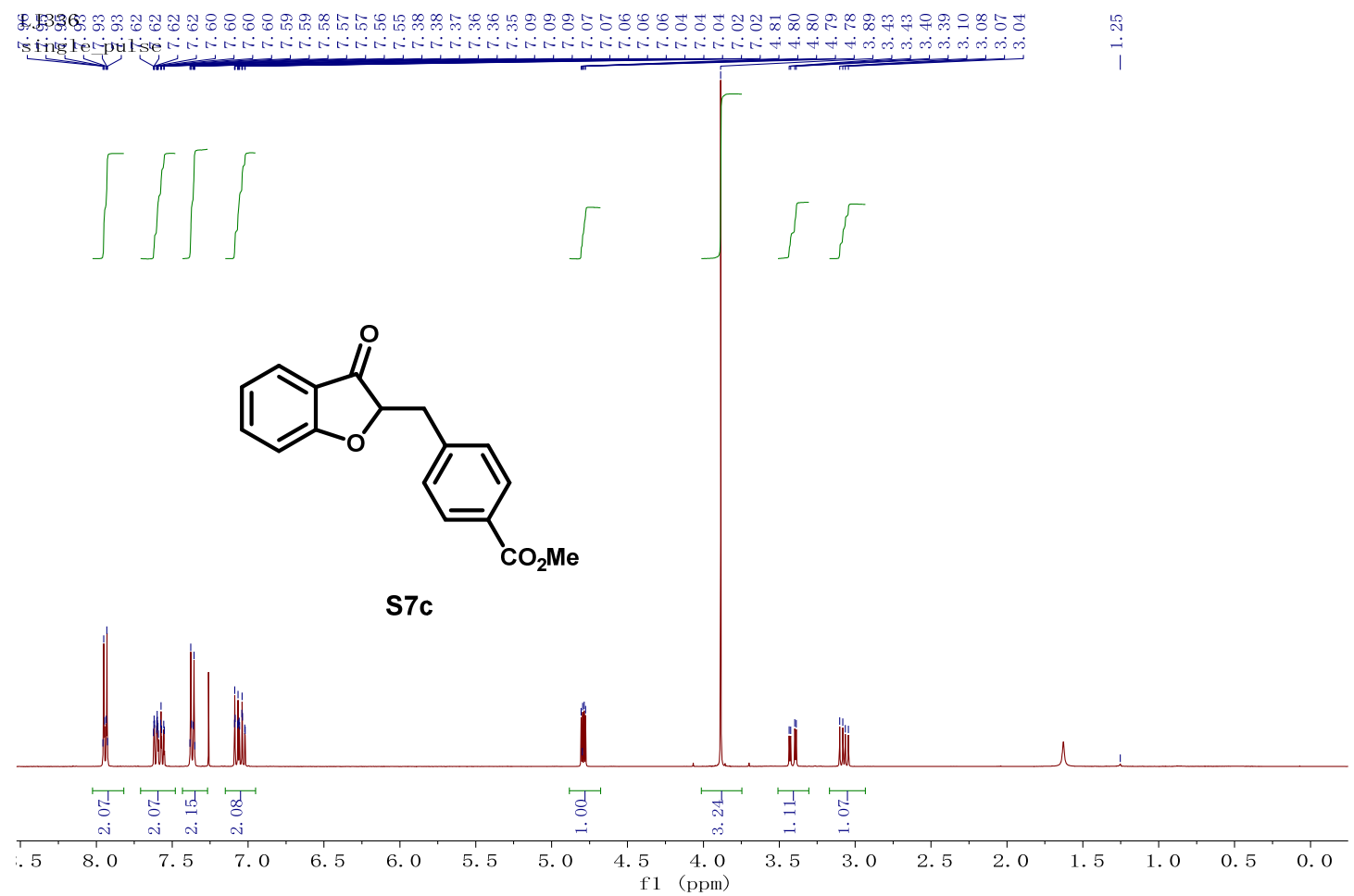

${ }^{13} \mathrm{C}$ NMR $\left(100 \mathrm{MHz}, \mathrm{CDCl}_{3} @ 77\right.$ ppm) 

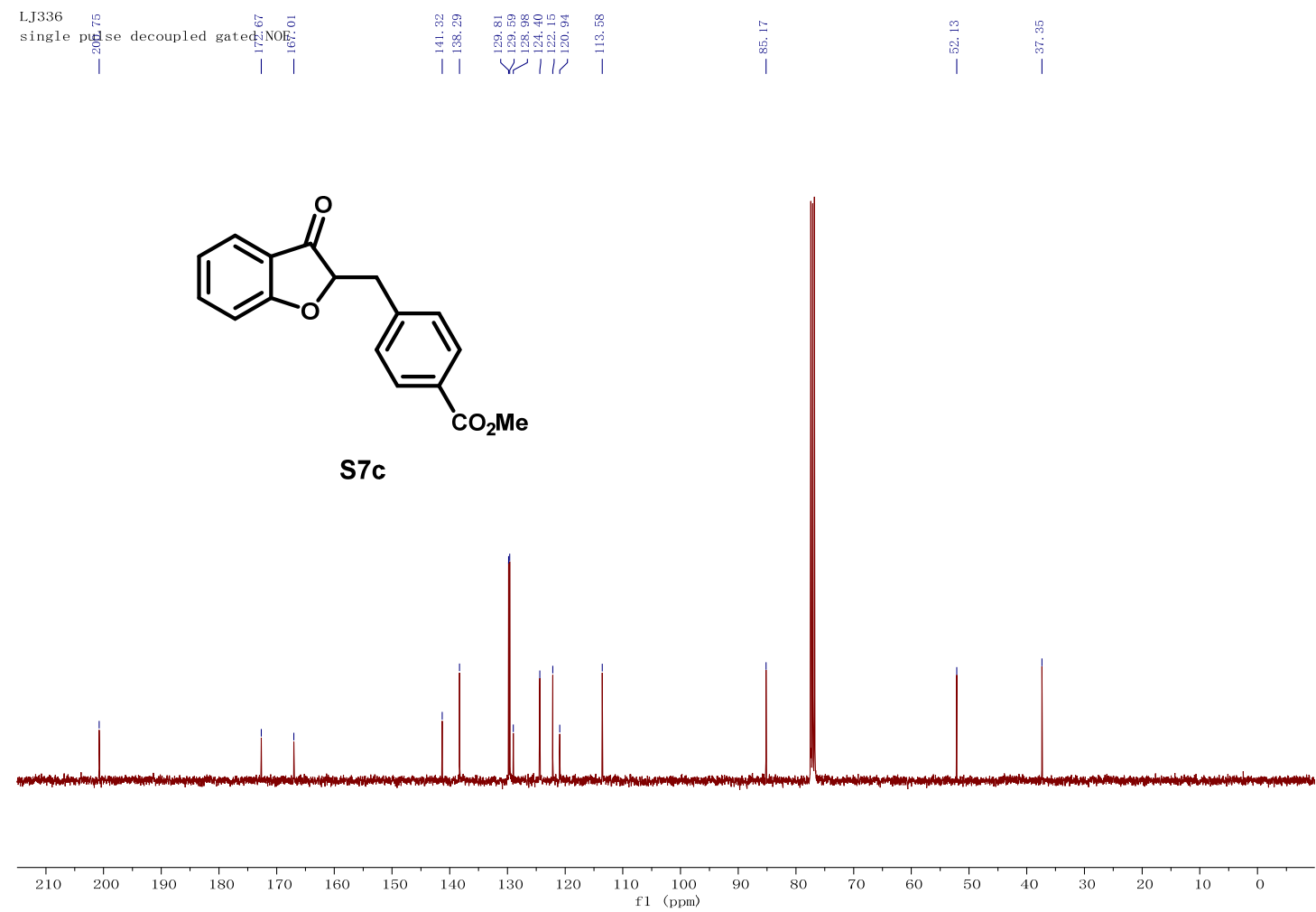

4-((3-Oxo-2,3-dihydrobenzofuran-2-yl)methyl)benzonitrile (S7d)

느 NMR $\left(500 \mathrm{MHz}, \mathrm{CDCl}_{3} @ 7.26\right.$ ppm)

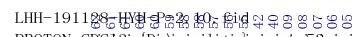

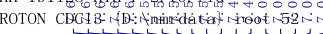

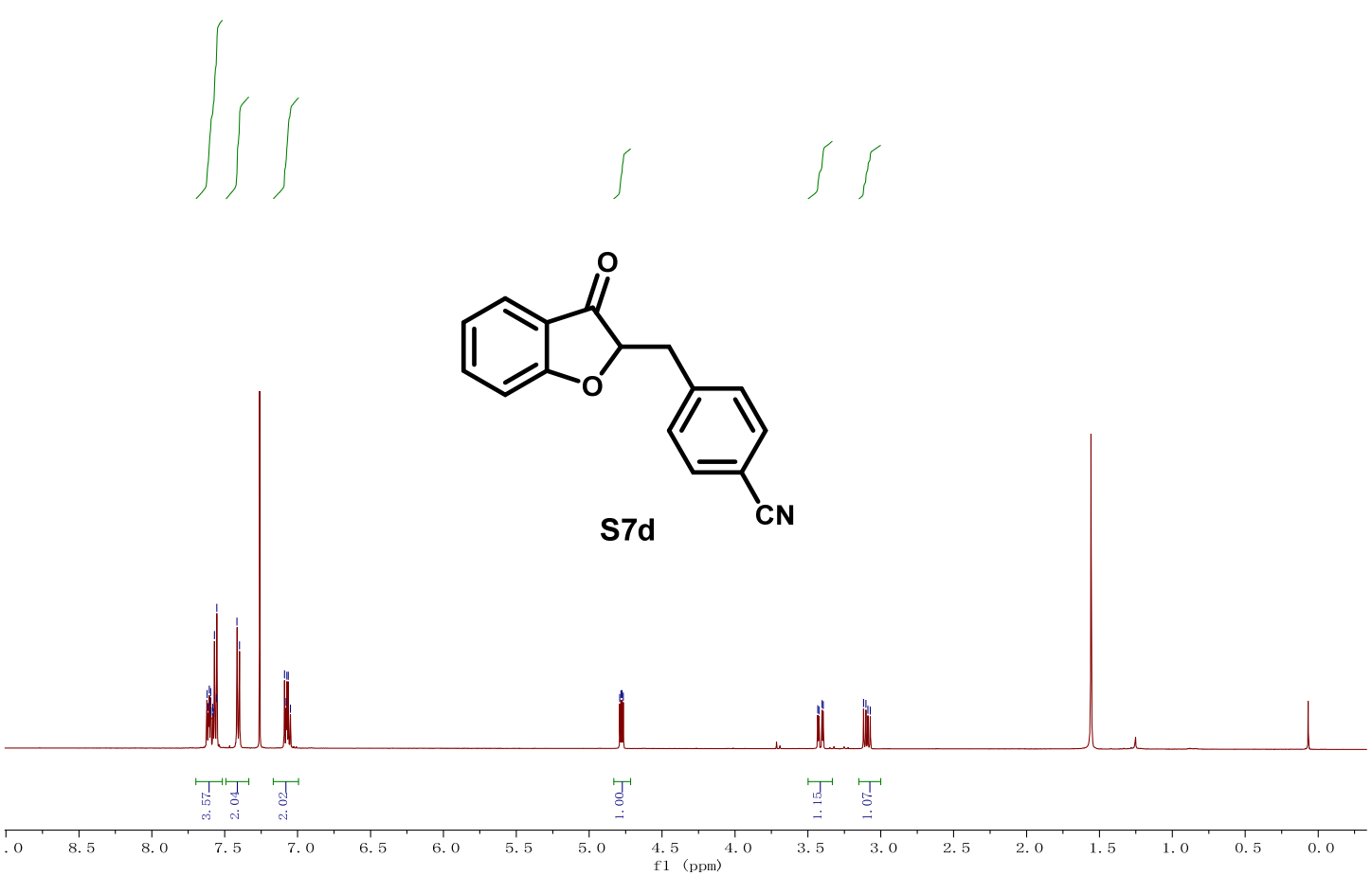



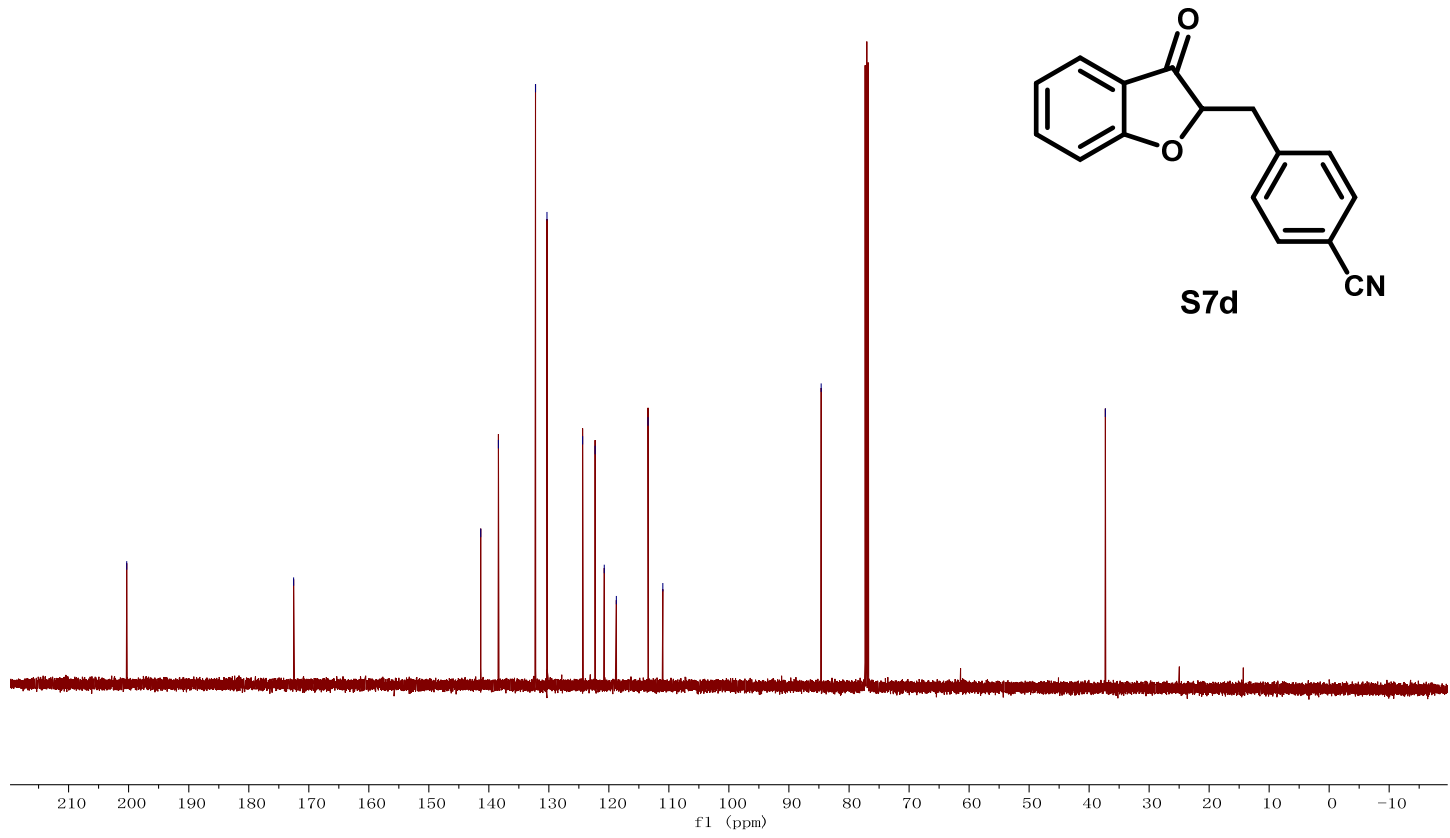

2-(4-(Trifluoromethyl)benzyl)benzofuran-3(2H)-one (S7e)

$\underline{{ }^{1} \mathrm{H} \text { NMR }}\left(500 \mathrm{MHz}, \mathrm{CDCl}_{3} @ 7.26 \mathrm{ppm}\right)$

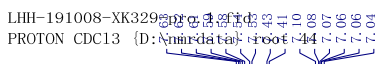
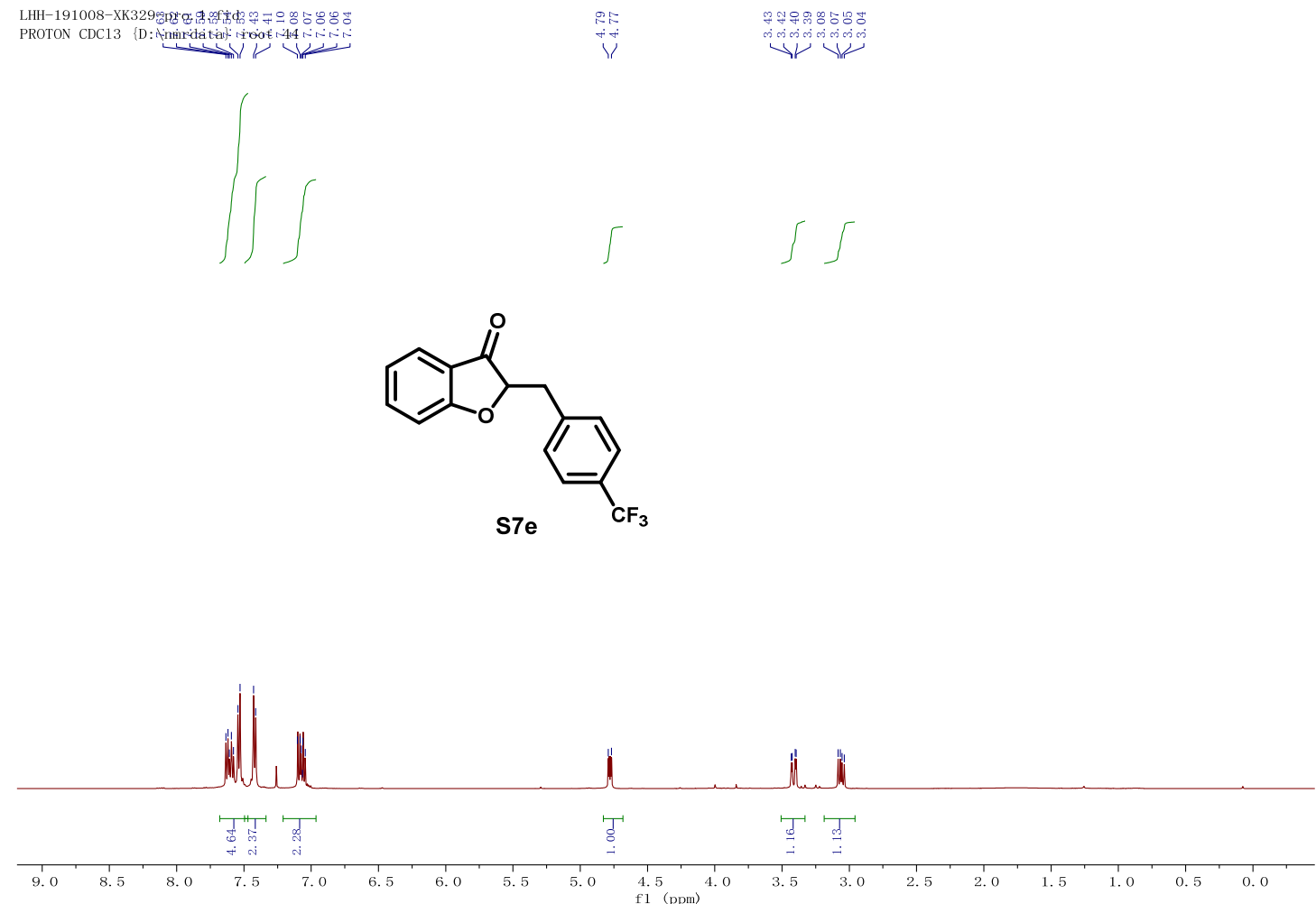
$\underline{{ }^{13} \mathrm{C} \text { NMR }}\left(125 \mathrm{MHz}, \mathrm{CDCl}_{3} @ 77\right.$ ppm)

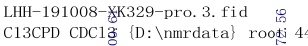

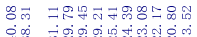

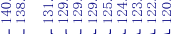

$\infty$
$\infty$
$\infty$
$\infty$
$\infty$
$\infty$
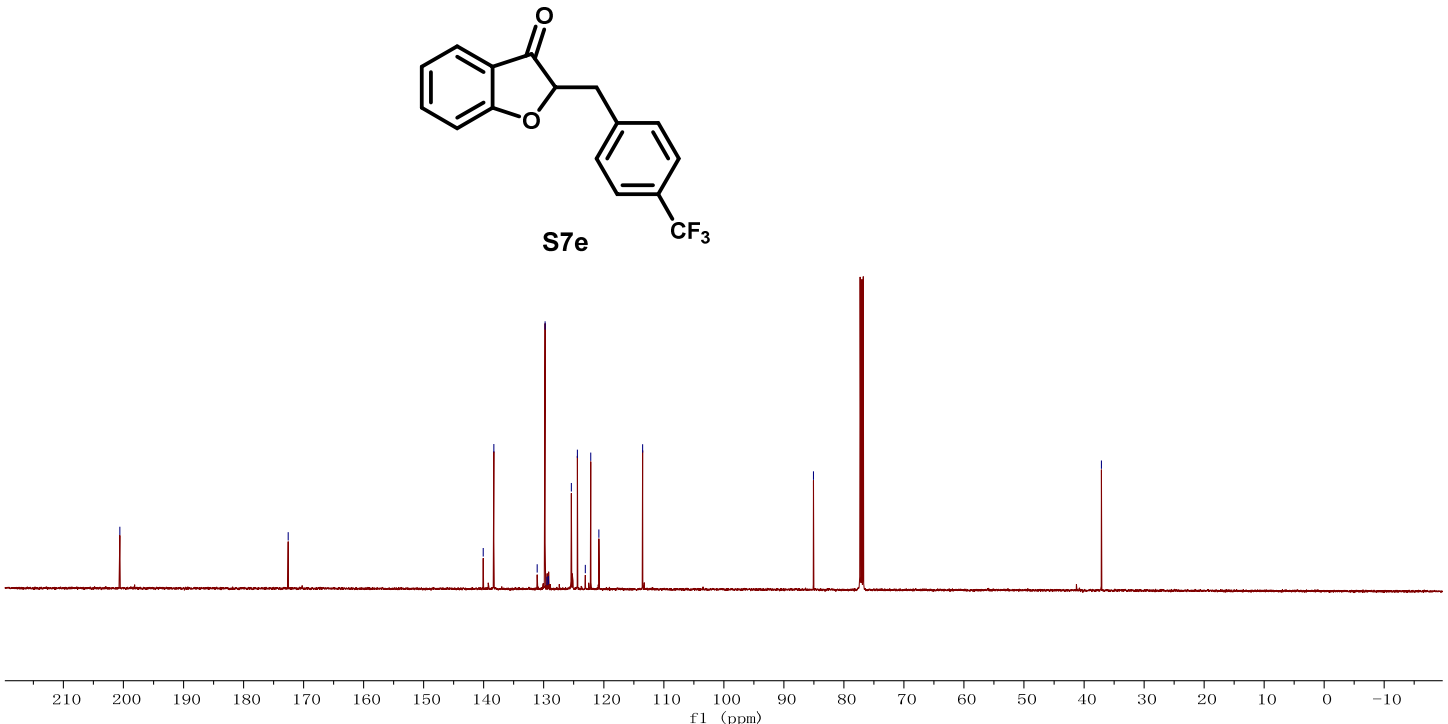

$\underline{{ }^{19} \mathrm{~F} N M R}\left(470 \mathrm{MHz}, \mathrm{CDCl}_{3}\right)$

LHH-191008-ХK329-pro. 2. fid

F19 CDC13 (D:) Inmerdata) root 44

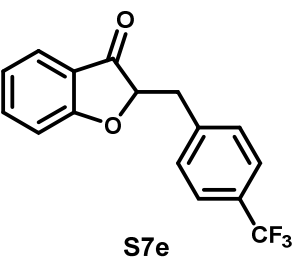

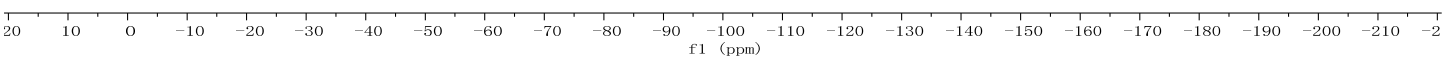

2-(4-Fluorobenzyl)benzofuran-3(2H)-one (S7f)

S128 
1H NMR (400 MHz, $\mathrm{CDCl}_{3} @ 7.26$ ppm)

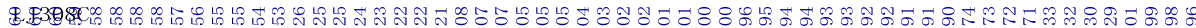

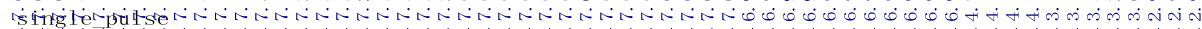

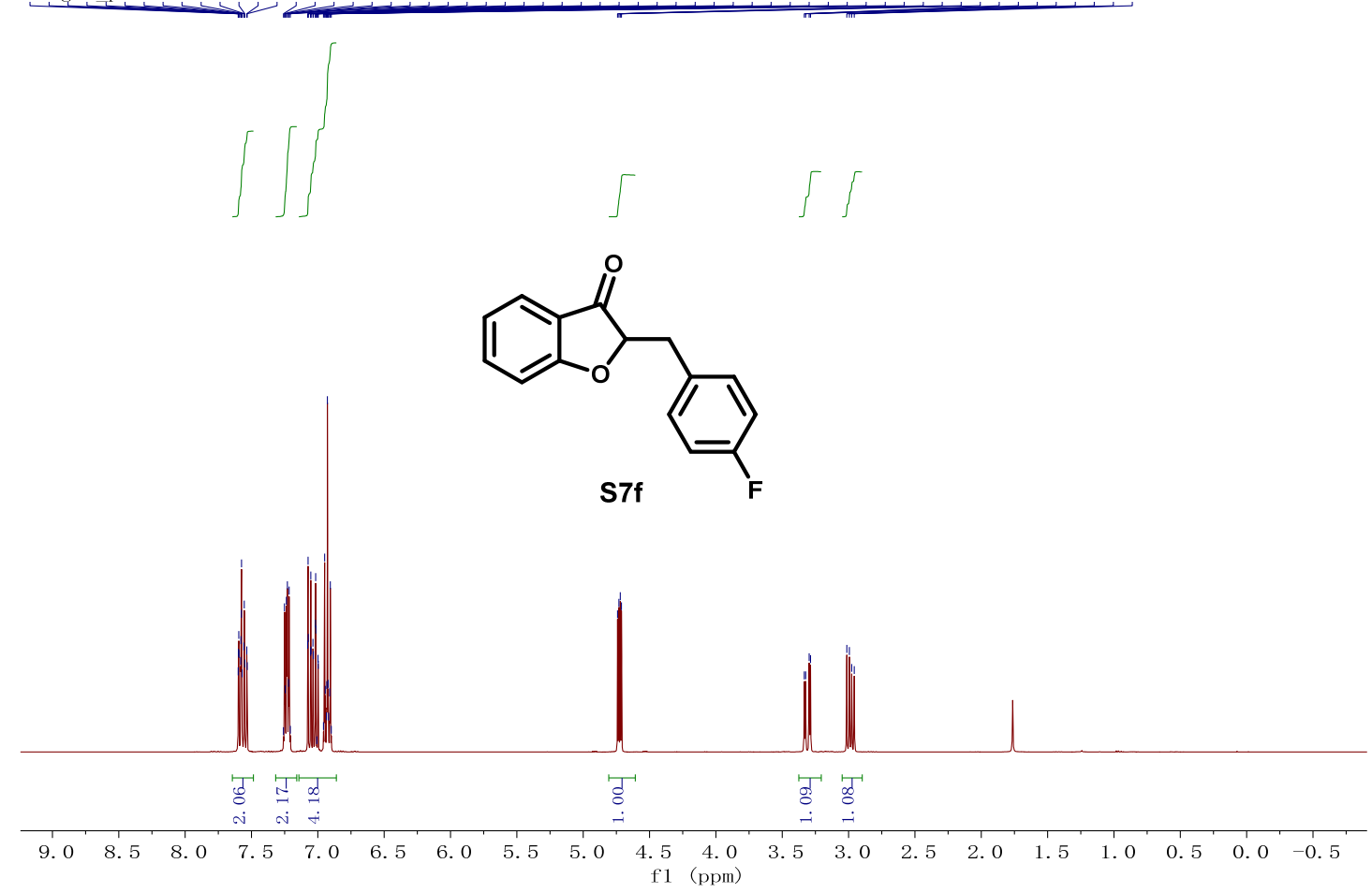

$\underline{{ }^{13} \mathrm{C} N M R}\left(100 \mathrm{MHz}, \mathrm{CDCl}_{3} @ 77\right.$ ppm)

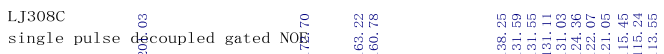

I

(1)
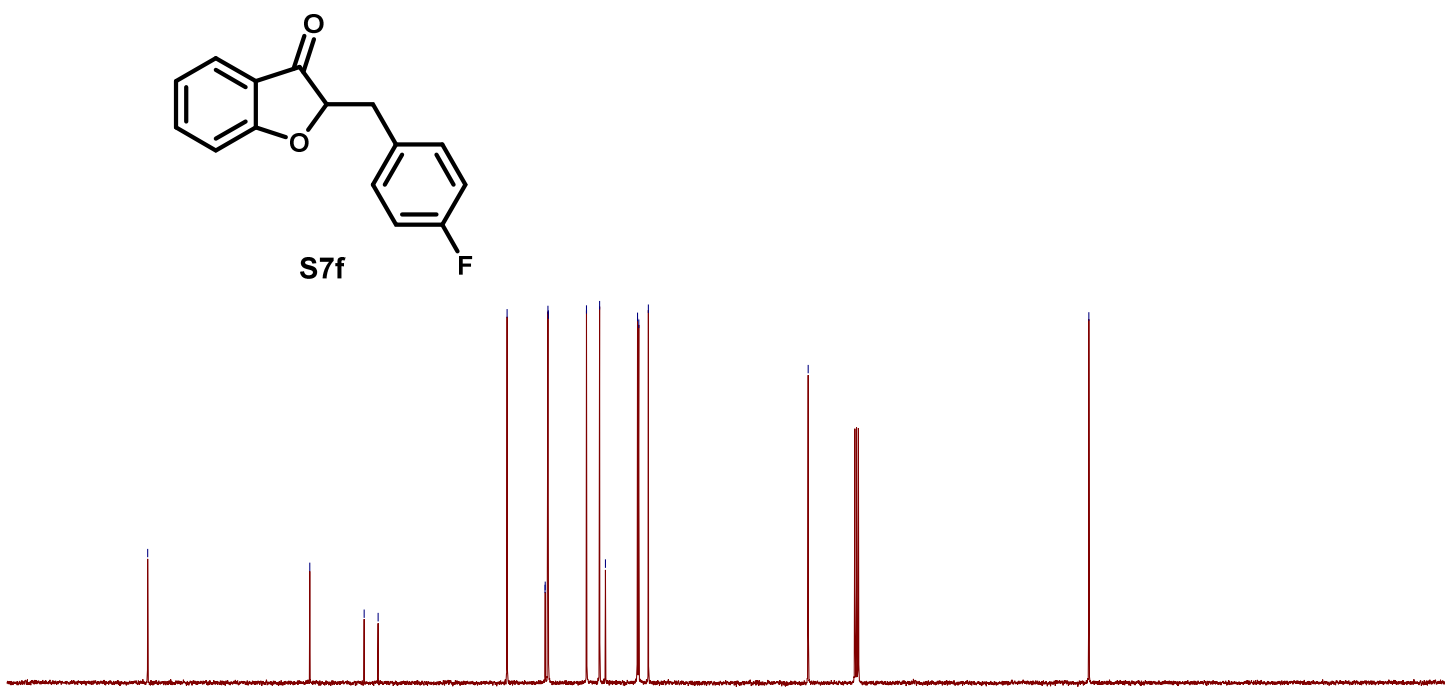

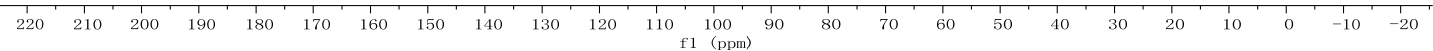


19F NMR (376 MHz, $\mathrm{CDCl}_{3}$ )

LHH-191128-LJ362-F. 10. fid

F19 CDC13 $\{\mathrm{D}: \backslash$ nmmrdata $\}$ root 47

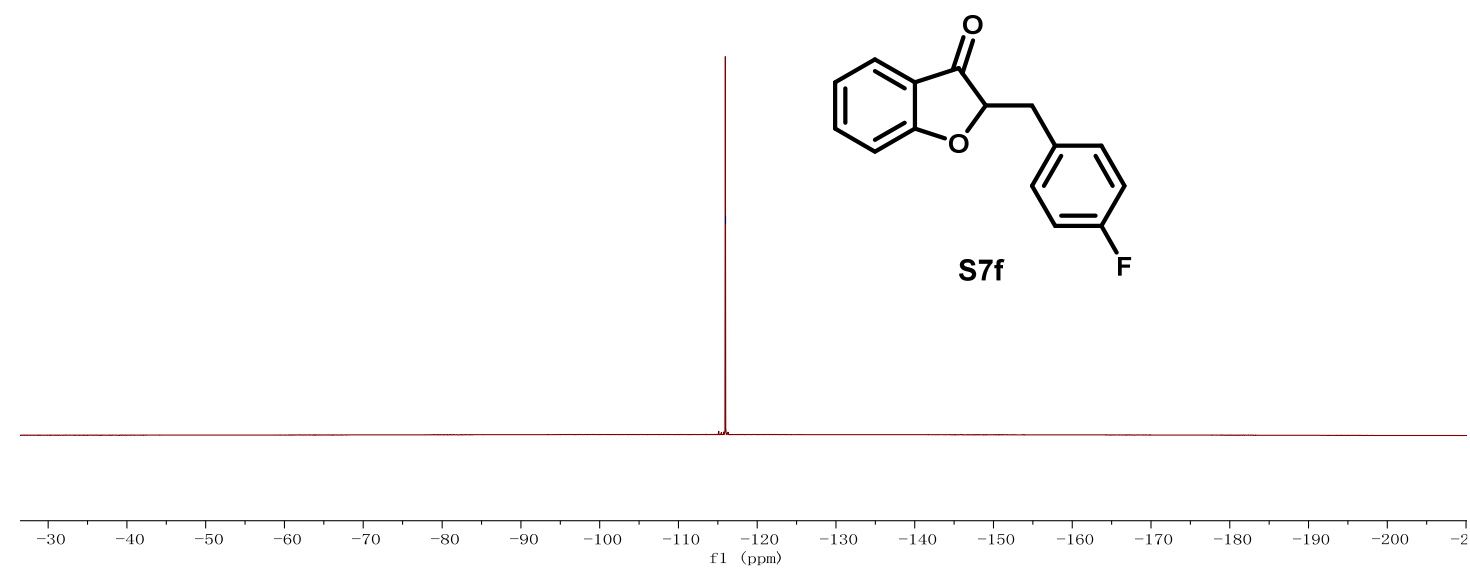

2-(4-Chlorobenzyl)benzofuran-3(2H)-one (S7g)

므 NMR $\left(500 \mathrm{MHz}, \mathrm{CDCl}_{3} @ 7.26\right.$ ppm) 

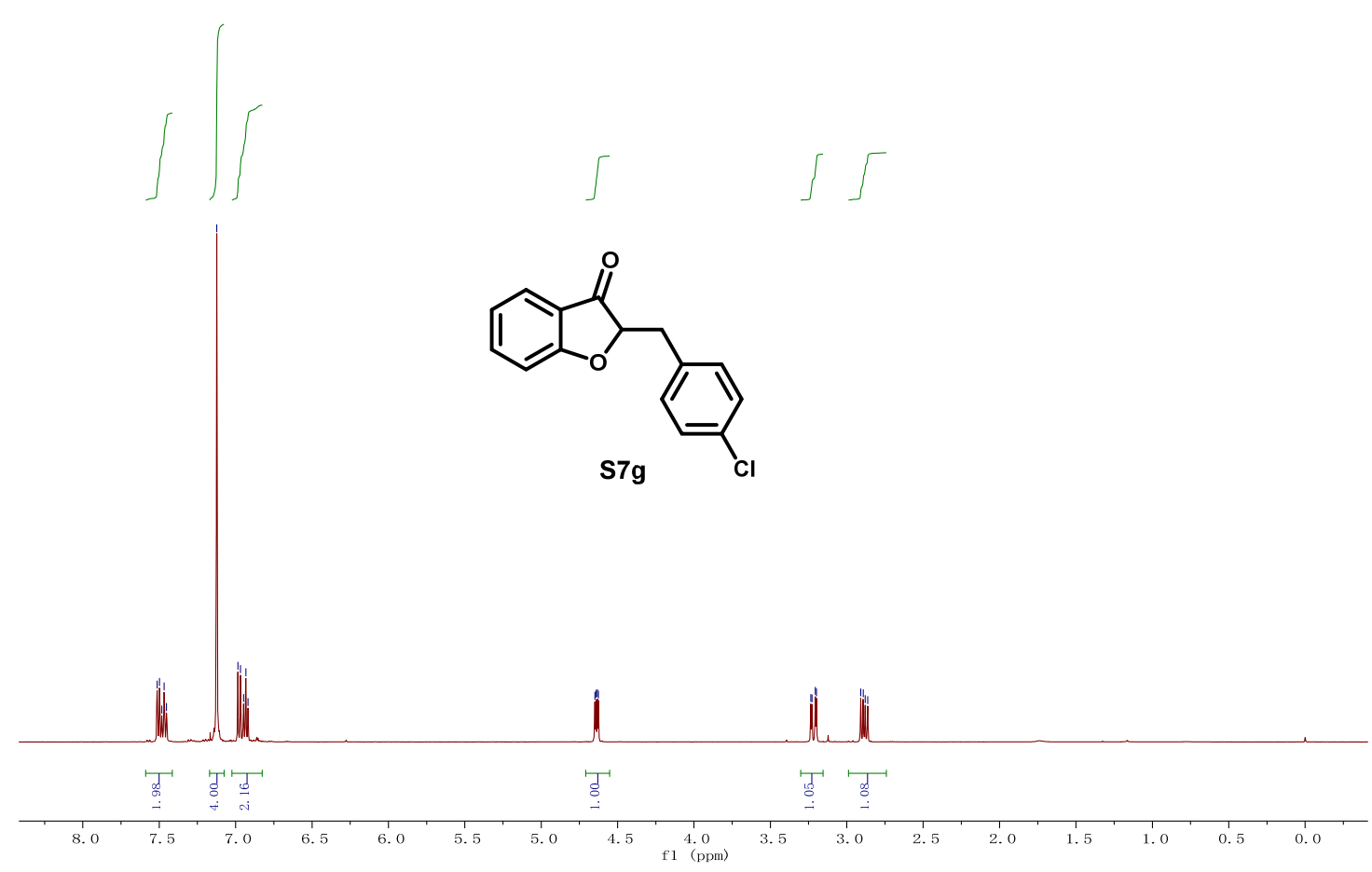

13. NMR (125 MHz, CDCl $@ 77$ ppm)

LHH-190923

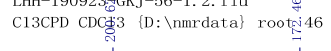
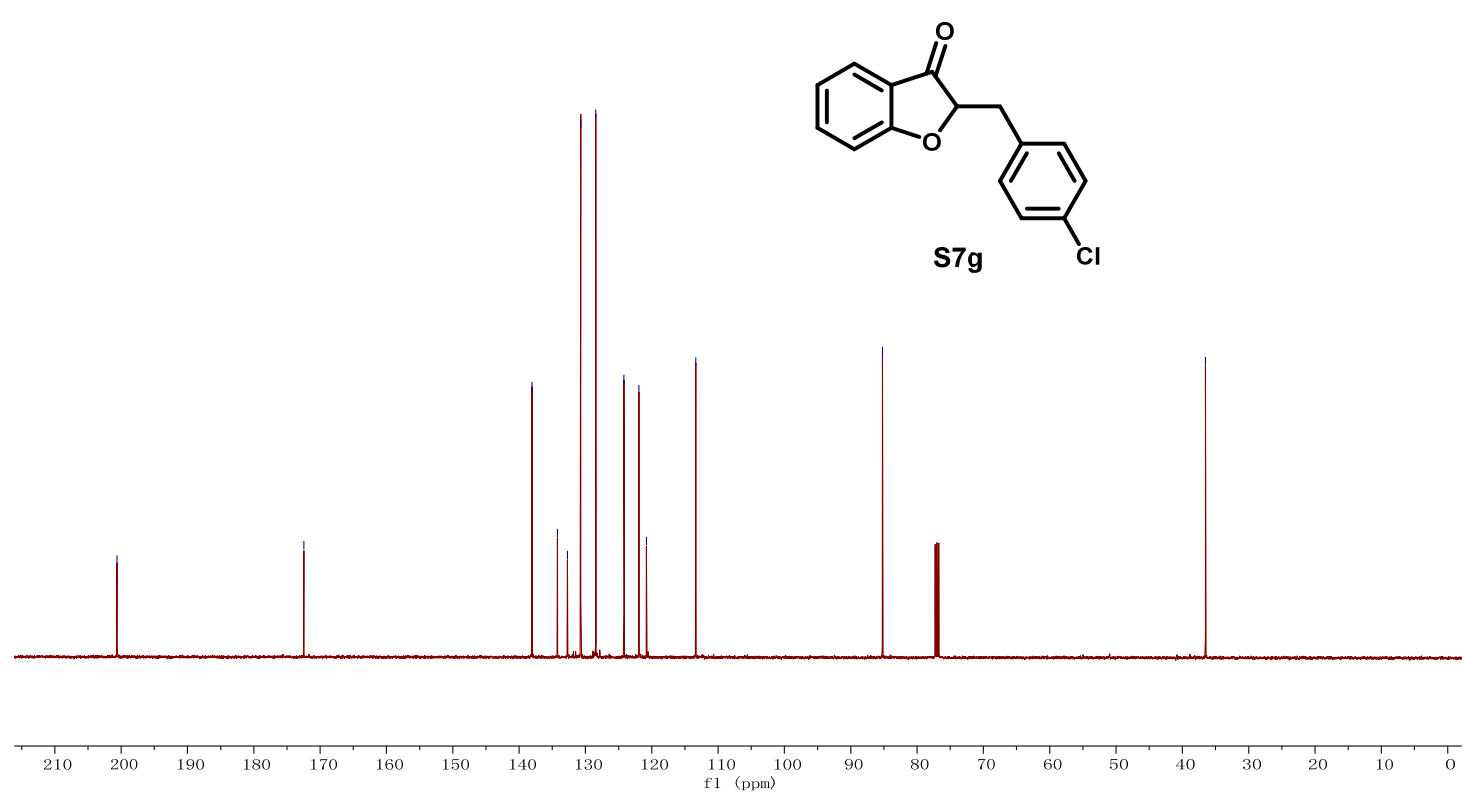

2-(4-Methoxybenzyl)benzofuran-3(2H)-one (S7h)

$\underline{{ }^{1} \mathrm{H} \text { NMR }}\left(400 \mathrm{MHz}, \mathrm{CDCl}_{3} @ 7.26\right.$ ppm) 


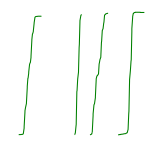<smiles>CC1Oc2ccccc2C1=O</smiles>

s7h<smiles>COc1ccc(C)cc1</smiles>

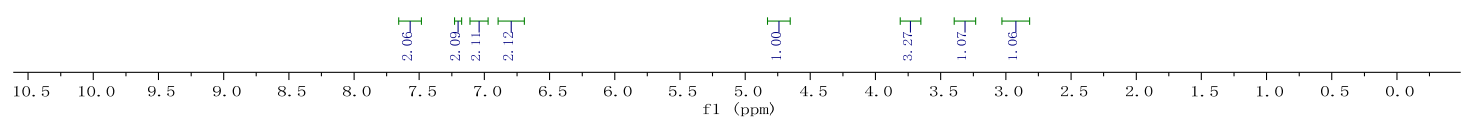

${ }^{13} \mathrm{C}$ NMR $\left(100 \mathrm{MHz}, \mathrm{CDCl}_{3} @ 77\right.$ ppm)
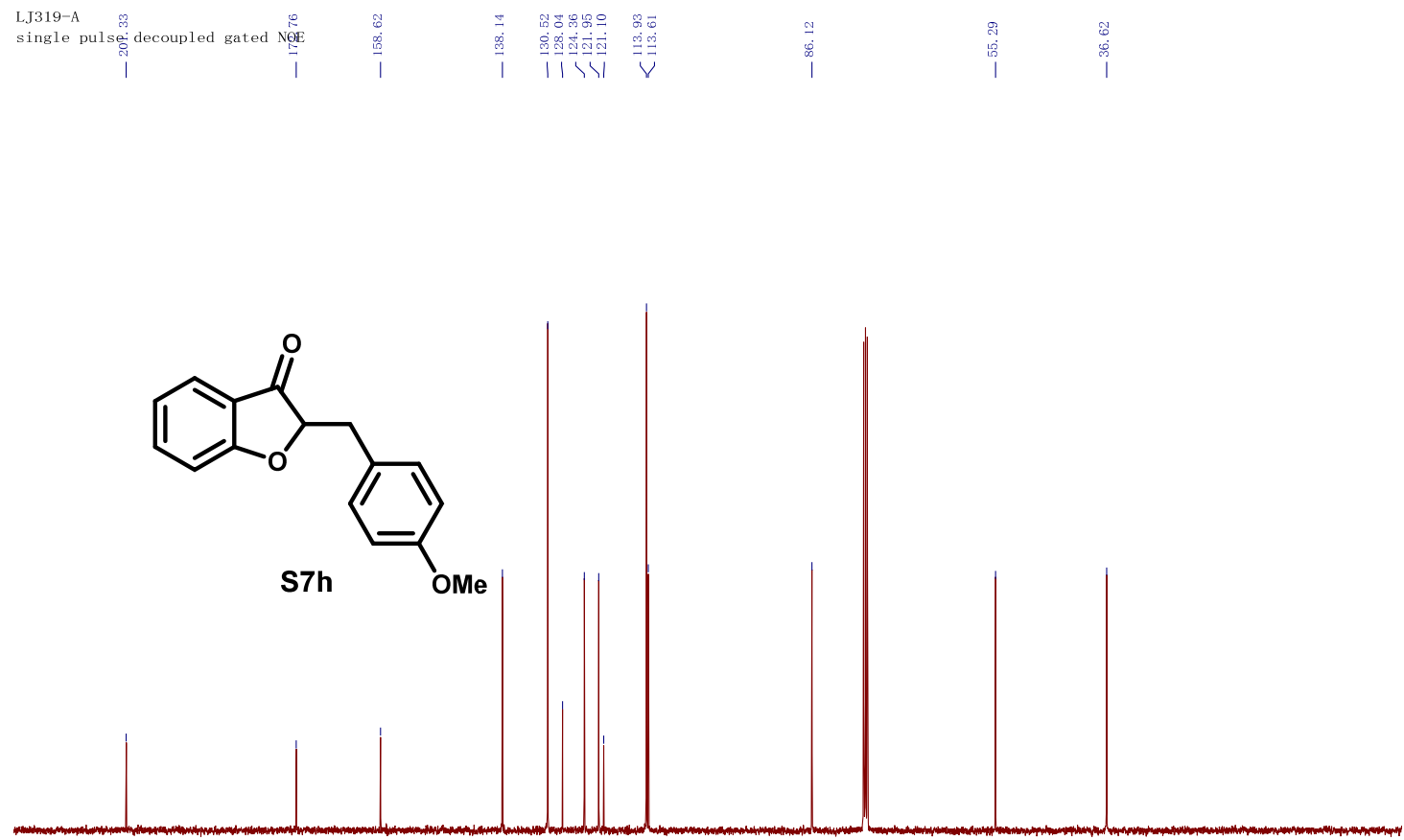

tert-Butyl (4-((3-oxo-2,3-dihydrobenzofuran-2-yl)methyl)phenyl)carbamate (S7i) 
$\underline{{ }^{1} \mathrm{H} \text { NMR }}\left(500 \mathrm{MHz}, \mathrm{CDCl}_{3} @ 7.26\right.$ ppm)

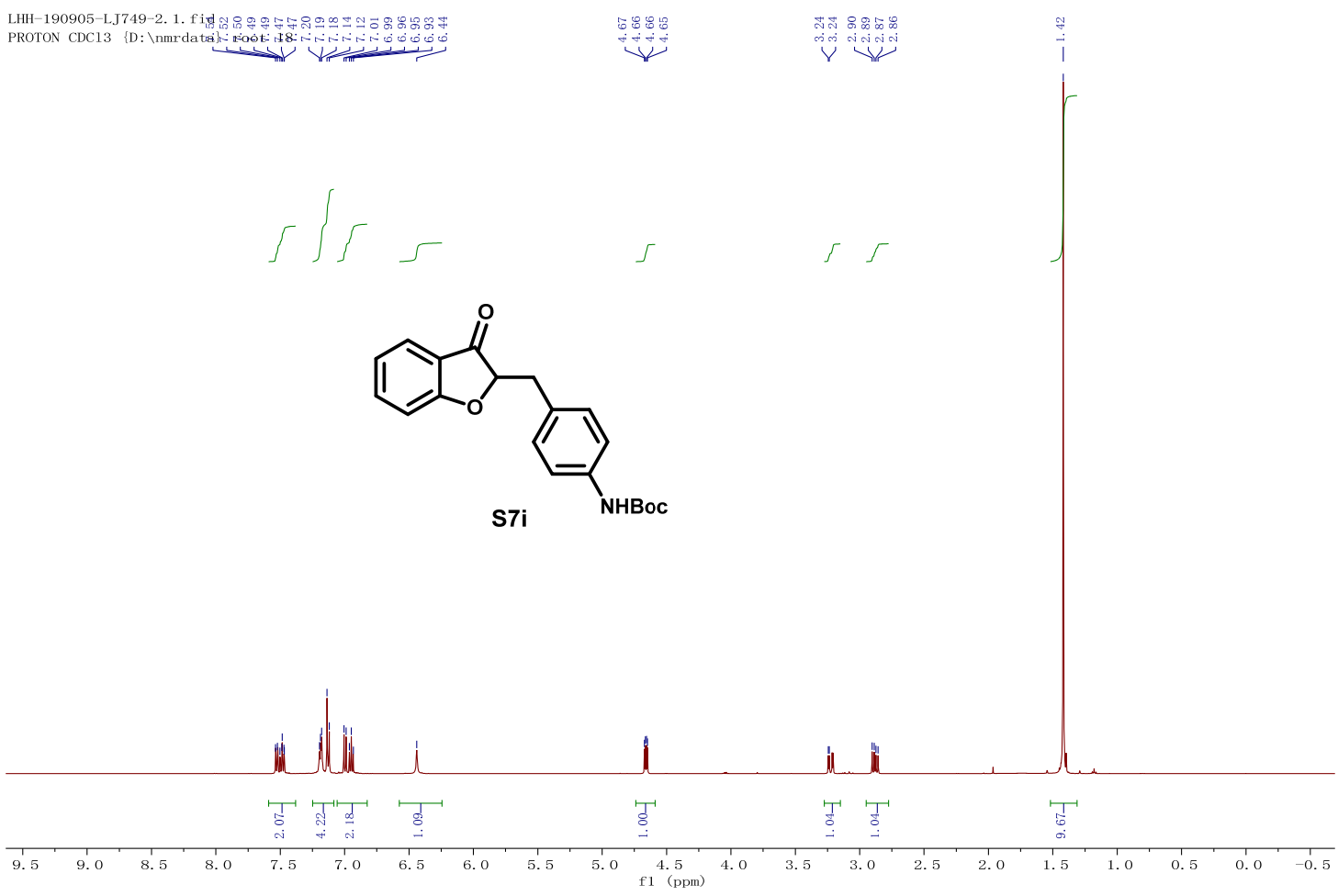

$\underline{{ }^{13} \mathrm{C} \text { NMR }}$ (125 MHz, $\mathrm{CDCl}_{3} @ 77$ ppm)

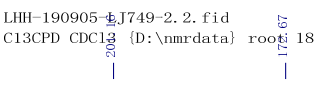

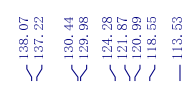

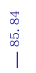

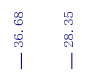
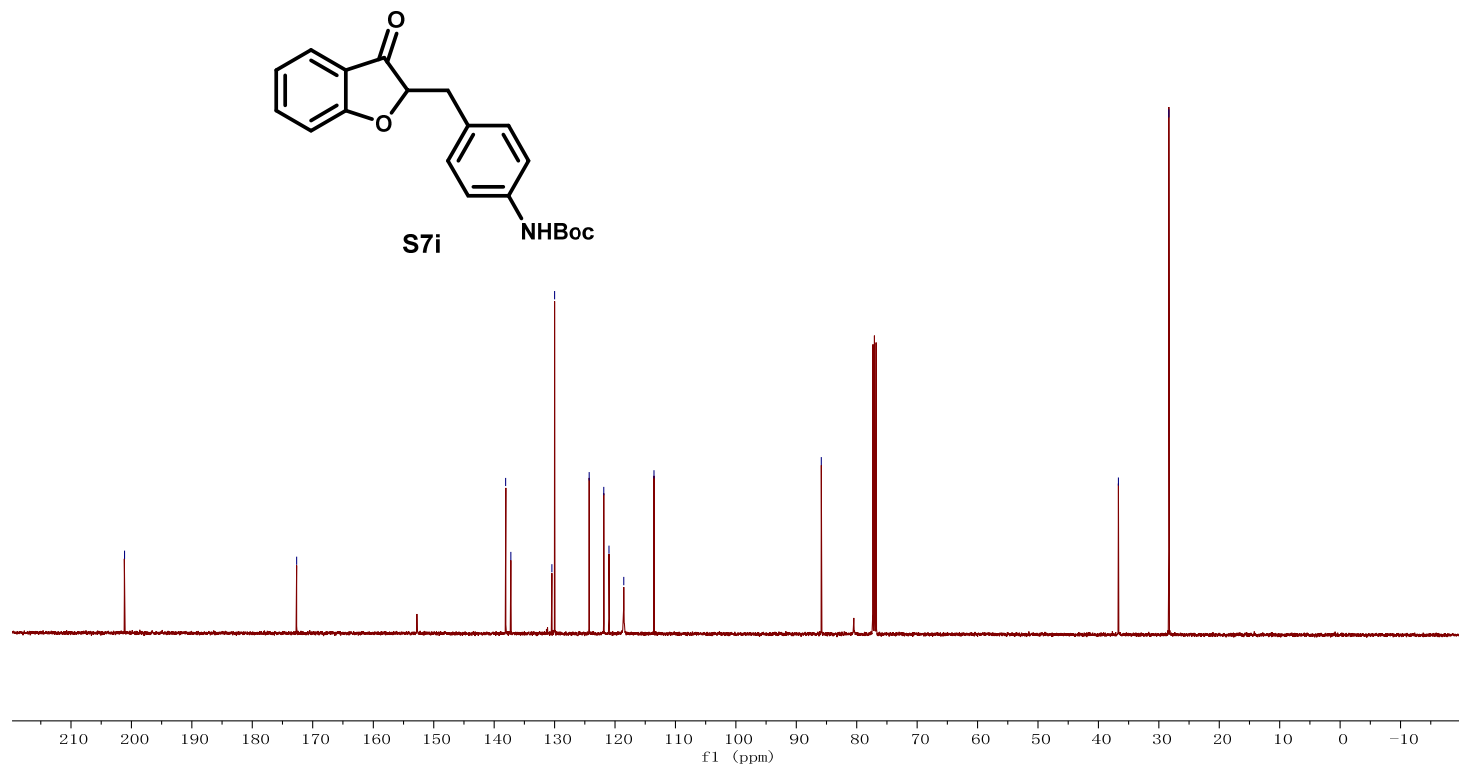

2-(3-Methoxybenzyl)benzofuran-3(2H)-one (S7j)

1․ NMR (400 MHz, $\mathrm{CDCl}_{3} @ 7.26$ ppm)

S133 


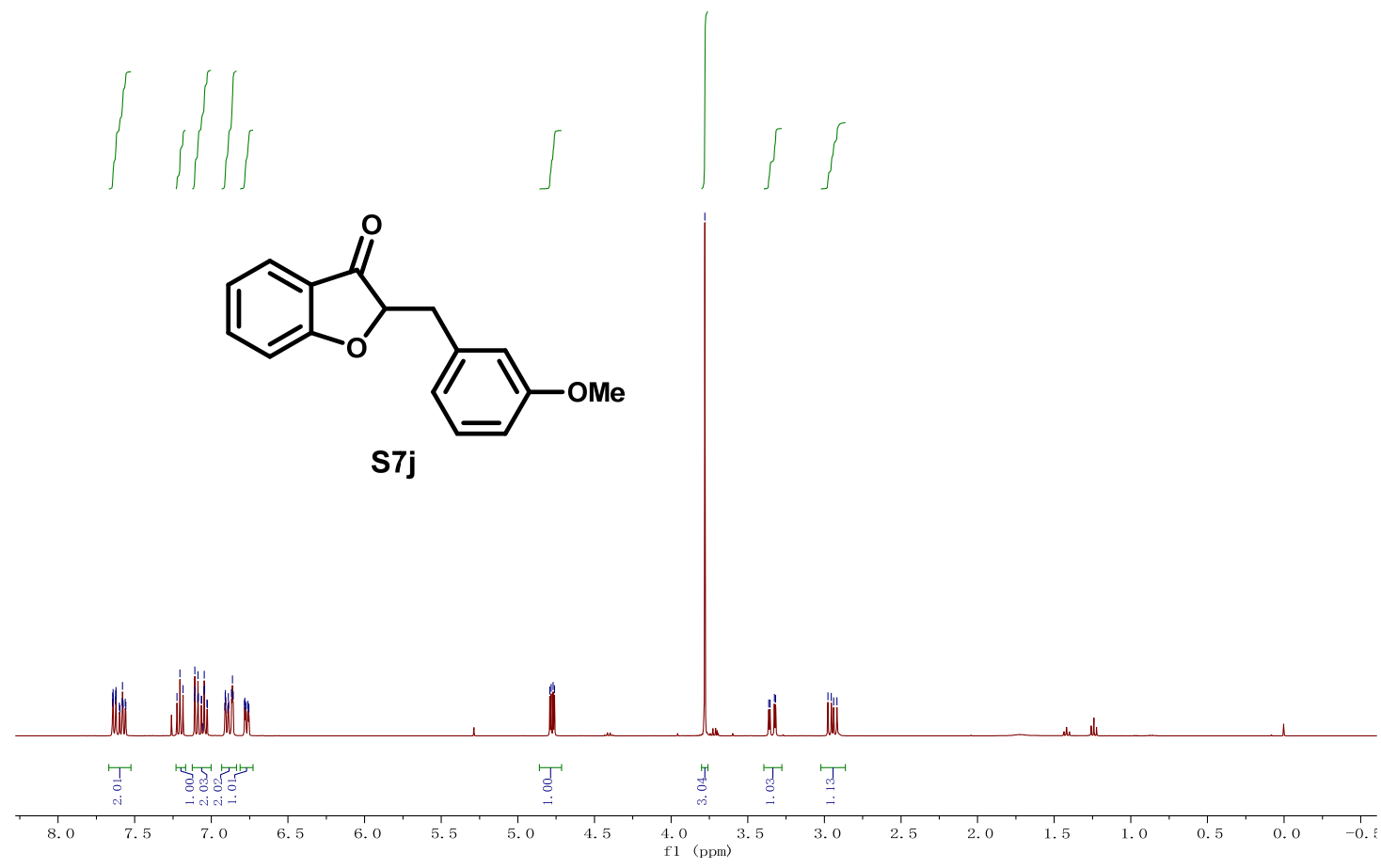

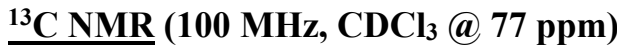
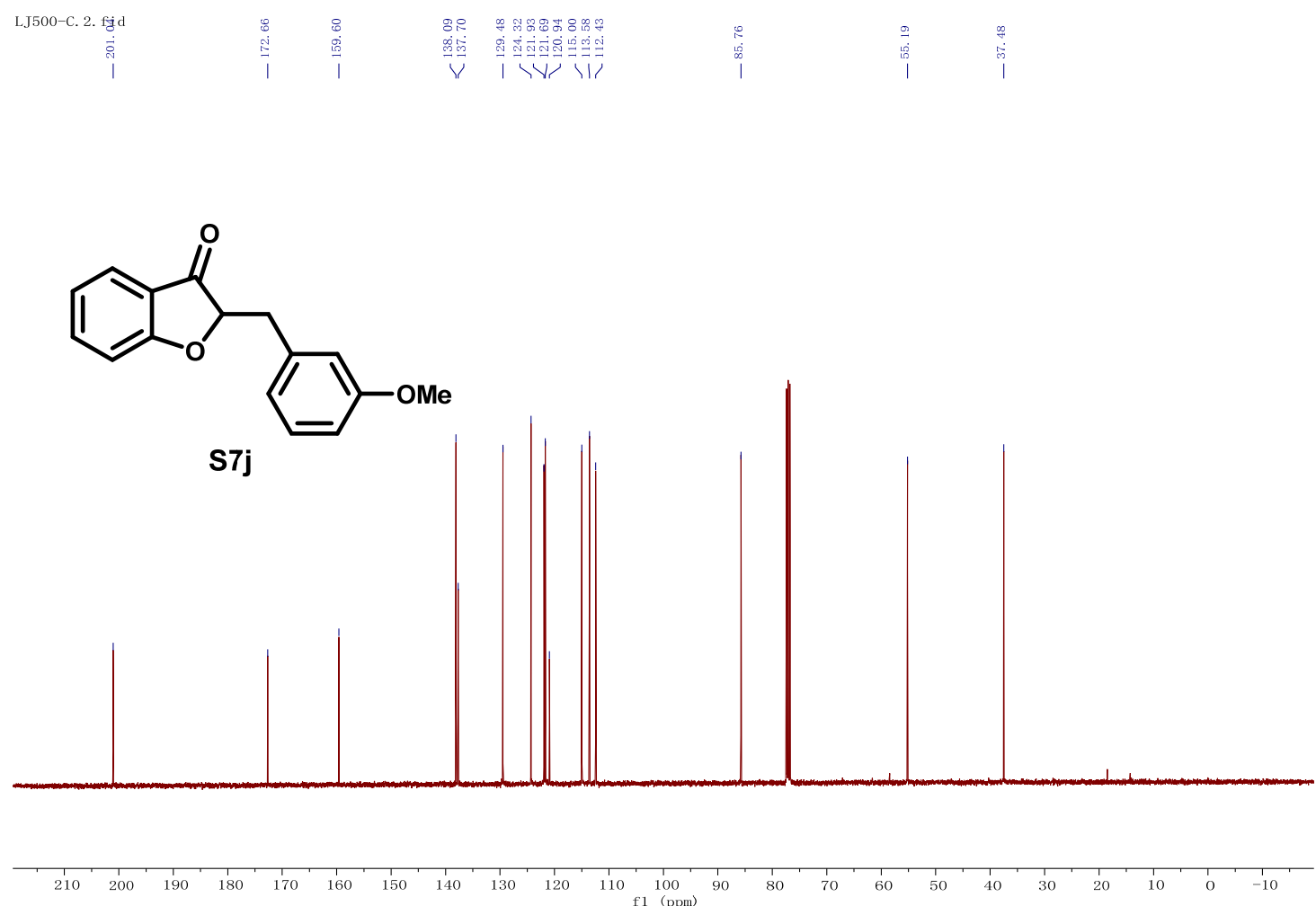

2-(2-Methylbenzyl)benzofuran-3(2H)-one (S7k) 
1 NMR (500 MHz, CDCl 3 @ 7.26 ppm)

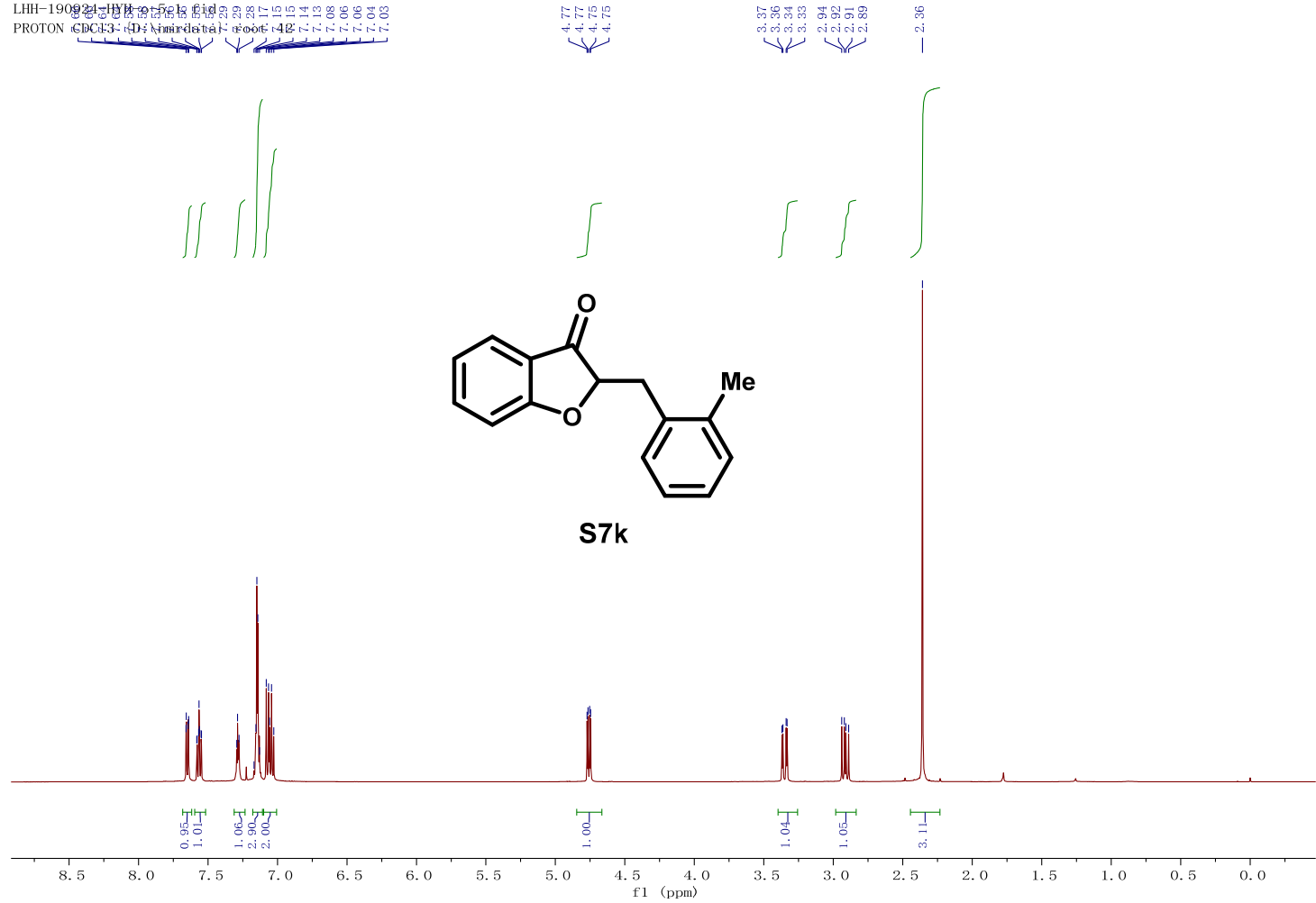

${ }^{13} \mathrm{C}$ NMR (125 MHz, $\mathrm{CDCl}_{3} @ 77$ ppm)

LHH-190924-4YH-o-5. 2. fid क्ष

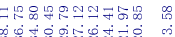

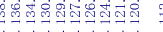

राप 41117

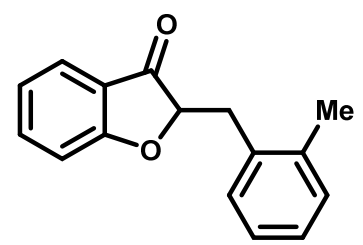

S7k

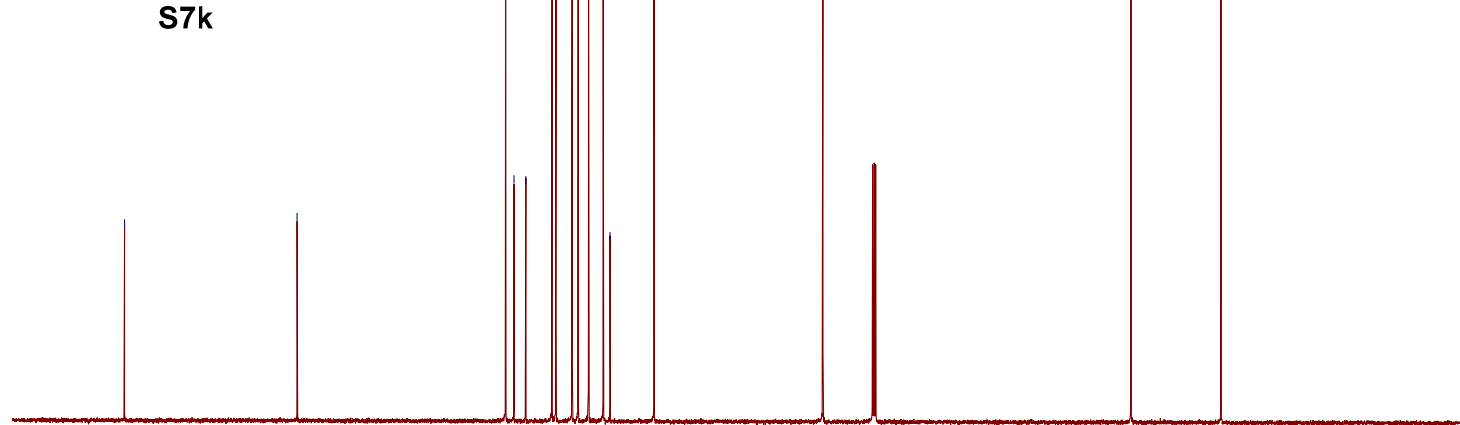

2-(2-Bromobenzyl)benzofuran-3(2H)-one (S7l) 


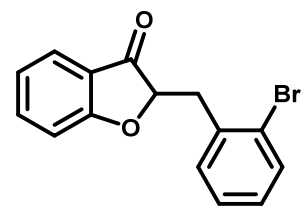

S7I

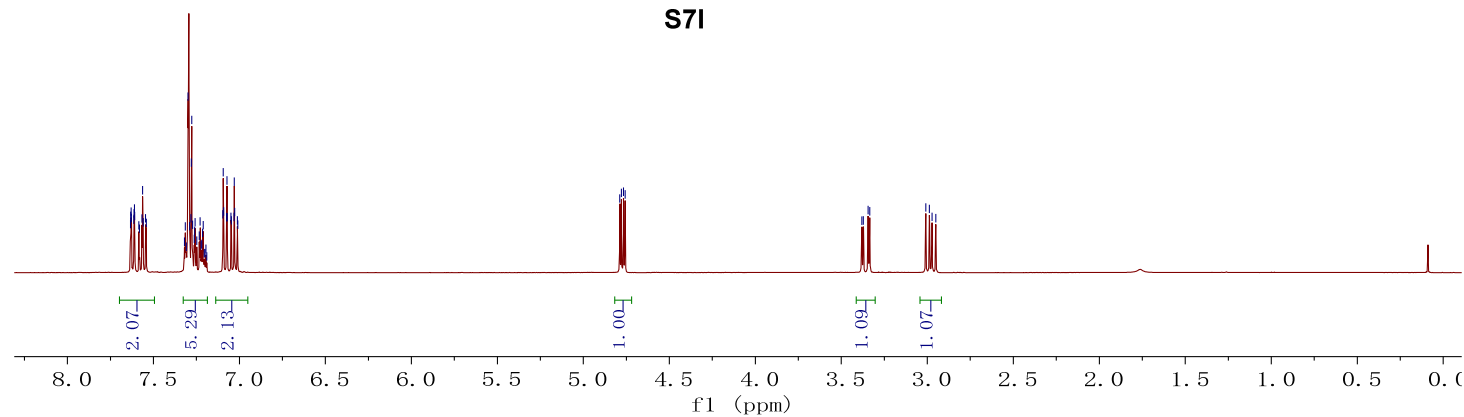

${ }^{13} \mathrm{C}$ NMR $\left(100 \mathrm{MHz}, \mathrm{CDCl}_{3} @ 77\right.$ ppm)

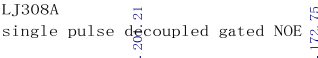

$\infty$
$\infty$
$\infty$

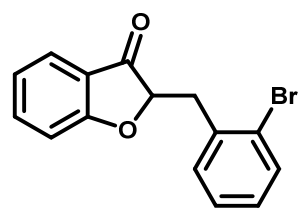

S7I

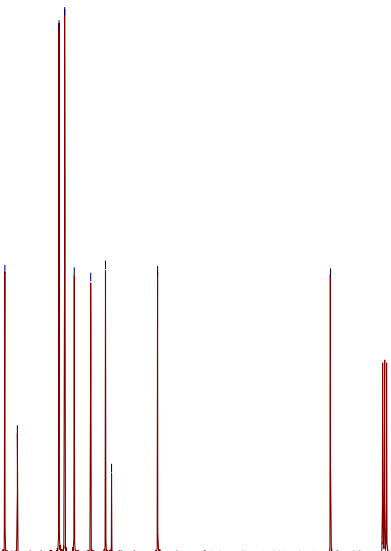

220

$20 \underset{110}{110} 100$ 
2-(2,4,6-Trimethylbenzyl)benzofuran-3(2H)-one (S7m)

1H NMR (500MHz, $\mathrm{CDCl}_{3} @ 7.26$ ppm)

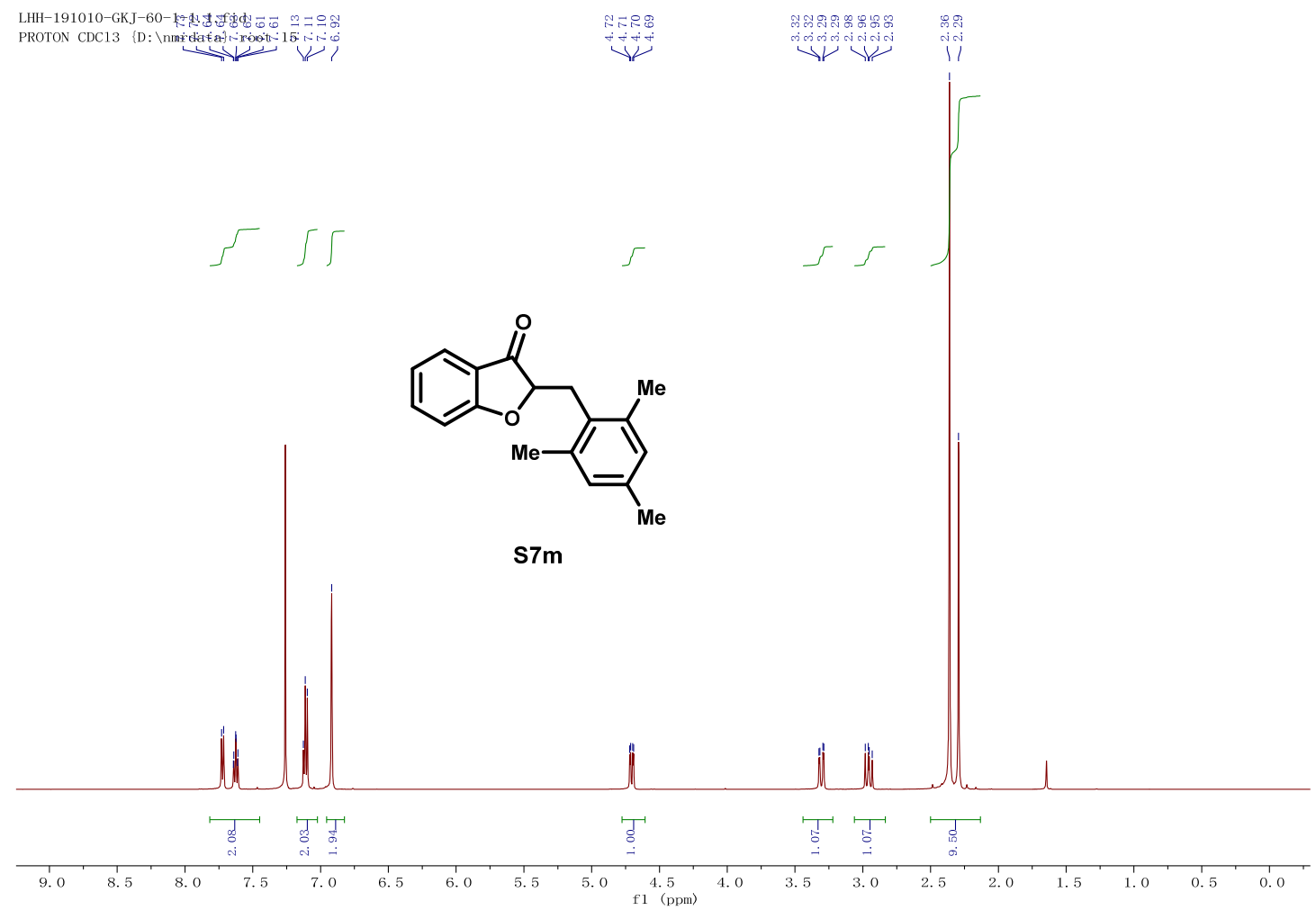

${ }^{13} \mathrm{C}$ NMR (125 MHz, CDCl $@ 77$ ppm)

LHH-191010-6 KJ-60-1-1. 2. fid ஜु C13CPD CDC13 $\{$ D: \nmrdata\} roo梠 15
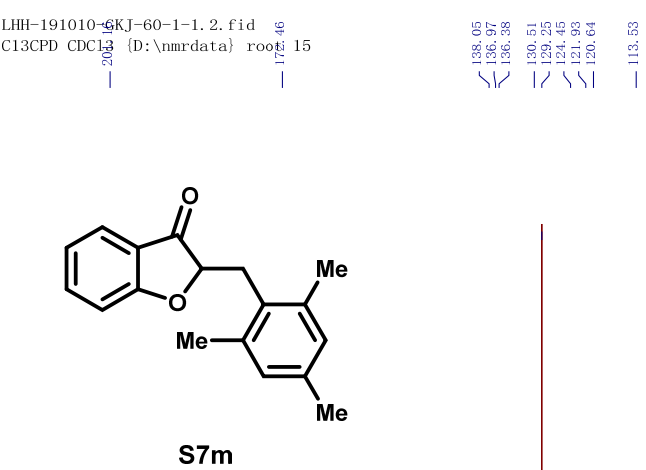

IV |l

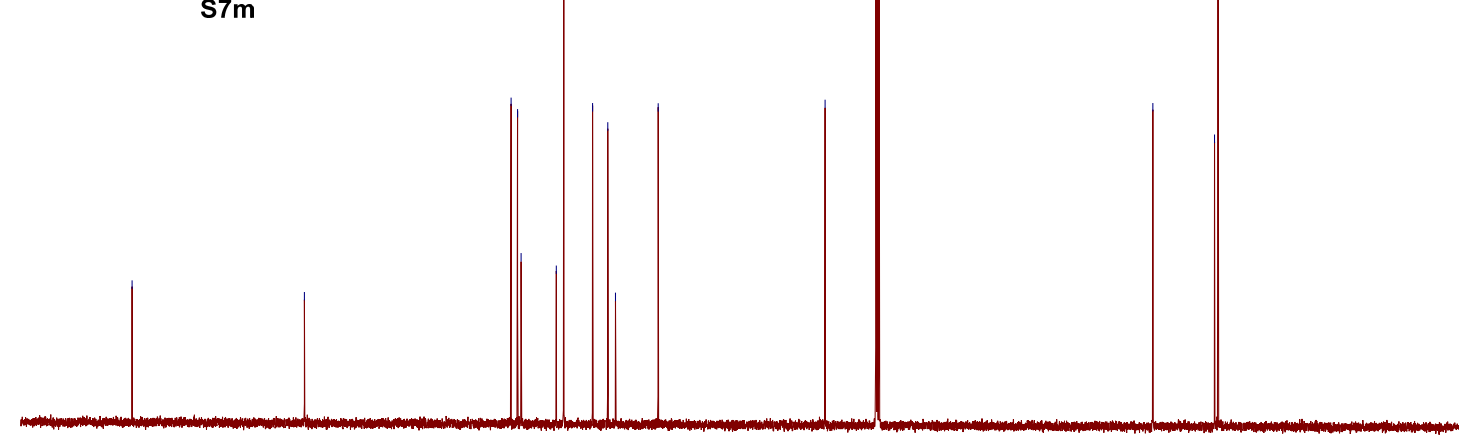

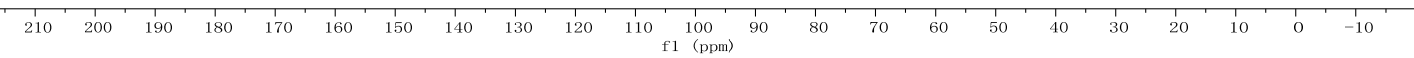


2-(Naphthalen-2-ylmethyl)benzofuran-3(2H)-one (S7n)

$\underline{{ }^{1} \mathrm{H} \text { NMR }}\left(400 \mathrm{MHz}, \mathrm{CDCl}_{3} @ 7.26\right.$ ppm)

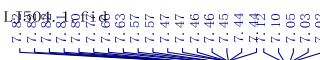
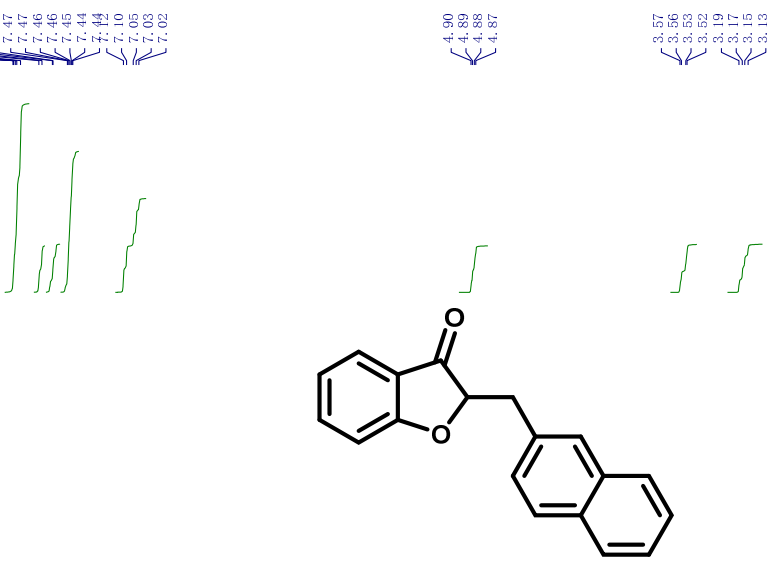

S7n

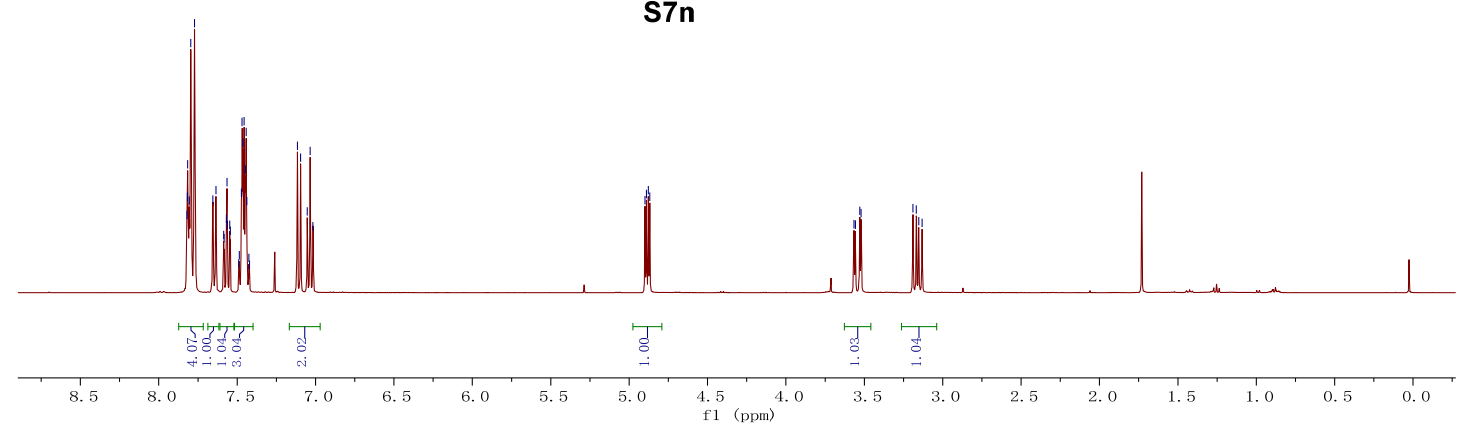

${ }^{13} \mathrm{C}$ NMR (100 MHz, CDCl 3977 ppm)

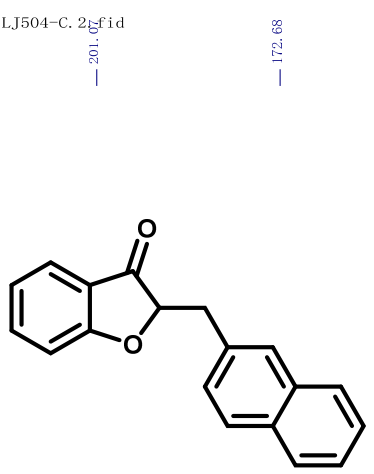

S7n
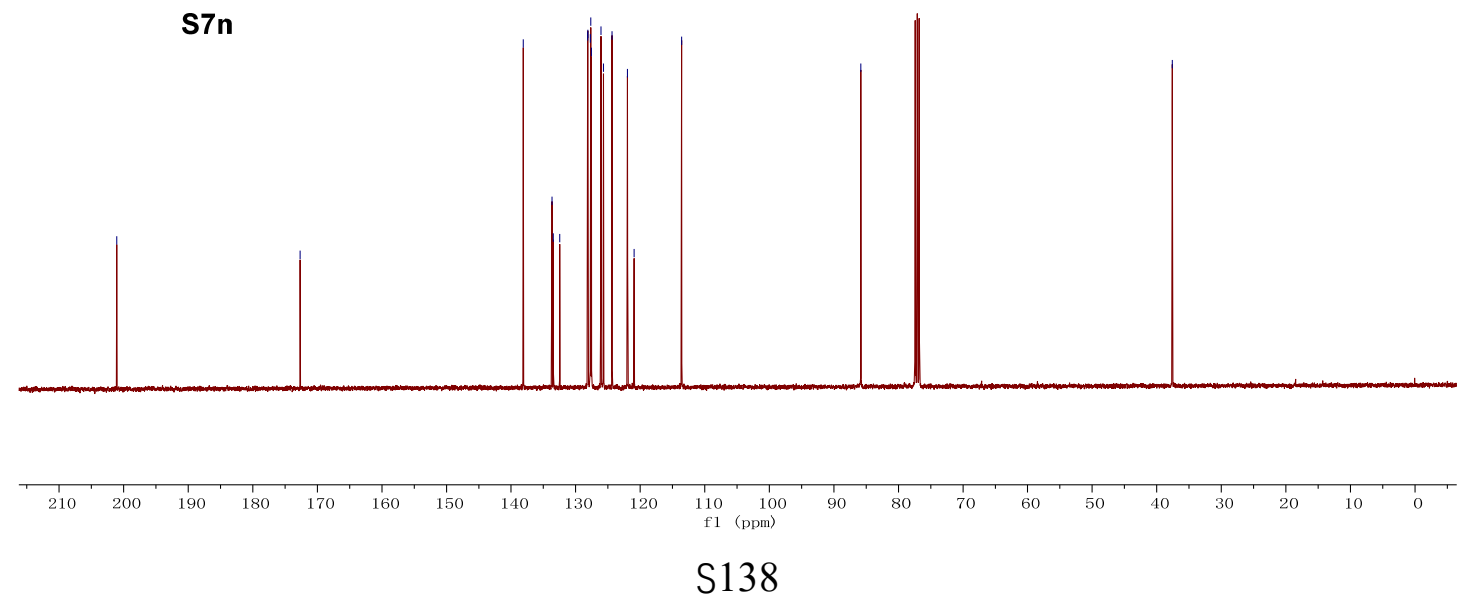
2-(Naphthalen-1-ylmethyl)benzofuran-3(2H)-one (S7o)

1H NMR (500 MHz, $\mathrm{CDCl}_{3} @ 7.26$ ppm)

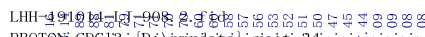

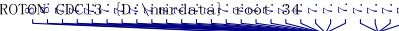

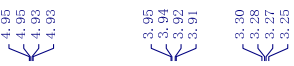

Suld
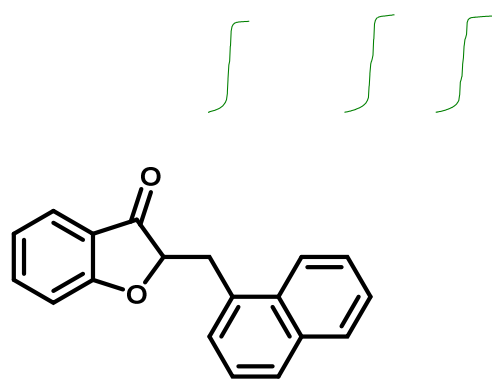

S7o
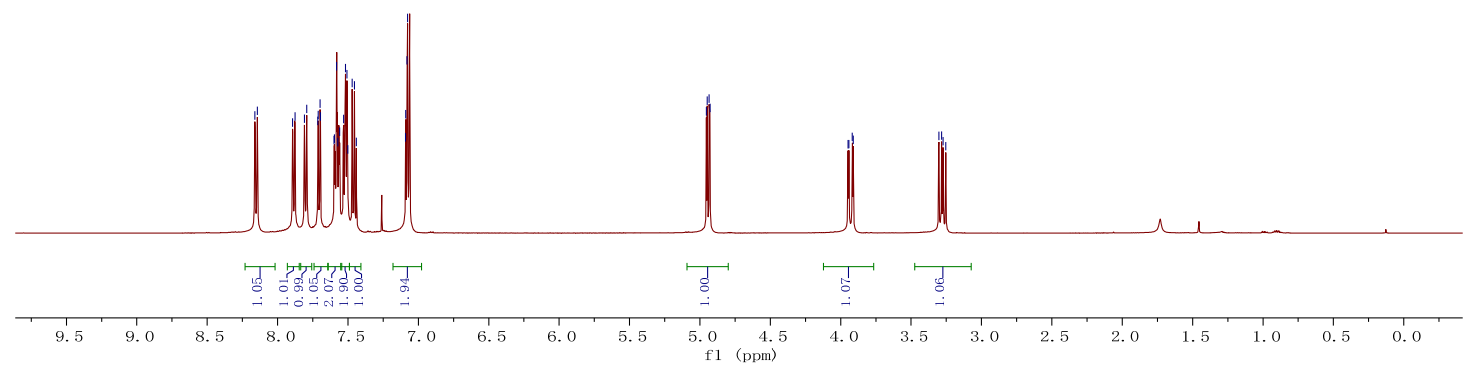

${ }^{13} \mathrm{C}$ NMR (125 MHz, $\mathrm{CDCl}_{3} @ 77$ ppm)

LHH-191044-LJ-908. 3. fid
C13CPD GDC13
\{D: \nmrdata\} rojot 34

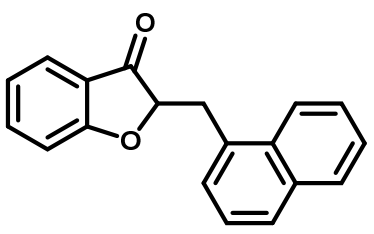

S7o

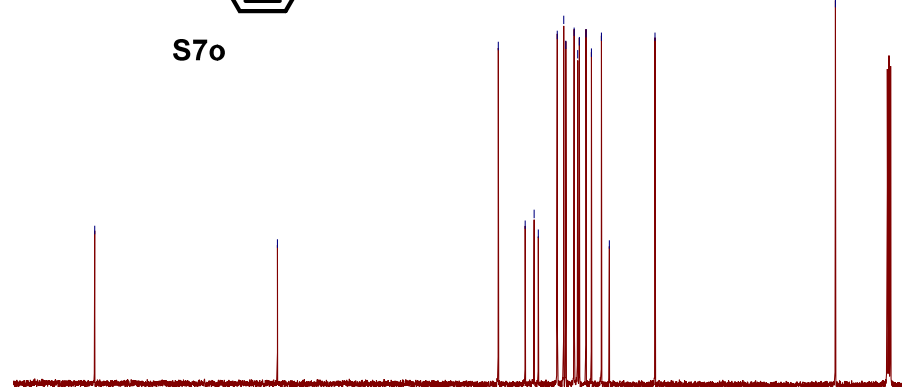

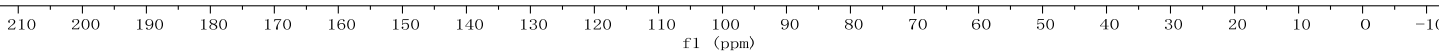




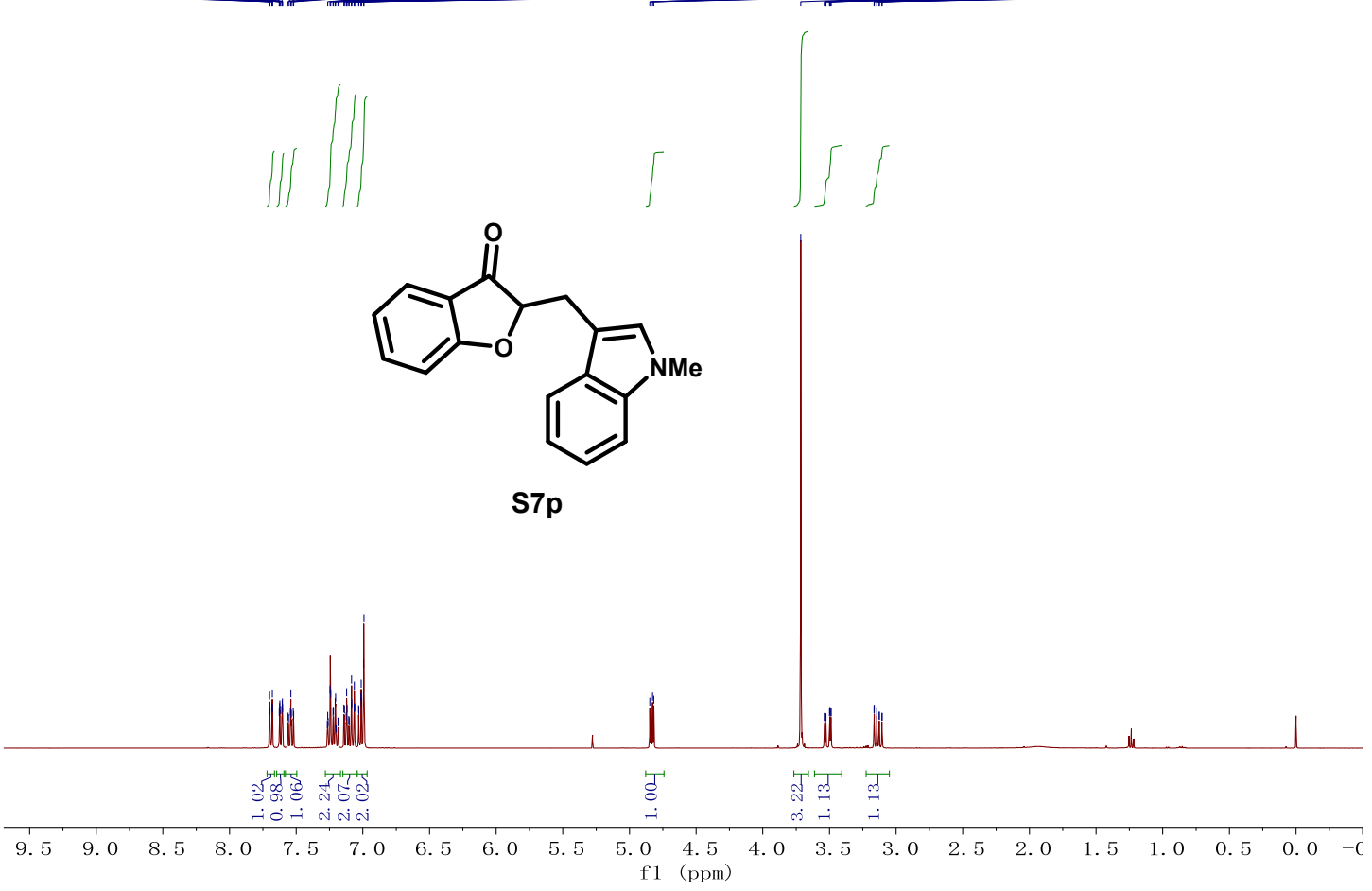

$\underline{{ }^{13} \mathrm{C} \text { NMR }}\left(100 \mathrm{MHz}, \mathrm{CDCl}_{3} @ 77\right.$ ppm)

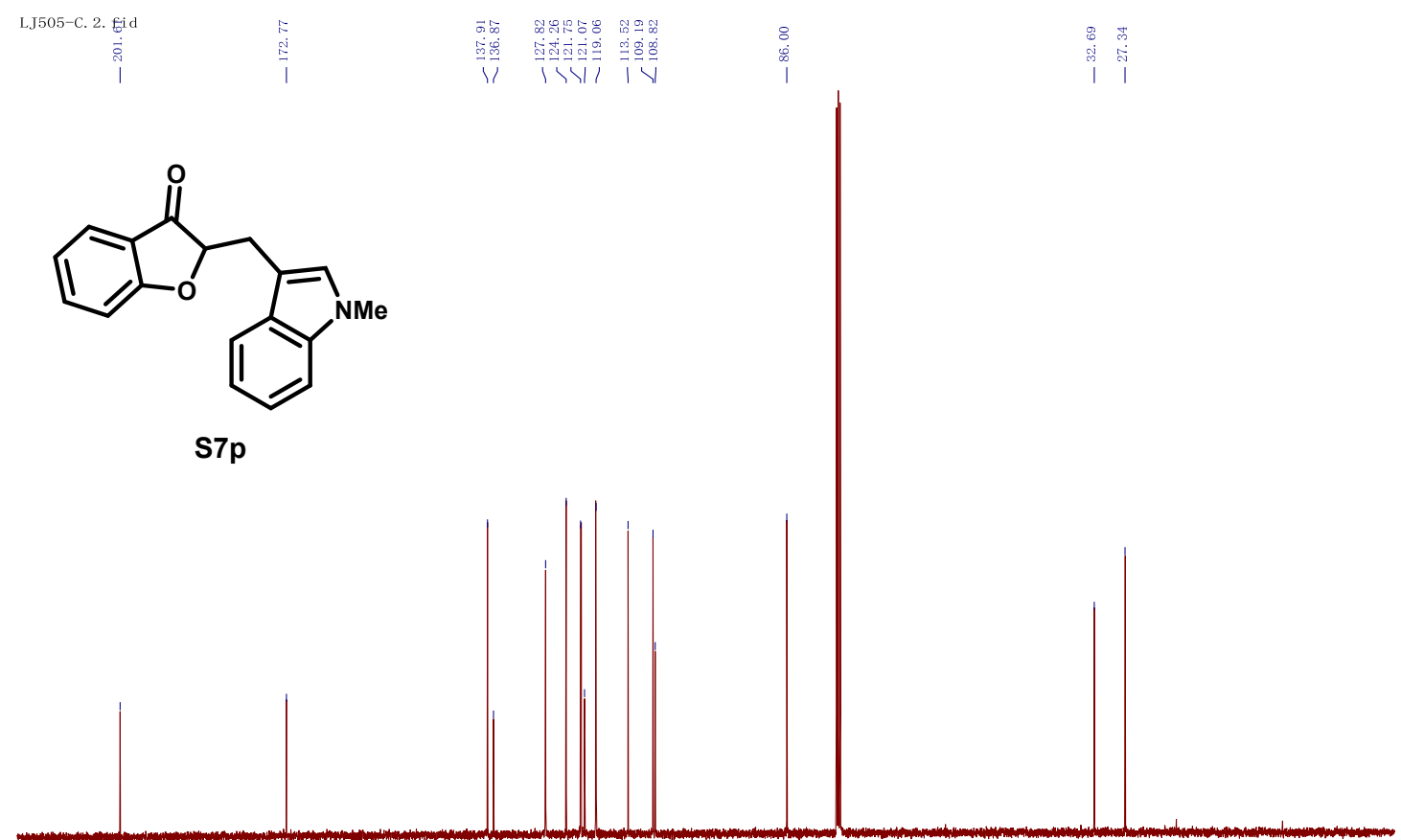

$\begin{array}{llllllllllll}210 & 200 & 190 & 180 & 170 & 160 & 150 & 140 & 130 & 120 & 110 & 100 \\ \mathrm{f} 1 & (\mathrm{ppm}) & 9\end{array}$ 
2-(Furan-3-ylmethyl)benzofuran-3(2H)-one (S7q)

1․ NMR (400 MHz, $\mathrm{CDCl}_{3} @ 7.26$ ppm)

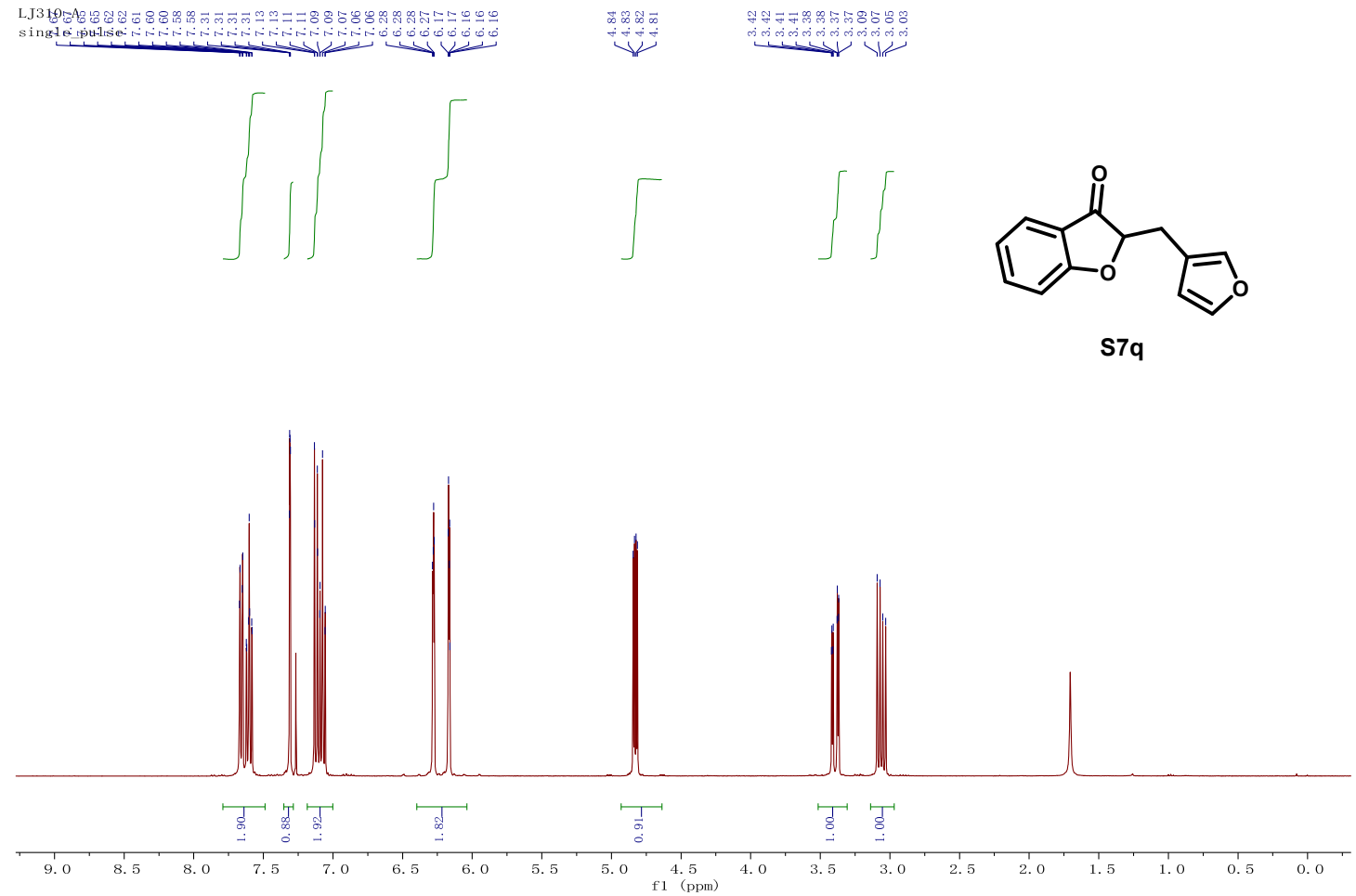

$\underline{{ }^{13} \mathrm{C} \text { NMR }}\left(100 \mathrm{MHz}, \mathrm{CDCl}_{3} @ 77\right.$ ppm)

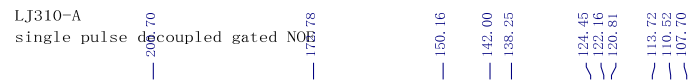
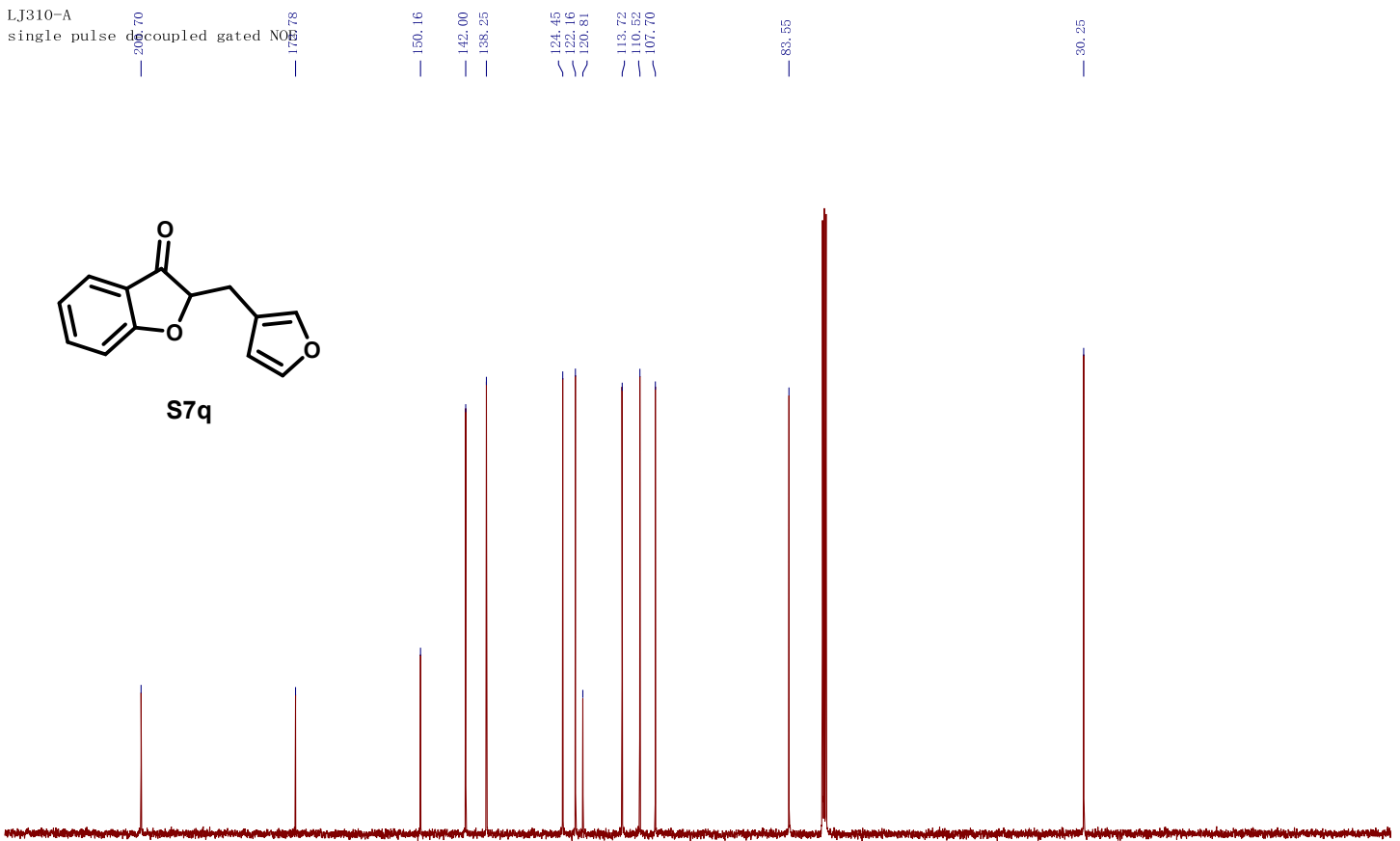

$\begin{array}{lllllllllllllllllllllllll}220 & 210 & 200 & 190 & 180 & 170 & 160 & 150 & 140 & 130 & 120 & 110 & 100 & 90 & 80 & 70 & 60 & 50 & 40 & 30 & 20 & 10 & 0 & -10 & -20\end{array}$ 
2-(Thiophen-3-ylmethyl)benzofuran-3(2H)-one (S7r)

$\underline{{ }^{1} \mathrm{H} \text { NMR }}\left(400 \mathrm{MHz}, \mathrm{CDCl}_{3} @ 7.26\right.$ ppm)

L

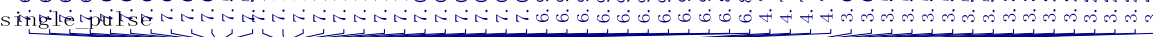
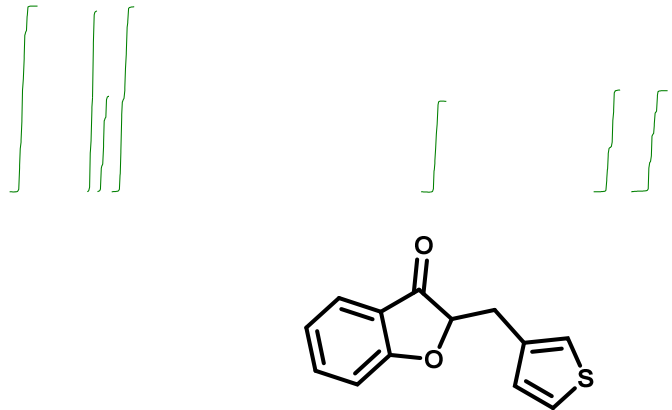

S7r
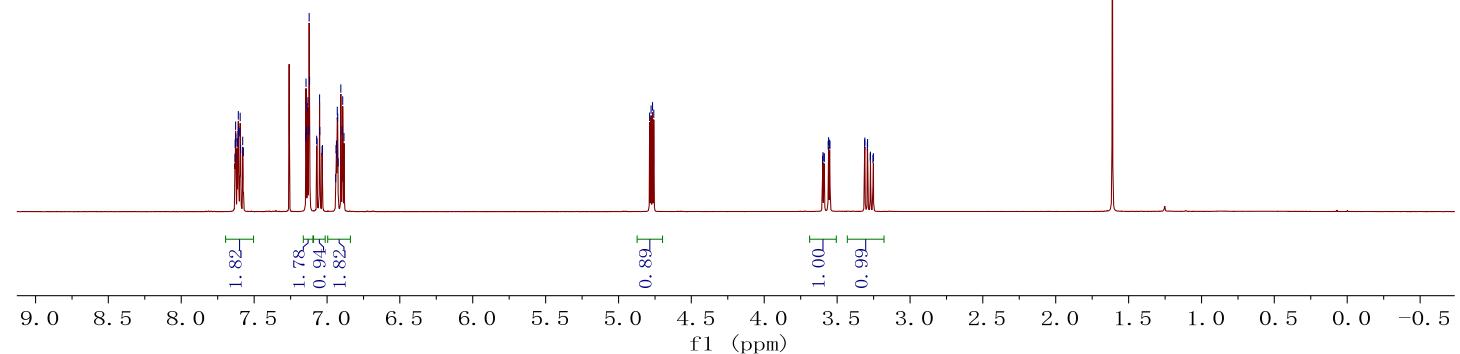

${ }^{13} \mathrm{C}$ NMR (100 MHz, CDCl $3 @ 77$ ppm)

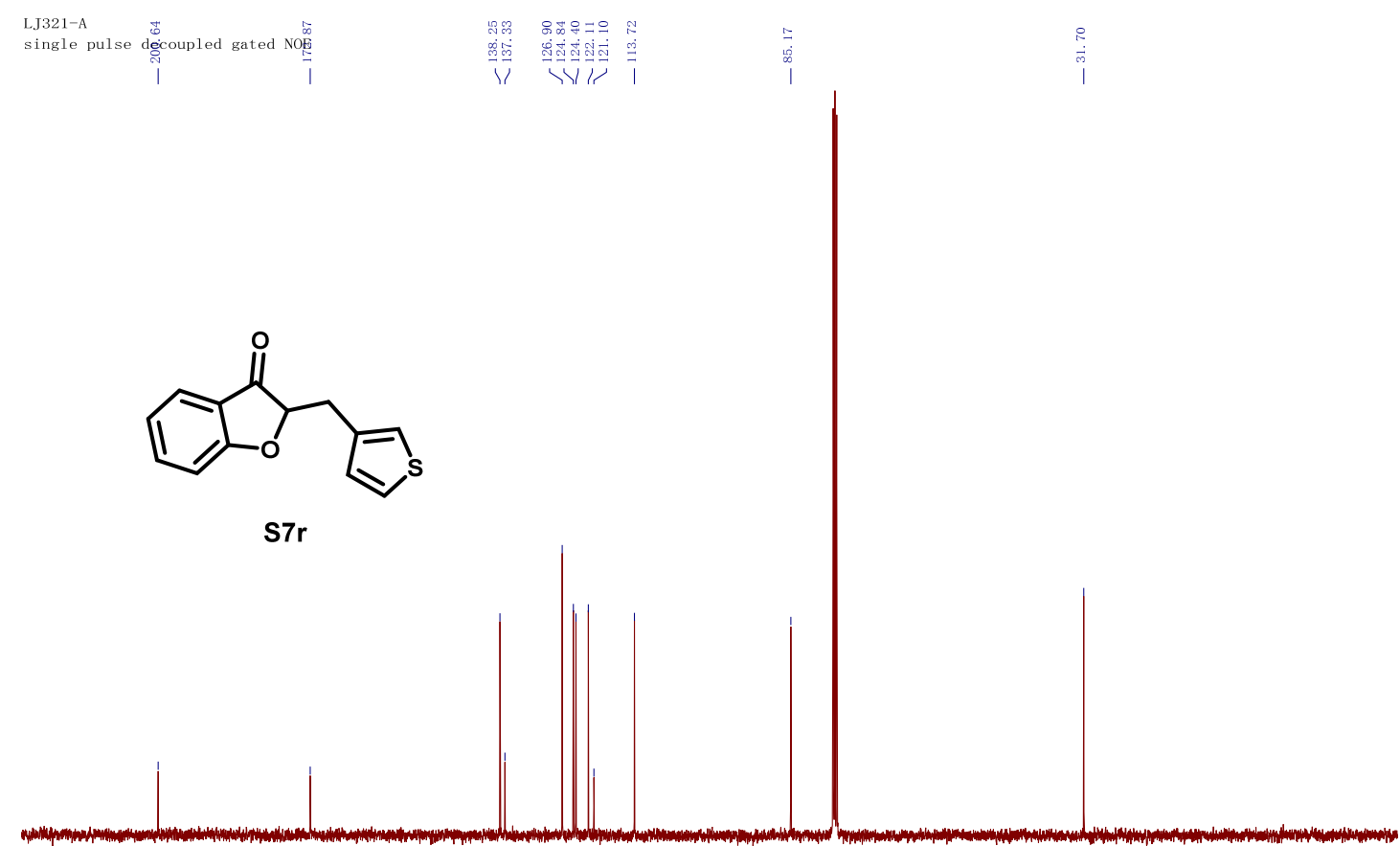

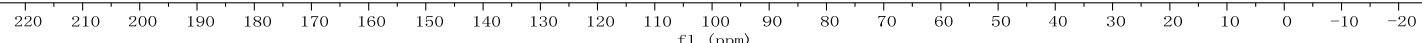


(E)-2-(3-Phenylprop-1-en-1-yl)benzofuran-3(2H)-one (S7s)

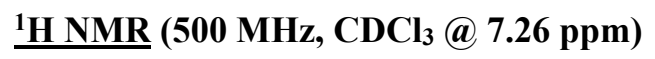

L HIIL

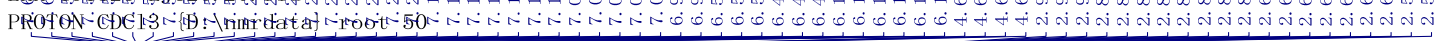

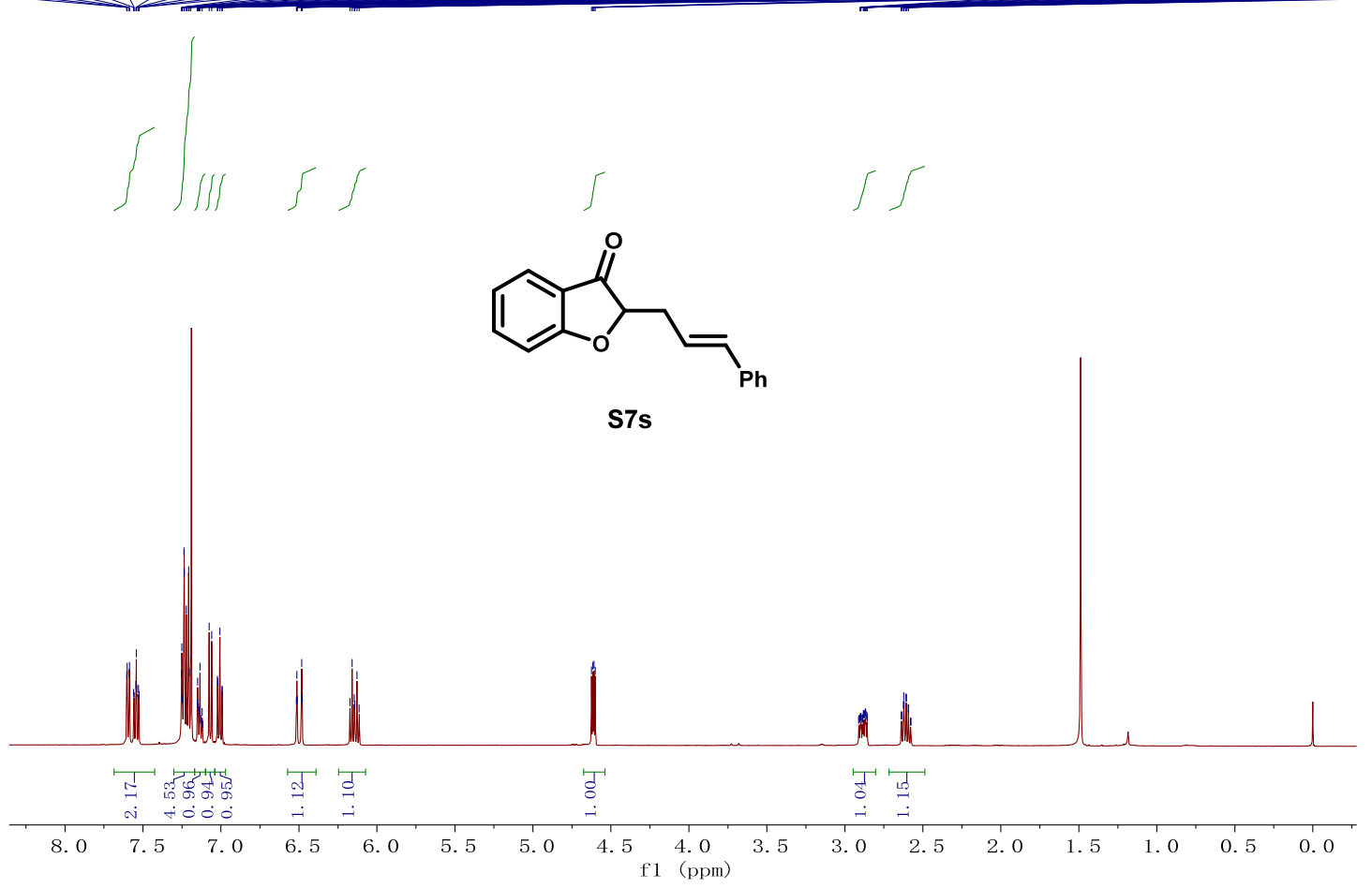

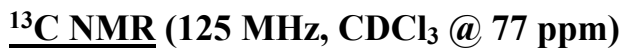

LHH-1909-13-LJ826. 3. fid

C13CPD GDC13 (D: \nmrdata\} raot 32

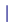

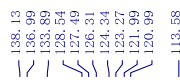
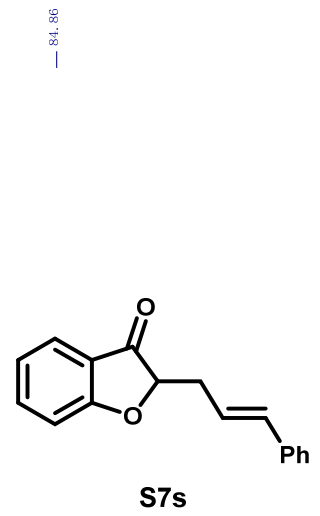

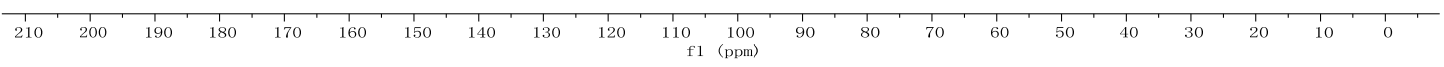

S143 
2-(3-Phenylpropyl)benzofuran-3(2H)-one (S7t)

$\underline{{ }^{1} \mathrm{H} \text { NMR }}\left(500 \mathrm{MHz}, \mathrm{CDCl}_{3} @ 7.26\right.$ ppm)

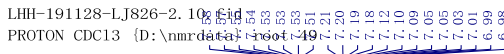

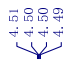

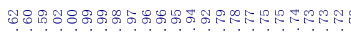
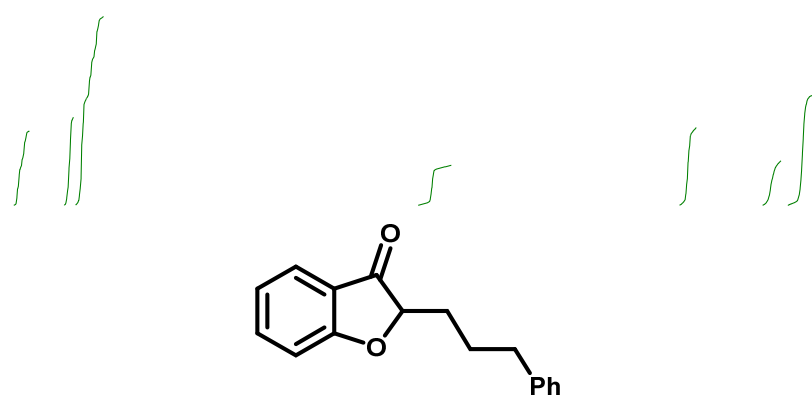

S7t

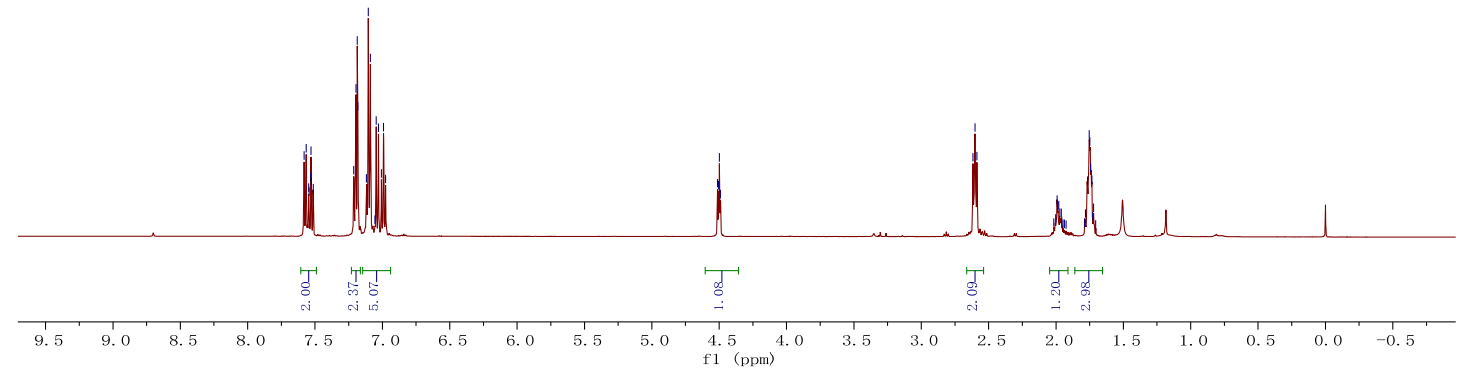

${ }^{13} \mathrm{C}$ NMR (125 MHz, CDCl $@ 77$ ppm)

LHH-191128- LJ826-2. 11. fid C13CPD CDCA3 $\{$ D: \nmrdata $\}$ roo通 49
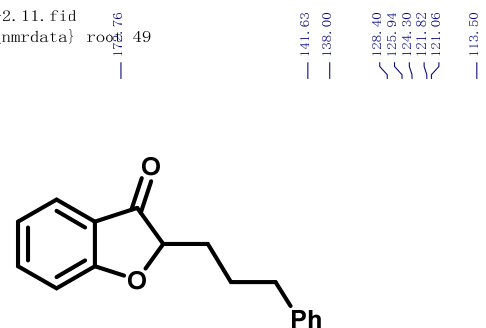

S7t

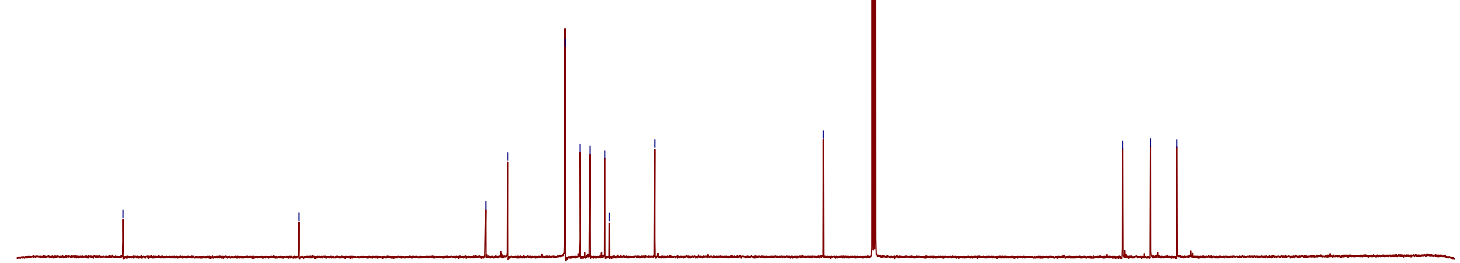


2-(Cyclopropylmethyl)benzofuran-3(2H)-one (S7u)

$\underline{{ }^{1} \mathrm{H} N M R}\left(500 \mathrm{MHz}, \mathrm{CDCl}_{3} @ 7.26\right.$ ppm)

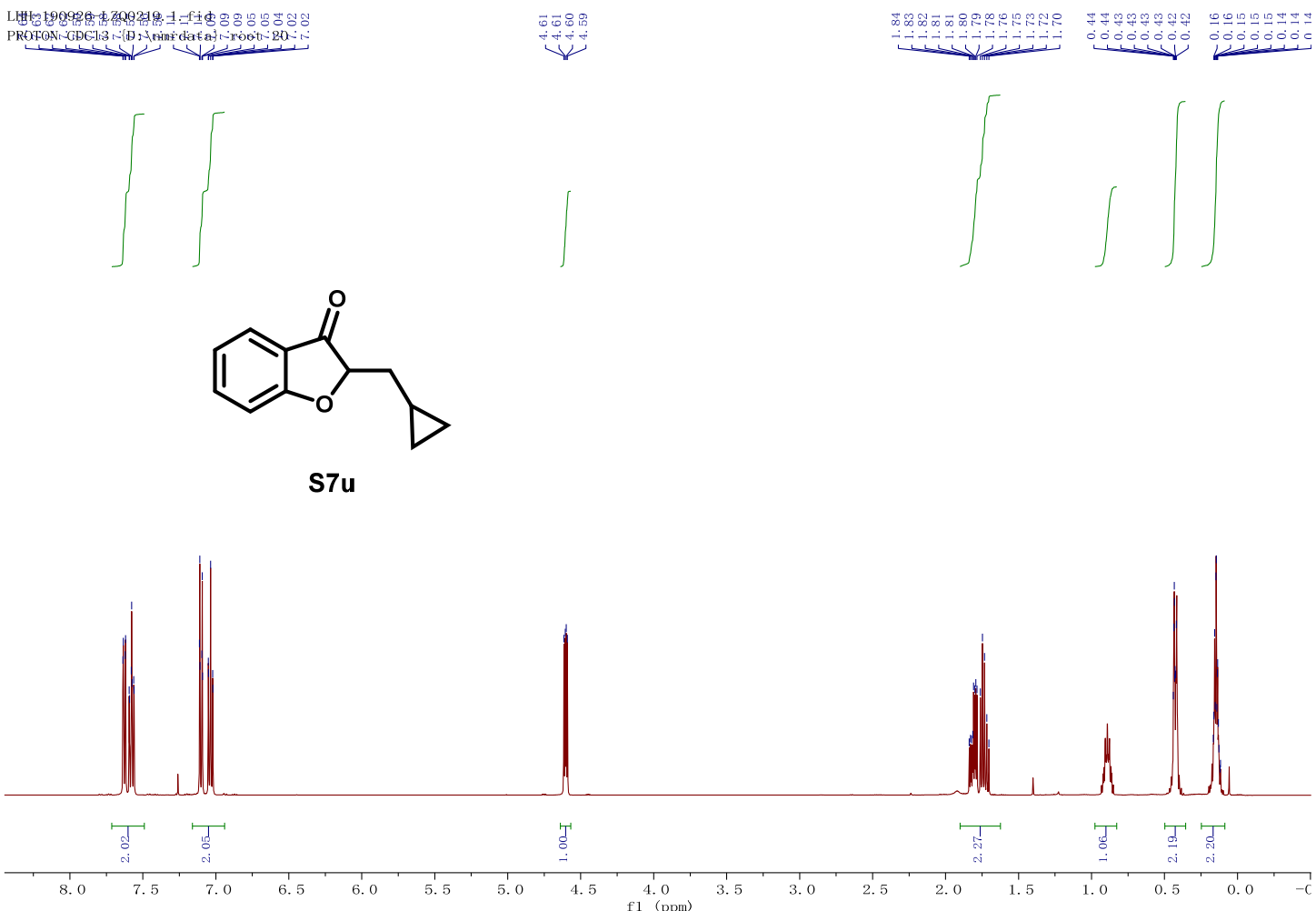

${ }^{13} \mathrm{C}$ NMR (125 MHz, CDCl $@ 77$ ppm)

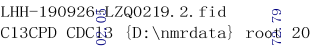

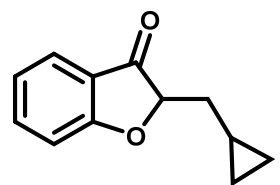

S7u

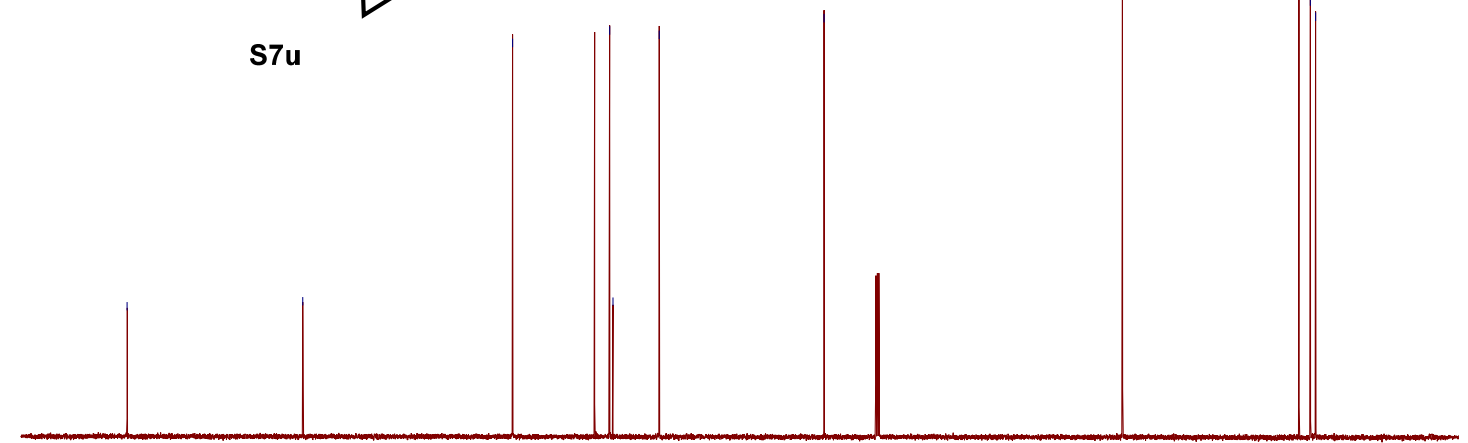

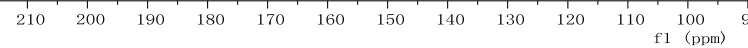


4-Methoxy-2-(naphthalen-1-ylmethyl)benzofuran-3(2H)-one (S7v)

1H NMR (500 MHz, $\mathrm{CDCl}_{3} @ 7.26$ ppm)

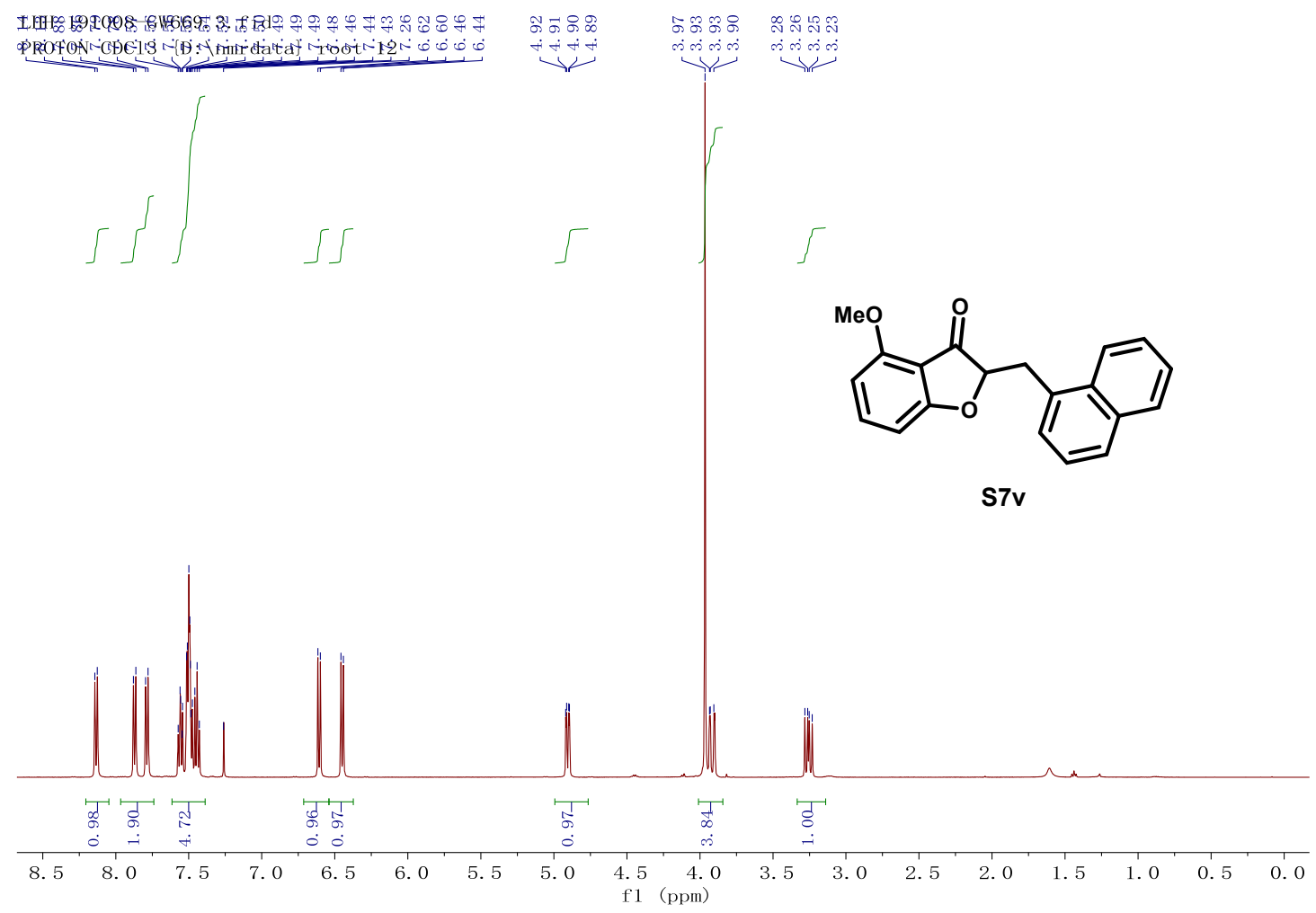

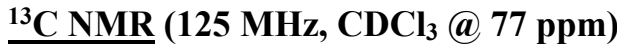

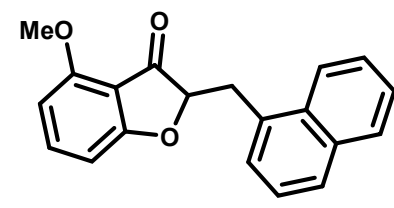

S7v

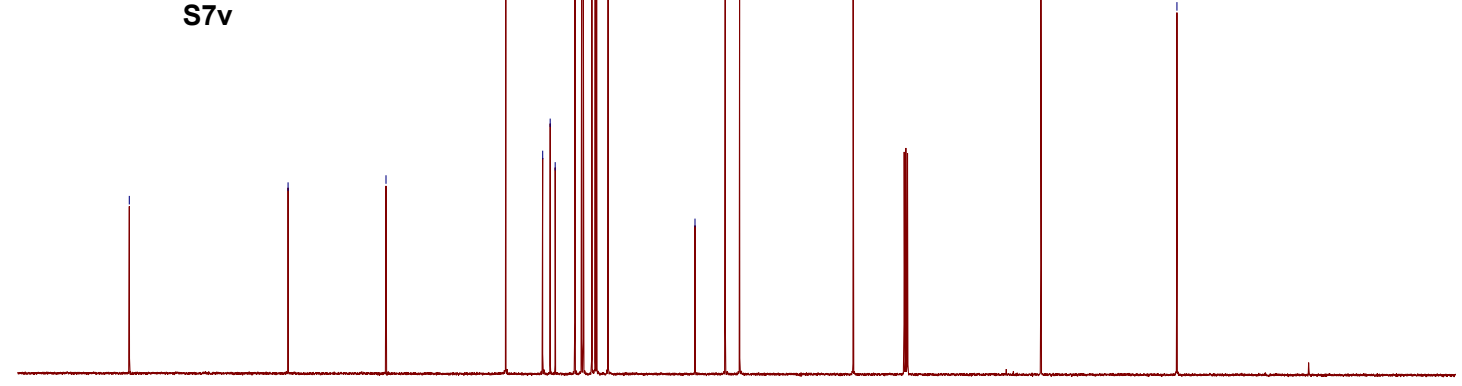

$\begin{array}{rrrrrrrrrrrr}110 & 200 & 190 & 180 & 170 & 160 & 150 & 140 & 130 & 120 & 110 & 100 \\ \mathrm{f} 1 & (\mathrm{ppm})\end{array}$ 
5-Methoxy-2-(naphthalen-1-ylmethyl)benzofuran-3(2H)-one (S7w)

$\underline{{ }^{1} \mathrm{H} N M R}\left(500 \mathrm{MHz}, \mathrm{CDCl}_{3} @ 7.26\right.$ ppm)

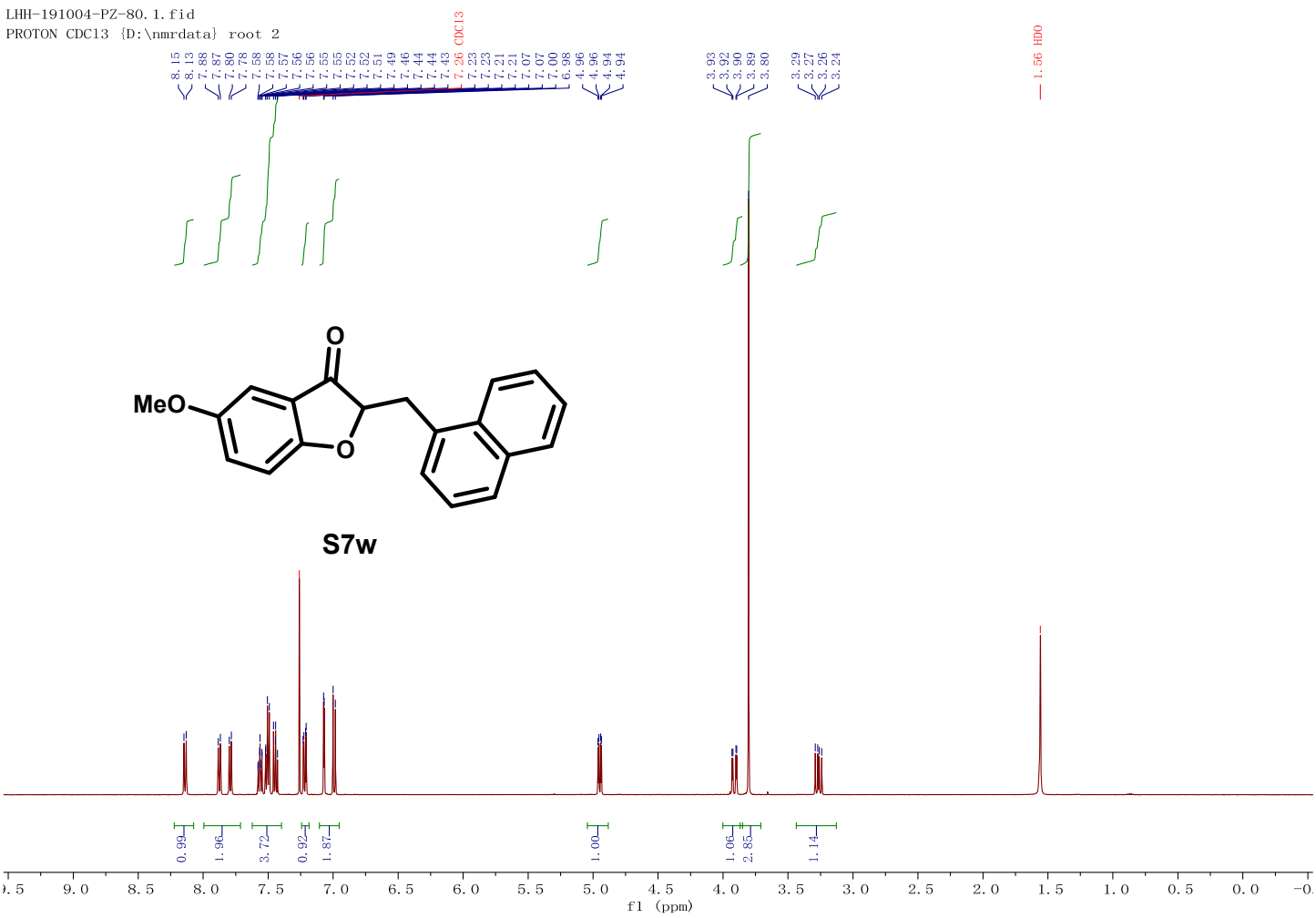

${ }^{13} \mathrm{C}$ NMR (125 MHz, CDCl 3077 ppm)

LHHњ191218-CMYstep1-

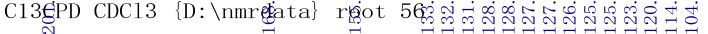

।

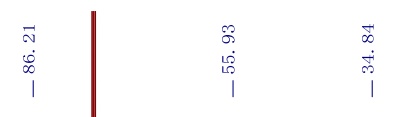<smiles>COc1ccc2c(c1)C(=O)C(Cc1cccc3ccccc13)O2</smiles>

S7w

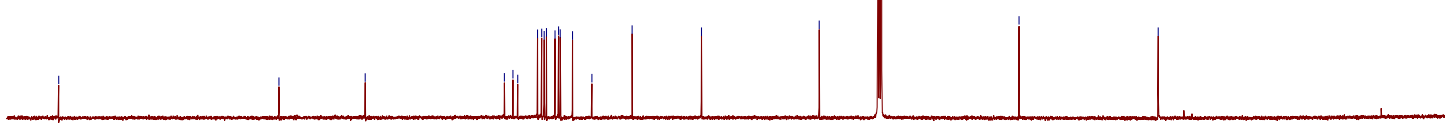

$\begin{array}{lllllllllll}200 & 190 & 180 & 170 & 160 & 150 & 140 & 130 & 120 & 110 & 100 \\ \mathrm{f} 1 & (\mathrm{ppm})\end{array}$ 
6-Methoxy-2-(naphthalen-1-ylmethyl)benzofuran-3(2H)-one (S7x)

1H NMR (500 MHz, $\mathrm{CDCl}_{3} @ 7.26$ ppm)

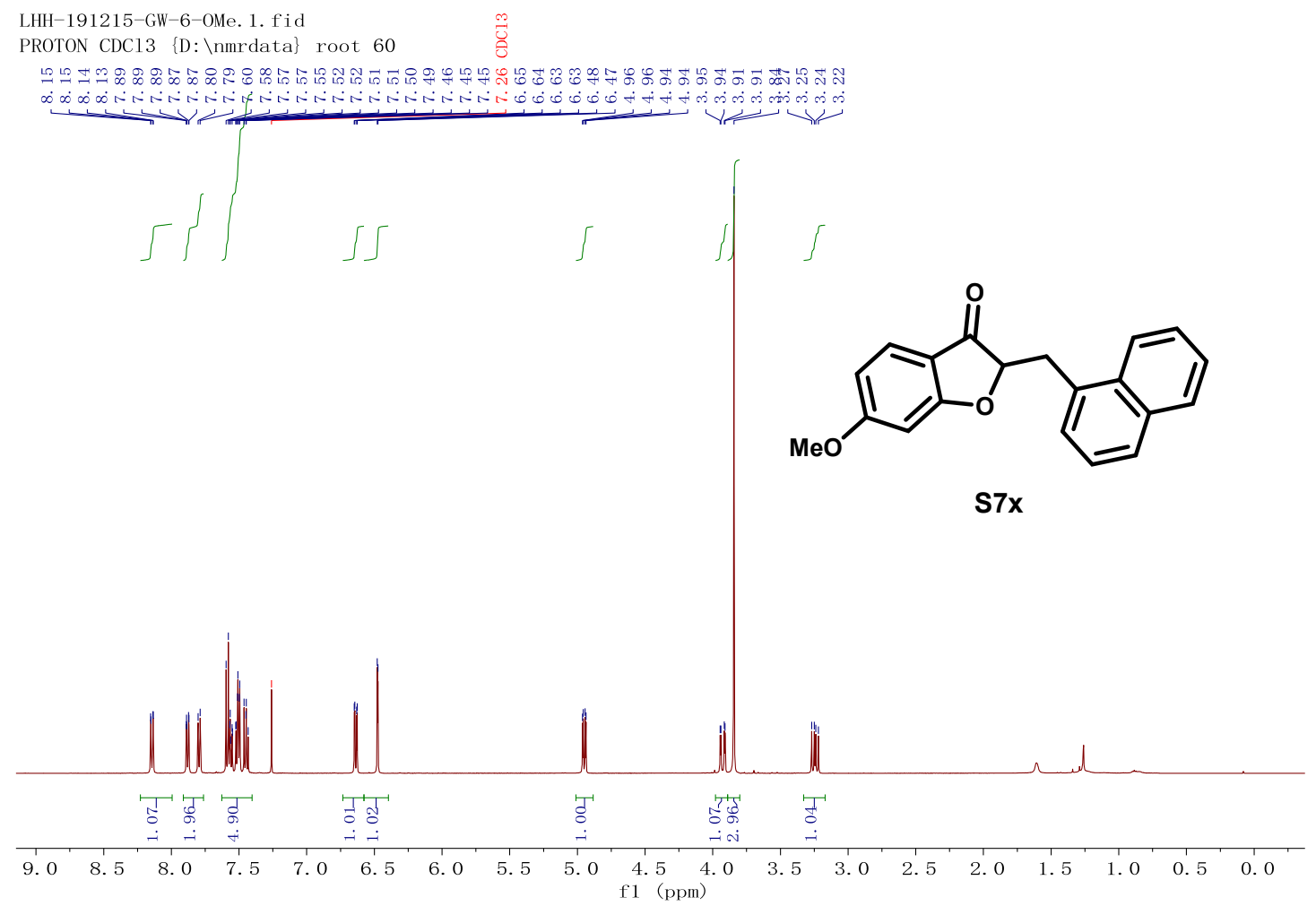

${ }^{13} \mathrm{C}$ NMR (125 MHz, $\mathrm{CDCl}_{3} @ 77$ ppm) 
<smiles>COc1ccc2c(c1)OC(Cc1cccc3ccccc13)C2=O</smiles>

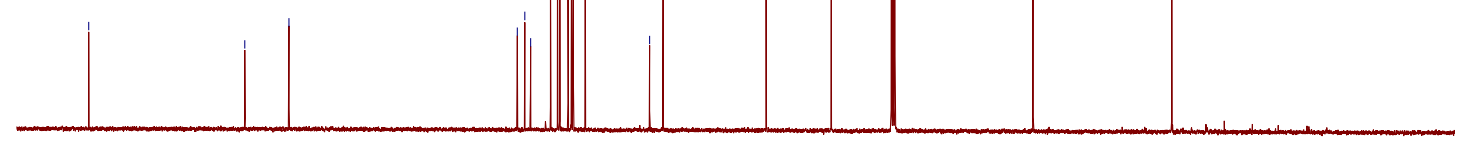

$\begin{array}{lllllllllllllllllllll}200 & 190 & 180 & 170 & 160 & 150 & 140 & 130 & 120 & 110 & 100 & 90 & 80 & 70 & 60 & 50 & 40 & 30 & 20 & 10 & 0\end{array}$

6-Bromo-2-(naphthalen-1-ylmethyl)benzofuran-3(2H)-one (S7y)

$\underline{{ }^{1} \mathrm{H} \text { NMR }}\left(500 \mathrm{MHz}, \mathrm{CDCl}_{3} @ 7.26\right.$ ppm)

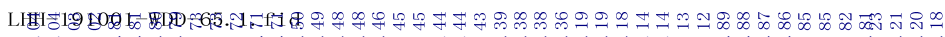

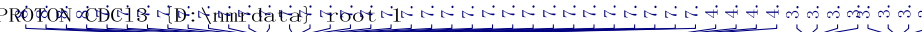
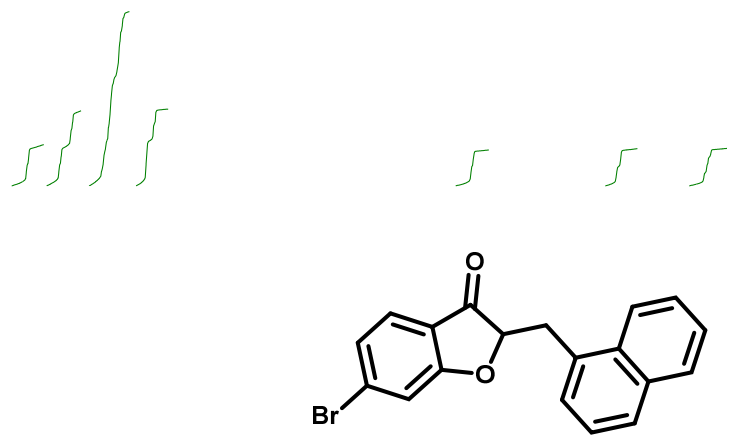

S7y

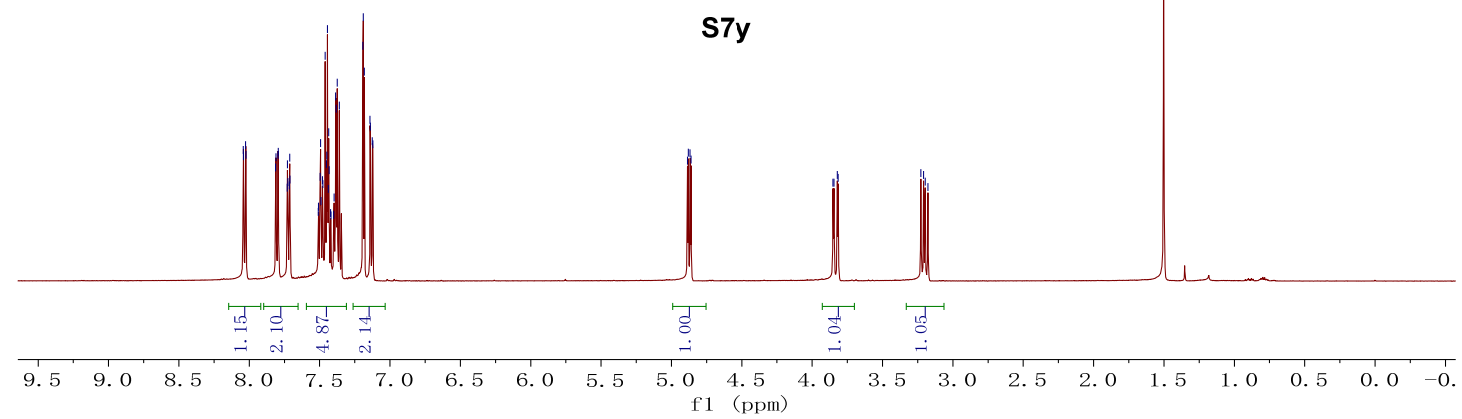

${ }^{13} \mathrm{C}$ NMR (125 MHz, $\mathrm{CDCl}_{3} @ 77$ ppm) 

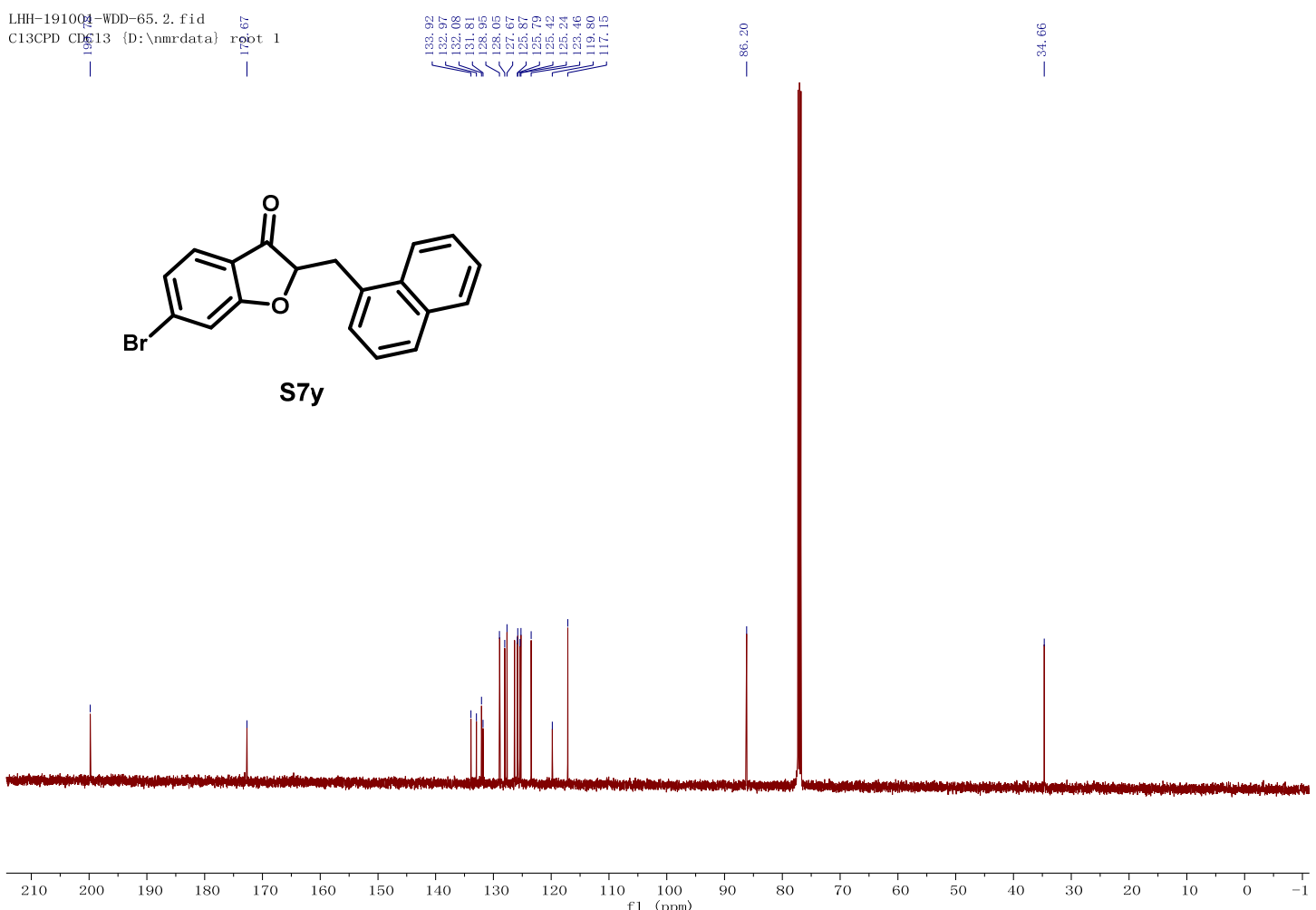

6-Fluoro-2-(naphthalen-1-ylmethyl)benzofuran-3(2H)-one (S7z)

매 NMR $\left(500 \mathrm{MHz}, \mathrm{CDCl}_{3} @ 7.26\right.$ ppm)

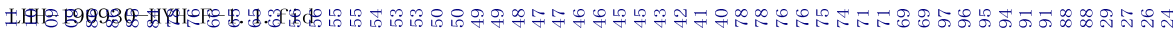

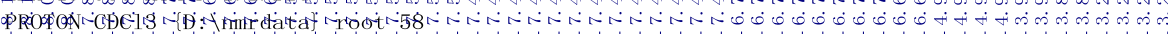
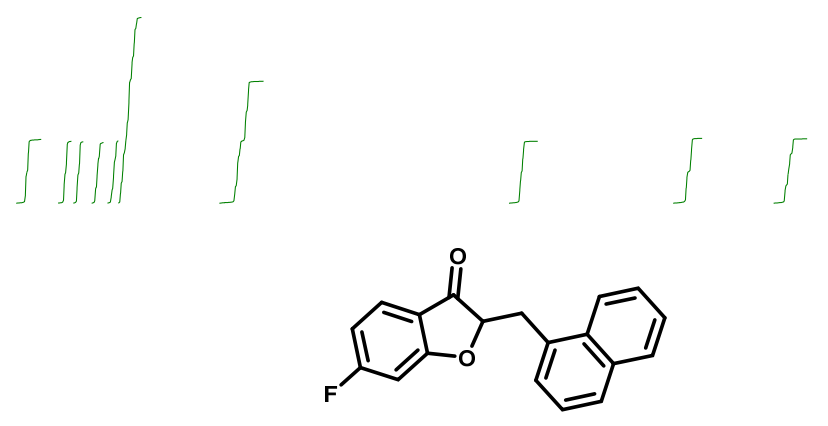

S7z

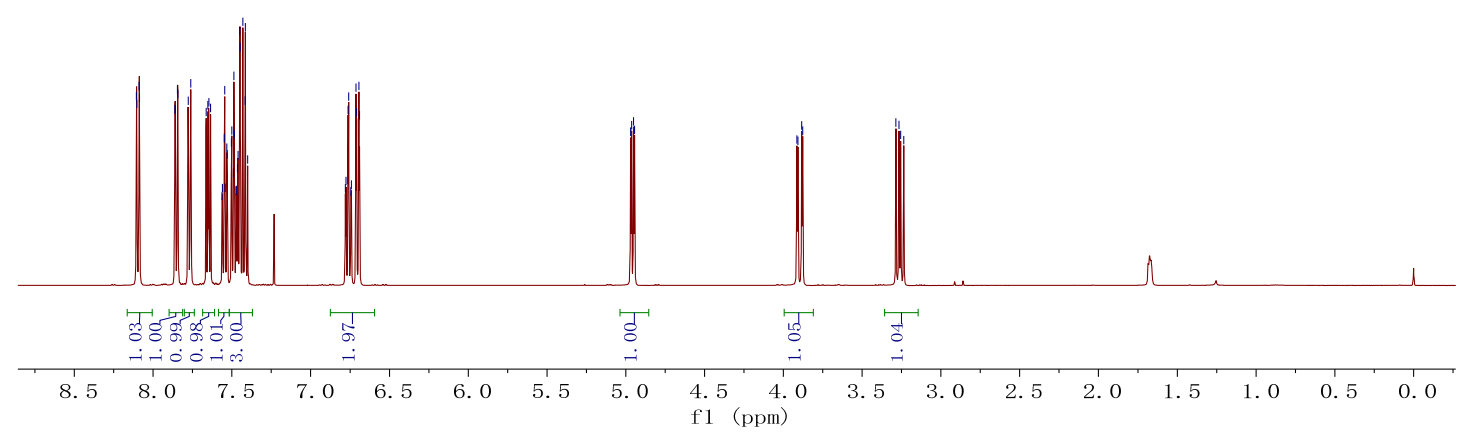

${ }^{13} \mathrm{C}$ NMR (125 MHz, $\mathrm{CDCl}_{3} @ 77$ ppm)

S150 


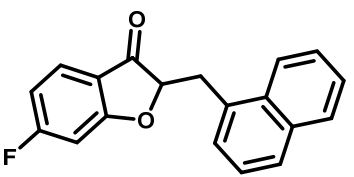

S7z

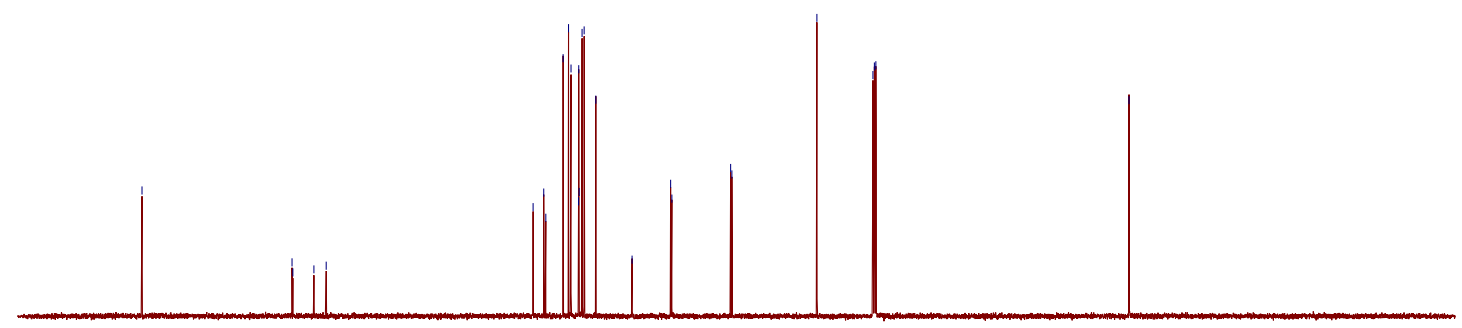

$\begin{array}{lllllllllllllllllllllllll}210 & 200 & 190 & 180 & 170 & 160 & 150 & 140 & 130 & 120 & 110 & 100 & 90 & 80 & 70 & 60 & 50 & 40 & 30 & 20 & 10 & 0 & -10\end{array}$

19 F NMR (470 MHz, $\left.\mathrm{CDCl}_{3}\right)$

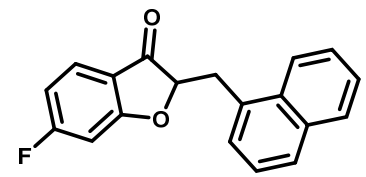

S7z

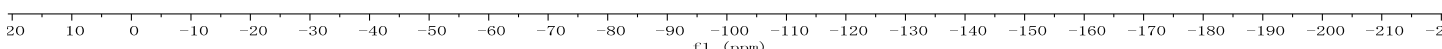

6-Morpholino-2-(naphthalen-1-ylmethyl)benzofuran-3(2H)-one (S7a')

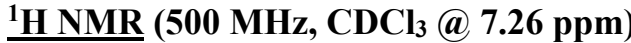




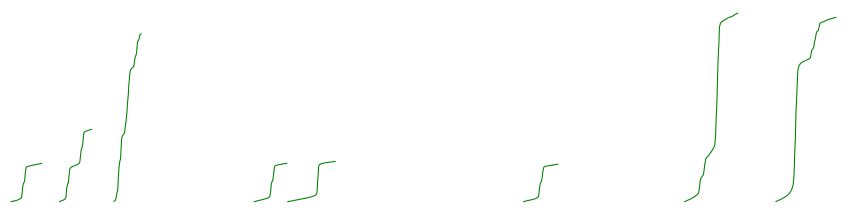

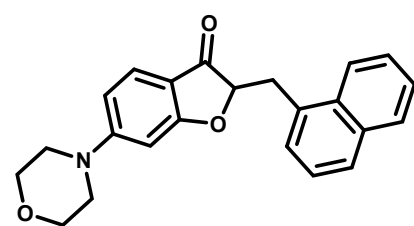

S7a'

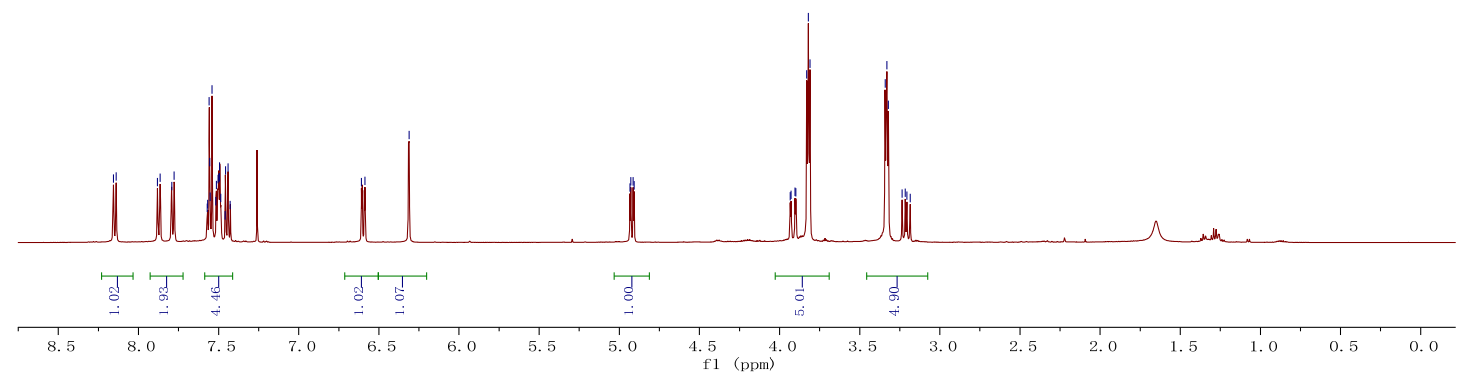

13. NMR (125 MHz, $\mathrm{CDCl}_{3} @ 77$ ppm)
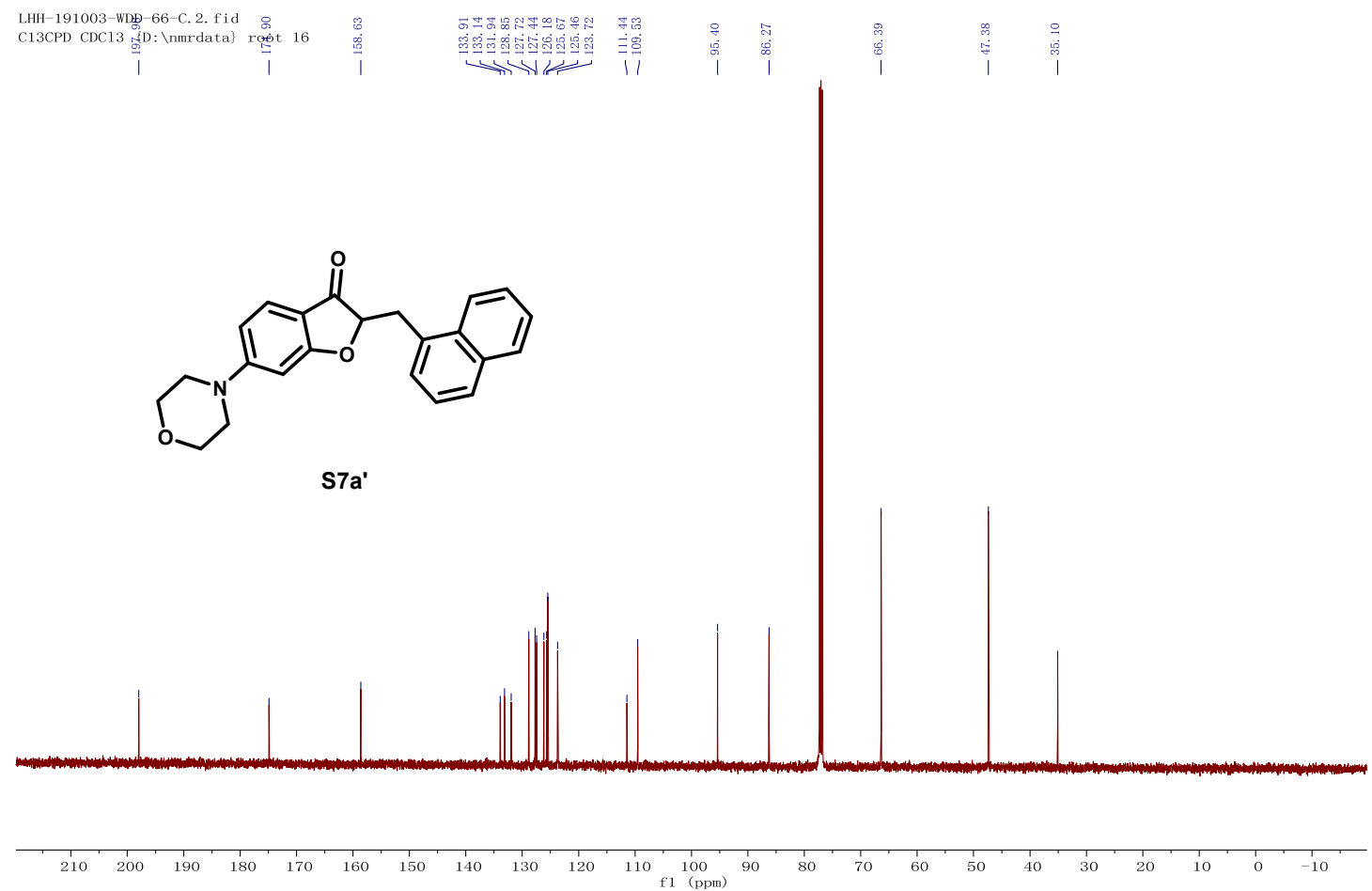

7-Methoxy-2-(naphthalen-1-ylmethyl)benzofuran-3(2H)-one (S7b')

1H NMR (500 MHz, CDCl $@ 7.26$ ppm) 


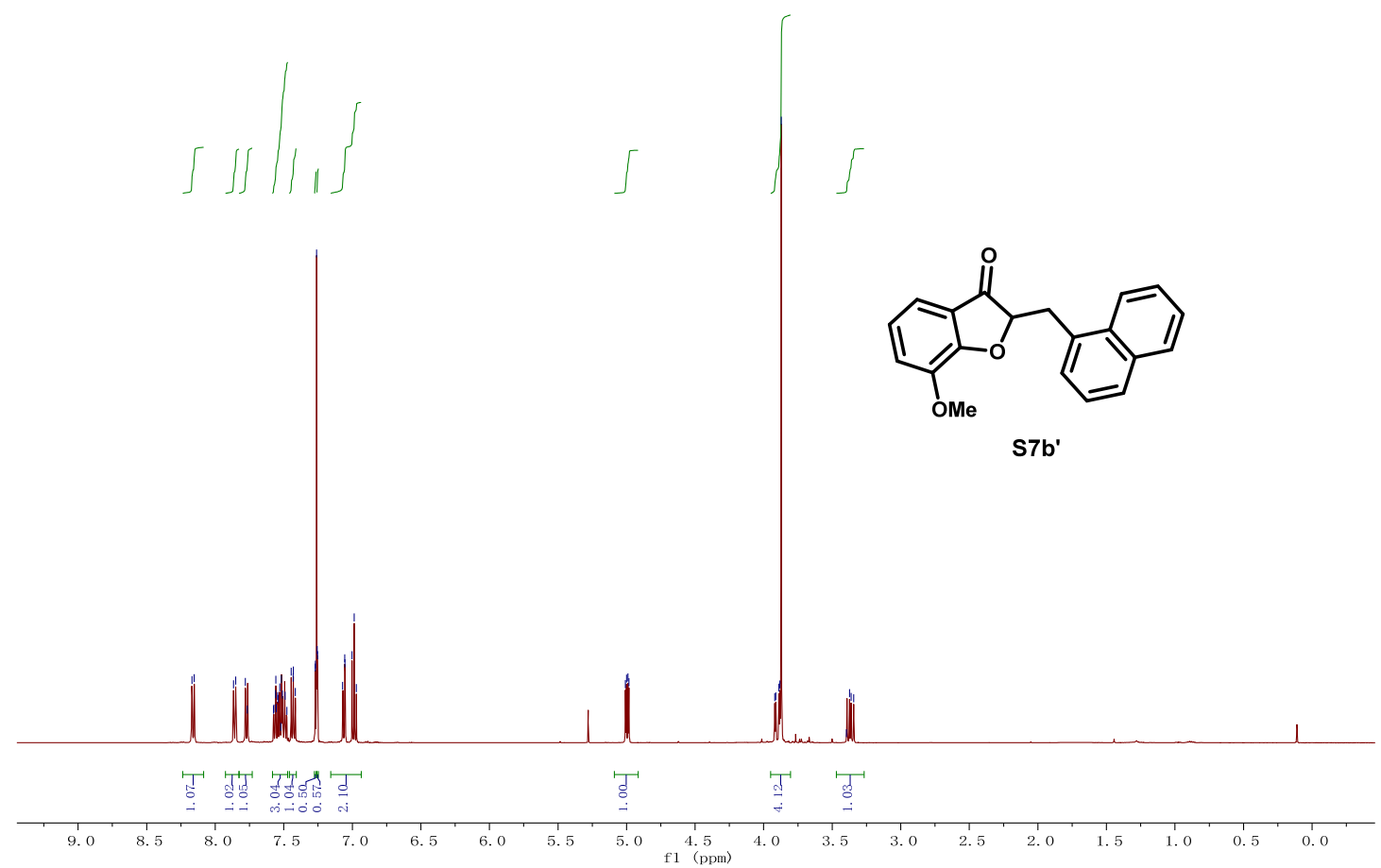

${ }^{13} \mathrm{C}$ NMR (125 MHz, $\mathrm{CDCl}_{3} @ 77$ ppm)

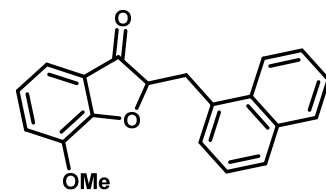

s7b'

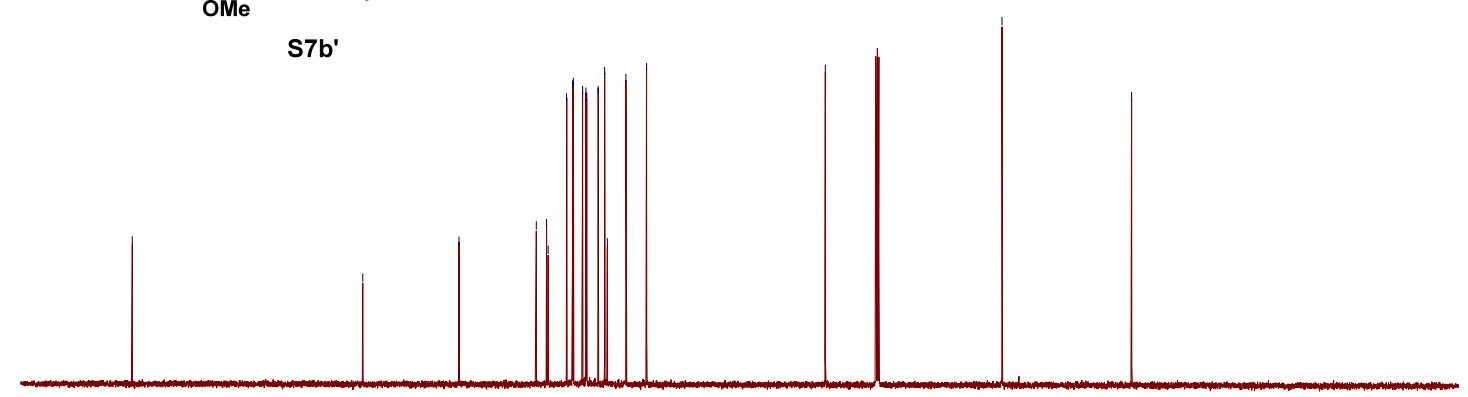

7-Bromo-2-(naphthalen-1-ylmethyl)benzofuran-3(2H)-one (S7c')

${ }^{1} \mathrm{H}$ NMR (500 MHz, $\mathrm{CDCl}_{3} @ 7.26$ ppm) 


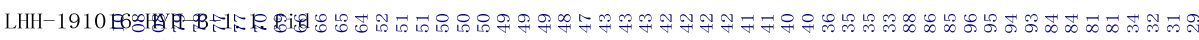

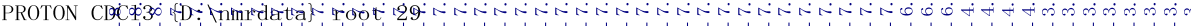
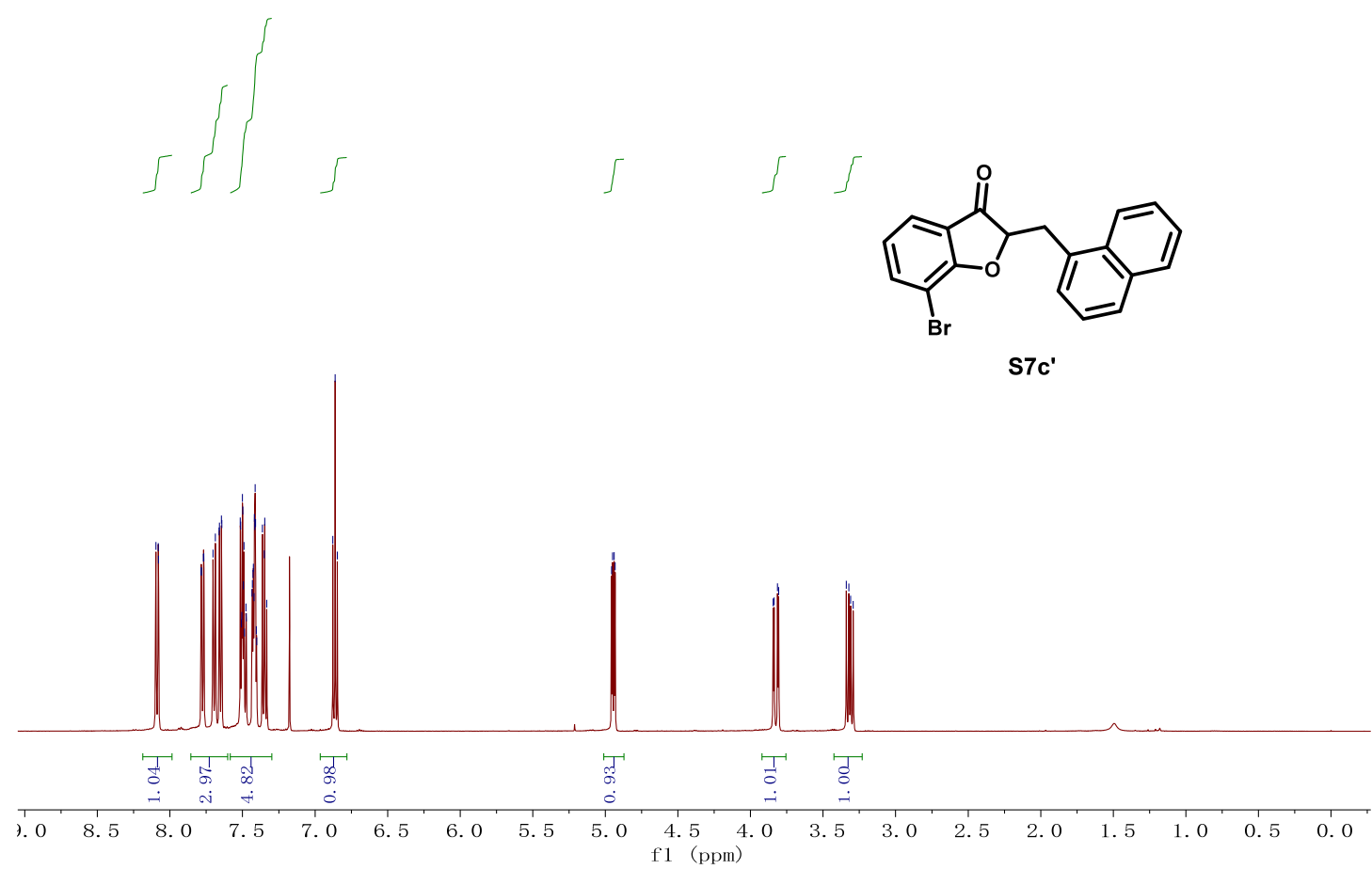

13. NMR (125 MHz, $\mathrm{CDCl}_{3} @ 77$ ppm)
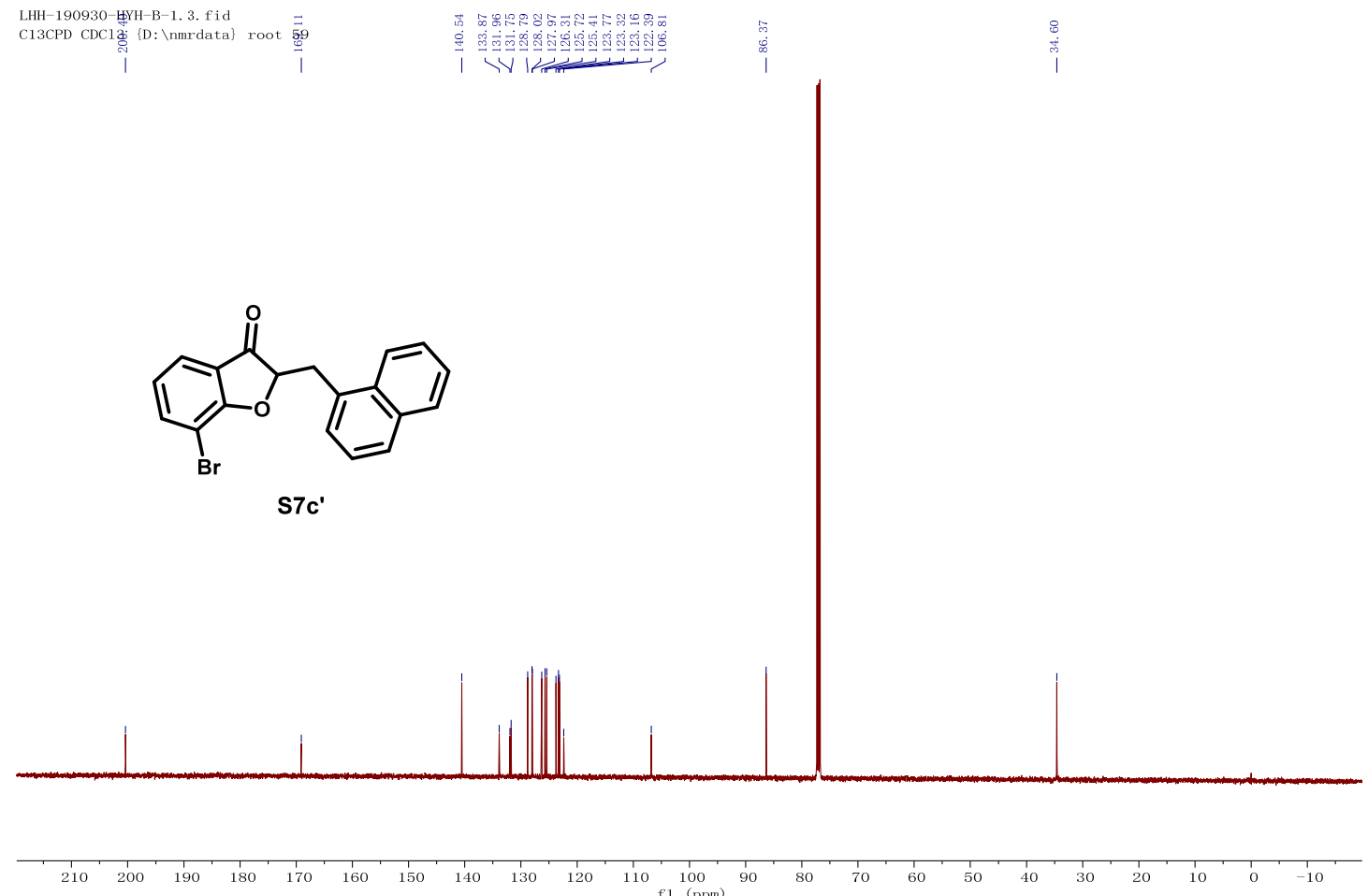

2-Benzylnaphtho[1,2-b]furan-3(2H)-one (S7d')

$\underline{{ }^{1} \mathrm{H} \text { NMR }}\left(500 \mathrm{MHz}, \mathrm{CDCl}_{3} @ 7.26\right.$ ppm) 

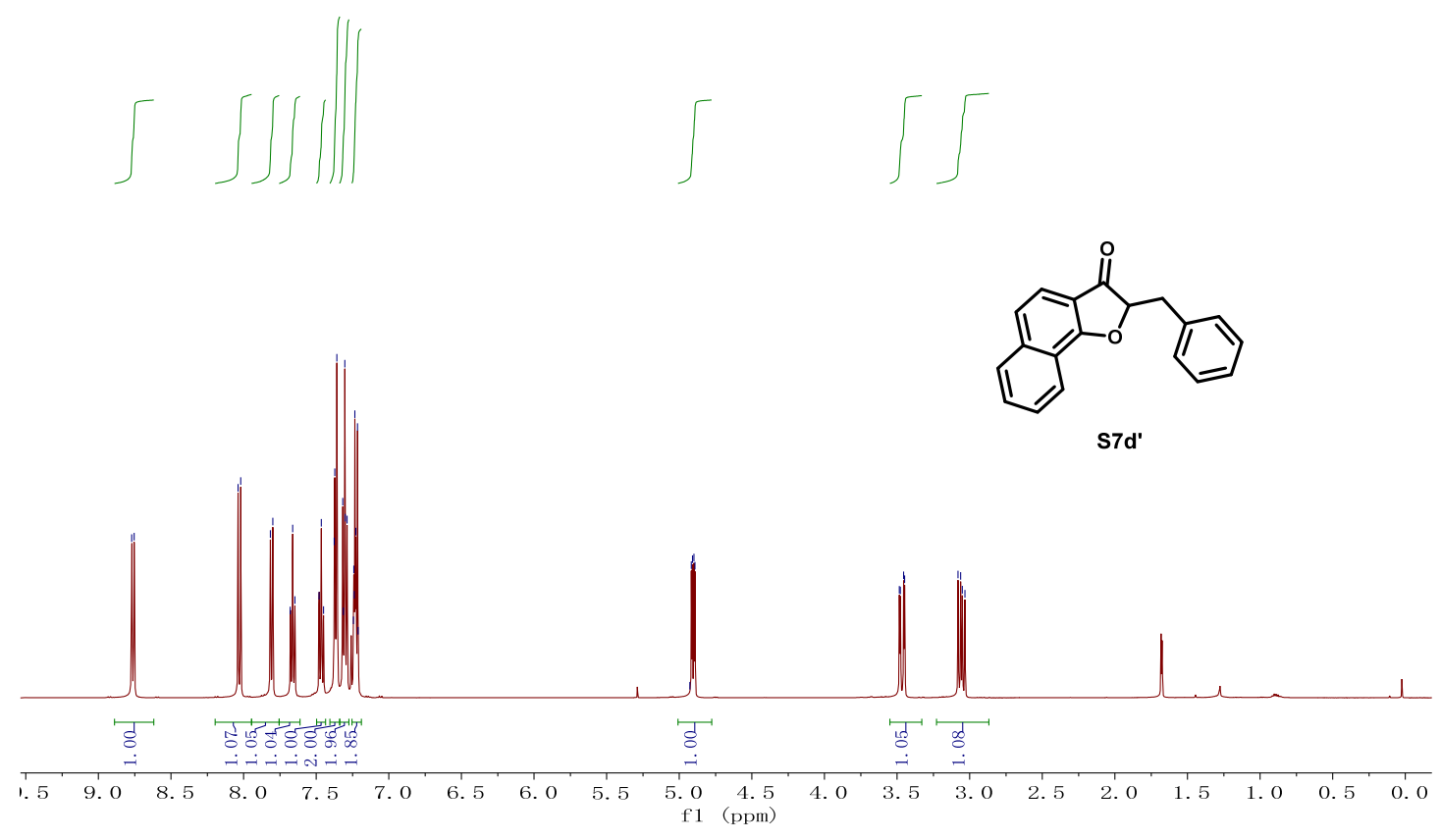

$\underline{{ }^{13} \mathrm{C} \text { NMR }}$ (125 MHz, CDCl $@ 77$ ppm)

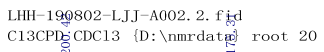

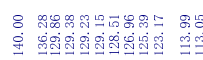

|

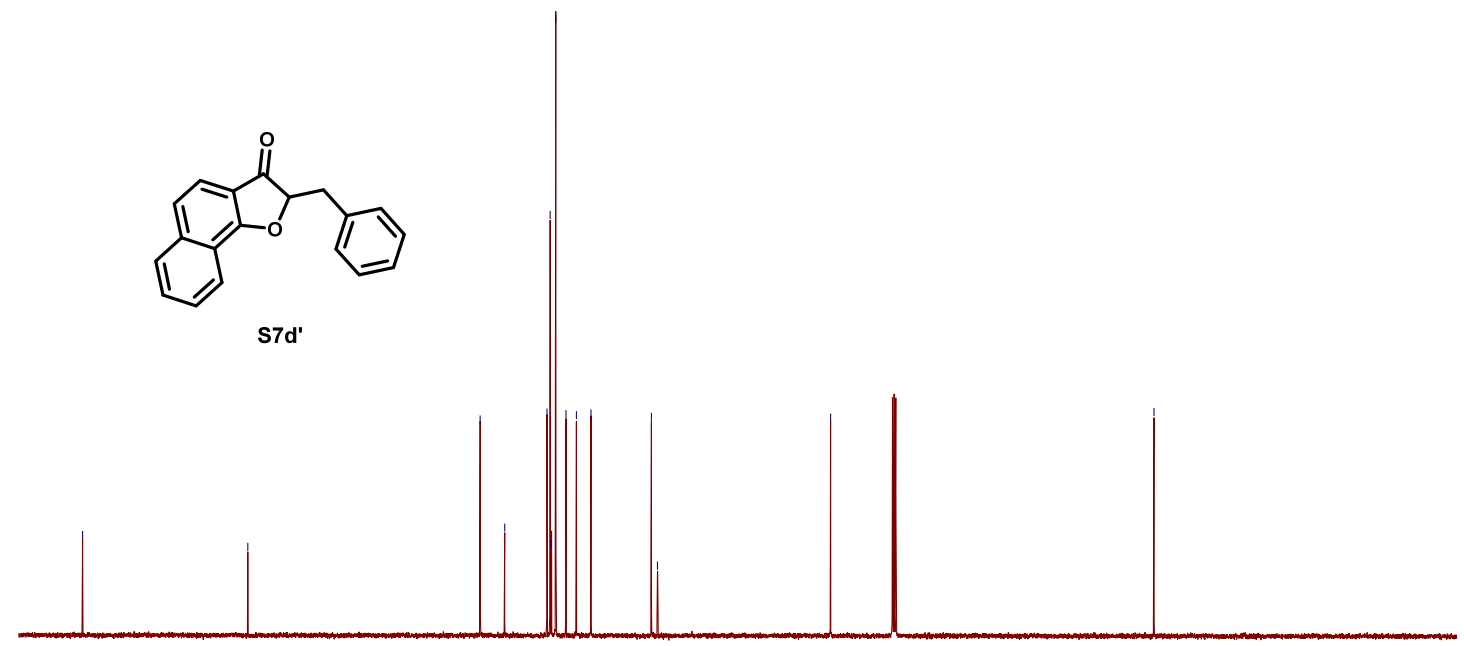

2-Benzylnaphtho[2,1-b]furan-1(2H)-one (S7e')

$\underline{{ }^{1} \mathrm{H} \text { NMR }}\left(500 \mathrm{MHz}, \mathrm{CDCl}_{3} @ 7.26\right.$ ppm) 


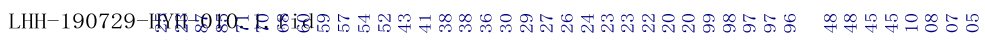

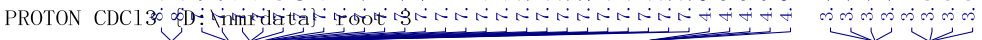
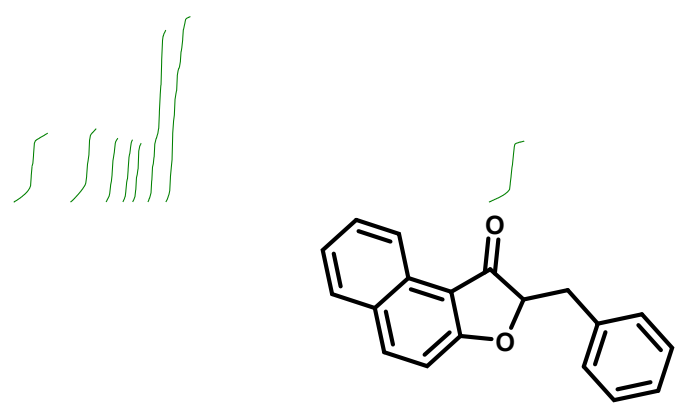

S7e"

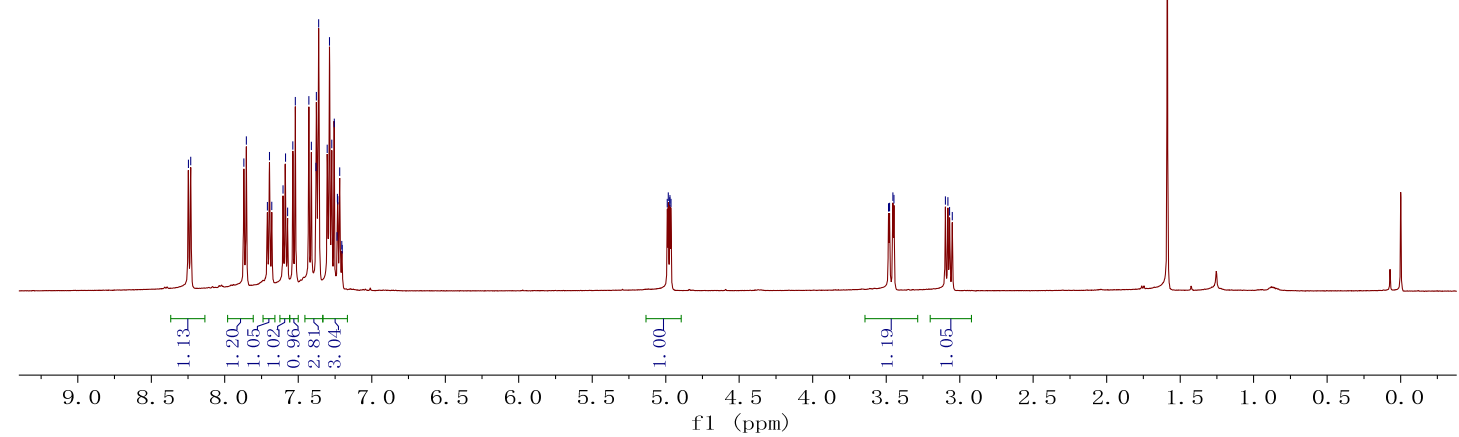

${ }^{13} \mathrm{C}$ NMR (125 MHz, $\mathrm{CDCl}_{3} @ 77$ ppm)

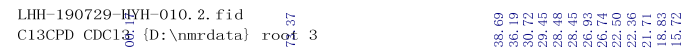

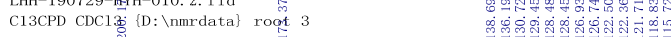

114,12

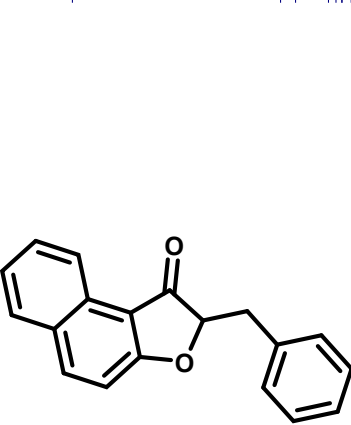

S7e'

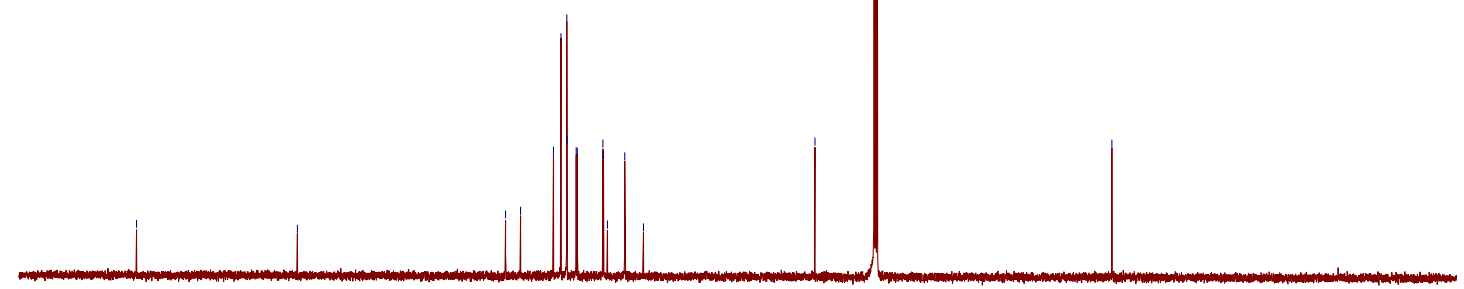

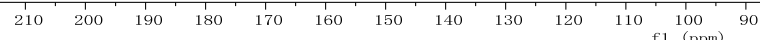

2-Benzylfuro[2,3-b]pyridin-3(2H)-one (S7f')

${ }^{1} \mathrm{H}$ NMR (400 MHz, CDCl 3 @ 7.26 ppm)

S156 


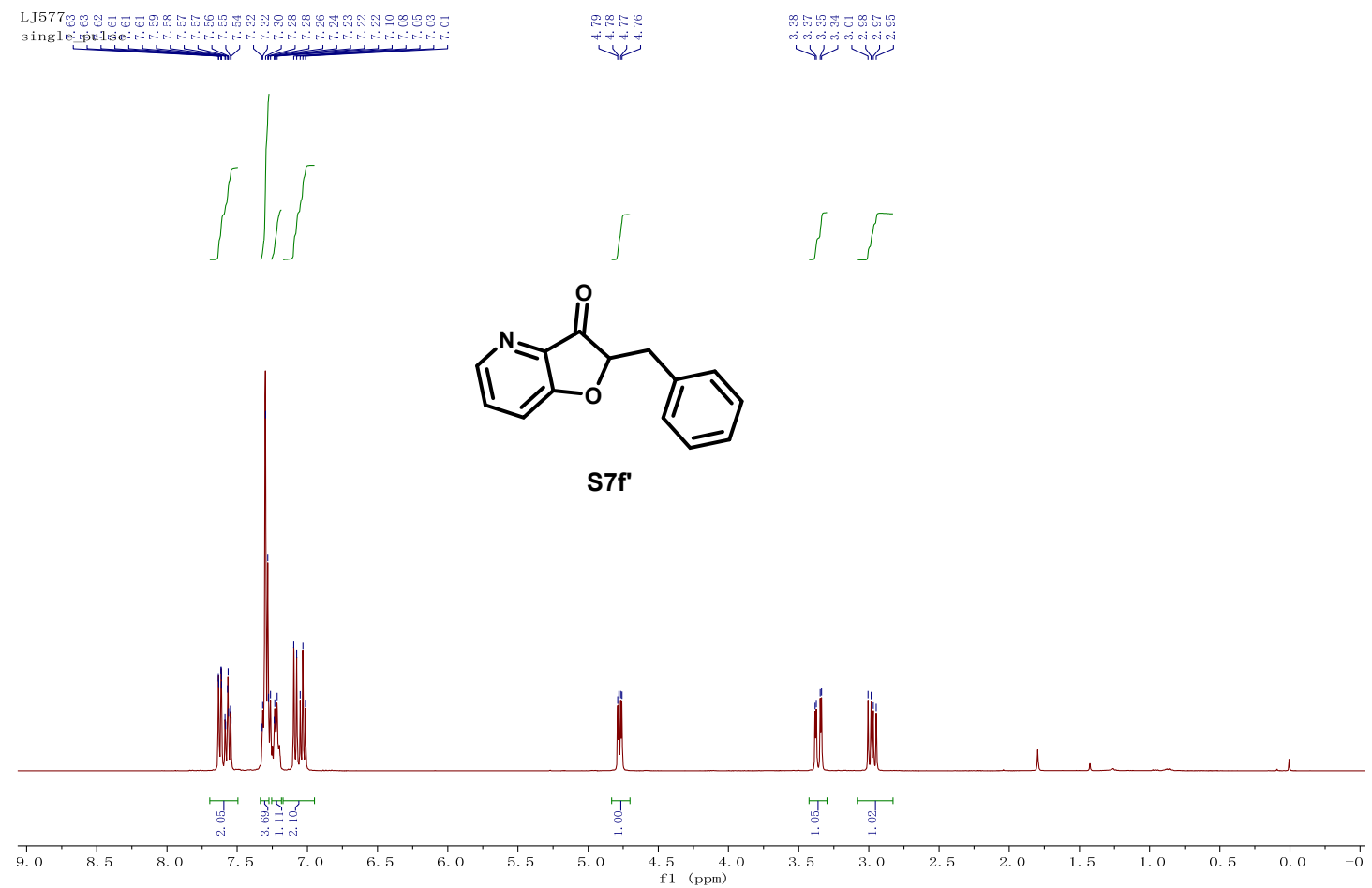

${ }^{13} \mathrm{C}$ NMR (100 MHz, CDCl 377 ppm)

LJ577
single pulse dedich

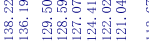

1)

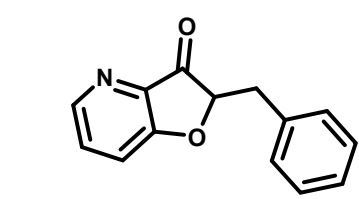

S7f'

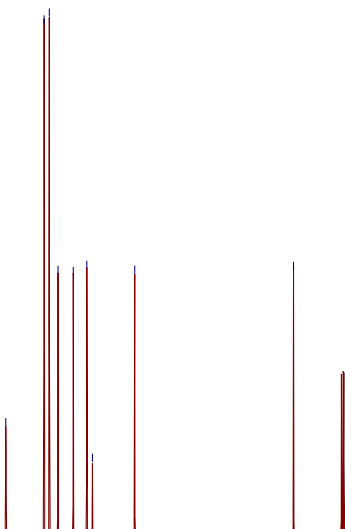

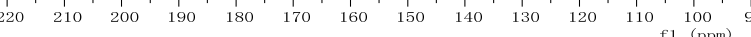

4,6-Dimethoxy-2-phenylbenzofuran-3(2H)-one (S7h')

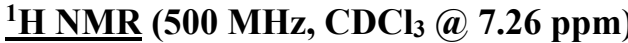



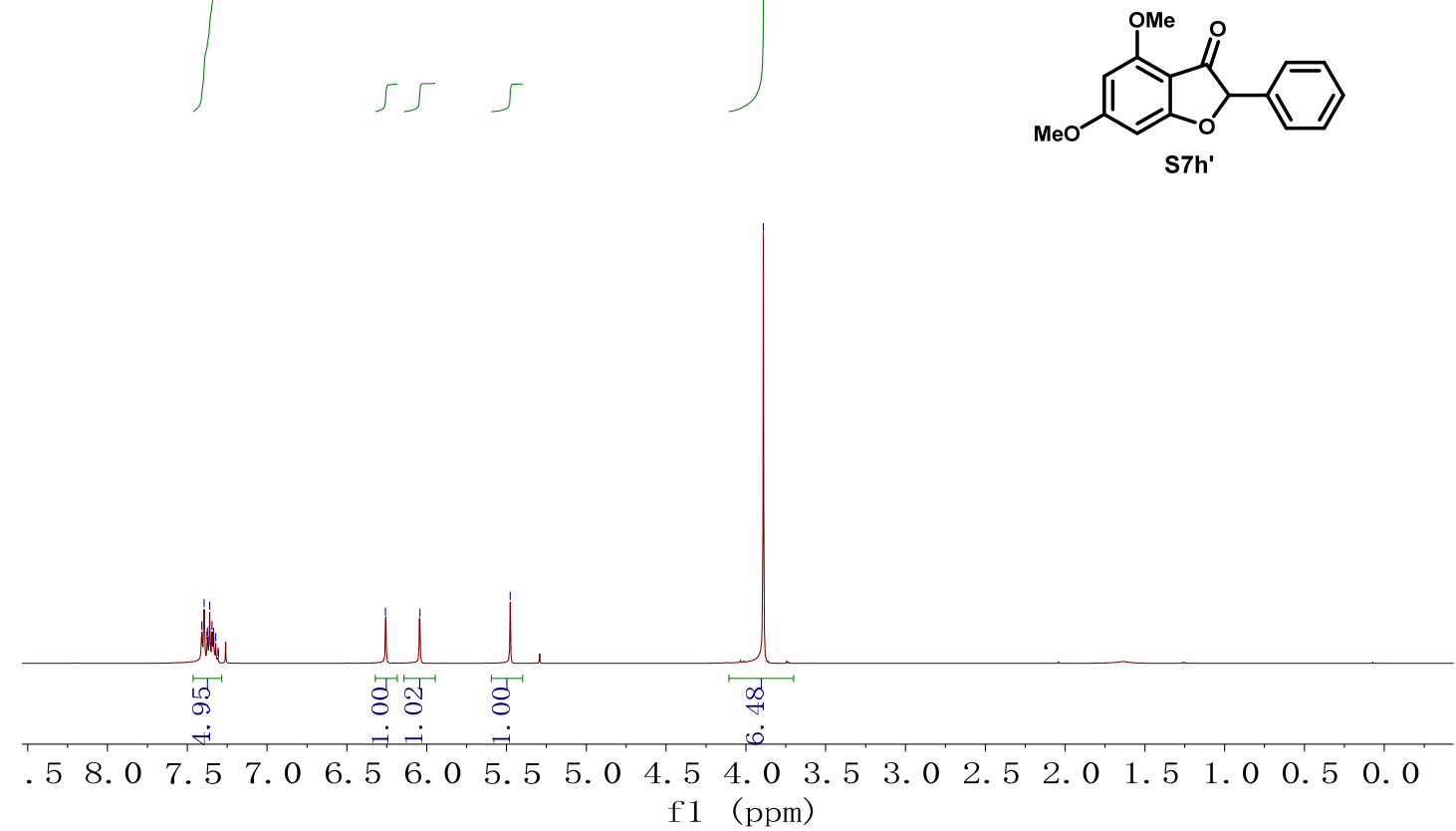

\section{${ }^{13} \mathrm{C}$ NMR (125 MHz, CDCl $@ 77$ ppm)}

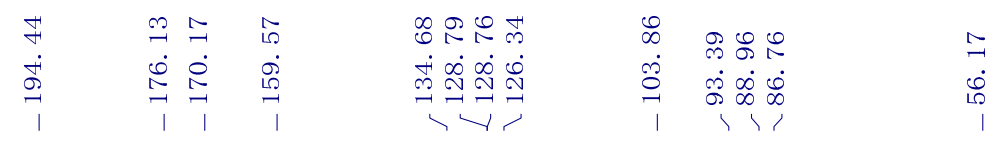
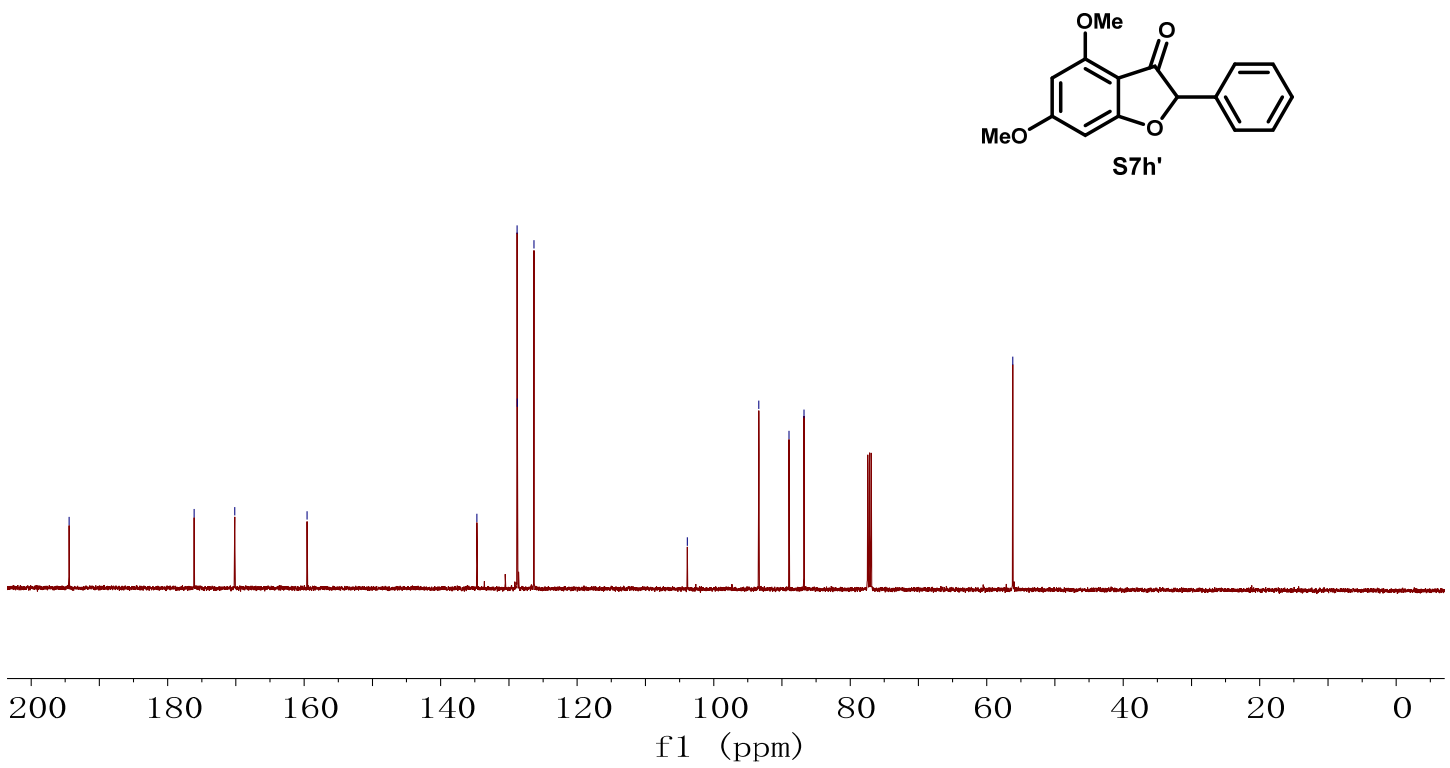

Allyl (2-benzylbenzofuran-3-yl) carbonate (12a)

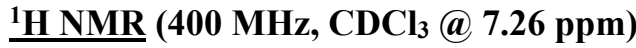




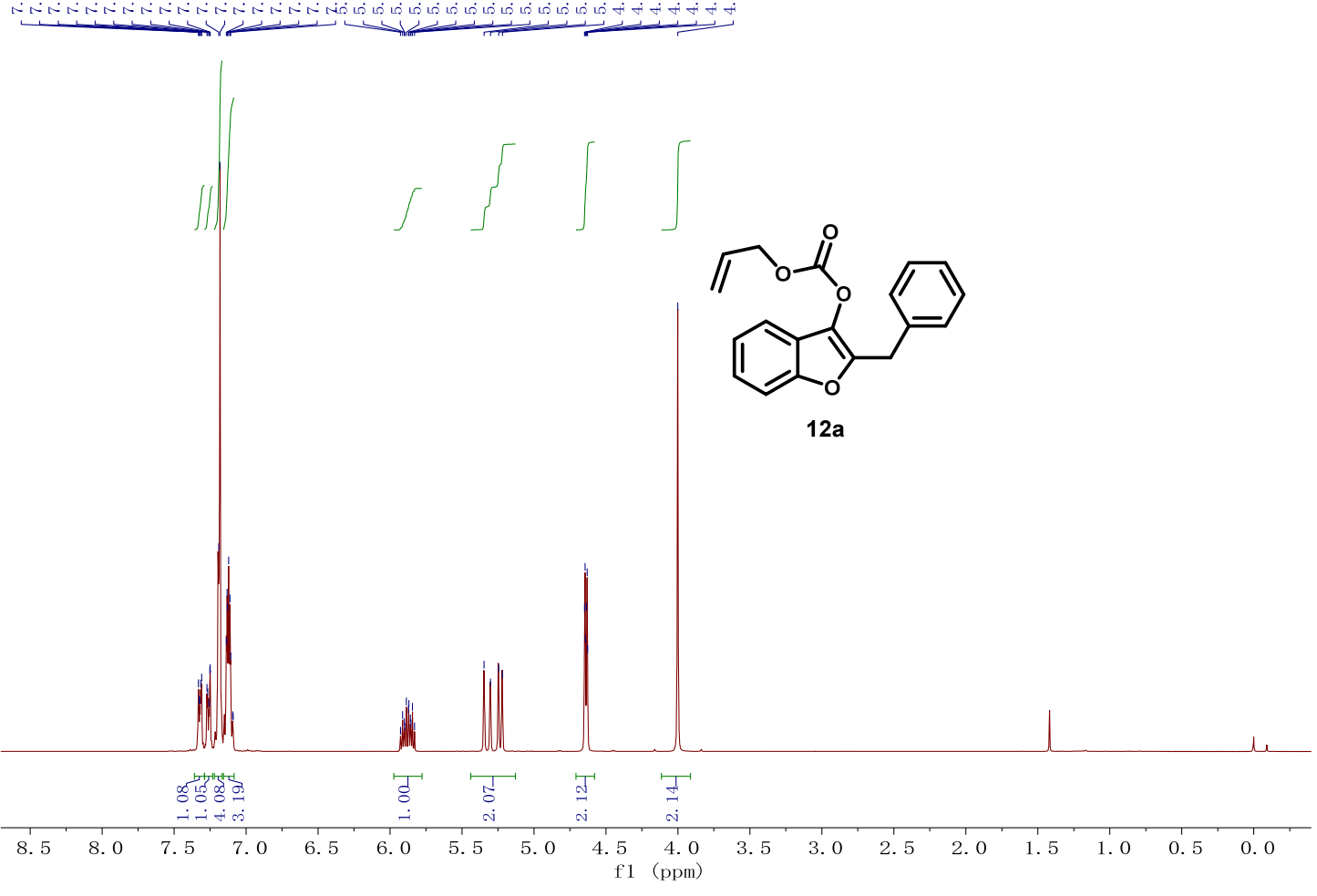

13. NMR (100 MHz, CDCl $@ 77$ ppm)
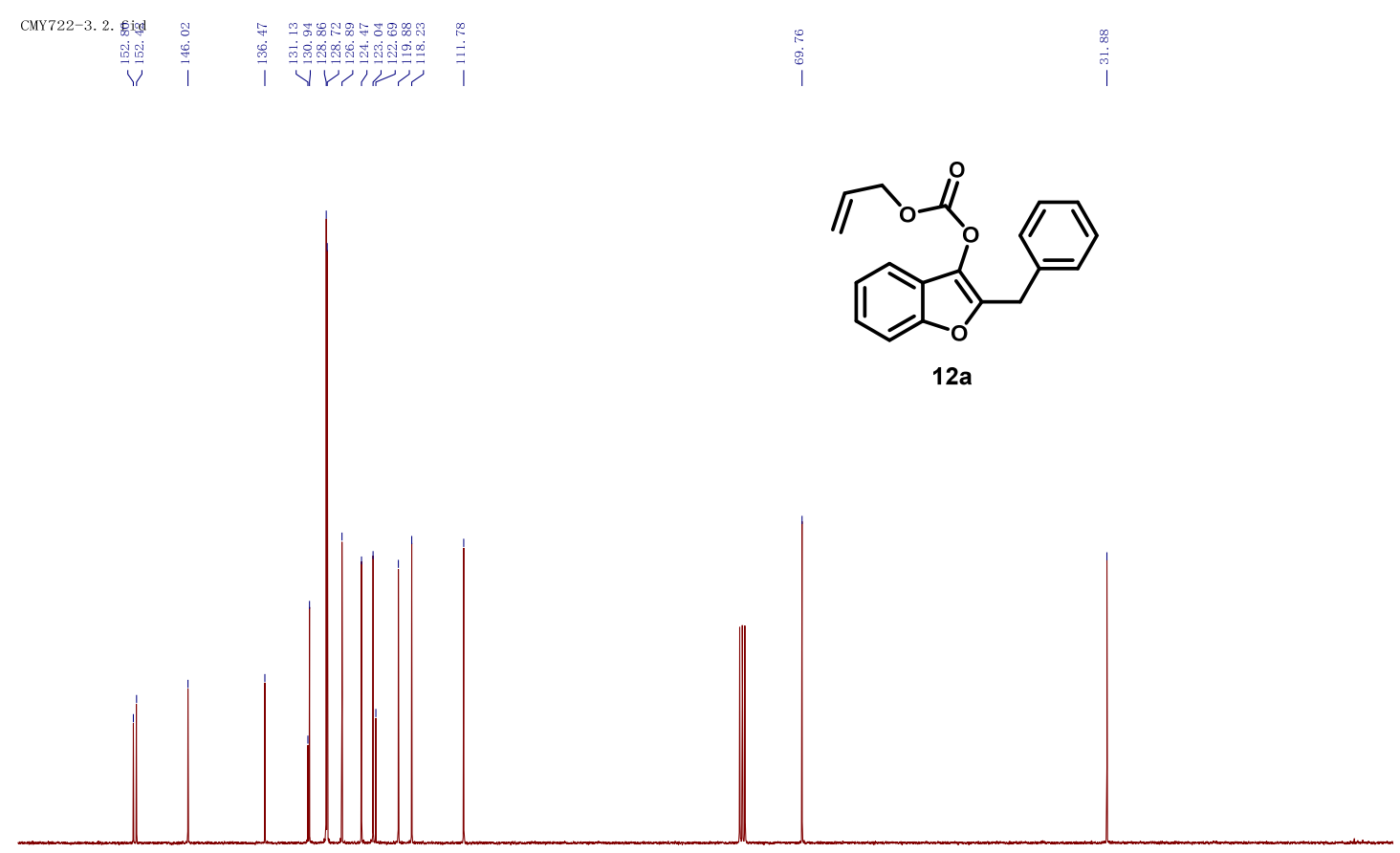

Acetyl-2-benzyl-1H-indol-3-yl allyl carbonate (12b)

$\underline{1}$ H NMR (500 MHz, $\mathrm{CDCl}_{3} @ 7.26$ ppm) 


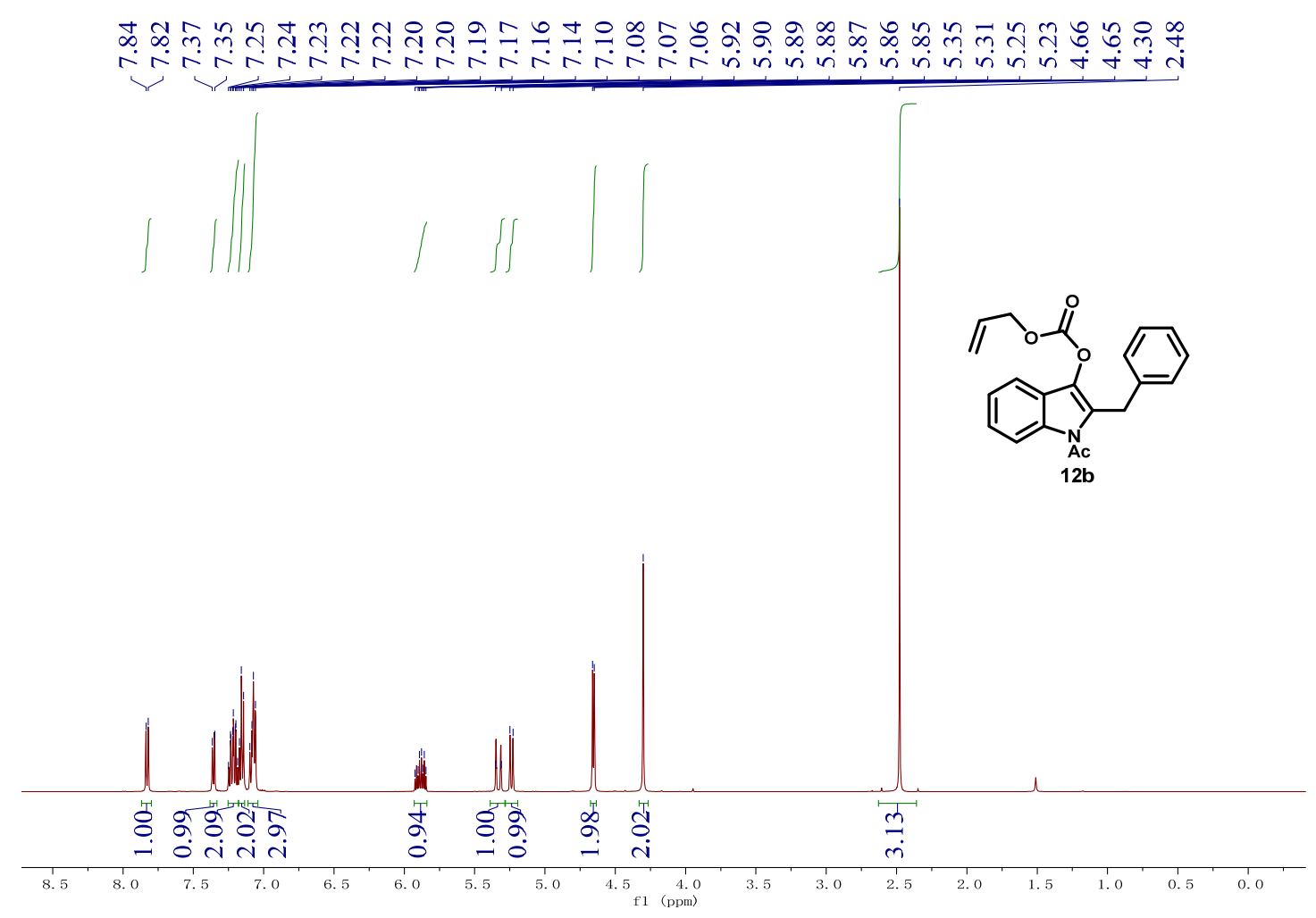

${ }^{13} \mathrm{C}$ NMR (125 MHz, $\mathrm{CDCl}_{3} @ 7.26$ ppm)

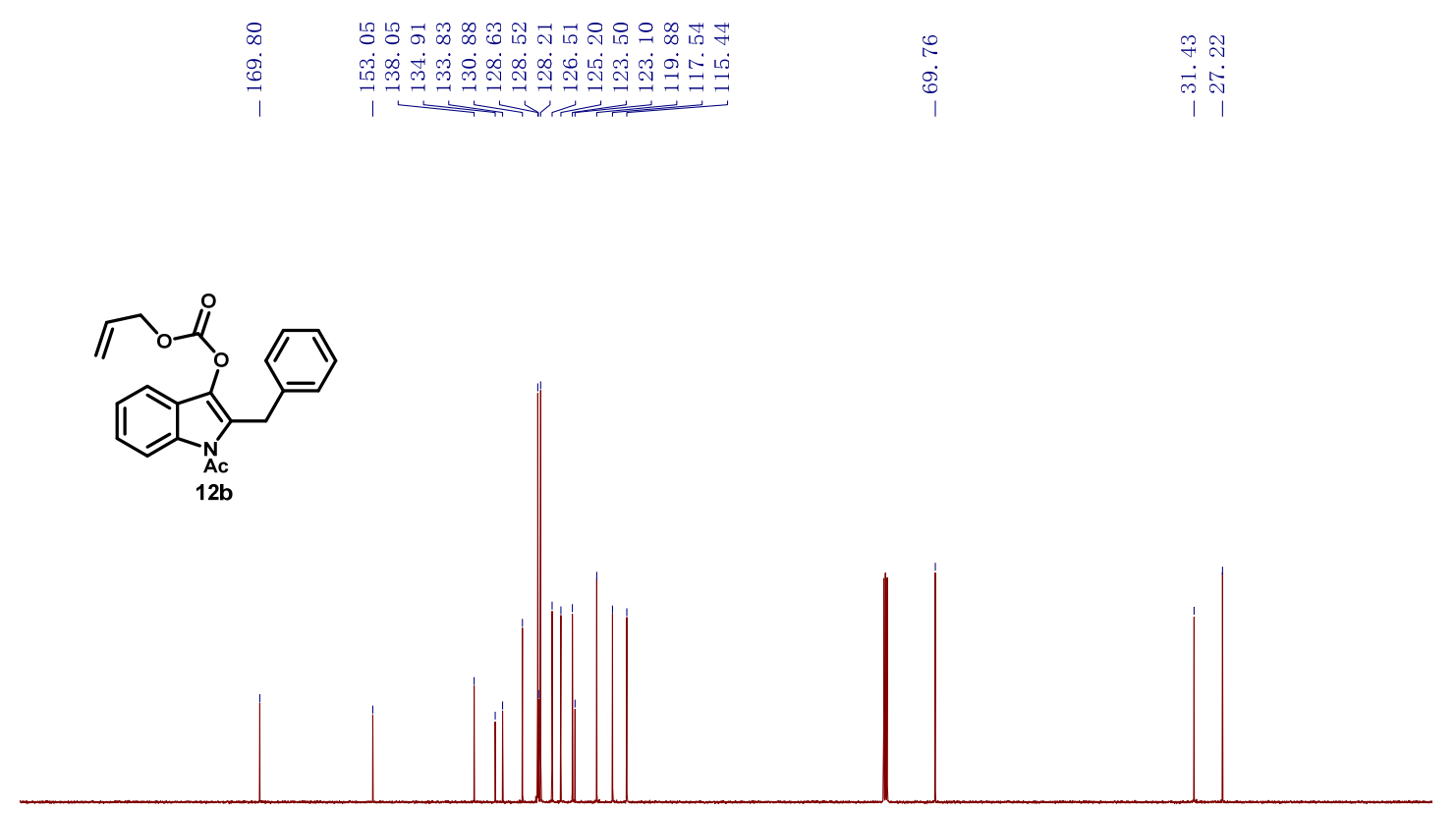

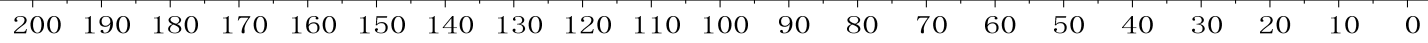
f1 (ppm)

Methyl 4-((3-(((allyloxy)carbonyl)oxy)benzofuran-2-yl)methyl)benzoate (12c) 
$\underline{{ }^{1} \mathrm{H} \text { NMR }}\left(400 \mathrm{MHz}, \mathrm{CDCl}_{3} @ 7.26 \mathrm{ppm}\right)$

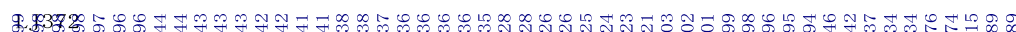

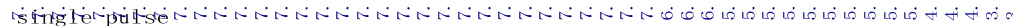

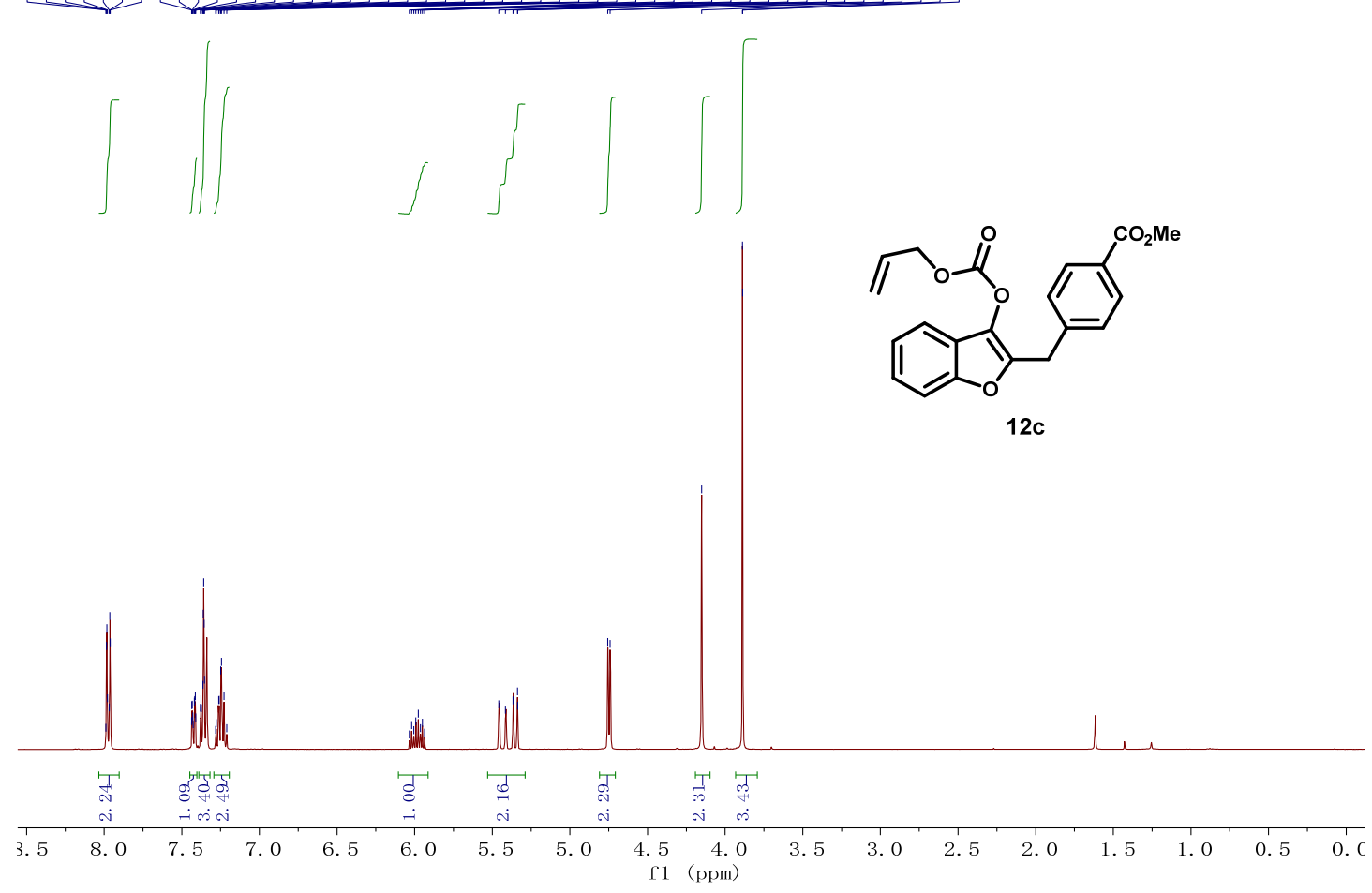

$\underline{{ }^{13} \mathrm{C} \text { NMR }}\left(100 \mathrm{MHz}, \mathrm{CDCl}_{3} @ 77\right.$ ppm)

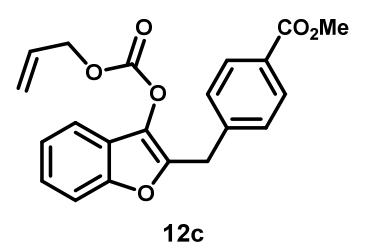

$12 \mathrm{c}$

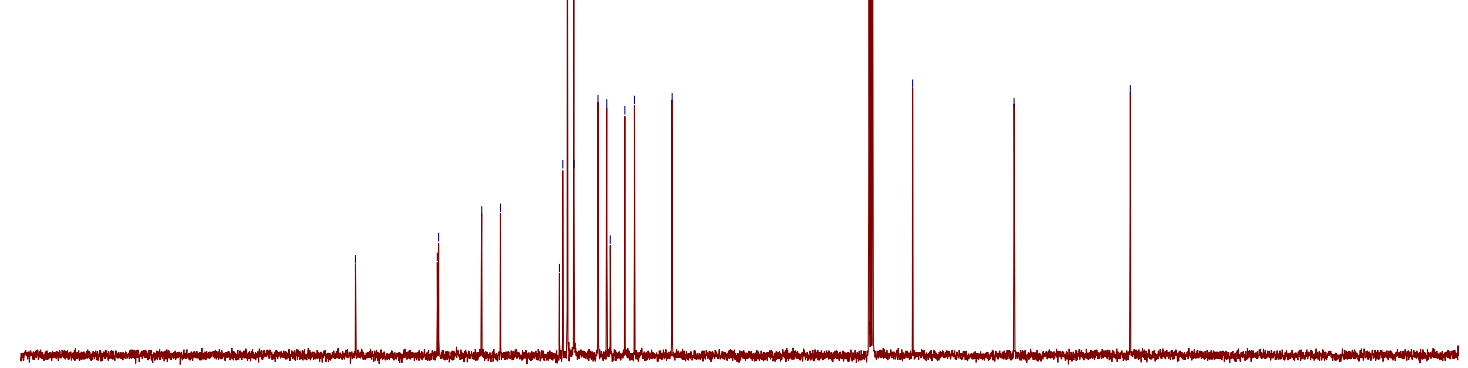

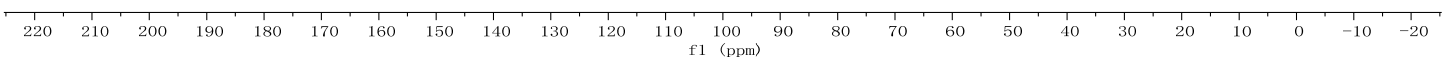

Allyl (2-(4-cyanobenzyl)benzofuran-3-yl) carbonate (12d)

S161 
$\underline{{ }^{1} \mathrm{H} \text { NMR }}\left(500 \mathrm{MHz}, \mathrm{CDCl}_{3} @ 7.26\right.$ ppm)

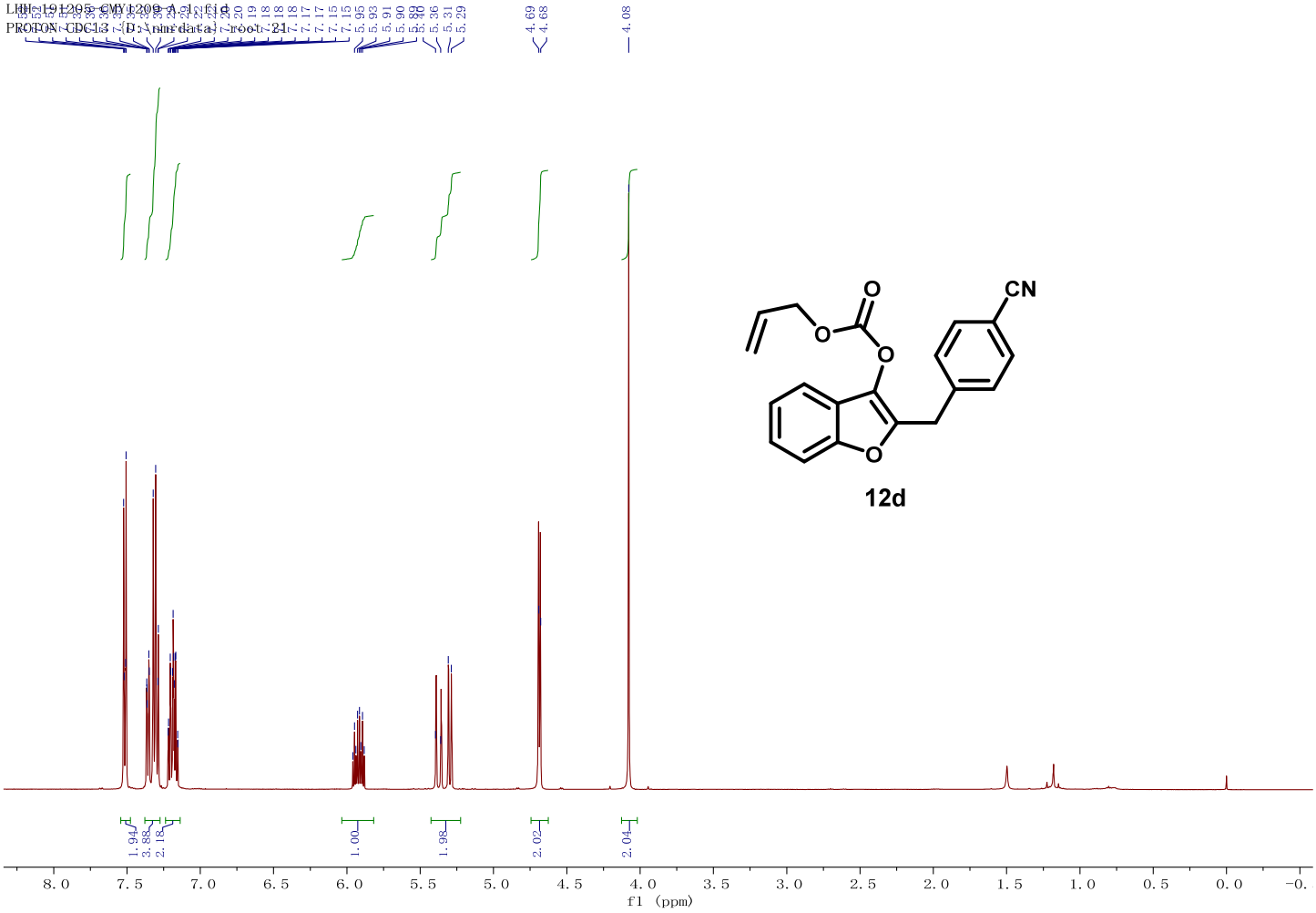

${ }^{13} \mathrm{C}$ NMR (125 MHz, $\mathrm{CDCl}_{3} @ 77$ ppm)

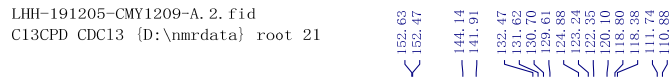
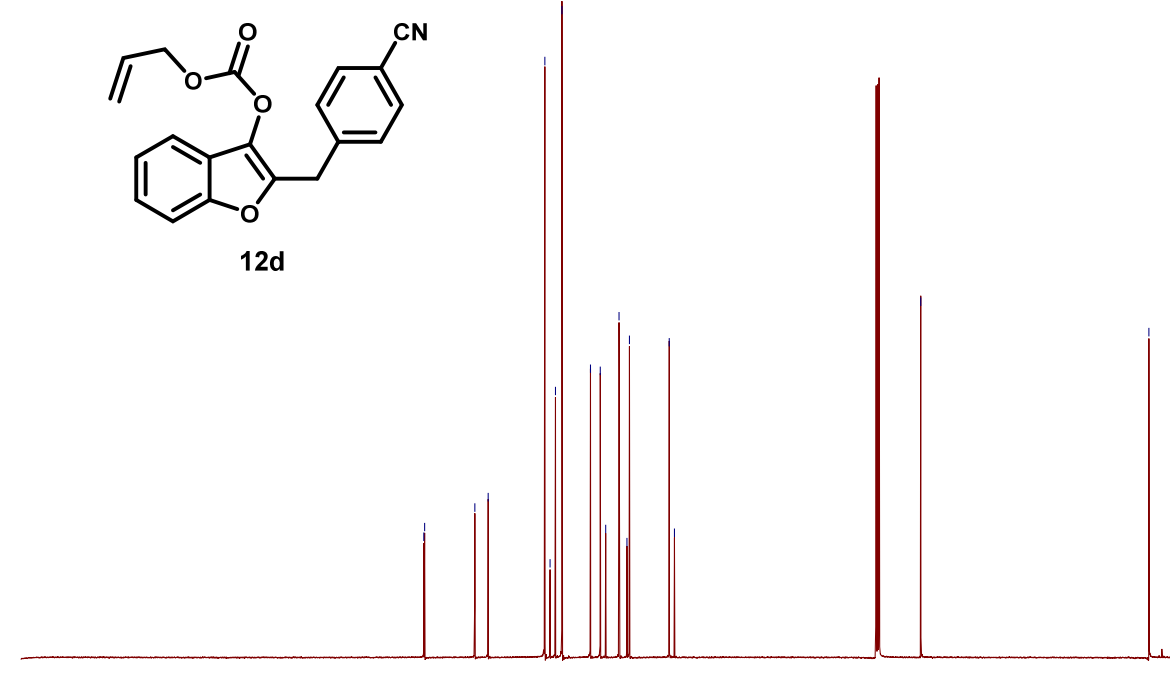
Allyl (2-(4-(trifluoromethyl)benzyl)benzofuran-3-yl) carbonate (12e)

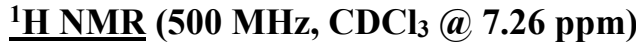

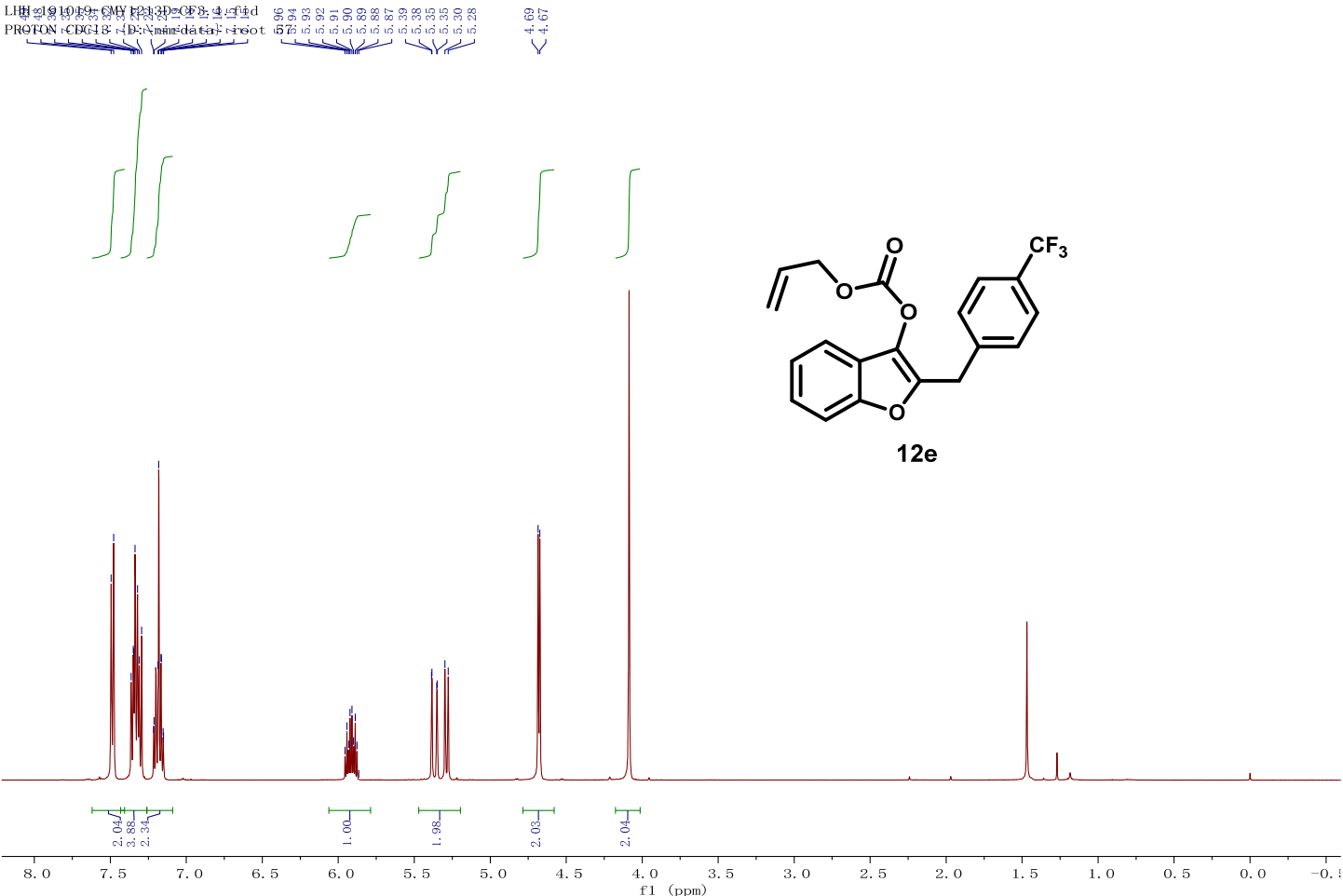

${ }^{13} \mathrm{C}$ NMR (125 MHz, $\mathrm{CDCl}_{3} @ 77$ ppm)

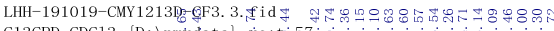

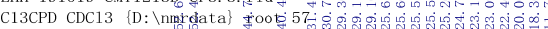

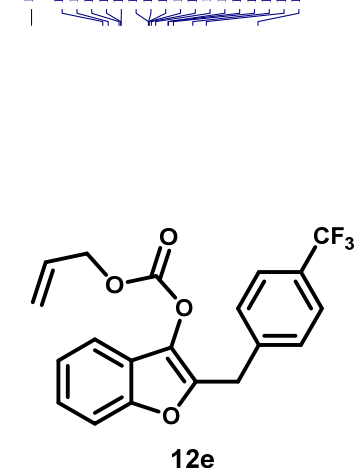

$12 e$

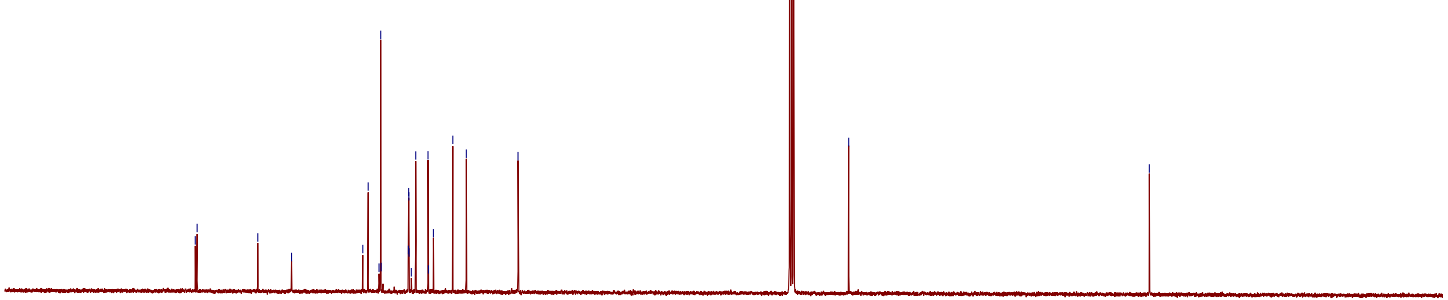

$\begin{array}{lllllllllr}170 & 160 & 150 & 140 & 130 & 120 & 110 & 100 & 90 & 80\end{array}$ 
LHH-191019-CMY1213D-CF3. 2. fid

F19 CDC13 \{D: \nmrdata\} root 57

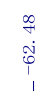

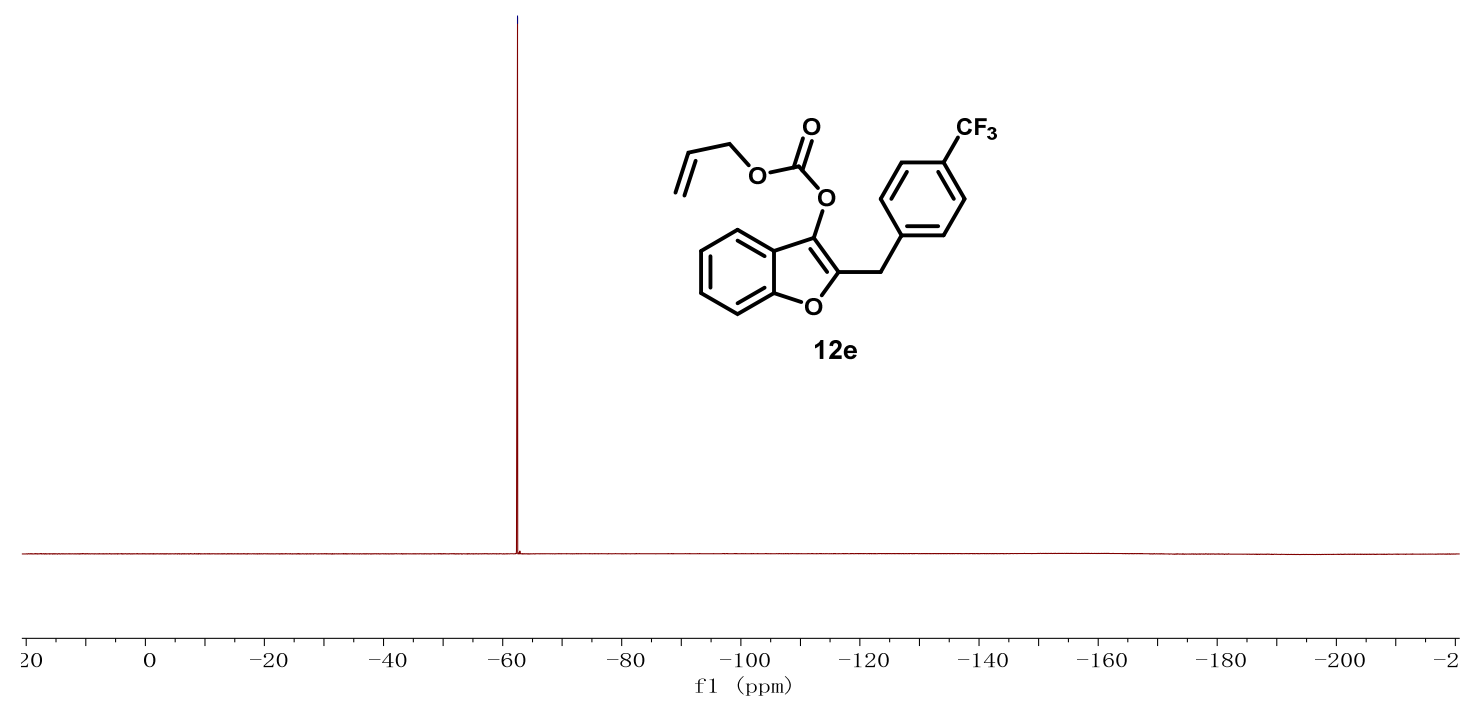

Allyl (2-(4-fluorobenzyl)benzofuran-3-yl) carbonate (12f)

매 NMR (400 MHz, $\mathrm{CDCl}_{3} @ 7.26$ ppm)

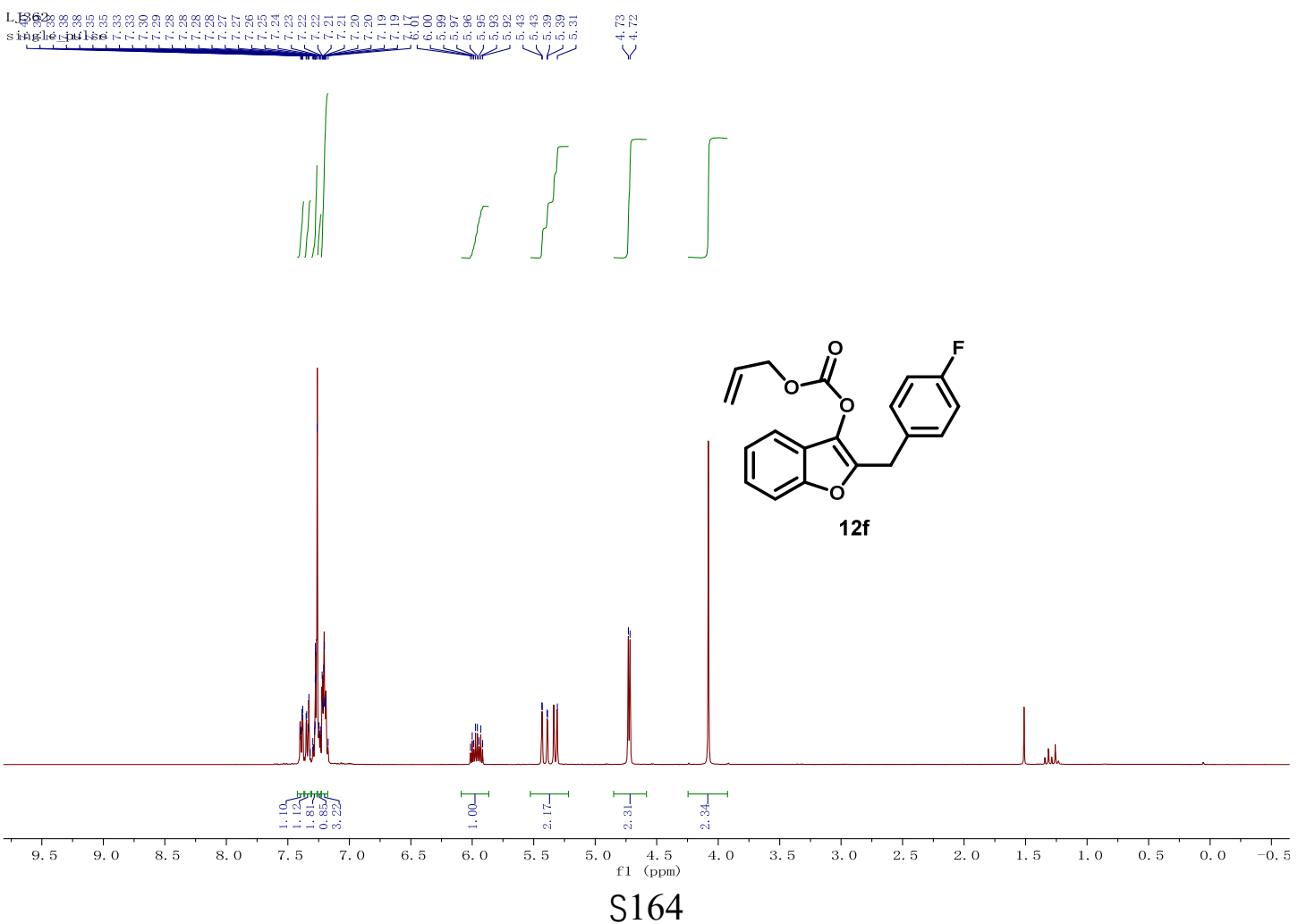


$\underline{{ }^{13} \mathrm{C} \text { NMR }}\left(100 \mathrm{MHz}, \mathrm{CDCl}_{3} @ 77\right.$ ppm)

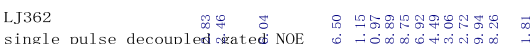

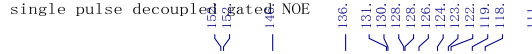
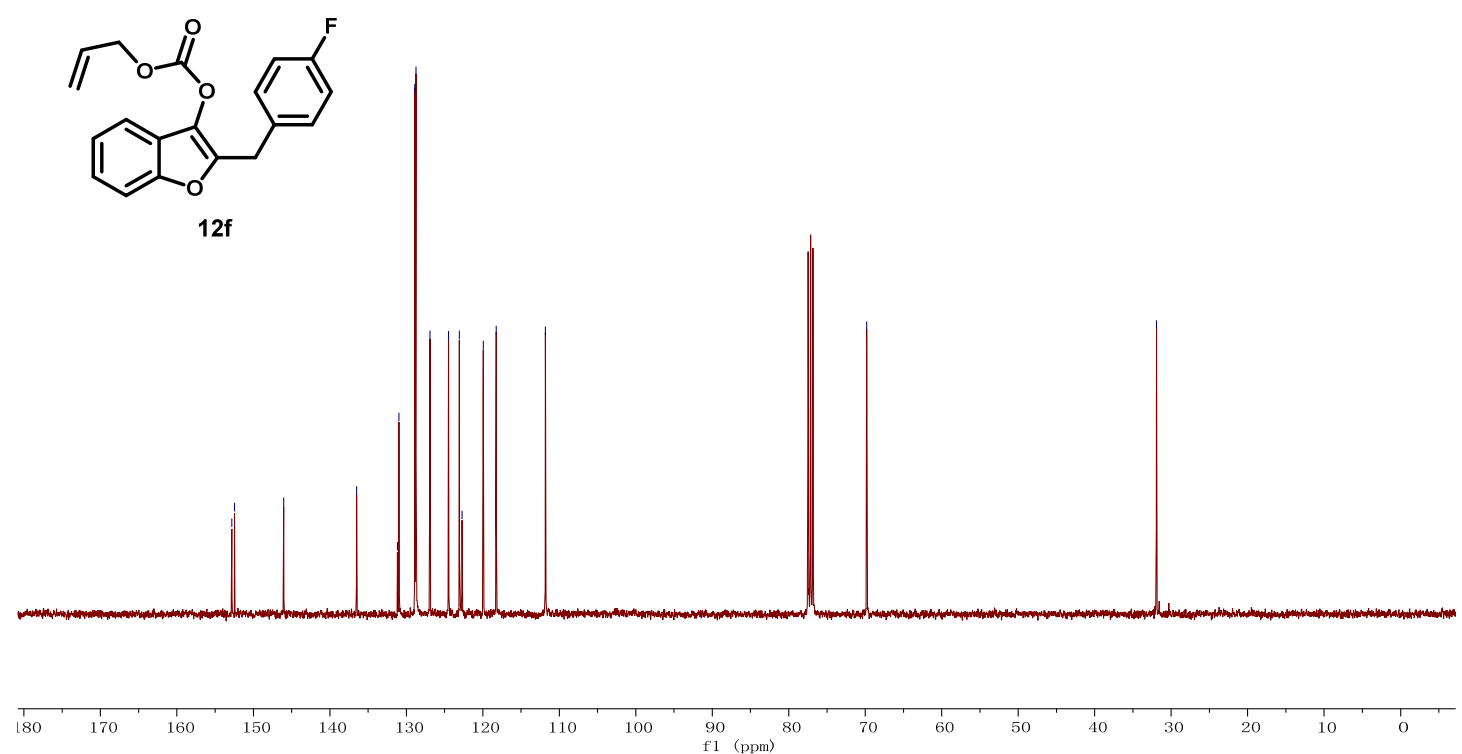

$\underline{{ }^{19} \text { F NMR }}\left(376 \mathrm{MHz}, \mathrm{CDCl}_{3}\right)$

LHH-191205-1 j-326-F. 10. fid (19)<smiles>C=CCOC(=O)Oc1c(Cc2ccc(F)cc2)oc2ccccc12</smiles>

$12 f$

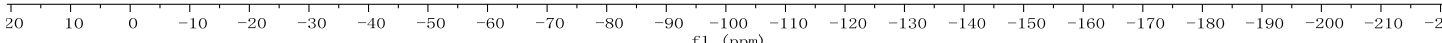

Allyl (2-(4-chlorobenzyl)benzofuran-3-yl) carbonate (12g)

S165 
$\underline{{ }^{1} \mathrm{H} \text { NMR }}\left(600 \mathrm{MHz}, \mathrm{CDCl}_{3} @ 7.26 \mathrm{ppm}\right)$

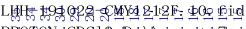

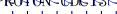

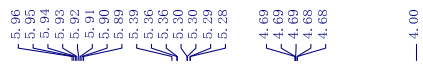
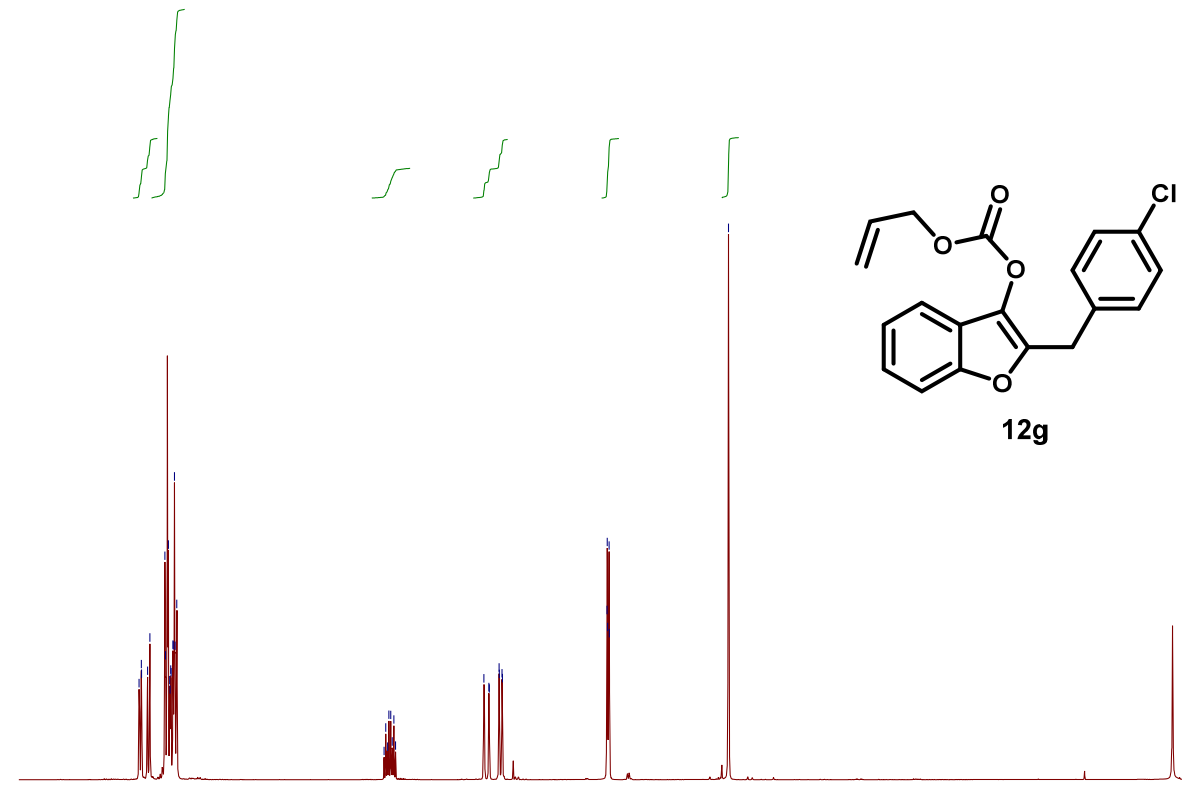

$12 \mathrm{~g}$

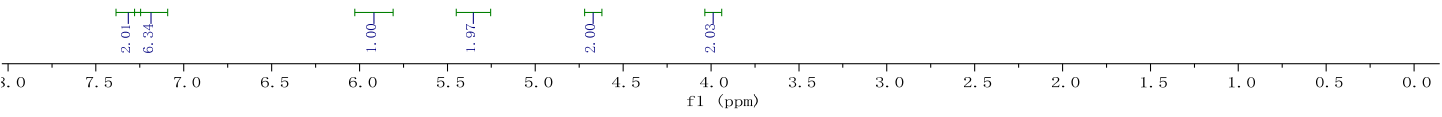

${ }^{13} \mathrm{C}$ NMR (150 MHz, CDCl 3977 ppm)
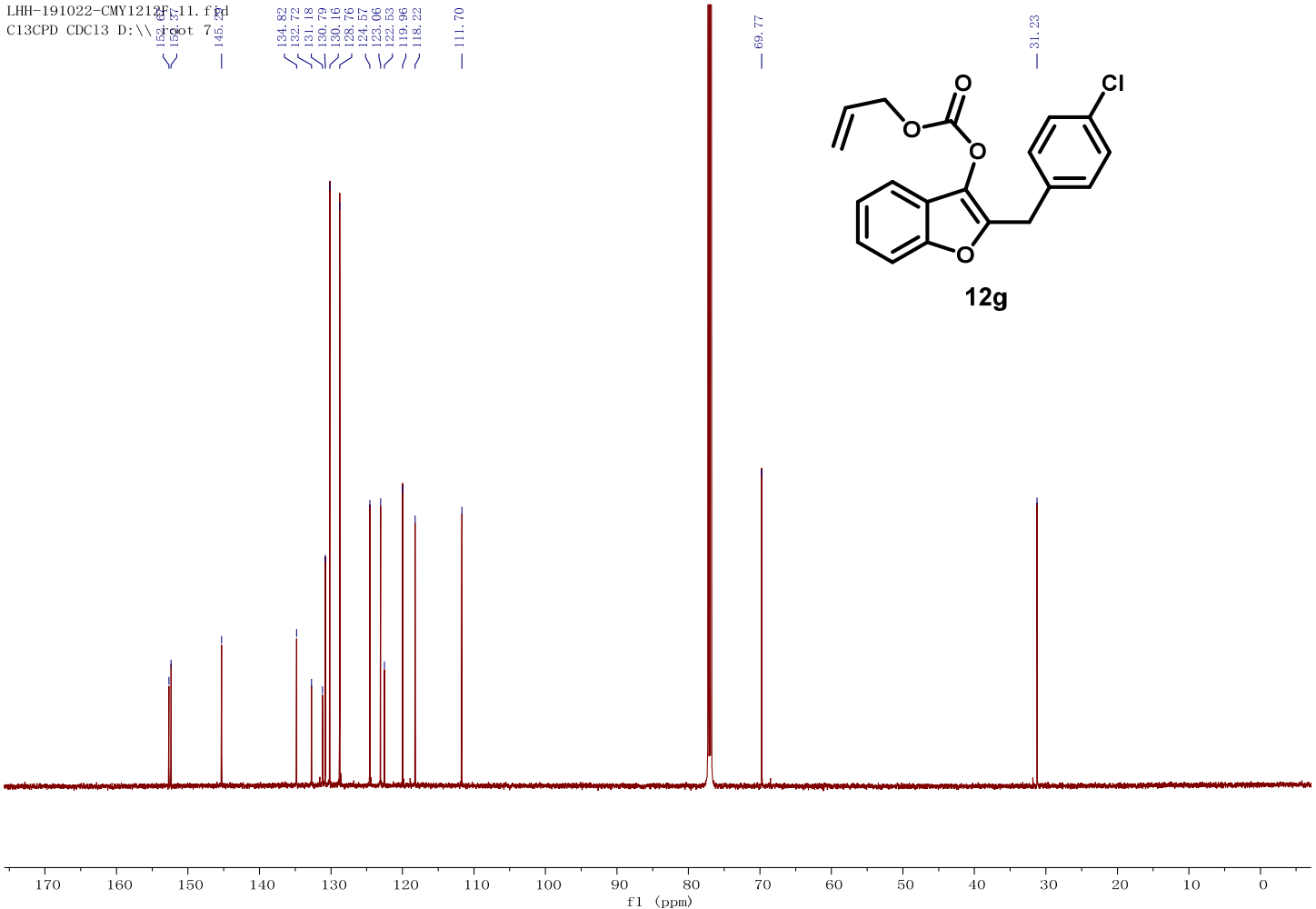

Allyl (2-(4-methoxybenzyl)benzofuran-3-yl) carbonate (12h)

S166 
$\underline{{ }^{1} \mathrm{H} \text { NMR }}\left(400 \mathrm{MHz}, \mathrm{CDCl}_{3} @ 7.26\right.$ ppm)

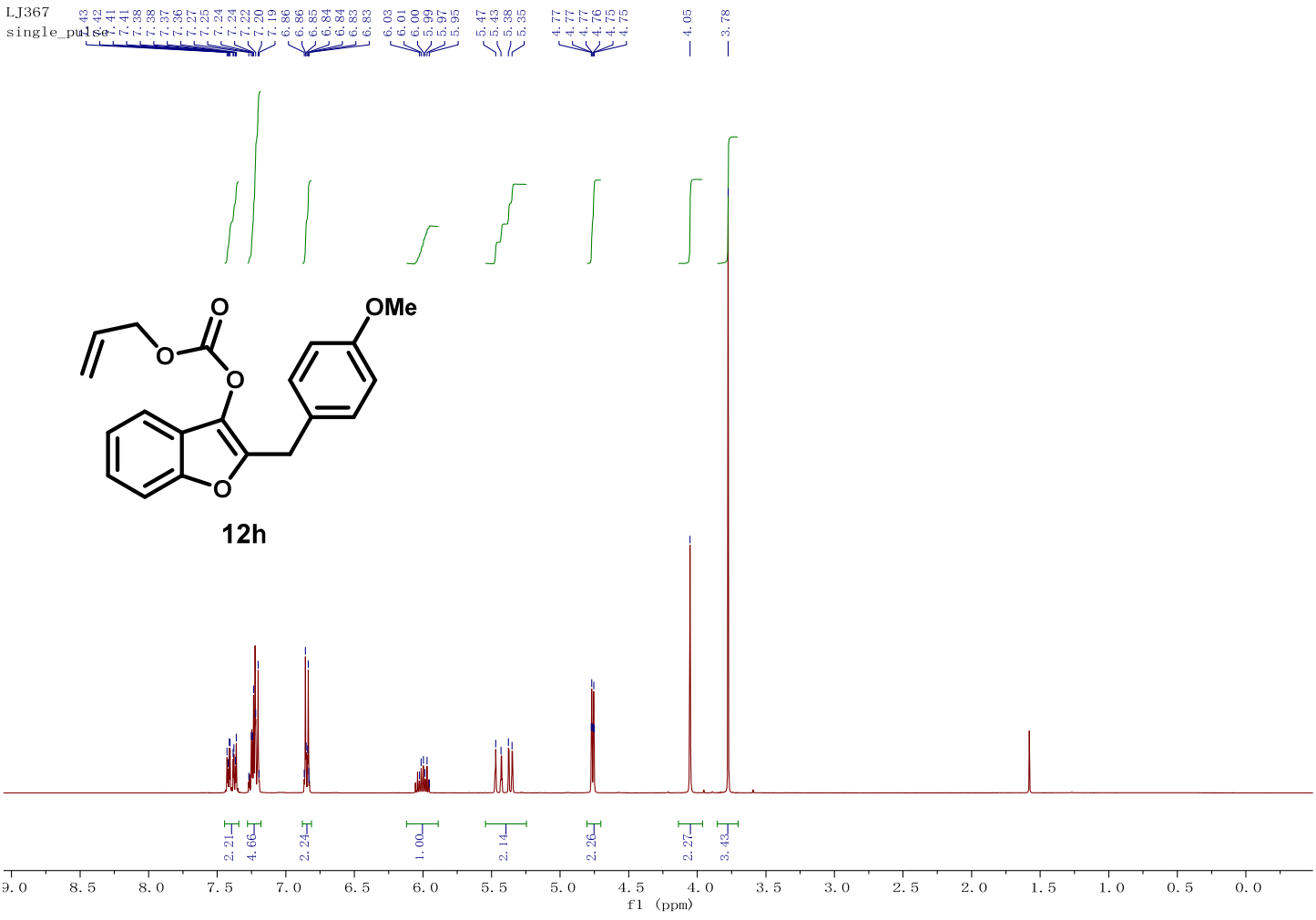

${ }^{13} \mathrm{C}$ NMR (100 MHz, $\mathrm{CDCl}_{3} @ 77$ ppm)

LJ367

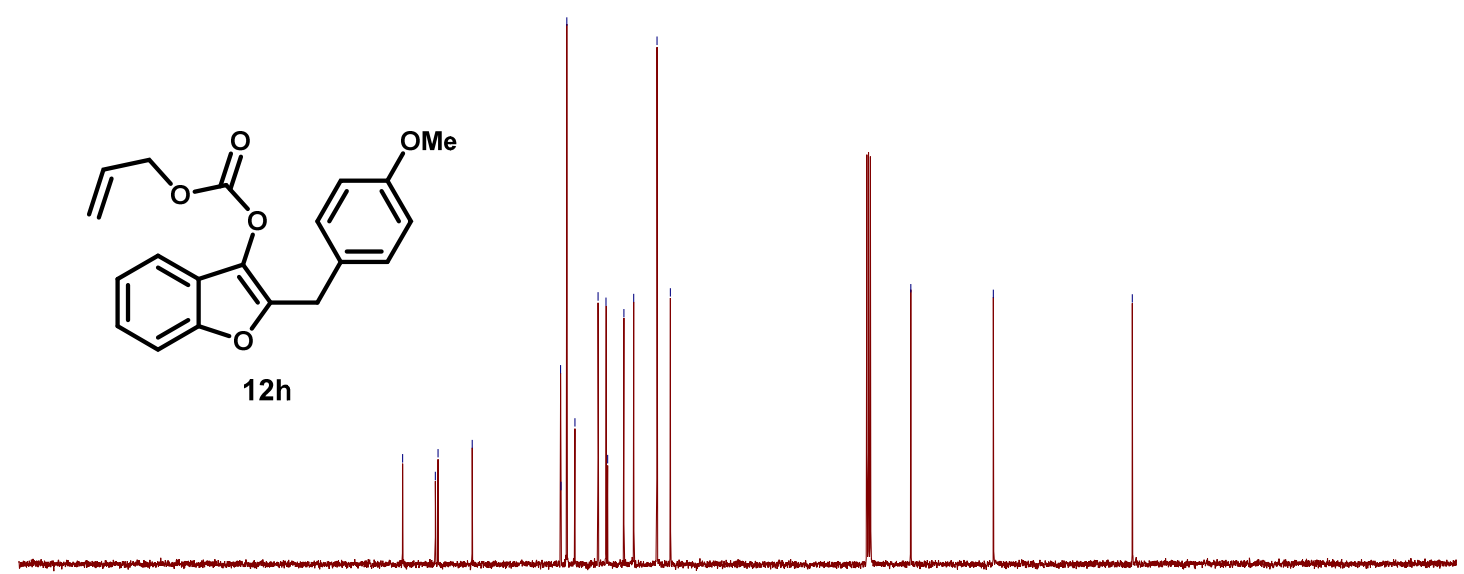

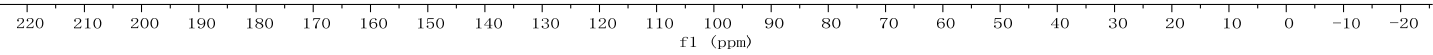

tert-Butyl (4-((3-(((Allyloxy)carbonyl)oxy)benzofuran-2-yl)methyl)phenyl)carbamate (12i) S167 
$\underline{{ }^{1} \mathrm{H} \text { NMR }}\left(500 \mathrm{MHz}, \mathrm{CDCl}_{3} @ 7.26 \mathrm{ppm}\right)$

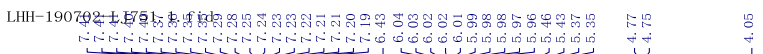
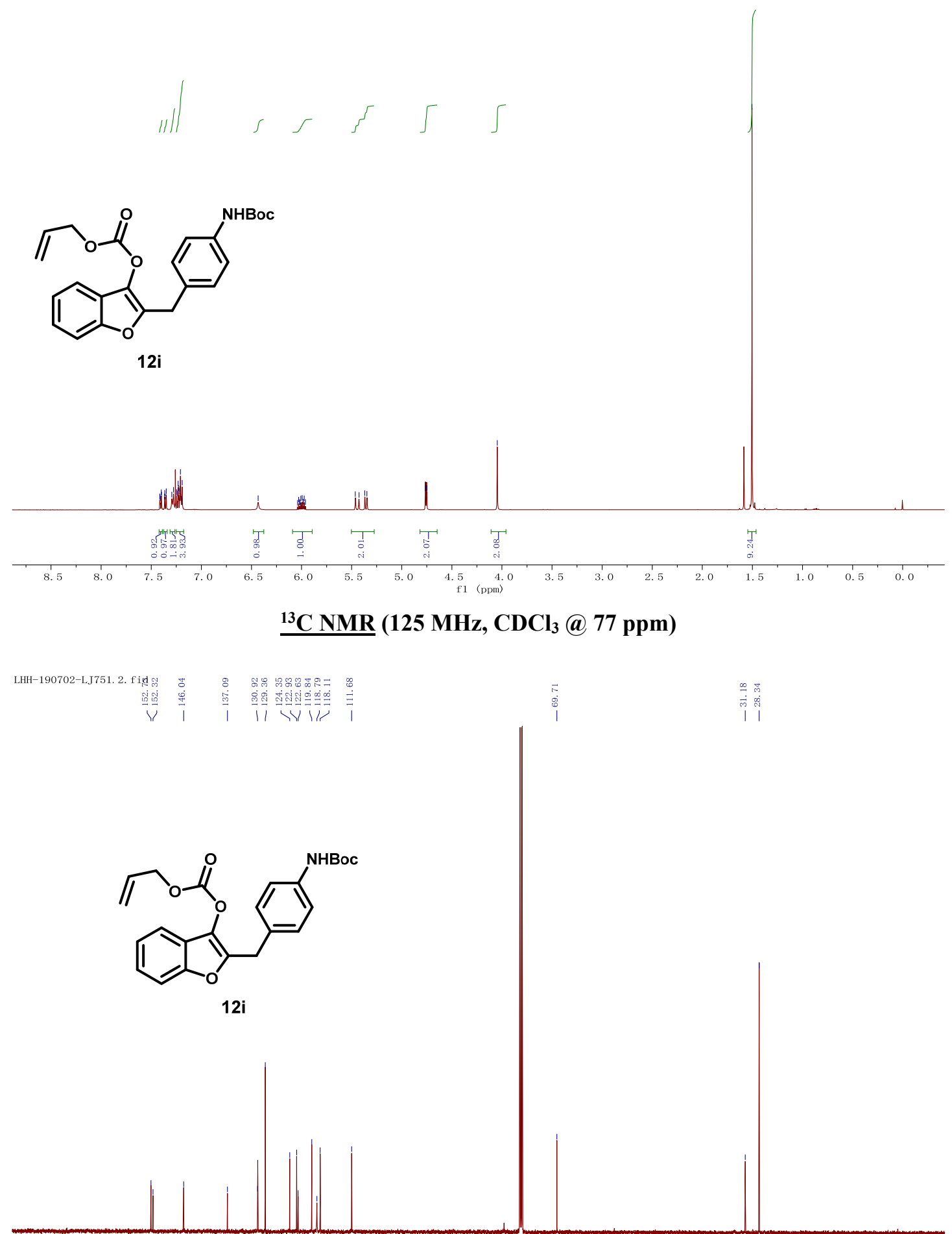

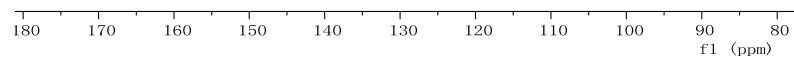

Allyl (2-(3-methoxybenzyl)benzofuran-3-yl) carbonate (12j)

S168 
$\underline{{ }^{1} \mathrm{H} \text { NMR }}\left(400 \mathrm{MHz}, \mathrm{CDCl}_{3} @ 7.26\right.$ ppm)

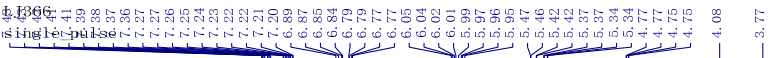

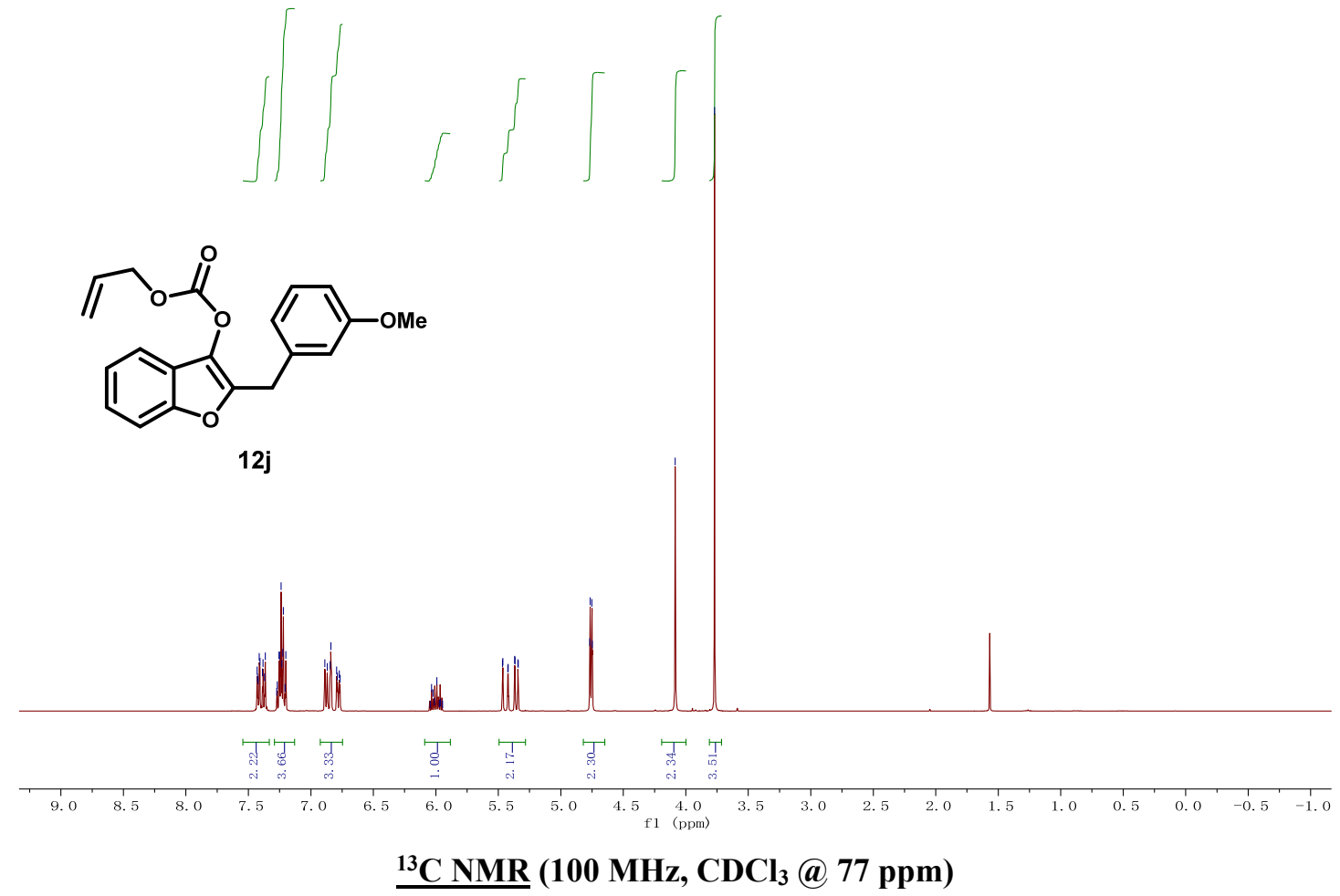

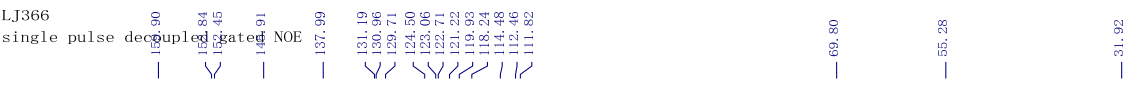

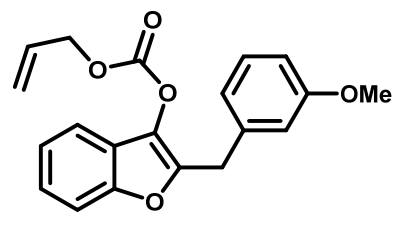

12j

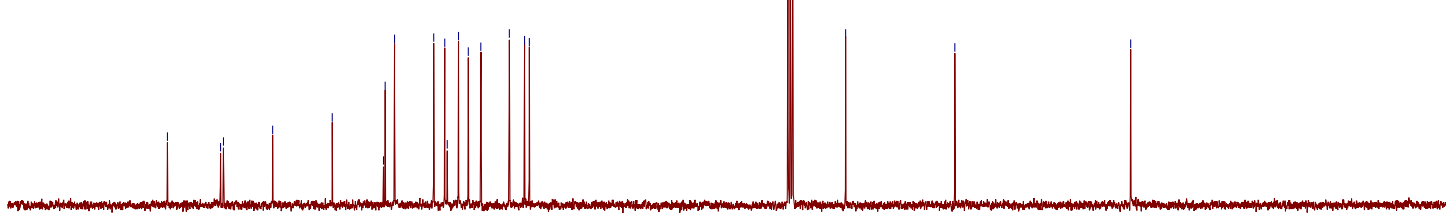

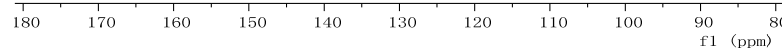


Allyl (2-(2-methylbenzyl)benzofuran-3-yl) carbonate (12k)

$\underline{1}$ H NMR $\left(500 \mathrm{MHz}, \mathrm{CDCl}_{3} @ 7.26\right.$ ppm)
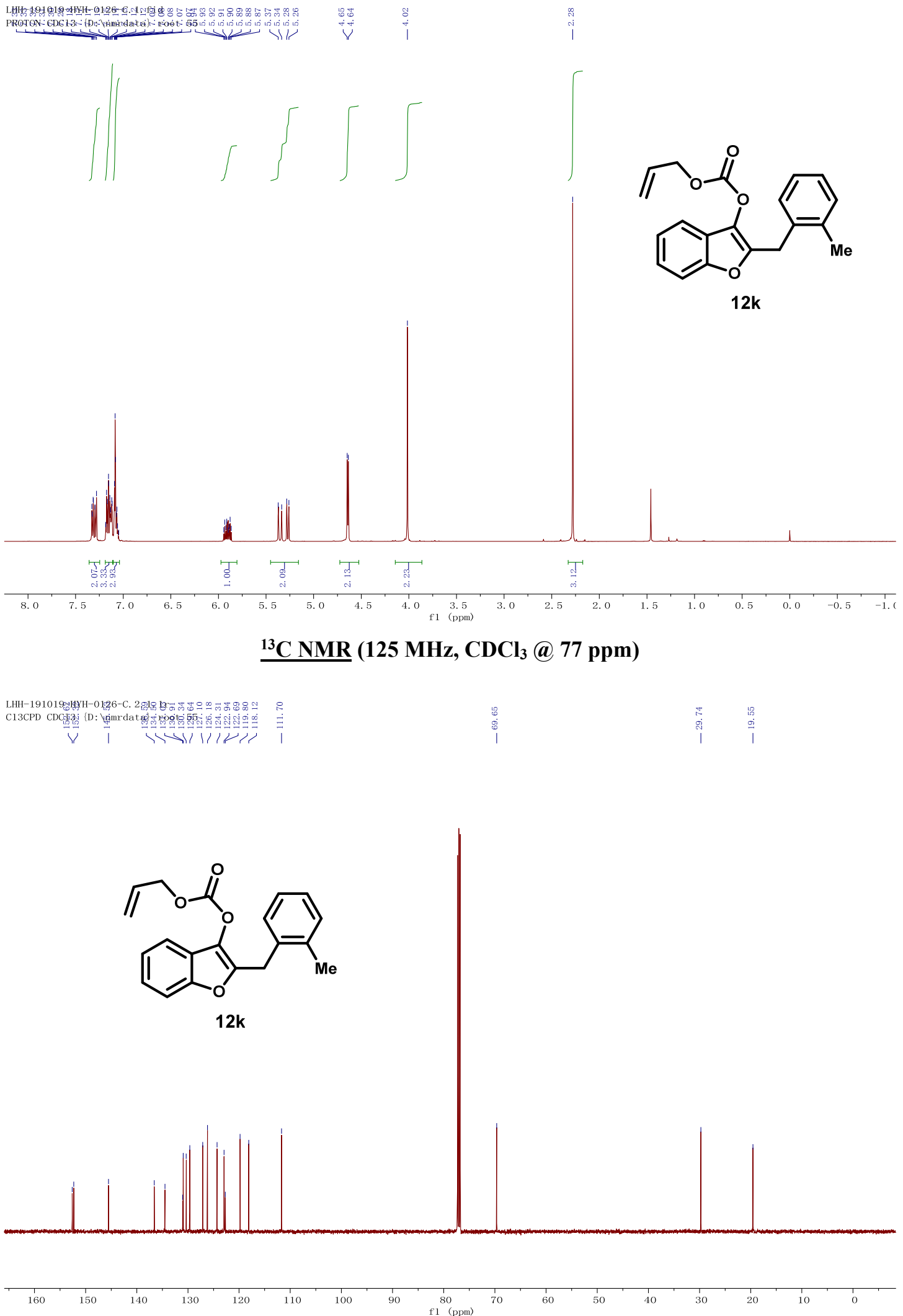
Allyl (2-(2-bromobenzyl)benzofuran-3-yl) carbonate (12l)

1 NMR (400 MHz, CDCl 3 @ 7.26 ppm)

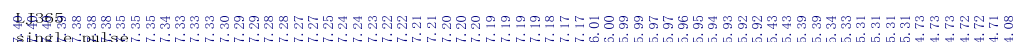

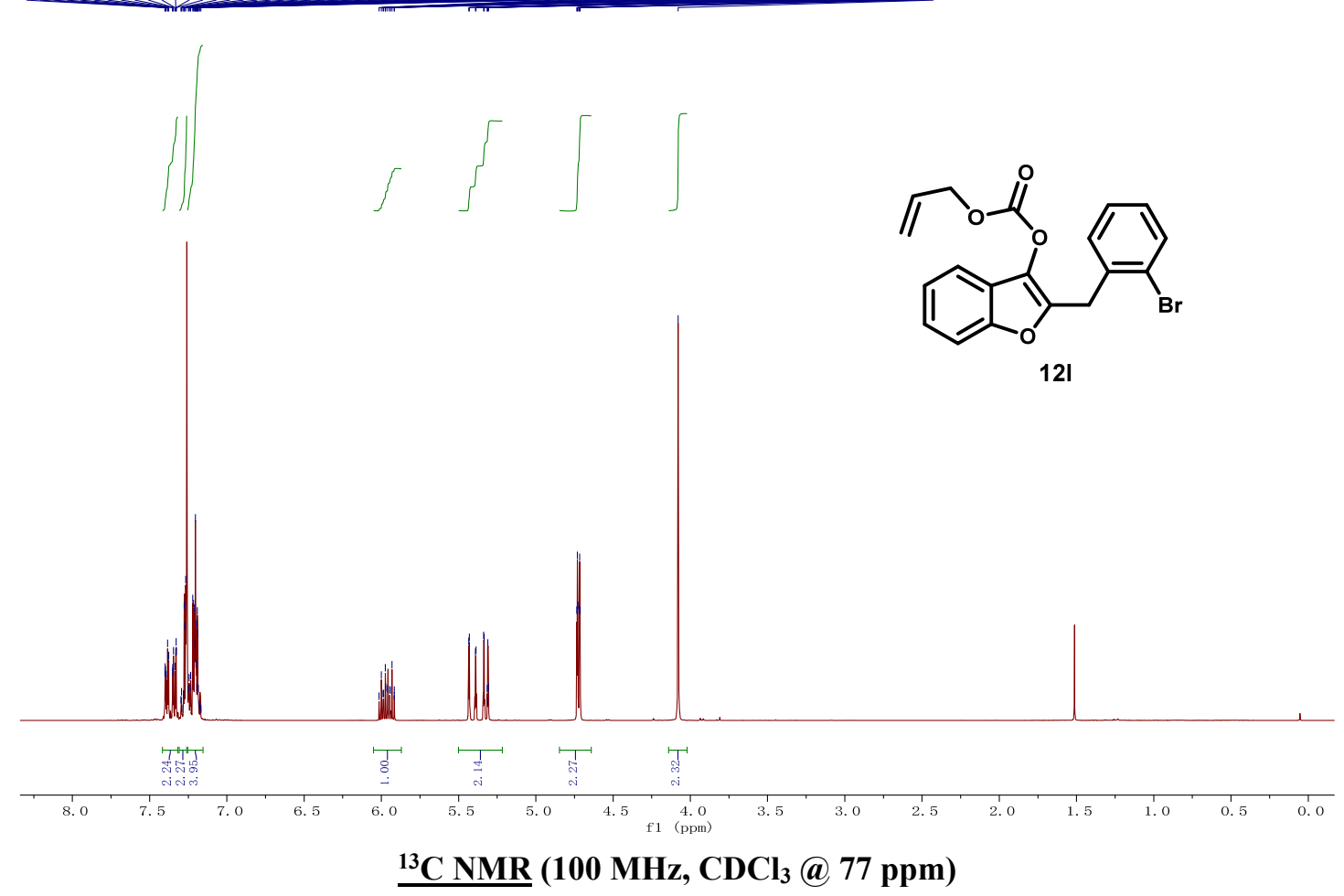

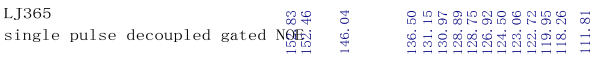

IVIIII)

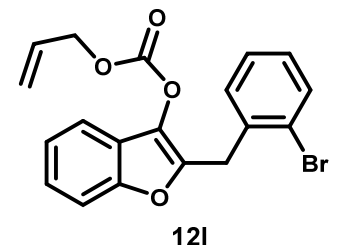

12I
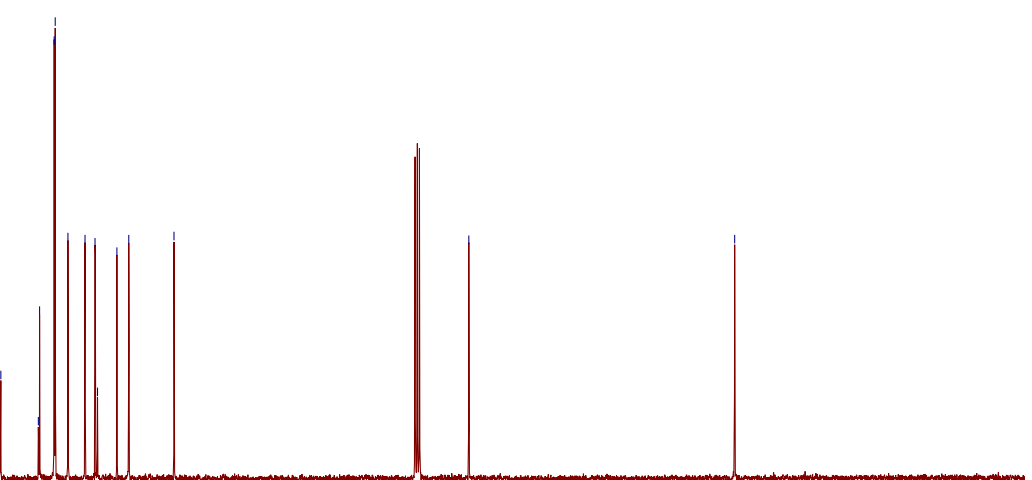
Allyl (2-(2,4,6-trimethylbenzyl)benzofuran-3-yl) carbonate (12m)

매 NMR $\left(600 \mathrm{MHz}, \mathrm{CDCl}_{3} @ 7.26\right.$ ppm)

4.

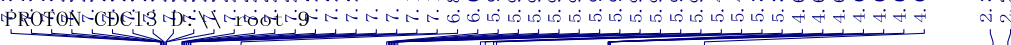

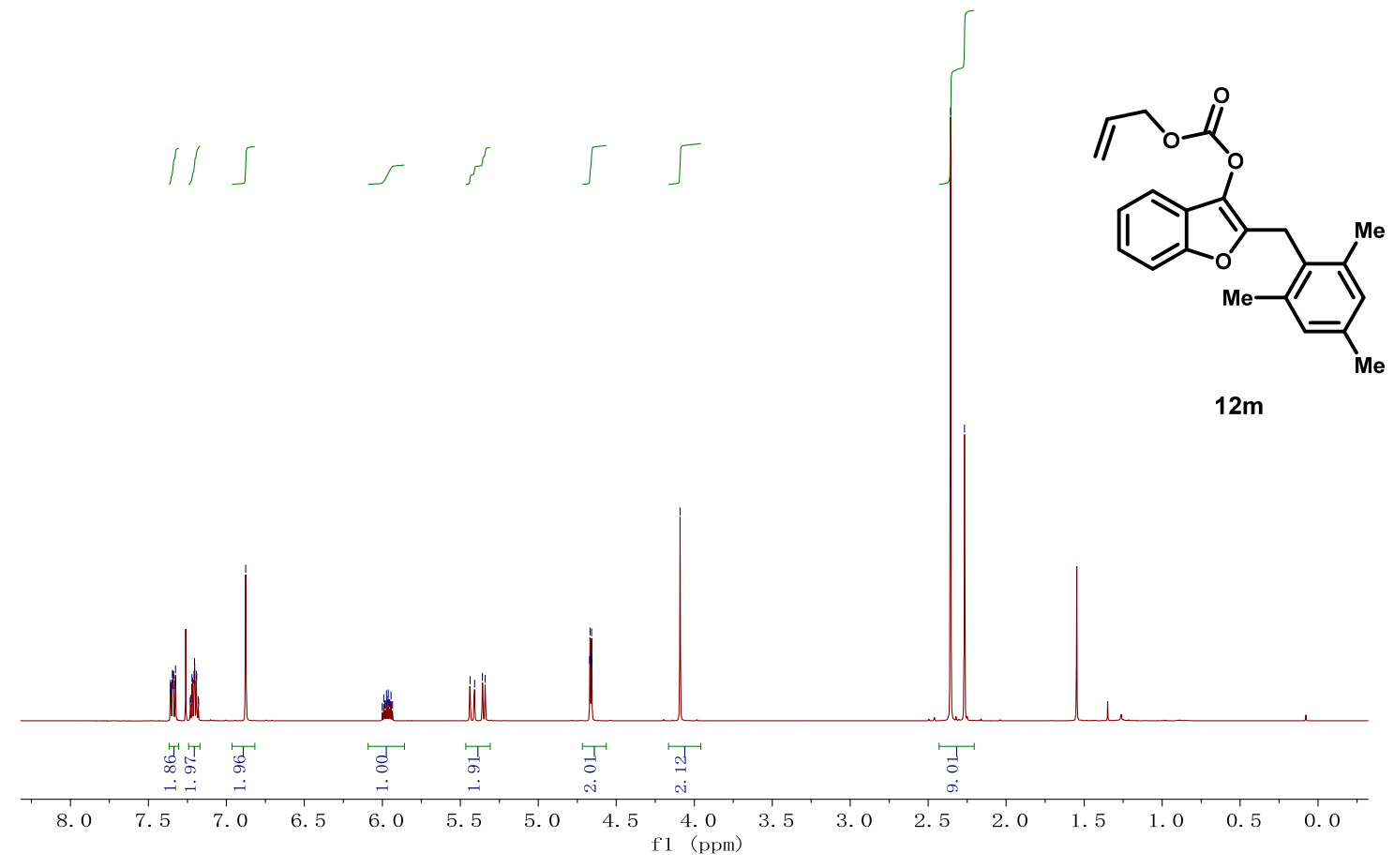

$\underline{{ }^{13} \mathrm{C} \text { NMR }}$ (150 MHz, $\mathrm{CDCl}_{3} @ 77$ ppm)

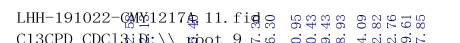

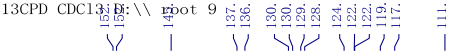

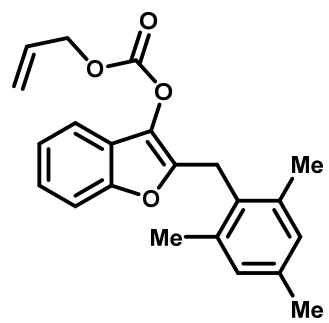

$12 m$
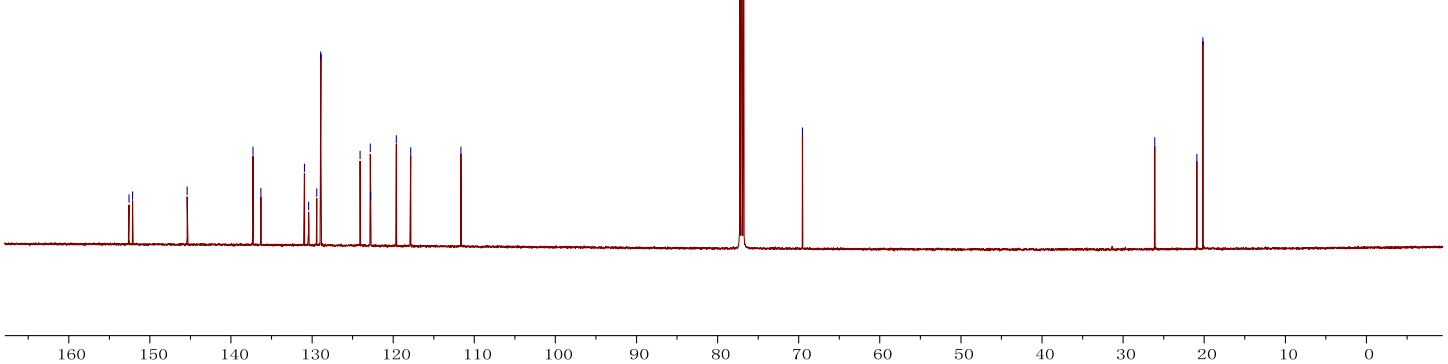

f1 $\stackrel{80}{(\mathrm{ppm})}$ 
Allyl (2-(naphthalen-2-ylmethyl)benzofuran-3-yl) carbonate (12n)

$\underline{{ }^{1} \mathrm{H} \text { NMR }}\left(400 \mathrm{MHz}, \mathrm{CDCl}_{3} @ 7.26 \mathrm{ppm}\right)$

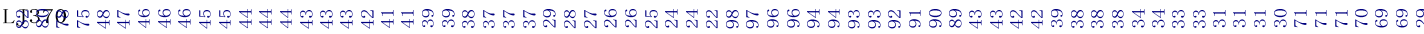

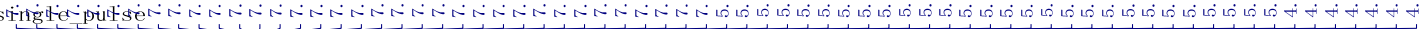

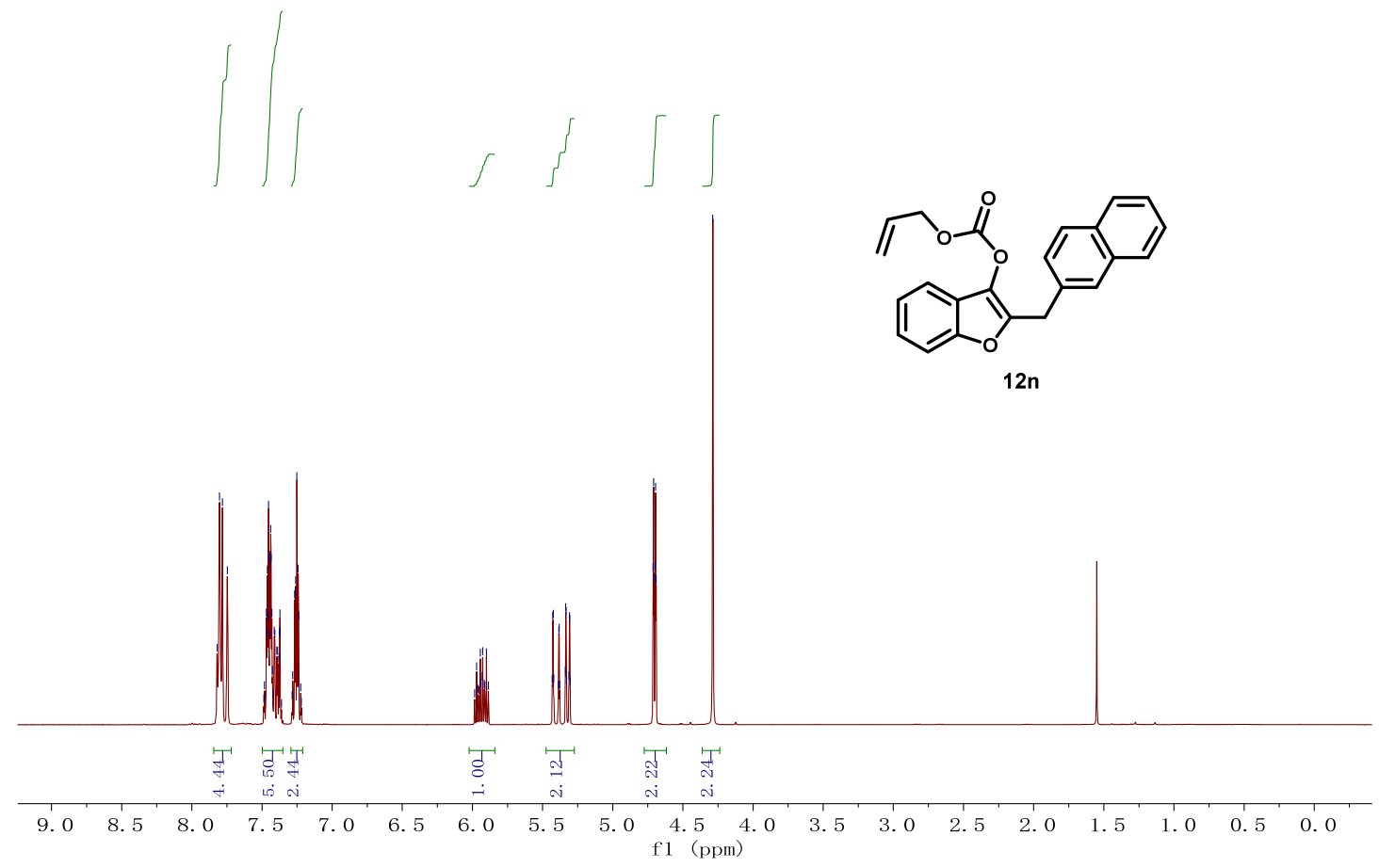

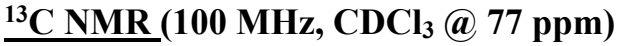

LJ370

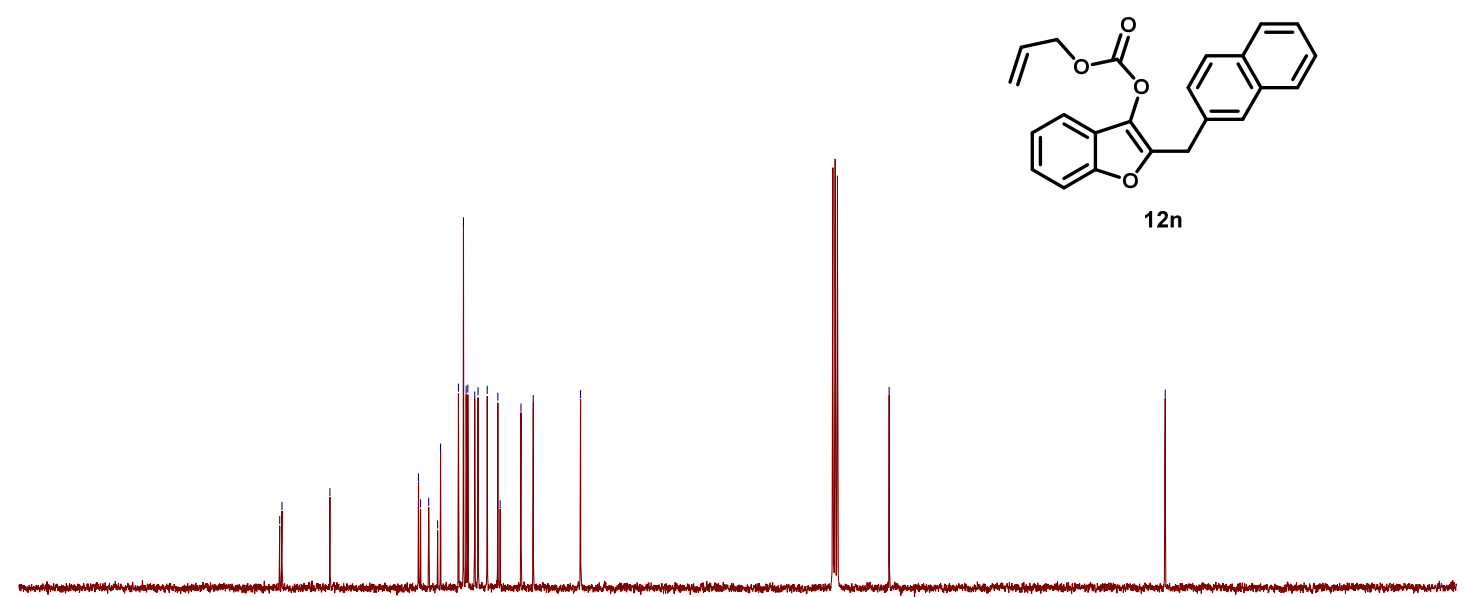




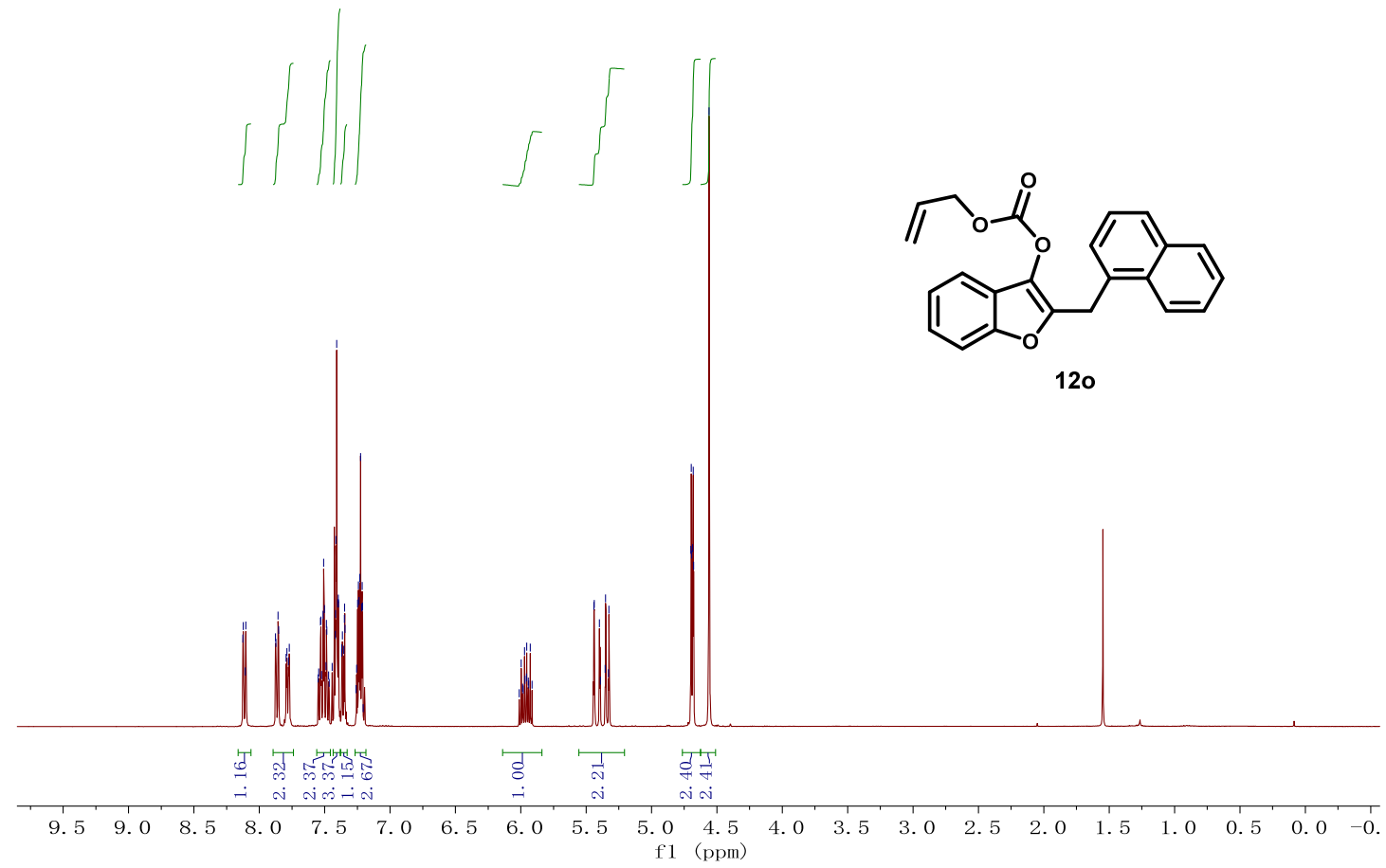

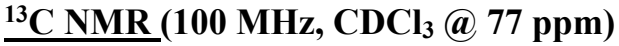

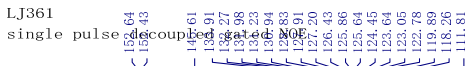

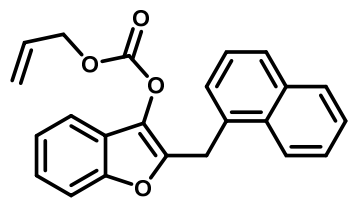

120 
Allyl (2-((1-methyl-1H-indol-3-yl)methyl)benzofuran-3-yl) carbonate (12p)

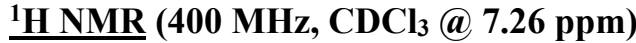

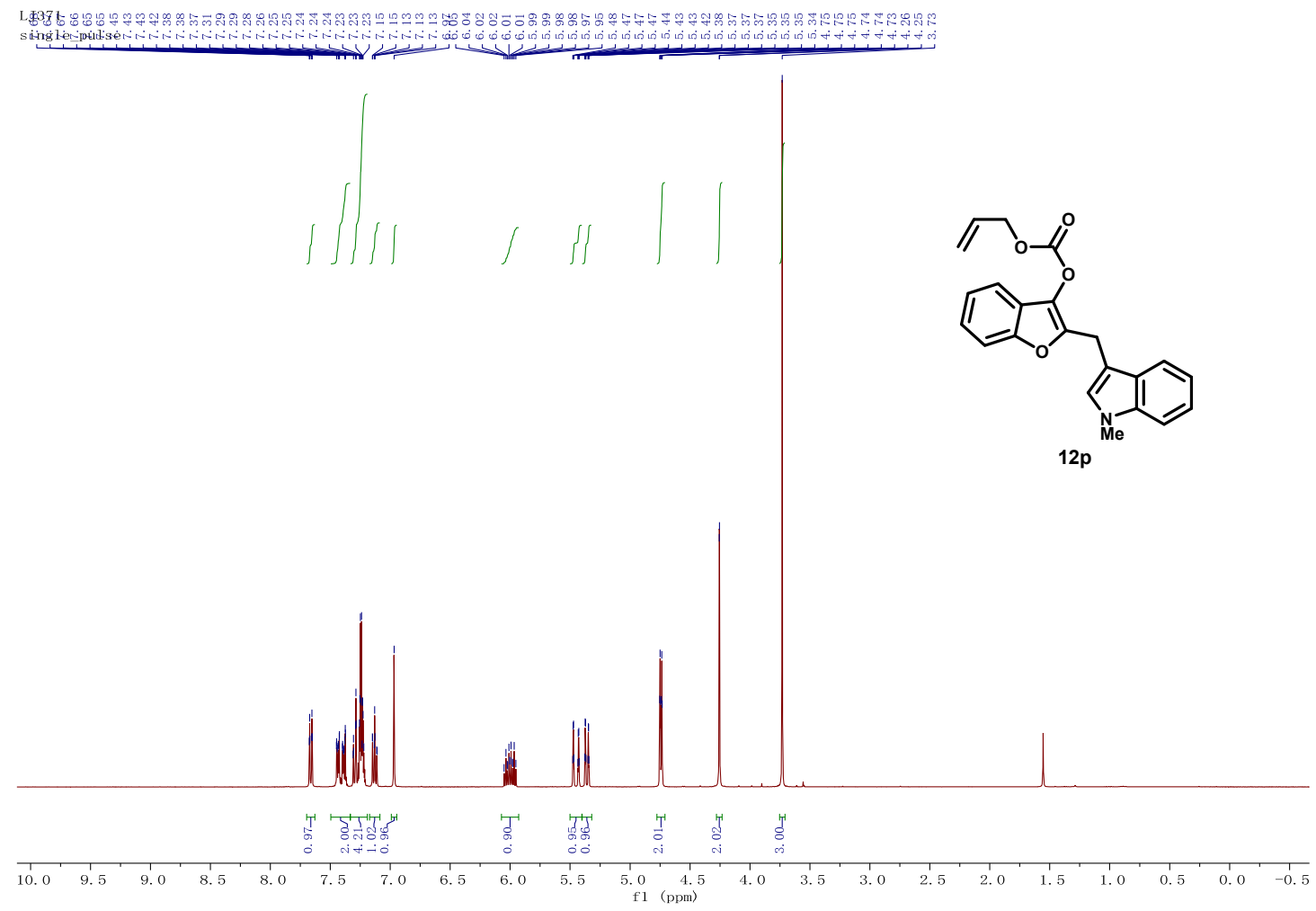

${ }^{13} \mathrm{C}$ NMR (100 MHz, $\mathrm{CDCl}_{3} @ 77$ ppm)

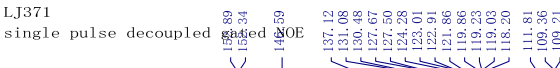

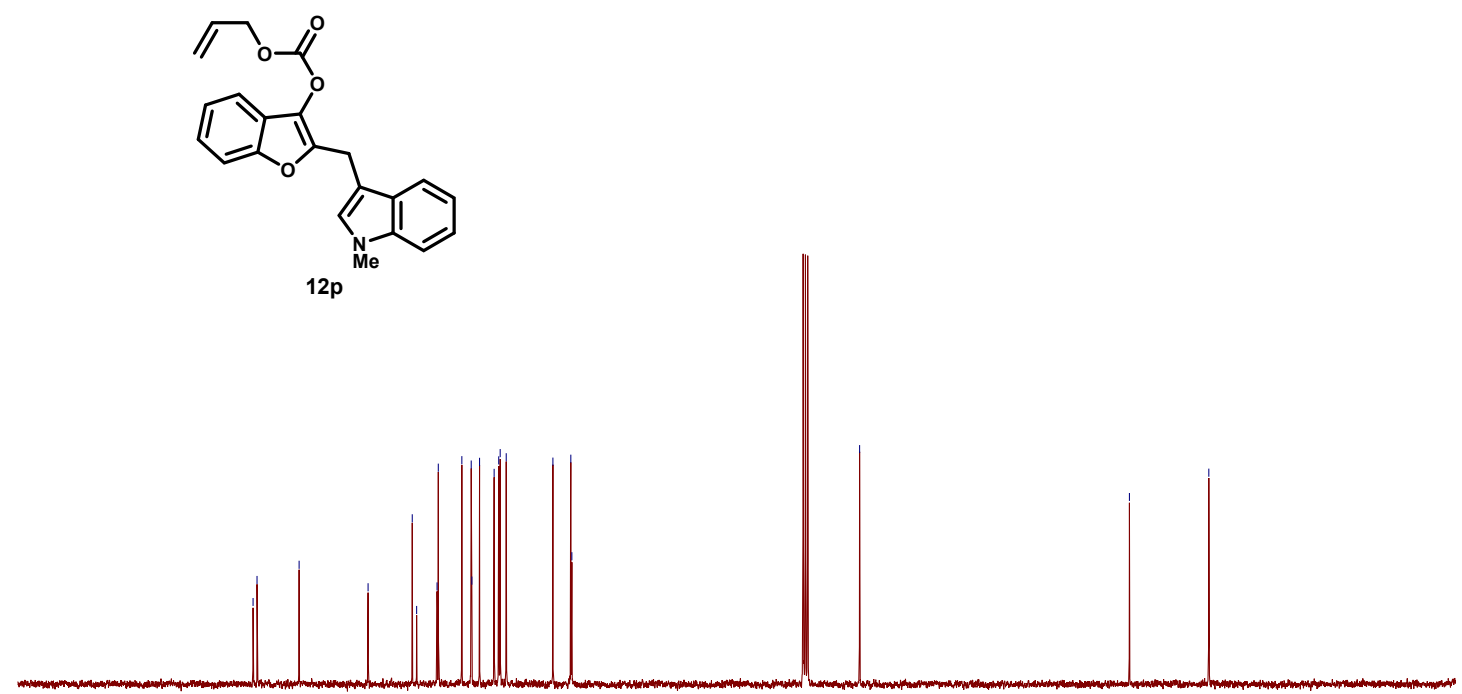

$12 p$

f1 (ppm) 80 
Allyl (2-(furan-3-ylmethyl)benzofuran-3-yl) carbonate (12q)

므 NMR $\left(400 \mathrm{MHz}, \mathrm{CDCl}_{3} @ 7.26\right.$ ppm)

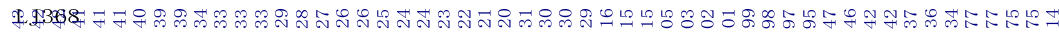

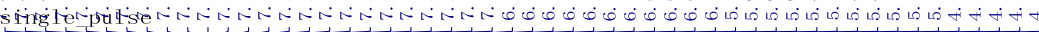
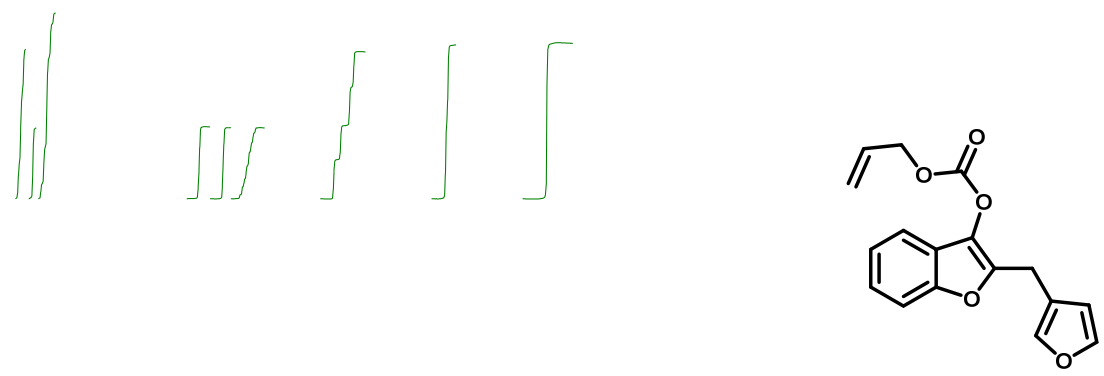

$12 q$

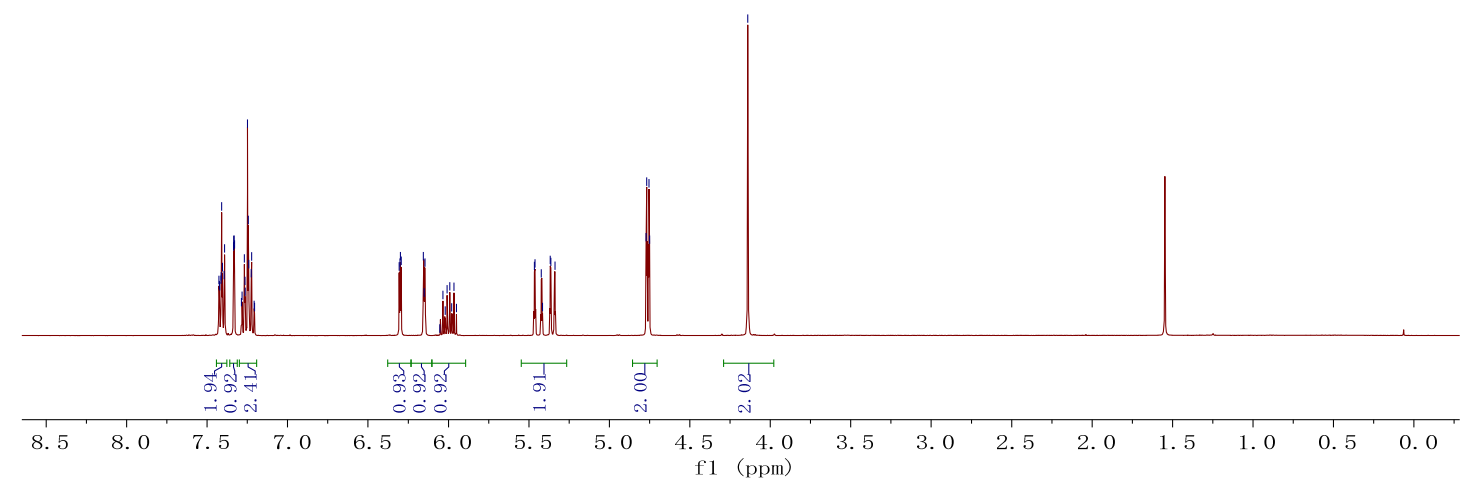

${ }^{13} \mathrm{C}$ NMR $\left(100 \mathrm{MHz}, \mathrm{CDCl}_{3} @ 77\right.$ ppm)

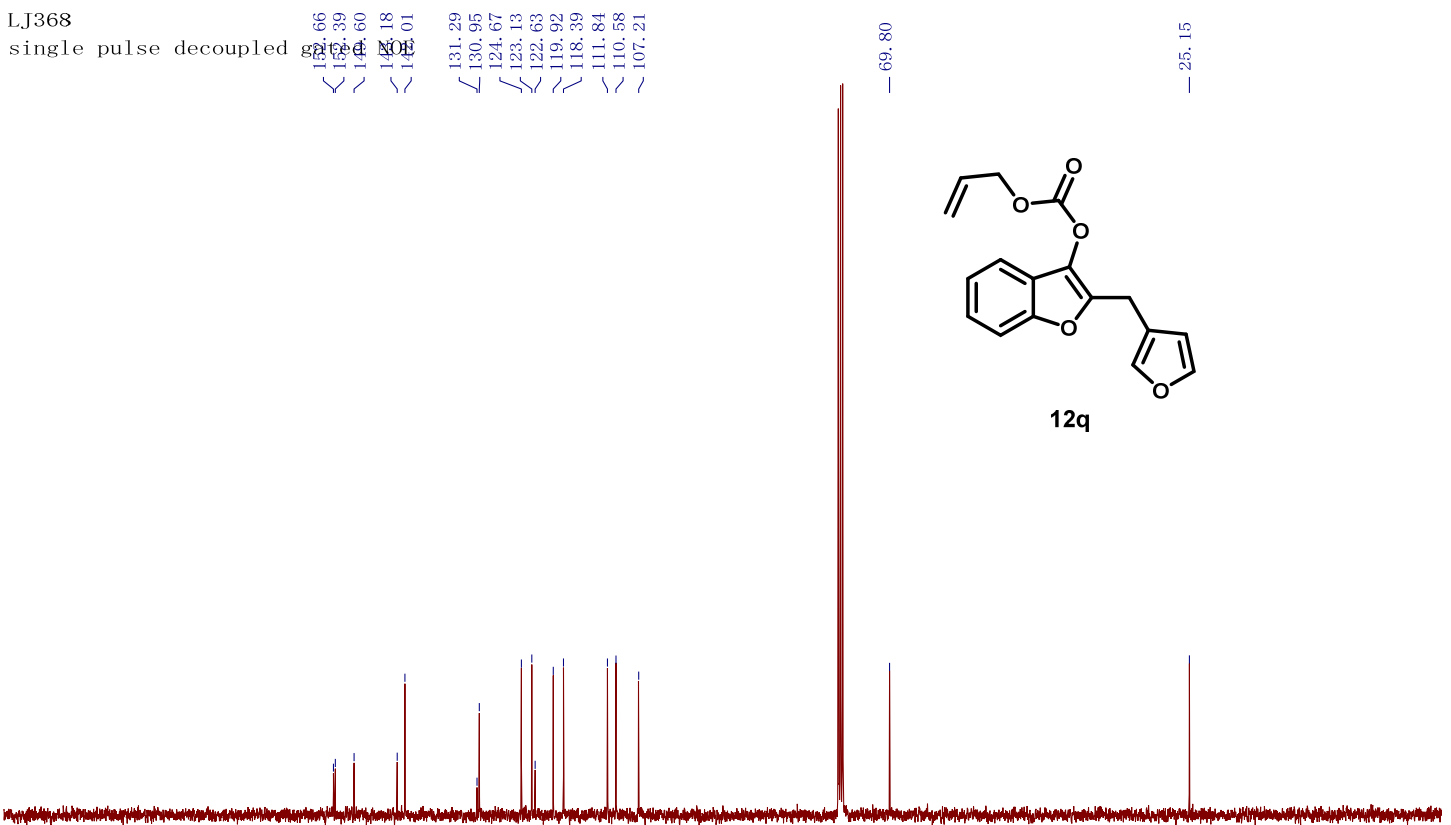

$\begin{array}{llllllllllllllllllllll}200 & 190 & 180 & 170 & 160 & 150 & 140 & 130 & 120 & 110 & 100 & 90 & 80 & 70 & 60 & 50 & 40 & 30 & 20 & 10 & 0 & -11\end{array}$ 
Allyl (2-(thiophen-3-ylmethyl)benzofuran-3-yl) carbonate (12r)

1H NMR (400 MHz, CDCl 3 @ 7.26 ppm)

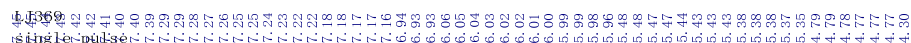

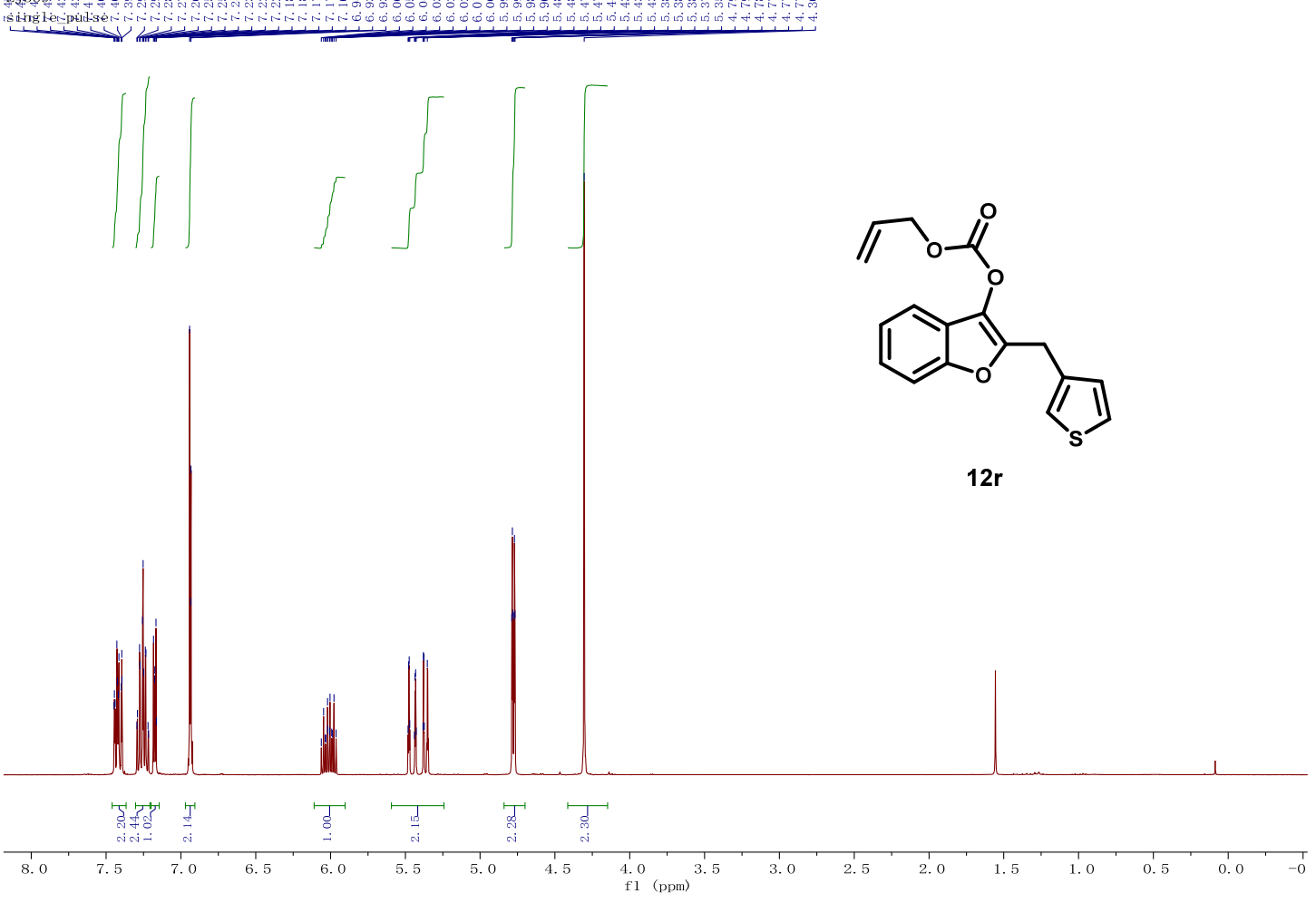

${ }^{13} \mathrm{C}$ NMR (100 MHz, $\mathrm{CDCl}_{3} @ 77$ ppm)

LJ369

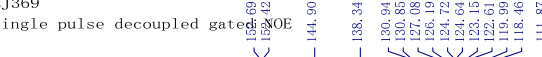

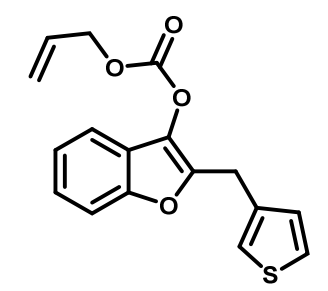

$12 r$

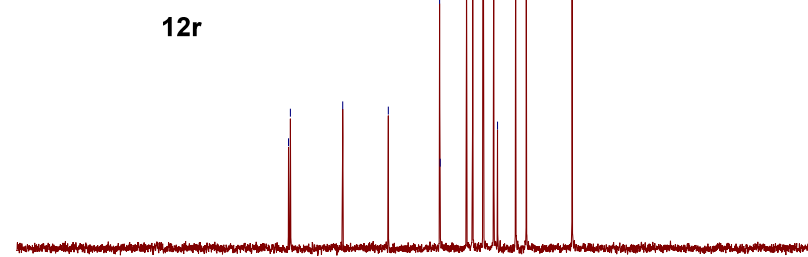

190

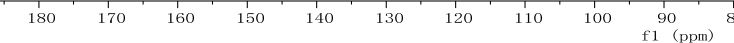




\section{(E)-Allyl (2-(3-phenylprop-1-en-1-yl)benzofuran-3-yl) carbonate (12s)}

느 NMR (500 MHz, CDCl $@ 7.26$ ppm)

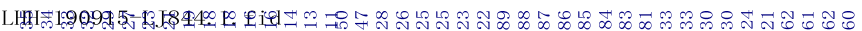

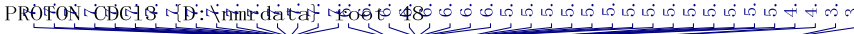
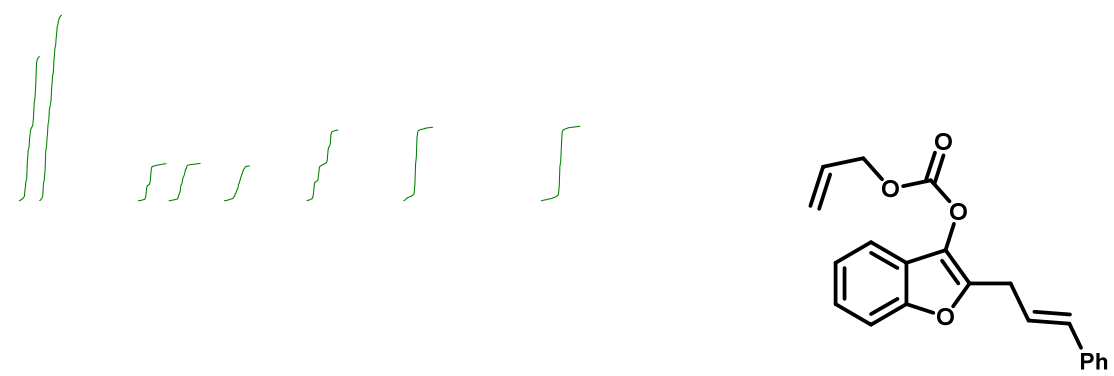

$12 \mathrm{~s}$

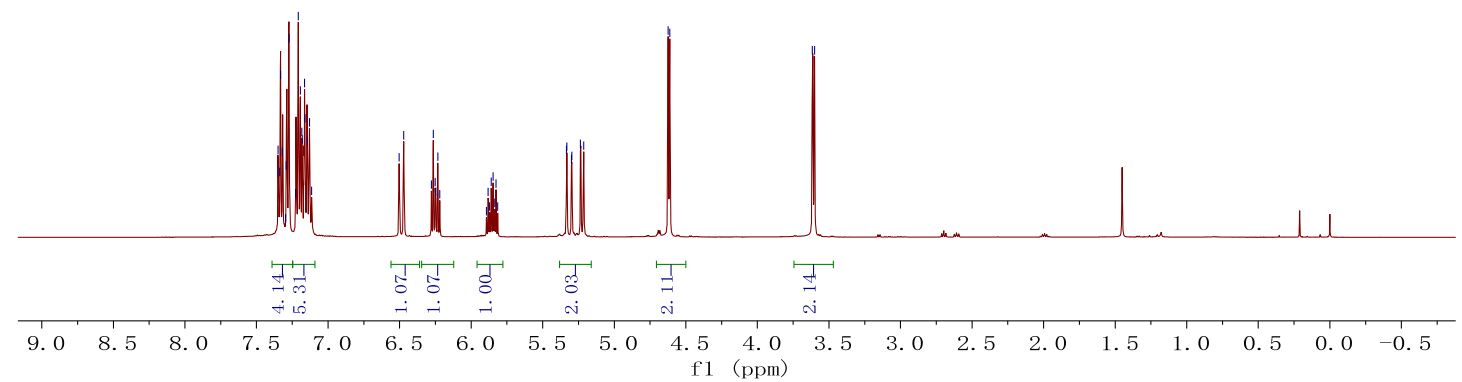

${ }^{13} \mathrm{C}$ NMR (125 MHz, CDCl $3 @ 77$ ppm)

LHH-190915-LJ844. 2. fid
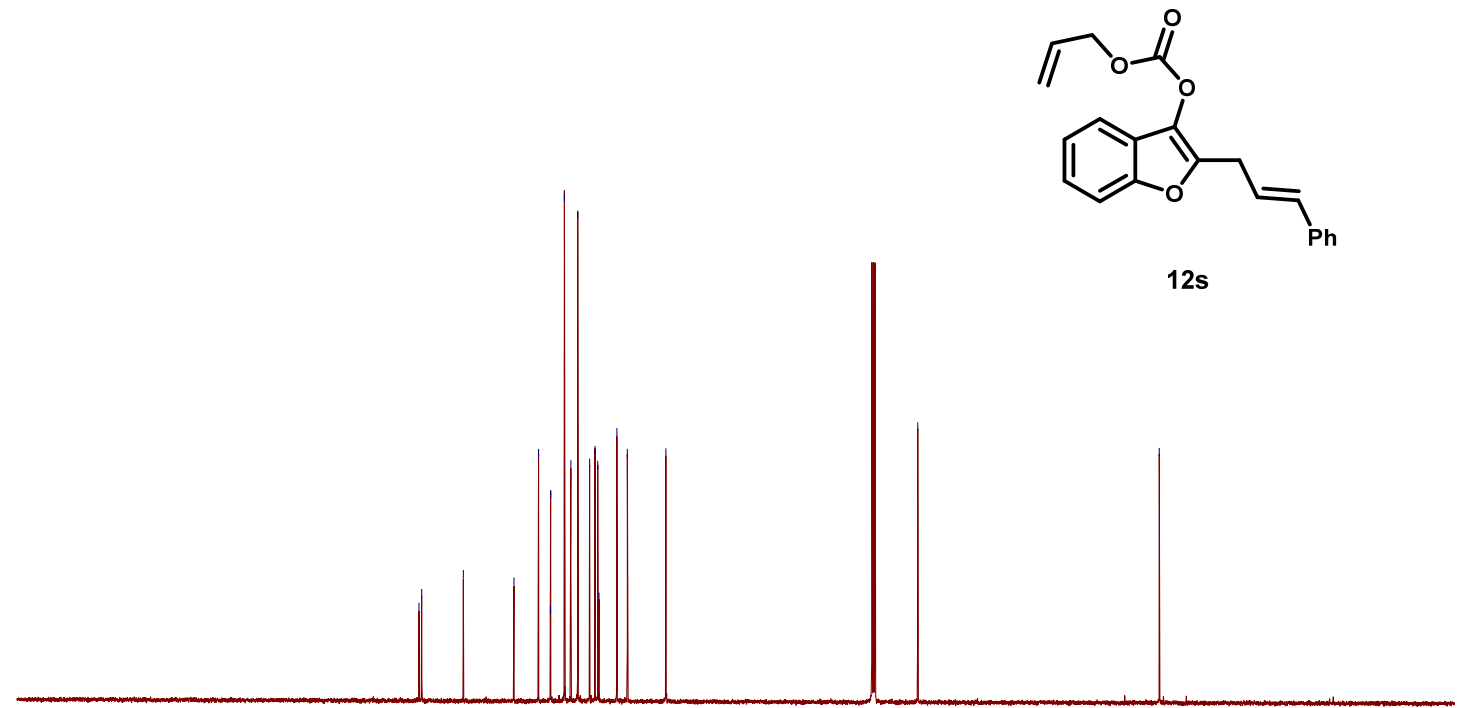

$\begin{array}{lllllllllllllllllllllll}210 & 200 & 190 & 180 & 170 & 160 & 150 & 140 & 130 & 120 & 110 & 100 & 90 & 80 & 70 & 60 & 50 & 40 & 30 & 20 & 10 & 0 & -10\end{array}$ f1 (ppm) 
Allyl (2-(3-phenylpropyl)benzofuran-3-yl) carbonate (12t)

1H NMR (500 MHz, CDCl 3 @ 7.26 ppm)

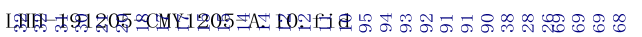

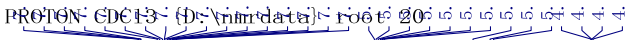

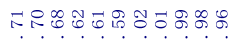

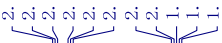
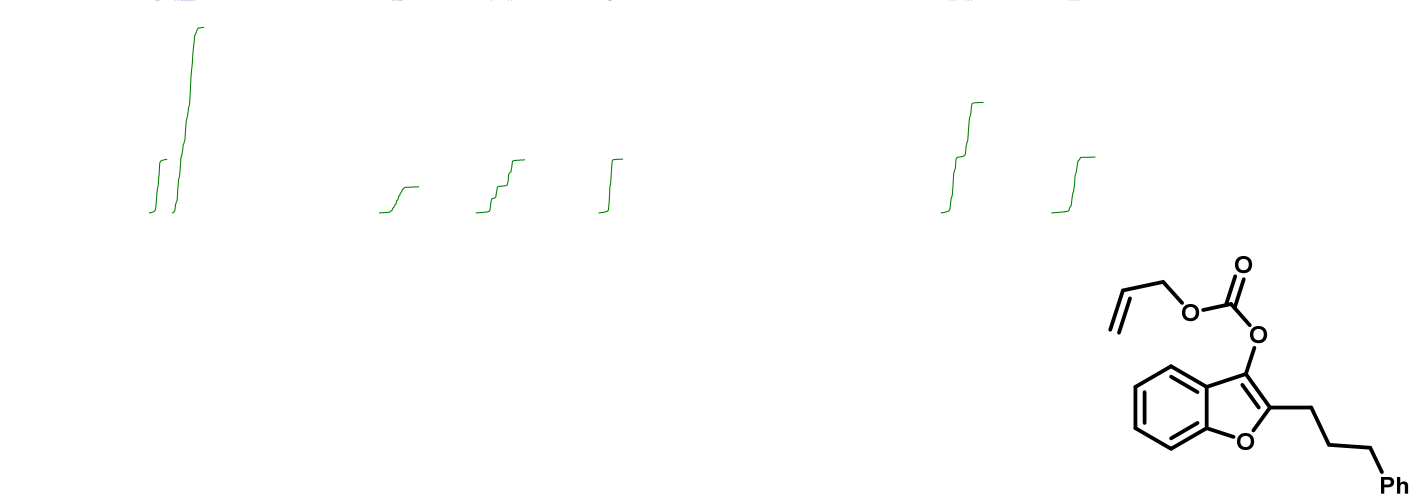

$12 t$

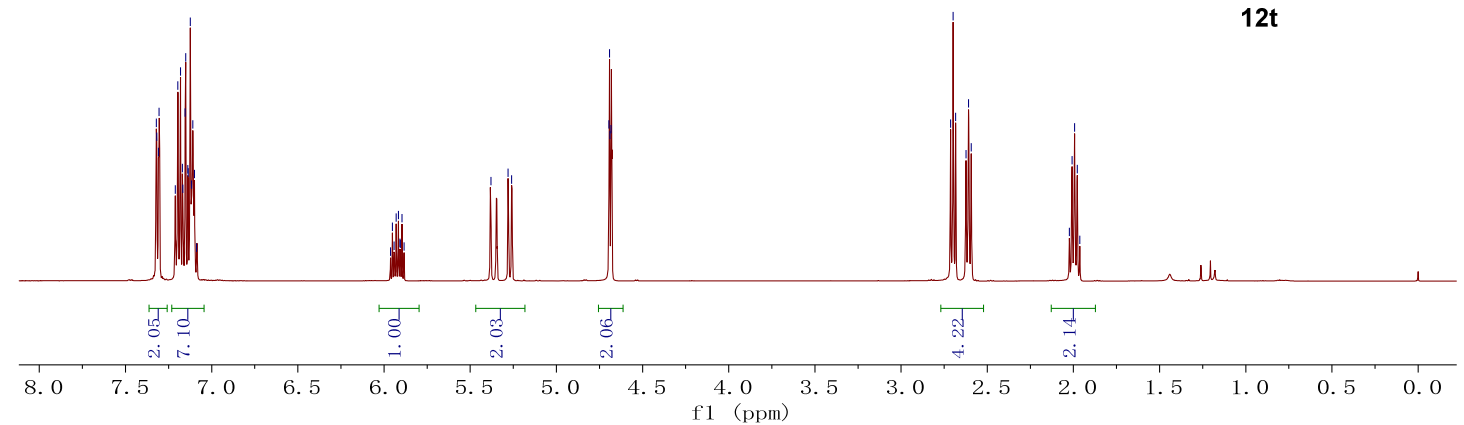

${ }^{13} \mathrm{C}$ NMR (125 MHz, $\mathrm{CDCl}_{3} @ 77$ ppm)

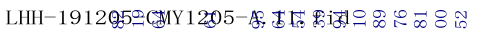

C13CPD CogC

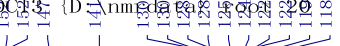
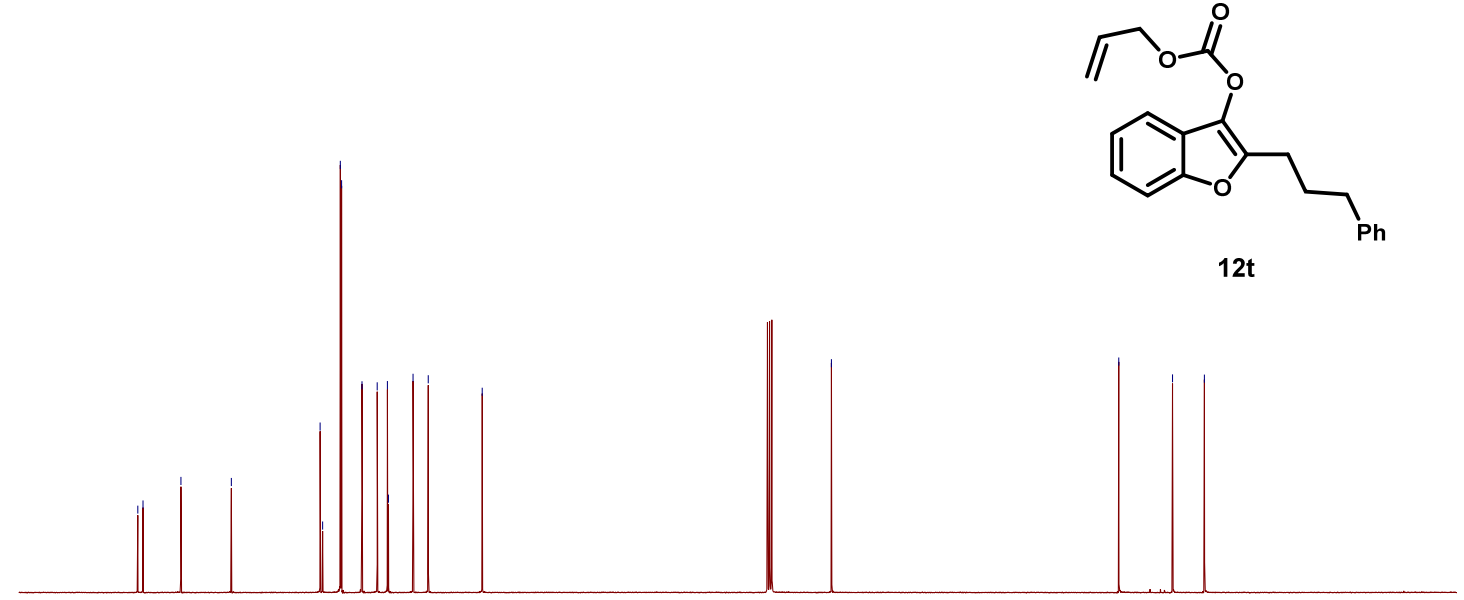

$12 t$

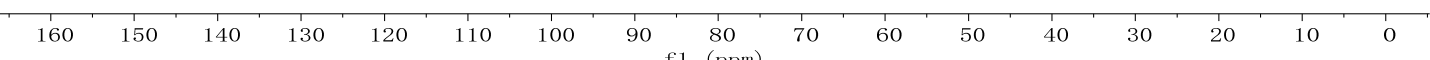

S179 
Allyl (2-(cyclopropylmethyl)benzofuran-3-yl) carbonate (12u)

1'

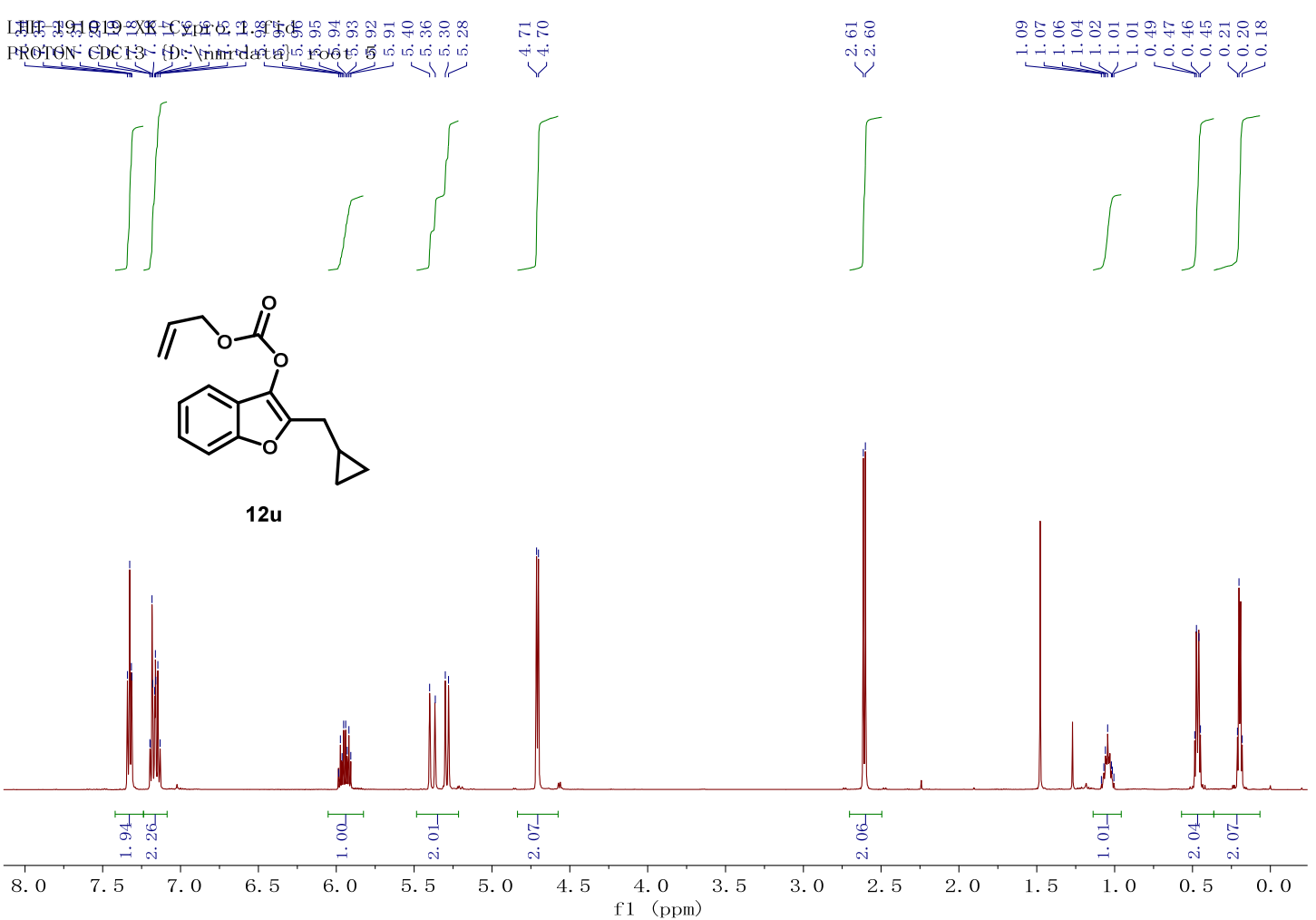

\section{${ }^{13} \mathrm{C}$ NMR (125 MHz, $\mathrm{CDCl}_{3} @ 77$ ppm)}

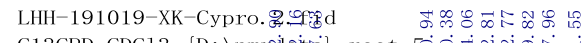

C13CPD CDC13 \{D:\nmriglath root 5
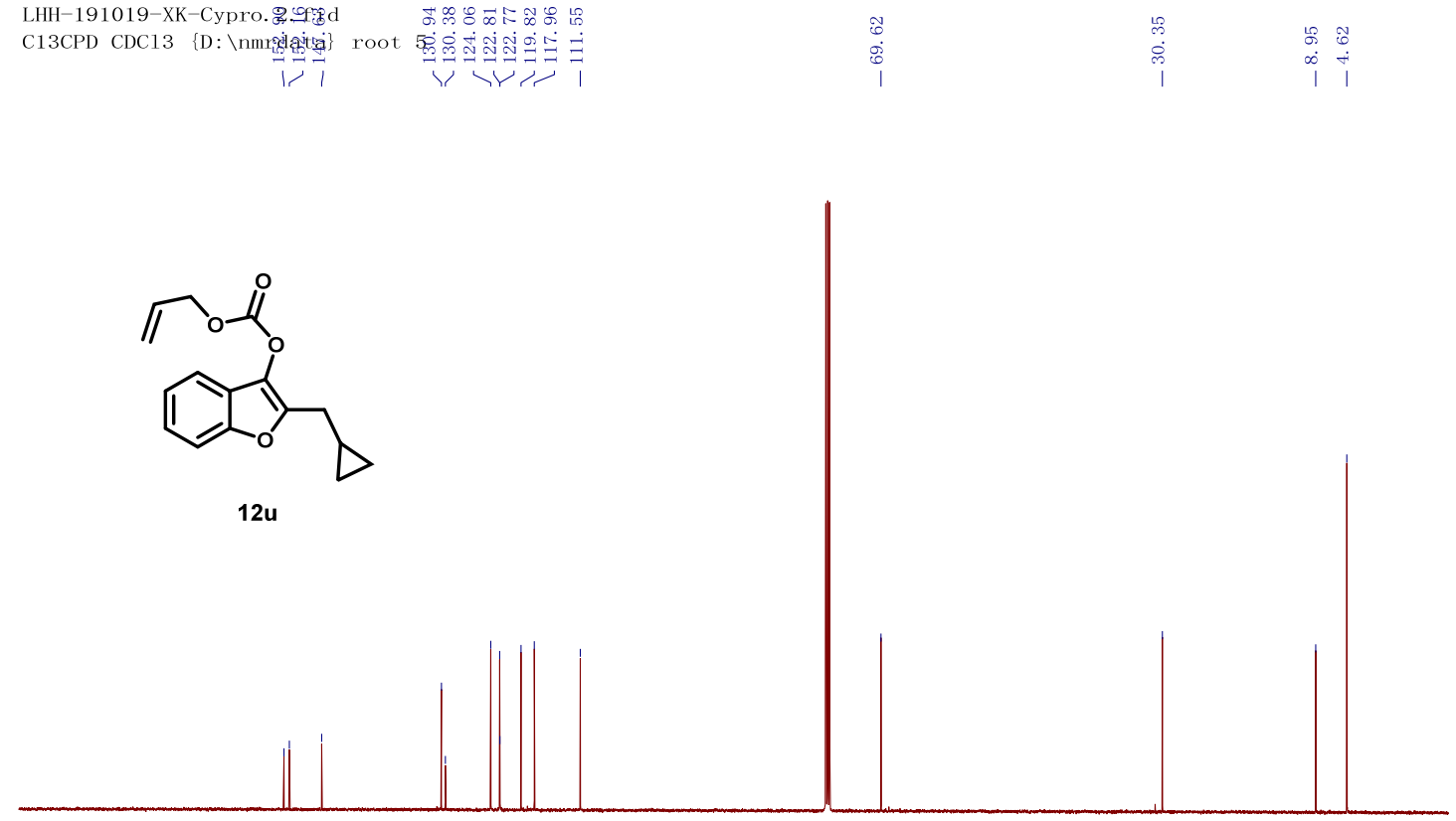

$\begin{array}{llllllllll}180 & 170 & 160 & 150 & 140 & 130 & 120 & 110 & 100 \underset{\mathrm{f} 1}{90}(\mathrm{ppm}) & 80\end{array}$ 
Allyl (4-methoxy-2-(naphthalen-1-ylmethyl)benzofuran-3-yl) carbonate (12v)

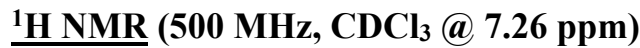

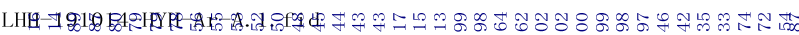

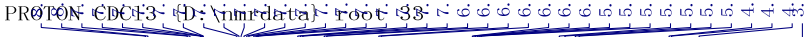
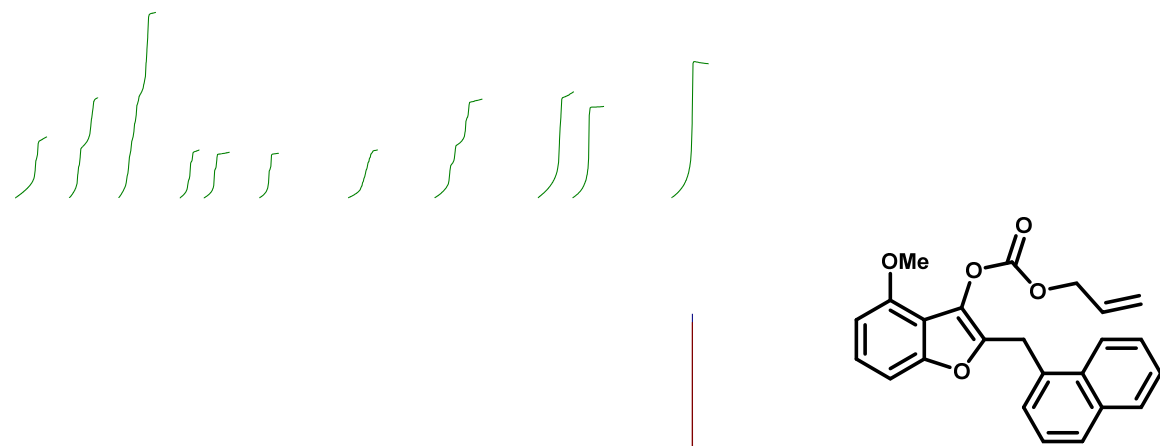

12v

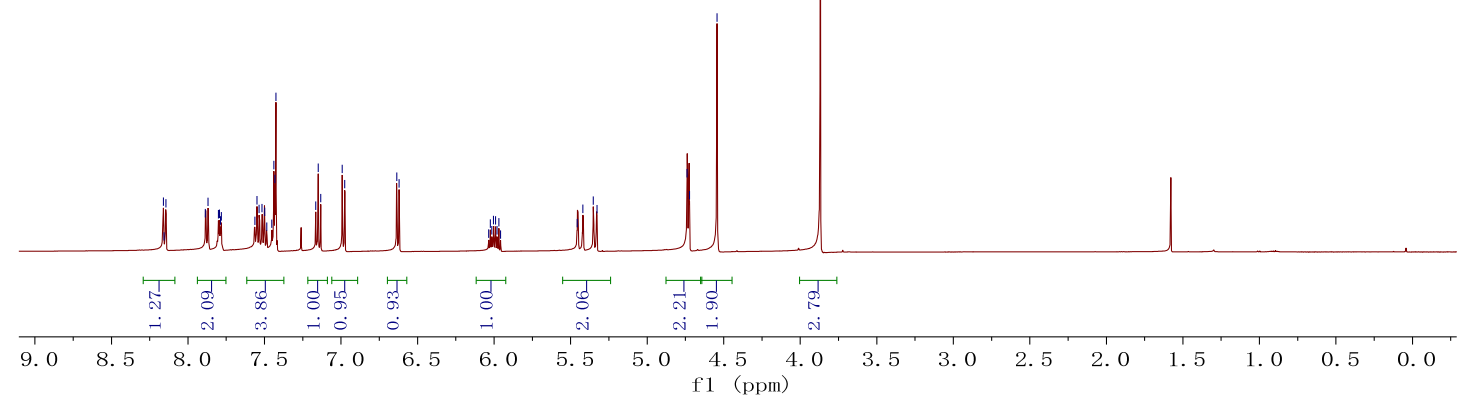

${ }^{13} \mathrm{C}$ NMR (125 MHz, CDCl 3 @ 77 ppm)

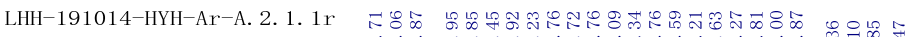
C13CPD CDC13 \{D:\nmrdata\} rogd

闰

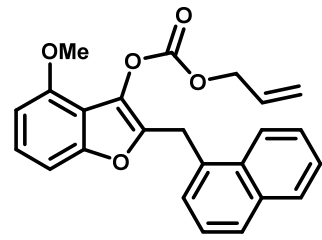

12v

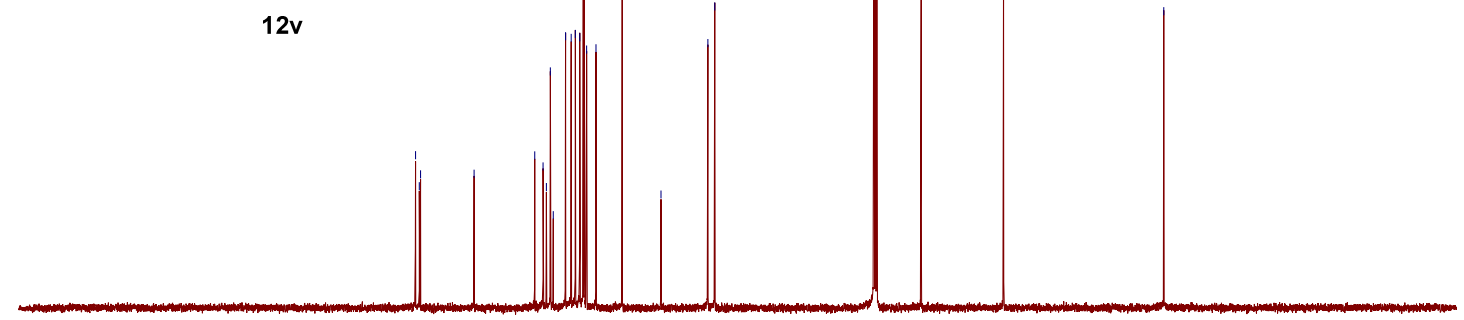

$\begin{array}{lllllllllllllllllllllll}210 & 200 & 190 & 180 & 170 & 160 & 150 & 140 & 130 & 120 & 110 & 100 & 90 & 80 & 70 & 60 & 50 & 40 & 30 & 20 & 10 & 0 & -10\end{array}$ f1 (ppm) 
Allyl (5-methoxy-2-(naphthalen-1-ylmethyl)benzofuran-3-yl) carbonate (12w)

1H NMR (500 MHz, CDCl 3 @ 7.26 ppm)

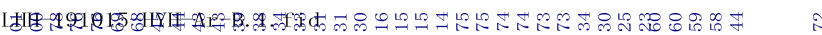

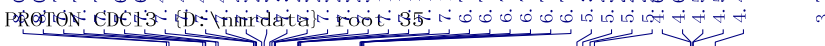

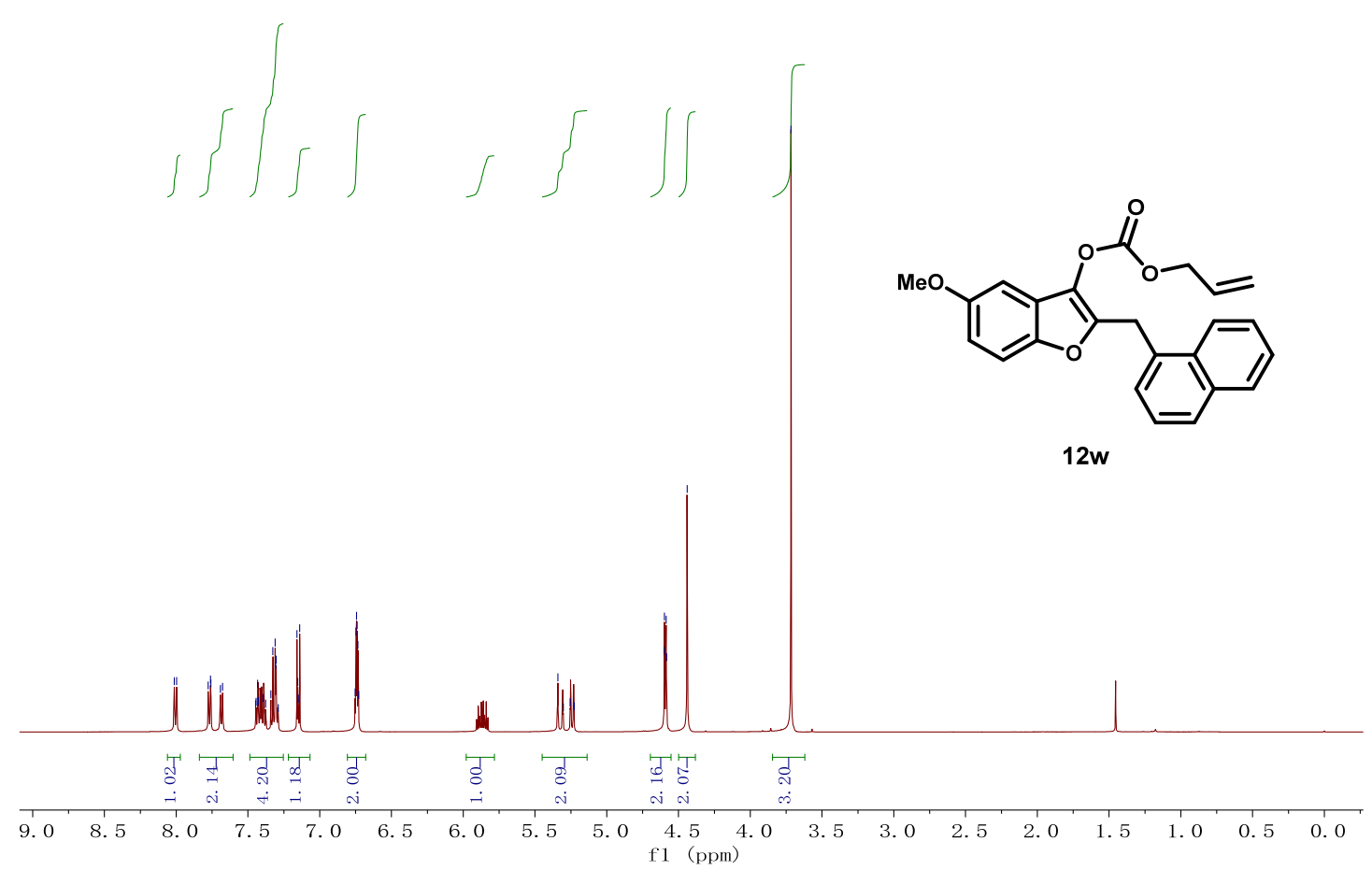

${ }^{13} \mathrm{C}$ NMR (125 MHz, CDCl 3 @ 77 ppm)

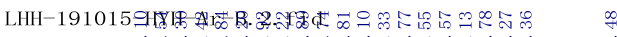
C13CPD CDC边

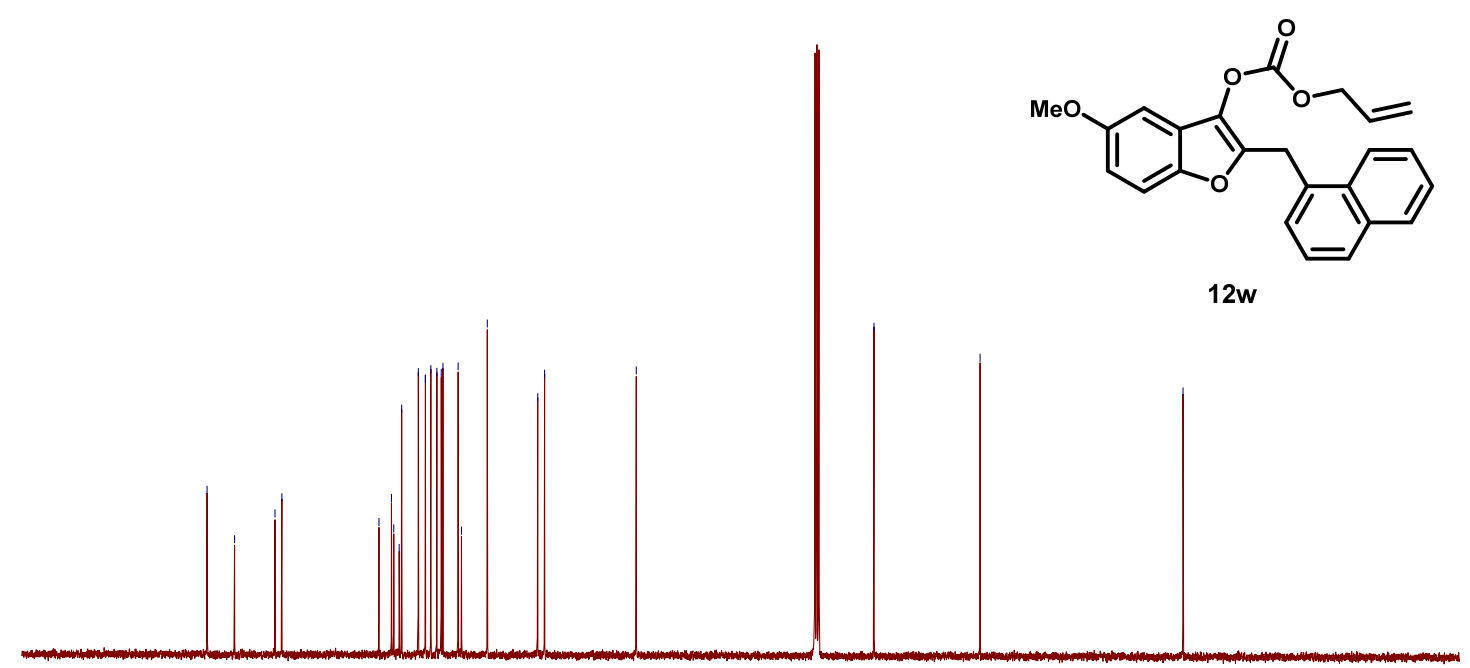

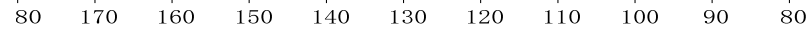

f1 (ppm) 
Allyl (6-methoxy-2-(naphthalen-1-ylmethyl)benzofuran-3-yl) carbonate (12x)

1H NMR (500 MHz, $\mathrm{CDCl}_{3} @ 7.26$ ppm)

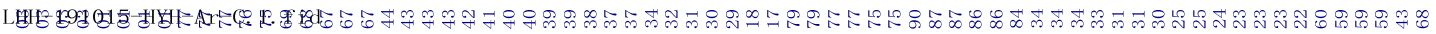

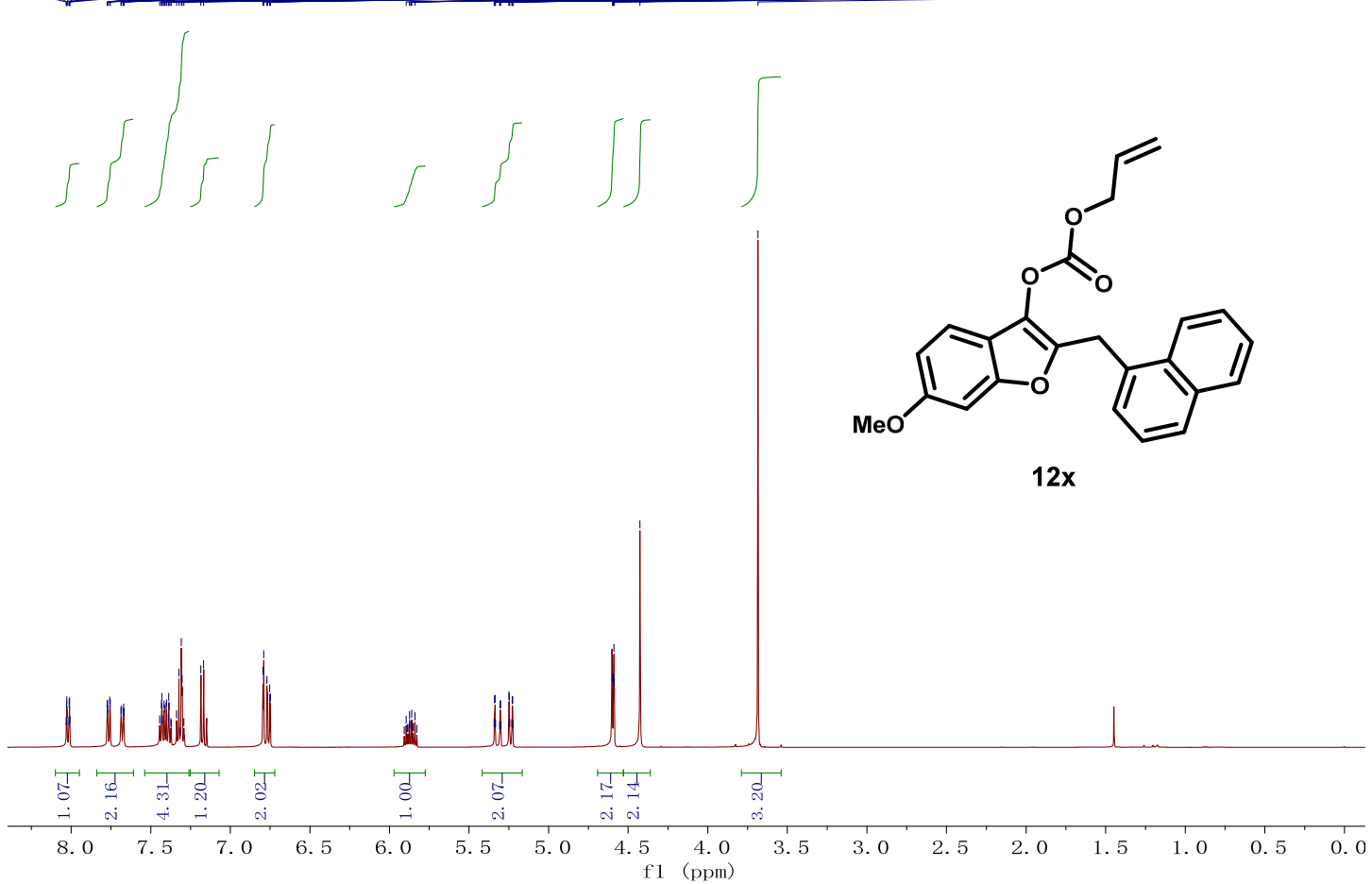

${ }^{13} \mathrm{C}$ NMR (125 MHz, $\mathrm{CDCl}_{3} @ 77$ ppm)

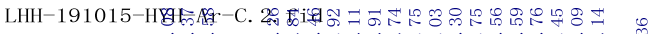

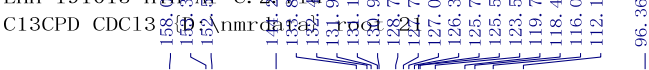

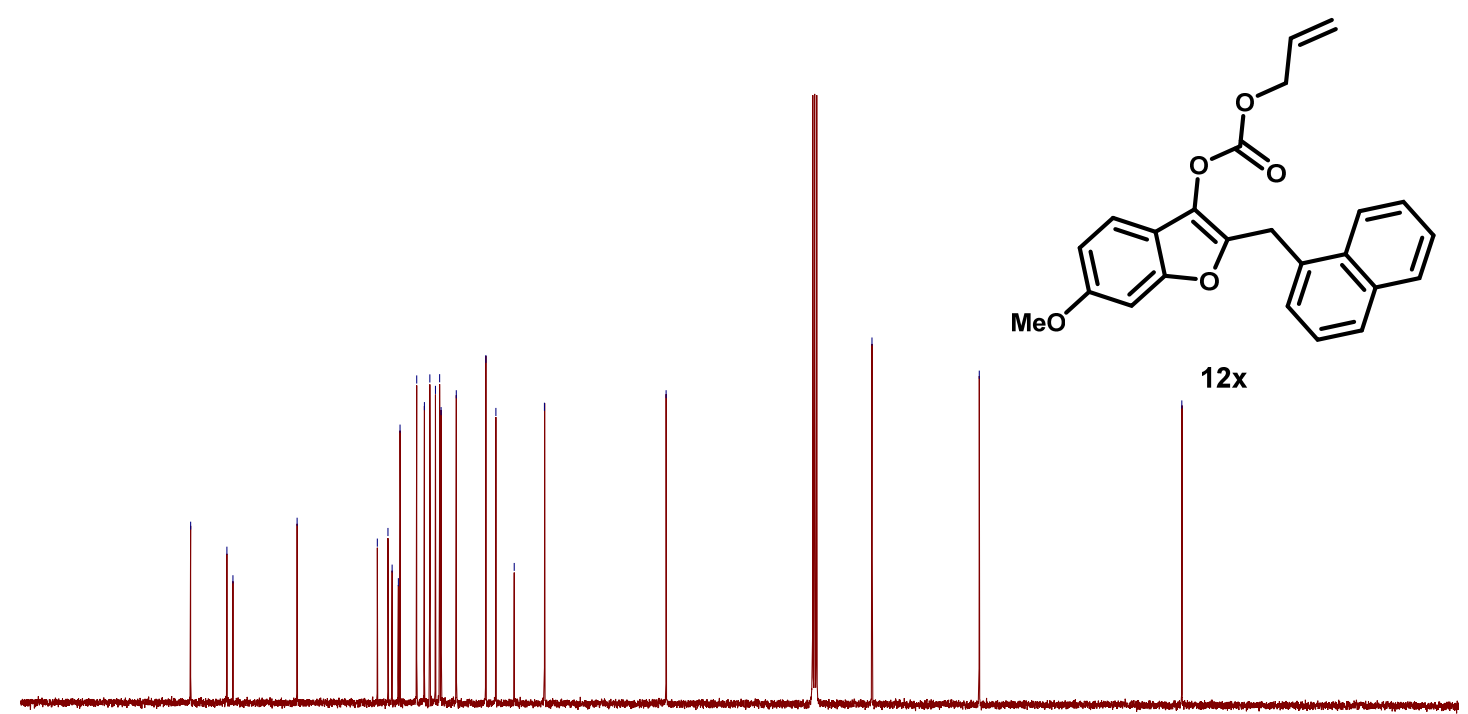

$\begin{array}{lllllllllll}80 & 170 & 160 & 150 & 140 & 130 & 120 & 110 & 100 & 90 & 80\end{array}$ 
Allyl (6-bromo-2-(naphthalen-1-ylmethyl)benzofuran-3-yl) carbonate(12y)

1 NMR (500 MHz, CDCl 3 @ 7.26 ppm)

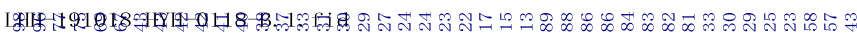

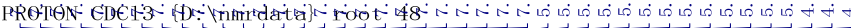

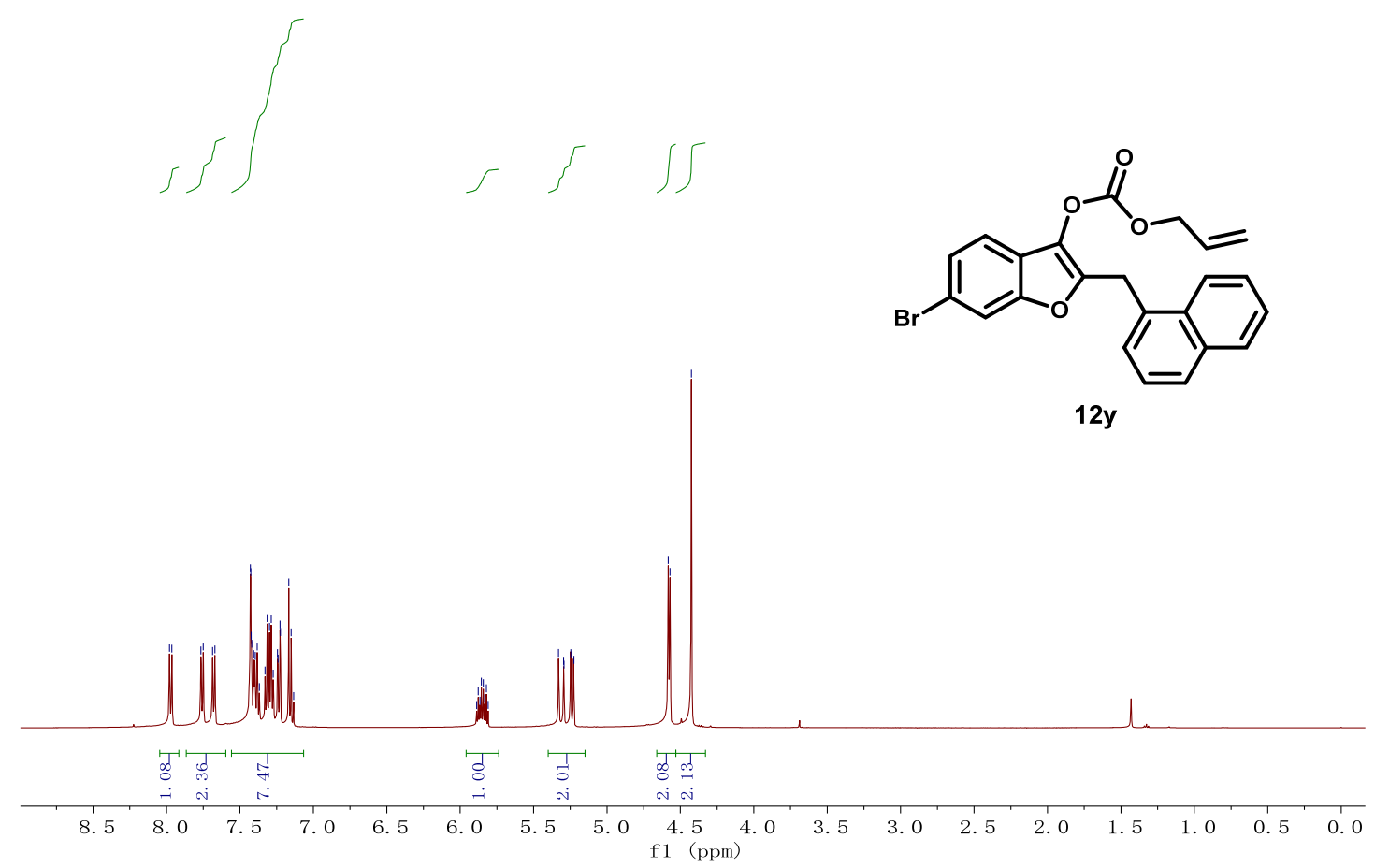

${ }^{13} \mathrm{C}$ NMR (125 MHz, $\mathrm{CDCl}_{3} @ 77$ ppm)<smiles>C=CCOC(=O)Oc1c(Cc2cccc3ccccc23)oc2cc(Br)ccc12</smiles>

$12 y$

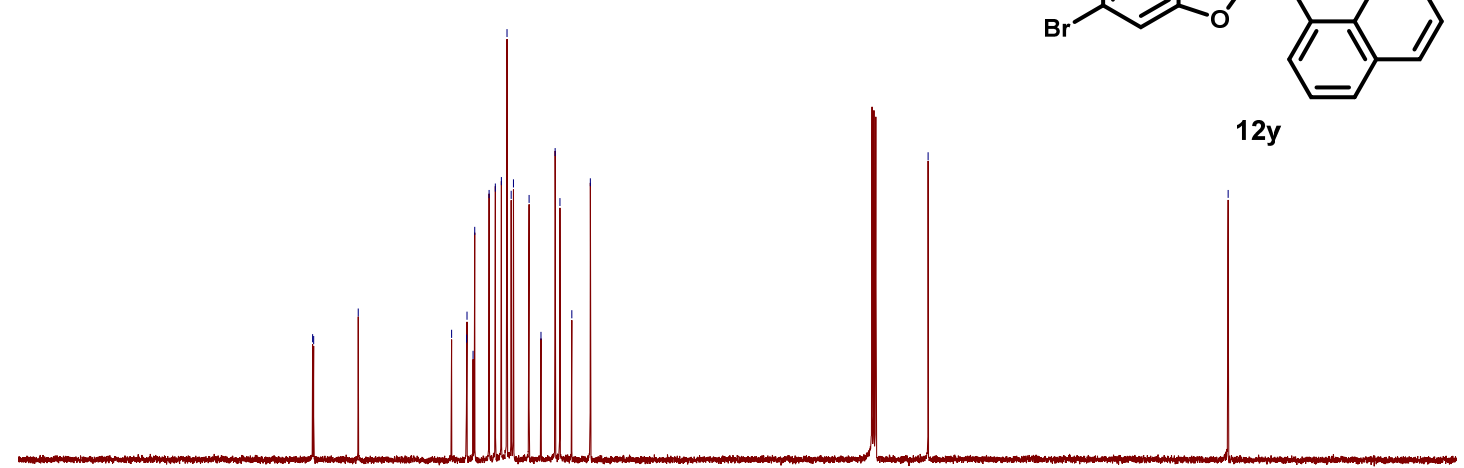

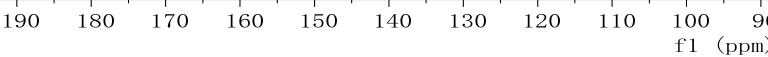


Allyl (6-fluoro-2-(naphthalen-1-ylmethyl)benzofuran-3-yl) carbonate(12z)

1H NMR (500 MHz, $\mathrm{CDCl}_{3} @ 7.26$ ppm)

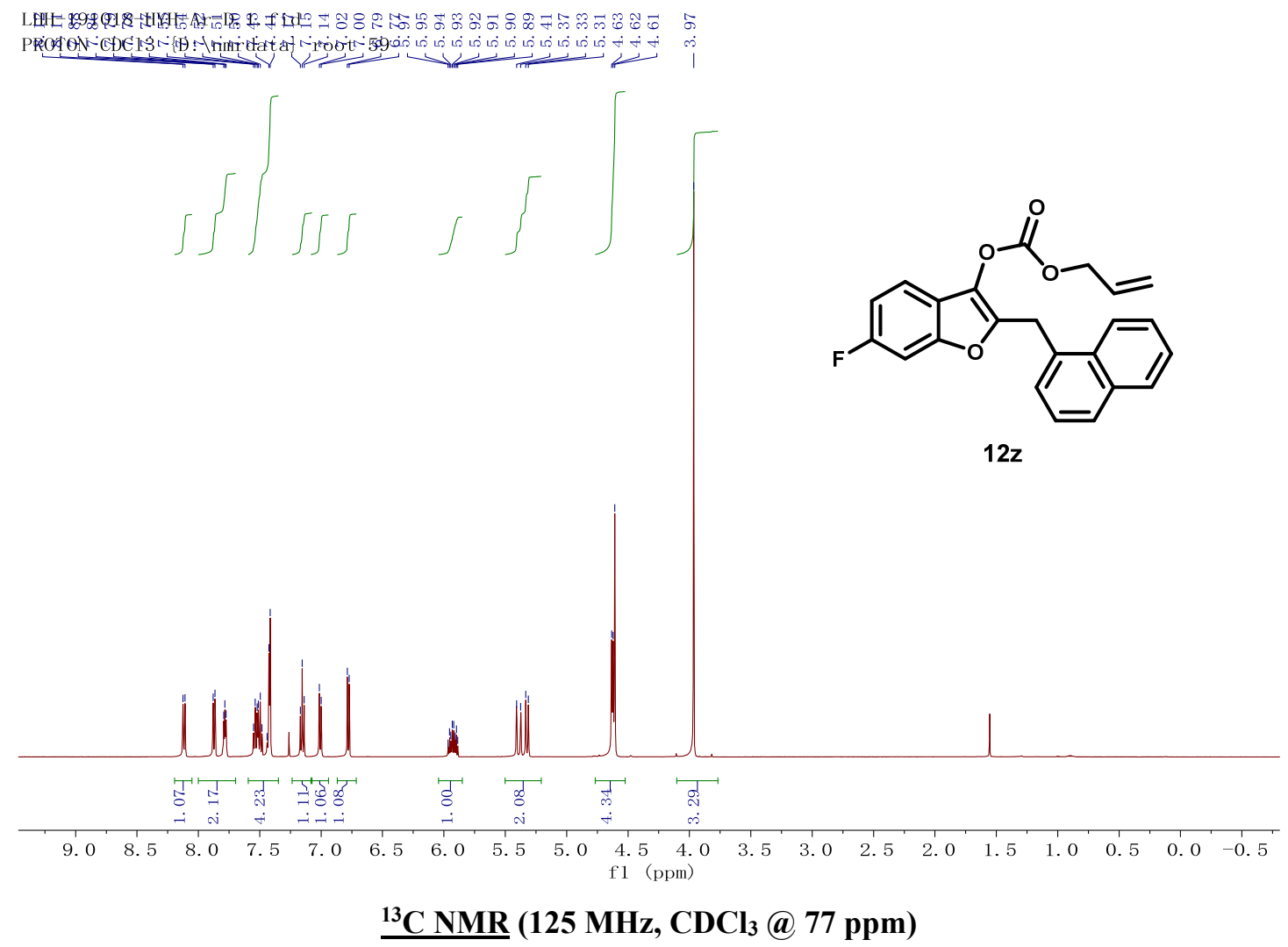




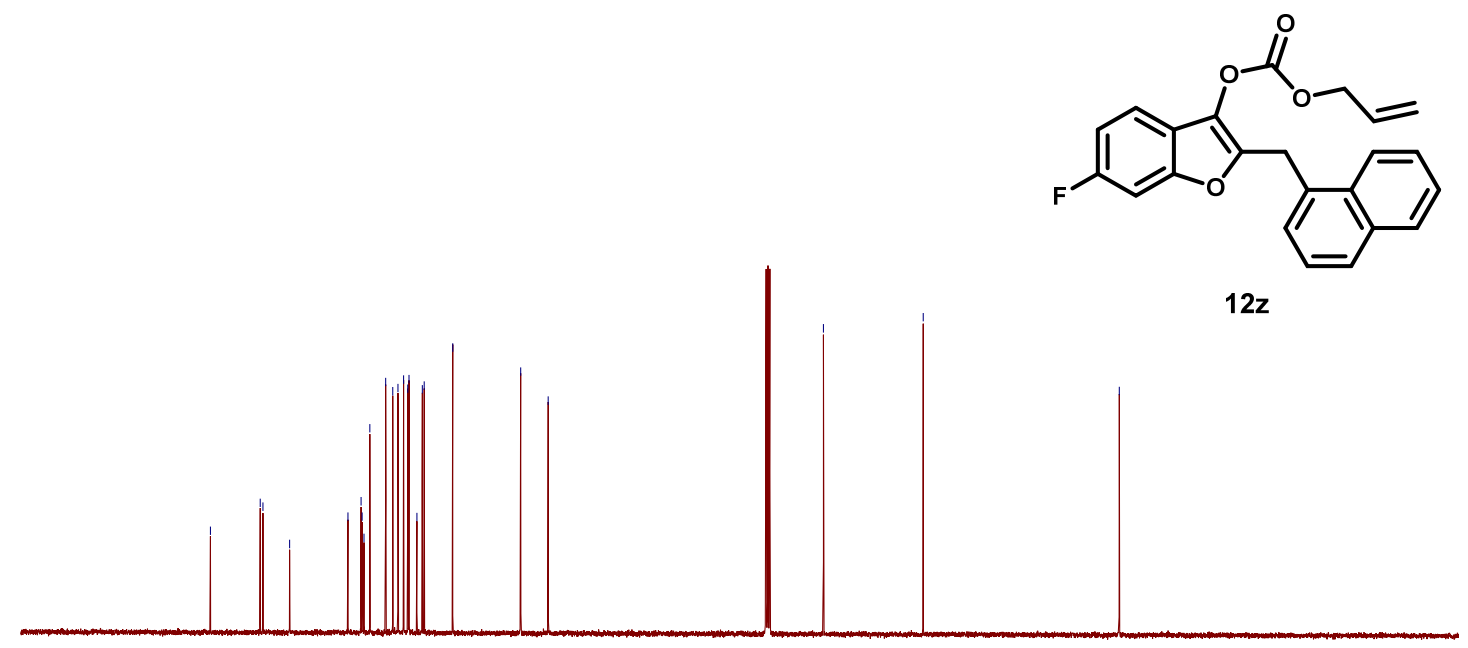

$\begin{array}{llllllllll}170 & 160 & 150 & 140 & 130 & 120 & 110 & 100 & 90 & 80 \\ \mathrm{f} 1 & & & \\ (\mathrm{ppm})\end{array}$

\section{${ }^{19}$ F NMR (470 MHz, $\left.\mathrm{CDCl}_{3}\right)$}

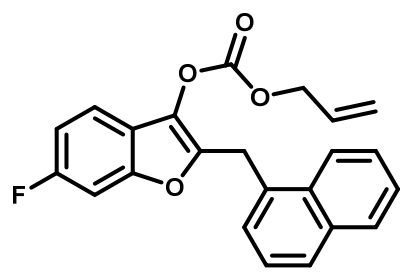

$12 z$

\begin{tabular}{|c|c|c|c|c|c|c|c|c|c|c|c|}
\hline 20 & 0 & -20 & -40 & -60 & -80 & $\begin{array}{c}-100 \\
\mathrm{f1} \quad(\mathrm{ppm})\end{array}$ & -120 & -140 & -160 & -180 & -200 \\
\hline
\end{tabular}

Allyl (6-morpholino-2-(naphthalen-1-ylmethyl)benzofuran-3-yl) carbonate(12a') 


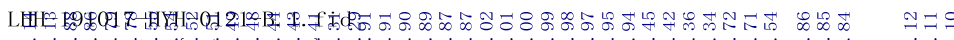

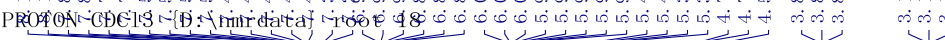

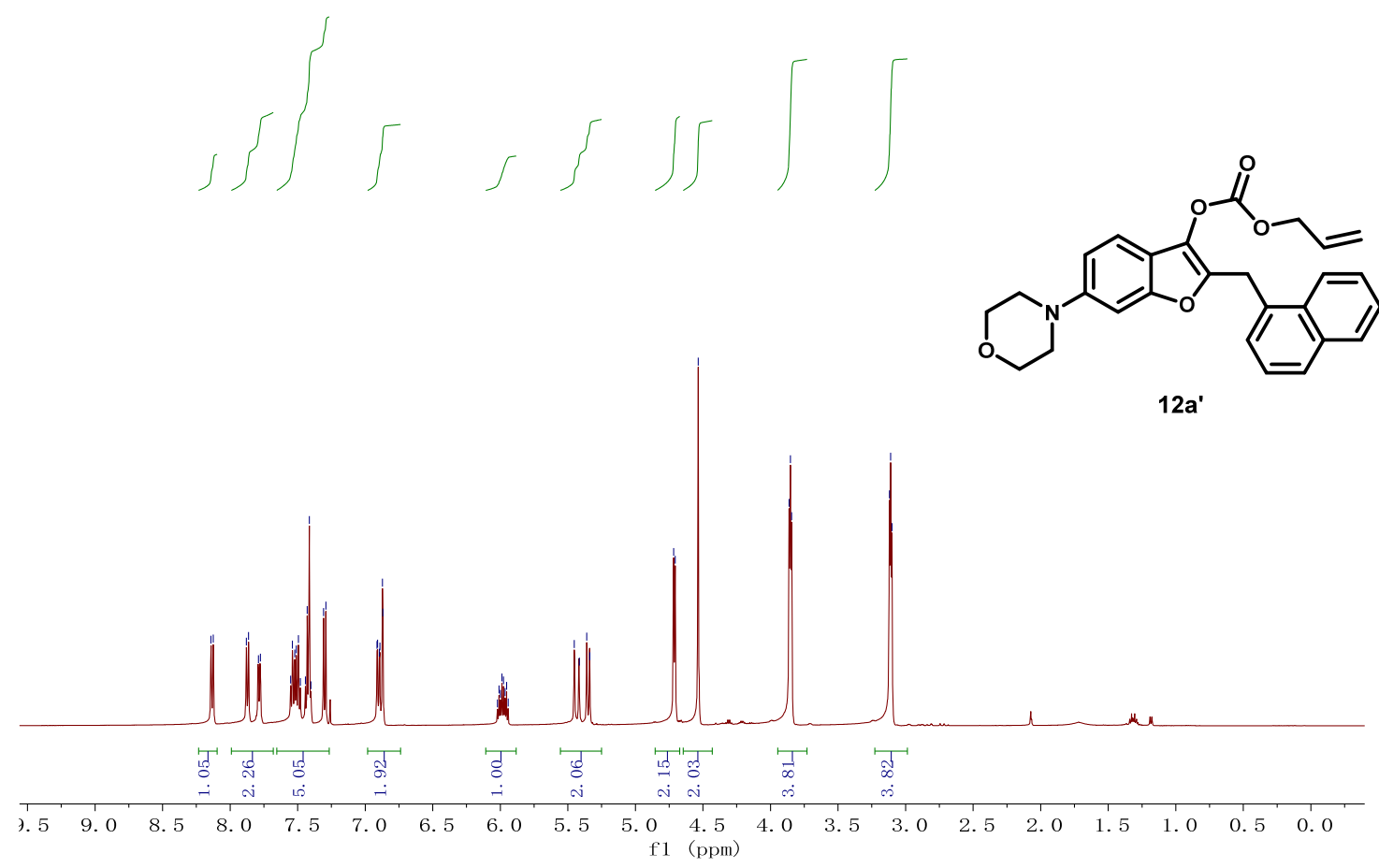

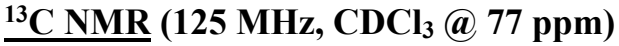

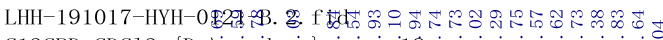

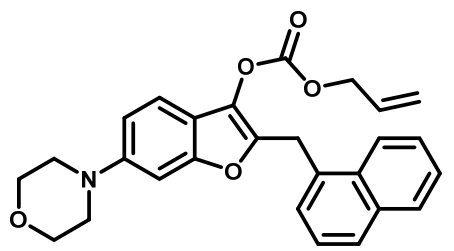

$12 a^{\prime}$

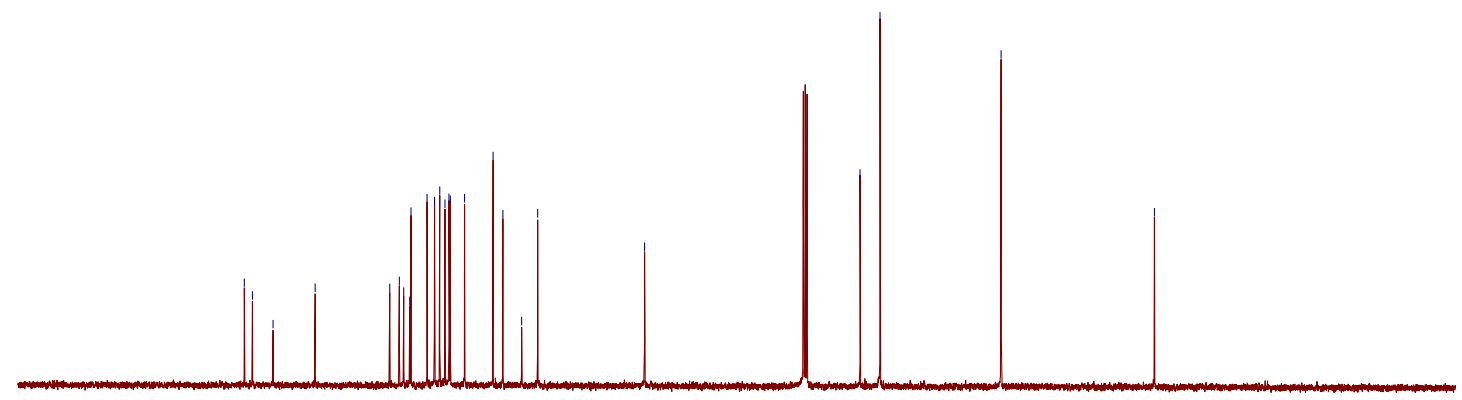

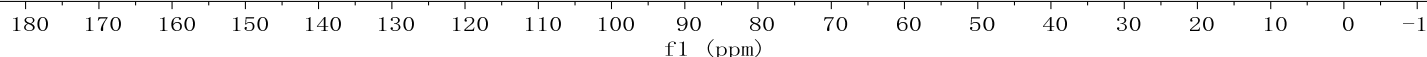

Allyl (7-methoxy-2-(naphthalen-1-ylmethyl)benzofuran-3-yl) carbonate (12b') 
$\underline{{ }^{1} \mathrm{H} \text { NMR }}\left(500 \mathrm{MHz}, \mathrm{CDCl}_{3} @ 7.26\right.$ ppm)

LHH世 1996

PROAON CDClos

\section{$7.26 \mathrm{ppm})$}

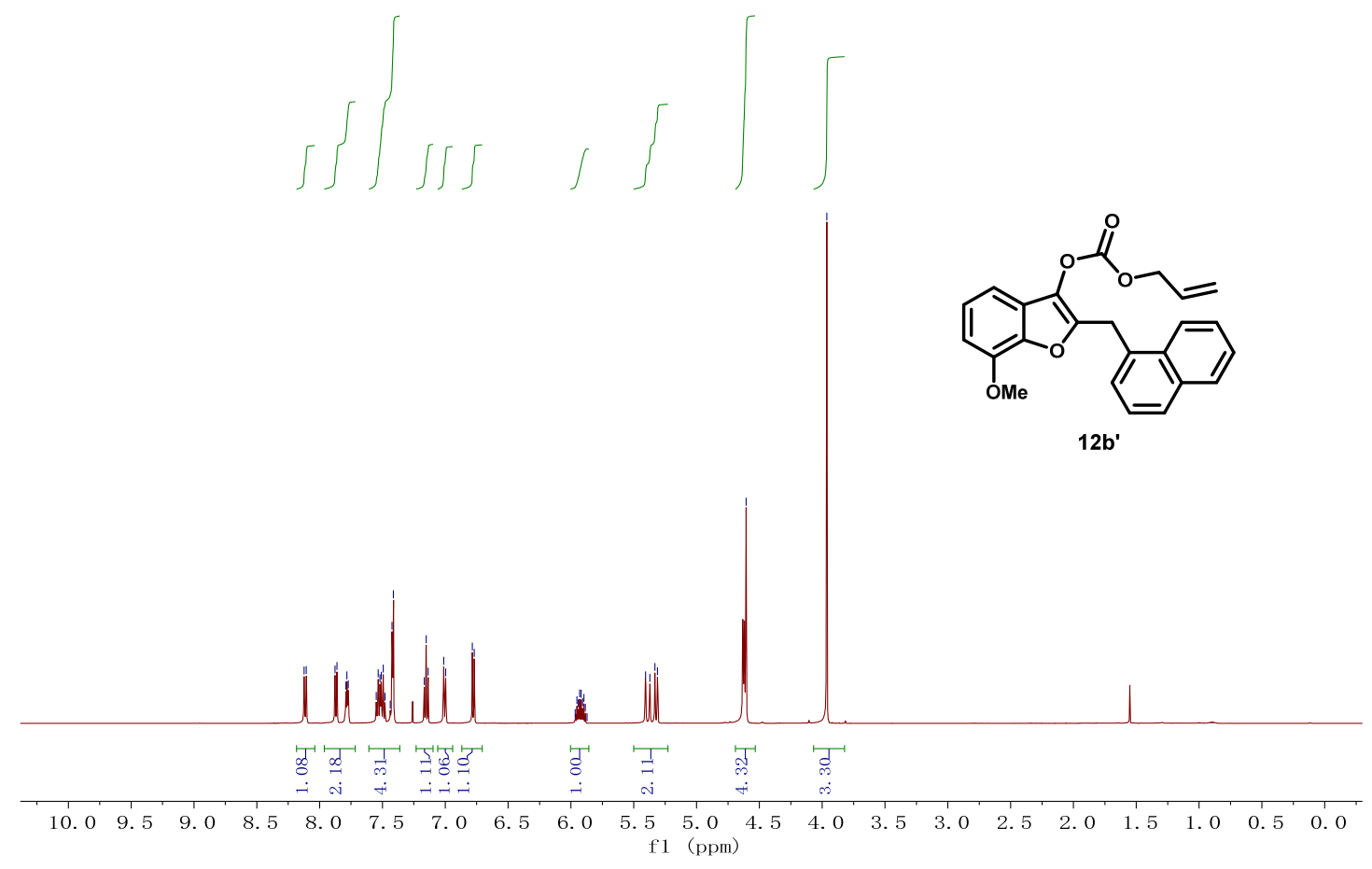

$\underline{{ }^{13} \mathrm{C} \mathrm{NMR}}\left(125 \mathrm{MHz}, \mathrm{CDCl}_{3} @ 77\right.$ ppm)

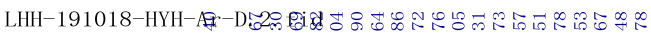

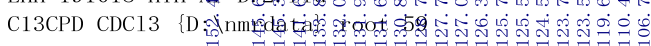

$\checkmark 1$
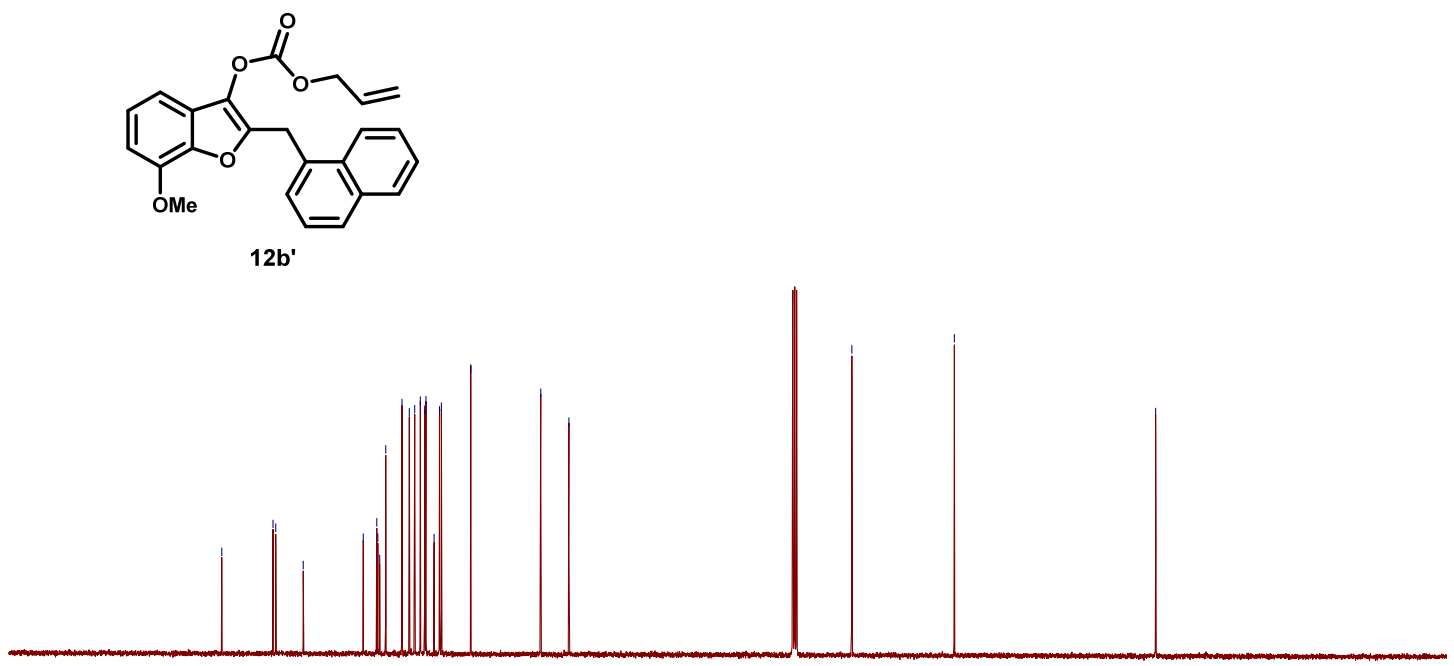

$\begin{array}{rrrrrrrrrr}80 & 170 & 160 & 150 & 140 & 130 & 120 & 110 & 100 & \begin{array}{c}90 \\ \mathrm{f} 1\end{array}\left(\begin{array}{c}80 \\ (\mathrm{ppm})\end{array}\right.\end{array}$ 
므 NMR $\left(500 \mathrm{MHz}, \mathrm{CDCl}_{3} @ 7.26\right.$ ppm)

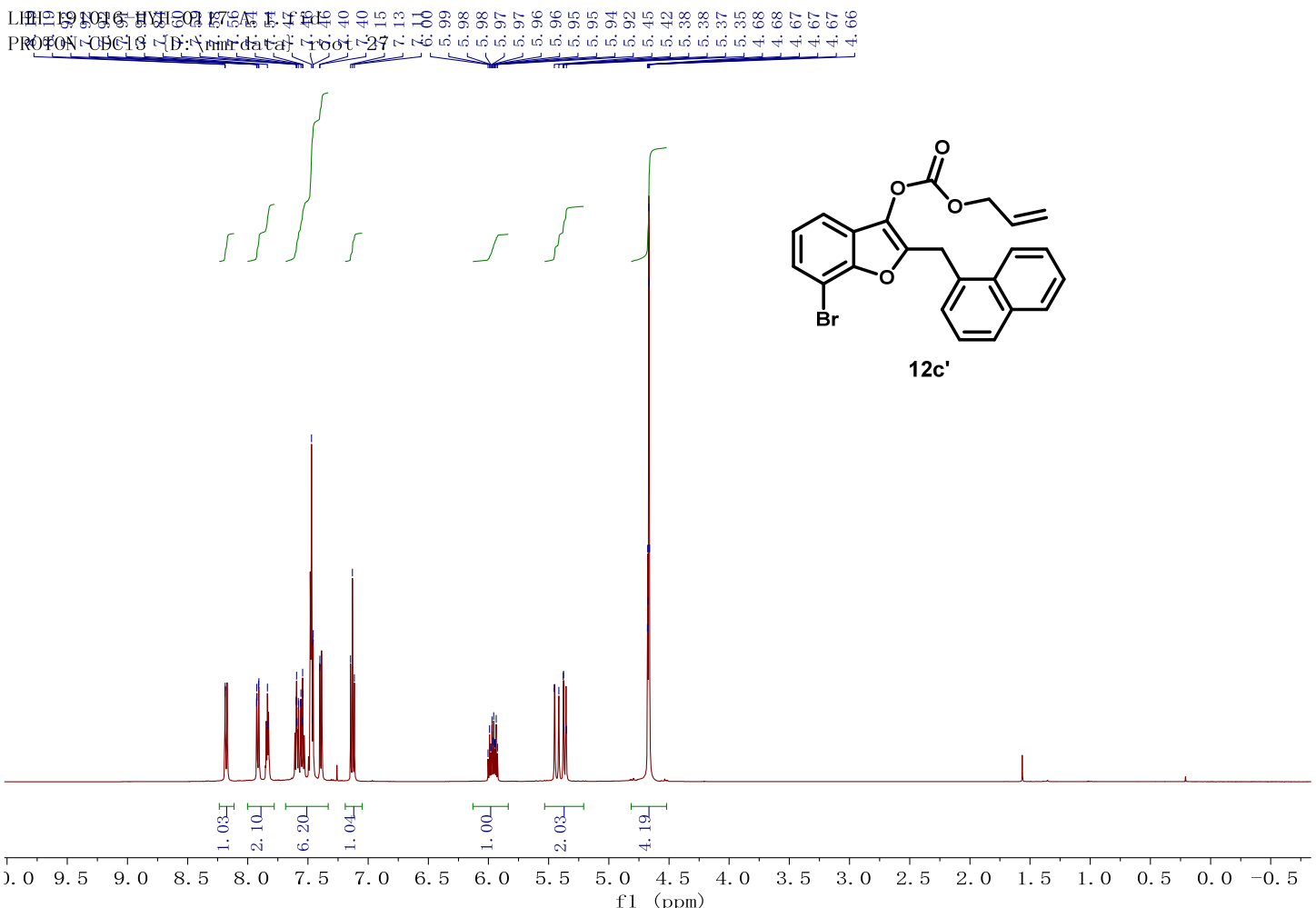

${ }^{13} \mathrm{C}$ NMR (125 MHz, $\mathrm{CDCl}_{3} @ 77$ ppm)

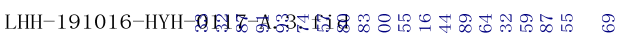

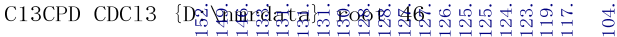

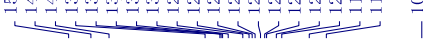<smiles>C=CCOC(=O)Oc1c(Cc2cccc3ccccc23)oc2c(Br)cccc12</smiles>

$12 c^{\prime}$

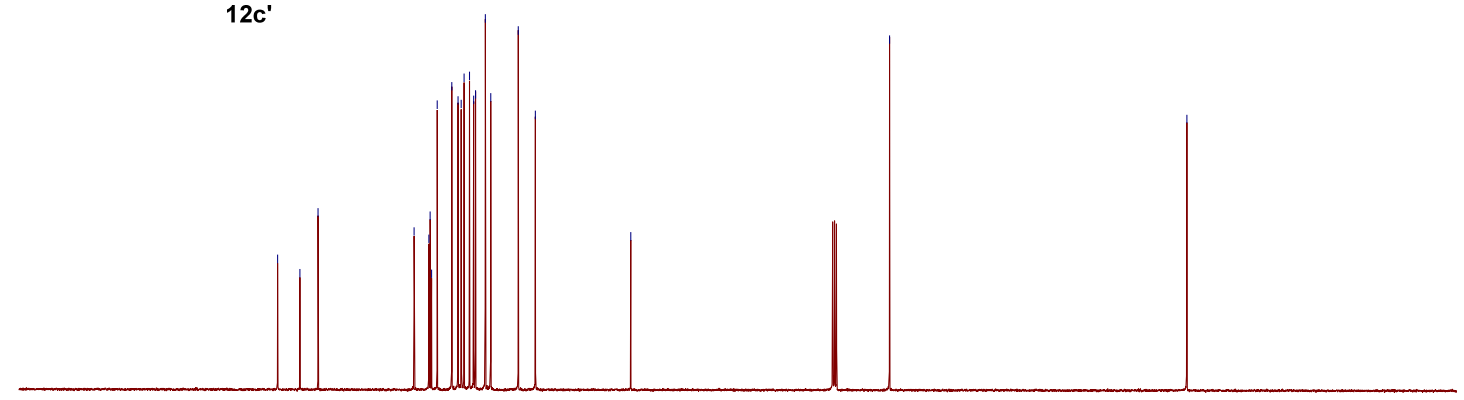

$\begin{array}{llllllllll}180 & 170 & 160 & 150 & 140 & 130 & 120 & 110 & 100 & 90 \\ \mathrm{f} 1 & & & (\mathrm{ppm})\end{array}$ 
Allyl (2-benzylnaphtho[1,2-b]furan-3-yl) carbonate(12d')

므 NMR $\left(500 \mathrm{MHz}, \mathrm{CDCl}_{3} @ 7.26\right.$ ppm)

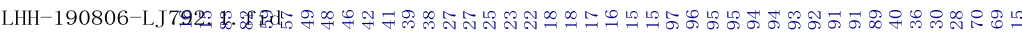

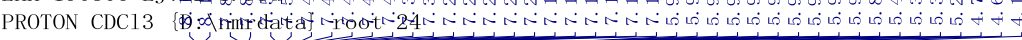
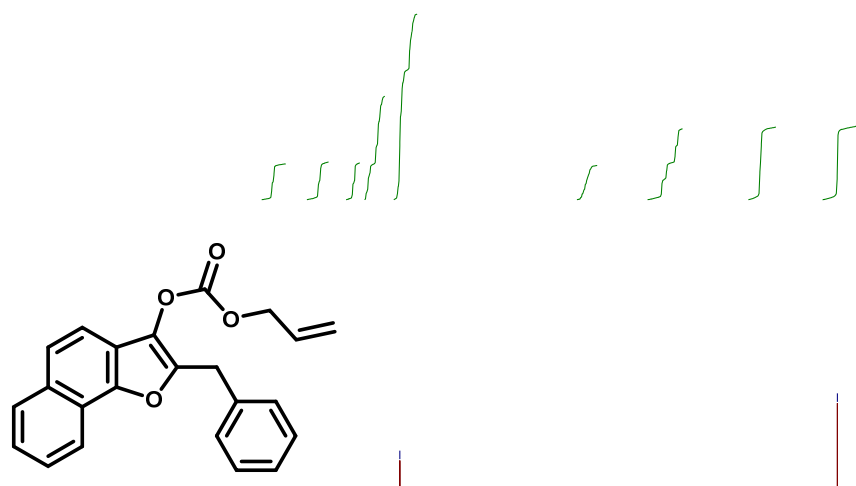

$12 d^{\prime}$

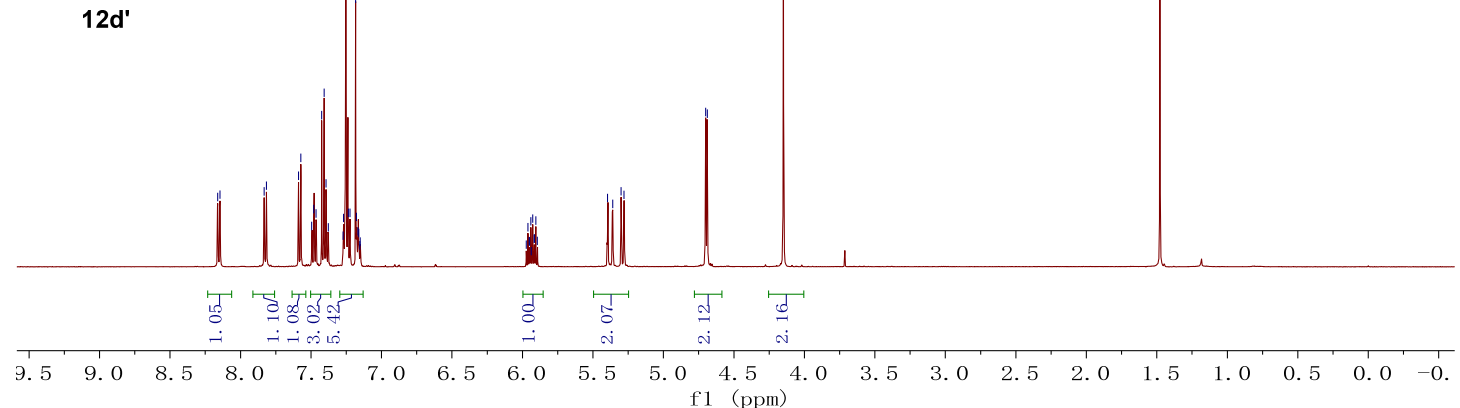

${ }^{13} \mathrm{C}$ NMR (125 MHz, CDCl 3077 ppm)

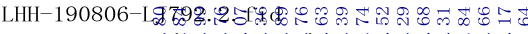

C13CPD CDC13

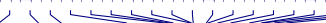

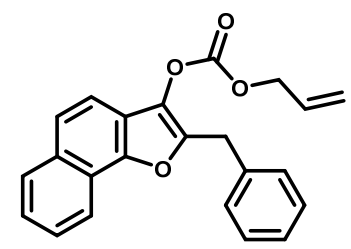

12d'

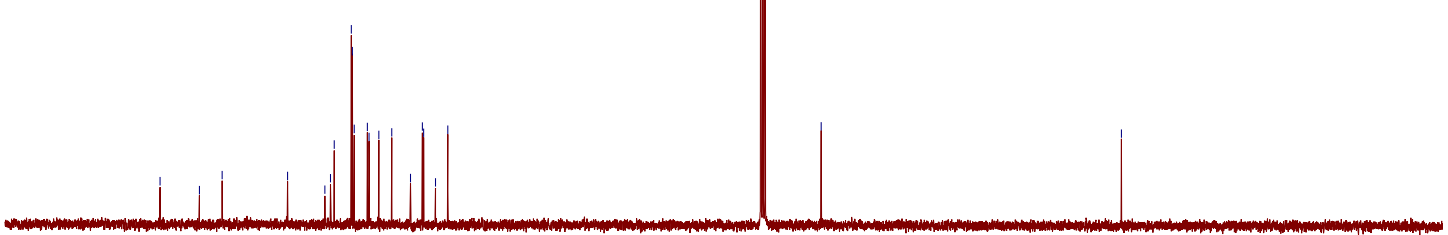

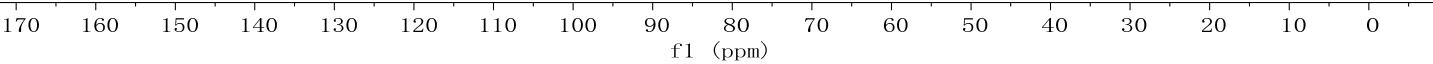




\section{Allyl (2-benzylnaphtho[2,1-b]furan-1-yl) carbonate(12e')}

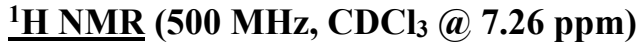

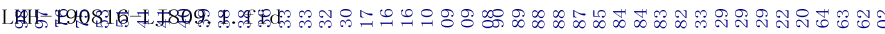

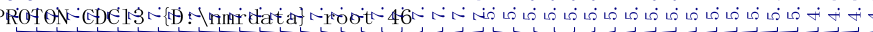

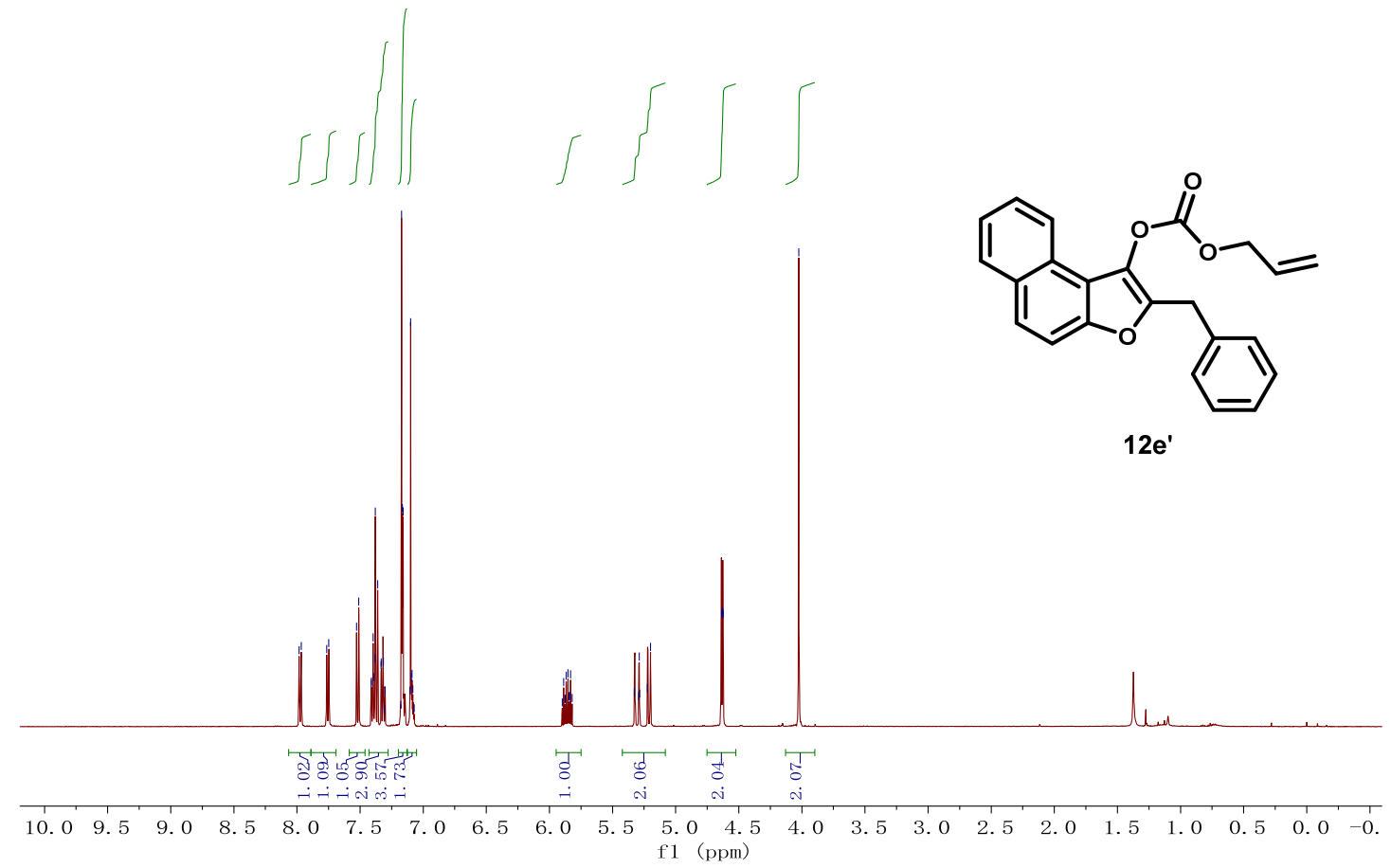

${ }^{13} \mathrm{C}$ NMR (125 MHz, $\mathrm{CDCl}_{3} @ 77$ ppm)

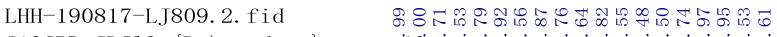

C13CPD CDC13 \{D:\nmrdata\} root

$\begin{array}{ll}\infty & \infty \\ \infty & \infty \\ 0 & \infty \\ 1 & 1\end{array}$

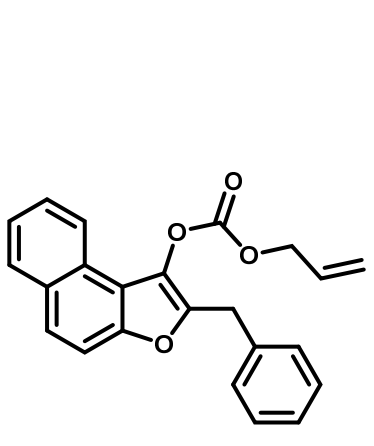

$12 e^{\prime}$

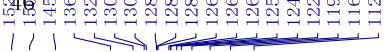

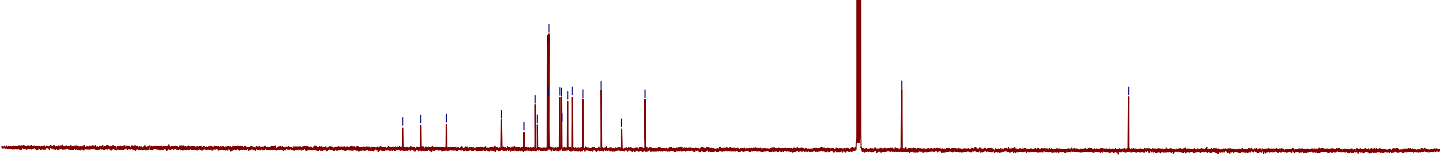

$\begin{array}{llllllllllll}210 & 200 & 190 & 180 & 170 & 160 & 150 & 140 & 130 & 120 & 110 & 100\end{array}$ 
Allyl (2-benzylfuro[2,3-b]pyridin-3-yl) carbonate(12f')

$\underline{{ }^{1} \mathrm{H} \text { NMR }}\left(400 \mathrm{MHz}, \mathrm{CDCl}_{3} @ 7.26\right.$ ppm)

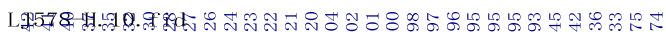

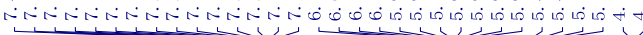

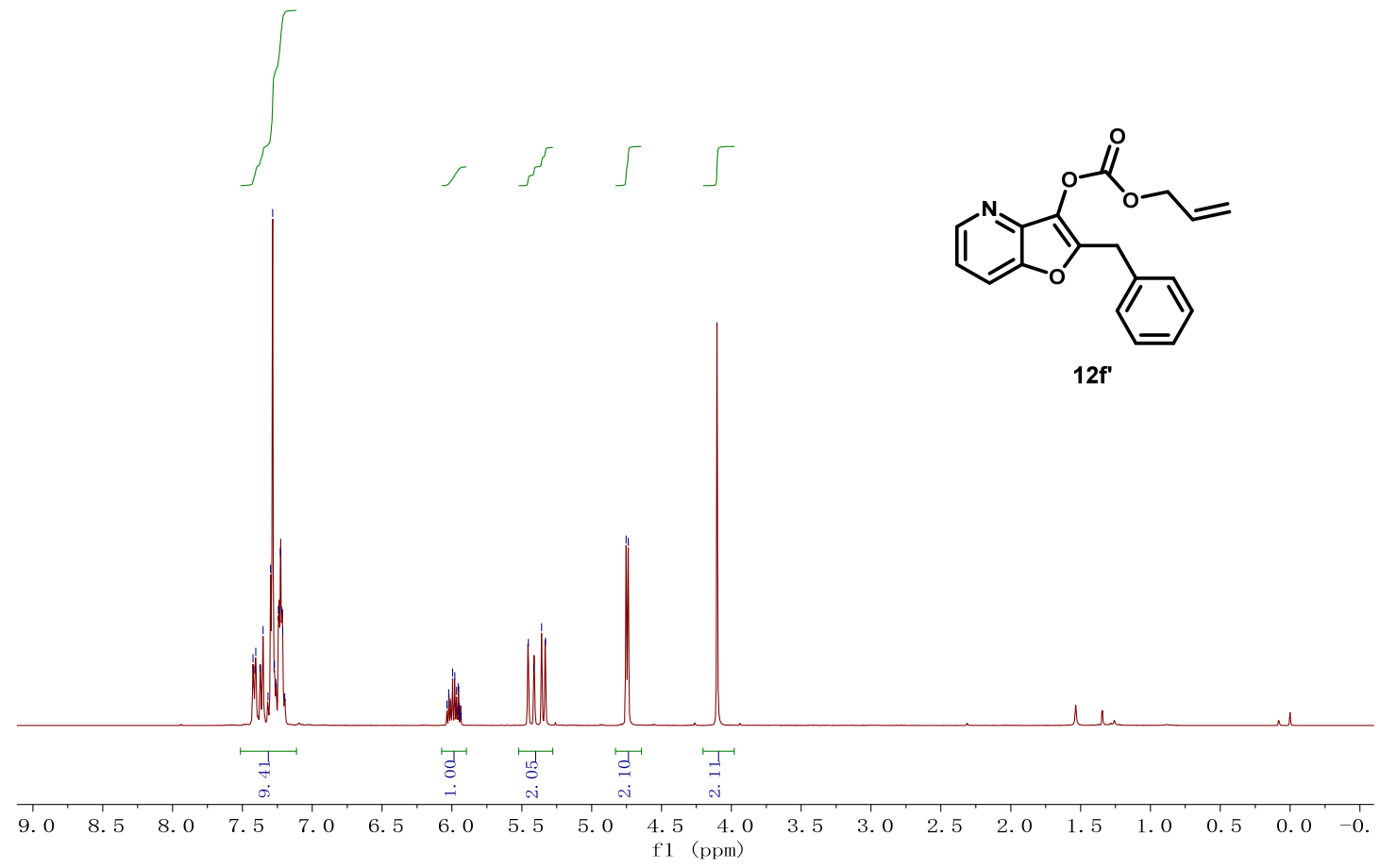

${ }^{13} \mathrm{C}$ NMR (100 MHz, $\mathrm{CDCl}_{3} @ 77$ ppm)

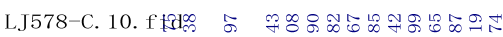

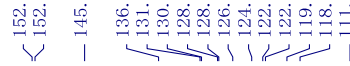
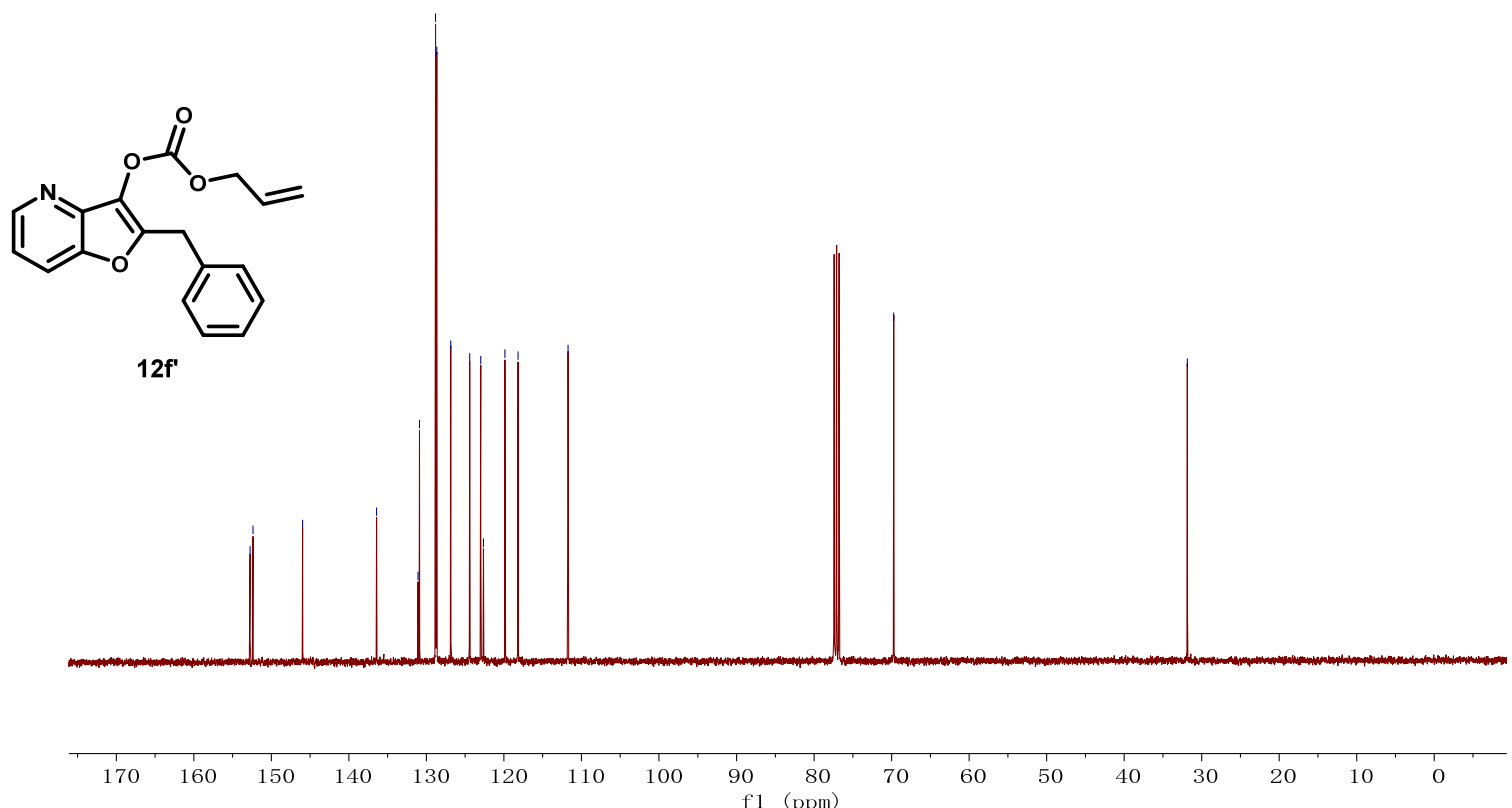

S192 
Allyl (4,6-dimethoxy-2-(4-methoxyphenyl)benzofuran-3-yl) carbonate (12g')

\section{$\underline{{ }^{1} \mathrm{H} \text { NMR }}\left(400 \mathrm{MHz}, \mathrm{CDCl}_{3} @ 7.26 \mathrm{ppm}\right)$}

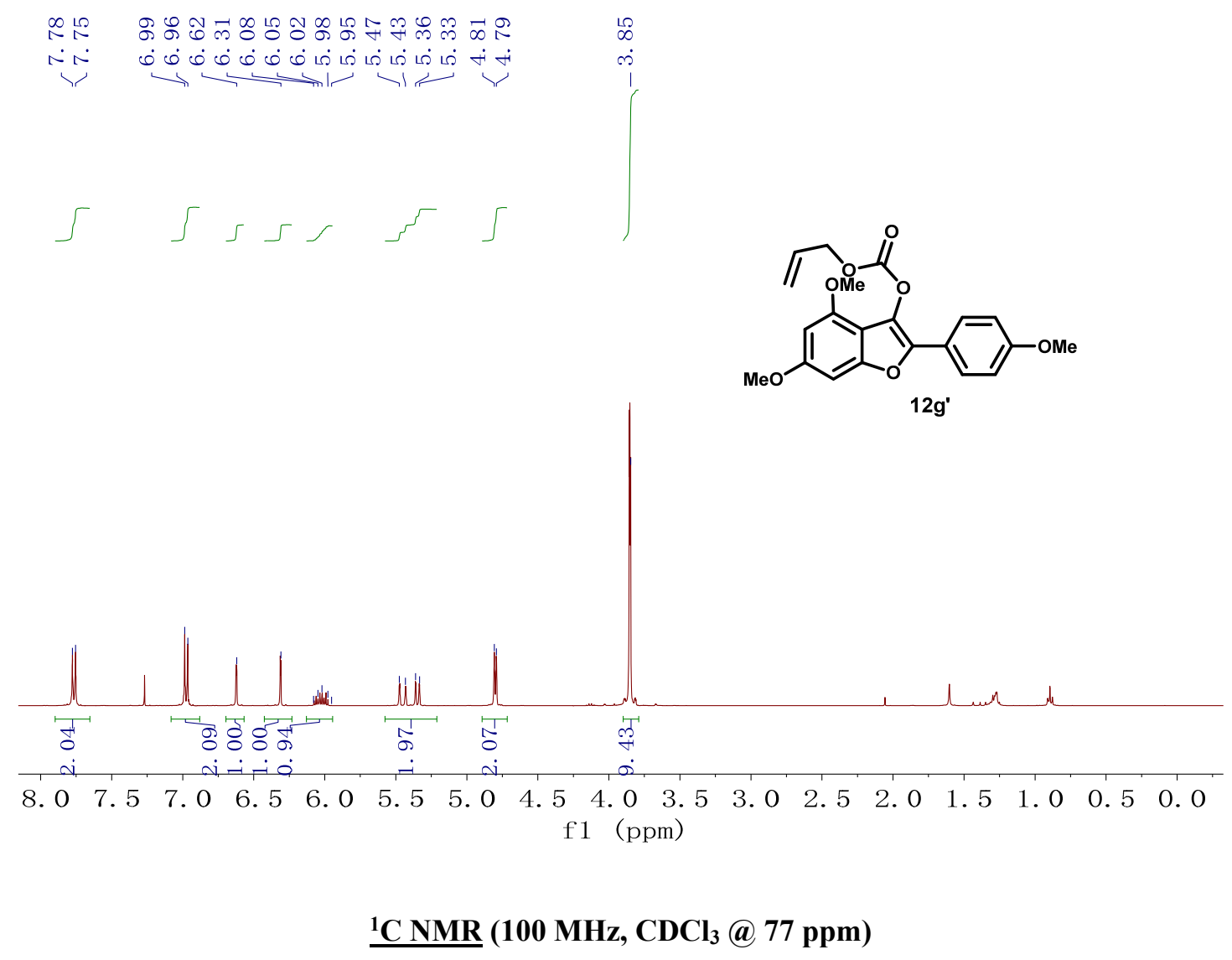




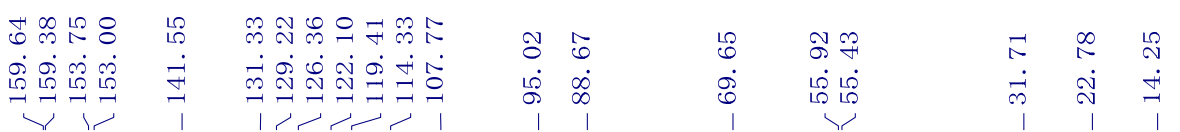
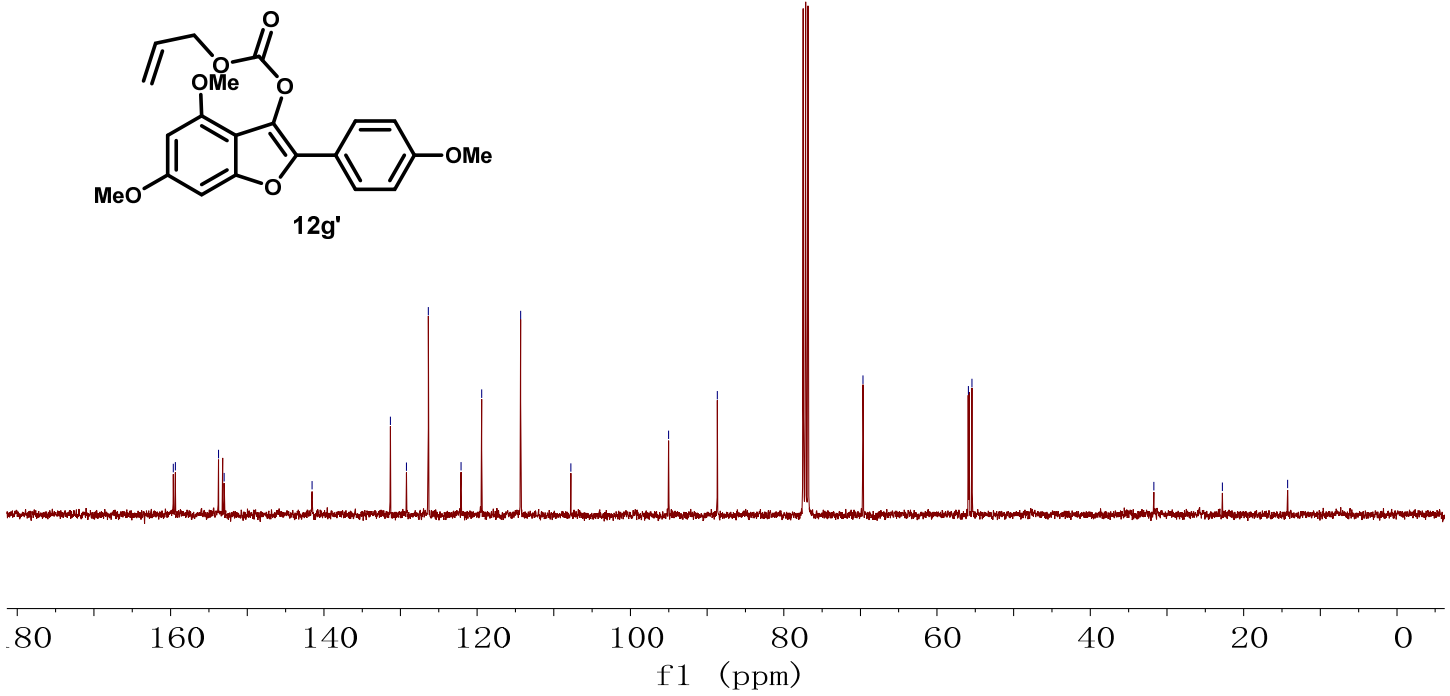

Allyl (4,6-dimethoxy-2-phenylbenzofuran-3-yl) carbonate (12h')

$\underline{{ }^{1} \mathrm{H} \text { NMR }}\left(500 \mathrm{MHz}, \mathrm{CDCl}_{3} @ 7.26\right.$ ppm)

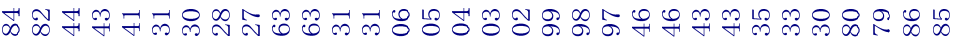

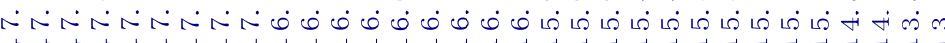

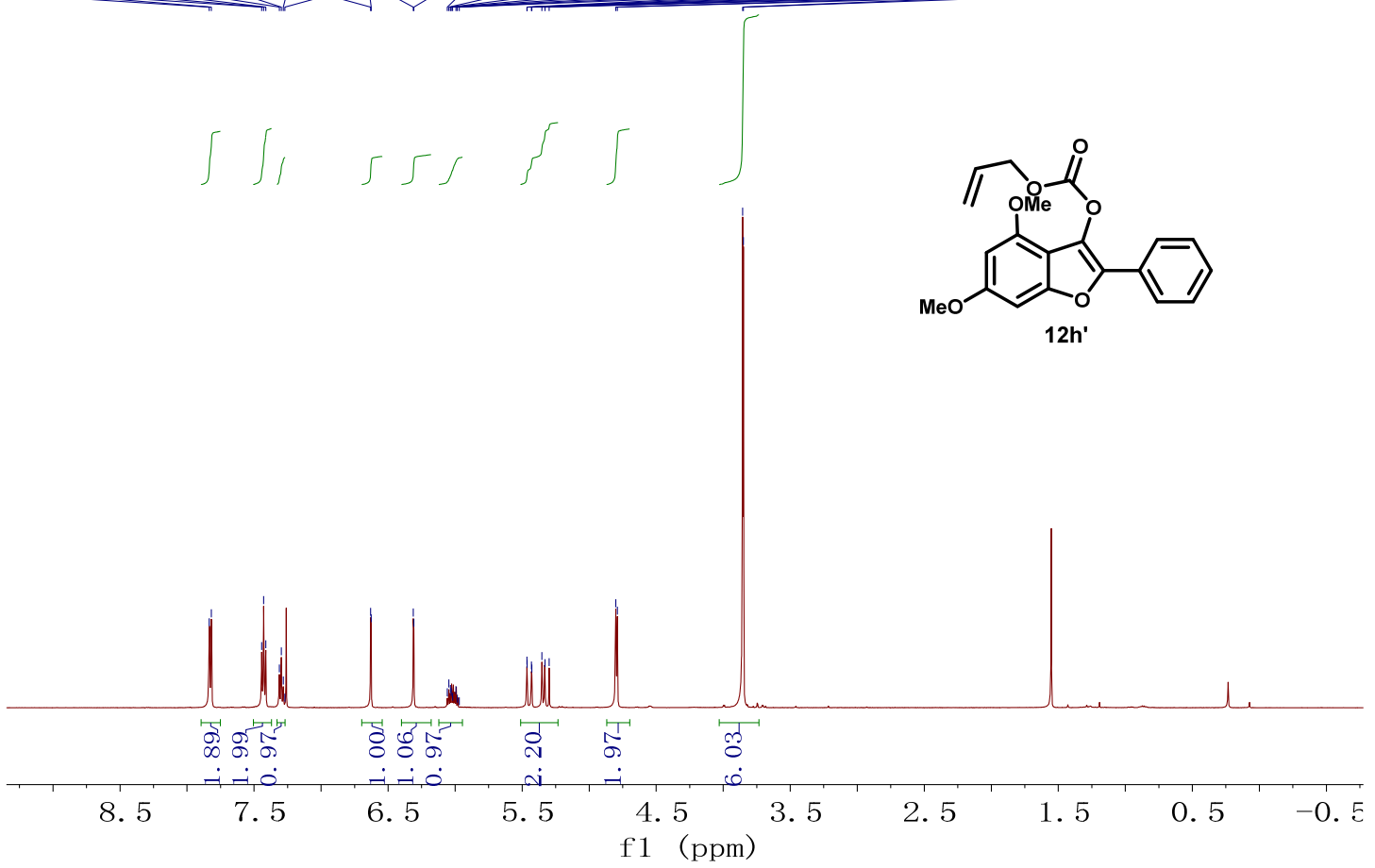

1 NMR (125 MHz, CDCl 3 @ 77 ppm) 


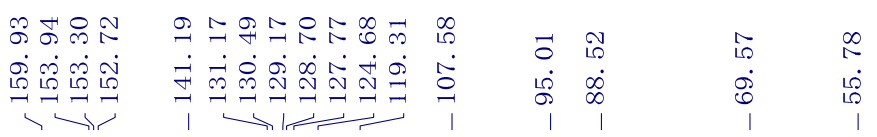

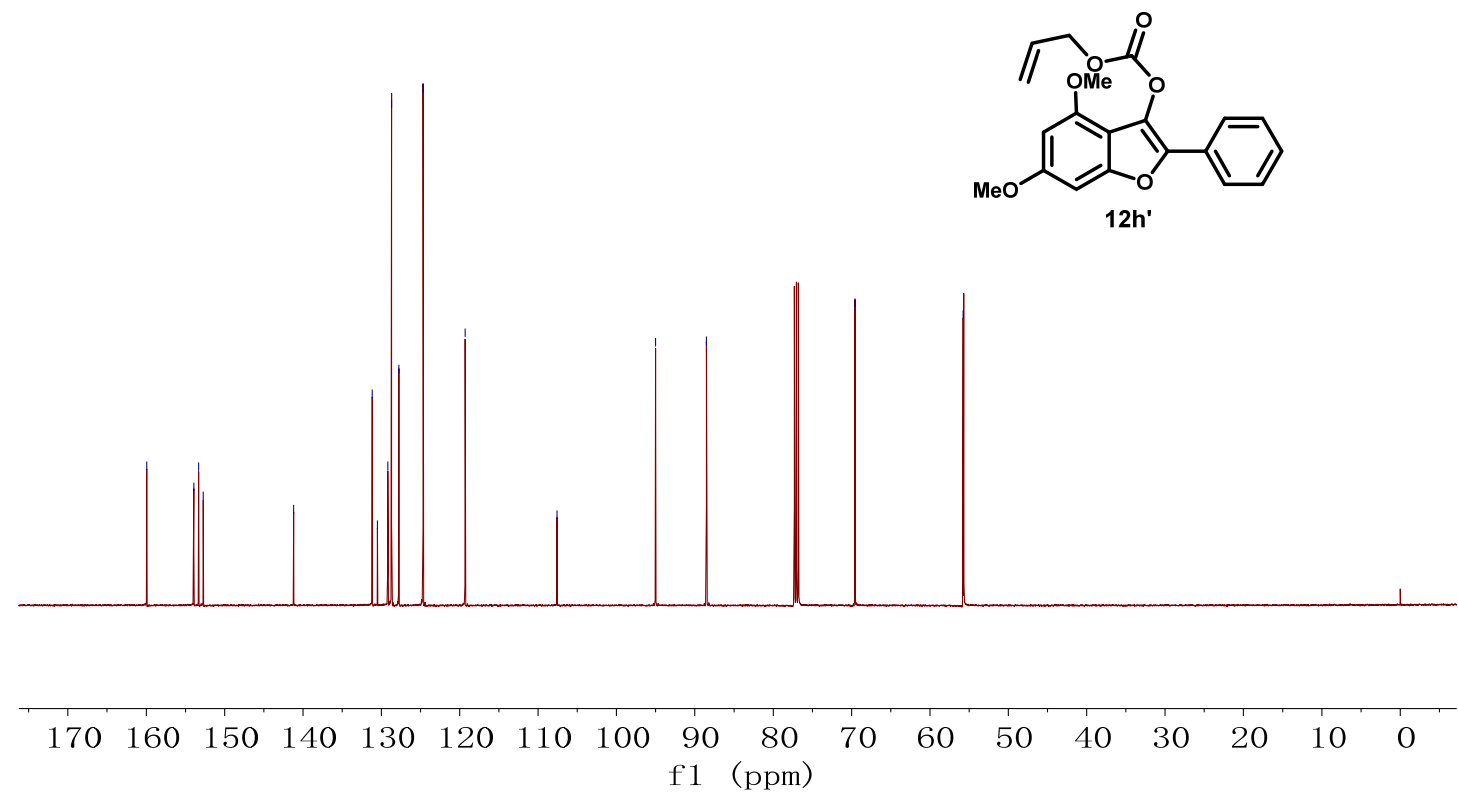

(S)-2-Allyl-2-benzylbenzofuran-3(2H)-one (10a)

${ }^{1} \mathrm{H}$ NMR (400 MHz, CDCl 3 @ 7.26 ppm)

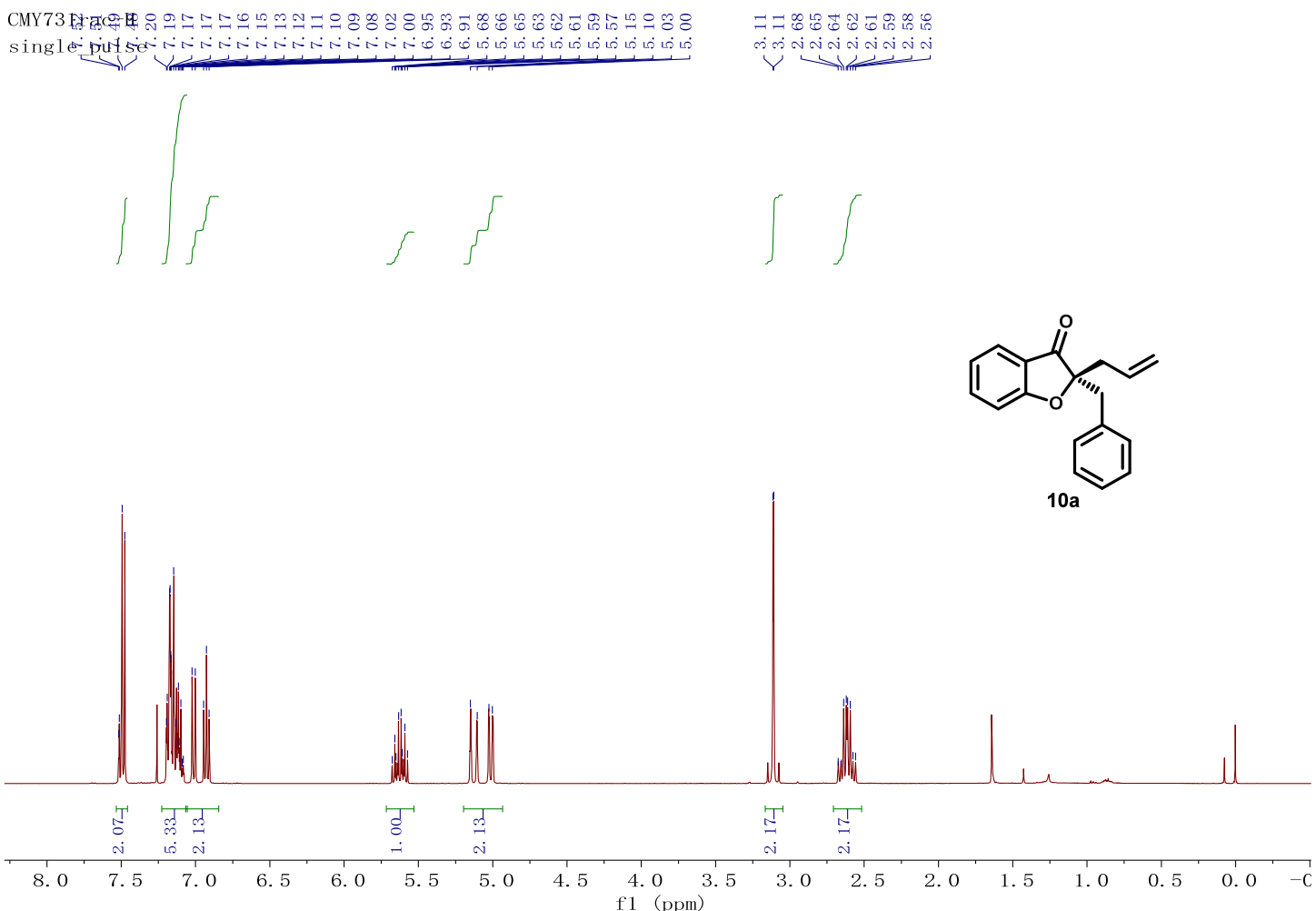


${ }^{13} \mathrm{C}$ NMR (100 MHz, $\mathrm{CDCl}_{3} @ 77$ ppm)

cmy-731ras

single pufise decoupled giated NOE

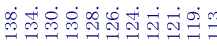

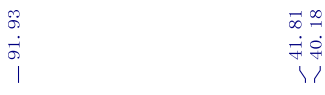

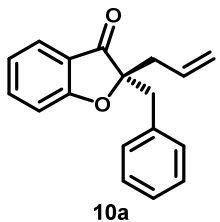

$10 \mathrm{a}$

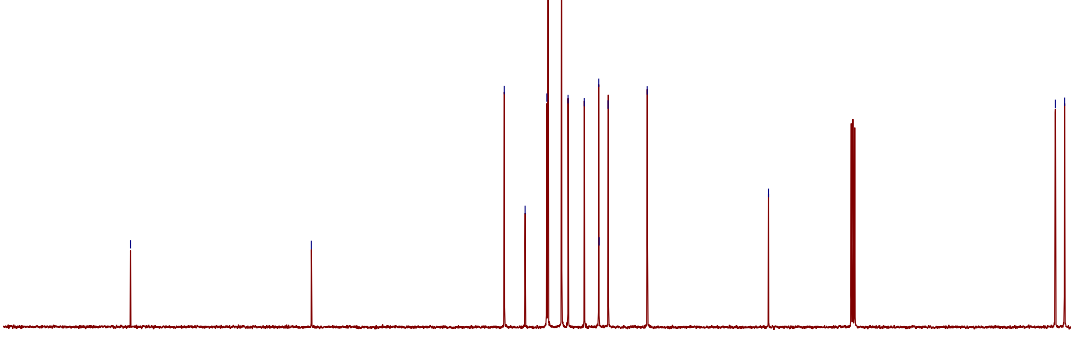

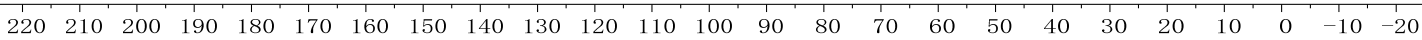

f1 (ppm)

(S)-1-Acetyl-2-allyl-2-benzylindolin-3-one (10b)

$\underline{{ }^{1} \mathrm{H} \text { NMR }}\left(500 \mathrm{MHz}, \mathrm{CDCl}_{3} @ 7.26\right.$ ppm)

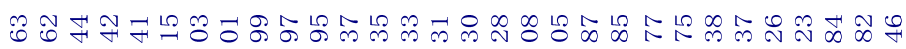

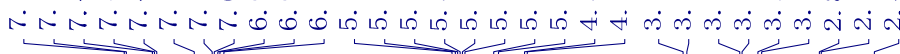
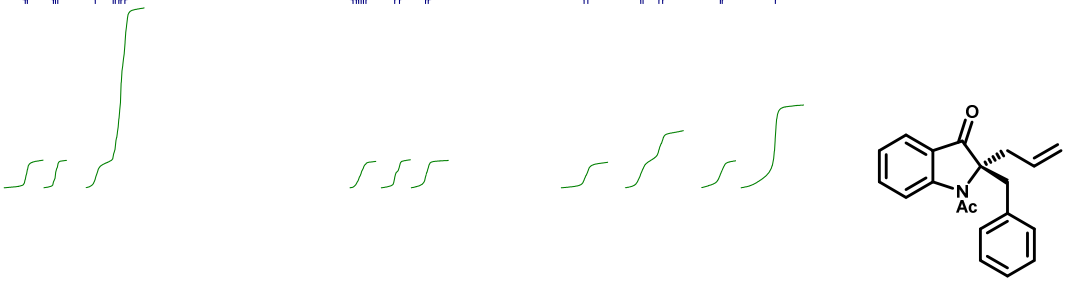

$10 \mathrm{~b}$

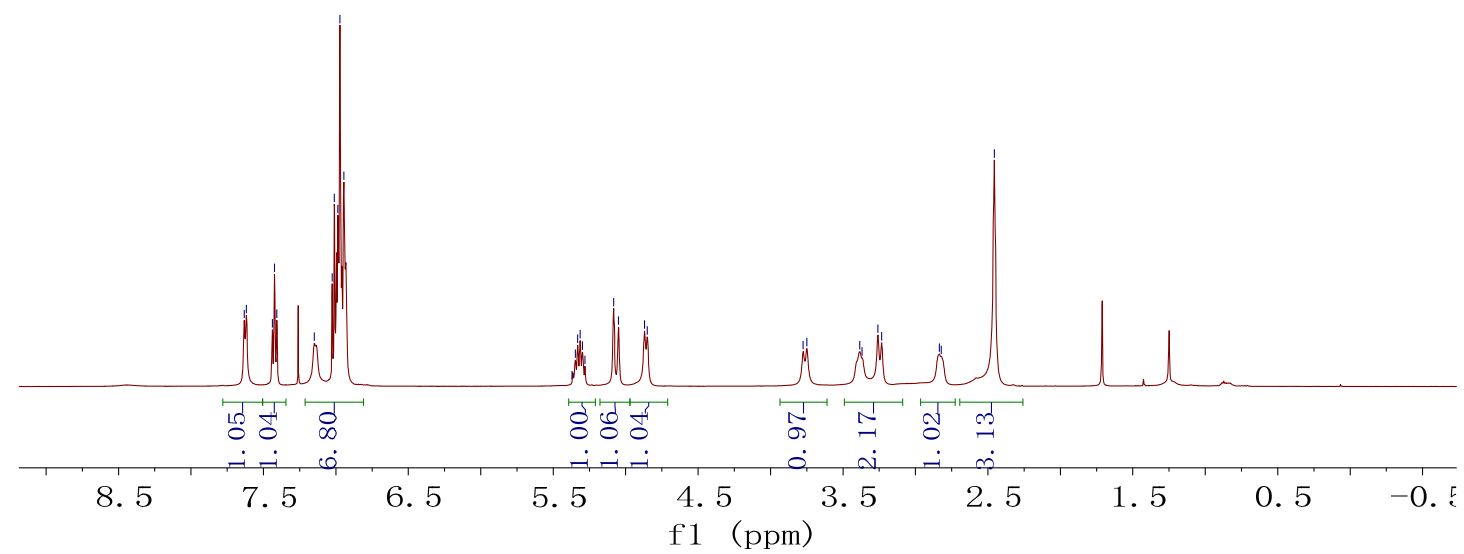




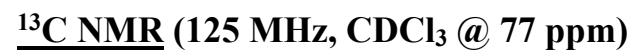

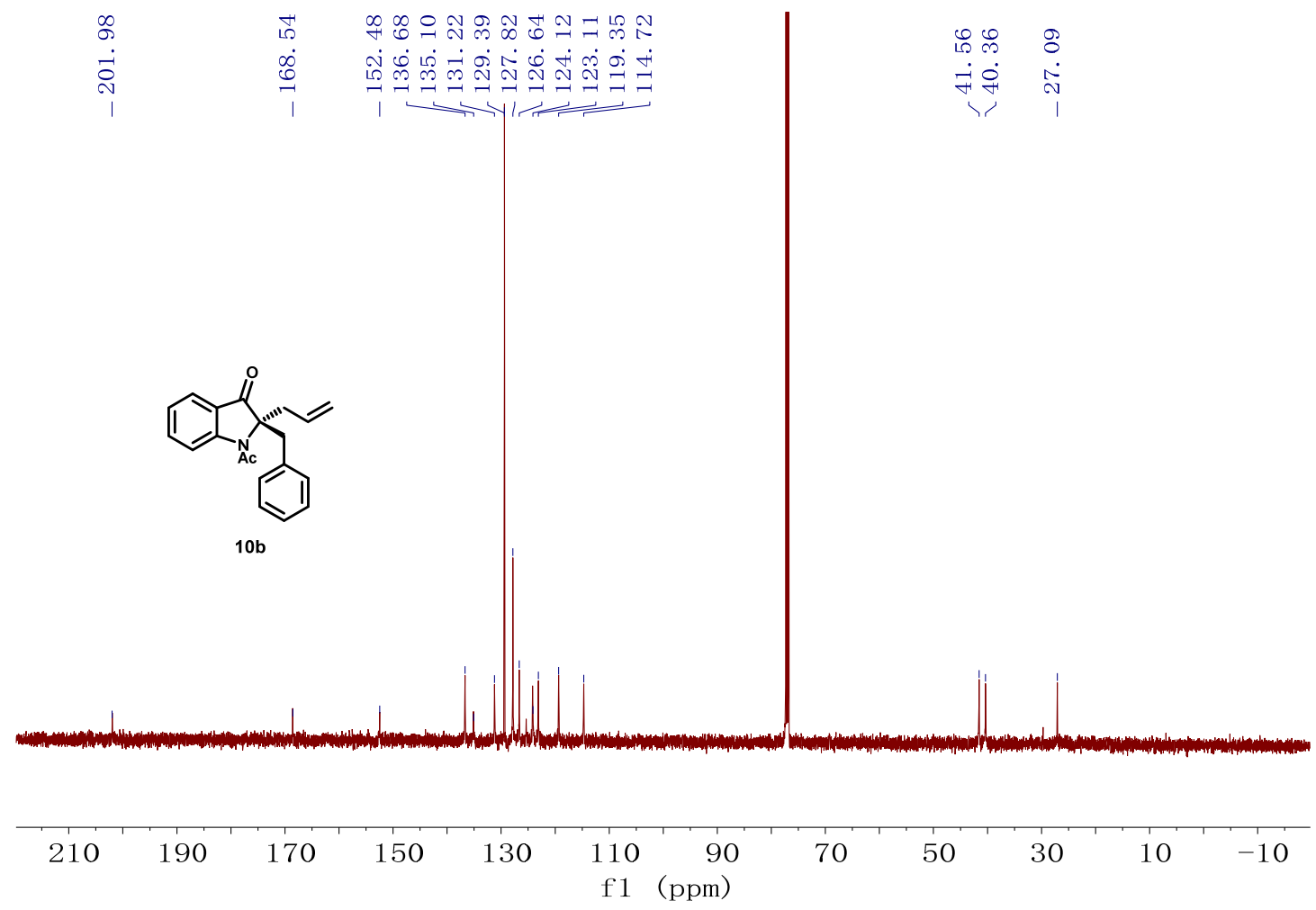

Methyl (S)-4-((2-allyl-3-oxo-2,3-dihydrobenzofuran-2-yl)methyl)benzoate (10c)

$\underline{{ }^{1} \mathrm{H} \text { NMR }}\left(400 \mathrm{MHz}, \mathrm{CDCl}_{3} @ 7.26\right.$ ppm) 


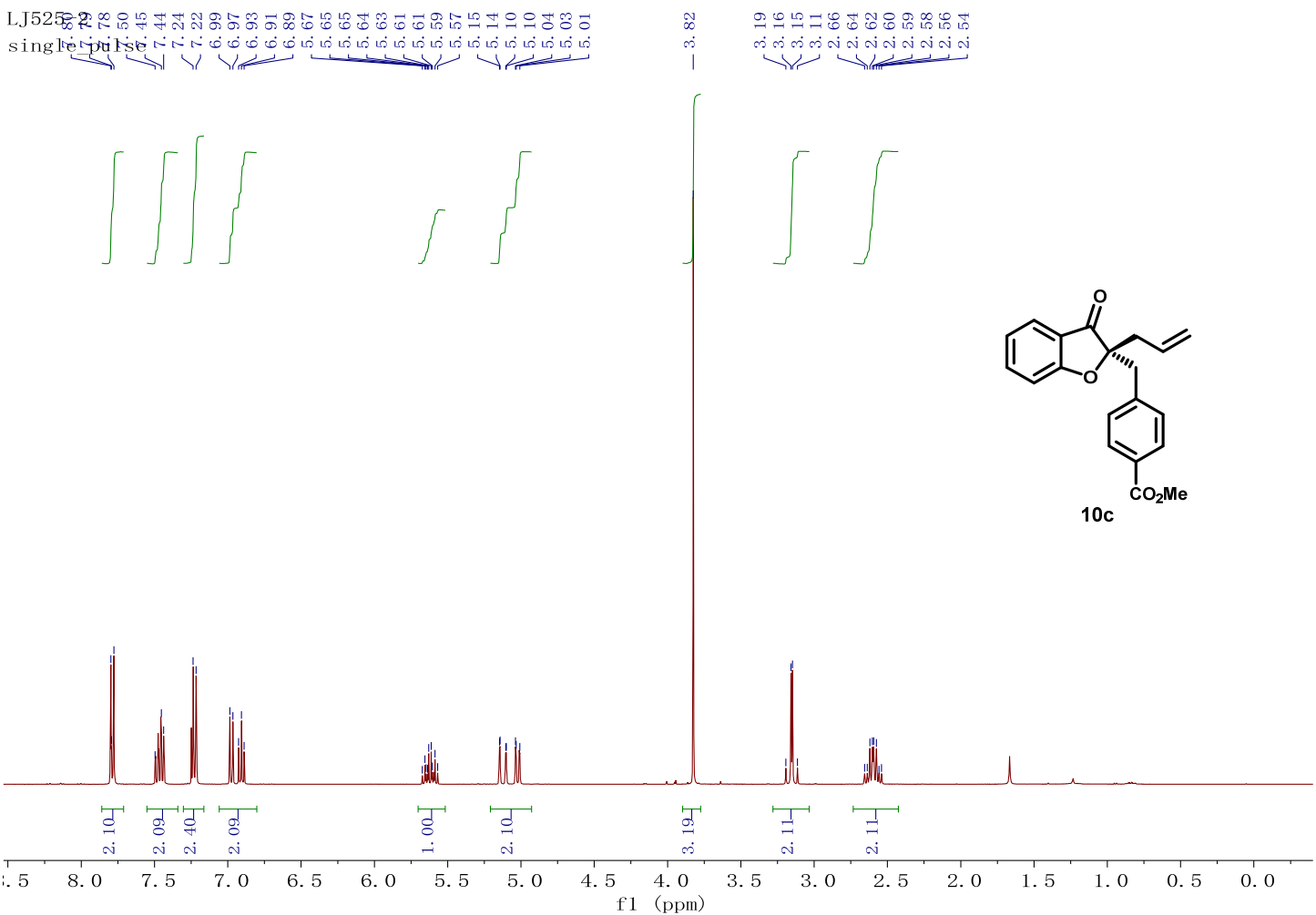

${ }^{13} \mathrm{C}$ NMR $\left(100 \mathrm{MHz}, \mathrm{CDCl}_{3} @ 77\right.$ ppm)

\begin{tabular}{|c|c|c|c|c|}
\hline 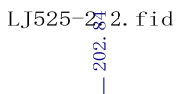 & 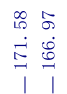 & 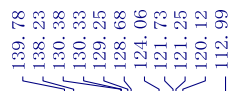 & $\stackrel{\text { 워 }}{\dot{\sigma}}$ & $\begin{array}{l}\text { D } \\
\text { id } \\
\text { if } \\
\text { i }\end{array}$ \\
\hline
\end{tabular}

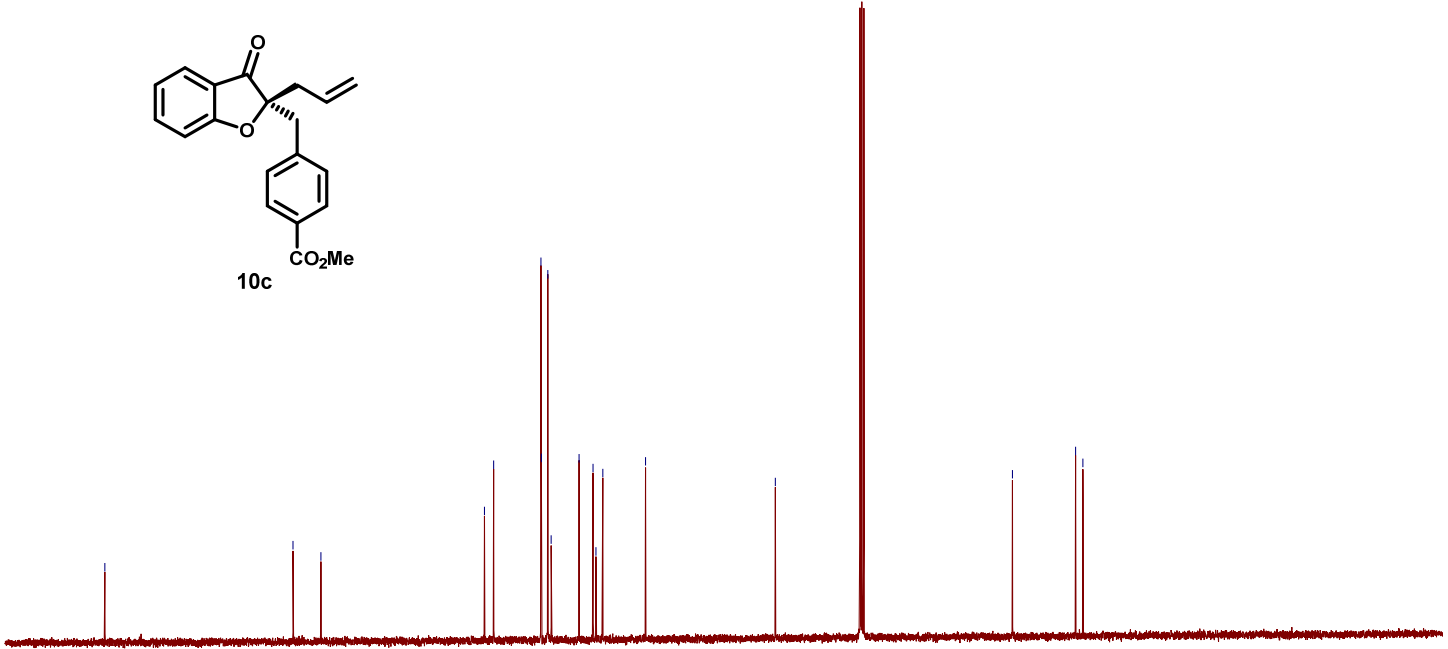

$\begin{array}{llllllllllllllllllllllll}210 & 200 & 190 & 180 & 170 & 160 & 150 & 140 & 130 & 120 & 110 & 100 & 90 & 80 & 70 & 60 & 50 & 40 & 30 & 20 & 10 & 0 & -10\end{array}$

(S)-4-((2-Allyl-3-oxo-2,3-dihydrobenzofuran-2-yl)methyl)benzonitrile (10d)

$\underline{{ }^{1} \mathrm{H} \text { NMR }}\left(500 \mathrm{MHz}, \mathrm{CDCl}_{3} @ 7.26 \mathrm{ppm}\right)$ 


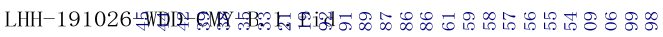

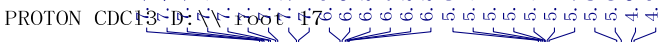
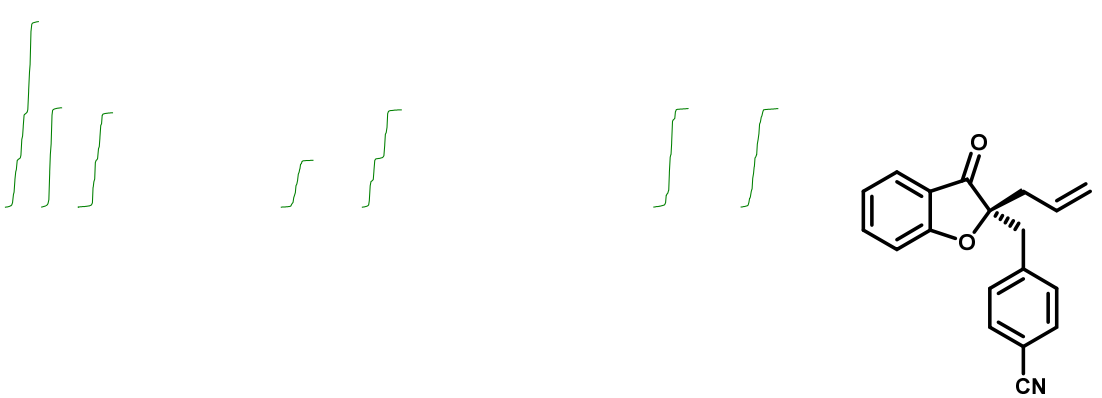

$10 d$

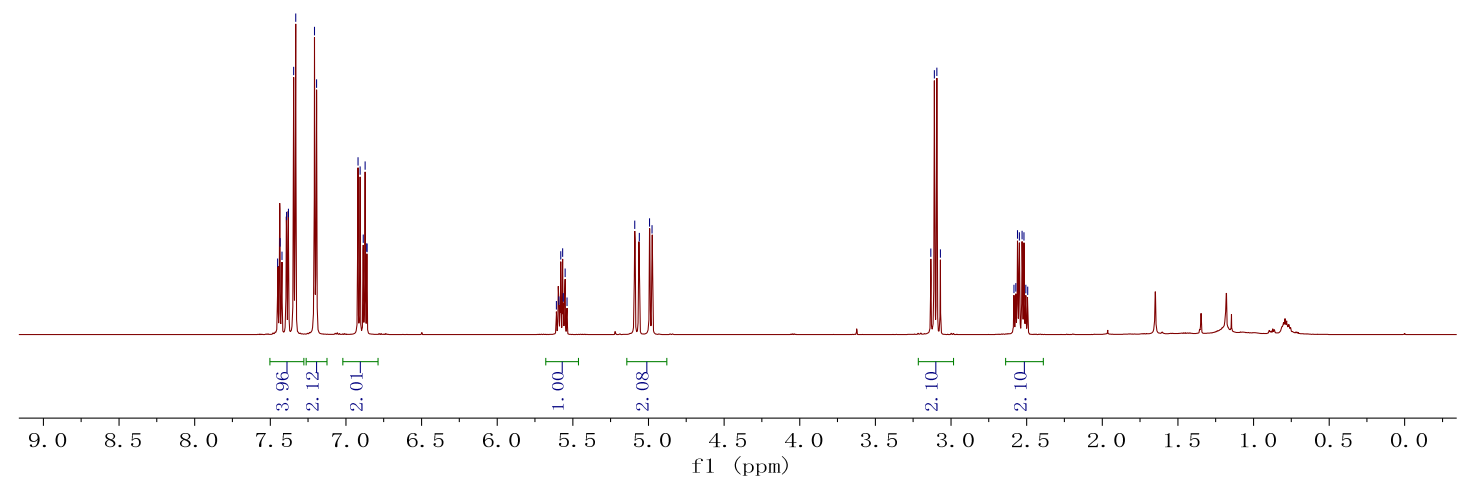

${ }^{13} \mathrm{C}$ NMR (125 MHz, $\mathrm{CDCl}_{3} @ 77$ ppm)

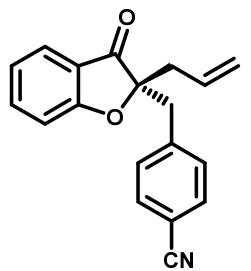

10d

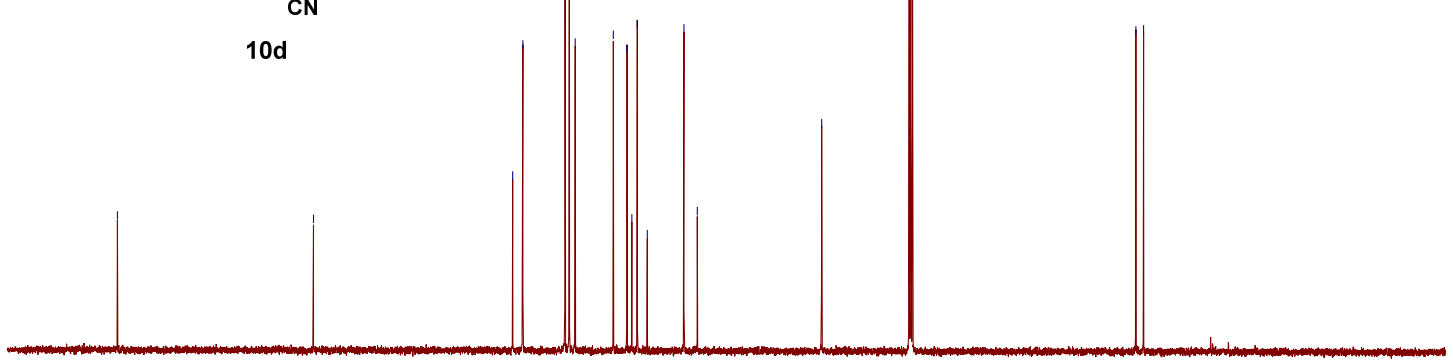

$\begin{array}{llllllllllll}210 & 200 & 190 & 180 & 170 & 160 & 150 & 140 & 130 & 120 & 110 & 100\end{array}$

(S)-2-Allyl-2-(4-(trifluoromethyl)benzyl)benzofuran-3(2H)-one (10e)

$\underline{{ }^{1} \mathrm{H} \text { NMR }}\left(500 \mathrm{MHz}, \mathrm{CDCl}_{3} @ 7.26 \mathrm{ppm}\right)$ 
LHH-XK-338-B.

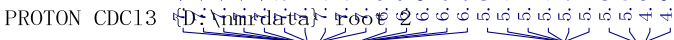
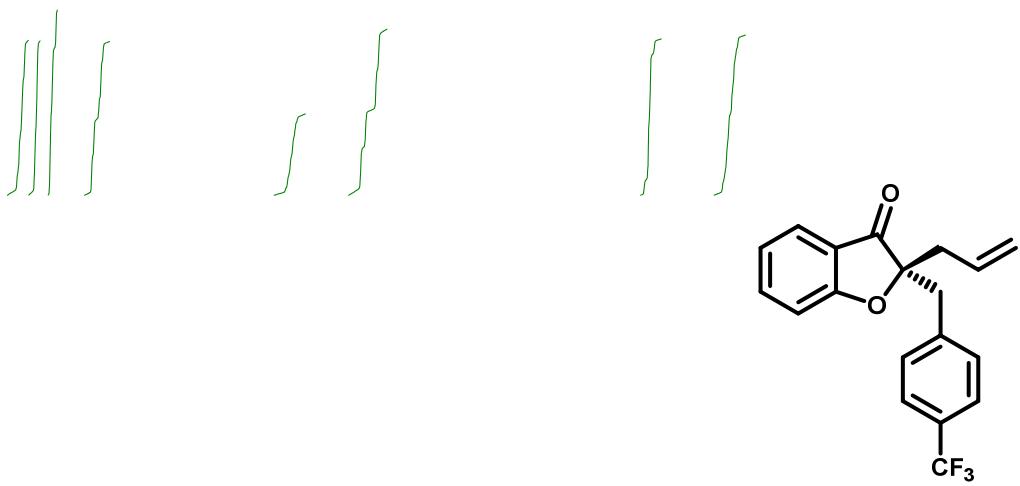

$10 \mathrm{e}$

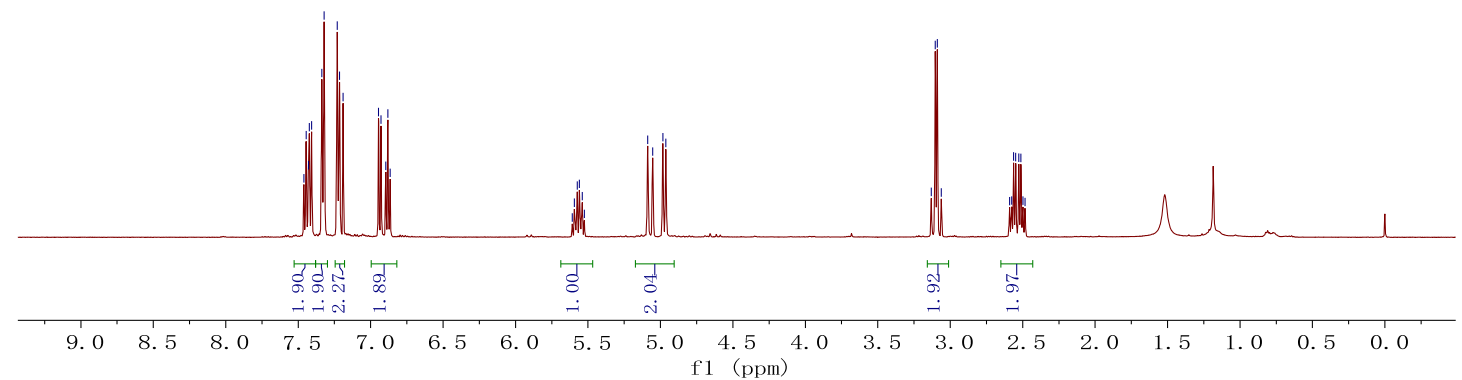

${ }^{13} \mathrm{C}$ NMR $\left(125 \mathrm{MHz}, \mathrm{CDCl}_{3} @ 77\right.$ ppm)

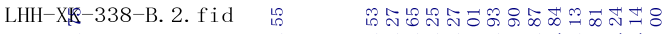

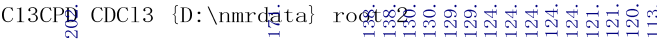

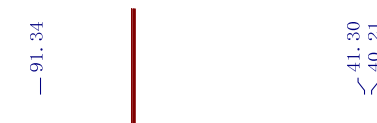<smiles>C=CC[C@]1(Cc2ccc(C(F)(F)F)cc2)Oc2ccccc2C1=O</smiles>

$10 \mathrm{e}$

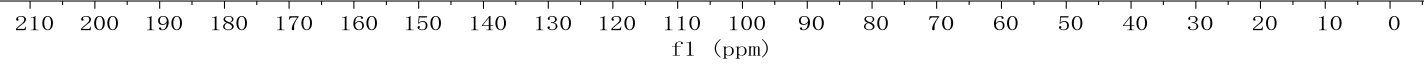

$\underline{19}$ F NMR (470 MHz, $\left.\mathrm{CDCl}_{3}\right)$ 


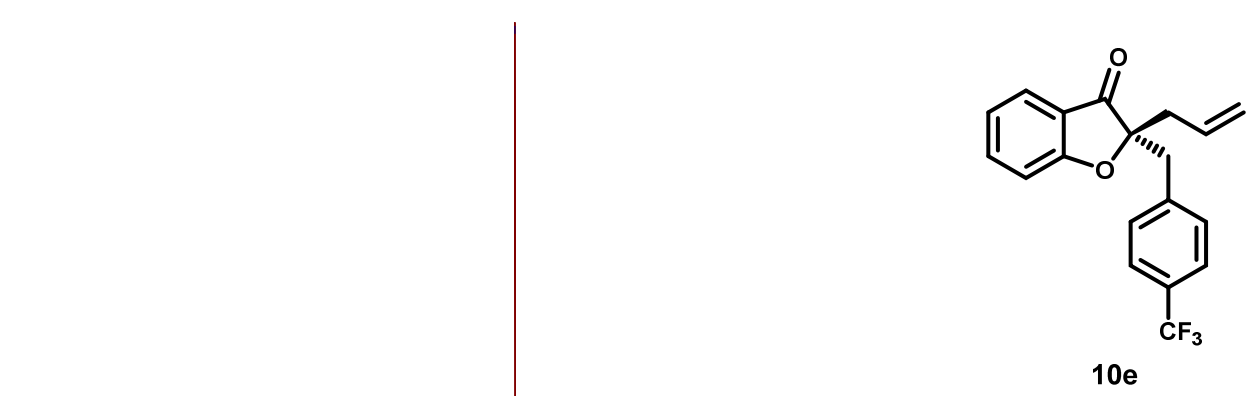

(S)-2-Allyl-2-(4-fluorobenzyl)benzofuran-3(2H)-one (10f)

$\underline{{ }^{1} \mathrm{H} \text { NMR }}\left(400 \mathrm{MHz}, \mathrm{CDCl}_{3} @ 7.26\right.$ ppm)

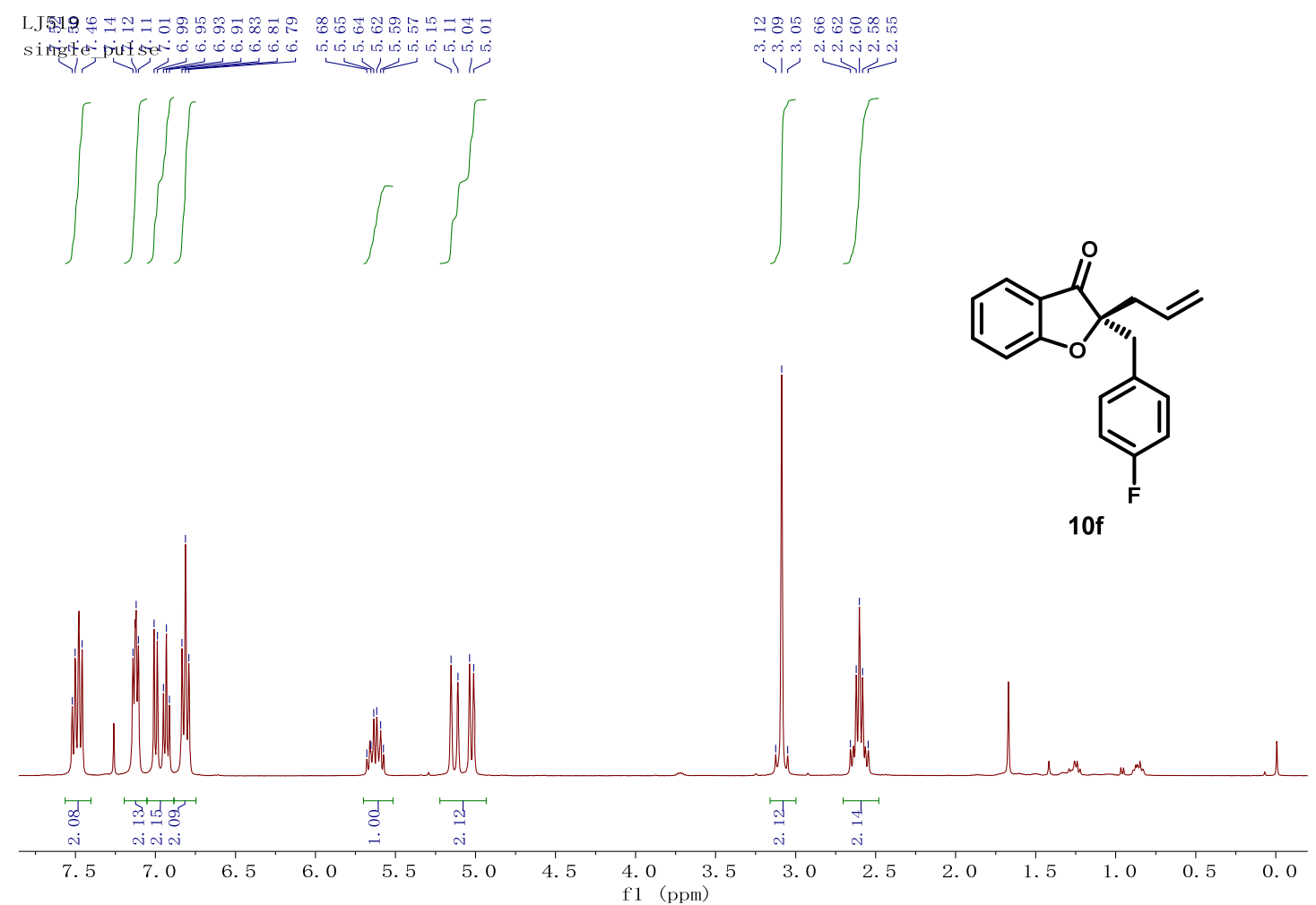

$\underline{{ }^{13} \mathrm{C} \text { NMR }}\left(100 \mathrm{MHz}, \mathrm{CDCl}_{3} @ 77\right.$ ppm) 


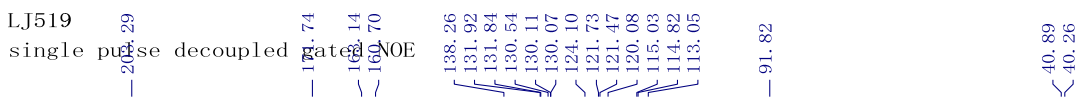

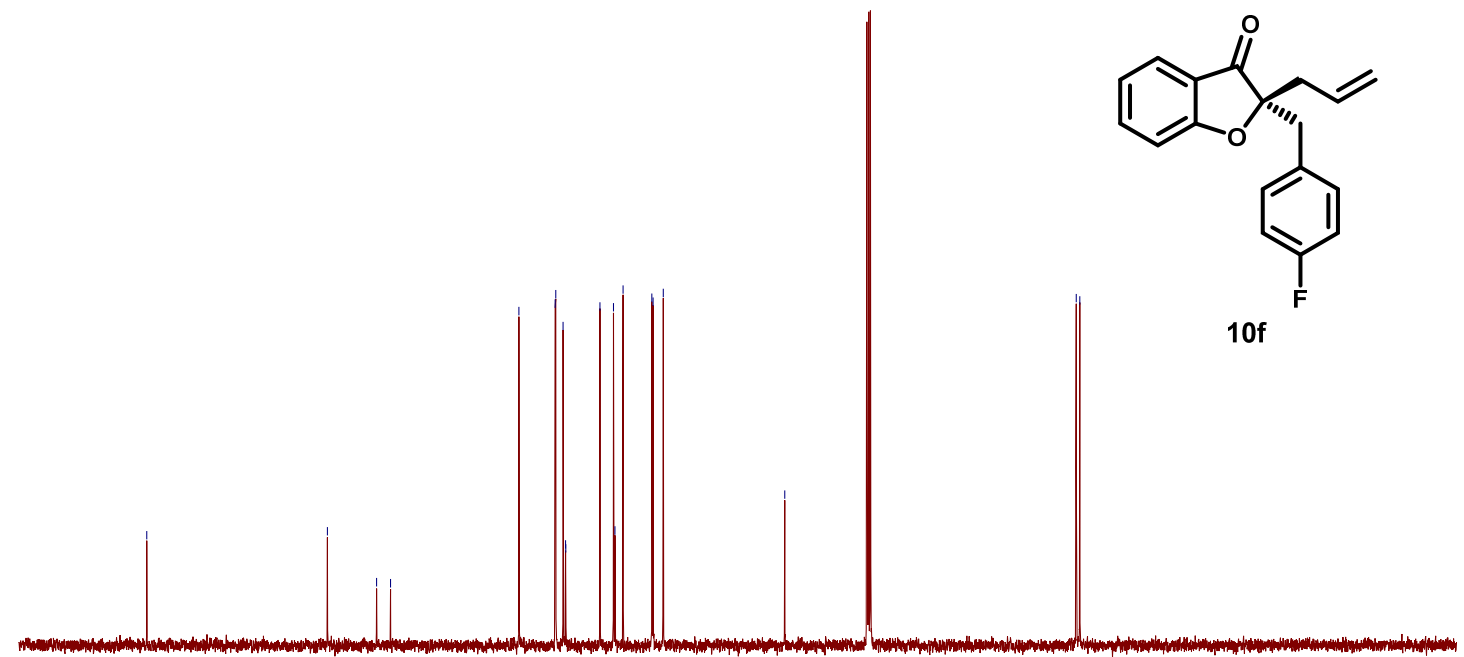

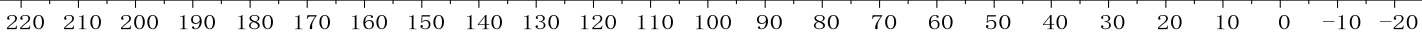
f1 (ppm)

${ }^{19}$ F NMR (376MHz, $\left.\mathrm{CDCl}_{3}\right)$

LHH-191205-1 j-519-F. 10, fid

F19 CDC13 \{D: \nmrdata\} root 25

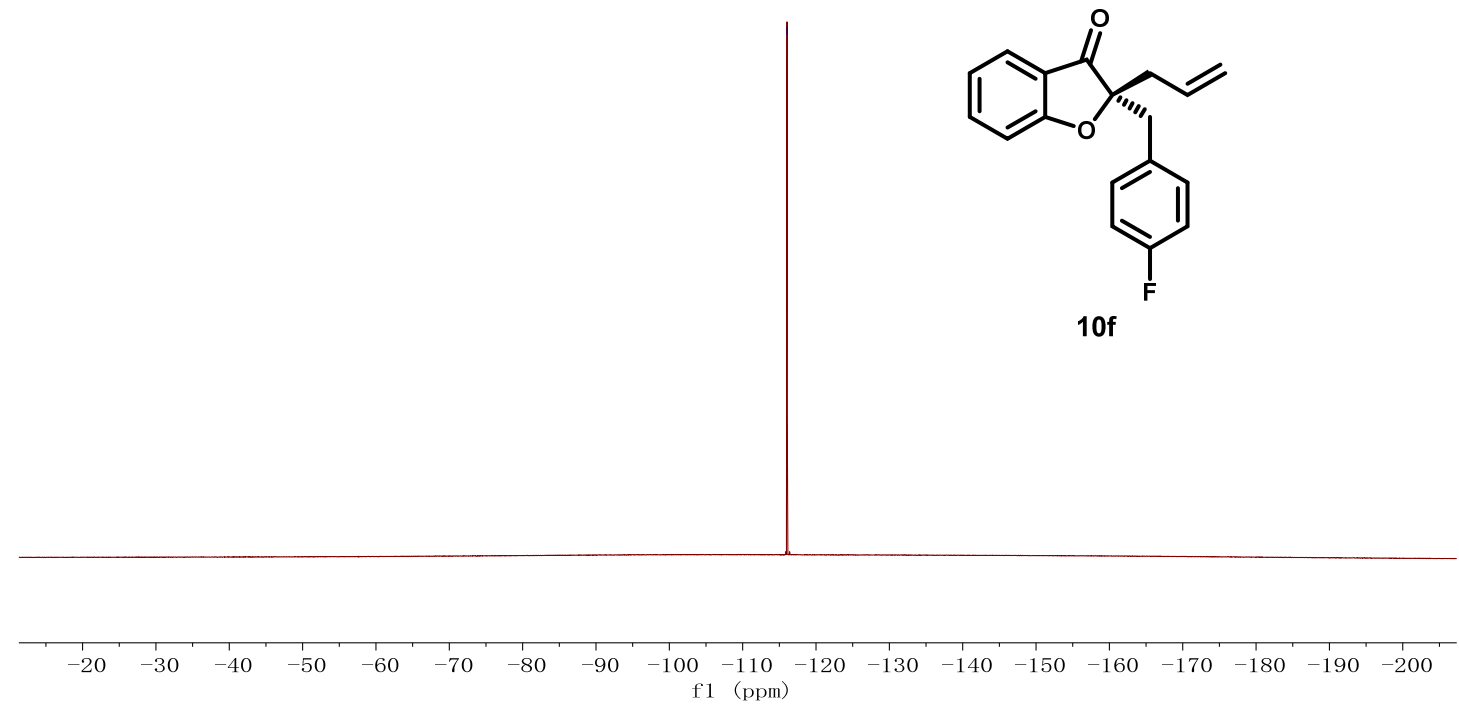

(S)-2-Allyl-2-(4-chlorobenzyl)benzofuran-3(2H)-one (10g) 
$\underline{{ }^{1} \mathrm{H} \text { NMR }}\left(500 \mathrm{MHz}, \mathrm{CDCl}_{3} @ 7.26 \mathrm{ppm}\right)$

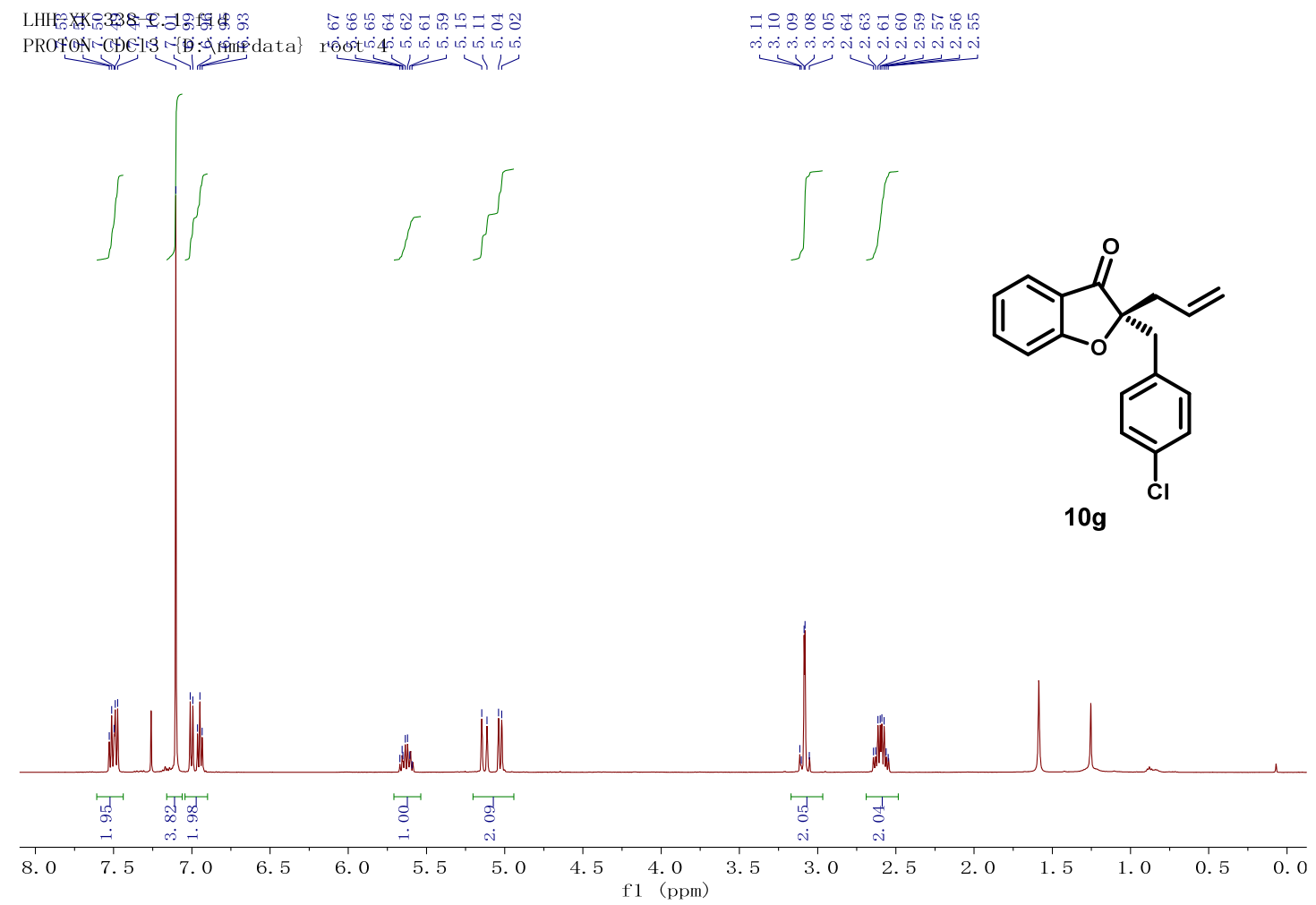

${ }^{13} \mathrm{C}$ NMR (125 MHz, $\mathrm{CDCl}_{3} @ 77$ ppm)

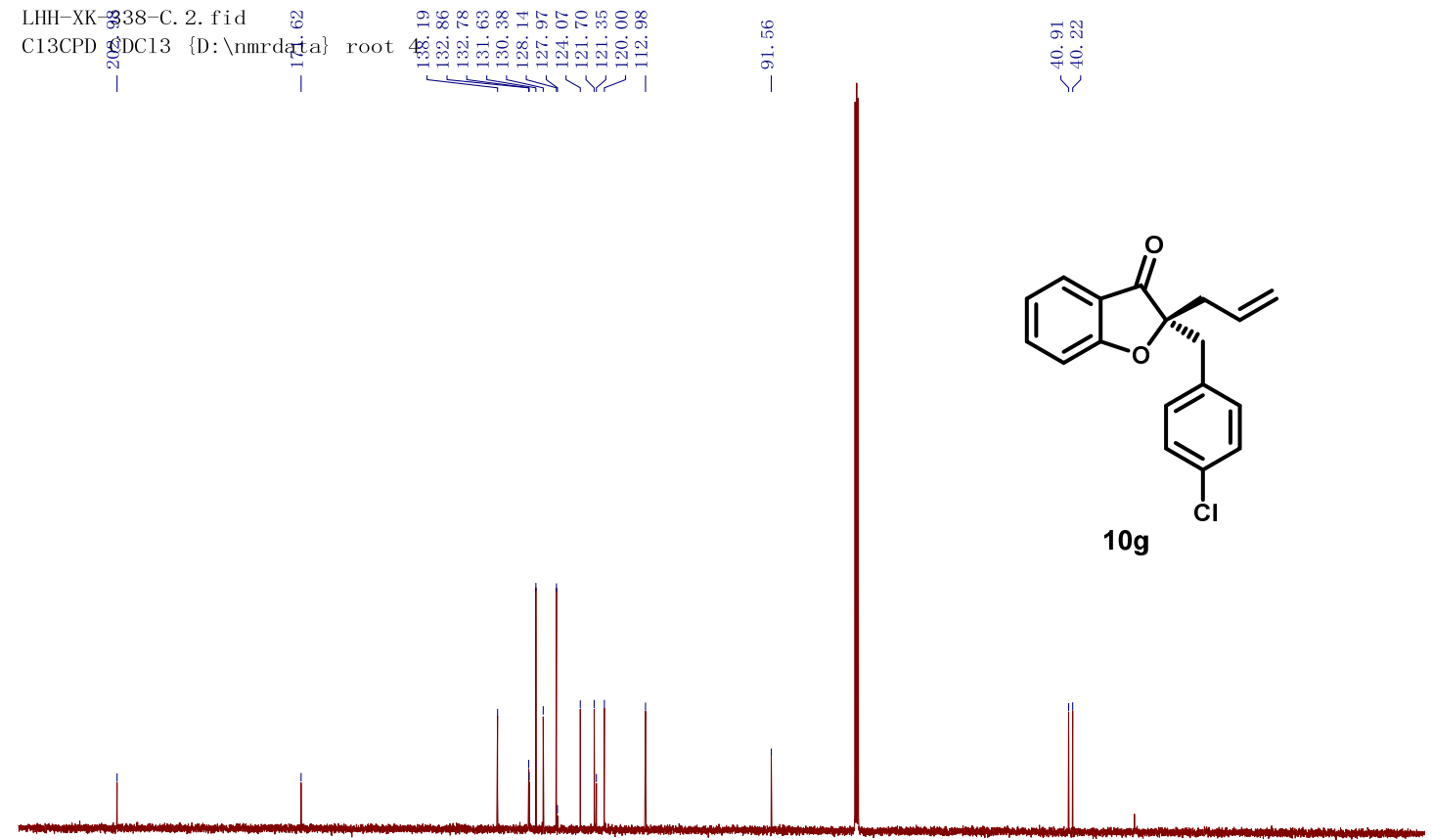

$\begin{array}{lllllllllllllllllllllllll}210 & 200 & 190 & 180 & 170 & 160 & 150 & 140 & 130 & 120 & 110 & 100 & 90 & 80 & 70 & 60 & 50 & 40 & 30 & 20 & 10 & 0 & -10\end{array}$

(S)-2-Allyl-2-(4-methoxybenzyl)benzofuran-3(2H)-one (10h) 
$\underline{{ }^{1} \mathrm{H} \text { NMR }}\left(400 \mathrm{MHz}, \mathrm{CDCl}_{3} @ 7.26 \mathrm{ppm}\right)$

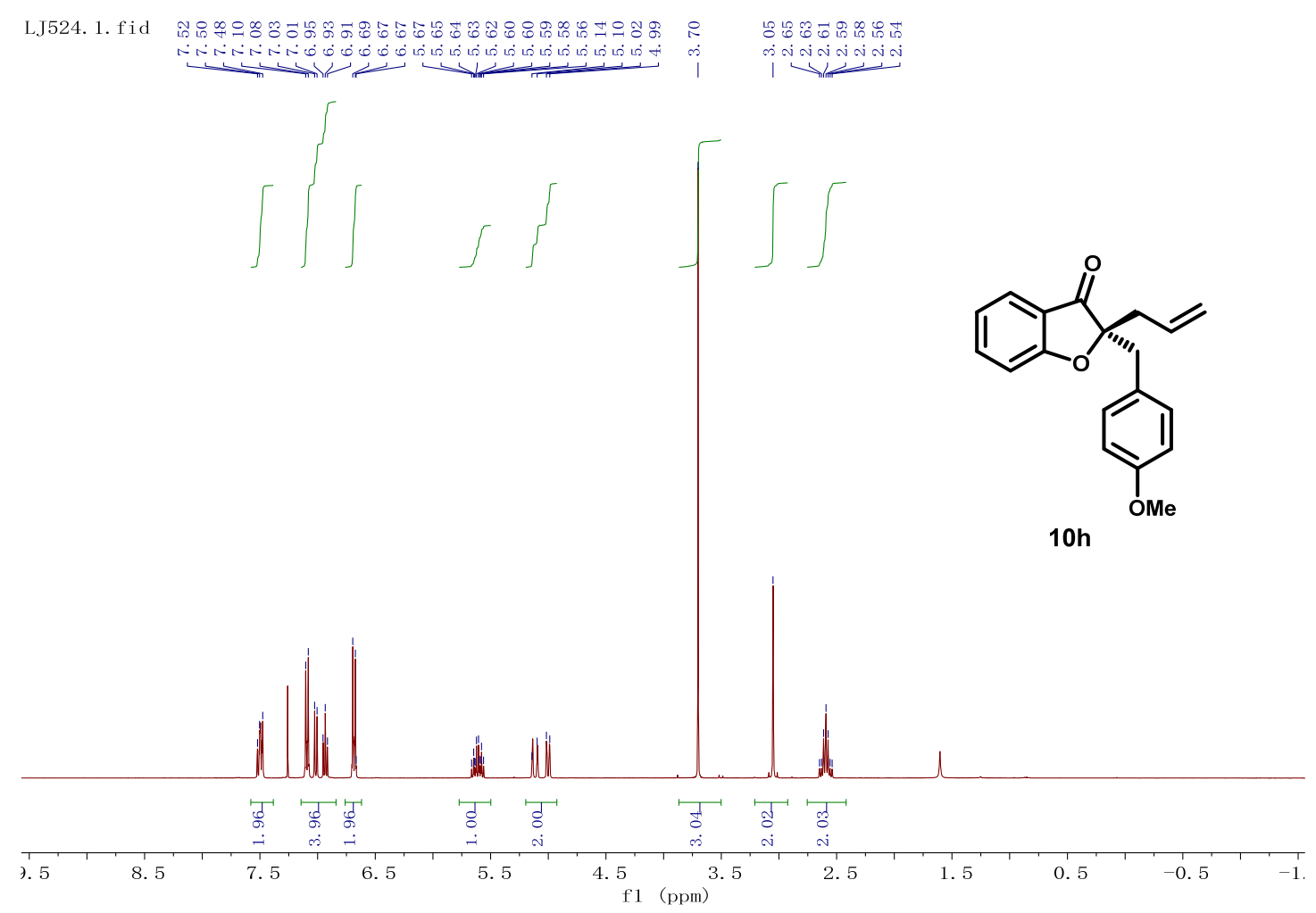

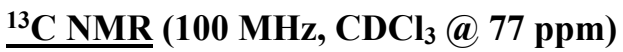

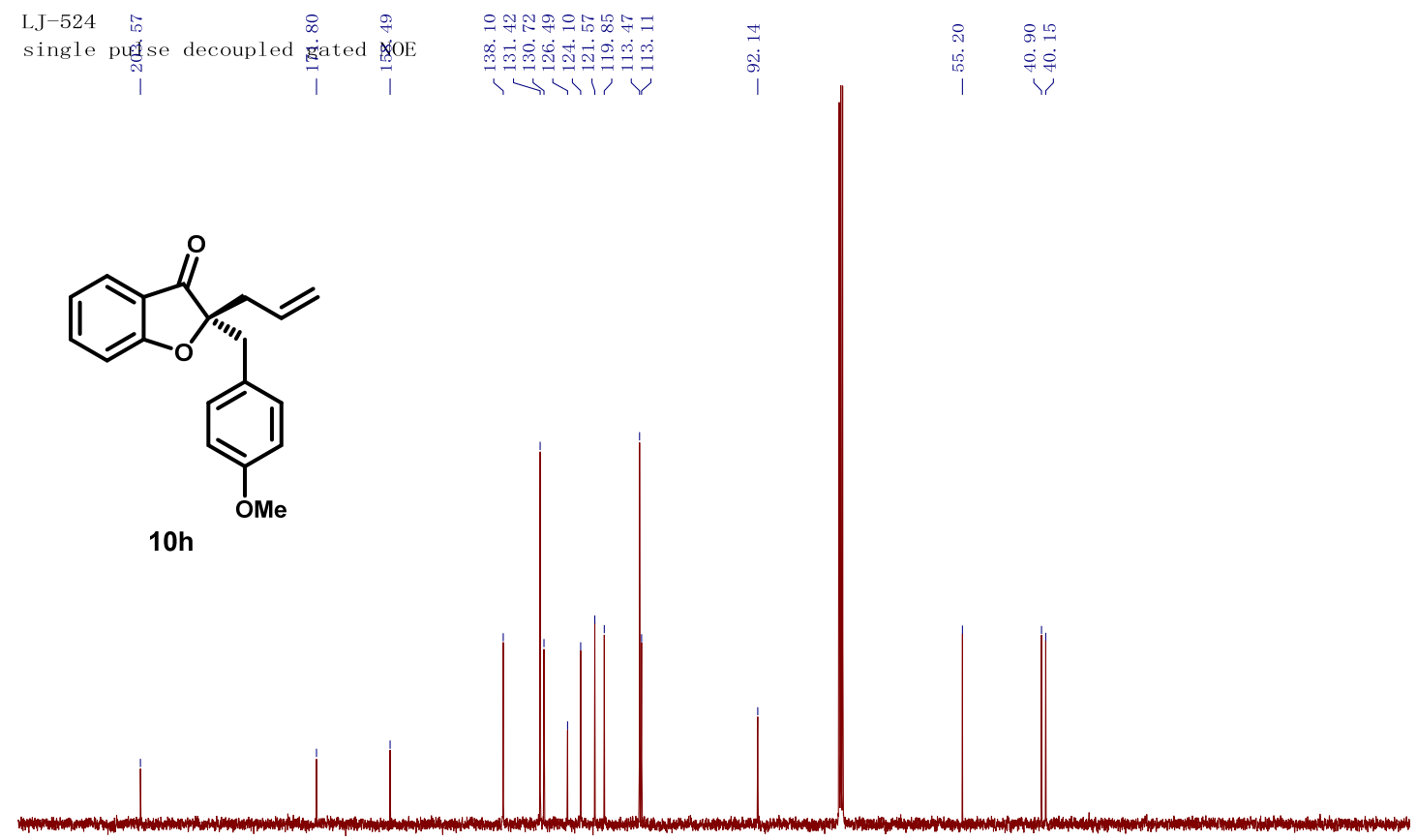

$\begin{array}{llllllllllllllllllllllllllll}220 & 210 & 200 & 190 & 180 & 170 & 160 & 150 & 140 & 130 & 120 & 110 & 100 & 90 & 80 & 70 & 60 & 50 & 40 & 30 & 20 & 10 & 0 & -10 & -20\end{array}$ tert-Butyl (S)-(4-((2-allyl-3-oxo-2,3-dihydrobenzofuran-2-yl)methyl)phenyl)carbamate(10i) S204 


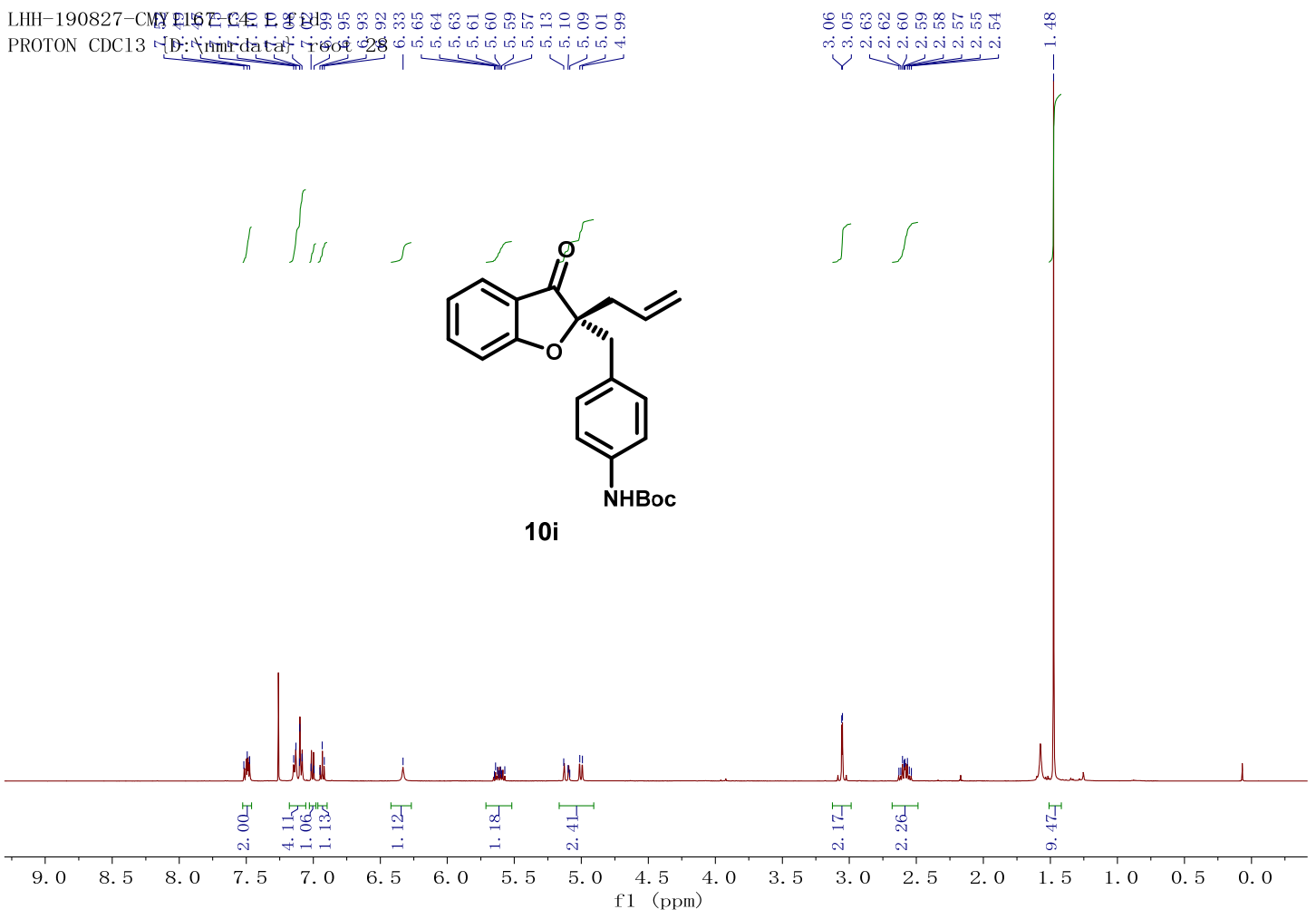

$10 \mathrm{i}$
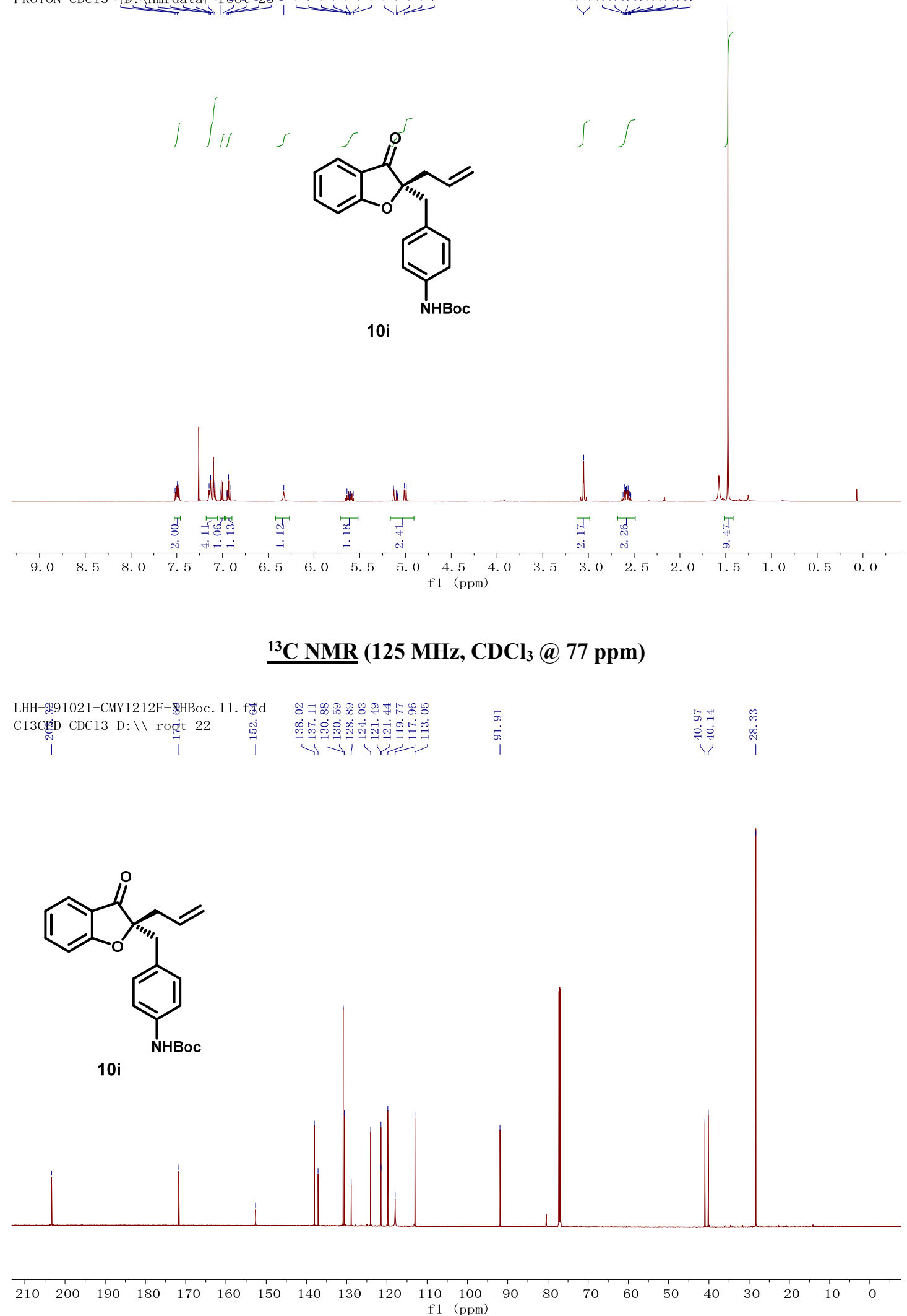

\section{$\underline{{ }^{13} \mathrm{C} N M R}\left(125 \mathrm{MHz}, \mathrm{CDCl}_{3} @ 77\right.$ ppm)}

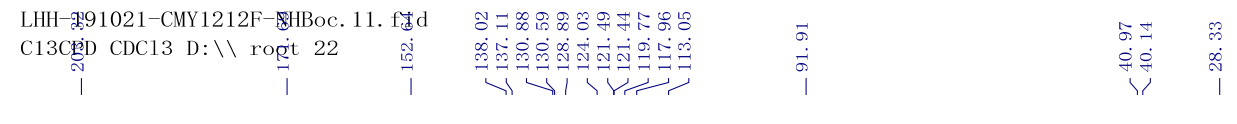

(S)-2-Allyl-2-(3-methoxybenzyl)benzofuran-3(2H)-one (10j) 
$\underline{{ }^{1} \mathrm{H} \text { NMR }}\left(400 \mathrm{MHz}, \mathrm{CDCl}_{3} @ 7.26\right.$ ppm)

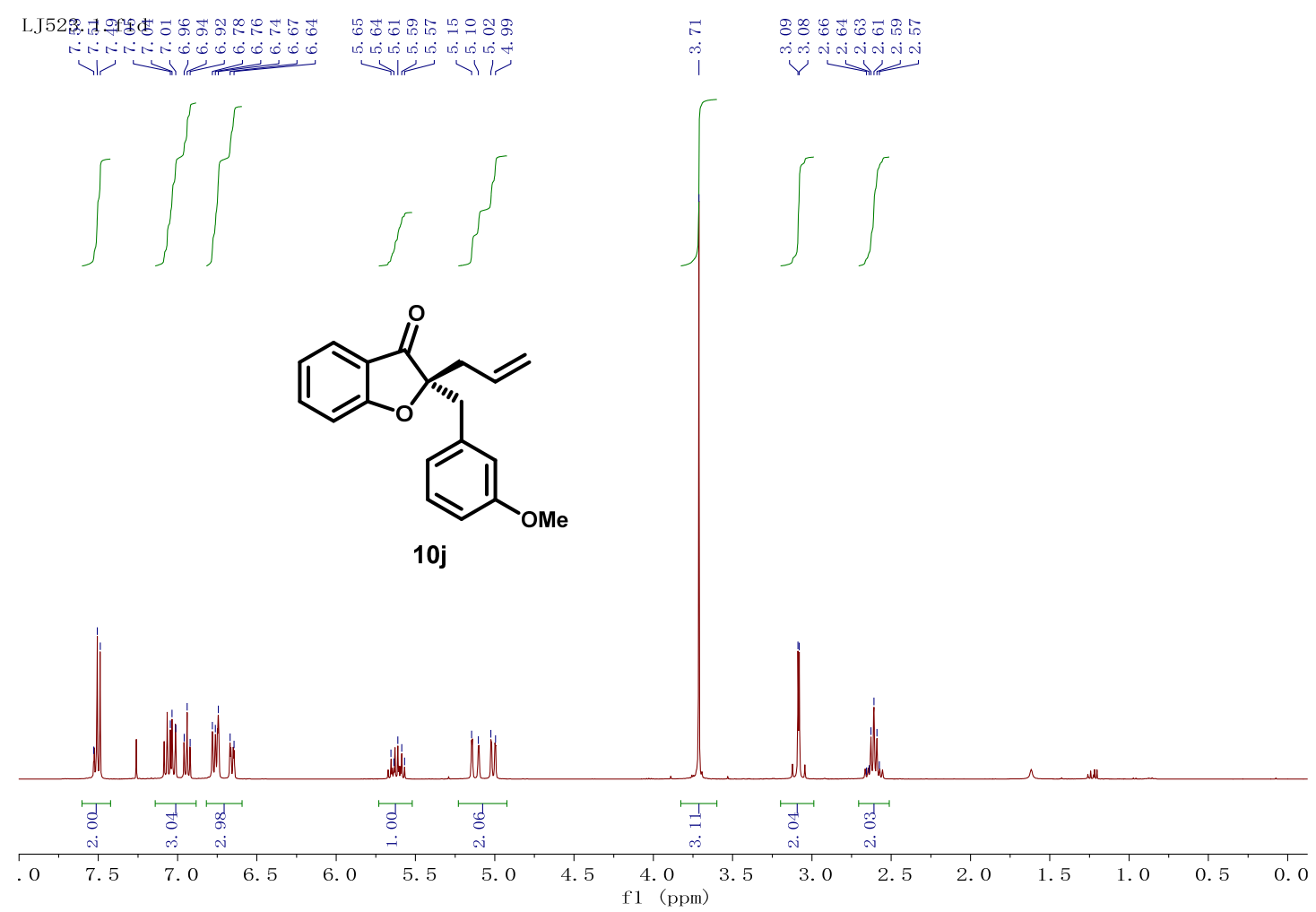

${ }^{13} \mathrm{C}$ NMR $\left(100 \mathrm{MHz}, \mathrm{CDCl}_{3} @ 77\right.$ ppm)

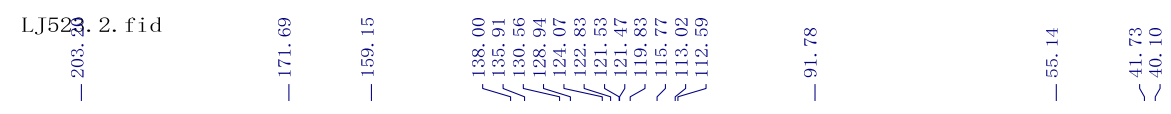<smiles>C=CC[C@]1(Cc2cccc(OC)c2)Oc2ccccc2C1=O</smiles>

10j

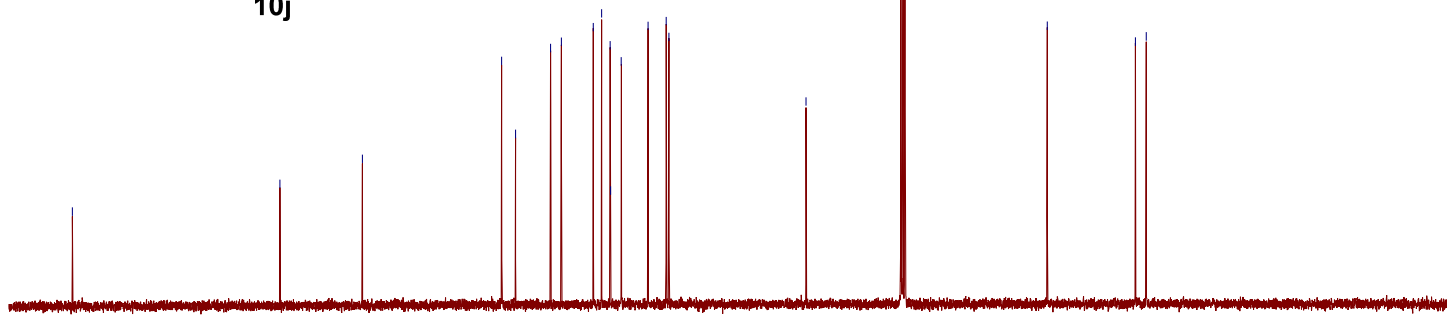

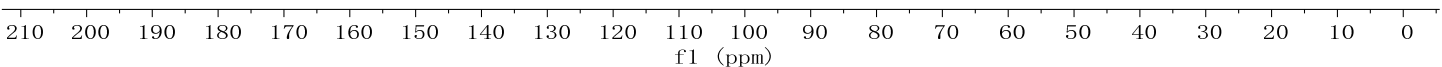

(S)-2-Allyl-2-(2-methylbenzyl)benzofuran-3(2H)-one (10k) 


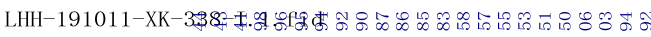

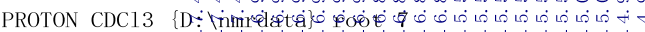

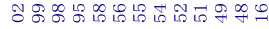

r

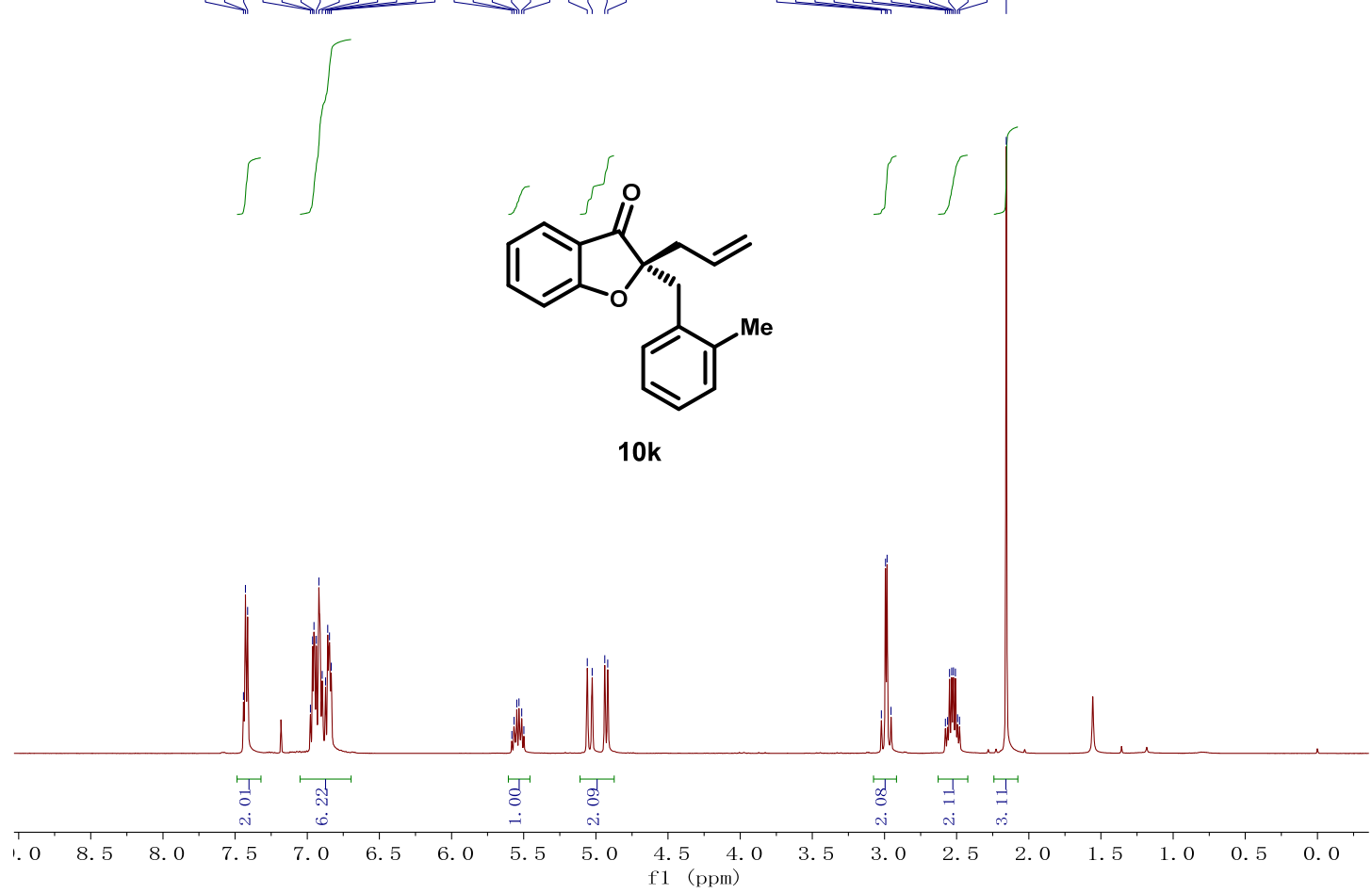

${ }^{13} \mathrm{C}$ NMR (100 MHz, $\mathrm{CDCl}_{3} @ 77$ ppm)

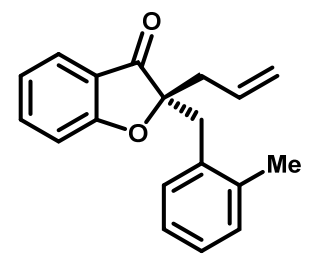

10k

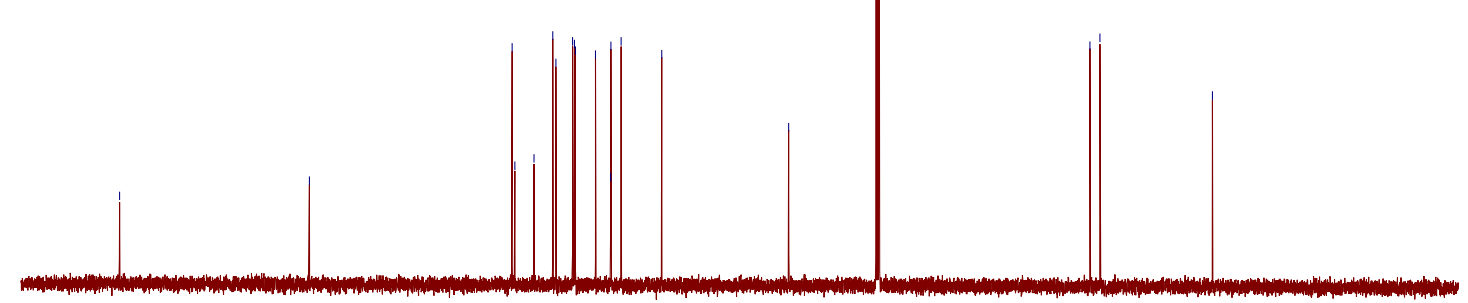

$\begin{array}{llllllllllll}210 & 200 & 190 & 180 & 170 & 160 & 150 & 140 & 130 & 120 & 110 & 100 \\ \mathrm{f} 1 & (\mathrm{ppm})\end{array}$

(S)-2-Allyl-2-(2-bromobenzyl)benzofuran-3(2H)-one (10I) 
$\underline{{ }^{1} \mathrm{H} \text { NMR }}\left(400 \mathrm{MHz}, \mathrm{CDCl}_{3} @ 7.26\right.$ ppm)

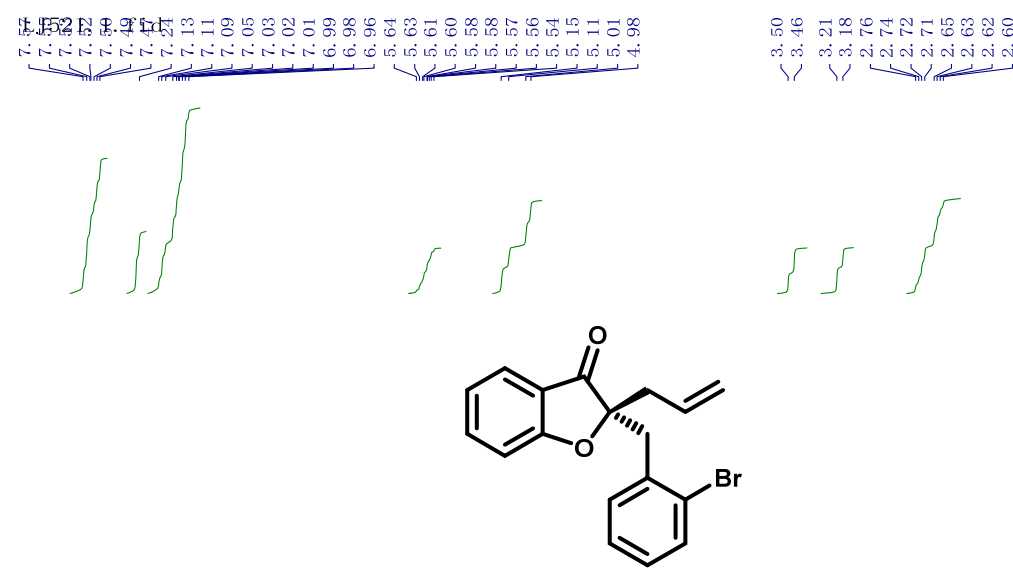

101

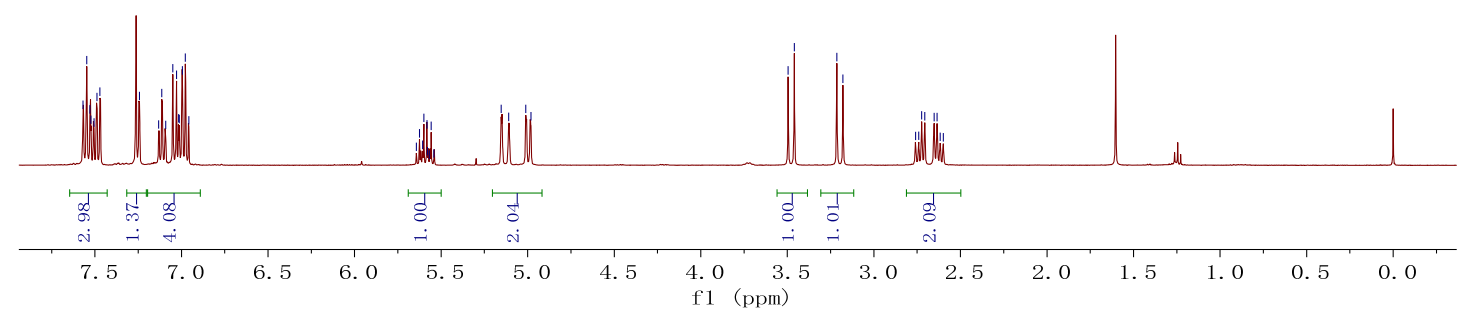

${ }^{13} \mathrm{C}$ NMR (100 MHz, $\mathrm{CDCl}_{3} @ 77$ ppm)

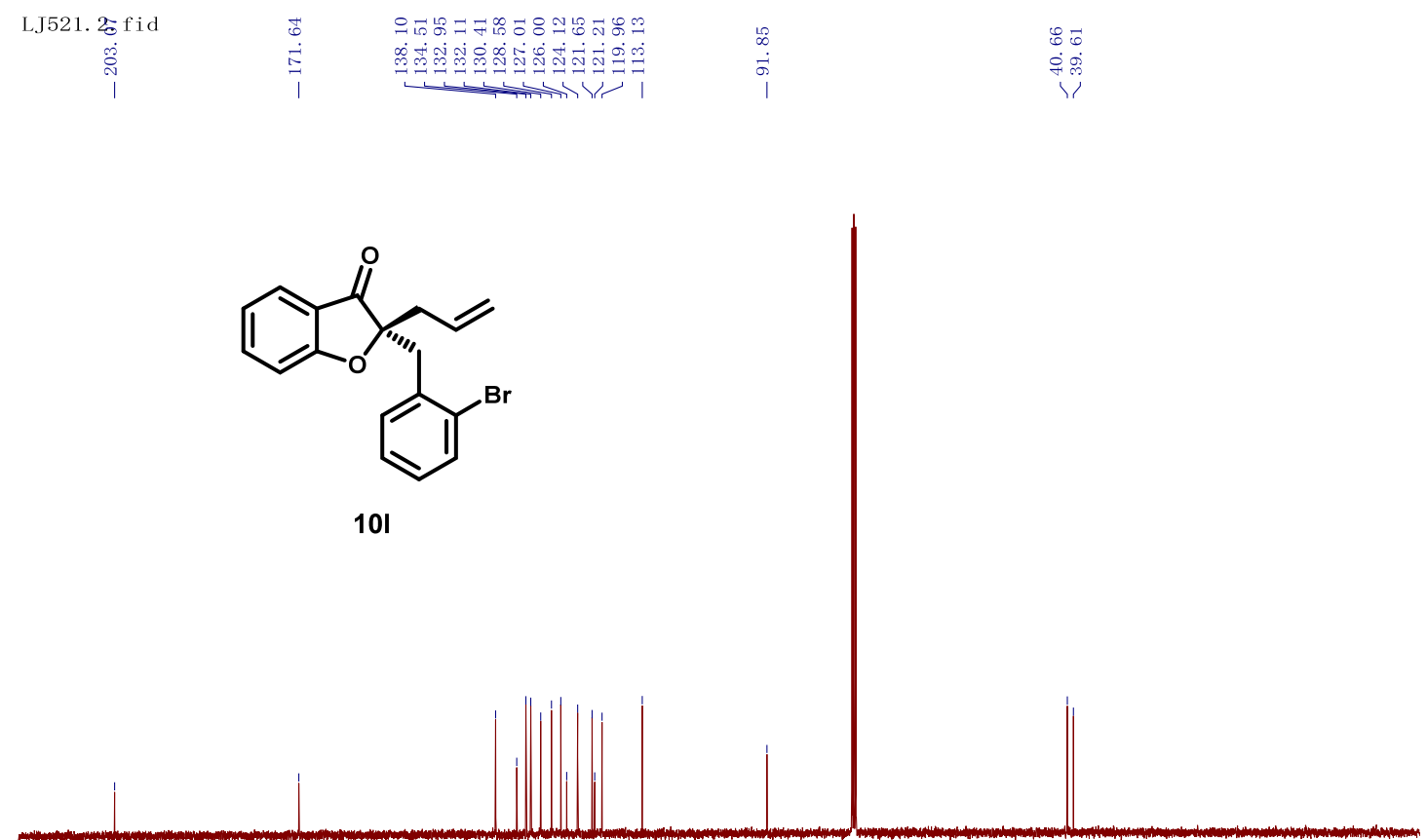

$\begin{array}{lllllllllllllllllllllllllllll}210 & 200 & 190 & 180 & 170 & 160 & 150 & 140 & 130 & 120 & 110 & 100 & 90 & 80 & 70 & 60 & 50 & 40 & 30 & 20 & 10 & 0 & -10\end{array}$

(S)-2-Allyl-2-(2,4,6-trimethylbenzyl)benzofuran-3(2H)-one (m) 


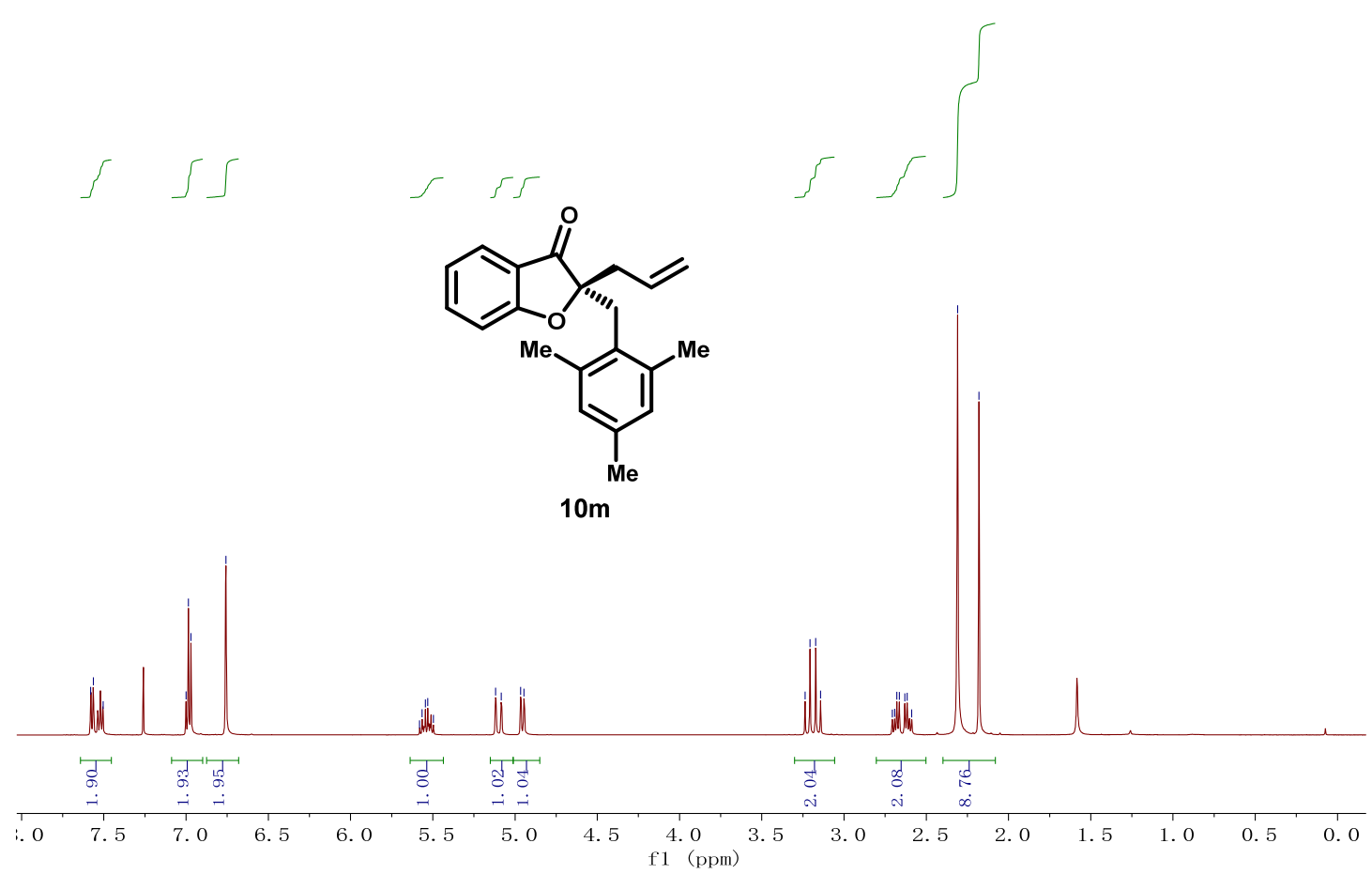

\section{${ }^{13} \mathrm{C}$ NMR (125 MHz, CDCl $3 @ 77$ ppm)}
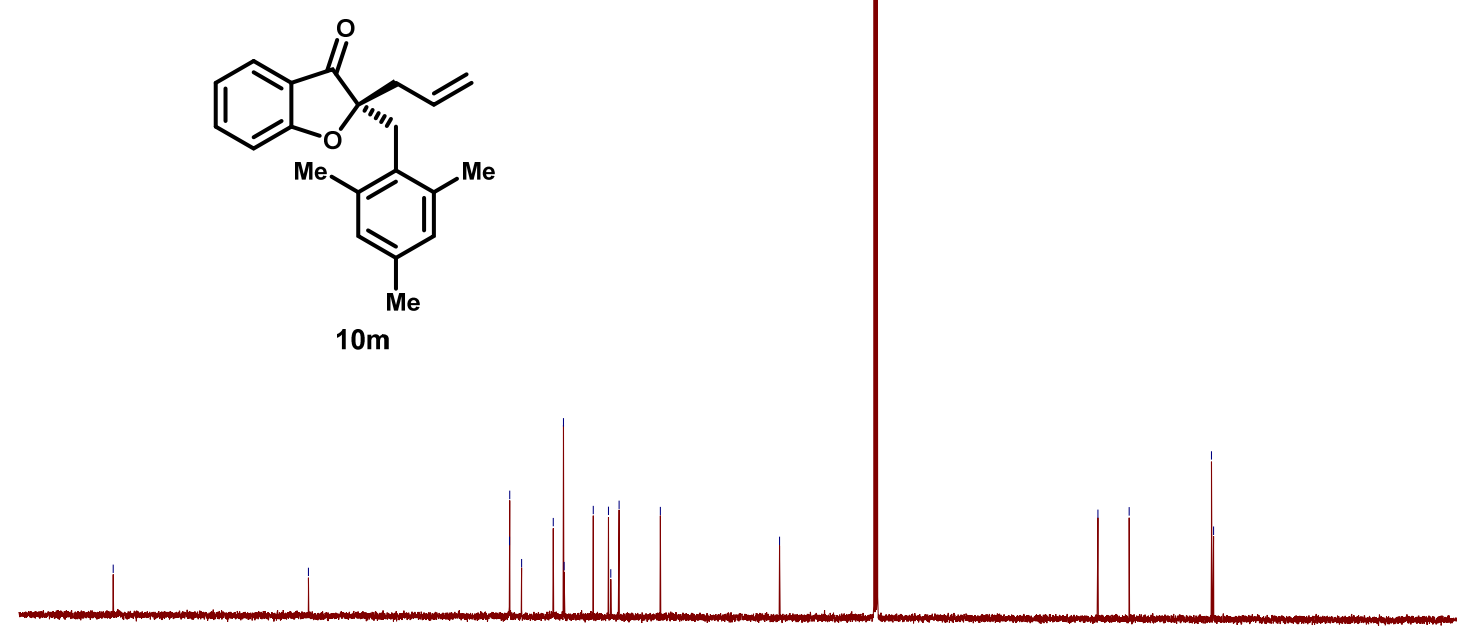

$\begin{array}{lllllllllllll}210 & 200 & 190 & 180 & 170 & 160 & 150 & 140 & 130 & 120 & 110 & 100 & 90\end{array}$

(S)-2-Allyl-2-((1-methyl-1 $H$-indol-3-yl)methyl)benzofuran-3(2H)-one (10n) 
$\underline{{ }^{1} \mathrm{H} \text { NMR }}\left(400 \mathrm{MHz}, \mathrm{CDCl}_{3} @ 7.26\right.$ ppm)

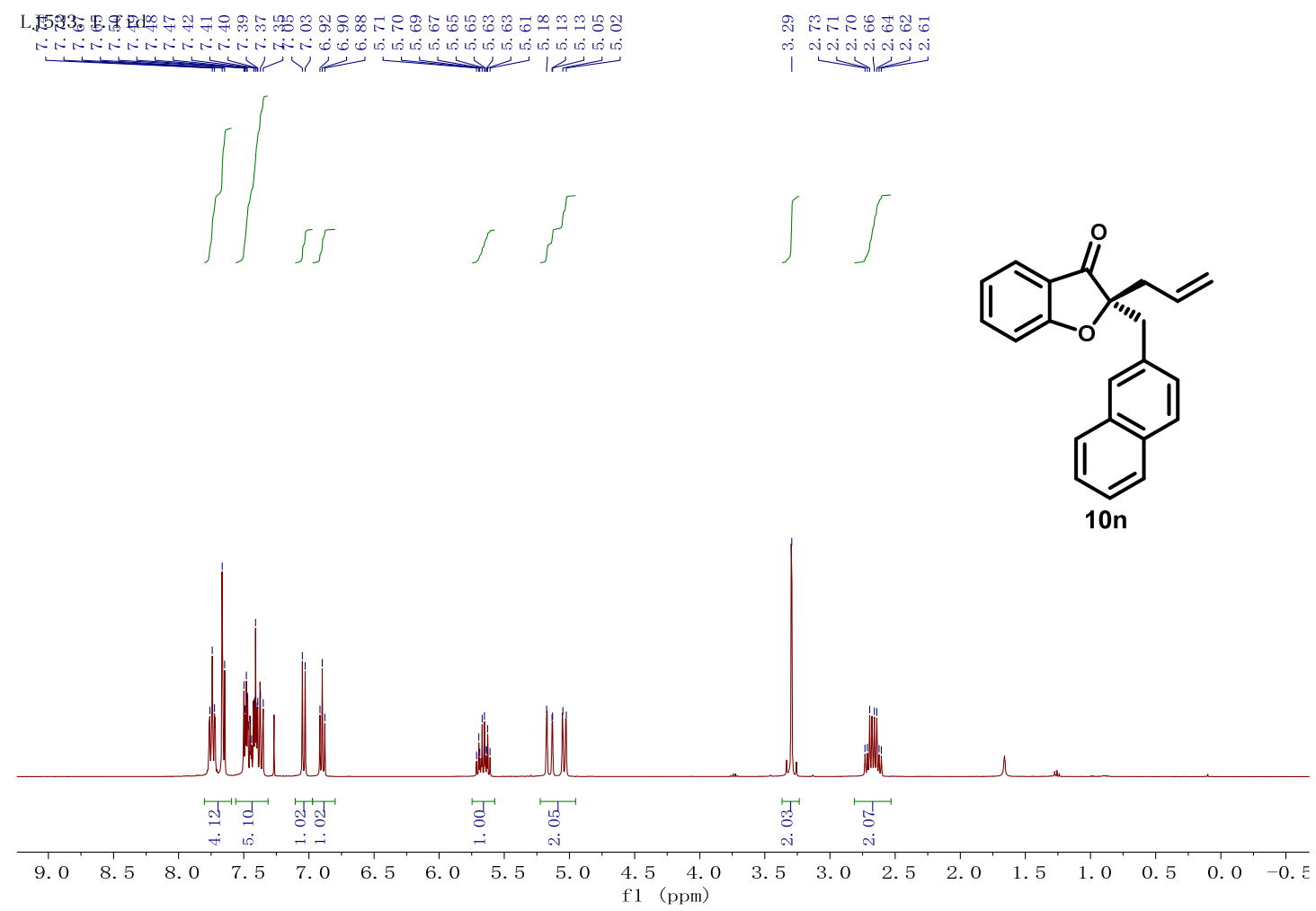

${ }^{13} \mathrm{C}$ NMR $\left(100 \mathrm{MHz}, \mathrm{CDCl}_{3} @ 77\right.$ ppm)
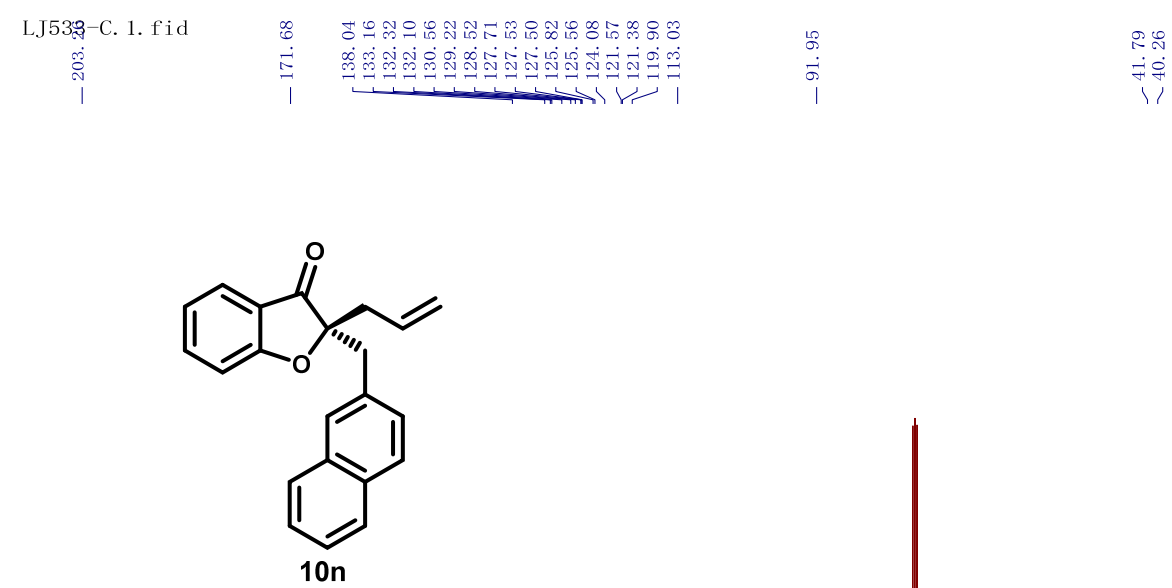

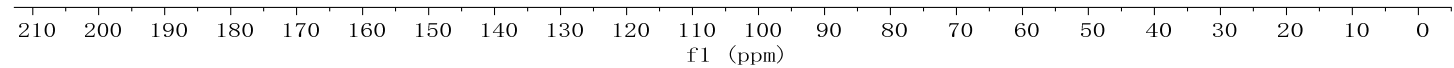

(S)-2-Allyl-2-(naphthalen-1-ylmethyl)benzofuran-3(2H)-one (10o)

S210 


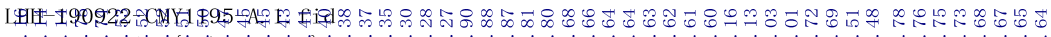

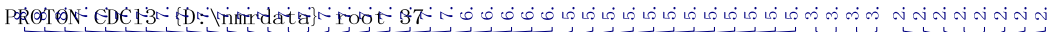

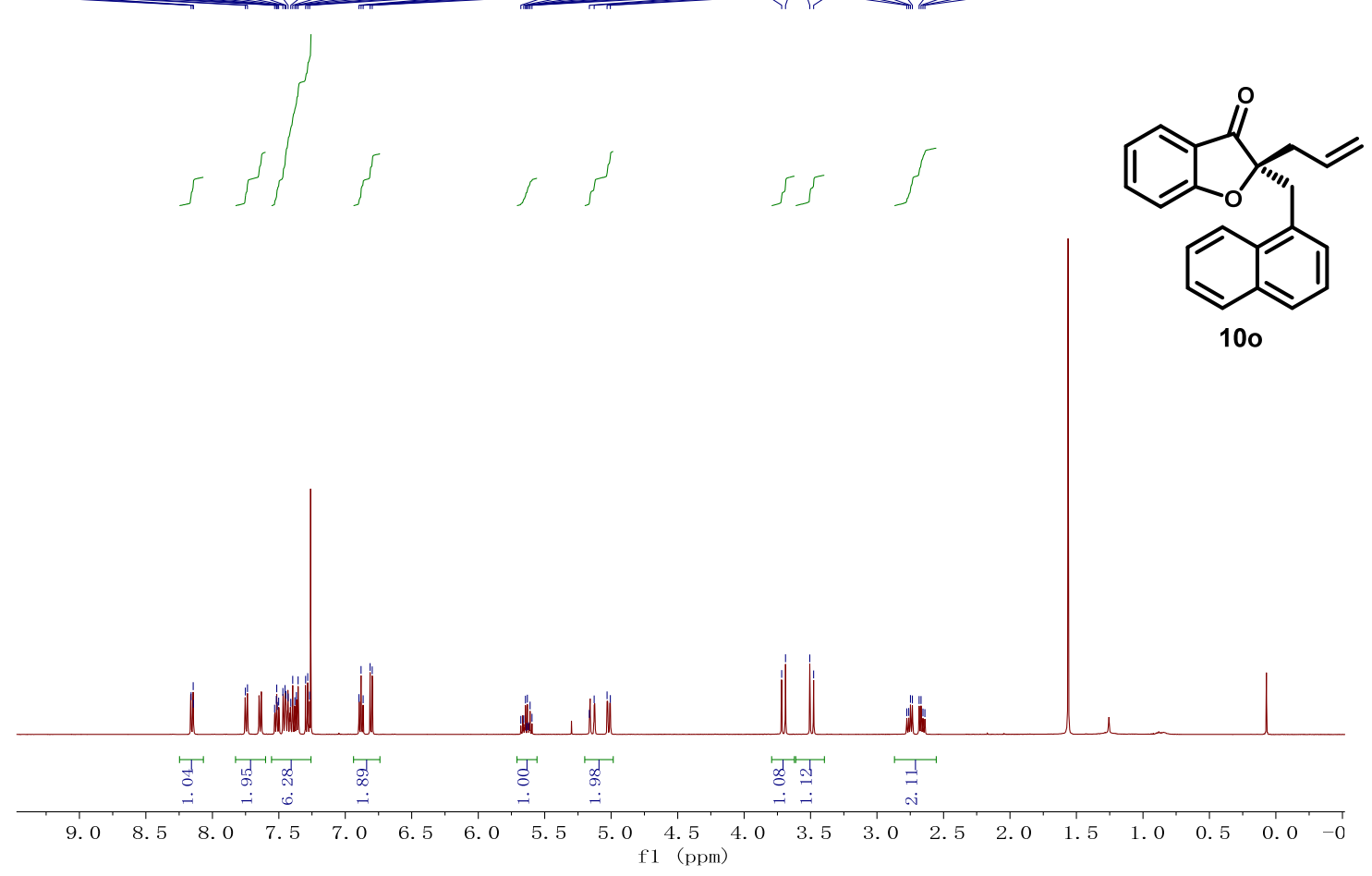

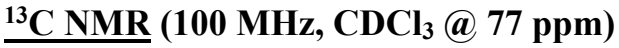<smiles>C=CC[C@]1(Cc2cccc3ccccc23)Oc2ccccc2C1=O</smiles>

100

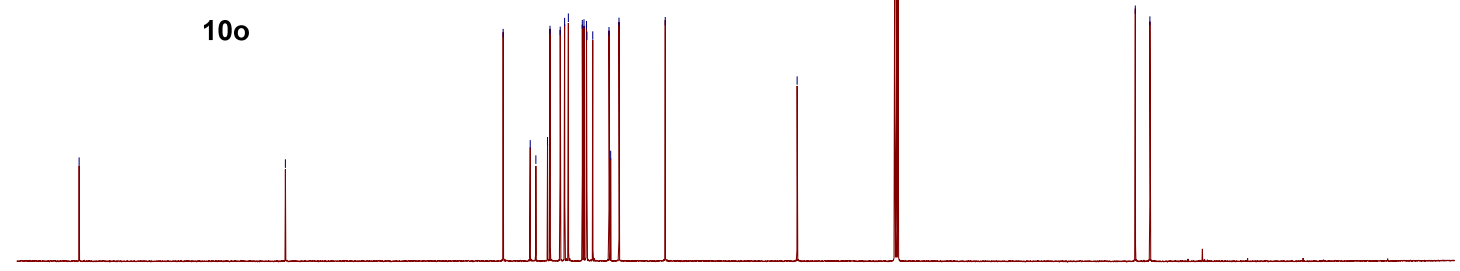

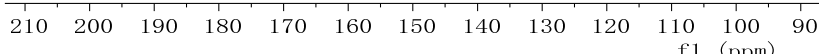


$\underline{{ }^{1} \mathrm{H} \text { NMR }}\left(400 \mathrm{MHz}, \mathrm{CDCl}_{3} @ 7.26\right.$ ppm)

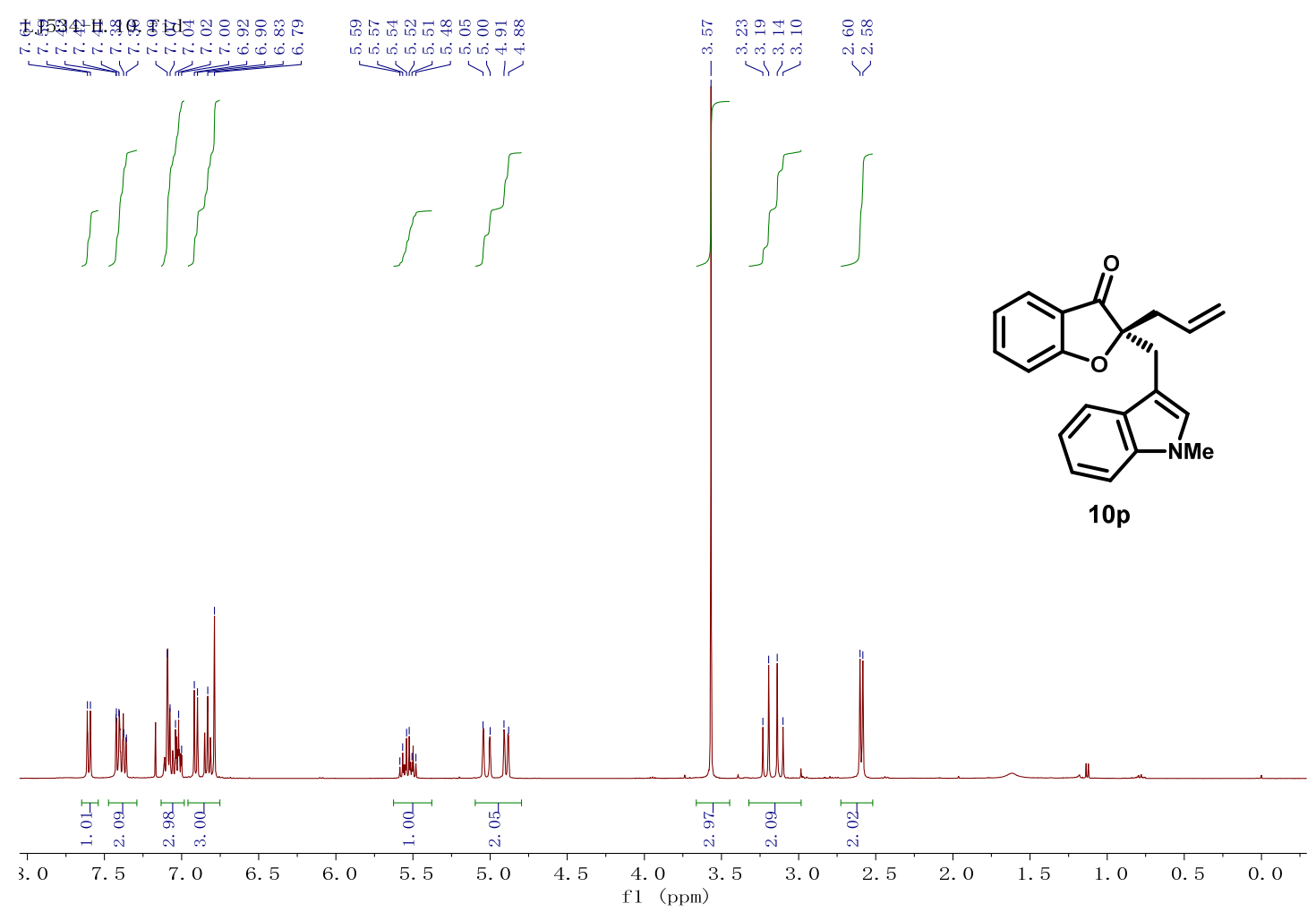

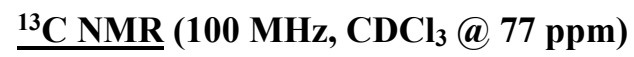

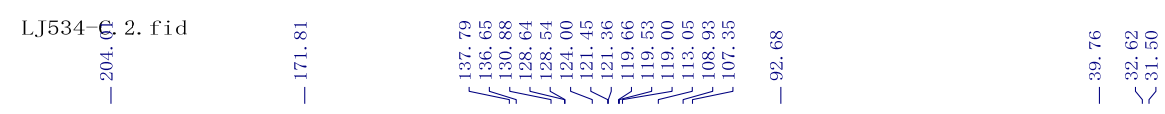

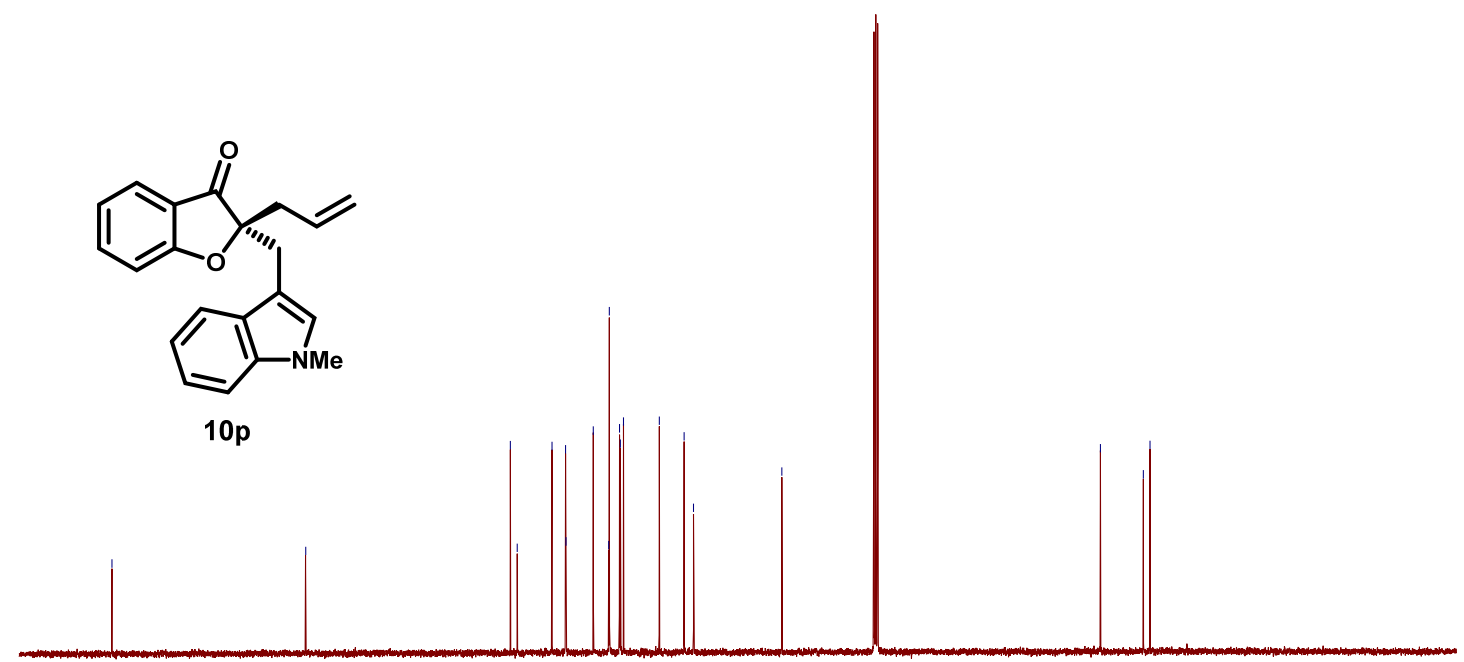

$\begin{array}{llllllllllllllllllllllllllll}210 & 200 & 190 & 180 & 170 & 160 & 150 & 140 & 130 & 120 & 110 & 100 & 90 & 80 & 70 & 60 & 50 & 40 & 30 & 20 & 10 & 0 & -10\end{array}$

(S)-2-Allyl-2-(furan-2-ylmethyl)benzofuran-3(2H)-one (10q)

S212 


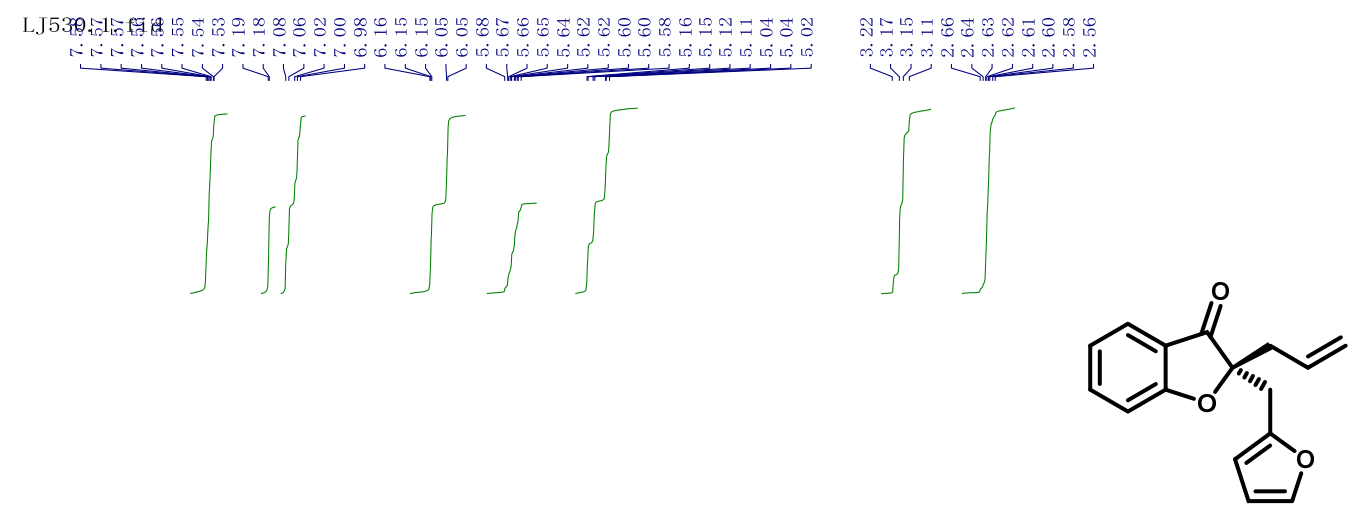

$10 q$

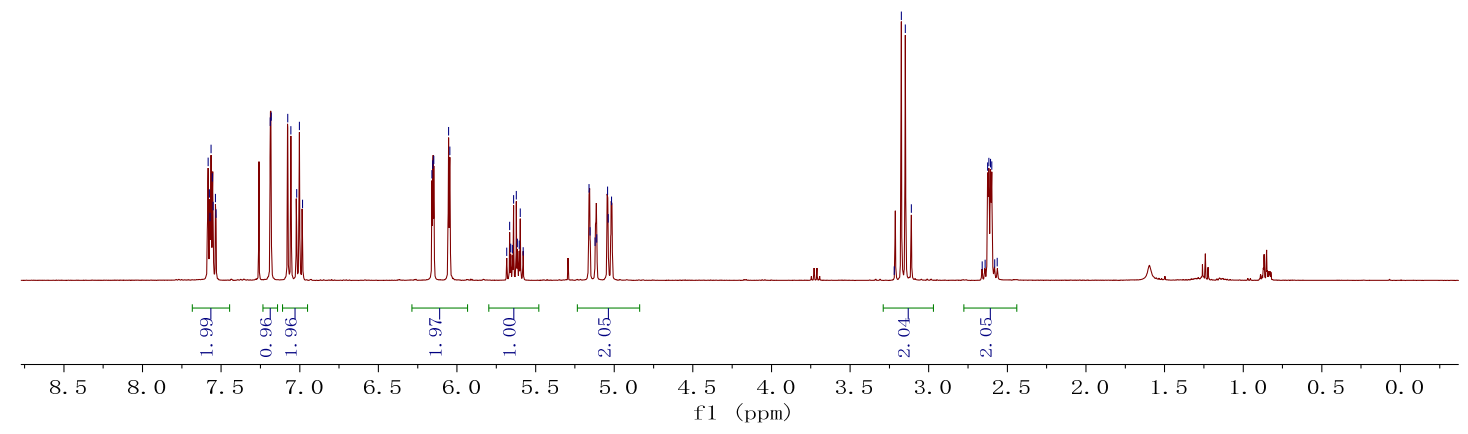

${ }^{13} \mathrm{C}$ NMR (100 MHz, $\mathrm{CDCl}_{3} @ 77$ ppm)

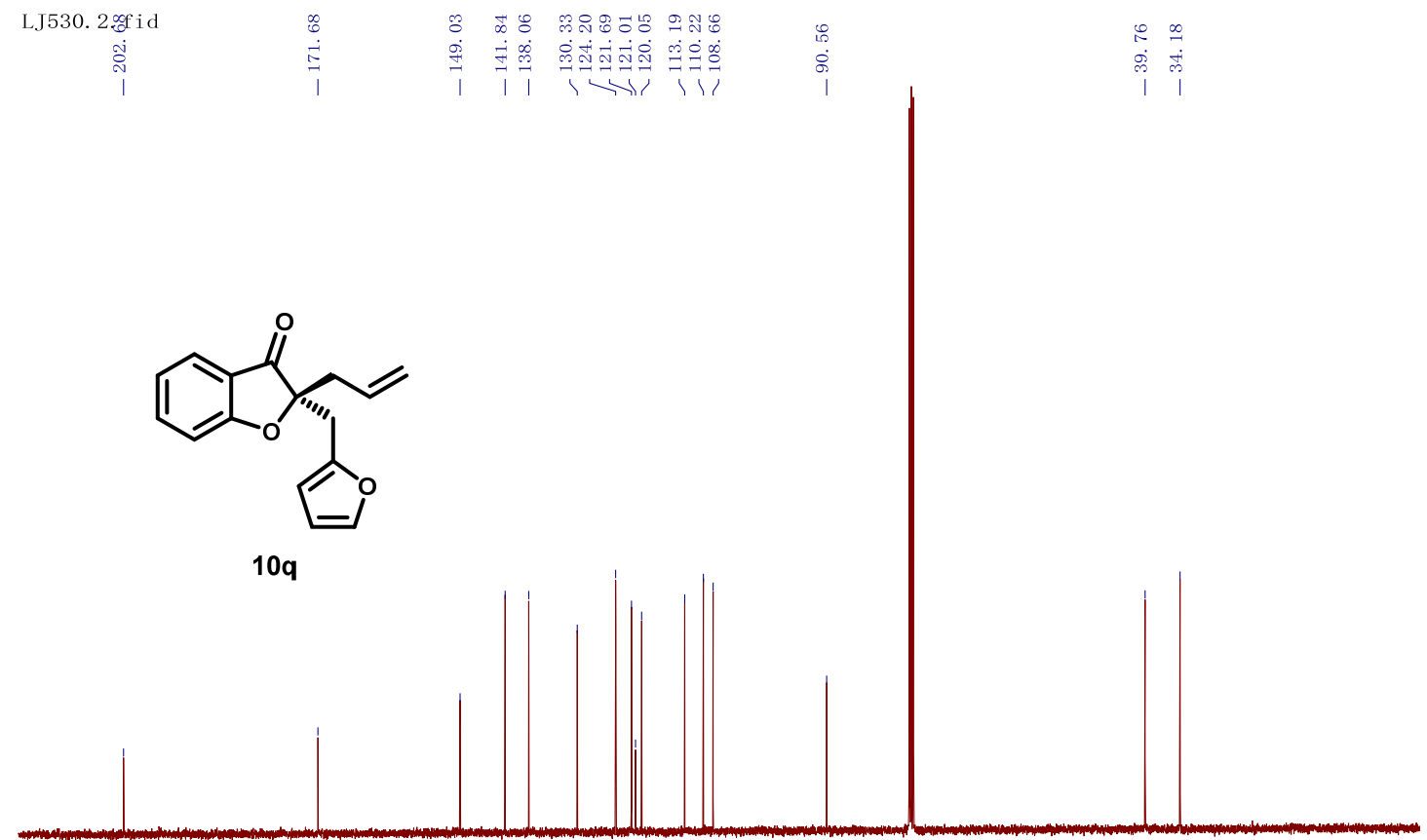

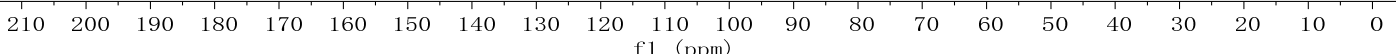

(S)-2-Allyl-2-(thiophen-2-ylmethyl)benzofuran-3(2H)-one (10r) 

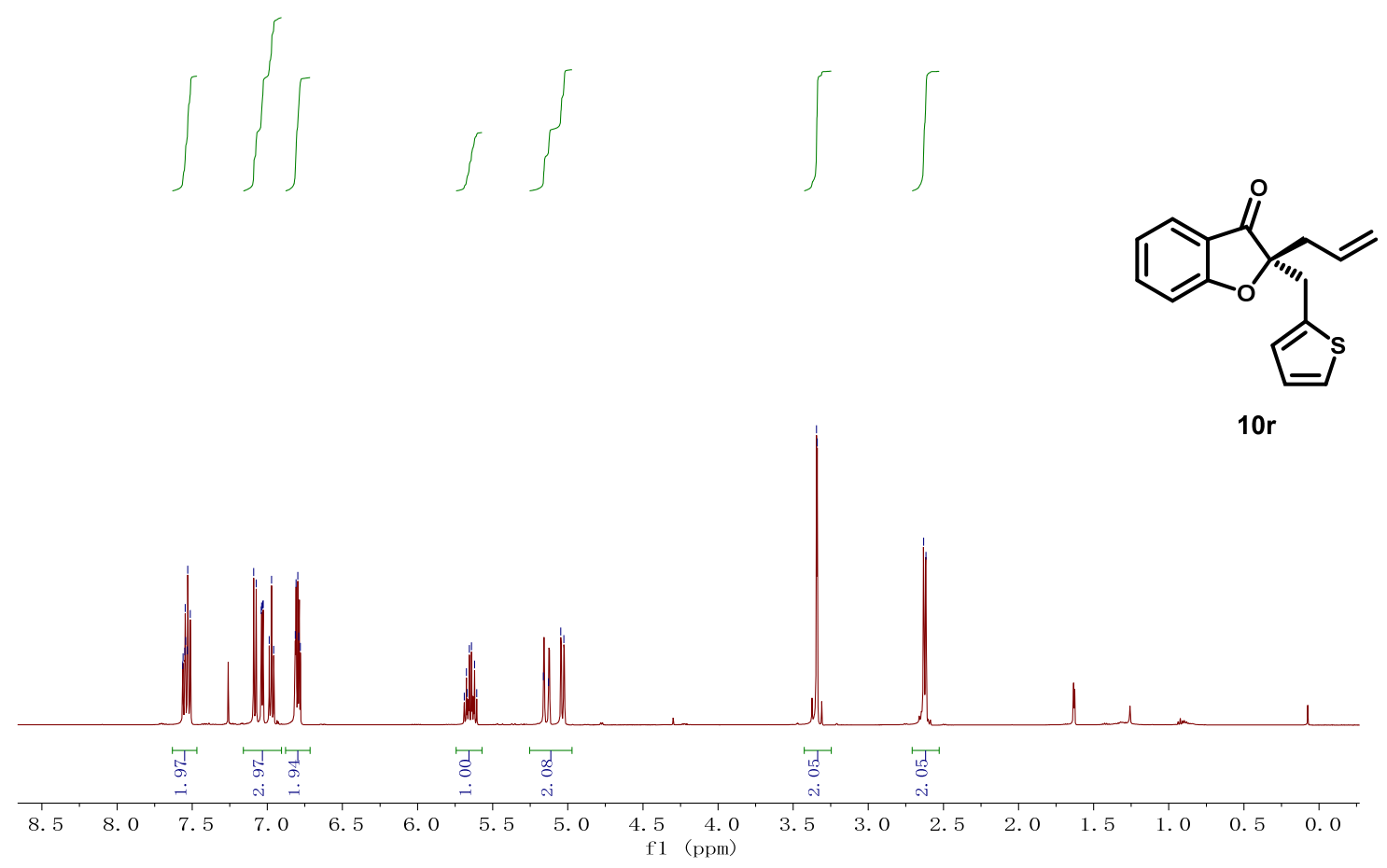

${ }^{13} \mathrm{C}$ NMR (125 MHz, CDCl $@ 77$ ppm)

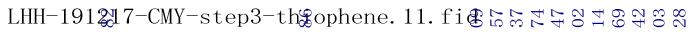

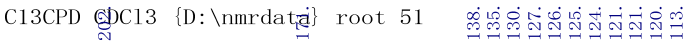

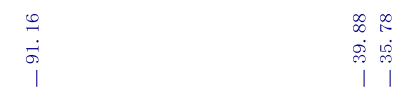

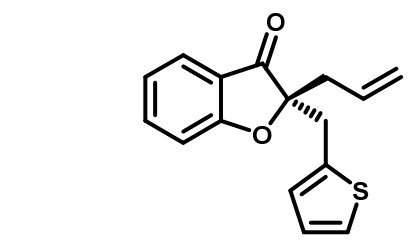

$10 r$

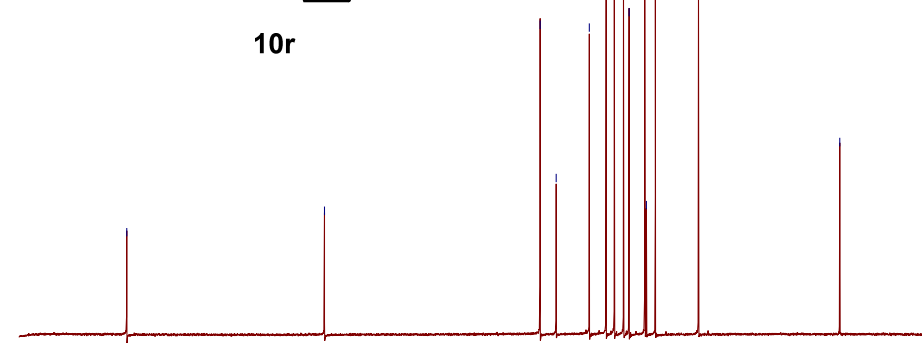

$\begin{array}{lllllllllllllllllllllllll}210 & 200 & 190 & 180 & 170 & 160 & 150 & 140 & 130 & 120 & 110 & 100 & 90 & 80 & 70 & 60 & 50 & 40 & 30 & 20 & 10 & 0\end{array}$

(S)-2-Allyl-2-cinnamylbenzofuran-3(2H)-one (10s) 
1H NMR (500 MHz, $\mathrm{CDCl}_{3} @ 7.26$ ppm)

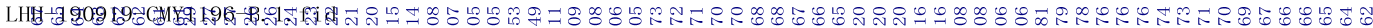

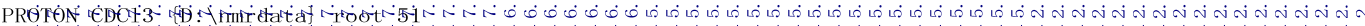

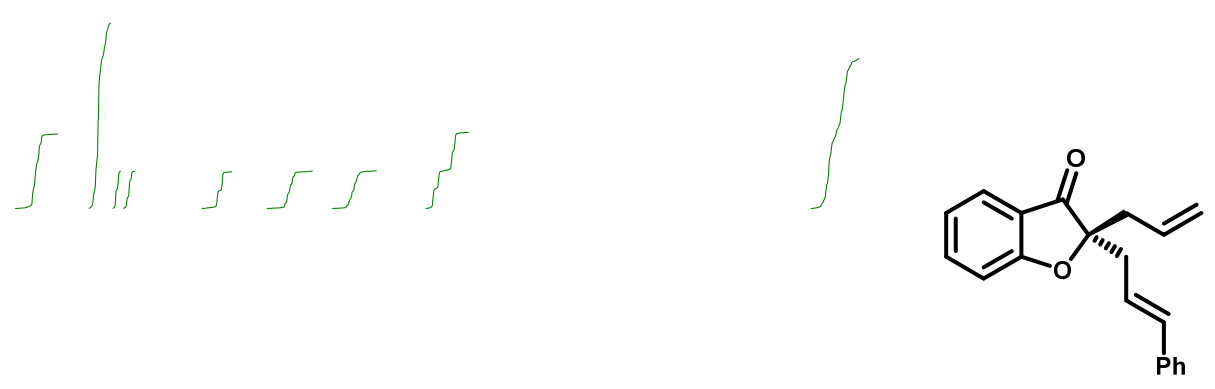

$10 \mathrm{~s}$

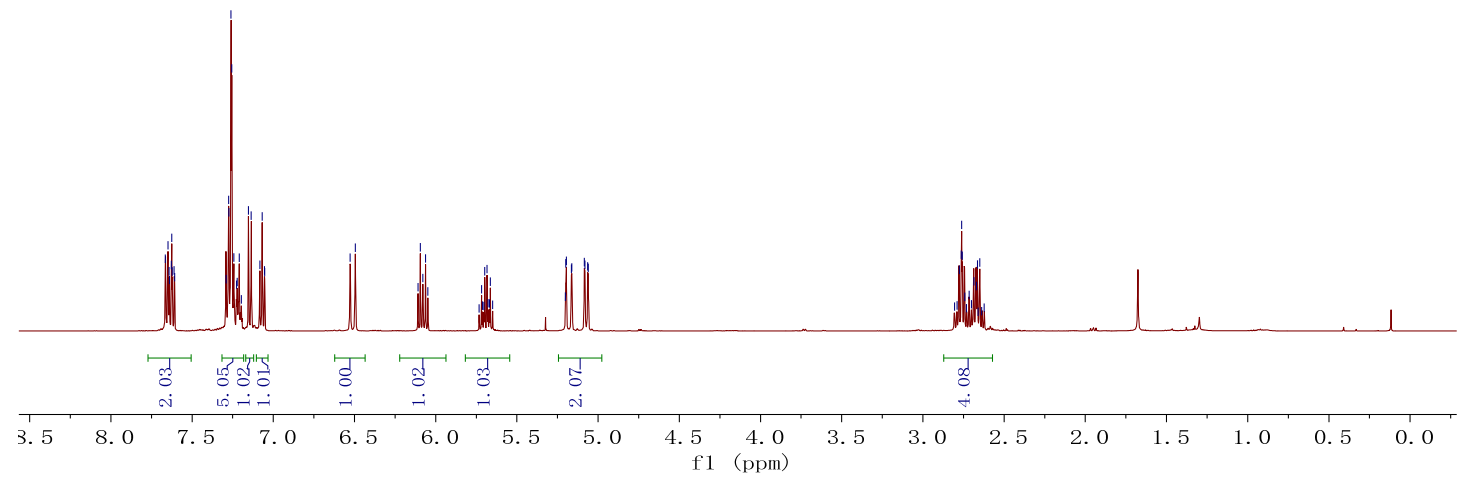

$\underline{{ }^{13} \mathrm{C} \text { NMR }}\left(125 \mathrm{MHz}, \mathrm{CDCl}_{3} @ 77\right.$ ppm)

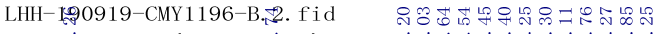

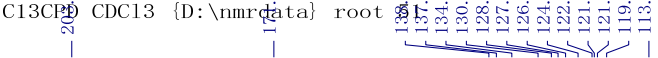

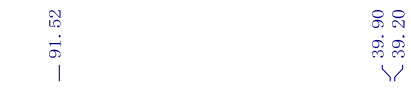

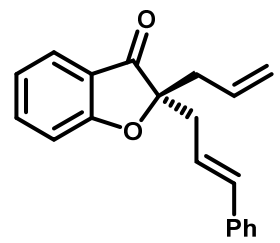

$10 \mathrm{~s}$

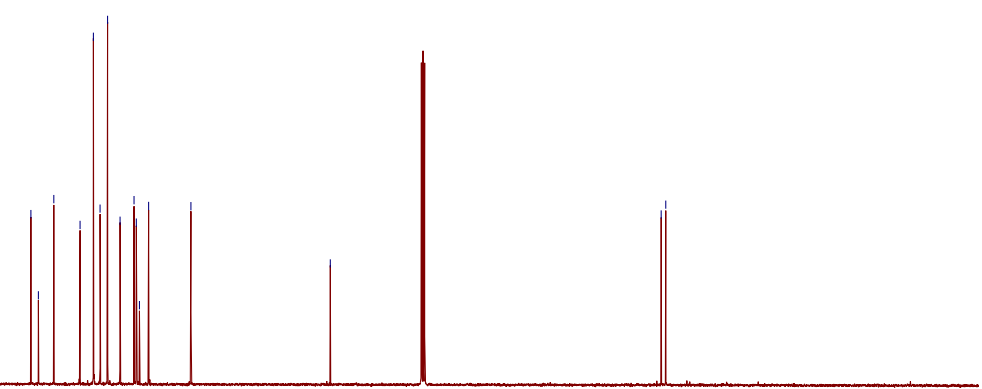

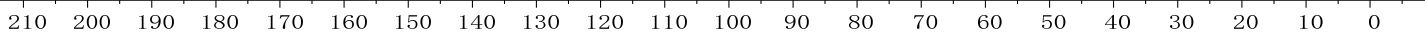
f1 (ppm) 
(R)-2-Allyl-2-(3-phenylpropyl)benzofuran-3(2H)-one (10t)

$\underline{{ }^{1} \mathrm{H} \text { NMR }}\left(500 \mathrm{MHz}, \mathrm{CDCl}_{3} @ 7.26 \mathrm{ppm}\right)$

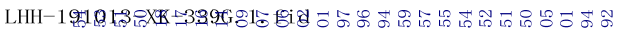

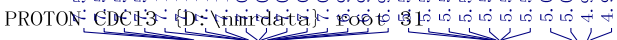

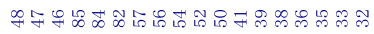

in
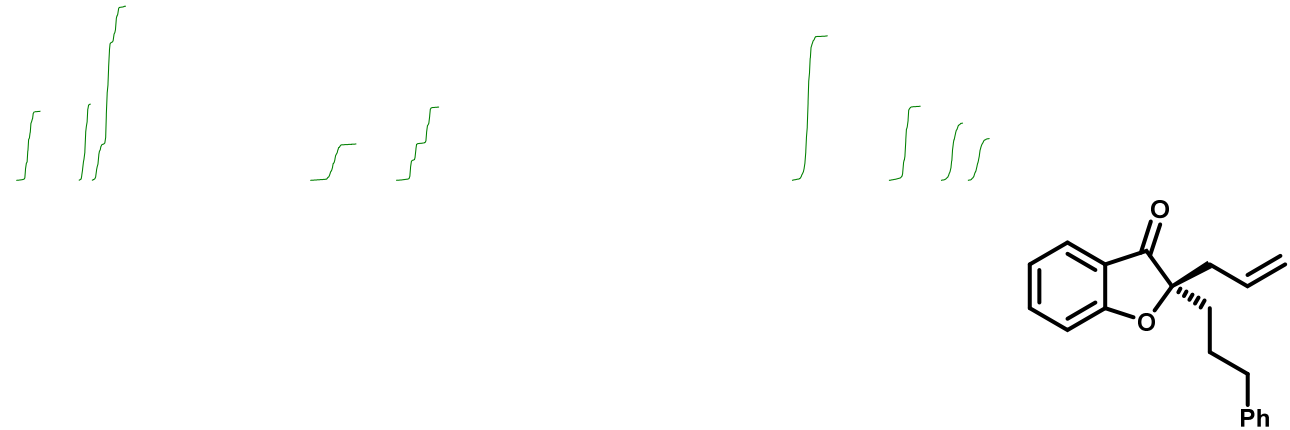

$10 \mathrm{t}$

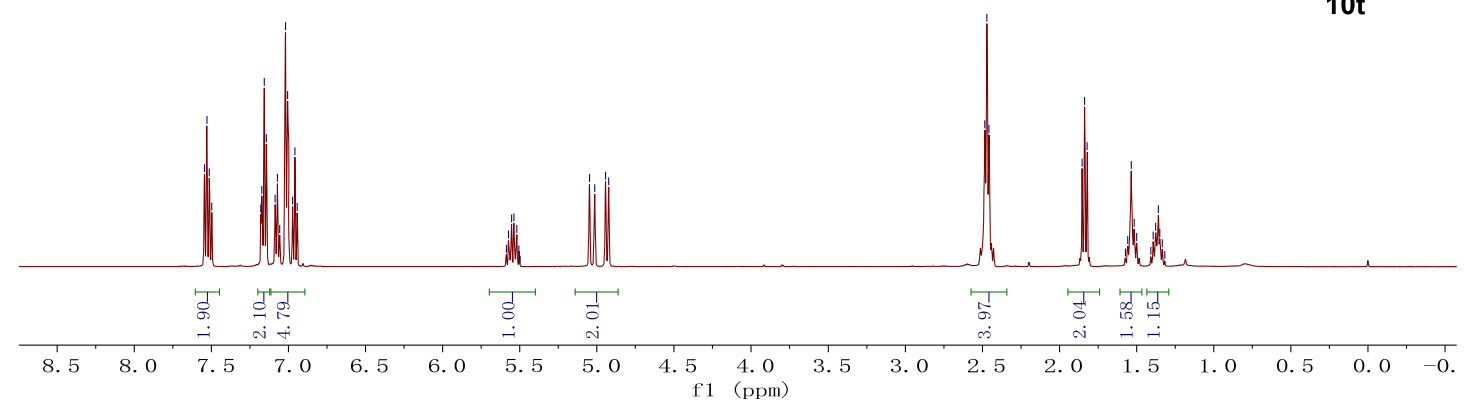

${ }^{13} \mathrm{C}$ NMR (125 MHz, CDCl 377 ppm)

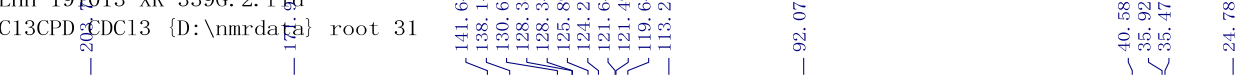

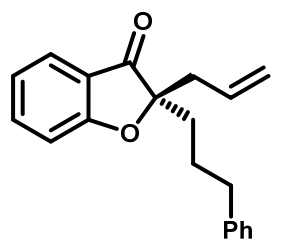

$10 \mathrm{t}$

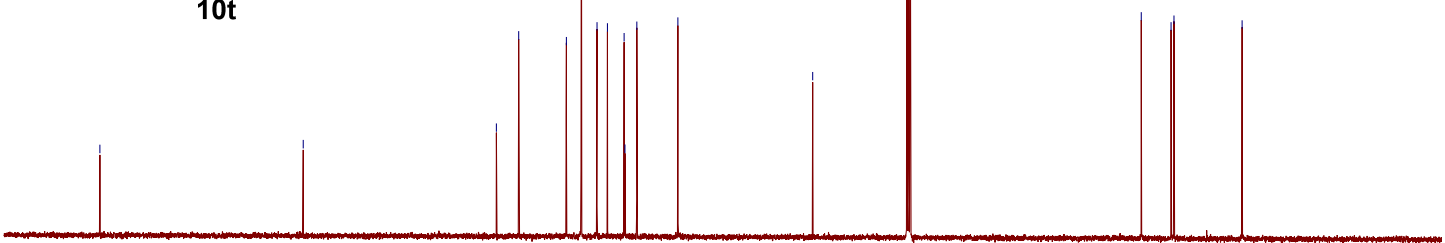

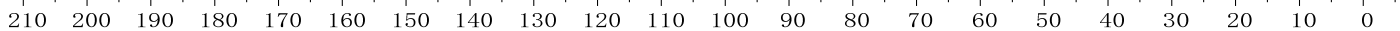
f1 (ppm) 
(S)-2-Allyl-2-(cyclopropylmethyl)benzofuran-3(2H)-one (10u)

$\underline{{ }^{1} \mathrm{H} \text { NMR }}\left(500 \mathrm{MHz}, \mathrm{CDCl}_{3} @ 7.26 \mathrm{ppm}\right)$

LHH-1911

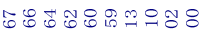

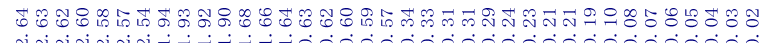

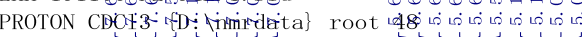

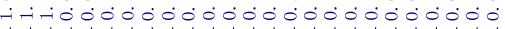
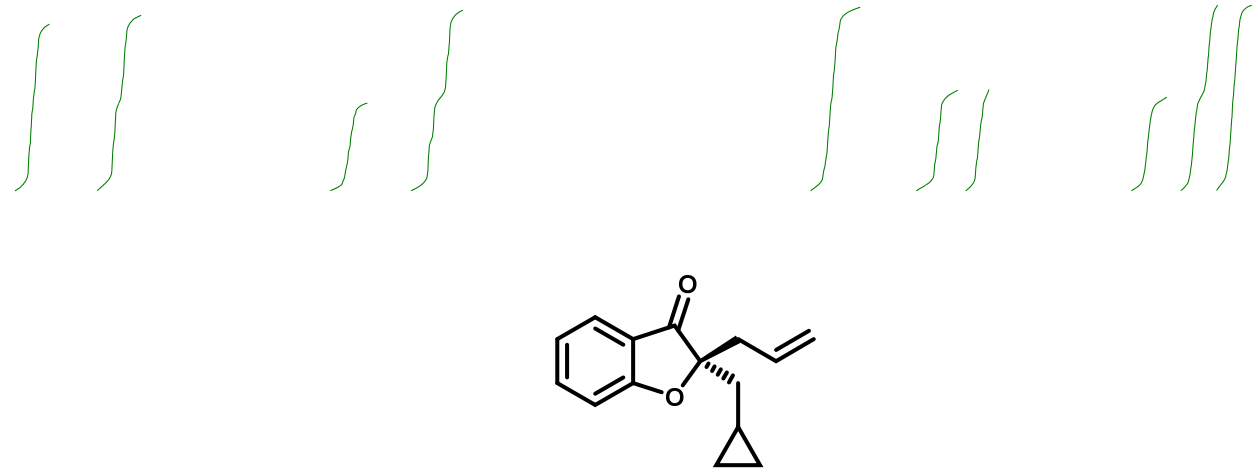

$10 \mathrm{u}$

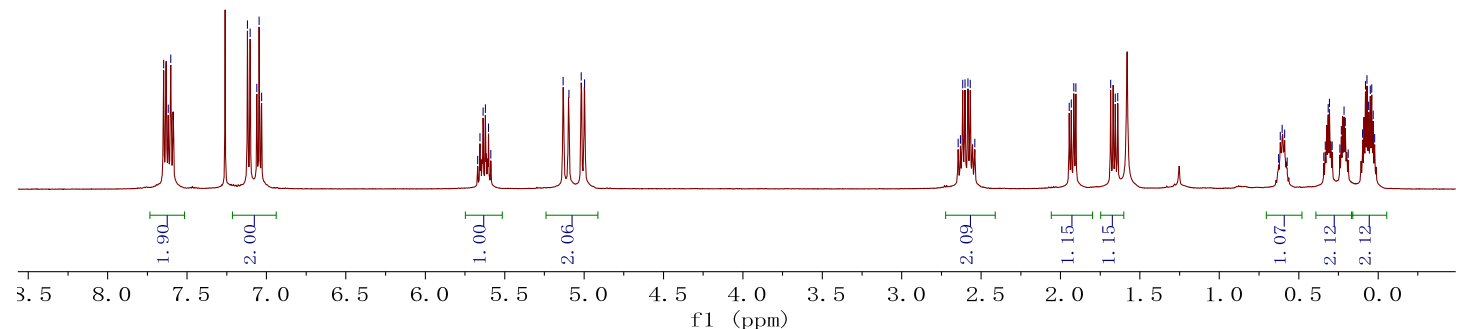

${ }^{13} \mathrm{C}$ NMR (125 MHz, CDCl 3977 ppm)

万人

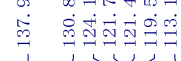

10
0
$\dot{\alpha}$
1<smiles>C=CC[C@]1(CC2CC2)Oc2ccccc2C1=O</smiles>

$10 \mathrm{u}$

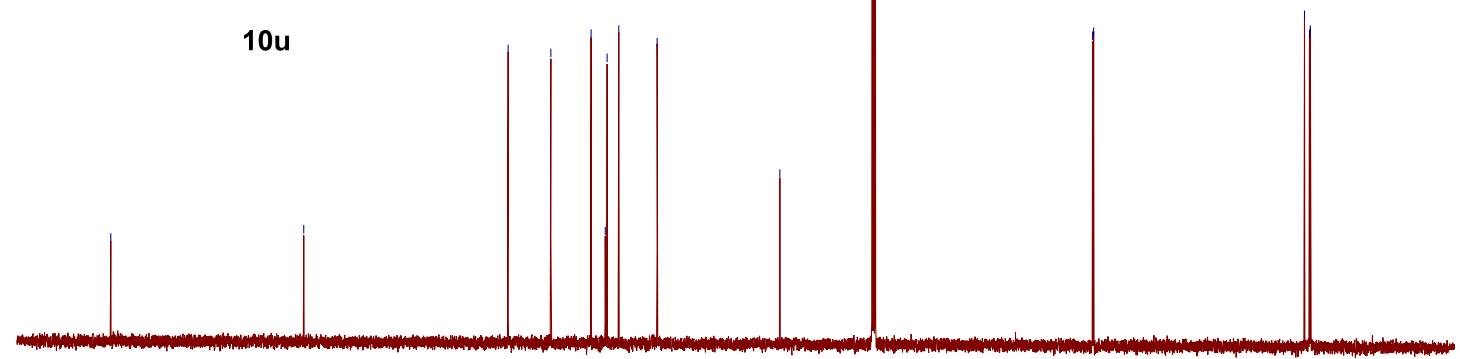

$\begin{array}{lllllllllllll}210 & 200 & 190 & 180 & 170 & 160 & 150 & 140 & 130 & 120 & 110 & 100 & 90\end{array}$ 
$\underline{{ }^{1} \mathrm{H} \text { NMR }}\left(500 \mathrm{MHz}, \mathrm{CDCl}_{3} @ 7.26 \mathrm{ppm}\right)$

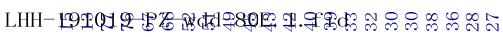

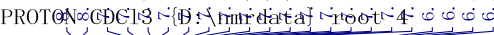

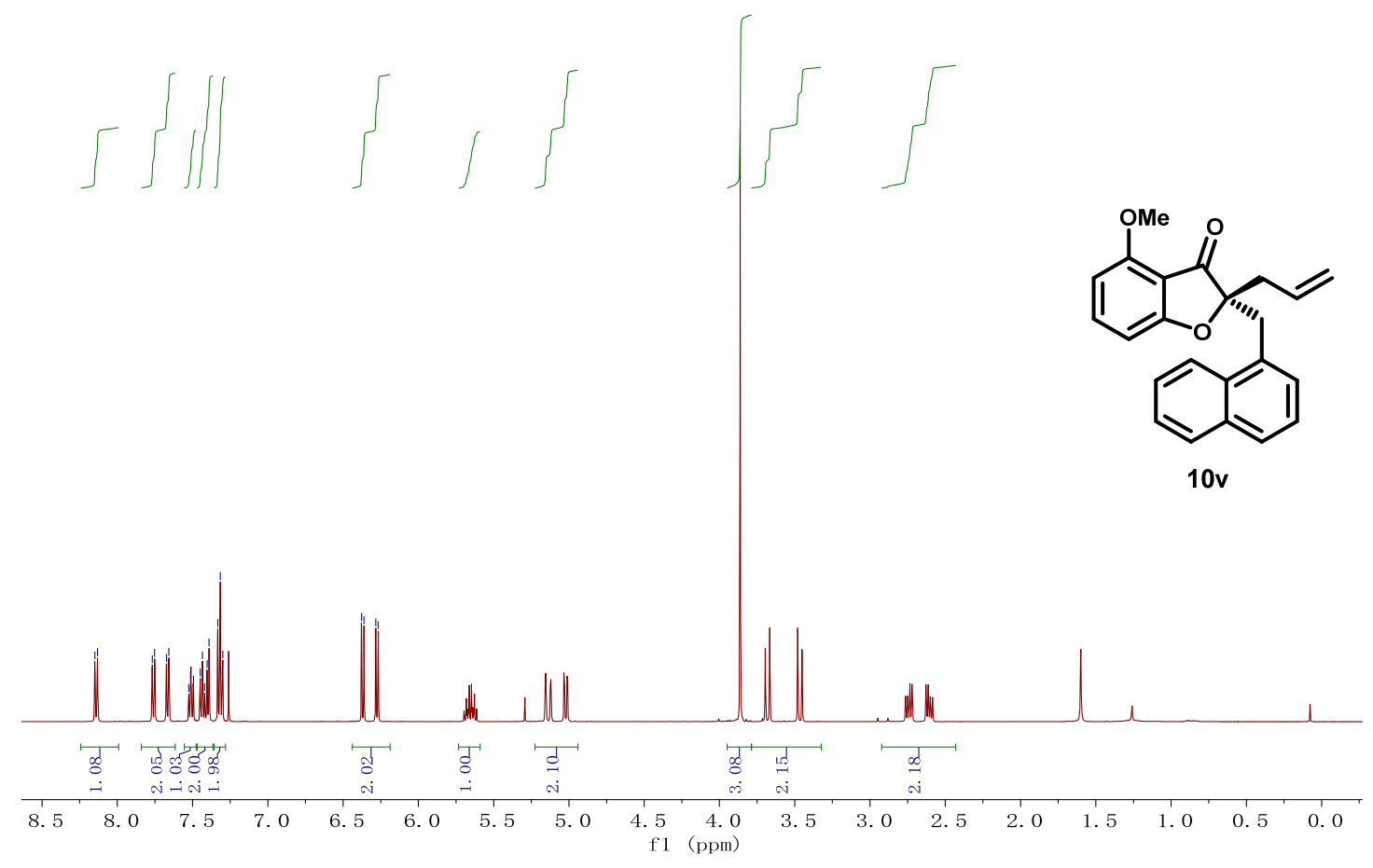

${ }^{13} \mathrm{C}$ NMR (125 MHz, $\mathrm{CDCl}_{3} @ 77$ ppm)

LHH-1910g9-PZ-wdd-80E⿱

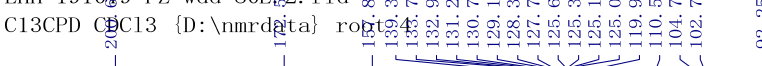

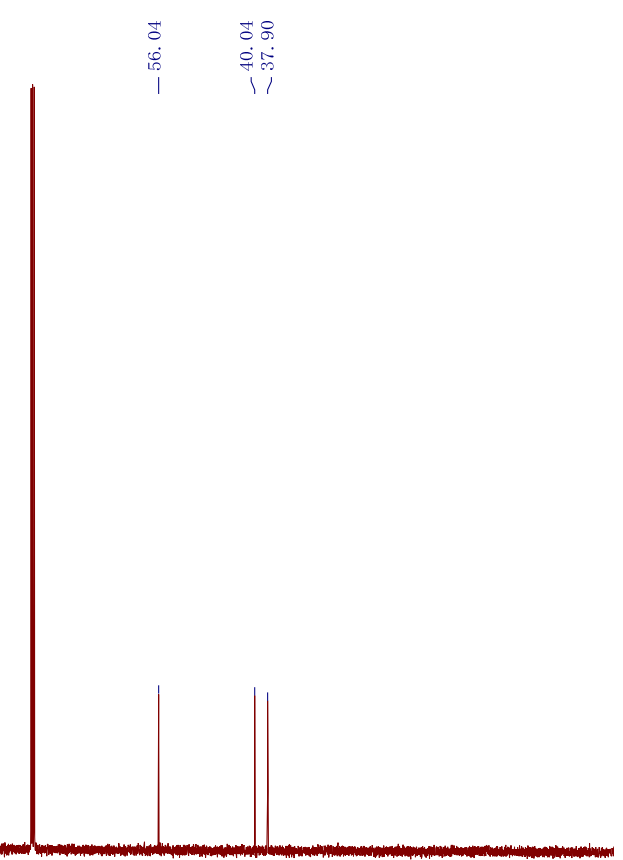

$\begin{array}{lllllllllllll}210 & 200 & 190 & 180 & 170 & 160 & 150 & 140 & 130 & 120 & 110 & 100 & 90 \\ \mathrm{f} 1 & (\mathrm{ppm})\end{array}$ 
$\underline{{ }^{1} \mathrm{H} \text { NMR }}\left(500 \mathrm{MHz}, \mathrm{CDCl}_{3} @ 7.26 \mathrm{ppm}\right)$

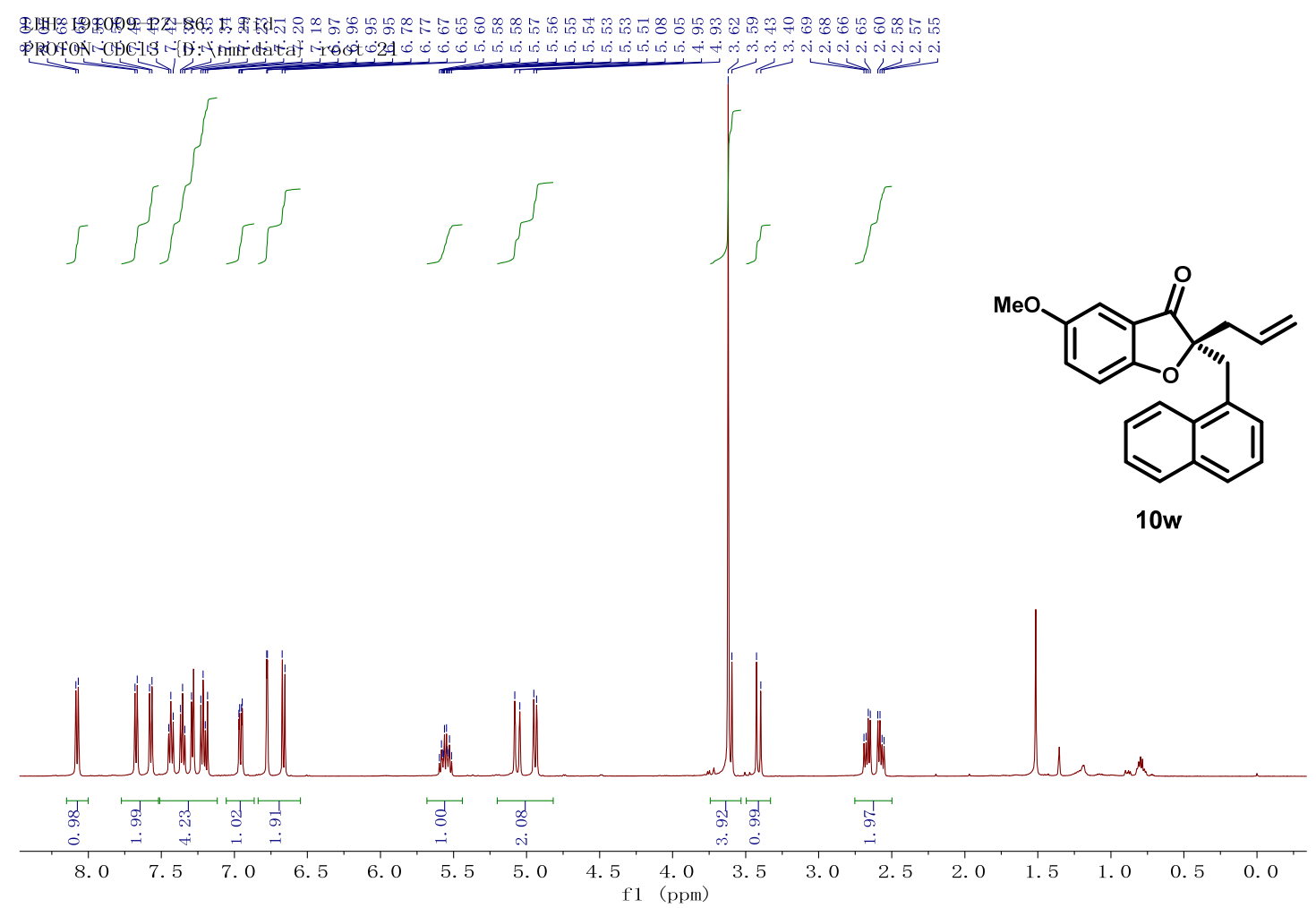

${ }^{13} \mathrm{C}$ NMR (125 MHz, $\mathrm{CDCl}_{3} @ 77$ ppm)

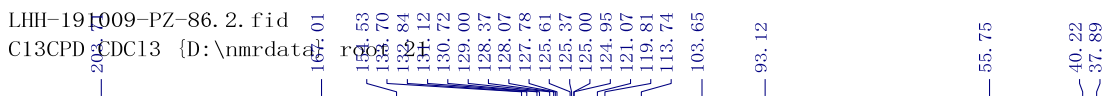<smiles>C=CC[C@]1(Cc2cccc3ccccc23)Oc2ccc(OC)cc2C1=O</smiles>

10w

$\begin{array}{lllllllllllll}210 & 200 & 190 & 180 & 170 & 160 & 150 & 140 & 130 & 120 & 110 & 100 & 9 \\ \mathrm{f} 1 & (\mathrm{ppm})\end{array}$ 
$\underline{{ }^{1} \mathrm{H} \text { NMR }}\left(600 \mathrm{MHz}, \mathrm{CDCl}_{3} @ 7.26 \mathrm{ppm}\right)$

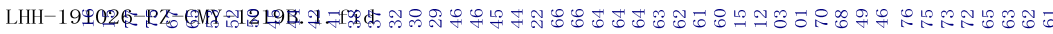

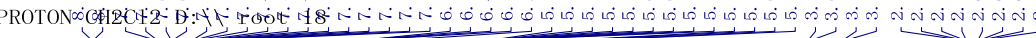

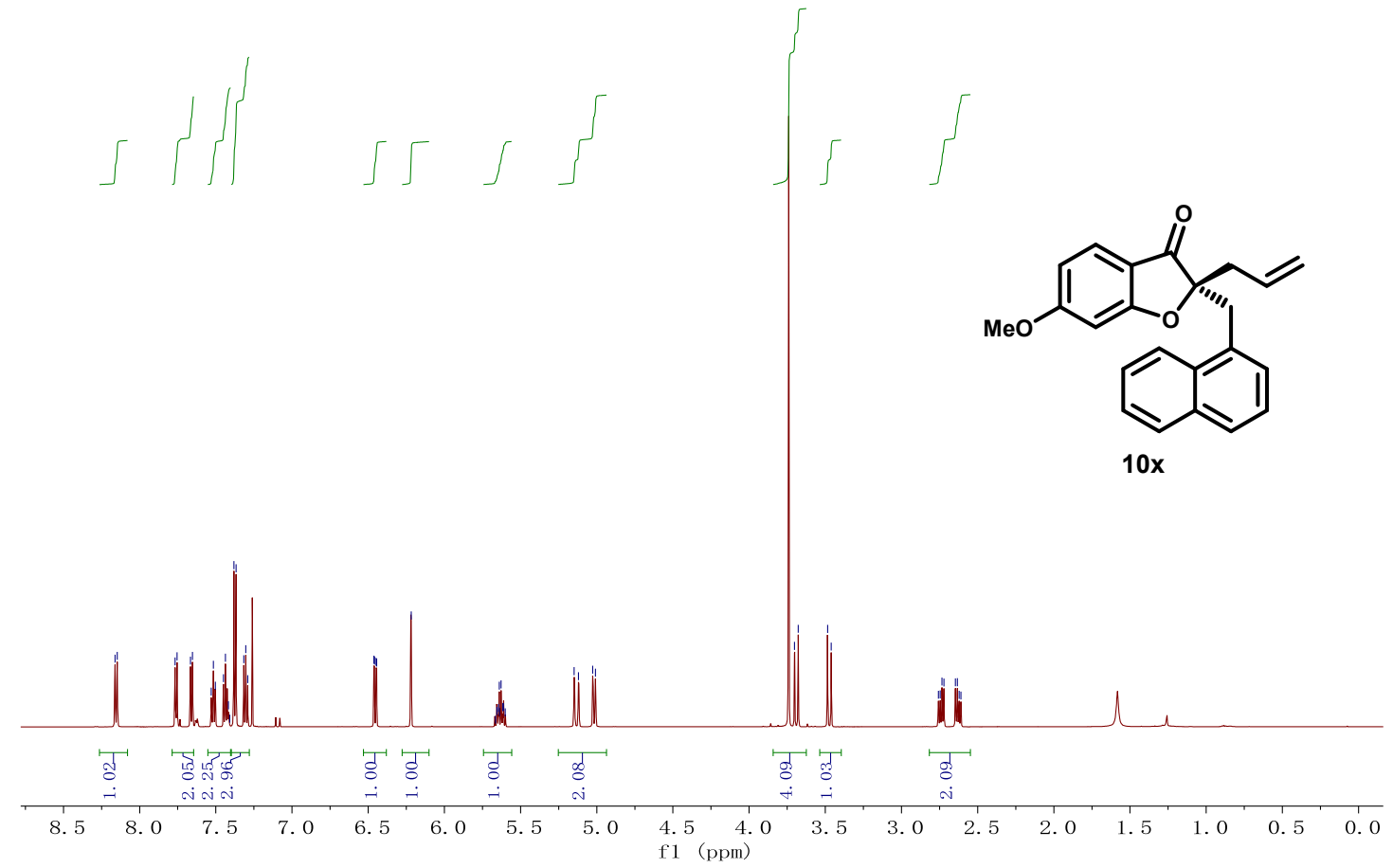

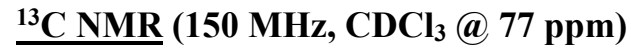<smiles>C=CC[C@]1(Cc2cccc3ccccc23)Oc2ccccc2C1=O</smiles>

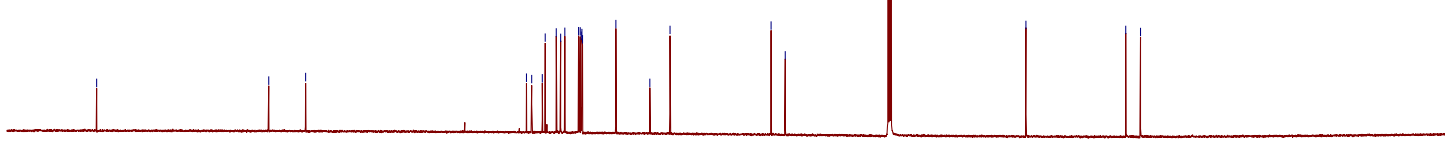


(S)-2-Allyl-6-bromo-2-(naphthalen-1-ylmethyl)benzofuran-3(2H)-one (10y)

$\underline{{ }^{1} \mathrm{H} \text { NMR }}\left(500 \mathrm{MHz}, \mathrm{CDCl}_{3} @ 7.26 \mathrm{ppm}\right)$

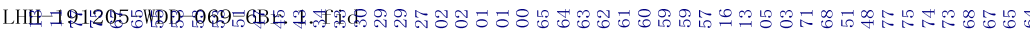

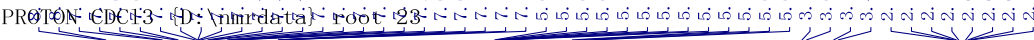

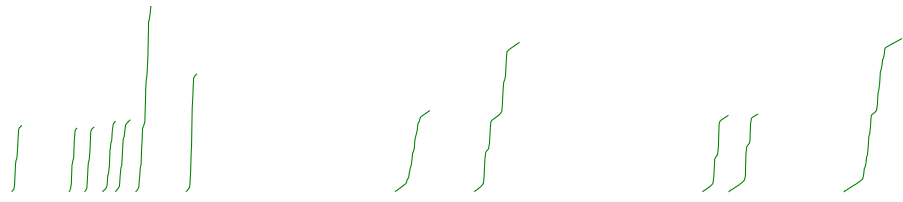<smiles>C=CC[C@@]1(Cc2cccc3ccccc23)Oc2cc(Br)ccc2C1=O</smiles>

10y

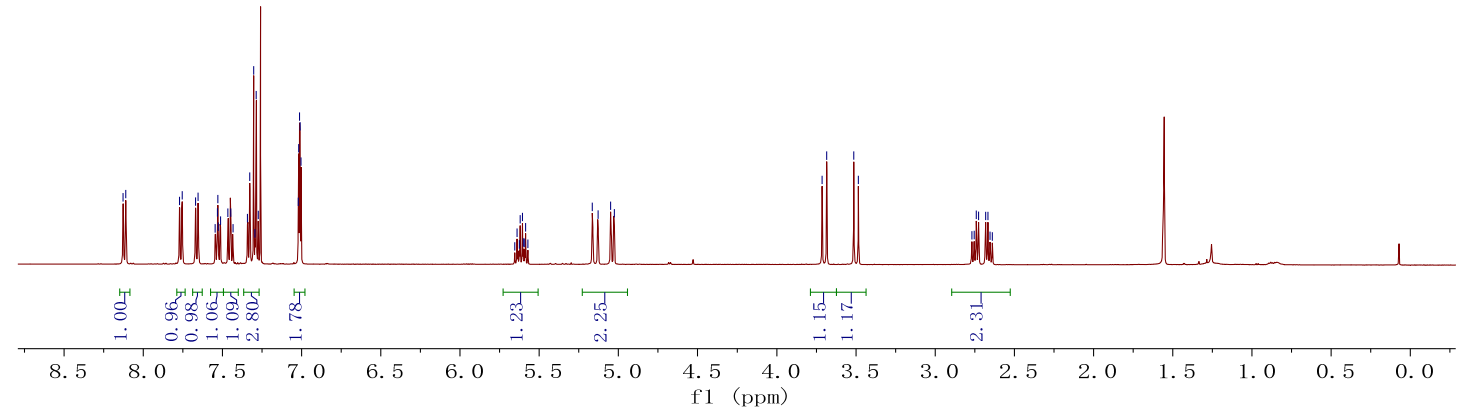

${ }^{13} \mathrm{C}$ NMR (125 MHz, CDCl 3 @ 77 ppm)

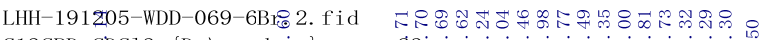

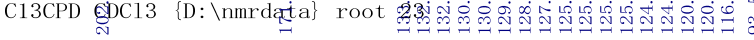<smiles></smiles>

10y 
$\underline{{ }^{1} \mathrm{H} \text { NMR }}\left(500 \mathrm{MHz}, \mathrm{CDCl}_{3} @ 7.26 \mathrm{ppm}\right)$

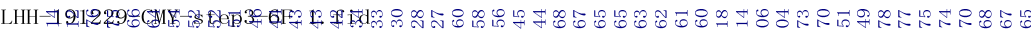

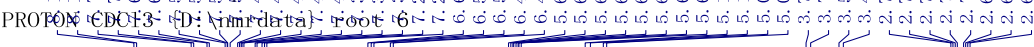

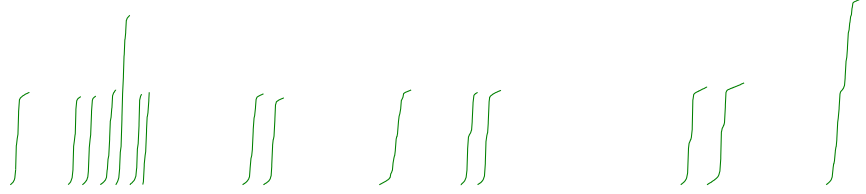<smiles>C=CC[C@]1(Cc2cccc3ccccc23)Oc2cc(F)ccc2C1=O</smiles>

$10 z$

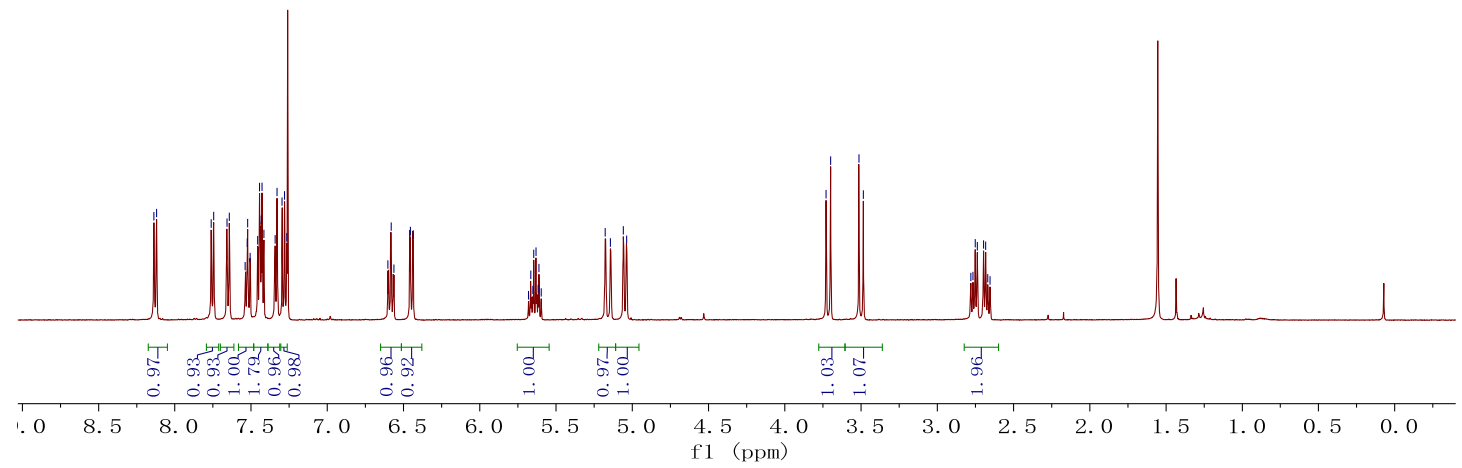

${ }^{13} \mathrm{C}$ NMR (125 MHz, $\mathrm{CDCl}_{3} @ 77$ ppm)

LHH-1910 6-WDD-PZ-9

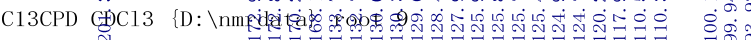

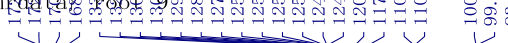<smiles>C=CC[C@]1(Cc2cccc3ccccc23)Oc2cc(F)ccc2C1=O</smiles>

$10 z$

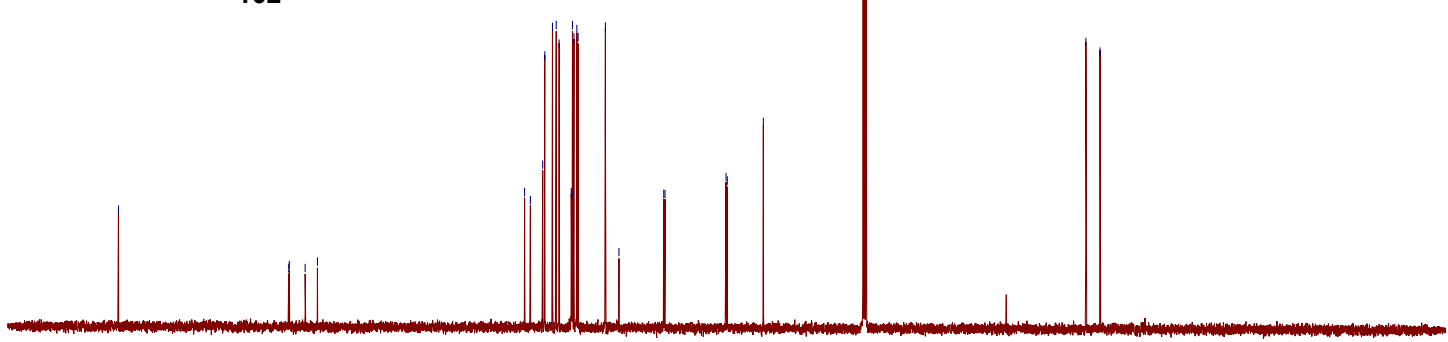

$\begin{array}{lllllllllllll}210 & 200 & 190 & 180 & 170 & 160 & 150 & 140 & 130 & 120 & 110 & 100 & 90\end{array}$ 
LHH-191217-CMY-6-f-STEP3. 10. fid F19 CDC13 $\{\mathrm{D}: \backslash$ nmrdata $\}$ root 55

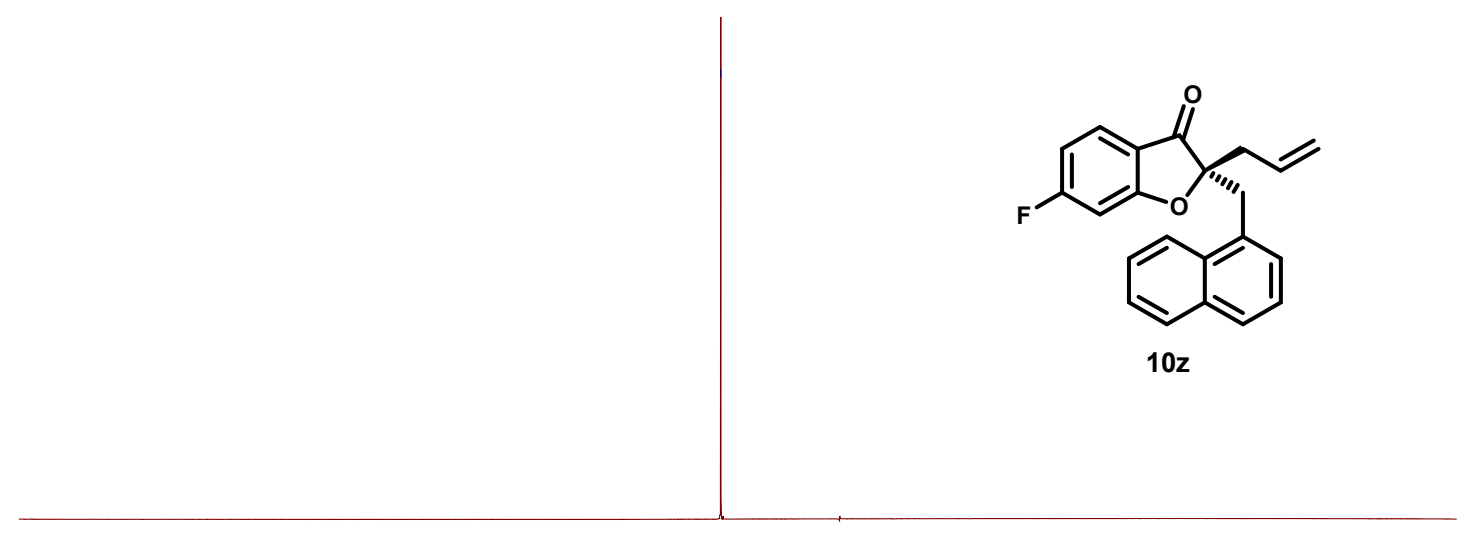

\begin{tabular}{|c|c|c|c|c|c|c|c|c|c|c|c|}
\hline 20 & 0 & -20 & -40 & -60 & -80 & 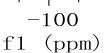 & -120 & -140 & -160 & -180 & -200 \\
\hline
\end{tabular}

(S)-2-Allyl-6-morpholino-2-(naphthalen-1-ylmethyl)benzofuran-3(2H)-one (10a')

$\underline{{ }^{1} \mathrm{H} \text { NMR }}\left(500 \mathrm{MHz}, \mathrm{CDCl}_{3} @ 7.26\right.$ ppm)

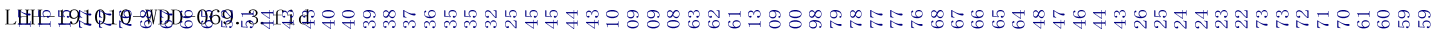
P央OA
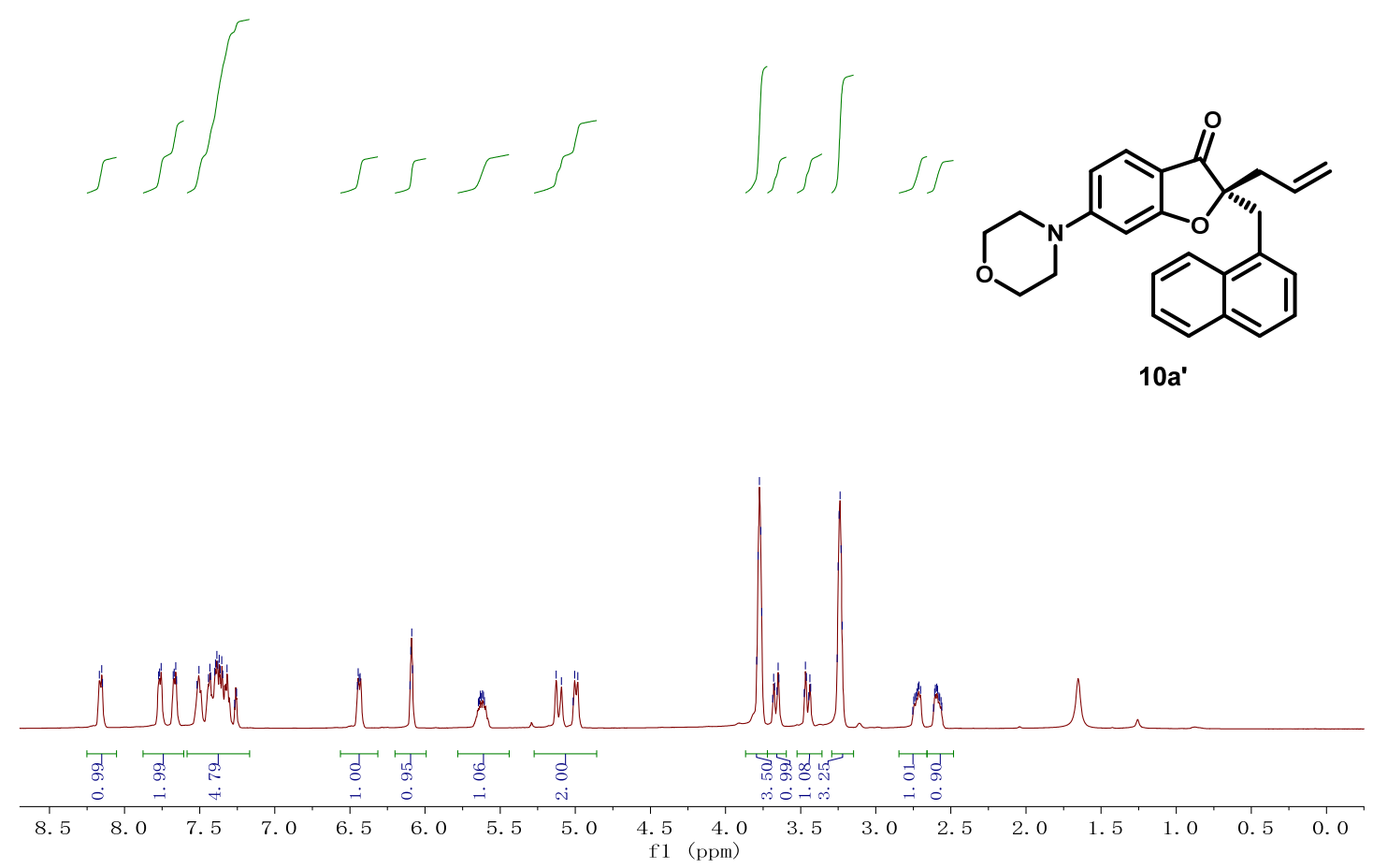
${ }^{13} \mathrm{C}$ NMR (125 MHz, $\mathrm{CDCl}_{3} @ 77$ ppm)

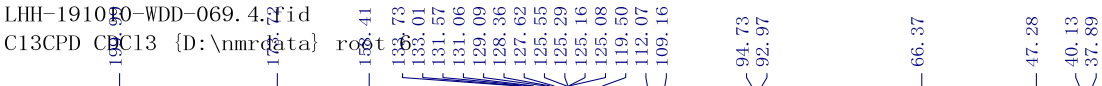<smiles>C=CC[C@]1(Cc2cccc3ccccc23)Oc2cc(N3CCOCC3)ccc2C1=O</smiles>

$10 a^{\prime}$

$\begin{array}{lllllllllllllllllllllll}210 & 200 & 190 & 180 & 170 & 160 & 150 & 140 & 130 & 120 & 110 & 100 & 90 & 80 & 70 & 60 & 50 & 40 & 30 & 20 & 10 & 0 & -10\end{array}$

(S)-2-Allyl-7-methoxy-2-(naphthalen-1-ylmethyl)benzofuran-3(2H)-one (10b')

$\underline{{ }^{1} \mathrm{H} \text { NMR }}\left(500 \mathrm{MHz}, \mathrm{CDCl}_{3} @ 7.26 \mathrm{ppm}\right)$ 


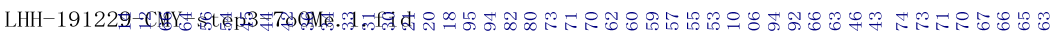

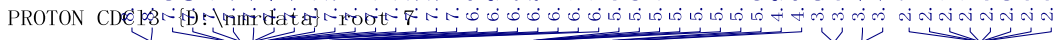

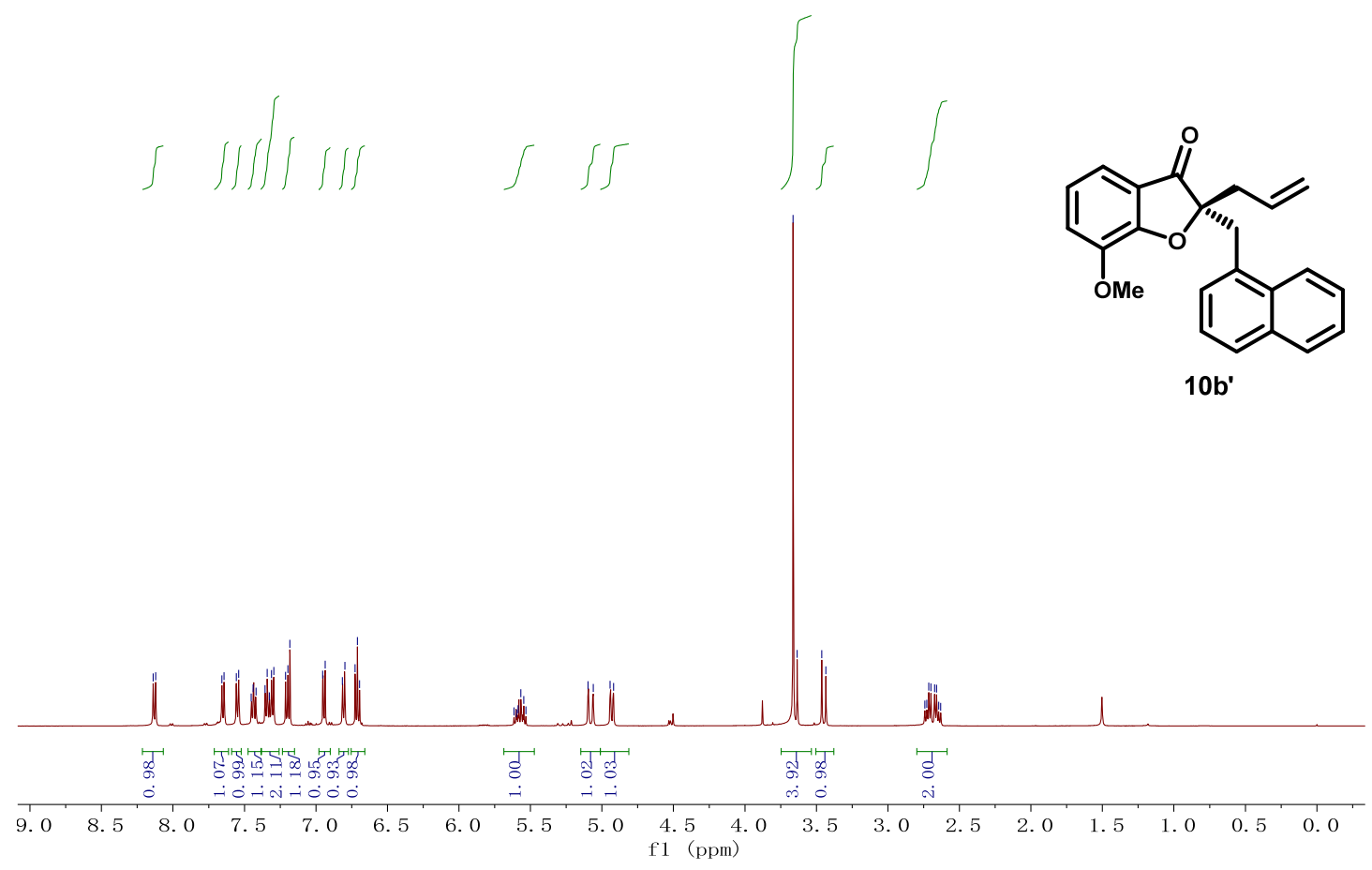

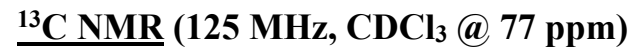

LHH-191922-WDD-PZ-D. 2. fid

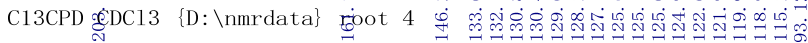

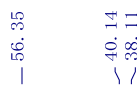

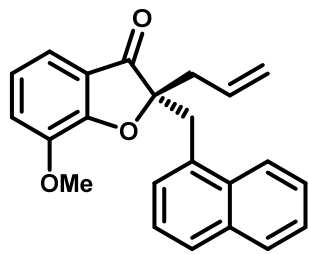

$10 b^{\prime}$

f1 (ppm)

(S)-2-Allyl-7-bromo-2-(naphthalen-1-ylmethyl)benzofuran-3(2H)-one (10c')

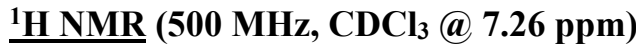




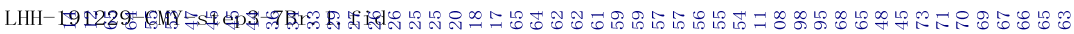

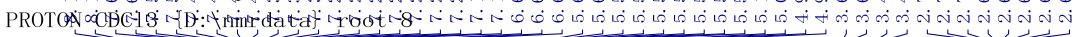
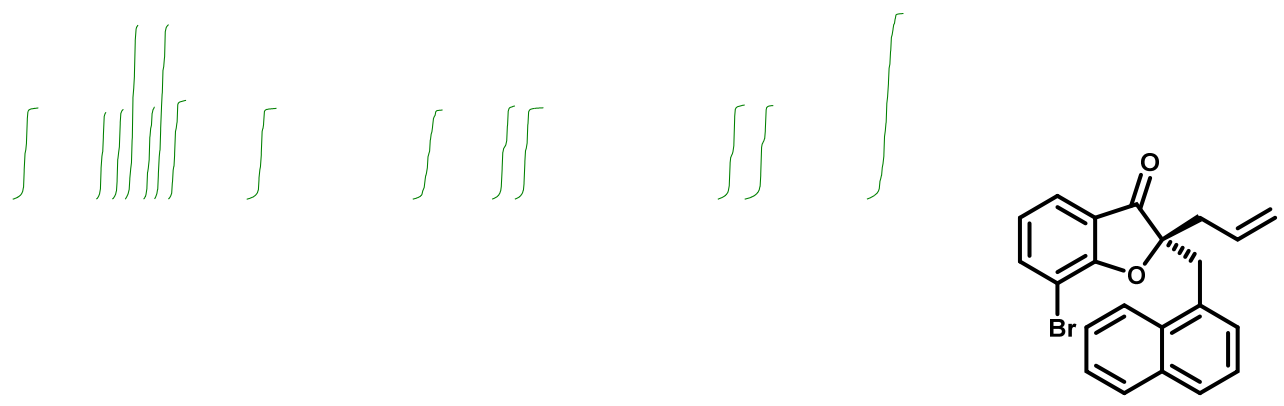

$10 c^{\prime}$

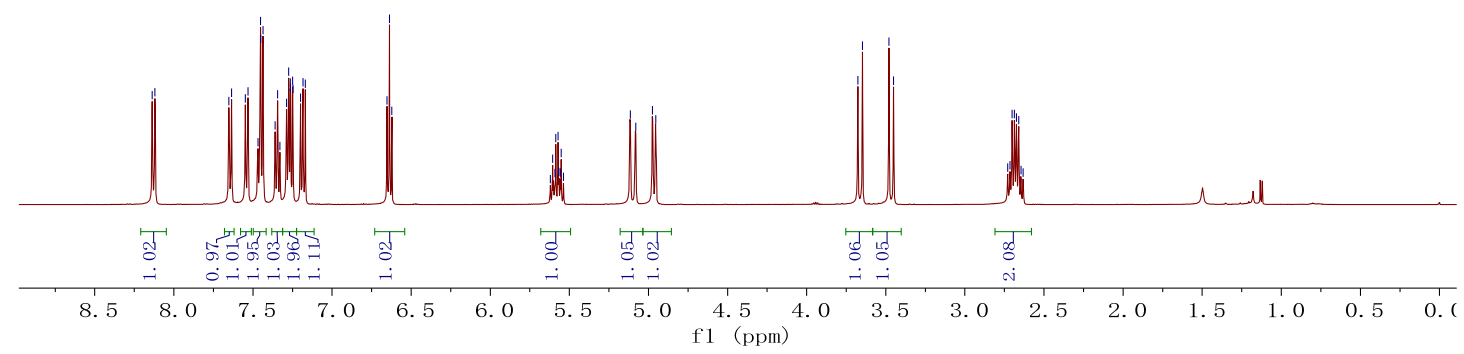

13 C NMR (125 MHz, CDCl @ 77 ppm)

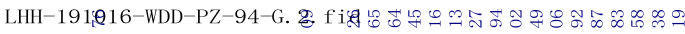

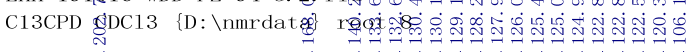

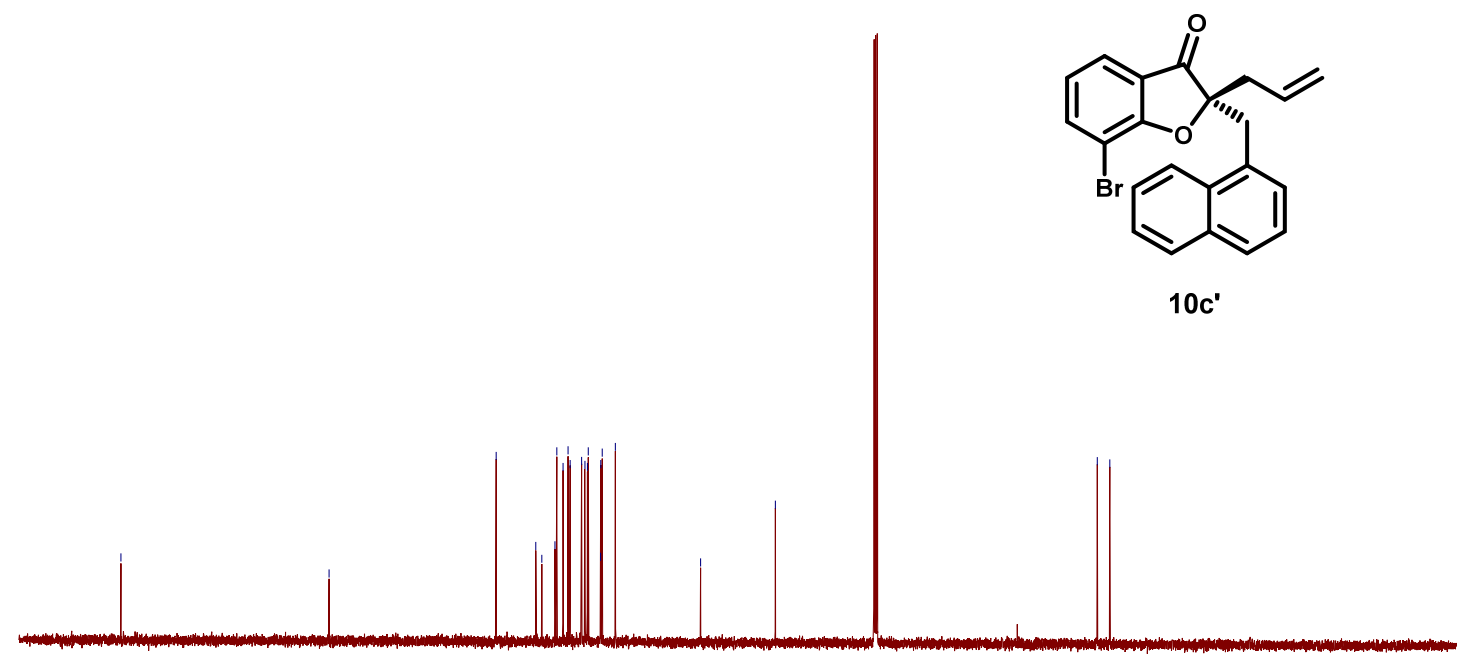

$\begin{array}{lllllllllllll}210 & 200 & 190 & 180 & 170 & 160 & 150 & 140 & 130 & 120 & 110 & 100 & 90\end{array}$

f1 (ppm)

(S)-2-Allyl-2-benzylnaphtho[1,2-b]furan-3(2H)-one (10d')

$\underline{{ }^{1} \mathrm{H} \text { NMR }}\left(500 \mathrm{MHz}, \mathrm{CDCl}_{3} @ 7.26\right.$ ppm) 


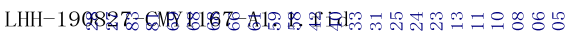

PROTON QCB̈CA

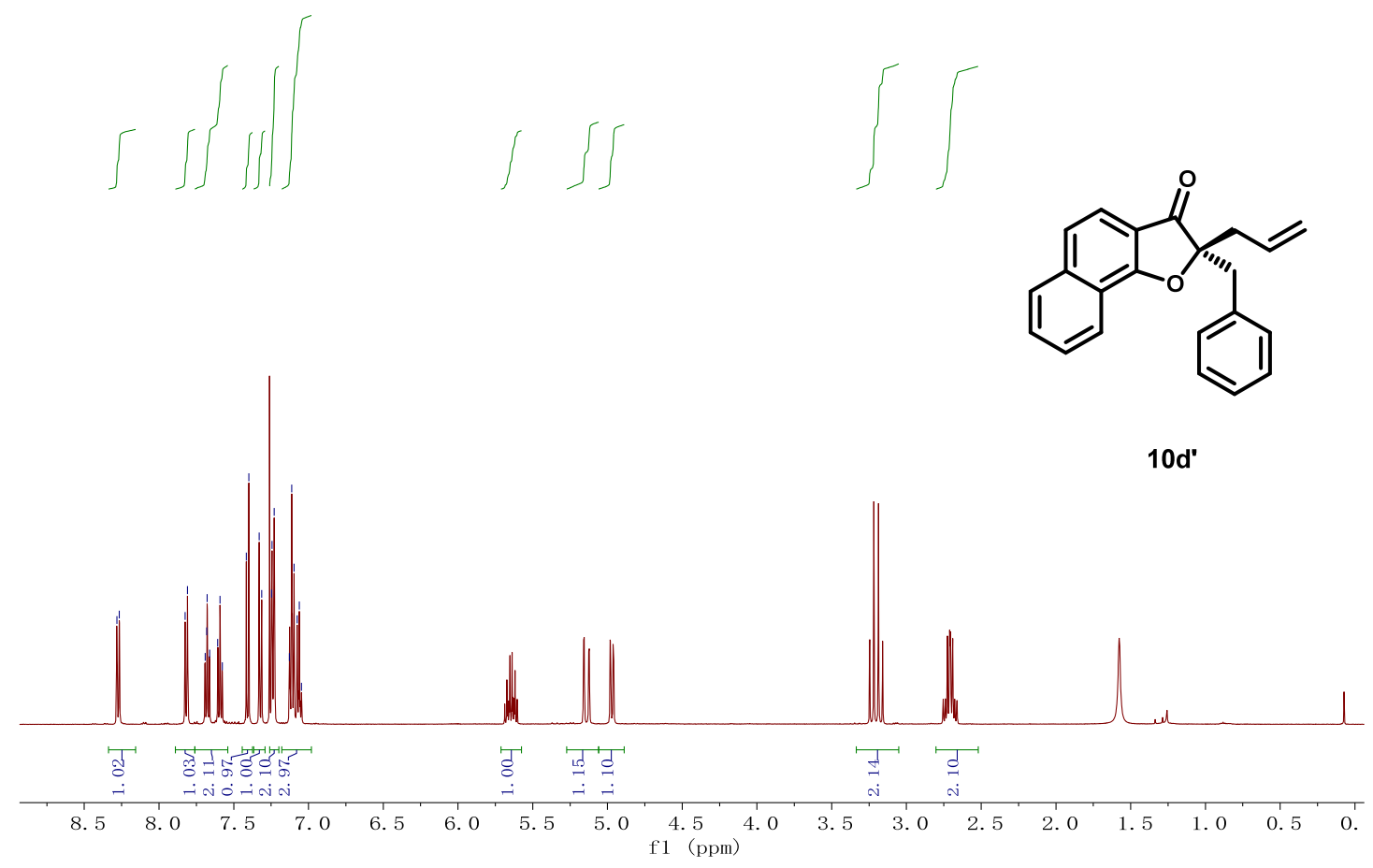

$\underline{{ }^{13} \mathrm{C} \mathrm{NMR}}\left(125 \mathrm{MHz}, \mathrm{CDCl}_{3} @ 77\right.$ ppm)

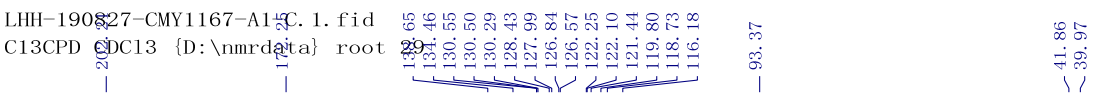<smiles>C=CCC1(c2ccccc2)Oc2c(ccc3ccccc23)C1=O</smiles>

10d"

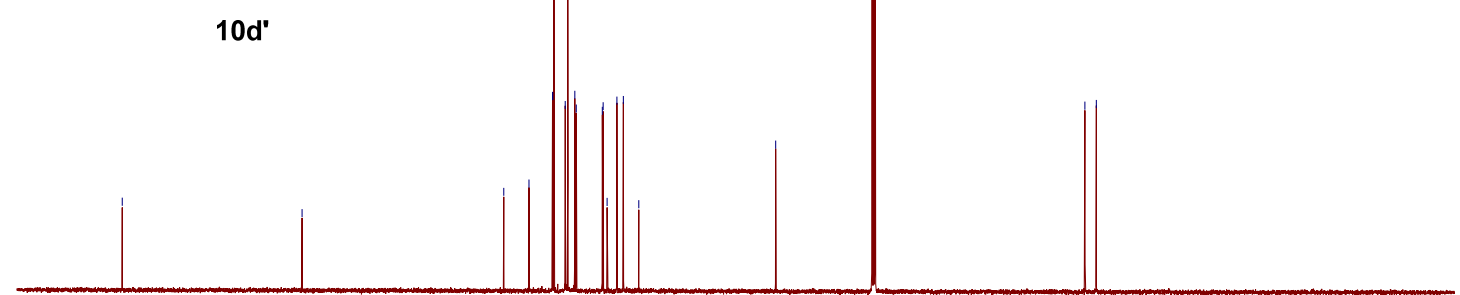

$\begin{array}{lllllllllllll}210 & 200 & 190 & 180 & 170 & 160 & 150 & 140 & 130 & 120 & 110 & 100 & 90\end{array}$

f1 (ppm)

(S)-2-Allyl-2-benzylnaphtho[2,1-b]furan-1(2H)-one (10')

매 NMR $\left(500 \mathrm{MHz}, \mathrm{CDCl}_{3} @ 7.26\right.$ ppm) 


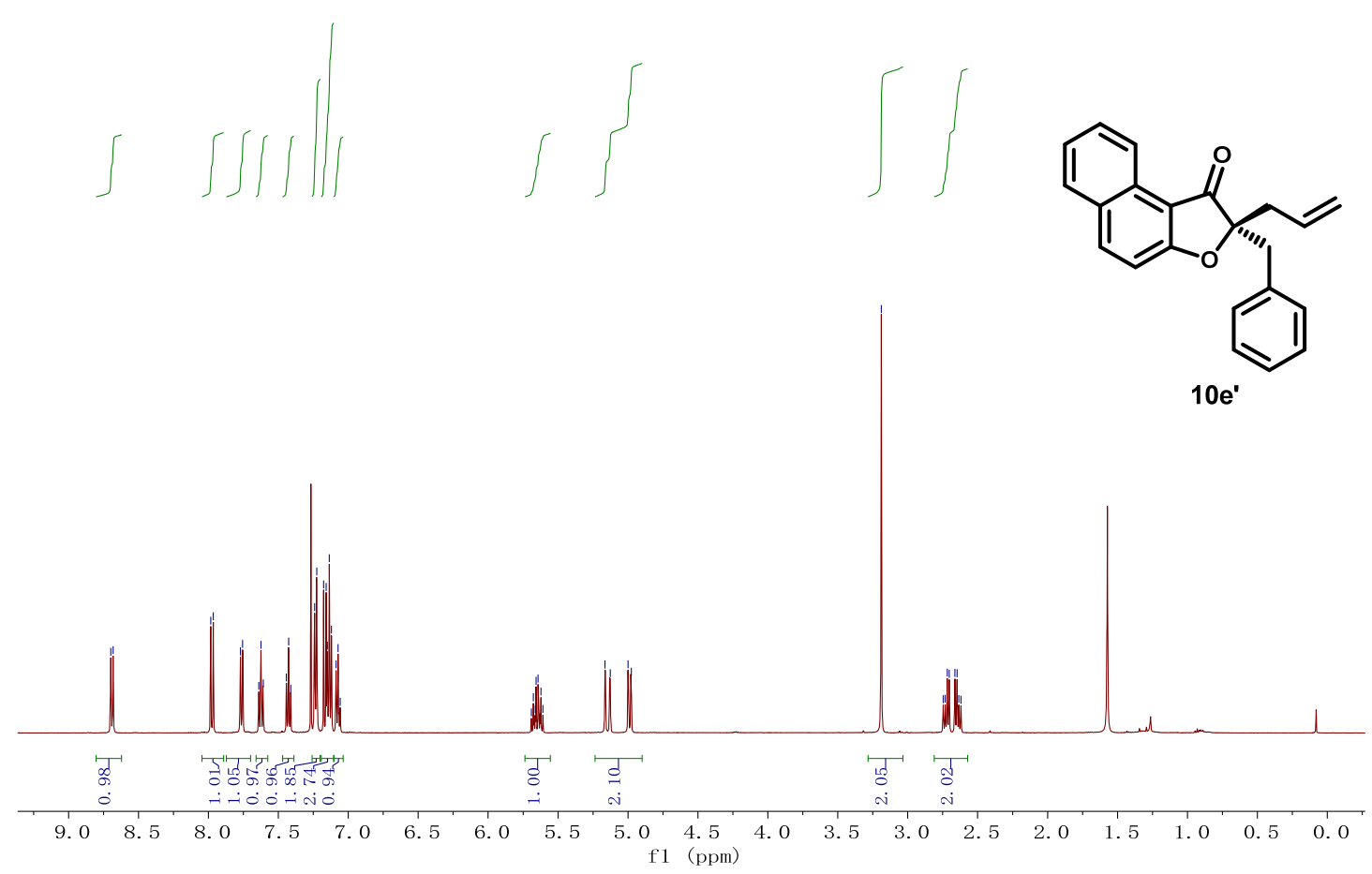

$\underline{{ }^{13} \mathrm{C} \mathrm{NMR}}\left(125 \mathrm{MHz}, \mathrm{CDCl}_{3} @ 77\right.$ ppm)

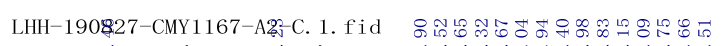

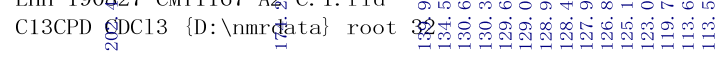

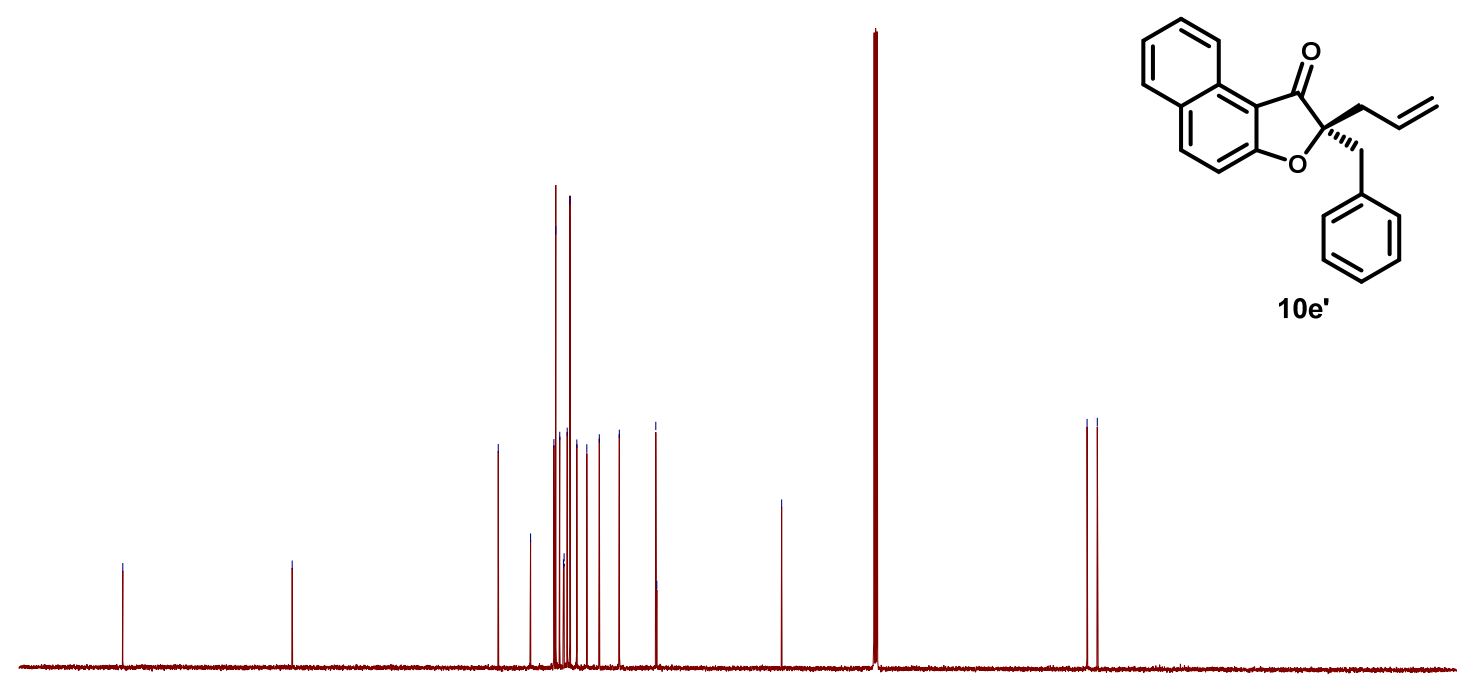

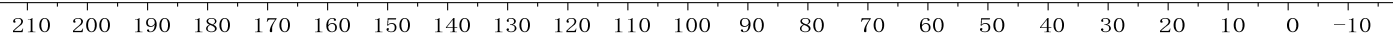
f1 (ppm)

(S)-2-Allyl-2-benzylfuro[3,2-b]pyridin-3(2H)-one (10f')

$\underline{{ }^{1} \mathrm{H} \text { NMR }}\left(400 \mathrm{MHz}, \mathrm{CDCl}_{3} @ 7.26\right.$ ppm) 


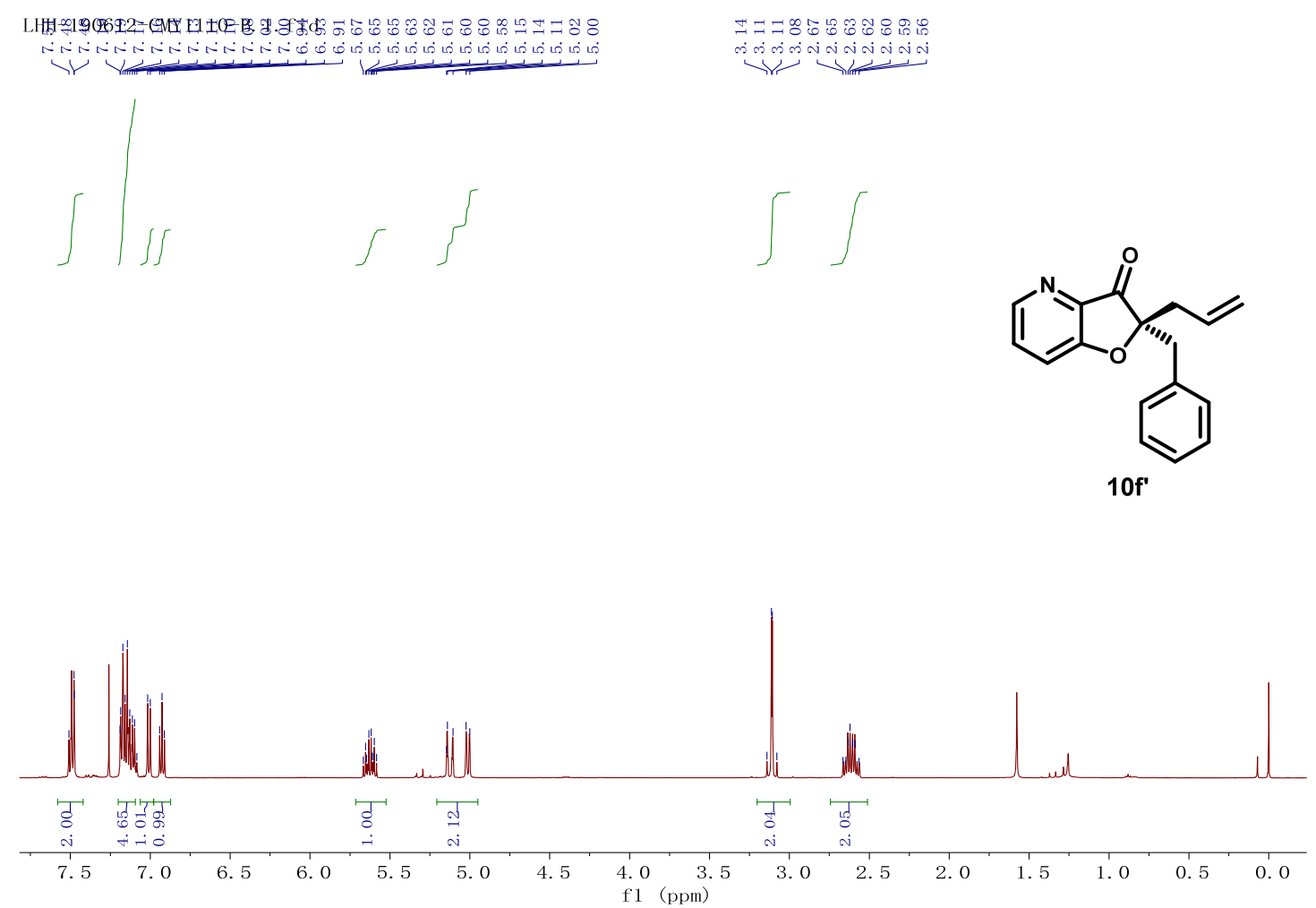

$\underline{{ }^{13} \mathrm{C} \mathrm{NMR}}\left(100 \mathrm{MHz}, \mathrm{CDCl}_{3} @ 77 \mathrm{ppm}\right)$

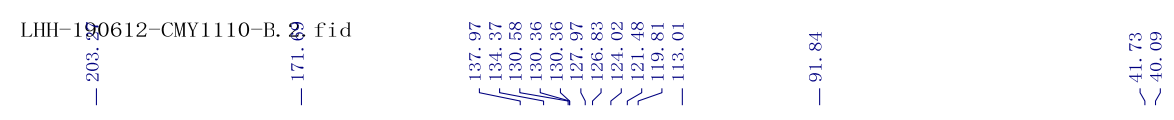

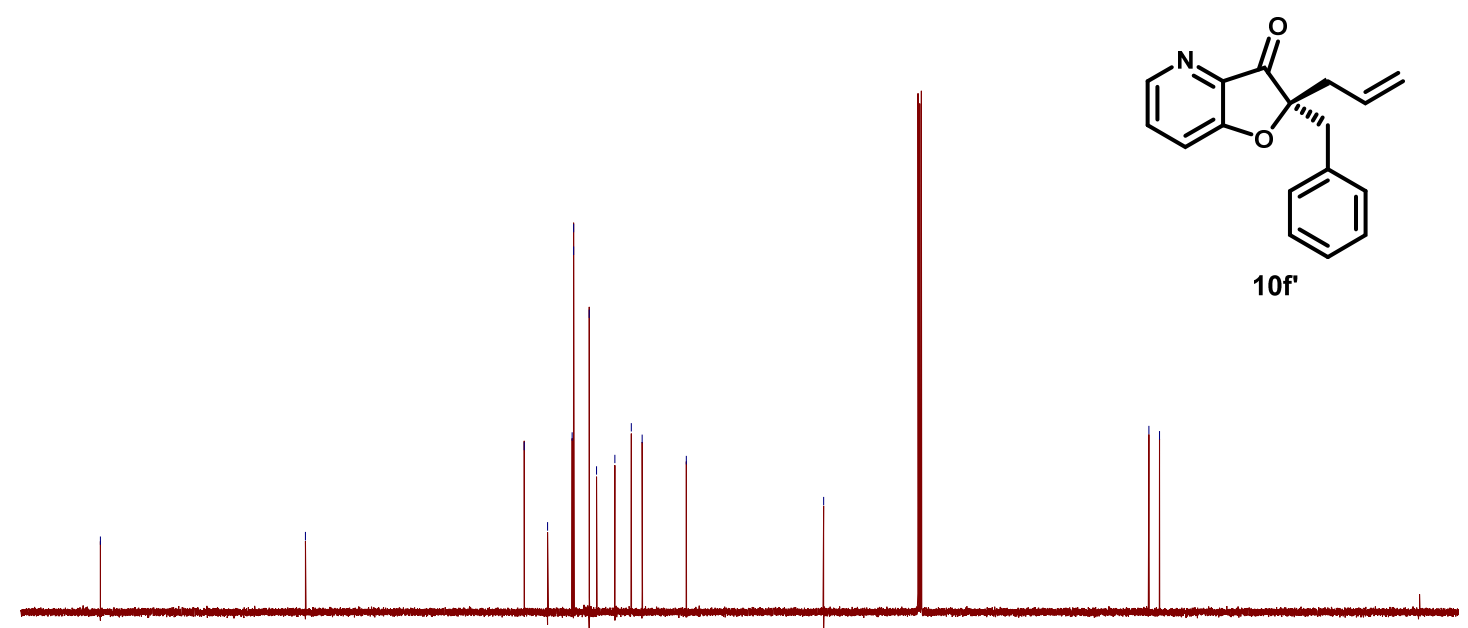

$\begin{array}{llllllllllllllllllllllllll}210 & 200 & 190 & 180 & 170 & 160 & 150 & 140 & 130 & 120 & 110 & 100 & 90 & 80 & 70 & 60 & 50 & 40 & 30 & 20 & 10 & 0\end{array}$

f1 (ppm)

(S)-2-Allyl-4,6-dimethoxy-2-(4-methoxyphenyl)benzofuran-3(2H)-one (10g') 


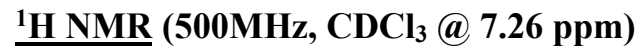

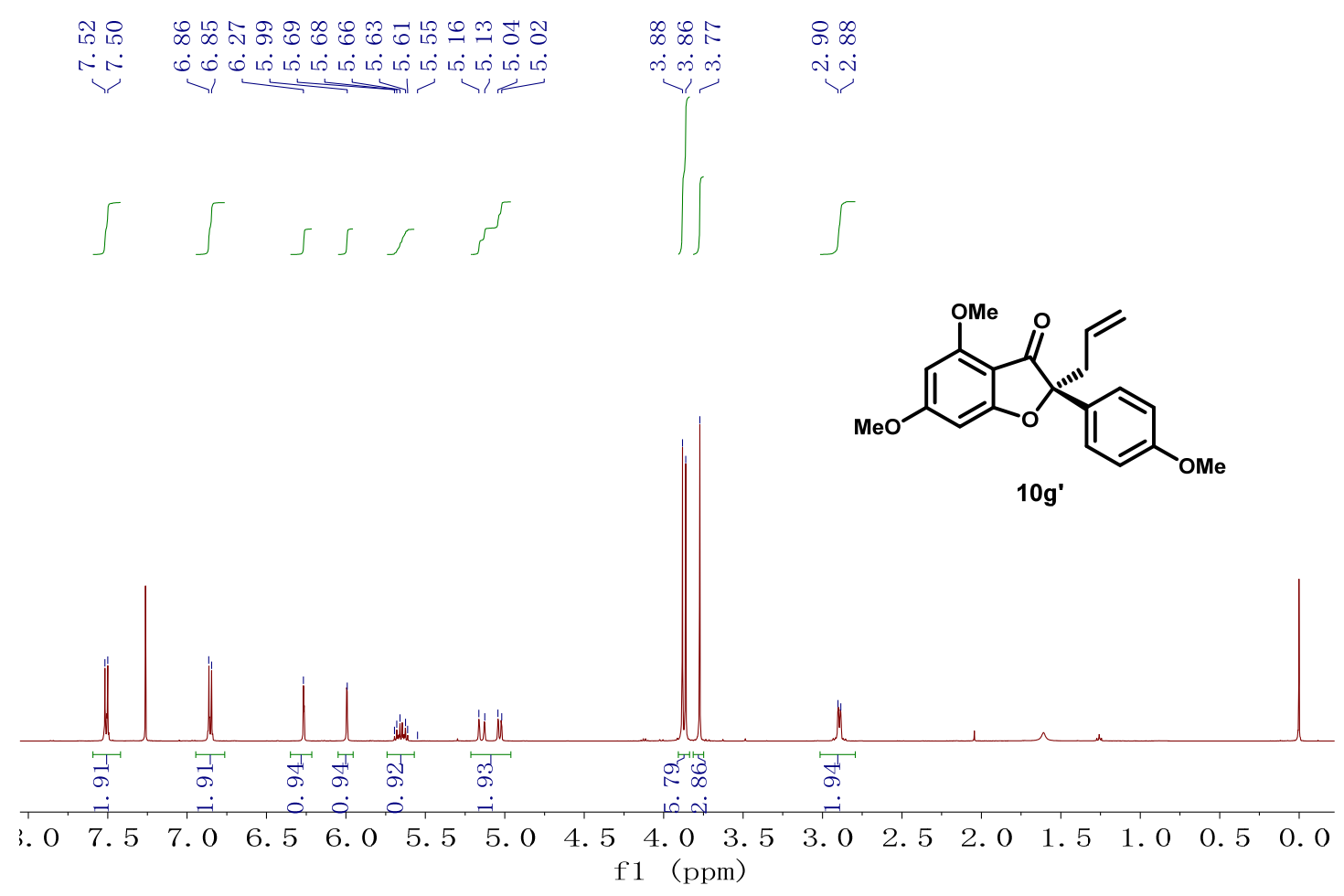

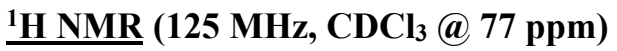

\begin{tabular}{|c|c|c|c|c|c|c|}
\hline 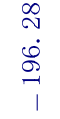 & 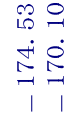 & $\begin{array}{l}\text { 우 } \\
0 \\
0 \\
\stackrel{0}{0} \\
1\end{array}$ & 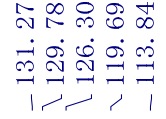 & $\begin{array}{l}\stackrel{+}{N} \\
\stackrel{8}{0} \\
\stackrel{0}{1}\end{array}$ & 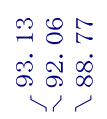 & 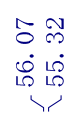 \\
\hline
\end{tabular}

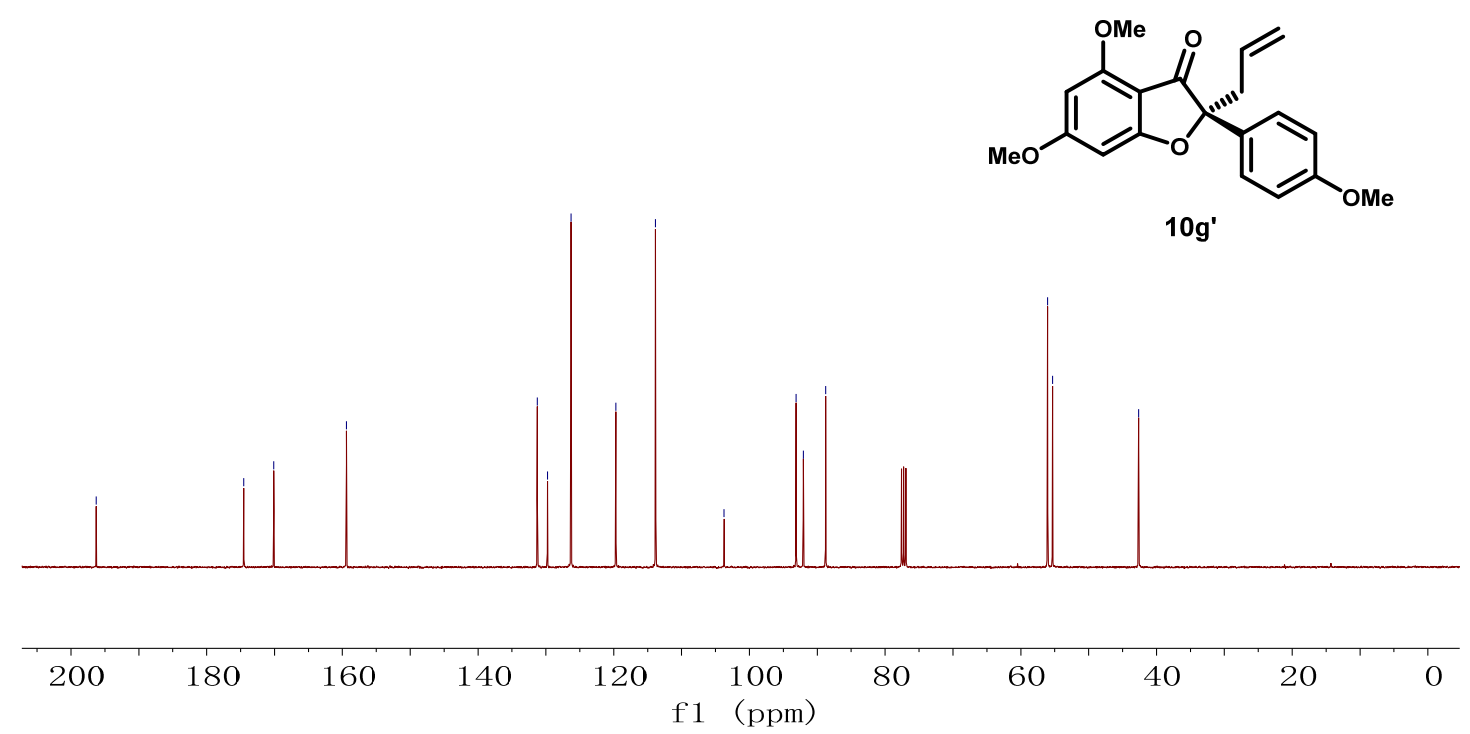

(S)-2-Allyl-4,6-dimethoxy-2-phenylbenzofuran-3(2H)-one (10 h')

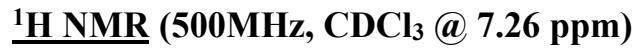




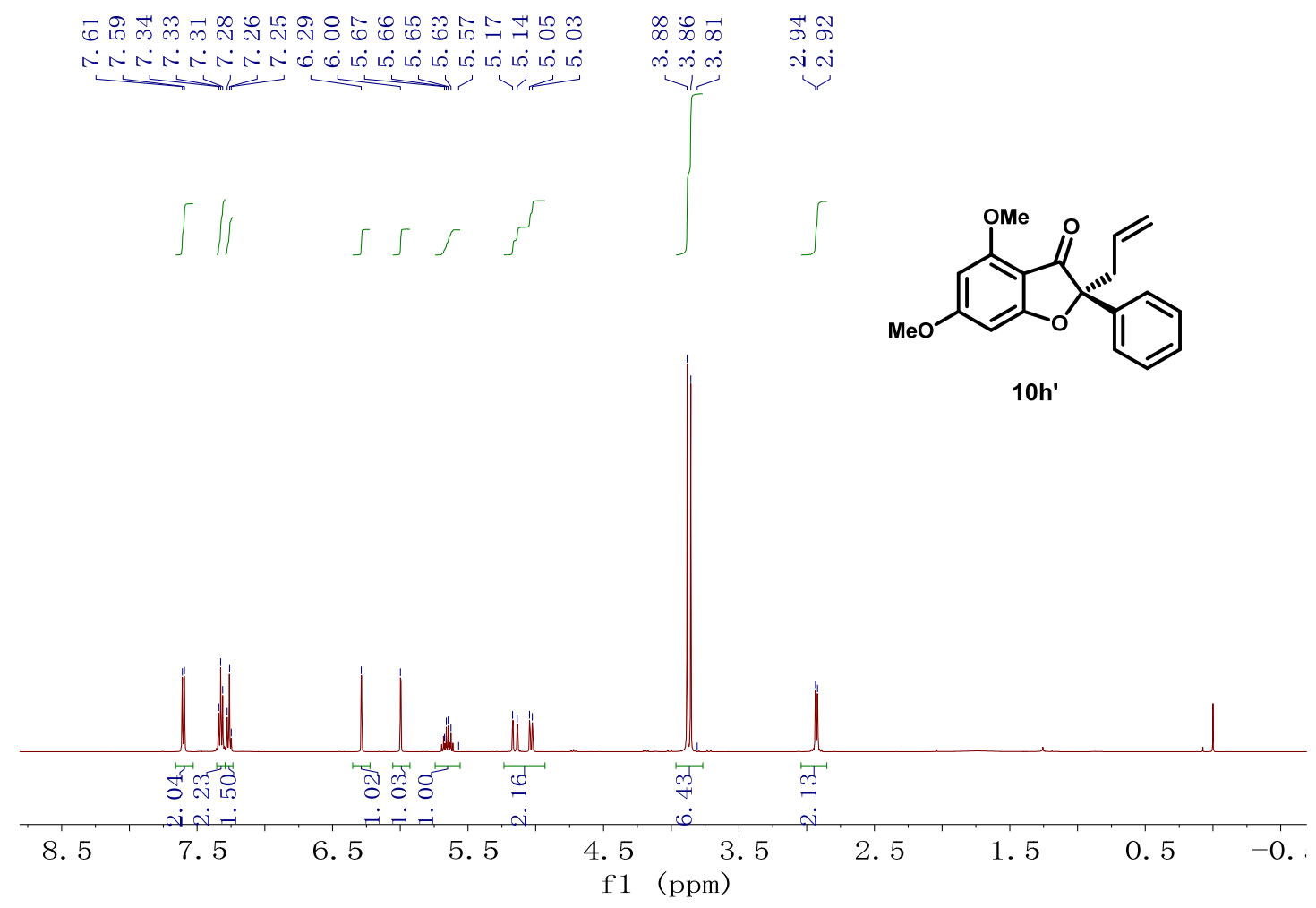

$\underline{{ }^{1} \mathrm{H} \text { NMR }}\left(125 \mathrm{MHz}, \mathrm{CDCl}_{3} @ 77\right.$ ppm)

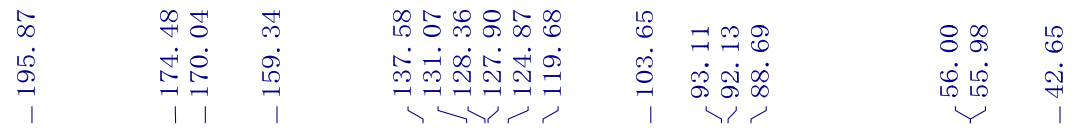<smiles>C=CCC1(c2ccccc2)Oc2cc(OC)cc(OC)c2C1=O</smiles>

$10 h^{\prime}$

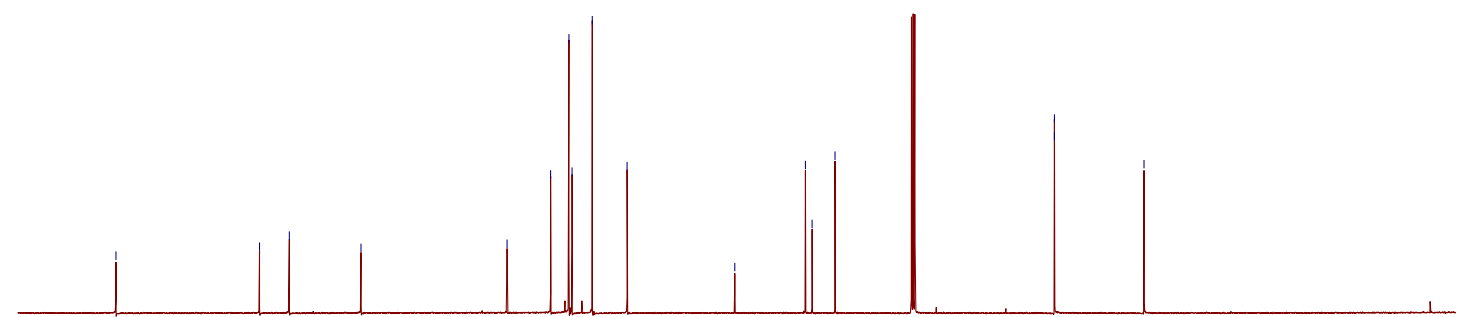

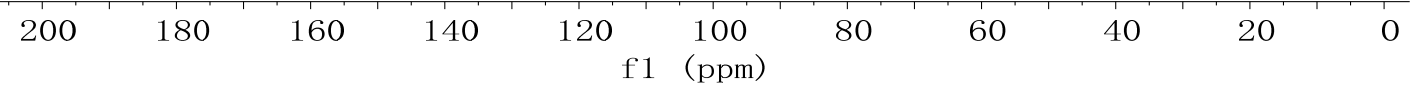

2R-2-Benzyl -2-(2-propen-1-yl)-3(2H)-benzofuranone (13) 
$\underline{1}$ H NMR (500MHz, $\mathrm{CDCl}_{3} @ 7.26$ ppm)

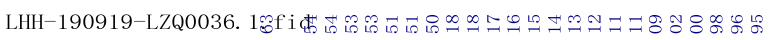

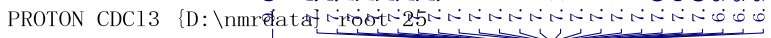

으음ำ

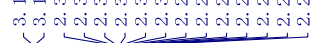
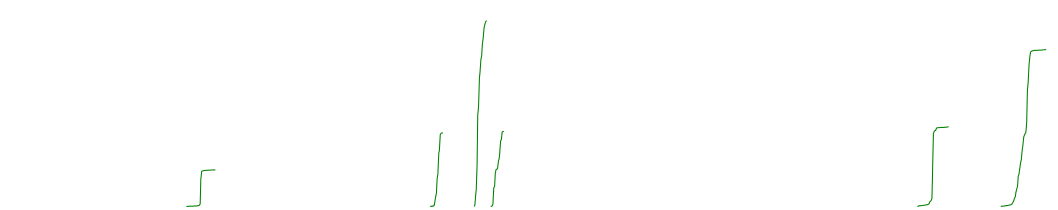

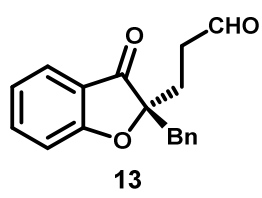

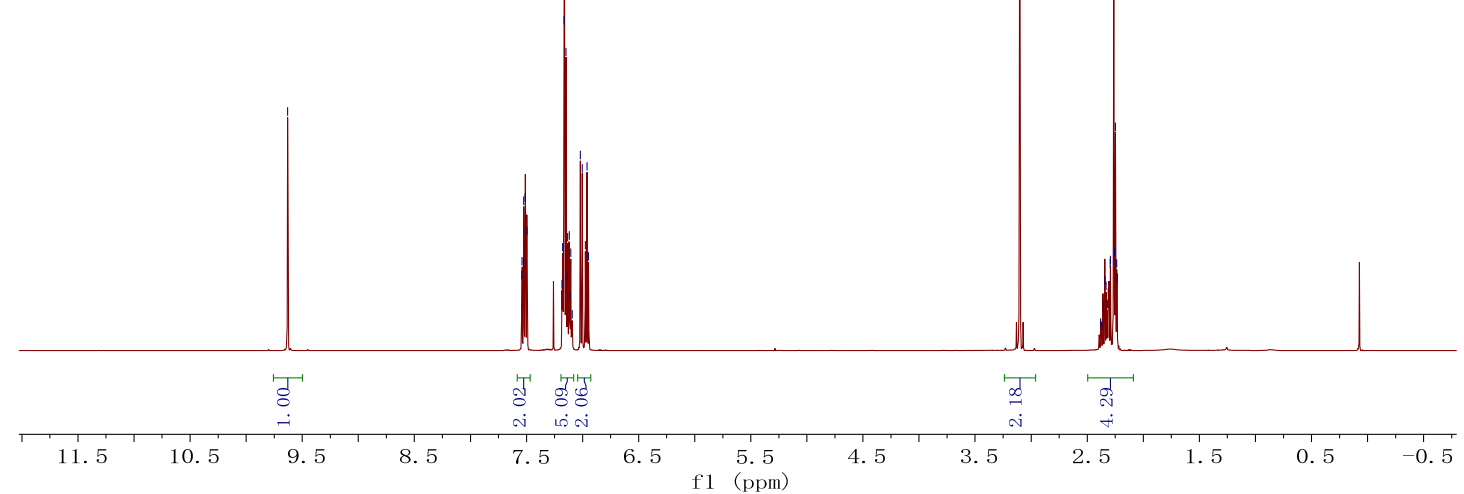

$\underline{{ }^{13} \mathrm{C} \text { NMR }}\left(125 \mathrm{MHz}, \mathrm{CDCl}_{3} @ 77 \mathrm{ppm}\right)$

LHH-1909 x9-LZQ0036. 2. 연d

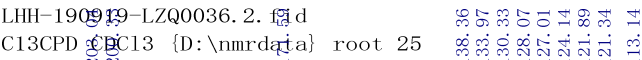

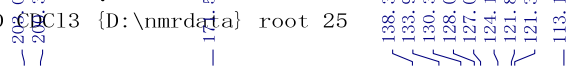
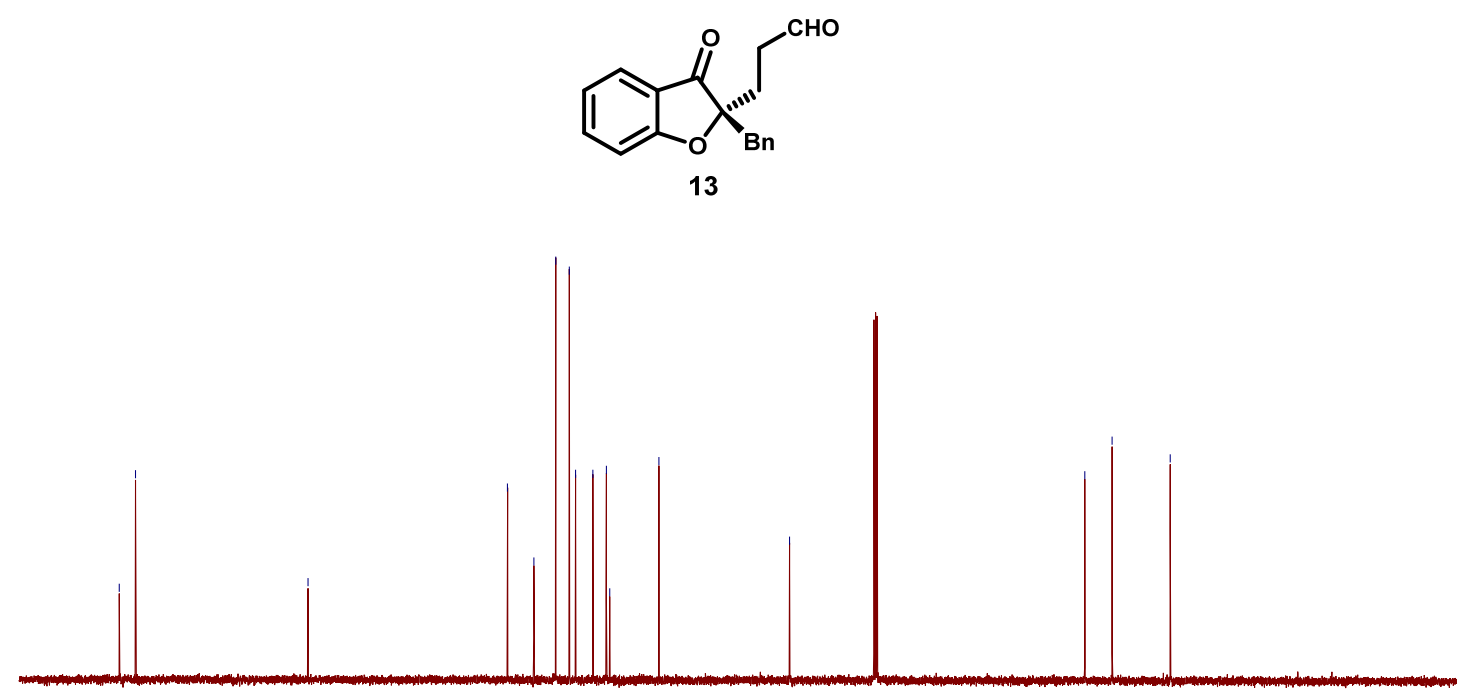

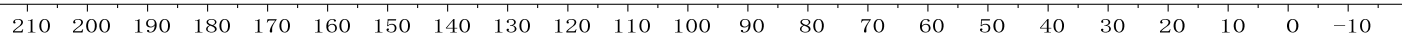

f1 (ppm)

$(3 \mathrm{a} R, 8 \mathrm{~b} R)$-2,3,3a,8b-Tetrahydro-8b-hydroxy-3a-benzyl-1 $H$-cyclopenta[b]benzofuran-1-one (15) 
LHH-190920-LZQ01900-

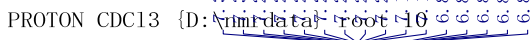
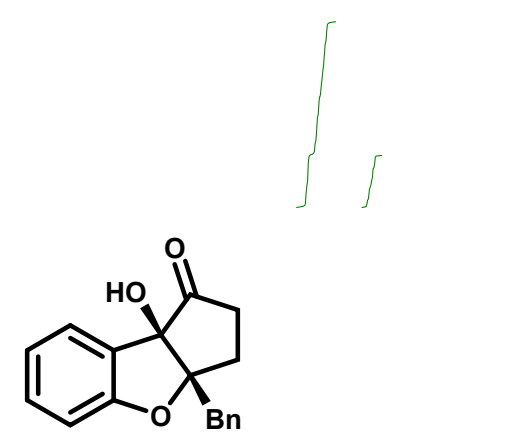

15

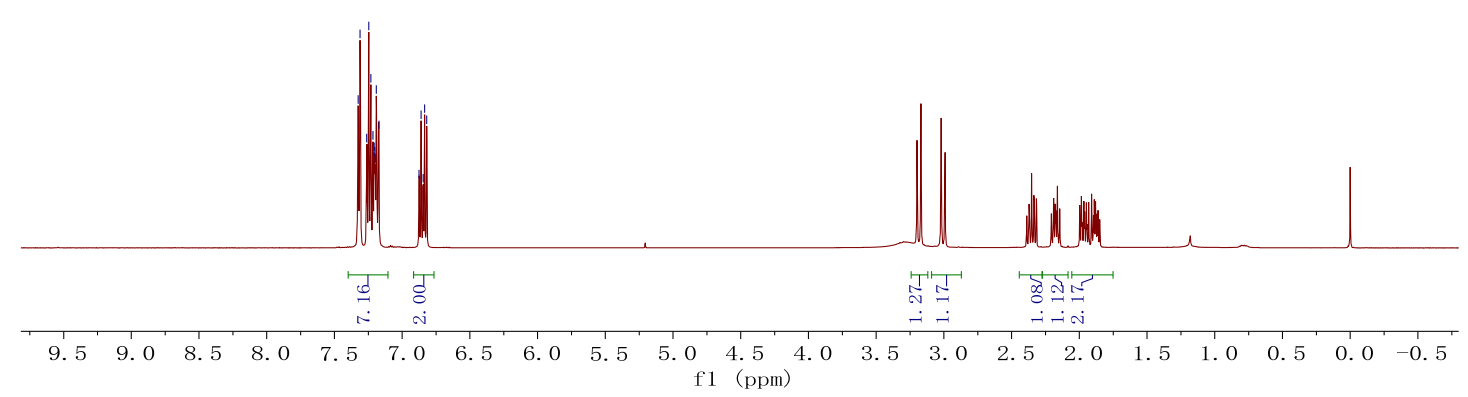

$\underline{{ }^{13} \mathrm{C} N M R}\left(125 \mathrm{MHz}, \mathrm{CDCl}_{3} @ 77\right.$ ppm)<smiles>O=C1CC[C@@]2(Cc3ccccc3)Oc3ccccc3[C@]12O</smiles>

15

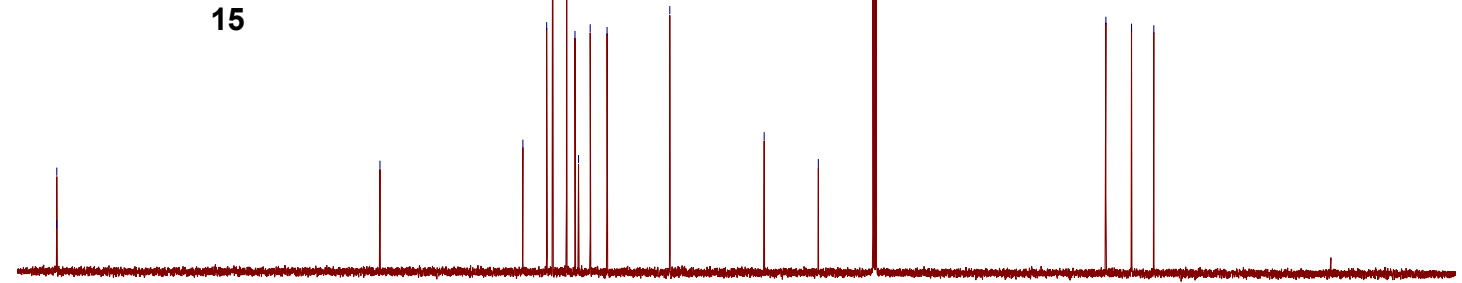

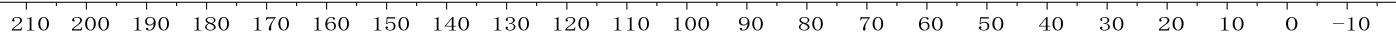

f1 (ppm)

(3aS,8bR)-3a-Benzyl-8b-hydroxy-3a,8b-dihydro-1H-cyclopenta[b]benzofuran-1-one (9a) 


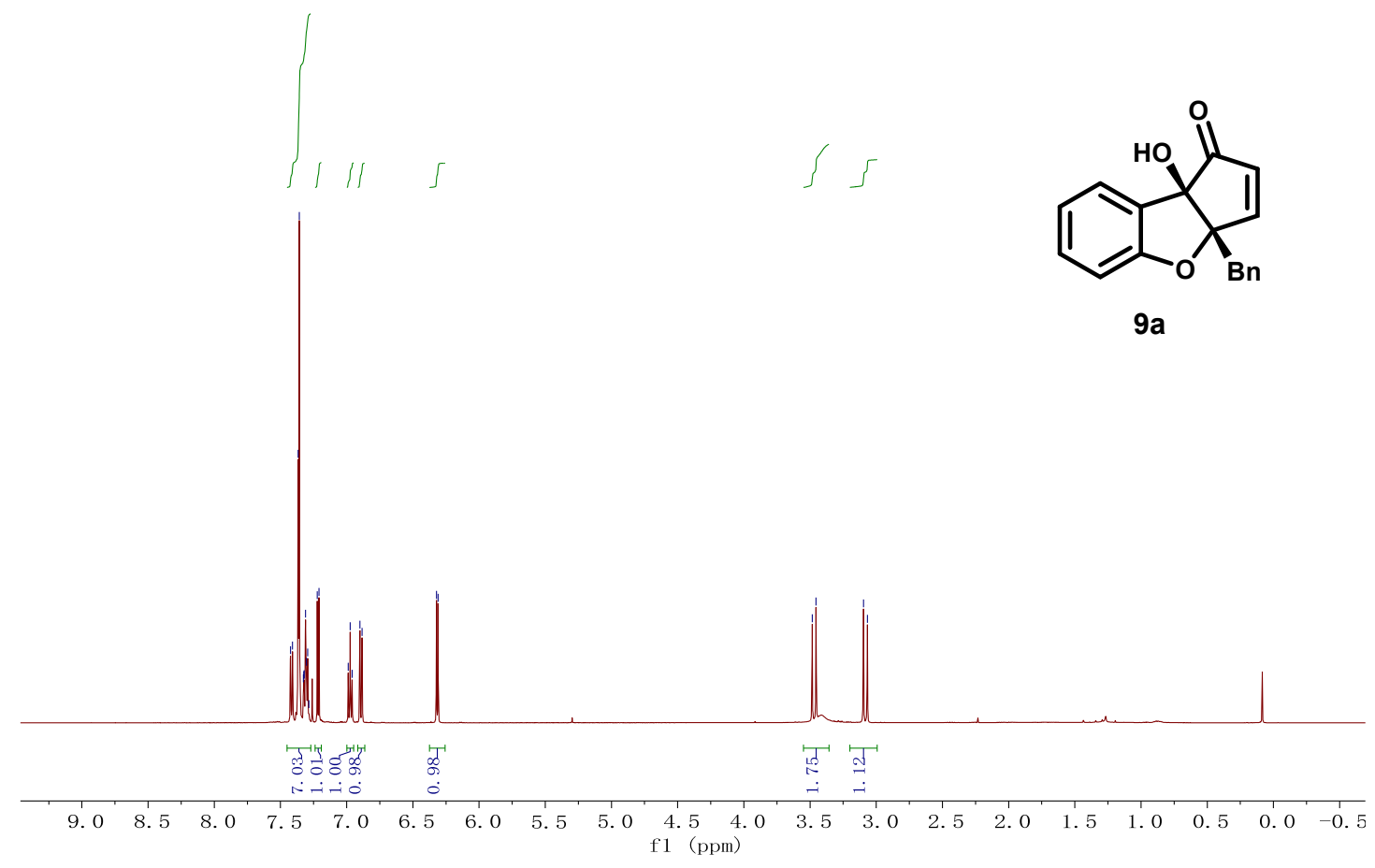

$\underline{{ }^{13} \mathrm{C} \text { NMR }}\left(125 \mathrm{MHz}, \mathrm{CDCl}_{3} @ 77\right.$ ppm)

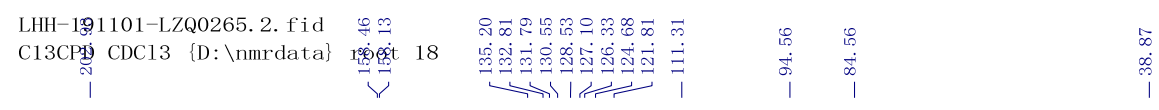

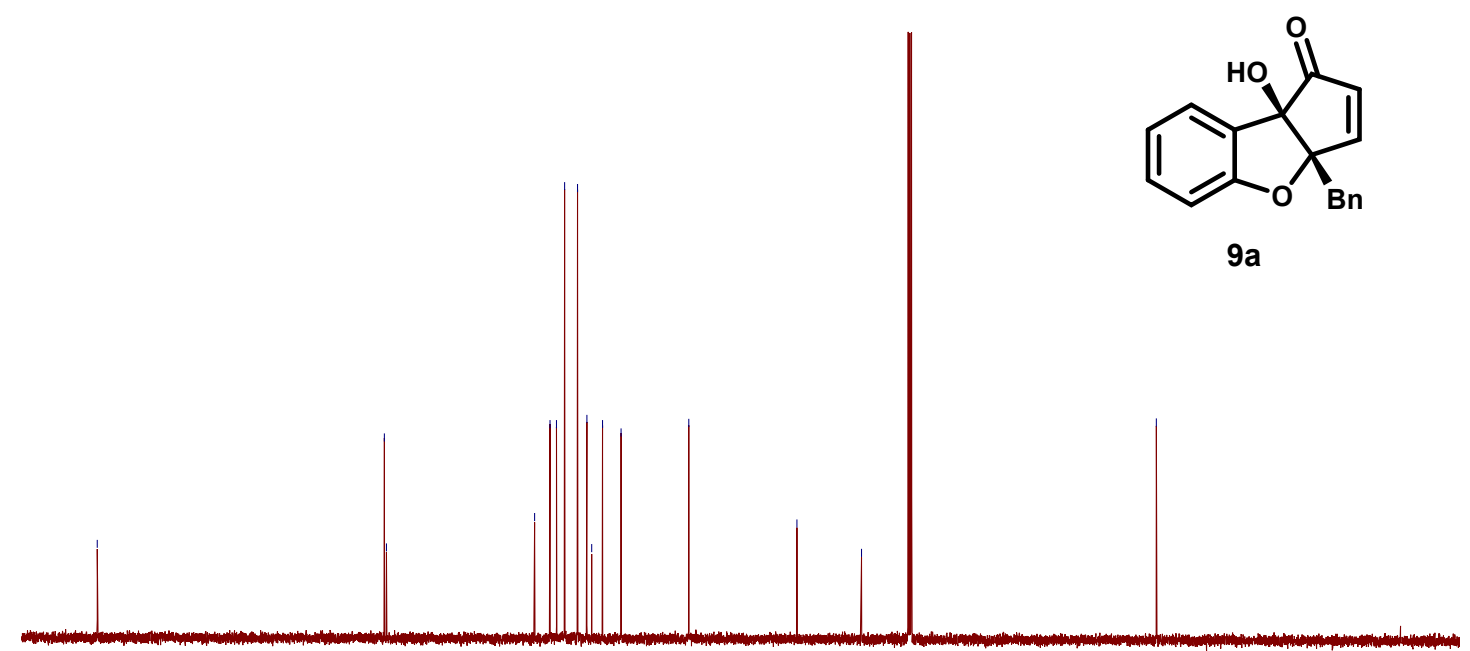

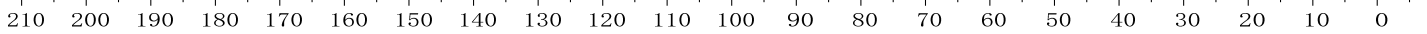

3aR,8bR)-8b-Hydroxy-6,8-dimethoxy-3a-phenyl-3a,8b-dihydro-1H-cyclopenta [b]benzofuran-1one $[( \pm)-9 b]$ 
$\underline{{ }^{1} \mathrm{H} N M R}\left(\mathrm{MHz}, \mathrm{CDCl}_{3} @ 7.26\right.$ ppm)

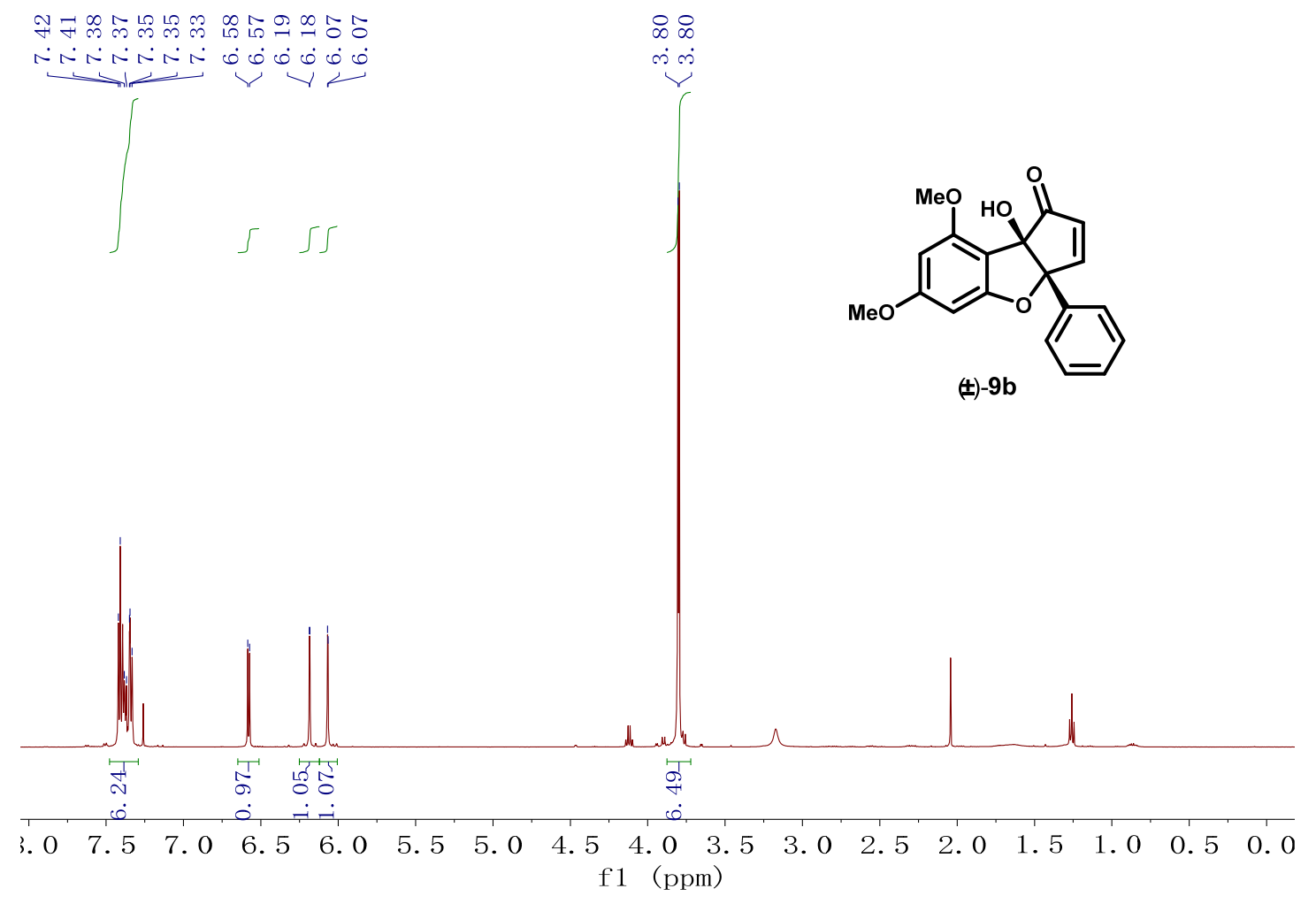

$\underline{{ }^{13} \mathrm{C} \mathrm{NMR}}\left(125 \mathrm{MHz}, \mathrm{CDCl}_{3} @ 77 \mathrm{ppm}\right)$
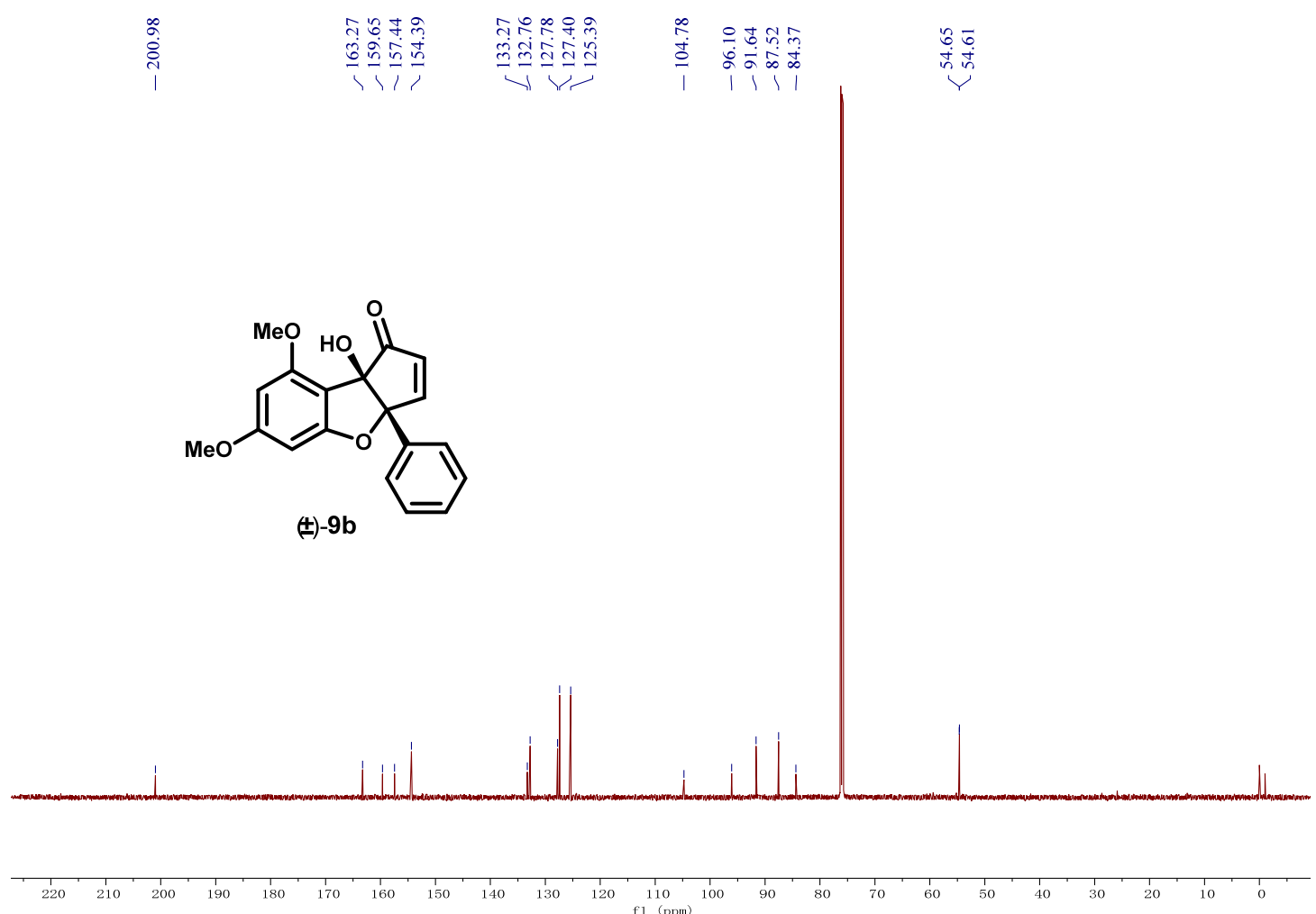

(3aS,8bR)-3a-Benzyl-8b-(benzyloxy)-3a,8b-dihydro-1 $H$-cyclopenta[b]benzofuran-1-one $[( \pm)-9 \mathrm{c}]$ 
1H NMR (500 MHz, CDCl $@ 7.26$ ppm)

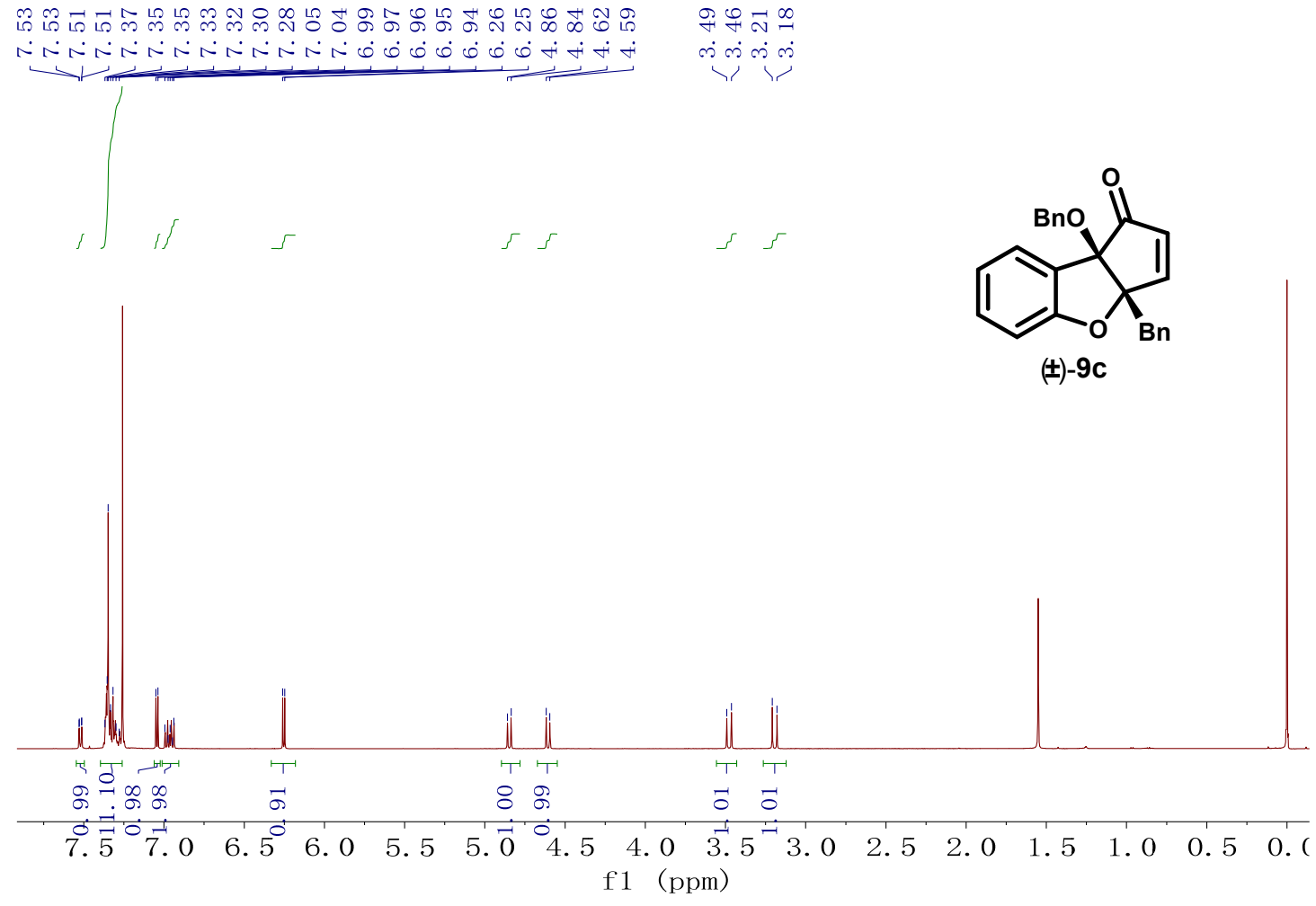

${ }^{13} \mathrm{C}$ NMR (125 MHz, $\mathrm{CDCl}_{3} @ 77$ ppm)

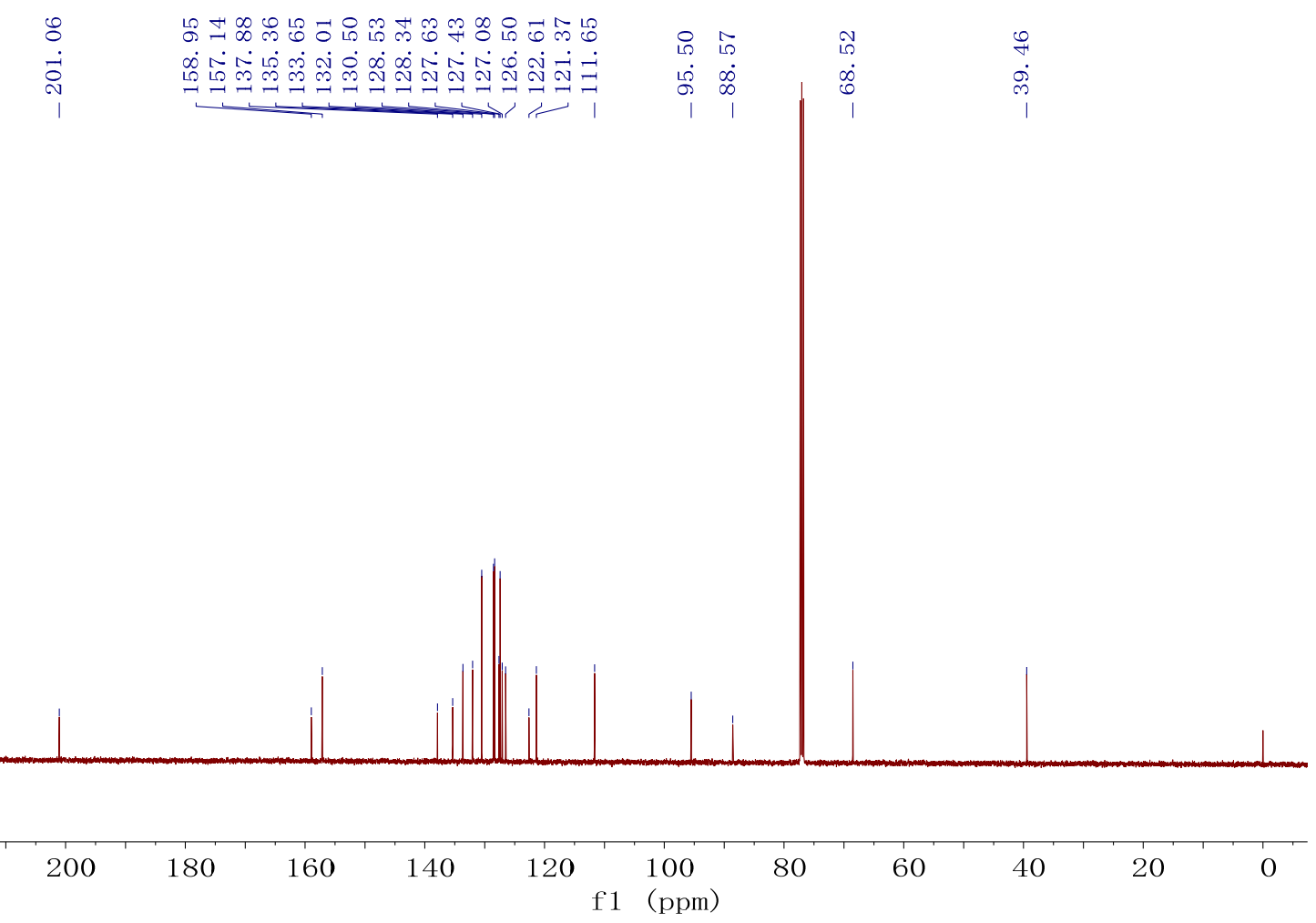

(3aS,8bR)-3a-Benzyl-8b-((tert-butyldimethylsilyl)oxy)-3a,8b-dihydro-1 $H$-cyclopenta[b]benzo furan-1-one $[( \pm)-9 d]$ 
1H NMR (500 MHz, $\mathrm{CDCl}_{3} @ 7.26$ ppm)

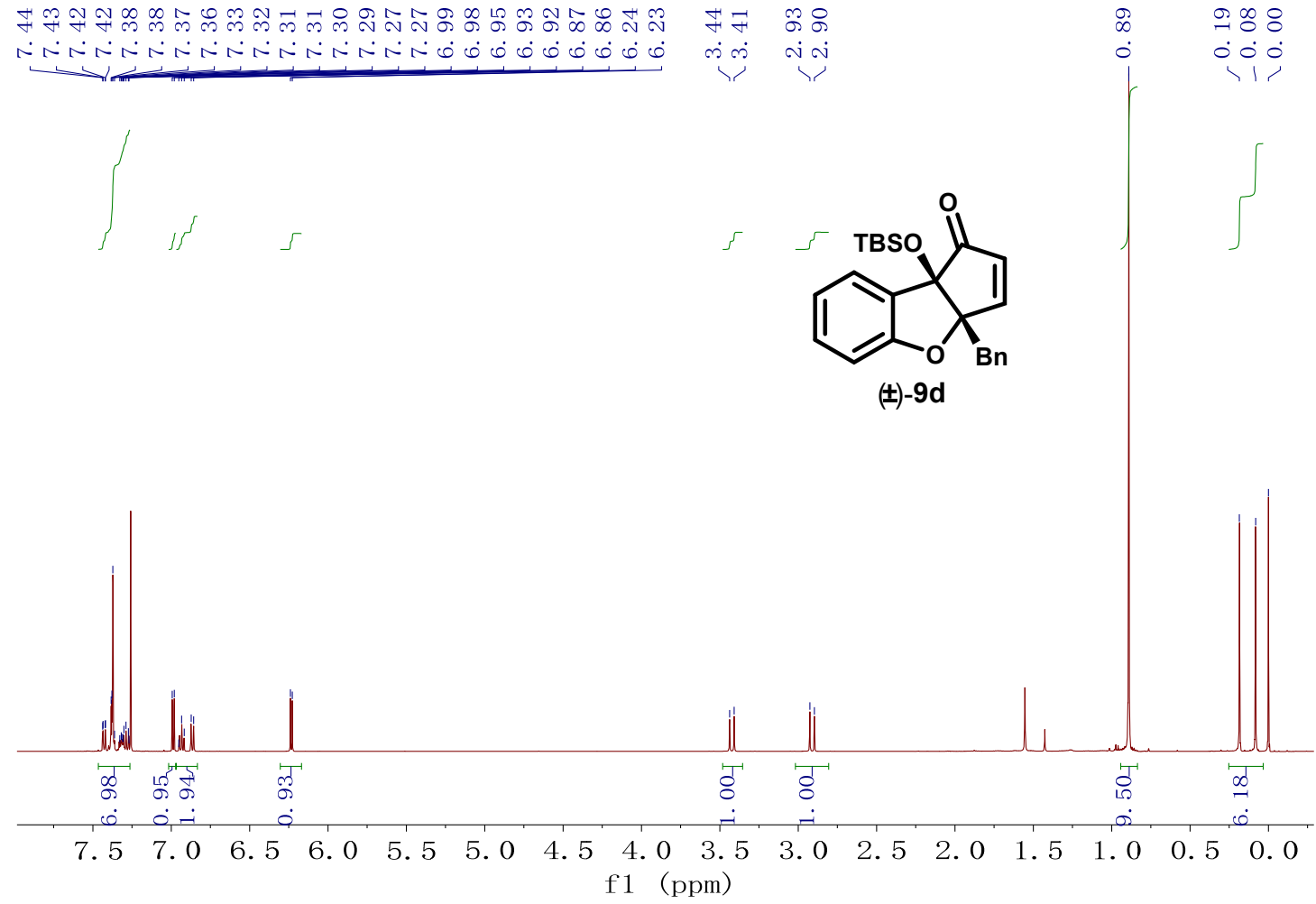

${ }^{13} \mathrm{C}$ NMR (125 MHz, CDCl 3 @ 77 ppm)

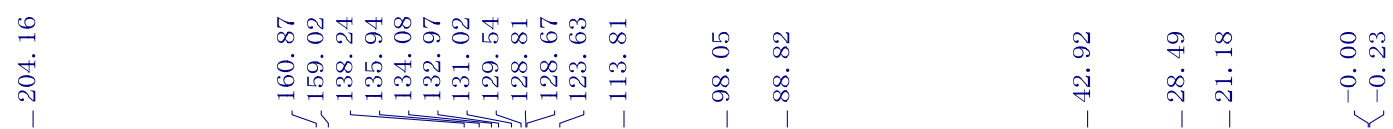

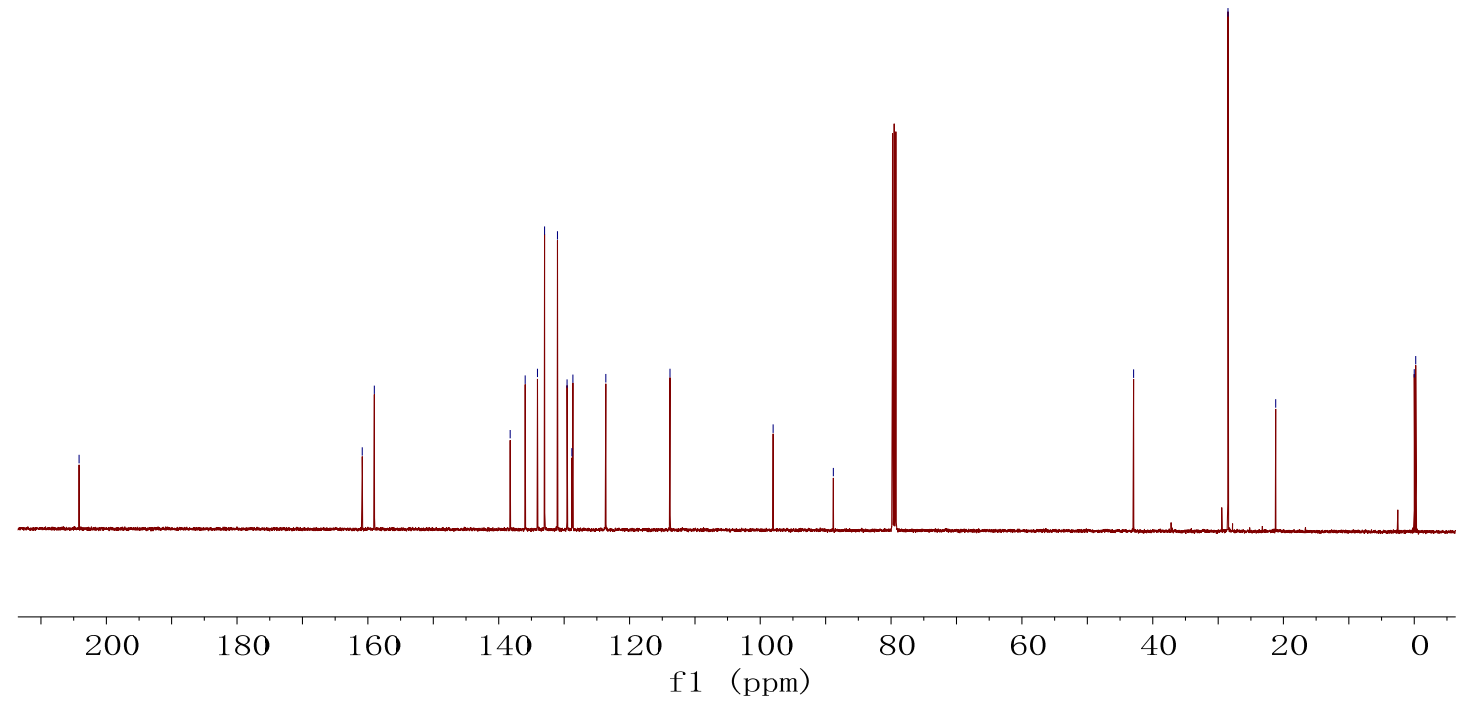

(3aS,8bR)-3a-Benzyl-2-bromo-8b-hydroxy-3a,8b-dihydro-1H-cyclopenta[b]benzofuran-1-one (16) 
$\underline{{ }^{1} \mathrm{H} \text { NMR }}\left(500 \mathrm{MHz}, \mathrm{CDCl}_{3} @ 7.26\right.$ ppm)

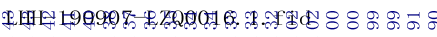

PROFOA

$\stackrel{\infty}{\rightarrow+} \stackrel{\infty}{\rightarrow} \stackrel{\infty}{N}=\infty$

मां
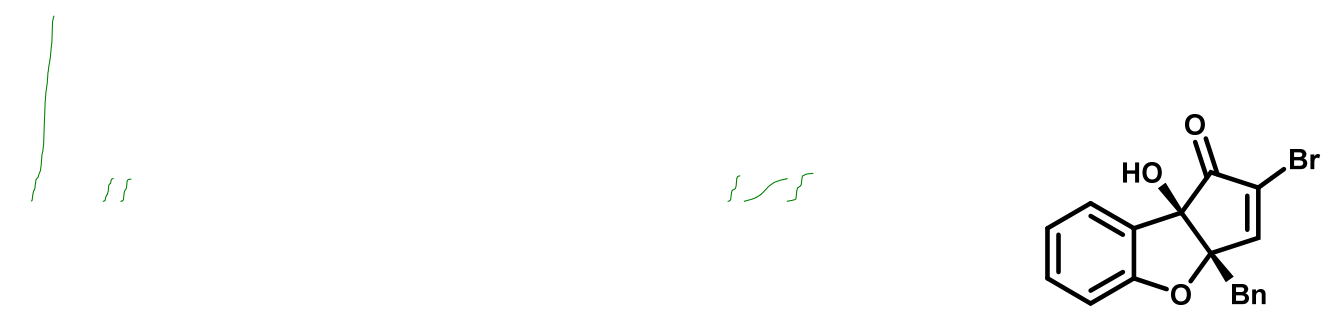

16

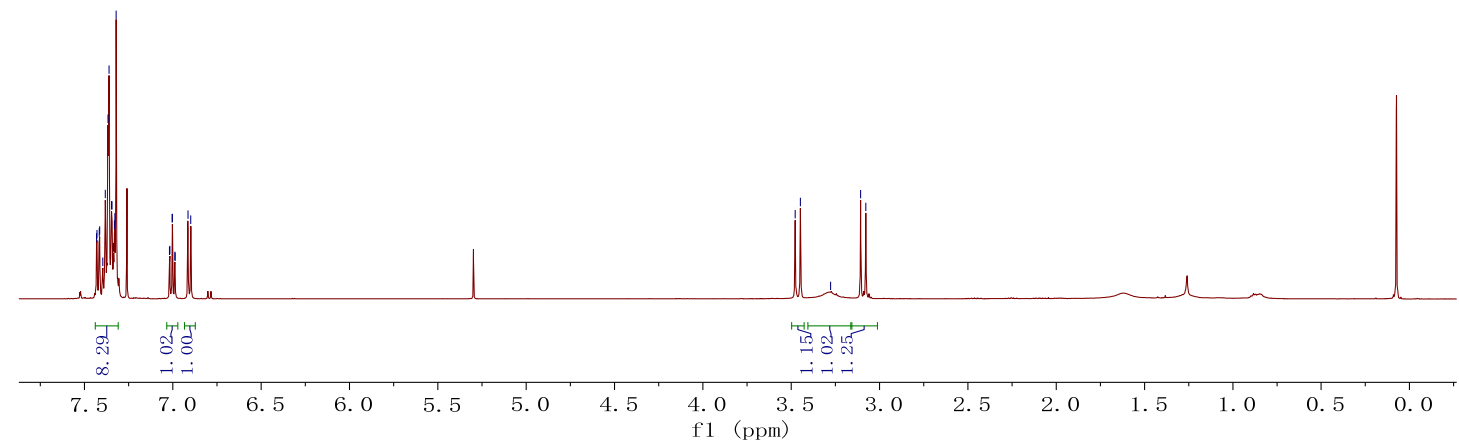

${ }^{13} \mathrm{C}$ NMR (125 MHz, $\mathrm{CDCl}_{3} @ 77$ ppm)

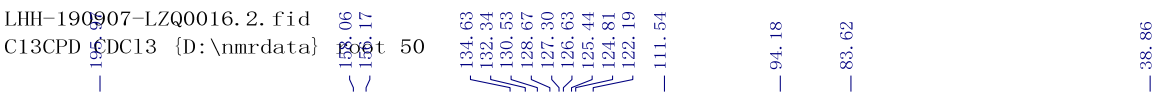

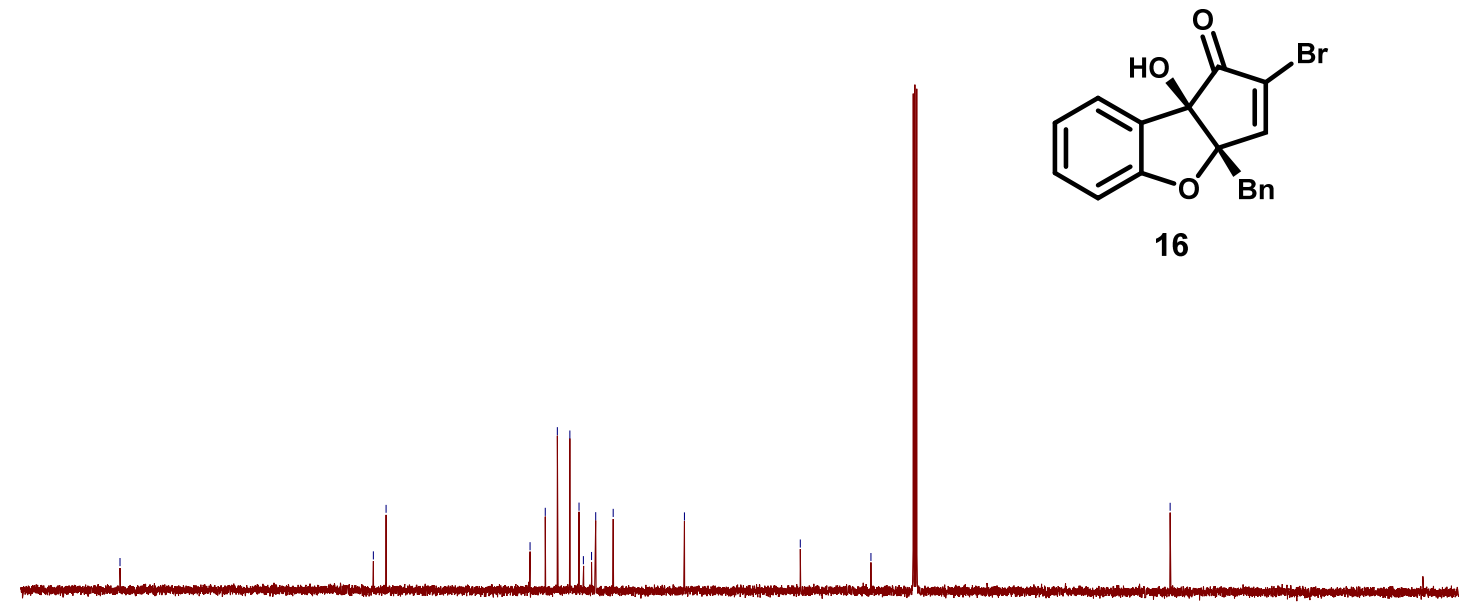

$\begin{array}{llllllllllll}10 & 200 & 190 & 180 & 170 & 160 & 150 & 140 & 130 & 120 & 110 & 100\end{array}$

$(3 R, 3 \mathrm{a} S, 8 \mathrm{~b} R)-3 \mathrm{a}-B$ enzyl-8b-hydroxy-3-isopropyl-2,3,3a,8b-tetrahydro-1H-cyclopenta $[b]$ benzofuran-1-one (17a) 
<smiles>CC(C)[C@H]1CC(=O)C2(O)c3ccccc3OC12Br</smiles>

$17 a$

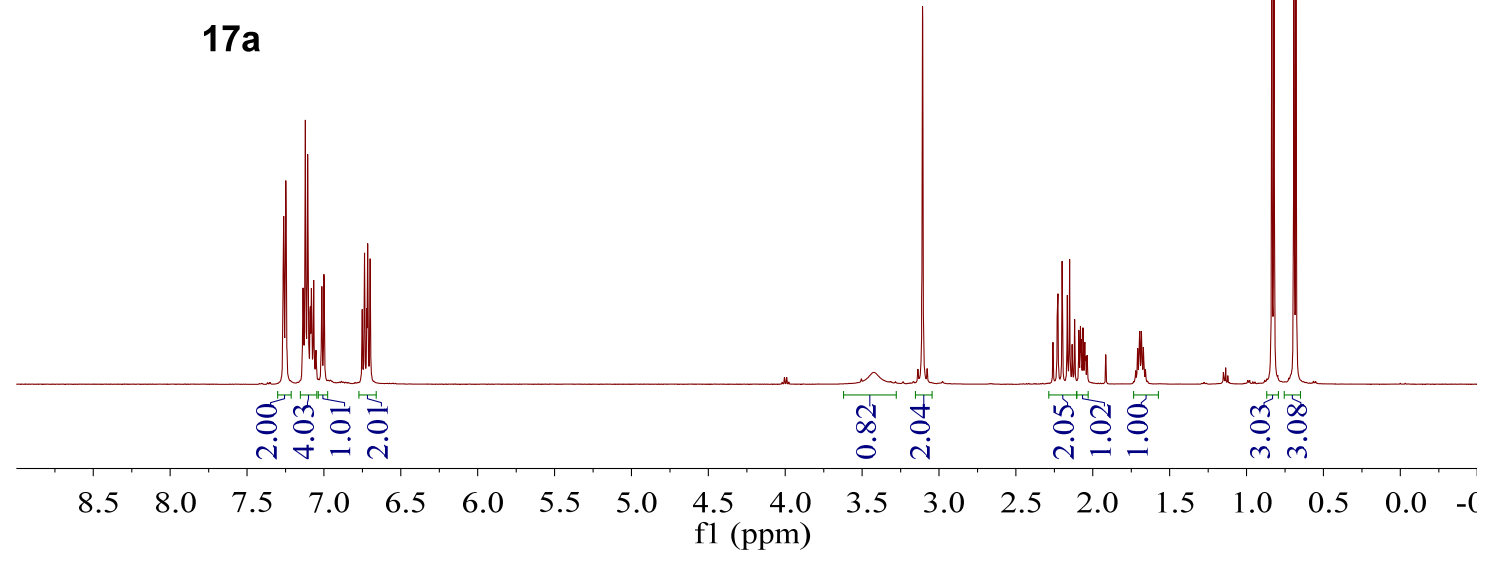

$\underline{{ }^{13} \mathrm{C} \text { NMR }}$ (125 MHz, $\mathrm{CDCl}_{3} @ 77$ ppm)
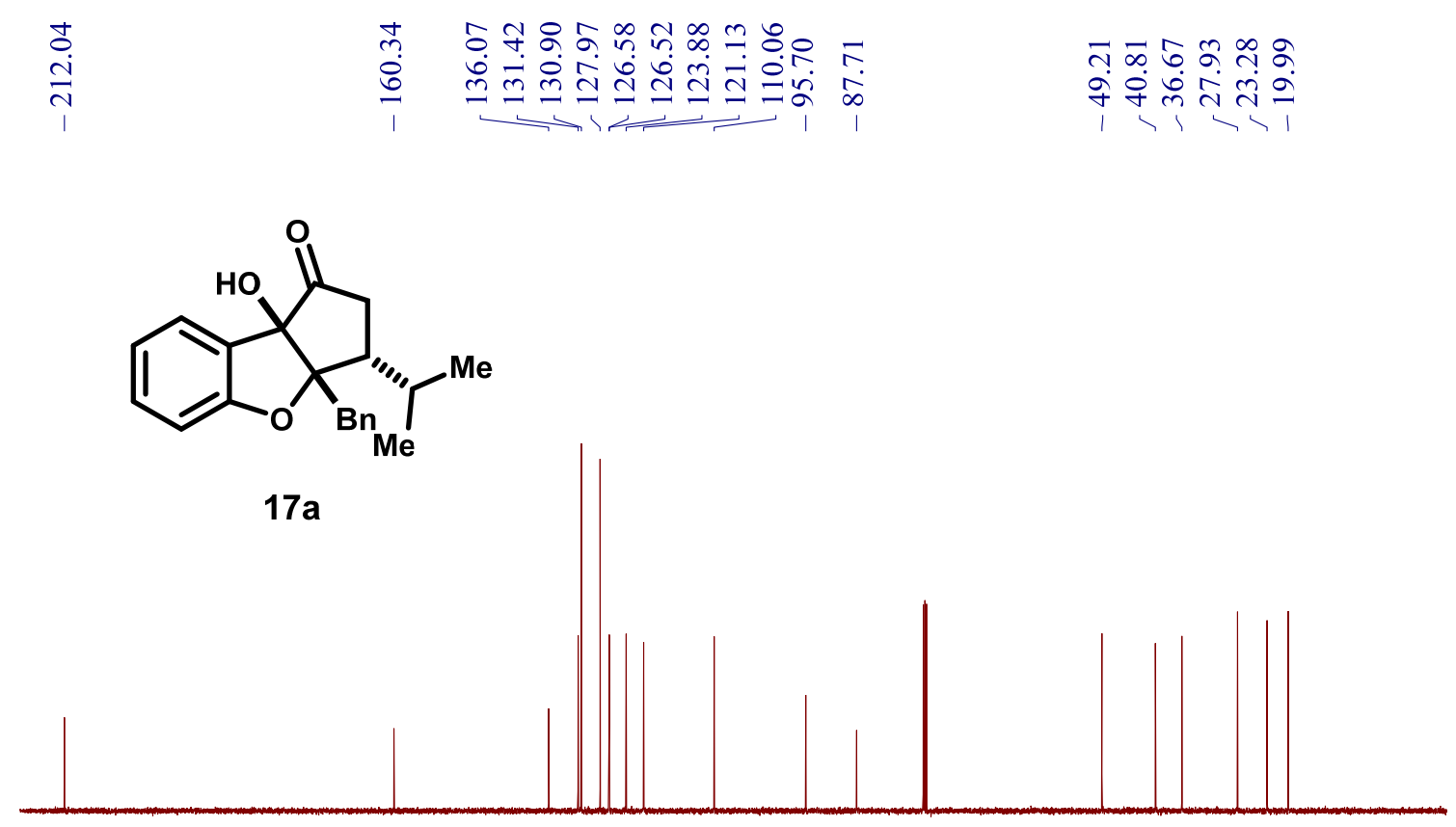

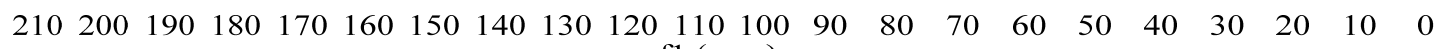
f1 (ppm)

( \pm )-(3S,3aS,8bR)-3a-Benzyl-3-ethyl-8b-hydroxy-2,3,3a,8b-tetrahydro-1H-cyclopenta[b]benzofuran1-one $[( \pm)-17 b]$ 


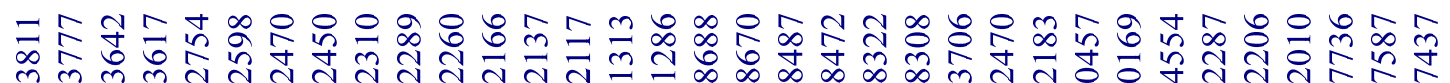

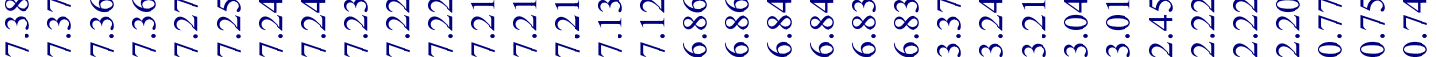

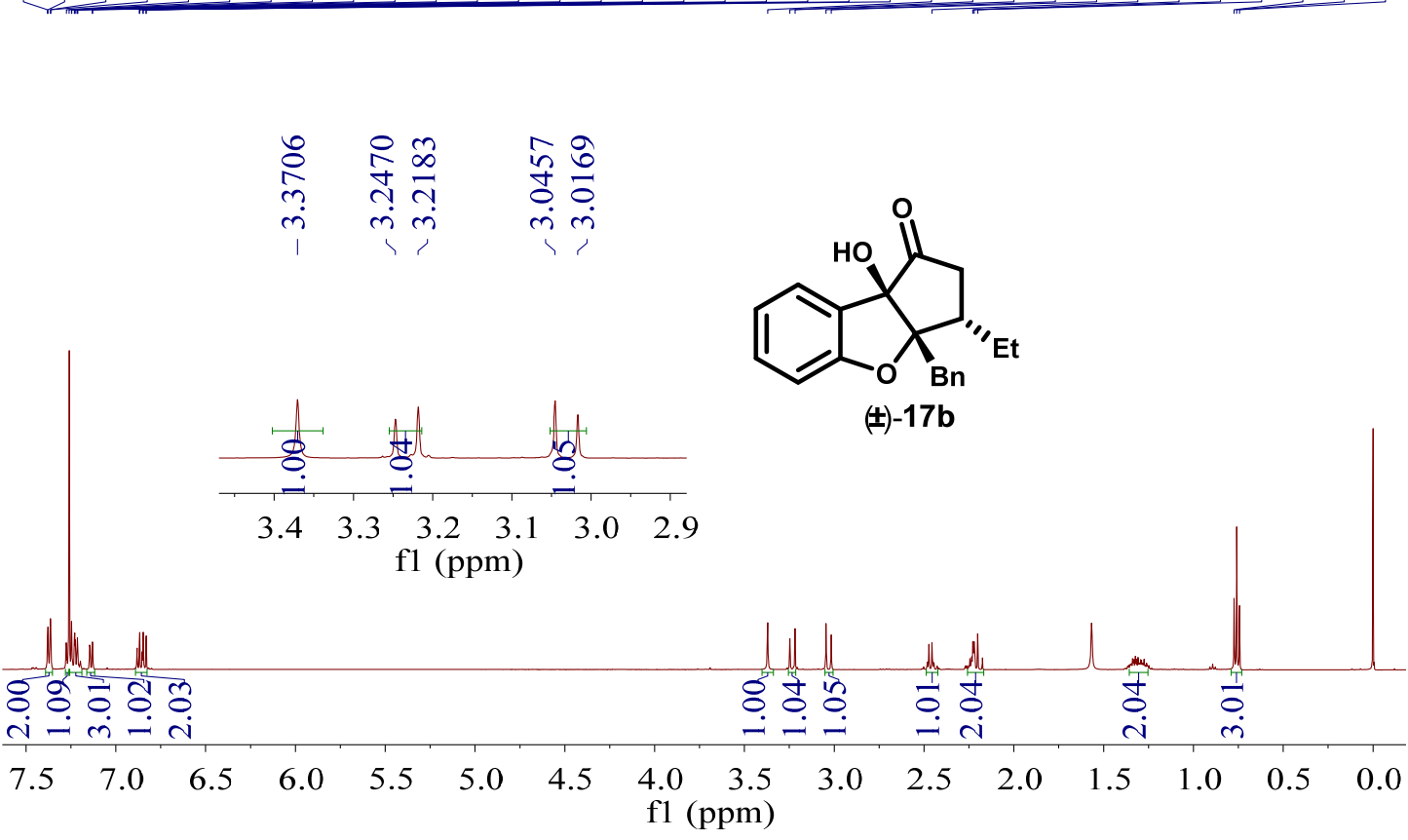

$\underline{{ }^{13} \mathrm{C} \mathrm{NMR}}\left(125 \mathrm{MHz}, \mathrm{CDCl}_{3} @ 77\right.$ ppm)
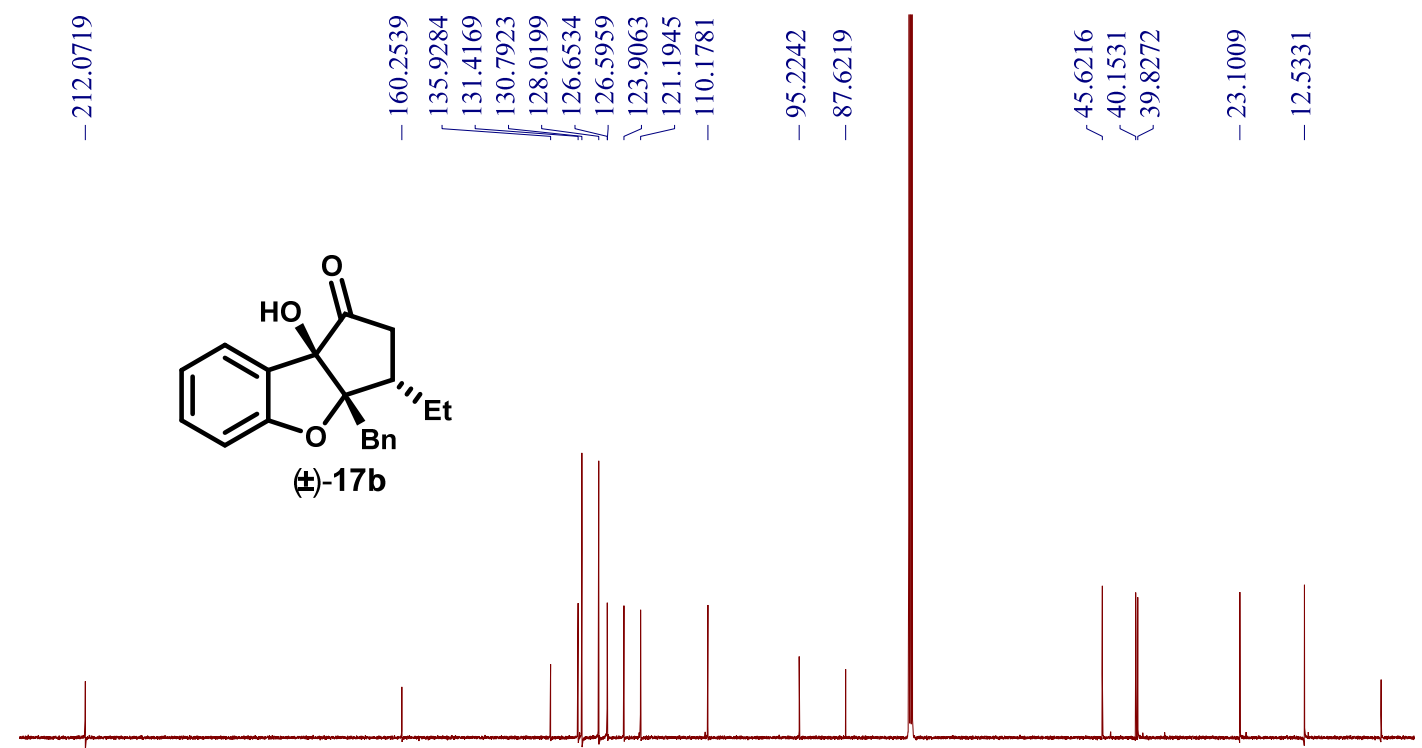

$\begin{array}{lllllllllllllllllllllll}220 & 210 & 200 & 190 & 180 & 170 & 160 & 150 & 140 & 130 & 120 & 110 & 100 & 90 & 80 & 70 & 60 & 50 & 40 & 30 & 20 & 10 & 0\end{array}$

( \pm -3-epi-(3R,3aS,8bR)-3a-Benzyl-3-ethyl-8b-hydroxy-2,3,3a,8b-tetrahydro-1H-cyclopenta[b] benzofuran-1-one $[( \pm)-3-$ epi-17b] 


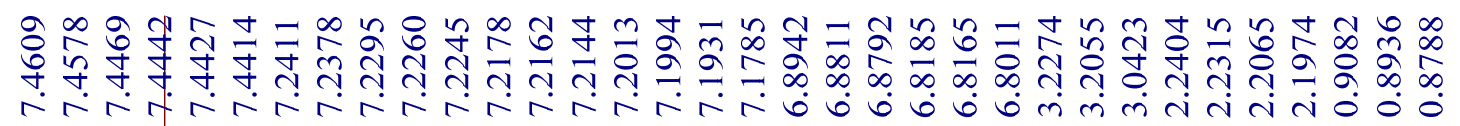

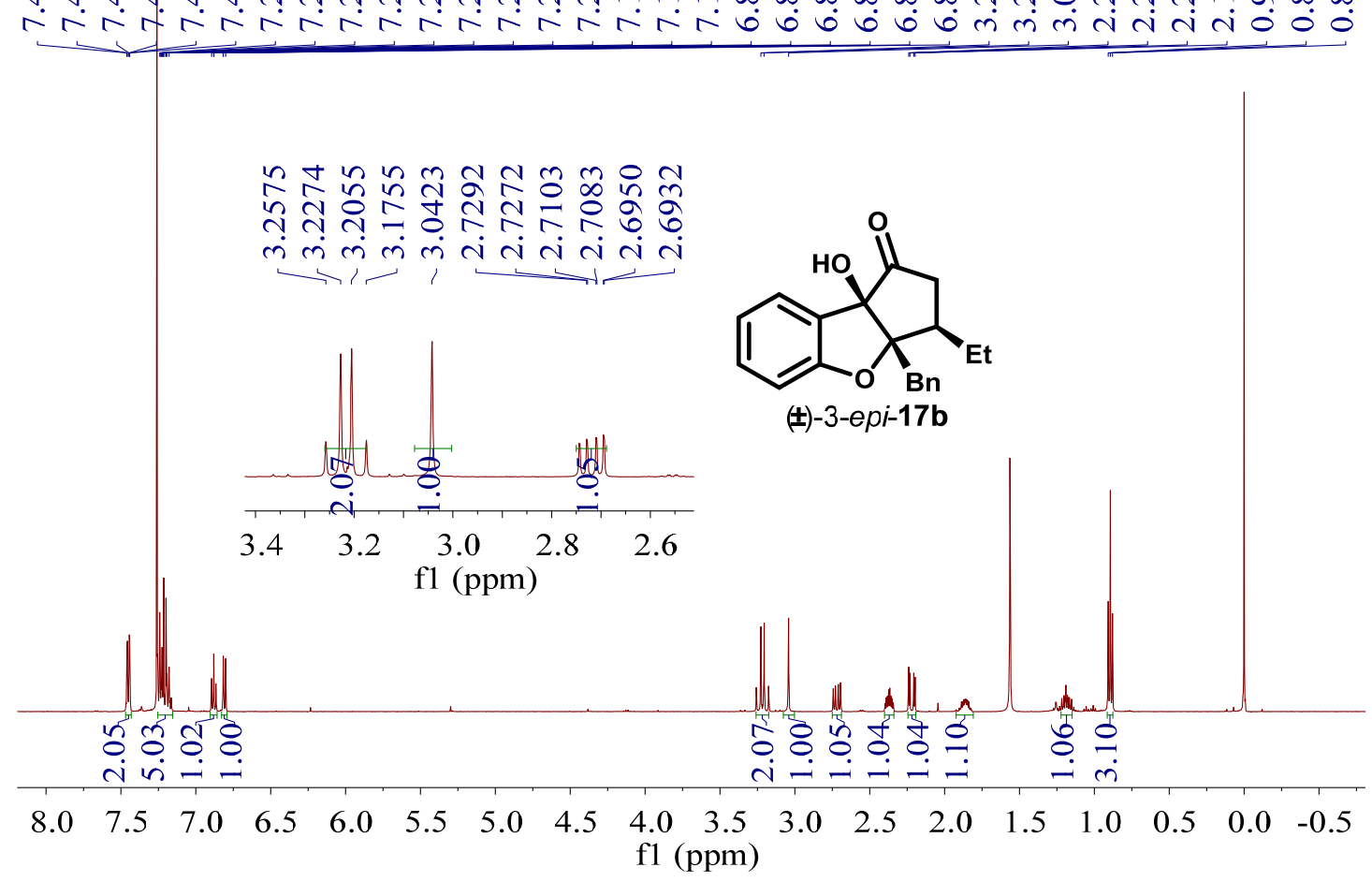

${ }^{13} \mathrm{C}$ NMR (125 MHz, CDCl 377 ppm)

$\infty$
$\stackrel{+}{0}$
$\stackrel{1}{1}$

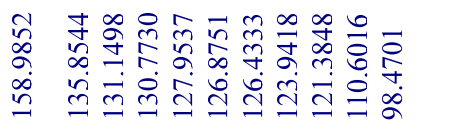

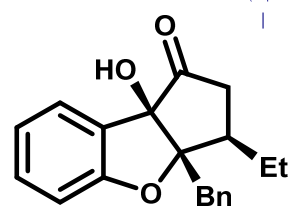

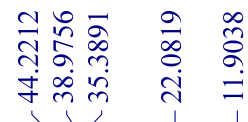

(士)-3-epi-17b

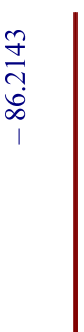


(3S,3aS,8bR)-3a-Benzyl-8b-hydroxy-3-methyl-2,3,3a,8b-tetrahydro-1 $H$-cyclopenta $[b]$ benzofuran-1one $(17 \mathrm{c})$

$\underline{1}$ H NMR $\left(500 \mathrm{MHz}, \mathrm{CDCl}_{3} @ 7.26\right.$ ppm)

I

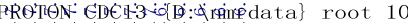

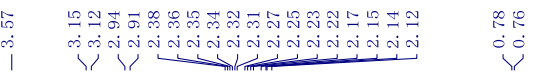
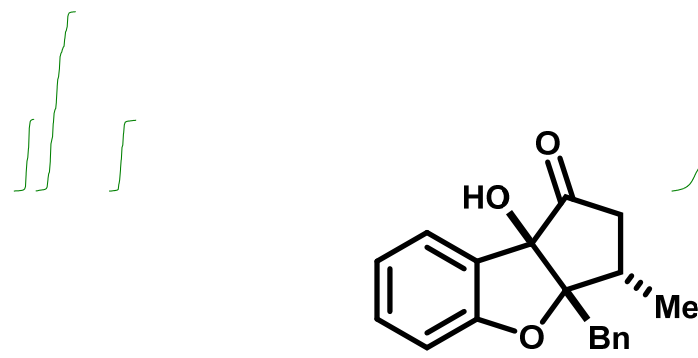

$17 \mathrm{c}$
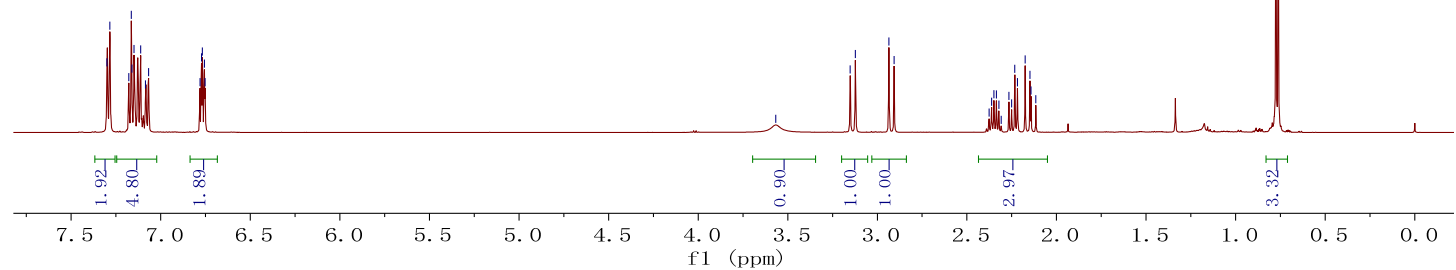

${ }^{13} \mathrm{C}$ NMR (125 MHz, CDCl $3 @ 77$ ppm)

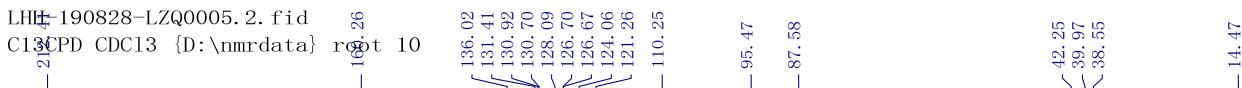

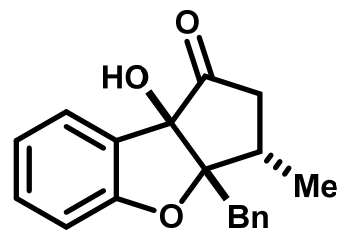

$17 \mathrm{c}$

$\begin{array}{lllllllllllll}210 & 200 & 190 & 180 & 170 & 160 & 150 & 140 & 130 & 120 & 110 & 100 & 90\end{array}$

( \pm )- (3R,3aS,8bR)-3a-Benzyl-8b-hydroxy-3-phenyl-2,3,3a,8b-tetrahydro-1H-cyclopenta[b]benzo- 
furan-1-one $[( \pm)-17 d]$

1․ NMR (500 MHz, $\mathrm{CDCl}_{3} @ 7.26$ ppm)

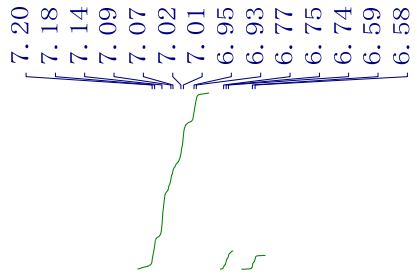

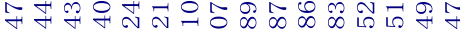

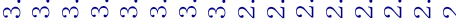

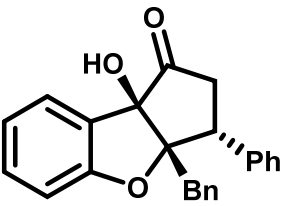

$( \pm)-17 d$

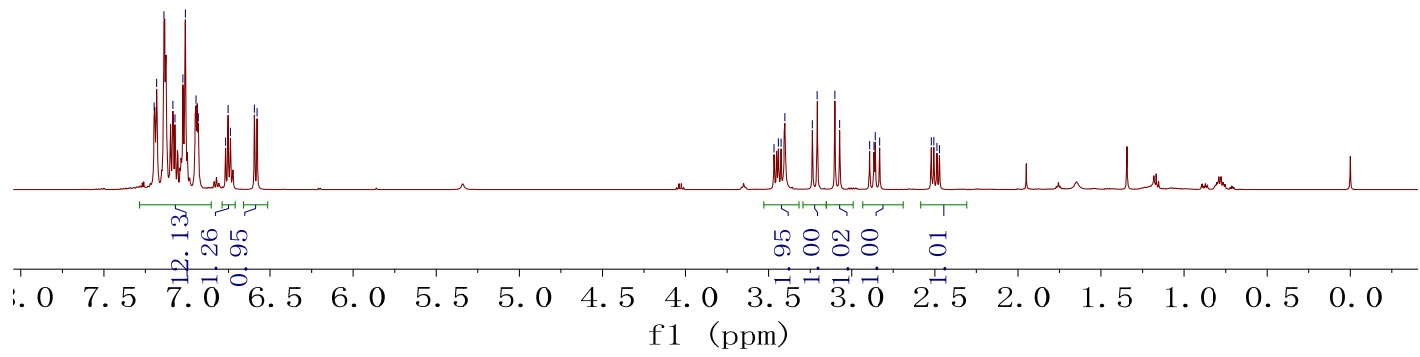

${ }^{13} \mathrm{C}$ NMR (125 MHz, CDCl 3 @ 77 ppm)
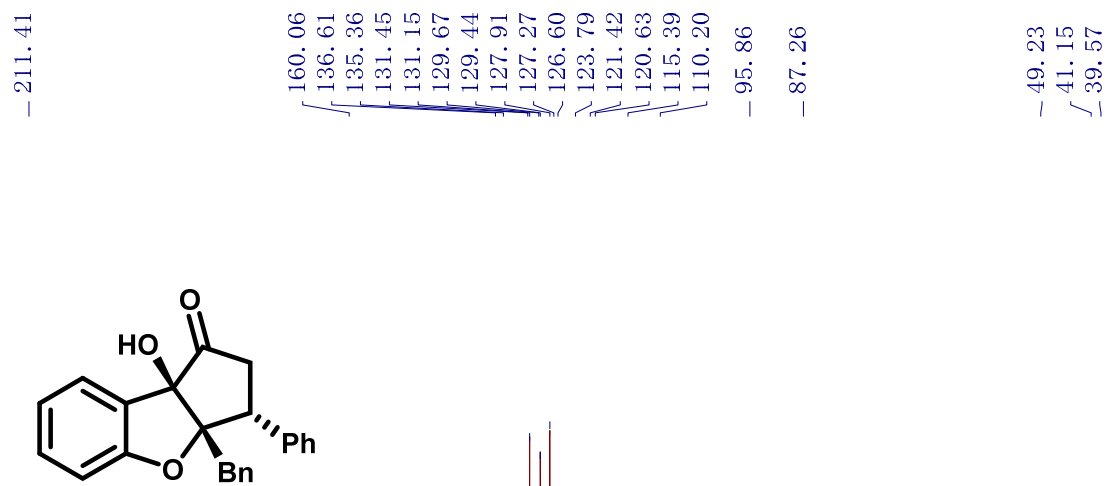

$( \pm)-17 d$

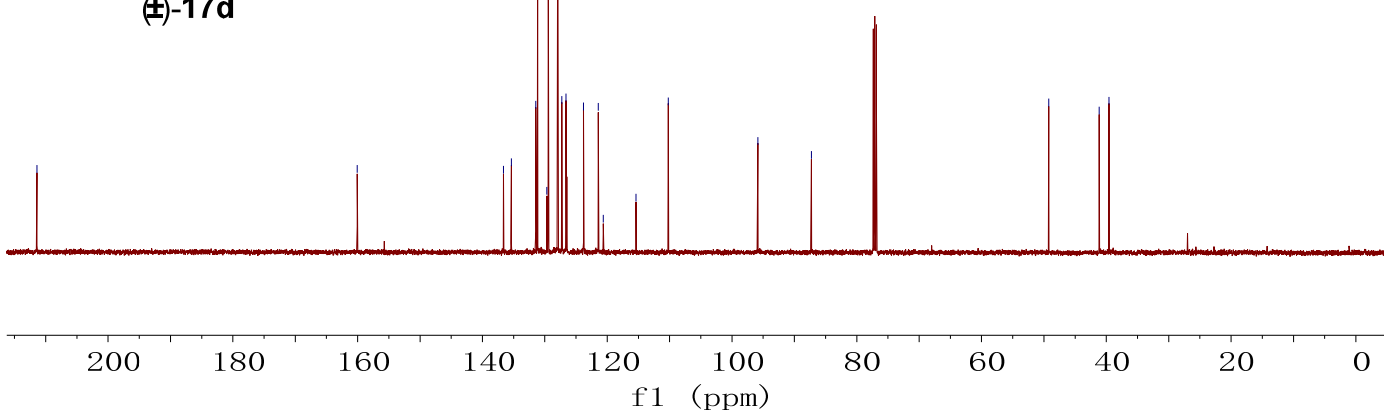

(3R,3aR,8bR)-8b-Hydroxy-6,8-dimethoxy-3,3a-diphenyl-2,3,3a,8b-tetrahydro-1H- 
cyclopenta $[b]$ benzofuran-1-one: $[( \pm)-17 \mathrm{e}]$

므 NMR $\left(500 \mathrm{MHz}, \mathrm{CDCl}_{3} @ 7.26\right.$ ppm)

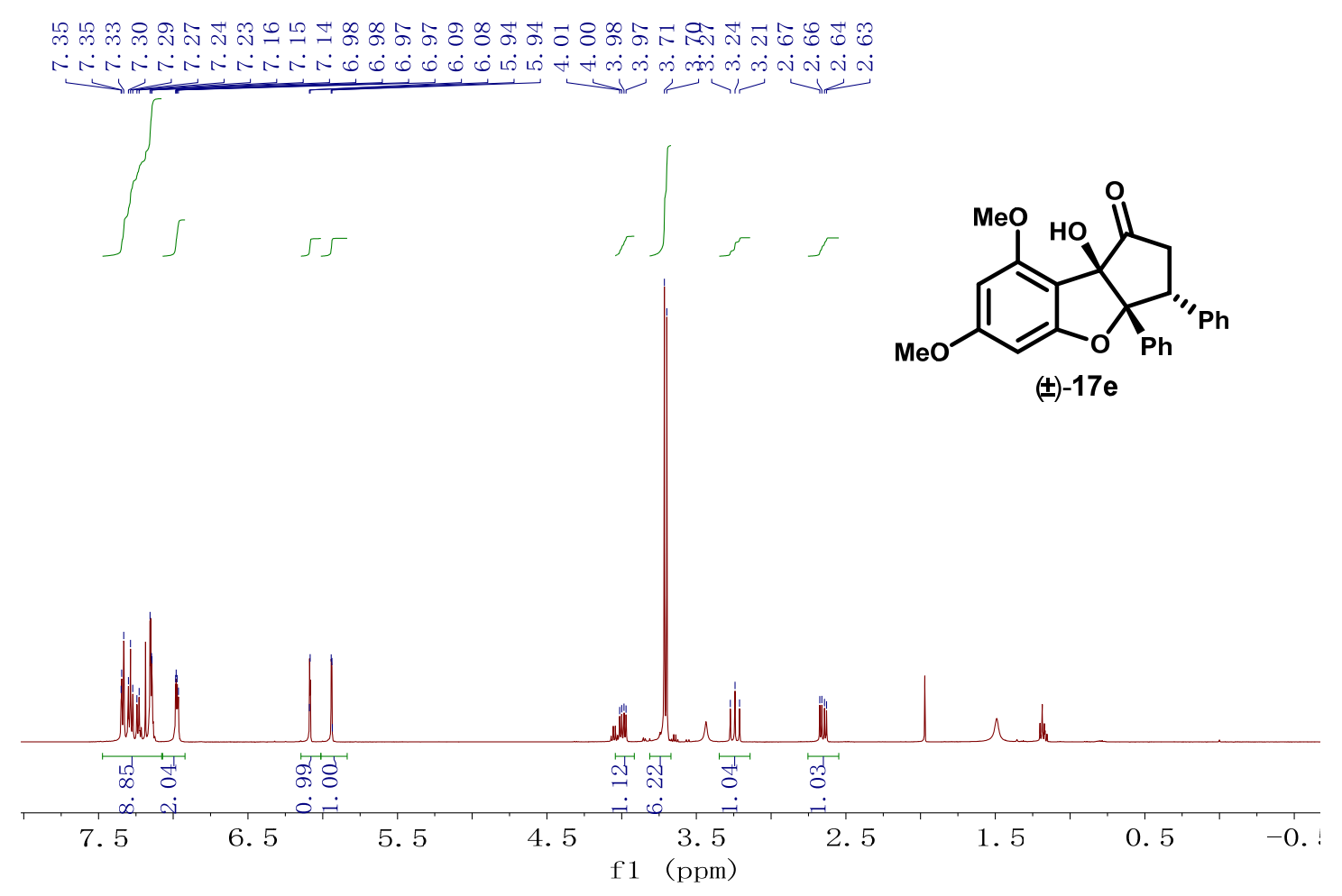

13. NMR (125 MHz, CDCl $@ 77$ ppm)

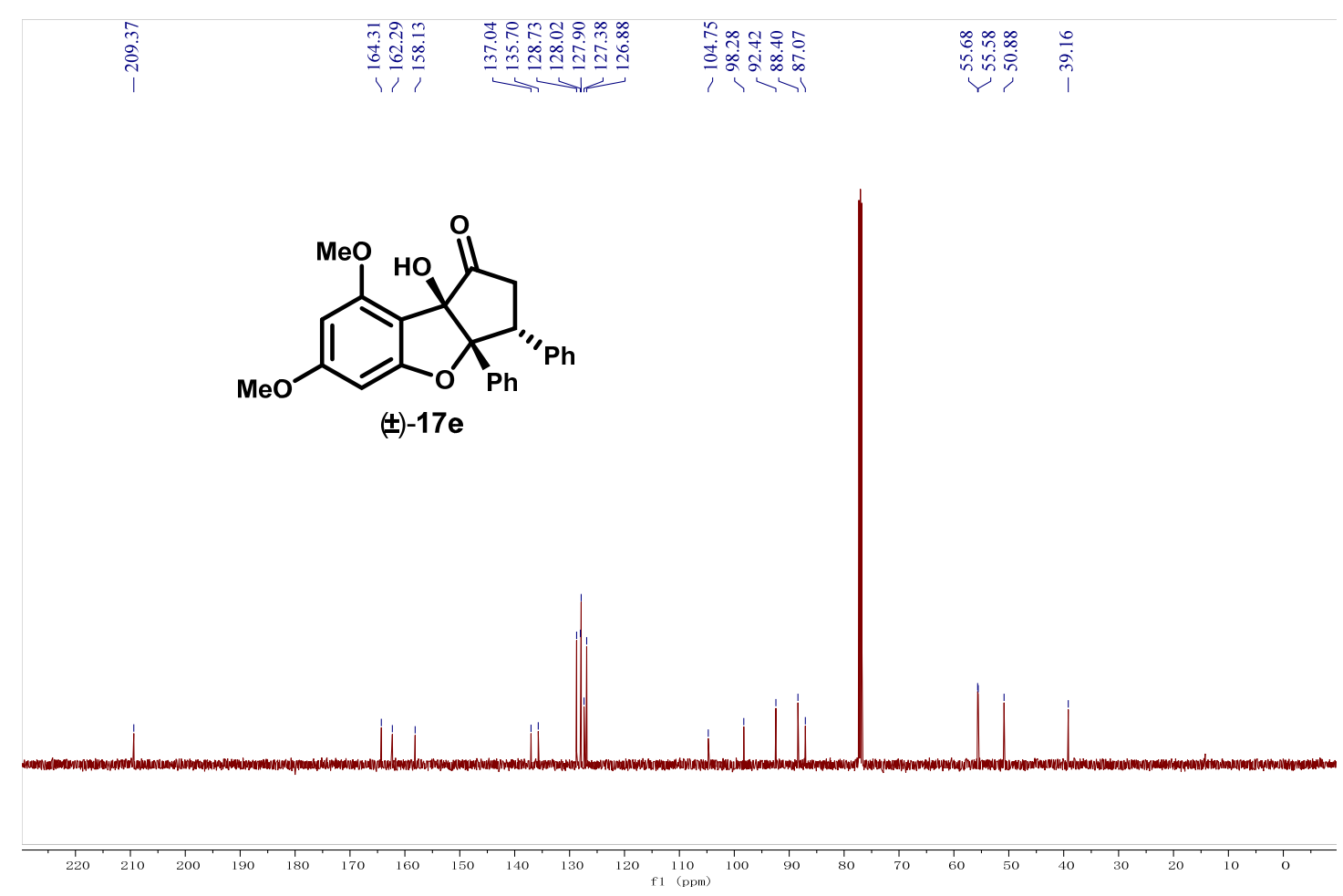


Methyl (3aS,8bR)-3a-benzyl-8b-hydroxy-1-oxo-3a,8b-dihydro-1H-cyclopenta[b]benzofuran-2carboxylate $[( \pm)-9 e]$

1H NMR $\left(500 \mathrm{MHz}, \mathrm{CDCl}_{3} @ 7.26\right.$ ppm)

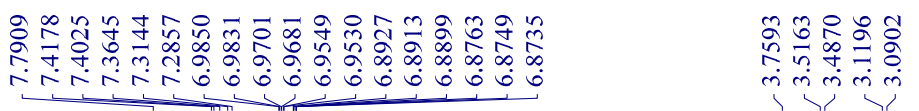

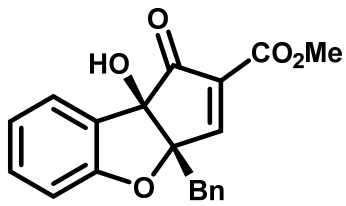

$( \pm)-9 e$

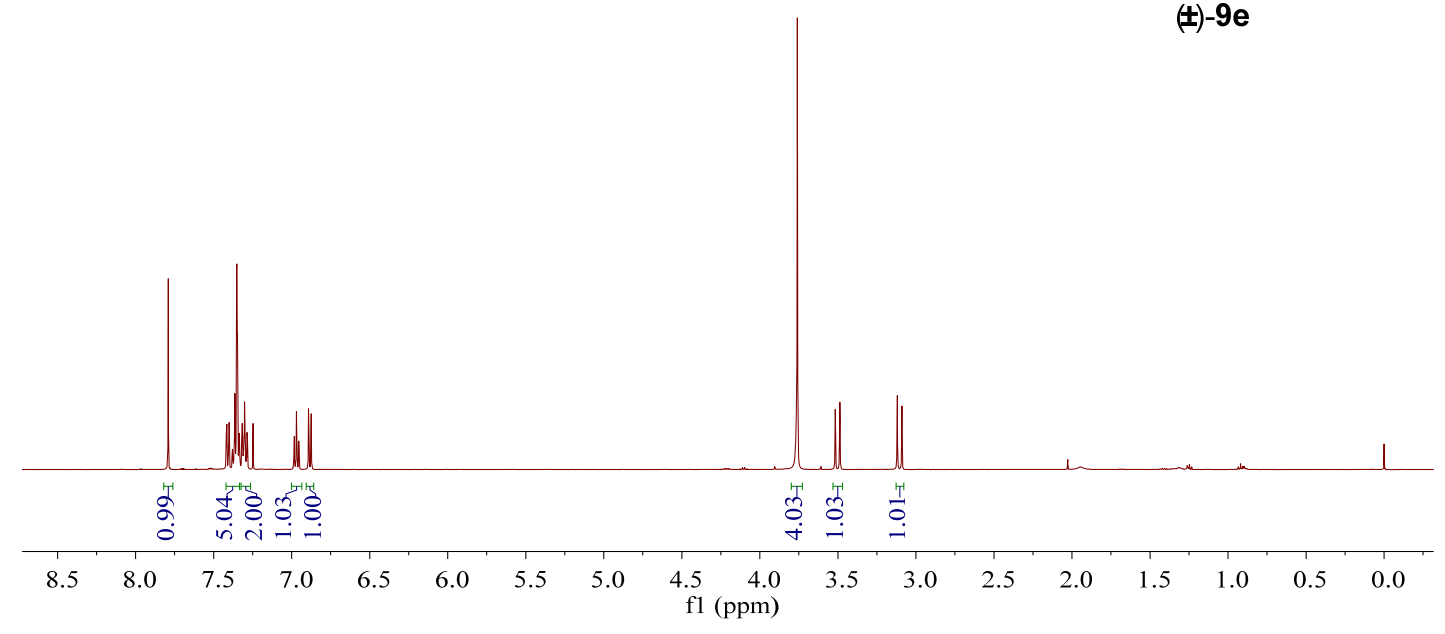

${ }^{13} \mathrm{C}$ NMR (125 MHz, $\mathrm{CDCl}_{3} @ 77$ ppm)
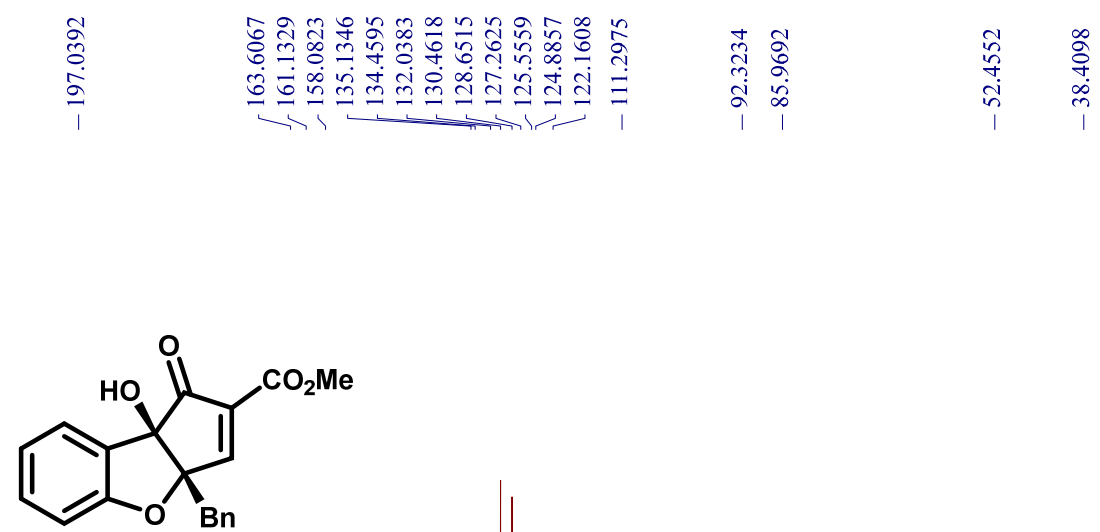

$( \pm)-9 e$

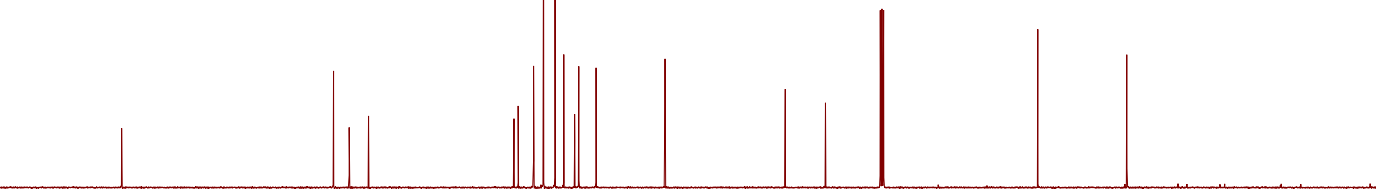

$\begin{array}{llllllllllllllllllllll}210 & 200 & 190 & 180 & 170 & 160 & 150 & 140 & 130 & 120 & 110 & 100 & 90 & 80 & 70 & 60 & 50 & 40 & 30 & 20 & 10 & 0\end{array}$ 
(3S,3aS,8bR)-3a-Benzyl-8b-hydroxy-3-isopropyl-2,3,3a,8b-tetrahydro-1H- cyclopenta[b]benzofuran-1one (3-epi-17b)

$\underline{{ }^{1} \mathrm{H} \text { NMR }}\left(500 \mathrm{MHz}, \mathrm{CDCl}_{3} @ 7.26\right.$ ppm)

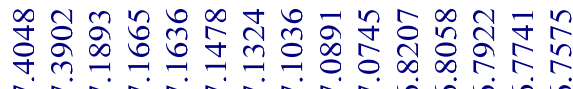

ปิ

m m

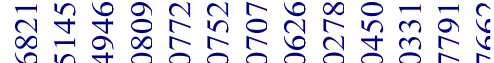

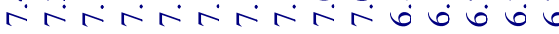

ina iuciari-joc

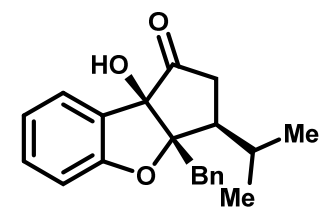

3-epi-17a

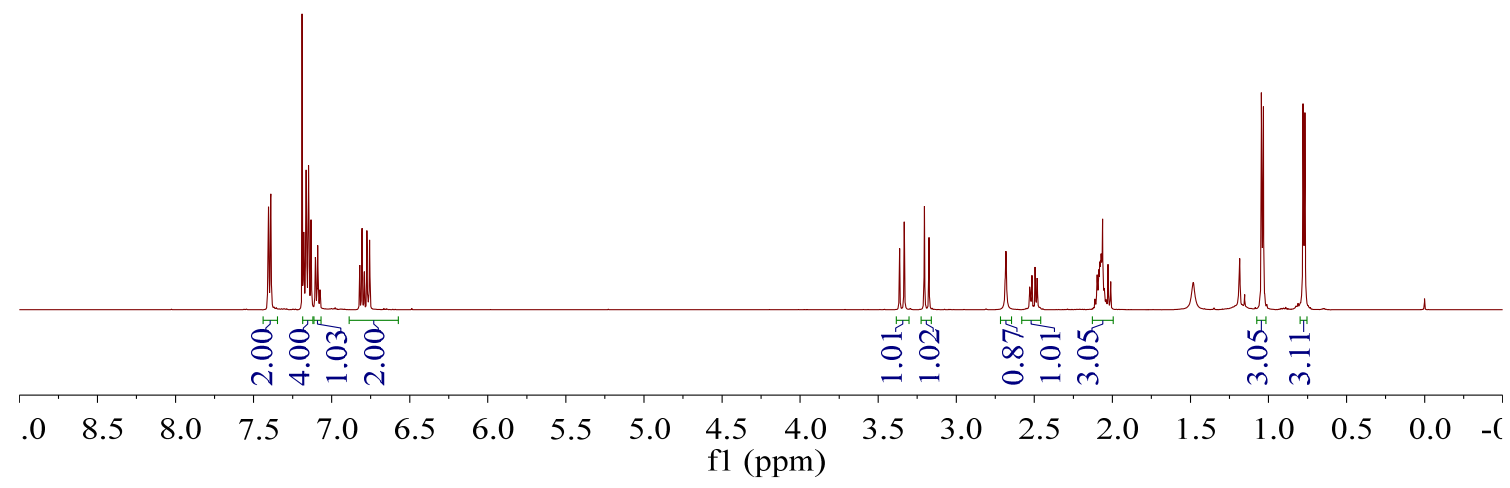

${ }^{13} \mathrm{C}$ NMR (125 MHz, CDCl $@ 77$ ppm) 


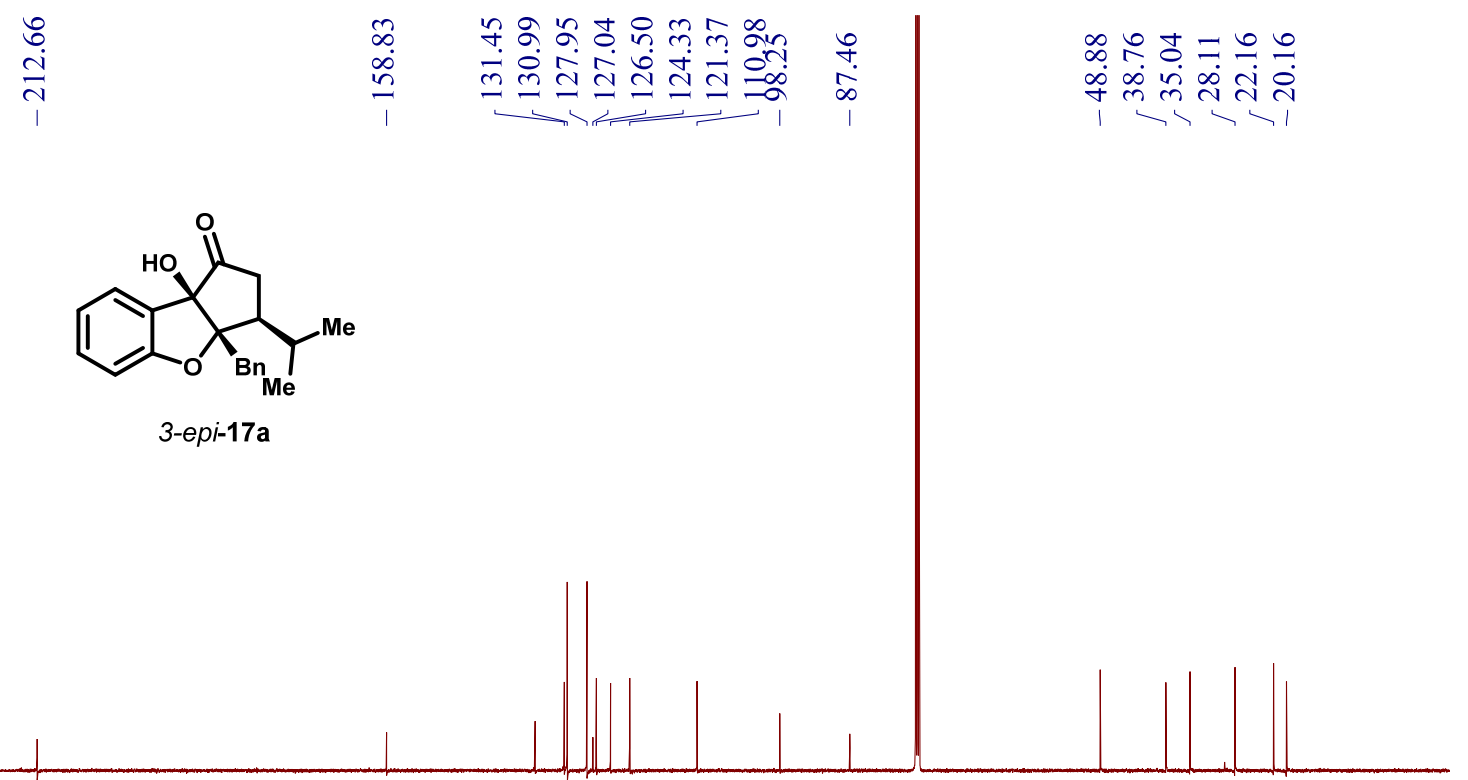

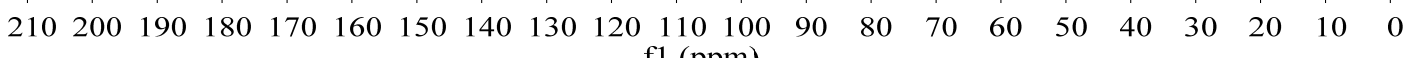
f1 (ppm)

$(1 R, 3 R, 3 \mathrm{a} S, 8 \mathrm{~b} S)$-3a-Benzyl-3-isopropyl-1,2,3,3a-tetrahydro-8b $H$-cyclopenta $[b]$ benzofuran-1,8b-diol (18)

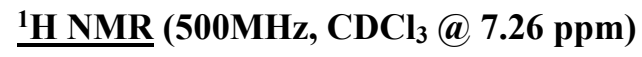

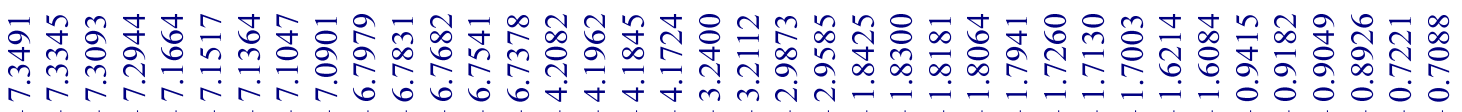

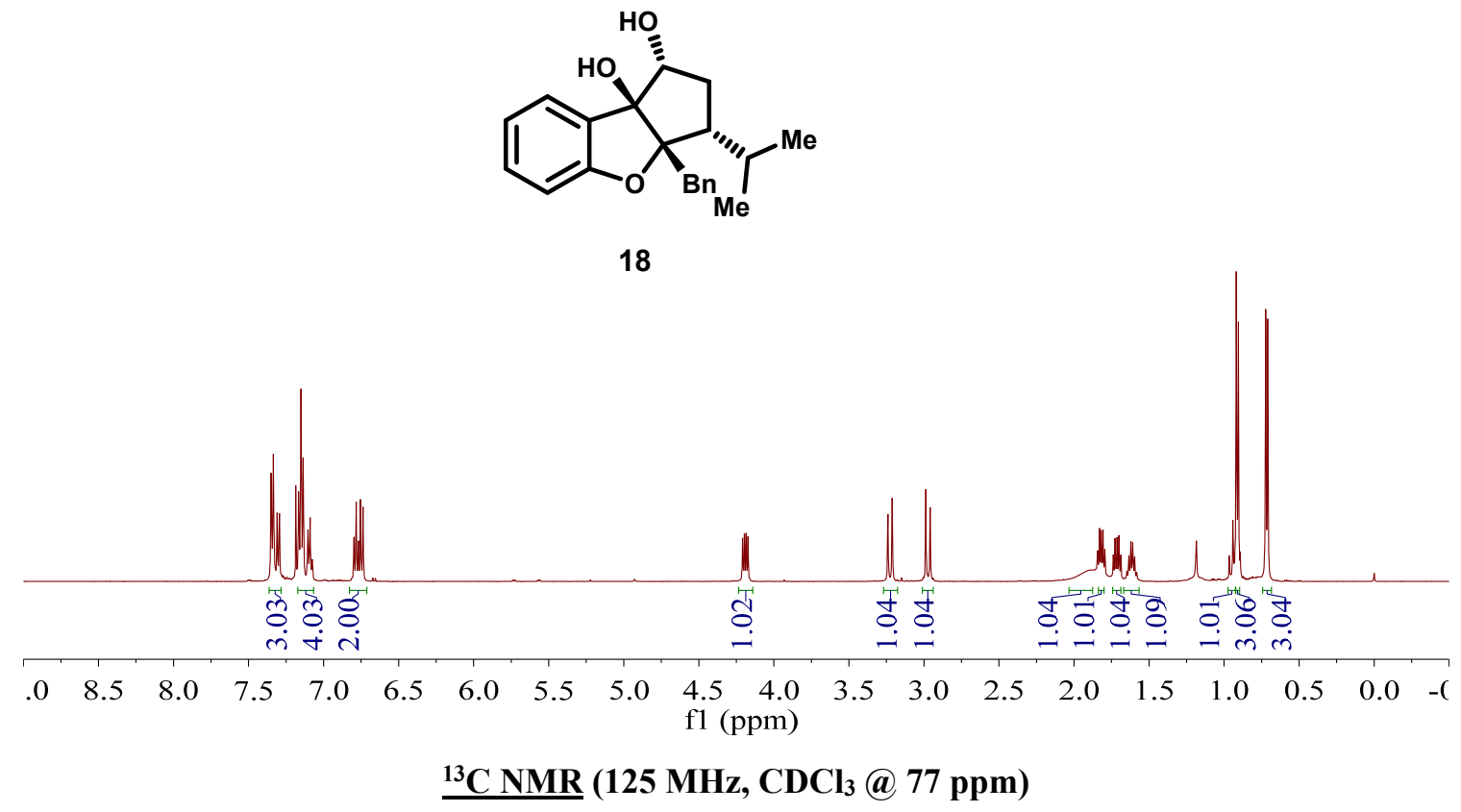



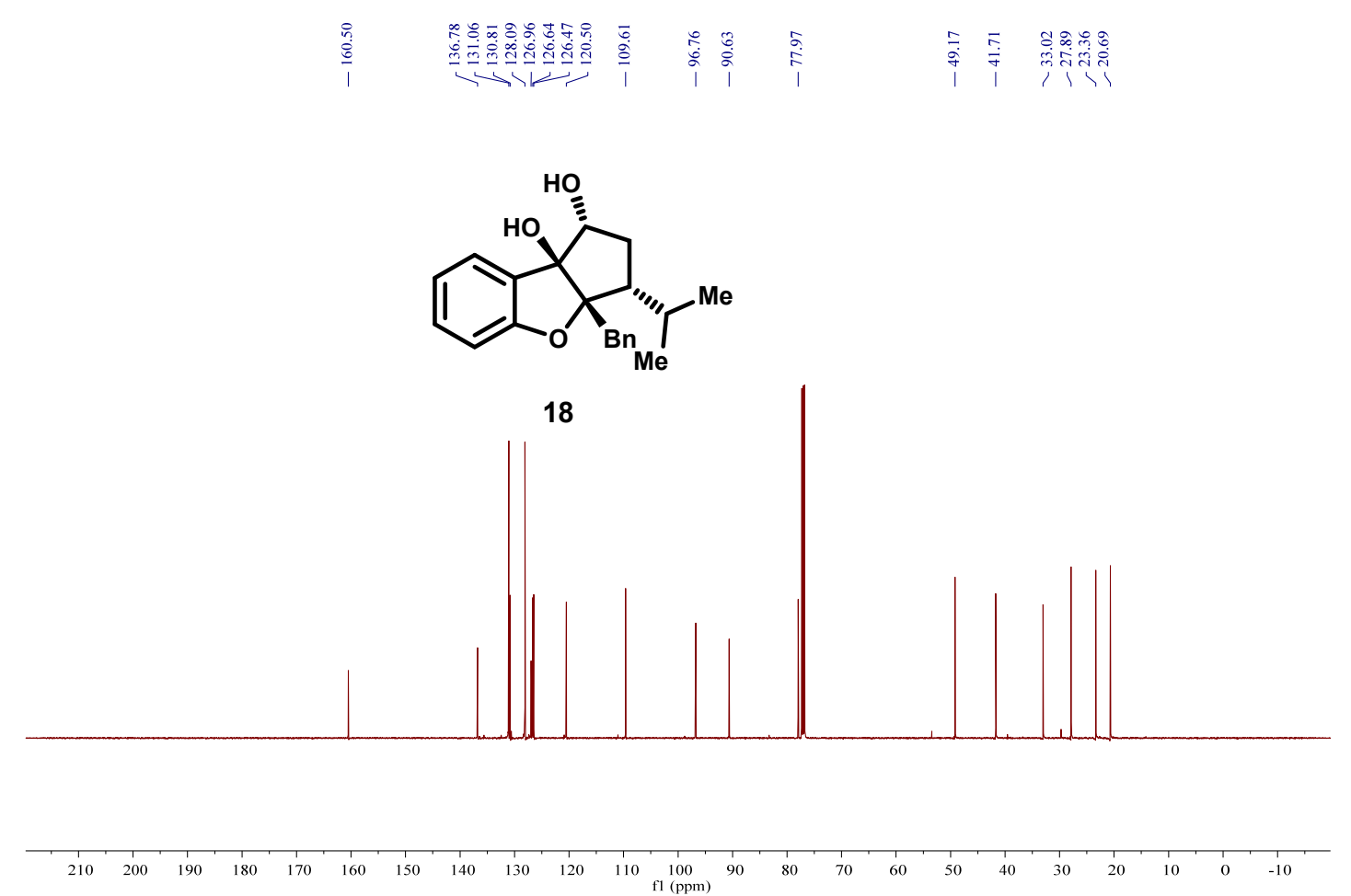

$(1 R, 2 S, 3 S, 3 \mathrm{a} S, 8 \mathrm{~b} S)$-3a-Benzyl-1,8b-dihydroxy-3-isopropyl-N,N-dimethyl-2,3,3a,8b-tetrahydro-1 $\mathrm{H}$ cyclopenta[b]benzofuran-2-carboxamide (20)

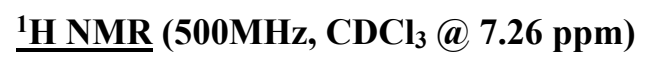

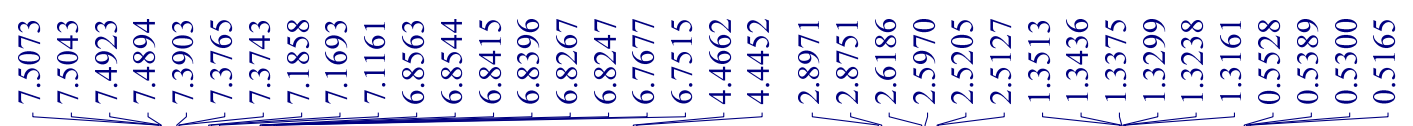

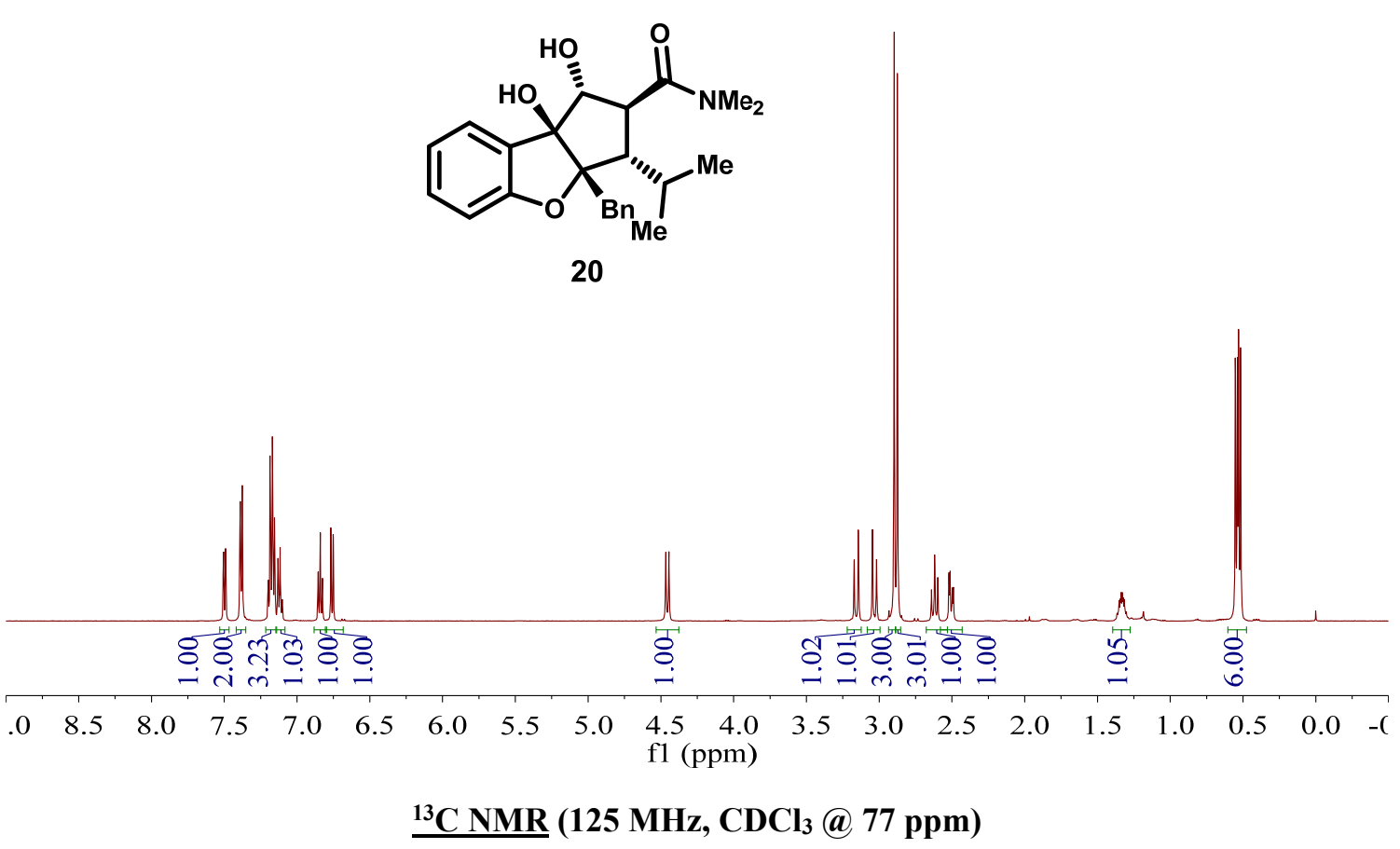



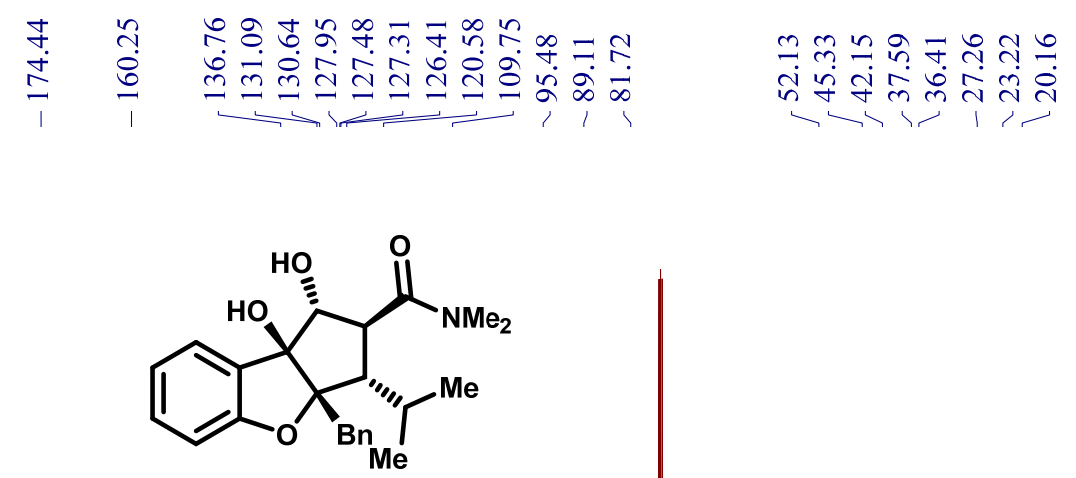

20

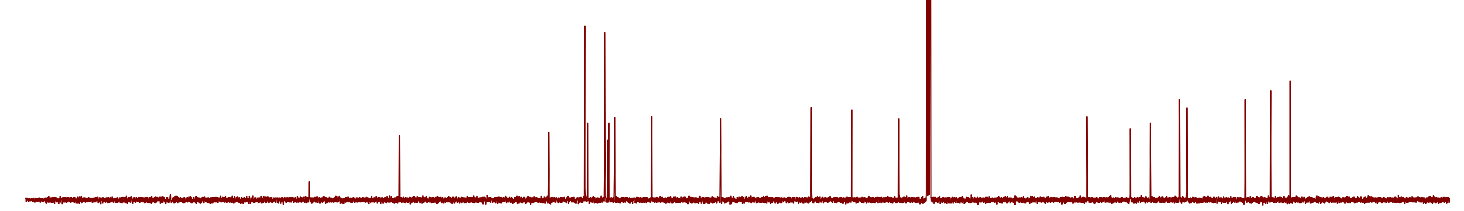

$\begin{array}{llllllllllllllllllllll}210 & 200 & 190 & 180 & 170 & 160 & 150 & 140 & 130 & 120 & 110 & 100 & 90 & 80 & 70 & 60 & 50 & 40 & 30 & 20 & 10 & 0\end{array}$ f1 (ppm)

$(1 R, 3 S, 3 \mathrm{a} S, 8 \mathrm{~b} S)-3 \mathrm{a}-B e n z y l-3-i s o p r o p y l-1,2,3,3 \mathrm{a}-$ tetrahydro-8b $H$-cyclopenta[b]benzofuran-1,8b-diol (21)

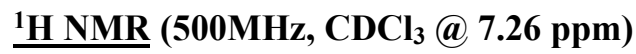

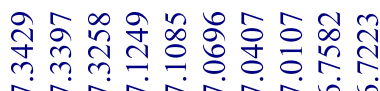

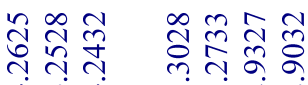

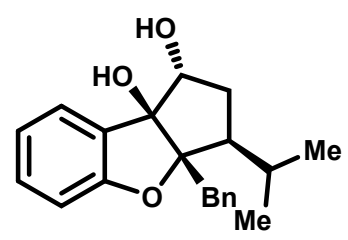

21

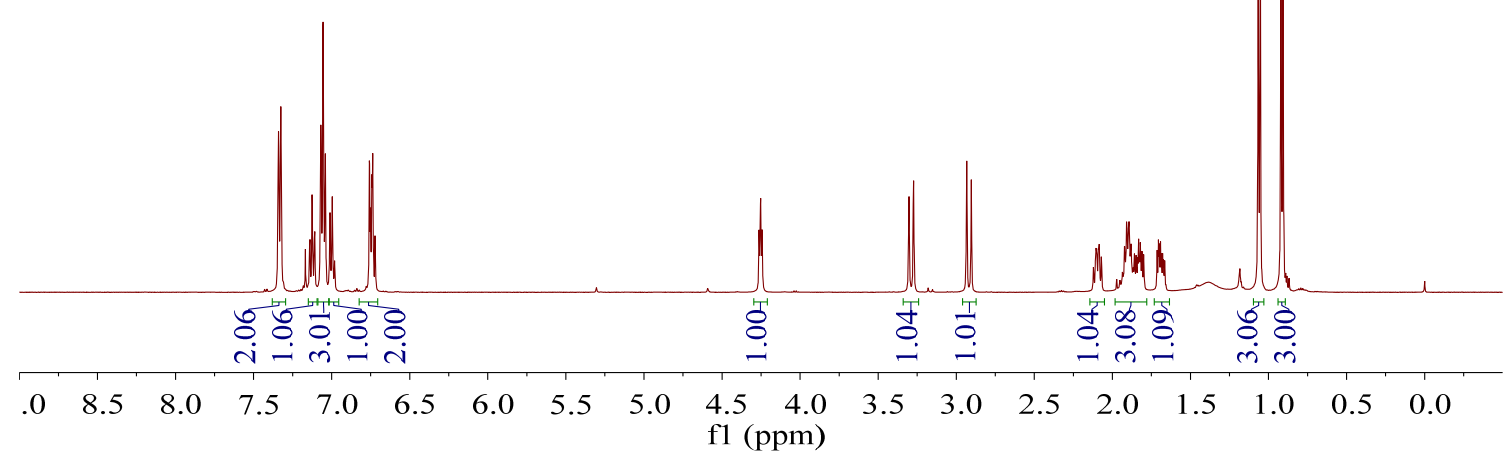

${ }^{13} \mathrm{C}$ NMR (125 MHz, CDCl 377 ppm) 


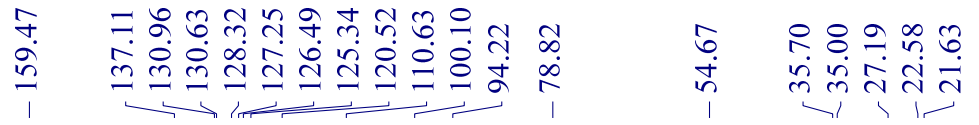

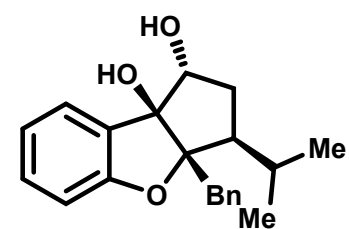

21

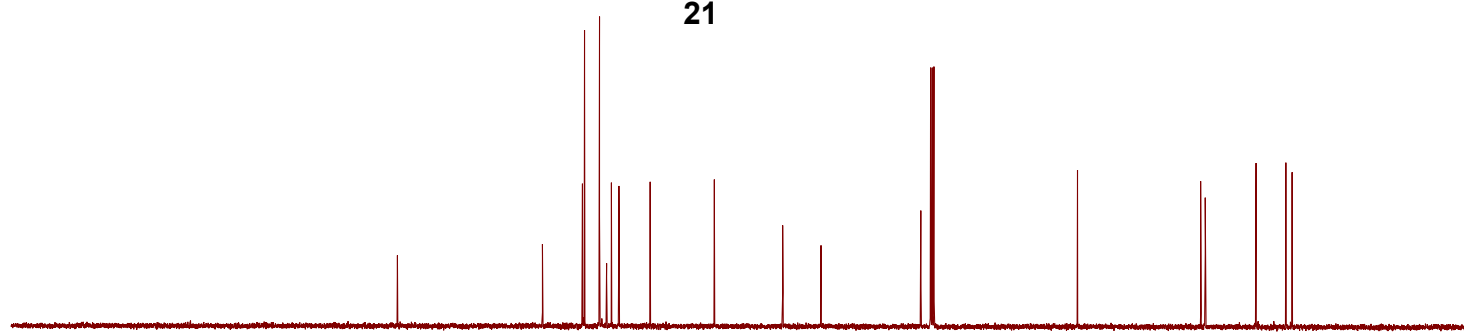

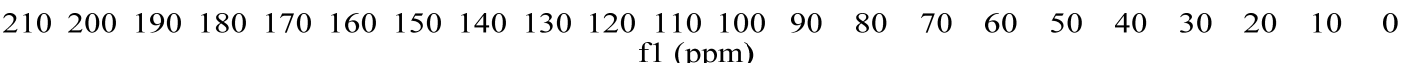

$(1 R, 2 S, 3 S, 3 a S, 8 b S)$-3a-Benzyl-1,8b-dihydroxy-3-isopropyl-N,N-dimethyl-2,3,3a,8b-tetrahydro-1Hcyclopenta[b]benzofuran-2-carboxamide (22)

\section{H NMR (500MHz, $\mathrm{CDCl}_{3} @ 7.26$ ppm)}

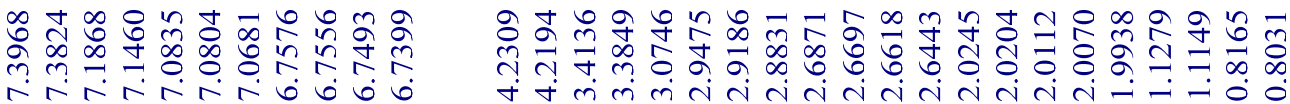<smiles>CC(C)C1[C@H](C(=O)N(C)C)[C@@H](O)C2(O)c3ccccc3OC12Br</smiles>

22

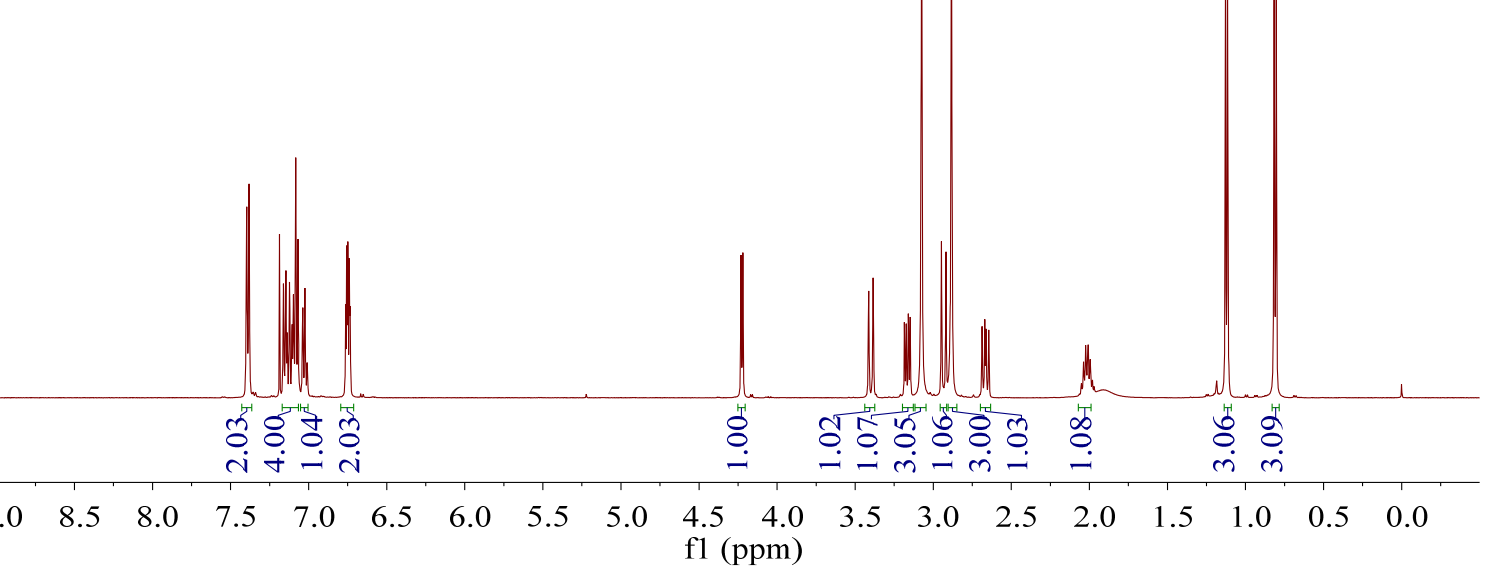


<smiles>CC(=O)[C@@H]1[C@H](C(C)C)[C@]2(Br)Oc3ccccc3[C@@]2(O)[C@@H]1O</smiles>

22

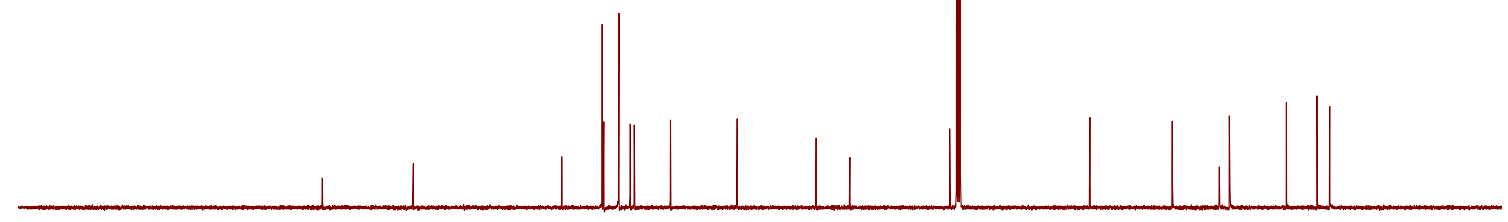

$\begin{array}{llllllllllllllllllllll}210 & 200 & 190 & 180 & 170 & 160 & 150 & 140 & 130 & 120 & 110 & 100 & 90 & 80 & 70 & 60 & 50 & 40 & 30 & 20 & 10 & 0\end{array}$ f1 (ppm)

(R)-3-(4,6-Dimethoxy-2-(4-methoxyphenyl)-3-oxo-2,3-dihydrobenzofuran-2-yl)propanal [( \pm )-S10a]

$\underline{1}$ H NMR (500MHz, $\mathrm{CDCl}_{3} @ 7.26$ ppm)

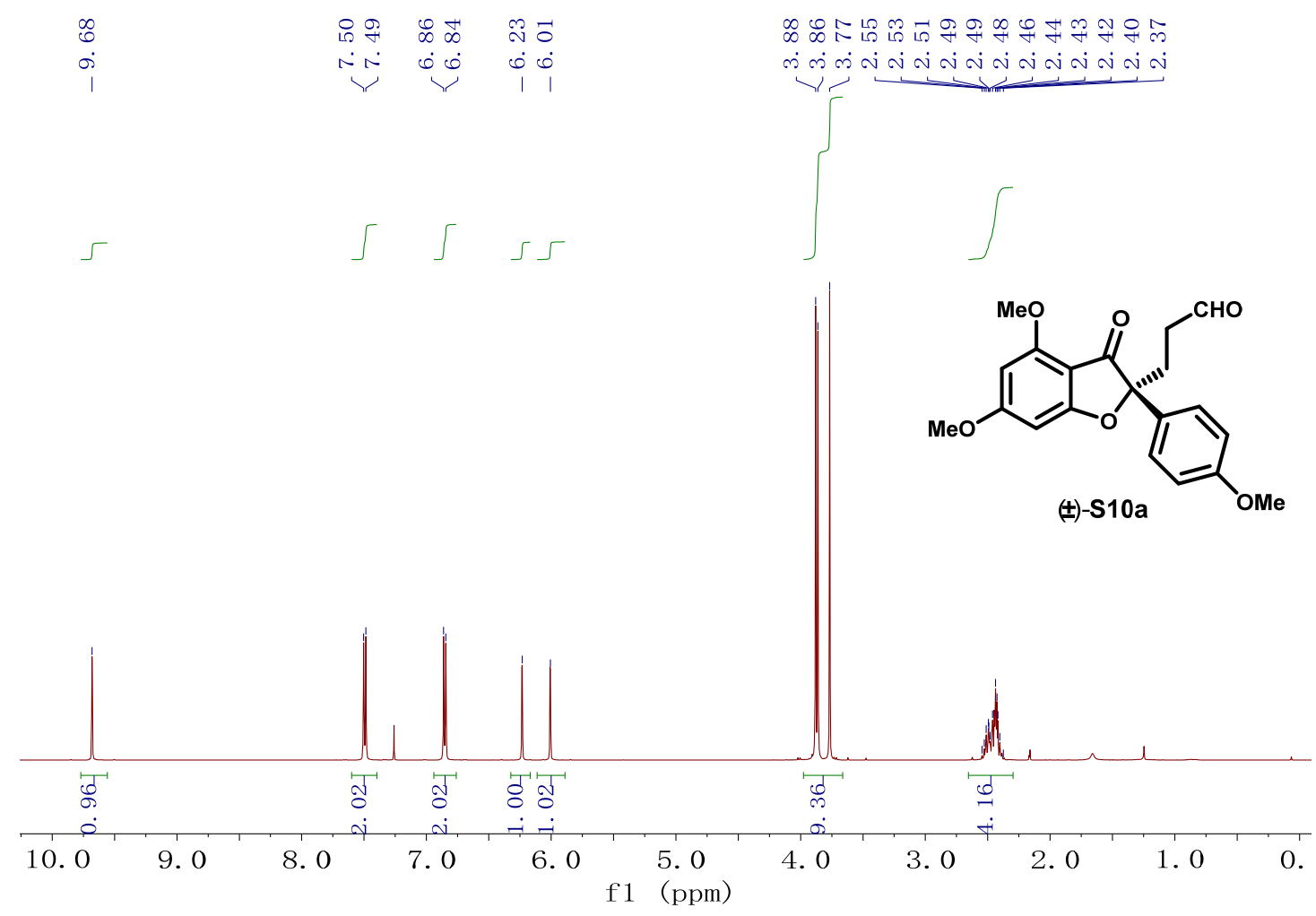


${ }^{13} \mathrm{C}$ NMR (125 MHz, CDCl 3 @ 77 ppm)

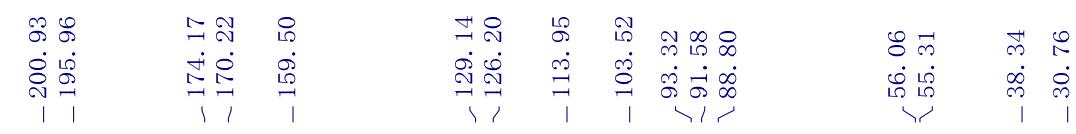
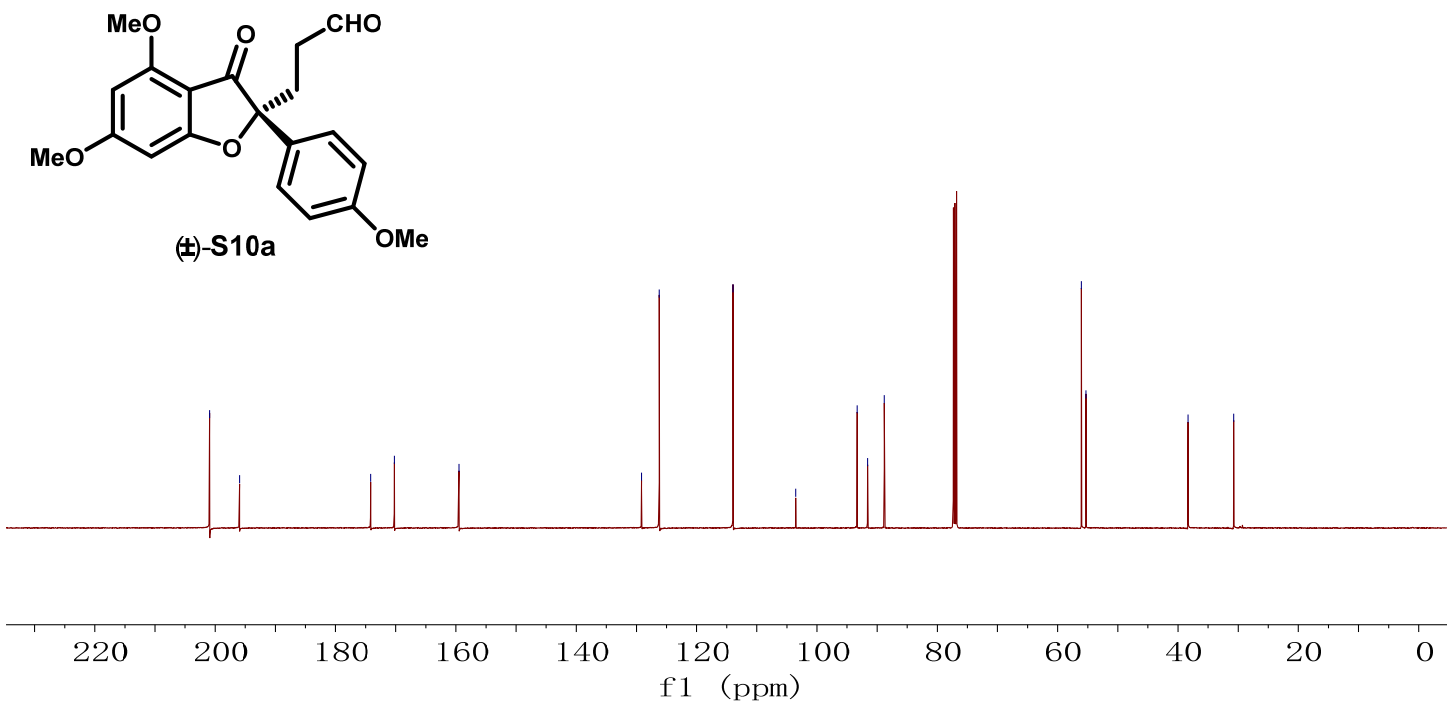

(R)-3-(4,6-Dimethoxy-3-oxo-2-phenyl-2,3-dihydrobenzofuran-2-yl)propanal [( $( \pm)-S 10 b]$

$\underline{{ }^{1} \mathrm{H} \text { NMR }}\left(600 \mathrm{MHz}, \mathrm{CDCl}_{3} @ 7.26 \mathrm{ppm}\right)$

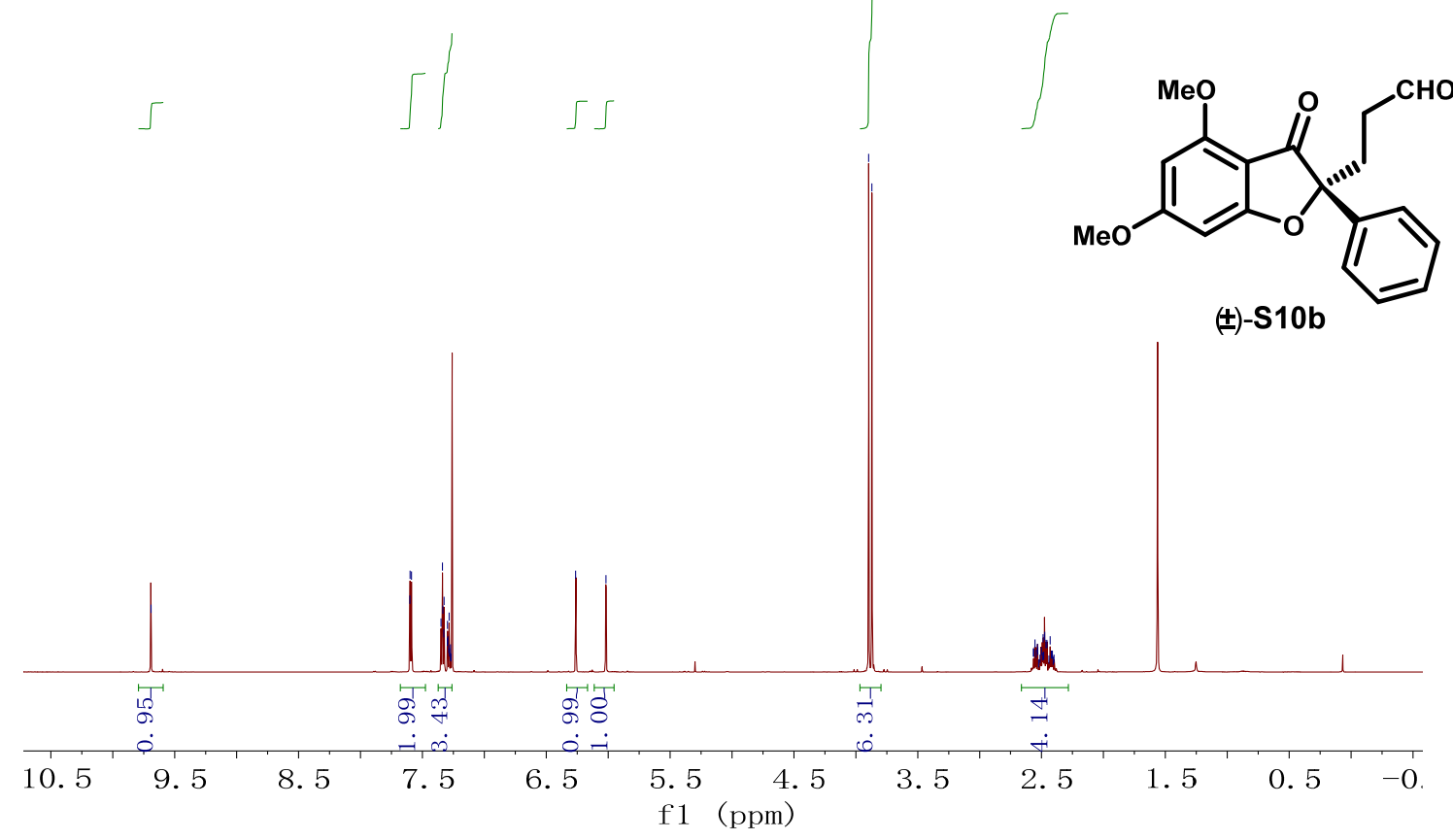


$\underline{{ }^{1} \mathrm{H} \text { NMR }}\left(150 \mathrm{MHz}, \mathrm{CDCl}_{3} @ 77\right.$ ppm)

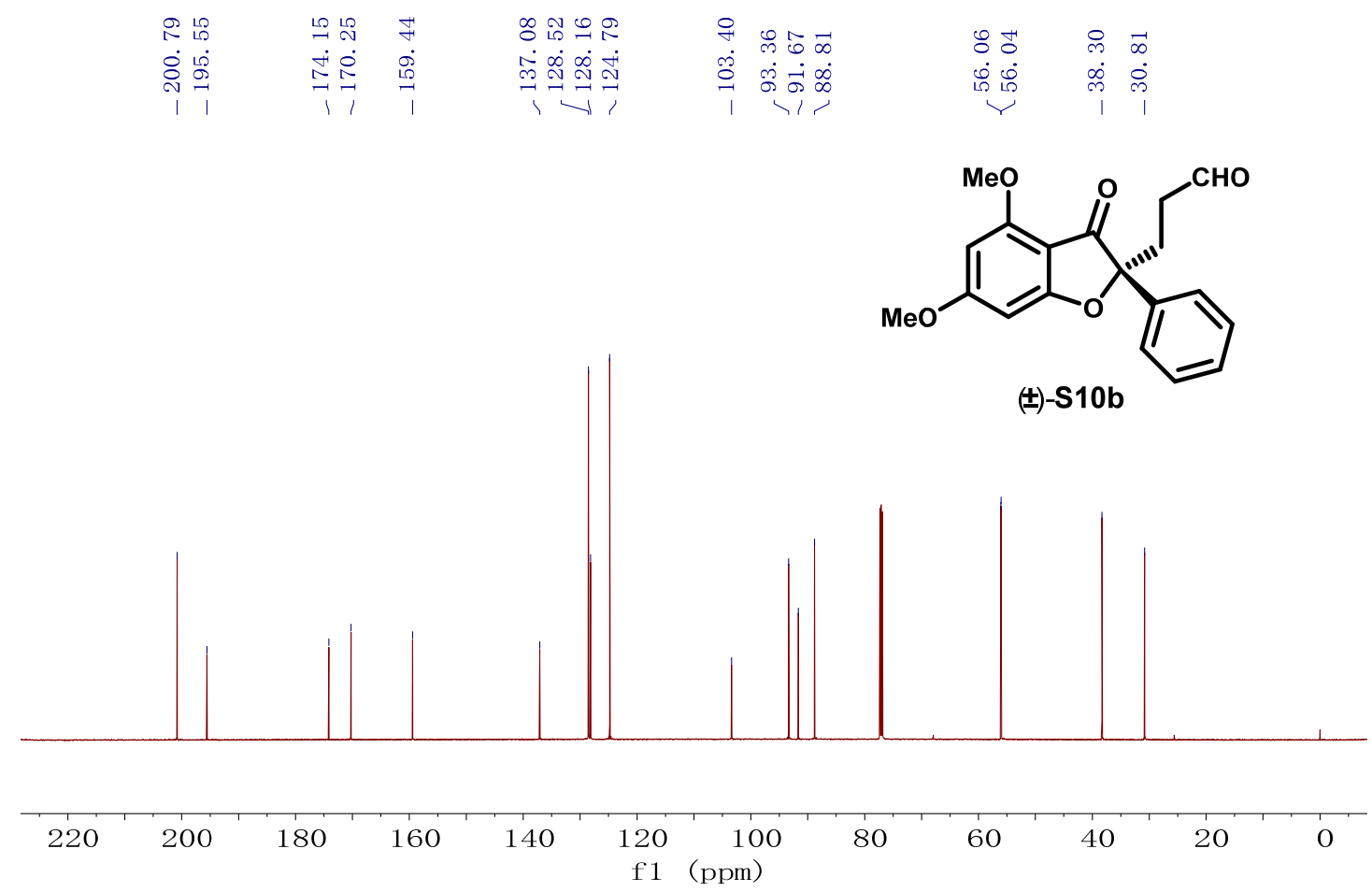

$( \pm)-(3 \mathrm{a} R, 8 \mathrm{~b} R)-8 \mathrm{~b}-\mathrm{hydroxy}-6,8$-dimethoxy-3a-phenyl-2,3,3a,8b-tetrahydro-1Hcyclopenta[b]benzofuran-1-one $[( \pm)-S 11 b]$

1H NMR $\left(500 \mathrm{MHz}, \mathrm{CDCl}_{3} @ 7.26\right.$ ppm)

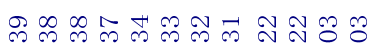
NNNNNNN00000

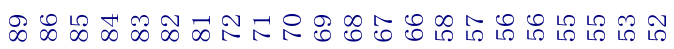

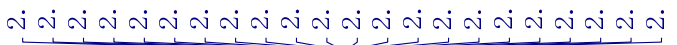
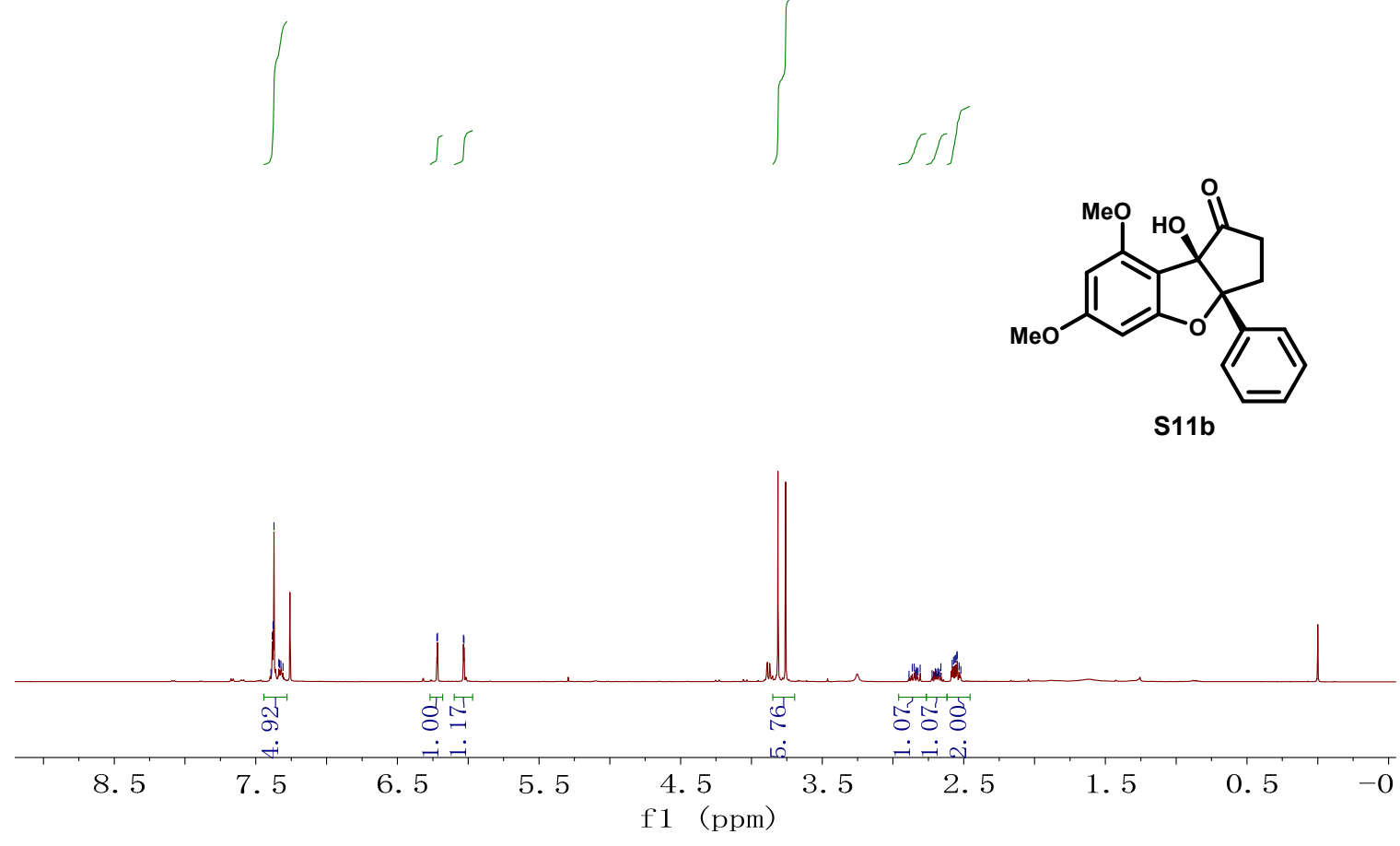


\section{C NMR (150 MHz, CDCl $@ 77$ ppm)}

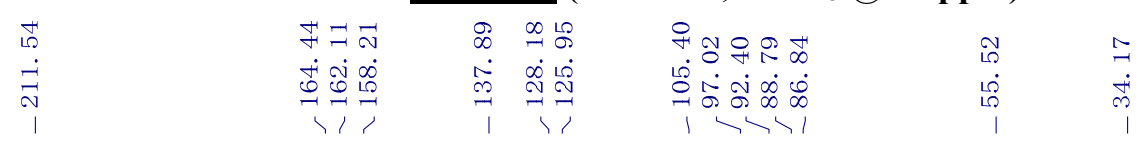

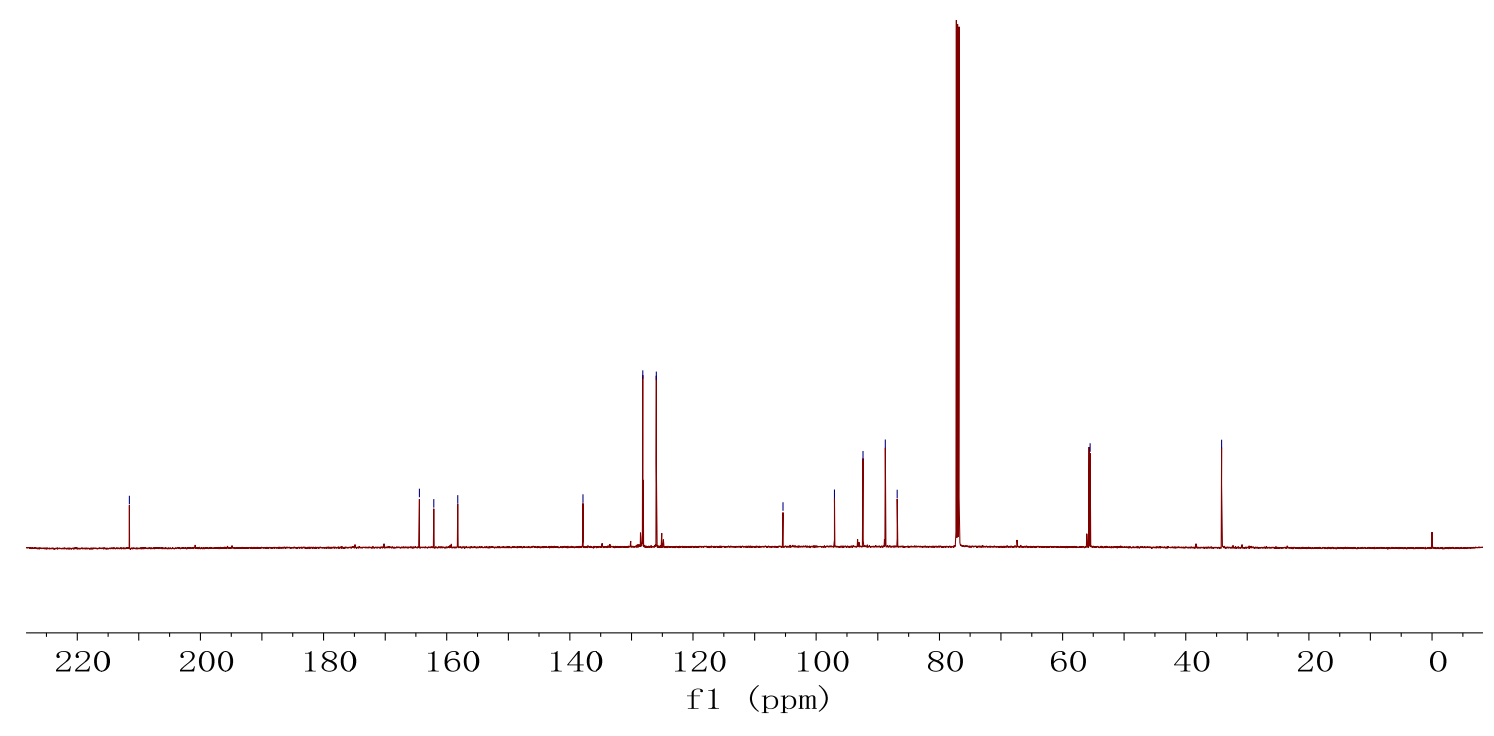

(3aR,8bR)-8b-Hydroxy-6,8-dimethoxy-3a-(4-methoxyphenyl)-2,3,3a,8b-tetrahydro-1Hcyclopenta[b]benzofuran-1-one $[( \pm)-S 11 \mathrm{a}]$

1H NMR (500MHz, $\mathrm{CDCl}_{3} @ 7.26$ ppm)

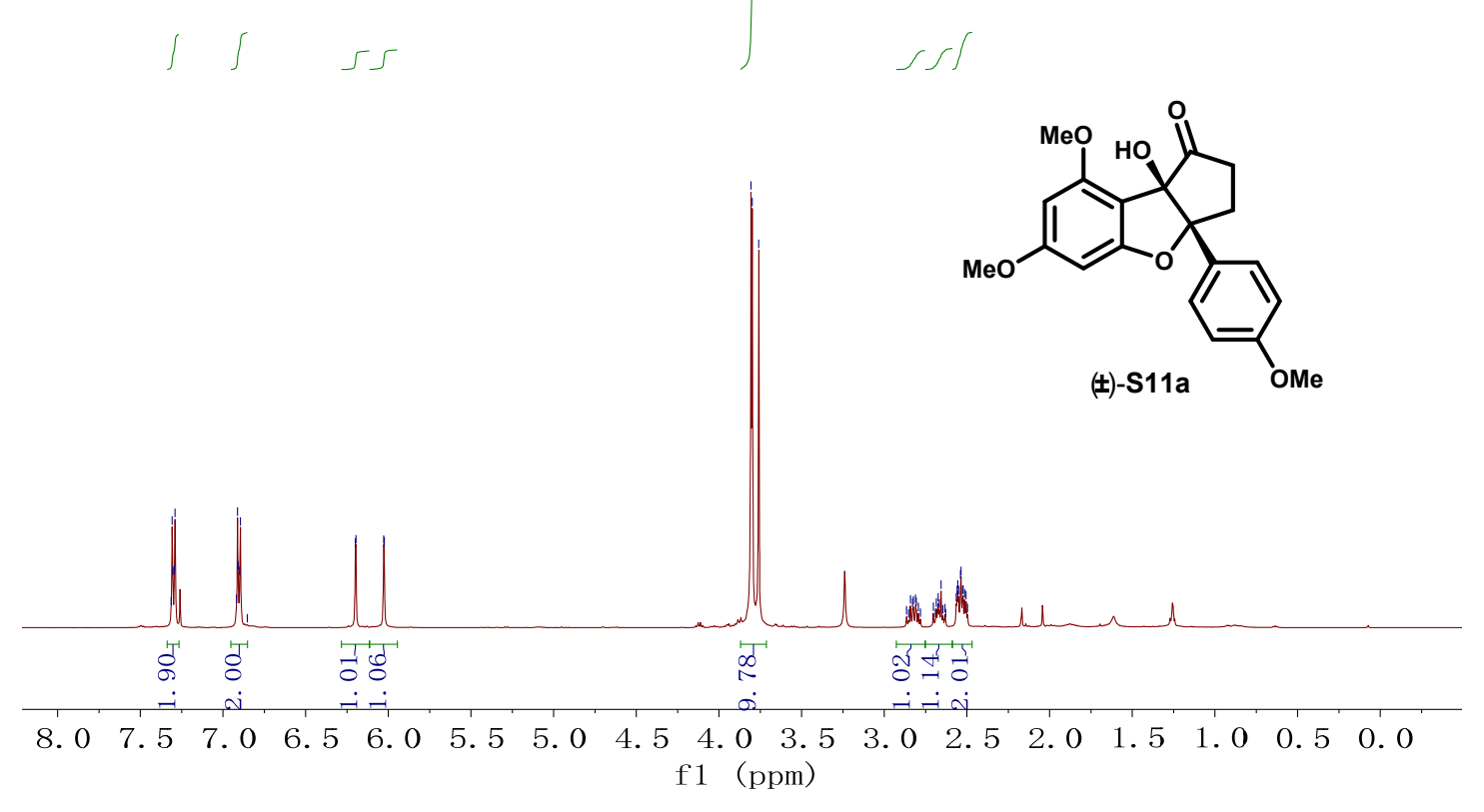


${ }^{13} \mathrm{C}$ NMR (125 MHz, $\mathrm{CDCl}_{3} @ 77$ ppm)

\begin{tabular}{|c|c|c|c|c|}
\hline I. & 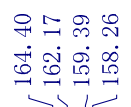 & 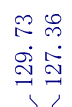 & 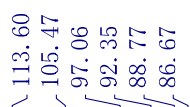 & 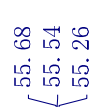 \\
\hline
\end{tabular}
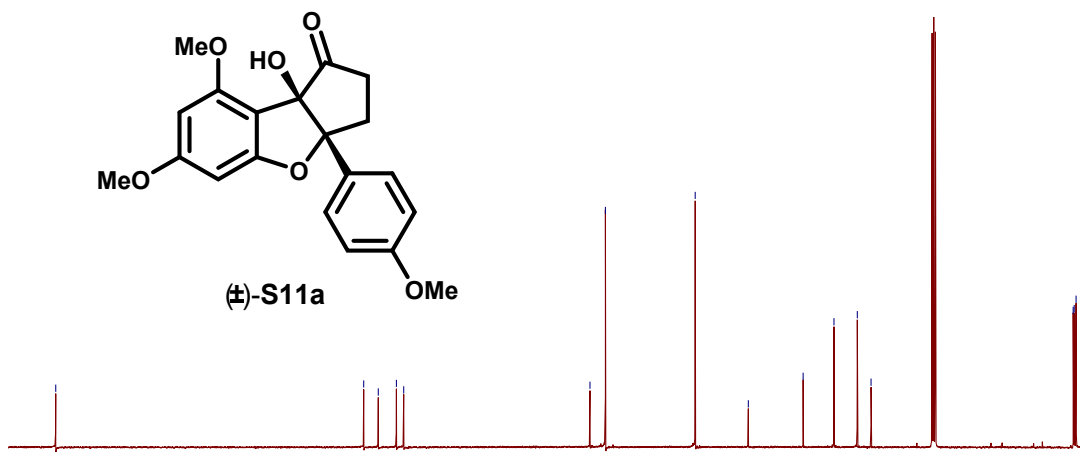

200

$180 \quad 160$

140

120

f1 (ppm)

( \pm )-Methyl (3aS,8bR)-8b-hydroxy-6,8-dimethoxy-3a-(4-methoxyphenyl)-1-oxo-3-phenyl-3a,8bdihydro-1H-cyclopenta[b]benzofuran-2-carboxylate [( $( \pm)-\mathrm{S} 12]$

$\underline{{ }^{1} \mathrm{H} \text { NMR }}\left(600 \mathrm{MHz}, \mathrm{CDCl}_{3} @ 7.26 \mathrm{ppm}\right)$
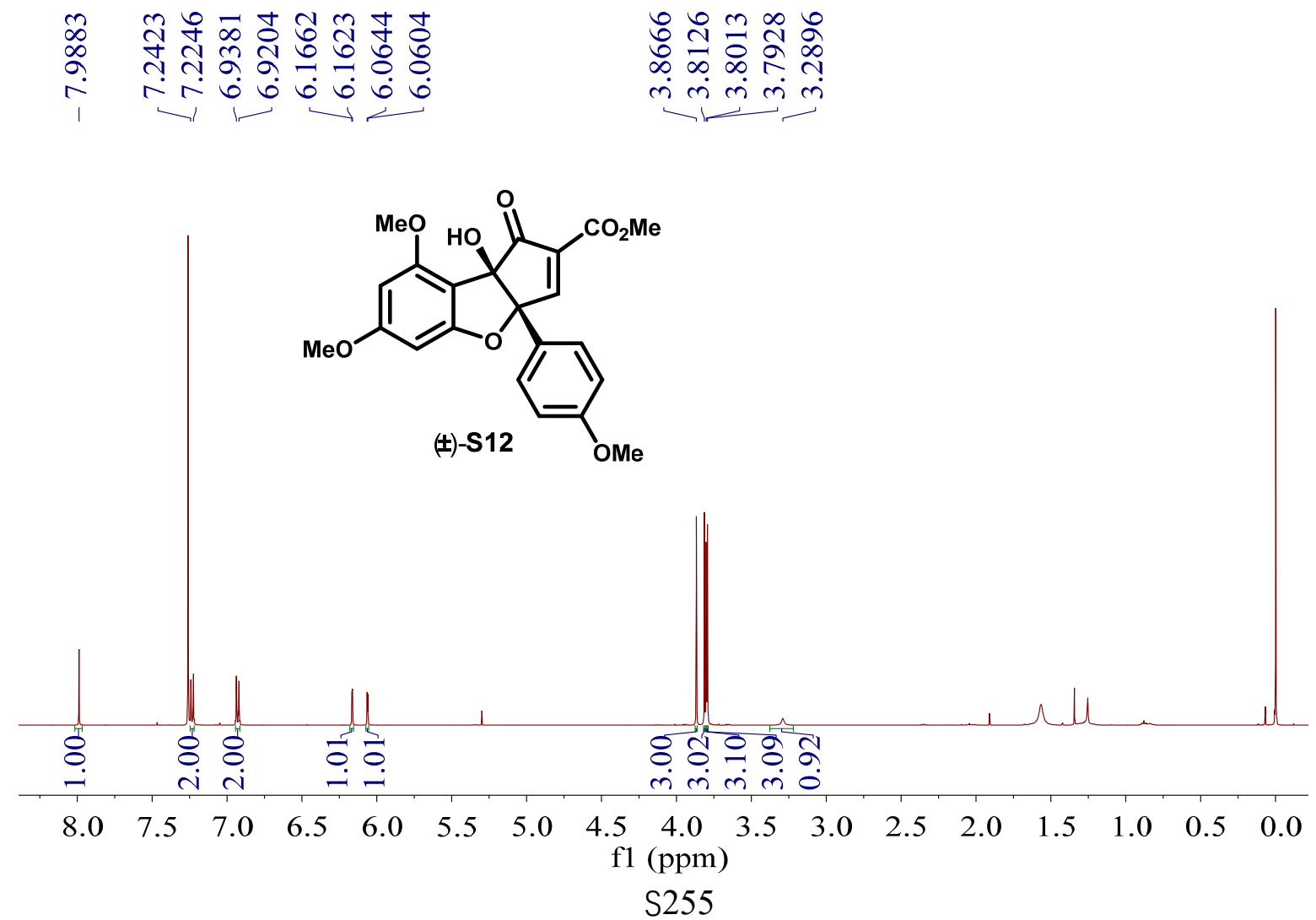


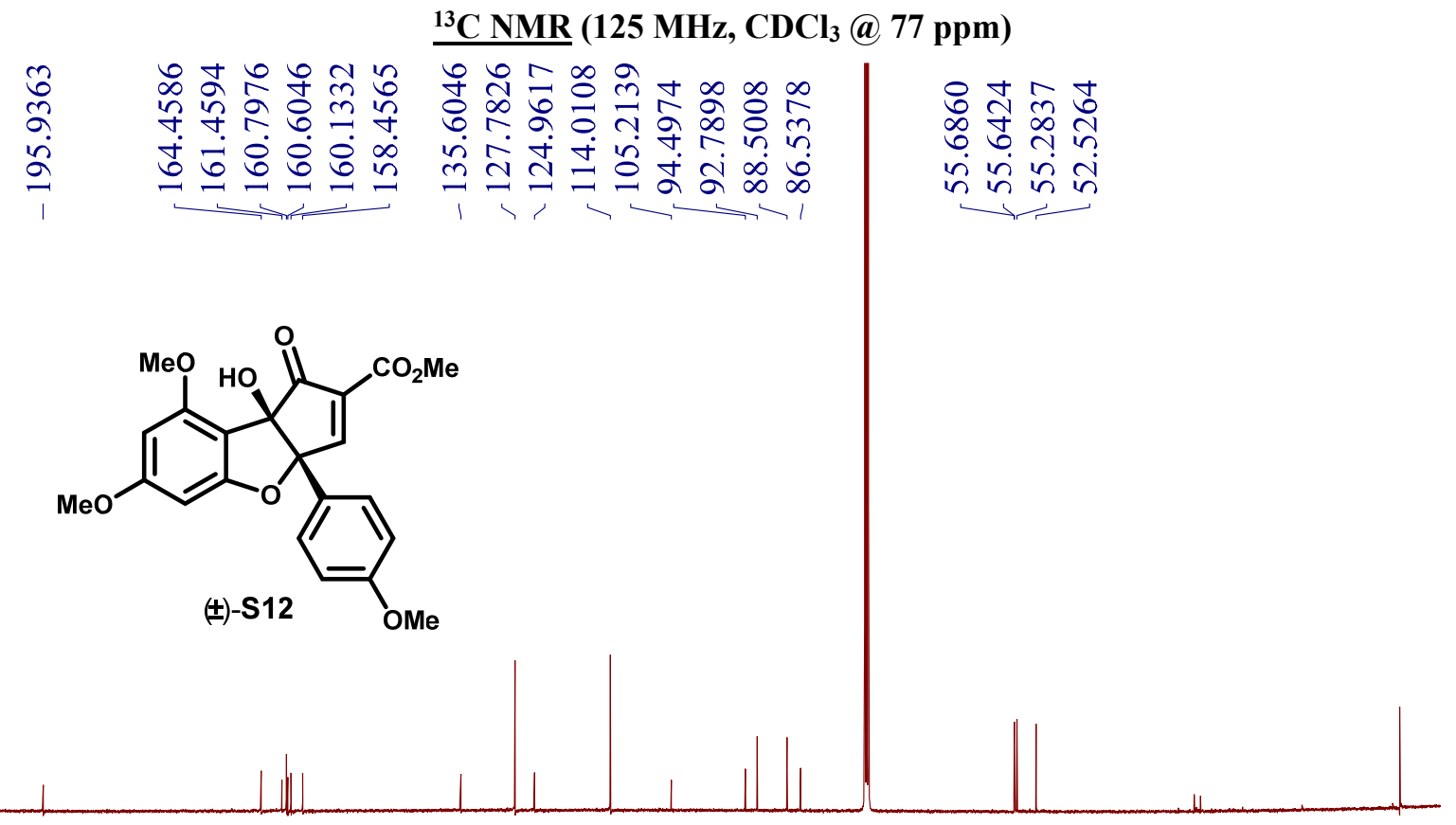

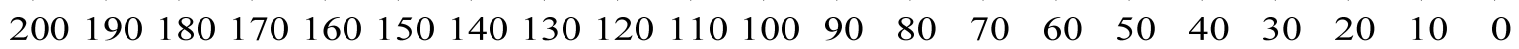
f1 (ppm)

Methyl (3aS,8bR)-8b-hydroxy-6,8-dimethoxy-3a-(4-methoxyphenyl)-1-oxo-3-phenyl-3a,8bdihydro-1H-cyclopenta[b]benzofuran-2-carboxylate [( $( \pm)-\mathrm{S} 13]$

$\underline{{ }^{1} \mathrm{H} \text { NMR }}\left(500 \mathrm{MHz}, \mathrm{CDCl}_{3} @ 7.26\right.$ ppm)
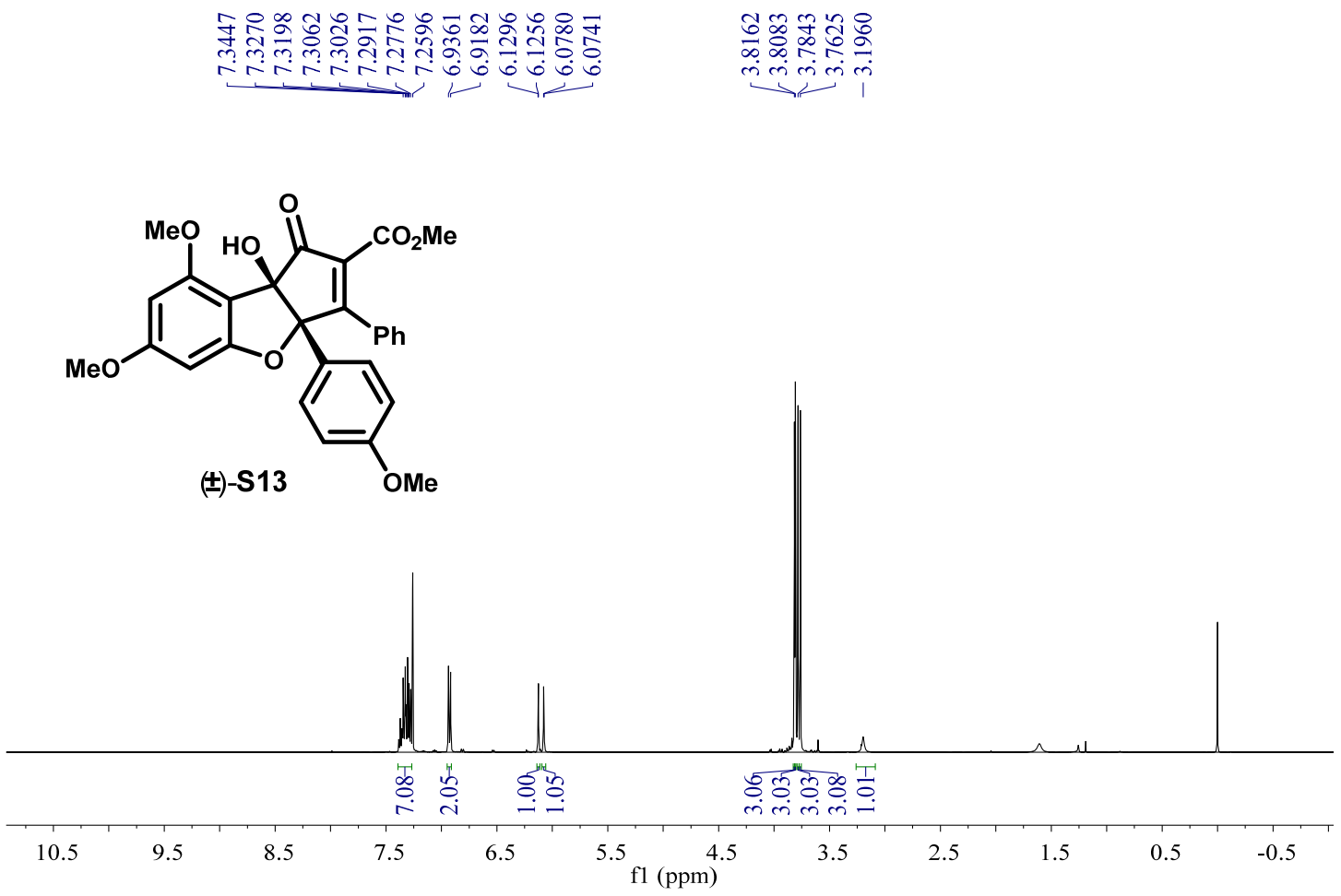


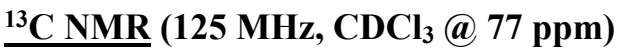

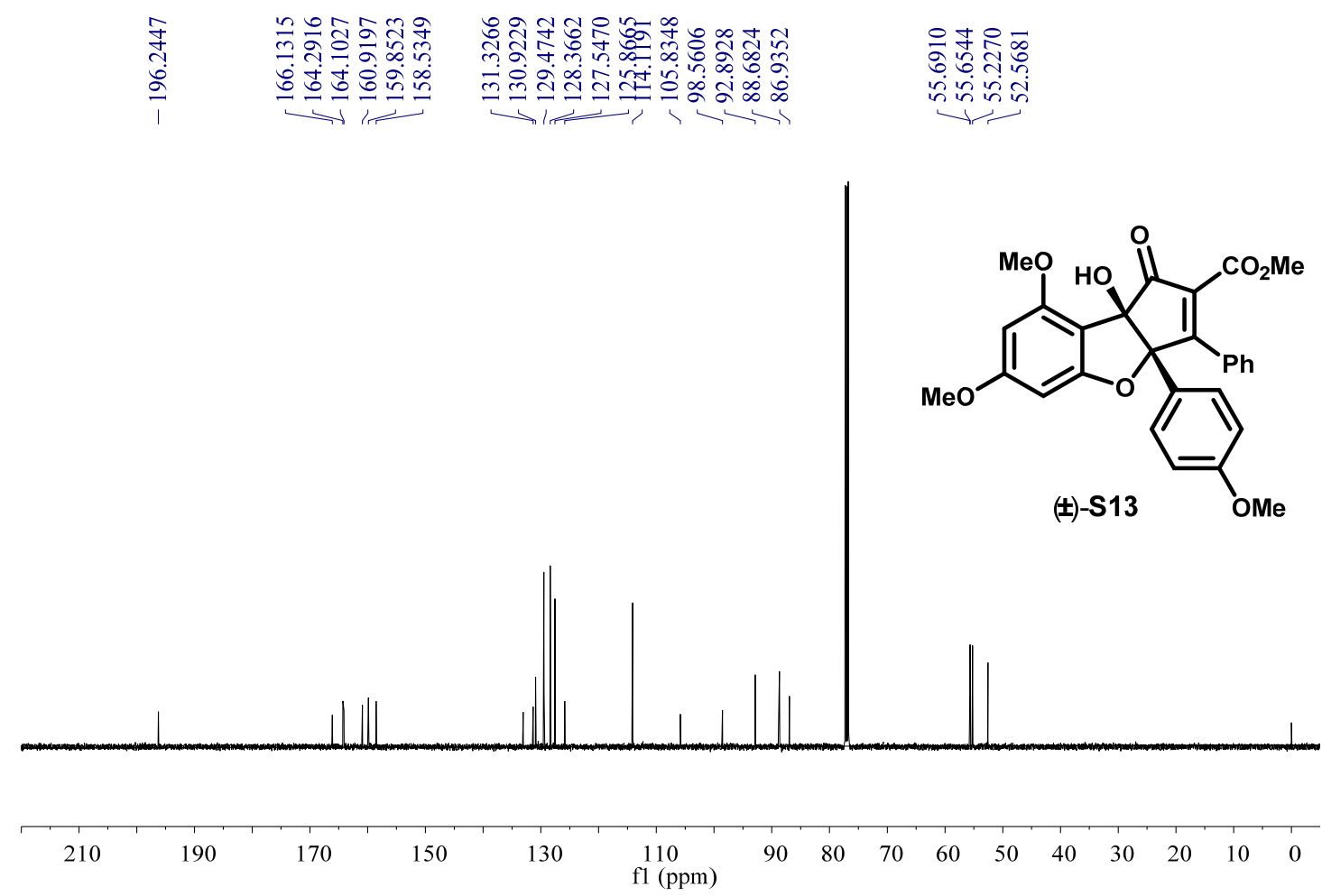

PHOX ligand L5 was prepared following a known method: Garcia-Fortanet, J.; Buchwald, S. L. Asymmetric palladium-catalyzed intramolecular $\alpha$-arylation of aldehydes. Angew. Chem. Int. Ed. 2008, 47, 8108-8111.

\section{$\underline{{ }^{1} \mathrm{H} \text { NMR }}\left(500 \mathrm{MHz}, \mathrm{CDCl}_{3} @ 7.26\right.$ ppm)}

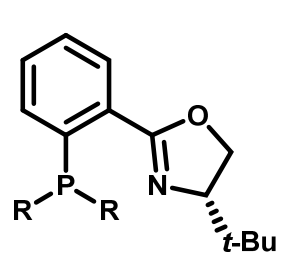

$$
\mathbf{L 5}(\mathrm{R}=c-\mathrm{Hex})
$$

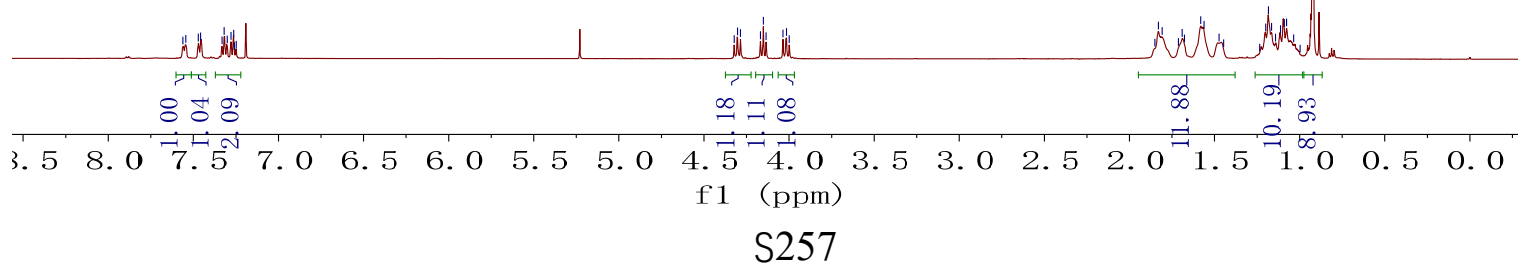


${ }^{13} \mathrm{C}$ NMR (150 MHz, $\mathrm{CDCl}_{3} @ 77$ ppm)

\& $\quad$ \&̊ำ

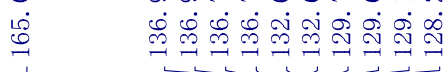

రิ

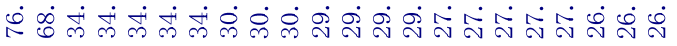

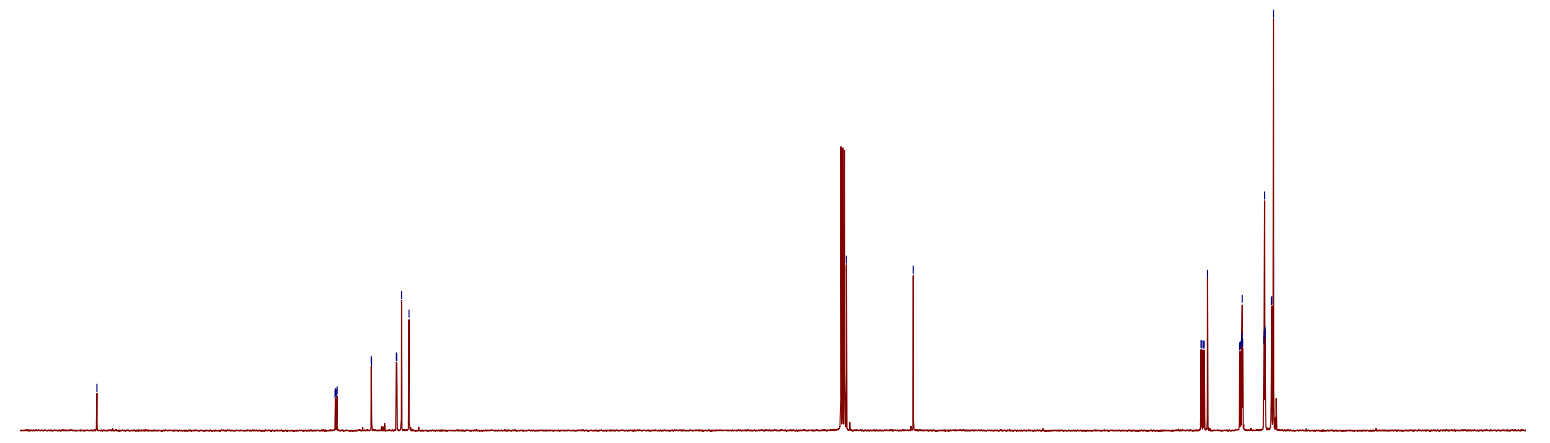

$\begin{array}{llllllllllllllllll}170 & 160 & 150 & 140 & 130 & 120 & 110 & 100 & 90 & 80 & 70 & 60 & 50 & 40 & 30 & 20 & 10 & 0\end{array}$ 31P NMR (202 MHz)

พิ ம்

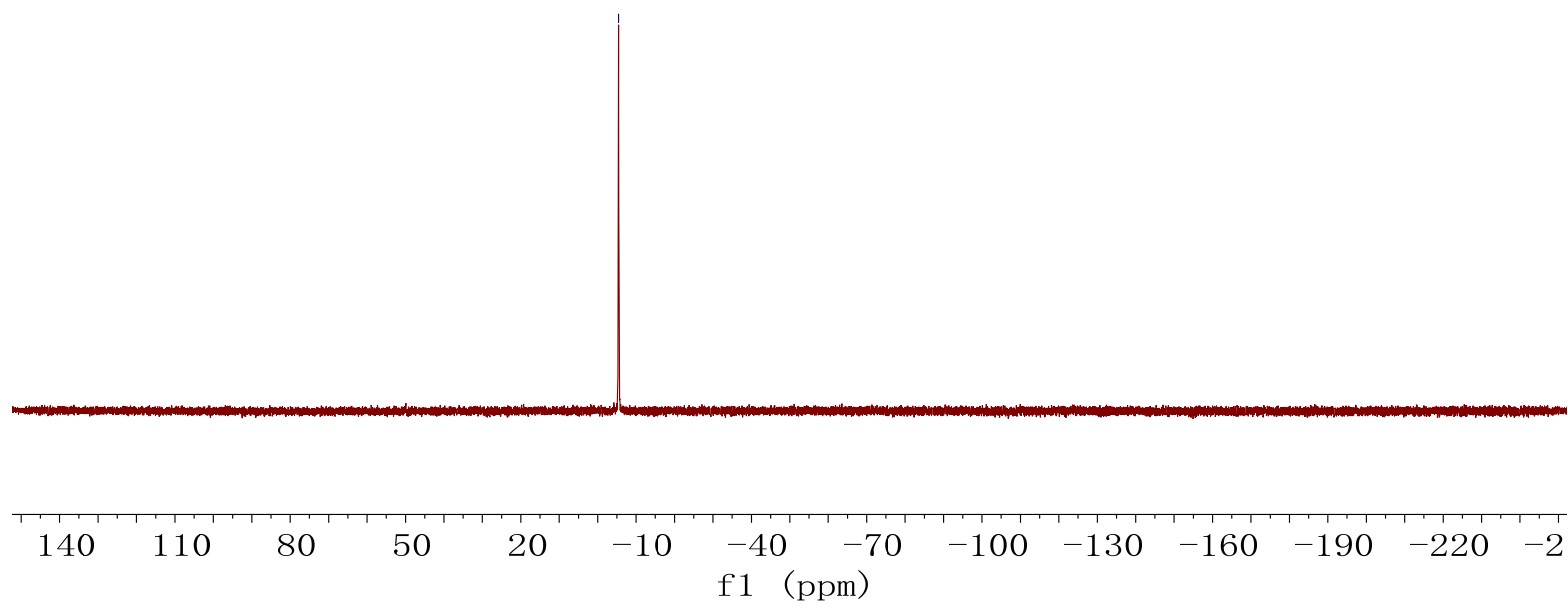




\section{HPLC Spectra}

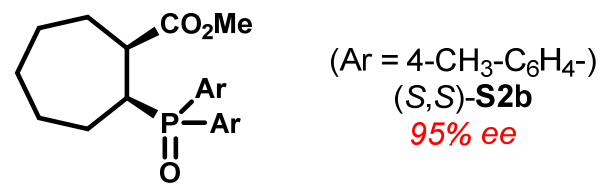

Daicel Chiralpak IA-3 column, n-hexane/i-PrOH (70/30), $1 \mathrm{~mL} / \mathrm{min} 210 \mathrm{~nm} 25.948 \mathrm{~min}$ (major enantiomer), $17.754 \mathrm{~min}$ (minor enantiomer).

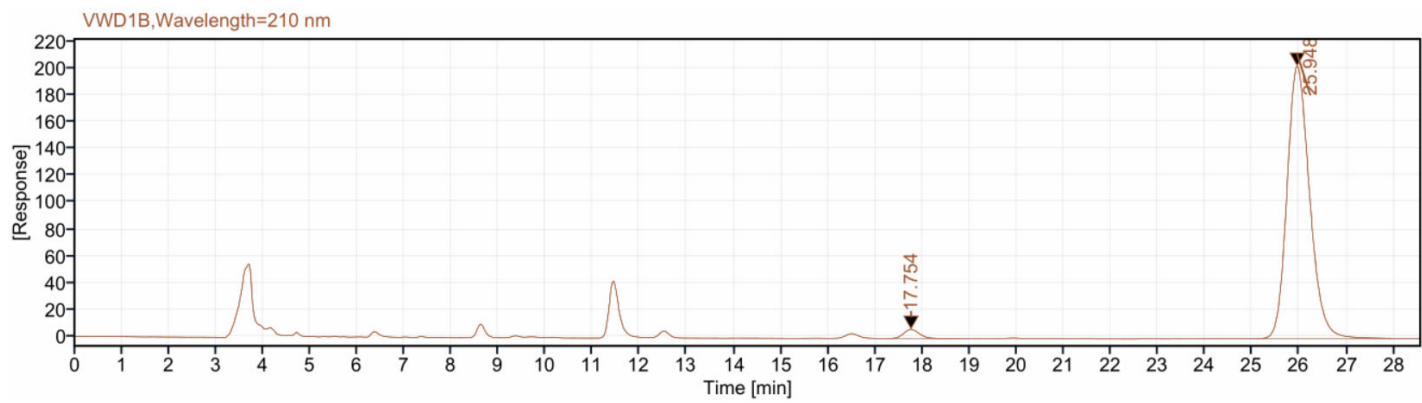

Signal: $\quad$ VWD1B,Wavelength $=210 \mathrm{~nm}$

$\begin{array}{rrrrrr}\text { RT [min] } & \text { Type } & \text { Width [min] } & \text { Area } & \text { Height } & \text { Area\% } \\ 17.754 & \text { BB } & 1.54 & 162.53 & 6.88 & 2.34 \\ 25.948 & \text { BBA } & 2.99 & 6794.78 & 203.49 & 97.66 \\ & & \text { Sum } & 6957.31 & & \end{array}$

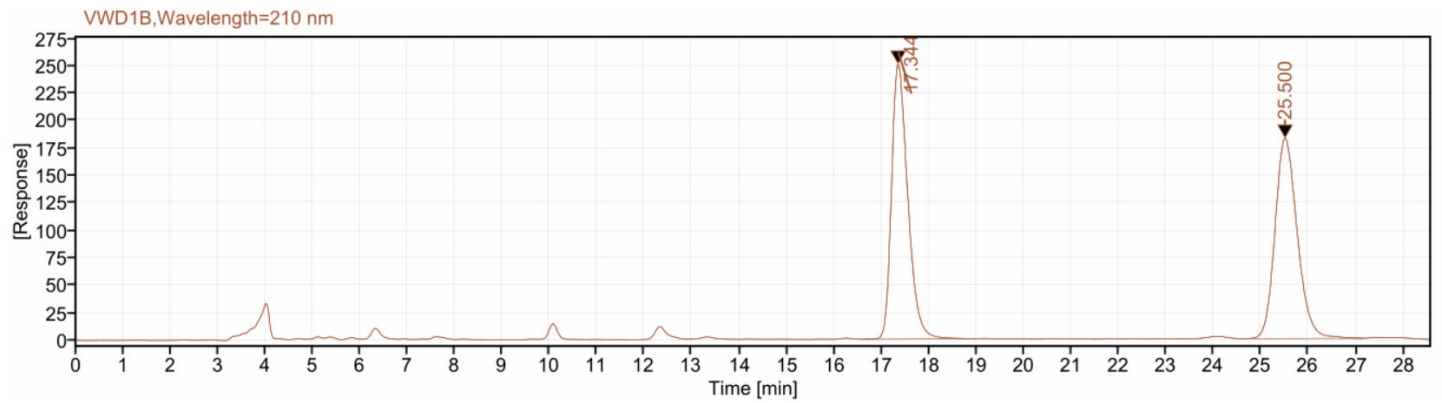

Signal: $\quad$ VWD1B,Wavelength $=210 \mathrm{~nm}$

$\begin{array}{rrrrrr}\text { RT [min] } & \text { Type } & \text { Width [min] } & \text { Area } & \text { Height } & \text { Area\% } \\ 17.344 & \text { BB } & 2.32 & 5954.82 & 250.35 & 50.03 \\ 25.500 & \text { BB } & 2.57 & 5947.22 & 182.42 & 49.97 \\ & & \text { Sum } & 11902.04 & & \end{array}$




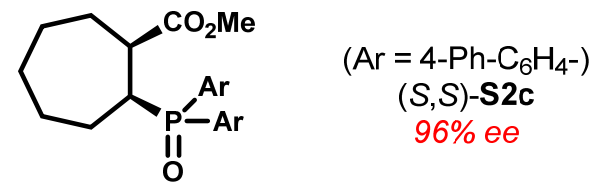

Daicel Chiralpak IC-3 column, n-hexane/i-PrOH (70/30), 1 mL/min $210 \mathrm{~nm} 35.329$ min (major enantiomer), $66.044 \mathrm{~min}$ (minor enantiomer).

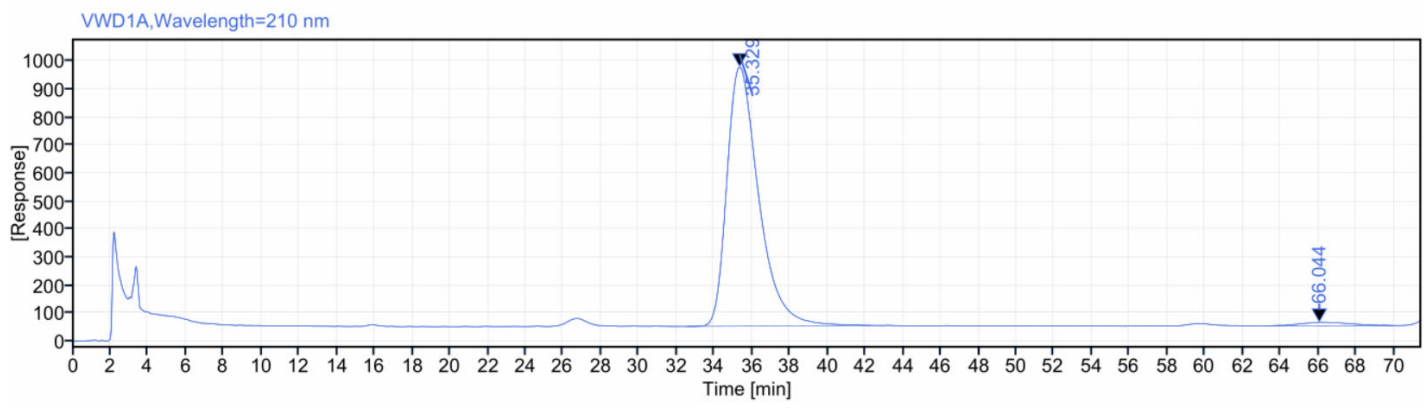

Signal: $\quad$ VWD1A,Wavelength $=210 \mathrm{~nm}$

$\begin{array}{rrrrrr}\text { RT [min] } & \text { Type } & \text { Width [min] } & \text { Area } & \text { Height } & \text { Area\% } \\ 35.329 & \text { MM m } & 1.78 & 108513.72 & 926.83 & 97.98 \\ 66.044 & \text { MM m } & 2.17 & 2236.99 & 12.46 & 2.02 \\ & & \text { Sum } & 110750.71 & & \end{array}$

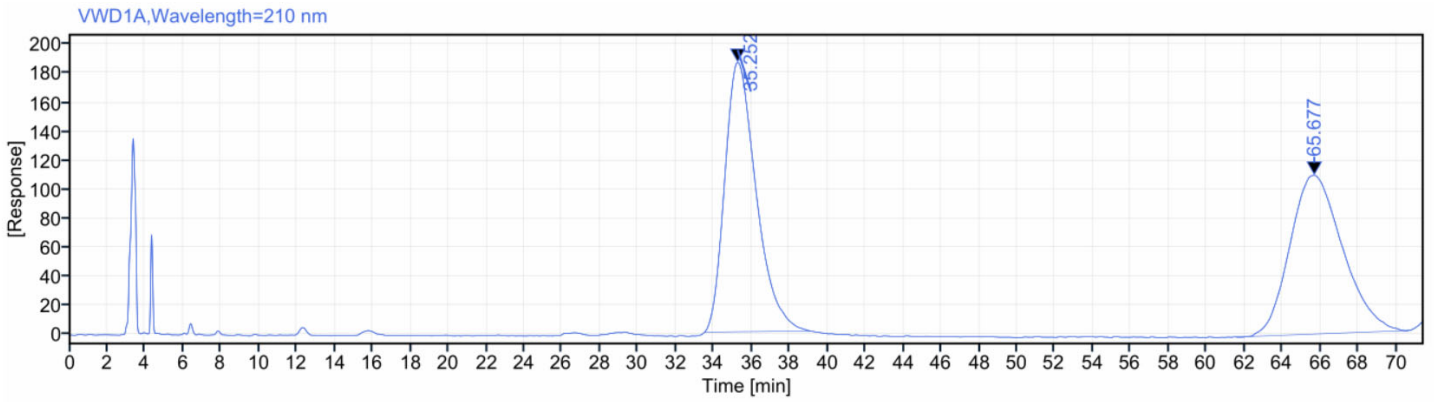

Signal: $\quad$ VWD1A,Wavelength $=210 \mathrm{~nm}$

$\begin{array}{rrrrrr}\text { RT [min] } & \text { Type } & \text { Width [min] } & \text { Area } & \text { Height } & \text { Area\% } \\ 35.252 & \text { MM m } & 1.76 & 21403.07 & 185.60 & 50.37 \\ 65.677 & \text { MM m } & 2.55 & 21089.19 & 109.40 & 49.63 \\ & & \text { Sum } & 42492.26 & & \end{array}$




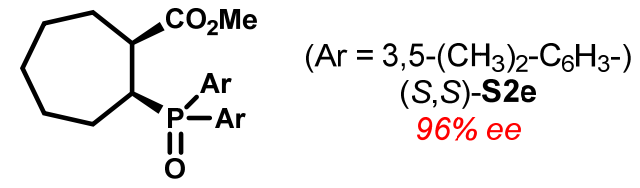

Daicel Chiralpak ID-3 column, n-hexane/i-PrOH (90/10), $0.5 \mathrm{~mL} / \mathrm{min} 210 \mathrm{~nm} 38.927 \mathrm{~min}$ (major enantiomer), $36.043 \mathrm{~min}$ (minor enantiomer).

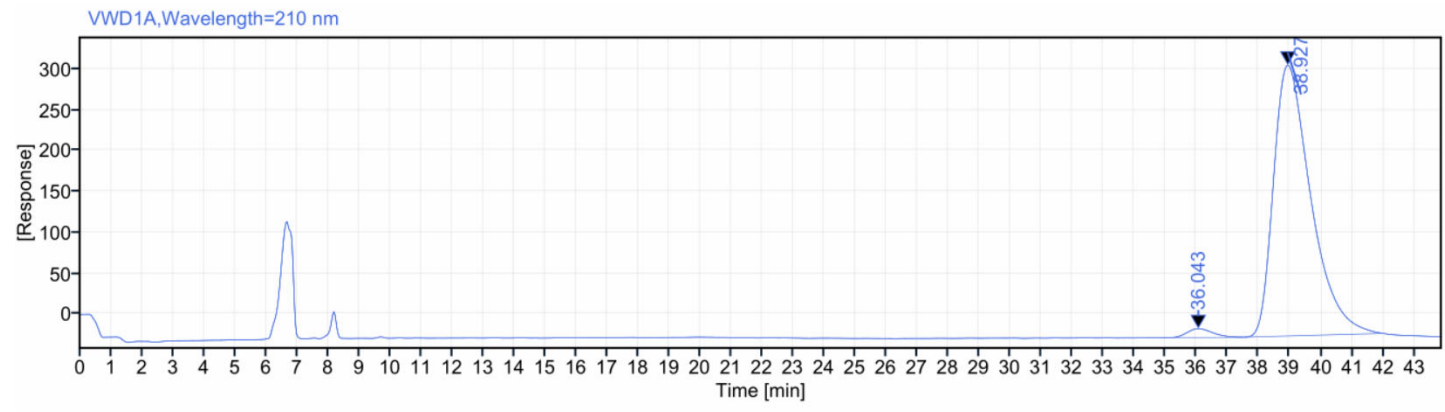

Signal: $\quad$ VWD1A,Wavelength $=210 \mathrm{~nm}$

$\begin{array}{rrrrrr}\text { RT [min] } & \text { Type } & \text { Width [min] } & \text { Area } & \text { Height } & \text { Area\% } \\ 36.043 & \text { MM m } & 0.87 & 620.85 & 10.90 & 2.19 \\ 38.927 & \text { BBA } & 4.48 & 27762.92 & 331.69 & 97.81 \\ & & \text { Sum } & 28383.78 & & \end{array}$

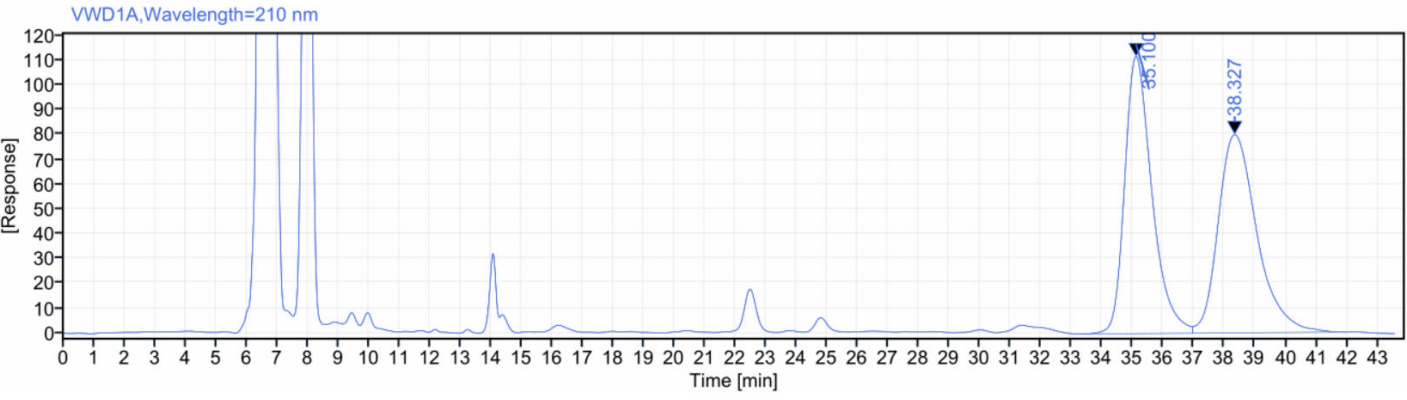

Signal: $\quad$ VWD1A, Wavelength $=210 \mathrm{~nm}$

$\begin{array}{rrrrrr}\text { RT [min] } & \text { Type } & \text { Width [min] } & \text { Area } & \text { Height } & \text { Area\% } \\ 35.100 & \text { BV } & 3.58 & 6939.19 & 111.72 & 49.67 \\ 38.327 & \text { VB } & 4.75 & 7030.49 & 79.98 & 50.33 \\ & & \text { Sum } & 13969.68 & & \end{array}$




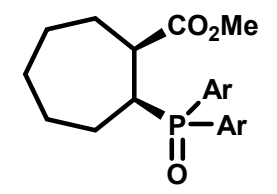

(Ar = 2-naphthyl)

$(S, S)-\mathbf{S 2 f}$

$98 \%$ ee

Daicel Chiralpak IC-3 column, n-hexane/i-PrOH (70/30), 1 mL/min $254 \mathrm{~nm} 87.703$ min (major enantiomer), $60.879 \mathrm{~min}$ (minor enantiomer).

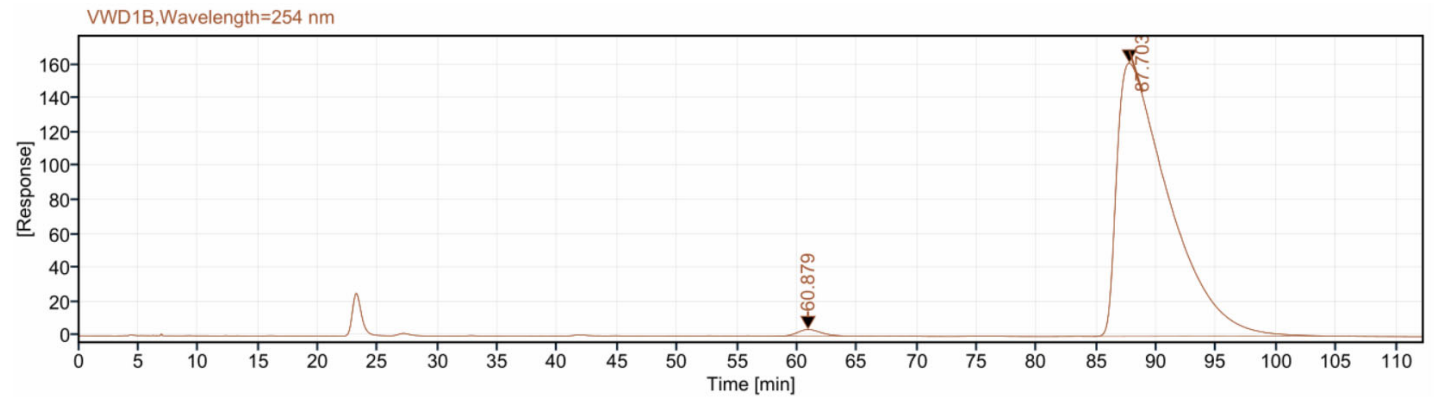

Signal: $\quad$ VWD1B,Wavelength $=254 \mathrm{~nm}$

$\begin{array}{rrrrrr}\text { RT [min] } & \text { Type } & \text { Width [min] } & \text { Area } & \text { Height } & \text { Area\% } \\ 60.879 & \text { MM m } & 1.74 & 513.49 & 3.81 & 1.05 \\ 87.703 & \text { MM m } & 4.07 & 48558.47 & 161.24 & 98.95 \\ & & \text { Sum } & 49071.96 & & \end{array}$

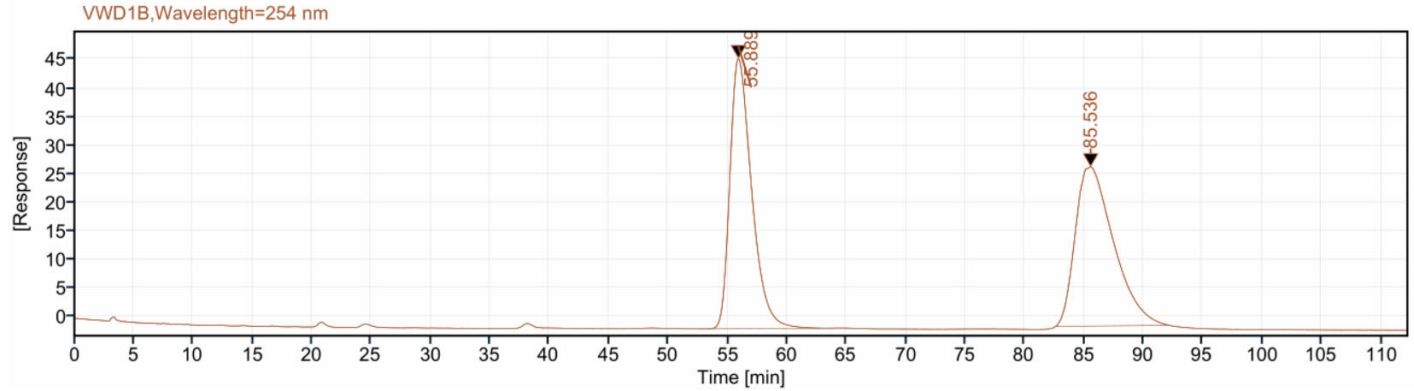

Signal: $\quad$ VWD1B,Wavelength $=254 \mathrm{~nm}$

$\begin{array}{rrrrrr}\text { RT [min] } & \text { Type } & \text { Width [min] } & \text { Area } & \text { Height } & \text { Area\% } \\ 55.889 & \text { BB } & 9.46 & 6021.88 & 47.15 & 49.14 \\ 85.536 & \text { MM m } & 2.76 & 6232.62 & 27.81 & 50.86 \\ & & \text { Sum } & 12254.50 & & \end{array}$




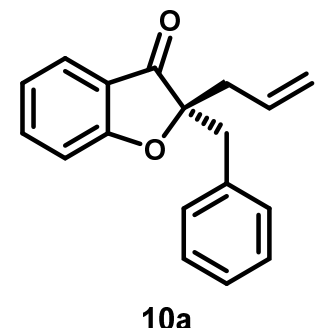

$89 \%$ y, $92 \%$ ee

Daicel Chiralpak IBN-3 column, n-hexane/i-PrOH (99.6/0.4), 0.5 mL/min 210 nm 16.969 min (major enantiomer), 15.970 min (minor enantiomer).

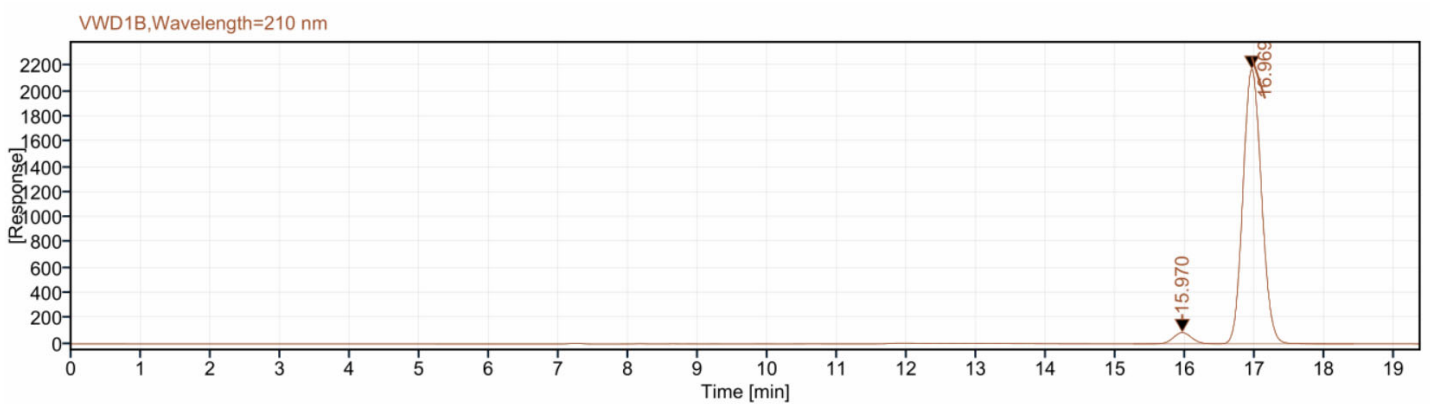

Signal: $\quad$ VWD1B, Wavelength $=210 \mathrm{~nm}$

$\begin{array}{rrrrrr}\text { RT [min] } & \text { Type } & \text { Width [min] } & \text { Area } & \text { Height } & \text { Area\% } \\ 15.970 & \text { BV } & 1.21 & 1664.85 & 89.57 & 3.97 \\ 16.969 & \text { VB } & 1.95 & 40279.94 & 2173.49 & 96.03 \\ & & \text { Sum } & 41944.79 & & \end{array}$

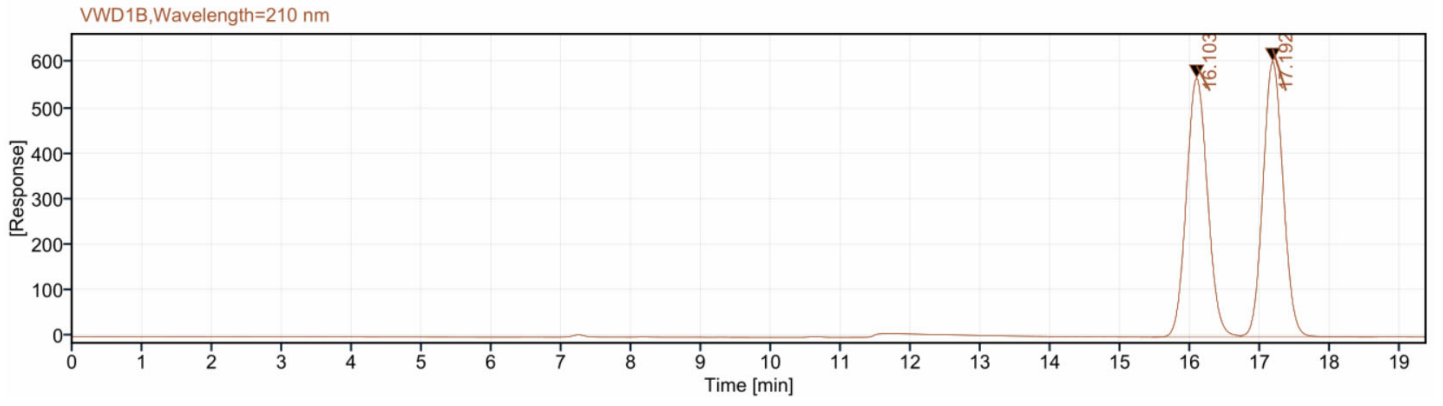

Signal: $\quad$ VWD1B, Wavelength $=210 \mathrm{~nm}$

$\begin{array}{rrrrrr}\text { RT [min] } & \text { Type } & \text { Width [min] } & \text { Area } & \text { Height } & \text { Area\% } \\ 16.103 & \text { BV } & 1.27 & 11976.03 & 566.16 & 49.71 \\ 17.192 & \text { VB } & 1.89 & 12117.88 & 602.78 & 50.29 \\ & & \text { Sum } & 24093.91 & & \end{array}$




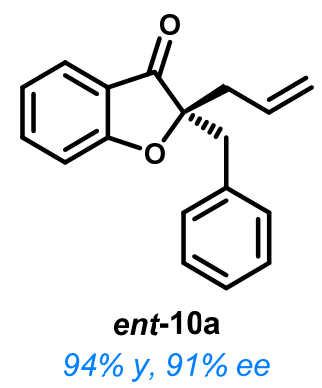

Daicel Chiralpak OD-H column, n-hexane/i-PrOH (99.4/0.6), $0.5 \mathrm{~mL} / \mathrm{min} 210 \mathrm{~nm} 18.554 \mathrm{~min}$ (major enantiomer), $21.159 \mathrm{~min}$ (minor enantiomer).

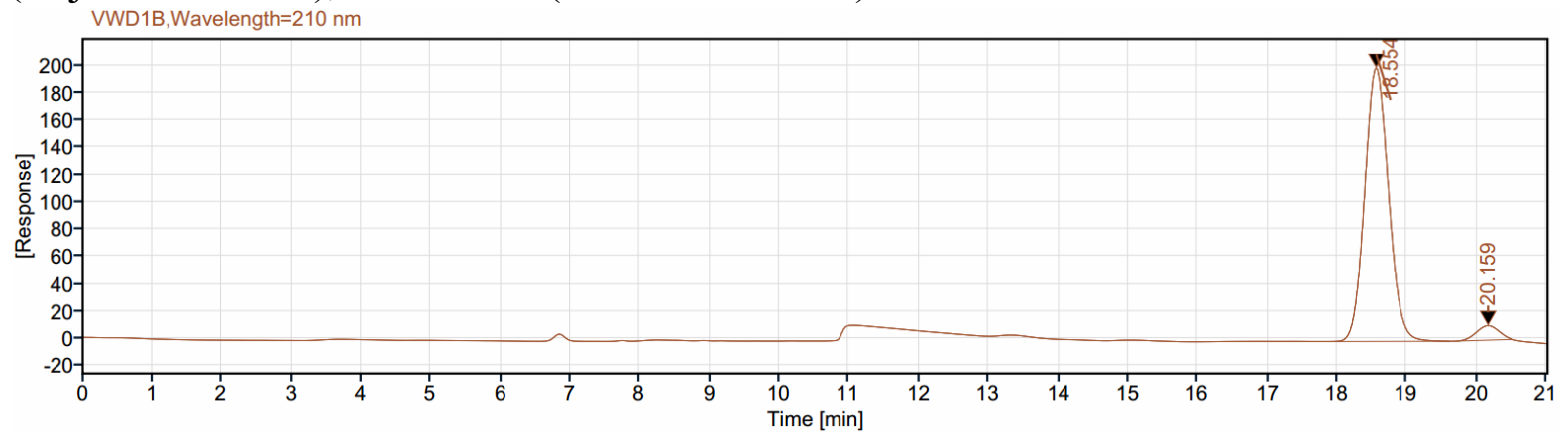

Signal: $\quad$ VWD1B,Wavelength $=210 \mathrm{~nm}$

$\begin{array}{rrrrrr}\text { RT [min] } & \text { Type } & \text { Width [min] } & \text { Area } & \text { Height } & \text { Area\% } \\ 18.554 & \text { BB } & 1.70 & 4761.61 & 199.81 & 95.26 \\ 20.159 & \text { MM m } & 0.36 & 237.12 & 10.53 & 4.74 \\ & & \text { Sum } & 4998.73 & & \end{array}$

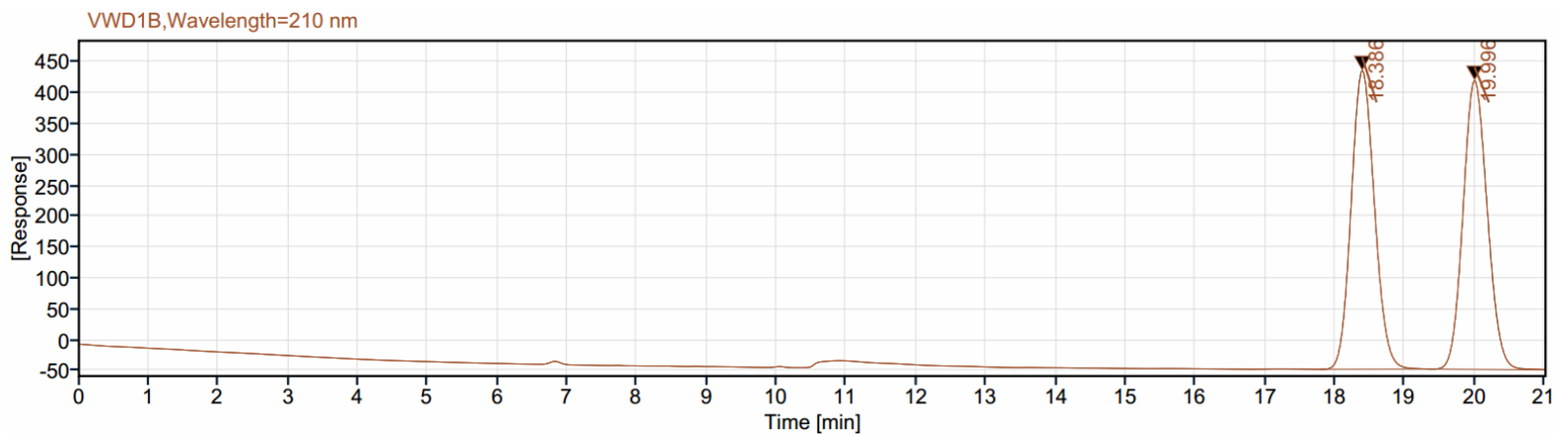

Signal: $\quad$ VWD1B,Wavelength $=210 \mathrm{~nm}$

$\begin{array}{rrrrrr}\text { RT [min] } & \text { Type } & \text { Width [min] } & \text { Area } & \text { Height } & \text { Area\% } \\ 18.386 & \text { BB } & 1.61 & 11225.73 & 483.57 & 49.78 \\ 19.996 & \text { BBA } & 1.88 & 11324.38 & 467.83 & 50.22 \\ & & \text { Sum } & 22550.11 & & \end{array}$




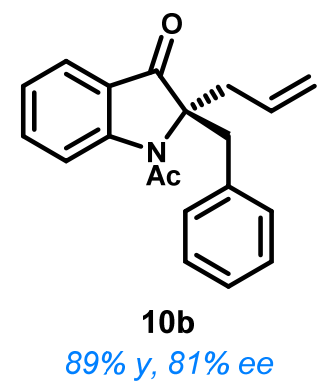

Daicel Chiralpak AD-H column, n-hexane/i-PrOH (95/5), $0.5 \mathrm{~mL} / \mathrm{min} 210 \mathrm{~nm} 23.788 \mathrm{~min}$ (major enantiomer), $19.623 \mathrm{~min}$ (minor enantiomer).

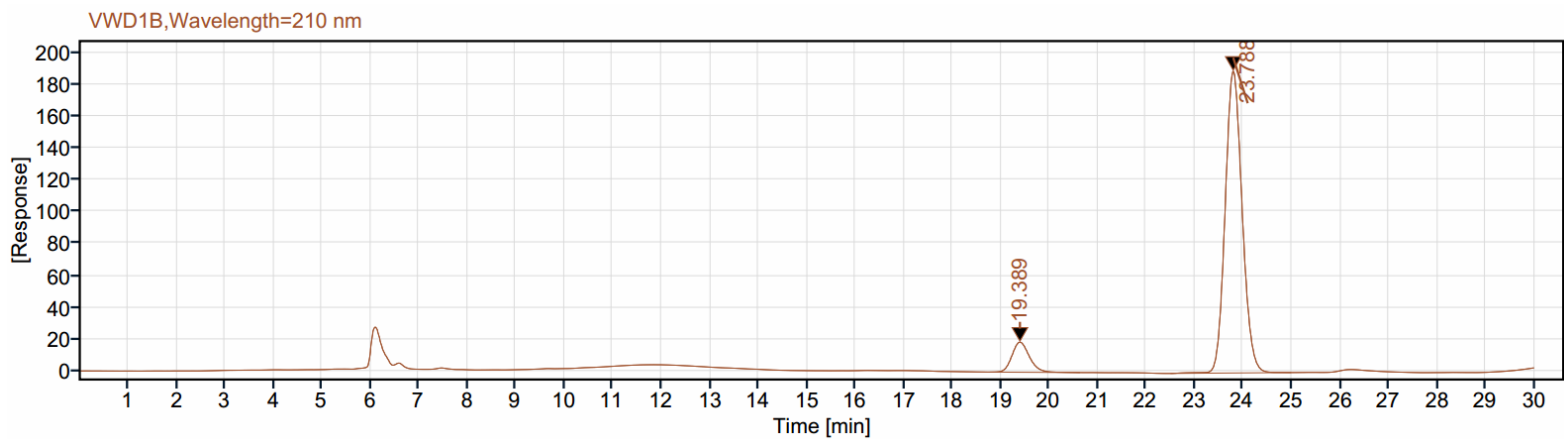

Signal: $\quad$ VWD1B,Wavelength $=210 \mathrm{~nm}$

$\begin{array}{rrrrrr}\text { RT [min] } & \text { Type } & \text { Width [min] } & \text { Area } & \text { Height } & \text { Area\% } \\ 19.389 & \text { BB } & 1.86 & 473.79 & 18.94 & 9.27 \\ 23.788 & \text { BBA } & 2.49 & 4637.94 & 190.13 & 90.73 \\ & & \text { Sum } & 5111.73 & & \end{array}$

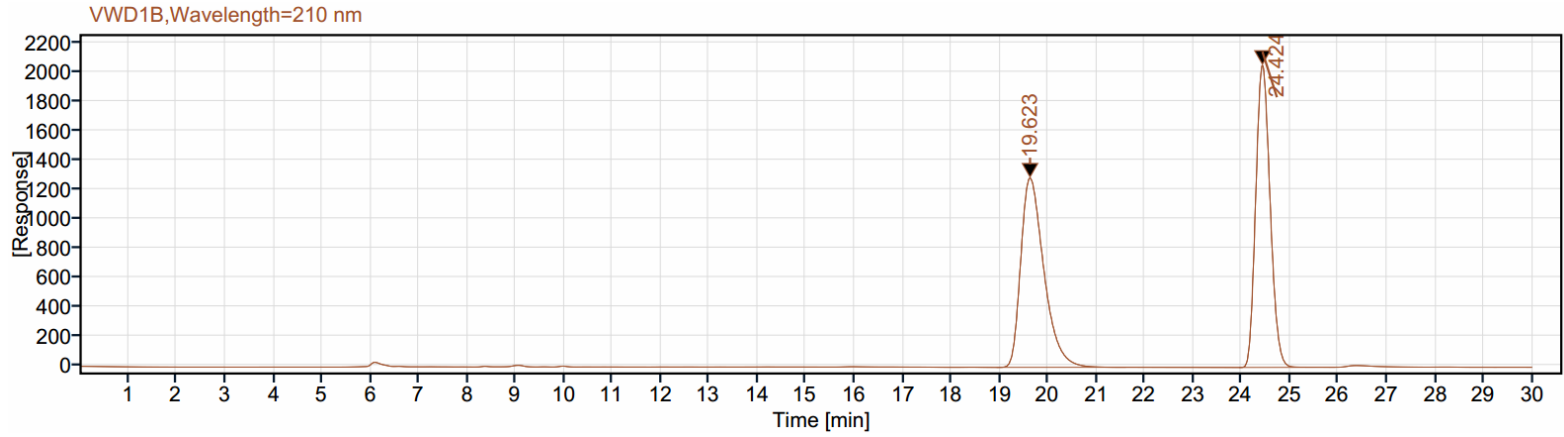

Signal: $\quad$ VWD1B,Wavelength $=210 \mathrm{~nm}$

$\begin{array}{rrrrrr}\text { RT [min] } & \text { Type } & \text { Width [min] } & \text { Area } & \text { Height } & \text { Area\% } \\ 19.623 & \text { BB } & 2.67 & 42612.75 & 1283.12 & 50.16 \\ 24.424 & \text { BV } & 1.99 & 42344.63 & 2047.87 & 49.84 \\ & & \text { Sum } & 84957.39 & & \end{array}$




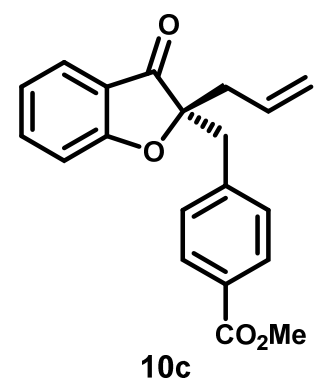

$99 \%$ y, $91 \%$ ee

Daicel Chiralpak OD-H column, n-hexane/i-PrOH (99/1), $0.5 \mathrm{~mL} / \mathrm{min} 210 \mathrm{~nm} 13.624 \mathrm{~min}$ (major enantiomer), $12.549 \mathrm{~min}$ (minor enantiomer).

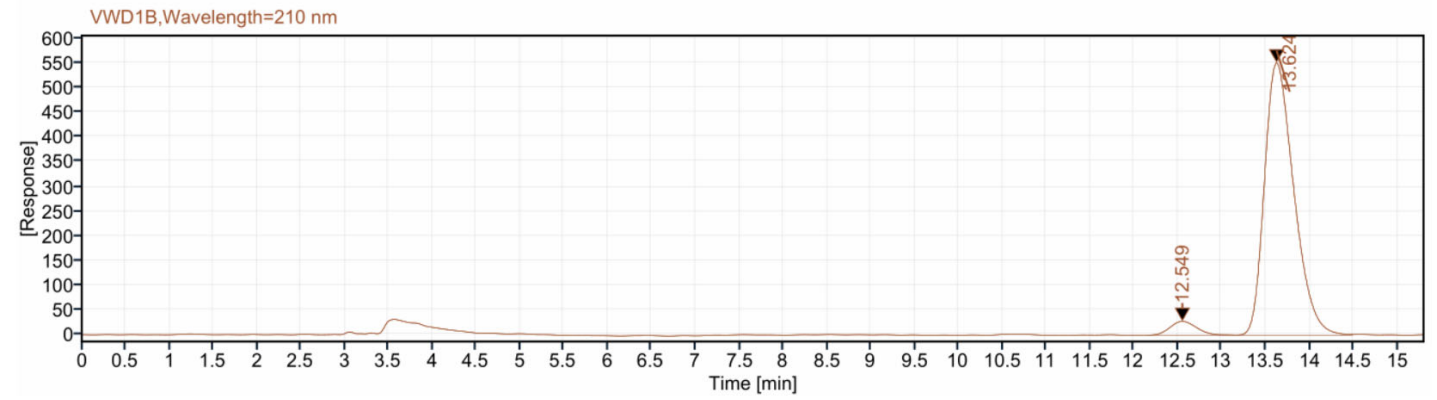

Signal: VWD1B,Wavelength $=210 \mathrm{~nm}$

$\begin{array}{rrrrrr}\text { RT [min] } & \text { Type } & \text { Width [min] } & \text { Area } & \text { Height } & \text { Area\% } \\ 12.549 & \text { VV } & 1.03 & 610.98 & 28.39 & 4.52 \\ 13.624 & \text { VV } & 1.33 & 12918.98 & 552.23 & 95.48 \\ & & \text { Sum } & 13529.96 & & \end{array}$

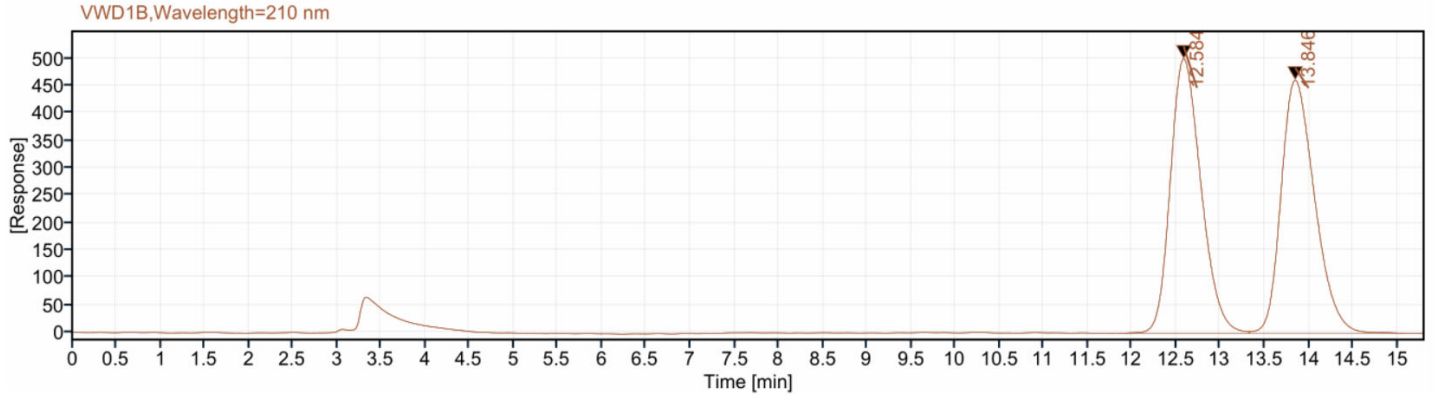

Signal: $\quad$ VWD1B, Wavelength $=210 \mathrm{~nm}$

$\begin{array}{rrrrrr}\text { RT [min] } & \text { Type } & \text { Width [min] } & \text { Area } & \text { Height } & \text { Area\% } \\ 12.584 & \text { BV } & 1.48 & 12115.55 & 500.87 & 49.79 \\ 13.846 & \text { VB } & 1.77 & 12216.67 & 462.15 & 50.21 \\ & & \text { Sum } & 24332.21 & & \end{array}$




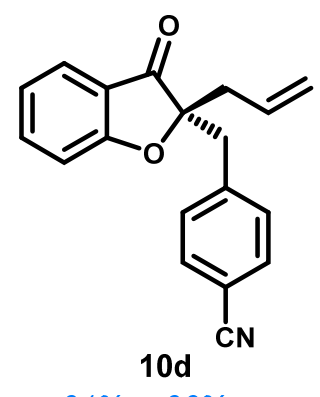

$91 \%$ y, $90 \%$ ee

Daicel Chiralpak AD-H column, n-hexane/i-PrOH (95/5), $1 \mathrm{~mL} / \mathrm{min} 250 \mathrm{~nm} 15.341 \mathrm{~min}$ (major enantiomer), 12.175 min (minor enantiomer).

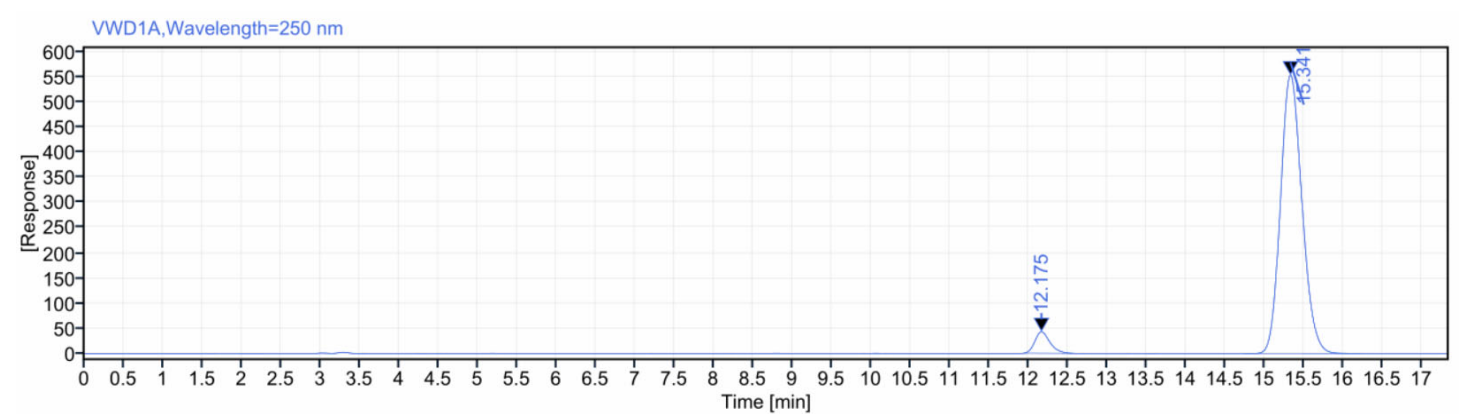

Signal: $\quad$ VWD1A,Wavelength $=250 \mathrm{~nm}$

$\begin{array}{rrrrrr}\text { RT [min] } & \text { Type } & \text { Width [min] } & \text { Area } & \text { Height } & \text { Area\% } \\ 12.175 & \text { MM m } & 0.20 & 568.85 & 42.85 & 5.12 \\ 15.341 & \text { MM m } & 0.30 & 10542.30 & 554.65 & 94.88 \\ & & \text { Sum } & 11111.15 & & \end{array}$

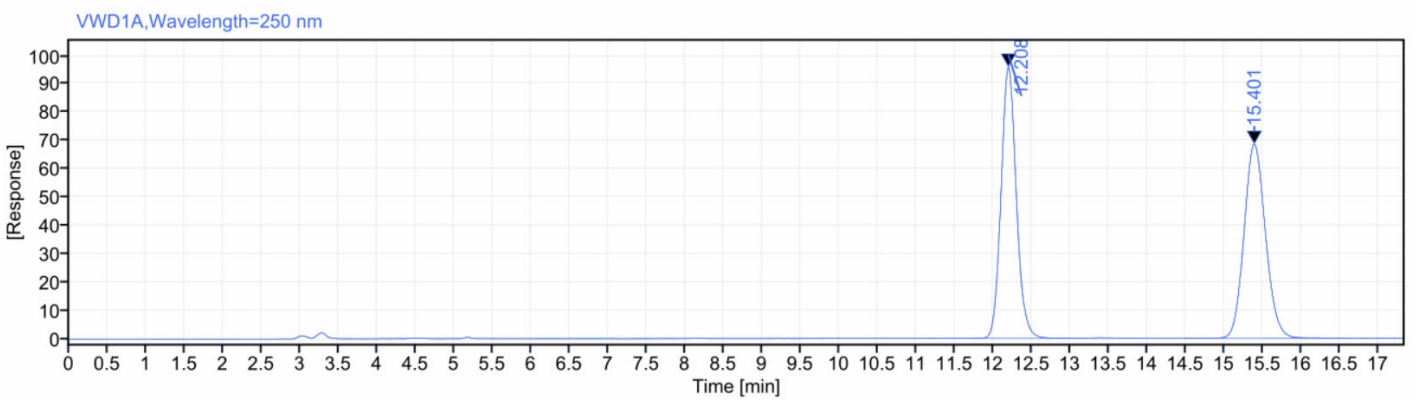

Signal: $\quad$ VWD1A, Wavelength $=250 \mathrm{~nm}$

$\begin{array}{rrrrrr}\text { RT [min] } & \text { Type } & \text { Width [min] } & \text { Area } & \text { Height } & \text { Area\% } \\ 12.208 & \text { BB } & 1.45 & 1317.32 & 95.78 & 49.96 \\ 15.401 & \text { BB } & 2.06 & 1319.41 & 68.44 & 50.04 \\ & & \text { Sum } & 2636.73 & & \end{array}$




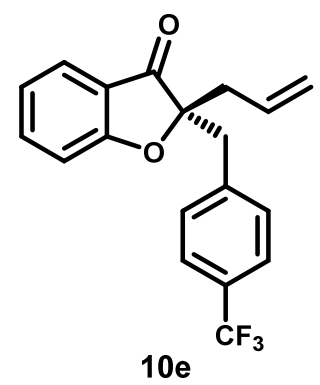

$92 \%$ y, $90 \%$ ee

Daicel Chiralpak AD-3 column, n-hexane/i-PrOH (99/1), 0.5 mL/min 210 nm 17.134 min (major enantiomer), $14.864 \mathrm{~min}$ (minor enantiomer).

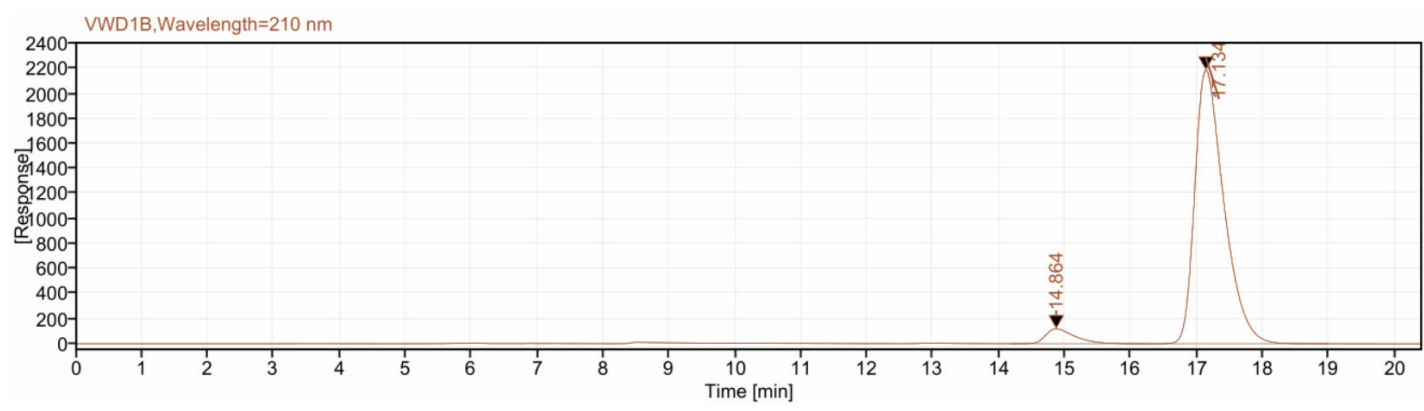

Signal: $\quad$ VWD1B, Wavelength $=210 \mathrm{~nm}$

$\begin{array}{rrrrrr}\text { RT [min] } & \text { Type } & \text { Width [min] } & \text { Area } & \text { Height } & \text { Area\% } \\ 14.864 & \text { BB } & 2.19 & 3698.92 & 120.63 & 5.20 \\ 17.134 & \text { BBA } & 2.63 & 67459.82 & 2192.51 & 94.80 \\ & & \text { Sum } & 71158.74 & & \end{array}$

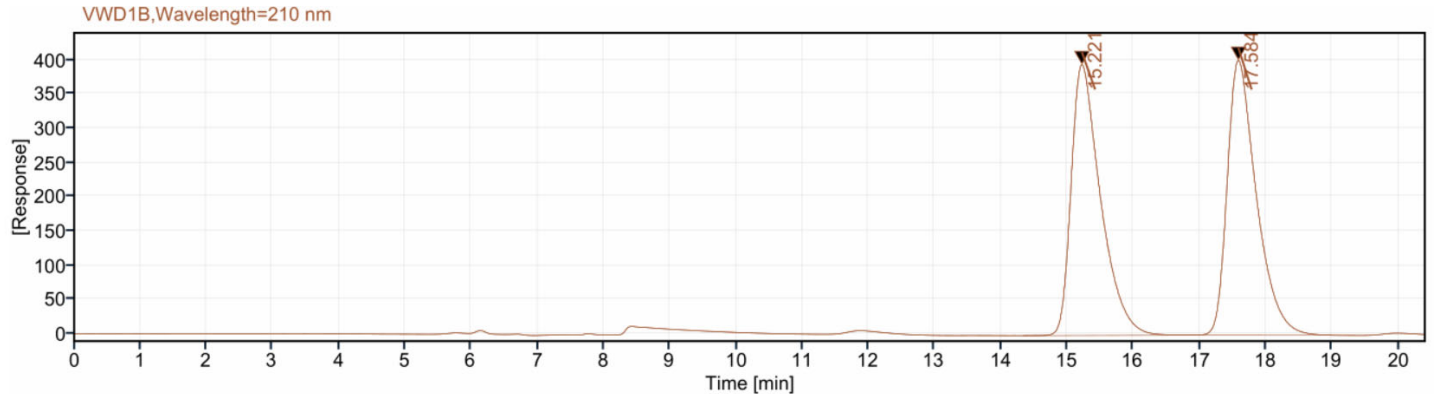

Signal: $\quad$ VWD1B,Wavelength $=210 \mathrm{~nm}$

$\begin{array}{rrrrrr}\text { RT [min] } & \text { Type } & \text { Width [min] } & \text { Area } & \text { Height } & \text { Area\% } \\ 15.221 & \text { BB } & 2.56 & 12228.58 & 395.74 & 49.82 \\ 17.584 & \text { BBA } & 2.11 & 12316.59 & 401.55 & 50.18 \\ & & \text { Sum } & 24545.17 & & \end{array}$




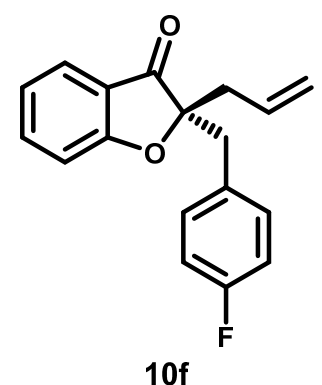

92\% y, $90 \%$ ee

Daicel Chiralpak AD-H column, n-hexane/i-PrOH (99/1), $0.5 \mathrm{~mL} / \mathrm{min} 210 \mathrm{~nm} 25.639 \mathrm{~min}$ (major enantiomer), 22.815 min (minor enantiomer).

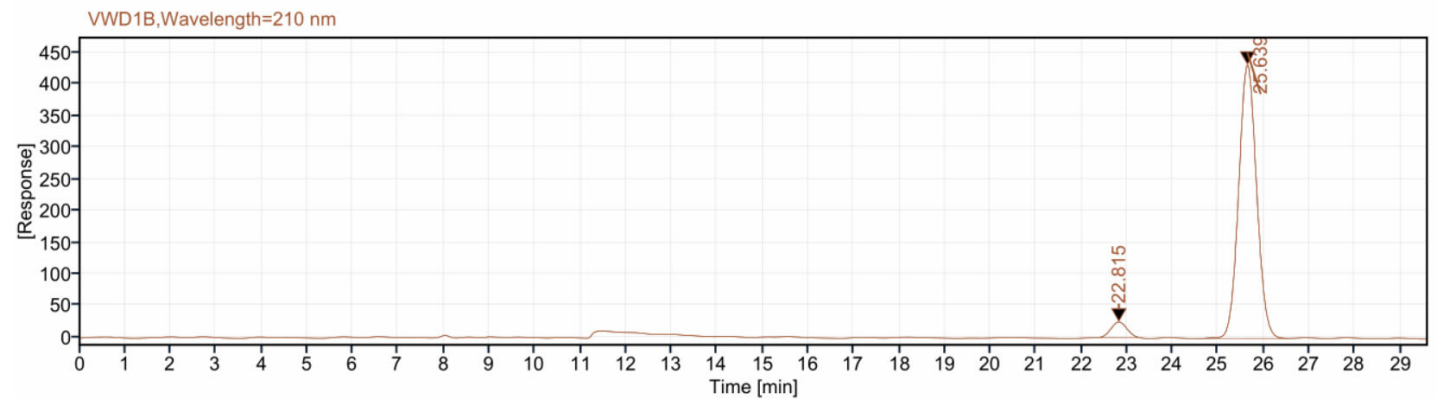

Signal: $\quad$ VWD1B,Wavelength $=210 \mathrm{~nm}$

$\begin{array}{rrrrrr}\text { RT [min] } & \text { Type } & \text { Width [min] } & \text { Area } & \text { Height } & \text { Area\% } \\ 22.815 & \text { MM m } & 0.39 & 604.14 & 24.73 & 4.83 \\ 25.639 & \text { BV } & 2.01 & 11892.51 & 431.66 & 95.17 \\ & & \text { Sum } & 12496.65 & & \end{array}$

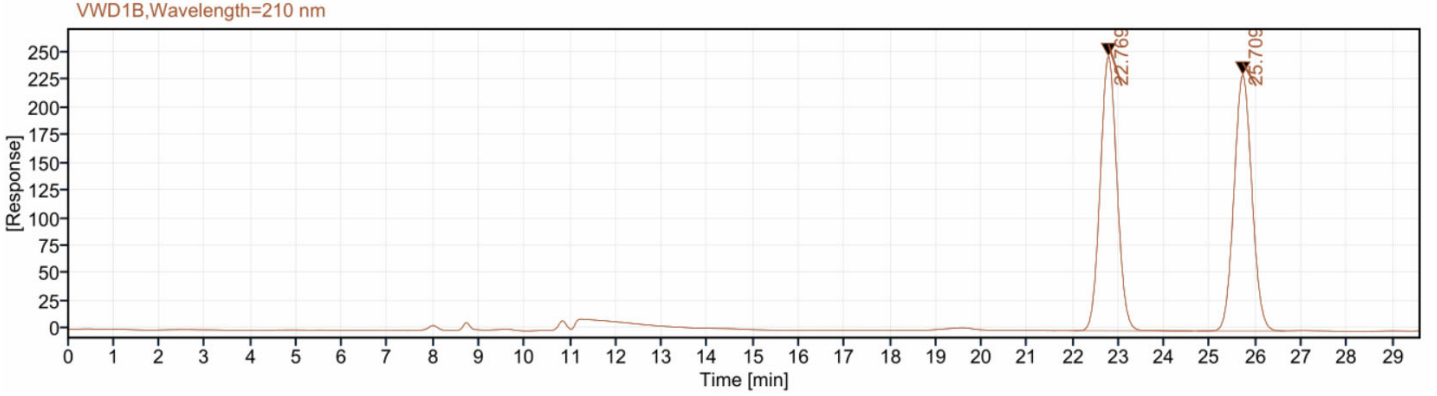

Signal: $\quad$ VWD1B,Wavelength $=210 \mathrm{~nm}$

$\begin{array}{rrrrrr}\text { RT [min] } & \text { Type } & \text { Width [min] } & \text { Area } & \text { Height } & \text { Area\% } \\ 22.769 & \text { BB } & 2.59 & 6296.60 & 247.71 & 50.05 \\ 25.709 & \text { BB } & 1.92 & 6283.40 & 231.11 & 49.95 \\ & & \text { Sum } & 12580.00 & & \end{array}$




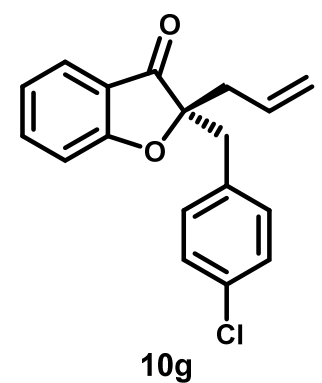

$93 \%$ y, $90 \%$ ee

Daicel Chiralpak AD-H column, n-hexane/i-PrOH (99.5/0.5), 0.5 mL/min $250 \mathrm{~nm} 25.754 \mathrm{~min}$ (major enantiomer), $23.530 \mathrm{~min}$ (minor enantiomer).

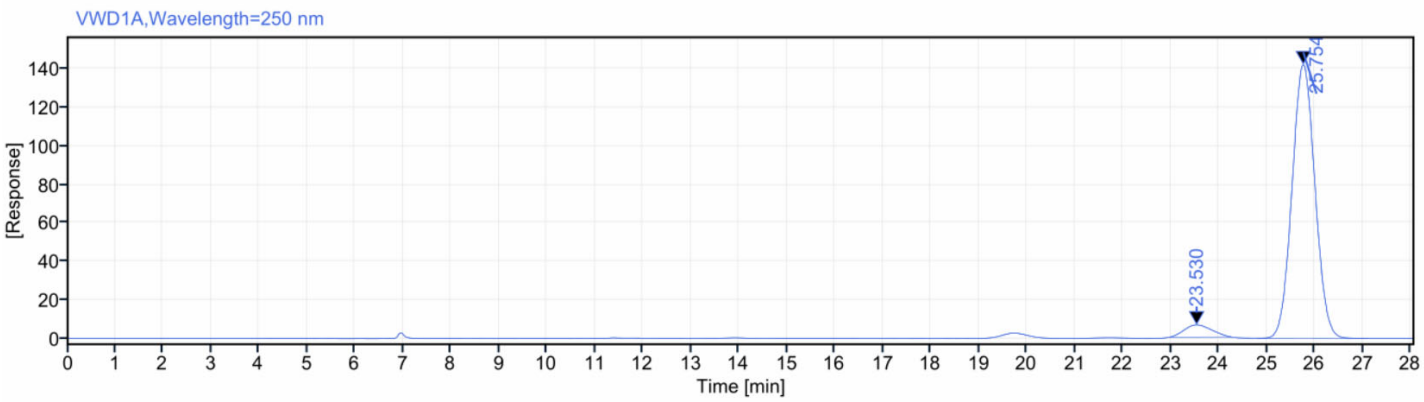

Signal: $\quad$ VWD1A,Wavelength $=250 \mathrm{~nm}$

$\begin{array}{rrrrrr}\text { RT [min] } & \text { Type } & \text { Width [min] } & \text { Area } & \text { Height } & \text { Area\% } \\ 23.530 & \text { MM m } & 0.63 & 255.50 & 6.41 & 5.12 \\ 25.754 & \text { MM m } & 0.52 & 4738.53 & 142.60 & 94.88 \\ & & \text { Sum } & 4994.03 & & \end{array}$

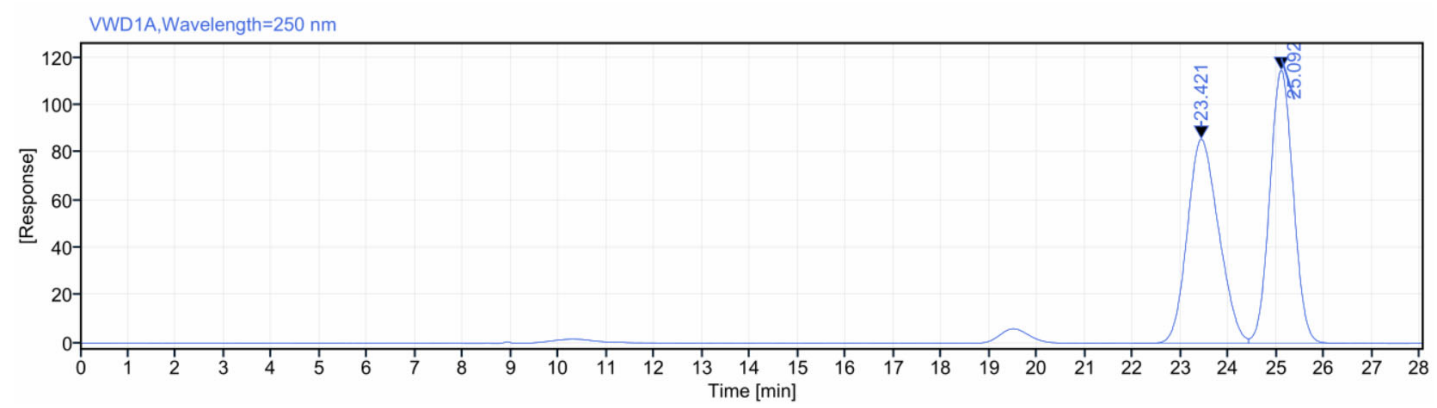

Signal: $\quad$ VWD1A, Wavelength $=250 \mathrm{~nm}$

$\begin{array}{rrrrrr}\text { RT [min] } & \text { Type } & \text { Width [min] } & \text { Area } & \text { Height } & \text { Area\% } \\ 23.421 & \text { BV } & 2.40 & 3915.89 & 85.84 & 49.99 \\ 25.092 & \text { VB } & 2.09 & 3918.24 & 114.75 & 50.01 \\ & & \text { Sum } & 7834.13 & & \end{array}$




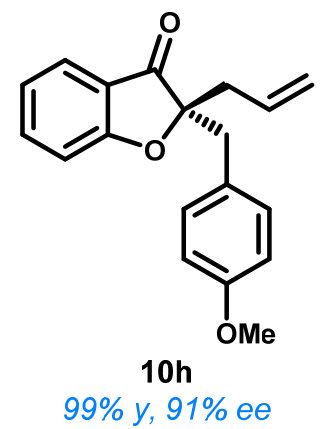

Daicel Chiralpak OD-H column, n-hexane/i-PrOH (99/1), $0.5 \mathrm{~mL} / \mathrm{min} 210 \mathrm{~nm} 23.343 \mathrm{~min}$ (major enantiomer), $21.681 \mathrm{~min}$ (minor enantiomer).

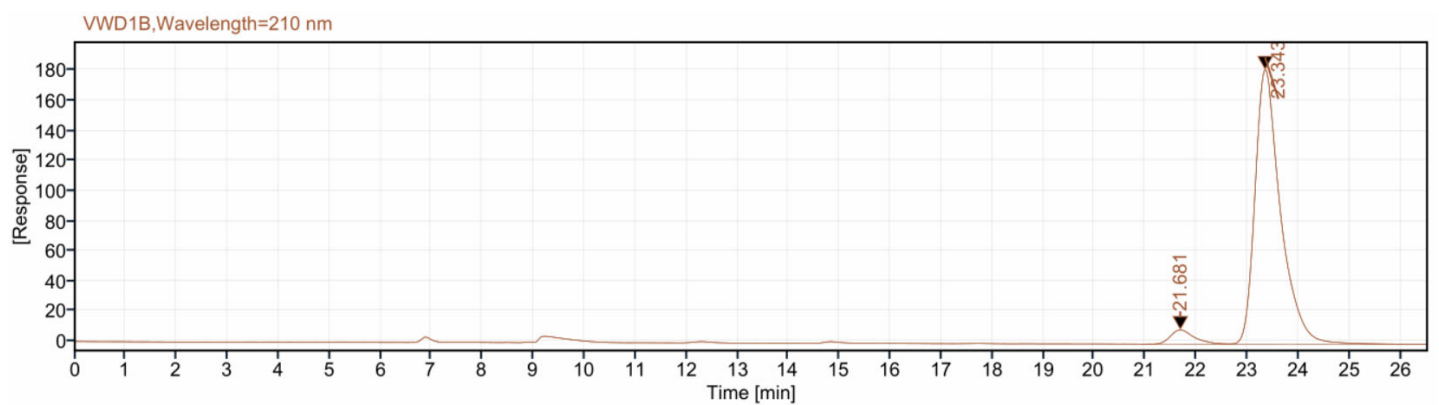

Signal: $\quad$ VWD1B,Wavelength $=210 \mathrm{~nm}$

$\begin{array}{rrrrrr}\text { RT [min] } & \text { Type } & \text { Width [min] } & \text { Area } & \text { Height } & \text { Area\% } \\ 21.681 & \text { BV } & 1.73 & 310.48 & 9.54 & 4.61 \\ 23.343 & \text { VB } & 4.43 & 6417.46 & 182.55 & 95.39 \\ & & \text { Sum } & 6727.94 & & \end{array}$

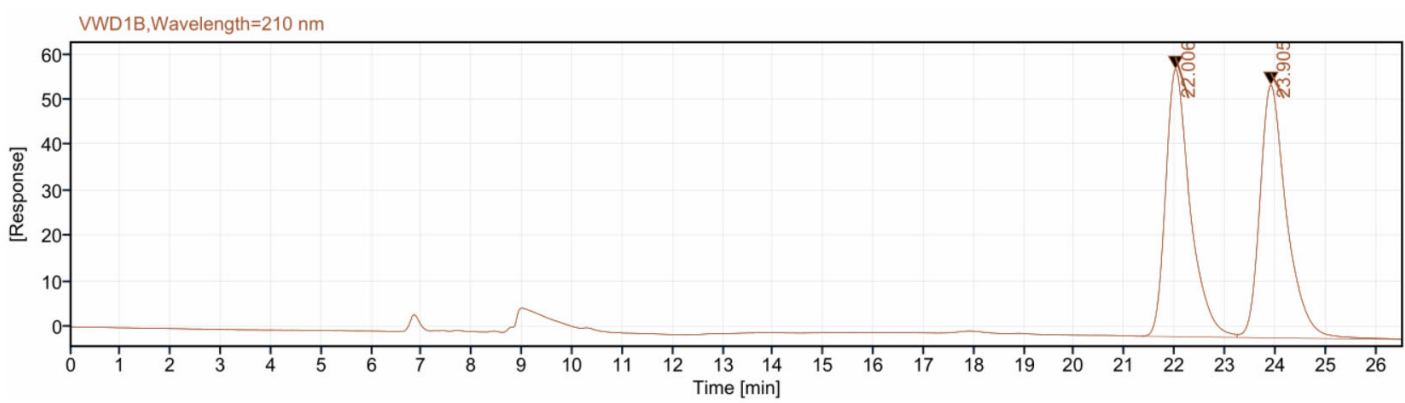

Signal: $\quad$ VWD1B,Wavelength $=210 \mathrm{~nm}$

$\begin{array}{rrrrrr}\text { RT [min] } & \text { Type } & \text { Width [min] } & \text { Area } & \text { Height } & \text { Area\% } \\ 22.006 & \text { BV } & 1.91 & 2003.10 & 58.90 & 49.35 \\ 23.905 & \text { VB } & 4.07 & 2056.19 & 55.67 & 50.65 \\ & & \text { Sum } & 4059.28 & & \end{array}$




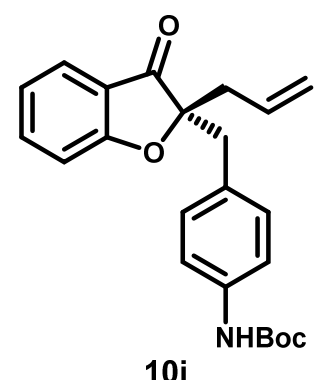

$88 \%$ y, $90 \%$ ee

Daicel Chiralpak OD-H column, n-hexane/i-PrOH (80/20), $1 \mathrm{~mL} / \mathrm{min} 250 \mathrm{~nm} 20.629 \mathrm{~min}$ (major enantiomer), 15.188 min (minor enantiomer).

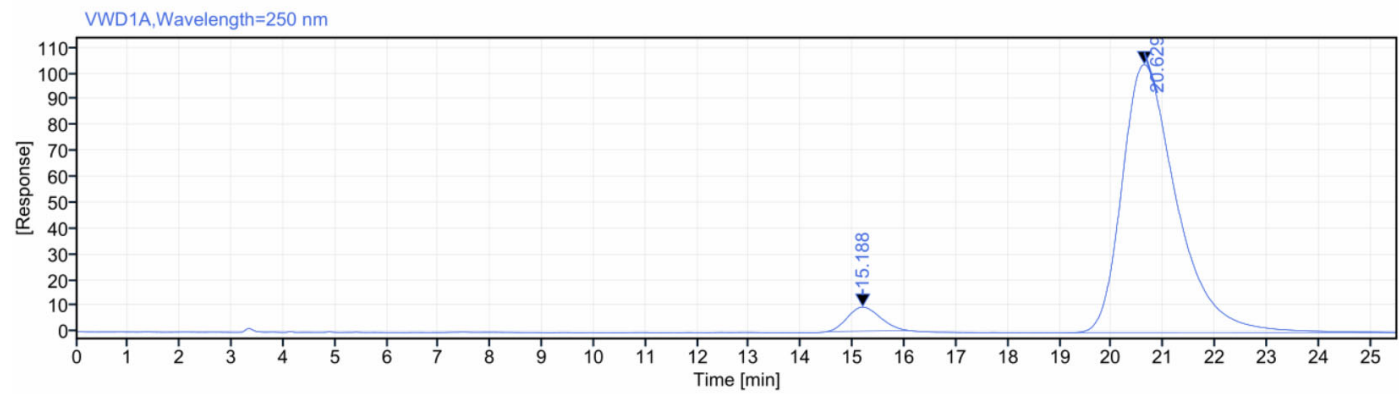

Signal: VWD1A, Wavelength $=250 \mathrm{~nm}$

$\begin{array}{rrrrrr}\text { RT [min] } & \text { Type } & \text { Width [min] } & \text { Area } & \text { Height } & \text { Area\% } \\ 15.188 & \text { MM m } & 0.69 & 411.18 & 9.35 & 5.12 \\ 20.629 & \text { BBA } & 6.87 & 7621.79 & 104.00 & 94.88 \\ & & \text { Sum } & 8032.97 & & \end{array}$

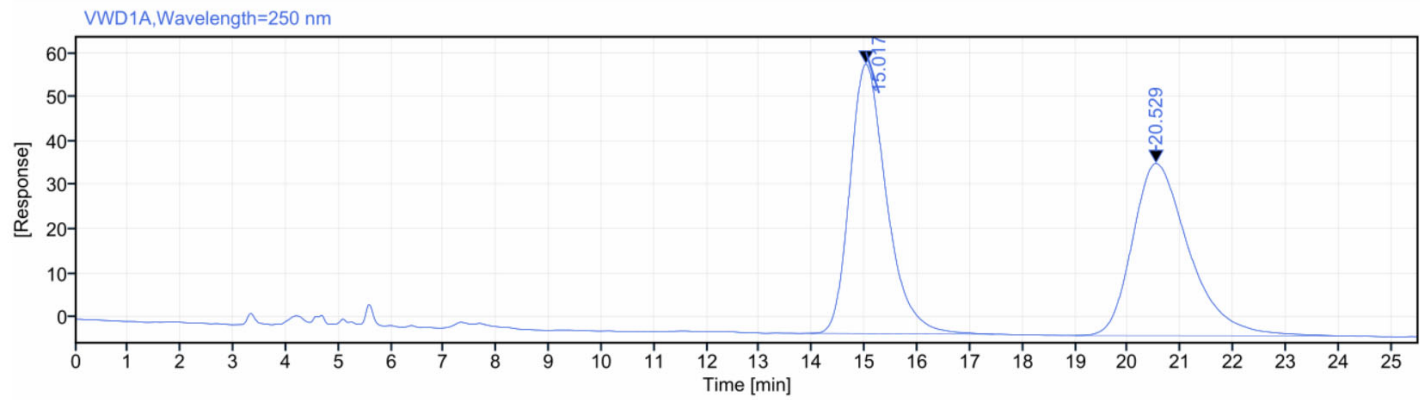

Signal: $\quad$ VWD1A,Wavelength $=250 \mathrm{~nm}$

$\begin{array}{rrrrrr}\text { RT [min] } & \text { Type } & \text { Width [min] } & \text { Area } & \text { Height } & \text { Area\% } \\ 15.017 & \text { BB } & 3.49 & 2962.11 & 61.17 & 50.44 \\ 20.529 & \text { BB } & 4.98 & 2909.89 & 39.08 & 49.56 \\ & & \text { Sum } & 5872.00 & & \end{array}$




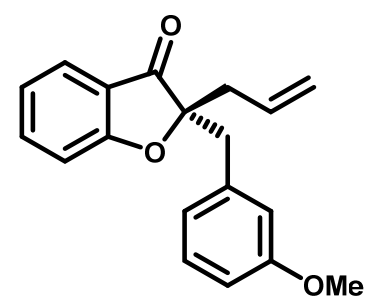

$10 \mathrm{j}$

$99 \%$ y, $91 \%$ ee

Daicel Chiralpak OD-H column, n-hexane/i-PrOH (99/1), $0.5 \mathrm{~mL} / \mathrm{min} 210 \mathrm{~nm} 18.113 \mathrm{~min}$ (major enantiomer), $21.104 \mathrm{~min}$ (minor enantiomer).

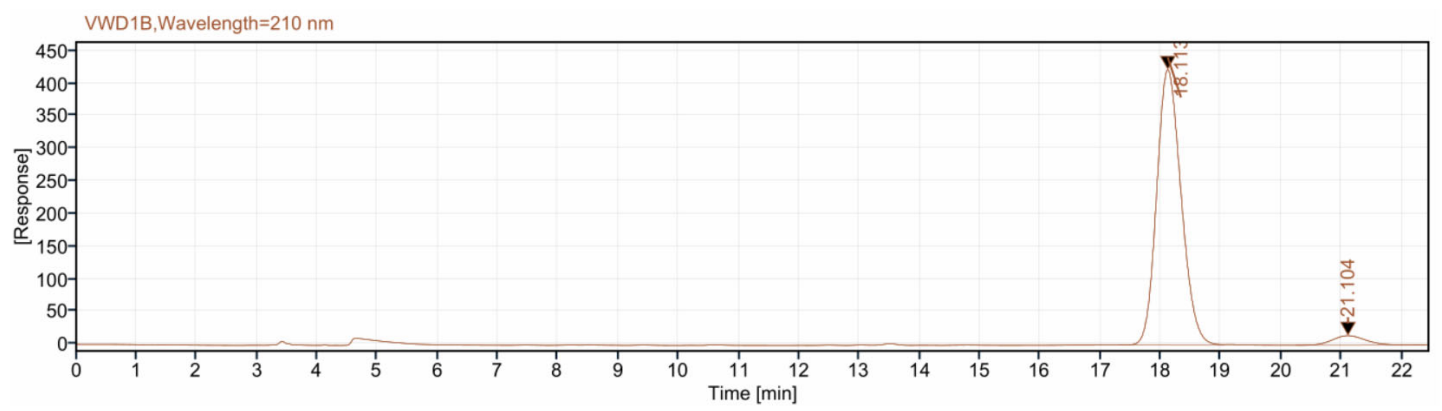

Signal: $\quad$ VWD1B, Wavelength $=210 \mathrm{~nm}$

$\begin{array}{rrrrrr}\text { RT [min] } & \text { Type } & \text { Width [min] } & \text { Area } & \text { Height } & \text { Area\% } \\ 18.113 & \text { BV } & 1.74 & 11919.66 & 422.24 & 95.49 \\ 21.104 & \text { BB } & 2.15 & 563.21 & 14.57 & 4.51 \\ & & \text { Sum } & 12482.86 & & \end{array}$

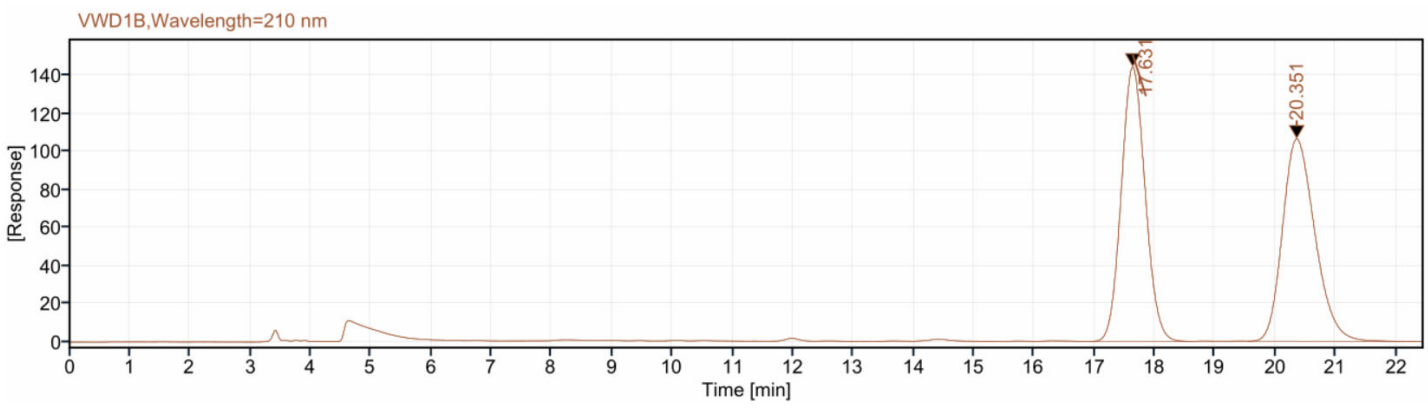

Signal: $\quad$ VWD1B, Wavelength $=210 \mathrm{~nm}$

$\begin{array}{rrrrrr}\text { RT [min] } & \text { Type } & \text { Width [min] } & \text { Area } & \text { Height } & \text { Area\% } \\ 17.631 & \text { BB } & 1.71 & 3973.42 & 144.14 & 50.00 \\ 20.351 & \text { BB } & 2.61 & 3972.75 & 106.30 & 50.00 \\ & & \text { Sum } & 7946.16 & & \end{array}$




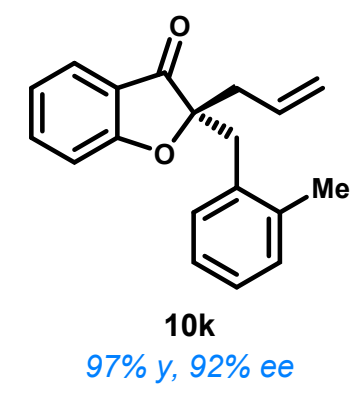

Daicel Chiralpak IG-3 column, n-hexane/i-PrOH (98/2), 1 mL/min $210 \mathrm{~nm} 8.466$ min (major enantiomer), $8.136 \mathrm{~min}$ (minor enantiomer).

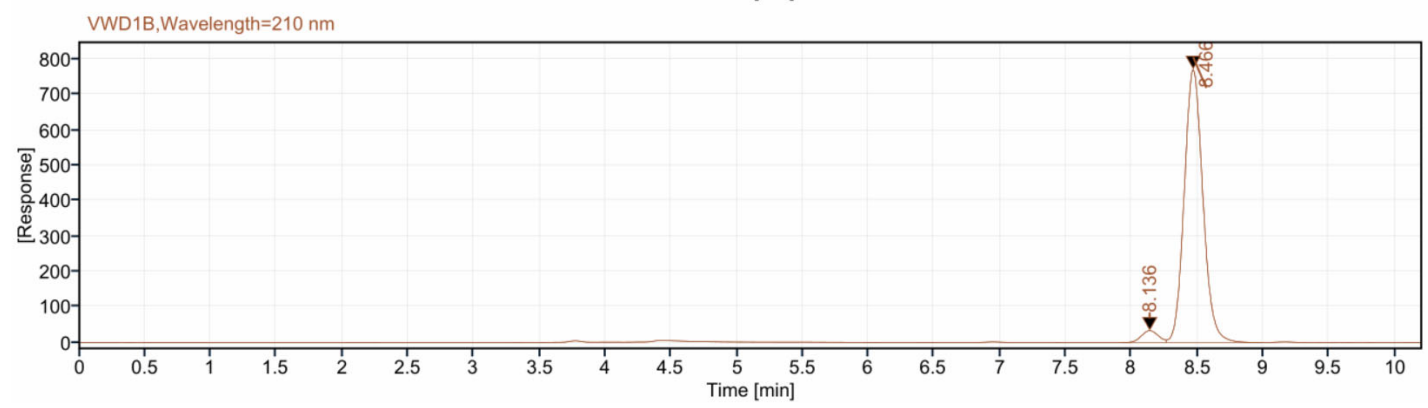

Signal: $\quad$ VWD1B, Wavelength $=210 \mathrm{~nm}$

$\begin{array}{rrrrrr}\text { RT }[\mathrm{min}] & \text { Type } & \text { Width [min] } & \text { Area } & \text { Height } & \text { Area\% } \\ 8.136 & \text { BV } & 0.35 & 302.27 & 33.73 & 3.81 \\ 8.466 & \text { VBA } & 0.73 & 7628.81 & 772.85 & 96.19 \\ & & \text { Sum } & 7931.09 & & \end{array}$

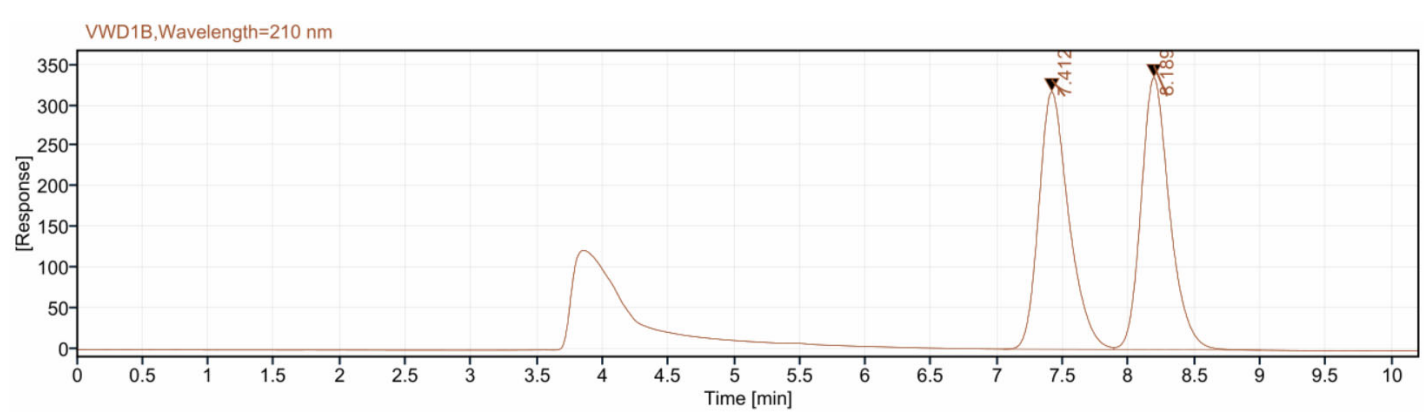

Signal: $\quad$ VWD1B, Wavelength $=210 \mathrm{~nm}$

$\begin{array}{rrrrrr}\text { RT [min] } & \text { Type } & \text { Width [min] } & \text { Area } & \text { Height } & \text { Area\% } \\ 7.412 & \text { BV } & 0.84 & 5023.19 & 317.03 & 49.83 \\ 8.189 & \text { VBA } & 1.12 & 5057.92 & 334.90 & 50.17 \\ & & \text { Sum } & 10081.12 & & \end{array}$




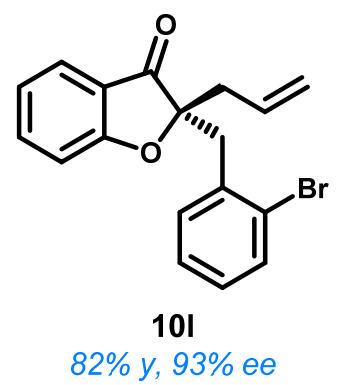

Daicel Chiralpak IC-3 column, n-hexane/i-PrOH (99.5/0.5), $1 \mathrm{~mL} / \mathrm{min} 210 \mathrm{~nm} 12.968 \mathrm{~min}$ (major enantiomer), $14.438 \mathrm{~min}$ (minor enantiomer).

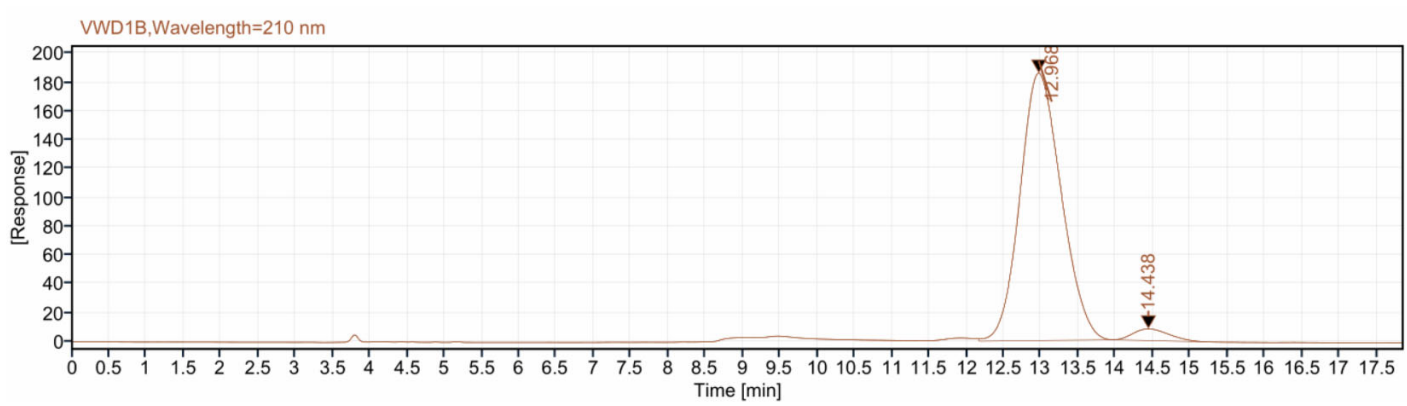

Signal: $\quad$ VWD1B,Wavelength $=210 \mathrm{~nm}$

$\begin{array}{rrrrrr}\text { RT [min] } & \text { Type } & \text { Width [min] } & \text { Area } & \text { Height } & \text { Area\% } \\ 12.968 & \text { VM m } & 0.60 & 7151.13 & 185.55 & 96.35 \\ 14.438 & \text { MM m } & 0.53 & 271.23 & 8.09 & 3.65 \\ & & \text { Sum } & 7422.35 & & \end{array}$

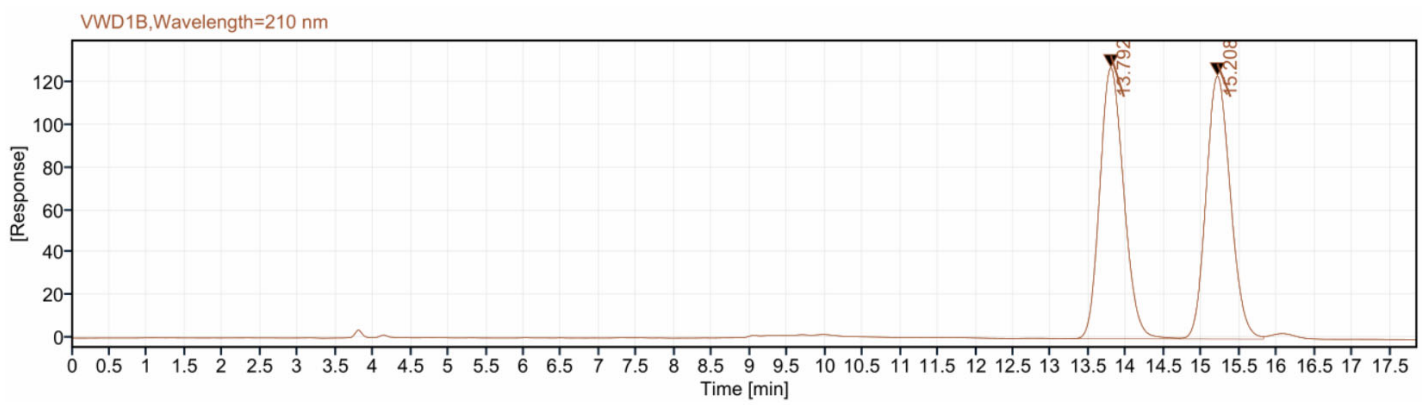

Signal: $\quad$ VWD1B,Wavelength $=210 \mathrm{~nm}$

$\begin{array}{rrrrrr}\text { RT [min] } & \text { Type } & \text { Width [min] } & \text { Area } & \text { Height } & \text { Area\% } \\ 13.792 & \text { BV } & 1.45 & 2801.51 & 126.77 & 50.30 \\ 15.208 & \text { VV } & 1.12 & 2767.85 & 123.04 & 49.70 \\ & & \text { Sum } & 5569.36 & & \end{array}$




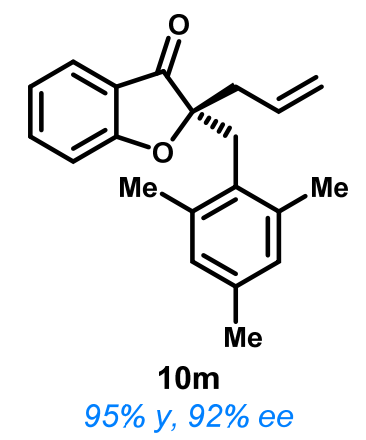

Daicel Chiralpak IA-3 column, n-hexane/i-PrOH (99.5/0.5), $0.5 \mathrm{~mL} / \mathrm{min} 210 \mathrm{~nm} 12.591 \mathrm{~min}$ (major enantiomer), $11.999 \mathrm{~min}$ (minor enantiomer).

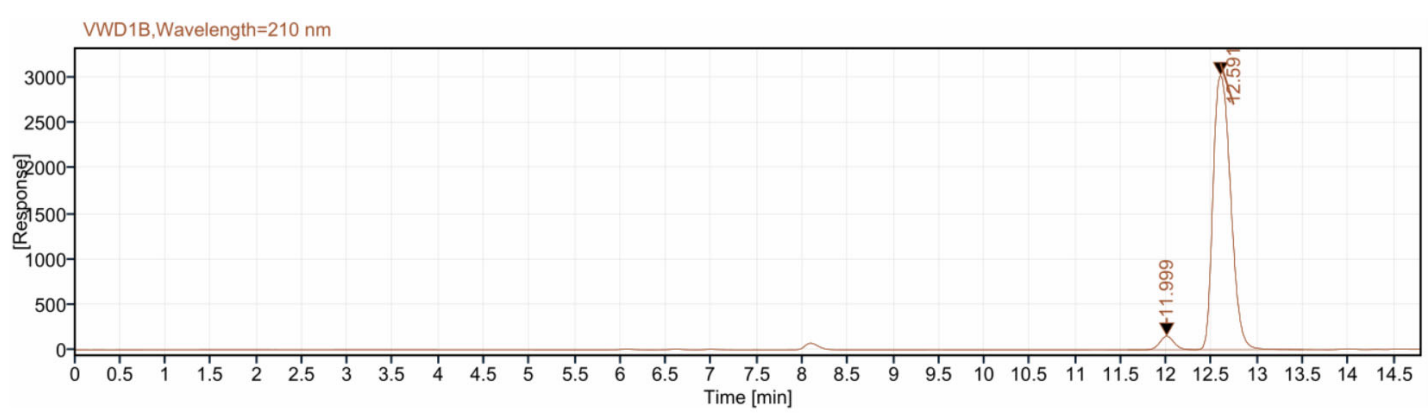

Signal: $\quad$ VWD1B,Wavelength $=210 \mathrm{~nm}$

$\begin{array}{rrrrrr}\text { RT [min] } & \text { Type } & \text { Width [min] } & \text { Area } & \text { Height } & \text { Area\% } \\ 11.999 & \text { BV } & 0.74 & 1637.94 & 149.58 & 3.85 \\ 12.591 & \text { VBA } & 1.19 & 40905.23 & 3030.12 & 96.15 \\ & & \text { Sum } & 42543.17 & & \end{array}$

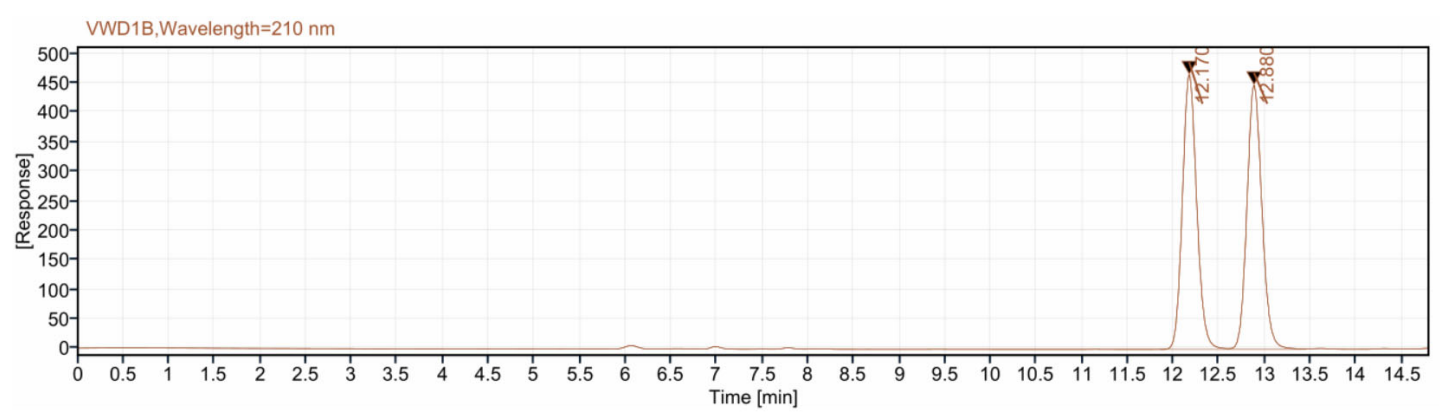

Signal: $\quad$ VWD1B,Wavelength $=210 \mathrm{~nm}$

$\begin{array}{rrrrrr}\text { RT [min] } & \text { Type } & \text { Width [min] } & \text { Area } & \text { Height } & \text { Area\% } \\ 12.170 & \text { BV } & 0.75 & 5089.56 & 466.13 & 49.89 \\ 12.880 & \text { VV } & 0.85 & 5112.22 & 448.24 & 50.11 \\ & & \text { Sum } & 10201.78 & & \end{array}$




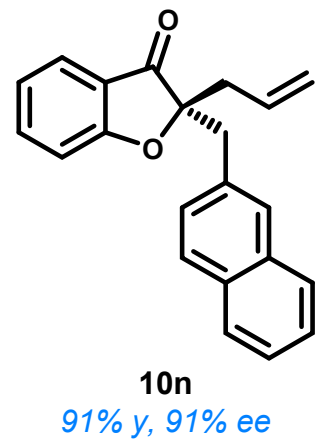

Daicel Chiralpak ID-3 column, n-hexane/i-PrOH (99/1), 0.5 mL/min $210 \mathrm{~nm} 19.658 \mathrm{~min}$ (major enantiomer), $25.978 \mathrm{~min}$ (minor enantiomer).

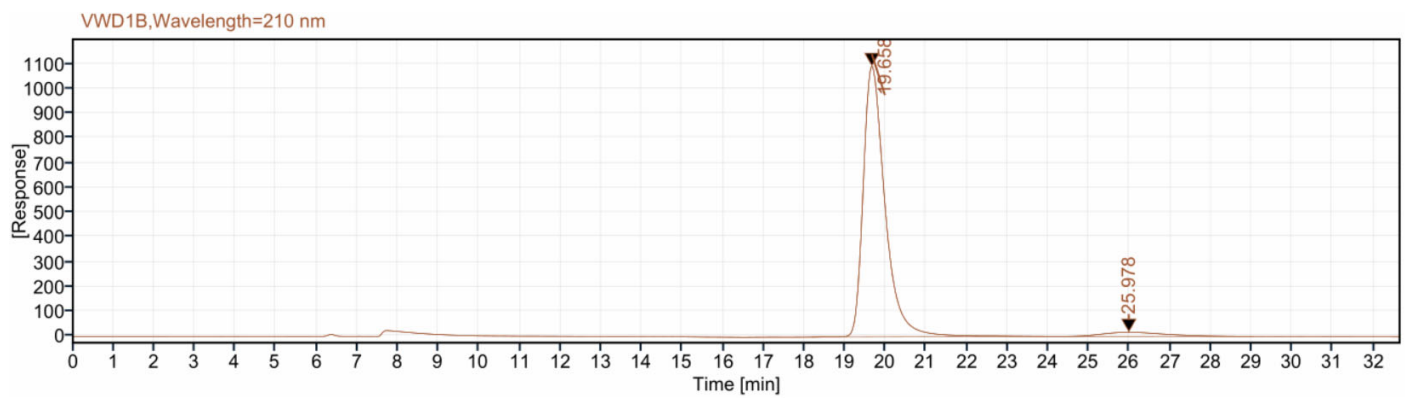

Signal: $\quad$ VWD1B,Wavelength $=210 \mathrm{~nm}$

$\begin{array}{rrrrrr}\text { RT [min] } & \text { Type } & \text { Width [min] } & \text { Area } & \text { Height } & \text { Area\% } \\ 19.658 & \text { MM m } & 0.57 & 40895.58 & 1090.22 & 95.61 \\ 25.978 & \text { MM m } & 1.65 & 1876.30 & 16.99 & 4.39 \\ & & \text { Sum } & 42771.88 & & \end{array}$

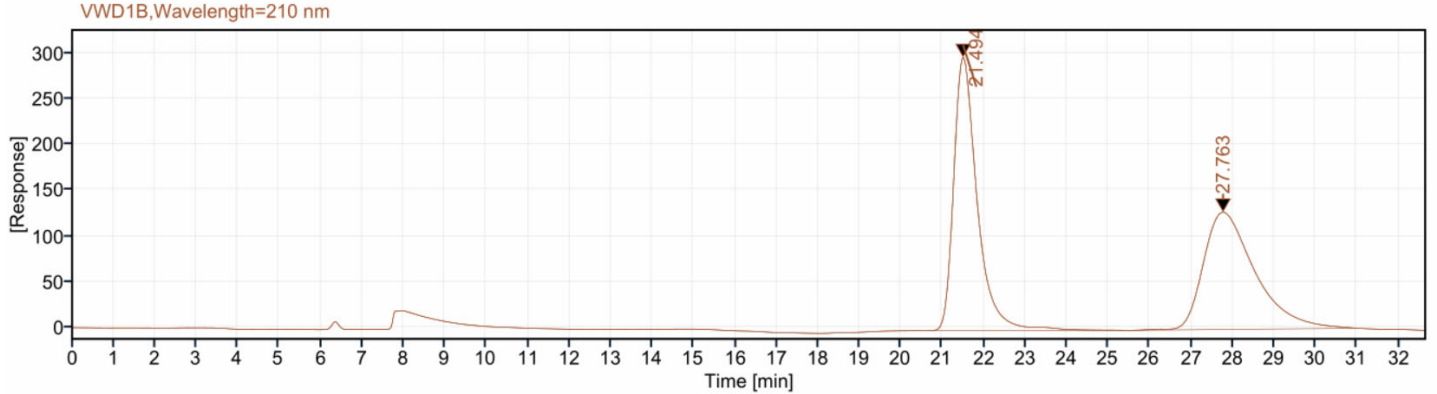

Signal: $\quad$ VWD1B, Wavelength $=210 \mathrm{~nm}$

$\begin{array}{rrrrrr}\text { RT [min] } & \text { Type } & \text { Width [min] } & \text { Area } & \text { Height } & \text { Area\% } \\ 21.494 & \text { BM m } & 0.59 & 11758.03 & 298.00 & 51.60 \\ 27.763 & \text { BBA } & 5.43 & 11028.95 & 127.95 & 48.40 \\ & & \text { Sum } & 22786.98 & & \end{array}$




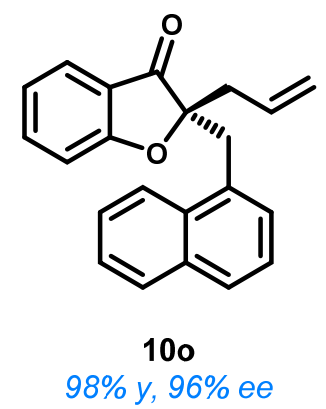

Daicel Chiralpak IA-3 column, n-hexane/i-PrOH (95/5), $0.3 \mathrm{~mL} / \mathrm{min} 210 \mathrm{~nm} 18.729 \mathrm{~min}$ (major enantiomer), $17.860 \mathrm{~min}$ (minor enantiomer).

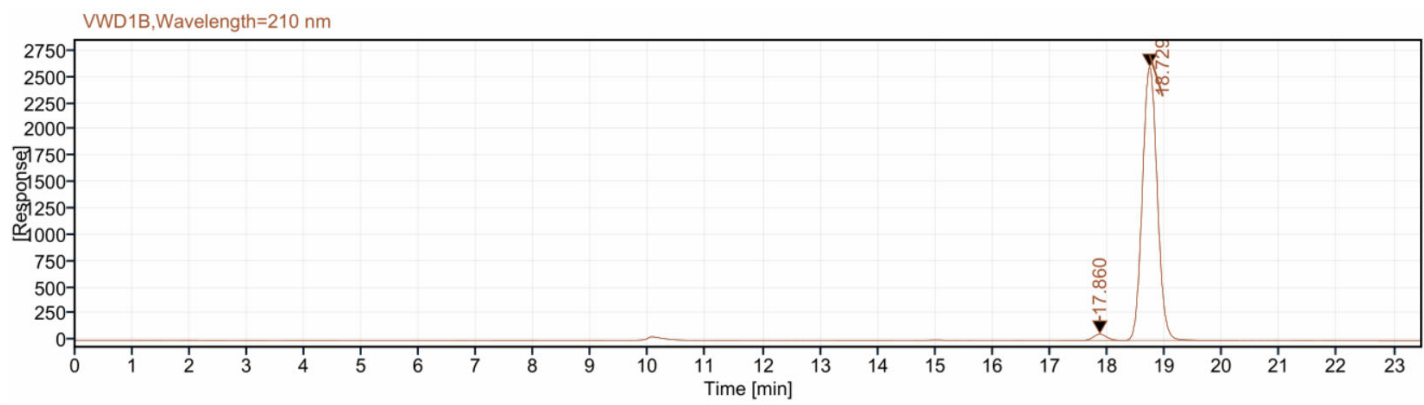

Signal: $\quad$ VWD1B, Wavelength $=210 \mathrm{~nm}$

$\begin{array}{rrrrrr}\text { RT [min] } & \text { Type } & \text { Width [min] } & \text { Area } & \text { Height } & \text { Area\% } \\ 17.860 & \text { BV } & 1.01 & 1056.90 & 62.19 & 2.21 \\ 18.729 & \text { VB } & 2.02 & 46765.22 & 2596.60 & 97.79 \\ & & \text { Sum } & 47822.12 & & \end{array}$

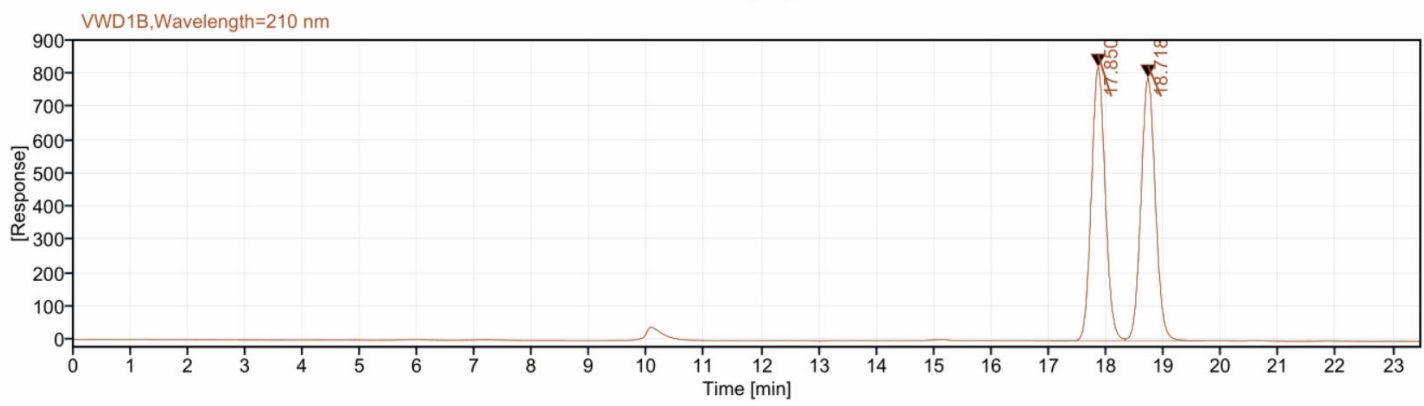

Signal: $\quad$ VWD1B,Wavelength $=210 \mathrm{~nm}$

$\begin{array}{rrrrrr}\text { RT [min] } & \text { Type } & \text { Width [min] } & \text { Area } & \text { Height } & \text { Area\% } \\ 17.850 & \text { BV } & 1.30 & 13812.83 & 824.69 & 49.88 \\ 18.718 & \text { VV } & 1.37 & 13877.24 & 792.39 & 50.12 \\ & & \text { Sum } & 27690.07 & & \end{array}$




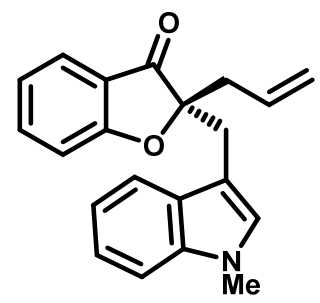

$10 p$

$99 \%$ y, $90 \%$ ee

Daicel Chiralpak AD-H column, n-hexane/i-PrOH (99.4/0.6), 0.5 mL/min $210 \mathrm{~nm} 19.685 \mathrm{~min}$ (major enantiomer), $17.348 \mathrm{~min}$ (minor enantiomer).

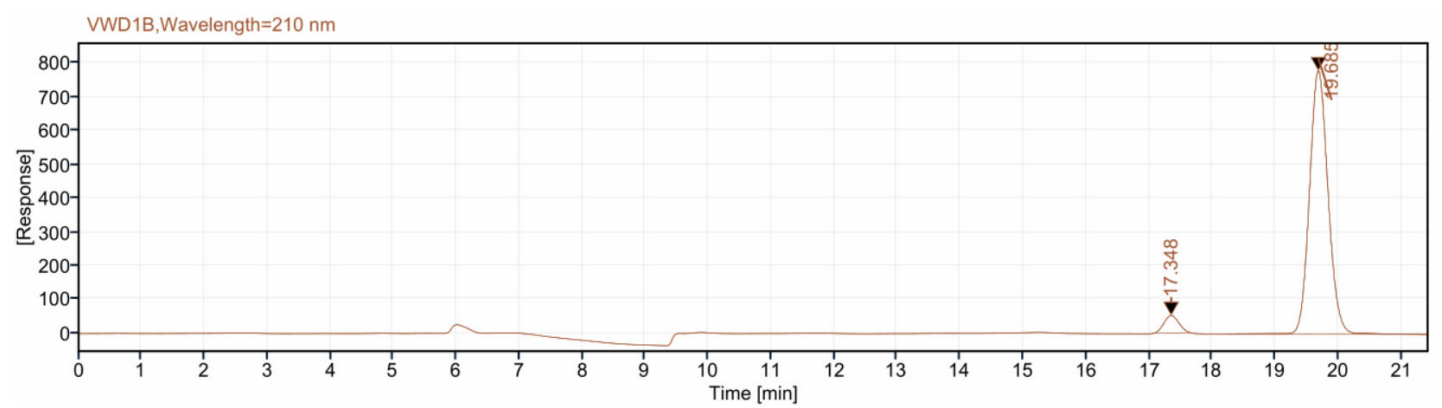

Signal: $\quad$ VWD1B,Wavelength $=210 \mathrm{~nm}$

$\begin{array}{rrrrrr}\text { RT [min] } & \text { Type } & \text { Width [min] } & \text { Area } & \text { Height } & \text { Area\% } \\ 17.348 & \text { MM m } & 0.26 & 850.80 & 51.70 & 5.04 \\ 19.685 & \text { BB } & 3.26 & 16034.08 & 780.16 & 94.96 \\ & & \text { Sum } & 16884.88 & & \end{array}$

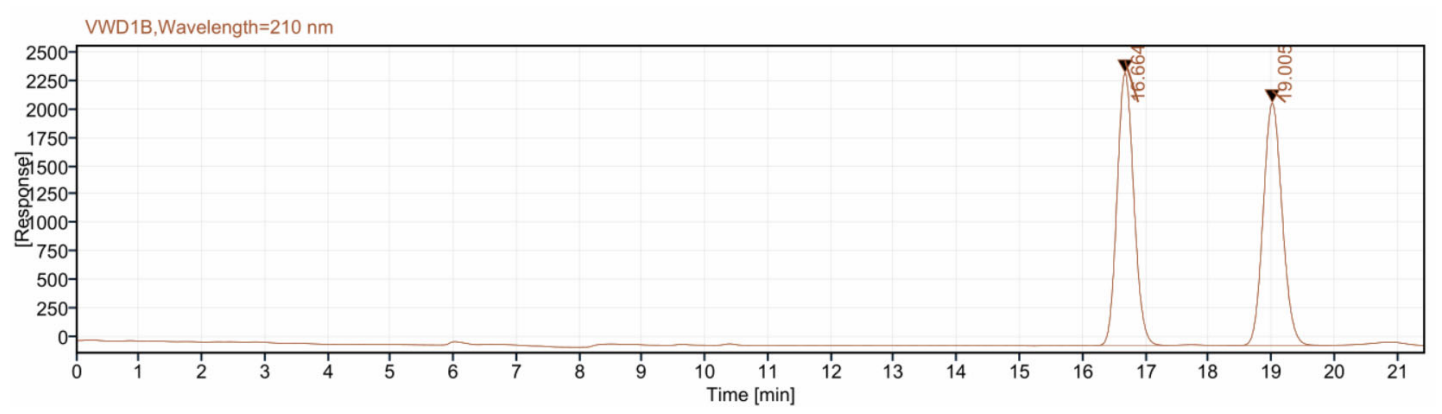

Signal: $\quad$ VWD1B,Wavelength $=210 \mathrm{~nm}$

$\begin{array}{rrrrrr}\text { RT [min] } & \text { Type } & \text { Width [min] } & \text { Area } & \text { Height } & \text { Area\% } \\ 16.664 & \text { BV } & 1.20 & 44376.91 & 2393.70 & 49.78 \\ 19.005 & \text { BV } & 1.66 & 44762.32 & 2131.15 & 50.22 \\ & & \text { Sum } & 89139.24 & & \end{array}$




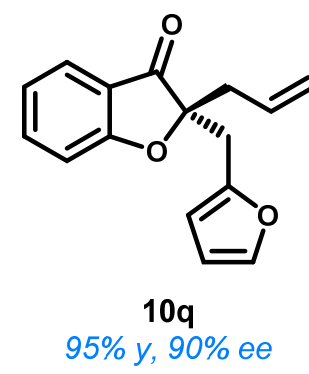

Daicel Chiralpak AD-H column, n-hexane/i-PrOH (99.4/0.6), 0.5 mL/min 210 nm 20.794 min (major enantiomer), 22.955 min (minor enantiomer).

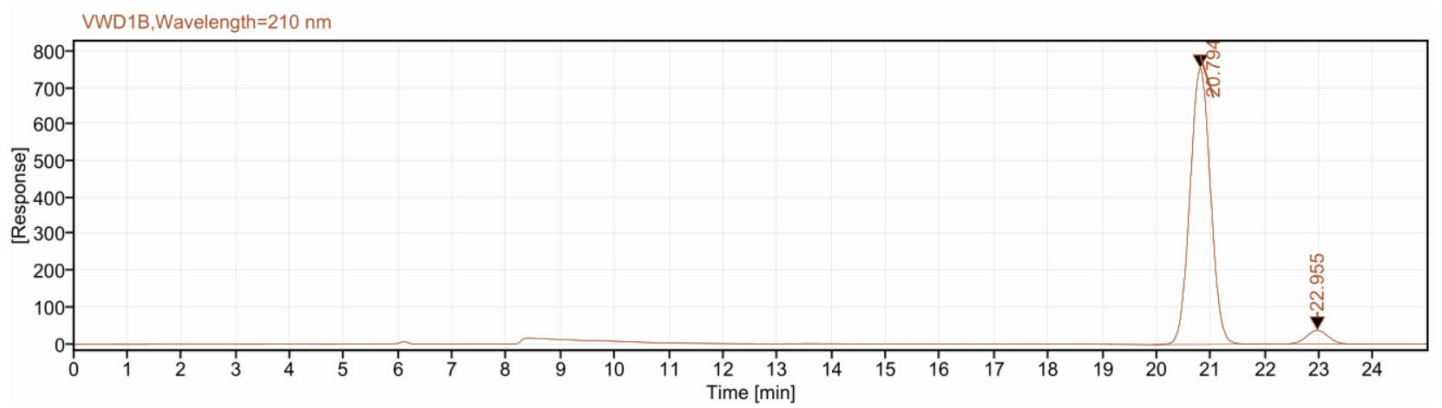

Signal: $\quad$ VWD1B,Wavelength $=210 \mathrm{~nm}$

$\begin{array}{rrrrrr}\text { RT [min] } & \text { Type } & \text { Width [min] } & \text { Area } & \text { Height } & \text { Area\% } \\ 20.794 & \text { BB } & 2.25 & 19874.20 & 754.18 & 95.16 \\ 22.955 & \text { MM m } & 0.43 & 1011.91 & 36.99 & 4.84 \\ & & \text { Sum } & 20886.11 & & \end{array}$

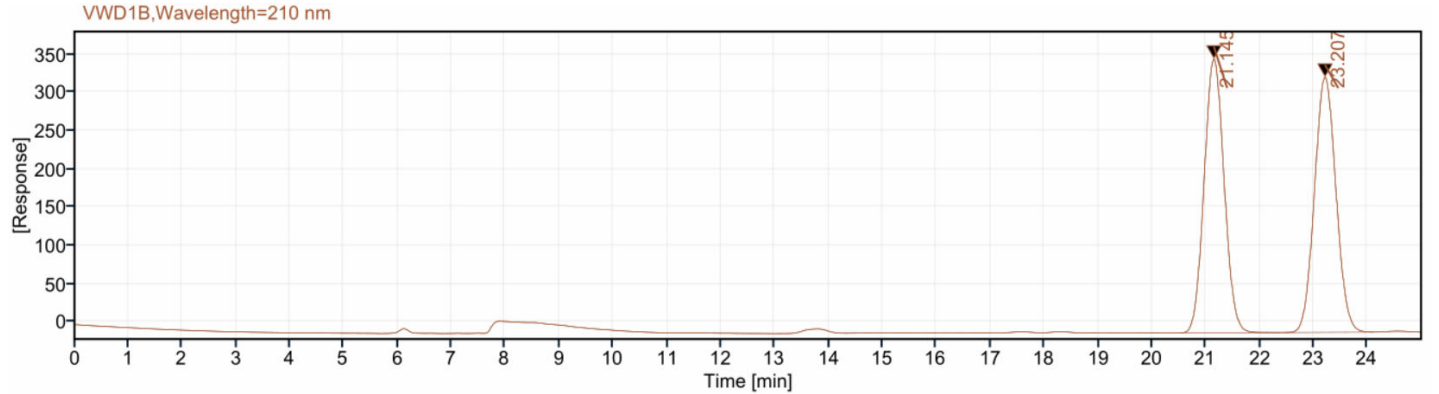

Signal: $\quad$ VWD1B,Wavelength $=210 \mathrm{~nm}$

$\begin{array}{rrrrrr}\text { RT [min] } & \text { Type } & \text { Width [min] } & \text { Area } & \text { Height } & \text { Area\% } \\ 21.145 & \text { BB } & 1.88 & 9265.43 & 358.61 & 49.98 \\ 23.207 & \text { BB } & 1.68 & 9273.23 & 334.12 & 50.02 \\ & & \text { Sum } & 18538.66 & & \end{array}$




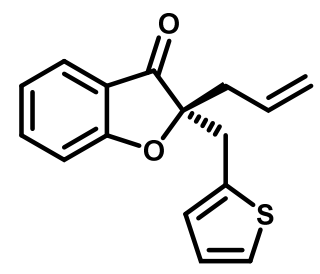

$10 r$

94\% y, 92\% ee

Daicel Chiralpak IC-3 column, n-hexane/i-PrOH (90/10), $0.5 \mathrm{~mL} / \mathrm{min} 210 \mathrm{~nm} 11.093 \mathrm{~min}$ (major enantiomer), $10.036 \mathrm{~min}$ (minor enantiomer).

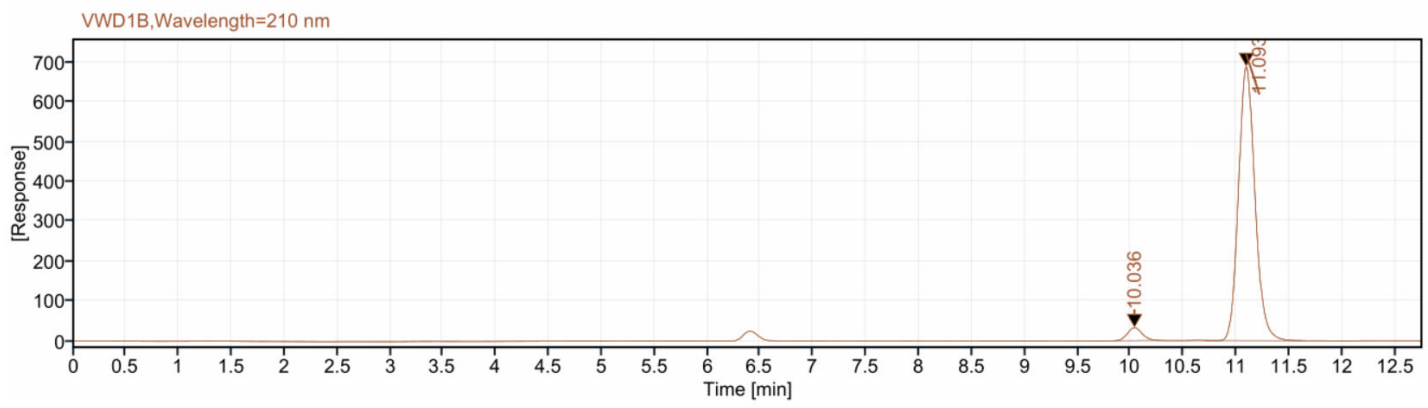

Signal: $\quad$ VWD1B,Wavelength $=210 \mathrm{~nm}$

$\begin{array}{rrrrrr}\text { RT [min] } & \text { Type } & \text { Width [min] } & \text { Area } & \text { Height } & \text { Area\% } \\ 10.036 & \text { MM m } & 0.15 & 310.43 & 32.21 & 3.98 \\ 11.093 & \text { MM m } & 0.17 & 7480.81 & 688.22 & 96.02 \\ & & \text { Sum } & 7791.24 & & \end{array}$

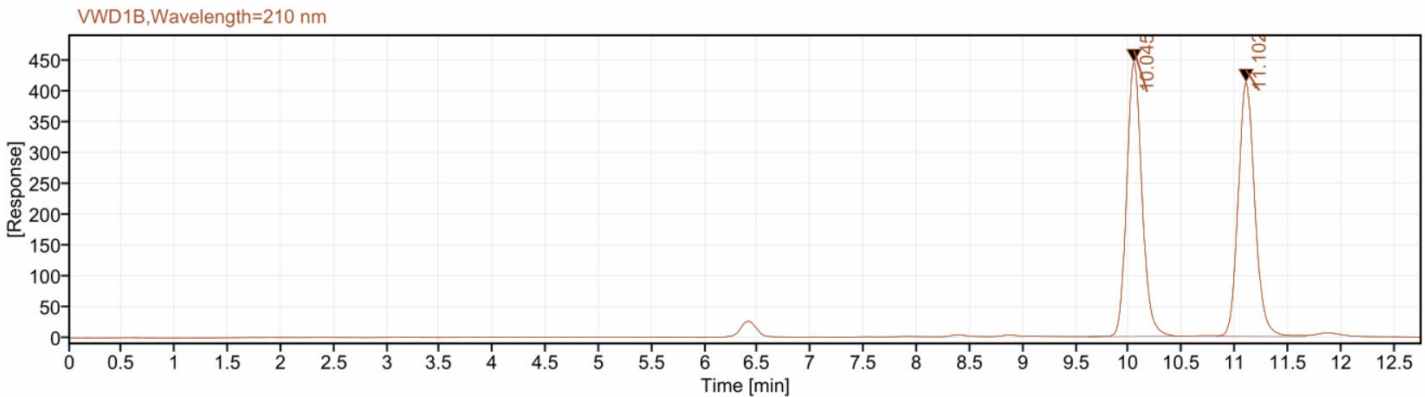

Signal: $\quad$ VWD1B,Wavelength $=210 \mathrm{~nm}$

$\begin{array}{rrrrrr}\text { RT [min] } & \text { Type } & \text { Width [min] } & \text { Area } & \text { Height } & \text { Area\% } \\ 10.045 & \text { BB } & 0.91 & 4488.01 & 445.95 & 49.75 \\ 11.102 & \text { VV } & 0.84 & 4532.77 & 413.62 & 50.25 \\ & & \text { Sum } & 9020.78 & & \end{array}$




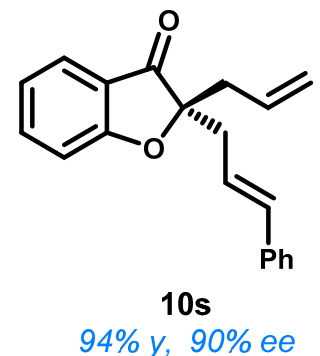

Daicel Chiralpak IA-3 column, n-hexane/i-PrOH (99.4/0.6), $0.5 \mathrm{~mL} / \mathrm{min} 250 \mathrm{~nm} 18.599 \mathrm{~min}$ (major enantiomer), $24.889 \mathrm{~min}$ (minor enantiomer).

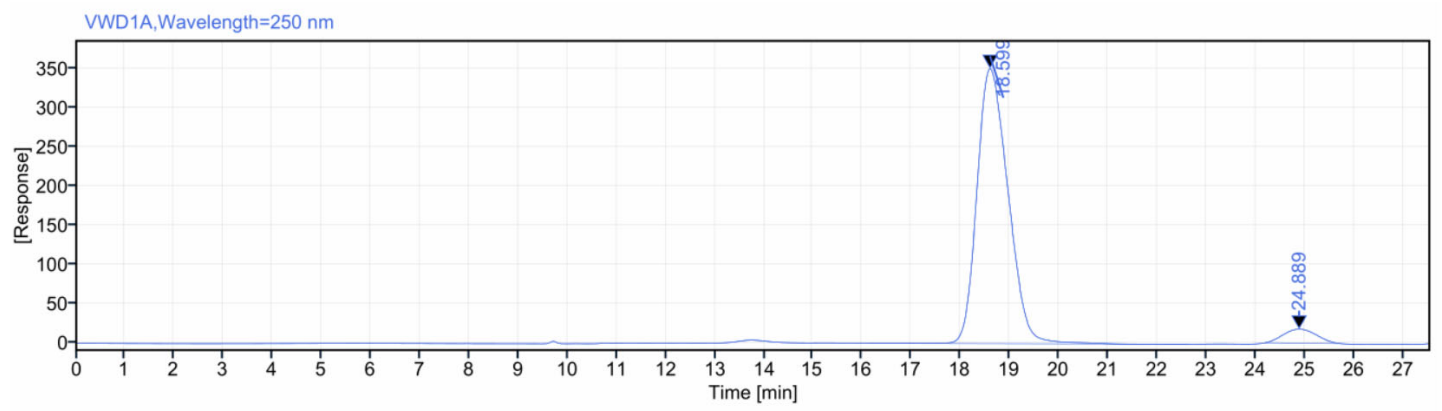

Signal: $\quad$ VWD1A,Wavelength $=250 \mathrm{~nm}$

$\begin{array}{rrrrrr}\text { RT [min] } & \text { Type } & \text { Width [min] } & \text { Area } & \text { Height } & \text { Area\% } \\ 18.599 & \text { MM m } & 0.69 & 15420.72 & 350.40 & 94.88 \\ 24.889 & \text { MM m } & 0.76 & 831.89 & 17.78 & 5.12 \\ & & \text { Sum } & 16252.60 & & \end{array}$

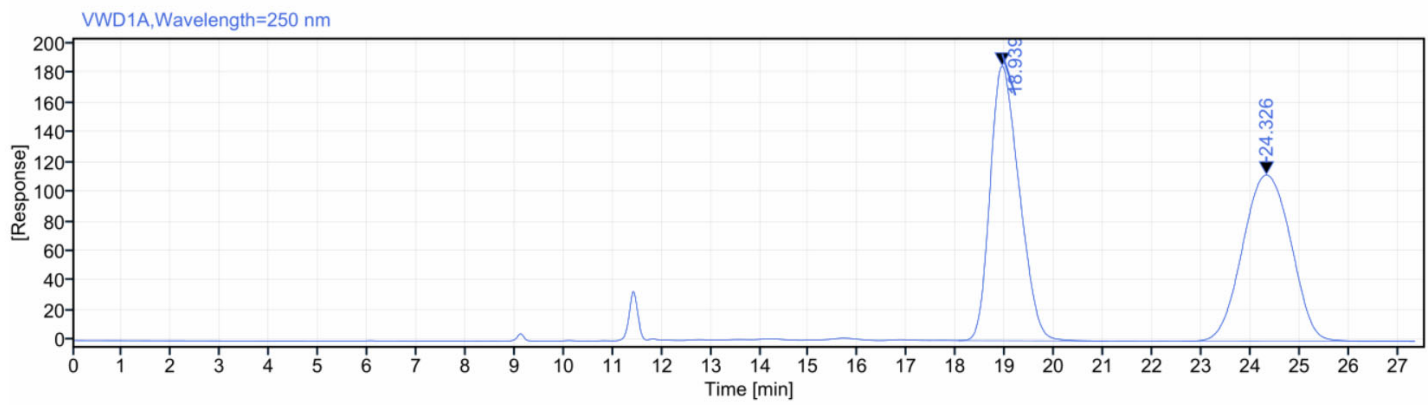

Signal: $\quad$ VWD1A,Wavelength $=250 \mathrm{~nm}$

$\begin{array}{rrrrrr}\text { RT [min] } & \text { Type } & \text { Width [min] } & \text { Area } & \text { Height } & \text { Area\% } \\ 18.939 & \text { BB } & 3.05 & 7623.32 & 184.58 & 49.89 \\ 24.326 & \text { BBA } & 4.75 & 7655.43 & 111.79 & 50.11 \\ & & \text { Sum } & 15278.76 & & \end{array}$




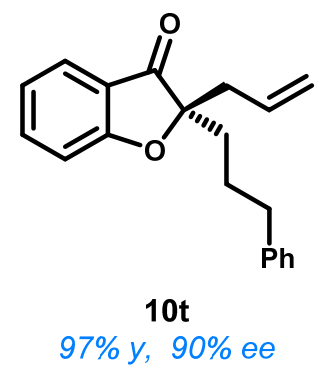

Daicel Chiralpak IC-3 column, n-hexane/i-PrOH (99/1), $0.5 \mathrm{~mL} / \mathrm{min} 210 \mathrm{~nm} 24.211 \mathrm{~min}$ (major enantiomer), $18.998 \mathrm{~min}$ (minor enantiomer).

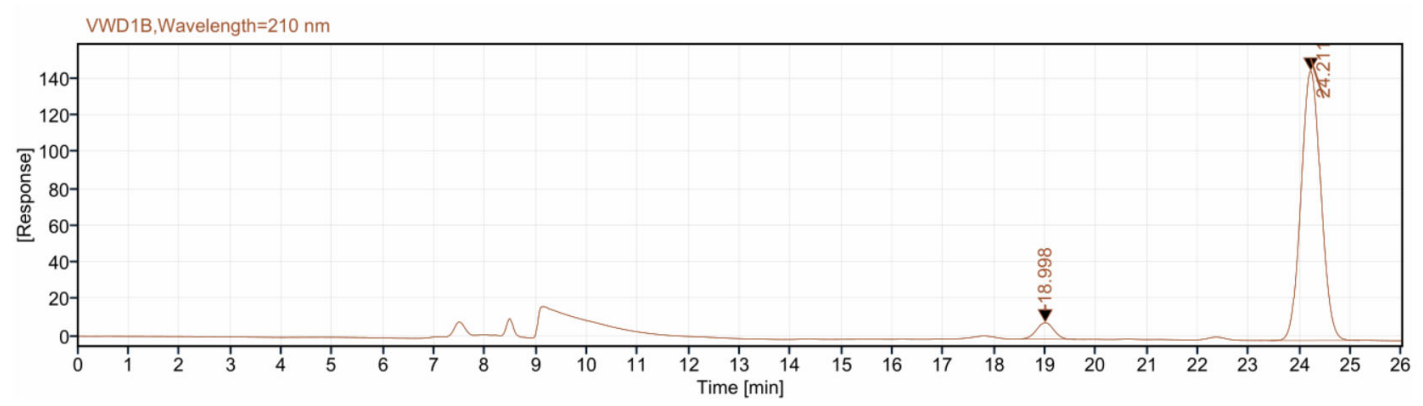

Signal: $\quad$ VWD1B,Wavelength $=210 \mathrm{~nm}$

$\begin{array}{rrrrrr}\text { RT [min] } & \text { Type } & \text { Width [min] } & \text { Area } & \text { Height } & \text { Area\% } \\ 18.998 & \text { MM m } & 0.37 & 211.00 & 8.90 & 5.06 \\ 24.211 & \text { BB } & 1.74 & 3957.99 & 146.09 & 94.94 \\ & & \text { Sum } & 4168.99 & & \end{array}$

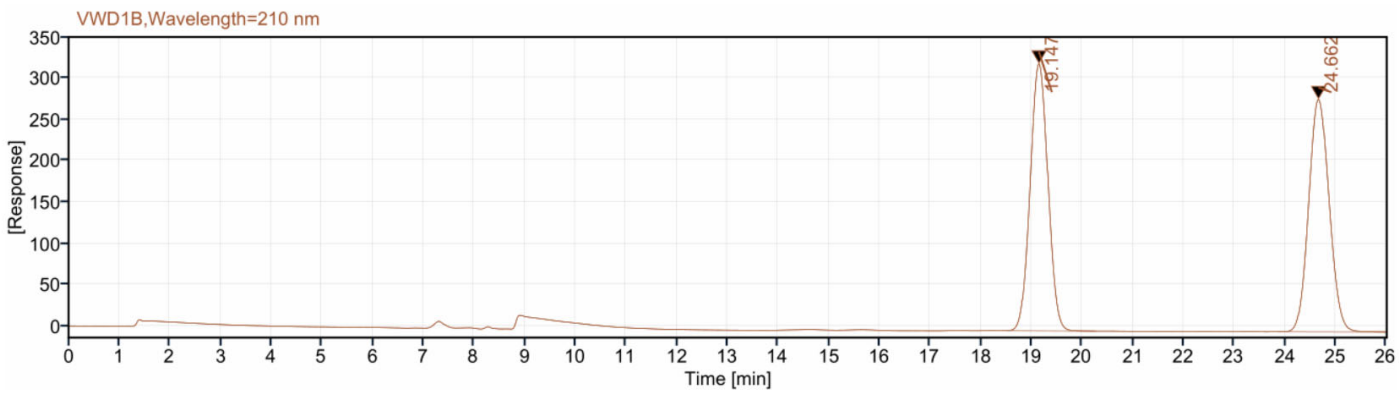

Signal: $\quad$ VWD1B, Wavelength $=210 \mathrm{~nm}$

$\begin{array}{rrrrrr}\text { RT [min] } & \text { Type } & \text { Width [min] } & \text { Area } & \text { Height } & \text { Area\% } \\ 19.147 & \text { BB } & 1.77 & 7948.40 & 324.17 & 49.94 \\ 24.662 & \text { BB } & 2.51 & 7968.79 & 282.16 & 50.06 \\ & & \text { Sum } & 15917.20 & & \end{array}$




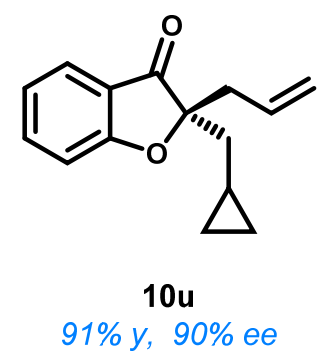

Daicel Chiralpak IC-3 column, n-hexane/i-PrOH (99.35/0.65), 0.5 mL/min $210 \mathrm{~nm} 18.407$ min (major enantiomer), $19.467 \mathrm{~min}$ (minor enantiomer).

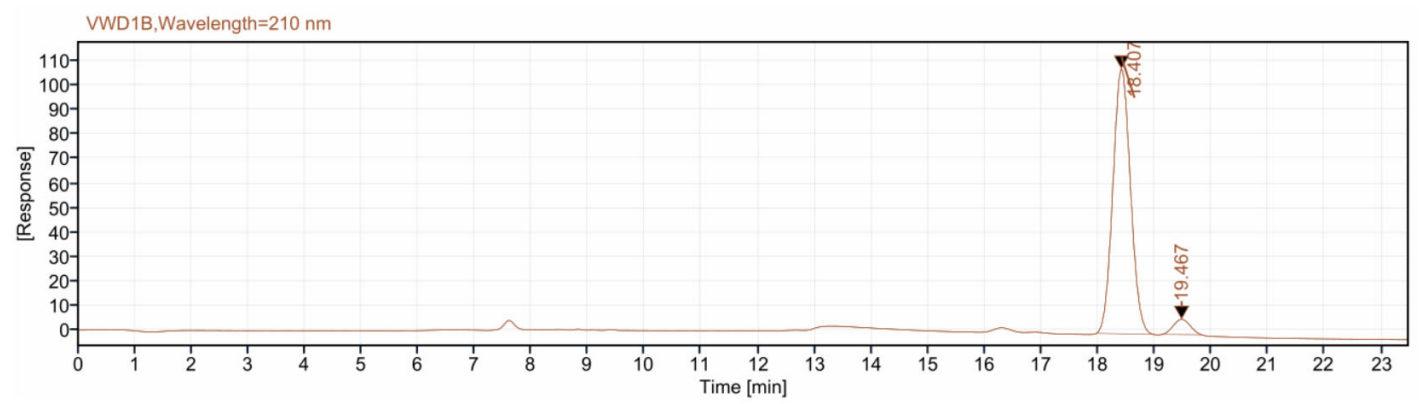

Signal: $\quad$ VWD1B,Wavelength $=210 \mathrm{~nm}$

$\begin{array}{rrrrrr}\text { RT [min] } & \text { Type } & \text { Width [min] } & \text { Area } & \text { Height } & \text { Area\% } \\ 18.407 & \text { MM m } & 0.34 & 2344.87 & 108.41 & 94.82 \\ 19.467 & \text { MM m } & 0.33 & 128.05 & 6.23 & 5.18 \\ & & \text { Sum } & 2472.91 & & \end{array}$

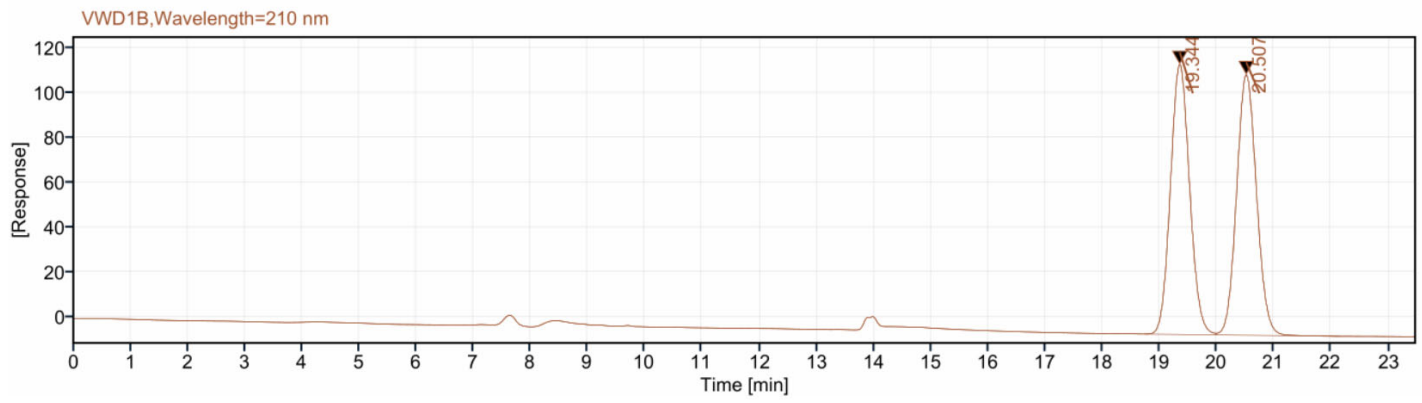

Signal: $\quad$ VWD1B,Wavelength $=210 \mathrm{~nm}$

$\begin{array}{rrrrrr}\text { RT [min] } & \text { Type } & \text { Width [min] } & \text { Area } & \text { Height } & \text { Area\% } \\ 19.344 & \text { BV } & 1.25 & 2801.54 & 120.17 & 49.92 \\ 20.507 & \text { VB } & 2.17 & 2810.80 & 115.98 & 50.08 \\ & & \text { Sum } & 5612.34 & & \end{array}$




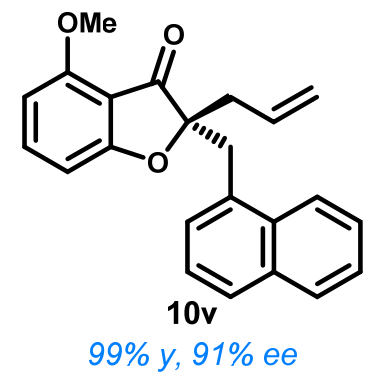

Daicel Chiralpak OD-H column, n-hexane/i-PrOH (95/5), $0.5 \mathrm{~mL} / \mathrm{min} 210 \mathrm{~nm} 27.511 \mathrm{~min}$ (major enantiomer), $43.943 \mathrm{~min}$ (minor enantiomer).

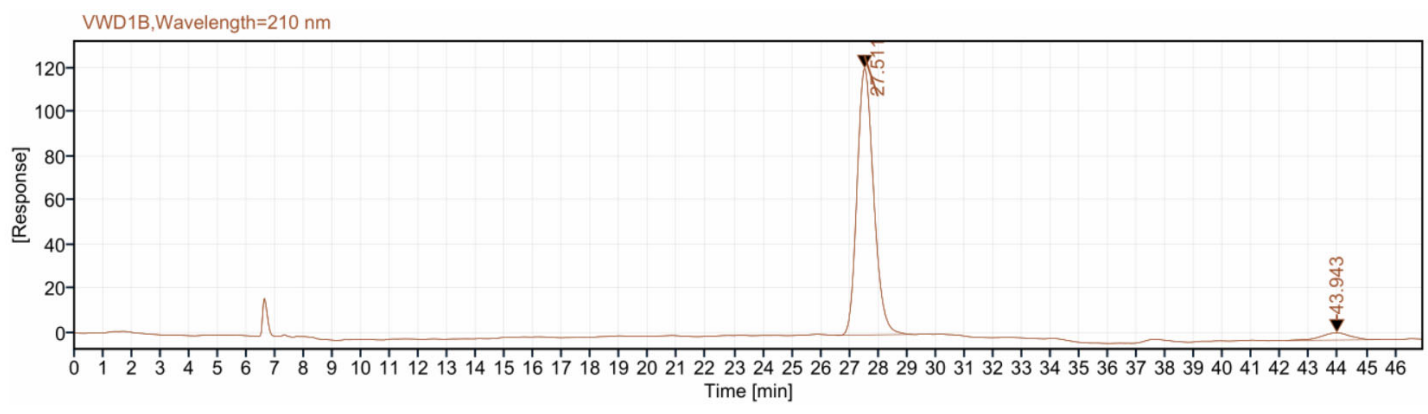

Signal: $\quad$ VWD1B, Wavelength $=210 \mathrm{~nm}$

$\begin{array}{rrrrrr}\text { RT [min] } & \text { Type } & \text { Width [min] } & \text { Area } & \text { Height } & \text { Area\% } \\ 27.511 & \text { MM m } & 0.62 & 4866.14 & 120.83 & 95.38 \\ 43.943 & \text { MM m } & 0.87 & 235.59 & 3.27 & 4.62 \\ & & \text { Sum } & 5101.73 & & \end{array}$

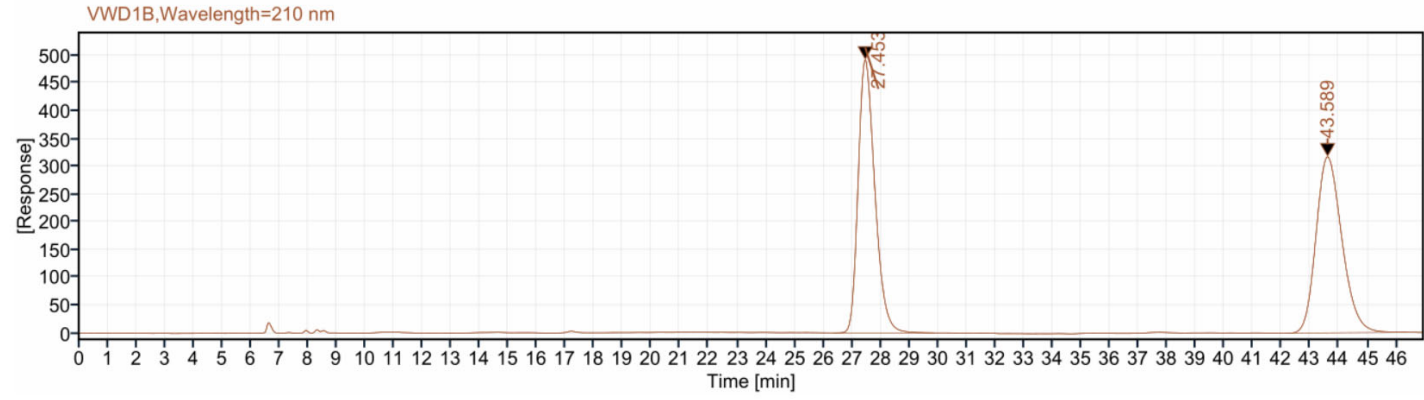

Signal: $\quad$ VWD1B,Wavelength $=210 \mathrm{~nm}$

$\begin{array}{rrrrrr}\text { RT [min] } & \text { Type } & \text { Width [min] } & \text { Area } & \text { Height } & \text { Area\% } \\ 27.453 & \text { BB } & 3.51 & 19951.67 & 491.26 & 50.09 \\ 43.589 & \text { BB } & 4.16 & 19879.00 & 316.82 & 49.91 \\ & & \text { Sum } & 39830.67 & & \end{array}$




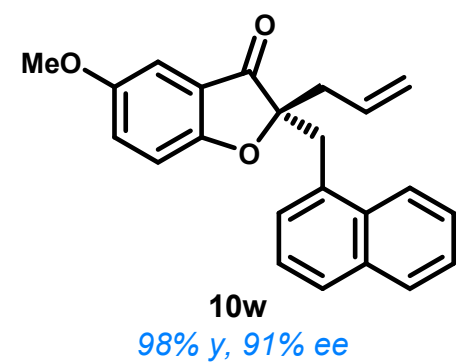

Daicel Chiralpak OD-H column, n-hexane/i-PrOH (95/5), $0.5 \mathrm{~mL} / \mathrm{min} 210 \mathrm{~nm} 18.349 \mathrm{~min}$ (major enantiomer), $20.719 \mathrm{~min}$ (minor enantiomer).

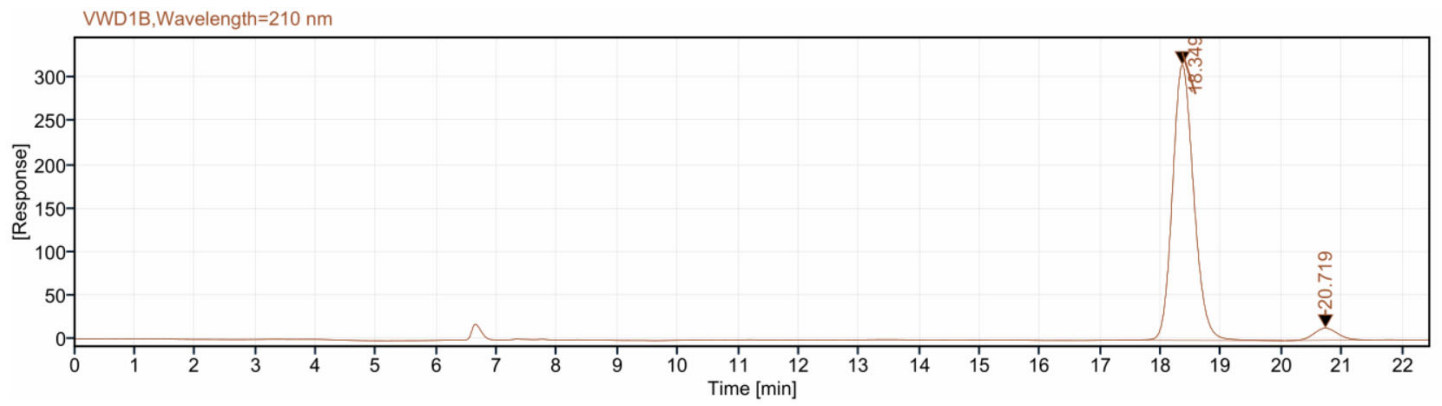

Signal: $\quad$ VWD1B,Wavelength $=210 \mathrm{~nm}$

$\begin{array}{rrrrrr}\text { RT [min] } & \text { Type } & \text { Width [min] } & \text { Area } & \text { Height } & \text { Area\% } \\ 18.349 & \text { BB } & 2.77 & 7741.37 & 316.02 & 95.25 \\ 20.719 & \text { BB } & 1.42 & 386.04 & 13.95 & 4.75 \\ & & \text { Sum } & 8127.41 & & \end{array}$

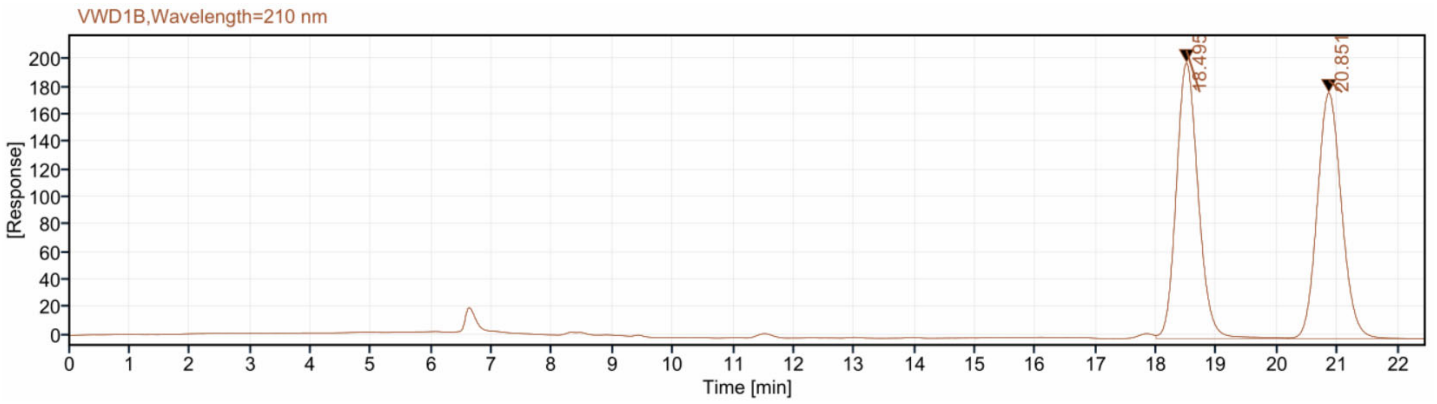

Signal: $\quad$ VWD1B,Wavelength $=210 \mathrm{~nm}$

$\begin{array}{rrrrrr}\text { RT [min] } & \text { Type } & \text { Width [min] } & \text { Area } & \text { Height } & \text { Area\% } \\ 18.495 & \text { VV } & 2.18 & 5067.38 & 200.09 & 50.36 \\ 20.851 & \text { VB } & 2.33 & 4994.30 & 178.46 & 49.64 \\ & & \text { Sum } & 10061.68 & & \end{array}$




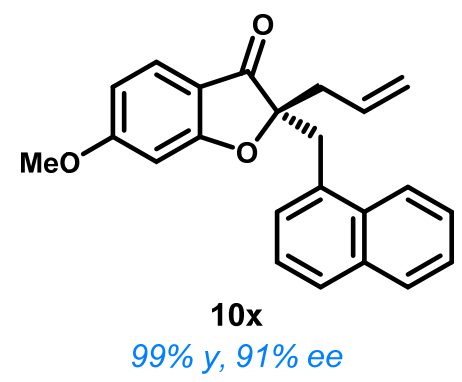

Daicel Chiralpak AD-H column, n-hexane/i-PrOH (95/5), $1 \mathrm{~mL} / \mathrm{min} 210 \mathrm{~nm} 13.434 \mathrm{~min}$ (major enantiomer), $11.781 \mathrm{~min}$ (minor enantiomer).

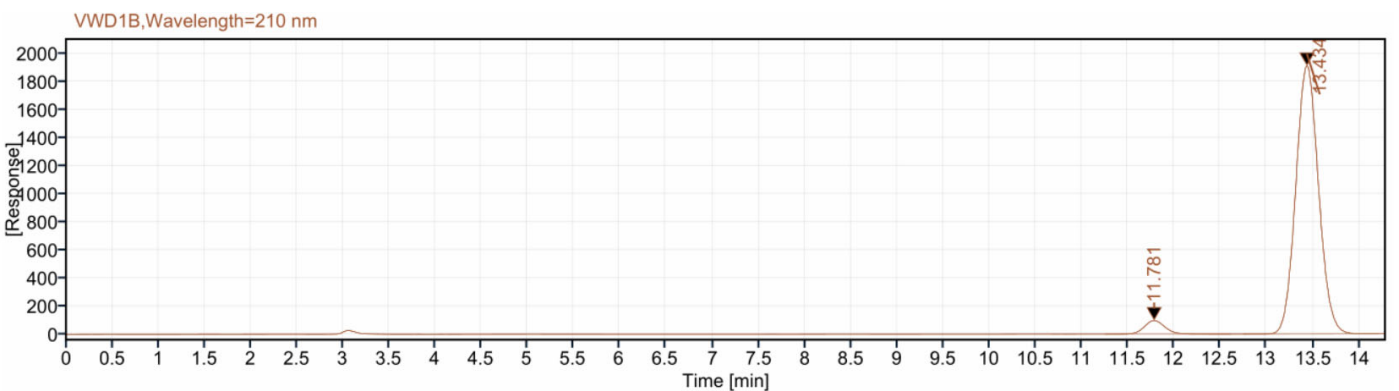

Signal: $\quad$ VWD1B, Wavelength $=210 \mathrm{~nm}$

$\begin{array}{rrrrrr}\text { RT [min] } & \text { Type } & \text { Width [min] } & \text { Area } & \text { Height } & \text { Area\% } \\ 11.781 & \text { BB } & 1.36 & 1483.11 & 96.82 & 4.31 \\ 13.434 & \text { BV } & 1.47 & 32945.43 & 1918.14 & 95.69 \\ & & \text { Sum } & 34428.54 & & \end{array}$

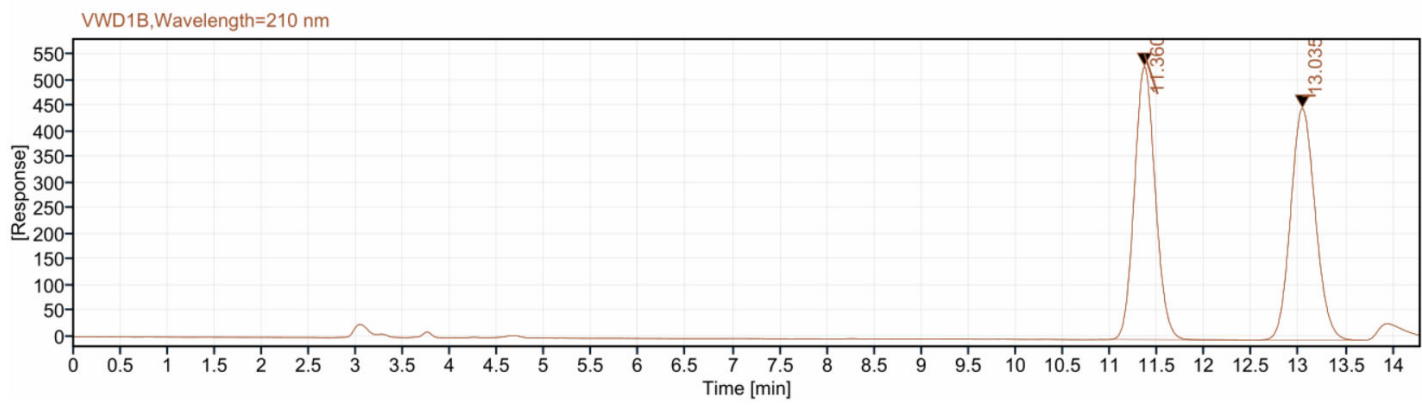

Signal: $\quad$ VWD1B,Wavelength $=210 \mathrm{~nm}$

$\begin{array}{rrrrrr}\text { RT [min] } & \text { Type } & \text { Width [min] } & \text { Area } & \text { Height } & \text { Area\% } \\ 11.360 & \text { BB } & 1.49 & 8183.06 & 531.87 & 49.93 \\ 13.035 & \text { BV } & 1.15 & 8206.07 & 451.21 & 50.07 \\ & & \text { Sum } & 16389.13 & & \end{array}$




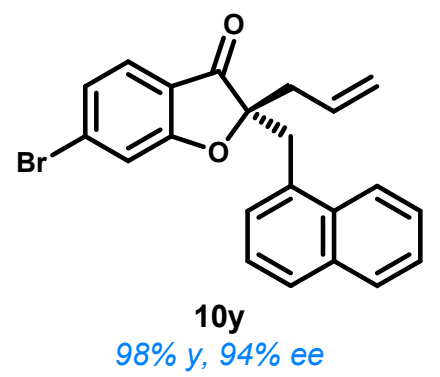

Daicel Chiralpak AD-H column, n-hexane/i-PrOH (95/5), $0.5 \mathrm{~mL} / \mathrm{min} 210 \mathrm{~nm} 12.888 \mathrm{~min}$ (major enantiomer), $10.363 \mathrm{~min}$ (minor enantiomer).

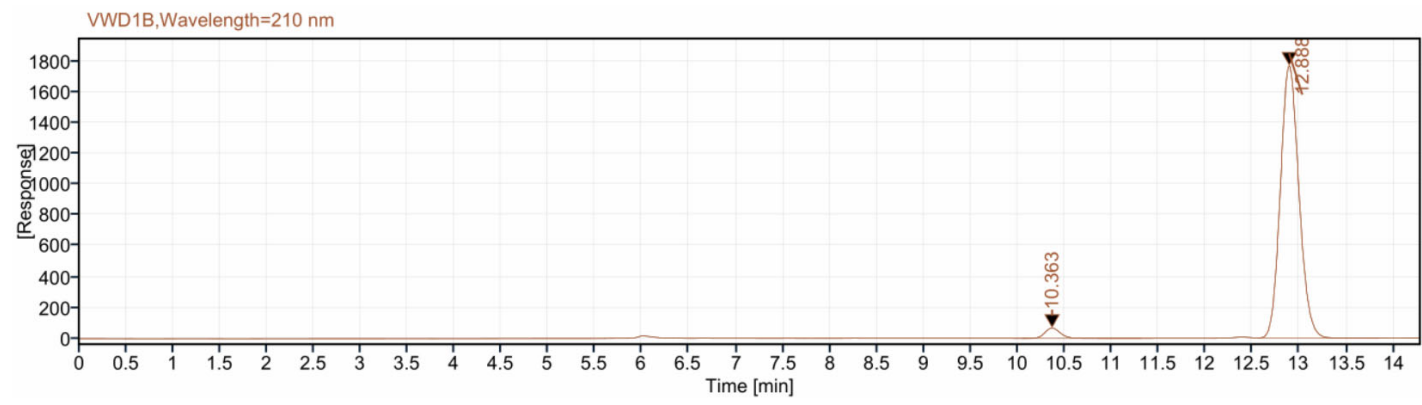

Signal: $\quad$ VWD1B,Wavelength $=210 \mathrm{~nm}$

$\begin{array}{rrrrrr}\text { RT [min] } & \text { Type } & \text { Width [min] } & \text { Area } & \text { Height } & \text { Area\% } \\ 10.363 & \text { BB } & 1.27 & 769.11 & 67.57 & 3.05 \\ 12.888 & \text { VB } & 1.28 & 24424.27 & 1768.77 & 96.95 \\ & & \text { Sum } & 25193.38 & & \end{array}$

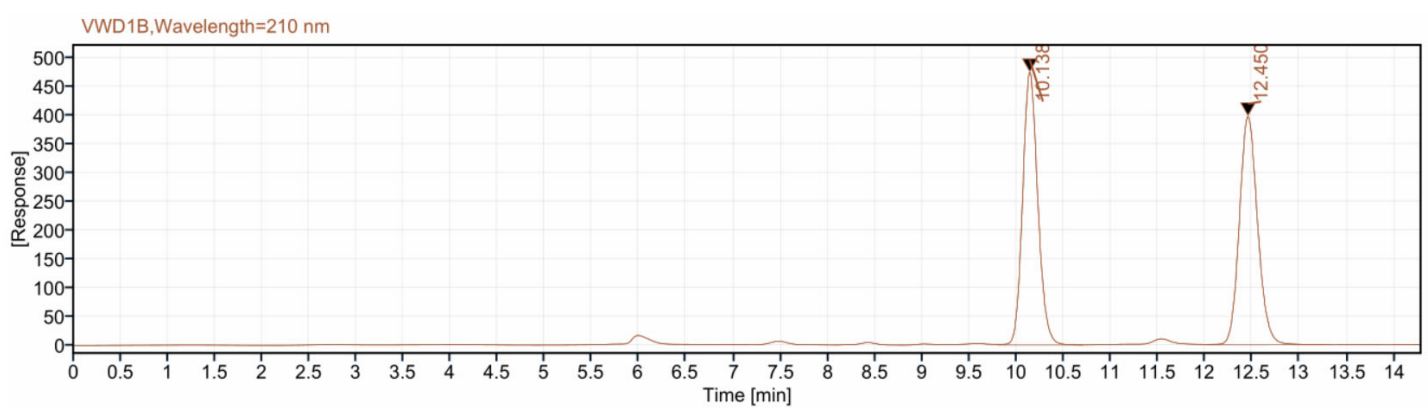

Signal: $\quad$ VWD1B,Wavelength $=210 \mathrm{~nm}$

$\begin{array}{rrrrrr}\text { RT [min] } & \text { Type } & \text { Width [min] } & \text { Area } & \text { Height } & \text { Area\% } \\ 10.138 & \text { VB } & 0.90 & 5308.84 & 474.33 & 49.80 \\ 12.450 & \text { VB } & 1.30 & 5351.36 & 397.61 & 50.20 \\ & & \text { Sum } & 10660.20 & & \end{array}$




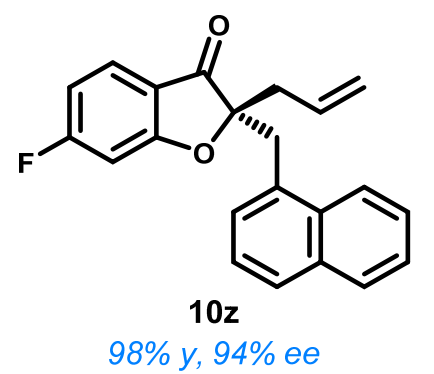

Daicel Chiralpak AD-H column, n-hexane/i-PrOH (95/5), $0.5 \mathrm{~mL} / \mathrm{min} 210 \mathrm{~nm} 13.758 \mathrm{~min}$ (major enantiomer), $11.597 \mathrm{~min}$ (minor enantiomer).

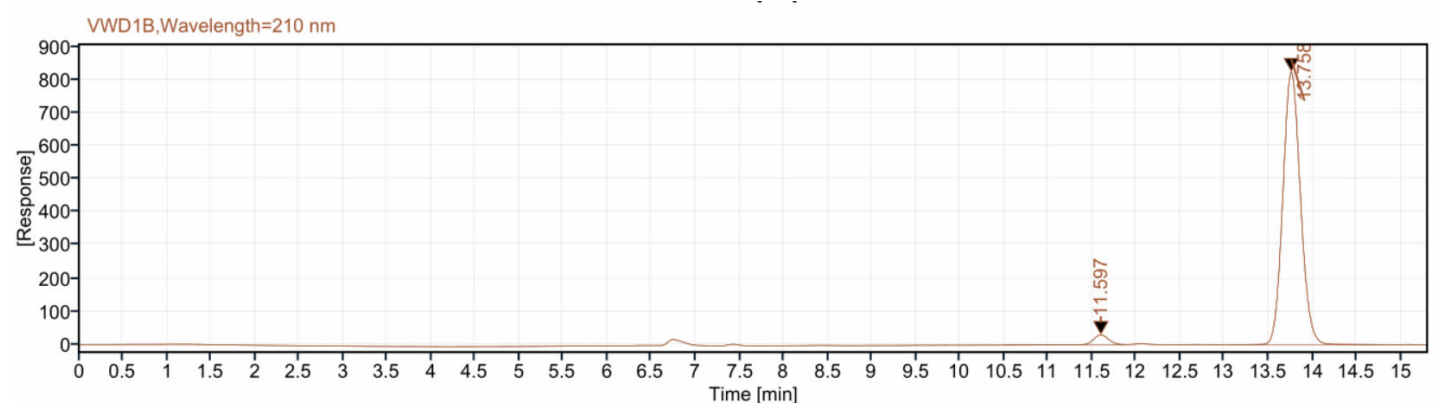

Signal: $\quad$ VWD1B, Wavelength $=210 \mathrm{~nm}$

$\begin{array}{rrrrrr}\text { RT [min] } & \text { Type } & \text { Width [min] } & \text { Area } & \text { Height } & \text { Area\% } \\ 11.597 & \text { BV } & 1.39 & 384.33 & 30.57 & 3.24 \\ 13.758 & \text { BB } & 1.78 & 11459.61 & 824.06 & 96.76 \\ & & \text { Sum } & 11843.93 & & \end{array}$

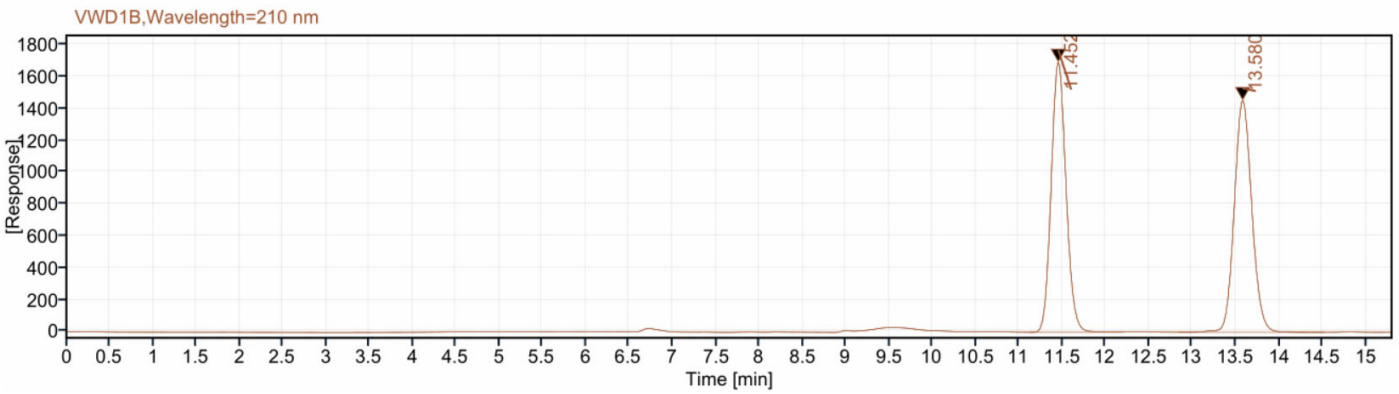

Signal: $\quad$ VWD1B,Wavelength $=210 \mathrm{~nm}$

$\begin{array}{rrrrrr}\text { RT [min] } & \text { Type } & \text { Width [min] } & \text { Area } & \text { Height } & \text { Area\% } \\ 11.452 & \text { BV } & 1.08 & 20242.26 & 1693.69 & 49.72 \\ 13.580 & \text { VB } & 1.69 & 20474.24 & 1454.12 & 50.28 \\ & & \text { Sum } & 40716.50 & & \end{array}$




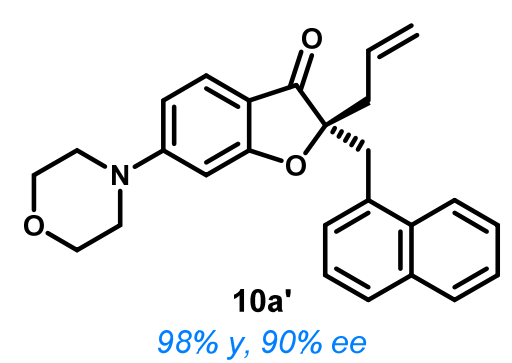

Daicel Chiralpak AD-H column, n-hexane/i-PrOH (80/20), 1 mL/min $210 \mathrm{~nm} 12.639 \mathrm{~min}$ (major enantiomer), 8.825 min (minor enantiomer).

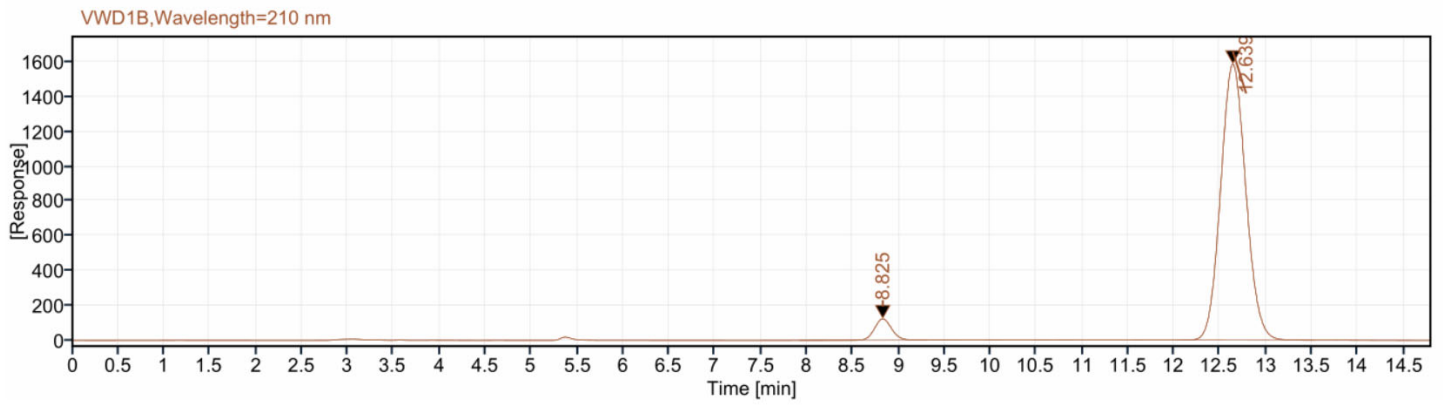

Signal: $\quad$ VWD1B,Wavelength $=210 \mathrm{~nm}$

$\begin{array}{rrrrrr}\text { RT [min] } & \text { Type } & \text { Width [min] } & \text { Area } & \text { Height } & \text { Area\% } \\ 8.825 & \text { BB } & 1.40 & 1594.72 & 123.15 & 5.00 \\ 12.639 & \text { BB } & 1.46 & 30312.34 & 1590.59 & 95.00 \\ & & \text { Sum } & 31907.05 & & \end{array}$

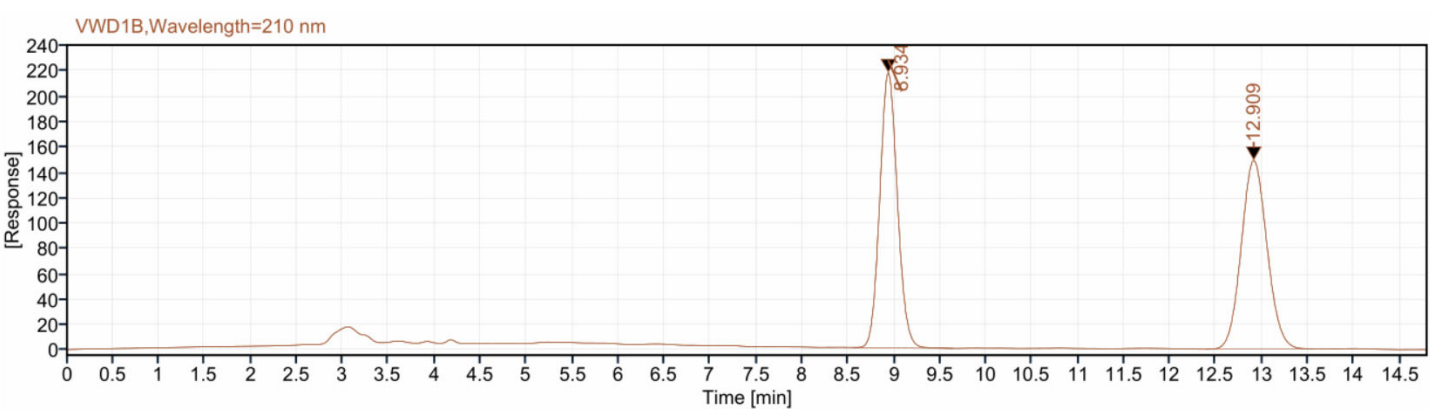

Signal: $\quad$ VWD1B,Wavelength $=210 \mathrm{~nm}$

$\begin{array}{rrrrrr}\text { RT [min] } & \text { Type } & \text { Width [min] } & \text { Area } & \text { Height } & \text { Area\% } \\ 8.934 & \text { BB } & 1.09 & 2890.28 & 216.79 & 49.98 \\ 12.909 & \text { BB } & 1.18 & 2892.11 & 148.65 & 50.02 \\ & & \text { Sum } & 5782.39 & & \end{array}$




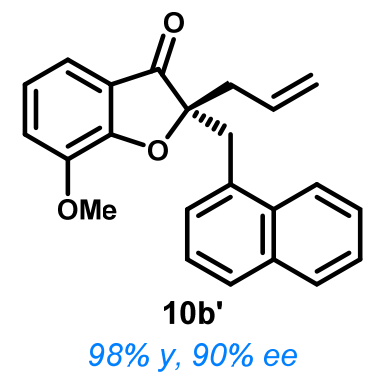

Daicel Chiralpak IC-3 column, n-hexane/i-PrOH (95/5), $0.5 \mathrm{~mL} / \mathrm{min} 210 \mathrm{~nm} 14.917 \mathrm{~min}$ (major enantiomer), $15.932 \mathrm{~min}$ (minor enantiomer).

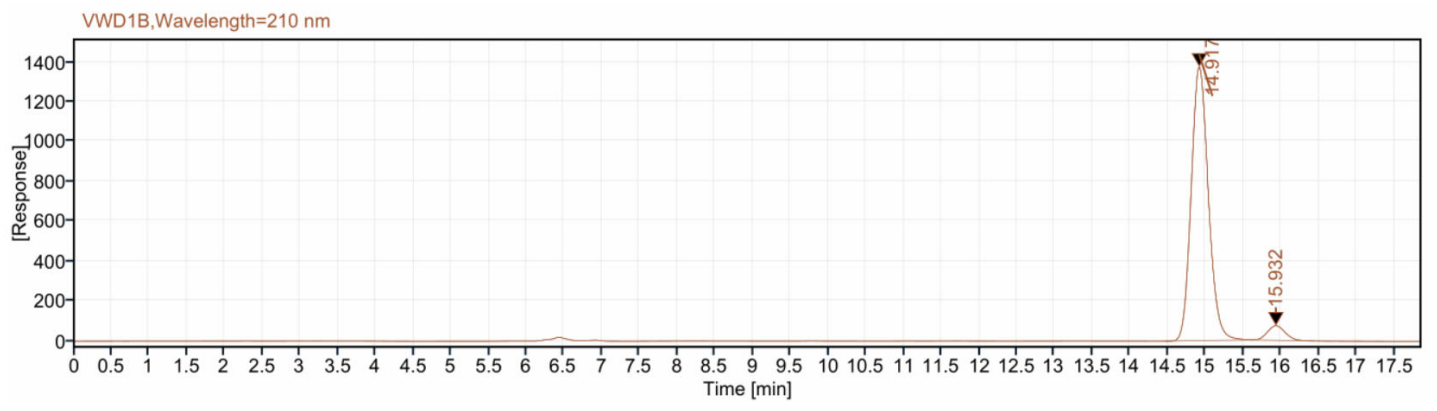

Signal: $\quad$ VWD1B,Wavelength $=210 \mathrm{~nm}$

$\begin{array}{rrrrrr}\text { RT [min] } & \text { Type } & \text { Width [min] } & \text { Area } & \text { Height } & \text { Area\% } \\ 14.917 & \text { MM m } & 0.25 & 21734.89 & 1373.01 & 94.76 \\ 15.932 & \text { MB m } & 0.26 & 1202.50 & 73.22 & 5.24 \\ & & \text { Sum } & 22937.39 & & \end{array}$

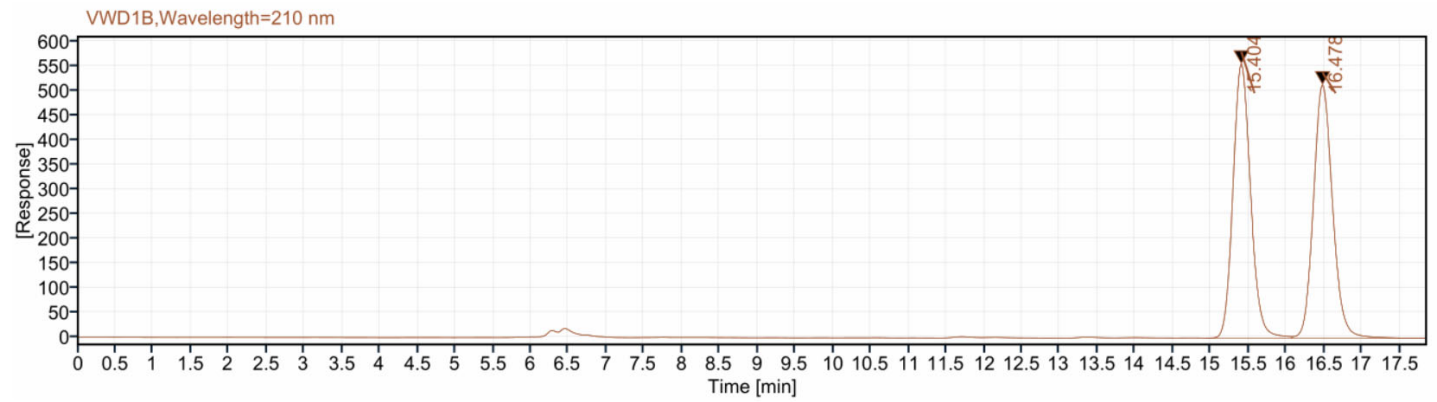

Signal: $\quad$ VWD1B, Wavelength $=210 \mathrm{~nm}$

$\begin{array}{rrrrrr}\text { RT [min] } & \text { Type } & \text { Width [min] } & \text { Area } & \text { Height } & \text { Area\% } \\ 15.404 & \text { VV } & 1.14 & 9022.39 & 555.30 & 49.84 \\ 16.478 & \text { VBA } & 1.93 & 9081.45 & 513.36 & 50.16 \\ & & \text { Sum } & 18103.84 & & \end{array}$




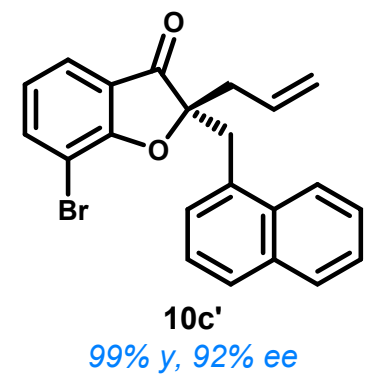

Daicel Chiralpak OD-H column, n-hexane/i-PrOH (95/5), $0.5 \mathrm{~mL} / \mathrm{min} 210 \mathrm{~nm} 21.950 \mathrm{~min}$ (major enantiomer), $17.393 \mathrm{~min}$ (minor enantiomer).

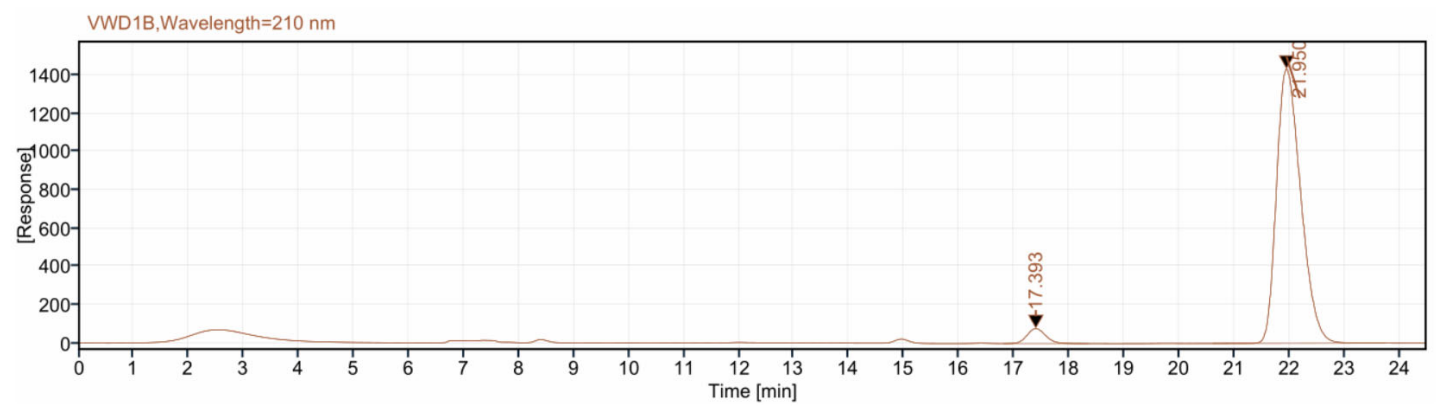

Signal: $\quad$ VWD1B,Wavelength $=210 \mathrm{~nm}$

$\begin{array}{rrrrrr}\text { RT [min] } & \text { Type } & \text { Width [min] } & \text { Area } & \text { Height } & \text { Area\% } \\ 17.393 & \text { VB } & 1.55 & 1855.45 & 78.84 & 4.04 \\ 21.950 & \text { BB } & 3.14 & 44026.37 & 1433.49 & 95.96 \\ & & \text { Sum } & 45881.82 & & \end{array}$

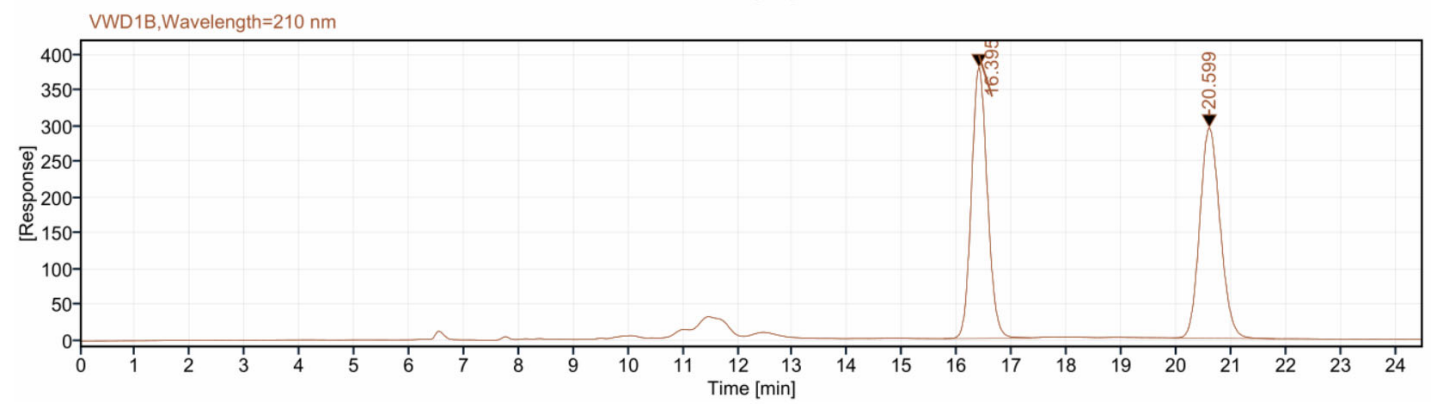

Signal: $\quad$ VWD1B,Wavelength $=210 \mathrm{~nm}$

$\begin{array}{rrrrrr}\text { RT [min] } & \text { Type } & \text { Width [min] } & \text { Area } & \text { Height } & \text { Area\% } \\ 16.395 & \text { BV } & 1.60 & 7659.35 & 378.12 & 50.24 \\ 20.599 & \text { BB } & 1.87 & 7587.63 & 293.59 & 49.76 \\ & & \text { Sum } & 15246.98 & & \end{array}$




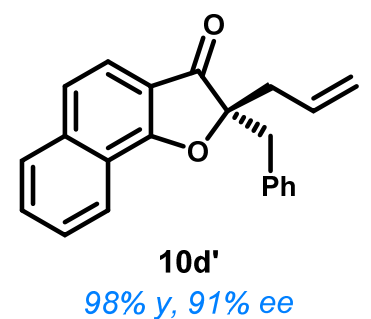

Daicel Chiralpak OD-H column, n-hexane/i-PrOH (99.4/0.6), 1 mL/min $210 \mathrm{~nm} 16.647 \mathrm{~min}$ (major enantiomer), $15.688 \mathrm{~min}$ (minor enantiomer).

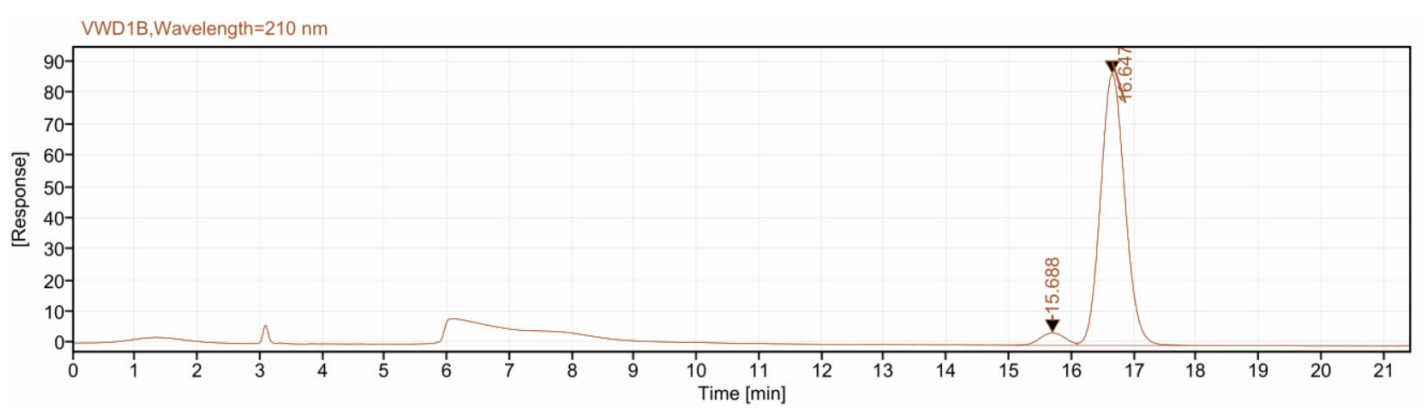

Signal: $\quad$ VWD1B,Wavelength $=210 \mathrm{~nm}$

$\begin{array}{rrrrrr}\text { RT [min] } & \text { Type } & \text { Width [min] } & \text { Area } & \text { Height } & \text { Area\% } \\ 15.688 & \text { BV } & 0.99 & 108.71 & 3.90 & 4.40 \\ 16.647 & \text { VB } & 1.73 & 2361.04 & 86.96 & 95.60 \\ & & \text { Sum } & 2469.75 & & \end{array}$

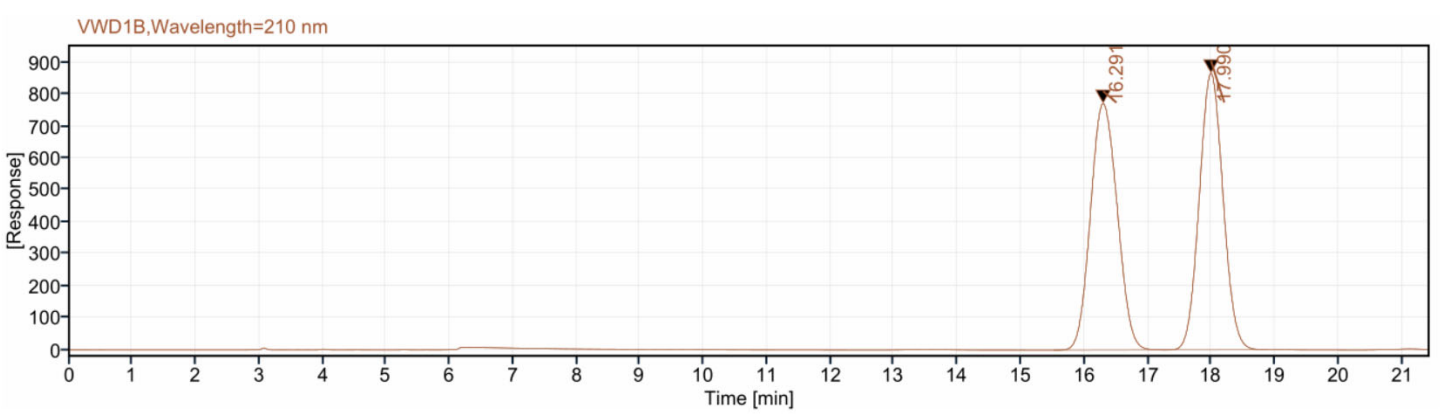

Signal: $\quad$ VWD1B,Wavelength $=210 \mathrm{~nm}$

$\begin{array}{rrrrrr}\text { RT [min] } & \text { Type } & \text { Width [min] } & \text { Area } & \text { Height } & \text { Area\% } \\ 16.291 & \text { BB } & 1.79 & 22596.32 & 771.46 & 50.02 \\ 17.990 & \text { BB } & 1.59 & 22582.19 & 865.49 & 49.98 \\ & & \text { Sum } & 45178.51 & & \end{array}$




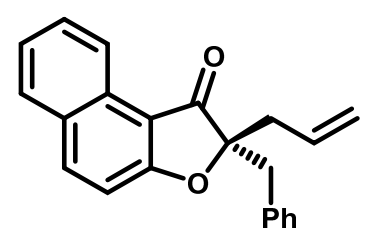

$10 \mathrm{e}^{\prime}$

$98 \%$ y, $94 \%$ ee

Daicel Chiralpak OD-H column, n-hexane/i-PrOH (99.4/0.6), 1 mL/min $210 \mathrm{~nm} 13.937 \mathrm{~min}$ (major enantiomer), $12.544 \mathrm{~min}$ (minor enantiomer).

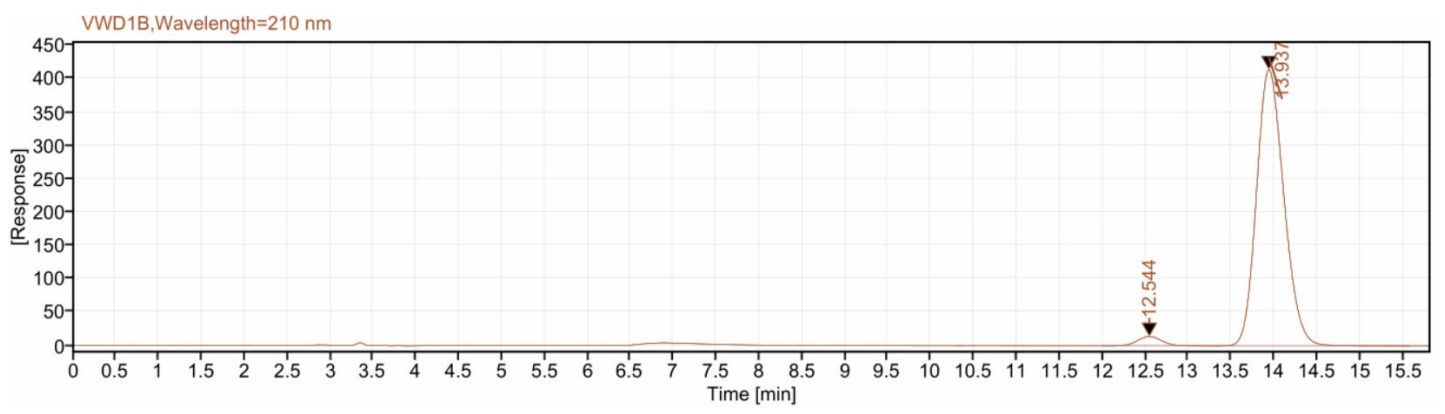

Signal: $\quad$ VWD1B,Wavelength $=210 \mathrm{~nm}$

$\begin{array}{rrrrrr}\text { RT [min] } & \text { Type } & \text { Width [min] } & \text { Area } & \text { Height } & \text { Area\% } \\ 12.544 & \text { BB } & 1.28 & 279.26 & 14.11 & 2.95 \\ 13.937 & \text { BB } & 2.31 & 9196.03 & 414.26 & 97.05 \\ & & \text { Sum } & 9475.29 & & \end{array}$

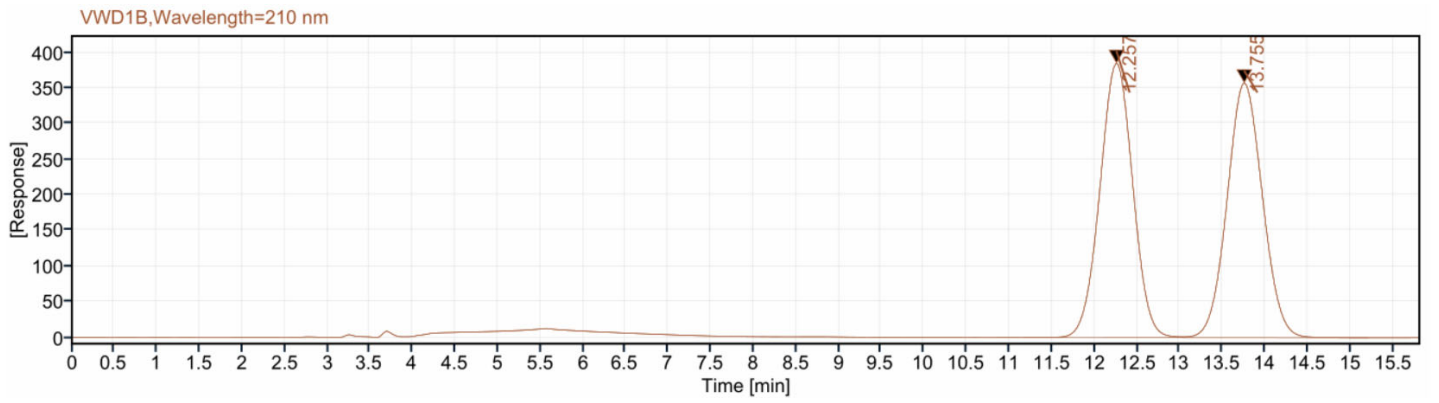

Signal: $\quad$ VWD1B,Wavelength $=210 \mathrm{~nm}$

$\begin{array}{rrrrrr}\text { RT [min] } & \text { Type } & \text { Width [min] } & \text { Area } & \text { Height } & \text { Area\% } \\ 12.257 & \text { BV } & 1.87 & 10307.94 & 384.63 & 49.95 \\ 13.755 & \text { VB } & 2.18 & 10326.65 & 357.24 & 50.05 \\ & & \text { Sum } & 20634.59 & & \end{array}$




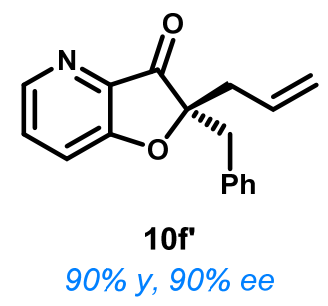

Daicel Chiralpak OD-H column, n-hexane/i-PrOH (99.4/0.6), 0.5 mL/min $210 \mathrm{~nm} 16.800 \mathrm{~min}$ (major enantiomer), $15.603 \mathrm{~min}$ (minor enantiomer).

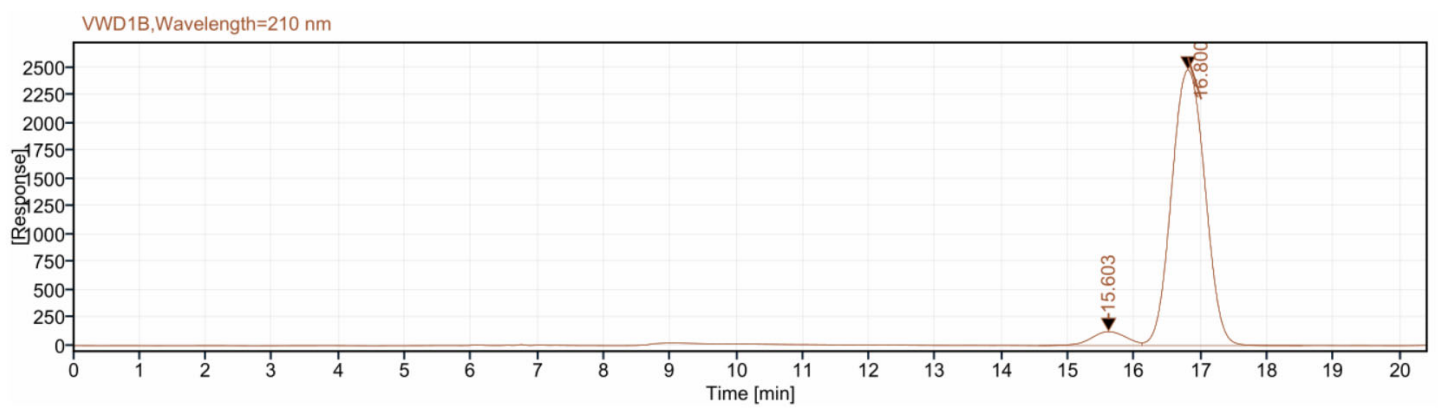

Signal: $\quad$ VWD1B, Wavelength $=210 \mathrm{~nm}$

$\begin{array}{rrrrrr}\text { RT [min] } & \text { Type } & \text { Width [min] } & \text { Area } & \text { Height } & \text { Area\% } \\ 15.603 & \text { BV } & 1.55 & 4620.11 & 125.53 & 5.05 \\ 16.800 & \text { VB } & 2.42 & 86813.66 & 2482.48 & 94.95 \\ & & \text { Sum } & 91433.77 & & \end{array}$

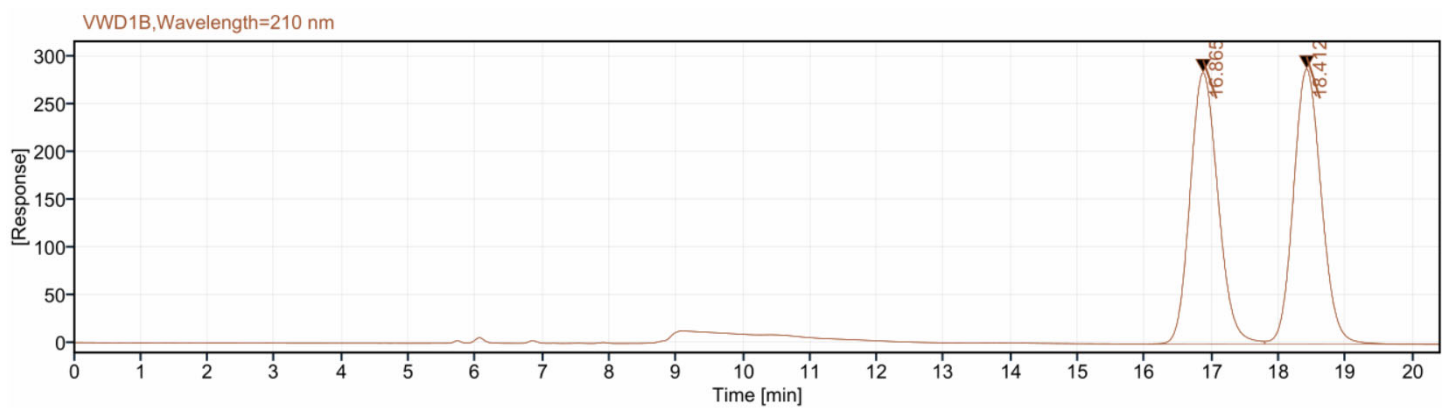

Signal: $\quad$ VWD1B,Wavelength $=210 \mathrm{~nm}$

$\begin{array}{rrrrrr}\text { RT [min] } & \text { Type } & \text { Width [min] } & \text { Area } & \text { Height } & \text { Area\% } \\ 16.865 & \text { BV } & 1.73 & 8264.20 & 284.17 & 49.78 \\ 18.412 & \text { VB } & 2.78 & 8336.04 & 288.17 & 50.22 \\ & & \text { Sum } & 16600.24 & & \end{array}$




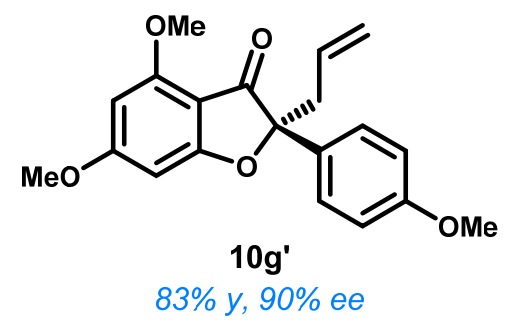

Daicel Chiralpak OD-H column, n-hexane/i-PrOH (80/20), $1 \mathrm{~mL} / \mathrm{min} 210 \mathrm{~nm} 20.787 \mathrm{~min}$ (major enantiomer), $14.378 \mathrm{~min}$ (minor enantiomer).

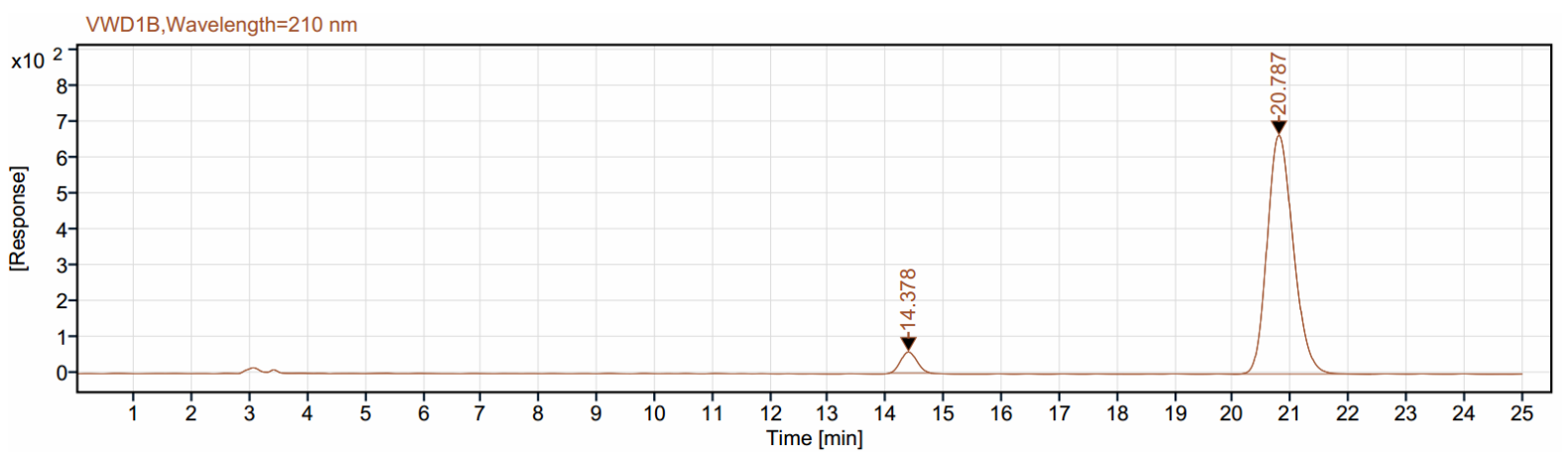

Signal: $\quad$ VWD1B, Wavelength $=210 \mathrm{~nm}$

$\begin{array}{rrrrrr}\text { RT [min] } & \text { Type } & \text { Width [min] } & \text { Area } & \text { Height } & \text { Area\% } \\ 14.378 & \text { MM m } & 0.31 & 1156.89 & 58.81 & 5.25 \\ 20.787 & \text { VB } & 2.39 & 20876.08 & 664.55 & 94.75 \\ & & \text { Sum } & 22032.97 & & \end{array}$

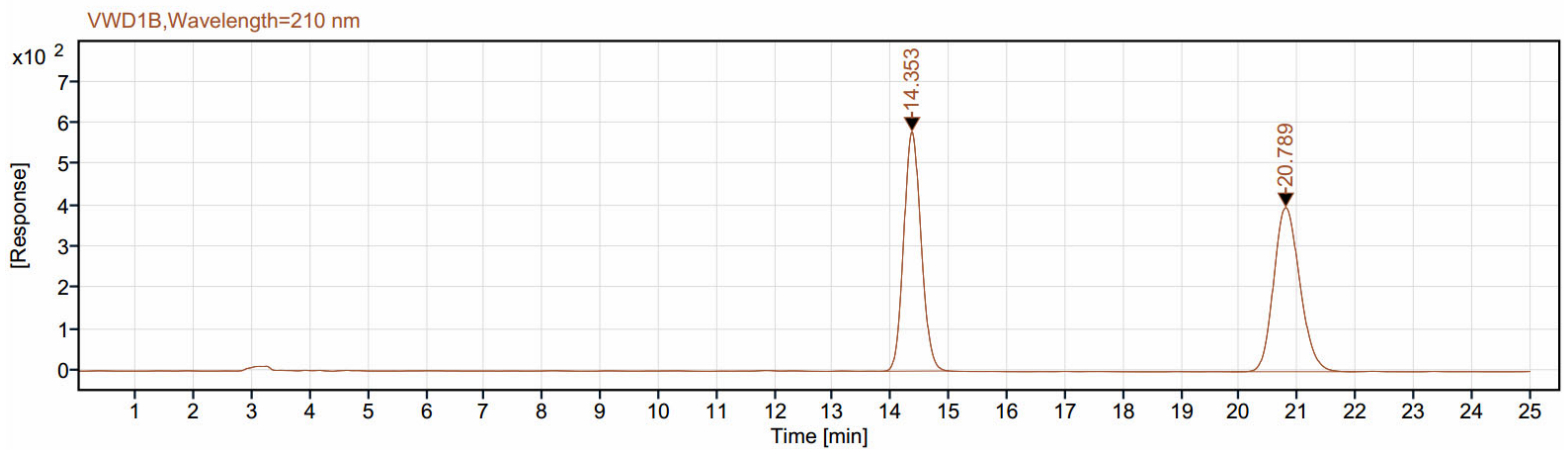

Signal: $\quad$ VWD1B,Wavelength $=210 \mathrm{~nm}$

$\begin{array}{rrrrrr}\text { RT [min] } & \text { Type } & \text { Width [min] } & \text { Area } & \text { Height } & \text { Area\% } \\ 14.353 & \text { MB m } & 0.32 & 12063.93 & 578.80 & 49.22 \\ 20.789 & \text { BV } & 2.13 & 12446.83 & 397.15 & 50.78 \\ & & \text { Sum } & 24510.76 & & \end{array}$




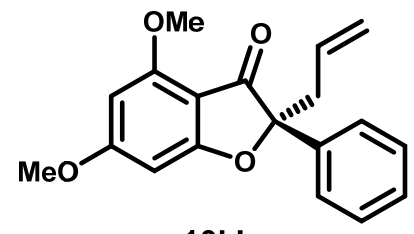

$10 \mathbf{h}^{\prime}$

$92 \%$ y, $90 \%$ ee

Daicel Chiralpak OD-H column, n-hexane/i-PrOH (80/20), $1.0 \mathrm{~mL} / \mathrm{min} 210 \mathrm{~nm} 7.394 \mathrm{~min}$ (major enantiomer), 6.457 min (minor enantiomer).

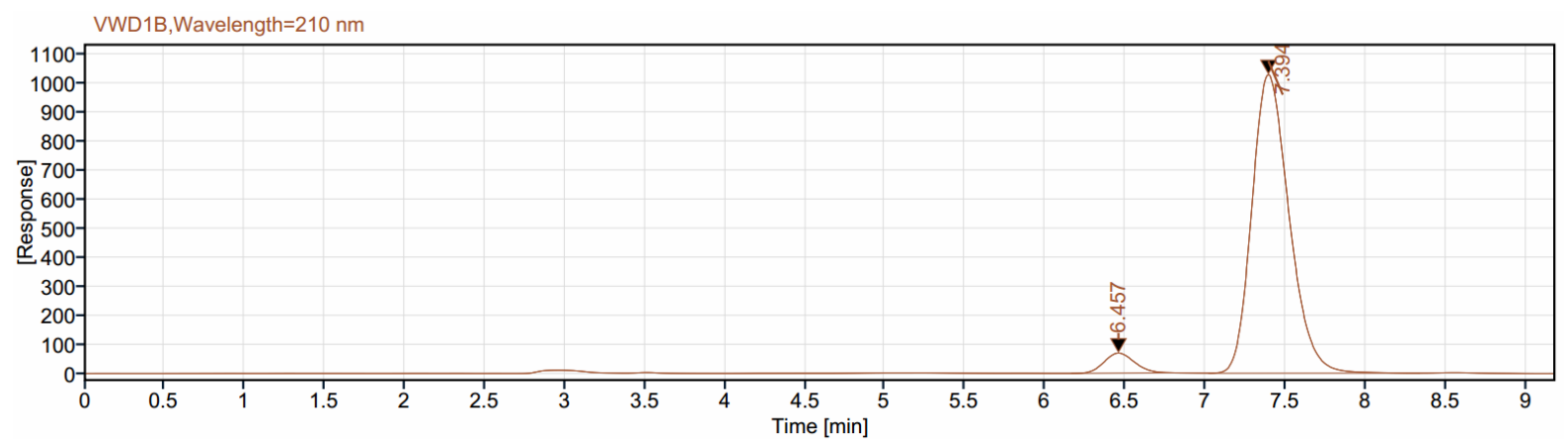

Signal: $\quad$ VWD1B,Wavelength $=210 \mathrm{~nm}$

$\begin{array}{rrrrrr}\text { RT [min] } & \text { Type } & \text { Width [min] } & \text { Area } & \text { Height } & \text { Area\% } \\ 6.457 & \text { MM m } & 0.21 & 912.01 & 69.16 & 5.17 \\ 7.394 & \text { VB } & 1.32 & 16719.40 & 1033.89 & 94.83 \\ & & \text { Sum } & 17631.41 & & \end{array}$

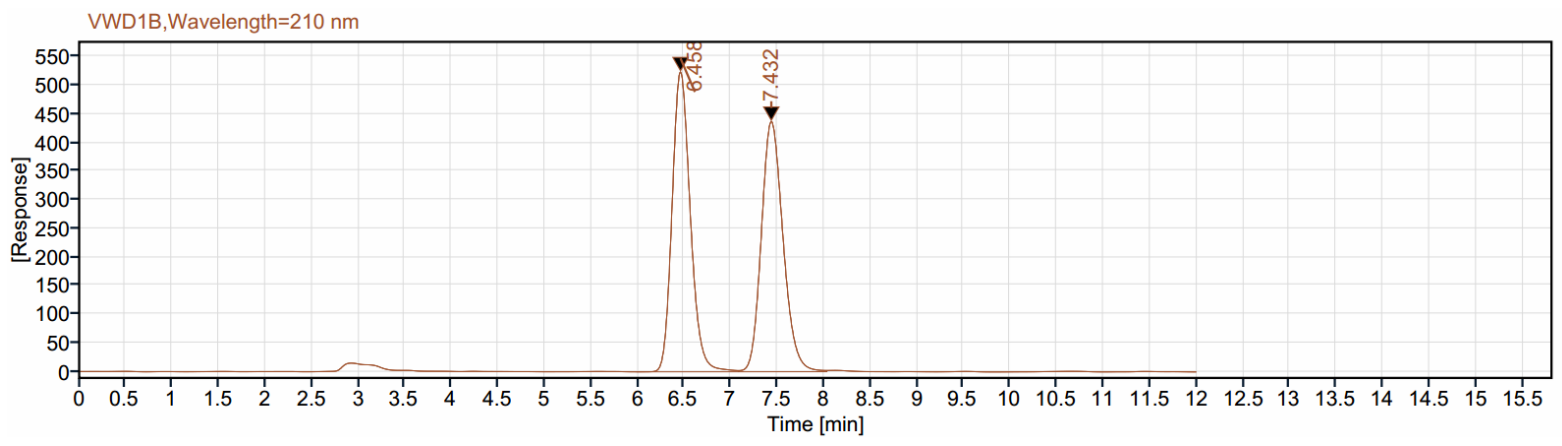

Signal: $\quad$ VWD1B,Wavelength $=210 \mathrm{~nm}$

$\begin{array}{rrrrrr}\text { RT [min] } & \text { Type } & \text { Width [min] } & \text { Area } & \text { Height } & \text { Area\% } \\ 6.458 & \text { BV } & 1.09 & 7148.81 & 524.82 & 50.18 \\ 7.432 & \text { VV } & 0.95 & 7098.89 & 438.00 & 49.82 \\ & & \text { Sum } & 14247.70 & & \end{array}$

